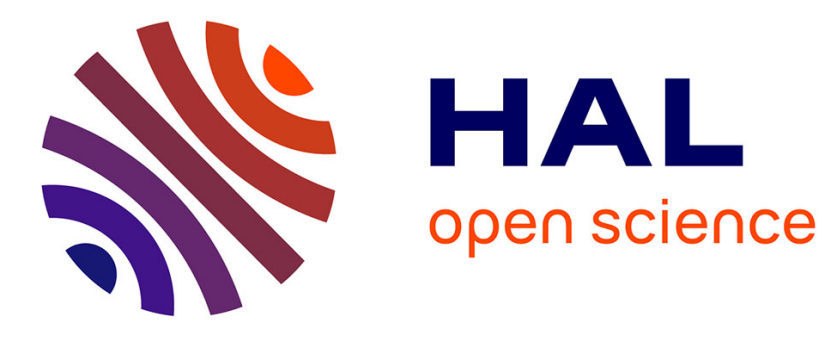

\title{
Les réalités de l'esclavage d'après le 'Digeste'
}

\author{
Marcel Morabito
}

\section{To cite this version:}

Marcel Morabito. Les réalités de l'esclavage d'après le 'Digeste'. Les Belles Lettres, pp.367, 1981. hal-01025208v2

\section{HAL Id: hal-01025208 https://hal-sciencespo.archives-ouvertes.fr/hal-01025208v2}

Submitted on 5 Dec 2017

HAL is a multi-disciplinary open access archive for the deposit and dissemination of scientific research documents, whether they are published or not. The documents may come from teaching and research institutions in France or abroad, or from public or private research centers.
L'archive ouverte pluridisciplinaire HAL, est destinée au dépôt et à la diffusion de documents scientifiques de niveau recherche, publiés ou non, émanant des établissements d'enseignement et de recherche français ou étrangers, des laboratoires publics ou privés. 


\section{Marcel MORABITO}

\section{LES RÉalités de l'esclavage}

\section{DAPRES LE DIGESTE}

ANNALES LITTERAIRES DE L'UNIVERSITE DE BESANÇON, 254

LES BELLES LETTRES 95, BOULEVARD RASPAIL PARIS VIe 


\section{Addenda et corrigenda}

p.3, n.2, ajouter : pour une analyse des textes juridiques dans une perspective sociologique, v. J. CAUTEPT, Catégories juridiques et classes sociales. In exemple : l'esclavage à Róne, Romanitas VIII (1967), p. 124 s.; plus rếcemment, M.I. FINLEY, Esclavage antique et idéologie moderne, tr. fr., Paris (1981).

p.5, 1.14 (après pouvoir), ajouter : n.16 bis: G. NELILLO, o.c., f. 18 s., 32; F. D'IPPOLITO, I giuristi e la città, Naples (1978), p.19.

p.10, n.40, 1.21, lire : le maitre de A. Namusa.

p.18, 1.5, lire : leurs fréquences.

p.21, n.9, 1.7, ajouter : D 15.4, Quod iussu.

p. 32, n.22, 1.28, lire: orationes principum.

p.49, n.2, ajouter : Sur la distinction entre causa et modus, v. J. MACQUERON, Droit romain, p. $394 \mathrm{~s}$.

p.73, n.45, 1.3 (au lieu de détention), lire : utilisation.

p.76, n.50, 1.2, 1ire : emphytéotiques.

p.105, n.191, 1.6, 1ire : BIEZUNSKA-'.ALOWIST

p.io7, 1.8 , lire : feminarum.

p.127, 1.9-10, lire : les vincula imposés au prisonnier étant moins importants.

p.142, 1.1, lire: naturellement.

p.144 (tableau, à Alfenus $\Gamma$ 50.16.203), lire : iunctores.

p.164 (tableau), ajouter sur n. 147 : Ulpien D 46.3.18.

p.164 (tableau, ̀̀ Marcien), lire : D 49.14.30 (et non D 19.14.30).

p.170, n. $480,1.1$, lire : risque.

p.171, n. 487, 1.9, 1ire : vingt biographies de grammairiens.

p.173, n.499, 1.12, lire : subscriptione.

p.194, n.572, 1.10, lire : III, 119 a.

p.203, 1.3, lire : produit.

o.204, n.629, 1.5 (et o. 385, n.623, 1.2), 1ire : res peculiaris.

p.239, n.39, 1.10, lire : affranchissement.

p.256, 1.9 , ajouter : on peut signaler que Numerius Negidius, nom conventionne du défendeur dans la procêdure formulaire, et Vindex, qui désigne tant le garant dans 1 'in ius vocatio que le protecteur dans la manus iniectio, ne sont en rien spécifiques à l'esclavage.

p.256, 1.24, ajouter : le reflet d'une telle mode n'est d'ailleurs pas exclu chez les juristes eux-mêtmes.

p.275, n.102, 1.4, lire : Aristo. p.354, 1.13, lire : iudicatum.

p.362, 1.5, lire : parenté par le sang.

p.440, n.852, 1.1-2, lire : signaler.

p.447, n.900, lire : privée.

p.500, n.1202, in fine, ajouter : sur le thème, D. DALLA, Senatus consuitur. Sizanianum, Milan (1980).

p.501, n.1203, 1.2, lire : p.495 s. (et non 49 s.)

p.504, n.1235, lire : in potestate.

p.679, $1.4,1$ ire : JALBERT P.

p.685, 1.29, lire : per le Scienze giuridiche. 
- INTRODUCTION -

Le Digeste, cette imnense compilation juridique erfectuée sur l'ordre de Justinien ( 1 ), a déjà fait l'ob.jet de nombreuses investigatinns en matière d'esclavage. C'est aux nnlutions du droit dans ce domaine que les historiens du droit se sont surtout intéressés. Si l'on fait une lecture attentive du Digeste, on n'est donc pas étonné de voir que de nombreux textes, moins importants que d'autres du point de vue de leur solution, n'ont jarais été cités. Cette perte d'information est tout-à-fait, regrettable,

Pourquoi ne pas considérer le Digeste avant tout comme une mine de renseignements sur l'esclavage, au même titre que les oeuvres d'un Varron ou d'un pline par exemple? La source juridique n'est-elle pas aussi une source de la réalité qu'il convient de décrypter, d'autant plus que l'intérêt que présentent les juristes par rapport aux auteurs lit.téraires est loin d'être mineur? Ils examinent en effet des questions ignorées des "littéraires", envisagent des cas beaucoup plus nombreux, plus variés. Le Digeste ne contient pas seulement des cas où les juristes sont consultés sur des litiges existants. Il renferme également des exposés théoriques généraux et des textes où l'esclave apparaît simplement comme un exemplum iuris, n'intervenant dans le passage qu'à titre purement accessoire pour illustrer une espèce ne le concernant pas, ce qui revêt un intérêt certain du point de vue idéologique. Faut-il laisser ces derniers textes dans l'oubli parce qu'ils sont"moins importants"au regard du droit ? Cela ne paraît pas souhaitable. Sans négliger les solutions du droit, il nous semble nécessaire d'examiner le contenu réel du discours des juristes sur l'esclavarge.

1 -Cf. A. GUARINo, Storia del diritto romano, Naples (1975), p. 575 et suiv.
Catte entreprise suppose non sevlement una gnalyso te l'esclavage, mais aussi la prise en considération des juristes. Comment ceux-ri rággiesent.ils par rapport à l'esclavage? Nous livront,-ils s?:n image fidèle ? Nous 13 cachent-ils ? C'est là un aspect qui $n$ 'a jamais été tròs dévelopṕn. En outre, bien que les élérents suivants ne se veuillent qu'indicatifs, il est utile de remarquer que la compilation compte 21001 paragraphes dont 5185 concernent l'esclavage, ce qui est considérable. Il est par ailleurs opportun de rappeler que l'éventai?. chronologique offert par ces textes s'étend de la fin de la République au début du Bas-Empire. Ce travail, dont il convient de mieux définir les termes pour en cerner plus précisément l'objet, était donc à faire.

Exploitation la plus cynique de l'homne par l'homme, l'esclavage, d'un point de vue juridique, peut être défini comme une institution ayant pour fin de procurer à certaines personnes l'activité d'individus leur appartenant, selon des modalités d'exploitation diverses (2). Il est dès à présent opportun de signaler que le terme"esclavage"sera pris dans un sens large, aprèsesclavage compris. En effet, l'afrranchissement, qui peut être considéré comme capital dans la vie de l'esclave, reste souvent théorique dans les faits (3). Et même sur un plan strictement juridique, la condition de l'esclave et celle de l'affranchi doivent etre analysóes conme deux formes dune mêne domination (') ).

L'esclavage a, plus que toute autre société, marqué la sociêté romaine (5). Selon l'état des connaissances, la Rome du début de l'époque

2- P. PETIT, Histoire générale de l'Empire romain, Paris (1974), p. 219. L'esclavage est défini juridiquement dans la société. Sur sa condition, v. W.L. WESTERMANN, The slave systems of Greek and Ronan Antiquity. v. W.L. WESTERMANN, The slave systems of Greek and Roman Antiquity
Philadelphie (1955), p. 77 et suiv.; J. GAUDEMET, Institutions de 1 Antiquité, p. 545; M. KASER, Das röm. Privatrecht $I_{2}$ (=RPR), Munich (1971), p. 283 et suiv. et II (1975),p. 124 et suiv. et $584^{2}$ et suiv.; F. DE MARTINO, Storia della costiłuzione romana, Naples ( 1974$), \mathrm{IV}_{2}$, p. 340 et suiv.; E. $M$. SCHTAERMAN et M.R. TROFIMOVA. La schiavitù nell Italia imperiale, Rome (1975), p. 210 et suiv. v. également les ouvrages plus anciens, mais toujours fondamentaux de H. WALLON, llistoire de l'esclavage dans l'Antiquité, III, Paris (1879), p. 46 et suiv. et W.W. BUCKIAND, Roman law of Slavery, Cambridge (1908) XIV + $735 \mathrm{p}$.

3- V. par exemple J. MACQUERON, Le travail des hommes libres dans I'Antiquité romaine, Aix-en-Provence (1958), p. 140.

4- K. VISKY, Le operae dei liberti, in Index 1 (1970) p. 221 et suiv., er sur P. Pescani, Le operae libertomom, Trieste (1967).

5- F. DE MARTINo, Storia IV, eit. p. 337 et suiv.; E.M. SCHTAERMAN, Die Blütezeic der Sklavenwirtschaft in der röm. Republik, Wiesbaden (1969), p. 278 et suiv. ; J. GAUDEMET, o. c., p. 543 ; P. PETIT, La paix romaine,
Paris (1971),p. 375. 
républicaine ne compte qu' un nonbre infime A'esclaves (6), mais la politique délibérée $d$ ' expansion dans laquclle elle se lance dès le 3ème siècle avant notre ère (7) va faire de la société romaine une société esclavagiste par excellence (8). Cependant, c'est seulement au cours du 2ème siècle avant notre ère, à $1 \mathbf{a}$ suite des grandes guerres contre 1 'Orient méditerranéen, que l'esclavage semble atteindre sa forme la plus classique et parvenir à son expansion maximale (9). Cette évolution, liée au développement de la grande propriété foncière, se traduit par une dégradation de la condition servile. Alors qu'il éteit jadis proche de la famille (10), l'esclave devient un matériel humain traité avec la plus froide détermination économique (11). Les révoltes serviles de la fin de 1 'époque républicaine sont le signe de cette dégradation (12).

L'avènement du Principat peut être analysé comme la corısolidation du système esclavagiste (13). Une phase de domination juridique fait alors suite à une phase davantage "physique". Mais le Haut-Empire, tout en voyant l'épanouissement de I'esclavage, connaît aussi sa crise et son effacement devent des structures pré-féodales (14). Le Digeste permet-il de dater cette crise avec précision?

6- F. DE MARTINo, Storia, cit. II, p. 6.

7- M. CLAVEL-LEVEqUE, Impérialisme, développement et transition, La Pensée $n^{\circ} 196(1977)$, p. 12

8- F. DE MARTINO, Storia, cit. II, p. 5, 289.

9- F. DE MARTINo, Storia, cit. II, p. 294. L'esclavage n'est cependant pas généralisé, M. CLAVEL-LEVEQUE, Impérialisme, cit., p. 19. Il faut sigraler que la condition de l'esclave n'est pas uniquement la base de la situation de producteurs. Les Romains cherchent de plus en plus à s'entourer d'une nombreuse familia d'esclaves donestiques, cette familia urbana ayant pour fonction d'assurer le standing du maitre (cf. H. WALLON, Histoire de 1'esclavage, cit. 1 , p. $166 \mathrm{~s}$. ; J. MARQUARDT et A. MAU, Vie privée, trad. de l'all. par V. HENRY, Paris (1892), p. 66 s. ; E. FAIRON, L'organisation du palais impérial a Rome, Le musée belge 4 (1900), p. $5 \mathrm{~s}$.; G. BOULVERT, Esclaves et affranchis impériaux, Naples (1970), p. $24 \mathrm{~s}$.).'Cela donne une prenière idée de l'hétérogénéî́té du groupe servile sur laquelle il sera revenu longuement.

10- Plut., Cato mai., XX, 5 ; Iuv., XIV, 166. F. DE MARTINo, Storia, cit., II, p. 8 .

11- varron, de re rust., I, 17, I ; Caton, de agr., LVI-LIX. F. DE MARTINo, Storia, cit. II, p. 295 et III, p. 119.

12- F. DE martino, Storia, cit. III, p. $117 \mathrm{~s}$.

13- F. DE MARTINO, Storia, cit. IV, p. 21.

14- Sur les causes et les modalités de cette transition, E.M. SCHTAERMAN, La schiavitũ, cit., p. $328 \mathrm{s.,} 343$; F. DE MARTINo, Storia, cit. V, p. 147 s. ; P. DOCKÉS, La libération médiévale, Paris (1979) ; P. ANDERSON, Les passages de 1'Antiquité au féodalisme, Paris (1977).
Répondre à une tej.le question suppose un classement chronologique préalable des juristes apparaissant dens le Digeste. Or, le Digeste ne nous livre pas moins de 86 ncms de juristes. Etait-il possible, au-delà de leur prise en considération individuelle, de périodiser leurs écrits en fonction des grands moments de l'évolution retracée ? Cette transposition repose sur un postulat qu'il nous faut justifier : l'interchangeabilité des juristes au sein de leur époque.

L'origine essentiellement aristocratique des juristes républicains conduit à envisager la période pré-classique de manière spécifique (15). Ce n'est qu'avec la crise de la respublica que les juristes semblent prendre du relief en tant que tels, l'activité jurisprudentielle n'étant pas jusqu'alors clairement distincte de l'action politique de la nobilitas (16). Quoi qu'il en soit, les juristes républicains sont dans une situation d'indépendance vis-à-vis du pouvoir . Avec l'instauration de I'Empire, la jurisprudence aura du mal à préserver cette autonomie.

15- A. GUARINo, Storia, cit., p. 304 ; G. MELILLo, Economia e giurisprudenza a Roma, Naples (1978), p. 18 s., 32.Selon cet auteur, les constructions jurisprudentielles républicaines se seraient édifiées, au-delà des petites controverses quotidiennes, à partir des intérêts économiques dominants (p. 19s.). Contra, $\mathrm{k}$. VOLTERRA, La base economica della elaborazione sistematica del diritto romano, in RISG, 3 a $\mathrm{S}$., 11 (1963-67), p. $239 \mathrm{~s}$.; G. GROSSO, Schemi giuridici e societa nella storia del diritto privato roG. GROSSO, Schemi giuridici e societa nella storia del diritto privato ro obbligazioni, Turin (1970), p. 49 s. MELILLO (p. 19 s.) remarque d'ailleurs 1 a "surprenante insuffisance" du traitement de faits êconomiques importants dans un contexte, pourtant, de cormerce international et d'activités financières complexes d'une part, et, d'autre part, la systématisation tardive du droit public. A ce propos, F. SCHULZ, I principi del diritto romano, tr. it. Florence (1946), p. $24 \mathrm{~s}$, pense qu'on ne peut considérer les auteurs de droit public, à l'époque républicaine, comme de véritables juristes. Contra, M. BRETONE, Tecniche e ideologie dei giuristi romani2, Naples (1975), p. 3 s.Il semble difficile de suivre MELILLO sur la voie d'une coincidence stricte droit et classe déterminée. Si les élites sociales italiennes furent sans doute les premières à profiter des progrès du droit privé républicain (notamment par les contrats comerciaux du ius gentium), l'histoire de cette période est aussi celle, à l'intérieur de cadres fixes il est vrai, d'une prodigieuse mobilité sociale.

16- A. GUARINO, Storia, cit., p. 308. 
Auguste, tout A'aburd, oprire un. disrrimination parmi les juristes par la concession du iug rospondonili ex auctoritate frincipis(17). Cetie concession semble avoir été accordée à certains juristes de l'ordre sénat.orial, pour être ensuite étendue à ceux de 1 'ordre équestre (18). Les responsa de ces juristes, jouissant d'une autoritó particulière, vont bientôt être contraignants pour les iuges (19). Quelles que soient les polémiques sur la matière, il est acquis que le ius publice respondendi, à

17 - v. Von LOBTON, in St. Arangio-Ruiz II, p. 369 et suiv.; F. DE MARTINo, Storia IV, cit., p. 491; A. GUAR1No, Storia del diritto romano, Naples (i975), p. 416-417, selon qui 1 'institution aurait seulement pris le nom de ius publice respondendi sous Tibère. Pour ces auteurs, les autres juristes, les non privilégiess, pouṿaient continuer a émettre des responsa, ceux-ci ctant toutefois de moindre valeur que les responsa de la jurisprudence of ficielle. Selon F. DE VISSCHER, in RHD (1936) p. 622, les responsa n'au-

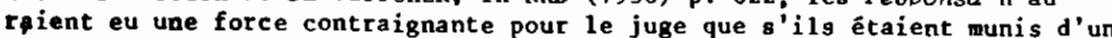
visa (auctoritas) d'Auguste. H. S1BER, in ZSS 61 (1941) p. 401 , pense même qu'une autorisation préalable était nécessaire pour chaque resnnonsum. Pour W. KUNREL, Rerkunft und soziale Stellung der röm. Juristen, Graz, Wien, Köln (1967),p. 281 n 596, et A. MAGDELAIN, Tus respondenai, RHD 28 (1950) p. 1 et suiv.., le ius respondendi aurait seulement contenu 1 'autorieation d'exercer l'activite consultative, et non le transfert d'une áuctoritas particulière aux responsa de juristes déterminés. Adhère aux vues des deux derniers cítés M. HORVAT, Note intorno allo ius respondendi, in Synteleía Arangio - Rui z 1 I, p. 710 et suiv. Sur le thème, v. également $F$. SChulz, Storia della giurisprudenza romana, trad. it., Florence (1968), p. 202 n 2 et 4; H. LEVY-BRUHL, Juge et prudent, RHD (1962), p. Set suiv.; G. PROVERA, Ancora sul ius respondendi, SDHI (1962), p. 342 et suiv.; M. BRETONE, Motivi ideologici dell'Enchiridion di Pomponio, Labeo (1965), p. 23 et suiv.; F. WIEACKER, Augustus und die Juristen seiner Zeit, Tijd. (1969) p. 331 suiv.

18 - Cf. Pomponius D 1.2.2.48; M. HORVAT, Note intorno allo im: respondinli cit. P, 7i2-713. Le fragment D 1.2.2. ne sera pas utilisé, dans 1 'analyse a venir des citations par renvoi (cr. chapitre préliminaire), comple tenu de sa valeur historique spucifique. Pomponius ne renvoie pas en effet à ses prédécesseurs sur une question de droit, mais simplement pour en retracer 1'évolution chronologique.

19 - Selon F. DE MARTINo, Storia IV cit., p. 492, c'est Tibère qui aurait le premier donné une force obligatoire aux responsa. L'auteur attribue a Hadrien une mesure prévoyant qu'en cas de contradiction entre responsa, les juges auraient été libres de leur choix. Cette idée est aussi développée par A. GUARINO, Storia del diritto romano, cit. p. 418. M. BRETONE, Techniche ideologie, cit.,p. 152 n 22 et 23 , ne penge pas que le ius respondendi ait subi des modifications quant à son contenu après le règne d'Auguste, mais plutôt que la force contraignante des responsa fut une conséquence inêluctable de la maior auctoritas des juristes privilégiés. L'auteur critique également l'idée selon laquelle le refus du ius respondendi aurait privé le juriste du droit de respondere (o.c. p. 153; contra, par exemple, M. HORVAT, Note intorno allo ius respondendi.cit.p. 713) et soutient que les reaponea des juristes non autorisés auraient fini par perdre toute valeur pratique. D'autre part, on peut se denander si les juristes n'ayamt pas obtenu le ius responderdi se distinguent riellement des priviligiës dans la mesure où ils n'ont peut-être pis perdu tout espoir de s'en voir gra-

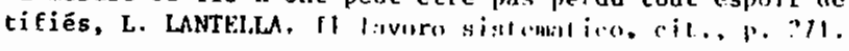

l'orizine reconnaissance de l'autorité personnelle des juristas at mnyen de la garantir, devient neu à peu une simple manifestation de l' indulgontia orincidis (20).

A vec Hadrien, 1a iurisprudence va même tendre æ̀ devenir un orpane de ronvernement (21), les juristes se voyant attirés dans le Consilium principis (22). Ainsi trouve-t-on Celsus fils et Neratius dans le Conseil

20- M. BRETONE, Tecniche e ideologie, cit., p. 154 et n. 27, p. 160 note que 1 'épisode des viri praetorii (cf. Pomponius $\mathrm{D}$ 1.2.2.49) indique une attitude plus restrictive d'Hadrien dans la concession du ius publice respondendi. Il est vrai qu'alors l'intégration des juristes dans le Conseil impérial va permettre au pouvoir impérial d'exercer un contrôle direct sur la jurisprudence (cf. M. HORVAT, Note intorno allo ius reapondendi, cit., p. 714). F. D'IPPOLITO, l giuristi e la citta, Naples (1978), p. 19, distingue trois sphêres ayant successivecitca, Maples (1978), p. Tignatio aux juristes 1 - le peuple ment concede la maxima dignatio ju $s$ exprine à travers quelques médiations dans la sphère de la culture et du pouvoir républicains : 3- elle est directement exprimée par le pouvoir impérial. C'est à tort, selon l'auteur (p. $20 \mathrm{ss}$ ), que Pomponius, dans son Enchiridion, s'efforce de retracer une histoire unitaire des juristes.

21- Sur 1a monarchie d'Hadrien comme tournant de l'évolution jurisfrüdentieile, F. PRINGSHEIM, JRS 24 (I934) p. 149 s. ; F. SCHULZ, History of Roman Legal Science 2 , Oxford (1953), p. $100 \mathrm{s.}, 103 \mathrm{~s},, 117$ B. D'ORGEVAL, L'empereur Hadrien, Oeuvre législative et administrative (1950), p. $59 \mathrm{~s}$.; F. WIEACKER, Studien zur hadrianischen Justizpolitik (Freiburger rechtsgeschichtliche Abhandlungen 5, 1935, 43-81) p. 79 s.Selon G. MELllLo, Economia e giurisprudenza, cit., p. 22,1 ' insuffisante absorption de la fonction jurisprudentielle dans les organes impériaux, de la fin de la République au milieu du zème siècle, conférerait a l'êlaboration juridique une situation particulière. Pour M BRETONE. Tecniche e ideologie, cit. P. 136 s. il culiere. Pour $M$, ne faut pas exagérer le rôle d'Hadrien dans les pratiques juridique du 2ème siècle : la complète "bureaucratisation" de la jurisprudence $n^{\prime}$ interviendrait que dans la dernière décennie du 2ème giècle, lorsque les juristes de rang équestre deviennent prépondérants par rapport a ceux de rang sénatorial, restés plus ou moins étrangers a la mentalitế bureaucratique (cf. W. KUNKEL, Herkunft und soziale Stellung, cit., p. 291 s., 298 s., 300 s.). Contre l'emploi du terme "bureaucratique" a propos de la jurisprudence, C.A. MASCHI, t,a conclusione della giurisprudenza classica all'eta dei Severi, Iulius Paulus, ANRW II. 15 (1976), p. 673.

22- F. DE MARTINO, Storia, cit. IV, p. 675 ; J. GAUDEMET, Institutions de 1 'Antiquité, cit., p. 480 ; A. GUARINo, Storia, cit., p. 418,457 
d' Hadrien (23), Vrlens et V. Verus dans celui d'Antonin le pieux (24), Screvola et T. Paternus dans celui de Marc-Aurèle (25), Tryphoninus et. Menander dans celui de Septime-Sévère (26). Certains juristes se maintiennent même alors que change l'empereur : Julien est présent dans les Conseils d'Hadrien, d'Antonin le pieux et de Marc-Aurèle (27), Marcellus et Maecianus dans ceux des deux derniers (28). Papinien, Paul et Ulpien sont non seulement membres du Conseil impérial mais, comme avant eux T. Paternus, épalement préfets du prétoire (29).

Dans ces conditions, est-il encore possible de parler de valeur autonome de la jurisprudence ? Il faut convenir que la culture juridique des premiers siècles de l'Empire ne peut se réduire à l'activité des juristes qui bénéficient des faveurs impériales, ceux-ci en constituant seulement le degré le plus élevé (30). Il subsiste une jurisprudence non officielle assez importante, mais les responsa des juristes la représentant étant privés de force obligatoire, comment pourrait-elle ne pas subir une évolution qui la dépas-

23 - Celsus (cf. SHA, Hadr., 18, 1; PIR', I 590; J. CROOK, Consilizm principis, Cambridge (1955), Index prosopographique $n^{\circ} 201$ ): Neratius (également membre du conseil de Trajan, ef SHA, Hadr. 18; D 37.12.5; PIR ${ }^{1}, N$ 46; A. GUARINO, Storia del diritto romano, cit. p. $474 n^{\circ} 224$; J. CROOK, Index $n^{\circ} 235$ ).

24 - Valens (cf. PIR $^{2}, F$ 526; J. CROOK, Index $n^{\circ} 154$; P. LAMBRECHTS, La composition du sénat romain de l'accession au trône d'Hadrien à la mort de Commode $(\mid 17-192)$, Anvers (1936), $\left.\mathrm{n}^{\circ} 175,632\right)$; Vindius Verus (cf. SHA, Pius 12 1; PIR, V 441; J. CROOR, Index $\mathrm{n}^{\circ}$ 343; P. LAMBRECHTS, o.c., $\mathrm{n}^{\circ} 138,503$ ).

25 - Scaevola (Cf. D 36.1.23; SHA, Marc, 11, 10; PIR ${ }^{2}$, C 681; J. CROOK, Index $n^{\circ} 87$; H.G. PFLAUM, Les carrières procuratoriennes équestres sous le HautEmpire romain, Paris, (1960-1961) 168 a); T. Paternus (cf. SHA, Marc., 11, 10

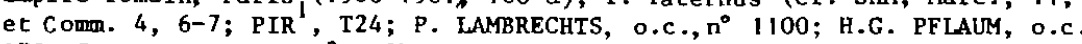
172; J. CROOK, Index $n^{\circ} 318$ ).

26 - Tryphoninus (cf. D 49.14,50; PIR ${ }^{2}$, C1045; J. CROOK, Index ${ }^{\circ} 100$ ); Menander (CF. D 4.4.11.2; PIR , A $1100 ;$ J. CROOK, Index $n^{\circ} 33$ ).

27 - CF. SHA, Pius 12, 1; PIR', S 102; P. LAMBRECHTS, o.c., n²4, 475; J. CROOK, Index $n^{\circ} 292$.

28 - Marcellus (cf SHA, Pius, 12, 1; D28.4.3; D 35.2.32.4; PIR!, V 556; J., CROOK, Index $\mathrm{n}^{\circ} 354$ ); Maecianus (cf. SHA, Pius, 12, 1; D 37.14.17; PIR', v. 657; H.G. PFLAUM, O.c.141; J. CROOK, Index $n^{\circ} 358$ ).

29- Papinien (cF. SHA, Cara. 8, z-3; PIR 2 A 388 ; J. CROOK, Index n¹5); Paul (CF. D 4.4.38; SHA, Alex. 68,1 ; PIRi, I 303 ; J. CROOK, Index $n^{\circ} 180$ ) ; Ulpien (cf. CI $4.65 .4 .1 ; 8.37 .4$; SHA, Alex. 26,5 et 68,1 ; PIR2, D 169; J. CROOK, Index $n^{\circ} 136$ ). Sur ces juristes, v. les articles de v. GIUFFrE (Papinien), C.A. MASCHI (Paul) et G. CRIFO (Ulpien), in ANRW II.15 (1976). se (31)?

Il est vrai que norme impériale et droit, jurisprudentiel ne sauraient être confondus, celui-ci se fondant sur la continuité d'une tradition juridique (32). Cette tradition collective, dont l'importance ressort des nombreux renvois des juristes à leurs prédécesseurs (33), tout en limitant la liberté d'"inventer le droit", donne à l'activité jurisprudentielle la force de l'autorité (34). Mais la certitude du droit dépend-elle plus de cette tradition que de la norme impériale (35)?

Quoi qu'il en soit, il semble que la jurisprudence officielle jouisse d' une certaine indépendance dans la mesure où elle peut influencer l'activité juridique des principes(36). Il paraît en effet acquis, au moins à partir de l'époque des sévères, que les véritables auteurs des rescrits impériaux furent les secrétaires a libellis (37), des juristes tels Papinien, Menander, ulpien, Modestin et Hermogénien.

31- Sur les controverses en la matière, cf. supra n. 17 et 19

32- M. BRETONE, Tecniche e ideologie, cit., p. 178, $224 \mathrm{~s}$.

33- L. LOMBARDI, Saggio sul diritto giurisprudenziale, Milan (1967), p. 27. Mais $P$. Iustus, juriste de la seconde poitié du zéme siècle, renvoie exclusivement à Ia législation impériale (cf. M. BRETONE, o.c., p. 228) et, sur 749 fragments de Papinien (relevés par 0. LENEL, Palingenesia Iuris Civilis I, Leipzig (1889), p. $803 \mathrm{s.}$ ), une centaine renvoie à la législation impériale (cf. v. GIUFFrE, Papiniano, ANRW II.15 (1976), p. 651 et n. 82 et 83 ).

34- L. LOMBaRdi, Saggio, cit., p. VII, VIII, $108,216,372$.

35- Selon M. BRETONE, o.c., p. 232, non seulement la norme impériale innove mais elle confirme et"légalise" le droit existant.

36- C.A. MASCHI, Iulius Paulus, cit., p. 674 ; A. GUARINo, Storia, cit., p. 453 ; T. HONORE, "Imperial" rescripts A.D. 193-305 : authorship and authenticity, JRS (1979), p. 51-64. Selon ce dernier' (p. 51), bien que $l^{\prime a d m i n i s t r a t i o n ~ d e ~} 1$ 'Empire ait été hautement centralisée, et que l'as sentiment de l'empereur fût même requis pour les actes les plus ordinaires, dans cette branche, le gouvernement impérial ne fut pas le fait d'un
dations seuI. Contra, M. BRETONE, o.c., p. 227, pour qui le droit tend de plus en plus à se résumer dans la voluntas impériale. F. CASAvola, Cultura e scienza giuridica neI II sec. d.C., ANRW II.15 (1976), p. 175 n. 105, parle d'un imposant processus idéologique de transformation et de réduction du ius a la lex. Sur le thème, G. ARCHI, Interpretatio iuris, zSS. RA 87 (1970), p. $13 \mathrm{~s}$.; F. CANCELLI, Sull'origine del diritto secondo un motivo riccorente in scrittori ellenistico-romani, e Cicerone, de re publica 5.3, SDHI 37 (1971), p. $328 \mathrm{~s} ., 333$.

37- T. HONORE, "Imperial" rescripts, cit., p. 55 (cf. Tryphoninus D 20.5.12 pr). Selon 1' auteur (p. 62-63), a la fin de l'époque classique (c'est- 
Qu'on nie s̀ la jurisprudence toute sutonomie ou qu'on lui reconnaisse au contraire une certaine indépendance vis-à-vis du pouvoir, aette deuxième attitude paraissant plus conforme à la réalité, dans un cas covme dans l'autre il reste que l'activité jurisprudentielle se présente à nous comme liée à son époque et doit donc être considérée comme telle (38).En d'autres ter mes, l'analyse chronologique doit prendre le pas sur l'analyse des individualités (39). Seule d'ailleurs une périodisation des écrits des juristes par tranches $d$ 'histoire relativement importantes peut rendre correctement compte de l'évolution qu'il nous faut retracer (40). Cela nous amène à mieux cerner 1 'objet du présent travail.

a-dire en 230 ou 240 , cf. R. ORESTANO, Introduzione allo studio stntico del diritto romano 2 (1961), p. $119 ;$ F. DE MARINI AVONZO, Critica testuale e studio del diritto2 (1973), p. 45), l'activité des juristes apparaît plus sous la forme de rescrits que de traités ou de responsa.

38- M. BRETONE, Tecniche e ideologie, cit., p. 231.

39- I1 ne $s^{\prime}$ agit pas d'affirmer pour autant, comne avait pu le faire LEIBNIZ, que les jurisconsultes des Pandectes semblent être tous un seul auteur (cf. F. STURM, Das röm. Recht in der Sicht von Gottfried wilhelm Leibniz (Recht u. Staat in Gesch. u. Gegenw/348/349) Tübingen (1968), p. 22 n. 43 ; G. CRIFO, U1piano, ANRW II.15 (1976), p. 709).

40- Cela au détriment de la prise en considération de l'origine sociale et géographique des juristes. Cette attitude diffère de celle adoptée dans un article paru dans 1 'Index $8(1978-79)$ sous le titre : Ricerche sulla schiavitù attraverso il discorso dei giuristi nel Digesto, alors que ce travail était seulement en chantier. A la page 285 , annonçant la démarche qui restait à entreprendre, nous écrivions : "occorrerà elaborare che qui restait à entreprendre, nous écrivions : "occorrerà elaborare una media delle attitudini dei giuristi in materia di schiavitù". Le
terms nous a fait prendre conscience du gigantisme d'une telle entreprise, que nous ne désespérons pas de mener à bien plus tard avec 1 'aide technique du Centre de Recherches d'Histoire Ancienne de Besançon. Mais, au fond, le contenu thématique de la jurisprudence connait-il des ruptures assez profondes pour envisager les écrits des juristes de manière individuelle ? Selon A. SCHIAVONE, Nascita della giurisprudenza? Bar (1977) , p. 3-68, on remarque une continuité thématique de la jurisprudence romaine par rapport au contextee culturel et materiel dang lequel dence romaine par rapport au contexte culturel et matériel dans lequel elle opère.11 n'est pas inintéressant à ce propos d'évoquer les nombreuses fillations intellectuelles existant entre juristes : ainsi, Rutilius fut 1 'élève de $P$. Mucius (cf. A. GUARINo, Storia del diritto romano, p. 311, $\left.n^{\circ} 153\right)$, Aquilius Gallus celui de Q. Macius (ibid. p. 311, n 154), Servius celui d'Aquilius Gallus (ibid. p. 311, $\mathrm{n}^{\circ} 154$ ). Servius fut le maître de la Namusa (ibid. p. $312, \mathrm{n}^{\circ} 154$ ), d'Alfenus (ibid, p. 312, $\mathrm{n}^{\circ}$ 154) et d'ofilius (ibid. p. $\left.312, n^{\circ} 154\right)$. Capito fut disciple d'Ofilius (ibid. p. $469, \mathrm{n}^{\circ} 222$ ). Labéon fut l'élève de Trebatius (ibid. p. $469, \mathrm{n}^{\circ}$ 222). Capito fut le maître de Sabinus, qui fut celui de Cassius, ce dernier ayant eu Caelius Sabinus pour élève (ibid. $p .471, n^{\circ} 223$ ). On. rencontre même des filiations par le sang : ainsi, chez les Proculiens, Nerva père et fils et Celsus père et fils (ibid. p. 472, n²24). Aristo fut I'élève de Cassius (ibid, p. $474, n^{\circ} 225$ ), Julien celui de lavolenus (ibid. p.
Celui-ci se propose non seulement de retracer l'évolution des textes du Digeste en matière d'esclavage, mais aussi de mettre à jour les réalités qu'il véhicule, et qu'une lecture superficielle du Digeste ne permet pas de dégager (41). Il ne s'agit pas d'un exposé de droit romain (42). Les solutions du droit seront certes évcquées, mais il s'agira surtout de réunir et d'analyser les informations concrètes que nous fournit le Digeste sur un phénomène social dont l'importance n'est plus à dérnontrer (43). N'est-il pas tout aussi fondamental d'apprendre ou réside 1 ' esclave, oul il travaille, $1 a$ manière dont il est nourri, que de savoir qu'il peut être institué héritier ou posséder un pécule? La recherche oscillera constamment autour de ces pôles.

Un effort enfin, qui ne doit épargner ni l' historien, ni le juris-

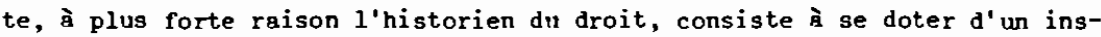
trument lui permettant un traitement objectif du matériel à analyser. La démarche, qui sera définie dans un chapitre préliminaire, procèdera systématiquement de la quantification. Il ne s'agira pas, par ce moyen, de prétendre fournir réponse à tout, mais plutôt de placer le lecteur devant des faiscenux d'arguments qui l'inciteront è réfléchir. Cet apport, original en droit romain, du présent travail n'est nullement le fruit du hasard, il est plutôt, pour paraphraser le titre d'un ouvrage célèbre, celui de la nécessité. Un essai d' histoire "scientifique", sans vouloir abuser de ce terme, suppose ici une analyse exhaustive du Digeste et non des seuls textes qui serviraient à vérifier une hypothèse $d^{\prime}$ ordre intuitif. Ainsi s'expliquent les nombreusès

$476, \mathrm{n}^{\circ} 226$ ) et Africain colui de Julien (ibid. p. 477, $\mathrm{n}^{\circ} 227$ ). Scaevolà fut le maître de Paul et de Tryphoninus (ibid. p. 481, $\mathrm{n}^{\circ} 229$ ). Enfîn, Modestín fut $1^{\prime}$ ealève d'Ulpien (ibid, p. 487, n⿳233).

41- Il sera procêdé a l'aide de l'Index thématique élaboré par le centre de Recherches de Besançon, présenté par F. FAVORY in Schiavitũ, manomissione e classi dipendenti nel mondo antico, L'Erma di BRETSCHNE IDER, Rome (1979), p. 143-159. S'appuyant sur R. ORESTANO, Introduzi one allo studio storico, cit., M. VILLEY, dans un article intitulé "Ce que l'histoire du droit doit à Comte", in Mél. Lévy-Bruhl (1959), p. 502, écrit: "Si l'histoire du droit prétend saisir 1 'expérience juridique concrète, c'est-à-dire totale, intégrale, avant d'en venir aux règles de droit et aux solutions judiciaires, elle nous décrira la manière dont le juet aux solutions judiciaires, elle nous decrira la manière dont le juriste perçoit le monde et ses modes de raisonnement". C'est animé de préocsupations identiques qu'il convient d'aborder ce travail : avan tout, dégager la manière dont l'esclavage est (réellement) perçu par le juriste.

42- L' oeuvre d'un BUCKLANd (Roman law, cit.) au début de ce siècle est déjà sur ce point considérable.

43- Cf. supra n. 2 ; v. aussi 1 'imposante 1 iste bibliographique dressée par 
pages de références au Digeste (44). Le lecteur sera sans doute indulgent devant la lourdeur de ces listes. Ce travail se fonde en effet sur une étude la plus minutieuse possible des textes. On comprendra également l'utilité de ces références pour les investigations ultérieures.

Cela dit, le Digeste est une source spécifique. Pour reprendre une terminologie qui a fait date, son analyse risque de se heurter à certains obstacles épistémologiques (45).

Il faut tout d'abord être conscient de ce que le Digeste peut ne pas être un reflet fidèle de la réalité. Conme tout recueil de textes juridiques, textes de lois ou surtout décisions de prudentes, il donne par définition le reflet du "mauvais revers de la médaille". Les juristes n'ont pas leur attention attirée par les esclaves travailleurs et dévoués, ou par les maitres indulgents et satisfaits de peu, mais, au contraire, par les tensions, les drames entre maîtres et esclaves. Le Digeste est une source de la "réalité clinique". Cette donnée n'empêche certes pas de rechercher les signes d'une évolution, mais il faudra l'avoir à l'esprit pour l'interprétation de certains résultats.

La source juridique est porteuse d'une deuxième difficulté. Le droit est une représentation spécifique de la réalité. A ce titre, il n'en est pas forcément un reflet égal. Il peut ainsi en constituer un prisme déformant lorsque celle-ci n' est pas en prise directe avec le droit, c'est notamment le cas si cette réalité est exclusivement économique.

Si ces difficultés tiennent à la nature juridique de la source en général, il en est qui sont plus précisément le fait-même du Digeste.

G. BOULVERT dans un article sur le droit de l'esclavage sous le HautEmpire romain, dont la conception nous est commune, à paraitre in ANRW.

44- Ces pages, compte tenu de leur lourdeur, devraient, selon les usages, se trouver en annexe, mais, par leur maintien, nous avons voulu montrer au lecteur 1 'important fondement de nos assertions.

45- C. PACHFT.ARD, La formation de 1'esprit scientifique, Paris (1972), 256 p.
Les compilateurs, vivant au 6ème siècle, sont-ils pleinement conscients des réalités de la période d'histoire dont ils nous livrent les textes? Les interpolations ne risquent-elles pas de trahir cette histoire (46) ? La jurisprudence n'est-elle pas en retard par rapport aux faits dont il nous faut tenter de retracer l'évolution? Ne se préoccupe-t-elle pas surtout des esclaves privilégiés, l'emploi de la force physique étant suffisant pour les autres? He risque-t-elle pas, toujours au détriment de la réalité, de surestimer les problèmes qui soulèvent le plus de difficultés juridiques ? C'est conscient de ces problèmes qu'il faut aborder.la lecture de ce travail.

$-0^{\circ}$

Il faut, pour déceler une crise de l'esclavage à travers le Digeste, se demander si celui-ci témoinne d'une évolution de l'exploitation esclavagiste. Après avoir fait subir au discours des juristes une périodisation, il conviendra de répondre aux questions suivantes.

Du point de vue économique, l'esclave est avant tout un outil qu'il est utile d'acquérir. Quels modes $d$ ' acauisition de la main-d'oeuvre servile les juristes nous indiquent-ils ? Ces modes subissent-ils une évolution? Mais ces interrogations sont encore insuffisantes, il faut aussi se pencher sur les formes d'exploitation de cette main-d'oeuvre : quelle est la situation de l'esclave en tarit que travailleur ? Cette situation est-elle uniforme ?

N'est-elle pas déterminante de la manière dont l'esclave vit concrètement son esclavage? Plus généralement, quelle inage les juristes nous livrent-ils de l'esclave dans les rapports sociaux ? Les textes permettentils de retracer une évolution de la condition servile qui autorise à dater la crise ?

46- On reviendra sur ce problème, mais on peut d'ores et déjà dire qu'il s'agira le plus souvent d'examiner, non les solutions, mais les réalités contenues par les texteg. De ce point de vue, strictement quan titatif, les interpolations $n^{\prime}$ apparaissent pas co, strictement quantitatif, les interpolations ' apparaissent pas coms genantes. Lorsqu'il faudra par contre etudier les solutions, nous signalerons bien entendu les interpolations êventuelles. Après une époque d'engouement pour les interpolations, on peut relever actuellement l'existence d'une tendance inverge. Dans ie sens, T HONORE, "Imperial" rescripts, cit., p. 63, se prononce pour $1^{\prime}$ authenticité du Digeste, les parallèles entre certaines phrases du Code de Justinien et du Digeste ne pou- 
Finfin, bien que l'idóolnpie ne soit certes pas absente des répnnses fournies par les juristes aux prócélientes questions, n'est-il pas intéressant aussi de s'interroger plus particulièrenent sur les manifestations de cette idéologie ? Celle-ci est-elle affectée par la crise ?

La progression suivie par ces questions nous conduira de l'explorá au moins exploré. C'est éviłement 1 a fin de cette progression qui constituera l'originalité du travail à entroprendre. En ce qui concerne les domaines que la romanistique a déjà explorés, bien qu'il ne soit pas inutile de retracer l'état des connaissances, il ne s'agira toutefois jamais de procéder à un simple rappel, la lémarche restant originale par sa méthode quantitative.

Nous tenterons de répondre aux questions posées on examinant dans une première partie : LA MAIN-D'OEUVRE SERVILF ; dans une deuxième partie LA PLACE DE L'ESCLAVAGE DAISS LES PFATIQIJS SOCIALES, et dans une troisième partie : LES JURISTES PORTEURS DE L' TDFOLOGIE ESCLAVAGISTE.

On cernera mieux encore l'utilité de cette progression après avoir dégagé l'importance de l'esclavage dans le discours des vuristes.

vant, selon lui, s'expliquer que par un profond respect des compilateurs nour les textes transmis.
CHAFITRE PRELIMINAIRE

\section{L' IMTORTANCE DE L' ESCLAVAGE}

DANS LE DISCOURS DES JURISTES ROMAINS

Il s'agissait d'opérer une auntification slobale du comportement verbal des iuristes romains (1)

I- Les juristes apparaissant dans le Digeste sont Sex.Aelius Paetus Catus (w. KUNKEL, Herkunft und soziale Stellung der römischen Juristen, Cit. p.8; 0. LENEL., Palingenesia Luris Civilis, Leipzig 1889, I p. 1-2) cos. 198 av. JC; M. Porcius Cato (Pal I p. 125-6; KUNREL p.9) cos. 195 av.JC; Manilius (Pal I p. 589-90; KUNKEL p. II) cos. 149 av. JC; Brutus (Pal I p. 75-6; KUNKEL p.12) praet. 142 av. JC; P. Mucius Scaevola (Pal I p. 755-6; 75-6; KUNKEL p.12) praet. 42 av. JC; P. Mucius Scaevola (Pal I p. 755-6;
KUNKEL p.12) cos. 133 av. JC; Drusus (Pal I p. 171-2; KUINKEL p. 14) cos. KUNKEL p.12) cos. 133 av. JC; Drusus (Pal I p. 171-2; KUINKEL p. 14) cos.
144 av. JC; Rutilius (Pal II p. 185-6; KUNKEL p. 15) cos. 105 av. JC; Q. Mucius Scaevola (Pal I p. 757-8; KUNKEL p. 18) cos. 95 av. JC; Aelius Gallus (Ciceronis fere aequalis selon Pal I p. 1-2); Gallus (Ciceronis aequalis praetor selon Pal I p. 55-6) praet. 66 av. JC (KUNKEL p.21); Servius (Pal II p. 321-2; KUNKEL p. 25) cos. 51 av. JC; Cascellius (contemporain d'Alfenus, ofilius et Trebatius selon Pal I p. 107-8; KUNKEL p. 25); Trebatius (sous César et Auguste selon Pal II p. 343-4; KUNKEL p. 28); Alfenus (Servii discipulus selon Pal I p. 37-8; KUNKEL p. 29) cos. suff. 39 av. JC; Ofilius (Serviz auditor, Caesaris amicus selon Pal I p. 795-6; KUNKEL p. 29); A. Namusa (Semii discipulus selon Pal I p. $75-6 \mathrm{n} \mathrm{l}$, ef ol. 2.2.44; KUNKEL p. 30); Ateius (Servii. auditor selon Pal I p. $71-2 \mathrm{nl}$, af.
1.2.2.44; KUNKEL p. 31) ; Cinna (Servii auditor selon Pal I p. 171-2; 1.2.2.44; KUNKEL p. 31) ; Cinna (Servii auditor selon Pal I p. 171-2;
KUNKEL p. 35); Tubero (Ofilius auditor selon Pal II p. 377-8; KUNKEL p. 37); Labéon (sous Auguste selon PaI I p. 501-2; KUNKEL p. 114); Capito (Pal I p. 105-6; KUNKEL p. 114) cos. 5 ap. J.C.; Blaesus (Trebatii auditor? selon Pal I p. 75-6; KUNKEL p. 1/5); Mela (contemporain de Labéon selon Pal I P. 691-2, of D33.9.3.10; D9.2.11 pr; KUNKEL $P$. 116); Sabinus (sous Tiber (sous Tiber I1); KEL p; 19 ; Nerva pater (Pal I p. 787-8; Kinke Minicius (Sabini auditor selon Pal l p. 699-700, cf D12.1.22; KUNKEL p. 121); Cartilius (Pal I p. 105-6; KUNKEL p. 122); Proculus (contemporain
de Nerva filius selon Pal II p. 159-160; KUNKEL p. 123); Atilicinus (Prode Nerva filius selon Pal II p. 159-160; KUNKEL p. 123); Atilicinus (Proculus aequalis selon Pal I p. 71-2 n1, cf D23.4.17; KINKEL p. 129); Nerva p. 109-110; KUNKEL p. 130) cos. 30 ap. JC; Caelius Sabinus (KUNKEI. p.131; Pal I p. 77-8) cos. 69 ap.JC; Pegasus (cos. et praef. urbi sous Vespasien. 
Cela posait des problèmes de méthode auxquels il convient de répondre avant d'exposer les résultats obtenus grâce à cette analyse quantitative.

Proculi successor selon Pal II p. 9-10; KUNKEL p.133); Plautius (contemporain de Pegasus) (selon Pa1 II p.13-14; KUNKEL p. 134); Fufidius (KUNKEL p. 136); Fulcinius (contemporain de Labéon, Mela et Atilicinus selon Pa1 I p. 179-180; KUNKEL p. 137); Celsus pater (Pal I p. 127-8; KUNKEL p. 137) cos.; Iavolenus (IUliani praeceptor selon Pa1 I p. 277-8; KUNKEL p. 138) legatus cos. 90 ap. JC; v. Lucul lus ( $\mathrm{Pa} 1$ II p. 1207-8; KUNKEL p. 140 - incertain); Aristo (Cassii auditor selon Pal I p. 59-60; KUNKEL p.

141); Neratius (sous Trajan et Hadrien selon Pal I p. 763-4; KUNKEL p. 144); U. Ferox (Pal II p. 1201-2; KUNKEL p. 145); Vivianus (sous Trajan selon Pal II p. 1225-6; KUNKEL p. 146); Celsus filius (Pal I p. 127-8; KUNKEL p. 146) cos. 129 ap.JC; Campanus (Pal I p. 105-6; KUNKEL p. 147 - incertain); Octavenus (sous Domitien, sous Trajan selon KARLOWA - RGI p. 702; Pa1 I p. 793-4; KUNKEL p.150); A. Valens (contemporain de Julien selon Pal II p. 120I-2; KUNKEL p. 151); v. Severus (cité par Julien selon Pal II p. 1207-8; KUNKEL p. 154); P. Clemens (sous Hadrien et Pius selon Pal I p. 803-4; KUNKEL p. 154); Julien (Flomit sub Hadriano et Pio selon Pal I p. 317-8; KUNKEL P. 157); Vindius Verus ( $\mathrm{Pal}$ II p.1223-4; KUNKEL P. 167) cos. 138 ap.JC; Pedius (KUNKEI p.168); L. Felix (sous Hadrien selon Pal I p. 557-8; KUNKEL p.I70); Pomponius (sous Hadrien, cf D1.2.2.49, sous Pius, Marc-Aurèle et Verus, cf D50.12.14; selon Pal II p.15-6; KUNKEL p.170) Africain (Iuliani iumis consulti aequalis minor natu selon Pal I p.i-2; KUNKEL p.172); Mauricianus (sous Pius, cf D31.57, selon Pal I p.689; KUNKEL p.176); T.Clemens (KUNKEL p.177); Publicius (Pa1 II p.185-6; KUNKEL p. 185); Gaius (Pa1 I p.181-2; KUNKEL p.186); Maecianus (sous Pius et divi fratres - interfectus est p.c. 125 selon Pal I p. 575-6; KUNKEL p.174) Venuleius (sous Pius et divi fratres selon Pal II p.1207-8; KUNKEL P. 181 184); Marcellus (sous Pius et Marc-Aurèle selon Pal I p.589-590; KUNKEL p. 213); P. Iustus (sous divi fratres selon Pal I p. 947-8; KUNKEL p.216); F1orentinus (Pa1 I p.171-2; KUNKEL p.217); Scaevola (sous divi fratres, Commode et Sêvère selon Pal II p. 215-6) (KUNKEL p. 217); T. Paternus (KUNREL p.219) praef. praet. 179 ap.JC; Papinien (sous Sévère et Caracalla interfectus est 212 pc selon Pal I p.803-4; KUNKEL p.224) praef. praet. 203 ap. JC; Messius (sous Sévère et Caracalla selon Pal I p.699-700); Tryphoninus (sous Sévèreet s.selon PalII.35I-2; KUNKEL p.231); Menander (sous Sévère et Caracalla, cf D4.4.1I.2; D.49.16.13.6 selon Pa1 I p.695-6; KUNKEL p.233); P.Fronto (Pa1 I p.947-8; KUNKFl F.235); Callistrate (sous Sé-

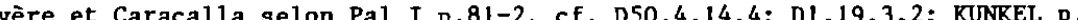
235) : Tertullien (sous SĆìn 235) ; Tertullien (sous sêvere et Caracall a selon Pal II p.341-2; KUNkEL $p$ 236); Arrianus (incertain selon Pal I p.69-70) KUNKEL p. 243); Puteolanus (incertain selon Pal II p.185-6; KUNKEL p.243); Paul (Pa1 I p. 951-2; KUNKEL p. 244) praef. praet. sous Alexandre - Sévère; Ulpien (interfectus est 228 pc selon Pal II p.379-380; contra J. MODRZEJEWSKI et T.ZAWADSKI , La date de la mort d'ulpien et la préfecture du prétoire au début du règne d'Alexandre Sévère, in RHD (1967), 565-611; KUNKEl p.245); praef. praet.; L. Rufinus (contemporain de Paul, cf. D40.13.4; selon Pal I p.559 n1; KUNKEl. p.255): Macer (sous Alexandre - Sévère selon Pal I p.561-2) (KUNKEL p.256); Marcien (contemporain de Paul et Ulpien, plus jeune qu'eux, écrit la majorité de ses livres après la mort deCaracalla, selon Pal I p.639 nl; KUNKEL p. 258; Morestin (Ulpiani discipulus selon Pal I p.701-2; KUNKEL p.259); I. Aquila (Pal I p.501-2; KUNKEL p.261); F. Anthianus (incertain selon Pal I p. 179-180; KUNKEL p.261); R.Maximus (incertain selon Pal II p.185-6 ; KUIRKEl p.261); Hermogénien (Pal I p.265-6; KUNKEL F.263); A. Charisius
L'analyse se voulant exhaustive, on devait procéder à une classification objective du matériel textuel. Ce matériel se présente dans le Digeste sous deux formes : soit le juriste auquel les compilateurs se réfèrent apparaît directement dans l'intitulé du fragment (2), soit il n'apparaît qu'aix deuxième degré à l'intérieur d'un paragraphe (3). Il est alors cité par celui dont le nom ressort dans l'intitulé du fragment. Il fallait donc opérer à deux niveaux : le premier concernant l'analyse normale ou directe, le second l'analyse des citations par renvoi à un auteur antérieur.

$4^{\circ}$ ap.J.C. selon Pal I p. 57-8.

I1 faut encore signaler Servilius (Proculo minor natu, maior $T$. Clemente, selon Pal II p.321-2) et Paconius (incertain selon Pal I p.803-4) qu'on n'a pu classer dans cette liste chronologique faute de précision des sources. Hormis quelques rares exceptions, le Digeste opère donc un concentration des textes juridiques du $1^{\circ}$ siècle avant notre ère au $3^{\circ}$ siècle après.

2-Par exemple D 1.14.3. Ulpianus libro trigensimo octavo ad Sabinum.

3-Par exemple, dans le même texte D 1.14.3, Pomponius cité par Ulpien. 


\section{Paragraphe 1 : L'analyse directe.}

Le Digoste se présente sous la forme d'un comportement verbal. La formation et 1a transmission des normes sociales, des valeurs, des attitudes collectives se manifestent en effet, en grande partie, par le moyen de communications verbeles. Il faut observer et ordonner ces actes verbaix, calculant leur fréquences, déterminant leurs interrelations. L'analyse de contenu permet, une telle investigation (4).

La première étape consistajt à définir un indice d'intégration des textes qui soit le plus proche de la róalité. Toutefois, certains textes ne contenant pas de référence explicite à l'esclavare mais concernant néanmoins un point de cette institution, il fallait dans une deuxième étape parvenir à les intégrer en vertu d'un critèro objectif.

\section{I - Le choix de I'indice : le paragraphe}

L'importance quantitative de l'esclavage étant l'unique variable à prendre en considération dans ce cadre, il a été procédé à une catégorisation dichotomique du matériel, suivant la présence ou l'absence d'une référence à l'esclavage (5). L'unité d'enregistrement était donc constituée par le terme référant à l'esclavene. On devait ensuite définir une unitó de contexte permettant de chiffrer l'évolution (6).

4- B. BERELSON, Content analysis in communication research, Glencoe, The Free Press (1952); D.P. CARTWRIGHT, L'analyse du matériel qualitatif, in Les méthodes de recherche dans les sciences sociales, publié sous la direction de L. FESTINGER et D. KATZ (tr. Fr. Paris 1974), p. 481-535.

5 - Le système de catégories est exhaustif, et les catégories sont mutuellement exclusives. Sur ce point, il respecte ce que P. LAZARSFELD et A.H.BARTON appellent les exigences d'une correction logique, in Quantitative measurement in the social sciences / Classification, typologies and indices, tiré de D. LERNER et H.D. L.ASSWELL, The policy sciences, Stanford (195i) p. 155-192.

6-Selon B. BERELSON, Content analysis, p.135, le plus large ensemble de contenu qui puisse être examiné lorsqu'on caractérise une unitê d'enregistrement.
La structure du Digeste offrait deux possibilités : lo frrement. nu le paraqraphe. Bien que d'une utilisation plus pratique, car incl'sif dי paragraphe et donc moins subdivisé, le fragment a finalement été rejeté, sa prise en considération risquant d'exagńrer 1 'importance de l'esclavage (7).

Cependant, écarter le fragment au bénéfice du paragraphe pouvait conduire à abandonner certains textes dont les termes ne font pas ressortir explicitement l'existence d'esclaves ou d'affrenchis.

\section{II - Construction d'un critère permettant l'intégration des paragraphes litigieux.}

Le terme désignant l'esclnve ou l'affranchi ne ressortant pas d'un paragraphe, il convenait d'opter pour une interprétation extensive lorsque ce paragraphe concernait tout de même l'esclavage. Toutefois, avant de trancher sur de tels paragraphes, il fallait les situer dans un ensemble plus large. Ainsi ont été distingués parmi les titres :

\footnotetext{
7 - Rappelons que le Digeste est divisé en livres, titres, fragments et paragraphes. Un fragment peut contenir un nombre élevé de pararagraphes parmi lesquels un seul concerne l'esclavage. Un exemple permettra de mieux cerner le problème. Ainsi D 47,10.5. Ce fragment contient douze paragraphes. Seu1 le dernier, D 47.10.5.11, concerne 1 'esclavage. I1 y avait donc avec le fragment un risque de surêvaluation de 1 'importance de 1 'esclavage. Le paragraphe, étant 1 'unité 1a plus subdivisée, est de ce point de vue plus proche de la réalitế.
} 
A - Ceux qui concernaient directement l'esclavage (8):

8- Il s'agit de D 1.10 - De officio consulis; D 7.7 - De operis servorm; D 11.3 - De servo corrupto; D 11.4 - De fugitivis - D 18.7 - De servis exportandis : vel si ita mancipium venierit ut manumittatur vel contra; D 29.5 - De senatus consulto silaniano et claudiano : quomon testamenta; ne aperiantur ; D 37.14 - De iure patronatus - D 38:1 - De operis liberne aperiantur ; D 37.14 - De iure patronatus - D 38.1 - De aperis liber-
tomm ; D 38.2 - De bonis libertomm D 38.3 - De libertis universitatiun ; D.38.4 - De adsignandis libertis;D 38.5 - Si quid in fraudem patroni factim sit;D 40.1 - De manzorissionibus;D 40.2 - De manzomissis vindicta; D 40.3 De manumissionibus quae sernis ad universitatem pertinentibus imponutur; D 40.4 - De manumissis testamento ; D 40.5 - De fideiconmissariis libertatibus ; D 40.6 - De ademptions libertatis ; D 40.7 - De statuliberis ; D 40.8-Qui sine manumissione ad libertatem perveniunt,

D 40.8- Qui sine manumissione ad libertatem perveniunt D 40.9 - Oui et a quibus manumise liberi non fiunt et ad legem A 2 liam
Sentiam ; 40.10 - De iure aureomm anulorm 040.11 - De natalibus restituendis ; D 40.12 - De liberali causa ; D 40.13 - Quibus ad libertatem proclamare non licet;D 40.14 - Si ingenuus esse dicetur ; D 40.15 Ne de statu defunctornon post quinquennizm quasratur; D 40.16 - De collusione detegenda ; D 43.29 - De homine libero exhibendo ; D 43.31 Utrubi ; D 45.3 - De stipulatione servorrm ; D 47.4 - Si is, qui testamento liber esse iussus erit, post mortem domini ante aditam hereditatem subripuisse aut corrupisse quid dicetur ; D 47.6- Si fomilia furtwm fecisse dicetur; $D$ 48.15- De lege Fabia de piagioriis.

D 43.29 et D 48.15 soulèvent un problème spécifique sur lequel il faut s'arrêter quelques instants. Ces titres exposent en effet le cas de 1 'home libre retenu en fait dans un état servile. Du point de vue qui nous préoccupe actuellement, il faut rapprocher de cette hypothèse le cas de 1 'homme libre qui sert de bonne foi. On reviendra en détail sur ces problèmes à propos de la reproduction du groupe servile. Quoi qu'il en soit, ces hypothèses font indirectement référence à l'esclavage, qu'il y ait du reste bonne foi ou non du détenteur de cet homme libre. Peu nous importe en définitive la qualité statutaire de cet individu. Ce qui nous préoccupe, c'est qu'il soit en fait dans un état servile. Un texte de Venuleius justifie ce choix. I1 est du reste assez êdifiant à cet égard. Ce juriste du $2^{\circ}$ siècle de notre ère écrit (in D 43.29.2) : nihil enim multum a specie servientim differunt, quibus facultas non datur recedendi, ce qui revient à dire, en situant ce passage dans son contexte, qu'il y a peu de différence entre un esclave et un homa libre a qui on ne laisse pas la $1 i-$ bertê d'aller et de venir.
$B$ - Ceux qui ne le concernaient que de manièrs secondaire (9).

C - Ceux qui ne le concernaient pas (10). catégories :

1. Ceux où apparaîssait un terme désignant le maître ou le patron (11).

9- Ces titres sont D 1.5 - De statu hominum ; D 1.6 - De his qui sui vel alieni iuris sunt; D 1.8 - De divisione reron et qualitate ; D 2.9 Si ex noxali causa agatur, quemadmodrom coveatur; 09.2 - Ad legem Aquilian;D 9.4 - De noxalibus actionibus ; D 14.4 - De tributoria actione ; D 14.5 - Quod cum eo, qui in aliena potestate est, negotizon gestion esse dicetur ; D 15.1 - De peculio ; D 15.2 - Quando de peculio actio annalis est ; D 15.3 - De in rem verso;D 21.1 - De aedilicio edicto et redhibitione et quanti minoris D 25.3 - De agnoscendis et alendis liberis vel parentibus vel patronis vel libertis ; D 33.8 - De peculio legato; D 37.15 parentibus vel patronis vel libertis ; D 33.8 - De peculio legato ; D 37.15
- De obsequiis parentibus et patmnis praestandis; $\mathrm{D} 48.18$ - De quaestionibus.

D 14.1 - De exercitoria actione et D 14.3 - De institoria actione n'ont pas été classés dans 1 'ensemble $B$, mais $C$, car ils visent non pas une alternative simple, du type esclave ou fils, mais complexe (cf. D 14.1.1.16 Ulpien:qui exercet ... pater familias, an filius fomilias, vel servus). Le problème est de mềne nature pour 1'actio institoria, car on peut être en présence d'un préposé étranger à la farrilia (cf. D 14.3.1 Ulpien où on a : servion ou hominem libemon, et non pas filizom).

D 48.18 - De quaestionibus a été rangé dana l'ensemble B,y. MOMMSEN Römi sches Stratfrecht - (tr. fr. J. DUQUESNE - Paris 1907) p. 416, selon qui l'interrogatoire avec torture est exceptionnellement appliqué aux qui 1 interrogatoire avec torture est exceptionnellement appliqué point, cf notament D 48.18.12 Ulpien (rescrit Hadrien) : Si quis, ne quaestio de eo agatur, libemom se dicat .... c'est-à-dire si quelqu'un se dit libre pour échapper à la quaestio).

10- Il s'agit de tous les autres titres, c'est-à-dire ceux qui n'ont pas été relevés dans les notes 8 et 9 . Bien que ses titres ne concernent pas 1 'esclavage, on peut $\mathrm{y}$ relever un noubre important de paragraphes visant cette institution. Par exemple, D 33.5 - De optione vel electione legata, contient 20. paragraphes concernant 1'esclavage sur un total de 31.

11-Hornis les paragraphes où dominus signifie propriétaire en général, et ne réfère pas alors à un rapport esclavagiste, et ceux où patronus vise seulement un rapport de clientèle.

Voici un relevé des textes dans lesquels dominus ne vise pas un rapport directement esclavagiste : D 1.8.6 pr Marcien ; D 3.3.8.3 Ulpien ; D 3.3.10 Ulpien ; D 3.3.12 Gaius; D 3.3.15 pr Ulpien; D 3.3.26 Paul; D 4.4.26 pr Paul ; D 4.6.20 Papinien'; D 7.2.3.1 ulpien (Celsus) ; D 8.2.25.1 Pomponius D 8.2.26 Paul ; D 9.1.1.15 U1pien ; D 9.2.27.14 U1pien ; D 10.3.19 pr Paul ; D 12.2.18 Ulpien ; D 12.3.8 Marcellus ; D 12.4.14 Pau1; D 12.6.33 Julien ; D 12.7.2. Ulpien; D 13.1.10.3 U1pien; D 13.1.14.2 Julien ; D 13.1.20 Try phoni nus; D 13.6.12.1 Ulpien; D 13.7.29 Julien; D 14.3.11.4 U1pien ; 
D 17.1.10.5 Ulpien ; D 17.2.51 pr Ulpien; D 19.1.11.2 Ulpien; D 19.1.13.25 Ulpien ; D 19.2.14. Ulpien : D 19.2.15.2-4-8 Ulpien ; D 19.2.19.3-5 Ulpien ; D 19.2.24 pr -1-2-4-8 Paul ; D 19.2.30 pr(Alfenus)-3 Paul D 19.2.34 Paul ; D 19.2.35 pr Africain ; D 19.2.51.1 Iavolenus ; D 19.2.55 pr Paul : D 19.2.60.5 Iav denus (Labéon); D 20.1.13.2 Marcien; D 20.1.16.1 Marcien; D 20.1.31 pr-1 Scaevola ; D 20.2.5.1 Marcien : D 20.5.12.1 Tryphoninus: D 20.6.1.2 Papinien; D 20.6.8.2 Marcien ; D 21.2.10 Celsus; D 23.3.66 Pomponius ; B 23.3.78 pr Tryphoninus ; D 26. 7.57.1 Tryphoninus ; D 28.2.11 Paul ; D 28.4.1 pr U1pien ; D 29.2.20 Ulpien ; D 30.41 .4 Ulpien; D 31.76 .2 Papinien; D 31.77 .8 Papinien; D 32.14.2 Gaius ; D 39.1.5.6-18 Ulpien ; D 39.1 .6 Julien ; D $39.1 .7 \mathrm{pr}$;
Ulpien ; D 39.1.21.5 Ulpien; D 39.1.23 Iavolenus ; D 39.2.5.1-2 Faul ; Ulpien ; D 39.1.21.5 Ulpien ; D 39.1 .23 lavolenus ; D $39.2 .5 .1-2$ Taul,

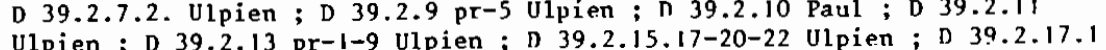
Ulpien ; D 39.2.13 pr-1-9 Ulpien; $\mathrm{D} 39.2 .15 .17-20-22$ Ulpien ; $D 39.2 .17 .1$ Ulpien ; D 39.2.18.4 Paul ; D 39.2.20 Paul ; D 39.2.22.1 Paul ; D 39.2.27 Paul ; n 39.2.31.1 Paul ; D 39.2.34 Paul ; D 39.2.39.2-3 Pomponius ; D 39.2.40.3-4 Ulpien ; D 39.2.47 Neratius ;

D $39.3 .4 \mathrm{pr}-2$ Ulpien; 139.3 .5 Paul ; $\mathrm{D} 39.3 .9 \mathrm{pr}-1-$

2 Paul D 39.3 .11 .2 Paul : D $39.3 .22 \mathrm{pr}^{-2}$ Pomponius ; D 39.4.11.2-4 Paul; D 39.6 i3 pr Julien: D 41.1 .53 Gaius : D 41.1.7-7-8-10-11-12 Gaius : D 41 Callistrate; D 41.1 .26 .1 Paul ; D 41.1.27.1-2 Pomponius ; D 41.1 .30 .3 t.25 Callistrate; D 41.1 .26 .1 Paul ; D $41.1 .27 .1-2$ Pompon
Pomponius ; D 41.1 .31 .1 Paul ; D 41.2 .6 .1 Ulpien ;

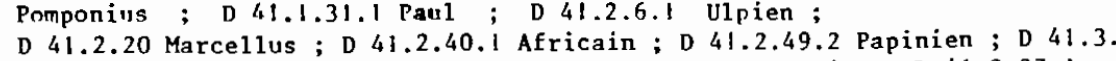
1 Gaius; D 41.3.4.6-12-25-27 Paul ; D 41.3.33.1-2 Julien; D 41.2.37.1 Gaius ; D 41.3.44.1 Papinien ; D 41.3.49 Paul ; D 41.4.7.6 Julien ; D 41. 4.13 Scaevola ; D $41.7 .2 \mathrm{pr}^{-1}$ Paul ; D 41.7.3Modestin ; D 41.8 .5 Iavolenus ; D 42.1.4 pr Ulpien ; D 43.10.1.1 Papinien ; D 43.16.3.8-10 Ulpien ; D 43.16. 8 Paul ; D 43.16.9.1 Paul ; D 43.16.10 Gaius ; D 43.16.12 Marcellus ; D 43. 17.3.3 Ulpien; D 43.18.1.1 Ulpien; D 43.19.3.5 Ulpien; D 43.20.3.5 Pomponius ; D 43.21.2 Paul ; D 43.24.11.4 (Julien)-14 (Julien) Ulpien ; D 43.24. 17 Paul ; D 43.24.18 pr Celsus ; D 43.25.1.4 Ulpien ; D 43.26.6 pr Ulpien ; D 43.26.22 pr Venuleius ; D 44.2.24 Julien ; D 44.2.30.1 Paul ; D 45.1.38.9 Ulpien : D 46.3 .13 Julien : D $46.3 .14 \mathrm{pr}$ Ulpien ; D $46.3 .58 \mathrm{pr}$ Ulpien ; D 46.3.78 Iavolenus; D 46.7.7 Gaius; D 46.8.1 Papinien; D 46.8.3.1 Papinien ; D 46.8.5 Scaevola ; D 46.8.6 Hermogénien ; D 46.8.8 pr-1 Venuleius; D 46.8.12.2-3 Ulpien; D 46.8.13 pr. Faul ; D 46.8.14 Paul ; D 46.8.16 pr-1 Pomponius ; D 46.8.18 Pomponius ; D 46.8.19 Paul ; D 46.8.22 pr-1-2-3-4-8 Julien ; D 46.8.25.1 Africain ; D 47.2.7.3 Ulpien ; D 47.2.12 pr-2 Ulpien; D 47.2.14.2 U1pien ; D 47.2.15.1-2 Paul ; D 47.2.20.2 Paul ; D 47.2.40 Paul ; D 47.2.43.1-5 U1pien ; D 47.2.46.7-8-9 U1pien; D 47.2.47 Paul ; D 47.2.48.7 Ulpien; D 47.2.52.8 Ulpien; D 47.2.54.3 Paul; D 47.2.57.4 Julien ; D 47.2.62.8 Africain; D 47.2.67 or Paul; D 47.2.77.1 Pomponius; Julien ; 47.2 .83 .1 Paul; D 47.2 .85 Paul ; D 47.2.87 Tryphoninus ; D 47.2 .91 IaD 47.2 .83 .1 Paul; D 47.2 .85 Paul ; D 47.2 .87 Tryphoninus; D 47.2 .91 Ia- 47 volenus; D $47.7 .7 \mathrm{pr}$ Ulpien ; D $47.7 .8 \mathrm{pr}-3$ Paul ; D 47.8 .6 Venuleius
$10.5 .4-5$ Ulpien; D 47.10 .23 Paul ; D 47.10 .44 Iavolenus ; D 49.1 .4 .5 10.5.4-5 Ulpien; D 47.10.23 Paul ; D 47.10.44 Iavol

Macer ; D 50.16.14pr Paul ; D 50.16.126 Proculus. Parmi ces textes, il en est qui opposent dominus a procurator (D 3.3 .8 .3 D 3.2.10; D 3.3.12; D 3.3.15pr ; D 12.2.18; D 12.4.14; D 17.1.10.5;

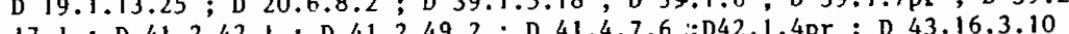
D 46.7 .7 ; 46.8 .3 ; $46.8 .5 ; 046.8 .8 p r-1 ; D 46.8 .12 .2-3 ; D 46.8$ 16pr-1; D 46.8.18; D 46.8.19;D 46.8.22pr-1-2-3-4-8; D 46.8.25.1 ; D 47.2 .43 .1 ). Le procurateur pouvant être libre ou esclave, 1 'opposition terminologique dominus / procurator ne pouvait permettre l'intégration du texte sans la possession d'une indication précise sur le statut du procurateur. Un seul texte est clair ł set égard. Il s'agit de D 15.1 .21 .1 Ulpien où il est question du pécule d'un procurateur, qui est donc esclave. Ce terte a évidement été intégré dans l'analyse des paragraphes. Un autre texte d'Ulpien D 21.1.31.14 contient la relation dominus/procirator, mais rien ne ressort sur la condition de ce dernier. Il a néanmoins été intégré, contrairement aux paragraphes précédemment Il a néanmoins été intêgrê, contrairenent aux paragraphes précédemment énumérés, en fonction du contexte (vente d'esclave/édit des édiles). Quant aux textes où pratronus semble seulement viser un rapport de
clientèle, ils sont au nombre de trois : D 26.3.1.1. Modestin et $\mathrm{D} 26$. 3.4 Paul où patronus est opposé a sxtraneus, ainsi que D 34.9.16.1 Papinien (cf MONIER, Droit romain, Tome ! Paris 1947, p. 308, selon lequel la femme qui vit en concubinage avec le patron est une ingénue). En dehors de ces textes, rien n'interdit de supposer que les textes oũ on a patronus visent un rapport patron/affranchi. Cette attitude est confor a patronus fortée par 0 . tronosve qui permet dintegrer les textes d'on ressort la relation terminologique parens/patronus. On a ainsi été amene à inclure dans cette analyse les paragraphes suivants : D 2.4.10.5-6-7-13 Ulpien ; D 2.4.4.1 Ulpien ; D 2.6.1 Paul ; D 2.6.2 Callistrate ; D 2.7.1.2 Ulpien ; D 2.8.8.5 Paul ; D 3.1.1.11 Ulpien ; D 4.6.26.2 Ulpien ; D 5.2.19 Paul ; D 25.7.1.3 Ulpien ; D 26.4.1.2 Ulpien; D 26.4.3.8 Ulpien ; D 26.4.5.1 Ulpien ; D 29.4.6.8 U1pien; D 29.4.19 Gaius ; D 37.4.4pr Paul ; D 38.2.3.15-17 Ulpien ; D 38.2.8.1 Ulpien; D 38.2.12pr-4 Ulpien ; D 38.2.16.10 U1pien ; D 38.2.19pr Ulpien ; D 38.2.20.3 Julien ; D 38.2.21 Julien ; D 38.5.3.4 Ulpien ; D 42.1.17 Ulpien : D 42.1.49 Paul : $044.1 .7 p r$ Paul : $\mathrm{D} 48.2 .1$ Pomponius : D 48.9.1 Marcien; 48.19 .28 .8 Callistrate ; D 49.14.40.1 Pomponius ; D 48.9.1 Marcier
Paul ; D 50.16.52 Ulpien.

12- Il peut $s$ 'agir du fils ou de l'esclave dans une hypothèse concernant le pécule ; ou bien de l'esclave ou d'un animal dans 1 'hypothèse de 1 'édit des édiles, pour se limiter à ces seuls exemples. Il faut cependant signaler un autre problème, celui posé par la désignation in potestate. On devait d'entrée écarter les textes oũ in potestate visait des cas de captivitê, mais on avait alors généralement in potestate hostizon. Le vécaptivité, mais on avait alors généralement in potestate hostizon. Le véou l'esclave ou englobait les deux. In potestate vise clairement le fils de famille dans D 1.6.8pr Ulpien; D 1.5.18 Ulpien; D 1.7.2.2. Gaius ; D 1.7.5. Celsus ; D 1.7.10 Paul ; D 1.7.12 Ulpien ; D 1.7.13 Papinien ; D 1.7.15pr Ulpien; D 1.7.28 Gaius ; D 1.7.31 Marcien ; D 1.7.40pr Modestin ; D 1.7.41 Modestin ; D 2.8.2.2. Ulpien ; D 3.3.8pr Ulpien ; D 4.4.38.1 Pau1 ; D 4.5.3pr Paul ; D 5.1.4 Gaius ; D 5.1.12.3 Paul ; D 5.2.7 Paul ; D 5.2.22pr Tryphoninus; D 6.1.1.2 U1pien; D 11.1.13pr Paul; Pap.D13.7.40.1: D 14.6.1pr-1 Ulpien; $\mathrm{D}$ 14.6.7.4-7 Ulpien; D 14.6.20 Pomponius ; D 15.2. 1.4; D 21.2.39.3 Julien ; D 21.2.71 Paul; D 22.3.8 Paui ; D 22.5.17 U1pien; D 23.1.10 Ulpien ; D 23.2.3 Paul ; D 23.2.17pr Gaius; D 23.2.19 Marcien ; D 23.2.44.3 Paul ; D 23.2.67.1 Tryphoninus; D 23.4.23 Africain ; D 24.1.3.4 Ulpien; D 24.1 .67 Faul ; D 24.3.29pr Ulpien; D $24.3 .34 \mathrm{Afri}$ cain ; D 24.3.45 Paul ; D 25.3.3.2-5 Ulpien ; D 25.3.5.1 Ulpien; D 26.2. Ipr-1-2 Gaius; D 26.2.2 Ulpien ; D 26.2.16.3 Ulpien ; D 26.3.1.1 Modestin ; D 26.5.21.1 Modestin ; -D 26.8.7.2 U1pien ; D 27.1.2.3. Modestin ; D 27.1.5 Ulpien ; D 28.1.20.1 Ulpien; D 28.2.28pr-1 Tryphoninus ; D 28.2. 29.10 Scaevola ; D 28.3.13 Gaius ; D 28.6.2pr Ulpien ; D 28.7.15 Papinien; D 29.1.28 Ulpien ; D 29.2.6.5-6 Ulpien; D 29.2.52pr Marcien; D 29.4.1.7 
3 - Ceux concernant une question générale de troit (13).

Ces paragraphes ont été intégrés lorsqu'ils se rapprochaient au moins accessoirement de l'institution de l'esclavage.

U1pien ; D 29.4.23 U1pien; D 29.9.1.7-8 U1pien ; D 30.25 Paul ; D $30.91 \mathrm{pr}$ Julien ; D 30.114.8 Marcien ; D 34.4.31.3 Scaevola ; D 35.1 .42 Africain ; D 36.1.13.5 vlpien; D 36.1.17.11 Ulpien; D 36.1.28.3 Julien ; D 36.1.52 Papinien; D 37.4.1.6-8 Ulpien; D 37.4.7 Gaius ; D 37.4.8.14 Ulpien ; D 37.4.13.1-2-3 Julien ; D 37.4.16 Pomponius ; D 37.4.20pr-1-2 Tryphoninus ; D 37.4.21 pr-1 Modestin ; D 37.5.15pr Paul ; D 37.5.16 U1pien;D37. 5.18 Africain;D37.5.25pr Marcellus;D37.6.1pr-3-4-5-8-24 U1pien; D37.6.2.1-3-5-7 Pau1; D37.6.3.2-4-6 Julien; D37.6.5pr Ulp.:D37.6.10 Scaevo 1 a ; D 37.6.11 Paul; D 37.6.12 Paul ; D 37.8.1pr-3-9-13-14 घ1 pien ;

D 37.8.3 Marcellus (nepos); D 37.8.4 Modestin (nepos) ; D 37.8.6 Scae

Dola ; D 37.10.8pr.Africain ; D 37.15.22 Gaius ; D 38.1.7 37.8 .6 Scaevola ; D $37.10 .8 \mathrm{pr}$ Africain ; D 37.15 .22 Gaius ; D 38.1.7.7 Ülpien ; D 38 4.3.2-3 Ulpien ; D 38.4.9 Modestin ; D 38.4.13.1 Pomponius ; D 38.6.5pr-1 Pomponius ; D 38.6.7pr Papinien (nepos) ; D 38.6.8 Papinien ; D 38.16.1.4 (nepos) -5-10 (consonguineos) U1pien; D 40.5.23.2 Papinien; D 40.12.1pr Ulpien ; D 41.2.50pr Hermogénien ; D 41.3.44pr Papinien; D 41.6.1.1 Paul; D 41.6.4 Pomponius; D 43.29.3.4 U1pien ; D 43.30.1pr-1-4-5 Ulpien; D 43 30.2 Hermogénien ; D 43.30.3.3-4-5 U1pien ; D 43.30.4 Africain ; D 45.1.14i. 2 (pupille)Gaius ; D 45.1.56.3 Julien ; D 45.3.20.1 Paul ; D 46.1.10.2 Ulpien ; D 48.5.15.2 Scaevola ; D 48.9.5 Marcien ; D 48.20.7.4 Paul ; D 48 10.15pr Callistrate; D 49.4.2.2 Macer ; D 49.15.12.1-3 Tryphoninus ; D 49. 15.25 Marcien ; D 50.1.21pr Paul ; D 50.4.2 U1pien ; D 50.4.3.4-16 U1pien ; D 50.16.56.1 Ulpien.

Bien que dans ces cas in potestate visât le fils, le paragraphe pouvait toutefois concerner par ailleurs 1 'esclavage : par exemple D 2.8.2.2 ; D 13.7.40.1; D 15.2.1.4; D 24.1.6.7; D 29.5.1.7 etc.

En revanche, in potestate vise 1 'esclave dans $D$ 1.1.4 Ulpien ; D 1.6.1.1 pr Gaius ; D 9.4.21.2-5 U1pien; D 9.4.22.3-4 Paul ; D 9.4.26.5 Paul ; D 9. 4.32 Callistrate; D 9.4.29 Gaius ; D 11.1.16pr Ulpien ; D 14.4.5.6 U1pien : D 28.5.23.1 Pomponius ; D 49.15.30 Paul : D 50.17.18 Pomponius . Enfin, in potestate peut avoir un sens général. Tantôt, la référence au fils et à 1 'esclave est explicite (par exemple in D 11.1 .9 .8 U1pien), tantôt elle est absente. On a néanmoins rangé les paragraphes relatifs à cette dernière hypothèse dans les paragraphes de la catégorie 2 (possibilité d'alternative), ceci dans la mesure oì vlpien, dont les définitions paraissent fiables, englobe fils et esclave dans le vocable in potestate. On a ainsi D 14.1.1.21 : In potestate autem accipiemus utriusque sexus, vel filios vel filias, vel servos vel servas; D 14.4.1.4 : Potestatic verbum ad omnem sexwm, item ad omnes qui sunt alieno iuri subiscti, porrigendrom erit ; D 15.1.1.5 : potestatis verbun conmuniter accipiendum est, tam in filio, quam in servo; D 24.1.3.3 : Verbion potestatis non solum ad liberos trahimus, vermon etian ad servos.

13- C'est-à-dire les modalités ou la finalité d'une action (v. par exemple D 21,1.45 Gaius).
Ainsi ont été intégrés les paragraphes de la catégorie 1, qu'ils appartiennent à l'ensemble $A, B$ ou $C(14)$. On a opéré de même pour les paragraphes 2 ou 3 lorsqu'ils provenaient de 1 'enserble A ou B (15). Ils ont au

14. Pour Al, il n'y avait pas de problème d'intégration; le titre réclamant de par lui-même cette intégration, de même pour Bl. Par contre, pour C1, c'est le paragraphe classé dans la catégorie I (contenant dominus ou - patronus) qui devenait déterminant par rapport au titre, celui-ci ne concernant pas I'esclavage.

Exemples de paragraphes classés Al : D 29.5.1.7-21 Ulpien ; D 38. 2.3.15-17 Ulpien; D 38.2.8.1 Ulpien; D 38.2.12pr-4 Ulpien ; D 38.2. 16.10 Ulpien ; D 38.2.19pr Ulpien; D 38.2.20.3 Julien ; D 38.2.21 Julien ; D 38.5.3.4 Ulpien.

Ont êté classés dans le groupe B1, D 9.2.11.6 U1pien ; D 9.4.21.5 Ulpien ; D 9.4.30 Gaius ; D 9.4.32 Callistrate ; D 14.4.7.4 Ulpien ; D 15.1.11.3 Ulpien; D 15.1.13 Ulpien; D 15.1.19.2 U1pien; D 15.1.21 pr-1-3 Ulpien ; D 15.3.4 Gaius ; D 21.1.31.14 U1pien

Dans le groupe $C 1$, on trouve $D$ 2.4.10.5-6-7-13 U1pien ; D 2.6.2. Callistrate ; D 2.4.4.1 Ulpien; D 2.6.1 Paul ; D 2.7.1.2 Ulpien ; D 2.8.8.5 Paul ; D 2.14.29 Ulpien; D 3.1 .1 . II Ulpien ;

D 4.6.26.2 Ulpien ; D 5.2.19 Paul ; D 12.2.14 Paul ; D 13.5.1.8 U1pien ; D 13.5.19.2 Paul; D 13.6.3.5 Ulpien; D 14.3.11.7 Ulpien ; D 14.3.5.15 U1p. ; D17.2.63.2 U1p.; D24.3.54 Paul; D 25.7.1.3 U1pien; D 26.4.1.2 ULP;D26.4. 3.8 Ulpien ; D 26.4.5.1 U1pien; D 29.4.6.8 U1pien ; D 29.4.19 Gaius : D 36.1.41.1 Paul; D 36.3.1.20 Ulpien ; D 37.4.4pr Paul; D $41.1 .63 \mathrm{pr}$ Tryphoninus (ce texte soulève un cas intéressant, 1a coexistence de deux hypothèses : $\mathrm{Cl}$ par domini et $\mathrm{C} 2$ par in aliena potestate; 1 'hypothèse la plus proche de l'institution de l'esclavage, c'est-à-dire $\mathrm{C1}$, a été choisie) ; D 42.1.17 U1pien ; D 43.4.1.7 Ulpien ; D 44.1.7pr Paul ; D 42.1.49 Paul ; D 47.2.62.7 Africain ; D 47.10.7.8 U1pien ; D 48.2.1 Pomponius ; D 48.9.1 Marcien ; D 48.19.28.8 Callistrate ; D 49. 14.40.1 Paul ; D 50.16.52 U1pien.

15- Pour les paragraphes classés $A 3$, le problème de 1 'intégration se posait de la même manière que pour $A 1$, le contexte du titre étant déterminant. Idem pour les hypothêses B2 et B3. Quant à 1 'hypothèse A2, elle n'apparait pas.

Dans le groupe A3, on relève D 11.3.5.2 U1pien ; D 11.3.9.2 U1pien ; D 11.3 .4 Paul ; D 11.3.6 Paul ; D 11.3.11pr Ulpien; D 11.3 .12 Paul ; D 11.3.13pr U1pien ; D 11.3.14pr-5 Paul ; D 29.5.1.8-9-10-20-23 U1pien ; D 29.5.3.19-20-21-22-23-24-25-26-27-28-30-31-32 U1pien ; D 29.5.5pr-1-3 U1pien ; D 29.5.24 U1pien ; D 29.5.8.1 Paul ; D 29.5.15pr-1 Marcien ; D 29.5. 18 Modestin ; D 29.5.20 Papinien ; D 29.5.21.1-2 Papinien ; D 29.5.23 Maecianus ; D 29.5.25.2 Gaius ; D 29.5.26 Scaevola ; D 29.5.27 Callistrate; D 38.1.7.7 U1pien; D 38.2.3.13-15 U1pien; D 38.2.8pr U1pien; D 38.2 .12 2-3-5 U1pien ; D 38.2.14.1-2-3 U1pien ; D 38.2.16.8 U1pien ; D 38.2.19.1 Ulpien ; D 38.4.1.4 Ulpien; D 38.4.13.1-2 Pomponius ; D 38.5.1.21-28 Ulpien ; D 38.5.3.1 Ulpien ; D 38.5.5.1 Paul ; D 38.5.7 Scaevola ; D 40. 5.4pr-1-8-9-10-11 Ulpien; D 40.5.32.2 Maecianus; D 40.5.51.5 Marcien; D 40.9.14.6 U1pien ; D 40.12.18pr-1U1pien ; D 40.12.20pr-1-2-3 U1pien ; D 40.12.22.1-2-4-6 Ulpien; D 40.15.1pr-1-2-3 Marcien: D 40.15.2.1-2 Papinien ; D 40.15.4pr-1 Callistrate ; D 40.16.2.1-4 Uipien ; $D 40.16 .3$ Papinien ; D 40.15.4pr-1 Callistrate ; D 40.16.2.1-4 U1pien ; D 40.16 .3 Callistrate; D $40.16 .5 \mathrm{pr}-1$ Hermogenien; D 45.3 .23 Paul ; D
tius ; D 47.4.1.12-13-15-16-17-18 U1pien; D 47.6.2 Julien.

On a dans le groupe B2, D 1.5.7 Paul; D 1.5.8 Papinien; D 2.9.1pr Ulpien ; D 9.2.7.1 Ulpien ; D 9.4.21.6 U1pien ; D 9.4.29 Gaius ; D 14.5.1 Gaius ; D 14.5.2pr Ulpien; D 14.5.3 Ulpien; D 15.3.1pr Ilpien. 
contraire étó rejetés lnrsqu'ils étaient issus de l'ensemble C (16). En outre ont áté écartés les naragraphes traitant du postiiminium, ceux-ri ne concernant pas l'institution spécifiquement romaine de l'esclavage (17).

Enfin, on note dans B3 la présence de D 9.2.2.1 Gaius ; D 9.2.7.3-6-7 Ulpien ; D 9.2.11.1-3 Ulpien; D 9.2.23.8-10 Ulpien : D 9.2.35 Ulpien; D 9.2.36 Pr Marcellus ; D 9.4.21.4 Ulpien ; D 14.4.4 Paul ; D 14.4.3.2 Ulpien ; D 14.4 .5 pr-5 Ulpien : D 14.4.7.3-5 Ulpien : D 14.4.9 pr Ulpien ; D 15.1.1.3 Ulpien ; D 15.1.34 Pomponius; D 15.1.35 Iavolenus : D 15.1.11.6-9 U1pien; D 15.1.20 Paul ; D 15.1.30.4-5-6-7 U1pien; D 15.1.32.1 Ulpien; D 15.1.10 Gaius ; D 15. 1.27.3 Gaius ; D 15.1.31 Paul; D 15.1.47.2 Paul ; D 15.1.55 Neratius ; D 15. 2.1.5-9 Ulpien; D 21.1 .1 pr-2-3-4-5 Ulpien: D $21.119 .2-3-5$ Ulpien:D 21.1 . $21 \mathrm{pr}$ Ulpien; D $21.1 .23 .1-5-7$ Ulpien; D 21.1 .25 .10 Ulpien; D 21.1 .28 Gaius $21 \mathrm{pr} U 1 \mathrm{pien}$; D $21.1 .23 .1-5-7$ Ulpien; D 21.1 .25 .10 Ulpien; D 21.1 .28 Gaius
D $21.1 .29 \mathrm{pr}^{-1}-2$ Ulpien; D $21.1 .31 .18-19-22$ Ulpien; D 21.1 .43 .4 Paul ; D 21 . D $21.1 .29 \mathrm{pr}^{-1-2}$ Ulpien; D 21.1.31.18-19-22 U1pien; D 21.1 .43 .4 Paul ; D 21 .
1.45 Gaius; D 21.1.48.2-5-8 Pomponius ; D 21.1 .60 Faul ; D 21.1 .62 Mndestin ; D 33.8.2 Gaius ; D 37.15.5.1 U1pien; D 37.15.7 pr-1-2-3-5 U1pien; 048.18 . 1.2-3-10-17-21-23-25 Ulpien; D 48.18.10 pr-1-3-5 A.Charisius; D 48.18.15.1 Callistrate ; D 48.18.16 pr-1 Modestin : D 48.18.18 pr-1-2-3-9 Pau1.

16- Les paragraphes classês C2 sont Dl1.1.5 Gaius ; D 27.6.9.1 Ulpien ; D 28.1.15 Ulpien; D 29.2.79 Ulpien; D 31.2 Paul ; D 31.6 Paul; D 34.3 .27 Trypboninus; D 36.3.3 U1pien; D 39.2 .17 pr Ulpien; D 41.3 .47 Paul; D 41 . 1.53 Pomponius : D 41.1.10.2 Gaius; D 41.2.49.1 Papinien; D 42.1.6.1 Ulpien; D 45.1.45.1 Ulpien; D 47.1.1.2 Ulpien; D 47.2.41.2-3 (malgré serrus qui vise une hypothèse de postliminizum) Ulpien ; D 45.10.15.17-19 Ulpien ; D 48.8.5 Paul (par rapport au contexte D 48.8.4.2). Sujvant la logique du critère, les paragraphes classés $C 3$ auraient tous dû être exclus. Cependant, certains ont étê intégrés exceptionnellement, le contexte immédiat du fragment plaidant dans ce sens (ainsi Paul D 4.4.10: D 4.8.8 Paul; D 2.4.10.9 Ulpien: D 6.1 30 Gaius; D 14.3 .17 .5 Paul ; D 24.3.64.9 Ulpien; D 42.8 .16 Paul; D 41.3 .37

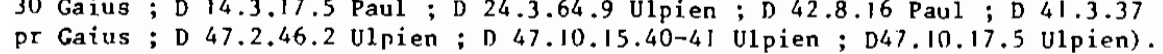
17- Même lorsque ces textes contenaient une rêférence terminologique à 1 'esclavage, mais concernaient au dêpart un citoyen romain (ex: D 26.1 .15 ulpien : D 28.1.20.1 Ulpien; D 41.1.7 pr Gaius; D II.7.36 Pomponius : D 37.1 .3 .6 Ulpien ; D 32.1.1 pr Ulpien; D 49.15.5.2 Pomponius). Cela dit, ont été intégrés les textes où il s'agissait d'un esclave ou d'un af franchi (D 15.2.2.1 Paul ; D 19.1.55 Pomponius; D 28.1.12 Julien; D $11.1 .16 \mathrm{pr}$ Ulpien ; D 28.5 32.1 Gaius ; D 29.2.71 pr U1pien; D 30.98 Julien ; D 35.2.43 Ulpien ; D 38 . 4.1.2 Ulpien; D 38.2.4.1 Pau1; D 40.7.6.1 Ulpien; D 40.7.6.2 Ulpien ; D 41. 3.11 Paul; D 44.2.11.4 U1pien; D 49.15.1 Marcellus ; D 49.15.12.7-8-9-10-1112-13-14-16-18 Trypboninus ; D 49.15.19.5-6-10 Paul ; D 49.15.27 Iavolenus : D 49.15.30 Paul ; pris aussi D 49.16.8 Ulpien: ingenui qui buna fide serviunt). L'hypotnèse du postíiminium peut ne pas viser directement l'esclave, bien que celui-ci soit par ailleurs concerné. C'est le cas lorsqu'il est question de l'esclave d'un prisonnier de.guerre (ex: D 41.3.15 pr Paul; D 41.3.44.7 Papinien; D 45.3.18.2 Papinien; D 49.15.12.1 Tryphoninus; D 49.15.22.1-3 Julien; D49.15.29 Paul). De tels textes ont été intégrês, de même que ceux où il est question de la captivité d'un patron (ex: D 23.2.45.6 Ulpien; D 26.4 . I.2 Ulpien: D 26.4.3.5 Ulpien). Ont été aussi inclus dans cette analyse les textes traitant de la réduction en servitude en général (ex: D 46.3.38 pr
Ces précisions átant faites, il ost. désormais possinle de russer à l'analyse des citations par rẹvoi

\section{Paragraphe 2 :L'analyse des citations par renvoi}

Il a paru opportun de distinguer les renvois portant sur un inriste de ceux portant sur un acte juridique impérial, ces deux typos de renvoi signifiant des réalités différentes (18).

Africain : D 44.7.30 Scaevola), le contenu de ces textes ayant une vocation plus large que ceux visant uniquement le post.liminizu, et pouvant aussi concerner la servitude de la peine. Contrairement a la captivité, l'esclavage pénal est une manifestation de l'institution typiquement romaine de l'esclavage. Les textes en traitant ont donc été retenus, et non seulement ceux où ressortait expressément la désignation servus poence mais aussi, en l'absence de cette référence ceux visant une peine rádui-

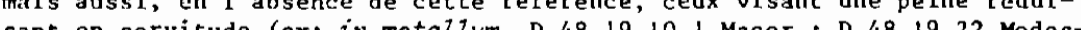
sant en servitude (ex: tin ; D 48.19 .23 ; D 48.19 .38 pr-3.Panl; nu ad bestias, D 28.3.6.10 U1pien : D 49.16.3.10 Modestin : D 49.16.4.1 Menander). Une autre peine en traine la reduction en servitude. Il $s$ agit de la peine de mort. Cependant, crimen capitalis ou pcena cmpitis a une signification plus large que peine de mort. Selon Callistrate (D $48.19 .28 \mathrm{pr}$ ), la peine capitale est graduée. On trouve au sommet 1 a mort : Srmom supplicium (qui entraîne immédiatement la perte du droit de cité et de la liberté, cf. Gaius D 48 . 19.29) ; ensuite, la peine la plus proche de la mort, la condamnation in metallum (qui, elle aussi, fait perdre la liberté, cf. Gaius D 28.1.8.4, ou encore Ulpien $D$ 48.19.8.4) ; enfin, la deportatio qui, contrairement aux peines précédentes, ne fait pas du citoyen romain un esclave. Il n'était donc pas possible d'intégrer les textes où on $n$ ' avait que la référence capitale punitus sans autre indication. Ont ainsi été rejetés $D 37.1 .13$

Africain ; D 48.19.15 Venuleius ; D 49.16.7 T.Paternus ; D 48.19.39 Tryphoninus : D 48.19.28.3-9-12-13 Callistrate; D 49.16.4.2-5; D 49.16.5.1-3; D 49.16.6.1-2-3-7 Menander ; D 46.1.53; D 48.2.21 Papinien ; D 39.4.11 pr2 ; D 47.11.1.2 ; D 48.1.2 ; D 48.19.38.1-11-12 Paul ; D 28.3.6.12; D 29. 1.11 pr ; D 39.6.7; D 46.3.45.1; D 47.11.9; D 48.19.8.1-3; D 48.21.1; D 48.24.1 ; D 49.14.26 Ulpien ; D 48.11.7.3; D 49.4.2.3 ; D 49.16.3.1I17-19 Macer; D 28.1.13.2; D 48.4.3; D 48.19.4 Marcien; D 48.3.14.2 ; D 48.8.16; D $48.9 .9 .1 ; 0$ (49.16.3.11-17-19 Modestin. Ont par contre pu être intégrés D 28.3.6.6 U1pien; D 37.14.9.1 Modestin ; D 38.2.15 Tryphoninus ; D 47.9.12.1 Ulpien; D 47.12.3.7 Ulpien; D 47.12.11 Paul : D 48.3. 2 pr Papinien; D 48.8.3.5 Marcien; D 48.8.4.2 Ulpien; D 48.10.1.13 Marcien ; D 48.10.8 Ulpien; D 48.15.1 Ulpien ; D 48.17.1.1 Marcien; D 48.18.1. 20 Ulpien ; D 48.19.2 pr Ulpien; D 48.19.5 pr Ulpien; D 48.19.6.2 Ulpien ; D 48.19.11.3 Marcien ; D 48.19.14 Macer; D $48.19 .28 \mathrm{pr}-10-14$ Callistrate : D 48.19.29 Gaius ; D 48.19.38.5 Daul ; D 48.20.5 pr Ulpien ; D 49.16.4.110 Menander ; D 49.16.11 Marcien, car contenant une référence à 1 'esclavage.

18- Cf. infra sur les résultacs de l'analyse quantitative globale. 
I- Les renvois opérés sur des juristes.

Les citations d'un juriste par renvoi à un de ses prédécesseurs devaient être envisagées séparément car elles revêtent un intérêt partieulier. Elles signifient en effet qu'à l'époque du juriste cité, le droit de l'esclavage, sur le point de référence, était déjà développé. Tout d'abord, il fallait opérer une sélection parmi les paragraphes contenant ces citations, un paragraphe pris globalement pouvant concerner l'esclavage, la citation n'étant pas effectuée sur un point de cette institution

(19). Ensuite, on pouvait trouver, dans un paragraphe, un auteur postérieur

19- Ont ainsi dû être rejetées les citations suivantes : Servius chez Ulpien D 2.4.4.3 ; Aristo chez Ulpien D 2.14.7.2 ; Celsus chez Ulpien D 2.14.7.2 ; Vivianus chez U1pien D 4.2.14.5; Pedius chez U1pien D 4.2. 14.5 ; Pomponius chez Ulpien D 4.3.7 pr ; Pomponius chez Ulpien D 4.2.7. 1 ; Pomponius chez U1pien D 4.4.11.4 ; Papinien chez U1pien D 5.3.13.6 ; Octavenus chez Pau1 D 6.1.6; Labéon chez U1pien D 6.1.13; Pomponius chez U1pien D 7.6.5.4 ; Proculus chez U1pien D 7.8.4 pr; Labéon chez Gaius D 9.2.2.2 ; Julien chez Pomponius D 12.1.12 ; Servius chez U1pien D 13.3.3 ; Celsus chez U1pien D 13.3.3; Fulcinius chez Paul D 13.1.13; Marcellus chez U1pien D 13.3.3 ; Julien chez Ulpien D 13.7.11.5 ; Nerva chez Paul D 18.1.1.1; Proculus chez Paul D 18.1.1.1; Cassius chez Paul D 18.1.1.1; Julien chez U1pien D 18.4.2.15; Trebatius chez U1pien D 19. 1.13.22 ; Labéon chez Paul D 19.1.42 ; Marcellus chez Paul D 22.1.11.1: Celsus chez Ulpien D 24.3.21; Aristo chez Pomponius D 26:7.61; Julien chez Ulpien D 28.6.10.7. Marcellus chez Ulpien D 29.5.1.8; Julien chez Chez U1Pien $D 28.6 .10 .7$; Marcellus chez Ulpien Papini 73 , 30.11 ; Sabinus D 33.7 .12 .3 ; Q.Mucius chez U1pien D 33.9 .3 pr ; Sabinus chez Paul D 33. 9.4,2 ; Q.Mucius chez Pomponius D 34.2.10 ; Sabinus chez Tryphoninus D 34.9.22 ; Aristo, Neratius et Julien chez U1pien D 35.1.7 pr ; Servius, Labéon, Sabinus et Cassius chez Pomponius D 35.1.6.1 ; Publicius chez Modestin D 35.1.51.1 ; Aristo et Julien chez Paul D 35.2.1.9; Papinien chez U1pien D 35.1.92; Julien chez Ulpien D 36.1.15.3; Papinien chez Ulpien D 36.1.18.6 ; Proculus et Servilius chez T.Clemens D 37.14.10 ; Pomponius chez Ulpien D 4l.2.!3 pr ; Pomponius chez Paul D 43.16.6 ; Julien chez Ulpien D 43.19.3.4; Julien chez Gaius D 44.7.5.5; Pedius chez Paul D 44. 4.5.4 ; Marcellus chez Paul D 45.1.8 ; Aristo chez Paul D 45.1.83.1 ; Pedius chez Paul D 45.1.83.5; veteres chez Paul D 45.1.91.3; Q.Mucius chez Papinien D 45.1.115.2; Julien chez Paul D 46.1.34; Servius chez Marcellus D 46.3.67; Alfenus chez Marcellus D 46.3.67; Labéon chez Papinien D 46. 3.95.7 ; Pegasus chez Papinien D 46.3.95.7 ; Julien chez Paul D 46.3.34; Labéon chez U1pien D 47.10.7.1; veteres chez Paul D 50.16.53 pr ; Celsus chez Paul D 50.17.23. En ce qui concerne les renvois à la législation iur périale, il a fallu de la même manière écarter Sévère et Caracalla chez Ulpien D 4.4.22 ; Hadrien chez U1pien D 5.2.8.16 ; Antonin le pieux chez U1pien D 5.2.8.16 ; Antonin le pieux chez Ulpien D 5.3.5 pr ; Sévère et Caracalla chez Ulpien D 19.2.9.1 ; Marc-Aurèle et Verus chez Marcien D 23. 2.57 (cas d'un rescrit qui, bien qu'adressé par 1 'intermédiaire d'un affranchi, ne concerne ni l'esclavage ni l'aprês-esclavage); Antonin le pieux chez Ulpien D $35.1 .7 \mathrm{pr}$; Hadrien chez Callistrate $D 47.9 .7$; Sévère à celui apparaissant dans l'intitulé (20). L'auteur le plus ancien a alors été intégré dens l'analyse des citations, et l'auteur le plus récent dans 1 'analyse "directe", cette attitude permettant de rendre compte à la fois de l'oeuvre originale et de son cormentaire.

Les problèmes posés par des renvois à des constitutions impériales étaient de nature différente.

\section{II - Les renvois à la législation impériale.}

Outre les difficultés occasionnées par la désignation parfois peu claire des empereurs (21), il a fallu prendre en considération un fait

chez Papinien D 35.1.92 ; Hadrien chez Ulpien D 37.10.3.5 ; Sévère et Caracalla chez Marcien D 48.2.13; Caracalla chez Marcien D 48.22.16 ; Sévère et Caracalla chez U1pien D 38.17.1.3; Trajan chez U1pien D 48.19.5 pr. 20- Par exemple Paulus notat dans un texte de Julien (D 5.1 .75 ; D 37.6 . 3.1 ; D 39.6 .15 ; D 40.2.4.2), ou Ulpianus notat dans un texte de Marcellus (D 20.1.27; D 26.7.28.1 ; D 29.7.9). On peut aussi 1 ire dans 1 'intitulé d'un paragraphe Apud Iuliamum $l i b$. Digest. Marcellus notat, ou bien Labeo lib. Fithanon a Paulo Epitomatoriom, ou bien encore Apud Scaevolam lib. Digest. Claudius notat, etc. Il s'agit de cas analogues.

21- Les difficultés surgissaient tout d'abord a propos de 1'appellation Antoninu6. Selon H.G. PFLAUM, Colloque CNRS sur les empereurs romains d'Espagne, Paris (1965), p. 717-736, les titulatures Imp. Antoninus Aug. et Antonime Imp. s'appliquent en principe à Antonin le pieux. Le prohleme senble plus delicat pour le discours des juristes qu'en matière d' cpigraphie. En plus delicat pour le discours des juristes qu'ente En effet, ladesignation Antonin le pieux, Marc-Aurele et Caracalla. Il n'y a pas dobstacle pour les contemporains d'Antonin le pieux. En revanche, on bute sur les juristes contemporains ou postérieurs à Caracalla. Il est vrai que ceux-ci lui attribuent quelquefois un nom distinctif (Magnus Antoninus, Magnus princeps, Antoninus Aug. noster, ou encore Imp. noster), mais en 1 'absence de cette indication, le problème reste posé. Ainsi, Antoninus seul désigne Caracalla chez Paul D 40.9.15 pr (cf. PIR ${ }^{2}$ (=Prosopographia Imperii Romani, 2ème éd. par GROAG, STEIN et PETERSEN, Berlin, depuis 1933) C 260), ou chez U1pien D 50.2.3.1 (cf. C.I. 10.61.1) et D 26.1.3.1 (cf. C.I. 5.70.1). En outre, divus Antoninus peut viser non pas Antonin le pieux mais Caracalla. C'est notamment le cas chez Marcien (D 48.21.3.4, cf. C.I. 9.50.1), chez Macer (D 49.13.1.1 ; D 49. 16.3.1) et chez Hodestin (D $27.1 .4 \mathrm{pr}-1$; D $27.1 .6 .9 \ldots$ ). En dehors de ce 16.3 .1 ) et chez Modestin (D $27.1 .4 \mathrm{pr}^{-1}$; D $27.1 .6 .9 \ldots$ ). En dehors de ce
cas 1 imite (et en 1 'absence d'autre indication), on a présumé Antcnin le cas limite (et en 1 'absence d'autre indication), on a présumé Antcnin le
pieux lorsque le texte donnait divus, ou encore T.Antoninus (cf. titulature de cet empereur). On a opté pour Marc-Aurèle lorsqu'on avait Antoninus et Verus. On a enfin choisi Caracalla dans les autres cas. Ainsi ont été attribués à Antonin le pieux par la désignation Títus Antoninus : Julien D 4.2. 
nouvesu. Le droit impérial ne se confond pas en effet avec la jurisprudence. C'est un droit ponctuel. Ainsi, il ne suffisait pas d'envisager

18 ; Callistrate D 4.4.45.1; Papinien D 12.6.3; D 36.1.57.1 ; D 36.3. 5.3 ; D 50.1 .11 pr. De même, par la désignation divus Antoninus, Paul D 1.15.3.2 (cf. PIR2 E 96, Erucius Clamus) ; Gaius D 1.6.1.2 ; Marcellus D 4.1.7 pr (cf. PIR' (=Prosopographia Imperii Romani, lère Ed. par DESSAU, KLEBS et ROHDEN, Berlin 1897), M 159, Marcius Avitus); Ulpien D 19 2.15 .5 ; Gaius D 31.56 ; D 32.96 ; Paul D 35.2.1.14 ; Gaius D 35.1 .90 ; D 36.1.65.5; Paul D 47.9.4.1 ; Ulpien D 48.18.1.5 (divus Antoninus et divus Badmionus). Pomponius D 50.12.14. Paul D 50.15.8.5. Enfin, par la simple appellation Antoninus il s agit ici, horais un cas (D 49.14 .

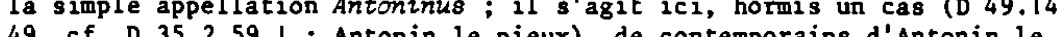
49. CI. D 35.2 .59 : Antonin le pieux), de contemporains d Antonin le pieux : Maecianus D 14.2.9 (cf. Eudaemon, PIR E 79) ; Gaius D 24.1 .42 Mauricianus D 31.57 (on a Foustina Augusta) et D 33.2 .23 ; Marcien D 36 . 1.31 .5 (Traionus et Hadrionus et Antuninus) ; Marcellus D 37.8 .3 ; Pomponius D 40.7.21.1. Ont été dédiés à Marc-Aurèle par 1 'association Antoninus et Verus D 2.14.37; D 8.2.14; D 8.3.17; D 18.1.71; D 39.4 .7 $\mathrm{Pr}-1$; D 42.1 .35 ; D 42.5.30; D 42.7.4; D 48.12.3 pr; D 48.16.18 pr; D 49.1.21 pr ; D 50.1.38 pr-2-6; D 50.2.13 pr ; D 50.8.11 pr; D 50.8. $12 \mathrm{pr}$; D 50.8.13; D 50.12.13 pr, tous de P.Iustus; D 50.1.24 de Scaevola. De plus, Antóninus a été présumé désigner Marc-Aurèle chez P. Iustus D 2.14.60. (ef. Avidius Cassius, SHA, Avid. 1,2), comme Antoninus Caesơn chez Marcellus D 28.4.3 pr-1. La désignation Antoninus seul par des contemporains de Caracalia nous a poussé à attribuer à cet empereur D 1.5.17 Ulpien; D 1.9.12 pr U1pien; D 8.4.2 U1pien; D 19.2.15.6 U1pien; D 22.1.6 pr Papinien; D 24.1.41 L.Rufinus ; D 24.3.2.2 Ulpien; D 26.1.3.1 (cf. C.I. 5.70.1) Ulpien ; D 27.1.6.2 Modestin ; D $31.70 \mathrm{pr}$ Papinien ; D 36.4.5.16 Ulpien ; D 40.9.15 pr (cf. PIR ${ }^{2}$ C 260, Calpurmius Crito) Paul ; D 40.12.34 Ulpien; D 40.7.34.1 Papinien ; D 40.5.31.4 Paul ; D 43.4.3.1 Ulpien; D 47.12.3.3 Ulpien; D 48.19.8.12 Ulpien; D 48.22.16 Marcien ; D 48.19.43 pr-1 Paul; D 50.2.3.1 (cf. C.I. 10.61.1) Ulpien. On a par ailleurs choisi Caracalla pour ditrs Antoninus dans D 49.1.4.1 Macer et D 50.12.2.1 Modestin car, chez ces auteurs, Antonin le pieux ressort toujours distinctement (cf. chez Macer D 49.16.13.6 ; chez Modestin D 27.1.6.7-8-10-19 ; D 42.1.20; D 48.8.11 pr ; D 48.19. 22 ; D $50.4 .11 \mathrm{pr})$.La deuxième difficulté provenait de la seule dénomination imperator ou princeps, le problème résidant dans le fait que de nombreux juristes ont été en activité sous plusieurs empereurs. On a néanmoins tenté, dans la mesure du possible, d'identifier ces empereurs. Pour Ulpien, la chose est aisée. Il s'agit de Caracalla (cf. D 38.16.1. 1 ; D 49.5.5.3 ; D 50.12.6.1 Imp. noster Antoninus Augustus, ou encore D 40.5.30.15 Antoninus noster. On relève aussi dans de nombreux cas "notre empereur et son père", par ex. D 12.3.4.1; D 23.2.45 pr; D 24.1.7. 5-6 : D 26.10.7.2 etc. On peut enfin lire imperatoris nostri et divi Severi). Ont ainsi été dédiés a Caracalla les textes suivants d'ulpien : D 3.6.1.3; D 11.7.14.7; D 12.5.2.2; D 13.7.11.6; D 16.1.4 pr; D 29. le paragraphe tel quel, mais il convensit de privilégier son contenu pour mieux coller à la réalité. La constituti in impériale a donc été prise ici camme nouvelle unité de contexte (22).

2.6.3; D 32.1.9; D 32.11.19; D 36.1.3.4; D 42.1.15.4). On a opéré de même pour Paul en fonction de $D 40.8 .7$ ("notre empereur et son père") et de $\mathrm{D}$ 50.15.8.6 (Imperator noster Antininus). On a donc attribué a Caracalla D 14.5 .8 ; D 16.2.24;D $4.4 .38 \mathrm{pr}$; D 17.2 .25 ; D 26.528 ; D 32 $27.2 ; \mathrm{D} 32.97 ; \mathrm{D} 36.1 .76 \mathrm{pr} ; \mathrm{D} 40.5 .38 ; \mathrm{D} 48.18 .20$. On a aussi opté pour Caracalla, pour Caracalla, a propos de Tryphoninus 0 (imperatore nrstro et divo Severo). Pour Papinien chez Tryphoninus (D 20.5.12 pr), on a choisi Sêvêre (rescriptum est ab imperatore libellos agente Papiniono, v. KUNKEL, o. c., p. 224) ; idem pour Papinien D 35.1 .72 .1 (cf. D 22.1.6.1). On a pris optimi maximique principes nostri (Papinien D 34.9.16.1) pour Sévère et Caracalla. En revanche, princeps (chez Papinien D 40,4.47 pr) vise Hadrien (cf. F.GROSSO, in Homm.Renard, II Latomus 1969, P. 307 a. 2). On a attribué divi principes (chez Marcien D 48.17.3) a Sévère et Caracalla. On a choisi Antonin le pieux pour Romponius D 32.85 et Gaius D 30.73 .1 ; et Trajan pour 1 a désignation Germanicus ehez Menander D 49.16.4.13 (cf. P.PETIT, Histoire générale de 1 'Empire romain, Paris (1974), p. 204). Selon E.M.SCHTAERMAN, La schiavitū, cit., p. 244, D 40.5.12.2 (Antoninus et Pertinax) viserait Sévère et $\mathrm{Ca}$ racalla.Cela dit, certains textes demeurent difficilement classables : Julien D 1.18 .8 (Caesar noster) : Marcien D 1.22 .2 (decreto principali) ; Atilicinus chez Paul D 8.3.35 (Caesarem) (selon T. HONORE," "Imperial" rescripts, cit., p. 52 n. 10 , il s'agirait de Claude ou de Neron et non d'Auguste, Atilicinus stant Proculi nequalie, cf. pal. I, p. 71-2 n. 1); Celsus D 22.3.13 (Caesar noster); Marcellus D 34.9 .6 (principe); Marcien D 37.14.5.1 (imperatoris nostri); ces juristes étant en activitê sous plusieurs règnes, de tels textes a'ont pu être attribués à un té sous plusieurs règnes, de tels textes a'ont pu être attribués à un empereur précis, mais ils ont quelquefois pu être pris en considération
pour le décompte global de la période, pourvu que le juriste ne chevauche pas la période en question.

22- Prendre ici le paragraphe comme unitê de contexte n'aurait pas correspondu à la réalité. En effet, une même constitution impériale peut apparaitre plusieurs fois dans un même fragment (ex. D $1.12 .1 \mathrm{pr}-4$ Ulpien, où il $s$ 'agit de la même epistula Severi), ou plus gênéralement dans un même titre (D 27.1.10.4-6 et $D$ 27.1.2.4-6 Ulpien, où il s'agit de la même constitution de Sévère). Le droit impérial étant ponctuel par rapport a la jurisprudence, il aurait été absurde de compter pour plusieurs références des paragraphes traitant d'un même acte juridique dans un même contexte; dans un même contexte car, au-delà du titre, le contexte juridique. n'étant plus le même, l'acte impérial devait être chiffré une nouvelle. fois. D'autre part, en fonction de cette attitude qui privilégie l'acte. fois. D'autre part, en fonction de cette attitude qui privilégie 1 acte
Juridique (le contenu) sur le peragraphe, il fallait compter pour plusieurs Juridique (le contenu) sur le peragraphe, il fallait compter pour plusi
références les actes juridiques distincts citês dans un mêtme paragraphe (ainsi deux rescrits de Trajan chez Ulpien $D 48.19 .5 \mathrm{pr}$; deux rescrits d'Antonin le pieux chez Ulpien 1 19.2.15.5). Ce nouveau critère explique les totaux des tableaux que $l^{\prime}$ on trouvera en annexe sur les renvois 3 la législation impériale. Ainsi, pour le livre $I$, on a pour Sévèré un total 
En đernier lieu, iI faut signaier que les compilateurs ont sans doute fait disparaître, dans certains textes, la référence à I'esclavage (23). Les chiffres proposés représentent donc un minimum. Du point de vue quantitatif, on peut observer de manière générale, en ce qui concerne les interpolations, que, dans de nombreux cas, même si elles sont probables et susceptibles de modifier le sens du texte, il n'en demeure pas moins que celui-ci se réfère à l'esclavage.

Ces éclaircissements méthodologiques étant apportés, il est désormais possible d'en exposer les fruits.

de $1 / 4$ car D 1.12 .1 pr-4 se réfèrent à la même epistula. Pour le Livre 19, on a sous Antonin le pieux un total de $0 / 2$ car D 19.2.15.5 contient deux rescrits de cet empereur. Pour le livre 26, on a pour Sévère et Caracalla un total de $1 / 8$ car D 26.6 .2 .6 et D 26.6 .4 concernent 1 a même constitution. Pour le Livre 27, on a pour Sévère un total de $3 / 6$ car D 27. 1.2.4-6 et D 27.J.10.4-6 visent 1a même constitution ; dans ce même livre, on a pour Sévère et Caracalla un total de I/9 car D 27.1.2.8-9 ; D 27.1.4 pr-1 ; D 27.1.6.9-11 ; D 27.1.8.10 ; D 27.1.13 pr-5-6-7-10-12 et D 27.1 .15 pr concernent la même constitution. Pour le Livre 29 , on a pour Trajan un total de $2 / 3$ car D 29.5.10.1 et D 29.5.11 se réfèrent à pour Traj. même constitution, et pour hadrien un total de $1 / 5$ car 11 s agit du même rescrit dans D 29.5 .1 .28-29. Pour le tivre 31 , on a un total de $0 / 1$ pour Alexandre-Severe car il s agit du meme rescrit dans $D$ 31.87.3-4. Pour le Livre 36, on a un total de $0 / 3$ pour Caracalla car c'est le même rescrit qui apparaît dans $\mathrm{D} 36.4 .5 .16$ et $\mathrm{s}$. Pour le Livre 40, on a un total de 13/13 pour Antonin le pieux car D 40.5.26.2-4 visent le même rescrit ; un total de $16 / 16$ pour Marc-Aurèle car $D 40.12 .38 \mathrm{pr}-1$ concernent la même constitution, de mêne qu'il s'agit d'une seule constitution dans $D 40.5$. 2 ; D 40.5.3 et D 40.5.4 pr a 23 ; un total de 6/6 pour Marc-Aurè le et Verus car D $40.1 .4 \mathrm{pr}$ à 14 et $\mathrm{D} 40.1 .5 \mathrm{pr}$ se réfèrent à la trême constitution; un total de $7 / 7$ pour Sévère et Caracalla car c'est la même constitution qui tal de qui ressort de $D$ 40.5.26.3 4 . Pour le Livre 46, on a un total de $0 / 1$ pour Hadrien car $D$ 46.1.26 et D 46.1 .27 .1 concernent la meme constitution. Pour le Livre 48, on a un total de $1 / 10$ pour Trajan car on a deux rescrits dan D $48.19 .5 \mathrm{pr}$. Enfin, pour le Livre 49 , on a un total de $1 / 4$ pour Trajan car toutes les citations de D 49.14 concernent le beneficium Traiani, et par aịlleurs D 49.16.4 pr-5 concernent le même rescrit. Quant aux orationes principium, elles n'ont pas été comptabilisées parce que d'une part apparaissant de manière trop diffuse (pratique régulière seulement à partir d'Hadrien, cf. P. PETIT, yistoire générale de 1 'Erapire romain, cit., p. 192-193), et d'autre part en nombre insuffisant pour avoir une valeur chronologique représentative.

23- Ainsi D 46.1.16.4 où eormm se référait à l'esclavage selon J. MACQUERON, Les obligations en droit romain, Aix-en-Provence (1975), p. 351
SECTION 2. - LES RESULTATS OBTENUS

On a justifié en introduction l'utilisation des juristes en tant qu'"éléments interchangeables" à I'intérieur d'une période donnée. Néanmoins, les proportions concernant chacun d'eux seront fournies pour expliciter les chiffres obtenus pour les périodes qu'on a distinguées de la manière suivante (24):

I - La République.

II - La phase de consolidation de l'Empire (27 av.JC-96 ap.JC).

III - L'apogée de l'Empire, ou les premiers Antonins (97-161).

IV - Les prodromes de la crise, ou les derniers Antonins (161-193).

v - La crise.

Les juristes ont alors été intégrés dans ces périodes en fonction de la chronologie donnée par W. KUNKEL (25).

Avant d'exprimer les conclusions permises par cette classification, il est nécessaire d'exposer le détail des tableaux.

Paragraphe 1: Les tableawx (26).

Il convient de reprendre la distinction auparavant annoncée entre analyse "directe" des paragraphes et analyse des citations par renvoi.

24- Sur cette division chronologique, v. P. PETIT, Histoire générale de 1'Empire romain, Paris (1974).

25- Herkunft und soziale Stellung, cit. Cependant, Maecianus et Venuleius ont été classés sous le règne de Marc-Aurèle, ces juristes écrivant encore à cette époque. Quant a Gaius, il n'est probablement pas mort avant le règne de Commode (cf. A.GuARINo, storia del diritto romano, cit. p. $478 \mathrm{n}^{\circ}$ 228), mais il est surtout représentatif du règne d'Antonin le pieux sous lequel il a donc été classé.

26- Le lecteur trouvera un décompte par livres de ces tableaux, avec indication des références justifiant les chiffres ici avancós, en annexe. Il s'agit d'un détail de chacune des analyses entreprises : analyse des paragraphes et analyse des citations par renvoi à des juristes et a des constitutions impériales. 


\section{I - L'analyse des paragraphes.}

juristes

paragraphe concernant

nombre de paragraphes

$$
\text { total }
$$

\section{Période I}

\section{Q.Mucius}

Aelius Gallus

Total

\section{Période II}

Proculus

Total

\section{Iavolenus}

Celsus filius

A.Valens

Africain

Meuricianus

T.Clemens

Gaius

Total

Période IV

Mecianus

Venuleius (27)

Marcellus

P.Iustus

Florentinus

Scaevola

T. Paternus

Total

Période V

Papinien

Menander
Tryphoninus (28)
Période III

Julien

$\begin{array}{ll}0 & 7 \\ 0 & 2 \\ 0 & 9\end{array}$

47

77
136
218
43
62
634
2
1172

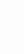

27- Pal. II, p. 1207, Q.Claudius Venuleius Saturninus, n.1 : Venulei Saturnini, cloudii Saturnini, Q. Satumini nomina quin ad unum eundemque spectent iuris consultum, non dubito. Ainsi, Quintus Saturninus (D 12.2.13.5; D 34.2.19.7; cf. Pal. II, p. 1224 Fr.77) et Saturninus (D 47.14.1.4, cf. Pal. II, p. 1224 ; p. $1216 \mathrm{Fr} .43$; et D 48.3.14.7, cf. Pal.II, p. $1224 \mathrm{Fr} .78$ ) visent le même juriste. 28- Les notes de Claudius ad Scaevolam sont attribuables a Tryphoninus (cf. Pal. II, p. 35I-2), et ont étê chiffrées dans l'analyse diøecte en ce qui concerne cet auteur, dans 1 'analyse des citations en ce qui concerne Q.Cervidius Scaevola (cf. supra n.20)

\section{Callistrate}

Tertullien

Paul

Upien

L. Rufinus

Macer

Marcien

Modestin

F.Anthianus

R.Maximus

Hermogénien

A.Charisius

Total

45
0
972
2140
7
17
166
127
2
0
1
41
7

223
10
3995
8983
18
129
618
615
2
3
1
163
49

3840

16192

II - L'analyse des citations par renvoi.

A/ - A des juristes.

$\begin{array}{lll}\text { juristes } & \text { citations } & \text { nombre } \\ \text { concernant } & \text { total de } \\ \text { l'esclavage } & \text { citations }\end{array}$

Période I

Sex. Aelius

M.Porcius Cato (29)

Manilius

Brutus (30)

P.Mucius

Drusus

Rutilius

Q.Mucius

Aelius Gallus

Gallus

Servius

Cascellius

Trebatius

Alfenus (31)

ofilius

A.Namusa (32)
$20 \%$
29- La rêférence a Catoniona regula in 034.7 .1 pr a été intégrée en fonction de Pal. I, p. 126 Fr. 5.

30- On a intégré un renvoi a Brutus, inapparent dans le texte, in D 9.2.27.23, selon Pal. I, p. 77 Fr. 5

31- Alfenus a entièrement été pris dans l'analyse des citations, et non dans 1 'analyse directe, car les textes de ce juriste qui $n$ 'ont pas été repris par Paul 1 'ont été par un auteur anonyme (lib. Digest. $a b$ anonymo epitomata, cf. Pal. I, p. $37-45)$.

32- Lorsque le texte ne donne que Aufidius, il s'agit d'Aufidius Namusa (cf. Pal. I, p. 75-76), le disciple de Servius (Servii auditor, D 1.2.2.44, cf. Pal, I, 
Période III

Iavolenus (42)

V. Lucullus

Aristo

Neratius (43)

U. Ferox

Vivianus (44)

Celsus filius (45)

Ḟerva pater (36)

Minicius

Campanus

Cartilius

Proculus

Atilicinus

Nerva filius
Cassius (37)

Caelius Sabinus

Pegasus

Plautius (38)

Fufidius

Fulcinius (39)

A. Yalens

Y. Severus

P.Clemens

Julien (46)

v.Verus (47)

Pedius

L.Felix

Pomponius

Africain (48)

p. 75 n. 1): C'est en vertu de cette qualité que LENEL le prend comme objet de trois renvois renvois d'où son nom ne ressort pas expressément (D 33.4.6.1 Iavolenus, cf. Pal. I, p. 75 Fr. 4 ; D 33.7.12.6 Ulpien, cf. Pal. I, p. 76 Fr. 5 ; D 39.3.1.6 Ulpien, cf. Pal. I, p. 76 Fr. 7).

33- La dénomination Ateius désigne le juriste de 1 'époque rêpublicaine C.Ateius, disciple de Servius, et non C.Ateius Capito, de l'époque d'Auguste (cF. Pal. I, p. $71 \mathrm{n}, 1 ; \mathrm{D} 1.2 .2 .44$; JORS, RE II, $1902 \mathrm{n}, 13$; KUNREL, O.c, P. 31 n.65). Ouant a l'appellation Antaeus, elle vise aussi ce juriste (v. D $39.3 .14 \mathrm{pr}$, cf. Qal. I, p. 55 a. 1).

34- Veteres vise les juristes de la période jurisprudentielle pré-classique, c'est-à-dire républicaine (cf. A. GUARINo, Storia del diritto romano, cit. P. $300 n^{\circ} 148$ ).

35- On a pris un renvoi d'Ulpien à Sabinus in D 9.2.27.11, sans que le aom de Sabinus apparaisse, en vertu de Pal. II, p. 194 Fr. 52 (cf. Coll 12,7,9).

36- Sur la distinction des textes a attribuer a Nerva pater et a Nerva filius, v. Pal. I, p. 787-790 et 791-792.

37- Dans D 24.3.59 Julien (cf. Pal. I, P. 116 Fr. 65), D 35.1 .54 pr Iavolenus (cf, Pal. I, p. $110 \mathrm{Fr} .9$ ) et D 46.3 .78 Iavolenus (cf. Pal. I, p. 110 Fr. 11). 1 'appellation Gaius vise en réalité Cassius.

38- Renvoi àplautius intégré en vertu de Pal. II, p. 13 Fr. 3 n. 3, opéré par Paul in D 20.4.13, bien que le nom de Plautius ne ressorte pas de ce texte, (quae sequintur esse Ploutii verba apparet ex nota a Paulo subiecta).

39- Idem pour un renvoi a Fulcinius, chez Ulpien D 25.1.3 pr (cf. Pal. I, p. 179 Fr. 4).

40- Sur la distinction des textes a attribuer a Celsus pater et a Celsus filius, v. Pal. I, p. 127-128 et 127-170.

41- Sur I'opposition entre Sabiniani ou Cassiani d'une part, et Proculiani de 1 'autre, v. A. GUARINO, Storia del diritto romano, cit. p. $458 \mathrm{~s}$. L'appellation Cassiani vieat du fait que Cassius, consul suffect en 30 , devenu après Sabinus le chef de l'école sabinienne, jouìt d'une telle réputation que les juristes se rattachant a cette école furent désormais désignés par un nôn tiré du sien (cf. P. KRUGER, Histoire des sources du droit rovain, Paris (1894) P. 205).

42- Il faut prendre Iavolenus pour Aulus in $D^{\circ} 28.5 .1 .17 .5$ (cf. Pal. II, p. 1229 Fr. 162 ; Pal. I, p. 300 n. 2).

43- Priscus in D 35.1.112.3 vise Neratius (cf. Pal. I, p. 784 ; p. 777 Fr. 98 n. 3).

44- Renvoi a Yivianus par Ulpien D 9.2.27.10, en vertu de Pal. II, p. 1225 Fr. 1 (ef. Coll. 12,7,8).

45- Iuventius chez Ulpien D 7.8.12.1 vise Celsus filius (cf. Pal. I, p. 152 Fr. 156).

46- Sur Africanus lib. Quaestionum, v. Pal. I, P. 2 n. 1, le renvoi a Julien étant explicite dans certains textes (ex. D 12.1.23; D 16.1.19.1), cette hypothèse n'a pas été comptabilisée lorsque le renvoi n'était pas clairement effectué. 47- Renyoi a vindius verus par vlpien in D 2.14.7.17 intégré en vertu de Pal. II, p. 1223 Fr. 2.

48- La confusion est possible sur 1'appellation Sextus Caecilius, cf. Pal. I, p. 35 n. 3 : non dubito quin Caecilius, qui saepius laudatur, Sex. Caecilius Africarus sit; exceptis tamen his Locis : D 33.9.3.9, ubi pro Caecilius leg. Aelius (v. Sex. Aelius Fr. 2 Pal. I, p. 1); D 21.1.14.10, ubi leg. Caelius (Sabinus) : D 15.2.1.7, D 24.1 .64 ubi pariter leg. Caelius (Sabinus), nisi altern fuisse malueris Caecilium iuris consultum Pegasi fortasse aequalem, ceterum ignotum, (cf. 0. RARLOWA, Römi sche Rechtsgeschichte I, Leipzig (1885), p. 711). Sextus iuris consultus tribus locis loudatus, D 29.5.1.27; D $30.32 \mathrm{pr}$; Gaius II , 
Total

Période IV

Maecianus (50)

Venuleius

Marcellus

Scaevola (51)

T.Paternis

Total

Période V

Papinien

Messius

Tryphoninus

Menander

P.Fronto

Tertulien

Arrianus

Puteolanus

Paul

Ulpien

L. Rufinus

Marcien

Modestin

Total

4
1
56
34
0

$\begin{array}{rr}20 & 20 \% \\ 5 & 20 \% \\ 231 & 29 \% \\ 79 & 43 \% \\ 1 & - \\ 336 & 31 \%\end{array}$

105

336

$31 \%$

151

$10 \%$

218, an et ipse intellegi debeat Sex. Caecilius Africanus, in incerto est, v. Th. MOMMSEN, Zeitschrift für Rechtgeschiellte, 7, 479). Ainsi, Sextus Caecilius désigne Sex. Aelius in D 33.9.3.9 ; Caecilius désigne Caelius Sabinus in D 15.2.1.7 (Pal. I, P. 81 Fr. 11), D 21.1 .14 .10 (Pal. I, p. 79 Fr. 2), D 24.1 .64 (Pal. I, p. 81-2 Fr. 12) ; Sextus Caecilius désigne Africain in D 24.1 .2 ; D 35.1.71 pr ; D $35,2.36 .4$; D 48,5.28.5. Quant à 1 a dénomination Sextus, il n'est pas possible de l'attribuer un juriste pré (Pal. Il P. 1229 n. 2 : verisimile pos Sextum illum, cui Celsum respondise Ulpionus re fert (D 42.4 .7 .17$)$, fuise eundem twn illwn, cui celsum respondisse ulpianus refert (D 42.4 .7 .17 ), fuisse eundem qui laudatur in fragmentis supra repetitis: quare nec de Sexto Pedio nec facile de Sexto Caecilio Africano Iulioni discipulo cugitaverim. Certe non esse intellegendum Sextum Pomponizm apparet (D $30.32 \mathrm{pr}$ ). L'incertitude pèse sur $D$ 29.5.1.27 et $D 30,32 \mathrm{pr}$.

49- In D 45.3.39, il s'agit bien de Gaius, cité par son contemporain Pomponius (cf. Pal. I, p. 25 i Fr. 482 n. 1 ; p. 266), contrairement a D 24.3.59; D 35.1 . $54 \mathrm{pr}$ et $\mathrm{D} 46.3 .78$ où Gaius est mis pour Cassius (cf. supra n. 37).

50- Renvois à Maecianus par Ulpien D 36.1.15.7-8 intégrés en vertu de Pal. I, p. $581 \mathrm{Fr} .32$.

51- In D 4.3.7.7 Ulpien, Quintus vise Q.Cervidius Scaevola (cf. Pal. II, P. $1229 \mathrm{n}$. 1). Pour distinguer ce juriste de Q.Mucius Scaevola, on 1'a simplement désigné par Scaevola, l'appellation Q.Mucius vísant Q.Mucius Scaevola.

\section{B/ -A des constitutions impériales.}

\section{Période II}

\section{Auguste}

Tibère

Vespasien

Titis

Domitien

Total

$\begin{array}{rr}2 & 12 \\ 1 & 2 \\ 4 & 7 \\ 1 & 5 \\ 0 & 4 \\ 2 & 3\end{array}$

\section{Période III}

\section{Nerva}

\section{Trajan}

Iladrien

Antonin ls pieux

Total

\section{Fáriode IV}

\section{Marc-Aurèle}

Marc-Aurèle et Verus

Marc-Aurèle et Cormode

Cormode

Pertinax

Total

$\begin{array}{rr}2 & 4 \\ 8 & 29 \\ 40 & 135 \\ 70 & 240 \\ 120 & 408\end{array}$

$50 \%$

Période V

\section{Sévère}

Sévère et Caracalla

Caracalla

Alexandre-Sévère

Total

$\begin{array}{rr}35 & 85 \\ 22 & 124 \\ 3 & 13 \\ 3 & 8 \\ 1 & 3\end{array}$

Il faut d'ores et déjà faire quelques remarques. La première concerne les renvois à la législation impériale. Les totaux des périodes II, III et $V$ seront sensiblement modifiés dans le tableau récapitulatif car des renvois manquant de précision et ne pouvant être attribués à un empereur sont intégrables dans le cadre plus général d'une période (52). En second lieu, les pourcentages livrés au lecteur sont arrondis à l'unité supérieure dès que $l$ 'on dépasse le niveau de $50 \%$ au-delà de la virgule (53). Enfin, on

52- Il s'agit de D 8.3.35 Atilicinus chez Paul (période II); de D 22.3. 13 Celsus (période III) ; de D 1.22.2 et 0 37.14.5.1 Marcien (période V). Les totaux sont ainsi modifiés : $10 / 34$ (période II); $120 / 409$ (période III) et $84 / 323$ (période V). Par contre, il n'a pas été possible d'intégrer D II. 18.8 Julien et D 34.9.6 Marcellus, ces juristes chevauchant les périodes III et IV.

53- Par exemple, Pomponius : $25,31 \%$ ramené à $25 \%$; Venuleius : $23,52 \%$ arrondi à $24 \%$, ces deux cas étant extraits de l'analyse directe des paragraphes. 
pe-t noter, de manière globale, que dans chacune de ces analyses (analyse directe des paragraphes et analyse des citations par renvoi à des juristes et à la législation impériale), on relève une proportion de textes concernant 1 ' esclavage de I'ordre de $1 / 4$ par rapport au nombre total de textes (54), ce qui montre objectivement à quel point l'esclavage occupe une place importante dans la société romaine.

\section{Paragraphe 2 : Les conclusions.}

On peut considérer le discours des juristes suivant un double point de vue, soit qu'il s'agisse d'analyser son évolution par rapport à la conjoncture, soit qu'on veuille rechercher un aspect structurel de ce discours en matière d'esclavage.

\section{I - Le discours des juristes en matière d'esclavage}

Reprenons les résultats obtenus par les précédents tableaux.

-Analyse des paragraphes

$\begin{array}{ccc}\text { paragraphes } & \begin{array}{c}\text { nombre total } \\ \text { de }\end{array} & \\ \text { concernant } & \% \\ \text { I'esclavage } & \text { paragraphes } & \\ 0 & 9 & - \\ 6 & 47 & 13 \% \\ 985 & 3581 & 28 \% \\ 354 & 1172 & 30 \% \\ 3840 & 16192 & 24 \%\end{array}$

-Analyse des citations par renvoi -à des juristes

$\begin{array}{lrrr}\text { I } & 128 & 525 & 24 \% \\ \text { II } & 347 & 1356 & 26 \% \\ \text { III } & 519 & 1712 & 30 \% \\ \text { IV } & 105 & 336 & 31 \% \\ \text { V } & 35 & 207 & 17 \% \\ \text {-à des constitutions } & \text { impériales } & & \\ \text { II } & 10 & 34 & 29 \% \\ \text { III } & 120 & 409 & 29 \% \\ \text { IV } & 64 & 233 & 27 \% \\ \text { V } & 84 & 323 & 26 \%\end{array}$

54- Pour 1'analyse directe, on a 5185 paragraphes concernant 1'esclavage sur un total de 21001 (25\%). Pour l'analyse des citations par renvoi a des juristes, on obtient 1134 citations concernant 1 'esclavage par rapport à un nombre total de citations de 4136 (27\%); si on ajoute a ces chiffres les auteurs inclassables dans une période (Sextus, $\mathrm{cf}$. supra $\mathrm{n}$. 48; Paconius et Servilius, cf. supra $n$. 1), on parvient à un total de $1135 / 4141$
Il faut tout d'abord faire des observations d'ordre général. En premier lieu, on notera l'importance numérique des textes sur lesquels se fonde cette étude. On dénombre en effet, répétons-le, 5185 textes visant esclave et affranchi dans le Digeste, ce qui justifie amplement I'intervention d'un instrument d'analyse statistique, instrument qu'on n'a utilisé qu'à un niveau élémentaire, descriptif.

En second lieu, on remarquera la sur-représentation de la période $v$ dans l'analyse des paragraphes. Cela entraîne une double objection. D'une part, il est vrai que les compilateurs ont donné une place importante, du point de vue numérique, aux textes des juristes du 3ème siècle, notamment à ceux de Paul et Ulpien. Cependant, le déséquilibre occasionné par ce choix $n$ ' est que relatif. En effet, il ne s'agit pas d'examiner les textes concernant l'esclavage du point de vue de leur importance numérique, mais de dégager une proportion de ces textes par rapport au nombre total de ceux qui sont relatifs à une période donnée. D'autre part, une observation superficielle pourrait amener à objecter que seul le pourcentage obtenu pour la période $V$ est pertinent, les périodes III et IV n'ayant respectivement qu'une valeur représentative de $22 \%$ et $7 \%$, du point de vue de leur importance numérique, par rapport à la période $V$. Ce n'est plus vrai si l'on approfondit l'analyse, la suite de l'étude le montrera. L'échantillonnage est pertinent, malgré les différences numériques, car Ia ventilation des thèmes, à l'intérieur de chaque période, est similaire.

En troisième lieu, on relèvera la faiblesse numérique flagrante des deux premières périodes dans $l$ 'analyse des paragraphes. De plus, pour la période II, les textes sont tous de Proculus. On voit ici epparaître la nécessité de 1 'analyse des citations par renvoi. Les périodes I et II sont correctement représentées dans cette analyse. Ce n'est pas le cas de la période $\mathrm{V}$, les juristes du 3ème siècle étant les derniers à avoir été retenus par les compilateurs (55). Ces deux analyses sont donc étroitement

(pourcentage inchangé de 27\%). Enfin, pour l'analyse des citations par renvoi a des constitutions imperiales, on compte 278 citations concernant I'esclavage sur un total de $999(28 \%)$. Ces chiffres diffèrent sensiblement de ceux donnés dans 1 'Index $8(1978-79)$ p. 283, mais ce travail était alors seulement en "chantier".

55- Sauf deux exceptions, Hermogênien et A. Charisius. 
complémentaires. en d'autre termes, si l'analyse des paragraphes est indispensable pour connatre "la fin de l'histoire", l'analyse des citations par renvoi l'est aussi pour en décourrir le dóout.

Cela dit, on relève la plus rette importance des textes concernant l'esclavage sous les Antonins (périodes III et IV), malgré le choix effectué par les compilateurs. C'est sans doute l'effet de l'intervention impériale dang le sens d'une amélioration du sort des esclaves et d'un adoucissement de leur exploitation (56). A partir des Sévères, la place de l'esclavage décroît (57), Les citations le confirment.

En ce qui concerne les constitutions impérieles, la diminution d'importance de l'esclavage est déjà sensible au cours de la période IV, alors que c'est précisément à cette époque que le discours des juristes réalise les totaux les plus élevés. Il y a donc une distorsion entre ce résultat et ceux relatifs aux seuls juristes, les citations de constitutions semblant refléter de manière plus sensible la crise de l'esclavage, conformément, bien entendu, à l'état de nos connaissances, $c^{\prime}$ est-à-dire la situant dès la deuxième moitié du 2ème siècle (58).

56- V. E.M. SCHTAERMAN, La schiavitù, cit. p. 228 s., selon qui la poli tíque des Antonins en la matière est mieux définie que celle de leurs prédécesseurs : notament, lutte avec plus d'énergie contre l'arbitraire dominical (p. 231) ; consolidation des "droits" des esclaves ordinarii sur le pécule de leurs vicarii (p. 233) ; prise en considération des liens familiaux serviles (p. 234) ; favor libertatis (p. $234 \mathrm{s.}$ )

57- Ceci est attesté aussi bien par $I^{\prime}$ analyse des paragraphes que par celle des citations de juristes. Pour les constitutions impériales, le déclîn quantitatif est antêrieur. En ce qui concerne ces dernières, il faut noter que les Sêvères ge sont maintenus sur la ligne tracêe par leurs prédécesseurs, E.M. SCHTAERMAN, o.c., p. 238 : renforcement des "droits patrimoniaux" de $l^{\prime}$ esclave (p. 242) ; favor libertatis (p. $244 \mathrm{~s}$.). On trouve une proportion de textes concernant 1 'esclavage plus faible pour les empeune proportion de textes concernant 1 'esclavage plus faible pour les empereurs de la période $V$ car le changement, la tentative de redefinition des rapports esclavagistes, s'est probablement effectué auparavant. C'est un point que l'on s'efforcera d'éclaircir par la suite. D'autre part, ce changement d'utilisation de 1 'esclave a posé des problèmes que dêjà les juristes de 1 'époque des Antonins se sont efforcés de résoudre.

58- Sur ce problème, v. notanment E.M. SCHTAERMAN, Die Krise der Sklavenhalterordnung im Westen des röm. Reiches (tr. a!1.), Berlin (1964); La shiavitũ, cit. P. $328 \mathrm{~s}$. ; F. DE MARTINO, Storia della costituzione romana $V$, cit. p. 147 s. ; M. JACOTA, Contributii la studiul crizei generale a sistemului sclavagist in imperial roman, Beiträge zur Eríafrung der allgemeine Krise des Sklavenhalter Systems im röm. Reich, in Studii Clasice 5 (1963), p. 191-210.
S'il est vrai que la législation impériale est la réponse ponctuclle aux exigences sociales les plus aiguës, on ne peut en dire autant dis l'activité jurisprudentielle (59). Le lien entre instances socieles et production jurisprudentielle peut apparaître discontinu. Le fait que is période IV, correspondant aux règnes de Marc-Aurèle et de Commode, atteigne le pourcentage le plus éleve, aussi bien dans l'analyse des paragraphes que dans celle des citations, s'explique-t-il par un retard des juristes par rapport à la pratique? Ou bien cette distorsion n'est-elle qu'apparente ? On manque encore d'éléments pour répondre à cette question, mais rien $n^{\prime}$ interdit de penser qu'une production impériale quantitativenent plus faible peut néanmoins être importante quant à son contenu et donc nécessiter de nombreux développenents jurisprudentiels. En d'autres termes, rien ne prouve que la baisse accusée par les constitutions impériales dans la seconde moitié du 2ème siècle soit forcément incompatible avec l'importance quantitative du discours des juristes de cette époque.

Il est aussi intéressant de tenter de déceler un aspect structurel du discours des juristes en matière d'esclavage.

\section{II - La continuité du discours des juristes en matière d'esclavage}

Les pourcentages concernant chaque juriste ayant été donnés, on se limitera dans ce cadre à opérer des associations par tranches de $10 \%$

59- Bien que l'activité législative impériale ne se confonde pas avec 1 activité jurisprudentielle, il existe entre elles des liens. On les a montrês en introduction. L. LANTELLA, Il lavoro sistematico, cit. p. 72, menant une analyse des formes du discours juridique, remarque que la ju risprudence emploie des formules "neutres" (caractère cognitif) tandis que ha legislation inperiale utilise des foraus "de pression" (car accertaine pression. 

Mauricianus, P. Iustus, T. Paternus, Tertullien, F. Anthianus.

Manilius, P. Mucius, Rutilius, Aelius Gallus, Aquilius Gallus, Ateius, Cinna, Blaesus, v. Severus, L. Felix, Publicius, T. Paternus, Messius, Tryphoninus, Tertullien, Arrianus, Puteolanus ipien, Marcien, Modestin (60). f., Menander, Macer, A.Charisius.

20 à 29\% Iavolenus, Pomponius, Gaius, Venuleius, Florentinus, $\mathrm{Pa}-$ pinien, Callistrate, Paul, Ulpien, Marcien, Modestin, Hermogénien.

Julien, Africain, Marcellus, Scaevola, Tryphoninus, L. Rufinus.

40 ลे $49 \%$ A. Valens, T. Clemens.
10 à 19\% Proculus, Neratius, Celsus
$50 \%$ et au-delà
Q. Mucius, Cascellius, Nerva p., Atilicinus, Fulcinius, Papinien, Paul.

M. Porcius Cato, Britus, Servius, Trebatius, Ofilius, Labéon, Sabinus, Proculus, Cassius, Iavolenus, Aristo, Celsus f., Pomponius, Maecianus, Venuleius, Marcellus, Menander, P. Fronto.

Alfenus, Tubero, Mela, Pegasus, Fufidius, Neratius, Vivianus, Julien, Pedius.

Nerva f., Plautius, Octavenus, v. Verus, Scaevola.

A. Namusa, Caelius Sabinus, Celsus p., U. Ferox, A. Valens, Africain, Mauricianus (62)
Des juristes représentatifs des diverses périodes apparaissent dans presque toutes les tranches. Ce phénomène s'estoupe au-delà d'un certain seuil : $40 \%$ dans 1 'analyse des paragraphes, $30 \%$ dans 1 'analyse des citations. Passé ce seuil, les juristes de l'époque des Antonins prévalent, les considérations chronologiques reprenant le dessus. Le seuil est plus bas pour les citations, les juristes du ßème siècle étant évidemment moins citês que leurs prédécesseurs.

A côté de ces premières constatations, on note que dans une même catégorie peuvent se retrouver Neratius et Macer, ou A. Charisius ; Pomponius, Gaius et Papinien, Paul, Ulpien, Marcien, Modestin, Hermogénien ; et dans les citations, Servius, Labéon, Aristo, Pomponius et Menander.

Outre un retard possible dans la prise en considération par les juristes des changements sociaux, cela n'est-il pas dû au fait que, souvent, ils ne traitent pas de l'esclavage pour trouver une solution à un problème le concernant, mais s'y réfèrent de manière seulement secondaire pour illustrer d'un exemple un cas ne concernant pas nécessairement l'esclavage ? $\nabla$ 'auraient-ils pas tout aussi bien pu se référer $a$ un autre élément patrimonial ? S'ils continuent à faire si grand cas de l'esclavage, ne seraitce que comme exemplum iuris, cela ne signifie-t-il pas que l'institution occupe toujours une place importante du point de vue ideologique?

$$
-\circ-
$$

Contrairement à $l$ 'évolution attestée par les constitutions impériales, l'esclavage connaît donc un regain d'intérêt chez les juristes de l'époque des derniers Antonins, probablement dû à une conception plus subtile de l'exploitation du travail servile. Malgré le déclin quantitatif relatif à la législation impériale, le discours des juristes se caractérise par la continuité, signe sans doute de la persistance de l'idéologie esclavagiste.

Toutefois, il ne s'agit là que d'hypothèses qu'il faut désormais tenter de vérifier à $l^{\prime}$ aide ''une analyse plus précise. Trois étapes, rappelons-le, seront utiles à l'investigation : LA MAIN-D'OEUVRE SERVILE (première partie), LA PLACE DE L'ESCLAVAGE DANS LES PRATIQUES SOCIALES (deuxième partie), et LES JURISTES PORTEURS DE $t$ 'IDEOLOGIE ESCLAVAGISTE (troisième partie).
60- Dans cette catégorie, on trouve aussi Servilius, dont 1 'époque est

61- I. Aquila et R. Maximus devraient apparaître dans cette tranche, mais indiquer dans ce tableau des juristes dont on ne possède respectivement que deux et un seul texte $n$ 'a pas paru opportun.

62- Les juristes qui n' étaient pas cités plus de deux fois ont été écarcullus, Campanus, P. Clemens, Gaius et , Capito, Minicius, Cartilius, V. Lu Sextus (auteur incertain, cf. Pal. II, p. $1229 \mathrm{r.2}$ ) et Paconius (dont l'époque est inconnue, cf. Pal. I, p. 803-4). 
PREMIERE PARTIE

IA MAIN-D'OEUVRE SERVILE 


\section{L'ESCLAVE, ELERENT PATRIMONLAL.}

Economiquement, l'esclave est avant tout un out,il qu'il est ut,ile d'acquérir pour en exploiter le travail. Cependant, sa situation professionnelle peut revêt,ir des formes très diverses, signes de l'hótérogénéité du groupe servile.
D'un point de vue général, l'acquisition de la main d'oeuvre servile peut se présenter sous deux aspects : soit elle nécessite une transaction juridique, soit elle est assurée pour ainsi dire automatiquement par $l_{a}$ reproduction du groupe.

\section{CHAPITRE I : LES FORMES D'ACQUISITION DE LA MAIN-D'OEUVRE SERVILE MOYENNANT} TRANSACTION JRIDIQUE

Fidèle à la démarche annoncée, nous dégagerons une analyse quantitative comparative de ces divers modes d'acquisition avant d'en envisager les réalités.

\section{SECTION 1. ANALYSE COMPARATIVE DES DIVERS MODES D'ACQUISITION}

L'acquigition de la main d'oeuvre servile pose un problème original dans la mesure où la force de travail est ici confondue avec le travailleur (1). Néanmoins, on ne peut limiter cette étude è la seule acquisition de l'esclave. Il existe d'autres formes, dénotent une exploitation par une autre personne que le mâ̂tre, qui tendent à l'utilisation de sa force de travail.

1- F. FAVORY, Présentation de 1' "index thếmatique" de Besançon consacr a l'esclavage et aux formes de dépendance, in Schiavitù, manomissione $e$ classi dipendenti nel mondo antico, L'ERMA di BRETSCHNEIDER, Rome (1979), p. 150. M. I. FINLEY, L'économie antique, Paris (1975), tr. fr., p. 82, note que les juristes romains eurent du mal a franchir les étapes conceptuelles allant de l'acquisition de l'objet a la simple acquisition de sa force de travail. v. J.A.C. THOMAS, Locatio and operae, BIDR. 64 (1961), p. 231-247. 
Différents contrats permettent de s'approprier un esclave.

La vente (3), contrat consensuel, permet à l'acheteur d'obtenir la

2- On n'envisagera ici que les modes d'acquisition de la propriété. Il sera question de la traditio, mode de transfert de la propriété, dans l'étude sur les rapports juridiques. C'est également alors qu'i l sera traité de la copropriété ét de la possession de l'esclave.

3- Traitent de 1a vente dans 1e Digeste, A1fenus (chez auteur anonyme) D 44.1 .14 ; Iavolenus D 9.2.38; D 15.1.33; D 18.6.17; D 21.1.53; D 24 . $1.50 \mathrm{pr}-1$; D 40.12 .42 ; D 41.3 .19 ; D 47.2.75; D 49.15 .27 (Ofilius, Trebatius, Labéon); Neratius D 19.1.31.I ; D 28.5.55 ; Celsus D 19.1.38 pr-1 (Sextus Aelius, Drusus) ; D 40.7 .23 pr ; D 47.2.68.3 ; Julien D 1.5.26; D 5.1.25; D 12.6.37; D 15.1.37.1-2; D 18.2.17; D 18.5.5.2 D D 19.1.24.1 (Sabinus, Cassius); D $21.1 .50 ; \mathrm{D} 21.2 .7 ; \mathrm{D} 21.2 .8 ; \mathrm{D} 21.2 .39 .1-3-4-5$; D 22.1 .25 .2 ; D 28.5.38.2-3-5; D 30.82.4-5; D 30.91.3-5; D 30.92.1; D 30. 94.1 ; D 39.5.2.7; D 39.6.18.3; D 41.1 .39 ; D 41.2 .38 .2 ; D $41.3 .33 \mathrm{pr}$; D 41.4 .7 .7 ; D 41.4 .8 ; D 41.4 .9 ; D $41.4 .40 ;$ D 43.24.14; D 43.33.1 pr D 44.2.25.1; Pomponius D 3.5.10 (Proculus); D 6.1.29; D 13.7.8.1; D 15 1.34 ; D 18.1.8 pr ; D 18.1.13; D 18.1.31; D 19.1 .55 (0ctavenus) ; D 21 . 1.16 ; D 21.1 .36 ; D 21.1 .46 ; D $21.1 .48 \mathrm{pr}-1-2-3-4-5-6-7-8$; D 21.1 .64 Pr (Labéoń)-1 (Labéon)-2 (Labêon); D 21.2.16.2 (Proculus); D 21.2.30; D 21 . $2.34 \mathrm{pr}-1$; D $24.1 .29 \mathrm{pr}$ (Fulcinius); D 24.1.31.3-5-10; D $30.45 \mathrm{pr}$ (OFilius Aristo, veratius) ; D 33.5.6: D $34.2 .10 ; 040.5 .20$ (Julien) (D 40 . lius, Aristo, Netion); D 40 (Trebatius); D 46.3.26 ; D 46.3.83; Africain D 3.5.48; D 12.1.23 (Julien) (Trebatius) ; D 46.3.26 ; D 46.3.83; Africain D 3.5 .48 ; D 12.1 .23 (Julien)

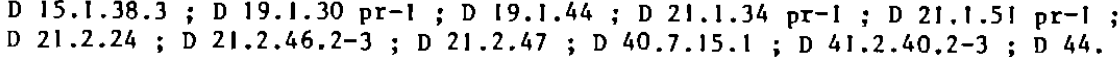
3.6.1 ; D 47.2.62 pr-2-4-5; D 50.16.207 (Mela); Gaius D 15.1.27.4 (Julien) -5 (Ju1ien)-6 (Julien)-7 (Julien) ; D 18.1.35.3; D 21.1.3; D 21.1.13; D $21.1 .18 \mathrm{pr}-1-2$; D 21.1 .20 (Caelius Sabinus); D 21.1 .22 ; D 21.1 .24 ; D 21. 1.26 ; D 21.1.28; D 21.1.32; D 21.1.45; D 21.2.54.1; D 30.70.2; D 35.1 . 17 pr ; D 39.4.13.2 ; D 40.1.18 ; D 41.1.43.2 ; D 47.10.2 ; Maecianus D 29. $4.28 \mathrm{pr}-1$; D 35.1.86 pr (Julien); D 40.4.55.2 ; D 40.5.32.1; Venuleius D 21 . $1.65 \mathrm{pr}-1$ (Cassius)-2 (Caelius Sabinus); D 41.1 .66 ; D 43.29.4.1 (Trebatius); Marcellus D 17.1.49; D 18.7.4 ; D 19.1.23 (Julien); D 28.5.40 (Julien); D 37.15.3 ; D 38.15.5.2 ; D 40.5.10 pr-1-2 ; Florentinus D 18.1.43 $\mathrm{pr}^{-1}$; Scaevola D 3.5.34.2; D 16.1.28 pr;D 21.2.69 pr-1-2-3 (Servius)-4-5; D 24 . 1.58 .2 ; D 28.6.48.2 ; D 36.1.80.12; D 40.5.41.5; D 44.3.14.2-4; Papinien D $17.1 .54 \mathrm{pr}$; D 17.1.57; D 18.7.5 ; D 18.7.6 pr (Sabinus)-1 (Sabinus); D 18.7.7; D 18.7.8; D 21.1.54; D 21.1.55; D 21.2.64 pr; D 21.2.66.2; D 21.2 .67 ; D $22.1 .4 \mathrm{pr}-1$; D 29.7 .12 ; D 31.73; D 38.2.41; D 40.1.19. D 40.1.20.2 ; D 40.4.5I pr ; D 40.5.21; D 40.7.35; D 41.3.44 pr; D 45.1. $118 \mathrm{pr}$; D 47.2.81 pr ; Tryphoninus D 9.4.37; D 18.7 .10 (Scaevola); D 2.50.1-2; D 49.15.12.7-8-9 (veteres)-10-11-12-13-14-16-18; Ca11iotid D D 21.2 .7 .2 ; 40.8 .3 (rescrit Marc-Aurele et Corme) D $4.7 .9 ; 0.4 .41 ; 012.1 .31 .1$ (Sabinus D 14.717 Dr D $14,4.10$; 15.143 (Laben) D 14.3.17 pr ; D 14.4.10; D 15.1 .43 (Labéon); D 15.1.47.3 (Proculus)-4 (Julien)-5-6; D 15.4.2.2 ; D 17.1.5.5; D 17.1.22.9; D 17.1.26.7 (Neratius)-8 Me1a); D 17.2.65.5 (Labéon, Proculus); D 18.1.1.1 (Sabinus); D 18.1.34 $\mathrm{pr}$ (Labéon) -6 ; D $18.1 .56 ; \mathrm{D} 18.4 .21 ; \mathrm{D} 18.6 .8 .1$ (Ju1ien); D 18.7.3 ; D 18

$7.9 ; \mathrm{D} 19.1 .4 \mathrm{pr} ; \mathrm{D} 19.1 .21 \mathrm{pr} ; \mathrm{D} 19.1 .42 ; \mathrm{D} 19.1 .43$ (Julien, U1pien) ;
D $19.1 .45 \mathrm{pr}$ (Julien, Africain)-1-2; D 19.1.54 pr (Labéon); D 19.4.2
(Aristo); D 20.6.10.1; D 2I.1.2; D 21.1.5; D 21.1.11; D 21.1.15; D 21 . $1.30 \mathrm{pr}-1$ (Aristo, Pedius) ; D 21.1.39; D 21.1.43.1-2-3-4-5-6-7-8-9-10; D $21.1 .44 \mathrm{pr}^{-1-2}$; D 21.1.47 pr (Labéon)-1; D 21.1.56 (Marcellus) ; D 21.1 . $57 \mathrm{pr}-1$; D $21.1 .58 \mathrm{pr}^{-1-2}$; D $21.1 .60 ; \mathrm{D} 21.2 .3 ; \mathrm{D} 21.2 .5$ (Labểon); $\mathrm{D} 21$. 2.9 (Sabinus) ; D 21.2.11.1; D 21.2.26; D 21.2.35; D $21.2 .41 \mathrm{pr}^{-1}$; D 21 . 2.42 ; D 21.2.56.2-3; D 22.3.4; D 24.1.28.1-3 (P1autius) -4 (Pomponius) -5 (Julien, Pomponius) ; D 24.1 .67 (Labéon) ; D 28.5.85.1-2; D $29.5 .8 \mathrm{pr}$;D 33.5 7;D34.4.15;D34.4.26pr;D34.4.27pr;D34.9.5.4;D35.1.28.1;D35.2.36.1;D35.2.48;D35. 2.63pr(Pedius);D40.1.9;D40.1.10;D40.1.23;D40.5.6;D40.5.25(Valens);D40.5.31.3-4 D40.5.39pr;D40.7.10;D40.8. 1 (rescrit de Marc-Aurè1e); D 40.8.9; D40.9.16pr;D40. 12.23.1; D 40.12.38 pr (constitution Marc-Aurèle)-1-2-3; D 40.13.4 (L. Rufinus) ; D 41.2.1.16 (veteres) ; D 41.3.4.18; D 41.4.2.6 (Neratius)-7 (Trebatius); D 41.10 .2 ; D 42.5.38 pr; D 44.4.3; D 44.4.5.4; D 46.3.35 (A1fenus) ; D 47.2.18 (cassiani); D 48.10.22.10; D 48.18.11; D 48.18. 18.6 ; D 48.19.38.4 ; D 49.14.45.3 ; U1pien D 1.12.1.1 ; D 2.4.10 pr (constitution Marc-Aurèle)-1 (Marcel1us) ; D 2.9.1.1 ; D 2.14.16.1; D $4.3 .7 \mathrm{pr}$ (Julien)-3 (Labéon) ; D 4.4.11.1 (Scaevola, constitution Marc-Aurèle)-4 (Marce11us) ; D 5.1.67 ; D 5.3.20 pr-1 (Julien) ; D 5.3.27 pr ; D 5.3.33.1 (JuIien); D 6.1.17 pr (Julien); D 6.2.11.2-3 (Julien) ; D 7.1.25.1 (Julien, Mauricianus, Marce11us) ; D 7.1.27.5 ; D 9.2.11.7 (Julien); D 9.4.7.1 (Pomponius) : $\mathrm{D} 9.4 .36 ; \mathrm{D} 9.4 .38 \mathrm{pr} ; \mathrm{D}$ 10.2.18.2 (Pomponius) ; $\mathrm{D} 10.2 .49$; D 11.3.1.1; D 12.4.5.2-3-4; D 12.6.26.12 (Celsus) ; D 15.1.7.6; D 15.1. 11.7 (Julien)-8 (Julien) ; D 15.1 .13 (Julien) ; D 15.1.21.2; D 15.1.30.5; D 15.1.32.1-2 (Neratius); D 15.3.3.8; D 15.3.5 pr-3; D 17.1.8.4 (Julien) -10 ; D 17.1.10.1; D 17.1.19 (Pomponius); D 17.2.58.3; D 18.1.7 (veteres) ; D 18.1.9 pr ; D 18.1.11.1 ; D 18.1.29; D 18.2.4.1 (Ju1ien)-2 (Ju1ien) D 18.4.2.15; D 18.7.1 (Papinien); D 19.1.11.5-7 (Neratius)-8 (Neratius)-12 (Neratius)-15 (Julien); D 19.1.13.1-2-3-4-13-18-22 (Trebatius, Labéon); D 20.1.21.1 (Ju1ien); D 20.6.4 pr ; D 21.1.1 pr (Labéon)-1-2-3-4-5-6-7 (Sabinus) $-8-9$ (Vivianus) -10 (Vivianus) -11 (Vivianus) ; D $21.1 .4 \mathrm{pr}-\mathrm{I}-2$ (Pomponius) -3 (Pomponius) $-4-5$ (Pomponius) -6 (Pomponius) ; D $21.1 .6 \mathrm{pr}$ (Pompo-

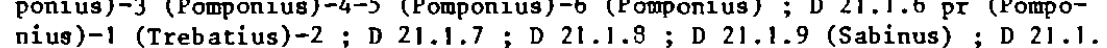

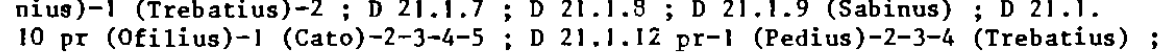
$10 \mathrm{pr}$ (Ofilius) -1 (Cato) $-2-3-4-5$ : D $21.1 .12 \mathrm{pr}-1$ (Pedius) $-2-3-4$ (Trebati
D $21.1 .14 \mathrm{pr}$ (Sabinus)-1-2-3 (Trebatius, Caelius Sabinus) 4 (Pedius) -5 D $21.1 .14 \mathrm{pr}$ (Sabinus) $-1-2-3$ (Trebatius, Caelius Sabinus) -4 (Pedius) -5
(Pedius) $-6-7-8-9-10$ (Caelius Sabinus); D $21.1 .17 \mathrm{pr}$ (Ofilius) -1 (Caelius (Pedius) $-6-7-8-9-10$ (Caelius Sabinus) ; D 21.1 .17 pr (Ofilius) -1 (Caelius
Sabinus)-2 (Cassius)-3 (Vivianus) 4 (Proculus) -5 (Vivianus) -6 (Caelius Sabinus)-7 (Caelius Sabinus) -8 (Caelius Sabinus)-9 (Caelius Sabinus)-10 (Caelius Sabinus)-11-12 (Labéon, Caelius Sabinus)-13 (Caelius Sabinus)-14 (Labéon)-15 (Labéon, Caelius Sabinus)-16 (Caelius Sabinus)-17-18-19-20; D 21.1.19 pr (Pedius)-1-2-3-4-5-6 ; D 21.1.21 pr-1 (Pomponius)-2 (Pomponius)-3 (Pomponius) D $21.1 .23 \mathrm{pr}$ (Pomponius) $-1-2$ (Pomponius) $-3-4-5-6-7$ (Julien) -8 (Julien) -9

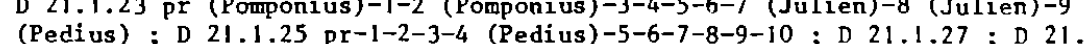
(Pedius) ; D 21.1 .25 pr-1-2-3-4 (Pedius) $-5-6-7-8-9-10$; D 21.1 .27 ; D 21 . $1.29 \mathrm{pr}-1-2-3$ (Julien) ; D $21.1 .31 \mathrm{pr}-1-2-3-4-5$ (Pomponius) -6 (Pomponius) -7
(Marcellus) -8 (Marcellus) -9 (Pomponius) $-10-11-12-13$ (Pedius) -14 (Pedius) 15 (Marcellus) -8 (Marcellus) -9 (Pomponius) $-10-11-12-13$ (Pedius) -14 (Pedius) -15 (Pedius)-16 (Julien)-17-18-19-20-21-22-23-24; D 21.1.33 pr (Pomponius) -1 D 21.1 .35 ; D 21.1 .37 ; D 21.1.38.2-3-7 (Ofilius, Caelius Sabinus)-10-14; D $21.1 .40 \mathrm{pr}$; D $21.1 .59 \mathrm{pr}-1$; D 21.1 .63 ; D $21.2 .4 \mathrm{pr} ; \mathrm{D} 21.2 .21 \mathrm{pr}-2$ (Julien)-3 (Julien) ; D 21.2.25; D 21.2 .31 ; D $21.2 .32 \mathrm{pr}^{-1}$ (Julien) ; D 21 . 2.33; D 21.2.37. I ; D 21.2.51.1 (Julien)-3; D 23.2.45 pr (rescrit Sévère et Caraca11a) ; D 24.1.19 pr (Julien)-1 (Julien, Marcellus) ; D 24.3.21; D 25.3.5.22 (Marce1lus); D 26.4.3.2 (Constitution Marc-Aurèle) ; D 26. 7.5 .9 ; D 27.3.13; D 28.5.6.3; D 28.5.9.16 ; D 28.5.51 pr ; D 28.6.18 pr

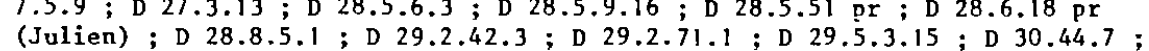
D $30.53 .4 ;$ D $32.73 .4 ;$ D 33.8 .8 .4 (Sabinus); D 38.1 .13 pr (Constitution Marc-Aurèle) ; D 38.2.3.3 ; D 38.16.3.3 (constitution Marc-Aurèle); D 40.1. 
vacua possessio de l'esclave, c'est-à-dire sa possession prisible et durable, moyennant un prix en espèces monnayées qu'il s'engage à livrer en propriété au vendeur (4). Proche de la vente on trouve l'échange, contrat par lequel une personne transfère la propriété d'une chose afin d'obtenir le transfert de la propriété d'une autre chose. Il s'agit donc encore d'une acquisition onéreuse et de caractère durable. Les Sabiniens s'efforcèrent vainement de faire entrer ce contrat du type do ut des dans les cadres de Is vente. Il sera reconnu plus tard compe un contrat autonome, souris aux

4 pr a 13 (constitution Marc-Aurêle et Verus) ; D 40.2.16.1; D 40.5 .7 ; D 40.5.24.6 (rescrit d'Antonin le pieux) -8 (Celsus)-12-14-15-16-17-18; D 40.5.26.2 (rescrits d'Antonin le pieux et de Sévère et Caracalla); D 40 $5.28 \mathrm{pr}$; D 40.5.52; D 40.7.3.7-17 ; D 40.7.6.3-5-6-7 ; D 40.7.9.1; D 40. 9.12.2 (Africain); D 40.9.30 pr (constitution de Marc-Aurele) ; D 40.12 14 Pr D 40.i2.16 Fr; D 40.12.20.4; D 41.2.13.2; D 41.3.10.2; D 42.5.9. 3 ; D 42.8.6.5; D 43.24.7.1 (Neratius); D 44.4.2.7 (Julien); D 44.4.4.5 (Labéon) ; D 46.6.2 ; D 47.2.17.2 (Sabinus) ; D 47.2.48.6 ; D 47.2.52.23 (Me1a); D 47.6.3 pr ; D 47.10.24; D 48.5.28.1; D 48.15.2 pr-1-2-3; D 48.18.1.6 (rescrit de Marc-Aurèle et Verus)-15 (rescrit de Sévère et Caracalla)-27 (epistula de Marc-Aurèle et Verus) ; D 50.16.195.3 ; L. Rufinus D 40.7.32; Macer D 48.19.10 pr; D 48.19.14 (Menander); Marcien D 18.1 .42 (rescrit de Marc-Aurèle et Verus); D 18.1.44;D 18;.2; D 21 . 1.52 ; D 28.5.39; D 28.5.52.1 (Ce1sus); D 34.8.3.2; D 39.4.16 pr (rescrit de Sévère et Caracalla)-3; $\mathrm{D} 40.1 .5 \mathrm{pr}$ (constitution de Marc-Aurềle et Verus) ; D 40.5.51 pr-2-10; D 40.8.6 (constitution de Marc-Aurèle) ; D 40. 9.9.2 ; Modestin D 21.1.62; D 27.1.14.3; D 37.14.7 pr (décret de Vespasien) ; D 37.14.8.1 ; D 40.5.15; D 40.7.25; D 40.7.27 (Julien) ; D 48 . 8.11 .1 ; Hermogénien D 5.1 .53 ; D 35.2.38.2. Certains textes ont été intégrés par extension : à comnencer par ceux traitant de la redemptio suis mumis de 1 'esclave qui, bien que présentant simultanément 1 'esclave comme acteur juridique, ne le situent pas moins comme objet d'une vente, de mêne certains textes ne contenant pas une rêférence explicite a l'escl avage, mais concernant tout de même cette institution par rapport au contexte du passage (par ex. textes classés in catégorie B 3 - cf. chapitre préliminaire - comme D 21. $1.1 \mathrm{pr}-2-3-4-5)$; ou encore des textes retenus parce que constituant $1 \mathrm{a}$ suite directe du fragment précédent, (cf. par ex. Pomponius D 15.1.34 par rapport a Iavolenus $D$ 15.1.33); de même des textes cancernant la vente d'un houme libre, mais par opposition explicite a une vente d'esclave (Paul D 40. 12.23.1 ; Ulpien D 40.12.14 pr ; D 40.12.16 pr ; D 40.12.20.4 ; Macer D 48. 19.14 (Menander) ; enfin, d'autres textes ont été intégrés par a contrario (ainsi Paul D 41.10.2; D 42.5.38 pr : bonis venditis excipiuntur concubina et liberi naturales).

4- v. J. MACQUERON, Histoire des obligations en droit romain, Aix-enProvence (1975), P. 99 ; sur l'édit des édiles, Id ., o.c., p. 135 s. : v. aussi W.W. BUCKLAND, Roman law, cit., p. $54 \mathrm{~s}$. L'édit des édiles (dans le Digeste D 21.1) sera utilisé par ailleurs, notament à propos de l'étude sur les "défauts" physiques et moraux de l'esclave. règles des contrats innommés (5). L'esclave peut enfin faire l'cbijet 1 'une stipulation, contrat verbal de la plus haute importance en troit romain (6).

On peut par ailleurs recevcir gratuitement et de manière durable un esclave par legs, fidéicommis ( 7 )

R. MONIER, Manuel élémentaire de droit romain, Paris (1947), Tome II, p. 190. Visent l'échange (permutatio) d'un esclave, dans le Digeste, deux textes de Paul, D 19.4.2 (Aristo) et D 41.3.4.17, et un d'Ulpien, D 15.2.1.6. Dans le même genre de contrat, du type do $u t$ des, on relève $\mathrm{D} 12.4 .16$ Celsus. debi tibi pecuniam, ut mihi Stichum dares, et Paul D 19.5.5.1 : Si scyphos tibi debi, ut Stichum mihi dares. Sur les rapports entre emptio venditio et permutatio, v. MEYIAN, Permutatio rerom, in Jus et Lex, Fs. Gutzviller (1959), p. 9s.

6- v. J. MACQUERON, o.c., p. $40 \mathrm{~s}$.; R. MONIER, TOme II, o.c., P. $21 \mathrm{s.}$ v. ARANGI0-RUIZ, Istituzioni di diritto romano, Naples (1976), p. $323 \mathrm{~s}$. A. GUARINO, Diritto privato romano5. Naples (1946), p. 747 s. La présence d'un cas de stipulation se trouve attestée par I'indicateur stipulatio, stipulator, stipulatus ou stipulari : Iavolenus D 45.1.105 (Sabinus) ; Julien D 30.76 ; D 30.82 pr ; D 34.5.13.2; D 40.9.5.2; D 44.7.18; D 45 . $1.54 \mathrm{pr}$; D 45.1.56.8; D 46.3.33 pr ; D 46.3.34.1-2-10; D 46.4.17; Pomr ponius D 17.1.47.1 (Julien); D 41.10.3 (Neratius); D 45.1.23; D 45.1.112 pr D 46.4 .10 ; Africain D 30.108 .4 ; Gaius D 12.6.63:D 44.7 .1 .9 . D 45 pr ; 46.4515 (Julan D 30.108 1.74 ; D 45.2 .15 (Julien); Venuleius D $45.1 .137 .1-4$; Harcellus D 45.1 .96 D $46.1 .38 \mathrm{pr}$; D $46.3 .72 .2-4-6$; D 46.8.17 Scaevola D 40.9 .6 (pris en fonction du contexte fourni par Julien D 40.9 .5 ; Papinien D 3.3 .66 ; 27 . D 45.1 .115 .2 (Pegasus, Sabinus) ; D 45.1 . 17 ; D 46.3.95.1; Paul D 2.14 .27 . 6 ; D 7.7.1 ; D 9.2.55; D 34.5 .21 pr ; D $41.2 .5 ; D 42.1 .8$; D 44.7 .44 .4
D $44.7 .45 ;$ D $45.1 .2 .1-3$; D 45.1 .8 (Sabinus, Proculus, Marcel1us) ; D 45 . 1.24 ; D 45.1.49 pr ; D $45.1 .73 \mathrm{pr}-1$; D 45.1.83.1-2-4-5-7 (Sabinus) ; D 45. I.85.4 ; D 45.1.88;D $45.1 .91 \mathrm{pr}-1$ (Julien) -6 (Julien) ; D 45.1.93; D 45. 1.128 ; D 45.3.20 pr ; D 46.1.34; D 46.2.32; D 46.3.98.6-8 (Celsus); D 47.2.67.5; D 47.2.86; Ulpien D 4.3.7.3 (Labéon); D 12.2.26.13 (Celsus, Marcellus)-14 (Ce1sus) ; D 13.4.2.2-3 (Scaevola) ; D 29.5.3.13; D 34.3.7. 1 (Julien) ; D 40.7.9.2 (Octavenus) ; D 45.1.1.5; D $45.1 .29 \mathrm{pr}-1$; D 45.1 . 1 (Julien) ; D 40.7.9.2 (Octavenus) ; D 45.1.1.5 ; D $45.1 .29 \mathrm{pr}-1$; D 45.1 32 ; D 45.1.51 ; D 45.1.69; D 45.1.75.1-4-8; D 45.3.9 Pr ; D 46.1.8.8 (Julien, Marcellus)-9 (Julien)-10; D 46.1 .33 ; D 46.2 .8 pr ; D 46.4 .13 .4 promissor : Julien D 12.6.32.3; D 13.5.23; D 46.3.33.1; D $46.3 .34 \mathrm{pr}$; Pomponius D 45.1.33; D 45.2.18; D 46.3.92 $\mathrm{pr}-1 ; \mathrm{D} 46.4 .15$; Gaius D 40 . 1.18 ; Marcellus D 46.3.67 ; Scaevola D 45.1.127; Papinien D 46.3.95 pr ; Paul D 4.3.18.5; D 9.2.22 pr ; D 13.5.21 pr ; D 17.1.46; D 45.1.49.3; D 45.1.73.2 ; D 45.1.91.3 (Celsus, Julien)-5 (Pomponius); Ulpien D 12.2. 34 pr (Marcellus) ; D 12.3.5.4 ; D 45.1.82.1 ; D 46.3 .29 ; Marcien D 22.1. 32.5. On peut enfin relever debere ou dare se opportere : Julien D 12.6 .32 pr : Pomponius D 12.6.19.3; $45.1 .16 \mathrm{pr}$; Marcellus D 46.3.72.1-5. Scaepr ; Pomponius D 12.6.19.3 ; D $45.1 .16 \mathrm{pr}$; Marcellus D $46.3 .72 .1-5$ i Scae-

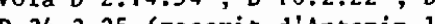

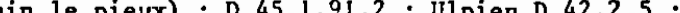
D $42.2 .6 .1 ; 0.36 .3 .1$.

7- v. V. ARANGIO-RUIZ, Istituzioni di diritto romano, cit., ch. XXVI, Legati e fedeconmessi, p. $564 \mathrm{~s}$. Traitent de legs d'esclaves Proculus $D 31$. $48 \mathrm{pr} ; \mathrm{D} 33.6 .6$; Iavolenus D $21.2 .58 ; \mathrm{D} 31.37 ; \mathrm{D} 11.38 ; \mathrm{D} 32.39 .3$ (Labéon); D 32.100 pr (Cascellius, Labéon); D 33.4.6.1 (Servius, Cascellius, béon); D 32.100 pr (Cascellius, Labéon); D 33.4.6.1 (Servius, Cascellius, tius D 47.2.65; Celsus D 30.63 (Servius) ; D 31.15; D $31.18 ;$ D 31.19 ; 
D $32.79 \mathrm{pr}-3$; D 33.8.13; Valens D 35.1.8.9; D $35.2 .37 \mathrm{pr}$; D 36.4 .15 ; Julien D 9.4.40; D 13.1.14 pr ; D 17.1.30 ; D 28.5.38 pr ; D 28.6.19; D 30.6 ; D 30.76; D 30.81.2-4-6-9-10; D 30.82 $\mathrm{pr}^{-4-6}$; D $30.84 .3-4-5-8-$ 9-10-11-12 ; D $30.86 \mathrm{pr}^{-1-2}$; D 30.91.2-3-4-5-6-7; D 30.94.2-3;D 30 . 96.2 ; D 30.97 ; D 30.98 ; D 30.99 ; D $30.101 \mathrm{pr} ; \mathrm{D} 33.5 .9 \mathrm{pr}-1-2(\mathrm{Q} . \mathrm{Mu}-$ cius) ; D 33.5.10; D 33.5.11; D 33.5.12; D 34.4.11 ; D 34.5.13.1 ; D 36 . $2.16 \mathrm{pr}-1$; D 40.4.15;D 40.5.47.3; D 40.5.48; D 40.9.7.1 (Proculus); Pomponius D 28.6.16 pr; D 30.8 .2 ; D 30.12 .1 (Proculus); D 30.13 (Neratius); D $30.20 ; \mathrm{D} 30.24 \mathrm{pr} ; \mathrm{D} 30.36 \mathrm{pr}$ (Labéon) $-1-3 ; \mathrm{D} 30.38 .1 ; \mathrm{D} 30$ $45 \mathrm{pr}$ (Ofilius, Áristo, Neratius) $-1-2 ; \mathrm{D} 30.46 ; \mathrm{D} 30.48 .1 ; \mathrm{D} 30.56 ; \mathrm{D} 31$. $45 \mathrm{pr}$ (Ofilius, Aristo, Neratius) $-1-2$; D $30.46 ; \mathrm{D} 30.48 .1$; D 30.56 ; D 31 .
11.1 (Julien) ; D 32.74 ; D 32.85 (Labếon); D 33.5 .6 ; D $33.5 .8 \mathrm{pr}^{-2}$; D 33 . $7.15 \mathrm{pr}$ (Servius)-2 ; D 34.2.10; D 35.1.1.3 ; D 40.4.11 pr; D 36.1.72. 1 (fidéiconmis d'hérédité) ; $040.4 .40 \mathrm{pr}$ (Julien)-1 (Ofilius) ; $\mathrm{D} 40.5$ 34.2 (Sabinus) ; D 45.1.23; D 46.3.92 pr ; Africain D 12.1.23 (Julien); D 30.107 .1 ; D $30.108 \mathrm{pr}-2-4-5-9-11$; D 30.110 ; D 40.4.20 ; D 40.5.49; T. Clemens D 33.5.17; Gaius D 7.1.76.1 ; D $30.65 \mathrm{pr}-1$; D $30.67 \mathrm{pr}$; D 30. 68.2-3 (Julien) ; D $30.69 \mathrm{pr}^{-1-4-5}$; D $30.70 \mathrm{pr}^{-2-3}$; D 35.1.17.1 ;

D 40.7 .37 ; D 40.9 .3 ; D 40.9.29.1 ; Maecianus D 32.95 (Aristo); D 36 . 1.71 (fidẹicominis d'hérédité) ; Venuleius D 4I.1.66 ; Marcellus D 9.2.34; D $9.2 .36 \mathrm{pr}$; D 29.1 .31 ; D 30.83 ; D 31.26 ; D 31.50 .1 ; D 32.69 .1 ; D 35 . 2.34 (Julien); D $35.2 .56 .3-4 ;$ D 46.3 .72 .4 ; Florentinus D 30.116 .2 ; Scaevola D 4.3.32 ; D 10.2.34.9; D 15.1.54; D 28.6.48.1 (Julien); D 31 88.7 ; D 32.37.7; D 32.41.2-4-5-10; D 32.93.2 ; D $32.101 \mathrm{pr}^{-1}$; D 35.5 . 18 (Neratius) ; D 33.5 .21 ; D 33.7.7; D 33.7 .20 pr-1-2-3-4-5-6-9 ; D 33. $7.27 \mathrm{pr}-1-3-4$; D 33.7.28; D 33.8.21 (Julien); D 34.1 .17 ; D 34.2.18 pr ; D $34.4 .31 \mathrm{pr} ; \mathrm{D} 36.1,80.12$; D 40.5 .41 .3 ; D 40.7.40.2-7; Papinien D 22.1.8; $331.65 \mathrm{pr}-3 ; \mathrm{D} 31.66 .3 ; \mathrm{D} 31.73 ; \mathrm{D} 31.77 .17 ; \mathrm{D} 32.91 \mathrm{pr}-2 ; \mathrm{D} 33.7 .3 \mathrm{pr}-1 ; \mathrm{D} 36.1 .55 ; \mathrm{D} 36.1 .40$ 4 et 6(fidéic.d'hếréd.);D36.2.25.1;D47.2.81.2;D50.17.77; Tryphoninus D34.1.15. (Scaevola);D49.15.12.18;Paul D9.2.14;D10.2.25.17;D11.3.8;D12.6.65.9; D 15.1. 47.6 ; D 19.5.5.2 (Julien) ; D 22.1.14 pr-1 (Neratius) ; D 29.1.40.2 ; D 29. 5.12 ; D $30.5 \mathrm{pr}^{-1}$ (Trebatius, Labéon, Cassius) ; D 30.35 ; $\mathrm{D} 30.52 \mathrm{pr}$;
$\mathrm{D} 30.62 ; \mathrm{D} 31.5 .1$; D $31.8 \mathrm{pr}-1$; $31.14 \mathrm{pr} ; \mathrm{D} 32.60 .1$ (A1fenus) 3 ; (Alfenus) ; D $32.61 ; \mathrm{D} 32.66 ; \mathrm{D} 32.78 \mathrm{pr}-1-2-3 ; \mathrm{D} 32.92 \mathrm{pr} ; \mathrm{D} 32.99$ (Alfenus) ; D 32.61 ; D 32.66; D $32.78 \mathrm{pr}^{-1-2-3}$; D $32.92 \mathrm{pr} ; \mathrm{D} 32.99$
$\mathrm{pr}-1-2-3-4-5 ; \mathrm{D} 33.5 .7$; D $33.5 .13 \mathrm{pr}^{-1}$ (Pomponius) ; D 33.7.13 pr (Neratius)-1 (Neratius); D 33.7.14; D 33.7.16.2 (A1fenus); D 33.7.18.1-4 (Scaevola)-5-6-7-8-9-10 (Cassius)-11-13 (Scaevola) ; D 33.7.19 pr-1 ; D 33. 7.22 .1 ; D 33.8.1 ; D 33.8.3 ; D 33.8.4; D 33.8.15 (A1fenus) ; D 34.2.7;

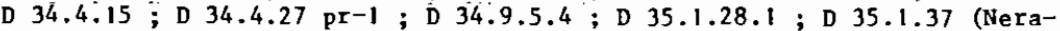
tius); D $35.2 .21 \mathrm{pr}-1$; D 35.2.24.1; D 35.2.33 ; D $35.2 .36 \mathrm{pr}-3-4$ (Africain) ; D 35.2.49 pr (Sabinus, Nerva, Atilicinus, Cassius, Plautius) ; D 36 1.68 .2 (fídéiconmis d'héréditê) ; D 38.2 .44 .1 ; D 40.1 .11 ; D 40.4 .10 .

I ; D 40.5.33.2 ; D 40.5.40 pr-1; D 40.7 .42 (Labéon) ; D 40.9.28; D 41. 2.1 .16 (veteres) $-17 ; \mathrm{D} 41.3 .4 .17 ; \mathrm{D} 48.10 .17$; D 48.10 .22 .7 ; U1pien D 2 . 14.7.2 (Julien, Mauricianus); D 4.3.7.5; D 4.3.9.1; D 9.2.13.3 (Julien) ; D 9.2.15 pr ; D 9.2.35 (par rapport au contexte fourni par Paul D 9.2.34) ; D 9.4.14.1; D 10.2.8 pr (Pomponius); D 10.2.18 pr (Julien); D 10.4.3.6-10; D 15.1.9.5 (Julien); D 15.2.1.7 (Pegasus, Africain); D 28. 1.21.1; D 28.5.35.3 (Q. Mucius); D 28.6.18.1 ; D 29.5.1.4-13 (Scaevola); D 30.32 .1 ; D $30.37 \mathrm{pr}$ (Cassius, rescrit de Sévère et Caracalla) ; D 30. $39 \mathrm{pr}$ (Julien, Africain)-1 ; D $30.44 \mathrm{pr}-7-8$; D 30.47.2-3 ; D 30.53.3-4-8-9 ; D $30.71 .1-3-4$; D $30.75 .3-4$ (Julien) B D 32.3 .1 (Julien) ; D $32.49 \mathrm{pr}$; D $32.68 .2-3$; D 32.71 ; D 32.66 ; D $32.73 \mathrm{pr}-1-2-3-5$ (Pomponius); D 33 4.1 .6 ; D 33.5.2 $\mathrm{pr}^{-1-2}$; D $33.7 .8 \mathrm{pr}-1$; D $33.7 .12 \mathrm{pr}$ (Servius) '(pris par extension : la nourriture des esclaves agriculteurs est comprise dans le legs de 1'instrumentum fundi) -2 (Alfenus)-3 (Labéon, Pegasus) (pris par on donation (8).

Il faut enfin signaler une forme particulière d'acquisition de l'esclave, la dot (9). Particulière en ce sens que le transfert de propriété

a contrario)-4 (Labêon, Neratius)-5 (Trebatius)-6-7-8-9-\{2-13-3\} (Ce1sus) -32-33-35 (Neratius)-37 ( Papinien)-38 (Papinien)-42 (Papinien) (intégré ainsi que D 33.7.12.37 par a contrario)-44 (Celsus)-45 (Papinien) 46 (Papinien) ; D 33.8.6.2 ; D 33.8.8.8 (Julien) ; D 33.8.24 ; D 34.4.12 ; D 36.1.11.2 (rescrit d'Antonin le pieux) ; D 36.1.17.7 (rescrit d'Antonin le pieux); D 36.1.23.3 (Neratius) (fidêiconunis d'hérédi té); D 36.2.7.6; D 36.2 .11 ; D $36.2 .12 .7-8$; D 36.2.23; D 36.4.5.8; D 40.1.2 ; D 40.1.4.' (constitution de Marc-Aurèle et Verus); D $40.2 .3 ; 040.4 .9 \mathrm{pr} ; \mathrm{D} 40$. 5.26.6-I1; D 40.5.45.2; D 40.5.46.5; D 40.7.2.2 (Julien); p 40.7.6.3; 5.26.6-11; D 40.5.45.2 ; D 40.5.46.5; D 40.7.2.2 (Julien) ; D 40.7.6.3;
D 47.6.3.1; D 50.16.160 (Marcellus); Marcien D 30.112.1.; D 30.114.1 ; D 47.6 .3 .1 ; D 50.16.160 (Marcellus); Marcien D 30.112.1.; D 30.114.1 ;
D $32.65 \mathrm{pr}$ (Labéon)-1-2-3 (Ce1sus); D 33.7 .17 .2 ; D $34.1 .2 \mathrm{pr}$ (rescrit de Sévère et Caracalla) ; D 35.1.33.4; D 37.14.3 (rescrit de Sêvère et Caracalla) ; D 38.4.6; D 40.2.14.1; D 40.5.50; Modestin D 31.31 ; D 31. $34 \mathrm{pr}$; D 31.35 ; D $32.81 \mathrm{pr}-1$; D 32.82 ; D 40.5.12 pr (rescrit de Caracal1a)-1 ; D 50.16.101.3 ; Hermogénien D 32.22.2 ; (auteur anonyme D 36.4. 16). L'association terminologique do, lego nous a conduit à intégrer dans cette rubrique les textes où on n'avait que le premier de ces deux termes (par ex. D 31.18; D 30.84.9).

8- P.F. GIRARD, Manuel élémentaire de droit romain, Paris (1924), p. $987 \mathrm{~s}$. les donations entre vifs, p. $988 \mathrm{~s}$. ; les donations à cause de mort, p. 999s. On ne distinguera pas ici s'il s'agit d'une donation entre époux (ex. D 24.1.18; D 24.1.31.5), ou entre concubins (D $24.1 .58 \mathrm{pr}-1$ ), ou entre fiancés (D $6.2 .12 \mathrm{pr}$ ), ou encore du père a sa fille (D 31.77 .17 ), etc. ou d'une donation a cause de tort (D 24.1.20; D 28.5.77). Ces différents cas se présentent à propos des esclaves, come en attestent ces références, mais le seul problème qui doive nous préoccuper est celui de 1 'esclave acquis par donation. Traitent de donations d'esclaves Iavolenus 0 24.1.20; Ju1ien D 30.84.11; D 39.6.18.3 ; Pomponius D 24.1.18; D 24.1.31.5 ; Gaius D 24.1.8; D 35.1.17 pr; D 39.5.11; Marcellus D 40.1.15; Scaevola D 24. $1.58 \mathrm{pr}-1$; D 32.37.3 ; D 41.6.5 ; D 45.1.122.2 ; D 45.1.135.3 ; Papinien D 28.5 .77 ; D 31.77 .17 ; D 34.4 .24 .1 ; D 39.5.31.2 ; D $40.1 .20 \mathrm{pr}$ (Zitterae de Marc-Aurèle); D 40.8.8 (constitution de Marc-Aurèle); D 41.2.48 (L'esclave ne constitue ici que 1 'accessoire de la donation : il s'agit de la donation d'un fonds cum servis), Paul D 6.2.12 pr ; D 12.4.12 ; D 24.1.38 pr (A1fenus) ; D 24.1.67 (Labéon) ; D 24.3.63; D 39.6.39; D $40.5 .40 \mathrm{pr}$; D 41.1 .57 (Julien); D 41.2.1.16 (veteres); D 41.3.4.17; D 47.2.54.4 (Julien); Ulpien D 6.2.11.3 (Julien) ; D 15.2.1.6 ; D 24.1.7.8 (Sabinus, Papinien, constitution de Marc-Aurèle) -9 ; D $24.1 .9 \mathrm{pr}^{-1}$ (Julien) ; D 24.1 17.1 ; D 24.1.19 pr (Julien)-1 (Julien, Marcellus) ; D 24.1.22; D 24.3. 24.4 ; D 24.3.62 ; D 32.68.1 ; D 39.5.18.1 (Aristo, Pomponius)-2 (Aristo, Pomponius) ; D 39.6.37.1 (Julien) ; $\mathrm{D} 40.2 .16 .1$; D 40.2.20.1 (constitution de Marc-Aurèle) ; D 44.4.4.9 ; D 47.6.3.1 ; D 49.17.6 ; Marcien D 30.112.1.

9- Cas d'esclaves dotaux in Proculus D $31.48 \mathrm{pr}$; Iavolenus D 24.3.31.4 (Labéon) ; D 24.3.66.3 (Labéon); Celsus D 23.3.58.1; Neratius D 25.1.15; Julien D 12.4.7.1 ; D 23.3.47; D 23.4.21; D 24.3.31.4 ; D 29.2.45.1 ; Pomponius D 23.3.18 (Labéon) ; D 23.3.65; D 24.3.67; Africain D 21.2.24; D 23.5.9.2 ; Marce11us D 46.3.72 pr ; Scsevola D 31.88.7 ; D 41.9.3; Papinien D 23.3.69.9 ; D 24.3.6I ; D 33.4.8 ; D 40.1.2I ; Paul D 15.1.47. 6; D 23.3.25; D 23.3.56 pr ; D 24.1.28.1;D 24.3.25.3; D 24.3.26; D 24.3.63; D 25.1.2; D 25.1.6; D 25.1.12; D 25.2.21.2; D 46.3.98 pr ; D 48.10.14.2 ; D 50.16.79.1 ; U1pien D 15.1.19.1 (Pomponius, Papinien) ; D 15.2.1.6; D 23.3.10.2-5; D 23.3.12.1 (Marcellus, Scaevola); D 24.3.7. $10-16$; D 24.3.24.4-5; D 24.3.62; D 24.3.64 pr-1-2-3-4-5-6-7-8-9 (Labéon) 
qu'elle opère n'est que provisoire, le mari átant soumis, selon certaines modalités, à une obligation de restitution à la fin du mariage (10).

Hormis ce demier cas, on $\mathbf{s}$ dónc affaire à une acquisition durable de l'esclave, tout du moins en prititipe. Cette assertion est en effet susceptible d'exceptions. Prenons exemple de la vente : un esclave peut être vendl à charge pour l'acheteur de l'afrranchir après un certain lélai (11). Il est certain qu'alors la vente perd son caractère d'acquisition durable. Il peut en être de même en matière de legs (12)

$-10 ; D$ 33.4.1.6; D 38.16.3.2; Modestin D 24.3.58.

10- H.H. BUCRLAND, Roman law, cit., servus dotalis, p. $262 \mathrm{~s}$. Bien que la dot fût, d'une manière effective, la propriété du mari, les jurisconsultes classiques semblent avoir admis qu'elle constituait une masse juridiquement distincte, dans le patrimoine du mari, et qu'elle était, dans une certaine mesure, $¥$ la feme, cf. R. MONIER, Droit romain, cit., p. 294, une certaine mesure, $A$ la fenme, cf. R. MONIER, Droit romain, cit., P. 294,
tome I. Dans ce sens, Tryphoninus D 23.3 .75 : quamvis in bonis mariti dos tome I. Dans ce sens, Tryphoninus D 23.3 .75 : quamvis in bonis mariti do
sit, mulieris tamen est. L'interpolation de cette phrase, soutenue par sit, mulieria tamen eat. L'interpolation de cet te phrase, soutenue par
ALBERTARIO, BESELER, GUARINO (in SDHI, 1939, p. 283 n. 28) et V. ARANGIORUIZ ne semble pas démontrêe selon 1 'auteur (o.c., p. 294, n. 1) ; v. aussi A. GUARINo, Diritto privato romano, cit., p. $536 \mathrm{~s}$.

11- Par exemple Papinien D 18.7.8; Tryphoninus D 18.7.10 (Scaevola) (1a

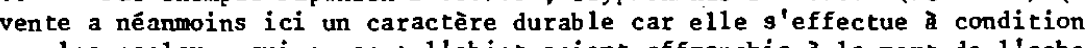
que les esclaves qui en sont 1 'objet soient affranchis a la mort de 1 'acheteur.) ; Callistrate D 40.8.3 (rescrit de Marc-Aurèle et Commode) ; Paul D 40.1.23; D 40.8.1 (rescrit de Marc-Aurèle); Ulpien D 29.2.71.1; D 40.8 . 4 ; Modestin D 37.14.8.1. I1 est parfois question de 1 a vente d'un esclave à condition de 1 'affranchir sans que rien n'apparaisse sur un délai : Marcellus D 18.7.4; Paul D 28.5.85.1 (constitution de Marc-Aurèle) ; D 40.8.9; D40.9.16pr;D40.12.38.1 (const.Marc-Aurè 1e)-3; U1 pien D2.4.10pr(idem);D4.4.11. 40.9. ; Darc- . de Marc-Aurêle) ; D 40.1.4.6 ; D $40.9 .30 \mathrm{pr}$ (constitution de Marc-Aurèle). D'autre part, la survenance d'un événement prévu dans le contrat de vente peut restreindre la durée de ses effets. On peut ainsi prévoir que si l'acheteur prostitue 1 'esclave vendue, elle obtiendra la libertê : Pomponius D 21.2 .34 Pr ; Paul D 18.7.9; Ulpien D 2.4.10.1 (Marcellus); Modestin (décret de Vespasien) $\mathrm{D} 37.14 .7$ pr. Sur un autre plan, la vente ne produit
bien entendu gu'un effet provisoire si elle a pour objet un statuliber(cf. W.W. BUCKLAND, Roman law, cit., p. $289 \mathrm{s.}$ ) ou un esclave a qui est due une liberté fidéicomissaire (cf. Paul D 21.2 .26 ). De même si un particulier doit, en vertu d'un fidéicommis, acheter un esclave pour l'affranchir (cf. W.W. BUCKLAND, o.c., p. 530 s.), (par ex. Paul D 40.5.6).

12- On peut recevoir le legs d'un esclave pour 1'affranchir : par exemple Julien D 40.4.15; D 40.5.48; Pomponius D 40.5.34.2 ; Africain D 40.4 20 ; D 40.5.49; Gaius D 40.7.37; Marcellus D 35.2.56.3 (délai de trois ans); Paul D 29.1.40.2; D 40.4.10.1; D 40.5.33.2; D 40.5.40 pr; D 48 . 10.17 ; Ulpien D 40.4.9 pr ; D 40.5.45.2 ; Marcien D 37.14 .3 (rescrit de Sévère et Caracalla). Un problème analogue, quant au caractère durable du Sévère et Caracalla). Un problème analogue, quant au caractère durable par l'héritier. Come en matière de vente, il a alors la possibilité de remplir la condition de sa liberté dans la personne de son nouveau maître (cf. Ulpien $\mathrm{D}$ 40.7.6.3). Si, par contre, un esclave est légué de la manière. suivante : Stichron Attio do lego.: et si ei numos centron dederit, liber esto, ou de donation (13).

Si on comptabilise ces differents modes d'acquisition de l'esclave pour roir quelle est leur place respective dans le Digeste, on obtient le tableau suivant (14):

- analyse des paragraphes

période vente échange stipulation ciégs et fómis donation dot

III 142 -

503

TOTAL

680

3

137 $-$

$\begin{array}{rr}- & - \\ - & 1 \\ 8 & 14 \\ 7 & 3 \\ 40 & 42\end{array}$

55

60

Ces chiffres permettent déjà de dégager la place essentielle que tient la vente. En effet, en additionnant ces différentes rubriques, on se rend compte que $50 \%$ des textes concernant des acquisitions d'esclaves traitent de la vente. En deuxième position viennent les legs avec 31 \%. Il est intéressant à ce titre de relever que les totaux de la période III

le problème ne se pose pas car, selon Iavolenus (D 40.7 .39 pr), la concession de la liberté conditionnelle éteint le legs.

13- Exemples de donation d'esclave pour être affranchi Gaius D 24.1.8; Scaevola D 45.1.122.2 ; Papinien D 40.1.20 pr (Zitterae de Marc-Aurèle) ; D 40.8.8 (constitution de Marc-Aurèle); Ulpien D 24.1.7.8 (Sabinus, Papinien, constitution de Marc-Aurèle)-9 ; D 24.1 .9 pr-1 (Julien) ; D 39. 5.18.2 (Aristo, Pomponius); D 49.17.6.

14- Les rếférences correspondant aux chiffres avancés avant été reproduites en note ne le seront pas dans les tableaux à suivre, ceci pour éviter des tableaux trop lourds. Certains textes traitent d'une aliénation en général de $1^{\prime}$ esclave. Il était difficile de les prendre en considération ici. En effet, si l'aliếnation peut viser la vente, ce qui est le cas le ici. En effet, si 1 aliénation peut viser la vente, ce qui est le cas
plus fréquent (ex. D $30.91 .3-5$ ), elle peut aussi concerner la donation (cf. D 40.1.15). On retrouvera ces textes à propos de $1^{\prime}$ étude portant sur les rapports juridiques, dans la rubrique "esclave, objet d'acte juridique". Toutefois, le doute n'existait qu'en l'absence d'un indicateur explicite permettant de ranger 1 'aliénation dans une rubrique juridique plus précise. Ainsi 1 'association alienatus... emptor autorisait-elle à ranger le texte dans la vente. Ont pu de cette manière être intégrés dans la vente : Julien D 30.91.3-5 ; Pomponius D 40.7.29.1 (Aristo, Celsus) ; Africain D 47. $2.62 \mathrm{pr}$; Maecianus D 35.1.86 pr (Julien); Scaevola D 28.6.48.2 ; Papinien D 40.5.21; Tryphoninus D 9.4.37; Paul D 34.4.15; $34.4 .26 \mathrm{pr}$; D $34.4 .27 \mathrm{pr}$; D $40.9 .16 \mathrm{pr}$; Ulpien D 17.2 .58 .3 ; D 28.5.9.16; D 40.5 . 24.8 (Ce1sus) ; D 42.8.6.5; D 43.24.7.1 (Neratius); Marcien D 28.5.52.1 24.8 (Ce1
(Celsus). 
sont à peu près identiques en iratì̀re de vente et de legs, et que la période IV contient plus de références aux legs qu'aux ventes d'esclaves, mais cela tient surtout à Scaevola qui intervient beaucoup en la matière (notamment dans D 33.7-. De instructo vel inotmomento legato). La stipulation joue de même un rôle non négligeable, (10\%), ce qui ne surprend pas, compte tenu du caractère formaliste du droit romain. Ensuite vient la donation avec $4 \%$. Quant à la dot, elle apparaît dans la même proportion.

Ces premières constatations étant faites, il convient de se replacer sur le terrain de l'analyse chronologique. Pour cela, il faut compléter ces données par celles que fournit l'analyse des citations par renvoi (15).

\section{- analyse des citations}

période venter échange

\section{stipulation}

$$
\begin{gathered}
\text { legs et } \\
\text { fidéicommis }
\end{gathered}
$$

donation dot

$\begin{array}{lrrrrrr}\text { I } & 21 & - & - & 19 & 2 & - \\ \text { II } & 60 & - & 4 & 23 & 2 & 4 \\ \text { III } & 99 & 1 & 16 & 37 & 10 & 1 \\ \text { IV } & 10 & - & 5 & 5 & 1 & 2 \\ \text { V } & 3 & - & - & 5 & 1 & 1 \\ \text { TOTAL } & 193 & 1 & 25 & 89 & 16 & 8\end{array}$

D'un point de vue purement quantitatif, l'analyse des citations confirme l'analyse directe. La vente occupe toujours une place prédominante (58\% des citations concernant l'acquisition d'un esclave), suivie par les legs et fidéicommis (27\%). Vient ensuite la stipulation ( $8 \%$ ), puis la donation (5\%). Enfin, 1 'esclave dotal ne ressort que de $2 \%$ des cas.

Pour dégager une évolution chronologique, il g'agit d'assembler les résultats obtenus grâce à ces deux analyses, et de dégager, pour chaque période, le pourcentage des chiffres précédemment obtenus par rapport au nombre total de paragraphes et de citations traitant des modes

15- Les citations par renvoi a la législation impériale ne seront pas reprises dans I'analyse des réalités. Elles perdraient la valeur comparative qu'elles ont sur le plan de l'analyse quantitative globale, seule une étude du Code de Justinien autorisant un parallèle plus détaillé, pour chaque thème envisagé.

\section{d'acquisition de l'esclave.}

Cette démarche nous conduit au tableau suivant :
Analyse deg paragraphes - vente

$\begin{array}{lclllll}\text { I } & - & = & - & \text { I } & 21 / 42 & =50 \% \\ \text { II } & 0 / 3 & = & - & \text { II } & 60 / 93 & =65 \% \\ \text { III } & 142 / 336 & = & 42 \% & \text { III } & 99 / 164 & =60 \% \\ \text { IV } & 35 / 113 & =31 \% & \text { IV } & 10 / 23 & =43 \% \\ \text { V } & 503 / 900 & =56 \% & \text { V } & 3 / 10 & =30 \%\end{array}$

$\begin{array}{lclllll}\text { I } & - & = & - & \text { I } & 21 / 42 & =50 \% \\ \text { II } & 0 / 3 & = & - & \text { II } & 60 / 93 & =65 \% \\ \text { III } & 142 / 336 & = & 42 \% & \text { III } & 99 / 164 & =60 \% \\ \text { IV } & 35 / 113 & =31 \% & \text { IV } & 10 / 23 & =43 \% \\ \text { V } & 503 / 900 & =56 \% & \text { V } & 3 / 10 & =30 \%\end{array}$

Analyse des citations

\section{- échange}

$\begin{array}{llllllll}\text { I } & - & & - & \text { I } & 0 / 42 & = & - \\ \text { II } & 0 / 3 & = & - & \text { II } & 0 / 93 & = & - \\ \text { III } & 0 / 336 & = & - & \text { III } & 1 / 164 & = & 0,60 \% \\ \text { IV } & 0 / 113 & = & - & \text { IV } & 0 / 23 & = & - \\ \text { V } & 3 / 900 & & 0,33 \% & \text { V } & 0 / 10 & =\end{array}$

\begin{tabular}{|c|c|c|c|c|c|c|c|}
\hline I & - & $=$ & & I & $0 / 42$ & $=$ & - \\
\hline II & $0 / 3$ & $=$ & & II & $4 / 93$ & $=$ & L㠰 \\
\hline III & $36 / 336$ & $11 \%$ & & III & $16 / 164$ & $=$ & $10 \%$ \\
\hline IV & $16 / 113$ & $14 \%$ & & $\Gamma$ & $5 / 23$ & $=$ & $22 \%$ \\
\hline $\mathrm{v}$ & $85 / 900$ & 10 & $(+)$ & $\mathrm{V}$ & $0 / 10$ & $=$ & - \\
\hline
\end{tabular}

\section{- gtipulation}

\begin{tabular}{|c|c|c|c|c|c|c|c|}
\hline I & - & $=$ & - & & I & $19 / 42$ & $=$ \\
\hline II & $2 / 3$ & $=$ & $67 \%$ & & II & $23 / 93$ & $=$ \\
\hline III & $136 / 336$ & $=$ & $41 \%$ & $(+)$ & III & $37 / 164$ & $=$ \\
\hline IV & $52 / 113$ & $=$ & $46 \%$ & & IV & $5 / 23$ & $=$ \\
\hline $\mathrm{v}$ & $227 / 900$ & $=$ & $25 \%$ & & $\mathrm{v}$ & $5 / 10$ & $=$ \\
\hline
\end{tabular}

\section{- legs et fidéicommis}

\section{- donation}

$\begin{array}{lllllcll}\text { I } & - & = & - & \text { I } & 2 / 42 & =5 \% \\ \text { II } & 0 / 3 & = & - & \text { II } & 2 / 93 & =2 \% \\ \text { III } & 8 / 336 & = & 2 \% & \text { III } & 10 / 164 & =6 \% \\ \text { IV } & 7 / 113 & = & 6 \% & \text { IV } & 1 / 23 & =4 \% \\ \text { V } & 40 / 900 & = & 4 \% & \text { V } & 1 / 10 & =10 \%\end{array}$

(+) 40,47\% arrondi à $41 \%$ pour obtenir un total de $100 \%$ pour la période

9,44 arrondi à $10 \%$ pour obtenir un total de $100 \%$ pour la période $v$. 
- $\underline{\text { dot }}$ I
II
III
IV
Y

$\begin{aligned}- & = \\ 1 / 3 & =33 \% \\ 14 / 336 & =4 \% \\ 3 / 113 & =3 \% \\ 42 / 900 & =5 \%\end{aligned}$

I
II
III
IV
V

$0 / 42$

$4 / 93$

$1 / 164$

$2 / 23$
$i / 10$

$5 \%$
$0,60 x$
$9 \%$
$10 \%$

On peut prósenter ces résultats de manière plus simple en ne reprenant que les pourcentages afférant à chaque période. Pour des raisons d'espace, "directs" remplacera analyse des paragraphes et "CPR" analyse des citations par renvoi.

périodes

\section{modes}

d'acquisition types

de l'esclave d'malyse

\begin{tabular}{|c|c|c|c|c|c|c|}
\hline - vente & $\begin{array}{l}\text { directs } \\
\mathrm{CPR}\end{array}$ & $50 \%$ & $65 x$ & $\begin{array}{l}142 \% \\
60 \%\end{array}$ & $\begin{array}{l}31 \% \\
43 \%\end{array}$ & $\begin{array}{l}56 \% \\
30 \%\end{array}$ \\
\hline - échange & $\begin{array}{l}\text { directs } \\
\text { CPR }\end{array}$ & - & - & $0,60 \%$ & $\overline{-}$ & $0,33 \%$ \\
\hline - stipulation & $\begin{array}{l}\text { directs } \\
\mathrm{CPR}\end{array}$ & - & $\overline{4} \%$ & $\begin{array}{l}11 \% \\
10 \%\end{array}$ & $\begin{array}{l}14 \% \\
22 \%\end{array}$ & $\begin{array}{l}10 \% \\
-\end{array}$ \\
\hline- legs et & $\begin{array}{l}\text { directs } \\
\mathrm{CPR}\end{array}$ & $-\overline{5} \%$ & $\begin{array}{l}67 \% \\
25 \%\end{array}$ & $\begin{array}{l}41 \% \\
23 \%\end{array}$ & $\begin{array}{l}46 \% \\
22 \%\end{array}$ & $\begin{array}{l}25 \% \\
50 \%\end{array}$ \\
\hline - donation & $\begin{array}{l}\text { directs } \\
\mathrm{CPR}\end{array}$ & $-\overline{5} \%$ & $\overline{2} \%$ & $\begin{array}{l}2 \% \\
6 \%\end{array}$ & $\begin{array}{l}6 \% \\
4 \%\end{array}$ & $\begin{array}{r}4 \% \\
10 \%\end{array}$ \\
\hline - dot & $\begin{array}{l}\text { directs } \\
\text { CPR }\end{array}$ & $\begin{array}{l}- \\
-\end{array}$ & $\begin{array}{r}33 \% \\
4 \%\end{array}$ & $\begin{array}{l}4 \% \\
0,60 \%\end{array}$ & $\begin{array}{l}3 \% \\
9 \%\end{array}$ & $\begin{array}{r}4 \% \\
10 \%\end{array}$ \\
\hline
\end{tabular}

Il faut d'emblée émettre une réserve à l'égard de la póricde II pour l'analyse des paragraphes. On ne relève en effet, dans cette période, que trois paragraphes, au demeurant tous de Proculus, ce qui n'est absolument pas représentatif de la période. A un niveau moindre, on ne compte pour la période $Y$ que dix citations. C'est un chiffre faible par rapport à celui réalisé par les périodes précédentes.

Utilisons les citations comme complément à l'analyse des paragraphes. A l'exception des legs, où la période $y$ accuse une baisse importante dans l'analyse des paragraphes, baisse toutefois atténuée par le pourcentage obtenu dans l'analyse des citations, l'impression qui émane de ce tableau est celle d'une grande continuité.

Prenons par exemple le cas de la vente. De nombreux textes où il est question de ventes d'esclaves concernent l'édit des Édiles. Or, on sait que cet édit apparaît déjà dans les comédies de Plaute, mort en 184 av. J.C. (16). Si l'on tient compte aussi de l'afflux de main-d'beuvre servile provoqué par les conquêtes $(17)$, on n'est pas étongé de rolever, pour les deux premières périodes, $c^{\prime}$ est-à-dire pour la période républicaine et le premier siècle de l'Empire, des pourcentages de l'ordre de 50 à $65 \%$ dans $l$ 'analyse des citations. Cependant, c'est sous la période $v$ que les textes traitant de ventes d'esclaves atteignent leur pourcentage maximal dans l'analyse des paragraphes, avec $56 \%$. Cela ne signifie pas que ce soit durant cette période qu'il y ait eu le plus de ventes d'esclaves, mais plutôt que ces ventes ont le plus préoccupé les juristes. Encore convientil de remarquer qu'on ne possède pas toutes les données en la matière. Il est ainsi possible que les juristes de cette époque aient simplement plus utilisé l'esclave comme un exemple destiné à illustrer des hypothèses de vente. Ils auraient donc pu être ainsi plus préoccupés par le contrat de vente que par le problème plus spécifique des ventes d'esclaves. Force est donc d'admettre à ce stade que le droit peut constituer un miroir dáformant de la réalité. Mais il permet de faire une constatation intéressante. Bien que la crise de l'esclavage soit alors certaine, les rentes d'esclaves continuent à alimenter les textes juridiques, et ce au-delà d'une moindre rentabilité supposée du travail servile (18).

Cet exemple de la vente peut être transposé aux autres catégories étudiées sauf la réserve émise pour les legs à propos de la période v. Les pourcentages obtenus à l'intérieur de chacune de ces rubriques sont du même ordre de grandeur.

Ainsi, les modes d'acquisition de l'esclave ne semblent aucunement affectés par la crise de l'esclavage. En est-il de même en matière d'utilisation du travail servile par un autre que le mâtre?

16- J. MACQUERON, Histoire des obligations, cit., p. 135.

17- v. E.M. SCHTAERMAN, La schiavitū, cit., p. $13 \mathrm{~s}$; J. KOLENDO, Il lavoro servile e $i$ mutamenti delle tecniche agrarie nell'Italia antica dal I secolo a.c. al I secolo d.C., in Storia sociale ed economica dell'età classica negli studi polacchi contemporanei, Milan (1975), p. 18 s.

18- Selon J. KOLENDO, o.c., p. 23, la thèse traditionnelle sur la moindre rentabilité du travail servile a l'époque impériale (par rapport a 1 'époque rêpublicaine) ne peut être démentie en l'état actuel des sources. 
Il s'agit d'une utilisation seulement provisoire de l'esclave. Toutefois, le délai d'exploitation peut, comme on le verra, être très important. Cette utilisation du travail servile sans acquisition de la personne du producteur peut apparaitre sous plusieurs formes.

D'une part, l'esclave peut faire l'objet d'un contrat réel, le prêt à usage ou commodat (19). Le commodant prête l'esclave gratuitement, mais le commodataire ne peut $s^{\prime}$ en servir autrement qu'il a été convenu sous peine de commettre un furtum usus (20). Le travail servile peut aussi faire l'objet a'un contrat consensuel, la locatio-conductio. La partie à laquelle le contrat procure un avantage (le conductor) doit payer à l'autre (le Locator) une rémunération (merces). Le conductor $n$ 'obtient en principe sur l'esclave reçu ni la propriété ni mème la possession, mais une simple détention (21). La locatio-conductio a d'abord revêtu l'aspect d'une locatio rei, lougge de l'esclave luj-mêrne (22) pour s'étendre ensuite à la locatio operamm (23).

19- Il est question d'un servus commodatus chez Africain D 13.7.31 (Julien) ; D 47.2.62.6 ; Gaius D 13.6.18 pr-2 ; Papinien D 41.2.47 (Nerva f.); Paul D 9.4.22 pr; D 13.6.22 ; Ulpien D 2.14.50; D 13.6.5.6 (veteres) -7 (Namusa, Mela)-13 (Cartilius); D 47.2.14.15

20- J. MACQUERON, Histoire des obligations, cit., p. 95 ; W.W. BUCKLAND, Roman law, cit. servus commodatus, p. 265. Les deux autres contrats réels que sont le depôt et le gage n'ont pu être intégrés a cette étude, le dépositaire n'ayant, pas plus que le créancier gagiste, le droit de se servir de 1 'esclave déposé ou engagé (J. MACQUERON, o.c., p. 94, $95 \mathrm{~s}$, $\mathrm{k} . \mathrm{K}$ BUCKIAND, o.c., servus depositus p. 265 s. et servus pigneraticius, p. 281 s.).

21- J. MACQUERON, o.c., p. 155. Cependant, F.M. DE ROBERTIS (Lavoro e lavoratori nel mondo romano, Bari (1963), p. 157 et Ancora sul receptim noutarrom, in Riv. dir. navigazione, 24 (1958), p. 254 a. 60) a montré la possibilitê d'un transfert de propriété occasionné par la locatio, et ceci tême 1 'époque classique, en se fondant sur $D$ 19.2.31. Dans ce fragment, on peut effectivement lire ces lignes d'Alfenus : remm locatomon duo geneo peut effectivenent lire ces lignes d'Alfens: remon locatarion duo genera esse, ut aut idem redderetur aut eiusdem generis... ce qui entraine : ex superiore causa rem domini manere, ex posteriore in creditum iri. Ulpien présente cette mutatio dominii come exceptionnelle, mais cependant toujours vérifiable : non solet locatio doninium mutare (D 19.2.39).

22- Concernant 1a Zocatio de 1 'esclave Iavolenus D 18.6.17; D 19.2.60.7 (Labéon) ; Africain D 41.2.40.3 ; D 47.2.62.5-6 ; Marcellus D 19.2.48.1 ; Papinien D 12.6.55 (par.a contrario : on a servus non locatus) ; Paul D 4.9.6.1-2 (Pomponius)-4; D 11.3.14.7; D 19.2.42; D 19.2.43; D 19.2 45.1 ; Ulpien D 2.14.50; D 16.3.1.10

23- Concernant la locatio des operue de 1'esclave Scaevo1a D 45.3.18.3 (Julien) ; Papinien D 33.2.2 ; Tryphoninus D 48.15.6.1 (rescrit d'Hadrien); Paul D 7.1.26 ; D 9.4.19.1 ; Ulpien D 7.1.25.2; D 9.2.27.34 (Mela) (servrm
On peut d'autre part profiter du travail servile gratuitement par la concession de I'usus fructus (24) ou de l'usus seulement (25). L'usufruit, en principe établi par testament, peut l'être aussi inter vivos, ce qui n'entraîne aucune différencé quant aux droits de l'usufruitier qui n'est jamais propriétaire du serrus fructuarius (26). L'usufruit peut être concéđé pour un certain délai, et même quelquefois à vie (27). Il est alors évident que le caractère viager de ce droit fait de l'usufruit

conduction ad mulum regentum); D 16.3.1.9; D 19.2.9.1 (Marcellus); D 24 3.7.10. Les operae de 1 'esclave peuvent bien entendu être louées par son maître (D 9.4.19.1; D 16.3.1.9). Elles peuvent aussi l'être par l'usufruitier (D 19.2.9.i), ou, ce qui est plus remarquable, par l'esclave lui-même (D 7.1.25.2; D 7.1.26; D 45.3.18.3; D 33.2.2). Dans ce dernier cas, le salaire va au maître, mais peut aussi tomber dans le pécule ( $\mathrm{J}$. MACQUERON, o.c., p. 157).

24- Traitent du serrus fructuarius Celsus D 46.3 .69 ; Neratius D 45.3. 22 ; D 45.3.24 ; Julien D 2.14.55; D 7.1.35.1 (Sabimus); D 15.1.37.3; D 19.1.24 pr ; D 21.2.39.5; D 40.5.47.1; D 41.1.37.2-5; D 41.3.35; D45.3. 1.5;Pomponius D9.4.18;D15.1.2;D21.1.48.6;D32.74;D33.2.20;D45.3.39(Gaius);Afri cain D7.1.36.2;Gaius D7.1.31;D7.1.45;D7.7.3;D7.7.4;D9.4.27pr-1;D9.4.30;D40.12 $9 \mathrm{pr}: \mathrm{D} 41.1 .10 \mathrm{pr}-3-4-5 ; \mathrm{D} 41.1 .43 .2 ; \mathrm{D} 41.3 .36 .1 ; \mathrm{D} 45.3 .28 \mathrm{pr}$; Maecianus D 49.17.

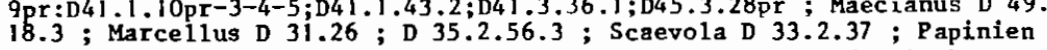
D 33.2.24.1; D 41.2.4. pr ; D 45.1.118 pr; D 45.3.18.3 (Julien); D 49.17 .15 .4 ; Tryphoninus D 41.1 .63 .3 ; Paul D $7.1 .24 ; \mathrm{D} 7.1 .26$; D 7.1 . 31 ; D 7.1.66; D 7.4.26; D 7.4.27; D 7.9.10; D 9.2.12; D 9.4.17.1; D $9.4 .19 .1 ; D 9.4 .26 .6 ; D 11.3 .14 .3 ; D 21.1 .43 .8-10 ; D 33.2 .21 ; D 35$ $1.96 \mathrm{pr}-1$; D 35.2.1.9(veteres) ; D $40.7 .7 ; \mathrm{D} 41 . \mathrm{I} .47 ; \mathrm{D} 41.1 .49 ; \mathrm{D} 41$. $2.1 .8 ; \mathrm{D} 45.3 .27$; D 45.3.31; D 45.3.32;D 45.3.33.1; D 46.3.63; D 46.3.98.7 ; D 46.4.11 pr ; Ulpien D 7.1.12.3 (Julien, Pomponius) 4

(Julien)-5 (Julien) ; D 7.1.15.1-2-3 (Labéon) ; D 7.1.17.1 (Cassius, Aristo) $-2-3$; D 7.1.21 (Labếon) ; D 7.1.22 ; D 7.1.23 pr (Julien)-1 (Sabinus, Cassius) ; D 7.1.25 pr-1 (Julien, Mauricianus, Marcellus)-2-3 (Julien)-4-5

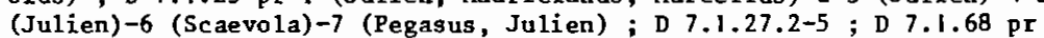
(Brutus); D 7.4.5.1 ; D 7.4.12.1 ; D 7.4.15; D 7.6.5.4 (a) -5; D 10.4 .3 . 12 (Pomponius); D 11.3.9.1; D $14.4 .1 .5 ; \mathrm{D}$ is.1.13 (Julien); D 15.1.15; D 15.1 .19 .1 (Marce1lus) -2 ;D $15.1 .32 \mathrm{pr}$ (Julien); D 15.2.1.9 (Pomponius) ; D 15.1 .19 .1 (Marce1lus)-2 ;D 15.1.32 pr (Julien); D 15.2.1.9 (Pomponius); ; D 24.1.3.8; D 28.5.9.20; D 28.7.2 pr; D 29.2.25 pr; D 29.5.1.1-2; D $32.73 \mathrm{pr}$; D 38.2.16.1 ; D 40.1.4.12 (constitution de Marc-Aurèle et Verus); D 40.2 .2 ; D 40.4 .6 ; D $40.12 .8 \mathrm{pr}-2$; D 42.1 .4 .8 (Ce1sus) ; D 47.2.43.12 ; D 47.2 .46 .1 ; D $47 \cdot 10.15 \cdot 37-47-48$; D 47.10 .17 .9 ; D 48.5.28.3-8-11 ; Hermogénien D 35.2.38.2; D 40.9.27.1.

25- Le cas du eervus usuarius ressort de Pouponius D 7.1.55; D 7.8.16.2 ; D 15.1 .2 ; T. Clemens D 7.7.5 (Julien) ; Marcellus D 7.8.20; Paul D 7.8. $15 \mathrm{pr}$; D 35.2 .1 .9 (veteres); D 45.3.23 ; D 46.4.11 pr ; U1pien D 4.3.7. 4 ; D 7.8.12.5-6 (Labéon, Sabinus, Octavenus) ; D 7.8.14 pr ; D 47.2.46.3 ; D 48.5.28.6. Il est d'autres textes où apparaît le mot usus, mais ne $s$ 'agissant pas d'un legs d'usage, il ne convenait pas d'en tenir compte (par ex. D 24.1.31.10; D 24.1.58.1).

26- W.W. BUCKIAND, Roman 1aw, cit., p. 356. Ainsi, un legs de "ses esclaves" n'inclut pas ceux sur lesquels on n'a qu'un usufruit, mais englobe par contre ceux dont on a attribué l'usufruit à un tiers (cf. D $32.73 \mathrm{pr}$; D 32.74$)$.

27- A. GUARINO, Diritto privato romano, cit., p. 657. 
un mode d'acquisition duratle du travail de l'esclave. Qunt all simple usage, il Jonne troit ǹ l'usus et non nu fmuctus, c'est-à-dire que l'1sanger peut acquérir ex re mais non ex operis, l'acquisition ex re étant une forme d'uti (28). Il a néanmoins droit anx operae de l'esclave qu'il peut utiliser non seulement dans son intérôt personnel, mais aussi pour les besoins de sa fomilia (20). Les operae servorum ressortent d'ailleurs des textes comse une sorte d'usus. Four Julien et T. Clemens, l'assimilation entre legs des travaux de l'esclave et legs d'usage est parfaite (30).

Ce n'est qu'à l'époque des sévères qu'n voit poindre une distinction dont Paul dénote encore la fragilité (31).

Comptabilisons, comme on l'a fait auparavant, pour les modes d' acquisition de l'esclave, les modes d'itilisation de sa force de travail.

- analyse des paragraphes

$\begin{array}{lcccc}\text { période } & \text { commodat } & \text { J.ourge } & \text { usufruit } & \text { usrge } \\ \text { I } & - & - & - & - \\ \text { II } & - & - & - & - \\ \text { III } & 4 & 5 & 35 & 4 \\ \text { IV } & - & 2 & 4 & 1 \\ \text { V } & 8 & 19 & 102 & 13 \\ \text { TOTAL } & 12 & 26 & 141 & 18\end{array}$

28- cf. D 7.8.14 pr : Fer servun usuarium si stipuler vel 'per traditionen' accipiom, an adquirom, qraeritur, si ex re mea vel ex operis eius, et si quidem ex operis eius, non valebit, quoniam nec locare operas eius possumus : sed si ex re mea, dicimus servwm usuarivon stipulantem vel 'per traditionem' accipientem mihi adquirere, cwn hac opera eius utor.

29- W.w. BUCKIAND, Roman law, cit., p. 369.

30- cf. D 7.7.5 : Operis servi legatis usw datum intellegi et ego didici et Iulianus existimat.

31- cf. D 35.2.1.9: ... Sed operis servi legatis cum neque usus neque usus fructus in eo legato esse videtur... L'incertitude persistante entre legs d'usage et legs des travaux de l'esclave nous a conduit assimiler ces deux notions, en intégrant donc les textes traitant de legs des operae (T. Clemens D 7.7.5 (Julien) (déjà pris par ušm datuon) ; Papinien D 33.2.2 ; Paul D 35.2.1.9 (veteres) (déjà pris par usus); Ulpien D 7.7.2; D 33.2.7) dans la rubrique concernant le legs d'usage. C'est un choix, il est vrai, quelque peu arbitraire car si, pour Julien et T. Clemens (D 7.7 .5 cit.), les quelque peu arbitraire car si, pour Julien et T. Clemens (D 7.7 .5 cit.), les
operae servorm sont une sorte d'usus, pour Gaius (D 7.7 .3 ; In hominis usu operae servomon sont une sorte d'usus, pour Gaius (D 7.7.3; In hominis usu
fructu operae sunt et ob operas mercedes) et Papinien (D 33.2.24.1 : Scorpum servom merm Semproniae concubinae meae servire volo. Non videtur proprietas servi relicta, sed usus fmuctus), il s'agit d'une sorte d'usufruit.

\section{- analysa des citations}

\begin{tabular}{|c|c|c|c|c|}
\hline période & commodat & louage & usufruit. & IS $3=0$ \\
\hline I & 2 & - & 2 & 1 \\
\hline II & 3 & 2 & 5 & 2 \\
\hline IIT & 1 & 2 & 18 & 2 \\
\hline IV & - & 1 & 5 & 1 \\
\hline$v$ & - & - & - & - \\
\hline TOTAL & 6 & 5 & 30 & 6 \\
\hline
\end{tabular}

Il ressort de ce tableau que le mode d'exploitation le plus courant de l'esclave par une autre personne que le maitre est l'usufruit : 72,6 dans 1 'snalyse des paragraphes et $64 \%$ dans celle des citations.' Quant aux autres modes, ils apparaissent en rapport dans une faible proportion : ainsi, l'usage regroupe $9 \%$ des paragraphes concernant 1 'exploitation du travail servile et $13 \%$ des citations effectuées sur ce même problème ; le louage en comprend respectivement $13 \%$ et $10 \%$, et le commodat $6 \%$ et $13 \%$

Penchons-nous désormais sur l'évolution chronologique de ces actes juridiques en suivant la même démarche qu'en matière d'acquisition de l'esclave, c'est-æ̀-dire en comparant le chiffre réalisé par une période sur une de ces catégories par rapport au nombre total de textes concernant l'utilisation de la force de travail servile pour cette période.

Analyse des paragraphes - conmodat

$\begin{array}{llllllll}\text { I } & - & & - & \text { I } & 2 / 5 & =40 \% \\ \text { II } & - & = & - & \text { II } & 3 / 12 & = & 25 \% \\ \text { III } & 4 / 48 & = & 8 \% & \text { III } & 1 / 23 & = & 4 \% \\ \text { IV } & 0 / 7 & = & - & \text { IV } & 0 / 7 & = & - \\ \text { V } & 8 / 142 & & 6 \% & \text { V } & - & = & -\end{array}$

Bien que ne résolvant pas explicitement la question, Justinien semble considérer les operae servormm coume quelque chose de distinct mais d'analogue à 1 usus (cf. A. GUARINO, Diritto privato romano, cit., p. 670, n. 67.6). C'est cette position qui a été suivie. 
Analyse des paragraphes

- louage

$\begin{array}{llllllll}\text { I } & - & & - & & \text { I } & 0 / 5 & = \\ \text { II } & - & & - & & \text { II } & 2 / 12 & =17 \% \\ \text { III } & 5 / 48 & = & 11 \%(+) \text { III } & 2 / 23 & =9 \% \\ \text { IV } & 2 / 7 & & =29 \% & \text { IV } & 1 / 7 & =14 \% \\ \text { V } & 19 / 142 & & 13 \% & \text { V } & - & =\end{array}$

- usufruit

\begin{tabular}{|c|c|c|c|c|c|}
\hline I & - & $=$ & I & $2 / 5$ & $40 \%$ \\
\hline II & - & $=$ & II & $5 / 12$ & $41 \%(+)$ \\
\hline III & $35 / 48$ & $73 \%$ & III & $18 / 23$ & $78 \%$ \\
\hline IV & $4 / 7$ & $57 \%$ & IV & $5 / 7$ & $72 \%(+)$ \\
\hline $\mathrm{v}$ & $102 / 142$ & $72 \%$ & $\mathrm{~V}$ & - & $=$ \\
\hline
\end{tabular}

- usage

$\begin{array}{lllllll}\text { I } & - & & - & \text { I } & 1 / 5 & =20 \% \\ \text { II } & - & = & - & \text { II } & 2 / 12 & =17 \% \\ \text { III } & 4 / 48 & = & 8 \% & \text { III } & 2 / 23 & =9 \% \\ \text { IV } & 1 / 7 & =14 \% & \text { IV } & 1 / 7 & =14 \% \\ \text { V } & 13 / 142 & = & 9 \% & \text { V } & - & =\end{array}$

ce qui entraîne

périodes

modes $d^{\prime}$ utilisation de la force
de travail servile

$\begin{array}{ll}\text { - commodat } & \begin{array}{l}\text { directs } \\ \text { CPR }\end{array} \\ \text { - louage } & \begin{array}{l}\text { directs } \\ \text { CFR }\end{array} \\ \text { - usufruit } & \text { directs } \\ \text { CPR } \\ \text { - usage } & \text { directs } \\ \text { CPR }\end{array}$

On remarque, et ceci vaut pour les deux analyses entreprises, une continuité de ces catégories juridiques durant les périodes envisagées. Ainsi, de même qu'en ce qui concerne les modes d'acquisition de l'esclave, il n'y a aucune évolution importante des modes d'utilisation de son travail. La crise de l'esclavage est donc inopérante à cet égard. Elle ne modifie pas les modes d'acquisition de la main-d'oeurre servile.

Pour avoir une idée plus précise de ce que représente l'acquisition de la main-d'ourre servile dans le discours des juristes, il est nécessaire de sortir du schéma dans lequel l'analyse a jusqu'à présent été confinée. Il faut y introduire un élément de comparaison. Autrement dit, l'esclave est-il plus ou moins fréquenment acquis qu'un autre élément patrimonial ? La société romaine étant principalement agricole (32), la terre,en tant que moyen de production, offrait une comparaison valable. Il faut cependant être conscient du fait que l'acquisition d'un fonds de terre et celle d'un esclave peuvent être concomitantes. C'est le cas dans le legs d'un fonds cum servia (33), mais cela ne créait aucun déséquilibre, le texte étant alors relevé dans les deux rubriques.

Comme l'esclave, le fonds de terre peut faire l'objet d'une vente (34)

32- v. M. MAZZA, Lotte sociali e restaurazione autoritaria nel III sec. d.C., Rome, 1973, p. 155 s. Selon cet auteur, la structure économique impériale repose sur trois fondements traditionnels : l'agriculture, l'artisanat spécialisé et la spéculation. Mais en réalité, la base productive essentielle est l'agriculture, et malgré la prêsence d'une certaine quantité de capital privé, il serait erroné de représenter l'économie romaine comme un système capitaliste. La seule véritable richesse, jouissant de la considêration sociale, est la richesse foncière (cf. le richissime affranchi du Satiricon de Pétrone, Trimalcion qui, dès qu'il le pouvait, investissait les capitaux gagnés dans $1^{\prime}$ acquisition de terres, $\nabla$. P. VEINE, Vie de Trimalcion, in Annales société, Economie et Civilisation (1961), p. 213247).

33- Eypothèse fréquente, notamment in $D 33.7$, De instructo ve 1 instrumento legato (ex. Scaevola D 33.7.20 pr-1-2-3); sur ce point, M. HORVAT, legation fundi e servi, in St. Volterra 5 (1971), p. 89-97.

34- Un fonds peut être désigné par plusieurs termes : principalement funcus et praedium, mais aussi par ager et même area, terra, saltus, solum, loctus. On peut aussi prësumer l'existence $d$ 'un fonds par silva ou horti.L'interpr tation des textes a donc été, comne pour l'esclavage, extensive. Fonds est objet de vente chez Alfenus (chez auteur anonyme) D 19.1.26; Proculus D $18.1 .68 \mathrm{pr}-2$; D 19.5.12 ; Lavolenus D $7.1 .54 ; 08.1 .19$ (Labéon) ; D 8. 4.5 (par solum) ; D 17.1.36.1-2-3; D 18.1.63.1 ; D 18.1.64 ; D 18.1.65; D 18.1 .77 (Tubero, Labéon) ; D 18.1 .78 pr (Labéon) (par fístulasicastelizum) -1 (Labêon) -2 (Labéon); D 18.1 .79 (Trebatius, Labẻon); D $18.1 .80 \mathrm{pr}$ (Labéon) -2 (Servius, Labéon) (par silva) ; D i $8.2 .19 ; \mathrm{D} 19.1 .51 .1$ (LaD 41.4.4 pr-1; D 47.7.12 (par agmom); Neratius D 17.1.35; D 18.3.5; 
D 21.2.48; Celsus D 6.1.38; D 8.6.12 (Sabinus, Neratius); D 18.1.59 (Q. Mucius) ; D 21.2.62.2; D 41.2.18.2; D 50.16.88; Julien D 3.3.75; D 4.4.41; D 8.3.31; D 18.1.41 pr; D 19.1.22; D 19.1.28; D 21.2.39 Pr -2 ; D 21.2.40; D 22.1.25.2 ; D 23.4.13 (fonds sous-entendu) ; $D 27.10$. 7.3; D 29.2.44; D 30.82.2 ; D 35.2.87 pr-1; D 41.3.33.1; D 41.4.7.1-2 -4-6 ; Pomponius D 7.8.22 pr (Hadrien otatuit) (par silva); D 8.3.25; D 8.6.19 pr-1 ; D 13.7.6 pr-1 ; D 16.1.32.2 ; D 18.1.6.1; D 18.1.18.1 (A1fenus) (par ager) ; D 18.1.20 (Sabinus) (par area) ; D 18.1.33 (par flumina); D $18.1 .66 \mathrm{pr}-1-2$ (Q. Mucius) (par a contrario) ; D 18.2.15 pr-1 ; D 18.3.2 ; D $19.1 .6 \mathrm{pr}^{-2}$ (par areim) -3 (par locum) -6 ; D 19.1.16; D 21.1.64 pr (La-

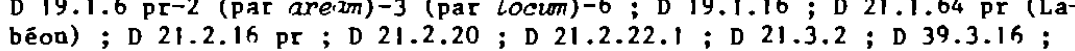
béon); D 21.2.16 Pr; D 21.2.20; D 21.2.22.1 ; D 21.3.2 ; D 39.3.15;

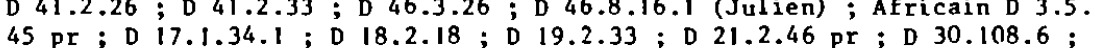

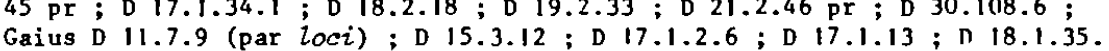
8 ; D 18.6.9; D 21.1.32; D 21.2.6; D $30.66 ; \mathrm{D} 39.3 .13$ (fonds sousentendu); Maecianus D 35.2.30.1 ; Venuleius D 21.2.75 (Q. Mucius, Sabinus) ; Marcellus D 8.6.13 ; D 13.7.34; D 18.1.60; D 30.82 .3 (Julien) ; D 30.92 pr (Julien) ; D 41.2.19 pr ; D 43.16.12 ; P. I ustus D 50.8 .11 .2 (rescrit de Marc-Aurèle et Verus) (par agros reipublicae); Scaevola D 2.14.47 pr ; D 4.4.39.1; D 4.4.47.1; D 8.5.20.1; D 10.2.39.5; D 13.7.43 pr (par Locum); D 18.1.81 pr-1; D $18.3 .6 \mathrm{pr}-1$; D 18.3.8; D 18.5.8; D 18.5.9; D $18.5 .10 \mathrm{pr}-1 ; \mathrm{D} 18.6 .11$ (Julien); D 19.1.48; D 19.1.52 pr-1; D 20.1. $31 \mathrm{pr}-1$; D 21.2.12; D 21.2.69.5-6; D 23.3.85; D 26.7.57.1; D 31.89.4-7

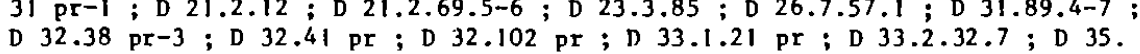
2.19 ; D 36.1.80.15; D 41.4.13 (par area); D 41.4.14; D 41.4.15; D 45 1.135 .2 (par hortos); D 46.1.45; D 42.8.21 ; Papinien D 3.3.67; D 6.1. $65 \mathrm{pr}$; D 16.1.27.1; D 18.1.58; D 18.1.73 pr (par locus)-1 (par hortis) ; D 20.1.1.2 ; D 20.5.2; D 20.6.11 ; D 21.2.64 pr-1 (par terrae)-2 (sousentendu) $-3-4$ (par 80 lom) ; D 21.2.66 pr-1-3 (par agri) ; D 23.5.12.1 D 31.77 .5 ; D 31.78 .1 (Sévère iussisset) ; D 32.91.3-5 (par hortum) ; D 34 . Tryphoninus D 23.3.78.3; D 32.38.5; D 36.1.80.6; Callistrate D 6.1.50 pr (par ager); D 21.2.72; Paul D 4.4.24.4;D 4.4.38 pr;D 6.1.37 (par area) (Julien)'; D 8.3.23.3 ; D 8.3.29; D 8.3.30; D 8.3.36; D 8.4.12; D 10.1 .12 ; D 10.2.25.8 (Pomponius) ; D 11.7 .11 (par locus) ; D 17.1.5.3; D 17.1 .15 ; D $17.1 .45 \mathrm{pr}$; D 18.1.34 pr ; D $18.1 .40 \mathrm{pr}-1-2$ (par ager)-3-4-5 : D 18.1.51; D 18.2.1; D 18.2.14.1 (Labéon, Sabinus, Nerva)

-2; D 18.6.7 pr-1; D 19.1.4.1 (par ager); D 19.1.8 $\mathrm{pr}^{-1}$; D 19.1.21.1-4 -5 ; D 19.1.27 ; D 19.1.42 (Labéon); D 19.1.45.1 (par area) ; D 19.1.53.1 (Labéon); D 19.1.54.1 (Labéon); D 19.2.20.2; D 20.4.17; D 20.5.10; D 21. $1.44 \mathrm{pr}$ (Pedius); D 21.2.11 ; D 21.2.13 (Proculus) (fonds sous-entendu) : D $21.2 .15 \mathrm{pr}$ (fonds sous-entendu) -1 ; D $21.2 .41 .2 ; \mathrm{D} 21.2 .45$ (A1fenus); D $21.2 .53 \mathrm{pr}$; $\mathrm{D} 23.5 .10$ (fonds sous-entendu); D 24.1.55 (par soli rem); D 27.9.13.1 (Papinien); D 27.9.14; D 39.2.18.7; D 39.3.7.1; D 39.3.9 pr ; D 39.3.12; D 39.3.14 pr (Ateius); D 41.3.13.2; D 41.4.2:6 (Neratius); D 42.8.7 (Proculus) ; D 50.16.169; D 50.16.205; U1pien D 1.21 .2 .1 ; D 2 . 14.52.1; D 2.15.8.15; D 3.5.16 (par area) ; D 5.3.20.2 ; D 6.1 .37 (Julien) (par area) ; D 6.1.72 ; D 6.2.11.9 (par aream) ; D 7.4.29 pr (Pomponius) -1) D 8.4.6.3 ; D 8.4.13 pr ; D 8.5.2.3 (Labéon, Julien, Pomponius) (fonds sousentendu) ; D 8.5.3 ; D 10.3.6.8 (Julien) ; D 11.6.1 pr (par ager) ; D 11. 7.8.1 (par Zocus) ; D 11.7.10; D 12.2.26.7 (Julien) ; D 13.7.11.4; D 16 . 1.8.1 (Papinien) ; D 17.1.8.6 ; D 17.1.10 pr-2 ; D 17.1.12.8 (Papinien); D $17.2 .52 \mathrm{pr}-7$ (Papinien) (par Zoci)-13 (Mela) (par arean); D 18.1.7.2 ; D $18.1 .9 \mathrm{pr} ; \mathrm{D} 18.1 .25 .1$; D 18.1.37; D 18.1.47; D 18.2.2 pr ; D 18.2.4. D $18.1 .9 \mathrm{pr} ; \mathrm{D} 18.1 .25 .1$; D 18.1 .37 ; D 18.1 .47 ; D $18.2 .2 \mathrm{pr}$; D 18.2 .4 .
3 (Marcellus) -4 (Julien) : D $18.2 .11 \mathrm{pr}$ (Sabinus, Julien) ; D 18.2.13 pr ; (Brutus, Q. Mučus. Lavéon, Sabinus, Celsus); D 18.3.1; D 18.3.4 pr (rescrit de Sévère et Caracalla)-3 ; D 19.1.1.1 (fonds sous-entendu) ; D 19.1.13.6 (Julien)-10 (par ager)-11 (par ager)-14 (Neratius)-15-17 (Ce1sus)-24; D 19.1.15; D 19.1.17 pr-2 (Trebatius) -6 (par harena) (Gallus Aquilius, Mela)-11; D 19.1.34;D 19.1.35; D 19.2.9 pr (Pomponius); D 21.1.61 Mela)-1I ; D 19.1.34; D 19.1.35 ; D 19.2.9 pr (Pomponius) ; D 21.1.61
(fonds sous-entendu) ; D 21.1.33 pr (Pomponius) ; D 21.2 .1 (par ager); (fonds sous-entendu) ; D 21.1.33 pr (Pomponius); D 21.2 .1 (par ager) ;
D 21.2 .14 (fonds sous-entendu); D 21.2 .23 (idea) : D 21.2 .51 .2 (par loD 21.2 .14 (fonds sous-entendu); D 21.2 .23 (idem): D 21.2 .51 .2 (par 20-
crm): D $21.3 .1 \mathrm{pr}$ (Marcellus)-1; D $26.1 .5 \mathrm{pr}$ (par ager); D 20.9 .2 ; D 23. 5.2 ; D 24.1.7.6; D 27.3.13; D 27.9.3 pr ; D 27.9.5.2-3-7-9-10-14 : D 27. 9.9 ; D 27.9.10; D 27.9.11; D 30.39.3 (Julien) ; D 30.47.6 (Labéon) D 30.49 .8 ; D 30.49.9 (fonds sous-entendu) ; D 42.1.15.12 ; D 43.18.1.1 (par soli) ; D 43.19.3 pr-2 (Pedius) (par locum)-7; D43.20.1.16 (Labéon) (par agrum) -24 ; D 44.2.11.9 ; D 44.4.4.12-32 ; Marcien D 20.6.8.7 (par agor) D23.5.17;D41.2.43pr (Julien); Modestin D10.2.30;D10.3.17;D13.7.39;D18.1.62pr; D19.1.39:D20.6.9pr;D21.2.63.1;D26.7.32.4;D41.3.18;D 50.1.36.1; Hermngênien P5-18.1.75. Fonds est objet de stipulation chez Iavolenus D 45.1.106 ; Julien D 45.1.56.7 ; D 45.1.58 : D 45.3.10; D 46.3.33 pr ; Pomponius D 45.1.9; Gaius D 45.1.74; Scaevola D 45.1.131.1 ; Papinien D 45.1.115 pr ; D 46.2. 28 ; Paul D 45.1.78.1; D 45.1.85.6; D 45.1.126.1; D 46.3.98.8 (par area) ; D 50.16.169; Ulpien D 27.9.5.6; D 45.1.38.21; D 45.1.72 pr (Tubero, Celsus) ; D 45.1.75.1-3-10; D 45.1.103 (a contrario) : D 45.1.114; D 46.4.13. $1-2-5$.

36- Fonds est objet de legs ou de fidéicoumis chez Proculus $D$ 50.16.126 ; Iavolenus D 7.1.54; D 31.10; D 31.39 (par area); D $31.41 \mathrm{pr} ; 032.30 .4$ (Labéon) ; D 32.39 .4 (Tubero, Labéon); D 33.7 .4 (Cascellius, Trebatius, Labéon); D 33.7 .25 pr (Tubero, Labéonł̀ (Trebatius, Labéon)-2 (sous-entendu) (Ofilius) ; D 33.7.26.1 (idem) (Cascellius, Ofilius, Trebatius, Labéon) D 35.1 .40 .1 (Cascellius, Ofilius, Cinna) 4 (Trebatius, Ofilius, Labéon) D 35.1 .55 ; D 35.1 .56 ; D 35.1 .67 ; D 35.2 .61 ; Celsus D 31.27 ; D 32 .

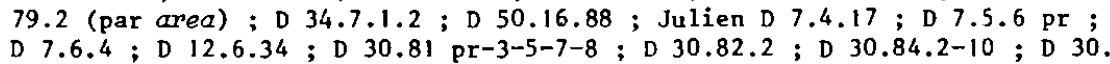
86.3-4 (par superficies) ; D 30.104 .4 ; D 33.2 .10 ; D 35.5 .11 ; D 34.5 . 13.1 ; D 35.1 .23 ; D 35.1.25; D 35.1 .30 ; D 35.2.86; D 39.6.20; D 40.4 . 16 ; Pomponius D $30.8 \mathrm{pr}$; D $30.24 .2-3-4$; D 30.38 .1 ; D 32.16 (par ager); D $32.44 ; \mathrm{D} 32.54 ; \mathrm{D} 33.7 .15 .2 ; \mathrm{D} 33.7 .21 ; \mathrm{D} 34.4 .2 \mathrm{pr}-1$ (fonds sous-) entendu) -2 ; D 35.1 .105 ; D $35.1,112.1-2$; Valens $D 36.4 .15$ (par locum); Africain D 7.2.9; D 30.108.6; D 30.109.1; D 32.64; D 38.2.26 ; D 41.1. 40 ; T. Clemens $D 31.54$; Gaius D 8.4.3; $30.66 ; 0.69 .3 ; 030.70 .1$ D $35.1 .17 .2-3$; Marcellus D 8.6.11.1; D $30.92 \mathrm{pr}$ ('ulien): D 31.23 ; D 33 . 2.15 .1 ; D 33.3.3 ; D 35.1.36.1 ; D 35.2.54; D 38.2.31 ; D 46.3.68; Florentinus D $30.116 .1-4$; Scaevola D 8.5.20 $\mathrm{pr}^{-1} ; \mathrm{D} 15.1 .54 ; \mathrm{D} 15.1 .58 ; \mathrm{D} 31$. 88.6-9 ; D 31.89.4; D 32.34.3; D 32.35.1-2; D 32.37 pr ; D 32.38 pr-1-7-8 D 32.39 .2 ; D $32.40 \mathrm{pr}$ (par agri)-1 (par hortis) ; D $32.41 \mathrm{pr}-3-5-9$; D 32 . 93.2-4-5: D $32.101 \mathrm{pr}^{-1}$; D 33.1.21 pr-1-5; D 33.2.17; D $33.2 .34 \mathrm{pr}$; D 33.2 .35 ; D $33.4 .10 ; \mathrm{D} 33.7 .6$; D $33.7 .20 \mathrm{pr}-1-2-3-4-5-6-7-9 ; \mathrm{D} 33.7$. $27 \mathrm{pr}-1-2-3-4-5 ; \mathrm{D} 33.7 .28$; D 33.5 .22 ; D $34.1 .16 .1 ; \mathrm{D} 34.4 .30 .1 ; \mathrm{D} 34$. $4.31 \mathrm{pr}-1$; D 35.1.108; D 35.2.23; D 35.2.25.1; D 36.1.80.13; D 36.2. 28 ; Papinien D 7.1.57 pr ; D 10.2.33; D 22.1.3.3; D $31.66 \mathrm{pr}$ (Jul ien) $-1-$ 2-4-6 ; D 31.67.3-4-5-6-7; D 31.69.1; D 31.76.2; D 31.77 pr-7-8-10-1113-15-27-28-32; D 31.78.1 (Sévère iussisset)-3 ; D $32.91 \mathrm{pr}^{-1-3-5}$ (par hortzom) ; D 33.4.9; D 33.7.2.1; D 33.7.3 pr-1; D 34.4.24.1 (par horti); D $34.5 .1 ; D 35.1 .72 .4-5 ; D 35.1 .73 ; \mathrm{D} 35.1 .77 \mathrm{pr}$; D 35.2.15.6; D 40 . $5.22 \mathrm{pr}$; D 50.15.5.1 (2itterae d'Antonin le pieux) -2 ; Tryphoninus D 31.88 12 (Scaevola) ; D 32.38.5 ; D 34.3.28.7 ; Callistrate D 33.10.14 ; D 35.1 . 
82 ; Paul D 7.1.50; D 8.1.18(fonds sous-entendu) (Papinien) ; D 11.7 .34 (par locus) ; D 23.5.3 px ; D 23.5.14.3 ; D 29.1.16 ; D 30.5.2 ; D 30.72 ; D 30.85 ; D 30.122 .2 ; 31.49 .2 (Trebatius, Labêon, Fulcinius)-3 (ProcuD ) D 31.82 pr-1 ; D $31.86 .1 ; 031.87,2$ D $32.27 .1 ; 032.60 .3$ (A1lus) ; D $31.82 \mathrm{pr}$; $\mathrm{D} 31.86 .1$; D 31.87 .2 ; $\mathrm{D} 32.27 .1$; 32.60 .3 (A1fenus) ; D 32.78 pr-1-2-3; D 32.89 ; D 33 (Par sal.tu) D 33.2.26 $\mathrm{pr}^{-1}$; D 33.7.1 $\mathrm{pr}^{-1}$; D 33.7.5 (Labéon) ; D33.7.9 (par sal
D 33.7.16.1 (Servius, Alfenus) (par vinea) -2 (Alfenus) ; D 33.7.18.3

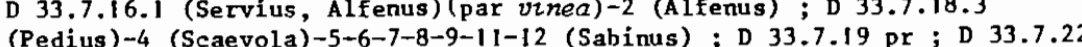
(Pedius) -4 (Scaevola) $-5-6-7-8-9-11-12$ (Sabinus) ; D $33.7 .19 \mathrm{pr} ; \mathrm{D} 33.7$
$\mathrm{pr}-1 ; \mathrm{D} 33.7 .24 ; \mathrm{D} 34.3 .25$ (rescrit d'Antonin le pieux) ; 34.4 .6 $\mathrm{pr}-1$; D 33.7 .24 ; D 34.3 .25 (rescrit d'Antonin le pieux) ; D 34.4 .6 ,

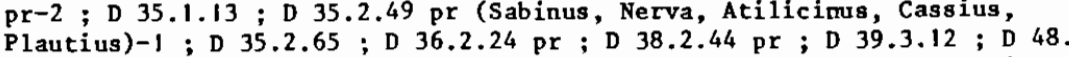
$14.40 \mathrm{pr}$; D 48.10.22.11; U1pien D 2.15.8.15; D 7.4.1.2 ; D 10.2.14.1; D 19.1.17.2 (Trebatius) ; D 23.5.13.4 (Scaevola) ; D 27.9.5.4-8; D 30.4 Pr (Pomponius); D 30.14 pr (Aristo, Celsus, Marcellus);D 30.34.4-12-14-15 ; pr (Pompon ius) ; D 30.14 pr-10; D 30.44.4 (par area) -9; D 30.47.6 (La-

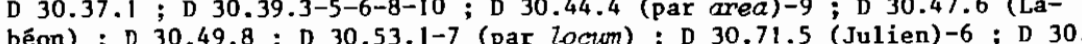
béon) ; D 30.49 .8 ; D $30.53 .1-7$ (par loctom); D 30.71 .5 (Julien) -6 ; D 30. 4.1 .14 (Mela)-15 (Me1a); D 33.4.2.1; D 33.7.8 pr-1; 033.7 .10 (fonds sous-entendu) : D $33.7 .12 \mathrm{pr}$ (par instmomento)-1 (par granaria et instrumenti)-2-3 (Labēon, Pegasus)-4 (Labéon, Neratius) (par saltuarioun)-5 (Trebatius) (fonds sous-entendu)-6 (Servius, Namusa)-7-8-9 (par instmonenti)-10 (par molas, machinas)-11 (Cassius)-12-13 (Sabinus, Cassius)-14-15-27 (Sabinus, Cassius) -28-29 (par horreo)-30 (Celsus)-31 (Celsus)-32-33-34-35 (Neratius)36-37 (Papinien)-38 (Papinien)-39 (Papinien) (par hortis)-41 (Papinien) -44 (Ce1sus) -46 (Papinien)-47 (Papinien (par hortis) ; 034.4 .3 pr-4-6-8; D 36 (Celsus) -46 (Papinien)-47 (Papinien (par hortis); 36.2 .14 .1 (Julien); D 36.4.5.7 (par vectigales agri)22 (par agros); D 37.6.1.12; D 40.5.24.20; Marcien D $30.112 \mathrm{pr}-2$;D 30.114. 22 (par agros) ; D 37.6.1.12 ; D 40.5.24.20 ; Marcien D 30.112 r r-2;D 30.14 3 (Marcellus) -4 (Marcellus) $-15-16$ (Marcellus) -17 ; D 32.13; D 32.67 (par
saltuon); D 35.2.91; Modestin D 22.1.42 (par terra) ; D 31.32.1; D 31 . 32.2 ; D 31.34 .1 ; D 32.82; D 33.2.19; D $34.1 .4 \mathrm{pr}$; Hermogënien D 35.2 . 40.1 .

37- Fonds est objet de donation chez Julien $\mathrm{D}$ 39.6.14; $\mathrm{D}$ 39.6.18.3; Pomponius D 24.1.31.2 (par area) ; D 41.2.26; Gaius D 35.1.17 pr; D 39. 3.13 (fonds sous-entendu); Marce1lus D 24.1.49; D 30.8.2.3 (Julien) ; Scaevola D 24.1.58 pr ; D 32.37.3; D 32.38.3 ; D 39.5.35.1 ; Papinien D 6.1. 48 ; D 20.1.1.4; D 31.77.10; D 41.2.48; Tryphoninus D 32.38.5; Paul D 22.1.28.3; D 31.87.4 (rescrit d'Alexandre-Sêvère) ; D 42.1.41.1 ; D 39.3 . 12 ; Ulpien D 6.1.77; D 17.1.12.8; D 24.1.7.6; D 43.19.3.6 ; Marcien D 44.4.10 (par area).

38- Fonds est objet de dot chez Proculus D 46.3.82 ; Iavolenus D 23.4.32 pr (Labéon); D 23.5.18 pr (Labéon)-1 (Labéon); D 35.1 .50 .4 (Trebatius, ofilius, Labéon) ; Neratius D 25.1.15 (par agrorım) ; D 25.1.16 (fonds sousentendu par colendi) ; Julien D 10.2.51 pr ; D 12.4.7.1 ; D 23.4.13 (fonds sous-entendu) ; D 23.4.22 ; D 23.5.7 pr-1 ; Pomponius D 23.3.32 ; D 23.3.66 ; D 24.3.9 (par agmom) ; Africain D 21.4.9.3; D 23.3.50 pr ; D 23.5.9 pr-1$2-3$; D 23.5.11; Gaius D 23.5.4; Maecianus D 49.17.18.2; Marcellus D 24. 3.57; Scaevola D 19.1.52.1; D 23.3.85; D 23.4.29; D 33.4.10; Papinien D 10.2 .35 ; D 23.3.69 pr; D $23.5 .12 \mathrm{pr}-1$; D 23.5 ; 15 ; D 24.3 .42 .1 ; D $10.2 .35 ; D 23.3 .69 \mathrm{pr}$; D $23.5 .12 \mathrm{pr}^{-1}$; D 23.5 .15 ; D 24.3 .42 .1 ;

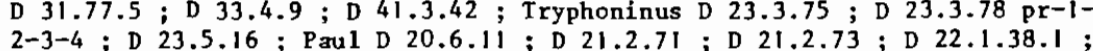
$2-3-4 ; D 23.5 .16$; Pal D 20.6.11 ; D 21.2.7I ; D 21.2.73 ; D 22.1.38.1
D 23.3.56.3 (Nerva, Scaevola) ; D 23.4.12.3-4; D 23.5.1 pr-1 ; D 23.5.3 D 23.3.56.3 (Nerva, Scaevola) ; D 23.4.12.3-4; D 23.5.1 $\mathrm{pr}^{-1} ; \mathrm{D} 23.5 .3$ $\mathrm{pr}^{-1}$; $\mathrm{D} 23.5 .8$ (AIfenus) ; $\mathrm{D} 23.5 .10$ (fonds sous-entendu) ; $\mathrm{D} 23.5 .14 \mathrm{pr}$ D 25.1.6; D 25.1.12 (par vineis) ; D 25.1.13; D 29.1.16; D 41.1.62; D 50.
16.79.1 (Fulcinus) ; Ulpien D 23.3.10.1; D 23.3.27; D 23.4.4; D 23.5.2 ; D 23.5.5 (Julien); D 23.5.13 pr-1-2-3 (fonds sous-entendu) 4 (Scaevola); D 24.3.5 ; D 24.3.7 pr (Scaevola)-1 (Papinien)-2-3 (par messes)-8 (par agri)-9-12-13-14 (par cretifodinae)-15-16 (Pomponius) (par agro) ; D 25.1. $3 \mathrm{pr}$ (par ager); D 25.1.5 pr (Pomponius) ; D 27.9.3.5 ; D 33.4.1.8-14 (Me1a) -15 (Mela); D 33.4.2.1 ; D 43.19.3.9; Macer D 2.8.15.3; Marcien D 23. 3.52 ; D 23.5.17; Modestin D 23.3.62; D 31.34 .7 .

39- Le terme désignant la locatio-conductio du fonds apparaît parfois clairement (par ex. in D 15.3.16 : functon... locavit), mais il arrive par contre asse $z$ souvent de ne trouver que le mot colonus. Or, on sait qu'avant d'acquérir le caractère d'un travail contraint, l'assimilant pratiquement à l'esclavage, le colonat a fonctionné à l'origine sur la base du contrat de locatio-conductio rei (B. LAPICKT, La transformation de la nature juridique du colonat romain, in St. Volterra 3 (1971), p. 359, 373). C'est pourquoi ces textes ont été intégrés dans cette rubrique. Il seront indiqués dans les références par le mot "colon" entre parenthèses. Ce choix est du reste aussi justifié par le fait que colonus ressort fréquement en compagnie d'un terme désignant clairement la locatio-conductio du fonds : c'est le cas in D 19.2.32; D 19.2.3; D 41.2.31; D 19.2.33; D 19.2.35 pr; D 47.2.68.5; D 47.2.62.8; D 19.2.25.1-2-3; D 43.16.12; D 19.2.61 pr ; D 19.2 .53 ; D 43.16.18 pr ; D 50.8.5 pr ; D 19.2.24.1-2-4; D 19.2.54.1; D $26.7 .46 \mathrm{pr} ; \mathrm{D} 41.2 .30 .6$; D $45.1 .89 ; \mathrm{D} 6.1 .77 ; \mathrm{D} 7.4 .29 \mathrm{pr} ; \mathrm{D} \mathrm{19.2.9.}$ 3 ; D 19.2.13.1I;D 19.2.14;D 19.2.15.2;D 19.2.19.2;D 24.3.7.3; D 33. $4.1 .15 ; D 43.26 .6 .2 ; D 47.10 .5 .4 ; D 41.2 .37$. Fonds est en fonction de cela objet de Locatio-conductio chez Alfenus (chez auteur anonyme) D 15.3. 16 ; D 19.2.29 (par silvam) ; Iavolenus D 18.1.65; D 18.1.79; D 19.2.21; D 19.2.51 pr : D 19.2.57 (Labéon) (par area); D 19.2.60.1 (Labéon) (colon)-5 (Labéon) (colon) ; D 20.6.14 (colon); D 32.30.1 (par hortos) ; D 33. 2.30.1 (colon); D 33.2.42 (colon); Celsus D 47.2.68.5 ; Julien D 2.14.56 (colon) ; D 7.1.34.1 (colon); D 19.2.32; D 41.5.2.1 (colon); D 43.33.1 pr (colon)-1 (colon)-2 (colon) ; D 43.33.2 (colon sous-entendu); Pomponius D 19.2.3 (Proculus) ; D 19.2.52; D 41.2.25.1 (colon); D 41.2.31; Af ricain D 19.2.33 ; D 19.2.35 pr (Servius)-1 (Servius) ; D 41.2.40.1 (colon); 2.34 (pris 2.34 (pris en fonct. du contexte, cf. Afric. D19.2.33);D20.4.11.2 (colon);D47.7 9 (colon); D43.18.2(solo); Venu1.D43.24.12(co1.);Marcel.D4l.2.19pr;D43.16.12; Scaev.D7.1.58pr (ager) (col.);D8.6.20(co1.);D19.2.61 pr; D 20.1.32

(colon) ; D 32.101.1 (colon); D 33.1.2I pr (colon) ; D 33.7.20.1 (colon)-3 (colon) ; D 33.7.27 pr (colon)-1 (colon) ; D 33.8.23.3 (colon) ; D 40.7. 40 pr (colon) ; Papinien D 19.2.53; D 32.91 pr (colon)-1 ; D 36.1.60.5; D 41.2.44.2 (par saltus) (colon); D 41.2 .45 (par saltus) (colon) ; D 43. $16.18 \mathrm{pr}$; D 46.1.52.2 (colon) ; D 50.8.5 pr ; Paul D 5.2.23.1 ; D 6.1. 74 (par solum); D 6.3.1 pr (par agri... vectigales) $-1 ; 0$ D 6.3 .3 (fonds sous-entendu) ; 12.286 (colon) 017.2 .46 (colon) $D 19.2$ (fonds

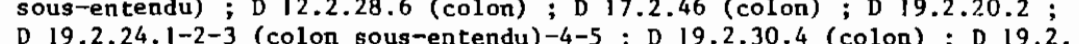
$54 \mathrm{pr}$ (colon)-1-2 (colon) ; D 19.2.55.1-2; D 26.7.46 pr ; D 13.1.16.2 $54 \mathrm{pr}$ (colon) $-1-2$ (colon) ; D 19.2.55.1-2; D 26.7.46 Pr ; D 13.7.16.2
(par praedizon vectigale); D 32.27 .2 (colon); D 32.78 .3 (colon); D 32.97 (colon) ; D 33.7.24; D 39.2.18.4 (par solit); D 39.3.5 (Labéon) (colon) ; D 41.2 .3 .8 (colon) -12 (colon) ; D 41.2 .30 .5 (colon) 6 ; D 41.3.31.3 (colon) ; D 43.16.20 (Labéon) (colon) ; D 43.24.19 (Sabinus) (colon) ; D 44.7.34.2 (colon) ; D 45.1.89; D 46.1.58 pr (colon); D 47.2.26.1 (colon) ; D 47.2.83. 1 (colon) ; D 47.2.86 (colon) ; D 49.14 .50 (colon); Ulpien D 5.3 .29 (colon) ; D 6.1.77 ; D 6.3.2 (fonds sous-entendu) ; D 7.4.29 pr; D 7.8.10.4 (colon) ; D 9.2.27.9 (Neratius) (colon)-11 (Proculus) (colon)-14 (Celsus); D 10.3.6.2 (Papinien); D 10.3.7.11 (colon); D 11.7 .14 .1 (Pomponius) (co-
100 ) ; D 12.1 .4 .1 (colon) ; D 19.1 .13 .11 (par ager) -30 (Servius, Tubero) 
ou seulement l'isage $(41)$. Far contre, rien ne ressert sur l'échange ou le commodat (42). Il est, vrai que, sur ces points, très peu de textes concernent l'esclave lui-même

Si on suit la même démarche, en distinguant l'acquisition Ju fonds de son utilisation dans un but d'exploitation par un autre que le propriétaire, on aboutit aux tableaux suivants (43) :

D 11.7 .43 ; D 31.76 .2 ; D 33.2.25; D 36.2.25.1 (legs des fruits); Tryphoninus D $7.1 .62 \mathrm{pr}$ (par saltibus) -1 (par vivariis); $\mathrm{D} 23.3 .78 \mathrm{pr}-1-2-3$; Paul D 2.14.27.8 ; D 7.1.11 (par arbores) ; D 7.1.16 (pris en fonction du contexte fourni par D 7.1.15.7 Ulpien) ; D 7.1.18 (par agri) ; D 7.1.48.1 ; D 7.1 .50 (Scaevola) ; D $7.1 .59 \mathrm{pr}$ (par arbores) $1-2$; 7.1 .60 .1 ; D 7.1 . 63 ; D 7.4 .9 (par 8olo) ; D 7.4 .13 (Labêon, Julien) ; D 7.4 .26 (par ager) ; D $7.9 .6 ; \mathrm{D} 8.2 .30 .1 ; \mathrm{D} 8.6 .21$ (fonds sous-entendu); D $10.1 .4 .9 ; \mathrm{D} 12$. D.12; D 21.2.15.1; D 33.2.12 (Alfenus); D 33.2.26 pr-1 ; D 33.2.28 ; D 45.1 .126 .1 (a contrario); D 47.7.5.2; Ulpien D $7.1 .7 \mathrm{pr}$ (par rei soli); D 7.1.9 pr (Celsus)-1-2- (par Lopicidinas)-3 (par metalla)-4 (Pegasus)-5 (par aucupiorwm, venatiorum)-6 (par agri) -7 ; $D 7.1 .12 \mathrm{pr}$ (par ager) (Labéon)-3 (Pomponius) (par ager) ; D 7.1.13.2 (Julien (par ager)-4-5 (par ager) -6 (idem) ; D 7.1.15.6-7 (Julien) ; D 7.1.17 pr (par locunt) ; D 7.1.20; D 7.1.27 pr-3 (par ager) 4 ; D 7.2.1.3 (Plautius, Celsus, Julien) ; D 7.2 . 3.1 (Celsus); $\mathrm{D} 7.2 .6 .2 ; \mathrm{D} 7.4 .1 \mathrm{pr}-2 ; \mathrm{D} 7.4 .5 .3$ (par area) ; 7.4 .8 D 7.4.10 pr-2 (par agri)-3 (par ager)-4 (par silva) ; D $7.4 .29 \mathrm{pr}$ (Pomponius) -1; D 7.6.1 pr (Labéon, Nerva, Julien, Marcellus); D 7.6.1.1 (par loco) -2-3 (fonds sous-entendu) -4 (idem) ; D $7.6 .5 \mathrm{pr}-1$ (Julien)-5; D 8.5.2.

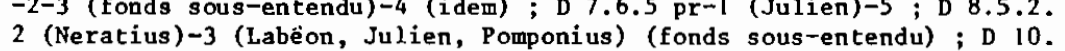
2.14.1; D 10.2.16.1 (Julien); D 10.3.6.10; D 11.7 .2 .1 (par agro) -7 (par locum) ; D 11.7.8.4 (par Zoci) ; D 19.2.9.1 (Marcellus, rescrit de Sévère et Caracalla) ; D 27.9.6; D 30.44.9; D 34.4.3.6; D 43.16.3.14-15 ; D 43.18.1.6 (par superficies) ; D 43.19.3.4 (fonds sous-entendu) -5 (Ju4.12 ; D 45.1.75.3; D 46.4.13.1-2; Marcien D 7.1.41.1 ; Modestin D 33.2. 19.

41- Fonds est objet d'usage chez Pomponius D $7.8 .16 \mathrm{pr}-1 ; \mathrm{D} 7.8 .22 \mathrm{pr}$ (Hadrien statuit) (par silva) ; Gaius D 7.8.11; D 43.16.10; Paul D 7.8.15 pr-1 ; Ulpien (Julien) D 7.1.13.2 (par ager) ; D 7.8.10.4 ; D 43.18.1.6 (par ouperficies).

42- Si le commodat a d'abord concerné les choses mobilières, il a vite été étendu aux imneubles (J. MACQUERON, Histoire des obligations, cit.,p. 95 ; A. GUARINo, Dirit to privato romano, cit., p. $776 \mathrm{n} \cdot 83-3$ expose 1 'idée selon laquelle l'expression commodare aurait été rêservée aux res mobiles et celle rem utendom dore aux res immobiles; sur le point, v. PASTORI, Il commodato nel diritto romano (1954), la synthèse de G. SCHERILLO in NNDI; A. POLACER, Commodato e furto : spunti d'interpretazione dialettica, in Labeo 19 (1973), p. $161 \mathrm{~s}$.).

43-- Les chiffres avancés dans ces tableaux se fondent sur les rêférences données dans les notes précédentes. 


\section{- snalyse des paragraphes}

$\begin{array}{lrrcrr}\text { période } & \text { vente } & \text { stipulation } & \begin{array}{c}\text { legs et } \\ \text { fidéicomis }\end{array} & \text { donation } & \text { dot } \\ \text { I } & - & - & - & - & - \\ \text { II } & 3 & - & 1 & - & 1 \\ \text { III } & 104 & 7 & 75 & 6 & 24 \\ \text { IV } & 52 & 1 & 74 & 6 & 6 \\ \text { V } & 204 & 18 & 209 & 14 & 77 \\ & & & & & \\ \text { TOTAL } & 363 & 26 & 360 & 26 & 108\end{array}$

- analyse des citations

$\begin{array}{lrrrrr}\text { I } & 14 & 1 & 23 & - & 3 \\ \text { II } & 31 & - & 28 & - & 8 \\ \text { III } & 29 & 1 & 17 & 1 & 2 \\ \text { IV } & 2 & - & 7 & - & 3 \\ \text { V } & 4 & - & 8 & - & 1 \\ \text { TOTAL } & 80 & 2 & 83 & 1 & 17\end{array}$

Utilisation du fonds

\section{- analyse des paragraphes}

$\begin{array}{lccc}\text { période } & \text { lounge } & \text { usufruit } & \text { usage } \\ \text { I } & - & - & - \\ \text { II } & - & - & - \\ \text { III } & 39 & 42 & 5 \\ \text { IV } & 15 & 19 & - \\ \text { V } & 122 & 110 & 5 \\ \text { TOTAL } & 175 & 171 & 10\end{array}$

44- Compte tenu des réserves déjà formulées à propos de la dot qui n'opère qu'un transfert de propriété provisoire.

45- Compte tenu des rêserves, formulêes aussi, notamment en ce qui concerne l'usufruit, dont le caractère parfois viager accentue la durée de la détention. Rappelons aussi que la locatio-conductio peut exceptionnellement entrainer une mutatio dominii (cf. Ulpien D 19.2.39; supra n. 21).

$\begin{array}{lccc}\text { période } & \text { louage } & \text { usufruit } & \text { usage } \\ \text { I } & 8 & 1 & - \\ \text { II } & 16 & 11 & - \\ \text { III } & 11 & 18 & 1 \\ \text { IV } & 3 & 4 & - \\ \text { V } & 3 & - & - \\ \text { TOTAL } & 41 & 34 & 1\end{array}$

Limitons-nous dans un premier temps à la place tenue par chaciune de ces institutions juridiques à l'intérieur de la seule problématique "fonds de terre". En ce qui concerne les modes a'grquisition du fonds, ce sont les legs ( $41 \%$ des paragraphes et $45 \%$ des citations) et 1 a vente ( $41 \%$ et $44 \%$ ) qui viennent en première position. On trouve ensuite la dot ( $12 \%$ et $9 \%$ ). La donation $n$ 'apparaît que faiblement ( $3 \%$ et $1 \%$ ), ainsi que la stipulation, dans les mêmes proportions.

En ce qui concerne l'utilisation du fonds, c'est le louage qui occupe la plus grande place ( $49 \%$ et $54 \%$ ), suivi de 1 'usufruit ( $48 \%$ et $45 \%)$. L'usage ne joue qu'un rôle restreint $(3 \%$ et $1 \%)$.

Par rapport aux résultats obtenus en matière d'esclavage, on constate donc une meilleure représentation des legs, de la dot et, surtout, du louage, mais cela en ne prenant en considération que les textes concernant un fonds. Il convient, pour rendre un meilleur compte de la comparaison, d'en mettre les deux termes en relation. On peut ainsi, pour chaque institution, additionner le nombre de textes concernant esclave et fonds pour en extraire ensuite un pourcentage de l'institution en ce qui les concerne respectivement. Par exemple, 680 paragraphes visent un esclave et 363 un fonds en maiière de vente. Sur un total de 1043 paragraphes, $65 \%$ concernent donc une vente d'esslave et $35 \%$ seulement une vente de fonds. 
I- acquisition du fonds ou de l'esclaye

\section{\$ des paragraphes}

\$ des citations

Esclave

Fonds

vente

$65 \%$

$35 \%$

Esclave

Fonds

stipulation

$84 \%$

$16 \%$

\section{legs}

$54 \times$

$46 \%$

$32 \%$

$36 \%$

$64 \%$

\section{$71 \%$}

$29 x$

$93 \%$

$7 \%$

$48 \%$

$52 \%$

dot
II - utilisation du fonds ou de l'esclave

\section{7 des paragraphes}

Esclave Fonds

louge

$13 \%$

$87 \%$

usufruit

$45 \%$

$55 \%$

$36 \%$
$\$$ des citations

Esclave Fonds

$11 \% 89 \%$

$47 \% 53 \%$

$86 \% \quad 14 \%$

Hornis trois cas, la dot, le louage et l'usufruit, il est proportionnellement plus question d'esclaves que de fonds, et cela aussi bien dens l'analyse des paragraphes que dans celle des citations, ce qui montre une fois de plus la fiabilité de l'instrument d'analyse. Mais arrêtonsnous tout d'abord sur ces trois cas.

C'est en matière de louage que l'écart est le plus important. Cela provient des textes traitant des colons. A 1'origine, les citoyens libres qui n'étaient pas propriétaires fonciers furent contraints par le développement des rapports économiques de mettre à profit les terres d'autrui et d'y fonder leur propre existence (46). L'sccroissement de la grande propriété et notamment des domaines impériaux, le déclin de l'esclavage, et $1 \mathrm{a}$ peine que les propriétaires avaient snurent en période difficile à percevoir les fermages en espèce (47) permirent la substitution progressive du

46- B. LAPICRI, La transformation de la nature juridique du colonat romain, cit., p. 362 ; v. M. JACOTA, Les transformations de 1 'économie romaine pendant les premiers siècles de notre ère et la condition de l'esclave agriculteur, in Et. J. MACQUERON, Aix (1970), p. 375-383.

47- Rareté du numéraire, mauvaises récoltes, arriérés fréquents, cf. $P$. PETIT, Histoire générale de l'Empire romain, cit.; p. 504. Pline le jeune (Ep. 3.19 et 9.37) montre, au début du règne de Trajan, que le prix des

colonat partiaire $(48)$ au travail servile et au fermage traditionnel an argent. Il est fondamental de remarquer que c'est précisément dans la deuxième moitié du 2ème siècle, époque à laquelle on peut discerner une crise de l'esclavage, que le discours des juristes prend le plus en considération les colons (49). Leur condition ira s'aggravant au उème siècle (50), mais il faudra attendre Constantin pour qu'un texte législatif

terres avait baissé et que les propriētaires tendaient à reuplacer le fermage en argent par le fermage en nature, qui faisait de l'exploitant un métayer, un colon partiaire beaucoup plus dépendant du propriétaire. Le même Pline (7.30.3) montre aussi qu'il était déjà difficile de trouver un colon solvable (colonus idoneus); v. R. MARTIN, Pline le jeune et les problèmes économiques de son temps, in REA (1967), p. 62-97.

48- cf. Pline le jeune, Ep. 9.37.3 ; Gaius D 19.2.25.6; sur ce point, v. E. CUQ, Le colonat partiaire, Paris (1897). Le colonus partiarius (a mifruit) est un colon pauvre, qui ne possède pas d'instruments d'exploitation, emprunte ceux-ci au propriétaire foncier, cum aestimatione. On trouve chez Proculus (D 19.2.3), et plus tard chez Paul (D 19.2.54.2) l'analyse de cas analogues. Or, le manque de fortune du colon contribue aggraver la situation du propriétaire foncier, étant donné que la récolte devient pour le tion du proprietaire foncier, étant donne que la recolte devient pour le fermage, ce qui pose des problèmes les années de sterilitas (B. LAPICKI, La transformation, cit., p. 365).

49- Les textes ou apparaît un colonus sont indiqués à la note 39. Excluons ceux qui concernent des esclaves. Le pourcentage de ces textes par rapport au nombre total de paragraphes relatifs à une période donnée est de : I $(0 / 9)=0 \%$; II $(0 / 47)=0 \% ; \operatorname{III}(25 / 3581)=0,69 \% ; \operatorname{IV}(14 / 1172)=$ $1,19 \% ; \mathrm{v}(77 / 16192)=0,47 \%$; Sur le problème, v. E.M. SCHTAERMAN, La schiavita, cit., p. 336. Sur 1'apparition plus rapide du colonat en Afrique, J. KOLENDO, La formation du colonat en Afrique, Recherches Internationales n. 84, 3 (1975), p. 129-157.

50- Au Haut-Empire déjà il existait une tendance à fixer le colon à la terre par des baux emphythéotiques ( $v$. SIRAGO, L'Italia agraria sotto Traiano, Louvain (1958), p. 164-165. Le fragment de Marcien D $30.112 \mathrm{pr}$ a été 1 'objjet de polémiques. On peut y lire : Si quis inquilinos sine praediis quibus adhaerent legaverit, inutile est legatum. Sed an aestimatio debeatur, ex voluntate defuncti statuendun esse, divi Marcus et Conmodus rescripsemunt. Ce texte est interpolé selon C. TOMULESCU, Colonatul in dreptul roman, Analele Univ. Bucuresti, Seria Stiinte sociale, Stiinte juridice (1956), p. 112 ; R. BRosz, Les changements sémantiques du mot colonus dans les sources du droit romain, Annales Univ. Scientiarum Budapestinensis, I (1959) p. 47 ; W.L. WESTERMANN, slave systems, cit., p. 146 ; FUSTEL DE COULANGES, Le colonat romain, in Recherches sur quelques problèmes d'histoire, Paris (1923), P. 65 ; 0. SEECK, Colonatus, RE IV, 1, vII Halbband (1900), col. 496 ; G. HUMBERT, DSDA, p. 1322 ; DONATUTI, Dal regime dei verba al regime della voluntas nei legati, in BIDR XXXIV (1925), p. 185, pense interpolé inutile est legatum : sed an aestimatio debeatur; SCHULIN, Geschichte des röm. Rechts, Stuttgart (1889), p. 273 ; KÜBLER, ZSS XXII, p. 208 ; Ch. SAUMAGNE, Le rôle de 1'origo et du census dans la formation du colonat romain Byzantion XIII (1937), P. 503 n. 3. Pour son authenticité, BANDI DI VESEE, 
entrave ouvertement leur liberté (51).

Il est, ensuite pius question de fonds que d'esclave dotal. Ce n'est pas vraiment surprenant. On sait que l'aliénation du fondsdotal italique, interdite au mari par la lex Iulia sans le consentement exprès de sa feme, a attiré l'attention des juristes classiques (52), mais surtout, dans une société ou la richesse première était la terre, il est normal que le fonds ait constitué un bien dont la dot se devait d'être assortie, compte tenu de la considération sociale qu'il entraînait (53).

C'est enfin en matière d'usufruit que les textes concernant un fonds sont plus nombreux. Cependant, l'écart est ici très faible. D'autre part, cette prépondérance ne joue pas, contrairement au louage et à la dot, pour toutes les périodes. Ainsi, dans l'analyse des citations, on dénombre plus de références opérées sur l'esclave aux périodes I et IV. Pour la période III, on relève autant de citations visant l'esclave que le fonds. Il s'agit donc d'une prépondérance toute relative.

En dehors de ces hypothèses, les juristes se préoccupent plus d'acquisitions d'esclaves que de fonds. A quoi cela tient-il ? Peut-on penser que, dans la réalité de la vie sociale romaine, les acquisitions d'esclaves ont été plus nombreuses ? Il faut être honnête. Les chiffres avancés ne permettent pas de trancher catégoriquement la question. Le discours des juristes n'est pas sur ce plan un reflet forcément fidèle de la réalité. Ce que l'on peut affirmer par contre, c'est que l'acquisition d'esclaves

ATO (1865-66) , 56. Pour M. JACOTA, Les transformations de l'économie romaine, cit., p. $380 \mathrm{s.,}$ il s'agirait d'un serrus inquilinus qui, come esclave, peut être attaché à me terre par une manifestation đe volonté du dominus.

51- Constantin décida en 332 que tout colon en fui te serait poursuivi, rendu à son maître et que le propriétaire qui 1 'aurait recueilli devrait payer la capitation du fuy ard (c. Th. v, 17, 1). C. TOMULESCU, Uber die lex a maioribus constituta, RIDA (1967), p. 429 s. estime que la lex exprimée par CI XI.51.1 ne peut être identifiée à celle de 332. Sur ce point, P. COLLINET, Le colonat dans 1 'Empire romain, in RecueiI de la Soc. J. BODIN, II, Le servage, (1959) et M. PALLASSE, Note complémentai re à "Le BODI, I1, Le servage, (1959) et M. PALLASSE, Note complémentai re à "Le colonat dans 1 'Empire romain", par P. COLLINET dans "Le servage". Plus généralement, sur le colonat, bibliogr. A. P IGANIOL, L'empire chrétien,

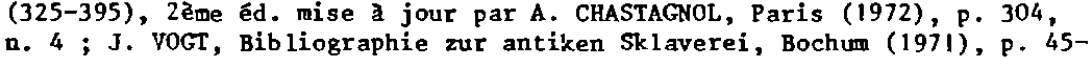
n. 4 ; J. VOGT, Bibliographie zur antiken Sklaverei, Bochum (1971), P. 45-
49 ; A.B.M. JONES, The roman Colonate, Past and present, 13 (1958), p. 113 ; J. GAGE, Les classes sociales dans l'Empire romain, Paris (1964), p. 399-404 ; F. DE MARTINO, Storia della costituzione romana, cit. v, p. $182 \mathrm{~s}$

52- Gaius, Inst. 2.63 ; R. MONIER, Droit romain, cit., I, p. 294.

53- P. DOCKES, La Libération, cit., p. 149. a incontestablement posé plus de problèmes aux juristes. Il suffit d'avoir à $l$ 'esprit la règlementation de l'édit des édiles sur les ventes d'esclaves (54). Il est possible également, en matière de vente, que les textes visent plus l'esclave que le fonds dans la mesure où la terre est plus stable, change rarement, de mains, stabilité n'existant pas pour une "denrée" aussi périssable que l'esclave qui de plus fait des "petits" que $l$ 'on peut vendre.

Selon le Digeste, la crise de l'esclavage n'a pas affecté les modes d'acquisition de l'esclave, ni même sa simple utilisation par un tiers en vue d'exploiter sa force de travail d'une part ; d'autre part, le problème de la main-d'oeuvre servile a plus préoccupé les juristes que celui de l'acquisition ou de l'utilisation d'un fonds. Il est vrai que l'exploitation de l'un est utile à l'exploitation de l'autre.

Ces résultats ayant été rappelés, il est perris de passer à une analyse des réalités de l'acquisition de la main-d'oeurre servile.

\section{SECTION 2. Les réalités de l'acquisition d'esclaves}

Combien d'esclaves un maître possède-t-il? Quel est le prix d'un esclave ? Voilà des questions sur lesquelles il nous faut interroger les textes du Digeste.

\section{Paragraphe 1: Le nombre d'esclaves possédés par un ma今ttre}

C'est au cours du Zème siècle avant notre ère, à 1a suite des grandes guerres contre l'Orient méditerranéen et de la politique de domination,

54- L'édit des édiles a suscité une littérature relativement abondante dont on peut relever VINCENT, Le droit des édiles (1922) ; DE SENARCLENS, La date de l'édit des édiles sur la vente des esclaves, in T. 2 (1923), p. $448 ; L$ 'extension de 1 'édit des édiles aux ventes de toute espèce de choses, in T. 5 (1926), p. $572 \mathrm{~s}$. ; La date de l'édit des édi les de mancipiis venduondis, in T. $4(1922-23), \mathrm{P} .384 \mathrm{~s}$. Plus rếcerment IMPALLOMEN I, L'editto degli edili curuli (1955); HONORE, The history of the aedilician actions, in St. De Zulueta (1959), p. $132 \mathrm{~s}$. 
que l'esclavage à Rome va connaitre san expansion maximale (55). Il semble. malheureusenent impossible d'avancer des chiffres précis pour la population de l'mpire ni même de proposer un pourcentage valable des libres et des esclaves (56). L'épigraphie ne permet pes d'investigation statistique, la conservation et la découverte des inscriptions étant le fruit du hasard, et la paurreté de la grande majorité des esclaves ne leur ay.nt guère permis les frais d'une inscription sur pierre (57). La littérature nous fournit cependant quelques renseignements.

Selon Galien, la population de Fergame aurait compris, au 2ème siècle de notre ère, $33 \%$ d'esclaves (59). Il paraît difficile d'avaliser telle donnée pour l'ensemble de la société romaine dans la mesure ou on ne peut démontrer que les conditions de cette société correspondaient strictement à celle de Pergame (59). D'autre part, les données les plus importantes concernent l'Egrpte ou le nombre d'esclaves ne dépassait pas 13,9\% de l'ensemble de la population (60). A partir de là ont été avancées les hypothèses les plus diverses quant au nombre d'esclaves en Italie (61).

55- F. DE MARTINO, Storia della costituzione romana, II, p. 294. Les seules guerres de Cesar en Gaule auraient procuré un million de prisonniers, cf. C. JULLIAN, Histoire de la Gaule, III, Paris (1923), p. 556558. Ce chiffre est sans doute exagéré, J. KoLENDo, Lavoro servile, cit., p. 19. Ainsi, W.L. WESTERMANN, Slave Systems, cit., p. 63, évalue a 150.000 le nombre de prisonniers capturés en Gaule. Sur la méthode des guerres et 1 'esclavage, v. J. MODRZEJEWSRI, Aut nascuntur aut fiunt, BIDR (1976), p. $1-25$.

56- J. GAUDEMET, Institutions de 1'Antiquité, cit., p. 543.

57- F.G. MAIER, Röm. Bevölkerungsgeschichte und Inschriftenstatistik, in Historia II (1954), p. 318-335.

58- Gal. V, p. 49, êd. C. KthH, v. F. DE MARTINo, Storia, cit. II, p. 291 ; W.L. WESTERMANN, Slave Sys tems, cit., p. 87.

59- F. DE MARTINO, Storia, cit. II, p. 292.

60- Selon M. HUMBERT et C. PREAUX, 13,7\% de la population urbaine et $10 \%$ de la population rurale; pour J.A. STRAuSs, respectivement $13,9 \%$ et $10 \%$; I. BIEZUNSKA-MALOWIST constate qu'il y a environ $10 \%$ d'esclaves dans le plat pays, c'est-ż-dire dans une zone où le rôle économique des esdans le plat pays, c'est-à-dire dans une zone où le rôle êconomique des es-
claves est très faible et oul leur utilisation est essentiellement domesticlaves est très faible et od leur utilisation est essentiellement domesti-
que, cf. P. LEVEQUE, Esclavage et exploitation du travail dans I'Egypte he lênistique et rotnaine, Revue des Etudes Grecques, XCII (1979), p. 236. 6i- J. BELOCH, Bevolkerung, p. 413 s. retient qu'en 28 avant J.C. il y aurait eu 2.200.000 esclaves en Italie; le rapport entre populations servile et libre aurait étê de trois esclaves pour cinq personnes libres dans 1'Italie centrale et méridionale, de trois pour dix dans la vallée du Po, et le rapport global de un pour deux (p. 434) en se fondant sur 1 'exemple de Pergame. Ces données ont été contestées ; v. les travaux de RORNEMANN, SEECK et NISSEN publiés in Biblioteca di storia economica, vol. IV ; BRUNT, Italian Manpower, (197l), p. $121 \mathrm{~s}$., qui pense que le nombre de $1 \mathrm{a}$ population globale était plus élevê et qui estime ainsi la population servile
Par voie de conséquence, le nombre d'esclaves fivant à Rome a fait l'objet de controverses.

Rome abritant la plus grande partie de la noblesse, le nombre d'esclaves devait sûrement y être plus élevé que dans les provinces (62). Néanmoins, l'assertion selon laquelle y auraient vécu 000.000 esclaves semble arbitraire (63). On ne peut ériger en règle les quelques exemples fournis par de grandes familles romaines employant un nombre important d'esclaves (64). Ils ne peurent constituer les éléments d'un calcul statistique sérieux (65). Quoi qu'il en soit, le nombre d'esclaves résidant à Rome était sûrement important, et, sur ce poin丸, Sćnèque nous donne une indication intéressante. Il mentionne en effot une proposition sénatoriale tendant à distinguer les esclaves des citoyens libres par le port d'un habit différent (66). Or, quel intérêt aurait pu avoir cette proposition si ce n'est d'éviter une confusion entre un esclave et un homme libre ? Et si cette confusion est possible, c'est, outre le fait que l'tmpire va développer une catégorie d'esclaves privilégiés, fait sur lequel on reviendra, la certitude que Rome abrite un grand nombre d'esclaves. Examinons à ce propos les textes du Digeste.

Il arrive qu'un homme libre passe pour être esclave, soit qu'il se croit (67) ou qu'on le croit tel (68). Ce problème est attesté par les

à 3.000.000; W.L. WESTERMANN, RE, Suppl. VI, p. 965 ; Slave Systems, cit., p. 67 ; P. ANDERSON, Les passages, cit., p. 67

62- F. DE MARTLNo, Storia, cit. IV, p. 339

63- Ce chiffre a été soutenu par J. MARQUARDT, Röm. Staatsverwaltung, (1884) Leipzig, II, p. 124 ; contra F. DE MARTINO, Storia, cit. IV, p. 23. 64- Front. de aq. 98 et 116 ; Tac. ann. XIV, 43, 4 ; Sen. de clem. I, 24,1 ; P1in. nat. hist. $\operatorname{XXXII}, 1$ (6), 26 ; Iuv. V, 66 ; Luc, nav. 22 ; Petr. Trim. 47 ; 37 et 53 ; v. aussi Sen. de vita beata XVII, 2 ; ep. CX, 17 ; Iuv. VI 141 ; 352 ; Apul. met. II, 2 ; Mart. II, 57

65- F. DE MARTINo, Storia IV, cit., p. 23.

66- Sen. de clem. I, 24,1 ; W.L. WESTERMANN, Slave Systems, p. 88 . Le motif pour lequel le sênat repoussa cette proposition est d'ailleurs très intếressant : Indista est aliquando a senatu sententia, ut servos a liberis cultus distingueret ; deinde apparuit quantum periculum immineret, si servi cultus distingueret; deinde appar

nostri numerare nos coepissent. statwon retineat); Africain chez Uipien (Si liber homo... condicionem sucm ignorat) D 48.5.28.5 ; Paul D 22.6.1.2 (Si quis... putans se servron esse). 68- Julien D 37.10.7.3 ; Porponius chez Paul D 29.2.22 (Si is... putaverit defunctum servon suzm esse... ; ... si quasi bbertini sui, cum ingenuu esset);Ulpien (Julien, rescrit d'Antonin le pieux) D 37.10.1.5; D 37.10. 
textes de juristes écrivant sous les Antonins et même les Sévères. Sont particulièrement significatifs deux fragments $d^{\prime} U \mathrm{l}^{\mathrm{ien}}$. Dans $l^{\prime}$ un, il est question d'un houme libre qui a été appréhendé comme fugitif (69), dans l'autre d'un individu qui frappe un home libre croyant qu'il s'agit de son esclave $(70)$.

Il arrive aussi qu'un eselave soit pris pour libre : soit il se conduit en homme libre, pleinement conscient de l'usurpation d'état (71) - parce qu'il a fui (72), ou bien par simple motif économique, par exemple pour s'affirmer débiteur valable (73) - ; soit il est in libertate avee l'accord de son maître (74) - on aboutit alors à des résultats assez surprenants, du genre : Igitur sciendum est et liberron posse dolo malo in libertate esse et servon posse sine dolo malo in libertate egse (75), c'est-à-dire : un homme libre peut être frauduleusement in libertate, tandis qu'un esclave peut se trouver sans fraude dans cet état ; soit, enfin, le texte nous apprend seulement que son entourage le croit libre (76).

1.6 ; D 40.5.24.3 (Si homini libero fuerit libertas per fideicommissum adscripta) ; D 47.10 .22 (Si liber pro fugitivo adprehensus erit) et D 47 . 10.3.4 (Si quis hominem liberun ceciderit, dwon putat semron sum).

69- D 47.10 .22 .

70- D 47.10 .3 .4 .

71- Pomponius D 46.3.19 (Fugitivus meus, cwo pro libero se gereret); Paul D 41.2.3.10 (Si. servus... pro libero se gerat, ut fecit spartacus) ; D 41.3.15.1 ( $\mathrm{Si}$ servus... fugerit, si pro libero se gerat) ; vipien (Nerva f.) D 15.1.3.8 (Si servus, cun se pro libero gereret); D 21.1.17.16 (Caelius Sabinus) (... pro libero se gerere); 0 47.2.52.15 (Servus, qui se liberum adfirmavit); D 47.10.15.45 (Mela) 6.. si pro libero se gerentem).

72- Pomponius D 46.3.19; Paul D 41.3.15.1.

73- Dlpien D 47.2.52.15.

74- Pomponius D 40.12.28 (... is enim demon voluntate domini in libertate est, qui possessionem libertatis ex voluntate domini consequitur). Ce texte, ainsi que $D$ 40.12.24.3 de Paul, visent des esclaves in libertate tuitione Praetoris, c'est-à-dire affranchis par un mode non formaliste, selon W.W. BUCKLAND, Roman law, cit., p. 446, n. 12 ; v. M. DE DOMINICIS, Les Latins Juniens dans la pensée du législsteur romain, RIDA 20 (1973), p. 311. V. sussi Ulpien D 40.12.10 (Alfenus, Julien); $40.12 .12 \mathrm{pr}-2-3$ 75- D1pien D 40.12.12 pr.

76- Julien D 28.5.41 (Si pater famizias Titium, quem ingenum esse credebat, heredem scripserit... deinde Titius, quia servus fuerat... ; Pomponius (constitution de Tibère) D 28.5 .42 (... Parthenii, qui tamquan ingenus heres scriptus adierat hereditatem, cum esset (aesaris servus); Marcellus D 23.3.59.2 (Eius nomine quae libera videbatur decem in dote deMarcellus D 23.3.59.2 (Eius nomine quae libera videbatur decem in dote de-
disti/... si manumissa nupserit) ; Ulpien D 26.2.22 (Si quis tutorem dederit filio suo servrom, quem putabat liberm esse, cum esset servus) ; D 29. 1.13.3 va encore plus loin puisqu'il s'agit d'un esclave que son propre
Cet entourage est du reste parfois très proche de l'intéressé. Papinien nous donne le cas d'un mari qui croyait sa femme libre alors qu'elle était esclave (77), et Uipien celui d'une ingénue qui épouse un esclave qu'elle croyait libre $(78)$.

Les barrières entre esclavage et liberté apparaissent donc fragiles dans la vie quotidienne, et la pratique domine une situation sur laquelle le droit ne peut rien, d'autant qu'il contribue lui-même au nivellement des classes inferieures (79). La confusion ne peut sans doute se produire entre n'importe quel esclave et n'importe quel homme libre. Pour que l'esclave puisse "jouer le jeu" de la liberté, encore faut-il qu'il en ait les moyens économiques. Pour qu'un bomme libre soit pris pour un esclave, ne faut-il pas qu'il soit dans une situation telle que la confusion scit possible $(80)$ ? Le phénomène $n$ 'en demeure pas moins intéressant, et il faut relever qu'il n'est pas l'apanage d'une période bien définie, mais qu'il ressort de toutes les périodes prises en considération (81).

maître croit libre (Si servun proprium, quem liberun esse credidisset, miles heredem sine libertate instituit) ; D 32.11 .16 (... out scit servom esse... aut ignoravit) ; D 40.5.24.9 (Marcellus, rescrit de Sévère et Caracalla) (... libermen putavit). v. D 23.3.67 Proculus où une ancilla ignore qu'elle est esclave; $v$. aussi $D$ 9.2.45.2; D 18.2.14.3; D 11.3.5.1.

77-- Papinien D 24.3.42.1 : Fructus ex praediis, quae in dotem data videbantur, bona fide perceptos et mulieris oneribus ante causam liberalem absimptos, quanvis osrvan fuisse postea constiterit...

78- Dlpien D 24.3.22.13 : Si mulier in condicione mariti exraverit putaveritque esse liberon, cum servus esset..

79- Ce nivellement provient aussi du fait - on reviendrs sur ce point que 1 'esclavage perd de sa spécificité économique (E.M. SCHTAERMAN, Sur la structure de clssse de la sociêté romaine, VDI (1969), 4, p. 37-59.).

80- v. par ex. J1pien D 40.12.12.1 : Infans subreptus bona fide in servituts fuit, cum liber esset, deinde, cum de statu ignarus esset, recessit et clam in libertate morari coepit : hic non sine dolo malo in libertate moratur. Bien que cet individu sit servi comme esclave de bonne foi, la solution d'Ulpien est tout de même singulière car elle revient plus globslement $a$ admettre la possibilitế qu'un home libre soit frauduleusement en possession de son état de libertê. Parallèlement, un esclave peut être sans fraude in libertate s'il a êté affranchi par un testament dont il ignore sans fraude in libertate s'il a été affranchi par un testament dont il maître, ou encore - le cas est intéressant - s'il a été élevé comme un houme libre (Ulpien D 40.12.12.2).

8I- Références a Alfenus (chez Ulpien D 40.12.10), à Tibère (chez Pomr ponius D 28.5.42), a Nerva Filius (chez Ulpien D 15.i.3.8), a Caelius Sabimus (chez Ulpien D 21.1.17.16), et des textes de Proculus, Julien, Pomponius, Marcellus, Paul, Ulpien (cf. notes supra) 
Mais venonsten au problème qui nous intéresse au premier plan, le nombre d'esclaves possédés par un maître. On sait par Tacite que Fedanius Secundus, préfet de la ville en 61 , possédait, 400 esclaves (82). On sait aussi que la lex Fufia Coninia (2 av. J.C.) fixait à 100 le nombre maximum des affranchissements testamentaires lorsque le maître avait plus de 500 esclaves (83). Il est donc certain que l'on pouvait posséder un grand nombre d'esclaves ( 84 ), mais cela était-il le lot du commun des Romains ? Il faut probablement se garder de toute exagération (85). C'est en tout cas ce à quoi nous incitent les indications extraites du Digeste.

Peu de textes malheureusement fournissent des renseignements précis à ce sujet et, de plus, la précision de ces textes ne porte que sur des cas extrêmes, mais dont l'intérêt n'est tout de même pas négligeable.e. Ainsi, Marcellus nous parle d'un maître qui n'a qu'un seul esclave (86), Julien et Scaevola d'un maître qui n'a pour tout bien que 2 esclaves (87), et Papinien d'un maître qui $n$ 'en possède que $3(88)$. Cn a sûrement affaire en 1 'espèce à des individus qui se situent à peine su-dessus du prolétariat (89), mais rien $n$ 'interdit de penser que de telles personnes étaient nombreuses à Rome (90). Paul signale par ailleurs qu'un légat peut avoir un seul esclave, maig aussi un grand nombre (91). Gaius nous apprend qu'un

\section{2- Tacite, Annales, XIV, 43,4.}

83- Gaius I, 43 : Suétone (Augustus 40,3) justifia cette loi par 1a volontê impériale de préserver le corps des citoyens romains d'une infiltration trop massive d'anciens esclaves. Cette loi est toujours opérationnelle a la fin du 3ème siècle (Scriptores historiae Augustae, Tacitus, nelle a la fin du 3ème

84- A ce sujet, on peut signaler 1'indication probablement exagérée de Pëtrone (Satiricon, 53) selon laquelle 30 garçons et 40 filles vermae seraient nésen un jour dans une des propriétếs de Trimalcion, et aussi le fait que moins d'un esclave sur dix connaisse son maître de vue.

85- Dans ce sens une décision d'Auguste (12 après J.C.) restreignant à 20 le nombre d'esclaves dont un maitre pourrait s'entourer durant son exil (Dio Cassius LVI, 27, 3).

86- D 35.2.56.2 : Is, qui in bonis unum drontaxat servion habebat.

87- Julien D 40.9.5.2; Si Titius nihil amplius in bonis quam Stichum et Pamphilion habeat. Scaevola (Julien) D 40.9 .6 constitue la suite de ce texte.

$88-\quad 40.5 .22 .1$

89- v. Iuv. Sat. III, $286 ;$ IX, 64-67; 142-147. Un vétéran sans esclave est mentionne in SHA, Hadr., XVII, 6

90- W.L. WESTERMANN, Slave Systems, cit., p. 89.

91- Paul (Cassius, Julien) D 5.1.24.2. tuteur doit fixer le nombre d'esclaves du pupille en fonction de sa iignitas et de ses facultés (92).

En dehors de ces cas, le degré de pertinence des textes est moindre. On ne trouve plus un nombre précis d'esclaves appartenant à un maître, mais une indication numérique fraqmentaire. Il est par exemple question du legs de 10 esclaves (93) ou de la promesse d'en remettre le même nombre (94), du legs de 8 lacticarii (95) ou de 8 esclaves ruraux (96), de 5 pueri (97), de 3 esclaves comédiens (98), de la vente de 3 esclaves (90) ou de 2 seulement (100), ou encore de 2 esclaves institués héritiers (101), mais rien ne ressort quant au nombre exact d'esclaves possédés par le maître (102). Pareillement on a des indications sur le nombre d'affranchis (103), mais dont le caractère incomplet est identique. Un texte se détache néanmoins des autres : il s'agit d'un fragment de Papinien ois 100 esclaves font l'objet d'une stipulation (104). C'est vraisemblablement, compte tenu du nombre élevé, une hypothèse d'école.

92- Gaius D 26.7.13 pr.

93- Gaius D 30.65 pr ; Scaevola D 33.5.21.

94- U1pien D 46.3.29.

95- Pomponius D 30.8.2.

96- Tryphoninus (Scaevola) D 34.1.15.1.

97- Scaevola D 32.41 .4 .

98- Modestin D 40.5.12 pr.

99- Paul (Julien, U1pien) D 19.1.43 (vente de trois esclaves dont deux houmes et une femrae.)

100- Pomponius D 24.1.31.3; Africain D 21.2.47; Tryphoninus (Scaevola, et les empereurs Hadrien et Marc-Aurèle) D 18.7.10 (vente de deux esclaves dont un horme et une ferme) ; Paul D 19.1.42; Paul (Pomponius) D 24.1.28. 4 ; Ulpien (Julien) D 18.2.4.2 ; D 2I.1.59.1.

101- Celsus D 28.5.61; Julien D 28.5.43; Ulpien (Ce1sus) D 28.7.2.1.

102- On a aussi d'autres indications in Julien D 47.2.57.5 (vol commis par deux esclaves); Pomponius $D$ 41.4.6.1 (achat de dix esclaves); Marcellus D 46.3.67 (promesse de remettre deux esclaves); $D$ 47.6.5 (deux esclaves communs); Paul D 10.3.31 (deux servi hereditarii doivent servir les pupilles) : Ulpien D 47.2.14.5 (deux esclaves donnés en gage); D 25.4.1.10 (six femmes esclaves se trouvant dans une chambre où une femme est en train d'accoucher). 103- Valens (Julien) D 32.94 (trois affranchis) ; Africain D 21.2.47 (quatre); Scaevola D 33.2.33.2 (trois) ; Tryphoninus (Scaevola) D 32.38. 5 (quinze) ; Paul D 17.2.71.1 (deux colliberti).

104- Papinien D 45.1.117: Si centum homines, quos ego heresve meus eligerem, stipulatus... 
En définitive, le Digeste n'apporte sur ce point qu' 'une certitude, même les pauvres peuvent avoir un esclave. Un texte de Paur est éloquent à cet égard : il s'agit d'un maître qui donne son escleve en gage et qui n'a pas assez d'argent, pour le récupérer (105). Cela montre à quel point l'esclavage est une institution importante de la scciété romaine.

On a vu que le nombre d'esclaves pouvait être une source de confusion entre esclave et libre. A plus forte raison en est-il une en ce qui concerne "ses" propres esclaves et ceux appartenant à autrui. Les textes juridiques se font l'écho de situations pour le moins inattendues. Le maître ignore parfois que tel esclave lui appartient. Julien nous soumet le cas $d^{\prime} u n$ individu qui achète un esclave, ignorant qu'il lui appartenajt (106). Il traite ailleurs d'un esclave volé qui est donné en gage à son maître, qui ignore qu'il est sien (107). On a dans le même sens un texte de Pomponius oul un maitre ignore qu'un tel est son esclave (108). Marcellus rappelle le cas d'um individu ayant acheté de bonne foi un esclave à qui n'en était pas propriétaire, son maître ne sachant pas qu'il lui appartenait (109). D'autre part, le maitre croit parfois que tel esclave est sien, alors qu'il n'en est rien. On trouve à ce propos chez Ulpien le cas d'un esclave affranchi par quelqu'un qui croyait être son maitre (110). Ces situations ne s'expliquent que dans la mesure où le mâेtre, en l'espèce, possède un nombre important d'esclaves, ce qui semble aussi ressortir des cas où i.l ignore le nom de ses esclaves (111).

\section{5- Paul D 9.4.22.1-2.}

106- Julien D 12.6.37 : Servrom merm insciens a te emi pecuniamque tibi solvi.

107- Julien D 41.4.7.7 : ... si igitur servum, qui tibi subreptus erat, ignoranti tibi tum esse pignori dedero...

108- Pomponius D 40.12.28 : Non videtur domini voluntate servus in libertate esse, quem dominus ignorasset surm esse.

109- Marcellus D 17.1.49: Servan Titii emi ab alio bona fide et possideo: mandatu meo ewn Titius vendidit, cum ignoraret surm esse.

110- U1pien D 40.12.12.2 : ... vel vindicta ei imposita est $a b$ eo, quem dominus esse putavit, cun non esset.

111- Ainsi Paul D 6.1.6 (... sed si nomen eius ignorem, demonstratione eius utendum erit : veluti 'qui ex illa hereditate est', 'qui ex illa natus est') ; Iavolenus D 34.5.28 : (sin autem ignota nomina servorion essent). La difficulté pour le maître de connaître le nom de ses esclaves peut être La difficulte pour le maitre de connartre le nom de ses esclaves peut être encore plus grande si plusieurs d'entre eux portent le même nom: Ulpien (Pomponius) D 6.1.5.5 (Si plures sint eiusdem nominis servi, puta plure Erotes....).
Les données juridiques sont-elles plus précises en ce qui concerne le prix de l'esclave?

\section{Paragraphe 2 : Le prix de l'esclave}

Le prix de l'esclave apparaît de deux manières : soit directement, soit par référence à la scame que doit verser l'esclave pour l'obtention de sa liberté (112).

L'apparition directe du prix peut elle même revêtir deux formes : le prix de l'esclave ressort seul ou bien d'une comparaison.

Pour ume meilleure présentation de l'information, les données seront présentées sous forme de tableaux, mais la valeur de ceux-ci ne sera ici qu'indicative.

\section{A - Le prix de l'esclave apparaît seul.}

\begin{tabular}{|c|c|c|c|}
\hline $\begin{array}{c}\text { juriste } \\
\text { auteur } \\
\text { du passage }\end{array}$ & $\begin{array}{l}\text { Juriste } \\
\text { cité }\end{array}$ & prix & Source \\
\hline Iavolenus & & 20 (aurei) & $\begin{array}{l}\text { D } 24.1 .50 \text { pr } \\
\text { D } 47.2 .75\end{array}$ \\
\hline Julien & & $\begin{array}{c}\text { (vicarius) } 10 \text { (aurei) } \\
\text { (vicarius) } 8 / 10 \text { (aurei) } \\
2 \text { esclaves vendus } 10 \text { chacun } ; \\
\text { un acheteur en propose } 30 . \\
20 \text { (aurei) } \\
10 \text { (aurei) } \\
5 \text { (aurei }\end{array}$ & $\begin{array}{ll}\text { D } & 10.3 .25 \\
\text { D } & 15.1 .37 .1 \\
\text { D } & 18.2 .17 \\
\text { D } & 30.81 .4 \\
\text { D } & 39.5 .2 .7 \\
\text { D } & 40.9 .5 .2 .\end{array}$ \\
\hline Pomponius & & $\begin{array}{r}5 \\
20\end{array}$ & $\begin{array}{ll}\text { D } & 24.1 .28 .4 \\
D & 40.5 .8\end{array}$ \\
\hline
\end{tabular}

112- H. WALLON, Histoire de $I$ 'esclavage, cit. II, p. $169 \mathrm{~s}$. , tient compe du prix de la liberté dans son analyse sur le prix de l'esclave (notanment p. $170 \mathrm{n}$. 1 et $171 \mathrm{n}, 1$ ). 
I B- Le prix de l'esclave ressort d'une comparaison

\begin{tabular}{|c|c|c|c|}
\hline $\begin{array}{c}\text { Juriste } \\
\text { auteur } \\
\text { du passage }\end{array}$ & $\begin{array}{l}\text { Juriste } \\
\text { cité }\end{array}$ & Prix & Source \\
\hline Africain & & $\begin{array}{l}\text { (vicarius) } \\
5 / 10\end{array}$ & $\mid \begin{array}{ll}D & 15.1 .38 .2 \\
\text { D } & 21.2 .47\end{array}$ \\
\hline Papinien & & $\begin{array}{l}10 \text { (ou moins) } \\
10 \text { (aurei) }\end{array}$ & {$\left[\begin{array}{ll}D & 38.2 .41 \\
D & 40.1 .20 .2\end{array}\right.$} \\
\hline Paul & $\begin{array}{l}\text { Mela } \\
\text { Plautius } \\
\text { Pomponius }\end{array}$ & $\begin{array}{c}\text { decem mili vm/viginti } \\
10 \\
10 / 20 \\
\text { decem/quinque milibus } \\
5 / 10 / 15 \\
10 \\
2 \text { esclaves pour 10 prix de } 10 .\end{array}$ & $\begin{array}{l}\text { D } 9.2 .55 \\
\text { D } 17.1 .5 .5 \\
\text { D } 17.1 .26 .8 \\
\text { D } 21.1 .57 .1 \\
\text { D } 24.1 .28 .3 \\
\text { D } 24.1 .28 .3\end{array}$ \\
\hline Ulpien & $\begin{array}{l}\text { Julien } \\
\text { Julien } \\
\text { Julien } \\
\text { Julien } \\
\text { Papinien }\end{array}$ & 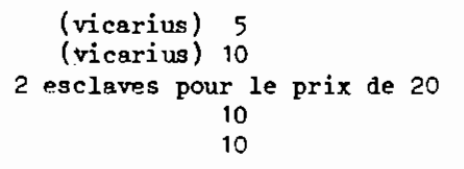 & $\begin{array}{ll}D & 15 \cdot 1 \cdot 11 \cdot 4 \\
D & 15 \cdot 1 \cdot 11.5 \\
D & 18 \cdot 2 \cdot 4.2 \\
D & 21 \cdot 2 \cdot 32.1 \\
D & 47.2 \cdot 14.5\end{array}$ \\
\hline
\end{tabular}

\begin{tabular}{|c|c|c|c|}
\hline $\begin{array}{c}\text { Juriste } \\
\text { auteur } \\
\text { du passage }\end{array}$ & $\begin{array}{l}\text { Juriste } \\
\text { cité }\end{array}$ & Priz & Source \\
\hline Iavolenus & $\begin{array}{l}\text { Servius, } \\
\text { Cascellius, } \\
\text { Ofilius, } \\
\text { Namusa, Labéon }\end{array}$ & $\begin{array}{l}\ldots \text { si servus alicui mort uus aut pro } \\
\text { eo centum legata essert. }\end{array}$ & D 33.4 .6 .1 \\
\hline Celsus & & Stichum aut decem. & D 31.15 \\
\hline Julien & & $\begin{array}{l}\text { Qui hominem aut decem debet } \\
\text { si hominea aut fundum non dederis, } \\
\text { centum dari spondes? } \\
\text { Qui hominem aut decem... dari pro- } \\
\text { misit. } \\
\text { Stipuiatus sum decem mihi aut hominem } \\
\text { Titio dari. } \\
\text { Si decem aut hominem dari stipula- } \\
\text { tus fuero. } \\
\text { Qui hominem aut decem stipulatus est }\end{array}$ & $\begin{array}{l}\text { D } 12.6 .32 .3 \\
\text { D } 34.5 .13 .2 \\
\text { D } 46.3 .34 \mathrm{pr} \\
\text { D } 46.3 .34 .2 \\
\text { D } 46.3 .34 .10 \\
\text { D } 46.4 .17\end{array}$ \\
\hline Pomponi us & & $\begin{array}{l}\text { Stichum/Stichum et Pamphilum... } \\
\text { decem/viginti. }\end{array}$ & D 46.4 .15 \\
\hline Gaius & Julien & $\begin{array}{l}\text { Decem/fundus Tusculanus/homo Stichus } \\
\text { Si... decem aut Stichum... stipulati } \\
\text { fuerint }\end{array}$ & $\begin{array}{l}\text { D } 45.1 .74 \\
\text { D } 45.2 .15\end{array}$ \\
\hline Marcellus & & $\begin{array}{l}\text { Si Titius mini aut decem debeat. } \\
\text { Stichum tibi, servo tuo decem lega- } \\
\text { vit vel contra tibi decem, servo tuo } \\
\text { Stichum } \\
\text { Qui decem debet.../servus. } \\
\text { Decem aut Stichum. }\end{array}$ & $\begin{array}{l}\text { D } 31.50 .1 \\
\text { D } 35.2 .56 .4 \\
\text { D } 46.3 .72 \mathrm{pr} \\
\text { D } 46.8 .17\end{array}$ \\
\hline Screvola & & Si debeas decem (milia) aut hominem & D 16.2 .22 \\
\hline Papinien & $\begin{array}{l}\text { Sabirus } \\
\text { Pegasus }\end{array}$ & $\begin{array}{l}\text { Si Pamphilum non dederis, centum dari } \\
\text { spondes. }\end{array}$ & D 45.1 .115 .2 \\
\hline
\end{tabular}


II - Le prix de l'esclave ressort de la somme due pour l'obtention de sa liberté

\begin{tabular}{|c|c|c|c|}
\hline $\begin{array}{c}\text { Juriste } \\
\text { auteur } \\
\text { du passage }\end{array}$ & $\begin{array}{l}\text { Juriste } \\
\text { cité }\end{array}$ & Prix & Source \\
\hline Paul & $\begin{array}{l}\text { Sabinus, } \\
\text { Proculus, } \\
\text { Marcellus }\end{array}$ & $\begin{array}{l}\text { Decem/Stichum. } \\
\text { Pro Sticho, quem mihi debes, decem } \\
\text { tibi doti erunt. } \\
\text { Sempronio decem aut... bominem Sti- } \\
\text { chum lego. } \\
\text { Titio usus fructus Stichi aut... } \\
\text { decem legata sunt. } \\
\text { Centum aureos... vel Stichum. } \\
\text { Si servo meo servus et mihi decem } \\
\text { legata fuerint. } \\
\text { Decem nut... hominem stipulor. } \\
\text { Si kalendis Stichum non dederis, de- } \\
\text { cem dare spondes ? } \\
\text { Si quis decem milia aut hominem } \\
\text { promiserit. } \\
\text { Te hominem et Seium decem mini dare } \\
\text { opportet. }\end{array}$ & $\begin{array}{l}\text { D } 13.5 .12 \\
\text { D } 23.3 .25 \\
\text { D } 31.8 .1 \\
\text { D } 33.2 .21 \\
\text { D } 34.3 .25 \\
\text { D } 35.2 .21 .1 \\
\text { D } 44.7 .44 .4 \\
\text { D } 45.1 .8 \\
\text { D } 45.1 .85 .4 \\
\text { D } 46.2 .32\end{array}$ \\
\hline Ulpien & $\begin{array}{l}\text { Celsus, } \\
\text { Marcellus. } \\
\text { Celsus } \\
\text { Julien } \\
\text { Julien, } \\
\text { Marcellus. } \\
\text { Julien }\end{array}$ & $\begin{array}{l}\text { Si decem aut Stichum stipulatus... } \\
\text { (suite) } \\
\text { Si quis Ephesi decem aut Capuae ho- } \\
\text { minem dari stipulatus experiatur. } \\
\text { Si quis ita stipulatus : Stichum aut } \\
\text { decem... } \\
\text {... Stichum aut decem deberet. } \\
\text {.. Stipulatur... decem vel hominem } \\
\text { Stichum. } \\
\text { Stichum aut decem fide tua iubes ? } \\
\text { Si is, qui hominem aut decém dari } \\
\text { stipulatus fuerat. } \\
\text { Decem aut hominem, utrum tu voles. } \\
\text { qui decem debet.../qui Stichum } \\
\text { debet. } \\
\text { Si Stichum aut decem sub condicione } \\
\text { stipulatus... }\end{array}$ & $\begin{array}{l}\text { D } 12.6 .26 .13 \\
\text { D } 12.6 .26 .14 \\
\text { D } 13.4 .2 .2 \\
\text { D } 30.75 .3 \\
\text { D } 30.75 .4 \\
\text { D } 45.1 .75 .8 \\
\text { D } 46.1 .8 .8 \\
\text { D } 46.1 .8 .9 \\
\text { D } 46.1 .8 .10 \\
\text { D } 46.3 .9 .1 \\
\text { D } 46.4 .13 .6\end{array}$ \\
\hline
\end{tabular}

\begin{tabular}{|c|c|c|c|}
\hline $\begin{array}{l}\text { Juriste } \\
\text { auteur } \\
\text { du passage }\end{array}$ & Juriste cité & Prix présumé & Source \\
\hline $\begin{array}{l}\text { Alfenus chez } \\
\text { euteur anony- } \\
\text { me }\end{array}$ & & 10 & D $40.7 .4 \mathrm{pz}$ \\
\hline Proculus & & 10 & D 12.6 .53 \\
\hline Iavolenus & $\begin{array}{l}\text { Q. Mucius, Servius } \\
\text { Ofilius, Labéon. } \\
\text { Trebatius, } \\
\text { ofilius, Labéon }\end{array}$ & $\begin{array}{l}100 \text { (numani) } \\
1000 \text { (nunumi) }\end{array}$ & $\begin{array}{l}\text { D } 40.7 .39 \mathrm{pr} \\
\text { D } 40.7 .39 .4\end{array}$ \\
\hline Neratius & & 10 & D 40.7 .17 \\
\hline Celsus & & 100 (en 5 ans) & D 40.7.23 pr \\
\hline Julien & & $\begin{array}{l}10 \\
10 \\
10 \\
10 \\
10\end{array}$ & $\begin{array}{l}\text { D } 28.5 \cdot 38.1 \\
\text { D } 30.91 \cdot 1 \\
\text { D } 40.7 \cdot 6.7 \\
\text { D } 40.7 \cdot 13.1 \\
\text { D } 40.7 \cdot 13.5\end{array}$ \\
\hline Pomponius & $\begin{array}{l}\text { Labéon, Aristo, Celsus } \\
\text { Q_Mucius }\end{array}$ & $\begin{array}{l}5 \text { (10 pour } 2 \text { esclaves) } \\
1000 \text { (nummi) (en } 3 \text { ans) } \\
10\end{array}$ & $\begin{array}{l}\text { D } 40.4 \cdot 11.1 \\
\text { D } 40.4 \cdot 41.1 \\
\text { D } 40.7 .29 .1\end{array}$ \\
\hline Africain & & $\begin{array}{l}10 \\
10 \\
10\end{array}$ & $\begin{array}{l}\text { D } 21.2 .46 .3 \\
\text { D } 40.7 .15 .1 \\
\text { D } 47.2 .62 .9\end{array}$ \\
\hline Mrecianus & Julien & 10 & D $35.1 .86 \mathrm{pr}$ \\
\hline Marcellus & & $\begin{array}{l}10 \\
10 \\
10\end{array}$ & $\begin{array}{l}\text { D } 39.6 .38 \\
\text { D } 40.7 .24 \\
\text { D } 46.3 .68\end{array}$ \\
\hline Scaevola & Servius & $\begin{array}{c}10 \text { (par an, pendant } 10 \text { ans) } \\
10 \\
10\end{array}$ & $\begin{array}{l}\text { D } 12.6 .67 \mathrm{pr} \\
\text { D } 21.2 .69 .2 \\
\text { D } 21.2 .69 .3\end{array}$ \\
\hline
\end{tabular}




\begin{tabular}{|c|c|c|c|}
\hline $\begin{array}{c}\text { Juriste } \\
\text { auteur } \\
\text { du passage }\end{array}$ & Jurigte eité & Prix présumé & Source \\
\hline & & 60 (en 5 mois) & D 40.7 .40 .2 \\
\hline Papinien & & $\begin{array}{c}20 \text { (aurei) } \\
20 \text { (solidi) } \\
10\end{array}$ & $\begin{array}{l}\text { D } 4.4 .31 \\
\text { D } 40.4 .47 \mathrm{pr} \\
\text { D } 40.7 .34 \mathrm{pr}\end{array}$ \\
\hline Tryphoninus & & $\begin{array}{l}\text { decem milia } \\
\text { decem milia.. }\end{array}$ & $\begin{array}{l}\text { D } 1.5 .15 \\
\text { D } 49.15 .12 . \\
11\end{array}$ \\
\hline Paul & $\begin{array}{l}\text { Plautius } \\
\text { Sabinus, Julien } \\
\text { Labéon }\end{array}$ & $\begin{array}{c}10 \\
10 \\
10 \\
10 \\
10 \text { (chaque année pend. } 3 \text { ans) } \\
10 \\
10 \\
10 \\
10 \text { (en operae) } \\
10 \\
10\end{array}$ & $\begin{array}{l}\text { D } 35.1 .44 .10 \\
\text { D } 40.7 .4 .3 \\
\text { D } 40.7 .4 .6 \\
\text { D } 40.7 .10 \\
\text { D } 40.7 .18 \\
\text { D } 40.7 .20 \mathrm{pr} \\
\text { D } 40.7 .20 .2 \\
\text { D } 40.7 .20 .3 \\
\text { D } 40.7 .20 .5 \\
\text { D } 40.7 .41 .1 \\
\text { D } 44.4 .3\end{array}$ \\
\hline Upien & $\begin{array}{l}\text { Sabinus } \\
\text { (rescrit de Sévère et } \\
\text { Caracalla) } \\
\text { (constitution de Marc- } \\
\text { Aurèle) } \\
\text { Pomponius } \\
\text { Julien }\end{array}$ & $\begin{array}{c}20 \text { (aurei) } \\
10 \\
10 \\
100 \\
10 \\
10 \\
10 \\
10 \\
10 \\
10 \\
10 \\
10 \text { (aurei) (chaque année pen- } \\
\text { dant } 3 \text { ans) } \\
10 \text { (chaque année pend. } 3 \text { ans) } \\
10 \\
10\end{array}$ & $\begin{array}{l}\text { D } 5 \cdot 2 \cdot 8 \cdot 17 \\
\text { D } 29 \cdot 4 \cdot 6 \cdot 10 \\
\text { D } 33 \cdot 8 \cdot 8 \cdot 3 \\
\text { D } 33 \cdot 8 \cdot 8 \cdot 7 \\
\text { D } 40 \cdot 5 \cdot 4.6 \\
\text { D } 40 \cdot 7 \cdot 3 \cdot 1 \\
\text { D } 40 \cdot 7 \cdot 3 \cdot 5 \\
\text { D } 40 \cdot 7 \cdot 3 \cdot 6 \\
\text { D } 40.7 \cdot 3 \cdot 7 \\
\text { D } 40.7 \cdot 3 \cdot 8 \\
\text { D } 40 \cdot 7 \cdot 3 \cdot 12 \\
\text { D } 40.7 \cdot 3 \cdot 13 \\
\text { D } 40 \cdot 7 \cdot 3 \cdot 14 \\
\text { D } 40.7 \cdot 6.5 \\
\text { D } 44 \cdot 4 \cdot 2 \cdot 7\end{array}$ \\
\hline L. Rufinus & . & 10 & D 40.7 .32 \\
\hline Modestin & $\begin{array}{l}\text { Paul } \\
\text { Publicius }\end{array}$ & $\begin{array}{l}20 \text { (aurei) } \\
10\end{array}$ & $\begin{array}{l}\text { D } 5.2 .9 \\
\text { D } 35.1 .51 .1\end{array}$ \\
\hline
\end{tabular}

\begin{tabular}{|c|c|c|c|}
\hline $\begin{array}{c}\text { Juriste } \\
\text { auteur } \\
\text { du paserge }\end{array}$ & Jurigte cité & Prix présumé & Solurce \\
\hline Bermogénien & & 10 & D 35.1.94 pr \\
& 10 & 35.1.94.1 \\
\hline
\end{tabular}

Plusieurs remarques g'imposent. Tout d'abord, le prix de la liberté peut ne pas être un indicateur valable du prix de I'esclave. On peut en effet vouloir avantager l'esclave et lui demander une some inferieure à sa valeur rélle (113). Néanmoins, les moyennes ressortant de cette rubrique sont identiques à celles relevées dans I'étude sur le prix de l'esclave, lorgque celui-ci apparaît dans une hypothèse autre que celle de la sonme à payer pour la liberté. Il peut aussi alors exister une distorsion entre la somme indiquée et le prix réel de l'esclave. Par exemple, Papinien traite de deux esclaves qui ont été donnés en gage pour 10 aurei. Or, l'un d'eix ne vaut pas moins de 10(114). Dans d'autres textes (115), il est question d'une somme remise par un individu dans le but d'affranchir l'esclave d'autrui. Là encore, la somme remise peut ne pas correspondre au prix réel de l'esclave (116). De même, on ne peut retenir les textes où il est

113- L'hypothèse inverse peut également se produire, cf. par ex. Africain D 21.2 .46 .3 .

114- Chez Ulpien D 47.2.14.5.

115- Ainsi Africain D 30.108 .14 (te, Titi, rogo, ut acceptis centum illwn servun manumittas) ; Papinien D 19.5.7 (Si tibi decem dedero, ut Stichuon manumittas) ; Id. D 40.5.22 pr (Si legatario fundi decem pretii nomine relicta sint in hoc, ut servion surm manumittat) ; Paul D 40.5.6 (Decem legata ount et rogatus est legatarius Stichum emere et marwonittere) ; U1pien D 28. 7.8.7 (Titio centuon ita lego, ut Stichrm manumittat) ; id. D 40.5.7 (Si cui legata sint centum ita, ut servion alienum redimat et manumittat).

116- Pomponius D 40.5.8 : Evon cui mille numi legati fuissent, si rogatus fuisset viginti servion manumittere, c'est-a-dire : un testateur lègue 1000 deniers pour que le legataire af franchisse un esclave qui vaut 20 (sous-entendu aure $i$, cf. H. WALLON, o.c., p. 169). Coupte tenu du fait que 1 aureus = 25 denarii (cf. H. MATTINGLY, Roman coins from the earliest times to the fall of the western Empire, Londres (1947), p. 122), le testateur lègue donc le double de la valeur réelle de l'esclave. 
question d'une somme sanctionnant l'inexécution d'un affranchissement (117), la peine pouvant entraîner une surévaluation du prix de l'esclave.

Contrairement aux sources littéraires et épigraphiques (118), on trouve un grand nombre de valeurs moyennes dans les questions traitées par les juristes ou tranchées par la loi. Les prix les plus élevés sont de

117- Ainsi Scaevola D 45.1.122.2 : Si hominem Stiohom, de quo agitur, quem hac dis tibi donationis causa manumissionisque dedi, a te heredeque two manumissus vindictaque liberatus non erit, quod dolo malo meo non fiat, poenae nomine quinquaginta dari stipulatus est...

118- A 1'époque de Caton 1'ancien, le prix d'un esclave semblait déja assez êlevé puisqu'il est disposé à en donner 1500 deniers (Plut., Cato maior IV, 5). Sous Auguste, Horace (Sat. II, 7, 43) parle de 500 drachmes $a$ propos d'un esclave bon warche, mais (Ep. II, 2,5) de 2000 deniers pour un esclave cultivé, connaissant le grec ; BGU IV, 1128,7 (14 gv. J.C.), 1000 drachmes d'argent équivalent a 1 'époque $a 1000$ deniers; BGU, IV, 1114,16 (5 av. J.C.), 1200 drachmes. Des prix variés sont aussi attestés plus tard : CIL IV, 3340, 49 (- FIRA III 407 n. 128 d.), 5300 sesterces pour deux esclaves veterconi, i.e. 662,5 deniers chacun. Petrone LXVIII, 7, indique la some de 300 deniers pour un enfant de talent, bon imitateur; il semble s'agir d'une "bonne affaire". Martial (VI, 66, 9) évalue a 600 deniers une prostituée de bas étage ; il indique ailleurs ( $\mathrm{x}, 31,1) 1$ 'achat $d^{\prime}$ un esclave pour 1300 deniers (W.L. WESTERMANN, S1ave Systems, cit., p. 100 et $P$. DE MARTINO, Storia, cit. IV, p. 26, n. 66, donnent 1200 bien que le texte inMARTINO, Storia, cit. IV, P. $26, \mathrm{n}$. 66, donnent 1200 bien que le texte indique 1300 Addixti servion numis, here, mille trecentis). Juvenal (IV, 26) donne 6000 sesterces (= 1500 deniers) pour un esclave pêcheur, mais i1 rappelle que le pêcheur peut coûter moins cher que le poisson 1 (potuit fortasse minoris piscator quom pisci emi). A ce sujet, Pline (nat. hist. $X$, 29 (43), 84) nous apprend qu'un rossignol blanc donne à Agrippine, feume de Claude, coûta 6000 sesterces. Columelle (III, 3, 8) évalue à 8000 sesterces (- 2000 deniers) un bon eaclave vigneron. On trouve par ailleurs des prix três élevés d'esclaves de luxe (Plin. nat. hist. IV, 12 (10) 56 200000 sesterces pour deux jumeaux se ressemb int parfaitement; Mart. III, 62,200000 . Mart. III (2) (autres donnees in Dow the Roman Empire, p. 349) Pour Te 2eme siecle, on nas a dindications sur le prix des esclaves a Róme, mais seulement dans les provinces. W.L. WESTERMANH, RE, Suppl, VI p. 1012 s., donne des prix d'enfants et d'adolescents entre 175 et 600 deniers dans les provinces orientales et en Egypte, et des prix d'adultes entre 350 et 700 de Ravenne a l'Orient. Une esclave expérimentée est payée 625 deniers par un marin a Ravenne. (F. PREISIGXE, Sammelbuch der griechischen papyri, $\mathrm{n}^{*} 6304$, tablette de cire du 2ème siècle, 2SS 42, 453). Un garçon grec est paye 600 deniers en 142 (CIL III, 941) ; Une fille de six ans 205 deniers en 139 (CII III, 937) ; les données concernant 1a partie occidentale de l'Pmire au 3 ìm siècle n'of fient des exemples que de prix tress très éleves, correspondant a des esclaves de luxe. SRA, Elagabalus, XXV, donne 1 exemple d'une prostituée achetée 100000 sesterces. Les prix pour cette perriode semblent plus utilisables en Egypte ( $v$. H.L. KESTERMANN, Slave Systems, cit., p. $101 \mathrm{n}, 131)$. CIL, VIII, 239561.14 indique le prix de 500 deniers ex forma censoria; sur la valeur de cette inscription, F. GROSSO, Pretiven servi ex forma censoria, in Latomus, Mélanges Renard, II, Bruxelles (1969), p. 302-310
I'ordre de 5000 deniers (119) - ce prix apparaît chez Paul (120) - à 2500 - prix que l'on trouve aussi bien chez Iavolenus (121), Celsus (122) et Julien (123) que chez Screvola (124) et chez Papinien (125), Tryphoninus (126), Paul (127)et Ulpien (128). Scaevola indique une somme de 1500 deniers qu'un esclave doit verser en $c$ inq mois pour parvenir à la liberté (129). Cependant, les prix contenus dans le Digeste sont, dans leur inmense majorité, plus bas. On relève le prix de 500 deniers chez Iavolenus (130), Julien (131), Papinien (132), Paul (133) et Upien (134), mais le prix le pluss fréquent est de 250 deniers (135). Paul nous donne le cas d'un esclave

119- Il faut raisonner sur la base de 1 aureus $=25$ denarii $=100$ sestertii, cf. H. Mattingly, Roman Coins, cit., p. 122

120- Paul 9.2.55 : Qunon Stichus esset decem (milivon), Pamphilus viginti. H. WALLON, Histoire de 1 'esclavage II, cit., p. $170 \mathrm{n} .1$, ëmet un doute quant gu fait de savoir s'il s'agit de mille sesterces ou d'aurei. Il est vrai que d'autant plus a craindre qu'elle ne change en rien la démonstration du juriste.

121- Iavolenus (Servius, Cascellius, ofilius, Namusa, Labéon) D 33.4.6.1, en interprétant (come le fait H. WALLON, O.c. II, p. $169 \mathrm{n}$. 1 pour Ulpien D 15.1.11.4) la somme donnée (100) en curei (= 2500 deniers).

122- Celsus D 40.7.23 pr (100).

123- Julien D 34.5.13.2 (100).

124- Scaevola D 16.2.22 (decem 'milia').

125- Papinien D 45.1.115.2 (100).

126- Tryphoninus D 1.5.15 et D 40.15.12.11 (decem milia).

127- Pau1 D 9.2.55; D 21.1.57.1 et D 45.1.85.4 (decem milia)/34.3.25 (100 ourei).

128- Ulpien D 33.8.8.7 (100).

129- Scaevola D 40.7.40.2 (60).

130- Irvolenus D $24.1 .50 \mathrm{pr}$ (20).

131- Julien D 30.81 .4 (20 aurei).

132- Papinien D 4.4.31 (20 aurei).

133- Paul (Mela) D 17.1.26.8 (20).

134- Ulpien D 5.2.8.17 (20 aurei).

135- C'est-ă-dire 10 aurei. Ce prix apparaît chez tous les juristes (cf. tableaux). 
ne sachant rien encore, acheté 10 (aurei) et revendu 20 au sortir de l'npprentissage (136). Le Digeste nous fournit encore des prix de vicarii ralant 250 (137), 200 (138) ou seulement 125 deniers (139). Il s'agit probablement $d$ 'hypothèses (140), mais rien n'interdit de penser que ces chiffres sont empruntés à la réalité. On pourrait nous opposer le prix excessivement bas ( 50 deniers) offert pour une esclave dans un texte de Iavolenus, mais il ne paut pas oublier qu'il s'agit d'une ancilla furtiva, et que le voleur l'a peut-être vendue $\mathrm{a}$ bas prix, pressé qu'il était de s'en débarrasser (141).

Cela dit, les textes les plus sûrs quant au prix moyen de l'esclave semblent être ceux qui contiennent ron plus des exemples choisis par les juristes, mais de véritables prescriptions (142). Plusieurs constitutions impériales ont préru le cas ol des esclaves, affranchis par une générosité imprudente ou par un acte invalidé, voyaient leur liberté compromise. Celle-ci était néanmoins maintenue contre remboursement au maître de 20 aurei (= 500 deniers) (143), prix qui apparaît donc comme valeur moyenne de l'esclave $(144)$.

\section{6- Paul (Mela) D 17.1.26.8.}

137-. Julien D 10.3.25 (10 aurei); Africain D 15.1.38.2 (10 aurei); v1piẹn D $15.1 .11 .5 \cdot(10)$.

138- Julien D 15.1.37.1 (8 aurei).

139- Ulpien D 15.1.11.4 ( 5 ).

140- H. WALLON, Histoire de 1 'esclavage, II, cit., p. 169.

$141-$ Iavolenus D $47,2.75$ (2 aurei).

142- H. WALLON, o.c., II, p. 170 s.; F. DE MARTINo, Storia IV, cit., p. 339, n. 6 .

143- Ulpien D 5.2.8.17: Plane si post quinquennizm inofficiosum dici coeptron est, ex magna et iusta causa, libertates non esse revocandas, quae competiement vel praestitae sunt, sed viginti aureos a singulis praestandos victori ; Papinien D 40.4.47 : Quum ex falsis codicillis per errorem libertas licet non debita, praestita tomen ab herede fuisset, viginti solidos a singulis hominibus inferendos esse heredi princeps constituit, cf. aussi $D$ 4.4.31 qui rappelle cette constitution d'Hadrien, qui est confinée a 1 'époque de Caracalla par CI 7.4.2, cf. 8.29.3 de 223 .

144- H. WALLON, O.c. II, p. 172 signale un autre texte qui ne concerne pas les esclaves, mais qui cependant paraît donner une moyenne de leur prix. Il s'agit de $D$ 9.3.1 pr ulpien, a propos du donmage causé par la chute d'un objet. L'édit du préteur, pour toute chose appréciable, fixe une réparation au double, et la porte, pour 1 'homme libre, a 50 aurei. Or, comme l'homme libre ne peut pas être estimé moins qu'un esclave, et que le préteur fixe une indemitê au double, le prix moyen de l'esclave devrait rarement dépasser 25 aurei (625 deniers). Ce serait là, pour 1 'auteur, une moyenne élevée.
Si le Digeste permet d'obtenir une valeur présumée moyenno de l'esclave, il ne permet en revanche de dégager aucune évolution. On estime généralement que la pin des captures massives a dû provoquer une augmentation du prix des esclaves (145). Cependant, aucune bausse des prix ne ressort de nos textes, que ce soit entre la fin de la République et le début de l'Bupire ou entre le premier et le second siècles de notre ère. On découvre certes des prix différents, mais ils correspondent à l'âge, à l'expérience, au degré d'instruction, ou encore aux attraits physiques de l'esclave (146). Le Digeste n'autorise donc pas à invoquer l'argument d'une bausse des prix de la main-d'oeurre servile pour affirmer que le nombre d'esclaves diminue au ler ou an zème siècle de notre ère (147).

145- J. ROLENDO, Lavoro servile, cit., p. 21, insiste aussi sur le fait que, suite a la pacification interne de 1 'Empire, la piraterie devint une source d'esclavage négligeable. Sur le thểe de la piraterie, v. H .A. ORMEROD, Piracy in the Ancient World, Liverpool, Londres(1924) ; E. MARORI, Die Rolle der Seerauber unter den Anhangern des Sextus Pompeius, Sozialökonomische Verhältnisse im Alten Orient und im Klassischen Altertum, Berlin (1961), p. 208-216 ; W.L. WESTERMANN, Slave Systems, cit., p. 85.

146- W.L. WESTERMANN, Slave Systems, cit., p. 100. De plus, les prix peuvent différer en fonction de 1 'endroit, $v$. pour 1 'Egypte $P$. RYLANDS, Papyrus 244 in Catalogue of the Greek papyri in the John Rylands library, Manchester (1915),2 : 244,10-16; v. aussi A. SEGRE, Circolazione monetaria e prezzi nel mondo antico ed in particolare in Egitto, Rome (1922), 173 ; sur la différence de prix entre un homme et une femme esclaves, BGU $4: 1128$, 7,15. J. CESKA, Diferenciace otracu . Italii, Praha, Brno (1959), p. 5865 , donne une table des prix approximatifs des esclaves aux deux premiers siècles de l'Empire, qui colle assez bien aux données fournies par les juristes : de 2500 a 5000 deniers, les esclaves hautement qualifiés; de 2000 à 2500 , les esclaves bien instruits; de $750 \mathrm{a} 2000$, les esclaves qualifiés, capables de travailler de maniêre autonome; les esclaves robustes, utilisables pour des travaux simples, coûtaient environ 500 deniers, et enfin, les esclaves moins utiles, les enfants, 250 deniers:

147- Pour une diminution du nombre, F. DE MARTTNO, Storia IV, p. 339, n. 6 ; W.L. WESTERMANN, RE, Supp1. VI p, $1012 \mathrm{~s}$, , qui reconnaît pourtant (Slave Systems, cit., p. 101) que la diminution du nombre des esclaves durant les trois premiers siècles de l'Emirire ne peut être établie grâce à un traitement scientifique des données. Contre 1'idée d'une diminution, J. CES$\mathrm{KA}$, (Diferenciace, cit.) pense que la thêorie selon laquelle le décilin de l'esclavage est dû à la forte hausse du prix des esclaves est infondée, les prix en vigueur aux deux premiers siècles étant les mêmes que ceux pratiqués durant les derniers siècles de la République. E.M. SclitaERMan, La schiavitù, cit., p. $26 \mathrm{~s}$. critique sévèrement W.L. WESTERMANN selon qui 1 a crise de 1 'économie esclavagiste serait due a la diminution quantitative des esclaves. D'autre part, 1 'idée soutenue par F. DE MARTINO (Storia IV, p. 339 s.) selon laquelle la diminution du nombre des esclaves serait prouree,e, au 2ème siècle, par les mesures législatives destinées à lui assurer un meilleur traitement, paraît fragile. Il faut rechercher ailleurs les causes de ces mesures (peur des maîtres, proportion importante d'esclaves fugitifs). On reviendra plus loin sur ce problème. Notons aussi que pour P. ANDERSON, 
D'ailleurs, les effets supposés de diminution de l'approvisionnenement ne se font sentir que très longtemps après la fin des conquêtes républicaines (148). De plus, si les prix avaient réellement augmenté, l'acquisition d'esclaves aurait perdu de son caractère économiquement rentable (149). Or, le discours des juristes est là pour nous rappeler qu'un tel investissement, soit pour l'utilisation directe de l'esclave, soit pour les salaires obtenus en louent ses travaux, reste valable (150).

Quoi qu'il en soit, l'arrêt de la politique de conquête systématique a évidemnent réduit l'afflux extérieur de main-d'oeuvre servile (151).

Les passages, cit., p. 102, les prix des esclaves bsisseraient au 3ème sieccle, compte tenu d'un effondrement de la demande; contra, E.M. SCHTAERMAN, La schiavitù, cit., p. 25, qui indique, pour le 3ème siẻcle, une augmentation des prix due à la dévaluation de la monnaie. Erfin, L. RUGGINI, Economia e societa nell'Italia annonaria. Rapporti fra agricoltura e cowmercio dal IV al VI secolo d.C., Milan (1961), p. 565, affirme que les prix se maintiennent approximativement au même niveau du zème au tềme siècle. malgré le tarissement des principales sources d'esclavage.

148- M.I. FINLEY, L'êconomie antique, cit., p. 113 ; E.M. SCHTAERMAN, La sohiavitù, cit., p. 4.

149- F. DE MARTINO, Storia, cit. IV, p. 26 s.; J. KOLENDO, Lavoro servile, cit., p. 21 .

150- W.L. WESTERMANN, Slave Sygtems, p. 101 s. On a montrế cette continuité dans la Section 1 (analyse comparative des divers modes d'acquisition).

151- A.H.M JONES, The Later Roman Empire, II Oxford (1964), p. 793, 851 ; E. CICOTTI, Il tramonto della schiavitù, Turin (1899), p. 282 ; R.B. BARROW, Slavery in The Roman Empire, Londres $(1928)$, p. 4 ; M. ROSTOVTZEFF, Social and Economic History of the Roman Empire (1926), p. 539, n. 41 ; Social and Econic the Roman Empire (1926), p. 539, n. 41 M. MAZZA, Lotte sociali, cit., p. 155 ; J. GAUDEMET, Institutions de 1 'Antiquité, cit., p. 534 ; W.L. WESTERYANN, Slave Systems, cit., p. 86, 97, 119 ; F. DE MARTINO, Storia cit. IV, P. 339 et V p. 148. La guerre pose le problème de la captivité et donc du postliminizum. On a déja indiqué les motifs du rejet de ces textes, hormis ceux où il est question d'un esclave, et qui se trouvent mentionnés supra Chapitre prêliminaire n. 17. Voici une liste complete de ces textes. Il s'agit de Proculus D $49.15 .7 \mathrm{pr}$; Iavolenus D 28.3.15; D 41.2.23.1 ; D 49.15.27 (Trebatius, Ofilius, Labêon); Julien D 1.5.26; D 23.1.1I ; D 24.2.6; D 28.1.12; D 28.6.28; D 30 . 98 ; D 30.101.1 ; D 49.15.22 pr-1-2-3-4; D 49.15.23 ; Pomponius D 9.2.43; D 11.1.36; D 19.1.55 (Octavenus); D $24.3 .10 \mathrm{pr}$; D 30.9 (Octavenus); D 38.4.13.2 ; D 38.17 .10 .1 ; D 49.15.5 pr-1-2-3 (i1 s'agit 1a d'un problème différent, c'est un ennemi qui a été fait prisonnier par les Romains, et qui devient esclave à Rome) ; D $49.15 .14 \mathrm{pr}-1$; D $49.15 .20 \mathrm{pr}-2$; Gaius D $28.1 .8 \mathrm{pr}$; D 28.5 .32 .1 ; D $41.1 .7 \mathrm{pr}$; Venuleius D 10.2.7; D 45.3.25 ; Marcel1us D 49.15.1 ; Florentinus D 49.15.26 ; Scaevola D 28.2.29.6-7-10-14 ; D 28.6.29 ; Papinien D 4.6.19; D 12.6.3 (rescrit d'Antonin 1e pieux) ; D 27.3 .8 ; D 36.3.5 pr ; D 38.16.15; D 41.3.44.2-7; D 45.3.18.2 ; D 49. $15.10 \mathrm{pr}-1$; D 49.15.11 $\mathrm{pr}-1$; Tryphoninus D $49.15 .12 \mathrm{pr}-1-2$ (Julien)-3-45-6-7-8-9 (veteres, Sabinus)-10-11-12-13-14-15-16-17 (constitution de Sévẻre et Caracalla) -18 ; Callistrate D 4.6.14; Paul D 3.5.18.5; D 3.5.20 pr (Servius, Alfenus) ; D 7.4.26; D 10.2.25 pr; D 15.2.2.1; D 23.2.60 pr ; D 23.4 .8 ; D 24.2.1; D 24.3.56; D 28.2.9.2; D 28.2.31; D 28.3.9;
Comment en assurer alors la reproduction?

\section{CHAPITRE II : LA REPRODUCTION DU GROUPE SERVIIE}

Après la cessation des conquêtes (152), le problène de la reproduction des forces productives s'est posé avec plus d'acuité. Dès lors, la naissance, en tant que mode d'asservissement, semble avoir pris de $1^{\prime}$ im portance, mais des entorses aux principes du droit romain allaient aussi consacrer la réduction en servitude d'hommes libres.

\section{SECTION 1. LA REPRODUCTION INTERNE, PAR LA NAISSANCE}

L'épigraphie fournit de très nombreux exemples a'unions durables ou sont impliqués des esclaves, unions constitutives de véritables familles de fait, et le vocabulaire utilisé par les conjoints est celui normalement

D 28.3.10 (Sabimus); D 28.7.9; D 29.1.39; D 29.2.33; D 35.2.1.1-4; D 35.2.18 pr; D 38.2.4.1-2; D 38.2.44.2;D 39.5.34.1; D 41.3.11; D 41 . $3.15 \mathrm{pr}$ (Julien, Marcellus); D 45.1.73.1; D 46.3.98.8; D 46.4 .11 .3 ; D 48 . 10.22 .1 ; D 49.15.8; D 49.15.13; D 49.15.17 ; D 49.15.19 pr-1-2-3-4-510.22 .1 ; D 49.15.8 ; D 49.15.13; D 49.15.17; D 49.15.19 pr-1-2-3-4-5-
$6-7-8-9$; D 49.15.10; D 49.15.28 (Labeon); D 49.15.29 (Labeón); U1pien D 2.11.4.3; D 3.1.1.6 ; D 3.3.19; D 3.5.19; D 4.6.1.1 ; D 4.6.15 pr-1-3; D 4.6.23.1-2 (Papinien)-3; D 11.1.16 pr; D 19.2.13.3; D 23.1.9.1 ; D 23. 2.45.6 (Julien) ; D 24.1.32.14; D 26.1.6.4; D 26.1.14.2; D 26.1.15; D 26.2.16.2 ; D 26.4.1.2 ; D 26.4.3.5; D 27.3.7.1; D 28.1.20.1 ; D 28.3. 6.1-4-5-12 ; D 29.1.10; D 29.2.71 pr ; D 29.5.1.12 (Scaevola); D 30.43 . 3 ; D 30.47.2-3 ; D 30.53.9 ; D 32.1 pr ; D 34.8 .4 .1 (Julien) ; D 35.2 .43 ; D 36.2 .7 .2 ; D 37.1 .3 .6 ; D $37.1 .12 \mathrm{pr}$; D 37.4.1.3 (Pomponius) -4 ; D 37 . $4.8 .9 ;$ D 37.6 .1 .17 ; D 37.8.1.8; D 37.9.1.8; D 38.4.1.2; D 38.7.2.3; $D$ 38.16.1.1 (rescrits de Marc-Aurềle et Verus et de Caracalia)-7; D 38.16 . 2 pr ; D 38.17.1.1-3 (rescrit de Sévère et Caracalla); D 38.17.2.3-7-30; D 40.4.30 (pour mémoire, il faut signaler un texte intéressant, bien que $s^{\prime}$ 'éloignant du problème : il s'agit de $D$ 40.5.24.2 qui nous apprend qu'on peut laisser une liberté fidéiconmissaire à l'esclave d'un ennemi, dans le cas où il devient notre alliê) ; D 40.7.6.1-2; D 42.5.22.1; D 43.5.1.9; D 43.29.3.3; D 44.2.11.4; D 44.3.1; D 46.6.4.5; D 47.2.41 pr-3 (Pomponius) ; D $47.2 .46 \mathrm{pr}$; D 49.15 .9 (rescrit de Sévère et Caracal1a) ; D 49 15.15 ; D 49.15.16 ; D 49.15.18; D 49.15.21 pr-1 ; D 49.15.24; D 49.16. 8; D 49.17.9; D 50.16.141; Marcien D 1.8.6 pr; D 49.14.3! (rescrit de Comonode); D 49.15.25 (rescrit de Sévère et Caraca11a); Modestin D 49. 15.4 ; Hermogénien D 38.7 .6 ; D 50.4 .1 .4 .

152- Néanmoins, cette source d'esclavage ne se tarit pas complètement (cf. Ios. be1, iud. VI, 9, 3 ; Chron. Pasc. I, 474 (Dind.) ; Hieron. ad Zach. XI, 5 ; ad Ierem. XXI, 15); v. R.H. BARROW, Slavery, cit., p. 4 s. J. GAUDEMET, Institutions de 1 'Antiquité, cit., p. 543, n. 6 ; E.M. SCHTAERMAN, La schiavitù, cit.. p. 13 g. 
employé pour les unions fondées sur le comubicon (153). Or, selon le droit romain, les esclaves ne peuvent se marier, que ce soit avec des eaclaves ou avec des personnes libres (154). Les enfants issus de ces mions sont donc illégitimes, ils suivent la condition de leur mère (155).

On peut constater sur ce point une libéralisation progressive. Alors qu'zar départ le droit romain, réaliste, prenait en consideration la condition de la mère au moment de la naissance, les juristes vont faire prévaloir des règles de plus en plus favorables à l'enfant (156). Ils sont par exemple divisés sur le point de savoir si, par favor libertatis, l'enfant naft libre lorsque sa mère a joui de la liberté pendant une période de la grossesse, mais est retombée en servitude lors de la naissance (157).

Le Digeste est assez riche en la matière. Le terme le plus usité pour désigner l'enfant de l'esclave est partus (158). On trouve aussi

153- Ex. CIL, VI, 8676, vie commune pendant quarante ans ; CIL, VI, 8409 a, $4847,8819,8827,22425$; R.H. BARROW, Slavery, cit., p. 158 s. Sur le caractère de pur fait de $1^{\prime}$ 'union des esclaves sous le Principat, E. POLAY, Caractère de pur fait de 1 union des esclaves sous le Princi
Die Sklavenehe im antiken Rom, Das Altertum 15 (1969), 86.

154- J. GAUDEMET, Institutions de l'Antiquité, cit., p. 549.

155- M. KASER, Partus ancillae, zSS 75 (1958), p. 156 s. ; G. FOTI, Contubernium, NNDI IV (1959), p. 769 ; le partus ancillae n'est pas considéré comme un fruit, M. BRETONE, Frutti (dir. rom.), NNDI vII (1968), p. $665 \mathrm{~s}$. Julien D 30.84.10 : partus matrem sequitur. (id. in Gaius 1, 78).

156- J. GAUDEMET, Institutions, cit., p. 544.

157- Gaius I, 90-91; I.J. 1.4 pr ; Marcien D 1.5.5.2-3.

158- Proculus D 31.48 pr ; Iavolenus (Labéon) D 24.3.66.3; Celsus D 23. 3.58.1; D 30.63 (Servius); Julien D 12.4.7.1; D 21.2 .8 ; D 24.3.31.4; D 30.82 .4 ; D 30.84.10; D 30.91.7; D 41.3.33 pr ; D 41.4.9; D 41.4.10; Pomponius D 15.2.3 ; D 18.1.8 pr ; D 18.1.31; D 23.3.18 (Labéon) ; Africain D 47.2.61; Gaius D 6.1 .20 ; D 20.1 .15 pr (Julien); D 20.4.11.3 ; D 22.1.28.1; D 35.2.73 pr; D 39.5.11; D 41.3.36.1; Maecianus D 35.2.30 pr ; Venuleius D 41.1.66; D 42.8.25.4-5 (Proculus) ; Papinien D 22.1.2 ; D 22.1.4.pr-1; D 22.1.8; D 23.3.69.9; D 31.73 ; D 31.77.17; D 33.7.3 pr ; D 35.2.9.1; D 36.1.60.4; D 41.3.44.2; Tryphoninus D $1.5 .15 ; \mathrm{D} 15.1$. 57.2 ; D 49.15.12.18; Paul D 6.1.16 pr; D 10.2.11; D 12.4.12 ; D 12.6 . 65.5 ; D 13.7.18.2 ; D 19.1.21 pr ; D 21.2.42; D 22.1.14 pr-1 (Neratius); D 24.1.28.5 (Julien, Pomponius) ; D 35.2.24.1; D 41.3.4.5-16 (Sabinus, Cassius) ; D 41.10 .2 ; D 45.1 .83 .8 ; Ulpien $\mathrm{D} 3.5 .3 .6$; D 4.2 .12 pr ; D 5.3.20.3 ; D 5.3.27 pr ; D 6.1.17.1 (Julien) ; D 6.2.11.2-3 (Julien) -4 (Julien) -5 (Pomponius) ; D 7.1.68 pr (Brutus) ; D 10.3.6.4 (Sabinus, Atilicinus); D 10.4.9.7 (Sabinus, Pomponius); D 12.2.11.1; D 16.3.1.24; D 18.2.4.1 (Julien)-2 (Julien); D 21.1.31.2-6 (Pomponius)-24; D 33.8.8.8 (Julien); D 36.1.23.3 (Neratius); D 36.4 .58 ; D 40.5 .26 .3 (rescrit de (Julien) ; D 36.1.23.3 (Neratius) ; D 36.4 .58 ; D 40.5.26.3 (rescrit de D $42.8,10.21 ; D 44.2 .7 .1-3 ; D 47.2 .48 .5-6$; D 47.4.1.11; D 50.16.26 (Scaevola); Marcien D 40.5.53 pr.
159- Lavolenus (Trebatius, Labéon) D 28.8.11; Julien D 42.8.17.1 ( $f$ il $i$ is naturalibus); Geius D 40.4.24; Maecianus D 40.5.54; Scaevola D 32.37.7; D 32.41 .2 (filizi naturales) -5 ; D 33.7.20.1-4 (filia) ; D 33.7.27.1 ; D 36 . 1.80 .2 (filizm naturalen) ; D 40.5 .41 .5 ; Papinien D $17.1 .54 \mathrm{pr}$ : Tryphoninus D 31.88.12 (filia naturalis) ; Paul D 9.2.33 pr (Pedius) (filizan naturalem) ; D 19.5.5 pr (naturalis meus filius); D 23.2.14.2 (filia); D 35.2 .63 pr (filius naturalis) ; 38.10 .10 .5 ; D 40.12.23.2; D 40.5 . 39.1 ; Ulpien D $1.7 .46 ;$ D 20.1.8 (filios naturales); D 21.1.35; D 32 . 68.1 ; D 34.5 .10 .1 (fili $a$ ) ; D 36.1 .11 .2 (rescrit d Antonin le pieux) ( $f i$ liam); D 37.15 .1 .1 (filizm) (il s'agit au départ d'un esclave, on a collicm) ; D 37.15.1.1 (filizm) (il s'agit au départ d'un esclave, on a col
libertos : matrem... filivon) ; D 38.17 .2 .3 ; D 40.2.11 (filius filiave naturalis) ; D 40.2.13 (filius, filia); D 40.2.20.3 (Marcellus) (filivon naturalem) ; D 40.4.13.3; D 40.12.3 pr. Sur 1'appellation filius pour l'enfant d'un esclave. Trebatius et Labéon chez Iavolenus D 28.8.11 : ... Trebatius negat, quia filii appellatione libertinus quoque continerstur : Labeo contra... Scaevola chez Tryphoninus D 31.88 .12 : ... quia creditur appellatione filiomon et naturales liberos, id est, in semitute creditur appellatione filiornot et naturales susceptos continemi. a eu alors qu'on etait esclave, elle peut ausi concerner 1 'enfant qu'a eu le maître de sa concubine esclave : dans ce sens, Paul D $42.5 .38 \mathrm{pr}$ : Bonis venditis excipiuntur concubina et liberi naturales. Sur le terme, J. PLASSARD, Le concubinat romain, Paris (1921), p. 128 ; E. DE RUGGIERO, D.E., III, art. filius, p. 85. Les textes concernant la filiation illégitime, mais n'ayant aucun lien avec l'esclavage ont bien entendu été écartés. 160- Scaevola D 34.1.20 pr-1; Tryphoninus (Scaevola) D 31.88.12; Paul D $30.62 ; \mathrm{D} 42.5 .38 \mathrm{pr}$; Hermogénien D 37.14.21.3; Modestin D 25.3.7.

161- Julien D 23.4.21; D 36.2.16 pr; D 43.33.1 pr ; Pomponius D 35.1.1. 3 ; D 40.13.3 ; D 41.10.4 pr (Trebatius) ; D 43.26.10 ; Gaius D 20.4.11:2 ; D 38.8.2 ; Scaevola D 32.37.3 (adgnata); D 32.41.10 (idem); D 33.5.21 (idem); Papinien D 36.2.25.1 ; Tryphoninus D 28.2.28.3; Paul D 7.7.1; D 12.6.16 pr; D 20.1.29.1; D 32.99.3; D 33.8.3; D 41.7 .8 ; Ulpien D 11. 4.1.5 (Labéon); D 21.1.1.1; D 38.17.13; D 38.16.3.12 (rescrit d'Antonin le pieux) ; D 40.5.24.6 (rescrit d'Antonin le pieux) ; D 40.11.1 ; D 43. $32.1 \mathrm{pr}$; D 45.1.75.4 ; D 50.15.4.9 ; Marcien D 1.5.5.1 ; D 40.5.53.1 ; D 40.11.2 ; D 40.15.1.4 (Marcellus) ; D 50.16.210; Modestin D 20.1.26.2 ; D 40.5.13. On trouve aussi editus, par ex. chez Paul D 40.13.3, chez U1pien D 23.3.10.2; D 37.10.1.5 (Julien, rescrit d'Antonin le pieux).

162- Paul (D 50.16.204) : Pueri appellatio tres significationes habet: unam, cion omnes servos pueros appellaremus : altercon, cum puemon con tramio nomine puellas diceremus : terticm, cum aetatem puerilem demonstraremus. La première acception est issue de la formule étrusque Gäipor, Lúcipor, Märcipor qui a donnê Gaipuer, etc., ce qui rappelle le français "garçon", (A. ERNOUT et A. MEILLET, Dictionnaire étymologique de la langue latine, Paris NOUT et A. MEILLET, Dictionnaire étymologique de la langue latine, Paris
$(1959-1960)$, p. 522, 543). Sur cette signification de puer, v. aussi M.I. $(1959-1960)$, p. 522, 543). Sur cette signification de puer, v. aussi M.t.
FINLEY, L'économie antique, cit., p. 108 . Quant aux deux autres, elles n'établissent aucune différence entre esclave et libre, puer, comé pue $l_{a}$, pouvant aussi bien concerner l'un que l'autre. Ont été retenus comne visant 1 'enfant esclave Alfenus D 9.2.52.2 ; Maecianus D 29.5.14; Scaevola D 32 . 41.4 ; Paul D 6.1 .6 ; D 6.1.27.5 (Proculus) ; D 24.1.28.1; D 24.1.38 pr (Aifenus) ; D 35.1.28.1 ; D 50.16.79.1 (Fulcinius) ; Ulpien D 9.2.7.7 ; D 9.2.27.28 (Vivianus) ; D 18.1.11.1; D 19.5.13.1 (Julien) ; D 21.1.17.5 (Vivianus); Modestin D $32.81 \mathrm{pr}-1$. 
'puella (163), ou alumnus (164), infans (165), servulus (166), parrulus (167), suboles (168), impubes (169), minor (170), ou encore virgo (171) ou nondun viripotens (172), et même novitius (173), paedagogia (174) sont autant d'allusions à des esclaves jeunes et donc des références indirectes à la naissance. De même, frater (175) peut viser un lien biologique servile. Le terme verma (176) qui désigne l'esclave né chez le maitre, n'apparait que tres. peu. Enfin, certain textés ne con- tiennent pas de référence explicite à l'enfant, mais traitent d'une

163- Papinien D 26.5.13.2 ; D 40.1.20.2 ; U1pien D $32.49 \mathrm{pr}$; Modestin D $32.81 \mathrm{pr}$.

164- Scaevola D 33.1.18.1; D 33.1.21.4; Paul D 40.5.38; Marcien D 40. $2.14 \mathrm{pr}$. Sur ce point, A. BERGER, Encyclopedic dictionary of Roman law, Philadelphie (1953), p. 36i.

165- Celsus D 47.2.68.2 ; Pomponius D 7.1.55 ; Gaius D 40.1.25 ; Pau1 D 6.2.12.5; D 13.1.13; Uipien D 7.1.12.3 (Pomponius) ; D 9.2.23.7 ; D 33.7 .12 .7 ; D 40.5 .26 .2 (rescrits d'Antonin le pieux et de Sévêre et

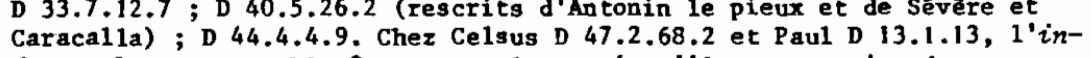
fans subreptus, semble être un esclave puisqu'il est question de son aestimatio.

166- Paul D 12.6.36; Alfenus D 40.1.7.

167- Marcien D 40.5.55.1 (Marcellus).

168- U1pien D 23.3.10.2.

169- Gaius D 29.2.57.2 ; Maeciamus D 29.5.14 ; Venuleius D 40.12.44.2 ; Papinien D 26.5.13 pr (rescrit d'Hadrien)-1 ; Paul D 6.1.31 ; D 41.2.1.13; Ulpien D 21.1.23.2 (Pomponius); D 29.5.1.32-33. On trouve aussi iuvenis Ulpien D 21.1 .23 .2 (Pomponit
chez Marcellus D 32.69 .1 .

170- vlpien D 7.7.6.1 (minor 5 anis... servus); Callistrate D 48.18.15. I (rescrit d'Antonin le pieux) et A. Charisius (rescrit du même) ; D 48. $18.10 \mathrm{pr}$ sont intégrables par quaestio (minore 14 annis).

171- Paul D 1.18.21 (devirginata); vlpien D 18.1.11.1; D 19.1.11.5; D 47.10.15.15; D 47.10.25; Modestin D 32.81.1 (assimile virgines a pueri).

172- D1pien D 29.5.1.32.

173- Un esclave sans expérience ne peut être qu'un esclave jeune. Le tertre apparaît chez Venuleius D 21.1.65.2 (Caelius Sabimus) ; Marcien D 39.4.16. 3 ; Ulpien D 21.1.37; Pomponius D 3.5.10 (Proculus).

174- Paedagogium signifie pension, école pour des esclaves destinés à des fonctions un peu hautes (Plin. Ep. VII, 27, 13) mais ausi les enfants qui fréquentent une école (Sen. Beat. XvII, 2; P1in. XXXIII, 152. Chez vlpien D 33.7.12.32, le terme a la seconde signification : Si instructum fundren legasset, ea paedagogia, quae ibi habebat, ut cum ibi venisset, prasesto essent in triclinio, legato continentur.

175- Papinien D 17.1.54 pr (frère du mâ̂tre) ; D 40.1.19 ; Faul D 21 1.39 ; Ulpien D 21.1.35; D 30.71 .3 ; D 40.2.11; D 48.18.1.10 (rescrit de Sévère et Caracalla). On trouve soror chez Paul D 23.2.14.2; Ulpien D 40 . 2.11 et gemelli chez Paul D 9.2.22.1.

176- Pomponius D 30.36 pr (Labéon)-1; Scaevola D 32.38.2 ; D 34.2.15; D 36.1.80.12; Paul D 32.99.5. esclave enceinte ou accouchant, ils out évidemment été pris en considération (177).

Avant de passer au décompte de ces textes, il faut encore dire que plusieurs d'entre eux ont été interprétés de manière extensive. Dans quelques cas, en effet, l'enfant de I'ancilza naît libre (178) ou doit être remis à sa mère pour être affranchi (179) ; ou bien encore il naft d'un père esclare mais d'une mère libre $(180)$; ou enfin on a affaire à une femme stérile ou plus généralement dens l'impossibilité d'enfanter (181). De ce fait, au sens strict, la reproduction du groupe n'est pas assurée. Néanmoins, on a intégré ces textes car ils visent tout de même l'hypothèse de la reproduction. Dans d'autres, on a affaire à l'enfant d'une femme qui

177- Pomponius D 30.24 pr (ancilla peperisset) (de pario, -is, peperi, partum, A. ERNOUT et A. MEHLET, Dictionnaire étymologique, cit., p. 483); Paul D 41.3.4.15-17-18; D 45.1.73 pr (quod in utero sit) ; vipien D 1.5. 16 ; D 9.2 .9 pr (Labéon); D 9.2.27.22 (Brutus); D 21.1 .14 pr (Sabinus)-1 (mulier praegnas) -2 (puerperam) ; D 40.7.3.16; D 40.7.6.1-2 ; D 40.7.16 ; D 48.19.3; Marcien D 1.5.5.2-3; D 40.5 .55 pr. I1 n'était par contre pas possible d'intégrer les textes où ancilza apparaissait seul, sans référence 3 la maternité (D 18.1.56; D 18.7.6 pr (serva); D 18.7.9; D 18.7. 10 ; D 19.1.43 (Arescusa) ; D 21.1.23.4; D $24.1 .19 \mathrm{pr}-1$; D 24.1 .31 .1 ; D 27.6.11.2; D 29.5.1.28; D 29.1 .40 .2 ; D 30.45 pr ; D $30.96 \mathrm{pr}$ (Pamphila); D 31.88.13; D 33.7.16.2; D 33.8.15;D 34.2.13).

178- Ex. Marcien D 40.5.53 pr : $S i$ quis rogatus ancillom manomittere moram fecorit, si interea enixa fuerit, constitutum est huiusmodi portwn libernon nasci et quidem ingemum; aussi U1pien $D$ 40.5.26.1-2 (rescrits d'Antonin le pieux et de Sévère et Caracalla)-4 (rescrits des mêmes) (récapitul.) ; pour une liste complète des cas où l'enfant de l'ancillza naît libre, w.W. BUCKLAND, Roman law, cit., p. 399 s. V. A. MONTEL, La condizione giuridica dei figli di schiava onorata di fedecomesso di liberta nati in periodo di mora o ritardo nella manomissione della madre, in St. Bonfante III, p. $631 \mathrm{~s}$. 179- Ex. Marclen D 40.5.53.1 : Sed si nondron debita libertate fideicommissa ancilla peperit, studio tomen heredis fuerit effectum, ut nondrm $l_{i-}$ bertas deberetur, veluti quod tardius adit hereditatem, ut qui nati sint ex ancilla servi eius fiant, placet manumitendos, sed 'tradi' matri oportere, ut ab ea manumitterentur st liberti potius matris fiant : nam quos indignus est heres servos habere, ne quidem libertos habebit; aussi, U1pien D 40.5 . 26.1-3 (rescrit de Sévere et Caracalla) ; Marcien D 40.5.55.1 ; Modestin D 40.5.13; L'enfant est affranchi par 1'héritier in Maecianus D 40.5.54 et Marcien D 40.5.55.1

180- Paul D 50.2.9 pr : Severus Augustus dixit : et si probaretur Titius in servitute patris sui natus, tanen, con ex libera muliere oit procreatus, non prohibetw decurio fieri in sua civitate.

181- Ulpien D 21.1.14 pr (Sabinus) : $\ldots$ muliere quae semper mortuos parit; D 21.1.14.3 (Trebatius, Caelius Sabinus): sterilis; D 21.1.14.7: mulieren ita artan, ut mulier fieri non possit, sanan non videri constat. 
s'est laissée vendre comme esclave (182) ou ailleurs, qui a été réduite en esclavage de la peine (183). Bien que la mère soit initialement libre et qu'en outre, d'autres formes d'asservissement apparaissent ici aux côtés de la naissance, 1 'enfant naît sans conteste esclave, et de tels textes devaient donc être également envisagés.

$\mathrm{Si}$ on considère le nombre de textes traitant de la naissance par rapport au nombre de textes concernant l'esclavage pour une période donnée, on parvient au tableau suivant (184):

182- Pomponius D 40.13.3: Eis, qui se prssi sint venire, ad libertatem proclamandi licentiam denegari. Quaero, an et ad eos, qui ex mulieribus, quae se passae sint venire, nascuntur, ita senatus consulta pertinent? dubitari non passae sint venire, nascuntur, ita senatus consulta pertinent ? dubitari non
potest, quin ei quoque, quae maior annis viginti venire se passa est, ad lipotest, quin ei quoque, quae maior annis viainti venire se passa est, ad
bertatem proclamandi licentia fuerit deneganda. His quoque danda non est, qui ex ea nati tempore servitutis eius erunt.

183- U1pien D 40.5.24.6 : Ex dormata in metallum concepto et nato fideicommissaria libertas dari poterit : quid mirum, com etinm venumdari eum posse quasi servum dirus Pius rescripsit ? Ce cas est différent de celui de la femme condamnée a l'ultimum supplicium qui, elle, donne la liberté a son enfant (U1pien D 1.5.18).

184- Suit la liste complète des textes relevés sur la naissance comme mode de reproduction interne du groupe servile : Alfenus $D$ 9.2.52.2-4; D 40.1.7; Proculus D $31.48 \mathrm{pr}$; Lavolenus D 24.3.66.3 (Labéon); D 28.8.11 (Trebatius, Labéon) ; Celsus D 23.3.58.1 ; D 30.63 ; D 47.2.68.2 ; Julien D 12.4.7.1 ; D 21.2 .8 ; D 23.4.21; D 24.3 .31 .4 ; D 30.82 .4 ; D 30.84.10; D 30.91.7; D $36.2 .16 \mathrm{pr}$; D $41.3 .33 \mathrm{pr}$; D 41.4 .9 ; D 41.4 .10 ;D 42.8 .17 .1 ; D 43.33.1 pr ; Pomponius D 7.1.55; D 15.2.3; D 18.1.8 pr ; D 18.1.31; D 23.3.18 (Labẻon) ; D $30.24 \mathrm{pr}$; D $30.36 \mathrm{pr}$ (Labéon)-1; D 35.1.1.3; D 40.13.3; D 41.10.4 pr (Trebatius) ; D 43.26.10 ; Africain D 47.2.61 ; Gaius D 6.1.20; D 6.1.28 ; D 20.1.15 pr (Julien) ; D 20.4.11.2-3; D 22.1.28.1 ; D 29.2.57.2 ; D 35.2 .73 pr;D 38.8.2; D 39.5.11 ; D 40.1.25; D 40.4.24; D 41.3.36.1; Maecianus Pr ; D 38.8 .2 ; D 39.5 ; $35.2 .30 \mathrm{pr} ; \mathrm{D} 40.5 .54$; Venuleius D 21.1 .65 .2 (Caelius Sabinus) D 40.12.44.2; D 41.1.66; D 42.8.25.4-5 (Proculus); D 48.2.12.4; Marcellus D 40.12 .44 .2 ; D 41.1 .66 ; D 42.8.25.4-5 (Proculus) ; D 48.2.12.4 ; Marcellus D 32.69 .1 ; D 37.15.3; Scaevola D $32.37 .3-7$; D 32.38 .2 ; D $32.41 .2-4-5-10$; 28 ; D $34.1 .20 \mathrm{pr}-1$; D 34.2 .15 ; D 36.1.80.2-3-12;D 40.4.60; D 40.5.41.5; Papinien D 17.1.54 pr ; D 22.1.2; D 22.1.4 pr-1; D 22.1.8; D 23.3.69.9; D 26.5 .13 pr (rescrit d'Hadrien)-1-2 ; D 31.73 ; D 31.77 .17 ; D 33.7 .3 pr ; D 35 . 2.9 .1 ; D 36.1 .60 .4 ; D 36.2.25.1 ; D 40.1.19; D 40.1.20.2; D 41.3.44.2 ; Tryphoninus D 1.5.15; D 15.1.57.2 ; D 28.2.28.3; D 31.88.12 (Scaevola); D 49. 15.12.18; Callistrate $D$ 48.18.15.1 (rescrit d'Antonin le pieux); Paul D 1.18 . 21; D 6.1.6; D 6.1.16 pr ; D 6.1.27.5 (Proculus); D $6.1 .31 ; D 6.2 .12 .5$; D 7.7.1 ; D 9.2.22.1 ; D 9.2.33 pr (Pedius); D 10.2.11;D 12.4.12; D 12.6. 15pr:D12.6.36;D12.6.65.5;D1311.13; D13.7.18.2; D 19.1.21 or : D 19.5.5 or-5; (Neratius); D 23.2.14.2; D 24.1.28.1-5 (Julien, Pomponius); D 24.1.38 pr (Alfenus) ; D 30.62; D 32.99.3-5;D 33.8.3;D 35.1.28.1; D 35.2.24.1 ; D $35.2 .63 \mathrm{pr}$; D 38.10.10.5;D 40.5.38;D 40.5.39.1;D 40.12.23.2; D 41 . $2.1 .13 ;$ D $41.3 .4 .5-15-16$ (Sabinus, Cassius) $-17-18: D 41.7 .8: D 41.10 .2:$ D 42.5 .38 $\mathrm{pr}$; D 45.1.73 pr ; D 45.1.83.8; D 48.23.4; D 50.2.9 pr (Sévềre) ; 050 . 16.79.1 (Fulcinus); Ulpien D 1.5.16;D 1.7.46;D 2.4.4.3 (Labéon) ; D 3. 5.3 .6 ; D 4.2.12 pr; D 5.3.20.3; D 5.3.27 pr; D 6.1.17.1 (Julien) : D 6.2.

\begin{tabular}{|c|c|c|c|c|c|}
\hline I & - & $=$ & I & $i 0 / 128$ & $=0$ \\
\hline II & $1 / 6$ & $=(17 x)$ & II & $19 / 347$ & $=$ \\
\hline III & $45 / 985$ & $5 \%$ & III & $24 / 519$ & $=$ \\
\hline$\Gamma \mathrm{V}$ & $33 / 354$ & $9 x$ & $\Gamma V$ & $7 / 105$ & $=$ \\
\hline$y$ & $200 / 3840$ & $5 x$ & $\mathrm{v}$ & $1 / 35$ & $=$ \\
\hline
\end{tabular}

Hormis les périodes II, dans l'analyse des paragraphes, et $V$, dans l'analyse des citations, qui menquent de représentativité, on remarque tout d'abord qu'il n'y a pas de différence notoire entre la République et l'umpire, ensuite que le pourcentage de textes visant la reproduction naturelle des esclaves n'est pas inférieur sous les Sévères à ce qu'il était sous les premiers Antonins, ce qui montre que la naissance est encore à cette époque une source importante d'asservissement. Comme on $1^{\prime}$ a observé en matière de prix, le Digeste ne permet pas de vérifier l'hypothèse d'une diminution brutele du nombre des esclaves (185). Pourquoi les juristes de l'époque des Sévères continueraient-ils donc à attacher autant d'importance que leurs prédécesseurs aux naissances d'esclaves si ceux-ci ne se reproduisaient plus ?

11.2-3 (Julien)-4 (Julien)-5 (Pomponius); D 7.1.12.3 (Pomponius); D 7.1. $68 \mathrm{pr}$ (Brutus) ; D 7.7.6.1 ; D 9.2.7.7 (Celsus); D 9.2.9 pr (Labéon) ; D 9. 2.23.7 ; D 9.2.27.22 (Brutus)-28 (Vivianus); D 10.2.12 pr (Sabinus); D 10 3.6.4 (Sabinus, Atilicinus) ; D 10.4.9.7 (Sabinus, Pomponius) ; D 11.4 .1 .5 (Labéon) ; D 12.2.11.1; D 16.3.1.24 ;D 18.1.11.1; D 18.2.4.1 (Julien)-2 (Julien) ; D 19.1.11.5; D 19.5.13.1 (Julien); D 20.1.8; D 21.1.1.1; D 21 . $1.14 \mathrm{pr}$ (Sabinus-1-2-3 (Trebatius, Caelius Sabinus)-7 ; D 21.1.17.5 (Vivianus); D 21.1.23.2 (Pomponius); D 21.1.31.2-6 (Pomponius)-21-24; D 21.1. 35; D 21.1.37; D 21.1.40 pr; D 23.3.10.2; D 29.5.1.32-33; D 30.71.3; D $32.49 \mathrm{pr} ; \mathrm{D} 32.68 .1 ; \mathrm{D} 33.7 .12 .7-32-33 ; \mathrm{D} 33.8 .8 .8$ (Julien); D 34.5 ; 10.1 ; D 36.1 .11 .2 (rescrit d'Antonin le pieux); D 36.1 .23 .3 (Neratius); D 36.4 .58 ; D 37.10 .1 .5 (Julien, rescrit d'Antonin le pieux); D 37.15 .1 .1 ; D $36.4 .58 ;$ D 37.10 .1 .5 (Julien, rescrit d'Antonin le pieux) ; D $37.15 .1 .1 ;$
D 38.16 .1 .1 (rescrits de Marc-Aurèle et Verus et de Caracal1a) ; D 38.16 .3 .12 (rescrit d'Antonin le pieux) ; D 38.17.1.3; D 38.17.2.3; D 40.2.11; D 40.2 . 12 ; D 40.2.13; D 40.2.20.3 (Marce11us) ; D 40.4.13.3; D 40.5.4.14; D 40. 5.24.6 (rescrit d'Antonin le pieux) ; D 40.5.26.1 (constitution de Sévère)-2 (rescrits d'Antonin le pieux et de Sévère et Caracalla)-3 (rescrit de Sévèr et Caracalla) -4 (rescrits d'Antonin le pieux et de Sévère et Caracalla) 5 ; et 40.7 .16 ; $40.76 \mathrm{pr}-1-2 ; \mathrm{D} 40.716$; $40.111 ;$ D 40.7 .3 .16 ; D $40.7 .6 \mathrm{pr}-1-2$; D 40.7 .16 ; D 40.11 .1 ; D 40.12.3 pr; D 41. 44.4.4.9; D 45.1.75.4; D 47.2.48.5-6; D 47.4.1.11; $\mathrm{D} 47.10 .15 .15 ; \mathrm{D} 47$. 10.25 ; D 48.18.1.10; D 48.19.3; D 50.15.4.9; D 50.16.26 (Scaevo1a) ; 10.25 ; D 48.18 .1 .10 ; D 48.19 .3 ; D 50.15 .4 .9 ; D 50.16 .26 (Scaevola) ;
Marcien D $1.5 .5 .1-2-3$; D 39.4 .16 .3 ; D $40.2 .14 \mathrm{pr}$; D $40.5 .53 \mathrm{pr}-1$; D 40. $5.55 \mathrm{pr}^{-1}$ (Marcellus) ; D 40.11.2 ; D 40.15.1.4 (Marcellus) ; D 50.16.210; Modestin D 20.1.26.2 ; D 25.3.7 ; D 32.81 pr-1 ; D 40.5.13 ; Hermogénien D 37.14 .21 .3 ; A. Charisius D $48.18 .10 \mathrm{pr}$. 
Le haut pourcentage réalisé par la période IV, correspondent aux règnes de Marc-Aurèle et Conmode, est sans doute dû à l'amorce de la reconnaissance par le droit des liens familiaux de l'esclave (186). C'est une entreprise que poursuivront les Sévères (187).

Est-ce à dire que le nombre de vernae, sous l'Bmpire, a proportionnellement été plus important que celui des esclaves achetés (188) ? Le Digeste ne permet pas de trancher. On a vu que l'apparition du terme verna dans les textes juridiques était très rare, mais surtout l'enfant esclave peut se trouver mêlé à des hypothèses de vente, soit par sa mère (189), soit directement (190).

Il est clair, sur ce plan, que l'achat d'enfants esclaves devait constituer un investissement rentable (191). Deux textes sont particulièrement

185- M. I. FINLEY, L'Économie antique, cit., p. 111,113 ; E.M. SCHTAERMAN, La schiavitù, cit., p. 4, 27. Contra, J. KOLENDO, Lavoro servile, cit., p. 17, qui pense que 1 'importance des esclaves nés sur le domaine ne dépend pas seulement de 1 'encouragement a leur reproduction, mais aussi de 1 'état des autres sources d'esclavage (guerre et piraterie). Selon l'auteur, l'élevage fournit une quantité constante d'esclaves, tandis que l'afflux provoqué par les guerres une quantité consta

est très variable. Pompeia de parricidits est applicable aux esclaves cum natura communis est, similiter animadvertetur (D 48.2.12.4).

187- E.M. SchtaER MAN, o. c., p. 241 ; cf. Paul, Sent. I, 13, 2, 1 g et v, 6, 16 ; Paul D 42.5.38 pr ; Ulpien D 20.1.8; Ulpien D 40.2.11 ; Paul D 21.1. 39 ; Ulpien D 21.1.35; Ulpien D 23.3.39.

188- RAHRSTED, Kulturgeschichte der rör. Kaiserzeit, p. 315 , donne ses faveurs à une prépondérance des vernae. Cependant, les listes des affranchissements de Delphes, attestées par des inscriptions (W.L. WESTFRMANN, RE Suppl. VI, p. 998) montrent au contraire qu'il y aurait eu une augmentation des esclaves achetếs vis-z-vis des vermae durant la période qui va de 50 av. J.C. jusqu'a 130 ap. J.C. par rapport au siècle précédent (cf. F'. DE MARTINo. jusqua l30 ap. J.C. par. rapport an siano, Storia cit. IV, p. 338 ). H.L. WESTERMANN (Slave Systems, cit., p. 86) pen
que le rapport numérique aurait été de 3 vermae pour 5 esclaves achetés.

189- Julien D 21.2.8; D 41.4.10; D 43.33.1 pr ; Papinien D 22.1.4 pr-1; Paul D 13.7.18.2 ; D 21.2.42; Ulpien D 18.2.4.1-2; D 21.1.1.1; D 21.1. $31 \cdot 2-6-24$.

190- Marcellus D 37.15.3; Venuleius D 21.1.65.2; D 40.1.20.2; Tryphoninus D 49.15.12.18; U1pien D 21.1 .37 ; D 40.5.24.6.

191- I. BIEZUNSRA - MALONIST, Les enfants-esclaves a la lumière des papyrus, in Hom. a M. Renard, Latomus II (1969), p. 91-96 ; id., Recherches sur 1 'esclavage dans 1 'Egypte romaine, in Comptes rendus de 1 'Acad. des Inscr. et Belles lettres, Paris (1959), p. 203-210; id., les esclaves nes dans la maison du maître et le travtil des esclaves en Egypte romaine, in Studii clasice, 3 (1962), p. 147-162 ; I. BIEZUNSRI-MALOWIST et M. MALOWIST, La procréation des esclaves coume source de l'esclavage, in Mélanges $\mathrm{K}$. Michalouski, Varsovie (1966), p. 275-280. éloquents à cet égard. Ils sont de Pomponius (192) et de Paul (193). Ces juristes y traitent de l'achat d'enfants non encore nés. Il s'agit en quelque sorte d'une véritable vente en l'état futur d'achèvement des travaux. Et si notre acheteur sur plan essuie parfois une déconvenue, on peut imaginer la surprise de celui qui avait acheté les enfants à venir d'une femme stérile, comme c'est le cas dans le texte de Paul.

On peut penser plus généralement que 1 ' "élevage" des enfants esclaves était d'un bon rapport (194). On les trouve liés non seulement à la vente, mais à d'autres institutions juridiques, legs (195), donation (196), dot (197)

192- Pomponius D $18.1 .8 \mathrm{pr}: \ldots$ Et tomen fructus et partus futuri recte ementur.

193- Paul D 19.1.21 pr : Si sterilis ancilla sit, cuius partus venit, vel maior anis quinquaginta, cum id emptor ignoraverit, ex empto tenetur venditor. 194- 1. BIEZUNSRA-MALOWIST, Les enfants-esclaves à la lumière des papyrus, cit.,p. 93 : "c'est précisément le nonbre relativement élevé d'enfants ci tés dans les actes d'achat et de vente qui permet de se rendre compte de 1 'importance accordée a l' 'élevage' des esclaves"; id., o.c.,p. 95: "les gens qui tance accordée a 1 'évage un enfant-esclave étaient stimulés par la possicouraient le risque d'élever un enfant-esclave étaient stimules par la possibilité de lui faire apprendre un métier à peu de frais, alors qu'ils devaient payer un prix élevé pour un esclave adulte, qualifié pour ce travail"; id., résultait entre autres du fait que la main-d'oeuvre constituée par les esclaves était employée par des personnes ayant une position sociale et matérielle assez modeste, pour qui il était plus facile de supporter les frais relativement peu élevés de l'entretien d'un esclave durant son enfance que d'acheter un esclave adulte". V. aussi P. DOckEs, La libération, cit., p. 166. L'auteur observe (p. 168) que l'élevage n'est pas incompatible avec le système de l'esclavage "p. 168) que ". Contra, M. WEBER, Agrarverhältnisse im Altertum, in Gesammel te Ausfsätze zur Ausfsätze zur Sozial und Hirtschaftsgeschichte, Tubingen (1924), p, in Annales sible) ; M. BLOCH, Comment et pourquoi finit l'esclavage antique, in Annales E.S.C (1947), p. 34 (êlevage trop délicat dans les conditions de la chiourme)
P. ANDERSON, Les passages, cit., p. 85 (utilisé temporairement après la fin de la traite massive, mais ne pouvant être une solution à long terme).

195- Ex. Proculus D $31.48 \mathrm{pr}$; Celsus D 30.63 (Servius); Julien D 30.82 . 4 ; D 30.84.10; D 30.91.7; D 36.2.16 pr ; Pomponius D 30.36 pr (Labéon)-1 ; D 35.1.1.3 ; Marcellus D 32.69.1 ; Scaevola D 32.41.4 ; D 33.5.21 ; D 33.7 . 28 ; D 32.37.3 (fidêicomanis); Papinien D 22.1.8 (fídéicoumis); D 31.77.17 ; D 33.7.3 pr ; D 36.2.25.1 ; Tryphoninus D 49.15.12.18; Paul D 22.1.14 pr (fidéiconmis) -1 (Neratius) (fidéicommis) ; D 30.62; D 33.8 .3 ; D 35.1. 28.1 ; D 35.2 .24 .1 (fidéicommis) ; Ulpien D 33.7.12.7-32-33; D 36.4.58; Modestin D $32.81 \mathrm{pr}-1$.

196- Ex. Gaius D 39.5.11; Paul D 12.4 .12 ; D 24.1.38 pr (Alfenus); Ulpien D 44.4.4.9.

197- Ex. Proculus D 31.48 pr ; Iavolenus D 24.3.66.3 (Labéon); Celsus D 23 3.58.1 ; Julien D 12.4.7.1 ; D 23.4.21; D 24.3.31.4 ; Pomponius D 23.3.18 (Labéon); Papinien D 23.3.69.9; Paul D 24.1.28.1; D 50.16.79.1 (Fulcinus); Ulpien D 23.3.10.2. 
et aussi usufruit (198). On trouve mêtme des partus ancillamum dans le pécule d'un esclave (199). Les juristes par ailleurs retracent des espèces où on promet la liberté à l'esclave mère de plusieurs enfants (200). C'était, il faut dire, pour le maitre, l'acquisition d'une main-d'oeuvre gratuite, d'autant plus que l'enfant travaillait très tôt (201). Du reste, aucune équivoque sur la fonction sociale de la femme esclave, c'est une reproductrice (202):

... marimum enim ac praecipuzon muras eminormun est cipere ac tueri conceptum.

Il faut encore signaler un texte qui, bien que s'éloignant de notre problématique, n'en est pas moins remarquable. C'est un frogment de Paul, on $y$ lit ceci :

Non sunt liberi, qui contra forman humani generis converso more procreantur (203).

Il s'en. dégage une mentalité esclavagiste peu commune. D'autant moins commune que l'enfant naît dans ce cas de parents libres. On relèvera en particulier l'assimilation entre condition servile et difformité.

198- Le legs de 1'usufruit d'un enfant est valable, bien qu'on ne puisse comencer à 1 'utiliser qu'au sortir de 1 'enfance, Pomponius 7.1 .55 et Ulpien D 7.1.68 pr.

199- Pomponius D 15.2.3.

200- Julien chez U1pien D 40.7.3.16: Arethusae libertas ita sit data, $3 i$ tres servos pepererit ; Tryphoninus D 1.5 .15 (ainsi que Ulpien D 1.5 .16 qui constitue la suite) : Arescusa, si tres pepererit libera esse testamento iussa, primo partu unum, secundo tres peperit.

201- Les textes montrent qu'un enfant de 5 ans est encore inutilisable. A ce sujet, comparaisons intéressantes de Pomponius (chez Ulpien D 7.1.12.3) : aegrotanti servo vel infanti, et Ulpien (D 7.7.6.1) : minor annis 5 vel aegrotanti servo vel infanti, et Ulpien (D 7.7.6.1) : minor annis 5 vel debilis servus. Cependant, les esclaves agés de 14 ans n'étaient plus considérés comme des enfants et commençaient probablement plus tôt à travailler pour leurs maitres,I. BIEZUNSKA-MALOWIST, Les enfants-esclaves, cit., p. $92 \mathrm{~s}$. F. LOT, La fin du monde antique et le début du Moyen-Age, Paris (1968), p. 118 , pense que 1 'élevage était dispendieux car jusqu'à 13 ans, 1 'esclave coûtait et ne rapportait pas. Les textes juridiques, parlant de 5 ans, incitent à abaisser la limite d'âge fixé par 1 'auteur.

202- Ulpien D 21.1.14.1. F. DE MARTINo, Storia economica, cit. II, p. 265, s'appuyant sur un autre texte d'Ulpien (D 5.3.27 pr), d'ailleurs suspecté d'itp., soutient que les propriétaires d' esclaves considéraient peu adroit d'acheter des ancillae pour les faire reproduire. Selon F. DE MARTINO (ibid.),

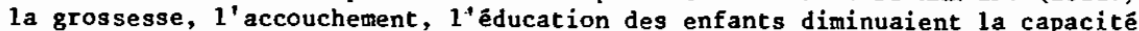
de travail de la mère. L'argumentation parâ̂t valable, mais n'est-elle pas en contradiction avec les encouragements à la reproduction d'un varron, d'un Columelle, encouragements dont on retrouve la trace même chez les juristes (cf. melle, encouragements dont on retrouve la tra

203- Paul D 1.5.14. Ce texte est à rapprocher de la disposition de Romulus permettant d'exposer les enfants difformes et monstrueux, $c f$. MONTESQUIEU, Esprit des lois, XXIII, XXII.
Cela dit, le nombre des naissances à l'intérieur du groupe servile $a-t-i l$ suffi à combler le vide provoqué par la cessation des conquêtes, et à maintenir la population servile à son niveau antérieur ? Il n'est pas possible d'être catégorique sur ce point. Cependant, si on tient compte des rélles conditions de travail des esclaves, surtout dans l'agriculture et les mines, malgré les encouragements prodigués (204), les unions ont-elles vraiment pu être prolifiques (205) ? Le reproduction du groupe à partir d'home libres signifie-t-elle que les naissances ont été insuffisantes pour maintenir l'effectif servile à un niveau satisfaisant pour la société ?

\section{SECTION 2. LA REPRODUCTION EXIEERNE, A PARTIR D' HCAMES LIBRES}

Elle va être assurée d'une part par la pratique, l'homme libre se trouvant alors dans une situation de fait assimilable à celle de l'esclave. Situation que le droit va parfois régulariser. Mais il ne va pas se contenter de réagir. Il va aussi intervenir directement pour consacrer la réduction en servitude d'homes libres.

\section{Paragraphe 1 : La force de 1 a pratique}

Si le droit lutte contre la détention violente ou dolosive d'un home libre, il va par contre doter d'effets juridiques la situation de celui qui est de bonne foi dans un état servile.

204- A 1'époque d'Auguste, Varron écrit que 1 'on doit encourager la vie de famille parmi les esclaves du domaine, en vue d'obtenir d'eux un meilleur rendement $(I, 17,5)$ (aussi Apul. VIII, 17, 22), cf. R. H. BARROW, slavery, cit., p. 152. Columelle parle d'exemption de travail pour les mères de trois enfants, et de concession de la liberté a celles qui en auraient davantage $(I, 8,19)$, cf. R.H. BARROW, O.c., p. 87 ; G. BOULVERT, Domestique et fonctionnaire sous le Haut-Empire romain, Paris (1974), P. 321. Sous Trajan, Pline le jeune est animé des mêmes préoccupations (Ep. VIII, 16).

205- F. DE MARTINO, Storia, cit. V, p. $148 \mathrm{~s}$. Selon T. FRANK, AHR XXI (1916), p. 689, moins de la moitié des inscriptions funéraires d'esclaves rappellent une union matrimoniale, et moins encore 1 'existence d'enfants. P.A. BRJNT (JRS (1958), p. 166) a soutenu que les naissances auraient suffi a maintenir le niveau antérieur de la population servile, en se fondant a maintenir le niveau antérieur de la population servile, en se fondant sur l'exemple américain ; aux Etats-Unis, jusqu'en 1860 , les esclaves im-
portés ne dépassaient pas le nombre de 600000 , alors que le ncmbre global de la population servile était de 4500000 . F. DE MARTINO (p. 148) critíque cette thèse en $s$ 'appuyant sur la diversité des conditions historiques et de 1 'organisation sociale et juridique de 1 'esclavage; contra M. I. FINLEY, L'économie antique, cit., p. 113 : "Qu'une population servile ne puisse se reproduire est une fiction, mais elle a la vie dure, en dépit de la preuve administrée par le Sud des Etats-Unis." 


\section{I - L'homme libre retenu de mauvaise foi}

Il n'était probablement pas rare que des personnages influents contraignent des jeunes gens à devenir leurs esclaves (206). Paul rappelle un cas d'asgervissement par destruction des preuves de la liberté (207). Le caractère fréquent de ces pratiques illégales est d'ailleurs attesté par la présence, dans le Digeste, de nombreux procès sur le status (208).

Le droit va réagir dès l'époque républicaine par la lex Eabia de plagiariis (209). Outre la volonté contraire de l'horme libre objet de l'appropriation dolosive (210), la lex Fabia exigeait expressément que le

206- E.M. SCHTAERMAN, La schiavitù, cit., p. 22

207- Paul D 4.2.8.1 : Si is accipiat pecuniam, qui instrumenta status mei interversumus est nisi dem, non dubitatur quin maximo meiu conpellat, utique si iam in servitutem petor et illis instrumentis perditis liber pronutiari non possum.

208- D 40.12 : De liberali causa. Le Digeste est par contre silencieux sur une autre pratique d'asservissement illégal. Quelquefois, des paurres, pressés par la nécessité, se vendaient au créancier ou cêdaient leurs propres enfants (.$M$. SCHTAERMAN , 1.c.). Sur cette pratique dans les provinces prientales, L. MITTES, Reichsrecht und Volkstecht, Leipzig (1891) p 358 Paul (Sent, 5, 1, 1) rappelle la segle telle vente ne saurait préjudicier a 1 'ingenuitas de 1 'enfant, car un home libre nullo pretio aestimatur. Il ajoute que le créancier qui l'accepterait en gage est passible de la relégation (D 20.3.5 : c'est la seule apparition du problème dans le Digeste). Caracalla se prononce aussi pour L'illicéité de cette pratique (CI 7.16.1). Il faudra attendre le Bas-Empire pour voir apparaitre des exceptions (les sanguinolenti, Vat. Fr. 34 ; W.W. BUCKIAND, Roman 1aw, cit., p. 421). Par ailleurs, E.M. SCHTAERMAN, o.c., p. 20, traitant des addicti pense que le texte d'Ulpien (D 23.5.2 : Si maritus fuerit in servitutem redactus, on dominus alienore hunc fundum non possit ? quod puto esse verius. Quare et si ad fiscum pervenerit, nihilo minus venditio fundi inpeditur, quamis fiscus semper idoneus successor sit et solvendo) fundi impeditur, quomvis fiscus semper idoneus successor sit et solvendo)
concerne le cas d'une personne qui préfère racheter sa dette par son propre travail en se laissant asservir.

209- La date de cette lex est incertaine. A. GUARINo, Storia del diritto romaro, cit., p. 270 , pense qu'elle est peut-être du $1^{\circ} \mathrm{s}$. av. J.C. G. LONGO (lex Fabia de plagiariis, in NNDI) soutient qu'on pourrait 1 'attribuer au (lex Fabia de plagiariz, in NNDI) soutient qu' on pourrait 1 'attribuer au consul Fabius Labeo. Ce qui est certain, c'est qu'elle est connue de Cicé-
ron (Pro Rab. perd. 3, 8). E. Costa (Crimini e pene da Romolo a Giustiniano, Bologne 1921 , p. 73 et. n. 2) a soutemu, sur la base du texte de Cicéron (... Bologne 1921 , p. 73 et. $n$. 2) a soutemu, sur la base du texte de Cicéron (... n'aurait concerné que les servi alieni et non les hommes libres ; contra A. BERGER, Lex Fabia de plagio (RE, Suppl. VII, p. 389) et R. REGGI, (liber homo bona fide serviens, Milan 1958, p. 159).

210- Callistrate D 48.15.6.2 : Lege Fabia cavetur, ut liber, qui horminem ingenum vel libertinum invituon celaverit... ; Th. MOMSEN, Le droit penal romain (tr. fr. J. DUQUESNE, Paris 1907), III, p. 90. coupable ait agi sciens dolo.malo (211). Ces paroles visent la conscience du tort commis, c'est-à-dire la connaissance du fait que la personne retenue est libre (212). De la formulation de Callistrate,

D 48.15.6.2 Lib. 6 de cognit. : Lege Fabia cavetur, ut liber, qui hominem ingenam vel libertinum invitum celaverit invictum habuerit emerit sciens dolo malo, quivi in earrm qua re socius erit...

il résulte qu'originairement la lex Fabia a expressément concerné le fait de cacher -celare-, d'enchaîner -vinciri-, d'acheter ou de vendre un home libre citoyen romain ou un affranchi, en ayent connaissance de son status (213). Gaius assimile à la vente la donation, la in dotem datio et l'échange (214). Le droit parviendra à une formulation plus générale des éléments de ce délit avec l'interdit quem libernon (215). Alors sera

211- PARTSCH, Aus nachgelassenen und kleineren verstreiten Schriften, Berlin (1931), p. 37 n. 91 , a soutenu que cet élément subjectif n'aurait pas étê requis par la Lex Fabria, sciens dolo malo étant selon lui le fruit d'une interpolation, mais A. BERGER, Note critiche ed esegetiche in tema di plagio, in BIDR 45 (1938), p. 279 s., a montré la faiblesge de cette thèse. 212- R. REGG, liber hamo, cit., p. 149. Ainsi, inversement, le possesseur de bonne foi, qui ignore que la personne qui est en sa possession est libre, ne commet pas de plagizm, cf. Marcien D 48.15 .3 pr : Legis Fabiae crimine suppressi mancipii bona fide possessor non tenetur, id est qui ignorabat suppressi mancipiz bona fide possessor non tenetur, id est qui ignorabat bona fide possessore ipsa lex scripta est : nan adicitur si sciens dolo malo hoc fecerit; et saepissine a principibus Severo et Antonino constitutuon est, ne bonae fidei possessores hac lege teneantur.

213- Les cas visés par les paroles includere et supprimere (cf. Callistrate D 48.15.6.1 ; Paul D 47.2.83.2 ; Ulpien D 17.2.51.1 et D 47.2.39; Marcien $\mathrm{D} 48.15 .3 \mathrm{pr}$ ) sont dus à un développenent ul térieur du contenu de la loi, R. REGGI, Liber homo, cit., p. 154.

214- Gaius D 48.15.4 : Lege Fabia tenetur, qui sciens libemon hominem donaverit vel in dotem dederit, item qui ex earm qua causa sciens liberum esse acceperit, in eadem causa haberi debeat, qua venditor et emptor habetur. Idem et si pro eo res perrmutata fuerit. Interpolation de sciens soutenue par PARTSCH, o. c., p. 37, n. 91 , et de Lege-tentur soutenue par H.NIEDERMETER, Crimen plagii und crimen violentiae, Zur Geschichte juristischer Begriffe, in St. Bonfante $2,397,59$.

215- Selon R. REGGI, Liber hamo, cit., p. 158, puisque 1 'interdit de hamine libero exhibendo se présente textuellement ainsi : Quem liberun dolo malo retines, exhibeas (0. LENEL, E.P. (3) 1261 p. 487), on peut penser, a partir de $l^{\prime}$ examen de $D 43.29 .1$. 1 et $D 43.29 .3$ pr Ulpien, aingi que de Paul, Sent. 5, 6, 14, que vinciri, supprimere, includere équivalent au génerique retinere. Ce terme signifie posséder, M. LAURIA, Possessiones, Naples (1953), I, p. 86. Sur le concours entre interdit de homine libero exhibendo et accusatio legis Fabiae cf. Ulpien D 43.29.3 pr (Neque hoc interdictum aufert legis-Fabiae exsecutionem; nam et hos interdicto agi poterit et nihilo minus accusatio legis Fabiae institui : et versa vice qui egit Fabia 
pris en considération non seulement le plagium commis par la force, mais aussi celui comnis par la tramperie, en cachant à la personne libre ignorant être telle son véritable état (216), ce qui montre à quel point, dans la pratique, la frontière entre esclavage et liberté est ténue.

Les textes traitant de ce problème dans le Digeste se répartissent par périodes de la manière suivante (217):

analyse des paragraphes

\begin{tabular}{|c|c|c|c|c|c|c|}
\hline I & - & $=$ & I & $0 / 128$ & $=$ & - \\
\hline II & $0 / 6$ & $=$ & II & $1 / 347$ & $=$ & $0,28 \%$ \\
\hline III & $7 / 985$ & 19 & III & $1 / 519$ & $=$ & $0,19 \%$ \\
\hline IV & $5 / 354$ & $1 \%$ & IV & $0 / 105$ & $=$ & - \\
\hline $\mathrm{v}$ & $32 / 3840$ & 1 & $\vec{v}$ & $0 / 35$ & $=$ & - \\
\hline
\end{tabular}

Ces textes ne sont certes pas assez nombreux pour permettre en euxmêmes un calcul statistique. Il s'agit simplement, conme pour les tableaux $\grave{a}$ venir sur ce même thène de la reproduction, de donner un aperçu indicatif

poterit nihito minus etiom hoc interdictum habere, praesertim cum alius interdictum, alius Fabiae actionem habere possit) et Paul Sent. 5, 6, 14 (Adversus evm, qui hominem liberwn vinxerit suppresserit incluserit operconve ut versus ew id fieret dederit, ton interdictum quon legis Fabiae super ea re actio redditur : et interdicto quidem id agitur, ut exhibeatur is qui detinetur, lege autem Fabia, ut etiam poena mumaria coerceatur). Ce dernier texte est suspecté par H. NIEDERMEYER, Crimen plagii, cit., p. 399 et n. 63 ; par contre, A. BERGER, Note critiche, cit., p. 277, et Lex Fabia de plagio, cit. p. 390 , pense qu'il est authentique.

216- R. REGGI, Liber homo, cit., p. $160 \mathrm{~s}$.

217- Il s'agit de Iavolenus D 41.2.23.2; D 45.3.34; Julien D 22.3.20; Pomponius D 18.1.6 pr (Celsus) ; Africain D 12.1.41 ; D 41.1.40; Gaius D 48.15.4; Venuleius D 43.29.2; D 43.29.4 pr-1-2-3; Callistrate D 4.6. 11; D 48.15.6.2; Paul D 4.2.8.1; D 18.1.34.2; D 41.2.1.6; D 41.2.30.1 Ulpien D 1.18.13 pr ; D 3.5.5.9; D 40.12.7.2-3; D 43.29.1 pr-1 ; D 43.29. 3 pr-1-2-3-4-5-6-7-8-9-10-11-12-13-14 (Labeoon)-15; D 48.15.1; Marcien D 48.15.3.1 (rescrit de Sévère et Caracalla); Hermogénien D 48.15.7. Ces deux derniers textes sont assez généraux, mais ont été intégrés du fait qu'ils traitent de la peine prévue par la lex Fabia. D'autre part, l'hypothèse de la vente de soi-même peut être rapprochée du problème qui nous préoccupe lorsqu'il y a scientia de l'acheteur. Il n'y a plus ni force, ni tromperie, 1 'home libre qui se vend sachant être tel. I1 n'en demeure pas moins qu'il y a mauvaise foi de 1 'acheteur puisqu'il a conscience cheter un homme libre. Cette hypothèse apparaît chez Paul D 40.12.17; $D$ 40.12. 33 ; D 40.13 .4 ; D 40.13.5 et Ulpien D 40.12.16.3-4; D 40.12.22.2-3-4-5 (Labéon). Néamoins, ces textes $n^{*}$ ont pu être intégrés, la personne libre se prêtant ici volontai rement au "jeu". De plus, elle se trouve alors dans une situation qui n'est plus seulement de fait, mais de droit. On peut aussi

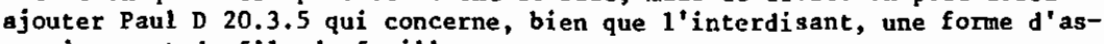
servissement du fils de famille. de l'importance du problème à une période donnée. On peut néanmoins extraire de ce tableau des indications intéressantes. En premier lieu, ancune róference n'est faite à la période républicaine. Il est vrai qu'on ignore la date exacte de la lex Fabia. Toutefois, le nombre infime de citations ne permet pas de déduire que les juristes républicains n'ont pas traité de ce problème. Du reste, preuve de l'intérêt du droit pour la question à cette époque, la lex Fabia elle-même. En second lieu, il est question d'hommes libres retenus de mauvaise foi à toutes les périodes prises en considération. En troisième lieu, et c'est la constatation la plus importante, si les juristes du 3ème siècle développent encore ce problème, cela signifie bien sûr une lutte du droit contre ce phénomène, mais aussi et surtout son impuissance. Les chiffres sont là. Trois siècles n'ont pas suffi au droit pour juguler cette pratique. D'ailleurs, il va régulariser une situstion voisine, celle du liber homo bona fide serviens.

\section{II - Le liber homo bona fide serviens : ignorance du status ou} servitude volontaire.

Cette situation (218) est parfois visée dans le Digeste par des 218- Si la possession de bonne foi ayant pour un objet un home libre est
communément admise en droit classique, sa possession de mauvaise foi semcommunément admise en droit classique, sa possession de mauvaise foi semInst. 2, 95 (Ex his apparet per liberos hominss, quos neque ium nostro subiectos habemus neque bona fids possidemus, item per alienos servos, in quibus neque usumfructum habemus neque iustam possessionem, nulla ex causa nobis adquimi posse. Et hoc est quod vulgo dicitur per estraneam personom nobis adquiri non posse) ; (sur ce passage, G. DONATUTI, Iusta possessio, in AIV, 80,1920,21, 1075 ; en sens critique, G. BESELER, Miscellanea critica, in ZSS $43,1923,421$ et Tijd. 10,1930, 181; contre ce dexnier S. PEROZzI, Istituzioni di diritto romano, Rome (1928), I, 868 et E. AL BERTARIO, Di alcuni riferimenti al matrimonio e al possesso in Sant'Agostino, in studi $1,241,5$; considèrent aussi ce passage interpolé BOzZA, Il no, in Studi $1,241,5$; considèrent aussi ce passage interpolé BozZA,
possesso, Parte prima, Naples (1936) 256, et s. SOLAZzI, Glosse a Gaio, possesso, Parte prima, Naples (1936) 256, et S. SOLAZZI, Glosse a Gaio,
II Puntata, in Per il XIV centenario delle Pandette e del Codice di GiustiII Puntata, in Per il XIV centenario delle Pandette e del Codice di Giusti-
niano, p. 361, 166 et Recenzione a DE ZULUETA, in IURA 4, 287, 2 ; pensent au contraire que ce texte est pur PRINGSHEIM, Acquisition of omership through servus fugitivus, in St. SOLAZZI, 626 ; M. LAURIA, Possessiones, cit., p. 104) ; Africain D 41.1 .40 (Non esse ait, ut hic bona jide possessor videatur, quando sciens liberwn possidere coeperit) (selon F. DE VISSCHER, Le régime romain de la noxali té, Bruxelles (1947), il faudrait attribuer 1a phrase entière aux compilateurs ; R. REGGT, liber homo, cit. p. 137, n. 29 , pense au contraire que $1^{\prime}$ idée qui y est exprimée est bien classique): Africain D 12.1.41 (... Utique si scierint eum sibi coheredem datum, quonian tunc non possunt videri bonae fidei possessores esse) et Paul D 41 . quoniam twe non possiont videm bonae fidei possessores esse) et Paul D 41 . 2.1 .6 (Sed et per erm, quem bona fide possidemus, quanis alienus sit vel
liber, possessionem adquiremus. Si mala fide eim possideamus, non puto adquimi nobis possessionem per ewn : sed nec vero domino aut sibi adquiret, 
formules telles que si quis quasi servus meus (219), liber homo cron tibi serviret (220), si homo liber serviat (221), ou encore si filius ab alio twrquam servus possideatur (222). Mais on trouve plus fréquemment les expressions bona fide possidere (223) ou bona fide servire, cette dernière étant la plus couramment employée, aussi bien à propos $d^{\prime}$ un homme libre seul (224) que d'un homme libre sssocié terminologiquement à un serrus

$q u i$ ab alio possidetur). Cette possibilité est par contre niée par Iavolenus (D 41.2.23.2 et $D$ 45.3.34) ; a partir de 1 a, 1 a grande majoríté de 1 a doctrine a penché pour l'impossibilitê d'une possession de mauvaise foi exercée sur un homme libre, notamment SAVIGNY, Traité de la possession, Bruxelles (1886), P. 108 ; P. BONFANTE, Corso di diritto romano, III. Diritti reali, Rome (1933), p. 214 ; E. ALBERTARTO, Corso di diritto romano. Possesso e quasi possesso, Milan (1946), p. 80 ; P. VOCI, L'errore nel diritto romano, Milan (1937), p. 148 ; G. GROSSO, Obbligazioni, Con tenuto e requisiti della prestazione, Obbligazioni al ternative e generiche, Turin (1947), p. 57. En revanche, M. LAURIA, Possessiones, I, cit., p. 83 et 104 , et R. REGGI, Liber homo, cit. p. 139, pensent qu'il s'agit d'une opinion personnelle de Iavolenus. Selon $R$. REGGI, la possession de mauvaise foi, c'est-z-dire celle qui repose sur la connaissance par le possesseur du status de la personne qui se trouve dans un état servile, bien qu'admise a 1 'époque classique, ne produit pas, contrgirement la possession de bonne foi, d'effets juridiques (p. 137).

219- U1pien D 3.5.5.7.

220- Celsus D 28.5.60 pr.

221- U1pien D 39.4.12.2.

222- v1pien D 41.2 .4 .

223- Cette expression apparaît seulement trois fois : Gaius D $41.1 .40 \mathrm{pr}$ ( $\Rightarrow$ Gaius 2, 86) et D 41.1 .10 .4 ( Gaius 2, 92); Paul D 4l.2.1.6. Elle est (a Gaius 2, 86) et D 41.1 .10 .4 (a Gaius 2, 92) ; Paul D 41.2.1.6. Elle est
plus utilisée à propos du servus alienus : G. DULCKEIT, Erblasserwille und Eluserbsuille bei Antretung der Erbschaft, Heimar (I934), cit., 15, 2, a relevé Gaius D 41.1.43 pr ; Paul D 9.4.12; D 45.3.20.1; vlpien D 29.5.1.2 ; D 47.2.17.3; D 47.8.2.18; D 47.10.15.47; Pomponius D 41.1.54.4 (REGGI, o.c., p. 10 attribue ce texte à Pomponius ; il a été suivi, Modestín ne semblant pas être I'auteur de ces extraits de commentaires ad Quintron Muciron, cf. A. GUARINO, Storia del diritto romano (cit. p. 463). R. REGGI, O. C., P. II, ajoute a cette liste un texte de Papinien $D$ 41.3.44.2 : partion eius mulieris, quom bona fide coepit possidere.

224- Cette expression n'apparait pas dans les Institutes de Gaius (cf. G. DULCKEIT, Erblasserwille, cit., p. 14 ; R. REGGI, Liber homo, cit. p. 11). Dans le Digeste, on la trouve par contre chez Iavolenus D 45.3.34 ; Julien D 29.2.45.4; D 41.4.7.8 ; Pomponius D 13.6.13.2; 41.1 .19 ; 41.154

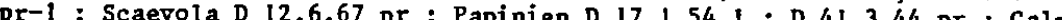
Pristrate 4.6 . D 45.1 .26 .2 ; 45.320 PT ; D 12.4 .3 .5 ; D 17.1 20 pr ; D 12.4.3.5; D 17.1.8.5; D 21.2.19.1; D 24.1.3.8; D 40.12.12.1-6; D 49. 16.8 (cf. G. DULCKEIT, o.c., P. 15, 1 ; CTULEI, Liber homo bona fide serviens, Paris (1941), p. 26, 1 ; R. REGGI, O.c., P. 12). alienus (225). Bona fide possiäere et bona fide servire sont également parfois employés simultanément (226).

L'usage terminologique de bona fide possidere, prépondérant à l'époque classique, marque l'attachement du droit à la bonne foi du possesseur, c'est-à-dire à son ignorance du véritable status de la personne traitée en esclave. A l'époque de Justinien, on relève en revanche une préférence pour l'expression bona fide oervire. Il saut voir là le signe d'une évolution du concept. C'est en effet l'ignorance par l'homme libre de son status qui devient alors déterminante (227).

L'emploi de ces expressions évoque donc deux réalités : si la servitude peut être le fruit de l'ignorance, elle peut aussi être volontaire.

\section{A) - L'ignorance du status.}

On a déjà vu que se produisaient Préquemment, en pratique, des confusions entre libres et esclaves (228). Mais comment un home libre peut-il concrètement ignorer sa condition ? Le Digeste apporte sur ce point plusieurs réponses. Il peut s'agir d'un enfant volé qui est resté par la suite dans un état servile, ignorant qu'il était libre par sa naissance. Le texte

225- Pomponius D 41.1.21 pr ; D 41.1.54.4; Africain D 12.1.41 ; Papinien D $45.1 .118 \mathrm{pr}$; Paul D 45.3.33 pr ; U1pien D 14.4.1.5; D 15.1.1.6; D 21 . 1.23 .6 ; D 21.i.25.2; D 41.1.23; D 41.2.13.3; D 47.8.2.14; D 47.10. 45.48 ; D 48.18 .1 .8 (cf. G. DULCKEIT, o.c., p. 15, 1 ; R. REGGI, o.c., p. 12).

226- Cet emploi simultané à propos d'un liber homo a lieu chez Poumponius D 41.1.54.4; chez Africain D 12.1.41; D 41.1.40.

227- C'est la position de R. REGGI, Liber homo, cit. p. 293. L'auteur rappelle et critique les différente précếdents doctrinaux : pour PERNICE, Labeo 2 , 1, Balle (1845), p. 372, suivi par J. IGLESIAS, Instituciones de derecho romano, I, Barcelone $(1950), p .46$, c'est la bonne foi de 1 'homme $1 i$ bre, il doit donc ignorer sa véritable condition, qui est déterminante ; bre, il doit donc igorer sa veritable condition, qui est determinante:

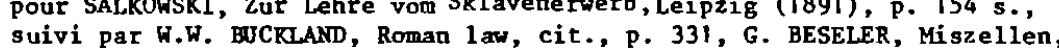
suivi par W.W. BUCKLAND, Roman law, cit., P. 331, G. BESELER, Miszellen,
ZSS 43 (1922), p. 554 et Miscellanea, zSS 44 (1924), p. 392-393, S. PEROZZI ZSS 43 (1922), p. 554 et Miscellanea, ZSS 44 (1924), P. 392-393, S. PEROZZI, blasserwille cit. p. 25, c'est au contraire la bonne foi du présumé maître qui est requise ; enfin, pour CIULEI, Liber homo, p. 28, et A. BERGER, Liber homo bona fide serviens, in Encycl. Diction. of Roman law, p. 562, la bonne foi des deux parties (présumé maître et présumé esclave) est nécessuire.

228- Il suffit de rappeler Paul (Pomponius) D 29.2.22 (si is... putcrerit defunctuon servrom sram esse...; si quasi libertini sui, cum ingenzus esset) et vlpien D 47.10.22 (Si liber pro fugitivo adprehensus erit). 
d'Ulpien qui rapporte ce cas mérite qu'on s'y arrête (229).

D 40.12.12.1 (230) U1pien 1ib. 55 ad edictum : Infans subreptus bona fide in servitute frit, cum liber esset, deinde, cum de statu ignarus esset, recessit et clan in libertate morari coepit : hic non sine dolo malo in libertate moratur.

Si plus tard cet individu, ignorant toujours sa véritable condition, deneure clandestinement - ¿̀ l'insu du prétendu maître - in libertate, $\mathrm{Ul}_{\mathrm{p}}$ en estime qu'il y a fraude. L'assimilation à la condition gervile est on ne peut plus nette.

Autre cas d'ignorance par l'boume libre de son status, l'enfent exposé (231). Celui-ci, abandonné dès son plus jeune âge par ses parents, bien $q u^{\prime} i l$ conserve en droit classique sa condition oxiginaire (232), peut se

229- Le cas est signalé par CIULEI, Liber homo, cit. p. $17 \mathrm{~s}$. 230- Il faut lire D 40.12.12.1 et non D 11.12 .12 .1 in E.M. SCHTAERMAN, La schiavitu, cit., p. 18 .

231- Sur I'expositio des enfants, MAU, Aussetzung, RE 2, p. 2588 s.: WEISS, Rinderaussetzung, RE $11, \mathrm{p} .463 \mathrm{~s}$. ; J. CARCOPINO, Le droit romain d'exposition des enfants et le Gnomon de I'Idiologue, in Mém. de la Soc.

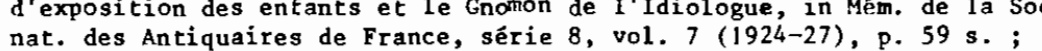
nat. des Antiquaires de France, série 8, vol. 7 (1924-27), p. 59 s. ;
FOURN IER, A propos des expositi, in RHD 5 (1926) p. 302 s. ; E. VOLTERRA, L'efficacia delle costituzioni imperiali emanate per le provincie e l'istituto dell'expositio, in St. Besta 1, p. $449 \mathrm{~s}$. ; LANFRANCHI, Il diritto nei retori romani, Contributo alla storia dello sviluppo del diritto romano, Milan (1938), p. $268 \mathrm{~s}$., et Ius exponendi e obbligo alimentare nel diritto romano classico, in SDHI $6(1940) \mathrm{p} .5 \mathrm{~s}$. ; WESTRUP, Introduction to

Early Roman Law, Comparative Sociological Studies, The Patriarchal Joint Family, vol. 1, Part. 1, The House Comminity, Sect. 1, Comminity of Cult, Copenhague, Londres (1944), p. 249 s.; S. SOLAZZI, Studi Romanistici II, C. Th. 5.9.1, e l'esposizione degli infanti, in RISG 86 (1949) p. $14 \mathrm{~s}$.; A. BERGER, Exponere filizm, in Encycl. Diction. of Roman Law, p. 464 ; SACHERS Potestas Patria, IX, Aussetzungsrecht, RE 22, p. 1089 s. ; w. w. BUCKLAND, Roman Law, cit., p. 402 ; L. MITTE IS, Reichsrecht und Volksrecht, cit., p. 361 , (sur la vente des enfants trouvés) ; N.L. WESTERMANN, Slave Systems, cit., p. 86 ; R.H. BARROW, Slavery, cit., p. 9.

232- Suet. De Gramal., 7, 21 ; P1ine, Litt. Trai., 65, 66 ; Scaevola D 40. 4.29 (Uxorem prasgnantem repudiaverat et aliom duxerat : prior enixa filizon exposuit hic sublatus ab alio educatus est nomine patris vocitatus : usque ad vitas tempus patris tam ab eo quam a matre, an vivorwon numero esset, ignorabatur: mortuo patre testamentoque eius, quo filius neque exheredatus neque heres institutus sit, recitato filius et a matre et ab avia paterna adgnitus hereditatem patris ab intestato quasi legitimus possidet. tum est, hi qui testamento libertatem accepermant utrom liberi an servi simt. Respondit filium quidem nihil praeiudicii passum fuisse, si pater eum ignoravit, st ideo, cum in potestate et ignorantis patris esset, testamentum non valere). Le reste du fragment (servi-fin) est interpolë, cf. R. tum non valere). Le reste du fragment (servi-fin) est interpolé, cf. R.
REGGI, Liber homo, cit. p. $97 \mathrm{n}$. 158 . Certains auteurs ont pourtant niế REGGI, Liber homo, cit. p. $97 \mathrm{n}$. 158 . Certains auteurs ont pourtant nié
le maintien de l'enfant exposé dans sa condition originaire, notamment GLôcK, trouver dans une situation servile de fait (233). Il est intéresgant de remarquer que, dans l'bypothèse ol un tel enfant est recueilli de bonne foi en tant qu'esclave par un particulier, le status est inconnu des deux parties.

Kotons aussi, en marge de ces considérations, que cette situation peut également viser un ancien esclave (234), mais ce dernier cas, ne concernant pas à proprement parler la reproduction du groupe à partir d'hommes

Commentario alle Pandette (tr. it.), Milan (1906), 22, 659, 47 ; DECLAREUIL Commentario alle Pandette (tr. it.), Milan (1906), 22, 659, 47 ; DECLAREu légale a Rome, in Mêl. Girard 1, 327, 6. Mais la majoritê des auteurs penche pour le maintien de la liberté (PAMPALONI, St.sul.ist. di erede ne diritto romano e odierno, in St. Senesi. Suppl. vol. V, 45,$20 ; P$. BONFANTE, Il ius vendendi del paterfamilias e la legge 2, Codice 4, 43, di Costantino, in Scritti 1,65 , et 66 ; E. COSTA, Il diritto privato nelle com medie di Plautio, Turin (1890), 88 ; w.W. BUCKLAND, Roman 1 aw, cit., p. 402 ).

233- Paul D 22.6.1.2 : Si quis nesciat se cognatum esse, interdion in iure, interdion in facto errat. Nan si et liberwm se esse et ex quibus natus sit sciat, iura outem cognationis habere se nesciat, in iure errat : at si quis forte expositus quorum parentium esse ignoret, fortasse et serviat alicui putans se servro esse, in facto magis quam in iure errat. Ce texte a été suspecté d'interpolation par VASSALLI, Iuris et facti ignorantia, in St. Senesi $30(1914), 10,27$ : G. BESELER, Beiträge zur Kritik der römischen Rechtsquellen, in zSS 66 (1948) 3,86 , et Unklassiche Wörter, in $2 S S, 56$ (1936) 69 pour-errare in; P. VOCI, Lerrore, cit. 216 et 217 ; G. DULCKEIT, Erblasgerwille, cit. 19, 2 ; CIULEI, Liber homo, cit. 19 ; G. DULCKEIT, Erblasserwille, cit. 19, 2 ; CIULEI, Liber homo, cit. 19;
VAN WARMELO, Ignorantia iums, in Tijd. 22 (1954) 20. Par contre, LANFRANVAN WARMELO, Ignorantitit. $37 \mathrm{~s}$. soutient que la totalité du fragment est authentique. R. REGGI, Liber homo, cit., 96 , estime que, quoi qu'il en soit, ce passage prouve que l'individu exposé conserve son status originaire.

234- L'hêritier peut par exemple posséder de bonne foi 1 'esclave dont il ignore 1 'affranchissement testamentaire, cf. Ulpien D 29.2.21.3: (Si quis partem ex qua institutus est ignoravit...) et Iavolenus D 45.3 .34 (Si servus testamento manumissus, crom se liberm esse ignoraret et in causa hereditaria maneret, pecuniam heredi stipulatus est, nihil debebitur heredibus, si modo scierint evon testamento manumisswn, quia non potest videri iustam servitutem, servisse iis, qui illum libermon esse non ignorabant. Distat ista cousa eius, qui liber emptus bona fide servit, quia in eo et ipsius et em torsa emptoris existimatio consentit. ceterm is, qui scit hominem libern esse, quamuis ille condicionem suan ignoret, ne possidere quidem ewn potest videmi) Sur ce dernier texte, le passage -distat-ceterm- a été retenu interpolé par G. BESELER, Miszellen, cit. p. 554 ; G. DULCKEIT, Erblasserwille, cit. p. 22 , n. 3 ; CIULEI, Liber homo, cit. p. 18 et 29 . Un esclave, par ailleurs, peut être affranchi conditionnellement dans un testament, et plus tard affranchi purement par des codicilles dont.il ignore 1'existence. I1 continue ainsi à vivre dans un état servile jusqu'à 1 'accomplissement de $1 \mathrm{a}$ condition de sa libertê, cf. Ulpien D 12.4.3.7 (Sed si servus, qui testamento heredi iusous erat decem dare et liber esse, codicillis pure libertotem accepit et id ignorans dederit heredi decem, an repetere possit ? ...); sur le texte, G. DONATUTI, Lo statulibero, Milan (1940), p. 167. Le statuliber peut encore ignorer la réalisation de la condition de sa iiberté, et ainsi demeurer dans son ancien êtat, cf. Paul D 29.2.74.3 (Similis est huic 
libres, n'a pas été retenu dans le calcul des textes sur le liber homo bona fide serviens.

L'ignorance par l'home libre de son status n'est cependant pas la seule base de la situation envisagée.

\section{B/- La servitude volontaire.}

$\mathrm{Si}$ on admet en effet que le critère déterminant est à l'époque classique la bonne foi du possesseur, un home libre peut se trouver en esclavage tout en ayant connaissance de sa condition (235). Cela conduit à intégrer dans nos calculs des textes où n'apparaît pas la bonne foi đu serviens, mais seulement celle de son possesseur (236).

statuliber, qui iussus ab herede adire hereditatem post condicionem listatuliber, qui iussus ab herede adire hereditate
bortatis existentem, cum hoc ignorcret, adiit).

Il $y$ a dans ces cas prise de possession du 1.h. par voie dérivée, alors qu'elle s'effectue par voie originaire dans le cas de l'enfant exposé, R. REGGI, Liber homo, cit. p. $84 \mathrm{~s}$, et $110 \mathrm{~s}$.

235- Cf. Pomponius D 41.i.54 pr : Homo liber hereditatem nobis adquirere non potest. Qui bona fide nobis servit, adquiret, si tamen sponte sua sciens condisionem suan adierit. SALKOWSKI, Zur Lehre vom Sklavenerwerb, cit.,
155,36 .

236- Ainsi Pomponius D 18.1.4 (Et liberi hominis et loci sacmi et religiosi, qui haberi non potest, emptio intellegitur, si ab ignorante emitur) : cependant, le passage $s i$ ab ignorante emitur ne serait pas classique, $P$. BONFANTE, Istituzioni di diritto romano, Turin (1946) 485, 2 ; BERTOLINI, Appunti didattici di diritto romano, Turin (1905) 450, 2 ; E. ALBERTARIO rec. ad Heldric, in AG 96 (1926) $111-112$ (Studi, II, 371); FERRINI, De iure sepulcrorm apud Romanos, in Opere IV, 12 ; P. VOCI, L'errore, cit. p. 149 ; v. ARANGIO-RUIZ, La compravendita in diritto romano, Naples (1954) p. 131 ; B. BIONDI, La vendita di cose fuori di commercio, in St. Riccobono 52,156 . L. Rufinus (Liberi hominis emptionem contrahi posse plerique existimavemmt, si modo inter ignorantss id fiat. Quod idem placet etion, si venditor sciat, emptor autem ignoret ous si mptor sciens liberum, emerit, nulla emtio tontrahitur) emerit, nulla entio contrahitur) ; P. VOCI, L'errore, cit. p. 150 pense que parmi les plemque, L. Rufinus aurait visé Sabinus. Scaevola D 21.2 $69 \mathrm{pr}$ (Qui libertatis causam excepit in venditione, aive iam turc cum traderetur liber homo fuerit, sive condicione quae testamento proposita fuerit impleta ad libertatem pervenerit, non tenebitur evictionis nomine) ; 0 . LENEL, Pa1 II, p. 273 ; P.F. GIRARD, L'auctoritas et 1 'action auctoritatis, Inventaire đ̛ interpolations, in Mélanges, 239, 2 ; G. DONATUTI, Lo statulibero, cit. 102, ont remplace la mention de la traditio par celle de 1a mancipatio, et par conséquent celle de 1'éviction par celle de l'autoritas pour le droit classique ; il s'agit selon R. REGGI, Liber homo, cit. p. 220 de la moncipatio d'une personme libre Paul D 41.230 .1 (Possessionem prittinus in em locom intulimo in ewm locin intulimus, quem possidebamus : nwane locion religiosum aut sacrum non possumus possidere, etsi contemnamus religionem et pro privato elm teneormus, sicut hominem libernon), bien que Paul y nie la possibilité de posséder un homal libre (cf. SEUFFERT, Praktisches Pandektenrecht,
Le Digeste ne fournit pas d'inaications précises sur ce thème. On sait par le Code de Justinien que parfois, pressés par la nécessité, les pauvres, bien que ce fût illégal, se vendaient à leur créancier ou lui laissaient en gage leurs propres enfants (237). Il est vrai que, dans une telle hypothèse, le créancier pouvait difficilement ignorer le status de celui qu'il recevait comme esclave, mais il en allait tout autrement si une personne, privée de ressources, se laissait vendre à un inconnu.

Il n'était pas facile pour le pauvre réduit à cette extrémité de retrouver sa liberté. Si l'affaire parverait devant un tribunal, il pouvait récupérer ses droits, mais le séjour en esclavage devait probablement, dans la réalité, être assez long (238). Il suffit de penser qu'en droit classique, une personne, même lorsqu'elle avait connaissance de son status, devait rester en servitude si elle ne réussissait pas à trouver un adsertor libertatis (239).

\section{$-0$}

Würaburg (1860), I \& 107, p. 135 ; WANGERar, Lehrbuch der Pandekten, 1 , Marburg et Leipzig (1876) i \& 201, p. 360 ; PUCHTA, Pandekten, Leipzig (1877) \& 128, p. 190; ARNDTS, Lehrbuch der Pandekten, Stuttgart (1874) 138, p. 212 ; CORNIL, Traite de la possession dans le droit romain, Paris (1905), p. 98 ; RUGGIERI, Il possesso e gli istituti di diritto prossimi ad esso, Parte prima, I1 possesso in diritto romano, Florence (1860), p. 237 ; RICCOBONo, Die Teminologie der Besitzverhältnisse (Naturalis possessio, civilis possessio, possessio ad interdicta), in zSS 31 (1910), p. 329) ; ce texte est en contradiction avec ce qu'il écrit in D 41.2.1.6 (Sed et per evm, quem bona fide possidemus, quamis alienus sit vel liber, possessionem adauiremis Si maia fide erm possidecomis...). Gaius D 44.7 .1 .9 ( $S i$ id, quod dari stipulemur, tale sit, ut dari non possit, palam est naturali ratione inutilem esse stipulationem, veluti si de homine libero... rali ratione inutilem esse stipulationem, veluti si de homine libero.. facta sit stipulatio inter eos, qui ignoraverint ewm hominem libern esse...) ; sur la signification de dari, BUSSMANN, L'obligation de délivrance du vendeur, Lausanne (1933), 20 ; 5. BIONDI, La vendita di cose
fuori di comercio, cit. 18 ; G. GROSSO, Obbligazioni, cit. $14 ; \mathrm{v}$. ARANGIONDIZ, La compravendita in diritto romano, cit. 161 ; G. DONATUTI, Le causae delle condictiones, in St. Parmensi 1 (1951), 45 et 64 . A en outre été intêgré U1pien D 50.17.118 (Qui in servitute est, usucopere non potest : nam cum possideatur, possidere non videtur) ; Sur ce passage, BARON, Zur Lehre vom Besitzwille, in Jahrbücher für die Dogmatik des heutigen römischen und deutschen Privatrechts, 29 (1890), 199 ; SALKOWSKI, Zur Lehre vom

Sklavenerwerb, cit. 168,11 ; W.W. BUCKLAND, Roman law, cit. p. 347, 5 ; G. DULCKEIT, Erblasserwille, cit. 31,2 ; s'il s'était agi d'un esclave, problème ne se serait pas posé : in servitute est vise donc une personne liproblème ne se serait pas posé :
bre vivant dans un état servile.

237- C.I. $4.10 .12 ; 4.43 .1 ; 7.16 .1 ; 8.16 .6$.

238- E.M. SCHTAERMAN, La schiavitū, cit., p. 22.

239- . R. REGGI, Liber homo, cit. p. 463. 
Cet home libre, nous dit Ulpien $(2 /, 0)$, est compris dans la familia. S'il a été acquis de bonne roi, l'interdit de homine libero exhibendo n'a pas lieu (241). Preuves encore de son assimilation de fait à un esclave (242), sa capacité juridique est limitée $(243)$, il ne peut acquérir de droits patrimoniaux $(24 h)$, ses acquisitions profitent à son maître présumé lorsqu'elles sont faites ex re domini vel ex operis suis (245).

En fonction de ce qui précède, ont été retenus par périodes les textes suivants $(246)$ :

analyse des paragraphes

\begin{tabular}{|c|c|c|c|c|}
\hline I & - & $=$ & - & I \\
\hline II & $0 / 6$ & $=$ & - & II \\
\hline III & $18 / 985$ & $=$ & 2 & II \\
\hline IV & $3 / 354$ & $=$ & $1 !$ & IV \\
\hline $\mathrm{v}$ & $49 / 3840$ & $=$ & 1 & $\mathrm{v}$ \\
\hline
\end{tabular}

240- D 21.1.25.2 : Fomiliae oppellatione omnes qui servitio sunt continentur etiam liberi homines, qui ei bona fide serviunt, vel alieni : accipe eos quoque qui in potestate eius sunt.

241- D 43.29.4.1 Venuleius : Trebatius quoque ait non teneri eum, qui liberum hominem pro servo bona fide emerit et retineat.

242- Il est plus précisément assimilé à un servus fructuarius, cf. Gaius 2, 86-91-92-93-94; R. REGGI, Liber homo, cit. p. 82 n. 102 ; E. M. SCHTAERMAN, La schiavitù, cit. p. 18 .

243- ALLARA, Le nozioni fondamentali del diritto civile, 1, Turin (1953) p. 189.

244- BETTI, Diritto romano, Padoue (1935), p. 82.

245- R. REGGI, Liber homo, cit. p. 365 s. : acquisitions ex operis, p. 371 s. ; acquisitions ex re, p. $380 \mathrm{~s}$. ; en revanche, lorsqu'il acquiert extra duas causas, le liber homo, contrairement a l'esclave, acquiert pour lui, p. $394 \mathrm{~s}$.

246- Iavolenus D 45.3.34; Celsus D 28.5 .60 pr (Trebatius, Labéon); Julien D 29.2.45.4; D 41.4.7.8; Pomponius D 13.6.13.2 (Celsus); D 18.1.4; D 41. 1.19 (Trebatius, Labéon, Áristo, v. Lucullus); D 41.1 .21 pr (Proculus); D 41.1.54 pr-1-2-3-4; Africain D 12.1.41; D 41.1.40; Gaius D 41.1 .10 pr4 ; D 44.7.1.9; Venuleius D 43.29.4.1 (Trebatius); Scaevola D 12.6.67 pr ; D 21.2.69 pr ; Papinien D 17.1.54.1; D 41.3.44 pr; D 45.1.118 pr ; Callistrate D 4.6.11 ; Paul D 2.14.19 pr ; D 3.5.18.2 (Labéon) ; D 3.5.35; D 11.1.13.1; D 13.5.6 ; D 18.1.5 (suite de D 18.1.4 Pomponius) ; D 22.6. 1.2 ; D 29.2.74.2 ; D 41.2.1.6; D 41.2.30.1; D 45.1.126.2 (a contrario); D 45.3.20 pr ; D 45.3.33 pr ; Ulpien D 3.5.5.7-9; D 9.2.13.1 (Julien);

D $11.3 .1 .1 ; 012.4 .3 .5$ (Neratius, Julien);D 14.4.1.5;D 15.1.1.6;

D 16.1 .26 ; D 16.3.1.27; D 17.1.8.5 (Julien); D 21.1.23.6; D 21.1.25.2;

D 21.2.19.1; D 24.1 .3 .8 ; D 29.5.1.2; D 39.4.12.2; D 40.12.7.2; D 40 .

12.12.1-6; D 41.1 .23 pr-1-2-3 (Julien, Scaevola); D 41.2.4; D 41.2.13.3;

D 47.8.2.14;D 48.18 .1 .8 ; D 49.16.8;D 50.17.118; L. Rufinus D 18.1.70.
La situation du liber homo bona fide serviens a posé des problèmes aux juristes de toutes les périodes prises en considération, sans qu'il y ait de variations importantes du discours d'une période à l'autre. Le droit a régularisé l'asservissement de fait de l'homme libre. Il lui a fait produire des effets juridiques en tant que situation transitoire (247). Pouvait-il en être autrement ? Les impératifs économiques et sociaux ont certainement poussé les juristes dans cette direction (248). De plus, 1 'home libre victime de pareille mésaventure était-il d' une situation économique reluisante? $N$ 'est-ce pas dans un tel sens qu'il nous faut interpréter ce passage de Paul ?

D 18.1.5 Paul lib, 5 ad Sabinum Quia difficile dinosci potest liber homo a servo.

Cependant, bien que les textes montrent que des cas de ce genre ont existé, on ne peut les considérer d'un point de vue statistique comme une source importante de travail servile $(248 \mathrm{bis})$. C'est, pourquoi ils ne seront pas comptabilisés dans $I$ 'analyse comparative des divers modes de reproduction du groupe servile. Ces cas ne correspondent qu'à des situations de fait, l'homme libre conservant en droit son status. Le droit est alors fidèle à ses propres principes :

D 40.12.37 Callistrate lib. 2 quaestionum - Conventio privata neque servum quemquam neque libertwm alicuius facere potest.

Il va pourtant permettre de véritables réductions en esclavage.

\section{Paragraphe 2 : Le droit intervient directement pour consacrer la réduction en esclavage d'hommes libres.}

Tantôt, l'homme libre va devenir l'esclave d'un particulier, tantôt, il va subir l'esclavage de la peine. 247- Il s'agit d'une situation de fait (B. BIONDI, La vendita di cose
fuori commercio, cit. p. 9 et 19) dotée toutefois d'effets juridiques (BETTI, Diritto romano, cit. p. 78) en tant que situation transitoire (car (BETTI, Dirit to romano, cit. p. 78 ) en tant que situation transitoire (car
il existe pour 1 'homme libre la possibilité de voir reconnaître sa véritable condition juridique).

248- E.M. SCHTAERMAN, La schiavitũ, cit. p. 22 ; R. REGGI, Liber homo, cit. p. 63. Un texte de Papinien témoigne de la fréquence des ventes d'homes libres, D 41.3.44 pr : ... nam frequenter ignorantia liberos emimus.

248 bis- Selon F. DE MARTINO, Storia economica, cit. II, p. 269 , on ne peut soutenir sur la base des textes, qui affirment que la fonction servile ne rend pas esclave (cf, CI $7.14 .2 ; 7.14 .14 ; 7.16 .10 ; 7.16 .16$ ), 1 'existence d'une pratique régulière selon laquelle celui qui engageait des travailleurs le servant pour une longue durée les assimilait à ses esclaves et en disposait à sa guise. 
I - La reproduction privée du groupe servile à partir d'hommes libres : La vente de soi-mêne

I1 n'est pas question dans le Digeste du s .c. Claudien qui infligeait la perte de la liberté à la fenme libre qui, après trois sommations adressées par le mâ̂tre d'un esclave, persistait à faire de cet esclave son gmant (249). Le but de ce s.c. était sans doute d'opérer une plus stricte démarcation de la barrière sociale existant entre libres et esclaves. Mais à long terme il a favorisé la création d'une sorte de source supplémentaire d'esclaves.

Les textes traitent en revanche d'une autre source d'asservissement, qui résulte au départ de la règlementation prétorienne (250), la vente de

249- Tacite, Ann. XII, 53, 1 ; Gaius I, 160 ; cf. B. ALBANESE, Appunti sul s.c. Claudiano, I1 circolo giuridico 22 (1951), p. 86-96 ; H.R. HOETINK, Autour du s.c. Claudien, Mế1. Lévy-Brub1 (1959), p. 153-162; T. RADo, I1 s.c. Claudian. Annales de la Fac. de doit d'Istabul 3 (1954), P. 44 s. Il fallait évidenment que cette fenme connaisse son vêritable état de fenane libre, cf. Paul, Sent. II, 21, A 12. Cette mesure ne concernait que la feme libre, cf. Paul, Sent. II, 21, A 12. Cette mesure ne concernait que la feme
sui iuris, cf. J. MACQUERON, Droit romain I (Aix-en-Provence 1954), p. 314, le s.c. n'ayant pas osé s'attaquer a la puissance paternelle. Sur le point de savoir si les sommations sont prévues par le s.c. ou s'il s'agit d'une élaboration jurisprudentielle, v. W.W. BUCKLAND, Roman law, cit. p. 412. Le s.c. autorisait à 1 'origine la citoyenne romaine qui avait des relations avec un esclave, avec 1 'assentissement du maître de celui-ci, à faire un pacte avec ce maitre en vertu duquel elle demeurait libre et ses enf ants naissaient esclaves, cf. J. CAUDEMET Institutions de l'Antiquité, cit. $p$. naissaient cit. $\mathrm{p}$ 544, n. les enfants seraient libres, cr. Gaius 1 , 84 . Vespasien fit cesser une inelegantia iums inverse en vertu de laquelle les fils nés d'un home $1 \mathrm{i}$ bre et d'une femme esclave, que celui-ci croyait libre, étaient libres, et les filles esclaves. Il décida que, quel que soit leur sexe, les enfants seraient esclaves, cf. Gaius I, 85 ; sur ce point, C. CASTELLO, La condizione del concepito da libero e schiava e da libera e schiavo, in St. Solazzi (1948) p. $232 \mathrm{~s}$. Enfin, quant aux enfants de la femme qui entretiendrait des relations avec un esclave sans 1 'assentiment du maître, ils naîtront fort logiquement esclaves, cf. Gaius $I, 91$.

250- Déjà une disposition de l'édit du préteur connue de Q. Mucius Scaevola interdisait de reconnaître la libertá de celui qui s'était frauduleusement fait vendre par un compère pour partager avec lui le prix de la vente, cf. D 40.12.23 pr Paul : Si usum fructum tibi vendidero liberi hominis et cessero, servum effici evm dicebat Quintus meus, sed dominium it (1) dem fieri mew, si bona fide vendidissm, alioquin sine domino fore, ACCARIAS, Précis de droit romain I, Paris (1886), p. 96 ; J. GAUDEMET,
Institutions de l'Antiqui té, cit. p. 544. Pour RARLOWA, Römische RechtsgesInstitutions de 1 'Antiquité, cit. p. 544. Pour RARLOWA, Rómische Rechtsges
chichte II, Privatrecht und Zivilprozess , Privatrecht, Leipzig (1901) p. 1117 , et R. REGGI, Liber homo, cit. p. 311 s, il ne $s$ 'agirait pas d'une vente ad pretium participandum, mais ad actum gerenium. R. REGGI (p. 313) invoque à 1 'appui de sa thèse deux motifs : le premier est qu'on a affair $a$ un vendeur de bonne foi, qui ne peut donc être le complice de celui qui soi-même. On distingue traditionnellement la vente ad pretium participancum de la vente ad actum gerendrom. Bien qu'un texte d'Ulpien évoque la deuxième forme (251), elle n'est pas classique. C'est seulement Constantin en 323 qui autorisera la réduction en esclavage de l'homme libre qui, majeur de vingt ans, et ayant connaissance de sa liberté, se laisse vendre pour remplir la fonction de serrus actor de l'acheteur, en d'autres termes pour en administrer le patrimoine (252).

Le classicisme de la première forme est lui aussi très discuté. L'homme libre, qui se laisse frauduleusement vendre par un conplice pour partager svec lui le prix de la vente, devient-il juridiquement esclave en droit classique ? On sait que cette situation a reçu une règlementation plus précise sous les Antonins (253), mais il ne semble pas que cet individu devienne alors esclave iure civili (254). Le préteur lui refuse

se laisse vendre (cf. NICOLAU, Causa Liberalis, Etude historique et comparative du procès de liberté dans les législations anciennes, Paris (1933), p. 266) ; le second est que dans le paragraphe suivant (D 40.12.23.1), il est dit que la rêduction en esclavage advient même dans le cas où 1 'hơne libre est objet de dot, de gage ou de donation, et on ne peut parler alors de partage du prix étant donné qu'il n'y a pas de prix a partager. Par contre, P. BONFANTE, Corso di diritto romano I, Diritto di famiglia, Rome (1925), p. 160, pense qu'il s'agit d'un cas de vente ad pretium participandiom

251- D 28.3.6.5 : Irritum fit testamentum, quotiens ipsi testatori aliquid contingit, puta si civitatem amittat per subitam servitutem, ab hostibus verbi gratia captus, vel si maior annis viginti venum se dari passus sit ad actum gerendum pretiume participandum. Sur le texte, ColI, Saggi ad actum gerendum pretiumve participandum. Sur le texte, Coli, Saggi critici sulle fonti del diritto romano, I, Copitis deminutio, Florence (1922) p. 16 ; RATTI, Studi sulla coptivitas, I, Libertà e cittadinanza,
in RISG I (1926) p. 205, n. 51 ; G. BESELER, Romanistiche Studien, in St. Riccobono, 1, p. 307 ; NICOLAU, Causa liberalis, cit. p. 265 ; AMIRANTE, captivitas e postliminium, Naples (1950), 26, 30, 73, 2 ; M. RASER, Zur Geschichte der Copitis Deminutio, in Iura 3 (1952), 51,13 ; W.W. BUCKIAND, Roman Law, cit., p. 433, n. 5. Alors que NICOLAU pense que le passage est authentique, M. RASER, suivant COLI, soutient qu'il est interpolé.

252- C. Th. 4,8,6 Imp. Constantinus (323 mai. 18). R.REGGI, Liber homo, cit. p. 318

253- Pomponius D 40.13.3 ; Venuleius D 40.14.2 pr ; E.M. SCHTAERMAN, La schiavitu, cit. p. 23, 237 ; W.W. BUCKLAND, Roman Law, cit. p. 433.

254- Celui qui s'est vendu pour avoir une part du prix devient esclave selon REIN, Das Privatrecht und der Zivilprozess der Römer von der ältesten Zeit bis auf Justinianus, Leipzig (1858), p. 123 (cas de copitis deminutio Zeit bis auf Justinianus, Leipzig (1858), p. 123 (cas de capitis deminutich maxima) : SALKOWSKI, Lehrbuch der Institutionen und der Geschichte des
Römischen Privatrechts, Leipzig (1880), p. 90 ; LEONHARD, Institutionen des Romischen Privatrechts, Leipzig (1880), P. 90 ; LEONHARD, Institution
römischen Rechts, Leipzig (1894), p. 175: BARON, Pandekten, Leipzig (1896), p. 50 (cas de copitis deminutio maxima) ; ROBY, Roman private 1 a in the times of Cicero and of the Antonines, I, Cambridge (1902), p. 43 ; SERAFINI, Istituzioni di diritto romano comparato col diritto civile patrio, 
simplement 1.action en revendication de sa liberté (255). S'il on nvait été autremont, la question du sort des enfants de 1a fenue qui s'était laissée vendre ad pretium participandion ne se serait probablement pas posáe (256). Il n'en demeure pas moins que si la diffórence est importante au regard du droit, elle est nulle au regard de la pratique, l'homme libre se trouvant quoi qu'il en soit dans une situation servile.

Si à l'ópoque de Gaius cette vente n'est pas encore classée parmi les causes de cupitie deminutic maxima (257) et entraîne serulement la

I, Rome (1920), p. 115 ; PACCHTONI, Corso di diritto romano, II, Turin (1920), P. 30; BRY, Principes de droit romain, I, Paris (1927), p. 39 ; SCIALOJA, Corso di Istituzioni di diritto romano, Rome $(1934)$, p. $74 ; j$. IGLESIAS, Derecho rnmano, Instituciones de dererho privado, I, Barcelone (1953), p. 47 ; DI MARZO, Mmuale Elementare di Dirittn Rnmano, Turin (1954). P. 42 ; SANF LlIPro, Istituzioni di diritto romano, Naples (1955), p. 115 .

255- R. REGGI, Liber homo, cil. p. 325 ; E. CUQ, Les Institutions juridiques des Romains, 2, Paris (1902), p. 74 (Hadrien refusa le droit de revendiquer la libertê); C. FERRINI, Manuale di Pandette, Milan (1908), p. 66 (une disposition très ancienne de l'édit du préteur entraîna l'impossibilitẹ de revendiquer sa liberté,"si tratta in sostanza di una denegatio actionis, che però la costituisce, di fatto, in condizione servile") (FERRINI, Pandette (1953) D. 55) ; P.F. GIRARD, Manuel élémentaire de droit romain, Paris (1911), P. 101 ; SOHM, Institutionen Gerchichte und Systen des römi

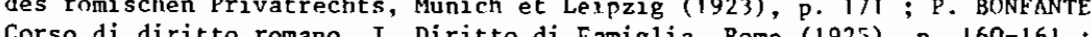
Corso di diritto romano, I, Diritto di Famiglia, Rome (1925), p. I60-161 GIFFARD, Précis de droit romain, Paris (1938), p. 212 ; V. ARANGIO-RUIZ, Istituzioni di diritto romano, Naples (1954), p. 482-483; P. VOCI, Istituzioni di diritto romano, Milan (1954), P. 94 ; MONIER-CARDASCIA-IMBERT, Histoire des institutions et des faits sociaux des origines a 1 'aube du Moyen Age, Paris (1955), p. 399 (le préteur, l'époque d'Hadrien, refuse 1 'action en revendication de la liberté ; l'individu est en fait esclave) ; G. SCHERILLO, Lezioni di istituzioni di diritto romano, Milan (1956), p. 142 (idem)

256- N1COLAU, Causa liberalis, cit. p. 272 ; 1'auteur remarque (p. 274): "Primitivement, la personne qui s'ëtait laissée vendre afin de partager le prix se voyait simplement refuser le moyen de faire valoir en justice la liberté dont ni le prêteur ni le sénat n'auraient pu la dépouiller. Le préteur en effet n'a jamais pu faire du droit civil, il $n$ 'a jamais eu le pouvoir de faire des heredes, à plus forte raison ne pouvait-il pas transformer de sa propre autorité un houne libre en esclave" ; (cf. Pomponius D 40.13.3).

257- Gaius I, 160: Maxima est copitis deminutio, cum aliquis simul et civitatem et libertatem amittit; quae accilit incensis, qui ex forma censuali venire iubentur : quod ius... ex lege... qui contra eam legem in urbe Roma domicilium habuerint; item feminae, quae ex senatoconsuto Claudiano ancillae fiunt eorum dominorm, quibus invitis et denuntiatibus cum servis eomm coierint. Dans les Institutes de Justinien, on 1 it en revanche : Ma-

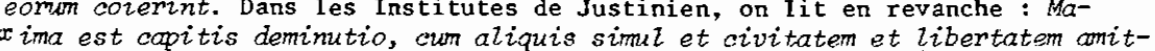
tit. Quod accidit in his, qui servi pnenae efficiuntur atrocitate sententiae, vel liberti ut ingrati circa patronos condemmati, vel qui ad pretium participondum se venumdari passi sunt (I, 1, 16, 1). La comparaison de ces denegatic adsertionis du préteur, une évolution semole s'être produite qu 3ème siècle. Marcien présente en effet la rente ad pretium participandiun comene un cas de perte de la liberté fondó sur le ius civile (258). Modestin afrirme d'autre part que l'individu qui s'est ainsi laissé vendre, s'il obtient la liberté, ne sera pas ingénu mais afranchi, comme un simple esclave (259). La date de ce changement reste néanmoins discutée (260).

Les textes sont flous quant à la limite d' âge de celui qui se vend. Certains n'en font pas mention (261). D'autres font. simplement état de maior et de minor(262). Il est difficile de retracer l'évolution qui a dû se produire en la matì̀re (2ó3). Papinien hésite entre vingt et vingt-cinq ans, Ulpien et Marcien parlant de vingt ans $(26 / 4)$. deux textes montre que la vente ad pretium participandwon est in cas de

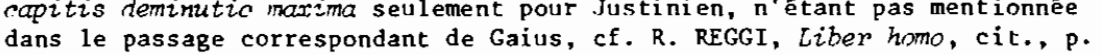
324 .

258- Marcien D 1.5.5.1: Semi autem in dominium nostmm rediguntur out ivre civili aut gentium : iure civili, si quis se maior viginti annis ad pretium participandum venire passus est : iure gentium servi nostri sunt, qui ab hostibus copiuntur aut qui ex ancillis nostris nascuntur. Sur ce passage, KARLOWA, Römische Rechtsgeschichte, cit. 2, p. 249, 257 et 1116 ; W.W. BUCKLAND, Roman $1 \mathrm{aw}$, cit. p. $427 \mathrm{~s}$.; Id., A textbook of roman law from Augustus to Justinian, Cambridge (1950), p. 71 ; S. PEROZZI, Istituzioni di diritto romano, cit. 1, p. 236 n. 3 , affirme que le texte est interpolé ; NICOLAU, Causa liberalis, cit., p. 266, estime au contraire que déjà à 1 'époque de Marcien, la vente ad pretium farticipandum est à considérer parmi les sources de 1 'esclavage.

259- Modestin D 1.5.21 : Homo liber, qui se vendidit, manumissus non ad sum statum revertitur, quo se abdicavit, sed efficitur libertinae condicionis. M. KASER, RPR I 2 , p. 292 n. 46 , se fondant sur ce texte et sur $D$ 1.5.5.l Marcien, pense que les juristes du 3ène siècle considèrent D' homo, cit. p. 325 , n. 78. Dans le même sens que Modestin, on trouve plus homo, cit. P. 325, n.
tard Hermogeñien $D$
40.12 .40 .

260- Certains auteurs considèrent qu'il n'interviendrait pas avant Justinien. C'est la position de R. REGGI, Liber homo, p. 326, de P. BONFANTE,

Corso di diritto romano, cit., p. $\{60-161$; de W.W. BUCKLAND, The Main Institutions of roman private law. Cambridge (1931), p. 49 ; de G. SCHEInstitutions of roman private lawi di istituzioni di diritto romano, cit., p. 142 ; et de RILLO, Lezioni di istituzioni di diritto romano, cit., p.
B. BIONDI, Istituzioni di diritto romano, Milan (1956), p. 114 .

261- Ainsi D 1.5.21 Modestin ; D 40.12.23 Paul ; D 40.12.33 Paul.

262- Ainsi D 40.14.2 pr Venuleius.

263- Selon E.M. SCHTAERMAN, La schiavitũ, cit. p. 237, Hadrien aurait fixé cette limite d'âge à 25 ans (D $40.14 .2 \mathrm{pr}$ ), mais ce texte ne semble pas très probant dans la mesure oû Pomponius parle, à propos de la fenme qui se vend comme esclave, d'une limite d'âge de vingt ans (D 40.13.3).

264- Ulpien D 40.13.1.1 ; Marcien D 1.5.5.1 ; de même Hermogénien D 40.12. 40. 
Si on relève tous les textes qui concernent la vente de soi-même (265), on obtient le tableau suivant : analyse des paragraphes

$\begin{array}{llll}\text { I } & - & = & - \\ \text { II } & 0 / 6 & = & - \\ \text { III } & 2 / 985 & = & 0,20 \% \\ \text { IV } & 2 / 354 & =1 \% \\ \text { V } & 44 / 3840 & =1 \%\end{array}$

analyse des citations

$\begin{array}{llll}\text { I } & 1 / 128 & = & 1 \% \\ \text { II } & 1 / 347 & = & 0,28 \% \\ \text { III } & 0 / 519 & = & - \\ \text { IV } & 0 / 105 & = & - \\ \text { V } & 2 / 35 & = & 6 \%\end{array}$

Comme dans les situntions précédemment envisagées, l'analyse quantitative montre la continuité du discours juridique en la matière. Le total important réalisé par la période $\mathrm{V}$ dans les citations est surtout dû au nombre faible de citations concernant l'esclavago chez les juristes de cette époque. Il est vrai que les juristes du 3ème siècle se sont préoccupés, lorsque cela leur a paru possible, de protéger le status de celui qui se laissait vendre. Ils ont ainsi prévr que l'acheteur de mauvaise foi, c'est-à-dire celui qui achète sciemment un home libre, ne pourrait s'opposer à l'action en revendication de liberté de celui-ci (266). De même, ils ont écrit que les majeurs de vingt ans qui se seraient laissés vendre pourraient revendiquer leur liberté pourvi qu'ils ne l'aient pas fait pour toucher une part du prix (267), et encore que les mineurs de ringt ans qui 1 'auraient fait dans un tel but pourraient réclamer leur liberté après avoir atteint cet âge (268). Il ne s'agit toutefois nullement d'une remise en cause de l'institution, seulement d'une définition plus précise.

265- Ponponius D 40.13.3 ; Gaius D 40.12.4 (il s'agit d'un affranchi); Venuleius D 40.14.2 Pr (constitution d'Hadrien); Marcellus D 40.5.10.2 ; Papinien D 4.4.9.4; Paul D 40.12.15; D 40.12.17; D 40.12.19; D 40.12. $23 \mathrm{pr}$ (Q. Mucius)-1; D 40.12.33 ; D 40.13.4 (L. Rufinus) ; D 40.13.5 ; U1pien D 28.3.6.5; D 40.12.7 pr-1-2-3; D 40.12.14 pr-1-2 ; D 40.12.16 pr$1-2-3-4$; D $40.12 .18 \mathrm{pr}-1$; D $40.12 .20 \mathrm{pr}-1-2-3-4$; D $40.12 .22 \mathrm{pr}-1-2-3-4-5$ (Labéon) -6 ; D 40.13.1 pr-1 ; Macer D 48.19.14 (Menander); Marcien D 1.5.5. 1 ; Modestin D 1.5.21 ; D 40.12 .21 pr-1 ; Hermogénien D 40.12.40. I1 est des textes oũ rien n'est dit sur la connaissance ou l'ignorance par 1 'homme libre de son status. Ils n'ont ainsi pas pu être intégrós dans cette rubrique (par ex. D 18.1.4 pris in possession de bonne foi ; D 18.1.6 pr in possession de mauvaise foi).

266- Paul D 40.12.33.

267- U1pien D $40.12 .7 \mathrm{pr}$.

268- Ulpien D 40.12 .7 .1 .
Bien que la vente de soi-même vise avant tout une fraude, le risque qu'elle comportait pour la personne aliénant sa propre liberté n'évoque-til pas l'état de pauvreté de celle-ci (269) ? Nous verrons que cette considération n'est pas étrangère à l'esclavage pénal qui, contrairement aux cas examinés jusqu'à présent, ne concerne plus la reproduction privée du groupe servile.

\section{II - L'esclavage de la peine}

Avant sa condamnation, le serrus poenae peut avoir été libre ou esclave. Seul le premier cas sera pris ici en considération, le second ne concernant plus le problème de la reproduction, mais uniquement celui de la répression (270).

Cette source d'esclavage se développe sous le Principat (271). Les $s \in r^{-}$ $v i$ poenae ne sont théoriquement les esclaves de personne (272). Mais la peine n'est pas un sujet de droit, c'est donc le peuple qui détient le droit de propriété sur ces esclaves (273). A l'époque des Sévères, divers textes tendent à opposer les servi poence aü servi Caesaris (274). Cette opposition est avant tout terminologique. On veut simplement ne pas conférer à des condamnés un titre porté par des esclaves impériaux, esclaves qu'il bonore (275). En effet, comme ceux-ci, les condamnés appartiennent au Fisc

269- J. GAUDERET, Institutions de l'Antiquité, cit., p. 545. La nisère n'est probablement pas étrangère par ailleurs à l'hypothèse de celui qu'on n'est probablement pas étrangère par ailleurs à
contraint à se vendre, cf. Ulpien D 40.12 .16 .1 .

270- Le cas de l'affranchì sera examiné. Il s'opère en effet pour lui un changement d'état. Il suffit de rappeler Ulpien D 50.17.209 : Servitutem mortalitati fere comparamus.

271- Th. MOMMSEN (Droit pénal III, p. 290) fait dater cette source du règne de Tibère.

272- R.H. BARROW, Slavery, cit. p. 13 ; G. DONATUTI La schiavitù per condanna, BIDR 42 (1934), p. 225 s. (servi sire domino).

273- G. BOULVER, Domestique et fonctionnaire sous le Haut-Empire romain, Paris (1974), p. 47.

274- Marcien D 34.8.3 pr ( $S i$ in metallwm donnato quid extra cousam alimentorum relictum fuerit, pro non scripto est nec ad fiscum pertinet : nam poence servus est, non Caesaris) et D 48.19.17 pr (Sunt quiaam servi poenae, ut sunt in metallum dati et in opus metalli : et si quid eis testamento datum fuerit, pro non scriptis est, quasi non Caesaris servo datum, sed poence. 275- Th. MOMMSEN, Droit pénal III, p. 290 n.5. 
puisqu'il est nócessaire à Antonin 1e pieux de décider que le Fisc ne doit pes profiter des legs faits en leur faveur (276).

La perte de la liberté découle d'une condamnation à une peine capitale (277). Il peut s'agir d'une condemnation aux travaux forrós, telle la peine des mines (278). Bien que eettc peine soit un cas typique d'esclavage pénal, la condarnation à aider les mineurs, ad ministemizm metalizicomum (279) ou même ad metallum, lorsqu'elle ne se voulait que temporaire, n'entrainait pas la servitus poenae (280). Les tertes parlent de condamnés in metallim ou ad opus metalli (281), les vincula imposées au prisonnier étant moins importantes dans le second cas (282). Les condamnations in

276- Callistrate D 49.14.12 : in metallum dammatis... magisque ait pcente ens furm fisci servos esse ; Marcien $034.8 .3 \mathrm{pr}$; cf. G. BOIRVERT, Domestique et fonctionnaire, cit. p. $48, n .285$; U. ZILLETTI, In tema di servitus pcens, SOHI 34, (1968), p. 44 ; w.W. BUCKLAND, Roman 1 aw, cit. p. 278.

277- Mais toute punition capitale ne réduit pas en esclavage. Elle peut simplement se traduire par une perte de la cimitas, cf. Paul D 48.1.2 : .. Capitalia sunt, ex quibus poena mors aut cxilium est, hoc est aquae et ignis interdictio: per has enim poenas eximitur caput de civitate. Nom cetera non exilia, sed relegationes proprie dicuntur : tunc enim civitas retinetur; Ulpien D $48.19 .2 \mathrm{pr}$ : Rei capitalis damatum sic accipere debemus, ex qua causa darnato vel mons vel stion civitatis amissio vel servitus contingit.

278- Contrairement à la peine des mines, 1 'opus publicum perpetuwn (C.I. 9.47.1) n'entraîne pas la perte de la liberté, r.f. W.W. BuckLAND, Ronan 1 aw, cit. p. 403 ; de même une condamnation aux vincula perpetua, car, bien que cette punition ne soit pas inconnue, elle n'en est pas moins illégale, cf. Ulpien D 48.19.8.13 (Sed sive in perpetua vincula fuerit domnatus serrus sive in temporalia, eius remanet, cuius fuit, antequam domaretur)à propos d'un esclave, et surtout Callistrate D 48.19.35 (Mandatis principalibus,

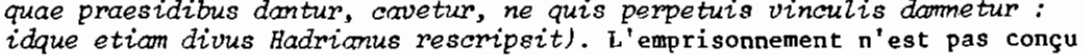

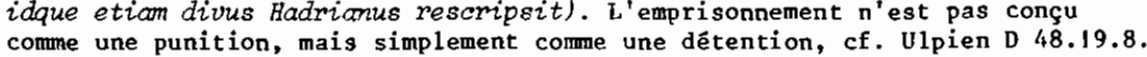
9 (Carcer enim ad continendos homines, non ad puniendos haberi debet).

279- Si l'on en croit Ulpien, c'est la peine à laquelle les femmes étaient habituellement condamnées, cf. D 48.19.8.8 : In ministerium metallicomm feminae (in perpetuum vel ad tempus) damnari solent.

280- Les enfants des femres ainsi condamnées naissaient libres, cf. Callistrate 0 48.19.28.6 (Divus Badrianus in haec verba rescripsit: 'In opus metalli ad tempus nemo domnari debet sed qui ad tempus domnatus est, etiansi faciet metallicum opus, non in metallom domatus esse intellegi debst : faciet metallicum opus, non in metallum dammatus esse intellegi debst :
huius enim libertas manet, quamdiu etian hi, qui in perpetuum opus dommantur.' Proinde et mulieres hoc modo damatae liberos pariunt).

281- La distinction terminologique apparaît par exemple chez Callistrate D 48.19.28.6 (supra $n, 280$ ).

282- Th. MOMMSEN, Droit pênal III, p. 294 ; W.W. BUCKLAND, Roman Law, cit. p. 404. Ulpien D 48.19.8.6: Inter eos autem, qui in metallum et eos, qui in opus metalli dormatur, differentia in vinculis tantum est, quod in metallum damantur, graviomibus vinculis premuntur, qui in opus metalli, levioribus, quodque refugae ex opere metalli in metallum dantur, ex metallo gravius coercentur. calcarion, in sulpurariom (dans les soufritres) (..53) ou in wa. saininamun (dans les salines) (284) étaient assimilśspar lo droit ì une sondamarion sux mines $(2 B 5)$. Les traveilleurs étaient marqués au fer rouge $\left(2 \mathrm{~B}_{0}\right)$ et on leur rasait la moitió de la chevelure (287). C'est la peine la plus rignureuse après la mort (288). Le rescrit d'Antonin le pieux visant à relâcher les travailleurs vieux ou infirmes est d'ailleurs noins inspiré par la pitié que par le manque de rentabilité de tels individus (2BG).

Les condamnés à mort sont aussi réduite nn esclavage pénal dans l'intervalle séparant. le verdict de la mort (290). Les textes parient souvent ie condamnations ad bestias (291) ou ad giadiun (292), mais la mort était

\section{3- Ulpien D 48.19.8.10.}

284- Pomponius D 49.15.6; W1pien D 48.19.8.8 (in sulinas).

285- Th. MOMMSEN, Droit pénal III, p. 293, n. 3 ; W.W. BUCKLAND, Roman $1 \mathrm{aw}$, cit. p. $404, \mathrm{n} .8$. Si la condamnation aux mines était prononcée dans un endroit où il n'y avait pas de mines, le condamné était expédié dans une région où il pourrait exécuter sa peine, cf. U1pien D 48.19.8.4: Metalla autem multa numero sunt et quadsm quidem provinciae habent, quaedom non habent : sed quae non habent, in eas provincias mittunt, quae metalla habent).

286- J. MARQuardt, Manuel Antiq. Rom. XIV, Vie privée des Romains, $p$. 216 (= Privatalterth, p. 184), cf. Suétone, Gai. 27 ; Pontius, Vita Cypriani, 7 ; C. Th. 9.40.2 (= C.I. 9.47.17).

287- J. MARQUARDT, vie privée, cit. p. 212, cf. Apul.; Metam. 9, 12 (capillum semirasi); Catul. 59,5 (semirasus ustor) ; Artemidor., Oneir., 1, 21 ; Petron. 103 ; Cyprian., Ep. 76, 2 ad Nemes. (semitonsi capitis capillus horescit). On tondait aussi les femmes, $\Lambda$ ch. Tat., 5, 17.

288- Callistrate D $48.19 .28 \mathrm{pr}$ : proxima morti poena metalli coercitio. Cette peine a vraisemblablement été introduite par imitation du régime en vigueur en Egypte où les travaux des mines étaient depuis une haute antiquité exécutés par des condamnés, cf. Diodore 3, 17 .

289- Modestin D 48.19.22 : In metallum damati si valetudine aut aetatis infirmitate inutiles oper faciundo deprehendantur, ex rescripto divi Pii a praeside dimitti poterunt, qui aestimabit de his dimittendis, si modo vel cognatos vel adfines habeant et non minus decem annis poenae suae functi fuerint.

290- Ulpien D 28.3.6.6 : Sed et si quis fuerit capite domnatus vel ad bestias vel ad gladiw... poenae servus efficitur; Gaius D 48.19.29: Qui ultimo supplicio damatur, statim et civitatem et libertatem perdunt.

291- La condamnation ad bestias est un mode de punition conmun à 1 'époque classique (Paul, Sent. 5 pass. ; Coll. 11 pass.) et plus tard (C. Th. 9.18.1 $=$ C.I. 9.20.16). Elle est encore mentionnée à l'époque de Justinien, W.W. BUCKC.AND, Roman law, cit. p. 405. Il s'agit d'une forme d'exécution dans une fête populaire, de là 1 'expression bestiis obici (cf. Ulpien D 47.9.12.1), Th. MOMMSEN, Droit pénal, III, p. 263 et n. 2. Selon cet auteur (p. 264), 1 'application de cette peine aux esclaves daterait de l'époque républicaine, car la lex Petronia, datant vraisemblablement du début de 1 'Empire (19 ap. J.C. ?) exigeait que la sentence du mâtre ait été confincée par un tribunal public, cF. Modestin D 48.8.11.2 : Post legom Eetronicm et senatus consulta 
généralement retardée de manière à extorquer aux condamnés, par la torture, certains témoignages (293). Il ne faut pas confondre ces peines arec le luchun venatorium, qui est aussi mentionné par le Digeste comme une forme d'esclarage pénal (29h), fréquemment appliquée aux jeunes gens qui encouraient une responsabilité capitale (295).

$$
\text { On a relevé dans le Digeste (296): }
$$
ad ean legen pertinentia dominis potestas ablata est ad bestias depugnandas
ouo arbitrio servos tradere : oblato tamen iudici servo, si iusta sit domini querella, sic poenae tradetur.

292- L'affectation d'un condamné à mort aux combats de gladiateurs pouvait aussi se présenter corume une forme d'exécution, cf. Sénèque, Ep. 7,3 ; C.

Th. 9.18.1:serrus vel libertate donatus bestiis primo quoque minere obiciatror, liber autem sub hac forma in ludum detur gladiatorium, ut antequam aliquid faciat, quo se defendere possit, gladio consumatur. Cette peine fut abolie en 325 par Constantin pour 1'Empire romain d'Orient (cf. C. Th. 15.12.1 = C.I. 11.44.1). Elle survécut en Occident jusqu'en 404, w.W. BUCKIAND, Rơnan 1 aw, cit. p. 405

293- W.W. BUCKLAND, Roman law, cit, p. 404 ; cf. Gaius D 48.19 .29 : Itaque praeoccupat hic casus mortem et nonmonquam longun tempus occupat : quod accidit in personis eorum, qui ad bestias domnatur. Saepe etiam ideo servam solent post donmationem, ut ex his in alio quaestio habeatur. Bien que le droit classique requière que 1 'exécution des condamnés ad gladiun ait lieu durant 1 'année de la condamnation (Pau1, Sent. 5.17.2), un laps de temps iroportant semble généralement s'écouler entre la sentence et la mort. Ainsi, Coll. 11.7 .4 nous apprend qu'après trois ans de luttes, le condamené peut obtenir la mudis, et après cinq ans, le pilleus, c'est-à-dire la liberté.

294- U1pien D 48.19.8.11 : Quicumque in lutuon venatorivo fuerint damati, videndum est, an servi poenae efficiantur : solent enim iuniores hac poena adfici. Utrum ergo servi poenae isti efficiontur an retineant libertatem, videnduan est. Et magis est, ut hi quoque servi efficiontur : hoc enim distant a ceteris, quod instituuntur venatores aut pyrricharii out aliam quon voluptatem gesticulandi vel aliter se movendi gratia; aussi D 48.19.8.12.

295- Ulpien D 48.19.8.11 (supra n. 294). C'est une peine plus légẻre, qui occupe le même degré que la peine des mines la moins grave. Th. MOMMSEN, Droit pénal, III, p. 298, cf. Pline, Ad Trai. 31; Paul, Sent. 5, 17, 2 ; C. Th. 15.12.1. HOMSEN (p. 297) pense qu'on devait éviter dans ce cas de soumettre le condamné à une lutte sérieuse. Situation distincte de celle de mettre le condamné a une lutte sérieuse. Situation distincte de celle de D 22.5.3.5; Ulpien D 3.1.1.6; Paul D 38.1 .37 pr.

296- Pomponius D 49.15.6 (in opus salinarum) ; Gaius D 28.1.8.4 (ad fermun, ad bestias, ad metallum) ; $\mathbf{0} 48.19 .29$ (ad bestias); Scaevola D 38.2. 48 (affranchi condamé in metailim); Tryphoni nus D 49.15.12.17 (constitution de Sévère et Caracalla) (in metallo); Henander D 49.16.4.1 (ad bestias)10; D 49.18.1 (ad bestias); Callistrate D $48.19 .28 \mathrm{pr}$ (summ supplicium : 10 ; D 49.18 .1 (ad bestias) ; Callistrate D 48.19 .28 pr (stomm supplicium ad furcom donnatio, vivi crematio, capitis amputatio; in metallumls metalliom)-6 (rescrit d'Hadrien) (in opus metalli, in metalliom)-10 (in
metallion)-14 (rescrit d'Hadrien) (in metallum)-15 (ad bestias) ; D 48.20 I pr ; D 49.14.12 (rescrit d'Antonin le pieux) (in metallion); D 50.13.5.3 (opus metalli/in metallum) ; Paul D 34.1.11 (in metallum) ; D 47.12.11 (in metallum) ; D 47.18 .2 (in metallion) ; D $48.19 .38 \mathrm{pr}$ (in metalliom) -2

$\begin{array}{llll}\text { I } & \overline{1} & = & - \\ \text { II } & 0 / 6 & = & - \\ \text { III } & 3 / 965 & & 0,30 x \\ \text { IV } & 1 / 354 & = & 0,28 x \\ \text { V } & 84 / 3840 & = & 28\end{array}$

Iéant

C'est a l'époque des Sépières que les juristes se sont le plus préoccupés de ce problzme (297). C'est une Évolution logique. Le processus de nivellement par le bas qui affecte les classes inferieures de la société (298) trouve son expression dans le droit pénal romain. Seul les houmes

(ad bestiisl-3 (in metallum)-5 (in metallum)-7 (in metallwn)-8 (in metallum)9 (in metalliom); D 48.23.4 (in metallim) ; Olpien D 1.6.7; D 1.12.1.10 (affranchi condaré a la matalli poena); D 28.3.6.6 (rescrit d'Badrien) (ad bestias, ad gladivon)-7-10 (ad bestias) ; D 29.1.13.2 ; D 29.2.25.3 (rescrit d'Antonin le pieux) (ad gladiwon, ad bestias, in motallion) ; 035. 1.59.2 ; D 36.1.18.6 ; D 37.4.1.9 (in mstallum) ; D 37.14.1 (affranchi condanse in matallion); D 37.14.21 pr (affranchi condame in metallum) ; D 38. 17.1 .4 ; D 38.17.2.3; D 40.5.24.6 (rescrit d'Antonin le pieux) (in mstal$l_{\mathrm{Lm}}$; : D 47.9.12.1 (beatiis obici); D 47.11.10 (in metallim); D 47.12.3. 7 (rescrit de sćvère) (poenom metali $i$ ) ; D 47.14.1.3 (Badrien) (metaili poencon, item operis vel etiom gladii); D 47.20.3.2 (opus metalii) ; D 48. 1.5 .1 (constitution de Ssvere et Caracalla) ; $\mathrm{D} 48.13 .7$ (ad bestias, poena motalli) ; 148.13 .8 .2 (édit A'Antonin le pieus) (metallo) : D 48.19.2 p D 48.19.5 pr (metallo) ; D 48.19.6.1-2; D 48.19.8.4 (in metallion, in opus metalli) -5 (epist. de cévère) (in metallum)-6 (in metalliem, in opus metalli) $\rightarrow$ (in opus metalli)-8 (in ministerivm mstallicorrm) (in salinas)-

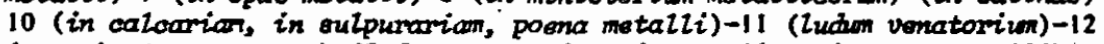
(rescrit de Caracalla) (ludum venatorium, in motallum, in opus metalli) ; D 48.19.9.11 (in metallwm, in opus metalli); D 48.20.5 pr ; D 48.23.1.1 (in metalliun) ; D 50.4.3.2 (in metallum); Hacer D 48.19.10.1 (in metal$Z_{2 \mathrm{~m})}$; D 48.19 .12 (ad bustias) ; Harcien D $34.8 .3 \mathrm{pr}$ (rescrit d'Antonin le pieux) (ir mstallion) ; D 34.3.3.1 (in metallsen) ; D 40.1.8 pr ; D 48.8.3.5 (bestiis oubioi) ; D 48.17.1.1 (in opus metalli) ; D 48.19.11.3 (bestiis obici) : D 48.19.17 pr (in metallum, in cpus mstaili); $\mathrm{D}^{\circ} 49.18 .3$ (ad bestias, obici) ; D 48.19.17 pr (in metallum, in cpus metalli) ; D 49.18 .3 (ad
in matalizen); Hodestio (constitution d'Radrien) D $48.9 .9 \mathrm{pr}$ (bestiio in mataltion) ; Hodestiv (constitution d'Radrien) D $48.9 .9 \mathrm{pr}$ (bestiis
obicitur) ; D 48.19 .23 (in metallum) ; D 48.19 .22 (rescrit d'Antonin le pleux) (in metallion) ; D 48.19.31 pr (ad bestios) ; D 49.16.3.1 (in motalLron, in opus metalii) -10 (ad bestias) ; Herwogênien D 37.4 .2 ; D 37.14. $21 \mathrm{pr}$ (in metallum); 048.15 .7 (in metallum).

297- Ce qui ne signtfie nullement que l'insticution ait été négligée durant les précédentes periodes de l'Empire. Du reste, le Digeste, en matiere de contitutions inperiales, fait apparaitre onze fois les Antonins (Eadrien chez Callistrate D 48.19.28.6 ; D 48.19.28.14; chez Ulpien D 28.3.6.6 ; D 47.14.1.3; D 48.13.8.2; chez Modestin D 48.9.9 pr ; Antonin le pieux chez Callistrate D 49.14.12 ; chez Olpien D 29.2.25.3 ; D 40.5.24.6 ; chez Marcien D 34.8.3 pr ; chez Modestin D 48.19.22) contre cinq fois ceulewent les Sévères (Sévére chez Ulpien D 47.12.3.7; D 48.19.8.5; Sévere et Caracalla chez Tryphoninus D 49.15.12.17; chez Ulpien D 48.1.5.1; Caracalla chez Ulpien D 48,19.8.12).

298- E.M. SCHTAERMAN, La schiavitù, cit. p. $238,250$. 
libres de basse condition sont passibles de cette peine. Décurions (299), soldats et vótérans (300), et plus généralement ceux que le drcit regroupe sous le rocable d'honestiores (301) y Echappent.

299- Selon Hadrien, la peine de mort ne s'appliquait paa a un décurion, hormis pour le parricide (D 48.19.15 Venuleius : Divus Hadrianus eos, qui in numero decurionum essent, capite puniri prohibuit, nisi si qui parentem occifissent) abstraction faite du crime de lèse-majesté, ajoute MOMASEN, Droit pénal III, p. 395. Quoi qu'il en soit, il fallait auparavant mol en reférer a 1 empereur ( $\mathrm{cF}$. Modestin $\mathrm{D} 48.8 .16$ :... Facilius hoc in decuriones fieri potest, sic tomen, ut consulto prius principe et iubente id fiat). Les décurions ne pouvaient être condamnés à des peines dégradantes (metallum, opus metalli, ministerizm metalli, furca, ad bestias...), cf. par ex. Ulpien D 48.19.9.11 (decuriones in metallum damari non possunt nec in opus metaliti, nec furcas subici vel vivi exuri...). Leurs ascendants et leurs enfants étaient pareillement protégés, cf. Ulpien D 48.19.9.12 (Parentes quoque et liberi decurionum in eadem causa sunt) et D 48.19.9.13 (Liberos non tantzom filios accipere debemus, verum ommes liberos). La protection couvrait même les enfants nés avant le dêbut du décurionat, et aussi apręs $s_{a}$ fin pourvu qu'ils aient été conçus avant, cf. Ulpien D 48.19.9.14 (Sed utrom hi soli, qui post decurionatum suscepti sunt, his poenis non (Sed utrion hi soli, qui post decumonatum suscepti sunt,

300- Soldats et vétếrans n'êtaient pas non plus punissables in metallum, in opus metalli ou ad bestias, cf. Modestin D 49.16.3.1 (nom in metallum aut in opus metalli non dabuntur...)-10 (Is, qui ad hostem conflugit et rediit, torquebitur ad bestiasque vel in furcam damnabitur, quamvis milite rediit, torquebitur ad bestiasque vel in furcam dannabitur, quanvis milite nihil eomum patiantur), Menander D 49.18.1 (Veteranorum privilegium inter cetera etian in delictis habet praerogativam, ut separentur a ceteris in poenis. Nec ad bestias itaque veter(mus datur nec fustibus caeditur) et Marcien $D$ 49.18.3, qui protège aussí les enfants des vêtérans (Veteronit.s et liberis veteronorum idem honor, habetur, qui et decurionibus : igitur nec in metallion dormabuntur nec in opus publicum vel ad bestias, nec fustibus eaedintur). Rien n'est dit sur les parentes, cf. W.W. BUCKLAND, Roman law, cit. p. 406 .

301- Th. MOMMSEN, Droit pénal III, p. 395 observe que bien que ces protections ne soient expressément établies que pour les décurions, ceux-ci constituant la catégorie la plus basse des privilégiés, elles concernent à plus forte raison les catégories les plus élevées. W.W. BOCKLAND, Roman law, cit. p. $406 \mathrm{n}$. 5 donne des cas de personnes qui peuvent difficilement devenir servi poenae. G. CARDASCIA, L'apparition dans le droit des classes d'honestiores et d'humiliores, in RHD (1950), p. $305 \mathrm{~s}$., établit une comparaison entre les peines de droit combun, dont est passible l'hromilior et les peines privilégiées, dont est passible l'honestior (p. 322). L'auteur souligne la privilégiées, dont est passible l'honestior ( $\mathrm{p}$. 322). L'auteur souligne la gamme plus étendue des peines concernant les hromiliores, ce qui ne correspond nullement a la probabilitê d'un taux de criminalité plus êlevé che ceux-ci. Lorsque 1'hromilior est passible de suma supplicia, l'honestior est puni caput (D 47.9.12.1 U1pien : bestiis obici/... copite puniuntur, ... in insulan deportantur) ou de deportatio (D 47.9.12.1 ; Paul D 47. 12.11 (sumo supplicio/in insulan deportantur) ; D 48.8.3.5 Marcien (bestiis subici/deportontur in insulam) ; D 48.19.38.2 Paul (in furcan tollvontur $\ldots$ bestiis obiciuntur/in insulam deportantur)). Lorsque 1 humilior risque ia beine des mines, 1 'honestior risque seulement la relegatio (D 47.12 .II 1a peine des mines, 1'honestior risque seulement la relegatio (D 47.12 . domnatur/in insulam relegantur aut in exilizon mittuntur); D 48.19.38.5
Il ne s'agit donc pas d'une reunise en cause de la snciét,á. Il n'est. pas question d'évaluer un home libre en argent. Tcuterois, au-delà des justifications de principe, le droit consacre ln réduction en esclavage de certains citoyens. Et c'est là une véritable sourse d'esclavage. Un rescrit d'Antonin le pieux rappelle en effet que les enfants conçus et nés de remmes condamnées in metallwm naissent esclaves (302).

D'un point de vise global, ne peut-on pas rapprocher cette situation de celles de l'homme libre retenu de mauvaise roi, du libor homo hona fide serviens, et même de celui qui se vend ad pretium partinifandum ? Toutes ces situations peuvent en effet présenter un point commun, la faiblesse éconnmique des individus qu'elles évoquent. Dans le cas de l'homme libre retenu de malvaise foi, parce qu'on imagine mal un membre d'une famille fortunée, ne serzit-re qu'aisée, être retenu de force comme esclave, et encore moins ignorer son propre état. Le liher homo bona fide serviens est le signe d'un statut douteux qui prouve que, dans la domas, homme libre et esclave se rapprochent. Il est vrai qu' on ne sait rjen sur ce qu'aurait été la situation de ce liber homo s'il ne s'était pas trnuvé dans une situation de fait servile, mais la confusion serait-elle possible dans une hypothèse autre que celle $d^{\prime}$ un individu démuni ? Ces cas, on l'a'vu, ne sont constitutifs que d'un esclavage de fait.

Quant à ceux dans lesquels le droit intervient, directement, la fraude de celui qui fejnt d'être esclave pour profiter du prix de sa vente n'implique pas nécessairement un état éccnomiquement décadent. Cependant, ce moyen

Paul (in metallum/in insulan... relegontur); $\mathrm{D} 48.19 .38 .8 \mathrm{Paul}$ (in metallum domatur/in perpetuun relegatur) : D 48.19.38.9 (in metailum damatur/ in insulan deportatur, mais Paul, Sent. 5, 25, 10 donne relegatur); aussi D 48.19 .38 pr Paul (poena metallilexilii punitur) ; D 47.17.1 Ulpien (metalli poena/relegationis); D 48.13.8.2 U1pien (metallo/exilio) ). U. BRASIELLO, La repressione penale in diritto romano, Naples (1937), p. 293. pense que la relegatio correspond au coput ; contra $G$ CARDASCIA, $0, c$, P. $323, \pi .4$. Aingi, gi le principe républicain est l' '́calité juridique . J23. des citoyens (Th. MaMSEN, Droit pênal III, P. 391), l'inégalité pénale des classes est la regle sous 1 Eupire (G. CARDASCIA, o.c., p. 307). Cel dit, les peines qui frappent les esclaves sont évidemment plus dures que celles qu'encourent les humiliores, cf. Paul D 47.9.4.1 : Divus Antoninus de his, qui praedam ex naufragio diripuissent, ita rescripsit : ... Ideoque si gravior praeda vi adeptita videbitur, liberos quidem fustibus caesos in triennizm relegabis aut, si sordidiores erunt, in opus publicum eiusdem temporis dabis : servos flagellis caesos in metalliom damabis.

302- Ulpien D 40.5.24.6 : Ex damnata in metallun concepto et nato fideicommissaria libertas dari poteri : quid mimom, cwm etian venumdari evm posse quasi servom divus Pius rescripsit 
de se procurer de $I^{\prime}$ argent n'était-il pas particulièrement tentant pour les pauvres? Enfin, seuls les non-honestiores sont susceptibles de devenir esclaves de la peine. Il est vrai que cet ensemble, composé de tous ceux qui ne font pas partie de la noblesse locale, ne comprend pas seulement des paurres, mais le droit n'écarte-t-il pas clairement de ce danger les citoyens les plus riches?

$$
-0^{\circ}
$$

Reprenons ces différents modes de reproduction en ne retenant que ceux que le droit a juridiquement consacrés : naissance d'une part, vente de soi-même et esclavage pénal de l'autre. Il est intéressant d'essayer de voir quel a été le rôle joué par chacune de ces sources dens l'alimentation du réservoir servile. Pour cela, il ne convient plus de se référer au nombre total de textes concernant l'esclavage, mais seulement au total, pour chaque période, de ceux qui traitent.d'un des modes de reproduction envisagés.

\section{analyse des paragraphes}

\section{- naissance}

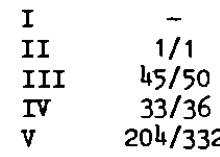

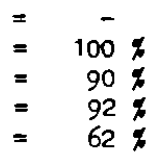

$00 \%$
$90 \%$
$92 \%$
$62 \%$

analyse des citations

\section{- vente de soi-même}

\begin{tabular}{|c|c|c|c|c|c|c|c|}
\hline I & - & $=$ & - & I & $1 / 11$ & $=$ & $9 \%$ \\
\hline II & $0 / 1$ & $=$ & - & II & $1 / 19$ & $=$ & $5 \%$ \\
\hline III & $2 / 50$ & $=$ & $4 x$ & III & $0 / 24$ & $=$ & - \\
\hline IV & $2 / 36$ & $=$ & $5 \%$ & IV & $0 / 7$ & $=$ & - \\
\hline v & $44 / 332$ & $=$ & $13 \%$ & $\mathrm{v}$ & $2 / 3$ & $=$ & $67 \%$ \\
\hline \multicolumn{8}{|c|}{ - esclavage pénal } \\
\hline I & - & $=$ & - & I & $0 / 11$ & $=$ & - \\
\hline II & $0 / 1$ & $=$ & - & II & $0 / 19$ & $=$ & - \\
\hline III & $3 / 50$ & $=$ & $6 \%$ & III & $0 / 24$ & $=$ & - \\
\hline IV & $1 / 36$ & $=$ & $3 \%$ & IV & $0 / 7$ & $=$ & - \\
\hline $\mathrm{v}$ & $84 / 332$ & $=$ & $25 \%$ & $\mathrm{v}$ & $0 / 3$ & $=$ & - \\
\hline
\end{tabular}

(+) 61,44 relevé à 62 pour arrondir à $100 \%$ le total de la période.
C'est la naissance à l'intérieur du groupe servile qui ressort le plus souvent des textes. Cependant, elle accuse ume légère baisse, proportionnellement aux autres modes de reproduction, à l'époque des Sévères. Bien qu'elle reste la source principale d'asservissement, la prépondérance de $l a$ vente de soi-même durant la période $V$ ne reposant que sur un nombre faible, donc peu fiable, de citations, les juristes du 3ème siècle se sont plus préoccupés que leurs prédécesseurs de la vente de soi-même et de l'esclavere pénal. Ces situations ont, il est vrai, probablement posé des problèmes plus délicats car elles s'attaquaient aux hommes libres, même si ceur-ci n'étaient que de condition modeste. Mais le fait qu'elles prennent de l'importance dans les textes juridiques du 3ème siècle ne peut seulement s'expliquer par une inaptitude de la reproduction familiale à maintenir l'effectif servile à un niveau satisfaisant pour la société. D'ailleurs, rien dans le Digeste ne dénonce clairement une telle insuffisance (303). Ce processus doit être plus globalement relié au nivellement des classes par le bas qui affecte alors la société romeine.

$$
-0^{-}
$$

Telles sont les considérations qu'inspire l'étude de l'acquisition de la main-d'oeuvre servile à travers le Digeste. Il est temps désormais d'examiner le rôle qui lui est dévolu.

303- V. T. ROVAL, "Sur quelques questions litigieuses dans 1 'article de S.L. OUTCHENRO et E.M. SCHTAERMAN", in V.D.I (1961) 3, P. 94-100, conteste que 1 'accroissement naturel du nombre des esclaves ait pu jouer un rôle important dans le processus de renouvellement de la main-d'oeuvre servile. Selon 1'auteur, pour des raisons économiques (1a faible productivité du travail servile rendait nécessaires la réduction des frals d'entretien et d'éducation des esclaves, ainsi que leur exploitation achamée), le groupe servile ne pouvait se renouveler que par des sources externes, c'est-à-dire par l'asservissement d'hommes auparavant libres, cf. M. RASROLNIROFF, L'apport de la recherche soviétique (1917-1965) a l'histoire des classes sociales dans le monde hellénistique et romain, II, Strasbourg (1972), P. 352. Néanmoins, et bien que les textes juridiques accusent une baisse d'intérêt au jème siècle, il faut voir dans la reproduction naturelle des esclaves la source principale de 1 'esclavage sous 1 'Empire. Les proportions qu'on a dégagées le montrent. Dans ce sens, E.M. SCHTAERMAN, La schiavi tù, cit. P. 17 ; M. I. FINLEY, L'économie antique, cit. P. 113 ; contra A.R. KORSOUNSKI, Le problème du passage révolutionnaire du régime esclavagiste au régime féodal, VDI (1964) 5, p. $97 \mathrm{n} .6$. 


\section{TITRE II}

\section{L'ESCLAVE TRAVAILLEUR}

Les egclaves ne forment pas un groupe homogène. Qu'y-a-t-il en effet de commun entre l'activité de l'ourrier et, celle du pédagogue, entre le producteur et le domegtique (304) ? Cette diversité engendre une grande variété des conditions de vie. Ainsi trouve-t-on des esclaves exploités très durement alors que d'autres sont à la tête de biens matériels.

\section{CBAPITRE I : LA NATURE DU TRAVAIL SERVILE:DE LA DEFERDANCE A L'AUTONOMIE RELATIVE}

Cotte diversité entrấne aussi un contact plus ou moins grand avec le monde juridique. Sur une même exploitation agricole, le droit est pour le mediastinus, le manoeurre, ce qui assure la soumission. Pour le vilicus, l'intendant ou pour le servus conductor, véritable colon, il est aussi ce qui va réglementer son statut d'agriculteur (305). C'est à propos d'un tel esclave qu'apparâ̂t une contradiction entre le défaut d'accès aux divers actes de la vie juridique et la nécessité de pouvoir valablement et

304- Ceux qui ne produisent pas et domestiques de niveau plus ou moins élevé constituent une frange dêtachée en fait de la classe des esclaves, spécialement considérée en ses éléments ruraux. Ils appartiennent a 1 'ordre, et non a la classe des esclaves, cf. E.M. SCHTAERMAN, Die Blütezeit der Sklavenwirts chaft in der römischen Republik, êd. all. Wiesbaden (1969), p. 168 ; G. BOULVERT, Domestique et fonctionnaire, cit. p. 331 ; contra M. BARTOSER, Le classi sociali nella Roma antica, in Et. J. MACQUERON, Aixen-Provence (1970) p. 78, 196. A. CARANDINI, L'ana tomia della scimia, La formazione economica della societa prima del capitale, Turin (1979), p. 158 s., parle d'une "classe animale", qu'il situe, non pas sur le plan social, mais sur le plan politique.

305- M. JACOTA, Les transformations de 1 'économie romaine pendant les premiers siècles de notre ère et la condition de l'esclave agriculteur. in Et. J. Macqueron, Aix-en-Provence $(1970)$, p. $376 \mathrm{~s}$. par sa seule activité modifier l'équilibre économique existant et stabilisé par le droit (306). D'où la nécessité de lui reconnaitre une certaine capacité.

On fera apparaître la gradation des activités serviles à travers une analyse des professions que nous livre le Digeste, puis par l'examen des differentes actions auxquelles ces astivités donnent lieu.

\section{SECTIOR 1. UNE HIERARCHIE DE LA DEPENDANCE : ANALYSE DES ACTTVITES PROFESSIONAELLES SERVILES}

Le critère apparement le plus simple à utiliser était celui distinguant fomilia urbona et familia matica (307). Il n'a cependant pas été possible de l'employer de manière égale car il ne permet pas un codage systématique des textes. La distinction entre fourlila rustica et urbana $n$ 'est en effet pas spécifique. Bien qu'apparaissant assez souvent, dans le Digeste (308), elle n'en est pas pour autant claire. Paul écrit que l'esclave urbain se distingue de l'esclave rural non par le lieu de résidence, mais surtout par l'usage qu'en fait le maitre (309). On lui

306- Sur ce problème d'intêressement de l'esclave à son travail, contradiction du sygtème esclavagiste, $M$. JACOTA, Les pactes de 1'esclave en son now propre, in RIDA 13 (1966) p. 206.

307- Ce n'est que lorsque la plupart des Romains abandonnèrent la campagne et trouvẽrent plus commode de vivre tour à tour en ville et dans leurs terres, ou même d'habiter constamment la ville (Colum. I, 13-20 ; XII, 9 ; Val. Max. VII, 5,2) que la fomilia se scinda en deux groupes, cf. J. MARQUAR DT, Vie privée, cit. p. 161.

308- Par exemple chez Pomponius D 50.16.166 pr ; Papinien D $31.65 \mathrm{pr}$; Scaevola D 32.41 .2 (mancipiis musticis vel urbanis); Paul D 32.99.2. D 33.7.18.13 (Scaevola) (manoipiis urbanis et musticis).

309- Paul D 32.99 pr (Servis urbanis legatis quidom urbana mancipia non Loco, sed opere separant, ut, licet in praediis rusticis sint, tamen si opus rusticum non faciant, urbani videntur. Dicendum autem est, quod ur bani intellegendi sunt, quos pater familias inter urbonos adnumerare solitus sit : quod maxime ex libellis fomilice, item cibariis deprehendi. poterit) i aussi, D 33.10.12 tabéon lib 4 pithanon a Paulo epitoutor potert sit a quod matirner (Quemad ita urbara penere usus, ita urbana perus et supellex ad usw usbann, non ad locum urbarm aut peregrinion dirigenda est, multumque interest, penus et supellex ea quae in urbe sit an urbana legetur vel promittatur). 
sert même une ncurriture différente (310), ce qui ne manque pas d'intérêt au niveau de la discrimination opérée entre les deux groupes. Il ajoute ailleurs que les muliones sont des esclaves urbains à moins que le testateur ne les ait destinés à un opus rurestre (311). En d'autres termes, si des esclaves urbains sont, exployés à des fonctions rurales, ils deviennent ruraux. Ils peuvent toutefois servir extra urbem et conserver leur caractère initial s'ils contimuent à remplir des fonctions urbrines ( 312 ). Scaevola évoque le cas de piscatores, pêcheurs, portés sur les registres urbains -urbicis rationibus- du testateur (313). Tryphoninus parle d'esclaves ruraux utilisés in ministerium urbarum (314).

Il fallait donc faire primer la fonction sur le lieu de travail. Parmi les fonctions serviles apparaissent des fonctions d' exécution mais aussi des fonctions de direction et de prestige. C'est dire que la dépendance même n'échappe pas à une hiérarchisation dont il convient de suivre la progression (315).

310- Paul D 32.99 pr et D 33.10.12 (Labéon) (supra n. 309) ; Paul D 32.99 .1 (Venatores et aucupes utrun in urbanis on in rusticis contineantur, potest dubitori : sed dicendron est, ubi pater familias moraretur et hos alebat, ibi eos numerari).

311- Paul D 32.99.2 (Ihuliones de urbano ministerio sunt, nisi proptor opus rurestre testator eos destinatos habebat). Alfenus donne une definition plus restrictive de la familia urbana, D 32.60 .1 lib. 2 digest. a Paulo epitomatorum (Servis et ancillis urbaris legatis agasonem mulionem legato non contineri respondi : eos enim solos in eo numero habe$\mathrm{mi}$, quos pater familias circum se ipse oui cultus causa haberet).

312- Labéon chez U1pien D 33.9.4.5 (sicuti urbica ministeria dicimus, et quae extra urbem nobis ministrare consueverunt).

313- Scaevola D 33.7.27 pr (Praedia maritima cum oervis qui ibi ernont et omoi instrumento et fructibus qui ibi exront et reliquis colonorw nutritori suo legavit. Quaesitum est, an servi piscatores, qui solebant in ministerio testatoris esse et ubicumque eum sequi et urbicis rationibus expungebantur nec mortis testatoris tempore in praediis legatis deprehensi fuerant, legati esse videantur...).

314- Scaevola D 34.1.15.1 (Testator concubinae mancipia rustica numero octo legavit... Claudius : ... vernon oi velut in ministerium urbonum $a b$ his transferentur...). Columelle $(1,12)$ signale aussi qu'on passait aisément d'une catégorie à 1 'autre.

315- P. DOCKES, La Liberation, cit. p. 80-89; J. KOLENDO, Lavoro servile, cit. p. $27 \mathrm{~s}$

\section{Paragraphe 1: Fonctions d'exécution.}

Il est ici possible d'opposer les fonctions murales aux fonctions urbaines.

\section{I - Fonctions d'exécution murales.}

Tantôt l'activité est indiquée de manière explicite, tantôt le texte ne fait que signaler la présence de l'esclave sur un fonds sans en donner la fonction.

\section{A/- Indicateur explicite.}

\begin{tabular}{|c|c|c|c|}
\hline $\begin{array}{l}\text { Juriste auteur } \\
\text { du passage }\end{array}$ & Juriste cité & Fonction & Source \\
\hline $\begin{array}{l}\text { Alfenus (chez } \\
\text { auteur anonyme) }\end{array}$ & & $\begin{array}{l}\text { fundum colendum } \\
\text { agrorum eolendum/operarii } \\
\text { rustici }\end{array}$ & $\begin{array}{l}\text { D } 15.3 .16 \\
\text { D } 50.16 .203\end{array}$ \\
\hline Iavolenus & $\begin{array}{l}\text { Trebatius, } \\
\text { Labeon - } \\
\text { Ofilius }\end{array}$ & $\begin{array}{c}\text { pastores } \\
\text { oviliones/pastores }\end{array}$ & $\begin{array}{l}\text { D } 33.7 .25 .1 \\
\text { D } 33.7 .25 .2\end{array}$ \\
\hline Scaevola & . & $\begin{array}{c}\text { servis culturae necessariis } \\
\text { coluit } \\
\text { piscatores }\end{array}$ & $\begin{array}{l}\text { D } 20.1 .32 \\
\text { D } 33.7 .20 .1 \\
\text { D } 33.7 .27 \mathrm{pr} \\
\end{array}$ \\
\hline Paul & Alfenus & $\begin{array}{c}\text { fundum coluerat } \\
\text { topiarius/opilionem } \\
\text { phasianarii/pastores } \\
\text { venatores/aucupes } \\
\text { rusticis operariis } \\
\text { bubulco } \\
\text { putatoribus } \\
\text { pastores/fossores } \\
\text { fundi colendi }\end{array}$ & $\begin{array}{l}\text { D } 18.1 .40 .5 \\
\text { D } 32.60 .3 \\
\text { D } 32.56 \\
\text { D } 32.99 .1 \\
\text { D } 33.7 .18 .5 \\
\text { D } 33.7 .18 .6 \\
\text { D } 33.7 .18 .7 \\
\text { D } 33.7 .18 .8 \\
\text { D } 33.7 .19 \mathrm{pr}\end{array}$ \\
\hline Ulpien & $\begin{array}{l}\text { Labéon } \\
\text { Sabinus } \\
\text { Servius } \\
\text { Papinien }\end{array}$ & $\begin{array}{c}\text { agri colendi } \\
\text { curate agros } \\
\text { qui agrum colunt } \\
\text { topiarii/pastores } \\
\text { cultorum } \\
\text { venatores/vestigatores } \\
\text { aucupes } \\
\text { topiarii/squarii }\end{array}$ & $\begin{array}{l}\text { D } 7.8 .10 .4 \\
\text { D } 32.68 .1 \\
\text { D. } 33.7 .8 \mathrm{pr} \\
\text { D } 33 \cdot 7.8 .1 \\
\text { D } 33.7 .12 \mathrm{pr} \\
\text { D } 33.7 .12 .12 \\
\text { D } 33.7 .12 .13 \\
\text { D } 33.7 .12 .42\end{array}$ \\
\hline Marcien & Labéon & $\begin{array}{c}\text { pescatoribus (praepositi) } \\
\text { topiarii }\end{array}$ & $\begin{array}{l}\text { D } 32.65 \mathrm{pr} \\
\text { D } 33.7 .17 .2\end{array}$ \\
\hline
\end{tabular}


Ces diverses tâches donnent un aperçu fragmentaire des fonctions d'exécution rurales (316). Elles fournissent néanmoins une idé de la diviaion du travail à la campagne. Parmi les esclaves passans, on relève le bubulcus, bouvier (317) et ceux qui cultivent la terre, désigoés dans les textes par fundum colendum ou servis culturae necessariis et autres appellations. Le terme operarii peut indiquer plusieurs fonctions (318). Les fossores, qui bêchent (319) et les putatores, qui emondent (320), travaillent dans les vignobles. On trouve ensuite les bergers, pastores (321) et opilionss (322). Les topiarii s'occupent des jardins (323), ainsi que les aquarii, préposés aux eaux (324), les phasianarii, faisandiers et les oucupes, oiseleurs (325), de la volaille. La fonilia rustica peut enfin comprendre des venatores, chasseurs (326), et des piscatores, pêcheurs (327).

Cependant, en pratique, un même esclave participait sans doute à diver ses activités. Entretenir un esclave qui ne connût qu'un seul de ces métiers n'était sûrement pas rentable (328). Le mediastinus, sorte d'home à tout faire est une illustration du problème (329).

316- Pour avoir une idée plus complète de ces fonctions, J. MARQUARDT, vie privée, cit., p. $163 \mathrm{~s}$;; F.M. DE ROBERTIS, L'organizzazione e la tecnica produttiva. La forza di lavoro e i salari nel mondo romano, Naples (1946), p. 28, donne, en sus de celles relevées dans le Digeste, les fonctions suivantes : asinarii, domitores, occatores, sarrinatores, runcatores, messores, foenisecae, leguli, factores, torcularii, capulatores, salictarii, vinitores, pastinatores, alligatores, pampinatores, vindematores, collectores, armentapastinatores, alligatores, pampinatores, vindemiatores, collectores, armenta-
rii, coprorii, suarii, equitiarii, hortulani, arboratores, alitores, apiarii, rii, caprarii, suarii, equitiarii, hortulani, arboratores, alitores, ap

317- L'existence de ces travailleurs est aussi attestée par les sources littéraires, cf. Cato 5,6 etc.

318- J. MARQUARDT, Vie privée, cit., p. 164.

319- Colum. III, 13,3; III, 15,2.

320- Colum. IV, 24,11.

321 - Varron (VII,6,9) leur oppose le magister pecoris, préposé général au petit bétail. Les pastores anserum de $D 32.66$ sont des gardeurs d'oies.

322- Varron II pr,4; Colura. VII,3,13.

323- Gicéron, Ad Q.fr., III,1,2\&5; sources épigraphiques ( $\mathrm{CIL}, \mathrm{VI}$,

4360 s., 4423, 6369 s., $7300,8639,8738,9943$ s.).

324- CIL, VI, 3935, 3936, $9131,9145$.

325- Varron, III, 3,4.

326- Varron (1.c.) ; une fomilia venatoria est mentionnée par CIL, V, 2541.

327- Varron, III, 17,6.

328- E.M. SCHTAERMAN, La schiavitù, cit., p. 36.

329- Colum. I,9,3; II,12,7. I1 y avait aussi des mediastini dans 1a familia urbana (Hor., Ep., $\mathbf{i}, 14,14$ ). Le nor désigne aussi les esclaves des médecins (Plin., N.H., XXIX,4). Dans D $7.7: 6 \mathrm{pr}$ et $\mathrm{D} 47.10 .15 .44$, rien ne dit's'il est rural ou urbain. Par contre, dans $D 4.9 .1 .5$, le contexte est urbain.
Bien que la chose ne ressorte pas elairement du Digeste, on sait que certains esclaves étaient contraints au port des chaínes (330). C'Était notamment le cas dans la viticulture (331), mais les chaines ne pouvaient être employees pour les bouviers, les bergers ou les esclaves travaillant dans la sphère de la villa rustica, ceux-ci devant être libres de leurs mouvements (332). On connaît donc, à l'intérieur même des fonctions d'exécution, une hiérarchie. Cet usage, très répandu en Italie à l'époque rópublicaine et au ler siècle de l'Empire (333), semble avoir subi un recul au début du 2ème siècle (334). Toutefois, les juristes du 2ème siècle et du début du 3ème parlent encore d' esclaves enchaînés comme d'un fait absolument normal, sans dire il est vrai à quels travaux ils sont employés (335).

Si les textes examinés jusqu'à présent fournissent des indications précises quant aux activités serviles rurales, d'autres nous renseignent seulement sur la présence d'esclaves à la campagne.

B/- Indicateur implicite.

\begin{tabular}{|l|l|c|c|}
\hline $\begin{array}{c}\text { Juriste auteur } \\
\text { du passage }\end{array}$ & Juriste cité & Passage & Source \\
\hline Iavolenus & Labéon & fundum... familia & D 18.1.78.1 \\
\hline Julien & & mancipia, quae in fundo & D 30.84.10 \\
\hline Gaius & & $\begin{array}{c}\text { fundum.... nata } \\
\text { mancipia quae in fundo sint }\end{array}$ & $\begin{array}{l}\text { D 20.4.11.2 } \\
\text { D 21.1.32 }\end{array}$ \\
\hline
\end{tabular}

330- Ainsi, Colum. $I, 7,1$. Des chaînes spéciales étaient prévues pour prévenir les tentatives de fuite (B. SAGLIo, Compes, DSDA I, P. 1428 ; H. THEDENAT, Ergastulum, DSDA II, p. 810 s.; A. MAU, Ergastulum, RE VI, col.431; R.R. BARROW, Slavery, cit., p. 83 ; W.L. WESTERMANN, Slave Systems, cit., p. 76 s. ; J. CESKA, Diferenciace, cit., p. 52-55).

331- Colum. I,9,4 s. M. WEBER, Die römische Agrargeschichte in ihrer Bedeutung für das Staat -und Privatrecht, Stuttgart (1891), p. $238 \mathrm{~s}$.

332- J. ROLENDO, Lavoro servile, cit., p. 30.

333- Cat. 56 ; Plaut., Mostel., 19 ; Tibul1., I,7,42 ; Ovid., Trist., IV, 1,5 ; Sen. Rhet., Gontrov., I, 26 ; v, 5 ; Sen., De ben., viI, 10,5 ; Luc.. Phars., vII,402 s. ; Iuv., Sat., XI,80; Mart., II, 2,4 ; Colum., I,6,3 ; I,7,1; I, 8,16 s. etc. ; App., B.C., IV, 130,180,195 ; F1or., II, 18 ; Diod., XXXIV, 2,36.

334- Plin., Epist., III,19,6. La date de la lettre de Pline est incertaine, cf. J. KOLENDO, Lavoro servile, cit., p. 51 n. 192 ; A.N. SHERWIN-WHITE, The Letters of Pliny, A historical and social commentary, oxford (1966), p. 253 ; selon v. SIRAGo, L'Italia agraria sotto Traiano, Louvain (1958), p.

115 n. 1 , il s'agirait de 110.

335- U1pien D 4.3.7.7 (Labéon, Scaevola); D 47.10.15.44. 


\begin{tabular}{|c|c|c|c|}
\hline $\begin{array}{l}\text { Juriste auteur } \\
\text { du passage }\end{array}$ & Juriste cité & Passage & Source \\
\hline $\begin{array}{c}\text { Scaevola } \\
!\end{array}$ & & $\begin{array}{c}\text { praedia praelegavit...cum servis } \\
\text { praedia legavit cum servis } \\
\text { mancipia...praedia tradidi } \\
\text { mancipiis rusticis } \\
\text { operis rustici } \\
\text { praedia... servis } \\
\text { mancipia... in eo fundo fuerint } \\
\text { homines in fundos legatos } \\
\text { servus... ex eo fundo } \\
\text { mancipiis... instrumento rustico } \\
\text { rusticis officis } \\
\text { fundum,...cum instrumento...et } \\
\text { familia } \\
\text { mancipiis, quae fundi causa erant } \\
\text { qui (pour esclave) coloni }\end{array}$ & $\begin{array}{l}\text { D } 15.1 .54 \\
\text { D } 15.1 .58 \\
\text { D } 32.37 .3 \\
\text { D } 32.41 .2 \\
\text { D } 32.93 .2 \\
\text { D } 32.101 \mathrm{pr} \\
\text { D } 32.101 .1 \\
\text { D } 33.7 .20 .5 \\
\text { D } 33.7 .20 .6 \\
\text { D } 33.7 .20 .9 \\
\text { D } 33.7 .27 .1 \\
\text { D } 33.7 .27 .3 \\
\text { D } 33.7 .27 .4 \\
\text { D } 33.8 .23 .3\end{array}$ \\
\hline Papinien & & $\begin{array}{l}\text { fundur instructum...partuum } \\
\text { servos praediorum } \\
\text { mancipiis hortorum } \\
\text { preedia cum servis }\end{array}$ & $\begin{array}{l}\text { D } 33 \cdot 7 \cdot 3 \mathrm{pr} \\
\text { D } 33.7 .3 .1 \\
\text { D } 34.4 .24 .1 \\
\text { D } 41.2 .48\end{array}$ \\
\hline Tryphoninus & Scaevola & $\begin{array}{l}\text { mancipia rustica } \\
\text { operis... in agro }\end{array}$ & $\begin{array}{l}\text { D } 34.1 .15 .1 \\
\text { D } 41.1 .63 .3\end{array}$ \\
\hline Paul & $\begin{array}{l}\text { Alfenus } \\
\text { Scaevola } \\
\text { Cassius } \\
\text { Labéon } \\
\end{array}$ & $\begin{array}{c}\text { servus coloni } \\
\text { servim... ex eo fundo } \\
\text { fundo...cum suis inhabitantibus, } \\
\text { id est, familia } \\
\text { operis rustici } \\
\text { mancipia...in (fundo) fuerunt } \\
\text { fundorum. . mancipiis } \\
\text { opus rusticum } \\
\text { mancipiis rusticis } \\
\text { mancipiorum (in fundo) } \\
\text { mancipia... instructi fundi } \\
\text { servus rusticus }\end{array}$ & $\begin{array}{l}\text { D } 19.2 .30 .4 \\
\text { D } 32.78 \mathrm{pr} \\
\text { D } 32.78 .1 \\
\text { D } 32.78 .2 \\
\text { D } 32.78 .3 \\
\text { D } 32.92 \mathrm{pr} \\
\text { D } 32.99 \mathrm{pr} \\
\text { D } 33.7 .18 .3 \\
\text { D } 33.7 .18 .9 \\
\text { D } 33.7 .18 .11 \\
\text { D } 33.10 .2\end{array}$ \\
\hline Ulpien & $\begin{array}{l}\text { Labéon } \\
\text { Proculus } \\
\text { Pomponius } \\
\text { Q. Mucius } \\
\text { Alfenus } \\
\text { Trebatius } \\
\text { Servius, } \\
\text { Ramusa. }\end{array}$ & $\begin{array}{c}\text { fundim... usuarium servur } \\
\text { posse ibi operari } \\
\text { coloni servi } \\
\text { servus... in praediis } \\
\text { in instrumento...mancipia } \\
\text { his quae ibi sunt } \\
\text { praedia... servis } \\
\text { hominibus... in fundo } \\
\text { familia rustica } \\
\text { rusticorum causa } \\
\text { uxores et infantes (eorum qui } \\
\text { supra enumerati sunt) } \\
\text { instrumenti... familiam } \\
\text { qui rure morari solebant } \\
\text { contubernales... instrueto fundo } \\
\text { praediis instructis...mancipis } \\
\text { servis qui in fundo morarentur }\end{array}$ & $\begin{array}{l}\text { D } 7.8 \cdot 16.2 \\
\text { D } 9.2 .27 \cdot 11 \\
\text { D } 10.4 \cdot 5.6 \\
\text { D } 21.1 .33 \mathrm{pr} \\
\text { D } 28.5 \cdot 35.3 \\
\text { D } 29.5 \cdot 1.30 \\
\text { D } 33.7 \cdot 12.2 \\
\text { D } 33 \cdot 7 \cdot 12.5 \\
\text { D } 33.7 \cdot 12.6 \\
\text { D } 33.7 \cdot 12.7 \\
\text { D } 33 \cdot 7 \cdot 12.10 \\
\text { D } 33.7 .12 .31 \\
\text { D } 33.7 .12 .33 \\
\text { D } 33 \cdot 7 \cdot 12.37 \\
\text { D } 33.7 \cdot 12.44\end{array}$ \\
\hline
\end{tabular}

\begin{tabular}{|c|c|c|c|}
\hline $\begin{array}{l}\text { Juriste auteur } \\
\text { du passage }\end{array}$ & Juriste cité & Passage & Source \\
\hline 1 & & $\begin{array}{c}\text { fundum... instructus... mancipi is } \\
\text { qui sunt in villis deputati } \\
\text { in agro } \\
\text { ex eo praedio in quo homines } \\
\text { fuerant. }\end{array}$ & $\begin{array}{l}\text { D } 33 \cdot 7 \cdot 12.46 \\
\text { D } 33.9 .3 .6 \\
\text { D } 40.9 .12 .1 \\
\text { D } 43.16 .1 .34\end{array}$ \\
\hline Modestin & & $\begin{array}{l}\text { mancipia, quae in praediis erant } \\
\text { fundo... servo } \\
\text { servus, qui in fundo morari } \\
\text { solitus erat. }\end{array}$ & $\begin{array}{l}\text { D } 20.1 .26 .2 \\
\text { D } 26.7 .32 .3 \\
\text { D } 32.82\end{array}$ \\
\hline
\end{tabular}

L'existence d'une fonction rurale ressort narurellement de la désignation servi ou mancipia rustica, ou encore de opus rusticum, sans qu'il soit toutefois possible de spécifier la fonction dont il s'agit. On a par ailleurs prósumé que l'esclave d'un colon remplissait une tâche rurale (336). Enfin, la présence d' enfants ou de femmes esclaves implique celle de travailleurs sur le fonds, mais leur métier n'apparaît pas.

Cette merge d'incertitude est encore aggravée par le fait que dans le legs d'un fonds cum instrumento ou instructus (337), problème qui concerne la plupart des textes relevés, les juristes incluent non seulement les esclaves exerçant une fonction rurale, mais également ceux qui ont un rôle de nature urbaine, comme le pistur, le tonsor, les lanificae, l'ostiarius, les fultones, les focariae (338). C'est là un témoignage du processus de concentration de main-d'oeuvre opéré par le système de la villa (330). Séparer l'es-

336- Ce n'est pas forcêment le cas, cf. Ulpien (Neratius) D 9.2.27.9 :fornacarius servus coloni.

337- Paul D 33.7.1 pr : Sive cum instrumento fundus legatus est sive instruc tus, duo legata intelleguntur. Sur le contenu de la distinction, en particulier Sabinus pour qui le legs d'un fonds instructus est plus important que le legs crom instrumento, cf. Ulpien D 33.7.12.27, car le testateur est censé léguer plutôt ses propres ustensiles que ceux du fonds; v. aussi Paul, selon qui, si le fonds a étê légué cum instrumento et qu'il ait été aliêné, le légataire ne peut rien revendiquer contrairement au cas où le fonds a êté léqué instructus, cf. D 33.7.5 Labeo lib. I Pithanon a Paulo Epitomaturum : $S i$ cui fundum et instrumentum eius legare vis, nihil interest, quomodo leges fundum cum instmonto an fundum et instrumentum an fundum instructum. PAULUS. Imo contra : nam inter ea legata hoc interest, quod, si fundo alienato morImo contra: nam inter ea legata hoc interest, quod, si fundo ailenato mor erit legatum, ex ceteris poterit instmumentum esse legatum. Sur le thème, A. GUARINo, Diritto privato romano, cit., p. 312 ; JOHN, Die Auslegung des Legats yon Sachgesamtheiten im römischen Rechts bis Labeo (1970), p. 8 s.;

HOLTHÖFER, Sachteil und Sachzubehör im römischen und im gemeinen Recht (1972). 338- Ulpien D 33.7.12.5 (Trebatius)-6 (Servius)-9.

339- La villa est autant autarcique qu'il est possible de l'être pour sa consommation, cf. P. DOCKES, La libération, cit., p. 159. 
clave dn fonds, G'átnit prendre le risqus de laisser une terro inculte, c'est pourqui les juristes des 2̀̀me et Jème siècles se sont inqéniọs à le lier au fonds et aux moyens de production (340), raisant aboutir la conception extensive de $l$ 'instrumentum fundi au détriment de la conception restrictive jadis soutenue par Alfenus ( 341$)$. Seuls les esclaves se trouvant sur le fonds de manière stable font partie de l'instrumentum (34a), mais

340- STEINWENTER, Fundus cum instrumento, SB. Akad. Wien, Phil. Hist. Klasse, 221.1 (1942), p. $25 \mathrm{~s} ., 82,100-101$, pense que les juristes, exerçant leur activité en ville, ne prirent pas en considération les nécessités de l'économie agraire et ne tinrent pas compte de la tendance de la législation impériale et de la pratique a fixer les travailleurs agricoles à la terre; contra, M. HORVAT, Legation fundi e servi, in St. Volterra 5 (1971), terre ; contra, M. Horvar, Legation fundi e servi, in St. Volterra 5 (1971), p. 90 s. Les institores font semblablement partie de l'instrumentum taber-
nae, D 33.7 .7 Scaevola ; D $33.7 .13 \mathrm{pr}$ Neratius chez Paul ; D $33.7 .15 \mathrm{pr}$ Servius chez Pomponius. Comme le constate justement E.M. SCHTAERMAN, La schiavitu, cit., p. 78 , le fait que les juristes mentionnés soient plus anciens que ceux qui apparaissent à propos du legs d'un fonds cum instrumento, englobant les esclaves qui y travaillent, implique que le lien entre travailleur et moyens de production a été réalisé plus tôt dans l'artisanat que dans l'agriculture.

341- Selon Alfenus (D 33.7.12.2), les esclaves ne font pas partie de 1 'instrumentum fundi "quia nihil animalis instrumentum esse". C'est la conception de Caton (de agr., I,15) pour qui l'instrumentum d' une villa rustica $n$ 'est composé que de 1 'attirail non vivant. Varron (de re rust., I, $17 \mathrm{s.}$ ) est le seul agronome faire exception à la règle: il distingue l'instmuest le seul agronome a laire exception a la regle ; tum (outils et machines). On trouve la conception extensive chez Paul (D 33. $7.19 \mathrm{pr}$ ) : ceterum instrumento fundi mancipia quoque colendi agri causa inducta contineri non ambigitur; le contenu de ce texte est classique selon inducta contineri non ambigitur; le contenu de ce texte est classique seld
M. HORVAT, o.c., p. $91 \mathrm{n} .2$; STEINWENTER, o.c., p. 79 . Cette conception est dêja celle de Sabinus (D 33.7.8 pr) : in instrumento fundi ea esse, quae fructus quaerendi cogendi conservandi gratia parata sunt... veluti homines qui agrum colunt...et même de Q. Mucius (chez Ulpien D 28.5.35.3) : cum fundus erat legatus vel cum instrumento vel cum his quae ibi sunt. Néanmoins, elle semble avoir eu du mal à l'emporter car Q. Cervidius Scaevola, au zème siècle, parle encore de l'obligation de mentionner les esclaves si on désire qu'ils soient compris dans 1'instrumentum (cf. D 33.7.20 pr-1-3-9), M. HORVAT, O.c., p. 91 .

342- Scaevola (D 20.1.32) distingue les esclaves se trouvant temporairement sur le fonds (temporis causa) de ceux qui y sont attachés en permanence (ut ibi prmetuo essent). Seuls les derniers font partie de l'instmune tum fundi, M. JACOTA, Les transformations de l' tum fund, M. JACOTA, les transfortit. p. 381 n. 17. M. HokvaT,o.c., $p$. 91 n. J, estime que $Q$. Mucius excluait déja de 1 instrumentum les esclaves qui n'étaient que temporairement sur le fonds (D 28.5.25.3) ; contra, E.M. SCHTAERMAN, Die Krise der Slavenhalter ordnung im Westen des römischen Reiches, Berlin (1964), p. 96. Cependant, Paul inclut dans 1 'instrumentum les esclaves qui se sont éloignés du fonds pour motif d'étude (D $32.78 \mathrm{pr}$ : Quaesitum est Stichum servum ex eo fundo. ante annum mortis testratoris abductum et disciplinae traditum, postea in eum fundum non reversum an deberetur. Responsum est, si studendi causa misisset, non quo de fundo eum aliorsum transferret, deberi) et Ulpien y com-

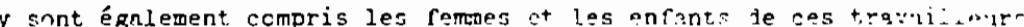
$(343)$.

L'incertitude est woindre à propos des fonstions d'exécution "rbaines.

II - Fonctions d'exécution urbaines.

\begin{tabular}{|c|c|c|c|}
\hline $\begin{array}{l}\text { Juriste auteur } \\
\text { du passage }\end{array}$ & Juriste cité & Fonction & Source \\
\hline $\begin{array}{l}\text { Nlfenus (chez } \\
\text { auteur snonyme) }\end{array}$ & & $\begin{array}{c}\text { ministratores/iuctores/cubicularii/ } \\
\text { coci. }\end{array}$ & D 50.10 .203 \\
\hline Isvolenus & Labéon & mulionem & D $19.2 .60 . \bar{i}$ \\
\hline Pomponius & Servius & $\begin{array}{l}\text { lecticarios } \\
\text { focariam }\end{array}$ & 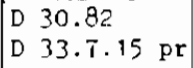 \\
\hline Africain & & Eurz....argenti & D 40.4 .22 \\
\hline Gaius & & $\begin{array}{l}\text { cocism } \\
\text { cocum } \\
\text { cocus }\end{array}$ & $\begin{array}{lll}D & 2 & 1.1 .18 .1 \\
D & 35.1 .17 .1 \\
D & 40.4 .24\end{array}$ \\
\hline Venuleius & & nomenculator & D 40.12 .44 .2 \\
\hline Scaevola & & $\begin{array}{l}\text { pedisequi } \\
\text { lecticarii } \\
\text { pedisequas }\end{array}$ & 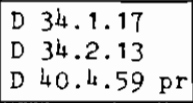 \\
\hline Papinien & & $\begin{array}{l}\text { lecticariis/pedisequis } \\
\text { cura (claves/anulum) (3h4) }\end{array}$ & 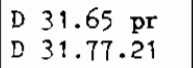 \\
\hline
\end{tabular}

prend ceux qui travaillent à l'extérieur, moyennant une merces, durant quelques mois de l'année (D 33.7.12.8 : Vel servi, si aliqua parte anni per eos ager colitur, aliqua parte in mercedem mittuntur", nihilo minus instrumento continentur).

343- U1pien D 33.7.12.7 : Uxores quoque et infantes eorum, qui supra enumerati sunt, credendum est in eadem villa agentes voluisse testatorem legato contineri, et D 33.7.12.33 : Contuberrales quoque servorum, id est uxores, et natos instructo fundo contineri verum est. L' esclave conductor ne faisant pas partie de l'instrumentum, $M$, JACOTA, o.c. p. 378, pense, a partir de D 33.7.12.7, que ses enfants en sont pareil p. 378 , pense, à partir de 133.7 .12 .7 , que ses entants en sont pareil lement exclus sur ce point, S. sorazz, I1 rispetto per la famiglia dello schiavo, sDH 15 (1949), p. 187-192. Les Sententlae de Paul nous apprennent que sa femne n'est pas comprise dans l'attirail du fonds (III, 6,40 : Uxores vero eomu qui mercedes praestare consueverunt, neque instructionis, neque instrumenti appeliatis continentur).

344- Il s'agit d'un affranchi. 


\begin{tabular}{|c|c|c|c|}
\hline $\begin{array}{l}\text { Juriste auteur } \\
\text { du passage }\end{array}$ & Juriste cité & Fonction & Source \\
\hline Paul & Neratius & $\begin{array}{l}\text { capsarios } \\
\text { mulionem/agasonem } \\
\text { ostiarium } \\
\text { muliones } \\
\text { lecticarii } \\
\text { cursores } \\
\text { balneator } \\
\text { fornacator }\end{array}$ & $\begin{array}{l}\text { D } 1.15 .3 .5 \\
\text { D } 32.60 .1 \\
\text { D } 32.61 \\
\text { D } 32.99 .2 \\
\text { D } 32.99 .4 \\
\text { D } 32.99 .5 \\
\text { D } 33.7 .13 .1 \\
\text { D } 33.7 .14\end{array}$ \\
\hline Ulpien & $\begin{array}{l}\text { Neratius } \\
\text { Mela } \\
\text { Q. Mucius } \\
\text { Trebatius } \\
\text { Servius, } \\
\text { Namusa. } \\
\text { Celsus } \\
\text { Papinien } \\
\text { Labéon }\end{array}$ & $\begin{array}{c}\text { diactarii } \\
\text { mediastinus/atriarii/focarii } \\
\text { balneator/(stercorandis latrinis) } \\
\text { fornacarius } \\
\text { servum conductum ad mulum regen- } \\
\text { dum. } \\
\text { agasonem } \\
\text { lecticarii/comites/ } \\
\text { (puellae exornant.) } \\
\text { scoparii . } \\
\text { focariam/mulieres quae } \\
\text { villam servent. } \\
\text { focariae } \\
\text { ostiarium/mulionem } \\
\text { suppellecticarios } \\
\text { in triclinio } \\
\text { ostiarii/diaetarii } \\
\text { nomenculator (345) } \\
\text { cubicularium (346) } \\
\text { capsarius } \\
\text { lecticariorum } \\
\text { comes }\end{array}$ & 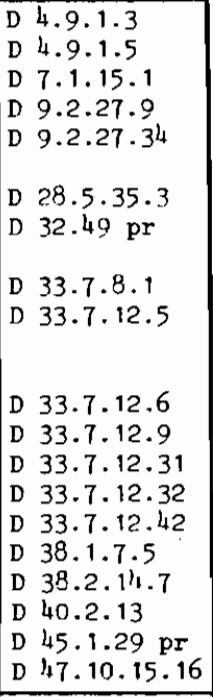 \\
\hline Marcien & Labéon & $\begin{array}{l}\text { cubicularios/obsonatores } \\
\text { lecticarius/cocus } \\
\text { coci/lecticarii } \\
\text { ornatricibus } \\
\text { balneator }\end{array}$ & $\begin{array}{l}\text { D } 32.65 \mathrm{pr} \\
\text { D } 32.65 .1 \\
\text { D } 32.65 .2 \\
\text { D } 32.65 .3 \\
\text { D } 33.7 .17 .2\end{array}$ \\
\hline
\end{tabular}

Le Digeste rend mieux compte de la division du travail en matière urbaine que rurale. Cette spécialisation ne s'explique pas, comme pour la familía rustica, par la diversité des besoins de l'exploitation, mais correspond aux exigences d'un luxe de plus en plus raf́finé (347).

\footnotetext{
345- Idem.

346- Idem.

347- J. MARQuARDT, Vie privée, cit., p. 166-167.
}

Chargés de l'entretien de la maison, le diaetarius, qui s'occupe des divers appartements (348), et le scoparius, balayeur, travaillent sous les ordres de l'atriensis (349), comme le portier, ostiarius (350). On trouve dans les grandes familles des suppellecticarii (351), qui prennent soin du mobilier.

Les cubicularii (352) remplissent la fonction de valets de chambre. Les ormatores (353), les omatrices (354), le capsarius (355), le balneator (356) et le formacator assurent la toilette du maitre ou de sa femme.

Les fonctions d'ordre alimentaire sont aussi à la charge de domestiques. On relève le cocus, cuisinier (357), ou la focaria, le formacarius, qui s'occupe du four, les ubsonatores, qui achètent les provisions (358). Les ministratores (359) servent le maitre à table.

348- Le diaetarius de D 4.9.1.3 est un gardien des cabines d'un navire, cf. Diction. GAFFIOT, p. 517 ; Diction. BENOIST et GOEL.ZER, p. 432.

349- Sur 1'atriensis, Cic., Parad,. V, 2,38. Selon Columelle (XII , 3,9), $c^{\prime}$ est la vilica qui exerce la surveillance générale du mobilier rustique. Les scoparii prennent généralement le nou d'atrarii, cf. D 4.9.1.5.

$350-$ v. Or. 5844,6333 ; CIL, VI, 3995 s., 8961 s. Il était anciennement attaché, comme un chien, cf. Suet., de reth., 3 ; Colum., I, 10 ; Ov., Am., $\mathrm{I}, 6,1$.

351- On trouve aussi dans les inscriptions a supellectili, cf. CIL, VI, $4035 \mathrm{~s} ., 4357$. L'esclave dont parle Africain (D 40.4.22), qui a la cura argenti, doit être situé à ce niveau.

352- Ce sont eux qui annoncent les visites, J. MARQUARDT, Vie privée, cit., p. 169 ; v. CIL, VI, 3956 s., 6254 s., 8774 s., 9295 s.

353- On sait par les 'littêraires' que les homenes aussi étaient coquets, cf. Gel1., I,5,2 ; Macrob., Sat., III,13,4 ; Cic., post red. in sen., VI,13;

VIr, 16 ; pro Sest., VIII, 18 ; Plut., Caes., 4.

354- Suet., Claud., 40 ; CIL, VI, 3993 s., 8957 s., 9726 s.

355- Il garde les vêtements. Il peut être soit amené par scn maître (cf.

CIL, vI, 3952, 6245, 7368 et Petron., 30), soit appartenir à l'établissement de bains (cf. CIL, VI, 9232). C'est le sens du terme dans Paul $D$ 1.15.3.5.

Il peut être aussi celui qui porte les livres des enfants à l'école, cf. Ulpien $\mathrm{D} 40.2 .13$.

356- CIL, VI, 6243, 8742, 9102. Il est encoro appelé thermarius, cf. CIL, $x, 6638$. Le fornacator fait office de chauffeur.

357- La cuisine peut être faite par une fccaria,cf. U1pien (Trebatius) D 33.7.12.5.

358- Sen., Ep., 47,8.

359- CIL, VI, 8915 s., 9641 s. ; Petron., 31. 
Enfin, assurent la suite du maitre les pedisequi ou pedisequae ( 360 ), qui le suivent à pied, les cursores (361), qui précèdent sa voiture, les lecticarii (362), qui portent sa litière, le nomenclator (363), les comites (364). Composent aussi cette suite des agasones, palefreniers (365), des muliones, muletiers (366) et des iumctores, postillons.

Quant au mediastinus dont parle Ulpien, il apparait nettement dans un contexte urbain (367).

Dans d'autres cas, la fonction n'apparaît pas précisément, mais on sait par le terme ministerium qu'il s'agit d'une fonction domestique (368) Il en va de même pour les operae officiales de l'affranchi (369). La fonc-

360- Pedisequus, Nep., Att., 13 ; CIL, VI, 4003 s., 6332 s.. 8992 s.; pedisequa, P1aut., Asin., 183 ; Aul., 807 ; Ter., Andr., I,1,96 ; CIL, VI, $4002,4355,5821,6335$ s., 7410 .

361 - Sen., Ep., 87,9;123,7

362- Sen., Ep., 31,10;80,8. Ce sont de préférence des Syriens ou des Cappadociens (sur les Syriens, Mart., IX,22,9 ; Iuv., VI, 351 ; sur les Cappadociens, Mart., VI,77,4.).

363- L'usage du nomenclator, qui accompagne le maître dans 1a rue, est signalé à l'époque républicaine (Cíc., ad $\Lambda t t .$, IV, 1,5; pro Mur., 36,77). Sous 1 'Empire, on a des nomenclateurs soit pour recevoir les visites (nomenclator $a b$ admissione, cf. Sen., ad Seren., 14,1; de tr. an., 12,6 ; Luc., de merc.cond., 10), soit pour les sorties ( $P 1$ ine le jeune a deux nomenclateurs, cf. Ep., II, 14,6). Le nomenculator qui apparaît chez Ulpien (D 38.1 $7.5)$ est un affranchi impubère.

364- Le comes peut être un howne ou une femme, 1ibre ou esclave, qui tient compagnie au maître en privé comme en public. Les paedagogi sunt des comites, cf. U1pien D 47.10.15.16.

365- Le stratox est aussi un palefrenier, cf. J. MARQUARDT, Vie privée, cit. p. $176 \mathrm{n} .2$, mais il n'apparaît pas dans le Digeste.

366- Paul, Sent., III, 6,72: Muliones inter urbana ministeria continentur; CIL, VI, 7409 .

367- Le contexte est celui de D 4.9 : Nautae caupones stabularii ut recepta restituant.

368- Ministerium signifie par exemple fonction domestique chez U1pien D 40 9.12 .1 (servw, qui extra ministeriwn eius mulieris fuit vel in agro vel in provincia) ou chez Papinien D 40.4.51.1 ( $a b$ hunore ministrandi, vel administrandae rei negotio), mais a une valeur terminologique plus large (fonction/ service) chez Africain D 41.1.40 (et circa servorum igitur operam ac ministerium eandem rationem sequend(cm). Pater familias sum instrumentum...faceret (Ulpien D 33.7.12.37) sous-entend aussi une fonction domestique, tout comme cultus cousa (Alfenus D 32.60.1) et officio (Marcien D 32.65.1).

369- Il en est question chez U1pien D 12.6.26.12 ( Celsus, Julien, Marcellus ); $\mathrm{D} 38.1 .6$ et $\mathrm{D} 38.1 .9 .1$. Sur le thème, et 1 'opposition entre operae officiales et operae fabriles, v. A. GUARINO, Diritto privato romano, cit., p. 291 et n. 24.2.1; K. VISKY, le operae dei liberti, cr. de P. PESCANI, le operae libertorum, Trieste (1967), in Index 1 (1970), p. 225. tion de mitrix (370), nourrice, est elle aussi domestique, wais elle n'est typrlogiquement ni urbaine ai rurale.

$-0^{\circ}$

On a ru qu'il était parfois difficile de distinguer avec précision les esclaves ruraux des esclaves urbains. La séparation entre domestiques et artisans $n$ 'est pas non plus, dans les textes juridiques, des plus claires. Il n'a pas été possible d'intégrer les artisans dans une catégorie franchement autonome par rapport aux domestiques pour trois raisons. En premier lieu, ils ont tous leur représentant dans la domesticité (371). En second lieu, les esclaves peuvent être mutés d'un emploi à-un autre, d'une fonction domestique à une fonction artisanale ou inversement (372). En troisième lieu, les domestiques reçoivent quelquefois une formation, tout coume les artisans (373).

La formation professionnelle de l'esclave est un problème important sur lequel il convient de s'arrêter quelques instants. Les textes sont unanimes sur un point : apprendre un métier à l'esclave lui donne une plus grande valeur financière (374). Cela peut constituer une source de spéculation, ou un moyen de subsistance pour le maitre peu aisé vendant son esclave un bon prix après l'avoir formé. L'esclave est quelquefois placé chez un magister. Sa condition d'apprenti est alors très dure. Julien estime en effet utile de préciser que le magister a le droit de le punir, sans coutefois aller jusqu'à l'estropier (375). Vivianus signale le cas d'un esclave qui s'enfuit de chez son pxaeceptor à cause de la cruauté de celui-

370- Scaevola D 34.1.20; Ulpien D 40.2.13.

371- J. MARquardT, Vie privée, cit., p. 184. P. LEVEQuE, Esclavage et exnloitarion du travail, cit., p. 236-237, remarque, a propoa de l'Egypte, qu'il ne faut pas se laisser abuser par le terule "domestique", le petit propriétaire se faisant aider par ses esclaves tant aux champs que dans un modeste artisanat familial.

372- Marcien D 32.65.1. / 373- Marcien D 32.65.3.

374- Par exemple, U1pien D 19.1.13.4: Si venditor d-lo fecerit, ut rem pluris venderet, puta de axtificio mentitus est aut de peculio, empti ewm iudicio teneri, ut prasstaret emptori, quanto pluris servum emisset, si ita peculiatus esset vel er artificio instructus; aussi Pomponius D 6.1. 29 ; Florentinus D 18.1.43 pr; Paul D 17.1 .26 .8 ; D 19.1.43 (Julien); Ulpien D 13.7.25.

375- Chez Ulpien D 9.2.5.3 : Si magister in disciplina vulneraverit servw vel occiderit, an Aquilia teneatur, quasi dammum iniuria dederit ? Et Iulianus scribit Aquilia teneri eum, qui eluscaverat discipulum in disciplina: nus scribit Aquilia teneri eum, qui elusciaverat discipulum in disciplina multr magis igitur in cccisn idem erit dicendwm. La suite concerne un fils le passage : Quia non faciendae iniuriae causa percusserit, sed monendi et docendi cousa. Cependant, la trop grande sévérité du praeceptor est réprimée,
Paul D 9.2 .6 . 
ci (376). Le possesseur peut se charger lui-même de la formation de l'esclave (377), de même que $1^{\prime a c h e t e u r ~(378), ~ o u ~ l e ~ m a r i ~ d a n s ~ l e ~ c a s ~ d ' u n ~}$ esclave dotal (379). L'usufruitier (380) et le créancier gagiste (381) peuvent aussi remplir cette fonction.

Les textes sont plus paurres quant aux métiers ainsi appris. On voit le possesseur d'un esclave lui enseigner la fonction de pictor ou de librarius (382), 1'acheteur en faire un agitator ou un pantomimus (383). Cependant, les esclaves placés spécialement à l'extérieur se trouvaient vraisemblablement le plus souvent chez des artisans.

Quoi qu'il en soit, 1'esclave qui avait effectué son apprentissage atteignait une situation appréciable car, si l'on en croit un passage de Valens, il pouvait se suffire à lui-mème (384).

Pour en revenir plus précisément à la distinction domestiques/artisans, il n'est done pas surprenant que les juristes romains parviennent à des conclusions assez inattendues. Ils comptent en effet parmi les artisans non seulement les cuisiniers, mais aussi les muletiers et mêne les porteurs de litière (385).

376- Chez Ulpien D 21.1.17.

377- Paul D 6.1.27.5.

378- D 19.1.43 Paul (Julien).

379- Paul D 25.1.6.

380- U1pien D 7.1.27.2.

381- Paul D 13.7.25 (selon 0. LENEL, Pal., le texte a été attribué par erreur à ulpien): le créancier gagiste peut se faire rembourser les frais occasionnés par la formation des esclaves engagés, mais seulement si le débiteur avait donné son accord ou avait comencé à apprendre à $1^{\prime}$ esclave le métier en question, ou bien, si aucune de ces conditions n'est remplie, s'il a appris a l'esclave un métier "utile".

382- Gaius D 6.1.28.

383- Paul D 19.1.43.

384- Valens D 32.12 : Stichus liber esto : et ut eum heres artificium doceat unde se tueri possit, peto. Pegasus inutile fideicomissum esse ait, quia genus artificii adiection non esset : sed praetor aut arbiter ex voluntate defuncti et aetate et condicione et natura ingenioque eius, cui relictwon erit, statuet, quod potissimum artificion heres docere eum sumptibus suis debeat.

385- Alfenus chez Paul D 32.60.1 : Servis et ancillis urbonis legatis agasonem mulionem legato non contineri respondi : eus enim solos in eo numero haberi, quos pater familias circum se ipse sui cultus causa haberet; Marcien D 32.65.2 : Si unus servus plura artificia sciat et alii coci legati fuerunt, alii textores, alii lecticarii, ei cedere servom dicendum est, cui legati sunt in quo artificio plemmque versabatur.
I1 était nécessaire d'adopter un critère qui rende plus objectivement compte de la distinction. Les fonctions domestiques urbaines ont été considérées comme étant par essence non productives. On a envisagé séparément les fonctions commerciales (386) et artisanales. Elles constituent une activité productive (387) dans 1a mesure ol elles contribuent à accroître directement $1 \mathrm{a}$ richesse du maître (388).

\begin{tabular}{|c|c|c|c|}
\hline $\begin{array}{l}\text { Juriste auteur } \\
\text { du passage }\end{array}$ & Juriste cité & Fonction & Source \\
\hline $\begin{array}{l}\text { Alfenus (chez } \\
\text { auteur anonyme) }\end{array}$ & & textores & D 50.16 .203 \\
\hline Iavolenus & Trebatius, Labéon & $\begin{array}{c}\text { figulos } \\
\text { fullonem/pistorem }\end{array}$ & $\begin{array}{l}\text { D } 33.7 .25 .1 \\
\text { D } 34.5 .28\end{array}$ \\
\hline Jurien & & pictor/faber (389) & D $38.1 .23 \mathrm{pr}$ \\
\hline Fomponius & Servius & $\begin{array}{c}\text { (operis ancillarum...vesti- } \\
\text { menta.) } \\
\text { textores } \\
\text { textores } \\
\text { pistores/(cauponio) }\end{array}$ & $\begin{array}{l}\text { D } 24 \cdot 1 \cdot 31.1 \\
\text { D } 30.36 \mathrm{pr} \\
\text { D } 30.36 .1 \\
\text { D } 33.7 .15 \mathrm{pr}\end{array}$ \\
\hline Gaius & Julien & $\begin{array}{c}\text { pictorem } \\
\text { sarcinatrix/textrix } \\
\text { sutor }\end{array}$ & $\begin{array}{l}\text { D } 6.1 .28 \\
\text { D } 15.1 .27 \mathrm{pr} \\
\text { D } 35.1 .17 .1\end{array}$ \\
\hline Marcellus & & (taberna) & D 7.8 .20 \\
\hline
\end{tabular}

386- Ont été relevês ici les textes concernant l'actio institoria lorsqu'ils contenaient une indication précise de la fonction remplie Fur le servus institor ou du lieu de son activité (par ex. couponium, taberna). Il ne s'agit pas d'opérer un traitement statistique mais simplement de donner une idée des diverses activités commerciales servìles. On reviendra plus tard de manière systématique sur les activités donnant lieu aux actions institoria et exercitoria, celles-ci olacant c'aîrement 1'esclave dans une situation privilégiêe.

387- Au sens large. Le travail productif est seulement celui qui produit du capital, celui qui augmente directement le capital. En tant que tel, il n'est admissible que dans le mode de production capitaliste, tel, il n'est admissible que dans le mode de production capitaliste,
D. MUST, Per una ricerca sul valore di scambio nel modo di produzione schiavistico, Ist. Gramsci (1978), p. 165. Le travail servile ne tend pas a la formation du capital car il se consume dans la formation d'un surproduit dont une grande partie est transformée en dépenses improductives, en oeuvres d'art, édifices religieux, travaux publics, D. MUSTI, o.c., p. 165-166.

388- C'est la définition malthusienne du travail productif, D. MUSTr, o.c., p. 165.

389- Il s'agit d'affranchis, ainsi que chez Scaevola D 31.88.3; D 38. 1.45 ; Tryphoninus D 32.38.5.(Scaevola); Paul D 34.2.4. 


\begin{tabular}{|c|c|c|c|}
\hline $\begin{array}{l}\text { Juriste anteur } \\
\text { du passago }\end{array}$ & Juriste cité & Fonction & Source \\
\hline Scaevoln & & $\begin{array}{c}\text { (mensae nummulariae) } \\
\text { (tabernan) } \\
\text { (tabornae ferrariae) } \\
\text { sut,orom } \\
\text { (vostiarii...negntiationem) }\end{array}$ & $\begin{array}{l}\text { D } 14 \cdot 3 \cdot 20 \\
\text { D } 20.1 .34 .1 \\
\text { D } 31.89 .3 \\
\text { D } 36.1 .80 .12 \\
\text { D } 38.1 .45\end{array}$ \\
\hline Papinien & & $\begin{array}{c}\text { (mensam) } \\
\text { (tahernam ourdurariam) } \\
\text { cerdonem }\end{array}$ & $\begin{array}{ll}D & 14.3 .19 .1 \\
\text { D } & 32.91 .2 \\
\text { D } & 38.1 .42\end{array}$ \\
\hline Tryphoninus & Scaevoln & (taberna) & D 32.38 .5 \\
\hline Paul & $\begin{array}{l}\text { Meln } \\
\text { Neratius }\end{array}$ & $\begin{array}{c}\text { (fabricam) } \\
\text { (taberna) } \\
\text { textoribus } \\
\text { (tathernar mauponina) } \\
\text { mul ioribus lanifiria } \\
\text { (nrte fabrica) } \\
\text { in Asialn misisset ad } \\
\text { purpuras... }\end{array}$ & $\begin{array}{ll}\text { D } & 17.1 .26 .8 \\
\text { D } & 19.2 .45 .1 \\
\text { D } & 32.61 \\
\text { D } & 33.7 .13 \\
\text { D } & 33.7 .16 .2 \\
\text { D } & 32.7 .19 .1 \\
\text { D } & 34.2 .4\end{array}$ \\
\hline vipien & $\begin{array}{l}\text { Labéon, } \\
\text { Octravenus. } \\
\text { Julien } \\
\text { Namusa } \\
\text { Labéon } \\
\text { Labéon } \\
\text { Labéon } \\
\text { Julien } \\
\text { Julien } \\
\\
\text { Mela } \\
\text { Trebrtius } \\
\text { Servius, } \\
\text { Namusa. } \\
\text { Servius }\end{array}$ & 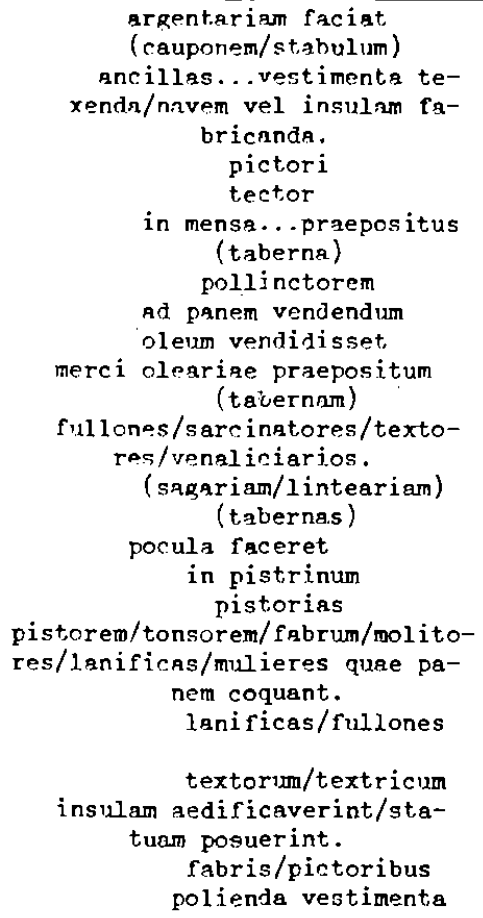 & 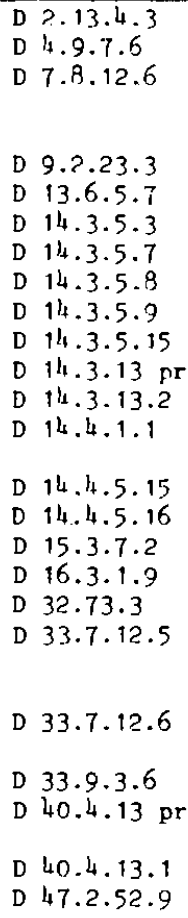 \\
\hline Marcien & & $\begin{array}{l}\text { textores } \\
\text { (cauponio) }\end{array}$ & $\begin{array}{l}\text { D } 32.65 .2 \\
\text { D } 33.7 .17 .2\end{array}$ \\
\hline
\end{tabular}

Travaillent dans l'habillement le teztor, la tertrix (390), tisserands, les lanificae (391), filsuses, les fullones, flulons, le sarcinator, la sarcinatrix (392), couturiers, le sutor (393), cordonnier. On trauve dans la construction le tector, couvreur ( 3 ls), et le pictor, peintre, mais l'activité de ce dernier peut aussi être artistique (335). on relère ailleurs des potiers, figuti (396), des ruvriers, fabri (39i), un combis (398). On note la présence de pistores, vinlangers (309), et

390- Varr., de re rust., I,2,21 ; Suet., de Gram., 23.

391- A la campagne, elles sont utilisêes pour fournir les vêtements aux esclaves ruraux, cf. D 33.7.12.5 Ulpien (Trebatius) (Ianificae quae fomilian mustican vestiunt) ; Paul. Sent., III,6,37 (ancillae quae vestimenta rusticis faciunt). Elles travaillent alors sous la direction de la vilizca, J. MARQUARDT, vie privée, cit., p. 183.

392- Sarcinator : CIL, VI, 6348 ; sarcinatrix : CIL, VI, 9039 b, 4467. Les termes vestifex (CIL, VI, 7467) et vestificus (CIL, VI, 8554) n'apparaissent pas dans le Digeste.

393- Paul, Sent., III, 6, 37 ; CIL, VI, 6355, 9050.

394- CIL, VI, 5985 (esclave impérial tector).

395- Le peintre "prêcieux" de D 9.2.23.3 (pretiuso picturi) est probablement un artiste, de même que celui qui apparaît à côté d'un librarius dans D 6.1.28. Par contre, les peintres qui, pour être affranchis, doivent peindre une pièce (membrom depinxissent) (cf. D 40.4.13.1) sont des travailleurs du bâtiment. Sur l'évaluation juridique du travail ardes travailleurs du bâtiment. Sur l'évaluation juridique du travail ar-
tistique, CALABI-LIMENTANI, Il lavoro artistico, Milan (1958), p. $80 \mathrm{~s}$. 396- Et des esclaves qui fabriquent des coupes (pocula), cf. Ulpien (Mela) D 15.3.7.2.

397- Ils exercent souvent chez le maitre, cf. Varr., I,16,4: si enim $a b$ fundo longius absunt uppida nut vici, fabros parant : quos habeant in villa ; Colum., XI,1,5. Les ouvriers signalés in $n 33.7 .12 .5$ sont dans la construction (fabri qui villae reficiendae parati sunt) ; ceux indiquếs par D 40.4.13.1 doivent construire un navire (si fabricassent navem). Paul, Sent., III,6,50, parle de fabri ferrarii, item tignarii. "Arte fabrica" (Paul $033.7,19.1$ ) vise aussi un esclave ouvrier; id. Paul (Mela) D 17.1 .26 .8 .

398- Le cerdo est un artisan, un "gagne-petit", cf. Iuv., IV,153. Le cerdo sutor est un savetier, cf. Mart., III, 59.

399- Selon Pline, N.H., XVIIr, 107, les pistores sont ceux qui broyent le blé. Plus tard, le pistor est l'esclave qui fait le pain, cf. Varr., cité par Gell., XV,19 ; Suet., Caes., 48 ; Paul, Sent., III,6,37. Ce métier peut être exercé par une fenane (cf. Ulpien (Trebatius) D 33.7,12. 5 : mulieres quae panem conuant) chez le maître, mais on ne relève pas de pistrix dans les pistrinae de la ville. Le nom de pistrix manque mêtne aux indices de DESSAU. Intéressante est la législation sur $1^{\prime}$ adultère qui assimilait les revendeuses libres aux prostituées, cf. Paul. Sent., 
de molitores, meuniers, dans 1'alimentation, puis de tonsores, berbiers (400). On remarque d'autres activités comerciales, celle de bnnquier (401), celle de venaliciorius, marchand d'esclaves, "métier" qui éclaire d'un jour sévère les rapports au sein du groupe servile. Scaevola évoque une taberna ferraria léguée pour moitié à un affranchi (402), U1pien un commerce de toiles et de sayons (403) tenu par un même esclave, ou encore deux boutiques dans des lieux différents à la tête desquelles se trouve un esclave (404). On voit aussi apperaitre des insti-

II,26, II : Quce mercibus vel tabernis exercendis procurant adulterium fieri non piacuit. 400- Mart., VI, 52 ; CIL, VI, 4359, 4474, $6366 \mathrm{~s}$; X, $1963 \mathrm{~s}$. J. CAR-
Copino, La vie quotidienne à Rome $\$ 1$ l'apogée de 1 'Empire, (1939) (réimpr. 1978), p. 187, signale les innombrables boutiques de tunsores qui se sont ouvertes au 2ème siècle de notre ère dans les tabernae de la ville. Bien que le tonsor puisse travailler chez le maître (cf. par exemple D 33.7.12.6), le fait qu'il exerce parfois à I'extérieur nous a amenê à considérer sa fonction coutune pouvant donner lieu à une activité commerciale. Sur les inconvénients des tonstrinae de plein air, (cf. Mela chez U1pien D 9.2.11 pr : si, cum pila quidem luderent, vehementius quis pila percussa in tonsoris manus ear deiecerit et sic servi, quem, tonsor habebat, gula sit croecisa sdiecto, cultello) ; toutefois, le liae selon Proculus.

401- Ulpien D 2.13.4.3: si servus argentariam faciat. Selon J. CARCOPINO, o.c., p. 213 , la démarcation était insensible entre les argentrrii qui vendaient des joyaux et ceux qui se chargealent de la banque et du change. L'argentarius de D 2.13.4.3 agit en son nom propre, I. BUTI, Studi sulla capacita patrimoniale dei servi, Camerino (1976), p. 129.

402- D 31.88.3.

403- D 14.4.5.15: servus...duas negotiationes exercebat, puta sagariom et lintearion ; on relève un affranchi marchand d'habits chez Scaeriam et lintear
vola $D 38.1 .45$.

404- Ulpien D 14.4.5.16: sed si duas tabernas eiusdem negotiationis exercuit et ego fui tabernae verbi gratia quan ad Bucinum habuit ratiocinator, alius eius quam trans Tiberim... tores (405) gérant une écurie, stabulum, une auberge, cauponi:m, un comptoir bancaire, mensa, une taberna purpuraria, ou plus généralement une taberma. Toujours générateurs de 1 'actio institoria l'esclave d'un pistor chargé de vendre le pain, des esclaves dans le commerce de l'huile, en particulier un merci oleariae praepositus à Arles, ou enfin un pollinctor, croquemort, qui, par son activité, soumet son employeur à une action quasi institoria (406).

I1 s'agit d'une catégorie seulement transitive, à un double titre. L'esclave qui travaille chez le maître est dans une situation de dépendance identique à celle d'un domestique (407). Par contre, s'il remplit sa tâche à l'extérieur, il jouit d'une certaine autonomie à l'image de celui qui, par son activité, soumet le maître à l'actio institoria, ou même tributoria ou de peculio (408). C'est dire l'impossibilité d'un traitement statistique de ces attirités somerciales en tant que telles, les textes étant rarement clairs sur le point de savoir si la fonction est exercée chez le maître ou à 1 ' extérieur (409).

405- I1 s'agit des textes suivants : Pomponius D 33.7.15pr (Servius); Marcellus D 7.8.20; Scaevola D 14.3.20; D 33.7.7; Papinien D 14.3.19.1 ; D 32.91.2 ;Paul D 33.7.13pr (Neratius); Ulpien D 4.9.7.6; D 14.3.5.3-7 (Labéon)-8 (Labéon) - 9 (Labéon)- 15 ; D 14.3.13pr (Julien)-2 (Julien) Marcien D 33.7.17.2.

406- Sur le probleme, G.LONGo, Actio exercitoria, actio institoria, actio quasi institoria, in St.Scherillo 2 (1972)p581s. ;CARRELLI, L'actio quasi insti trmia, in St.Scorza, Rome (1940).

407- L'esclave dont le métier a un caractère artisanal ou commercial fait partie de l'instromentum fundi s'il exerce a la campagne, (cf supra n 338); partie dait partie de l'attirail de la maison s'il l'exerce dans la propriété urbaine du maitre, (cf D 33.2.12.42 Ulpien). Mais il est viai que même l'institor qui se trouve dans une situation d'indépendance de fait par rapport à son maître, est coupris dans 1 'instrumentum tabernae ( $\mathrm{cf}$ supra $\mathrm{n}$ 340).

408- Cf D 14.4.1.1; D 14.4.5.15; D 15.1.27pr ;. D 15.3.7.2; sur les institores, (supra n405).

409- L'esclave exerce aussi son activitê à 1'extérieur lorsqu'il est question dans le texte d'une merces, comme c'est par exemple le cas chez Scaevola (D 36.1.80.12) a propos d'un sutor; chez Paul (D 33.7.19.1) a propos d'un ouvrier qui verse une somme annuelle à son maitre (servom vero arte fabrica peri-
twn, qui anmuam mercedem praestabat, instmonento viilae non contineri), celui- 
Il faut rapprocher des activités artisanales de l'esclave les textes où 1a seule indication est constituée par le terme artificizm (410), de même que ceux or il est question des operae fabriles vel pictorias de l'affranchi (411).

$$
-0^{-}
$$

Plus gentralement, les operae servi et liberti constituent aussi un critère d'activité économique, sans qu'il soit toutefois possible de deterainer avec précision la nature du métier exercé (412).

ci n'étant pas compris dans l'instrumentzon villae; ou encore chei Ulpien D 42.5.8.2.

410- Neratius D 38.1.50pr (affranchi) ; Valens (Pegasus) D 32.12 ; Pomponius D 6.1.29; Florentinus D 18.1.43pr; Caliistrate D 38.1 .38 .1 (affranchi) : Paul D 6.1 .31 ; D 13.7.25; Ulpien D 7.1.17.1 (Cassius, Aristo) : D 7.7.6pr (Mela) ; D 10.4.11.1 (Labéon) ; D 19.1.13.4; D 21.1.17.20; D 21.1.19.4; D 33.7.12.42 (Papinien) : D 50.15.4.5 ; Modestin D 6.1.32 ; D 48.19.31pr. On trouve Également le wot ars indiquant une activité servile chez Papinien D 37.15 .11 (affranchi) et Paul D 25.1.6 (... servos artes docuerit). Le terme professio (cf Marcien (rescrit d'Antonin le pieux) D 39.4.16.9) a une valeur cout-à-fait générale.

411- Chez Julien D 38.1.23pr, on relàve fabrilibus vel pictoriis operis, mais Également faber/pictor, ce qui explique que le texte ait étê auparavsn intégré dans le tableau concernant l'artisanat. Il faut ajouter à ce texte D 12.6.26.12 Ulpien (Celsus, Julien, Marcellus) (operas... fabriles, veluti pictorias) ; D 38.1.6 (operas fabriles) ; D 38.1 .9 .1 (fabriles). D 19.5.25 Marcien concerne des operae fabriles servi. Sur $1^{\prime}$ aff ranchi, A.GUARINO, Diritto privato cit. p291 (ou dans 1 a note 24.2 . 1, il faut lire $D 12.6 .26 .12$ et non $D$ 12.26.2) R.VISXY, Le operae, cit.,p226. Les operae officiales sont 1 iées personnellement au patron (P. PESCANI, Le operae libertorum, cit.p79), tandis que les operae au patron (P. PESCAN, Le operae libertorum, cit.p79), Candis que les operae fabriles, de nature technique, peivent etre effectueses pour d'autres avec -1 ' accord du patron (iubente patrono), étant donné qu'elles sont susceptibles d'une évaluation en argent. Les premiẻres ne nassent pas à 1 ' héritier du patron, contrairement aux secondes (E.M.SCRTAERMAN, La schiavitu, cit. p113), (cf D 38.1 .6 Fabriles (perce... ad heredem transeunt, officiales vero non transeunt). Ne anmoins, le recours a une formule stipulatoire diverse (mihi liberisque meis) de 1 a formule habituelle (mihi aut Titi.) pouvait permettre aux fils du patron de les acquérir (P.PESCANI, O.c.,p69s ; D 38.1.5). La distinction entre overae fabriles et officiales serait post-classique, selon C. COSENTINI, Studi sui liberti 2 vol. Catania, (1948-1950) p125s., mais classique selon G.LAVAGGI, Nuovi studi sui liberti, in St. De Francisci 2 (1956) p91s.

412- Certains textes concernant les operae n'ont pas été relevếs ici $\mathrm{c}$ ar
On a vu que les operae sorvi étaient généralement ssoimilées par les juristes de l'Époque classique à une sorte d'usus (413). Quelquefois, elles apparaissent come une condition d'obtention de la lilerté (414), mais

ils contiennent un indicateur plus pertinent de l'activité de l'esclave ou de 1 'affranchi. Il eat aingi des textes ou il est question des operae servi, wis qui réferent également soit au terae artificium (Ulpien D 7.7.6pr (Hela) et D 10.4.11.I (Labéon), soit au pécule de l'esclave (Julien D 15.1.37.3 ; Pomponius D 18.1.31; D 35.1.57; Tryphoninus D 15.1.57.2; D 49.15.12.11; U1pien D 15.1.19.1; D 19.1.13.13; D 44.4.4.17). De la même maniàre, des textes concer nant les operae liberti renferment une indication plus précise : c'est le cas de ceux ou il est question des operae nfficiales et fabriles ou pictoriae de 1 'affranchi (D 38.1 .24 Julien, $f a b r i l_{8 \theta}$ ) ou de ceux qui indiquent un oftier

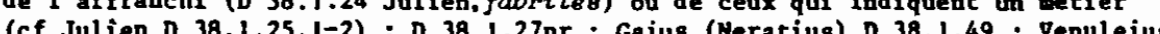
(cf Julien Do D 38.1 .38 pre vec précision, ou seulement un artificium (cf Callistrace D 38.1.38.1).

413- cf supra n30 et 31 . Les droits de jouissance des operae sont semblables a ceux de $1^{\prime}$ usus, sauf sur différents points; aucun texte ne réfảre à l'ópera si ce n'est corme créée par un legs: le bénéficiaire peut louer les operae et autoriser 1 'esclave à faire de même (Papinien D 33.2.2) ; 1'opera

n'eat pas perdue par capitis deminutio ou par non-usage (Papinien D 33.2.2 ; n'est pss perdue par capitis deminutio ou par non-usage (Papinien D 33.2 .2 ; Paul D 7.7.1); íle n'est pas perdue par la mort du bénéficiaire, mais passe a 1 'héritier (Papinien D 33.2.2) ; elle est perdue par contre si un tiers usucape jusqu'au dies venit (Paul D 7.7.1), W.H.BUCKCAND, Roman lav, cit. p370-371.

414- Par exemple D 40.7.39.3 : Dama servus cion heredi meo onnorum septen operce solverit, liber esto. Compte tenu de tels textes, la liste des références où operae constitue le seul critēte d'activité servile est la suivante : Alfenus (chez auteur anonyme) D 10.3.26; D 40.7.14pr ; Lavolenus D 40.7.39.3 (Servius, Labéon) -5 ; D 45.3.36 ; Neratius D 45.3.24 ; Julien D 22.1.25.1-2 ; D 29.2.45.1 ; D 45.1.54.1 : Pomponius D 33.1.2 ; D 35.1.57; D 43.24.21.1 (opus) ; D 45.3.39; Africain D 7.1.37; D 41.1.40 ; T.Clemens D 7.7.5 (Julien) ; Gaius D 7.7.3 ; D 7.7.4; D 7.8.13 (Labéon) ; D 10.3.11 ; D 41.1.10.3; D 45.3.28.1; Marcellus D 35.2.56.3 ; D 40.7.24 ; Scaevola D 33.7.20.2 ; Papinien D 12.6.55; D 22.2.4.1; D 33.2.2 ; D 41.2.49pr; D 45.3.18.3 (Julien) ; Tryphoninus D 41.1.63.3 ; Callistrace D 48.15.6.1 (rescrit d'Hadrien) ; Paul D 7.1.26; D 7.7.1; D 9.4.19.1; D 24.1.28.2; D 35.2.1.9 (veteres, Arigto); D 35.2.36.4 (Africain); D 40:7.4.4. D 40.7 .20 .5 ; D 40.7.41 pr (Labéon); D 41.1.47; D 41.2.1.8; Ulpien D 7.1 .21 (Labéon); D 7.1.25.2-5 (Julien) -7 (Pegasus, Julien); D 7.7.2; D 7.7.6.1-2-3 D 16.3.1.9 ; D 19.1.13.18; D 19.2.9.1 (Marcel1us) D 24.3.7.10 ; D 30.39.1 : D 33.2.7; D 39.4.12.2; D 40.1.4.10; D 40.7.3.3-8; D 40.12.12.5;D 41.1.23pr; D 43.24 .7 .1 (Neratius) 
elles ne se confondent pas alors avec les operae liberti que l'esclave, une fois affranchi, doit fournir è son vatron (415).

Ces operae doivent être exécutées lorsque le patron le désire (416)

415- Operae constitue le seul indicateur d'activité économique de 1 'affranchi dans les textes suivants : Iavolenus D $38.1 .21 ; 038.1 .33$; franchi dans les textes suivants : Lavolenus $\mathrm{D} 38.1 .21$; D 38.1 .33 ; D 38.1 .47 (Campanus) ; Julien D 38.1.11; D 38.1.23.1 ; D 38.1.25 pr-3-4; D 45.1.54.1; Pomponius D 38.1.3. pr-1; D 38.1.4 (Aristo); D 38.1.8 pr (Labéon)-1 ; D 38.1.10 pr-1 ; D 38.1.12; D 38.1.34; D 45.3.38 (Celsus); Africain D 41.1 .40 ; T. Clemens D 38.1 .14 ; $\mathrm{D} 40.9 .32 .1-2$ (nctavenus) ; Gaius D 38.1.19; D 38.1.22 pr-1 (Julien)-2; Venuleius D 40.12.44.1; Marcellus D 37.15.3 ; Scaevola D 38.1 .44 ; Papinien D $36.1 .57 \mathrm{pr}$; D 37.10 .11 ; D 38.1 .40 ; D 38.1.41 ; D 38.1.43; D 5n.1.17 pr ; Tryphoninus $D$ 37.15.10 (par extension) ; Paul D 12.2.30.4; D 12.6.65.8 ; D 37. 14.6.1 ; D 38.1.1 ; D 38.1.16 pr-1 ; D 38.1.17; D 38.1 .18 (Sabinus) ; D $38.1 .20 \mathrm{pr}-1$ (Proculus) ; D 38.1.28; D 38.1.35; D $38.1 .37 \mathrm{pr}^{-1-2}$ (Pomr ponius)-3-4-5-6 (Julien)-7-8:D $38.1 .39 \mathrm{pr}-1: \mathrm{D} 38.1 .51: \mathrm{n} / \mathrm{n} .5 .33 \mathrm{pr}$; D $45.1 .73 \mathrm{pr} ; \mathrm{D} 46.1 .56 \mathrm{pr} ; \mathrm{D} 50.16 .53 \mathrm{pr}-1 ; \mathrm{D} 50.16 .70 ;$ U1pien D 22 . $3.18 \mathrm{pr}$ (Julien); D 24.1 .9 ; (Julien); D $24.34 .2 ; \mathrm{D}$; 95 ; D 38.1 .2 $\mathrm{pr}-1$; D 38.1 .5 ; D $38.1 .7 \mathrm{pr}-1$ (Celsus) $-2-3-4$ (rescrit d' $\mathrm{Ha}$ $\mathrm{pr}-1$; D $38.1 .5 ;$
drien)-6-7-8-9; $38.1 .7 \mathrm{pr}-1$ (Celsus) $-2-3-4$ (rescrit d $\mathrm{Ha}^{-}$ drien) $-6-7-8-9 ; D$
rẽle) 1 (idem) $-2-3-4-1.9 \mathrm{pr} ; \mathrm{D} .38 .1 .13 \mathrm{pr}$ (constitution de
D $38.1 .15 \mathrm{pr}-1$ (Celsus, Papinien)

D 38.1 .29 ; D 38.1 .36 (Ĺabéon); D 38.2.1.1; D 38.2.37 pr (Julien) ; D 40.12 .12 .5 ; D 44.5.1.7; D 46.4.13 pr ; Marcien D 12.6.40.2; D 38 . $2.29 \mathrm{pr}-1$; Modestin D 38.1.31; D 38.1.32; D 38.2.33; D 46.3.77; Hermogénien $\mathrm{D} 38.1 .48 \mathrm{pr}-1-2$. Sur les operae liberti, J.N. LAMBERT, Les operae libert $i$, Contribution à 1 'histoire des droits de patronat, Paris (1934) ; G. LAVAGGI, La successione dei liberi patroni nelle opere dei liberti, in SOHI 11 (1945), p. 237-7.78; id. Nuovi studi sui liberti, in St. De Francisci 2 (1956) p. $91 \mathrm{~s}$.; A.E. GIFFARD, La portée de l'édit de operis libertorm (D 38.1.2.) in RHD 17 (1938) D.92-05; P. JAIJBERT, La lex Aelia Sentia et la locatio conductio des operae liberti, in RHD 43 (1965), p. 5-21; M. KASER, Die Geschichte des Patronatsgewalt über Freigelassene, in ZSS.RA 58 (1938), p. $92 \mathrm{~s}$. ; J.A.C. THOMAS, Locatio Freige lassene, in ZSS. RA 58 (1938), p. $92 \mathrm{~s}$. : J.A.C. THOMAS, Locatio
and opeme, in BInR $64(1961)$, p. $231-248$; c. COSENTINI, Studi sui $1 \mathrm{i}-$ and operae, in BINR $64(1961)$, P. $231-248$; C. COSENTINL, Studi sui li-
berti $1(1948), 2(1950)$, p. 105 s. et Liberti in NNDI ; F.M. De ROBERTIS Operae (d.r.) in NNDI. Sur le iusiurandzon liberti, c. TOMMLES, Sulla forma del iusiurandrom liberti, in RIDA 15 (1968) D. 461-471; G. SECRE, Sulle formule relative alla negotiorum gestio, in St. Moriani 2 (1906) p. $334 \mathrm{s.}$; id, Mutuo e stipulatio, in St. Simoncel1i (1917) p. 344 n3 ; B. BIONDI, Iudicizon operamo, in AUPE (1914): P. KRÏICER, Verweisungsedikte im prätorischen Album, in ZSS 37 (1915) p. $230 \mathrm{~s}$. ; DESSERTEAUX, Capitis deminutio 2 (1919) 1, p. 289 s. ; CHEVRIER, Du serment promissoire en droit romain (1921) p. 89 s. ; O. LENEL, EP. (3) p. $338 \mathrm{~s}$. ; E. ALBERTARIO, Sui negozi giuridici conch iusi dal liberto ontrandae libertatis causa (1916), in St. 3 (1936) p. 391 s.; C. COSENTTNI, Studi, cit. p. 160 s., $176 \mathrm{~s}$.; A. GUARINO, Dirit to privato romano, cit. p. $745 \mathrm{~s}$. Au ler ou $2 \mathrm{e}$ siècle de notre ère, on ajoute au iusiurandrom une stipulatio operamm, K. VISKY, Le operae dei liberti. cit. p. 222.

416- Gaius D 38.1.22 pr : Cun patronus operas stipulatus sit, tunc scilicet comittitur stipulatio, cum poposcerit nec libertus praestiterit. Nec interest, adiecta sint haec verba 'cum poposcero' an non sint adiecta. alind enim est de operis, aliud de ceteris rebus. Com enim operarum editio et au lieu de sa résidence (417). L'affranchi peut être tenu de tournir des operae relatives à un métier qu'il a appris depuis son affranchissement (418). Il doit pourvoir à sa nourriture et à son habillement (419). Cependant, il ne s'agit pas, comme pour les operae servi, d'une exploitation illimitée au profit du dominant. I'engagement ae l'affranchi ̀̀ des operae a déjà été limité sous la Réoublique (420). Cette orotection va être accrue sous l'Empire. Dans le cadre de sa politique nataiiste, Auguste exonère de l'obligation de fournir les operae les affranchis ayant au moins deux enfants (421). Hadrien écarte de cette obligation les esclaves affranchis par fidéicommis (422), et Marc Aur̀̀le ceux qui,

nihil aliud ait quam officii praestatio, absurdum est credere alio die deberi officiun quam quo is vellet, cui praestandum est. Les operae constituent un diumum officiom (Paul D 38.1 .1 ) et doivent donc être exécutées de jour en jour et durant la journée entière (Pomponius $D$ 38.1.3.1) Si 1 'affranchi est commum, les patrons devaient s'accorder pour déterminer le moment auquel l'affranchi exécuterait ses obligations vis-à-vis de chacun d'eux (Pomponius D 38.1.8 pr).

417- Iavolenus D 38.1.21 : Operae enim loco edi debent ubi patronus moratur, sumptu scilicet et vectura patroni.

418- Paul D 38.1.16 pr : Eius artificii, quod post manumissionem didicerit libertus, operas debebit praestare, si haec sint, quae quandoque cerit libertus, operas debebit praestare, si haec sint, quae quandoque honeste et sine periculo vitae praestantur, nec semper hae, quae
sionis tempore praestari debuerunt; Callistrate D 38.1.38.1.

419- Paul D 38.1.18 : Suo victu vestituque operas praestare debere libertum Sabinus ad edictum praetoris urbani libro quinto scribit: quod si alere se non possit, praestanda ei a patrono alimenta. L'obliga-
tion des operae est distincte de celle de fournir des aliments au patron indigent, Papinien D 38.1.41.

420- Ce fut 1 'objet de réformes prétoriennes, J. MACnUERon, Le travail des houmes libres dans 1 'Antiquité, Aix-en-Provence (1958) p. $110 \mathrm{~s}$.; $\mathrm{J}$. GAUDEMET, Institutions, p. 562 .

421- Paul D 38.1.37 pr (1ib. 2 ad legem Iuliam et Fapian) : Qui libertinus duos pluresve a se genitos natasve in sua potestate habebit praeter eum, qui artem ludicram fecerit quive operas suas ut cum bestiis pugnaret locaverit : ne quis eomm opera doni moneris aliudve quicauan libertatis causa patrono patronae liberisve eorm, de quibus iuraverit vel promiserit obligatusve erit, dare facere praestare debeto. L'affranchi qui n'a qu'un seul enfant de cinq ans est semblablement exonéré des qui n'a qu' un seul enfant de
operae, cf. Paul D 38.1.37.1.

422- U1pien D 38.1.7.4 : Rescriptum est a divo Hadriaro et deinceps cessare operarum persecutionem adversus evm, qui ex causa fideicommissi ad libertatem perductus est; (c'est D 38.1.7.4 qu'il faut li re chez E.M. SCHTAERMAN, La schiavitù, cit p. 236 , et non $\mathrm{D} 38.1 .6$ qui ne concerne pas ce problère, mais celui des operae officiales et des operae fabriles). Dans le même sens Valens (Campanus) D 38.1 .47 et Marcien D 38.2.29 pr. Cependant, Marcien (D 38.2.29.1) admet une réserve : Sed si defunctus filio suo legavit servom et rogavit, ut exm manumittat, ea mente, ut plenum ius patroni habeat, defendendom est posse evon operas iure imponero. 


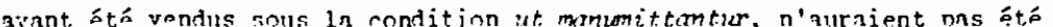
affranchis par l'acheteur of seraient ainsi narvenus ì la liherté ox constitutione (h?3). De même, le sormus suis numis omptus (hah) ne doit pas non plus d'operie (has).

La situation des affranchies est dans quelques cas flus enviahle aue celle des affranchis. L'affranchie qui a plus te cinquente ans n'est pas tenue aux operae (426), de même que colle qui s'est marióo avec l'accord de son patron (427) oll qui vit, en concubinage avec celui-ci (429). Cependant, l'affranchie est débitrice des operce envers sa natronne même après son mariage car cela n'est pas contraire à la bienséance (4zo), ̀̀ noins qu'elle ait atteint une position soriale élevée (430). Flle est alors exonóráe tans tous les cas.

Il est prócigó ágaloment que l'nn ne doit, prs tron charger l'affranchi, ni exiger de lui un travail incommatible avec sa dignité, son

423- Ulpien D 38.1.13 pr : si. mis hac lege emptus sit, ut manmittatur et ex constitutione divi Marci por"enerit ad lihertatem, operce ei imoositae nulizon effection hahebunt. De même, une constitution de Marc-Aurèle interdit à celui qui s'est porté adjudicataire des biens d'une succession, pour protéger les 1 ibertés accordées aux esclaves, de leur réclamer des pour protéger les libertés acrord
operae, cf. Ulpien D 38.1 .13 .1 .

424- Il n'apparaît nulle part comme étant af franchi ex decreto ou ex constitutione. C'est donc à tort que E.M. SCHTAERMAN; La schiavitì, cit P. 236 voit en D $38,1.13$ pr un cas de suis nummis redermptio ; il s'agit (supra) d'une vente à condition d'être aff fanchi, \%. H. BUCKLAND, git (supra) d'une vente à condi

425- Sévère et Caracalla, CI.6.3.3; Alexandre-Sévère, CI.6.3.7 ; v.W. BUCKLAND, Roman law, cit, p. 640. Cette exonération a dû restreindre considérablement 1 'usage des oporcre, la 1 iberté n'étant que très rarement concédée gratis (à tel point que l'affranchi spếcifie s'il reçoit la

liberté gratuitement, G. BOULVERT, Domestique et fonctionnaire, cit. p. 98).

426- Pau1 D 38.1.35 (1ib. 2 ad legem Iuliam et Pariam) : Liberta maior quinquaginta annis operas praestare patrono non sogitur.

427- Hermogénien D $38.1 .48 \mathrm{pr}$. Cependant si le mariage auquel le patron a consenti est nul, il pourra exiger les operae, cf. Hermopénien $D 38.1$. 48.1 .

428- Valens D 38.1 .46

429- Hermogénien D 38,1,48.2.

430- Pomponius D 38.1.34. mode de vie et sa spácialisation professionnelle (431). Les dépenses occasionnées pour se rendre à la demeure du patron afin d'y fournir les operae sont à la charge du patron (432). Si l'affranchi n'arrive olus à subvenir à ses propres besoins, le patron doit l'aider sous peine de roir s'éteindre son droit de patronat (433), corme celui qui norterait à tort une accusation capitale contre son effranchi (434) ou qui voudrait indûment transformer en argent les operae (435)

L'Empire donne en effet au devoir d'operae un caractère strictement personnel. La lex Aelia Sentia ne permet pas an patron de louer l'affranchi ou d'exiger de lui une redevance pécuniaire (436). Cette mesure, nrise dans le but de protéger l'affranchi, fut probablement gênante ì la fois pour les patrons peu aisés et pour lẹs affranchis les plus riches qui

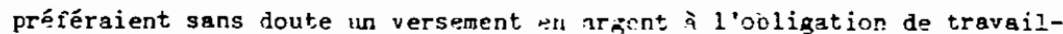
ler pour leur ancien maitre $\left(h_{37}\right)$. C'est poizrquoi la jurisprudence ra s'efforcer de la remettre en question. L'interdiction est en orrtie

43!- On ne peut imposer des operae qui portent atteinte à la dignité, (cf. par exemple D 38.1 .38 or Callistrate) ou à la vie de l'afframchi, (cf. D $38.1 .38 \mathrm{pr}$ : Paul D $38.1 .16 \mathrm{pr}$ ). On doit pareillement tenir combte de 1 'age et de la santé de 1 'affranchi, (cf. Paul D 38.1.16.1; $\mathrm{B} 38.1 .17$ ). Le patron doit permettre a l'affranchi de se soigner convenablement, (cf. Gaius D 38.1.22.2, de même que Neratius D 38.1.50.1). On doit aussi respecter la spécialisation professionnelle de l'affranchi, (cf. Neratius D 38.1.50 pr). Par ailleurs, il est préru qu' on ne doit pas interdire à 1 'affranchi d'exercer la même profession que son patron dans la même ville que 1ui, (cf Scaevola D 37.14.18 et D 38.1.45).

432- Pau1 D 38.1.20.1 : Ex provincia Libertum Romam venire debere ad reddendas operas Proculus ait : sed qui dies interea cesserint, dron Romam venit, patrono perire, dumodo patronus tanauan vir bonus et diligens pater familias Romae morretur vel in provinciam proficiscatur : ceterum si vagari per orben termarm velit, non esse iniumgendam necessitatem liberto ubique eum sequi.

433- D 38.1.18 Paul (supra n419) ; Marcien D 37.14.5.1 : Imperatoris nostri rescripto cavetur, ut, si patronus libertum suom non aiuerit, ius patroni perdat.

434- Ulpien D 38.2.14 pr : Qui, cum maior natu esset quam viginti quinoue annis, libertum capitis accusaverit out in servitutem petierit, removetur a contra tabulas bonorion possessione.

435- T. Clemens D 40.9.32.1-2 (Octavenus) : 1 - Non prohibentur lege Aelia Sentia patroni a libertis mercedes capere, sed obligare eos : itaque oi sponte oua libertus mercedem patrono praestiterit, nullion huius legis praemiten consequetur. 2 - Is, qui operas out in singulas eas certom sumam promisit, ad hanc legem non pertinet, quoniam operas praestando potest liberari. Idem Octavenus probat et adicit : obligare sibi libertion, wt mercedem operurum capiat, is intellegitur, aui hoc solum agit, ut utiaue. mersedem capiat, etiomsi sub titulo operamon pan stipulatus fuerit ;

J. GAUDERET, Institutions, cit, p. 564 .

436- P. JAUBERT, La lex Aelia Sentia et la locatio-conductio des operae liberti RHD (1965) p. 5s ; J. GAUDEMET, Institutions, cit, $\mathrm{p} .563$.

437- E.M. SCGTAERMNN, La schiavitù, cit, p. 236. 
tournée par Octavenus qui admet que le patron peut se faire promettre moyennant stipulation soit les operae, soit de l'argent (438). T. Clemens affirme que la location des operae est possible pourvu que ce soit l'affranchi qui ait demandé au patron de les louer (439). Dans ce cas, Julien soutient que la contrepartie n'a pas valeur de merces, mais représente seulement le prix des operae $(440)$. Cependant, le 3ème siècle voit ressurgir la vieille méfiance à l'égard de l'obligation à une redevance pecuniaire (441). Il est' possible que la crise économique qui secoua. alors 1'Empire ne soit pas étrangère à ce retournement, les patrons risquant de souffrir d'un paiement effectué en argent dévalué (442).

Voilà quels sont les traits essentiels de la protection de 1'affranchi. Il n'est pas étonnant de constater qu'un tiers environ des textes traitant des operae liberti concerne ce problème, dont 1'importance quantitative augmente dans la jurisprudence à partir du zème siècle (443). En effet, ces mesures sont aussi favorables aux patrons. Les operae existent dans leur intérêt économique. Il n'est nurlement dans le sens de l'institution d'imposer à l'affranchi des charges vexatoires ou de l'empêcher de se procurer grâce à son activité sa propre subistancé.

Si les derniers critères examinés manquent de précision quant $\mathfrak{i}$ 1a place des fonctions dans la hiérarchie des activités serviles, il ressort clairement de certains textes que I'esclave occupe, de par sa profession, le sommet de la pyramide.

438- Chez T. Clemens D 40.9.32.2 (supra n435).

439- D 40.9.32.1 (supra n435).

440- D 38.1.25.4 : Nonnonquan autem ipsis libertis postulantibus patroni operas locant : quo facto pretiun magis operarom quan mercedem capere existimandi surt.

441- Par exemple, Alexandre-Sévère CI.6.3.7.

442- R. VISKY, Le operae dei liberti, cit, P. 228 : R. BROSZ, Nen teljes jogu polgarok a romai jogforrasokban (=Die nichtvollherechtigten Bürger in den römischen Rechtsquellen) (avec résumé all.) Budapest (1964) p. 104. 443- Neratius D 38.1.50 pr-1 ; Celsus D 38.1 .30 pr ; Valens D 38.1.46 ; D 38.1.47 (Campanus); Julien D 38.1.25.4; Pomponius D 38.1 .34 ; T. Clemens D 40.9.32.1-2 (Octavenus) ; Craius D 38.1.19; D 38.1.22.1 (Julien)-2; mens D 40.9.32.1-2 (Octavenus) ; Caius D 38.1 .19 ; D 38.1 .22 .1 (Julien) (Sabinus); D 38.1.20 pr-1 (Proculus); D 38.1.35; D $38.1 .37 \mathrm{pr}-1-2$ (Pomponius) $-3-4-5-6$ (Julien)-7-8; D 50.16.53 pr; Ulpien D $38.1 .2 \mathrm{pr}$ :
Paragraphe 2 - Fonctions privilégiées : direction et prestige.

I - Les fonctions de direction.

Ce sont essentiellement des fonctions à caractère financier. Mais on trouve également à un moindre niveau des fonctions de surveillance.

A - Fonctions à caractère financier et de gestion.

\begin{tabular}{|c|c|c|c|}
\hline $\begin{array}{l}\text { Juriste auteur } \\
\text { du passage }\end{array}$ & juriste cité & fonction & source \\
\hline $\begin{array}{l}\text { Alfenus (chez } \\
\text { auteur anonyme) }\end{array}$ & & $\begin{array}{c}\text { dispensator } \\
\text { dispensatores/vilici/insularii } \\
\end{array}$ & $\begin{array}{l}\text { D } 11.3 .16 \\
\text { D } 50.16 .203 \\
\end{array}$ \\
\hline Julien & & $\begin{array}{c}\text { dispensator } \\
\text { actor } \\
\text { actus }\end{array}$ & $\begin{array}{l}\text { D } 14.3 .12 \\
\text { D } 34.3 .12 \\
\text { D } 40.7 .13 .2\end{array}$ \\
\hline Pomponius & $\begin{array}{l}\text { Servius } \\
\text { Labéon }\end{array}$ & $\begin{array}{c}\text { institores(443 bis) } \\
\text { disponsator } \\
\text { dispensator/insularius/vilico }\end{array}$ & $\begin{array}{l}\text { D } 33.7 .15 \mathrm{pr} \\
\text { D } 40.7 .21 \mathrm{pr} \\
\text { D } 50.16 .166 \mathrm{pr}\end{array}$ \\
\hline Africain & . & $\begin{array}{c}\text { institoria a. } \\
\text { kalendario praeposuerat } \\
\text { magistrum navis } \\
\text { institoria a. }\end{array}$ & $\begin{array}{l}\text { D } 4.4 .4 \\
\text { D } 12.1 .41 \\
\text { D } 14.1 .7 \mathrm{pr} \\
\text { D } 14.1 .7 .2\end{array}$ \\
\hline Gaius & & $\begin{array}{l}\text { a, institoria/exercitoria } \\
\text { actor } \\
\text { dispensator/cellarius }\end{array}$ & $\begin{array}{l}\text { D } 14.5 .1 \\
\text { D } 20.6 .7 .1 \\
\text { D } 40.4 .24\end{array}$ \\
\hline Maecianus & Cassius & dispensator & D 40.5 .35 \\
\hline Marcellus & & institor & D 7.8 .20 \\
\hline Scaevola & & $\begin{array}{c}\text { institoris } \\
\text { actori/vilicus } \\
\text { actoribus } \\
\text { vilico } \\
\text { institoribus } \\
\text { vilicis }\end{array}$ & $\begin{array}{ll}\text { D } & 14.3 .20 \\
\text { D } 20.1 .32 \\
\text { D } 32.41 .2 \\
\text { D } 32.41 .5 \\
\text { D } 33.7 .7 \\
\text { D } 33.7 .20 \mathrm{pr}\end{array}$ \\
\hline
\end{tabular}

D 38.1 .7 .4 (rescrit d'Hadrien); D 38.1 .13 pr (constitution de Marc-Aurèle)I (idem) ; D 38.2.1.1 ; D 12.6.26.12 (Celsus, Julien, Marcellus) ; Marcien D 12.6.40.2; D 38.2.29 pr ; Modestin D 38.1.31; D 38.1.32; D 38.1.33; D 38.1 .36 (Labéon); D 44.5.1.7 ; Hermogênien D 38.1.48 pr, ce qui représente 45 paragraphes sur un total de 131 (cf. supra n. $411,412,415$ ).

$443 \mathrm{bis}-$ Sont également mentionnées parmi ces fonctions celles renvoyant aux actions institoria et exercitoria, celles-ci étant prises pour le moment dans un sens matériel, les esclaves qu'elles concernent se trouvant dans une situation privilégiée. On s'intéressera plus loin aux problèmes juridiques que ces actions soulèvent, 


\begin{tabular}{|c|c|c|c|}
\hline & & $\begin{array}{l}\text { vilicorum/actore } \\
\text { actore } \\
\text { actores } \\
\text { vilicum/actorem } \\
\text { actores } \\
\text { actus } \\
\text { actus } \\
\text { vilicus }(-a) / \text { dispensator } \\
\text { arcarius } \\
\text { actor } \\
\text { vilicis } \\
\text { actoribus } \\
\end{array}$ & $\begin{array}{l}\text { D } 33.7 .20 .3 \\
\text { D } 33.7 .20 .4 \\
\text { D } 34.1 .18 .3 \\
\text { D } 34.4 .31 \mathrm{pr} \\
\text { D } 40.5 .41 .4 \\
\text { D } 40.5 .41 .11 \\
\text { D } 40.5 .41 .13 \\
\text { D } 40.5 .41 .15 \\
\text { D } 40.5 .41 .17 \\
\text { D } 40.7 .40 .3 \\
\text { D } 40.7 .40 .5 \\
\text { D } 40.7 .40 .8 \\
\end{array}$ \\
\hline Papinien & & $\begin{array}{l}\text { institoriae a. } \\
\text { institorem } \\
\text { pecuniis... faenerendis praepo- } \\
\text { situs } \\
\text { ad negotiationem praepositi } \\
\text { artore } \\
\text { institor } \\
\text { actor } \\
\text { actores } \\
\text { institoribus } \\
\text { actori (444) } \\
\text { actor }\end{array}$ & $\begin{array}{l}\text { D } 3.5 .30 \mathrm{pr} \\
\text { D } 14.3 \cdot 19.1 \\
\text { D } 14.3 \cdot 19.3 \\
\text { D } 16.1 .27 .1 \\
\text { D } 16.3 .24 \\
\text { D } 26.7 .37 .1 \\
\text { D } 26.7 .39 .18 \\
\text { D } 32.91 \mathrm{pr} \\
\text { D } 32.91 .2 \\
\text { D } 33.1 .10 .3 \\
\text { D } 46.3 .94 .3\end{array}$ \\
\hline Paul & $\begin{array}{l}\text { Julien } \\
\text { Pomponius } \\
\text { Proculus } \\
\text { Neratius } \\
\text { Scaevola } \\
\qquad \\
\text { Julien }\end{array}$ & $\begin{array}{c}\text { actor } \\
\text { navem exercuerit } \\
\text { institorem } \\
\text { magistrum navis } \\
\text { navem exercebit } \\
\text { navem exercuerit } \\
\text { exerceat navem } \\
\text { merci praepositus } \\
\text { vilicus } \\
\text { institorem } \\
\text { institore } \\
\text { praeposito } \\
\text { mutuis pecuniis dandis... } \\
\text { institoria a. } \\
\text { actor } \\
\text { actore } \\
\text { institores } \\
\text { vilico } \\
\text { actoris } \\
\text { actoris } \\
\text { dispensatori } \\
\text { dispensator } \\
\text { navem exerceat } \\
\text { pecuniis exigendis praepositus }\end{array}$ & $\begin{array}{ll}\text { D } & 1.18 .21 \\
\text { D } & 9.4 .19 .2 \\
\text { D } & 12.1 .29 \\
\text { D } & 14.1 .5 \mathrm{pr} \\
\text { D } & 14.1 .5 .1 \\
\text { D } & 14.1 .6 \mathrm{pr} \\
\text { D } & 14.1 .6 .1 \\
\text { D } & 14.3 .14 \\
\text { D } & 14.3 .16 \\
\text { D } & 14.3 .17 .1 \\
\text { D } & 14.3 .17 .3 \\
\text { D } & 14.3 .17 .4 \\
\text { D } & 14.5 .8 \\
\text { D } & 15.1 .47 \mathrm{pr} \\
\text { D } & 26.7 .24 \mathrm{pr} \\
\text { D } & 32.97 \\
\text { D } & 33.7 .13 \mathrm{pr} \\
\text { D } & 33.7 .18 .4 \\
\text { D } & 33.7 .22 .1 \\
\text { D } & 44.4 .5 .3 \\
\text { D } & 46.3 .51 \\
\text { D } & 46.3 .62 \\
\text { D } & 47.2 .42 \mathrm{pr} \\
\text { D } & 47.2 .67 .3\end{array}$ \\
\hline Ulpien & Labéon & $\begin{array}{l}\text { (servus exercitoris) } \\
\text { navem exercuit } \\
\text { institorem }\end{array}$ & $\begin{array}{l}\text { D } 4.9 .3 .3 \\
\text { D } 4.9 .7 .6 \\
\text { D } 5.1 .19 .3\end{array}$ \\
\hline
\end{tabular}

444- Il s'agit d'un esclave affranchi : libertis dari volo quae viva praestaban : et habitatio praestabitur : sumptus iumentorum non debebitur, quem actori domina praestare solita fuit utilitatis causa...

\begin{tabular}{|c|c|c|c|}
\hline & $\begin{array}{l}\text { Pomponius } \\
\text { Pomponius } \\
\text { Julien } \\
\text { Marcellus } \\
\text { Labéon } \\
\text { Labéon } \\
\text { Labéon } \\
\text { Julien } \\
\text { Julien } \\
\text { Marcellus, } \\
\text { Marc-Aurèle } \\
\text { rescrit de } \\
\text { Sévère et } \\
\text { Caracalla. } \\
\text { Sabinus } \\
\text { Papinien } \\
\text { rescrits de } \\
\text { Marc-Aurèle } \\
\text { et de Sévère } \\
\text { et Caracalla }\end{array}$ & $\begin{array}{c}\text { actor } \\
\text { actori } \\
\text { pecuniis exigendis praepositus } \\
\text { magister (navis) } \\
\text { qui exercet... } \\
\text { navem exercet/institorum } \\
\text { (exercitoria a) } \\
\text { navem exercuit } \\
\text { magistro (navis)/exercitore } \\
\text { navem exerceat } \\
\text { navem exercuit/magistro } \\
\text { servus...exercuit } \\
\text { institorem } \\
\text { praepositum } \\
\text { institoris } \\
\text { institorian a. } \\
\text { (institoria a.) } \\
\text { institor } \\
\text { institor } \\
\text { institorem } \\
\text { institor/exercitoriae a. } \\
\text { merci praepositum/mutuis } \\
\text { accipiendis praepositus. } \\
\text { institorem/exercitorum } \\
\text { procurator } \\
\text { actoribus } \\
\text { societati..praepositi } \\
\text {..praepcsuerint } \\
\text { negotiatoren servum(444bis) } \\
\text { actorem } \\
\text { vilici } \\
\text { cellarium } \\
\text { actor } \\
\text { calculator (4h5) } \\
\text { procurator ( } 446 \text { ) } \\
\text { institoria a. } \\
\text { actus } \\
\text { actor } \\
\text { actor }\end{array}$ & 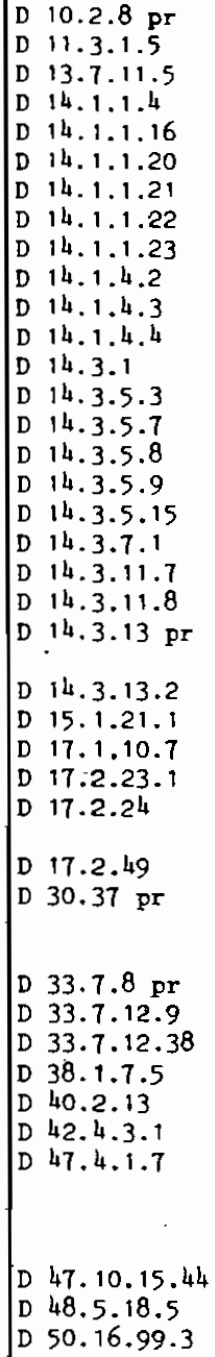 \\
\hline Marcien & $\begin{array}{l}\text { rescrit de } \\
\text { Sévère et } \\
\text { Caracalla }\end{array}$ & actores & D 19.14 .30 \\
\hline
\end{tabular}

444 his- Sur.les negotiatores, v. E.M. SCHTAERMAN, La schiavitù, cit., p. 50.

445- Ce calculator est un affrancbi, cf. D 38.1 (de operis libertomu).

446- De même que le procurator de $\mathrm{D}$ 40.2.13 : si in hoc manumittatur, ut procurator sit.

447- L'hypothèse est intêressante. Elle montre la continuitê de 1 a fonction d'esclave à celle d'affranchi : si quis servo pecuni $i$ s exigendis, proeposito solvisset pnst manumissionem, si quidem ex contractu domini, lien). Ces textes (D 33.1.10.3; D 38.1.7.5: D 40.2.13; D 46.3.18; cf.

n. $444 \mathrm{~s}$ )) ont été intégrés car ils concernent toujours, quoique de ma- 


\begin{tabular}{|l|c|c|c|}
\hline & Labéon & $\begin{array}{c}\text { negotiatoribus } \\
\text { institorem }\end{array}$ & $\begin{array}{l}\text { D } 32.65 \mathrm{pr} \\
\text { D } 33.7 .17 .2\end{array}$ \\
\hline Modestin & & actores & D 40.14 .8 \\
\hline Hermogénien & & actores & D 49.14 .46 .7 \\
\hline
\end{tabular}

Ces fonctions ont été données en bloc car, bien qu'il soit clair quelquefois que le travail est exercé à la camnagne (448), c'est notamment le cas du vilious (449), les textes sont souvent flous sur ce point.

On doit situer au sommet de la iiérarchie le procurator, fondé de pouvoir du mâtre, qui exerce le contrôle suprême (450). Vilici et actores doivent lui rendre des comptes de l'exploitation du domaine qu'ils régissent (451). Paul distingue le vilicus ordinaire, chargé de recueillir les fruits mais non d'en tiror profit. de celui qui se trouve dans une posi tion d'institor, doté de la possibilité de conclure des affaires économiques (452). Cet esclave peut soit cultiver la terre de son mâtre fide dominica, soit en prendre à bail une parcelle : c'est alors un sorvus conductor (453). Screvola est consulté an sujet d'un vilicus nour savoir

nière dérivếe, la fonction servile; E.M. SCHTAERMAN, La schiavitù,cit.p. 84, donne l'exemple d'un esclave définissant une aśfranchie comme sa conserva, compagne de servitude.

448- Par exemple, 1'actor dont il est question in $\mathrm{n}$ 20.1.32. (..eorum praediorion pars sine colonis fuit eaque actori suo colenda debitor ita tradidit adsignatis et servis culturae necessariis...) ou in D 32.41 .2 tradidit adsignatis et servis culturae necessariis...) ou in D 32.41 .2
(...Quaesitum est, com Eros et Stichus servi in diem vitae testatoris in (...Quaesitum est, cum Eros et Stichus servi in diem vitae testatoris in à 1 a campagne. Mais on sait par ailleurs qu'il peut résider en ville, cf. Scaevola D 33.7.20.4 (Idem quaesitit in actore legato, an uror et filia legato cedant, cum actor non in praediis, sed in civitate moratus sit...).

449- J. MARQUARDT, vie privée, cit, p. 153 ; E.M. SCHTAERMAN, La schiavitĩ, cit, p. 38, signale qu'i1 n'était pas bon de donner cette charà à un esclave urbain de peur qu'il regrette les plaisirs de la ville et qu'il corrompe ainsi la fomilia, cf. Hor., Ep., I, 14.

450- Colum, I, 6, 7 : P1in., Ep., III, 19, 2.

451- J. MARQUARDT, Vie privée, cit, p. 162-163; G. BOULVERT, Esclaves et affranchis impériaux sous le Haut-Empire romain, Naples (1970) : aucun dispensator ou arcarius $n$ 'est homme libre alors que les vilici le sont parfois (p. 198) : il s'agit 1à de vĩici impériaux.

452- D 14.3.16 : Si com vilico alicuius contractum sit, non datur in dominum actio, quia vilicus propter fructus percipiendos, non propter quaestion praefonitur. Si tumen vilicum distrahendis quoque mercibus praepositum habuero, non erit iniqum exemplo institoriae actionem in me competere; sur les pouvoirs du vilicus, F.M. DE ROBERTIS, Lavoro, cit, p. $110 \mathrm{~s}$ 453- E.M. SCHTAERMAN, La schiavitù, cit, p. 43 ; M. JACOTA, Les transformations de 1 'économie romaine, cit, p. $376 \mathrm{~s}$. Un tel esclave se trouve à la tête de moyens de production, E.M. SCHTAERMAN, o. c. D. 339. Sur le thème,

F. DE HARTIHO, Storia ecoromica, cit. II, p. 285 . s'il fait partie de l'instrumention frondi (454). Si l'esclave viizicus cultive moyennant une certa pensio payée à son maitre, il n'en fait nas partie. La solution est différente pour celui qui cultive fide dominica (455). Ce sont des considérations sociales qui fondent le shoix du juriste, et cela incite à rapprocher des textes donnés dans le tableau ceux où il est question d'un esclave conductor (456) ou quasi colonus (457).

Bien qu'actor et vilious désipnent dans un texte de Scaevola deux personnes différentes (458), les deux termes sont généralement synonymes (459); comme le vilicus, l'actor est parfois simple administra-

454- Scaevola chez Paul D 33.7.18.4 : Con de vilico ouareretur et an instrmento inesset et dubitaretur, scaevola consultus respondit, si non pensionis certa quantitate, sed fide dominica coleretur, deberi.

455- Dans ce cas, il n'y a pas de convention entre les parties, mais seulement un acte de volonté unilatéral du dominus, M. JACOTA, Les transformations de l'économie romaine, cit, p. 379. L'opposition ressort aussi de $D$ 33.7.20.1 Scaevola : ... Respondit, si non fide dominica, sed mercede, ut extranei coloni solent, fundum coluisset... Sur $1 \mathrm{e}$ problème, M. HORVAT, ut extroidi ervi, in st Volterra 5 (1971) P. 89-07, spécialement

p. $95-96$.

456- A1 fenus D 40.7.14 pr : ... si fundron a domino conduxisset ; et D 15.3.16: Quidam fundron colentum servo suo locavit... Du point de vue économique, cette opération entre le maître et son esclave se présente sous 1a forme d'une locatio, mais il ne s'agit pas d'un contrat, $1^{\prime}$ 'esclave étant juridiquement incapable, M. JACOTA, Les transformations de l'économie romaine, cit, p. 376 .

457- D 33.7.12.3 U1pien : Quaeritur, an servus, qui quasi colonus in agro erat, instrumento legato contineatur. Et Labeo et Pegasus recte negavemunt, quia non pro instrumento in fundo fuerat, etiamsi solitus fuerat et fomiliae imperare. M. I. FINLEY, L'économie antique, cit, p. 81, fuerat et folliae imerase. $M$ i colonus ou du tiher homo bona fide semiens parle, a propos du servif quasi colonus ou du tiker ho

p. 339 .

458- D 20.1.32 : Debitor pactus est, ut quaecromque in pratdia pignori data inducta invecta importata ibi nata paratave essent, pignori essent eorum praediomm pars sine colonis fuit eaque actori suo colenda debitor ita tradidit adsignatis et servis culturae necessariv : quapitur on et ita tradidit adsignatis et servis culturae necessaris : quaemitur, an et Stichus vilicus et ceteri servi ad culturam missi et Stichi viearii obliuati essent. Respondit eos dumtaxat, qui hoc animo a domino inducti essent, non temporis causa accomodarentur, obligatos. Sur ce texte, F. DE MARTINO, Storia economica, cit. II, p. $282 \mathrm{~s}$.

459- Par exemple, du même scaevola, D $34.4 .31 \mathrm{pr}:$... sed cum in altero ex fundis filio praelegatis cognovisset vilicum nor esse, Stichon misit et tan rei rusticae quam rationibus fundi praefecit : ... Stichw his praediis, in quae translatus est, actorem cedere. Les deux mots ne sont pas synonymes originairement, J. MARQUARDT, Vie privée, cit, p. $163 \mathrm{nl}$. 'actor est un chargé d'affaires, un caissier. Paul (D 44.4.5.3) fait llusion a cetce fonction. Actorig qui exigendis pocunis praencsitus est. etion a ceterio dans cette rubrique les textes oil il est question d'un esclave exicendis 
teur, mais il peut aussi être sır le fonds en tant que locataire (460).

Le dispensator occupe de même in poste imbortant (461). Il est chargé d'opérations financières et commerciales (462). Il exerce ses fonctions aussi bien en ville qu'à la campagne (463). Le cellarius, dont le rôle est de contrôler l'exactitude des comptes (464), lui est subordonné (465). L'arcarius, simple caissier, agit aussi sous sa responsabilité (466).

Quant '̀ l'insularius, sa tâche est proche, selon Pomponius, de celle de vilicus. Il entretient ct exploite les insulae possédées par son maître à Rome (467).

pecuniis praepositus (cf. tahleau). Sur les actores de domaines impériaux, G. BOULVERT, Esclaves et af franchis impériaux, cit, p. 434-435, donne des exemples ; n355, thermogénien D 49.14.46.7; Modestin D 49.14.8 sur ce point, G. LE BRAS, L'évolution génêrale du procurateur en droit privế romain des origines au IIIIème siẹcle, Paris (1922), p. 75.

460- Dans D 33.7.20.3 (Scaevola : Praedia ut instructa sunt com dotibus et religuis colonorm et vilicorwon et mancipiis et pecore ommi legavit et peculizis et crem actore...), l'actor est un administrateur tandis que les vilici interviennent en tant que locataires, tout comsne les colons, cf. E.M. SCHTAERMAN, La schiavitù, cit, p. 44 ; mais dans d'autres cas 1 es actores louent le fonds, ainsi D 32.91 pr Papinien (Praediis per praeceptionem filiae datis cuon reliquis actorom et colonorm ea reliqua videntur legata, quae de reditu praediomon in eadem causa mansuernint...). L'auteur (ib. p. 45) pense qu'au début du 3ème siècle, actor et colon sont devenus deux figures juridiques identiques.

461 - E.M. SCHTAERMAN, La schiavitù, cit, p. 82, estime qu'ils sont au sommet de la hiérarchie domestique, cf. Sen., De Ben., III, 28.

462- C'est un caissier par excellence, Gaius I, 122 ; Pétrone, Sat., $\mathrm{xxx}$, 9. Sa fonction de receveur explique sa condition. Etant esclave, i1 n'a pas de personnalité juridique, et la réception des paiements à laquelle i1 procède produit des effets directement dans la personne de son maître, G. BOULVERT, Esclaves et affranchis impériaux, cit, p. 430 ; Th. MOMMSEN, sous CIL, V, 83, explique au contraire son statut par la volonté de pouvoir livrer a la quaestio le dispensator infidèle. Il n'est pas ordonnateur, Gaius III, 160 ; Paul D 46.3.51 et 62 .

463- Pomponius D 50.16.166 pr : Urbana familia et mustica non loco, sed genere distinguitur : potest enim aliquis dispensator non esse servorm urbanorm nomero : veluti is, qui rusticarion remom rationes dispenset ibique habitet.

464- Ulpien D 33.7.12.9: Cellararizon quoque, id est ideo praeposition, ut rationes salvae sint...

465- J. MARỌUARDT, Vie privée, cit, p. 182 n/4.

466- G. BOULVERT', Esclaves et affranchis impériaux, cit, p. 429 et

n 347 ; F. FUCHS, D.E. I, art, arcarius, p. 634.

467- Pomponius D 50.16.166 pr : ... Non multron abest a vilico insularius; autem urbanorim numero est.
Dans le prolongement de ces fonctions de direction, le Digeste nous indique quelques fonctions de surveillance.

\section{B - Ponctions de surveillance}

\begin{tabular}{|c|c|c|c|}
\hline $\begin{array}{l}\text { juriste auteur } \\
\text { du passage }\end{array}$ & juriste cit & fonction & source \\
\hline $\begin{array}{l}\text { Alfenus (chez } \\
\text { auteur anonyme) }\end{array}$ & & atrienses & D 50.15 .203 \\
\hline Pomponius & & $\begin{array}{c}\text { mulier villae custos.../ } \\
\text { agri } \\
\text { custodiam/saltuarius }\end{array}$ & D 33.7 .15 .2 \\
\hline Gaius & Neratius & custodiae domus $(1+68)$ & D 33.1 .49 \\
\hline Sca evola & & $\begin{array}{c}\text { saltuariis } \\
\text { custodiam templi }\end{array}$ & $\begin{array}{l}\text { D } 33.7 .20 .1 \\
\text { D } 34.1 .27 \\
\end{array}$ \\
\hline Paul & $\begin{array}{l}\text { rescrit } \\
\text { d'Antonin } \\
\text { le pieux } \\
\text { Alfenus }\end{array}$ & $\begin{array}{l}\text { servis custoribus (cella/ } \\
\text { armarium/arca) } \\
\text { saltuarjus }\end{array}$ & $\begin{array}{l}\text { D } 1.15 .3 .2 \\
\text { D } 32.60 .3\end{array}$ \\
\hline Ulpien & $\begin{array}{l}\text { Sabinus } \\
\text { Labéon, Nera- } \\
\text { tius } \\
\text { Neratius }\end{array}$ & \begin{tabular}{|c} 
atriensem \\
monitores \\
saltuarii/atrienses \\
saltuarius \\
custodiam(ve) villae
\end{tabular} & $\begin{array}{l}\text { D } 7.1 .15 .1 \\
\text { D } 33.7 .8 \mathrm{pr} \\
\text { D } 33.7 .8 .1 \\
\text { D } 33.7 .12 .4 \\
\text { D } 33.7 .12 .35\end{array}$ \\
\hline Marcien & Labéon & saltuarius & D 33.7 .17 .2 \\
\hline
\end{tabular}

L'atriensis est chargó de surveiller 1 'entretien de la maison et l'ameublement (469). Les monitores appartiennent à la fomilia mustica (470). Ils sont l'équivalent des decuriones (471) placés ì la tête des decuriae, subdivisions de la domesticité lorsqu'elle est nombreuse (472). Les

468- Il s'agit d'un affranchi.

469- J. MARQUARDT, Vie privêe, cit, p. 167.

470- Colum, I, 9,4 et 7 ; Paul, Sent., III, 6, 35.

471- Sen., Ep., XuVII, 9 ; Colum. I,9,7 ; sur la familia urbana, Petr., Sat. 47 ; Suet., Dom., 17 ; CIL, VI, 8773, 8914, 9093.

472- J. MARQUARDT, vie privêe, cit, p. 180-181, selon qui, sous l'Empire, les grandes familles organisent leur domesticité sur le modèle des collèges urbains (P1in., Ep., VIII, 16,2). Chaque classe a un chef (magister dans la fami lia mustica, Colum, I,8,17, qui n'apparaît pas dans nos sources) et se divise en decuriae si le nombre l'exige. 
saltiarii, moins importants, sont chargés de surveiller la produc-

tion (473).

Peu nous importent les fonctions visant la garde ou l'entretien de biens mobiliers ou immobiliers. Il s'agit là de fonctions domestiques ne soulevant pas de problèmes particuliers. La surveillance du personnel servile est par contre du plus grand intérêt, et elle évoque uniquement la vie rurale. On peut d'ailleurs légitimement penser que les maitres ont rencontré plus de difficultés à la campagne qu'en ville, où l'exploitation était plus "libérale" (474).

On est surpris de prime abord par la faible représentation du travail de surveillance, mais ce n'est qu'une apparence. Il ne fant pas oublier en effet que travail de direction et travail de surveillance se situent du même côtế de la division sociale du travail (475) et que, plus précisément, le régisseur du domaine, le vilicus, exerce la surveillance au plus haut niveau (476). C'est même dans le système esclavagiste que le travail de surveillance atteint son maximum, l'opposition y étant la plus grande entre le producteur direct et le propriátaire des moyens de production (477). Cette surveillance est telle que l'exploitation rurale, le fait ne manque pas d'interêt, peut fonctionner en $l$ 'absence $d$ 'hommes libres $(478)$ !

Toutefois, pour que la surveillance s'exerce dans de honnes conditions, mieux vaut avoir affaire à un esclave "soumis". Les juristes nous

473- A la différence des gardiens qui doivent protéger les limites de la propriété, cf. Ulpien D 33.7.12.4 : saltuarizm autem Labeo quidem putat evon demum contineri, qui fructuon servandorum gratia paratus sit, eum non, qui finium custodiendomm causa.

474- Au ler siècle de notre ère, époque à laquelle il semble que le travail des esclaves atteigne son niveau maximal dans la campagne italienne (E.M. SCHTAERMAN, La schiavitù, cit, p. 31), Columelle se fait l'écho des difficultés des propriétaires (vols, I, I ; appauvrissement de la terre par négligence des esclaves, I, 8).

475- N. POULANTZAS, Les classes sociales, cit, p. 240 s.

476- Cf. Colum, I, 8 et XI.

477- Sur le problème de savoir si les frais de surveillance n'altèrent pas la rentabilité du système, P. DOCKES, La libération, cit, P. $160 \mathrm{~s}$

478- P. DOCKES, La libération, cit, p. 89. apprennent d'ailleurs qu'il est préférable d'acheter un esclave débutant, novicius, plutôt qu'un esclave expórimenté, veterator (479). Le savoir fait peur (480).

\section{II - Fonctions intellectuelles et fonctions de prestige}

\section{A - Fonctions intellectuelles}

\begin{tabular}{|c|c|c|c|}
\hline $\begin{array}{l}\text { juriste auteur } \\
\text { du passage }\end{array}$ & juriste cité & fonction & source \\
\hline Pomponius & & interprete (481) & D 49.15 .5 .3 \\
\hline Gaius & & $\begin{array}{l}\text { librarium } \\
\text { librarius (482) }\end{array}$ & $\begin{array}{l}\text { D } 6.1 .28 \\
\text { D } 38.1 .49\end{array}$ \\
\hline Venuleius & Caelins Sabinus & liberalibus st,udiis erudjt tus & D 21.1 .65 .2 \\
\hline Maecianus & Cassius & paedagogus & D 40.5 .35 \\
\hline Florentinus & & litteratum & D $18.1 .43 \mathrm{pr}$ \\
\hline Scaevola & & notarium & D 40.5 .41 .3 \\
\hline
\end{tabular}

479- On trouve une définition de ces termes dans D 21.1.65.2 venuleius Servus tam veterator quam novicius dici potest. Sed veteratorem non spatio serviendi, sed genere et causa aestimandron Caelius ait : nam quicronque ex venalicio noviciomom emptus alicui ministerio praepositus sit, statim eum veteratomom numero esse : novicium autem non tironicio animi, sed condicione servitutis intellegi. Nec ad rem pertinere, Latine sciat nec ne : nam ob id veteratorem esse, si liberalibus studiis eruditus sit. On n'est pas veterator du seul fait de son érudition dit la fin du passage : c'est par veterator du seul fait de son érudition, dit la fin du passage : c'est par a contrario un facteur a prendre en considération. Mais plus intéressant est 0 . novicius constitue une bien meilleure affaire, car il est encore maléable : Praecipiunt aediles, ne veterator pro novicio veneat. Et hoc edictum fallaciis venditorion occurrit : ubique enim curant aediles, ne emptores a venditoribus circumveniontur. Ut ecce plerique solent mancipia, quae novicia non sunt, quasi novicia distrahere ad hoc, ut pluris vendant : praesumptrom est enim ea mancipia, quae rudia sunt, simpliciora esse et ad ministeria aptiora et dociliora et al onme ministerium habilia : trita vero nor cipia et veterana difficile est reformare et ad suos mores formare. Quia igitur venaliciarii sciunt facile decurri ad noviciorum emptionem, idcirco inter polant veteratores et pro noviciis vendunt. Quod ne fiat, hoc edicto aediles denuntiant : ot ideo si quid ignorante emptore ita venierit, redhibebitur.

480- La stupidité est voisine de la soumission, 1'intelligence rique au contraire de favoriser les méfaits, cF. encore au 4 ème siècle Palladius, rapporté par E.M. SCHTAERMAN, La schiavitù, cit., p. 40.

481- Il s'agit d'un ennemi capturê par les Romains.

482- Ce librarius est un affranchi (D 38.1.49). 


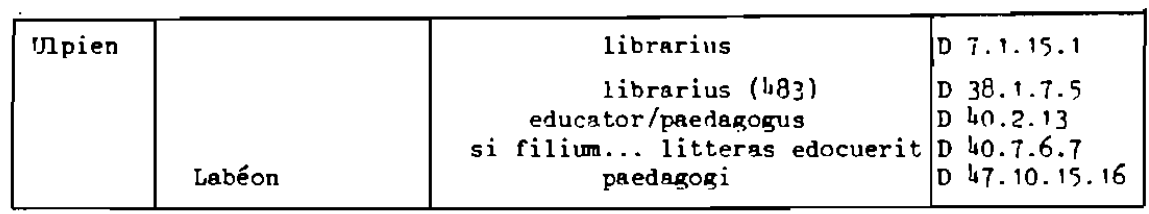

Bien que le travail de l'esclave soit la plubart du temps physique, l'esclave est capable de travail intellectuel (48b), rais son activité est considérée, même dans ce cas, comre un travail physique (489). Malgré sa valeur supérieure, son travail est en effet rabaissé par sa condition d'esclave (486).

L'enseignement, grammaire et rhétorique, a été tout d'abord le fait d'étrangers, grecs notament, parmi lesquels beaucoup d'affranchis et d'esclaves (487). Il ne s'agit pas d'un ars liberalis, qui seul est digne

483- de même que celui de D 38.1.7.5. On a vu que le travail obligatoire de l'affranchi pour son ancien maître concerne toujourg 1 'esclavake. C'est la conséquence de son ancien état d'esclave. Ce travail présente d'ailleurs les mêmes caractéristiques que celui de $1^{\prime}$ esclave, A. PERNICE, Parerga, zSS 9 (1888), p. 243 ; G. HUMBERT, Artifices, in D.S. I p. 446 ; A. BERNARD, La rémunération des professions libérales en droit romain classique, Paris (1935), p. 121 ; L. MITIEIs, Operae officiales und operae fahriles, 2ss 13 (1935), p. 121 ; L. MITTEIS, Operae officiales und operae fahriles, 2SS 13
(1892) p. 158; 0. RARLOWA, Römische Rechtsgeschichte, II, Leipzig (1901) (1892) p.
p. 642 .

484- Selon E.M. SCHTAERMAN, La schiavitu, cit., p. 5, le nombre des intellectuels d'origine servile aurait diminué sous l'Empire. La faible représentation du thème ne permet pas de vêrifier cette assertion. 485- Ce n'est que lorsque le travail des esclaves se fit prépondérant
que le mépris des houmes libres pour le travail physique devint plus fort, que le mépris des houmes libres pour le travail physique devint plus fort,
$\mathrm{J}$. MACOUERON, Le travail des hommes libres dans l'Antiquité romaine, AixJ. MACOQUERON, Le travail des hommes libres dans l'Antiquité romaine, Aix
en-Provence (1954-55), p. 54-55; cf. Cicêron, De officiis, I, 42, 150, ou Pro F1acco, 8, 17. L'homme libre qui s'oblige a un travail salarié, même provisoirement, se place dans une situation d'assujettisement comne un esclave, R. JHERING, Der Zweck im Recht, Leipzig (1884), vol. I p. 107 ; J. MACQUERON, o.c., p. 193 ; E. CUQ, Honororizm, D.S. V, p. 23\%g. Le salaire qu'il reçoit, meroes, exprime la servitude, F.M. DE ROBERTIS, Lavoro, cit, p. 81-82; DE MAGALHAES - VILHENA, Essor scientifique et technique et obstacles sociaux a la fin de 1 'Antiquité, Paris (1966), p. 9-10 ; cf. Cicéron, De officiis, $I, 42,150$.

486- K. VISKY, Esclavage et artes liberales a Rome, RIDA 15 (1968) p. 474. 487- Suétone, De gramm. et rhet., 5.1, 6.1, 10.1, 11.1, 12.1, 13.1, 15.1 $16.1,17.1,18.1,19.1,20.1,23.1,27.1$; A. BERNARD, La rémunération des professions libérales, cit, p. 25s. Ceci explique sans doute le mêpris des Romains pour 1 'enseignement (Cicéron, De oratore 48,212 ); R. VISKY, Esclavage et artes liberales, cit, p. 479 ; J. MAROUARDT (sur i'enseignement), Das Privatleben der Römer, Leipzig. (1886), p. 105-106 ; gur le thème, J. KOLENDO, Intellectuels et couches serviles : le cas des gramr mairiens chez Suétone, in Index $8(1978 / 79)$, p. 214, qui souligne que, sur vingt biographies des grammairiens rêunies par Suétone, quinze ou seize vingt biographies des grat
sont celles d'esclaves. d'un home libre (428), mais d'un artificion (480).

On peut aussi considérer les secrátaires, librarius, ratarius (loon), come exerçant une fonction intellectuelle. Leur travail rosuiert ies artitudes intellectuelles évidentes : il faut au moins savoir lire ot, érrire. Il n'est pas inutile de s'attarder quelque peu sur cet, asnect du probl ̀̀me.

Pour toutes les fonctions comptables auparavant signalóes, il est certain que l'esclave sait compter et, il serait. superflu de dóvelopoer ce point (401). Par contre, dans de nombreux textes sù n'apparâ̂t pas directement l'activité professionnelle de l'esclave, on possède une indication permettant de savoir qu'il détient sette frculté. Il en est ainsi lorsqu'il

488- Cicéron, De officiis, I, 150-151; Sénèque, Ep. ad Lucilium, 88.1. 2.20 ; Quintilien, Oratoriae institutio 12.1. Proem. 1 ; Ulpien D 50.13.1 pr : ... Liberalia autem studia amipimus... : rhetores continebuntur, grcomatici, geometrae ; ce texte est tenu pour interpolé par H. SIBER, operae iiberales, Jherings Jahrbücher für die Dogmatik des bürgerlichen Rechts $(1939-40)$ p. 189 ; F. KÜHNERT, Allgemeinbildung und Fachbildung in der Antike, Berlin (1961), p. 28-29, compte parmi les artes liberales la gramaire, la dialectique, la rhétorique, la musique, l'arithmétique, la grammaire, la dialectique, la rhétorique, la musique, l'arithmétique, la
géométrie et l'astrologie, à partir des écrits de Cicêron (De or. I, q-ıl; géométrie et l'astrologie, à partir des écrits de
III, 58 et 127 ; De fin. I, 26 et 72 ; III, 3-5).

489- Gaius, Inst. I, 29 ; Paul D 17.2.71 pr : Duo societatem coierunt, ut grommaticcon docerent et quod ex eo artificio quaestus fecissent, conmune erorum esset... Seul i'enseignement portant sur la culture de la grammaire et de la rhétorique à un degré plus élevé, en particulier dans les universités, était considéré comme ars líberaliss, K. VISKY, Esclavage et artes liberales, cit, p. 479 ; A. GUDEMAN, Grammatik, RE VII (1911), p. 1211 ; A. STEINVENTER, Rhetorik und Zivilprozess, ZSS 65 (1947) p. 72 ; H. PARKER, The seven liberal arts, The English Historical Review, 5 (1890) p. 453.

490- Sur notarius, Pline, Ep. IX, 36, 2 ; Auson., Epigr., 146 ; CIL, VI, 9704 s. ; X, 1932, 4789. Sur lihrarius, Plin., N.H. VII, 91 ; CIL VI, 8435, 8450b. On trouve aussi d'autres désignations : amanuenses (Paul, Sent. III, 6, 70 ; Suet., Ner. 44, Tit. 3 ; CIL VI 9542) ; a manu servi (Suet., Caes. 74 ; Or. 2733, 2931, 5394, 6651, 7189 ; CIL, vI8886 s., $9534 \mathrm{s.}$ ) ; ad manion (ib. 4449); a comentariis (ib. 8623 s.) ; a studiis (0r. 719, 2958) ; librarius a manu (CIL, VI, 6314, 9524) ; librarius ad maniom (ib. $9523=0$ r. 2874), mais elles n'apparaissent pas dans le Digeste. Sur librarius, BIBABEL, RE XIII p. $137 \mathrm{~s}$. ; D.E. IV art. librarius p. $956 \mathrm{~s}$. Chez les empereurs, on relève les fonctions de a libellis et de $a b$ epistulis, J. MARQUARDT, Vie privée, cit, p. $177 \mathrm{n} 4$; L. FRIEDLANDER, Darstellungen, I (5) p. 94s, et 157s.

491- Il est bien entendu qu'actor, dispensator, vilicus, institor, et en général celui qui possède un pécule ont cette faculté, de même que l'esclave dont parle Ulpien (D 11.3.1.5) qui tient les rationes dominicas; de même $D$ 9.2.23.4 (Labéon) et D 10.4 .3 .14 (Julien); aussi D 14.4.5.13 (Labéon) où l'esclave tient le registre de la boutique. 
doit rendre les comptes à son maître, notamment pour parvenir à la liberté (492), lorsqu'il calcule la dette de son maître (493) ou est capable de décourrir une fraude du tuteur (hgh), ou encore lorsqu'il est employé dans la perception des impôts (495) ; de même s'il altère les comptes de son maitre (496). De plus, il est probable que l'esclave qui vole le registre des comptes de la succession (497) et celui qui fabrique de fausses pièces d'or (498) savent pareillement compter. Ces esclaves savent très probablement lire et écrire, mais on relève d'autres textes d'oì ces aptitudes ressortent clairement (499). Particulièrement intéressants

492- Celsus D 40.7.23.1 ; Iavolenus D 40.7.28 pr ; Julien D 40.4.17.1 ; D 40.5.47.2 ; D 40.7.12; D 40.7.13.2 ; Pomponius D 35.1.111; D 35.1.112. 3 (Neratius) ; $\mathrm{D} 40.7 .5 \mathrm{pr}$ (Aristo, Neratius); de même D 40.4.8 ( $\mathrm{si} \mathrm{rq}^{-}$ tiones diligenter tractasse) et $\mathrm{D} 40.7 .21 \mathrm{pr}$ (veteres, Labéon) (idem) ; Africain D 35.1.32; D 40.4.22 (rationes argenti) ; Gaius D 30.69 .4 (à propos d'un legs), ainsi que D 40.7.31 pr ; Scaevola D 21.2.69.4; D 33.8.23 pr;D 34.3 .31 .1 (affranchi); D $34.4 .31 \mathrm{pr} ; \mathrm{D} \mathrm{40.4.59.2;} \mathrm{D} \mathrm{40.5.18;}$ D 40.5.41.7; D 40.5.41.8-9 (deductio peculit) ; D 40.5.41.10-11-12-13-16D 40.5.41.7; D 40:5.41.8-9 (deductio peculiz) ; D 40.5.41.10-1)-12-13-1517 ; D 40.7.40 pr-4-6-7-8 ; Papinien D 40.5.23 pr ; D 40.7.34.1 (rescrit de Caracalla) ; Callistrate D 35.1 .82 ; Paul D $35.1 .81 \mathrm{pr}$; D 40.4 .53 ; Ulpien D 18.1.7 pr (veteres) (à propos d'une vente); D 27.3.1.3 (décret de Sévère) (ut rationes a servis qui rem gesserant proferantur) ; D 33.8 . 8.7 (rescrit de Sévère et Caracalla) ; D 35.1 .50 (Antonin le pieux) ; D 40.4.13.2 (Julien) ; D 40.5.37 (rescrit de Marc-Aurèle) ; D 40.7.6.7; D 40.12.34 (constitution de Caracalla) ; Marcien D 30.119 ; D 40.1.5.1; Modestin D 35.1.53 ; D 40.7.26 pr-1 ; aussi 1e texte de Papinien D 33.8.19 pr (où l'esclave est affranchi s'il fait une déclaration des effets composant son pécule), et Tryphoninus (Scaevola) D 34.3.28.7 par a contrario (Sticho testamento mantomisso functom instructum et alia iegavit et haec verba adiecit : quem rationem reddere veto...).

493- Scaevola D $46.3 .89 \mathrm{pr}$.

494- Iul ius Aquila D 26.10.12.

495- Ulpien D 39.4.1.5; D 39.4.3 pr ; D 39.4.12.2, cF. D 39.4 : De publicanis et vectigalibus et comissis.

496- Upien D 11.3.11.1; v. aussi Julien D 17.1.30.

497- Gaius D $30.67 \mathrm{pr}$.

\section{8- Ulpien D 48, 10.8.}

499- Dans deux textes signalés à propos de la faculté de compter, il apparaît explicitement que 1 'esclave sait également écrire : scaevola D $46.3 .89 \mathrm{pr}$ (... Qunesitum est, an ex ceteris chirographis, quae non Stichus cavit...) et Ulpien (Julien) D 10.4.3.14 (... Servus, inquit, uxo$r i s$ meae rationes meas conscripsit...). Les fonctions indiquées dans le tableau parlent d'elles-mêmes, tes autres textes oủ ces facultés apparaissent sont D 46.3.102.2 Scaevola : (Vaterius Lucii Titii servus scripsit...) Callistrate D 48.10 .15 pr (édit de Claude) : (... scribere autern sibi legatwm videri non solum oum qui manu sua id facit, sed etiam qui por semmon tum videri non sol.rm oum qui manu sua id facit, sed etiam qui por semnon sum vel filiw, quem in potestate habet, dictante testatore legato hono-
ratur) ; D 48.10.15.1 (Plane constitutionibus principalibus cavetur, ut, ratur) ; 1 itator specialiter subscriptions sua declaraverit dictasse servo alicuius...) ; D 48.10.15.2 (Item senatus censuit, ut, si servus domini sui iussu testamento ondicillisve libertatem sibi adscripserit...) ; $\mathrm{D} 48.10 .15$. sont les paragraphes de Callistrate, ol̀ le juriste śvoque les escificos rédigeant sous la dictée le testament de leur mâ̂tre (500). Ils prennent du relief si on les rapproche d'un texte de paul dans lequel il ́́voque un maître ne sachant pas écrire (501). Une telle situation ne rófòre tonc pas forcément à la confiance qu'a le maître en son esclave, mais ì l'ignorance de ce maître, probablement "nouveau riche", dépossédé de la culture au profit de celui qu'il possède. Certains passages, qui concernent l'instruction des esclaves, sont aussi une allusion à leurs capacités intellectuelles (502).

Comme les fonctions intellectuelles, les fonctions de prestige occupent une place appréciable dans la hiérarchie domestique.

\section{B - Fonctions de prestige}

Il faut distinguer les fonctions liées aux plaisirs du maître de celles qui ont un rôle beaucoup plus utilitaire.

3 (rescrit d'Antonin le pieux) (...Nan servos, cum dominis suis parent, necessitate potestatis excusari, si tamen accedat domini auctoritas subscribentis se ea dictasse et recognovisse...) ; D 48.10.15.4 (Matri quoque, cui per servum sum dictante filio legatum scriptum esset...) ; Paul D 45. cui per servm sum dictante filio legatwo scriptum esset...) ; Paul D 45 . 9 (...Servus, qui alieno testamento fideicommissam libertatem sibi adscripsit): Ulpien D 47.2.52.23 (Mela) (Si quis servo meo persuaserit, ut sit) : Ulpien D 47.2.52.23 (Mela) (Si quis servo meo persuaserit, ut
nomen surm ex instmomento puta emptionis tolleret); D 47.2 .52 .24 (Sed si nomen sulm ex instmonento puta emptionis tolleret) ; D 47.2.52.24 (Sed
servo persuasum sit ut tabulas meas describeret) ; Macer D 48.10.10 pr servo persuasw sit ut tabulas meas describeret) ; Macer D 48.10.10 Pr
(...si filius servusve sibi adscripsissent); Marcien D 48.10.1.8 (Inter filium et servum et extronew testamentw scribentes...) ; D 48.10.1.13 (Poena falsi vel quasi falsi deportatio est et omnium bonorm publicatio : et si servus eorm quid admiserit, ultimo sipplicio adfici iubetur).

500- D 48,10,15.1-3-4 (supra n499).

501- Il s'agit d'un maître muet. Il peut donner 1 'ordre à son esclave d'accepter une succession par un simple signe, mais il est préférable qu'il sache écrire car le message sera alors transmis à l'esclave sans qu'il sache écrire car le message
difficultê, cf. Paul D 29.2.93.1.

502- Scaevola D 33.7.20.6 : ... Quaesitum est, si Stichus servus, ex eo fundo ante annom mortis testatoris abductus et in aisciplinom traditus, postea in evm fundum non reversus sit, an debeatur. Respondit, si studend $i$ causa misisset, non quo a fundo ewn aliorsum transferat, deberi ; Paul causa misisset, non quo a fundo ewn aliorsum transferat, deberi ; Paul emptor fecit... ; Ulpien D 19.5 .13 .1 (Julien) : ... si puertm docendion... tibi dedero; $\mathrm{D} 33.7 .12 .33$ : paedagogia. 
1/ Fonctions liáes aux plaisirs du maître

\begin{tabular}{|c|c|c|c|}
\hline $\begin{array}{l}\text { juriste auteur } \\
\text { du passage }\end{array}$ & juriste cité & fonction & source \\
\hline Celsus & & chorus & D $33.79 \mathrm{pr}$ \\
\hline Julien & & $\begin{array}{l}\text { pantomimus/archimimus (5n3) } \\
\text { artem pantomimi ( } 504)\end{array}$ & $\begin{array}{l}\text { D } 38.1 .25 .1 \\
\text { D } 38.1 .27\end{array}$ \\
\hline Africain & & $\begin{array}{c}\text { comoedi/chorus } \\
\text { comoedos }\end{array}$ & $\begin{array}{l}\text { D } 21.1 .34 \text { pr } \\
\text { D } 21.1 .34 .1\end{array}$ \\
\hline Venuleius & & histrio & D 40.12.44.2 \\
\hline Paul & Julien & $\begin{array}{l}\text { comoedis/symphoniacis } \\
\text { agitatorem/pantomimum }\end{array}$ & $\begin{array}{l}\text { D } 9.2 .22 .1 \\
\text { D } 19.1 .43\end{array}$ \\
\hline Ulpien & $(506)$ & \begin{tabular}{|c|} 
histrio/symphoniaco/palaestra \\
histrio \\
pantomimum (505) \\
tragoedi/mimi \\
histrionicas \\
voluptatis artifex/histrio \\
\end{tabular} & $\begin{array}{ll}\text { D } & 7.1 .15 .1 \\
\text { D } & 7.4 .12 .1 \\
\text { D } & 12.4 .3 .5 \\
\text { D } & 21.1 .38 .14 \\
\text { D } & 32.73 .3 \\
\text { D } & 38.1 .7 .5 \\
\end{array}$ \\
\hline Modestin & $\begin{array}{l}\text { rescrit de } \\
\text { Caracalla }\end{array}$ & tragoedos & D $40.5 .12 \mathrm{pr}$ \\
\hline
\end{tabular}

Il y a peu à dire sur ces acteurs, comédiens, mimes, musiciens et danseurs sinon qu'il est de bon ton, dès la fin de la République, de posséder de tels esclaves, au même titre que les lettrés (507). Mais Galien atteste un déclin des goûts artistiques an 2è̀me siècle (508). La place de ces esclaves est néanmoins importante, elle correspond à celle que tient le plaisir dans la société romaine. A ce propos, il faut aussi signaler un texte de Callistrate qui śvoque un harenarius (509).

\section{3- Il s'agit d'un affranchi.}

504-. Idem.

505- C'est le pantomime de Domitia, la fille de Náron.

506- I1 s' agit d'un affranchi. On remarquera une nouvelle fois 1 'identité des fonctions d'esclave et d'affranchi. Voluptatis artifex vise un un cas de prostitution masculine.

507- J. MARQৃUARDT, Vie privée, cit, p. 177-178; P1in., Ep., VII, 24, 5. 508- Galien X, p. $3 \mathrm{~K}$.

509- Le contenu du passage est à relever : on ne peut pas demander à un combattant $\mathrm{du}$ cirque, harenarius, qu' on affranchit, de fournir ses operae conbattant ducircue, harenar iu qu affrant en exerçant cette fonction, car fait que, le juriste le prêcise n'est pas ininteressant, on ne peut demander à une prostituée, mexetrix, a qui on a donné la llberté, de s'acquitter de ses operce envers son patron en lui prodiguant ses "services", Callistrate D $38.1 .38 \mathrm{pr}$.
Plus utiles, vitaux même, sont les esclaves ou les affranchis qui veillent sur la santé du maître ou du patron et de ses dénendants :

\section{2/ Autres fonctions}

\begin{tabular}{|c|c|c|c|}
\hline $\begin{array}{l}\text { juriste auteur } \\
\text { du passage }\end{array}$ & juriste cité & fonction & source \\
\hline $\begin{array}{l}\text { Alfenus (chez } \\
\text { auteur anonyme) }\end{array}$ & & $\begin{array}{r}\text { medicus } \\
\text { medicinam }\end{array}$ & $\begin{array}{l}\text { D } 38.1 .26 \mathrm{pr} \\
\text { D } 38.1 .26 .1\end{array}$ \\
\hline Julien & & $\begin{array}{c}\text { medici servos eiusdem artis. } \\
\text { medicinam }\end{array}$ & $\begin{array}{l}\text { D } 38.1 .25 .2 \\
\text { D } 38.1 .27\end{array}$ \\
\hline Pomponius & & $\begin{array}{l}\text { sernis. . . demonstrandis } \\
\text { finibus agri }\end{array}$ & D 18.1 .18 .1 \\
\hline Scaevola & & $\begin{array}{l}\text { medicus } \\
\text { medicos }\end{array}$ & $\begin{array}{l}\text { D } 34.1 .16 .1 \\
\text { D } 1.0 .5 .41 .6\end{array}$ \\
\hline Faul & & medicus & D 33.7 .18 .10 \\
\hline Ulpien & Pomponi us & mensor (sous-entendu) & D 11.6 .3 .6 \\
\hline
\end{tabular}

Selon toute vraisemblance, D 19.1.18.1 de Pomponius, qui traite d'un esclave chargé de faire voir, littéralement, les limites d'un terrain, vise un arpenteur, mensor. Cette fonction jouit de considération sociale car, dans 1 'Antiquité, c'est aussi une activité religieuse relevant des augures (510). Fonction prestigieuse aussi que celle du médecin. Parmi les références données à ce sujet, deux textes seulement concernent des esclaves (511), les autres visent des affranchis qui continuent $\grave{\imath}$

510- K. VISKY, Esclavage et artes liberales, cit, p. 482 ; A.A.F. RUDORFF, Das Aekergesetz des Spurius Thorius, Beilage I. Über die Feldmesser, Zeitschrift für geschichtliche Rechtswissenschaft, $X / 1$ (1839), messer, Zeitschrift für geschichtliche Rechtswissenschaft, $X / 1$ (1839), p. $413-414$; E. CUQ, Honorarizon, cit, p. 241. Selon les veteres, 1'arnen tage ne pouvait faire 1 'objet d'une locatio-conductio, cf. D $11.6 .1 \mathrm{pr}$ Ulpien (sur les interpolations, A. PERNICE, Parerga, ZSS Rom. Abt. cit, vo1. II $/ 2$ p. 173 ; E. LEVY, Privatstrafe und Schadenersatz im Klassischen. römischen Recht, Berlin (1915) p. 56 s., B. KÜBLER, Die Haftung für Verschulden in den Schuldverhä1tnissen, ZSS. Rom. Abt. 39 (1918) p. 209 ; A. WATSON, The law of obligations in the later roman Republic, Oxford (1965) p. 109-110. Mais il est question plus tard, chez Paul, et non chez Gaius comme 1'écrit K. VISKY, o. c. p. 482, d'un contrat de travail avec un agrimenseur, cf. D 10.1.4.1 (... si mensor ab altero solo sonductus sit...).

511- Paul D 33.7.8.10 et Julien D 38.1.25.2 ; dans ce dernier texte, les esclaves médecins parviennent à la liberté. 
travailler comme métecins pour leur patron (512). C'est une caractéristique de l'époque impériale (513). Initialement, c'était le pòre de famille qui était lui-même médecin de la maison (514), mais sous 1'Fmpire, l'activité médicale peut faire l'objet d'une locatio-conductio (515).

Reprenons maintenant les principales données. Compte tenu des difficultés annoncées, un traitement statistique global serait inefficace. Il faut d'une part. écarter de I'analyse les operae et les fonctions exercées dans le petit commerce ou I'artisanat pour les raisons qui ont auparavant été mentionnées. D' autre part, il convient de n'envisager que les rubriques dont les indications sont suffisamment explicites pour permettre de dégager des résultats valables.

$\mathrm{Si}$ on regroupe sous le titre "fonctions privilégiées" les fonctions de direction et les fonctions intellectuelles et de prestige (515bis), par opposition aux fonctions d'exécution, la main-d'oưre dépendante ressort du discours des juristes de la manière suivante :

5I2- A ce propos, il faut relever un passage d'Alfenus (D 38.1.26 pr) quí réfère $a$ un medicus libertus qui se fait accompagner de ses affranchis, médecins aussi, et ne leur permet pas d'exercer leur profession dans l'espoir d'ainsi augmenter sa propre clientèle. Il s'agit d'un cas original, d'operae liberti négatives, en ce sens où les operae ne sont pas requises mais empêchées par le patron.

513- Varron, De re rustica, I, 16, 4 ; Sénèque III, 24 ; S. REINACH, Medicus, D.S. vol. 3 p. 1673 ; R. HERZOG, Artz, Reallexikon für Antike und Christentum, vol. I (1950) p. 722. La référence donnée par K. VISKY, o. C., p. $483 \mathrm{n} 2$ doit être corrigée, il s'agit de D 34.1 .16 .1 et non de D 34.1 .13 .1 .

514- Plut., Cat. mai., 23 indique que Caton connaissait des médecins d'origine grecque à Rome vers 219 (Plin., N.H., XXIX, 12), mais aussi qu'il avertissait de s'en garder (1.c. 14); J. MAROUUARDT, vie privée, cit, p. $183 \mathrm{nB}$.

515- D 9.2.7.8 Ulpien (Proculus) ; D 19.5.27 Pomponius. K. VISKY, o.c. p. 483 ; J. MACQUERON, Le travail des hommes libres, cit, p. $182 \mathrm{~s}$. Au 4ème siècle, une constitution de Constantin (CI 10.66.1) mentionnera les médecins parmi les artisans.

515bis- A 1'exception des fonctions liêes aux plaisirs du mấtre, qui manquent de pertinence au niveau d'une comparaison. analyse des paragraphes

$\begin{array}{lllllll}\text { I }- & = & - & \text { I }- & = & - \\ \text { II }- & = & - & \text { II }- & = & - \\ \text { III } 21 / 30 & = & 70 \% & \text { III } 9 / 30 & = & 30 \% \\ \text { IV } 28 / 35 & = & 80 \% & \text { IV } 7 / 35 & = & 20 \% \\ \text { V } 98 / 151 & = & 65 \% & \text { V } 53 / 151 & = & 35 \%\end{array}$

analyse des citations

\begin{tabular}{|c|c|c|c|c|c|}
\hline I & $6 / 18$ & $33 \%$ & I & $12 / 18$ & $67 \%$ \\
\hline II & $15 / 24$ & $62,5 \%$ & II & $9 / 24$ & $37,5 \%$ \\
\hline III & $13 / 16$ & $81 \%$ & III & $3 / 16$ & $19 \%$ \\
\hline IV & $3 / 3$ & $100 \%$ & IV & $0 / 3$ & $=$ \\
\hline v & $1 / 3$ & $33 \%$ & $\mathrm{v}$ & $2 / 3$ & $67 \%$ \\
\hline
\end{tabular}

L'analyse des citations montre I'existence de fonctions privilégiées au dernier siècle de la République, mais les juristes se préoccupent plus alors des fonctions d'exécution. Au ler siècle de notre ère, les fonctions privilégiées prennent de I'importance. Il faut cependant attendre les Antonins, spécialement les derniers, pour les voir devenir nettement prépondérantes. C'èst un point fondamental.

Le chapitre préliminaire a montró que l'esclavage occupait la place la plus importante dans le discours des juristes an 2ème siècle, plus précisément dans la deuxiène moitié de ce siècle. Une des causes de cette progression quantitative est donc le changement d'utilisation de l'esclave, qui se traduit par une plus grande importance des fonctions privilégiées dans les développements jurisprudentiels. Au 3ème siècle, on observe une diminution sensible de ces fonctions. Cette érolution s'explique par le fait que de nombreux textes de la période sont relatifs à I'attachement des esclaves aux moyens de production, attachement qui, bien que n'épargnant pas les privilégiés, affecte dans une proportion beaucoup plus considérable les simples exécutants ( 516 ).

516- On a vu que l'attachement des esclaves aux moyens de production affectait aussi les privilégiés, notanment à propos de l'actor. Il faut ajouter que non seulement les esclaves en sont victimes, mais également les affranchis. C'est ainsi qu'il faut interpréter les legs de terres faits par des patrons à leurs affranchis, avec interdiction de les aliéner. La pratique se dêveloppe à partir du 2ème siècle (cf. par exemple Scaevola D 33.1.18 pr : Codicillis testamento confimatis funcum libertis legavit eumque alienari vetait... ; ou aussi D 36.1.80.13 : Plumibus heredibus institutis, in quibus et libertis tribus ex dodrante, eisdem frondos per praeceptionem dedit et ab his petit, ne eos alienarent...). Sur $1 \mathrm{e}$ thème, E.M. SCHTAERMaN, La schiavitii, cit, p. $53 \mathrm{~s}, 114 \mathrm{~s}$ 
Pour avoir une vue plus complète de la place économique de l'esclavage dans le discours des juristes, il est nécessaire de ne plus se limiter à l'analyse des activités professionnellos, dont on a vu les faiblesses, dues à l'imprécision des textes en matière artisanale ou commerciale, et d'examiner les actions auxquelles le waitre peut être soumis par l'activité de son esclave (517).

\section{SECTION 2 - UNE INDEPETDANCE GRADUEE : ANALYSE DES ACTIONS AUXOUELLES} DONIE LIEU L'ACTIVITE ECONOMIOUE DE L'ESCLAVE (517 bis)

L'édit du préteur, à l'époque rópıblicaine, a articulé la représentation du maître par son esclave sur deux axes, l'un dohnant lieu à une responsabilité illimitée du maitre, au moyen des actions exercitoria, institoria et quodiussu, l'autre entraînant une responsabilité limitée de celui-ci, par la mise en oeurre d'une institution préexistante, le pécule.

\section{Paragraphe 1-L'indépendance de fait de l'esclave : actions}

\section{exercitoria, institoria, quod iussu.}

L'actio exercitoria s'applique lorsque l'exercitor navis, généralement houme libre, própose son esclave (ou son fils, ou gême plus tard un extraneus) au commandement d'un navire, en qualité de magister novis (518), le chargeant d'accomplir les opérations économiques liées à cette fonction (519). Mais un esclave, de la même manière qu'il peut être préposé à

517- Dans un droit ignorant la reprégentation parfaite entre homes libres, la représentation dụ maître par l'esclave est pratique car directe : exemple in G. BOULVERT, Nouvelles Tabulae Pompeianae, Note sur un affranchi de Tibère et son esclave, RHD 1 (1973), p. 60-61, qui montre conment un affranchi impérial peut"faire des opérations économiques, sans négliger le service du prince, par le moyen de son esclave.

517 bis- Il ne s'agira ici que d'esclaves.

518- La désignation magister navis à propos d'un esclave ressort de D 14.1.7 pr Africain ; D 14.1 .5 pr Paul et D 14.1.1.4 Ulpien. L'esclave est fait capitaine du navire par celui qui en est l'exercitor, qu'il s'agisse du dominus, ou d'un extraneus, (ou même d'un esclave armateur), cf. D 14.1.1.4 : Cuius autem condicionis sit magister iste, nihil interest, utrom liber an semrus, et utrun exercitoris on alienus : sed nec cuius aetatis sit, intererit, sibi imputaturo qui praeposuit. Sur le thème,

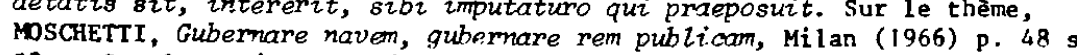
59 s. Sur le magister navis, aussi A. CUARINo, Magister e gubermatr" havis, Labeo 11 (1965) p. $36 \mathrm{~s}$; ROUGE, La justice 3 bord du navire, St. Volterra 3 (1971) p. $173 \mathrm{~s}$.

519- A. GUARINo, Diritto privato romano, cit, p. 383-384. Outre D 14.1 une activité commerciale en tant que servus institor, neut ôtre rhargé par son maître d'exercere un navire (520). Il remplit alors lui-mème la fonction d'armateur. Un texte d'Ulpien (5P1), lui reconnaît même la faculté d'exercer cette activité en son nom et, pour son propre compte (522).

Si la prósence d'un esclave dans un commerce maritime ressort clairement du Digeste par la référence des juristes à l'actio exercitonria (523), on peut la présumer, en l'absence de cette mention, à deux reprises : par transmarinas negotiationes chez faius (5.h) at par pecioniam nautioam chez

520- Cf. supra tableau p. $162 \mathrm{~s}$.

521- D 14.1.1.20 : Licet autem detur actin in ewm, cuius in potestate est qui navem exerset, tamen ita demom dotur, si voluntate eius exerceat. Ideo autem ex voluntate in solidrom tenentur oui habent in potestate exercitorem, quia ad swomam rem publisom naviom exercitio pertinet. At institoriom non idem usus est $\therefore$ ea propter in tribution domtazat vocantur, qui contraxemunt cwo eo, qui in merce peculiari sciente domino negotiatur. Sed si sciente drontaxat, non etiam volente cum magistro contraction sit, utrom quasi in volentem domus actionem in solithom an vero exemplo tributoriae dabimus? In re igitur dubia melius est verbis servire et neaue scientam solam et nudam patris dominive in navibus onerare, neque in peculiaribus mercibus voluntantem extendere ad solidi obligationem. Et ita videtur et Pomponius significare, si sit in aliena potestate, si quidem volintate gerat, in solidim ewn obligari, si minus in peculium. Le passage est interpolé selon l'Index itp. ; F. DE MARTINO, Studi sull'actio
exercitoria, Riv. dir. nav. 7 (1941) p. $25 \mathrm{~s}$; A. BERGER, In dubiis beniexercitoria, Riv. dir. nav. 7 (1941) p. $25 \mathrm{~s}$; A. BERGER, In dubizs beni
gniora, (D 50.17.56), in Atti Congr. intern. dir. rom. Verona (1948), 2 (Milan 1951), p. 202, dêfend l'authenticité de in re igitur-servire, notant que les compilateurs n'auraient pas fait référence aux verha edicti ; WESENER, Actiones ad exemplum; ZSS 75 (1958) p. $243 \mathrm{n} 127$, considère interpolé le passage sed si sciente-dabimus; G. PUCLIESE, In tema di actio exercitoria, Labeo 3 (1957) p. 323 s ; G. LON toria, actio institoria, actio quasi institoria, St, Scherillo, Milan (1972) P. 598, soutient l'authenticité du passage dans lequel il releve (1972) p. seulement la glose ideo-pertinet, considerant la phrase quasi-in dabimu comme un ajout de l'époque de Justinien ; en ce sens, I. BUTI, Stud sulla capacità patrimoniale dei servi, Camerino (1976), p. 127 nl 132. 522- Le début du passage, en soulignant que l'action contre celui qui a l'exercitor en sa puissance n'a lieu que dans le cas où le second exercet par la volontê du premier, semble impliquer qu'un esclave puisse être exercitor sans la volontê de son maître. Puis, la fin du passage précise que si l'exercitor voluntate gerat, le dominus est tenu in soliaum, et que dans le cas contraire il est seulement tenu in peculizom : ceci confirme que l'esclave peut accomplir de manière autonome la tâche d'exercitor, I. BUTI, o.c., p. 127-128.

523- Africain D 14.1.7 pr ; Gaius D 14.5.1 ; Paul D 9.4.19.2; D 14.1.5 $\mathrm{pr}^{-1}$; D $14.1 .6 \mathrm{pr}-1$; D 47.2.42 $\mathrm{pr}$; Ulpien $\mathrm{D} 4.9 .3 .3$; D 4.9 .7 .6 ; $\mathrm{D} 14$. $1.1 .4-16-20$ (Pomponius) $-21-22-23$ (Julien) ; D 14.1.4.2-3-4;D 14.3.7.1; D 14.3.13.2 (Julien).

524- D 40.9.10. 
Scaevola (525). Par zilleurs, bien que ne concernant plus l'esclave magister, ni exercitor, certains textes indiquent la présence d'esclaves sur un navire, en qualité de marins notamment (526). Il convenait de le signaler.

Quant à l'actio institoria, elle a lieu dans 1 'hypothèse ờ le maître a préposé ouvertement (527) son esclave (528) à la gestion d'une boutique ou de toute autre entreprise en dehors d'un commerce maritime (529). Les juristes se réfèrent plus souvent à cette action qu'à 1 'actio exercitoria (530). Un texte d'ulpien est éloquent quant à la situation économique de certains esclaves. Il y est question d'un esclave qui prépose son institor à une activité commerciale (531).

Enfin l'actio quod iussu est accordée au créancier de l'esclave (ou du fils) contre le maitre (ou le père) dans l'hypothèse oì celui-ci

525- D 45.1.122.1.

526- Nauta chez Paul D 9.4.19.2 ; U1pien D 4.9.7 pr. La présence d'esclaves sur un navire est attestée par Paul D 4.9.6.1-2 (Pomponius) ; Ulpien $D$ 47.5.1.5.

527- De manière à ce que soit clair pour tous, A. GUARINO, Diritto privato romano, cit, p. 384-385.

528- Le pater familias peut aussi préposer son fils ou un extraneus (servus atienus ou hotme 1 ibre) ou encore une ancilla, cf. D 14.3.7.1 U1pien:

529- Outre D 14.3 (De institoria actione), v. Gaius IV, 71.

530- Julien D 14.3.12 ; Pomponius D 33.7.15 pr (Servius) ; Africain D 4.4.4; D 14.1.7.2 ; Gaius D 14.5.1; Marcellus D 7.8.20; Scaevola D 14.3.20; D 33.7.7; Papinien D 3.5.30 pr ; D 14.3.19.1-3; D 26.7.37.1; D 32.91.2; Paul D 12:1.29 (Julien); D 14.3.14; D 14.3.17.1-3 (Pomponius)4 (Proculus) ; D 14.3.16 ; D 15.1 .47 pr ; D 33.7 .13 pr (Neratius); U1pien D 5.1 .19 .3 (Labéon) ; D 14.1.1.20 (Pomponius); D 14.3.1 (Marcellus) ; D 14. 3.5.3-7 (Labéon)-8 (Labéon)-9 (Labéon)-15; D 14.3.7.1; D 14.3.11.7-8; D 14.3.13 pr (Julien)-2 (Julien) ; D 42.4.3.1 ; Marcien D 33.7.17.2. Il $n$ 'est pas certain que les autres textes traitant de 1 'actio institoria concernent des esclaves : le problème est le même pour 1'actio exercitoria (cf. supra $\mathrm{n} 9 \mathrm{ch}$. prélim.). Sur procurator, cf. supra nll Ch. Prélim. 531- D 14.4.5.3 : Item parvi refert, cum ipso servo contrahatur an cum institore eius. Il est parallèlement question, dans un autre passage d'Ulpien, d'un servus vicarius qui navem exercuit, cf. D 14.1.1.22: $S i$ tatem servus peculiaris volente filio familias in cuius peculio erat, vel servo vicarius eius navem exercuit, pater dominusve, qui volrmtatem non accomodavit, drontaxat de peculio tenebitur, sed filius ipse in solidim. Plane si voluntate domini vel patris exerceant, in solidum tenebrontur et praetera et filius, si et ipse voluntatem accomodavit, in soiziom exit obligatus; sur ce passage, F. DE MARTINo, Studi sull'actio exerciexit obligatus ; sur ce passage, F. DE MARTINo, Studi sull actio exercitoria, cit, p. $26 \mathrm{~s}$; G. LONGO, Actio exercitoria, cit, p. 600, qui pro-
pose 1 élímination de vel servus vicarius; G. PUGLIESE, In tema di actio exercitoria, cit, p. 324 s ; I. BUTI, Studi, cit, p. 128, n133. a manifesté son iussum (532). L'action a lieu chaque fois que l'opération a été faite par la volonté du maître (533), indépendament du mode par lequel elle s'est manifestée : ordre donné au tiers ou à l'esclave, autorisation, mandat ou simple ratificstion (534).

Chaque fois qu'apparaît dans les textes un iussim (535) ou un acte de préposition à une activité commerciale déterminée (536), l'esclave n'agit pas en nom propre, mais pour le compte de son maitre. Les

532- Outre D 15.4 (Quod iussu), v. Gaius IV, 70. Sur le problème, 0 . LENEL, EP (3) \& 278 ; LEMOSSE, L'affranchissement par le cens, RH 27 0. LENEL, EP (3) 278 ; LEMOSSE, Laffranchissement par le cens, RH 27

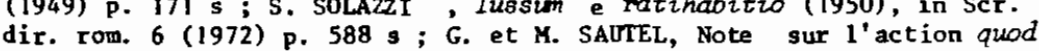
iussu et ses destinées post-classiques, in Mél. Lévy-Bruhl (1959) p. $257 \mathrm{~s}$. 533- Ulpien D 15.3.5.2 : Quod servus domino emit, si quidem voluntate eius emit, potest quod iussu agi.

534- Exemples de iussrom adressé a l'esclave, D 12.4.3.6 Ulpien ( $S i$ quis quasi statuliber mihi decem dederit, crom iussus non esset condicere evm decem Celsus scribit); de mandat, D 15.4.1.3 Ulpien (Sed et si mandaverit pater dominusve, videtur iussisse) ; de ratification, D 15.4.1.6 ulpien ( $S i$ ratur habuerit quis... quod iussu in evm actio datur); mais le iussum le plus courant semble être celui qui implique me autorisation. le iussion le plus courant semble être celui qui implique une autorisation, adressée au tiers, de contracter avec 1 'esclave, du type Quod voles E. BETTI, Diritto romano t (Padoue 1935), p. 394. s, considère cortune un élément essentiel pour le iussum le fait qu'il soit connu du tiers; dans le mête sens, BONIFACI0, Iussum, in NNDI IX (Turin 1965), p. 394. RABEL, Ein Ruhmesblatt Papinians, Die sogennannte actio quasi institoria, in Festschr. Zitelmann, Munich (1913) = Gesamme1te Auf sätze 4., Tübingen (1971) p. 291 s, suivi de G. et M. SAUTEL, Note sur 1 'action quod iusau cit, p. 257 s, soutient que le iussum, pour donner lieu a une action quod iussu, doit être une invitation adressée au tiers ; contra, I. BUTt, Studi, cít, p. 149 in 5 .

535- A 1'exception des textes où il s'agit d'un iussom du mâtre à l'esclave pour qu'il accepte une succession dont il a été institué héritier, clave pour qu'il accepte

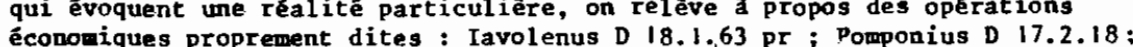
économiques proprement dites : ravolenus $\mathrm{D}$ ( 45.3 .6 (Ofilius, Sabinus, Cassius); Africain $\mathrm{D}$ 15.3.17.1; Gaius $\mathrm{D} 14$. D.1; D 15.1 .27 Pr (Julien) : D 46.4.22; Marcellus D 7.8 .20 ; Tryphonius 5.1; D 15.1.27 pr (Julien) ; D 46.4.22; Marcellus D 7.8.20; Tryphonius
D 41.1.63.2; Paul D 12.1.29 (Julien); D 15.4.2 pr-1-2 ; D 15.4.5 pr-1 ; D 41.2.1.13-19; D 45.3.33 pr-1; Ulpien D 12.4.3.6 (Celsus) ; D 14.5.2 pr ; D 15.1.1.1; D 15.3.5. 2 ; D 15.4.1 pr-1-2-3-4-5 (Marcellus) -6-7-8 (Marcellus)-9 (Labéon); D 15.4.3; D 15.4.4 (Pomponius); D 17.2.63.2 ; D 41. 1.23 .3 (Scaevola) ; D 42.4.3.1 ; D 45.3.5; D 45.3.7 pr-1. I1 faut assimiler a ces textes Pomponius D 43.26.13 (two mandato); Africain D 21.1.51 pr (mandatu domini)-1 (domino mandati) ; Paul D 17.1.5.4 (servo... dominus si praeceperit...); D 41.2.1.10 (si servrom miseris...); Ulpien D 2.13.4. 3 (voluntate domini) ; D 4.9.3.3 (voluntas danini); D 15.3.3.4 (voluntatem) -6 (volenti) (GAY, 'L' in rem versum à 1 'époque classique, Etudes de tem) -6 (volenti) (GAY, L'in rem versum à l'époque classique, Etudes de
droit romain 2 (Paris 1956) p. 210 , estime que la mention de la volonté droit romain 2 (Paris 1956) p. 210 , estime que la mention de la volonté
dominicale dans ce dernier texte n'est pas authencique ; contra, I. BUTI, Studi, cit, p. 149 n5); D 50.12.2.1 (auctoritate domini).

536- Sur les problèmes relatifs à la praepositio, VAliNo, Las actiones adiecticiae qualitatis y sus relaciones basicas en derecho romano, in AHDE 37 (1967) p. $356 \mathrm{~s}$; sur la praepositio du magister navis, G. LONGO, Actio exercitoria, cit, f. 587 s ; sur la praepositio institoria, CARrell 
actions exercitoria, institoria et quod iussu, qui entrainent une responsabilite in oolidren du maitre, sont le signe de l'absence d' initiative de l'esclave (537).

Cependant, bien que faisant ces opérations au non du maitre, l'esclave se trouve en fait dans une certaine indépendence. Ainsi, le magister navis peut être amené à se trouver très loin de son maître. L'institor exerce son activite hors du dowaine. Ouant à l'esclave qui agit en vertu d'un iussum, il a la possibilité de contracter avec des homes libres.

Or, les hommes libres qui traitent avec un tel esclave ont intérêt a ce que les opérations accomplies produisent les effets attendus (538). Le maitre lui-même perdrait une grande partie de l'intérêt qu'il a d'ainsi utiliser son esclave s'il lui fallait sans cesse corriger les "actes" de celui-ci. C'est pourquoi les juristes vont s'efforcer, sans pour cela remettre en question 1 'institution elle-mêre, de reconnấtre aux esclaves une certaine capacité dans les affaires.

\section{Paragraphe 2 - L'autonomie relative de l'esclave : actions de peculio, tributoria, de in rem verso.}

Il convient d'examiner la notion d'autonomie économique relative de l'esclave avant d'óvoquer les progrès qu'elle connut sous l'Empire.

\section{I - La signification de l'autonomie rolative.}

Il n'y a pas d'ordre ou de prénosition du maitre dans les actions qui entrainent pour lui une responsabilité seulement limitée. C'est le cas de l'actio de peculio (530) et de l'actio tributoria (540). Le maitre est alors tenu dens les limites du pécule car l'activité commerciale a été accomplie par l'esclave de sa propre initiative et dans son propre inté-

L'actio quasi institoria, cit, p. $143 \mathrm{~s}$, et ANGELINI, Osservazioni in tema di creazione dell'actio ad exemplion institoriae, in BIDR 71 (1968) p. 230248. Du cas typique du semus tabernae propositus est dérivêe une extension ultêrieure à d'autres hypothèses (cF. D 14.3.5 pr-14).

537- I. BUTI, Studi, cit., p. 148.

538- M. JACOTA, L'esclave créancier, Studi Grosso 2 (1968), p. 216. rêt (541). L'actio de in rem verso caractérise une opération accomplie par l'esclave de sa propre initiative mais dans l'intérēt du maitre (542). La particularité de cette action réside dans le fait que si la versio in rem domini a éțe supérieure à la valeur du pécule, le maître pourra être poursuivi dans les limites de son enrichissement (543). Ces trois actions existent sur le fọdement du pécule (544), sorte de quasi-patrimoine à travers lequel les juristes vont progressivement dégager une autonomie relative de 1 'esclave en matière éconorique.

La concessio peculii resulte à l'origine d'une manifestation

539- A. GUARTNo, Diritto privato romano, cit, p. 386 ; P.F. CIRARD, Droit romain, cit, p. 103 ; sur les liens chronologiques existant entre les actions précédement examinées et 1 'actio de peculio, S. PERozzI, Istituzioni di diritto romano, I cit, p. 216 ; S. SOLAzZI, L'eta dell'actio exercitoria, in Scritti 4 (Naples 1963) p. $243 \mathrm{~s}$. Selon cet auteur, 1 action quod iussu aurait été précédée par l'action exercitoria et suivie par l'action institoria et l'action de peculio. En ce sens, G. PUGlese, In

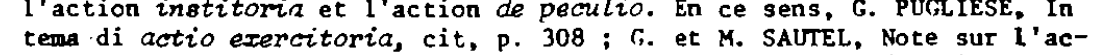
tema di actio exercitoria, cit, p. 308 ; G. et M. SAUTEL, Note sur l'ac-
tion quod iussu, cit, p. 262, et G. LoNco, Actio exercitoria, cit, p. 581 s ; v. aussi $\varepsilon$. VALINO, Las actiones adiecticice qualitatis, cit, p. $343 \mathrm{~s}$.

540- Par l'actio tributoria, qui a lieu lorsque les dettes de l'esclave sont nées dans l'exercice d'un comerce connu du maître mais non autorisé par celui-ci, les créanciers obtiennent que le mâ̂tre partage le pếcule avec eux, J. MACQUERON, Les obligations, cit, p. 402. Sur le thème, E. VALINO, La actio tributoria, in SDRI 33 (1967) p. $103 \mathrm{~s} ;$ E. ALBERTARIO, Responsabilita fino al limite dell'arricchimento nell'actio tributoria e nelitactio de peculio, (1913), in St. 4 (1946) p. $3 \mathrm{~s}$, selon lequel il faut attribuer aux compilateurs la tendance consistant a réduire la responsabilité aux limites de l'enrichissement : sur ce problème, E. BETTI, Responsabilita nossale e responsabilità del pater (domims) nei limiti dell'aricchimento, in ATO (1915-15).

541- I. BUTI, Studi, cit., p. 152. Pour A. BURDESE, cr. de Buti, in Iura 27 (1976), il serait exagéré de parler de "capacitê patrimoniale" de l'esclave, l'intêrêt de celui-ci n'étant pris en considération qu'en fonction de l'intérêt dis mâ̂tre, (p. 210$)$.

542- Le fait que l'activité qui produit une versio in rem domini soit accomplie de manière autonome par l'esclave est confirmé par la possibilité de voir le maitre devenir débiteur de l'esclave, cf. D 15.1.7.6 (síforte (servus) in domini rationem impendit et dominus ei debitor manere voluit...). 543- R. MONIER, Droit romain, cit, I. p. 212.

544- Sur les origines de l'institution, I. BiTI, Studi, cit, p. $13 \mathrm{~s}$. Selon R. BROSZ, Peculizm servi, in Acta Antiqua Acad. Scientiarum Hu naricae, $18(1970)$ p. $307 \mathrm{~s}$, les textes en matière de pécule auraient été altérés dans la compilation. Au départ, seul le pécule de l'esclave aurait existé. Celıi du fils de famille serait né avec les dispositions sur le pécule castrense et se serait développé sous l'influence de l'hellénisme, tandis que le pécule servile aurait servi à rentabiliser le travail de l'esclave. Dans le sens aussi de l'antériorité du pécule de l'esclave par rapport à celui du fils de famille, R. MONIER, Droit romain, cit. I, p. 251. 


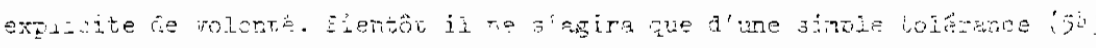
Pour Tubero, la volonté expresse du maître est non seulement requise pour que le pécule existe, mais aussi pour qu'il subsiste (5la). Il est suivi par Sabinus, Cassius, Celsus et Pedius $\left(5^{\prime}, 7\right)$. On note une atténuation de ce principe chez Pomponius, pour lequel la concessio est nécessaire dans le cas où le pécule provient du mâtre et non de tiers (548). Julien semble être pour un simple permissum, même implicite (549). U.pien va encore plus loin. Selon lui, la volonté du maître est présuóe à moins qu'il ne manifeste une intention contraire, par I'ademptio (550). Or, la capacité pour I'esclave de s'obliger ne découlant pas de la concessio peculii (551) mais de la simple existence du pécule (552), la volonté du maŝtre ne revêt plus qu'un rôle de second rang. Cela revient à dire qu'en matière contrac-

\section{5- I. BUTI, Studi, cit, p. 18-36.}

546- Chez Ulpien D 15.1.5.4 : Peculium autem Tubero quidem sic definit, ut Celsus libro sexto digestomon refert, quod servus domini permissu separation a rationibus dominicis habet, deducto inde si quid domino debotur.

547- Sabinus et Cassius chez Paul D 41.2.1.5; Celsus chez U1pien D 15.1. 7 nr-1; Pedius chez vipien D 15.1.7.3.

548- Pomponius D 15.1.4 pr : Peculii est non $i d$, cuius servus seorsum a domino rationem habuerit, sed quod dominus ipse separaverit suam a servi domino rationem habuerit, sed quod dominus ipse separaverit suan a servi minuere dominus possit, animadvertendzm est non quid servus, sed quid dominus constituendi servilis peculii gratia fecerit, ; $15.1 .49 \mathrm{pr}$ : Non solion id peculizm est, quod dominus serro concessit, verton id quoque, auod ignorante quidem eo adquisitum sit, tamen, si rescisset, passurus erat esse in peculio.

549- Julien (D 40.7.13.1) parle en effet de peculizm quocumque modo adquisition. Même si, par cette expression, il semble vouloir signifier avant tout que le pécule peut provenir du maître ou de tiers, on peut en déduire, selon I. BUTI, Studi, cit, p. 29 , que Julien ne requiert pas une manifestation explicite de volontê de la part du maître.

550- Ulpien D 15.1.3.4 : ... nam ot huius semus peculiwn habere potest, non si fuerit concessum, ut habeat, sed si non fuerit prohibition, ne habeat ; D 15.1.7.1 : Ego autem puto non esse opus concedi peculium a domino servum habere, sed non adimi, ut habeat.

$551-$ I. BUTI, Studi, cit, p. 37 ; contra A. BURDESE, Autorizzazione ad alienare in diritto romano, Turin (1950) p. 34 , selon lequel la capacité pour 1 'esclave de contracter et d'aliéner découlerait de la concessicn du pécule; ID., cr. de Buti, cit., p. 204.

552- La concessio peculii, comme nous 1 'avons vu en retraçant son évolution jurisprudentielle, n'est pas considérée par tous les juristes come un acte explicite et nécessaire. De plus, la possibilité pour l'esclave de contracter ne découle pas d'une autorisation du maître, car celui-ci est tenu de peculio même lorsqu'il a interdit à l'esclave de contracter, cf. Gaius D 15.1.29.1 : Etiansi prohibuerit contrahi cum servo dominus, erit in eum de peculio actio.

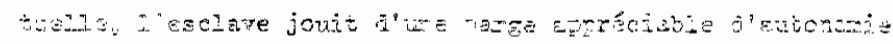

Cependant, la simple existence du pécule est insưfisante pour que l'esclave puisse disposer des biens péculiaires, spécialement s'il s'agit de biens immobiliers ou de biens mobiliers de valeur (553). Alfenus et Proculus requièrent une autorisation du maître pour chaque acte de disposition fait par l'esclave (554). Mais cette pratique a dû paraître gênante avec le dévelōppement des relations commerciales. Ainsi s'explique la teidance, dès le ler siècle, à comprendre dans la concessio I'autorisation d'accomplir des actes de disposition (555). Ce nouveau procéde fut à son tour abandonné du fait de I'affinement des capacités techniques des juristes (556) qui en vinrent à distinguer l'administratio peculii

553- Il est probable que le maître, en concêdant le pécule, donnait à l'esclave la faculté de disposer des biens mobiliers de faible valeur, I. BUTI, Studi, cit., p. 38 . Selon A. BURDESE, cr. de Buti, cit., p. 204 , ce n'est pas entre biens qu'il faudrait distinguer, mais entre types d'aliénation.

554- Alfenus D 41.3.34: Si serrus insciente domino rem peculiorem vendidisset, emptoren usucapere posse. Cela signifie que pour $1^{\prime}$ efficacité' des actes de disposition, la volonté du maître est nécessaire, car si l'esclave a vendu a son insu, l'acheteur n'acquiert pas directement la propriété de la chose, mais seulement après 1 'avoir usucapée, I. BuTr, Studi, cit, p. 40 ; contra WASTSON, The law of Persons in the Later Ronan Republic (Oxford 1967) p. $179 \mathrm{~s}$, qui considère que rem peculiarem est interpolé. Proculus D 12.6.53 : Dominus testamento servo suo libertatem dedit, si decem det : servo ignorante id testamentum non valere data sunt mihi decen : quacitur, quis repetere potest. Proculus respondit : oi ipse servus peculiares mumos dedit, cum ei a domino id permissun non esset, manent numi domini eosqus non per condictionem, sed in rem actione petere debet. Si domini eosque non per condictionem, sed in rem actione petere debet. Si servi, cuivs nomine dati siont, per condictionem petere potest : sed tom benignius quan utilius est recta via ipsum qui numos dedit sum recipere. La fin de la phrase, a partir de oed, est considérée comne l'oeuvre des compilateurs, cf. Index itp. ; G. DONATUT, Lo gtabulibero, Milan (1940), p. 165 ;.RRAMPE, Proculi epistulae, Eine frühklassische Jurigtenschrift, Rarlaruhe (1970) p. 68 . Sur les aspects relatifs a la vindicatio d'une somme d'argent, NIEDERLANDER, Die Bereicherungshaftung im Klassichen rösome d'argent, NIEDERLANDER, Die Ber eicherungshaftung im Klassichen
mischen Recht, Weimar (1953), p. $76 \mathrm{~s}$; F. SCHWARZ, Die Grundlage der

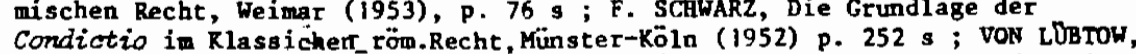

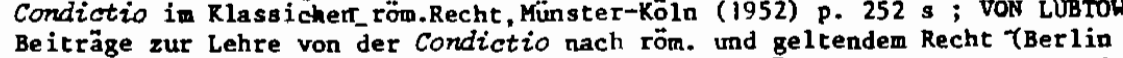
1952) p. 31 n44, p. $59 \mathrm{nl}$; M. KASER, Das feld in rômischen Sachenrecht, in T. 29 (1961) p.-173 s ; L. LABRUNA, Rescriptum divi Pii, Gi attil del pupillo oine tutoris auctoritate. Naoles (1962) p. $92 \mathrm{~s}$.

555- Julien D 46.1 .9 (0. LENEL, Pal., Iu1., p. 866 n2, attribue le respondit à Minicius ; de même G. MICOLIER, Pécule et capacité patrimoniale, Lyon (1932), p. 527.)

556- L'autorisation d'accomplir des actes de disposition, comprise dans la concessio peculit, a dô paraitre incompatible avec le fait que le pécule pouvait naitre sans un acte explicite de concession de 1 a part du maître, I. BuTI, Studi, cit., p. 48. 
de 1 a roncessin peculit (557).

Ces coneopts de concessio pemilii et, l'adninistratio peculiti sont porteurs d'une même exigence : la protection des intárêts du maitre. En effet, présumer la concession, c'est encourager l'esclave à participer à la vie des affaires (558) pour faire fructifier son pécule, c'est-à-dire juridiquement le patrimoine du maître.

Capable de s'obliger et d'obliger de façon sutonome, pouvant ainsi devenir aussi bien créancier que dóbiteur, l'esclave peut., selon faius et Paul, libérer le débiteur en acceptant. un paiement de sa part (550). Malgré l'incapacité processuelle de l'esclave, les juristes parlent d'acions intentées servi nomino $(560)$. Fn outre, le protuit de ces actions profite au pécule (561). Cela signifie q’’’en réglité, c'est bien la créance de l'esclave qui est sanctionnóe. Co qu'il est, important de saisir.

557- L'individualisation de l'administration peoulii soulève des discussions dont voici les grandes lignes. Selon la doctrine dominante (r. LONGo, Il concetto classico e il concetto giustinianeo di administratio peculii, in AG 100 (1928) p. $367 \mathrm{~s}$, et Libera administratio peculii, Il limiti e lo spirito di una innovazione giustinianea, in Ricerche romanistiche (Milan 1966) p. $387 \mathrm{~s}$; E. ALBERTARIO, Libera administratio peculii, in RIL 61 (1928) p. $139 \mathrm{~s})$ le concept d'a thinistratio poculii anrait été distinpué de celui de concessio peculii par les compilateurs. Adhèrent à cette thèse F. BONIFACIO, La novazione nel diritto romano, Naples (1959) p. I54 s ; F. BONIFACIO, La novazione nel diritto romano, Naples (1959) p. I54 s;
F. SERRAO, Sulla mutui datio da parte del servo comune, in St. ArangioRuiz 3, Naples (1953) p. 66 n38. A. BURDESE, La nozione classica di naturalis obligatio, Turin (1955) p. $43 \mathrm{n} 36$, nie aussi le classicisme de 1 'individualisation de 1 'administratio peculi $i i$. G. MICOLIER, Pécule et capacité patrimoniale, cit, p. 500, s'oppose à cette thèse ; selon lui, cette distinction date de 1 'époque des Sévères, à partir de Papinien, Paul et Ulpien. Enfin, I. BUI, Studi, cit, p. $68 \mathrm{~s}$, soutient que cet te individualisation de $i$ 'administratio est classique, et fait remonter $l$ 'élaboration du concept à Julien.

558- I. BurI, Studi, cit, p. $73 \mathrm{~s}$.

559- Paul D 12.2.20: Servus quod detulit vel iuravit, servetur, si peculii administrationem habuit...; Gaius D 12.2.21 : ... huicenim solvi quoque recte potest et novandae obligationis ius habuit. G. MICOLIER, Pécule et capacité patrimoniale, cit, p. 509 , nie qu'il s'agisse d'une thèse classique. Ces paiements faits à l'esclave peuvent être soit des solutiones de dettes envers le mâtre, qui accroissent le patrimoine de celui-ci, soit concerner des nomina peculiória dont le paiement augmente le pécule, I. BUTI, Studi, cit, p. 161; contra, A. BURDESE, cr. de Buti, p. 205.

560- v. Pomponius D 44.7.56.

561- U1pien D 15.1.7.5 : Sed et si quid furti actione servo deberetur vel alia actione, in peculivon computabitur : hereditas quoque et legatron, ut Labeo ait. G. SEGRE, Obligatio, obligare, obligari nei testi della giusrisprudenza classica e del tempo di Diocleziano, in Scr. vari di Dir. rom., Turin (1952) p. $272 \mathrm{n} 60$, doute de 1 'authenticité de vel alía actione. c'est que l'arfectation au pécule du produit de l'action tend avant tout à protéger les créanciers néculinires (56a). En matiòre de dettes serviles, $l$ 'inspiration jurisprudentielle est identique. Il s'agit de protéger les tiers qui contractent avec l'esclave. Cette protection, bien que tournés contre le maitre, est en fait la sanction de l'obligation servile. C'est ce qui ressort du mécanisme de l'actio et de la deciuctio de deculio (563).

Relevons les textés concernant pécule ou action de peculio (564).

562- I. BUTI, Studi, cit, p. 174.

563- On peut trouver confimation de ce fait dans la qualification servi nomine de l'action de peculio (I. BUTI, O.C. p. 189), dans sa structure avec fictio libertatis de l'esclave (p. 190), dans sa limitation à l'actif du pécule (p. 191), dans le lien étroit entre l'issue de l'actio de peculio et l'obligation de l'esclave, par le fait que peuvent coexigter une fideiussio en faveur du maitre et une autre en faveur de l'esclave une fideiussio en faveur du maitre et une autre en faveur de l'esclave
(p. 198). La deductio de peculio semble aussi prouver que le maitre ne (p. 198). La deductio de peculio semble aussi prouver que le maitre ne
paie pas sa dette, mais celle de 1 'esclave (p. 201). En outre, si l'esclave paie sa dette, le maître est libéré de l'actio de peculio (cf. Paul D 15.3. i1). Quant a la decuctio de peculio opérée par le maître lorsque l'esclave est débiteur envers lui, elle ne peut être considérée corme une simple émanation de la dominica potestas ( $p, 211$ s). Sur ce dernier point, contra : A. BURDESE, La nozione classica, cit., p.65 et cr. de Buti, p. 207 ; P. CORNIOLEY, Naturalis obligatio Essai sur l'origine et l'évolution de la notion en droit romain, Genève (1964), p. $152 \mathrm{~s}$.

564- L'existence du pécule présume celle de l'action de peculio même lorsque celle-ci n'apparaît pas expressément dans le texte. N'ont ainsi été in tomia de toria et de in rem verso. Concernent pecule ou action de peculio, Alfenus (chez auteur anonyme) D 15.3.16; D 33.8.14; D 40.1.6 ; Proculus D 12.6.53 ; D 33.6 .6 ; D 46.3.84 ; Iavolenus $\mathrm{D} 15.1 .33$; D 15.1.35; D 19.2.60.7 (Labéon) ; D 33.8.17; D 33.8.22 pr (Labéon)-1 (Trebatius, Labéon) ; D 40.7 .28 1 (Cassius); D 40.7.39.2 (Trebatius, Labéon); D 41.2.24; Neratius D 15. I.55; D 15.3.18; D 40.7.17; Celsus D 15.1 .6 (Tubero, Labéon) : D 19.1.38 pr ; D 33.8.13; D 33.8.25; D 40.7.23.1 ; D 47.2.68.4; Julien D 10.3.25: D 13.7.28.1;D 14.3.12;D 14.4.12;D 15.1.12;D 15.1.14 $\mathrm{pr}-1 ; \mathrm{D} 15.1$. 28 ; D 15.1.37.1-2-3; D $19.1 .24 \mathrm{pr}-1$ (Sabinus, Cassius) ; D 21.2.39.1 ; D 33.8.12;D 40.7.13.1; D 41.1.37.1; D 41.4.7.2; D 44.7.16;D 45.3.1. I-2; D 46.1.19; D 46.3.32;D $47.2 .57 .2-3 ; D 49.15 .22 .3$;

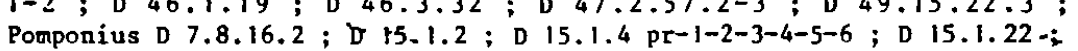
Pomponius D 7.8.16.2; D H5.1.2 ; D 15.1.4 pr-1-2-3-4-5-6; D 15.1.22-;
D 15.1.23; D 15.1.25; D 15.1.34; D $15.1 .49 \mathrm{pr}-1-2 ;$ D 18.1.31; D 24.3. -67 ; D $30.48 \mathrm{pr}$ (Atilicinus); D 33.8.10; D 35.1 .57 ; D 35.1.110; D 41 . $1.54 .4 ; \mathrm{D} 43.26 .13 ; \mathrm{D} 44.2 .21 .4 ; \mathrm{D} 46.1 .3$; D 46.3.19; Africain D 10. 3.9 ; D 15.1.38.2-3; D 15.3.17.1; D 19.1.30 pr; D 21.1.51 pr ; D 33.8. 16 pr-1 ; D 46.3.38.2-3 ; D 47.2.62.9; Gaius D 2.14.28.2 (Julien) ; D 2. 14.3n.1 (Julien) ; D 12.7.21: D 14.4.11: D 14.5.1; D 15.1.10 : D is. $1.2 \% \mathrm{pr}$ (Julien) $-1-2-3$ (Julien) -4 (Julien) -5 (Jul ien) -6 (Julien) -7 (Julien)-8 (Julien) ; D 15.1.29 pr-1 ; D $21.1 .18 \mathrm{pr}-2$; D 35.2.76 pr ;

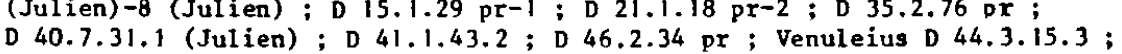
D 45.2.12.1 (Julien); Marcellus D is.1.16 (Julien) ; D 19.1.23 (Julien); D 35.2.56 pr-1; Florentinus D 15.1.39; D 46.2.16; Scaevola D 10.2.39.4; D 15.1.51; D 15.1.54; D 15.1.58; D 33.7.20.3 ; D 33.8.21 (Julien) ; D 33.8.23 pr-1-2-3; D 34.1.18.3; D 39.5.35 pr; D 40.5.41.8-9; D $40.7 .40 .1-3$; Papinien D 15.1.50.2-3; D 16.2.19;D 20.1.1.1; D 26.7. 
D 41.2.44.1 : D 41.3.45.1 ; D 45.1.118 pr: D 46.3.94.3; Tryphoninus D 15.1 .57 pr-1 (Julien)-2; D 34.3.28.7 (Scaevola); D 49.15.12.11; Paul D 3.5.17 (Proculus, Pegasus, Neratius); D 3.5.18 pr ; D $3.5 .40 ; \mathrm{D} 3.5$. $41 ; \mathrm{D} \mathrm{4.3.20} \mathrm{pr} \mathrm{(Labéon);} \mathrm{D} \mathrm{4.4.24.3;} \mathrm{D} \mathrm{4.8.32.8} \mathrm{(Octavenus);} \mathrm{D} \mathrm{5.3.}$ 41 ; D 4.3.20 $\mathrm{pr}$ (Labéon) ; D 4.4.24.3; D 4.8.32.8 (Octavenus); D 5.3. 34.1 (Julien) ; D 5.3.36 pr (Proculus, Julien, Mauricianus) ; D 6.2.10 ; D 9.4.19.2; D 10.3.8.1-4; D 11.5.4.1; D 12.1.2.4; D 12.1.31.1 (Sabinus, Cassius, Julien) ; D 12.2.20; 012.2 .22 ; D 12.6.13 pr (Pomponius) ; D 13.5.19.2 ; D I3.7.18.4; D14.1.6 pr ; D 14.3.17.4 (Proculus); D 14.4. 2 ; D 14.4.6;D 15.1.8; D 15.1.18;D 15.1.20; D 15.1.26; D 15.1.31; D 15.1.43 (Labéon); D 15.1.47 pr-1 (Sabinus)-2-3 (Proculus)-4 (Julien) -5$6 ; \mathrm{D} 15.148 \mathrm{pr}-1 ; \mathrm{D} 15.1 .52 \mathrm{Pr} ; \mathrm{D} 15.1 .53 ; \mathrm{D} 15.1 .56 ; \mathrm{D} 15.2 .21$.

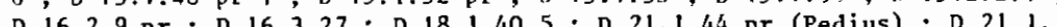

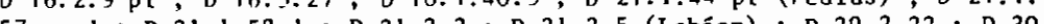
$57 \mathrm{pr}-1$; D $21.1 .58 .1 ;$, $21.2 .3 ;$ D 21.2 .5 (Labeon) ; $29.2 .22 ; 0$; 30. $52 \mathrm{pr}$; D 32.97; D 33.7.22.1; D 33.8.1 ; D 33.8 .5 ; D 33.8.9 pr-1 (Marcel1us)-2 ; D 33.8.15 (A1.fenus) ; D 40.2.24; D $40.4 .10 \mathrm{pr} ; \mathrm{D} 40.7 .20 \mathrm{pr}$ D 40.9.18.1; D 41.2.1.5 (Sabinus, Cassius, Julien)-22 (Nerva filius); D 41.2.3.12; D 41.2.14 pr: D 41.2.32.2 ; D 41.3.4.7 (Labéon)-9 (Pomponius); $041.3 .8 \mathrm{pr}$ (Labéon, Neratius, Julien); D 41.3.31.3: D 41.3. 34 (Alfenus) ; D 41.3 .44 .7 ; D $41.4 .2 .10-11$ (Celsus) -12 (Pomponius) 13 (Ce1sus) ; D 42.1.19 pr ; D 44.7.4.9; D 45.3.20 pr ; D 45.3.27; D 46.1. 35 (Alfenus) ; D 46.3.62; D 46.4.11.1; D 47.2.42 pr ; D 49.15.29 (Labéon); Ulpien D 2.13.4.3; D 3.2.4.3 (Pomponius); D 3.3.33 pr (Labéon) ; D 3.5. 5.8 (Labéon, Pomponius)-9 (Pomponius) ; D 3.5.13: D 4.3.7 pr (Julien) ; D 4.3.9.4 (Labéon); D 4.4.3.11 (Marcelius); D 4.9.3.3; D 4.9.7.6; D 6 $1.41 .1 ; 0.3 .1 .8 ; D 11.1 .9 .8 ; 011.6 .3 .6$ (Pomponius) ; D 12.1 .11 .2 ; D 12.1.i3.2 (Papinien); D 12.4.3.8 (Ce1sus) -9 ; D 12.6.11; D 13.5.1.8; D 12.1 .13 .2 (Papinien); D 12.4.3.8 (Celsus) -9; D 12.6.11; D 13.5 .1 .8 ; D 14.4.1.2; D 14.4.3 pr (Julien)-1 (Pomponius); D 14.4.5.1 (Pomponius)11; D 14.4.9.1; D 14.5.2 pr ; D 15.1.1 pr-1-2-3-4-5-6; D 15.1.3 pr-1 (Labéon) -2-3 (Pedius) -4-5 (Celsus)-6 (Julien)-7 (Julien)-8 (Nerva filius)-9 (Sabinus, Cassius)-12 (Labéon) ; D 15.1.5 pr-1-2-3-4 (Tubero, Ce1sus) ; D 15.1.7 pr (Tubero, Ce1sus)-1 (Julien, Marcellus)-2 (Pomponius)-3 (Pedius)4-5 (Labêon)-6-7; 15.1 .9 pr-1 (Neratius, Pomponius)-2-3 (Servius)-4 4-5 (Labéon) $6-7$, 15.1 .9 pr-1 (Neratius, Pomponius) 2 (Servius) -4 (Pedius) 4 (Julien)-6-7-8; Deratius, Julien)-4 (Julien) 5 (Julien)-6-7 (Julien) (Julien)-g (Julien) ; D 15 1.13 (Julien); D 15.1.15; D 15.1.17 (Servius, Proculus, Atilicinus) ; D 15.1.19 pr-1 (Pomponius, Marce11us, Papinien) -2 ; D 15.1.21 pr (Mela)-1
(Pomponius)-2-3; D 15.1.24; D 15.1.30 pr (Proculus, Pegasus)-1-2-3-4-5-6(Pomponius)-2-3; D 15.1.24; D 15.1.30 pr (Proculus, Pegasus)-1-2-3-4-5-67 ; D 15.1.32 pr (Julien)-1-2 (Neratius) ; D 15.1.36 (Pomponius) ; D 15.1.41
D 15.2.1pr-1-3-4-5-6-7 (Pegasus, Caelius Sabinus)-8 (Scaevola)-9 (Pomponius); D 15.3.1 pr-1 (Labêon)-2 (Julien, Pomponius); D 15.3.3 pr-1 (Pom ponius)-5 (Labéon, Pomponius) ; D 15.3.5.3; D $15.3 .7 \mathrm{pr}^{-1}$; D 15.3.10.68 (Pomponius); D 16.3.1.28-42 (Julien); D 17.1.8.5 (Julien) (sur le liber homo hona fide samiens); D 17.2.24; $18.1 .29 ; 0$ 18.4.2.6-12 (Julien)13 (Marcellus) ; D 19.1 13.4-13;D 21.1.31.4; D 21.1.33 Pr ; D 21.3.1.4; 13 (Marcellus); D 19.1.13.4-13; D 21. 31 ; D 21.1 .33 pr; D 21.3.1.4 ; (rescrit d'Antonin le pieux); D 29.2.20.4 (Julien, Papinien); D 33.4.1.10; (rescrit d'Antonin le pieux) ; D 29.2.20.4 (Julien, Papinien) ; D 33.4
D 33.6.9.3; D 33.8.6 pr-1-2-3-4 (Pegasus, Atilicinus, Nerva) (rescrit D 33.6.9.3 ; D 33.8.6 pr-1-2-3-4 (Pegasus, Atilicinus, Nerva) (rescrit
de Sévère et Caracalla2-5; $\mathrm{D} 33.8 .8 \mathrm{pr}$ (Pegasus)-1-2-3 (Sabinus)-4 (Sabide Sévère et Caracalla-5; $\mathrm{D} 33.8 .8 \mathrm{pr}$ (Pegasus)-1-2-3 (Sabinus)-4 (Sabi-
nus) -5 (Labéon)-6-7 (rescrit. de Sévère et Caracalla)-8 (Julien); $\mathrm{D} 33.8 .11$; D 33.8.24 ; D 38.5.1.22; D 39.6.36; D 40.1.4.1; D 40.7.3.1-2 (Servius, Labéon, Cassius) $-7-12$; D 40.7.6.6 ; D 41.2.13.8; D 42.4.3.1; D 42.4.7. 15 ; D 42.8.6.12 (Labéon) ; D 44.4.2.7; D 44.4.4.17; D 46.1.3; D 46.3. 18 ; D 47.2.52.9-26; D 50.16.17 pr ; D 50.16.182 ; Marcien D $15.1 .40 \mathrm{pr}$ (P. Fronto) 1 (veteres) ; D 20.3.1.1; D 20.6.8.5; D 30.119; D 33.8.18 D 33.8.20; D 39.4.16.2 (rescrit de Sêvère et Caracalla) ; Modestin D 26.

7.32.3; D 34.3.19. action tributoria (565) et action de in rom verso $(565)$

Reprenons maintenant, les t,ext,es concernant. indopondance de fait et autonomie relative de l'esclave et essayons de dógager une évolution historique du discours des juristes en ces domaines. Quand le droit semble-t-il produire les effets les plus complets ? Pour parvenir à un résultat valable du point, de vue statistique, il faut éviter les doubles emplois. Un mêne texte peut, en effet renvoyer à diffórentes actions concernant un thème identique, qu'il $s^{\prime}$ agisse de l'indépendance de fait ou de l'autonomie relative. Il faut donc, pour opérer une comparaison pertinente des deux thèmes envisagés, ne compter un tel texte qu'une seule fois (567).

565- Visent 1'actio tributoria Julien D 14.4.8; D 14.4.12; raius D 14. 4.11 ; D 14.5.1 ; D 15.1.27 pr (Julien); Paul D 14.1.6 pr;D 14.4.2;14.4.4; D 14.4.10 ; U1pien D 14.1.1.20 ; D 14.3.11.7; D 14.4.1pr-1 (Pedius)-2-34-5; D 14.4.3 pr (Julien)-I (Pomponius)-2 ; D 14.4.5.1 (Pomponius)-2-3-45-6-7 (Labéon) -8-9-10-11-12-13 (Labéon)-14-15-16-17-18-19; D 14.4.6; D 14.4.7 pr-1 (Pedius)-2-3-4-5; D 14.4.9 pr-1-2 (Labéon, Pomponius).

566- Traitent de 1 'action de in rom verso Alfenus (chez auteur anonyme) D 15.3.16 ; Iavolenus D 15.3.2 ; D 19.2.60.7 (Labéon); Neratius $D$ 15.3. 18 ; Julien D 14.3.12 ; Pomponius D 43.26.13 ; Africain D 15.3.17 $\mathrm{pr}^{-1}$; Gaius D 15.3.4 ;D 15.3.12 : Scaevola D 15.3.20.1 ; D 34.3.31.1; Tryphoninus D $15.3 .6 ;$ Paul D $3.5 .41 ; \mathrm{D} 4.3 .20 \mathrm{pr}$ (Labéon); D 14.3.17.4

(Proculus); D 15.3.8 (Pomponius) ; D 15.3.11: D 15.3.14 (Julien, Marce1lus); D 46.4.11.1; Ulpien D 2.13.4.3; D 3.5.13; D 15.1.1.1; D 15 i.3.1 (Labéon) ; D 15.3.1 pr-1 (Labéon)-2 (Julien, Pomponius) ; D is.3. $3 \mathrm{pr}^{-1}$ (Pomponius) -2-3 (Labéon)-4-5 (Labêon, Pomponius)-6 (Labéon)-7-8-910 ; D 15.3.5 pr (Pomponius)-1 (Pomponius)-2-3; $\mathrm{D} 15.3 .7 \mathrm{pr}^{-1-2-3}$ (Labêon) -4 (Labéon) ; D 15.3.10.5 (Marcel1us) -6-7 (Pomponius)-8 (Pomponius)-9 (Pomponius) ; D 15.3.13 (Julien) ; D 18.4.2.6 ; D 38.5.1.22 : D 42. 4.3.1; D 42.8.6.12 (Labéon)

567- Action de peculio ou pécule apparaît aux côtés de 1'action de in rem verso dans Alfenus (chez auteur anonyme) D 15.3.16; Iavolenus D 19.2. 60.7 (Labéon) ; Neratius D 15.3.18; Julien D 14.3 .12 ; Pomponius D 43. 26.13; Africain D 15.3.17.1; Paul D 3.5.41; D 4.3.20 pr (Labéon); D 14.3.17.4 (Proculus) ; D 46.4.11.1; Ulpien D 2.13.4.3 ; D 3.5.13; D 15.1.3.1 (Labéon) ; $\mathrm{D} 15.3 .1 \mathrm{pr}^{-1}$ (Labéon)-2 (Julien, Pomponius) ; D 15.3.3 pr-1 (Pomponius)-5 (Labéon, Pomponius) ; D 15.3.5.3 ; D 15.3.7 $\mathrm{Pr}^{-1}$; D 15.3.10.6-8 (Pomponius); D 19.4.2.6; D 38.5.1.22;D 42.4.3.1; D 42.8.6.12 (Labéon). Action de peculio ou pécule ressort avec action tributoria dans Julien D 14.4.12; Gaius D 14.4.11; U1pien D $14.4 .3 \mathrm{pr}$ (Julien); D 14.4.5.1 (Pomponius); D 14.4.9.1. Ces textes $n$ ' ont donc été considérés que pour une seule référence. Le problème des doubles emr plois se pose de la même manière en ce qui concerne 1 'indépendance de fait de 1 'esclave. Ainsi Caius D 14.5.1 vise à la fois 1 'action exercitoria, 1 'action institoria et 1 'action qund iussu. U1pien D 14.1.1.20 (Pomponius), D 14.3.7.1;0 14.3.13.2 (Julien) visent les a. expreitoria et institoria. D 12.1 .29 (Julien) ; U1pien 0 42.4.3.1. Enfin U1pien 04.9 .3 .3 renvoie à 
Le respect de cette précaution méthoiologique nons conduit au tahleau suivant :

\section{Indépendance de fait}

- analyse des paragraphes.

\begin{tabular}{|c|c|c|c|c|c|c|c|}
\hline I & - & $=$ & - & I & - & $=$ & - \\
\hline II & $0 / 3$ & $=$ & - & II & $3 / 3$ & $=$ & $(100 \%)$ \\
\hline II I & $15 / 127$ & $=$ & $12 \%$ & III & $112 / 127$ & $=$ & $88 \%$ \\
\hline IV & 3/29 & $=$ & $10 \%$ & IV & $26 / 20$ & $=$ & $90 \%$ \\
\hline v & $86 / 460$ & $=$ & $19 \%$ & $\mathrm{v}$ & $374 / 460$ & $=$ & $81 \%$ \\
\hline \multicolumn{8}{|c|}{ - analyse des citations. } \\
\hline I & $2 / 18$ & $=$ & $11 \%$ & I & $16 / 18$ & $=$ & $89 \%$ \\
\hline II & $9 / 68$ & $=$ & $13 \%$ & IT & $50 / 68$ & $=$ & $87 \%$ \\
\hline III & $9 / 92$ & $=$ & $10 \%$ & II I & $83 / 92$ & $=$ & on 6 \\
\hline IV & $5 / 1 / 1$ & $=$ & $36 \%$ & TV & $9 / 1^{1 / 4}$ & $=$ & fis $\%$ \\
\hline v & $0 / h_{1}$ & $=$ & - & v & $4 / 1$ & $=$ & $100 \%$ \\
\hline
\end{tabular}

Les problèmes péculiaires sont déjà une réalité largement prise en considération par les auteurs rópublicains et ceux du ler siècle, ainsi que l'indiquent les fréquents renvois dont ils sont l'objet.

I1. faut bien entendu émettre une réserve sur les $100 \%$ obtenus pal 1 a période II, dans l'analyse des paragraphes, et par la période $V$, dans celle des citations, la base numérique de textes étant dans les deux cas insuffisante.

Surtout, on notera que l'analyse directe montre une prédominance du thème de l'autonomie relative sous les Antonins, plus spécialement sous les derniers de ces empereurs, malgré le faible total réalisé par les juristes de la période $T$ dans $l$ 'analyse des citations, total qui ne signifie nullement une baisse d'intérêt de ces juristes pour les problèmes péculiaires, mais simplement que leurs successeurs se rófèrent moins à leurs solutions qu'à celle de leurs prédécesseurs. Sela confirme notre analyse sur les activités professionnelles. On observe un intérêt croissant des juristes pour les esclaves privilégiés dès le zème siècle, et de manière plus nette encore, dans la deuxième moitié de ce siècle.

1 'action institoria et à l'action quod iussu par voluntas domini. Ces textes n'ont aussi été pris en considération qu'une seule fois. Cela dit, lorsqu'un texte référait simultanément à $1^{\prime}$ action institoria et à $1^{\prime}$ 'action de peculio par exemple, il devait être compté deux fois car concertion de peculio par exemple, il devait être compté deux fois car concer-
nant deux thèmes différents (indépendance de fait et autonomie relative) et non plus deux formes d'un mêtre thème.
En outre, on remarquera que le poursentage obtenu par la póriode v, qui débute, rappelons-le, avec les sévères, reste élevá. Le frit est important. On a vu en effet que la place de l'esclavage dans le discours des juristes diminuait à cette époque. Qune le chiffre obtenlu en matiòre d'autonomie relative soit élevé montre que les próoccupations essentielles des juristes de l'époque des Sévères portent sur les formes "les olus achevées" de l'esclavage.

Ce développement par le droit d'une autonomie économique relative de l'esclave, en vue $d$ 'une moilleure rentabilisation de son travail (568), va lui-même tendre à se trouver dépassé. On s'aperçoit en effet, surtout au 2àme siècle, que les concessions accordées sont insuffisantes car l'esclave peut, pour son activité économique, avoir besoin de sortir des relations fondées sur le pécule.

\section{II - Les progrès de l'autonomie relative}

La jurisprudence a tout d'abord développé la notion d'obligation naturelle, mais l'intérêt du comerce a imposé la reconnaissance de sanctions plus énergiques.

La notion d'obligation naturelle n'est pas apparue comme une innovation considérable. Elle n'a rien d'une pure construction de l'esprit : naturatis signifie conforme ì la nature des choses, dicté ou imposé par elles (560).

Alors qu'auparavant, l'apparition de l'esclave sur la scène juridique se faisait en représentation du maître, tenu de peculio aut de in rem verso et bénéficiant des droits nés du fait de l'esclave, désormais,

568- Sur la rentabilitê de l'esclavage en "chiourme", v. P. DOCKES, La libération, cit, p. $157 \mathrm{~s}$.

569- P. CORNIOLEY, Naturalis obligatio, cit, p. 62 ; Ph. DIDIER, Evolution des notions de liens naturels dans 1 a jurisprudence romaine classique, Paris (1967) p. 15, rapproche ce terme des expressions naturalis datio, naturalis cognatio, naturalis possessio, et donne à l'obligation naturelle le sens d'obligation de fait. Les idées de cet auteur sont reprises dans 1 'article : les obligations naturelles chez les derniers Sabiniens, in RIDA 19 (1972), p. $239 \mathrm{~s}$. C'est à propos de $1^{\prime}$ esclave qu'est apparue la notion d'obligation naturelle, 0. GRADENWITZ, Natur und Sklave beì der naturalis obligotio, (1900), p. 133-179; R. MONIER, Droit romain, cit. II, p. 252. Conme pour le pécule, le fils a donc été "à la remorque" de l'esclave. Il semble cependant que les développements concernant le prenier aient été plus incisifs et aient relégué l'o.n. au second plan (cf. en matière de capacité processuelle, A. GUARINO, Diritto privato romano, cit. p. 711 ; R. VILLERS, Rome et le droit privé, París (1977), p. 237.). 
arec l'obligation naturelle (570), ce n'est plus le maître qui est tenu à travers l'apperence d'obligation de l'esclave, l'obligation est relstive à l'esclave lui-même. Cette rotion semble être apparue dès avant Iavolenus selon la doctrine dominante (571). Prenant naissance à propos de relations relatives au pécule, elle va bientôt, pour répondre aux besoins de la pratique, se détacher de celui-ci. La survie de l'obligation naturelle au pécule semble avoir été préconisée par les disciples de Julien (572). Les effets essentiels de l'institution, la soluti retentio,

570- Selon Ph. DLDIER, Evolution, cit, P. 266, naturale debitum et naturalis obligatio seraient deux notions distinctes, la première visant un acte moralement souhaitable, la seconde donnant un nou au debitzom servi vel filizi. Cet auteur pense que le parallèle doit être établi non entre civilis obligatio et natumlis obligatio, mais entre naturalis obligatio et naturale debitum. Contra, I. BUII, Studi, cit, p. $271 \mathrm{n} 120$, relève que et naturale debitum. Contra, délence entre naturale debitun et devoir d'équité chez Julien est 1 'équivalence entre naturale debitum et devoir d'équité chez Julien est
fondée sur la dernière partie de $D$ 15.1.11.2 vlpien (... naturalia enim fondée sur la dernière partie de $\mathrm{D} 15.1 .11 .2$ vlpien (... naturalia enim
debita spectamus in peculii deductione : est autem natura aequon liberari filium vel servrom obligatione eo quod indebitum videtur exegisse) généralement considérée comne interpolée (J. VAZNY, Natruaís obligatio, in St. Bonfante 4 (Milan 1930) p. $149 \mathrm{~s}$, a partir de naturalia enim; mais la doctrine dominante pense que 1 ' interpolation porte seulement sur est autem, fin du passage, cf. G.E. LONGO, Concet to e limiti dell'obbligazione natura dello schiavo nel diritto romano classico, in SDHI 16 (1950) p. $105 \mathrm{n} 49$, et Ricerche sull'obligatio naturalis, Milan (1962) p. 105 n160: P. CORN1OLEY, Naturalis obligatio, p. 173 ; A. BURDESE, Dubbi in tema di naturalis obligatio, in St. Scherillo, Milan (1972) p. 149.) D'autre part, toujours selon I. BUTI, la comparaison ne semble opérable qu'entre debition (ou obligatio) naturale et obligatio civilis, come le montrerait D 35.1.40.3 Iavolenus (...ego puto secundim mentem testatoris naturale magis quan civile debitrom spectandwom eser)

571- En ce qui concerne la date d'apparition de l'expression naturalis obligatio servi , la doctrine est loin d'être monolithique. Selon P. CORNIOLEY, Naturalis obligatio, cit, p. $157 \mathrm{~s}$, la natraralis obligatio servi aurait été admise seulement par Papinien et Paul. Contra, M. RASER, cr. de CORNIOLEY, in ZSS. RA 83 (1966) p. 469 ; L. LABRUNA, cr. de CORNIOLEY, In IURA 16 (1965) p. $416 \mathrm{~s}$; G.E. LONGO, $\mathrm{cr}$. de CORNIOLEY, in Labeo 12 (1966) p. $383 \mathrm{~s}$. et A. BURDESE, Dubbi, cit, p. 499. Y adhèrent G. BROGGINI, Obligatio naturalis, Milan (1966) p. 502 s. et L.B. MER, cr. de CORNIOLEY, in RH (1966) p. 281 . I. BUTL, Studi, cit, p. $265 \mathrm{~s}$. soutient que la notion d'obligatio naturalis avec référence a l'esclave énergerait que la notion d'obligatio naturalis avec référence a l'esclave énergera
déjà chez Sabinus ou peut-être Labéon, de toute façon avant Iavolenus. RUNTZE, Cursus des röm. Rechts (Leipzig 1879) p. 444, et J. VAZNY, Natura$l_{i s}$ obígatio, cit, p. 145, pensent que $\mathrm{D} 35.1 .40 .3$ indiquerait que Labéon a employé le premier le terme de naturalem debitrm. Des éléments en ce sens chez J. GAUDERET, cr. de BURDESE, in Labeo 12 (1966) p. 112.

Sur les rapports entre monde moral et monde juridique à propos de robligation naturelle, v. A. MANTELLO, Beneficium servile-debitum naturale I, Rome (1979), spéc. p. $72 \mathrm{~s}$.

572- Selon Julien, sauf si le pécule est légué, l'obligation naturelle ne survit pas laffranchissement. Ce sont les disciples de Julien qui ont admis la survie de l'obligation naturelle au pécule. Paul cite Pompoaius et non Julien (D 12.6 .13 pr : Natumliter etian servus obligatur : et ideo, si quis nomine eius solvat vel ipse manumissus, ut Pomponius scribit, ideo, si quis nomine eius solvat vel ipse manumssus, ut Pomponius ocribit,

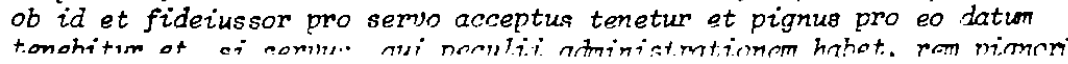

impossibilité de répéter la some payée en vertu de l'obligation, et la validité de la fideiuasio garantissant 1'obligation (573), sanctionnent aussi bien les dettes que les créances serviles, au point de faire de I'esclave le créancier naturel de son propre maître (574), avant de pouvoir devenir celui d'extranei au début du 3ème siècle (575).

in id quod debeat dederit, utilis pigneraticia reddenda est) come ayant établi la possibilité pour 1 'affranchi, indépendamment du cort de gon ancian pécule, de payer sa dette après son affranchíssement. Tout ce que Juliea a pu admettre, c'est la survie de l'obligation du fidéjusseur à 1 a digparition du pécule, G. BOULVERT, cr. de DIDIER, in Index 2 (1971) p. 35. da conception de l'obligation naturelle selon Gaius (III, 119 a) est scricta conception de ${ }^{\prime}$ obligation naturelle selon Gaius (III, 119 a) est stric tement conforme a celle de Julien, G. BOULVERT, La validité de 1 'obliga-
tion principale dans le cautionnement verbie, in Et. Audinet (Aix-en-Protion principale dans le cautionnement verbie, in Et. Audinet (Aix-en-Provence 1966 ) p. 33. Sur ce texte, L. LABRUNA, Interpretazione di Gaius.
III, $119 \mathrm{~s}$, in Gaio nel suo tempo, Naples (1966) p.55 s. G.E. LONGo, ConcetIII, $119 \mathrm{~s}$, in Gaio nel suo tempo, Naples (1966) p. $55 \mathrm{~s}$. G.E. LONGO, Concet-
to, cit, p, $108 \mathrm{~s}$. et Ricerche, cit, p. $47 \mathrm{~s}$, tout en admettant que l'obligation naturelle de l'esclave ne soit pas liée a l'existence effective d'un pécule, rattache celle-ci, à l'époque classique, a l'existence théorique d'un moyen de coercition lié au pécule. Contra, A. BURDESE, in Iura 14 (1963) p. $267 \mathrm{~s}$; G. DONATUTI, in BIDR 64 (1962) p. $311 \mathrm{~s}$; L. LABRUNA, in labeo 10 (1964) p. 286, et G. BOULVERT, in Et. Audinet p. $33 \mathrm{~s}$. Pour I. BUTI, Studi, cit, p. 273, la qualification de naturalis a propos de la 1. BUIT, Studi, cit, p. 273, la qualification de naturalis a propos de 1 a créance de 1 esclave serait nêe avec la référence à des rapports extrapeculiaires (cf. D 35.1.40.3), le terme debitum, en dehors des relations péculiaires, ayant dû paraître inadéquat. Mais le terme aurait très tôt englobé les obligations péculiaires. L'auteur ajoute (p. $273 \mathrm{n} 125$ ) que $1 \mathrm{a}$. notion de naturalis obligatio servi est née par référence à des dettes qui, contractées en présence d'un pécule, devaient être définies à partir du moment où le pécule venait à inanquer, mais ceci $n$ 'exclut pas 1 'hypothèse plus Eréquente de l'obligation naturelle lorsque l'esclave était pourvu du pécule. Il est en effet évident que, hormis de rares exceptions, seuls les esclaves pcurvus d'un pécule pouvaient contracter des obligations.

573- Julien D 46.1.16.3-4.

574- Iavolenus D 35.1.40.3: Dominus servo aureos quinque eius legoverat: heres meus Sticho servo meo, quem testcmento libernom esse iussi, cureos quinque, quos in tabulis debeo, dato. Nihil servo legation esse Namusa Servium respondisse scribit, auia dominus servo nihil debere gotuisset : ego puto secuordon mentem testatoris naturale magis quam civile debitum spectandum sase, et eo iure utimur. Tryphoninus D 12.6.54 : Si quid dominus sexvo debuit, manumisso solvit, quamvis existimans ei aliqua teneri actione, tomen repetere non poterit, quia naturale adgnovit debitim : ut enim libertas naturali iure continetur et dominatio ex gentium iure introducta est, ita debeti vel non debiti ratio in condictione naturaliter intelligenda ita debeti vel non debiti ratio in condictione naturaliter intelligenda
est. Ce dernier texte est tenu pour interpolé de ut enim à la fin par est. Ce dernier texte est tenu pour interpolé de ut enim à la fin par
A. BURDESE, la nozione, cit, p. $56 \mathrm{~s}$; G. DONATUTI, cr. de Lengo, cit, p. A. BURDESE, La nozione, cit, p. $56 \mathrm{~s}$; G. DONATUTI, cr. de Lengo, cit, p.
314 ; G. BROGGINI, Obijgatio naturalis, cit, p. 499 ; P. CORNIOLET, Naturalis obligatio, cit, p. 171 s. M. KASER, cr. de CORNIOLEY, cit, p. 464 , pense que dans la partie finale du texte, on peut relever des traits classiques.

575- L'extension aux créances de l'esclave envers un extraneus ne semble se faire qu'au début du 3ème siècle, vlpien 0 44.7.14 (Servi ex delictis quidem obligantur et, si monumittantur, obligati remanent : ex contractibus autem civiliter quidem non obligantur, sed naturaliter et obligantur et obligant. Denique si servo, qui mihi mutuan pecuniam dederat, manumiaso solvan liberor). Le passage, retenu interpolé par H. SIBER, Naturalis obligatio, 
Mais l'obligation naturelle, telle qu'elle existe à l'ópoque classique (576), est loin de comprendre toutes les opérations de l'esclave en nom propre.

Les textes du Digeste paraissent montrer que les opérations aluxquelles prennent part les esclaves peuvent avoir d'autres offets que ceux que nous venons de rencontrer. Jusqu'à la fin du ler sip̀cle, apràs l'affranchissement, les créances de $l$ 'ancien esclave envers son ancien maitre semblent ne pas produire d'effet juridique (577). Cependant, au zème siècle, on relève une tendance à reconnaitre certains effets de droit à de telles créances de l'esclave lorsque ces effets ne sont pas ouvertement opposés au principe de l'incapacité. Ils ne sont toutefois admis que dans des cas d'espèce où les circonstances sont de nature à faire

Leipzig (1925) p. 35, est considéré conme authentique par 0. GRADENIJITZ, Leipzig (1925) p. 35, est considéré comme authentique par o. GRADENIJIT,
Natur und Sklavebei der Naturalis obligatio, in Fest. J. Th. Schirmer, Natur und Sklave bei der Naturalis obligatio, in Fest. J. Th. Schirmer
Xönigsberg (1900) p. 171 ; B. B IONDI, Actiones noxales, Ann. Palerme (1925) p. 175 ; v. DEVILLA, in St. Sassaresi 17 (1939) p. 237. La doctrine majoritaire exclut 1 'existence a 1 'époque classique des créances naturelles de l'esclave. G.E. LONGO, Concetto, cit, p. $91 \mathrm{n} / 2$, nie toute existence des créances serviles envers des extranei et n'admet l'existence de créances de l'esclave envers son maître qu'a l'époque de Justinien.

p. CORNIOLEY, Naturalis obligatio, cit, p. 170 soutient aussi que la notion de créance servile envers un tiers est étrangère au droit classique.

A. BURDESE, La nozione, cit, p. $53 \mathrm{~s}$, pense que la créance de l'esclave envers son maître est classique. I. BUTI, Studi, cit, p. $247 \mathrm{~s}$, soutient que ces dewx types de créances serviles sont classiques, étant donnée 1 'impossibilité pour les juristes, lorsqu'ils parlent de dettes ou de créances serviles, de se référer a une autre notion qu'a celle de naturalis obligatio. 576- La recherche actuelle semble restreindre de plus en plus le champ
des obligations naturelles relativement a leur étendue et à leurs effets ; par exemple E. NARDI, In tema di confini dell'obligazione naturale, in par exemple E. NARDI, In tema di confini dell obligazione naturale, in St. De Francisci, Milan ( 1956$)$ p. 585 , exclut la rétention des effets de
l'obligation naturelle. A nous en tenir rigoureusement aux opinions exprimées par les classiques, le champ des obligations naturelles est beaucoup plus, restrejnt qu'on ne $l^{\prime}$ imaginait autrefois, M. JACOTA, L'esclave créancier, in St. Grosso 2 (1968) p. $228 \mathrm{n} 13$.

577- Fondamental sur le problème, M. JACotA, L'esclave créancier, cit, p. 215 s. Ulpien D 33.8.6.4 (cite Nerva et Pegasus) : Sicut autem aes alienon, hoc est quod debetur domino, minuit legatum peculizm, ita per contrarizon id quod dominus dobet servo augere dobet. Sed huic sententiae adversatur rescriptum imperatoris nostri et patris eius, quod ita est : com peculizm servo legatur, non etiam id conceditur, ut petitionem habeat pecuniae, quam se in rationem domini impendisse dicit. Quid tamen si haec voluntas fuit testatoris? Cur non possit consequi? Certe corpensari debet hoc quod impendit cum eo quod domino debetur. An et quod dominus scripsisset se servo debere, peculio legato cederet ? Pegasus negat : idem Nerva. C'est une conséquence du principe du ius civile sur l'incapacité de l'esclave. admettre une telle décision (578). On observe un certain parallélisme de l'évolution jurisprudentielle en matière de dettes de l'esclave (57?).

Le milieu du zème siècle senble avoir vu le point culminant des solutions dans ce domaine (580). Au début du 3ène siècle, tout en continuant à se préoccuper, comme on l'a vu, des formes achevées de l'esclavage, on en revient à des effiets plus modestes des opérations de l'esclave en son nom propre (581). Hors ces espèces où il s'agit d'une sorte de prolongation chronologique du pécule, les juristes des zème et jème siècles (582) reconnaissent aux pactes de liberté entre l'esclave et son maître ou entre $l$ 'esclave et un tiers certains effets substantiels grâce à l'action de dol (583), l'actio in faction (584) ou une simple intervention

578- Scaevola parle d'une consuetudo domua selon laquelle on restitue a l'esclave les sommes qu'il a déboursées pour couvrir les dépenses de son maitre, cf. D 33.8.23.1. Décision en sens inverse avec un rescrit de Septime-Sévère et Caracalla, chez Ulpien D 33.8.6.4 (cité supra n577), selon lequel les créances de l'esclave n'augmentent pas le pécule.

579- Proculus et Pegasus admettent come solution d'espèce que l'esclave, affranchi entre vifs, qui a reçu son pécule, peut être poursuivi dans la mesure du pécule pour les dettes qu'il a contractées pendant l'esclavage, cf. D 3.5.17 Paul. Lavolenus admet, bien qu'indirectement, que l'esclave, affranchi testamentaire, légataire du pécule, peut être tenu pour les dettes contractées pendant l'esclavage, cf. D 33.8.17.

580- M. JACOTA, L'esclave créancier, cit, p. 234.

581- Marcien D 33.8.18: Si servo monumisso peculizm legation fuerit, in eum sine dubio creditoribus peculiariis actiones non competunt : sed non alias heres peculium praestare debet, nisi ei caveatur defensu iri aciversus creditores peculiarios. Selon Marcien, l'esclave ne peut être poursuivi par les créanciers. C'est i'héritier qui est poursuivi en raison des dettes du pécule. Il admet cependant de manière indirecte une certaine responsabilité de l'esclave par le fait que l'héritier ne remet le pécule à l'affranchi que $s^{\prime}$ il donne caution de 1 'indeumiser pour ce qu'il paierait aux créanciers.

582- Sur ce point, autre article important de M. JACOTA, Les pactes de 1 'esclave en son nom propre, in RIDA 13 (1966) p. $205 \mathrm{~s} ; \mathrm{cr}$. A. GUARINO, in Labeo 13 (1967) p. 295 qui, exprimant son assentiment à l'idée présidant à cet article, émet quelques réserves quant à l'argumentation de 1 'auteur.

583- Ulpien D 4.3.7.8 : Servus pactionis pro libertate rewn domino dedit ea condicione ut post libertatem transferatur in eum obligatio : monomissus non patitur in se obligationem transferm. Pomponius scribit locum habere de dolo actionem, sed si per patronion stabit, quo minus obligatio transferatur, dicendum ait patronum exceptione a reo sumovendum. Ego moveor: quemadmodum de dolo actio dabitur, cum sit alia actio? Nisi forte quis queat, quonion exceptione patronus sumoveri potest, si agat cum reo, debere dici, quasi nulla actio sit quae exceptione repellitur, de dolo decernendici, quasi nulla actio sit quae exceptione repellitur, de dolo decemendam : atquin patronus tunc sumovetur, si nolit expromisaorem ipsum manumissum accipere. Expromissori plane adversus monumisszon dari debebit de signale les interpolations ego moveor-manumissum accipere (cf. Index itp.). Le passage dans sa totalité a été abrégé et considérablement remanié, mais la solution contenue dans expromissori plane... domino dabitiv est classique 
du govverneur de province (585;. Ie pacte ut minus soivitup conclu par l'escluve héritier ante aditam hereditatem avec les créanciers de la succession va finir par être consiaéré comme valable (586).

Ces solutions d'espèce auraient pu aboutir ̀̀ la reconnaissance d'une capacité conventionnelle de l'esclave si l'évolution économique n'avait rendu moins utile une telle capacité (587). Elles allaient cependant dans l'intérêt des maîtres. En effet, la promesse de la liberté, la possibilité d'acquérir un gain, de l'utiliser pour obtenir la liberté, quoi de p.lus stimulant pour l'activité de l'esclave et son respect de la hiérarchie sociale (588)? De plus, ces innovations se caractérisent par leur aspect conservateur. Admises dans la seule mesure oì elles sont indispensables, elles demeurent dans le cadre des formes traditionnelles (589), elles constituent tout au plus des exceptions. Enfin, ce changement n'aen définitive eu lieu que pour une minorité du groupe servile,

au fond, M. JACOTA, Les pactes de 1 'esclave, cit, p. $209 \mathrm{n} 12$. Sur les abréviations, P. BONFANTE, Histoire du droit romain ( $t r$. fr.) Paris (1928) II p. 154 ; J. GAUDEMET in RIDA (1957) p. 259 s. et RIDA (1960) p. 399 s. Sur le passage aussi A. GUARINO, cr. JACOTA, Labeo 13 (1967) p. 295.

584- Al ex. Sévère en 222 , CI $4,14,3$.

585- $D^{\prime}$ après une constitution de Marc-Aurèle et Verus, si le redemptor refuse d'affranchir l'esclave racheté suis nummis, celui-ci a la faculté de porter plainte devant le préfet de la ville à Rome, et devant le gouverneur dans les provinces, cf. D 40.1 .5 pr Marcien. V. aussi CI 4.6.9.

586- Selon Vindius Verus, consul en 138 , ce pacte n'est pas valable, mais Marcellus 1 'admet et Ulpien suit 1 'avis de celui-ci, cf. D 2.14.7.18 U1pien : Sed si semus sit qui paciscitur, priusqucm libertatem et heredita tem apiscatur, quia sub condicione hereo scriptus fuerat, non profuturn tem apiscatur, quia sub condicions harso scriptus fuerat, non profutumom

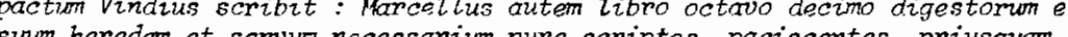
sum hereden et servon necessarium pure scriptos, paciscentes, priusquan se immisceant putat recte pacisci, quod veriom est. Le passage est jugé interpolé, cf. Index itp. ; F. PRINGSHEIM, Subsidiaritat und Insolvenz, in ZSS RA 41 (1920) p. 252 (quia sub-fuerat et pure scriptos). Sanction : exceptic pacti, M. JACOTA, Les pactes de 1'esclave, cit, p. 217. Dans un autre domaine, le pacte de l'esclave ne a se peteretur est nul au temps de Paul, cf. D 2.14.21.1 : Quod si sorvus, ne a se peteretur, pactus fuerit, nihil valebit pactum : de doli exceptione videamus. Et si in rem paciscatur, proderit domino et heredi eius pacti conventi exceptio : quod si in personom pactum conceptim est, tunc domino doli suparast excoptio. $L$ exception de dol (de doli exceptione videamus) envisapee dans le texte est sans de dol (de doli exceptione videaruino doti superast exceptio. doute interpolé (cf. Index itp.). On ignore a quelle epoque cette solution a été admise. Il semble que Justinien, par ses interpol ations, $n$ ait fait que reconnaitre une pratique plus ancienne, M. JACOTA, ibid., p. $219, \mathrm{n} 22$. 587- ibid., p. 226 s.

588- ibid., p. 206

589- M. JACOTA, L'esclave créancier, cit, p. 236. les privilégiés, auxquels certains suteurs vont jusqu ì feconra: une capacité processuelle (500).

Il est vrai qu'en ce domaine aussi le principe de l'incapacité de l'esclave a dû être gênant. Lorsqu'un esclave faisait des opérations avec son pécule dans une province lointaine, comment exercer l'actio de peculio contre son maitre si celui-ci était en Italie? Il est possible que l'action fût alors intentée directement contre l'esclave titulaire du pécule (591). Toutefois, juristes et empereurs ont soutenu avec force le principe de l'incapacité processuelle de l'esclave (592) contre une pratique relevant du droit vulgaire. La reconnaissance de la capacité processuelle eut constitué un point de non retour dans l'admission d'une véritable capacité gervile.

On voit donc les juristes s'intéresser particulièrenent aux fonctions serviles privilégiées au 2ème siècle, de manière plus importante encore à partir de la deuxième moitié de ce siècle. Le choix d'un critère d'investigation juridique (593) confirme sur ce point l'analyse menée sur les activités professionnelles de l'esclave. Dès le ler siècle, les juristes se préoccupaient de mieux réglementer l'activité écononique de l'esclave dans le sillage de son maitre, mais, sous les Antonins, ils vont plus loin et lui permettent dans certains cas d'agir en nom propre. c'est le point culminant d'une évolution qui correspond à une nouvelle définition des relations esclavagistes, plus prócisément à la substitution d'une exploitation subtile à $L^{\prime}$ exploitation exclusivement brutale du travail servile. Cela explique que les textes traitant des esclaves privilégiés soient quantitativement importants. La situation de ceux-ci pose évidemment beaucoup plus de problèmes aux juristes que celle des esclaves exploités par la force. Les pourcentages font apọarâtre

590- A. BISCARDI, La capacità processuale dello schiavo, in Labeo 22 (1976) p. $143 \mathrm{~s}$. Il faut noter que dans les espèces jusque 1 à envisagées, c'est affranchis que leg esclaves font les procès.

591 - J. MACQUERON, cité par BISCARDI, o.c. p. $160 \mathrm{n} 111$.

592- Ibid. n64 et 65. Mais cela, selon A. BISCARDI, o.c. p. 162 s., n'est que le résultat d'une évolution : il est en effet démontré par cet auteur que pour 1'esclave agissant pour le compte du dominus, certains juristes, et plus précisément les Proculiens, ont eu tendance à admettre la capacité de $l$ 'esclave à être partie dans un procès.

593 - Une comparaison entre les activités qualifiées de dépendantes et toutonomes n'est pas possible, ces catégories se chevauchant. Par exemple, l'esclave astor a gênéralement in jécule. 
de semblables préccupations à l'époque des SEvères. Ce n'est que par la suite que ces constructions juridiques connaîtront un arrêt, faute d'intérêt, compte tenu sans doute du rôle de plus en plus restreint de l'esclave dans la production sociale (594).

Oo peut certes parler de changement puisqu'on en est venu à reconnaître à l'esclave une certaine "capacité patrimoniale", lui permettant d'agir de façon relativement autonome, "dans son propre intérêt". Mais cette meilleure utilisation de la capacité de l'esclave à représenter son maître ne tend-elle pas directement à augmenter les profits de celui-ci ? En outre, les esclaves que le droit prend en considération dans ce domaine ne sont-ils pas eux aussi des propriétaires d'esciaves, et en tout cas totalement intégrés au système ?

\section{CHAPITRE II - L'ESCIAVE A LA TETE DE BIENS MATER IELS}

Il est nécessaire de répondre à deux questions. De quoi le pécule est-il concrètement composé ? Est-il souvent légué à l'esclave affranchi ? SECTION 1 - LA COMPOSITION DU PFCULE

Peuvent sé trouver dans le pécule des biens meubles comme immeubles (595).

On relève un fonds (596), une maison (597), mais aussi de l'or ou de l'argent (598), un vêtement (599), du vin (600), des animaux (601). Florentinus nous apprend que le pécule peut aupgnenter par les Économies de l'esclave ou ce qu'il acquiert par son travail (602). Celsus parle d'un pécule d'une valeur de "dix" (603), mais cette estimation n'est

594- M. JACOTA, Les pactes de l'esclave, cit, p. 229.

595- Upien D 15.1.7.4: In peculio autem res esse possunt omes et mobiles et soli. Plus loin, ce juriste a joute : hoc amplius et nomina debitorm. On a vu (cf. supra n561) qu'étaient également affectés au pécule le produit des actions intentées servi nomine ainsi que celui des legs et successions.

596- Julien D 19.1.24 pr : Si servus... fundum emerit ; D 41.4.7.2 : Servus meus Titio mandavit, ut fundum ei emeret; Ulpien D $33.8 .6 \mathrm{pr}$ (Celsus, Julien) : Si peculivm legetur st sit in corporibus, puta fundi... ; D 6.1. 41.1 : Si servus... furdzon vendidit.

597- Pomponius D 15.1.22 : aedizon peculiarman; vlpien (Celsus, Julien) D $33.8 .6 \mathrm{pr}: \ldots$ vel aedes.

598- vlpien D 14.4.5.13: Cetera tomen, quae extra haec in peculivm habuit, non tribuentur, ut puta argentum habuit vel oumon.

599- Pomponius D 15.1.25: Id vestimentron peculii esse incipit, quad ita dederit dominus, ut eo vestitu servion perpetuo uti vellet eoque nomine ei traderet, ne quis alius eo uteretur idque $a b$ eo eius usus gratia custodiretur. Sed quod vestimention servo dominus ita dedit utendum, ut non semper, sed ad certion usion certis temporibus eo uteretur, veluti cum sequeretur sed ad sive cenanti ministravit, id vestimentron non esse peculii. Si le maitre a renis a l'esclave un habit potị qu'il s'en serve tous les jours, il fait vraisemblablement qu'indicative. On notera toutefois, et le rapprochement est intéressant, qu'il s'agit généralement du prix, toujours indicatif il est vrai, demandé à l'esclave pour l'obtention de sa liberté (504)

Le pécule peut même comprendre des esclaves, les vicarii. C'est de ces derniers dont il est 1 e plus question dans le Digeste (605). Les ordinarii peurvent intégrer à leur pécule un ou plusieurs esclaves par legs (606), achat (607) ou autre forme d'acquisition (608). On a vu,

partie du pécule. Si en revanche il ne le lui a remis qu'occasionnellement. par exemple pour le suivre ou pour le servir à table, il n'en fait pas partie.

600- Upien D 33.6.9.3 : Vino autem paterno legato id demm legatim videtur, quod testator vini numero habuit, non quod pater. Item si peculiare vinum legation sit, id continebitur, quod servi habuerunt. Cur tan diverse? Guod paternum vinum ion coepit usus ipsius testatoris esse, at peculiare in usu servorion remansit. La distinction a son importance : il ne faut pas confondre le vin rëservé au mầtre avec celui qui est destiné aux esclaves. La discrimination sociale s'opère même en atière de boisson.

601- Pomponius D 15.2.3: pecomon fetu; Tryphoninus D 15.1.57.2 : fetus pecudion; Ulpien (Julien) D 33.8.8.8: fetus pecomom.

602- Florentinus D 15.1.39.

603- Cel sus D 19.1.38 pr.

604- Cf. supra étude sur le prix de l'esclave.

605- Alors que 8 textes seulement se réfèrent à des choses diverses

(fonds, maison, vêtement, or, argent, vin, animaux), 64 visent des esclaves. H. ERMAN, Servus vicarius, L'esclave de l'esclave romain, in Recuei publié par la Faculté de Droit de l'Université de Lausanne à l'occasion de l'exposition nationale suisse (Genève et Lausanne 1896). $\mathrm{Ch}$. 1 \& 4 , explique l'appellation vicarius par la pratique de permettre à des esclaves de se procurer d'autres esclaves pour agir a leur place dans les services qu'ils devaient rendre au maître. Ch. LECRIVAIN, vicarius, D.S.D.A. v p. 825 et n9, estime que c'est pour remplir une condition mise a son afranchissetnent que l'ordinarius achette et forme un vicarius qu'il doit laisser comme remplaçant.

606- Le legs peut être fait par un tiers, cf. Marcellus D 35.2.56.4 Paul D 35.2.21.1. Le maître lègue que lquefois à son esclave, lors de $1^{\prime}$ affranchissement, son pécule avec les vicarii qui en font partie, mais le problème est ici différent : d'une part, le vicarius avait déjà été acquis par l'esclave puisqu'il faisait partie de son pécule ; d'autre part, l'esclave une fois affranchi va devenir le véritable maître du vicarius, c'està-dire non plus seulement le titulaire de fait, mais le titulaire de droit. Traitent d'un legs de pécule avec les vicorii fait à l'esclave : Iavolenus D 33.8.22.1 (Trebatius, Labêon) ; Celsus D 33.8.25 ; Africain D 15.1.38.2 D 33.8.16 $\mathrm{pr}^{-1}$; Paul D 15.1.18; D 33.8.15 (Alfenus); Ulpien D 33.8.6.2 ; Que 1 e dominus soit le veritable nropriataire (Ales vicari fait qu'il peut les léguer à un tiers Selon Pomponius (chez Ulpien D 73.5) le leus des esclaves n' inclut pas les vicarii, mis W.W. BuCKLAND. 73.5), le legs des esclaves $n$ inclut pas les vicari, mais W.W. BUCKLAND, mus n'est, cit, p. 240, pense qu 11 n en était pas toujours ainsi. Le vicarius n'est pas juridiquement considéré consune un accessoire : si un esclave est légué cum vicariis le legs des vicarii est valable même dans le cas où 'esclave est mort (cf. Gaius D 33.8.4). 
à propos du prix de l'esclave, que les textes fournissaient des estimations de vicarii allant de 125 à 250 deniers (609), ce qui constitue une moyenne relativement basse. Il se peut néanmoins que le vicarius ait une valeur supérieure à celle de l'ordinarius dans le pécule duquel il se trouve $(610)$. Les activités des vicarii sont multiples : on relève un institor (611), un exercitor ravis (612), un magister navis (613), un marin $(61 /)$; ailleurs, on remarque à la campagne des vicarii d'un vilicus (615). Ulpien signale un esclave louant les operae de son vicarius à un tiers (616). Pomponius traite d'un esclave qui prostitue les ancillae qui sont dans son pécule (617). Le fait que certains textes parlent d'une responsabilité contractuelle encourue vicarii nomine suggère que les vicarii appartiennent généralement à des esclaves tels que les dispensatores ou les institores, dont ils agissent corme les employés (618).

607- Cf. par exemple Julien D 15.1.37.1 ; D 19.1.24.1 (Sabinus, Cassius) ; Africain D 21.1.51 pr ; Jlpien D 15.1.11.4 (Julien).

608- v. Ch. LECRIVAIN, D.S.D.A. V,p. 824 n20.

609- 125 deniers : Ulpien D 15.1.11.4 (Julien); 200 deniers : Julien D 15.1.37.1 ; 250 deniers : Julien D 10.3.25 ; Africain D 15.1.38.2 ; U1pien D 15.1.11.5.

610- Paul D 21.1.44 pr: :.. nom et plerromue plus in peculio est quam in servo, et nonmomquam vicarius qui accedit pluris est quam is servus qui venit. W.W. BUCRLAND, Roman law, cit, p. 240 , écrit qu'il doit s'agir d'un esclave âgé qui a amassé un pécule important.

6I1- Ulpien D 14.3,11.8 : Si a servo tuo operas vicarii eius condurero et evon merci meae institorem fecero... ; 0 14.4.5.3 ; Item parvi refert, com ipso servo contrahatur an ow institore eius.

612- Ulpien D 14.1.1.22:Si tomen servus peculiaris volente filio

familias in cuius peculio erat, vel servus vicarius eius naven exercuit.. On a vu (supra n53I) que G. LONGo, Actio exercitoria, cit, p. 600, proposait d'éliminer du passage vel servus vicarius.

613- U1pien D 14.1.1.23: Quanguam autem, si cum magistro eius gestrom sit, dumtaxat polliceatur praetor actionem, tcmen, ut Iulionus quoque scripsit, etiansi cum ipso exorcitore sit contractron, pater dominusve in solidum tenebitur.

614- Paul D 9.4.19.2 : Si servus tuus navam exercuerit eiusque vicarius et idem nauta in eadem nave dammum dederit...

615- Scaevola D 20.1.32 : Stichus vilicus... Stichi vicarii.

616- Ulpien D 14.3.11.8 (supra n611).

617- Ulpien D 3.2.4.3 : Fomponius et evm, qui in sorvituto peculiaria mancipia prostituta habuit, notari post libertatem ait.

618- H. ERMAN, sorvus vicarius, cit, 54,5 , arrive à cette conclusion a partir des inscriptions.
Si l'on en croit le Digeste, il est fréquent qu'un esclave ait plusieurs vicarii (619). Ceux-ci peuvent même avoir leur pécule, distinct de celui de l'ordinarius (620). Dans ce pécule peuvent également se trouver des esclaves : des vicarii de vicarii (621). Le fait est remarquable. On observe un phénomène de mimétisme social en cascaje. En effet, l'esclave dont la position économique est confortable tend à acquérir des esclaves, c'est-à-dire à devenir lui-même propriétaire d'esclaves : le cas de celui qui prostitue les ancillae de son pécule est particulièrement édifiant. Les privilégiés ne cherchent absolument pas à se démarquer des pratiques esclavagistes ; iI est vrai qu'ils en retirent les "fruits". Plus surprenante est a priori la situation du vicarius qui a lui-mêne ses propres sous-esclaves, mais elle ne rósiste pas à l'analyse : il s'agit là encore d'un privilégié, comme le montrent quelques-unes des fonctions précédement exposées.

Une évolution chronologique de ce ohénomène est-elle verceptible? On a affaire à deux sortes de textes : dans certains, la référence au terme vicrrius apparaît expressément ou il est clairement indiqué qu'il

619- La présence de plusieurs vicarii dans le pécule d'un esclave ordinarius ressort de : Celsus D 33.8.25 ; Gaius D 33.8.4 ; Scaevola D 20. 1.32 ; U1pien D 3.2.4.3 (Pomponius) ; D 15.1.17 (Servius, Proculus, Ati1icinus); D 14.4.5.14 ; Marcien D 15.1.40.1 (veteres). II en va de même des textes traitant des partus ancillarmo se trouvant dans le pécule : Pomponius D 15.2.3 ; Tryphoninus D 15.1.57.2 ; Upien D 33.8.8.8 (Julien). 620- Selon Ch. LECRIVAIN, vicarius, D.S.D.A. V, p. 825, le vicarius est en droit la propriété du maître qui seul a le droit de $1^{\prime}$ 'affranchir, soit isolément, soit en même temp̣s quue l'esclave ordinaire, ou d'autoriser 1 'affranchissement; sur ce point, v. A. BERGER, Nota minima sur semins vicarius, Iura 8 (1957) p. 123. En fait, cependant, avec le consentenent implicite du mâ̂tre (cf. Jlpien D 15.1.5.4 (Tubero, Celsus) ; D 40.7.6.6), l'esclare ordinceius profite des acauisitions du vicarius pour son propre 1 esclave ordinarius profite des acauisitions du vicarius pour son prop pecule (ce. Poponius D 18.1.31, Paul D 9.4.19.2; Ulpien D 14.3.11.8), (cf. Celsus D 15.1.6 (Tubero, Labéon); Ulpien D 15.1.5.4 (Tubero, Celsus)) Ce pécule obéit aux mêmes règles que le pếcule ordinaire (cf. Pomponius Ce pécule obéit aux mêmes règles que le pécule ordinaire (cf. Pomponius
D 15.1.4.6 ; Ulpien D 15.1 .17 (Servius, Proculus, Atilicinus) ; D 33.8.6. 2-3). Par ailleurs, aucun texte ne vise une acquisition faite par un vicarius, a l'exception de D 18.1.31 Pomponius : sed ot si quid postea accessit peculio, reddendwn est venditori, veluti, partus et quod ex operis vicarii perception est,et encore s'agit-il d'une allusion sans importance comme le soul igne W.W. BUCKLAND, o. c. p. 241, F. AFFOLTER, ZSS 23, p. 61, soutient qu' aucune action directe de peculio ne peut être intentée contre le dominus; contra u. ordinarii, les dettes du vicarius envers le dominus on un conservus peuvent être déduites, mais seulement du pécule du vicarius car elles n'ont pas d'existence en dehors de lui (cf. D 15.1.17 Ulpien cite Servius, Proculus, Atilicinus : D 15.1.38.2 Africain). Quant aux dettes de l'ordirarius envers le vicarius, elles ne sont pas déduites (cf. D 15.1.17).

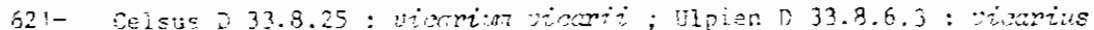
viscrit. 
s'agit d'un sous-esclave (6â2) : dans d'autres, il est simplement question d'un esclave légué $\mathrm{a}$ un autre esclave ou acheté par lui. La pramière hypothèse ne soulève pas de difficultés, le produits des legs étant affecté au pécule (623). En ce qui concerne la seconde, il n'est possible de relever que les textes où il est spécifié que l'achat est effectué à partir du pécule (624), l'esclave n'intervenant ailleurs que compe un intermédiaire ponctisel de l'opération faite au profit du dominant (625).

analyse des paragraphes

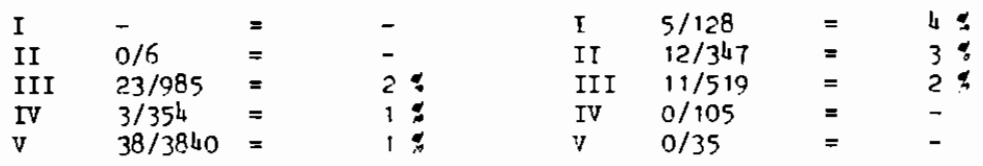

Les textes concernant, des vicarii sont peu nombreux, leur valeur statistique est donc limitée. Cela ne signifie pas qu'il y a peu de sousesclaves, mais seulement qu'ils ne sont pas importants du point de rue

622- Iavolenus (Trebatius, Labéon) D 33.8.22.1; Celsus D 15.1.6 (Tubero, Lab.): D 33.8.25:Jul. D10.3.25; D $14.3 .12: D$ 15:1.37.1 : Pomnoniug D 15.1 .4$. 6 ; D 15.1.23; D 18.1.31 ; D 50.16.166 pr ; Africain D 15.1.38.2 ; D 15 3.17.1 ; D 33.8.16.1 ; Gaius D 33.8.4 ; Scaevola D 20.1.32; D 33.8.21 (Julien); Paul D 9.4.19.2; D 15.1.18; D 21.1.44 pr (Pedius); D 21.2.5 (Labéon) ; D 40.4.10 pr ; Ulpien D 14.1.1.22; D 14.3.11.8; D 14.4.5.3 (Pomponius) ; D 15.1.7.4 ; D 15.1.11.4 (Julien)-5 (Julien) ; D 15.1.17 (Servius, Proculus, Atilicinus) ; D 15.1.19 pr ; D 21.1.17.7 (Caelius, Sabinus); D 32.73.5 (Pomponius) : D 33.7.12.44 (Celsus); D 33.8.6.2-3 ; Marcien D 15.1.40.1 (veteres). A côté de ces textess on trouve d'autres qui, malgré 1 'absence de référence à vicarius, ne soulèvent pas de problèqui, malgré car il est clairement indiqué par ailleurs qu'il s'agit d'un sousmescar il est clairement indique par ailleurs qu' esclave : Pomponius D 15.2.3 (oncillarm partubus); Africain D 33.8.16 pr
(Pamphilum) ; Tryphoninus D 15.1.57.2 (Partus ancillarm); Paul D 12.1.31. 1 (Sabinus, Cassius, Julien) (hominem) ; D 17.1.22.9 (Mela) (servos); D 21.1 .57 .1 (hominem); D 33.8.9 pr (servo); D 33.8.15 (Alfenus) (ancilla); Ulpien D 3.2.4.3 (Pomponius) (mancipia); D 14.1.1.23 (magistro) ; D 14. 4.5 .3 (institore)-14 (mancipia) ; D 21.1.33 pr (Pomponius) (mancipia) ; D 33.8.8.8 (partus concillamm) (Julien): D 47.10.15.44 (ordinarius sousentend vicarius).

623 - Ont ainsi été pris en considération Julien D 30.99 ; D 30.101 pr ; D 33.5.10; Marcellus D 35.2.56.4; Paul D 35.2.21.1; chez Ulpien D 7.8. 12.5 , il s'agit seulement d'un legs d'usage.

624- Julien D 19.1.24.1 (Sabinus, Cassius); D 21.2.39.1 ; Africain D 21.1.51 pr; Gaius D 41.1.43.2 ; Paul D $21.1 .57 \mathrm{pr}$; Ulpien D 40.7.6.6. I1 faut rapprocher de ces hypothèses $D$ 33.8.8.4 Ulpien (Sabinus) (un statuliber vend un esclave à l'héritier ; celui-ci est retranchế du pécule) et $\mathrm{D}$ 40.1.4.8 Ulpien (esclave acheté suis nummis par un autre esclave).

625- Ainsi Ulpien D 7.1.25.1 (achat d'un esclave ex re fructuarii) ou D 24.1.19.1 (ex re mariti). De même, il n'est pas possible de savoir si on a affaire a un vicarius dans les textes qui traitent d'un point de vue général de 1 a vente d'un esclave par un autre esclave (par exemple Ulpien juridique (626). L'analyse des paragraphes montre une prómondírance relative de la période III, corresponjant aux premiers Antonins. Cepondant, $s i$ on prend en considération les citations, on constate une baisse progressive de la période I à la période III. Cette baisse ne doit nas surprendre. Elle n'est pas due ì une diminution du nombre des vicorit, la persistance des privilógiés le prouve, mais an fait que les probl'mes qu'ils ont posés ont étá en grande partie rósolus par le drnit.

L'évolution obtenue par l'analyse des fonctions privilógiąes trouve-t-elle plus ample confirmation dans la fróquence des legs du nécule?

\section{SECTION 2 - LA FREOIIENCE DES LEGS DF PEC,ULE}

Lorsque l'esclave est affranchi entre vifs, que ce soit par vindicta ou de manière informelle, il obtient, son pácule, à moins que colui-cj ne soit expressément retenu (6:7). Par contro, 1 'affranchi testamentaire ne l'obtient que si cela a été clairement próvu dans le testrment (6ą). Mais le pécule peut aussi être légué à un tiors ( 29$)$.

Il ne s'agit pas d'exposer les problèmes juridiques occasionnós par le legs du pécule (630), mais dans l'optique d'une étude des privilégiés, de faire apparaître si le pécule ressort des textes du Digeste comme étant plus souvent légué à l'esclave qu'à un tiers, ou inversement, en fonction de la période envisagée. Pour cela, il convient de comparer le chiffre obtenu pour une catégorie, par exemple le legs du pécule à l'esclave, au nombre total de textes traitant du legs de pécule par póriode.

626- W.W. BUCKLAND, Roman law, cit, p. 240.

627- Paul D 15.1.53: Si Sticho perulium cum manumitteretur ademption non est, videtur concessum. Ch. LECRIVATN, D.S.D.A. V, p. 823.

628- Ulpien D 33.8.8.7 : ... Imperator igitur noster mom patre rescripsit, peculium quidem non nisi legatum deberi... Dans un autre cas, celui du legs de l'esclave, le pécule ne passe pas non plus au légataire à moins que ce ne soit expressément prévu, cf. Ulpien D 33.8.24 : Si legatus fuepit servus, peculium excipere non est necesse, quia non sequitur, nisi legetur.

629- Julien établit à ce sujet une distinction : le segs du pécule à l'esclave inclut toutes les acquisitions jusqu'au dies sedens ; par contre, s'il est légué à un tiers, les accroissements du pécule survenus depuis la mort ne vont pas au légataire, à moins qu'il ne s'agisse des accroissements ordinaires de la res peculiares, CF. D 33.8.8.8 Ulpien.

630- V. notament w.W. BUCKLAND, Roman law; p. $189 \mathrm{~s}$. 
Si la personne du légataire est indiquée avec précision dans la plupart des textes, il en est certains dont le caractère général imọlique la possibilité des deux rapnorts : legs à l'esclave ou legs à un tiers (631). Ces textes ont été retenus dans les deux rubriques, de même que ceux où les deux rapports ne sont pas seulement dossibles mais apoparents $(632)$.

\section{Pécule légué à l'esclave (633)}

- à un tiers $(634)$

analyse des oaragraphes

$\begin{array}{lll}\text { I } & - & = \\ \text { II } & 0 / 1 & = \\ \text { III } & 8 / 13 & = \\ \text { IV } & 8 / 10 & = \\ \text { V } & 32 / 46 & =\end{array}$

$$
\begin{aligned}
& 62 \% \\
& 80 \% \\
& 70 \%
\end{aligned}
$$

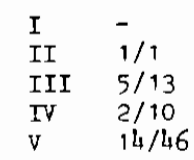

$(100 \%)$

$38 \%$

$20 \%$

$30 \%$

analyse des citations

$\begin{array}{llllllll}\text { I } & 5 / 5 & = & 100 \% & \text { I } 0 / 5 & = & - \\ \text { II } & 12 / 12 & = & 100 \% & \text { II } 0 / 12 & = & - \\ \text { III } 5 / 8 & = & 62,5 \% & \text { III } 3 / 8 & = & 37,5 \% \\ \text { IV } & 2 / 3 & = & 67 \% & \text { IV } 1 / 3 & = & 33 \% \\ V & - & = & - & V & V & = & -\end{array}$

Ecartons la période II dans l'analyse des paragraones, elle n'est pas pertinente compte tenu de l'unique texte relevé. Cette précision faite, on remarque que, quelle que soit la période envisagée, il est toujours plus question de legs du pécule à l'esclave qu'à un tiers. En second lieu, l'analyse des citations montre l'intérêt pour le problème des juristes des deux premières périodes. En troisième lieu, c'est le point le plus

631- Peuvent théoriquement aussi bien concerner le leg̣s du pécule an un esclave qu'à un tiers $\mathrm{D}$ 33.8.6.5 Ulpien (Non solum autem quod domino debetur peculio legato deducitur, sed et si quid heredi debitum fuit) et 0 33.8.9.1 Paul ( $S i$ conservom suum vulneraverit servus et viliorem fecerit, Marcellus non esse dubitondum deduci ex peculio, quod domino interesset (nom quid non esse dubitondum deduci ex peculio, quod domino interesset (nam quid interest, conservion vulneret an scindat aliquid vel frangat an
quo casu sine dubio minuitur peculium), sed non ultra simplim).

632- Ulpien (Julien) D 33.8.8.8.

633- Pécule léguẻ a 1 'esclave : Alfenus (chez auteur anonyme) D 33.8.14; D 40.1.6 ; Tavolenus D 33.8.22 pr (Labéon)-1 (Trebatius, Labéon) ; D 40.7 . 28.1 (Cassius) ; Celsus D 33.8.25; Pomponius D 33.8.10; Africain D 15.1. 38.2 ; D 33.8.16 pr-1 ; Scaevola D 33.8.21 (Julien); D 33.8.23 pr-1-2-3 ; D 34.1 .18 .3 ; D $39.5 .35 \mathrm{pr}$; D 40.7 .40 .3 ; Papinien D $33.8 .19 \mathrm{pr}-1$; Try phoninus $D$ 15.1.57. (Julien); D 34.3 .28 .7 (Scaevola); Daul D 15.1 .18 D $15.1 .53 ; 032.97 ; 033.4 .1 .10 ; 0$ 33.8.9.1 (Karcellus) 033.8 .15

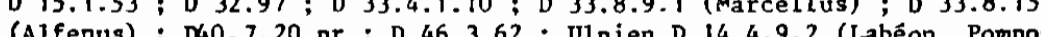
(Alfenus) ; D40.7.20 pr ; D 46.3.62 ; Ulpien D 14.4.9.2 (Labéon, Pomponius); D 21.3 .1 .4 ; D 23.3.39 pr; D 33.8.6.1-2-3-4 (Nerva, Atilicinus,
Pegasus/rescric de Sévère et Caracalla)-5; 033.8 .8 pr (Pegasus)-1 (Julien)2-3 (Sabinus) -4 (Sabinus) -5 (Labéon) $-6-7$ (rescrit de Sévère et Caracalla) -8 important, l'analyse directe confirme les résultats antérieurs sur I'intérêt culminant des juristes pour les esclaves orivilágiós dans la deuxiòme moitié du zème siècle. Enfin, on notera le pourcentage élevé réaliśs var la période $V$ dans $l$ 'analyse des paragraphes. C'est aussi une confirmation, celle de l'importance des formes les plus acheváes de l'esclavage dans les textes de l'époque des Sévères. Cette dernière póriode correspond d'ailleurs à un certain renforcement des "droits" patrimoniaux de l'esclave, plus précisément à un assouplissement de la règle selon laquelle l'esclave affranchi par testament ne recevait pas son pécule ì moins que le testament ne l'ait ọrérd de manière explicite (53̄5).

\section{Conclusion de la première partie}

Les sources de l'esclavage connaissent sous l'Emoire une évolution à laquelle sont insensibles les différents modes d'acquisition de la main-d'oeuvre servile. La cessation des conquêtes engendre le besoin d'encourager les esclaves à se reproduire. Cependant, la reproduction naturelle, bien que restant la source d'asservissement la plus importante, accuse elle-mêne une baisse au 3ème siècle proportionnellement aux modes

(Julien) ; D 33.8.11; D 40.7.3.2 (Servius, Labéon, Cassius) ; Marcien D 33.8.18.

634- Pécule légué à un tiers : Proculus D 33.6.6 ; Tavolenus $\mathrm{D} 15.1 .35$; D 33.8.17; Julien D 33.8.12 ; Gaius D 33.8.2; D 33.8.4 ; Scaevola D 10.2. 39.4 ; D 33.7.20.3 ; Papinien D $31.65 \mathrm{pr}$; Tryphoninus D 15.1.57.2 ; Paul D $30.52 \mathrm{pr}$; D 33.7.22.1 ; D 33.8.1; D 33.8.5; D 33.8.9 pr-1 (Marcellus)2 ; D 40.4.10 pr; Ulpien D 33.8.6 pr (Celsus, Julien) -5 ; D 33.8.8.8 (Julien); D 33.8.24.

635- Assouplissement néanmoins limitê : si le testateur n'avait pas laissé de dispositions précises à cet égard, il fallait, pour que le pécule aille à l'esclave affranchi, qu'il ait écrit qu'on devait lui laisser la libertể après qu'il ait rendu les comptes ou renis une somme à l'héritier, conditions que l'esclave n'aurait pu satisfaire sans le pécule, E.M. conditions que l'esclave n'aurait pu satisfaire sans le pécule, E.M.
SCHTAERMAN, La schiavitũ, cit, p. 242 , cf. D 33.8.8.7 vlpien : Interấon SCHTAERMAN, La schiavitũ, cit, p. 242 , cf. D 33.8 .8 .7 UlDien : Interaion
etsi non sit legatum peculium, velut legatiom sic accipitur, id est in huiusmodi specie : quidam servo libertatem, si rationes reddidisset, dederat, et $i$ heredibus centum intulisset. Imperator igitur moster com patre rescripsit, peculizon quidem non nisi. legation deberi: vemon, inquit, si condicionibus praescriptis paruit servus, testatorgm voluisse eum retinere peculium interpretamur : videlicet ex eo, quod ex peculio elm iusserat centum inferre. 
de reproduction d" groupe servile ̀̀ partir d'hommes libres. C'est le signe du nivellement de la société romaine par le has.

L'esclave casé dans une boutique ou sur une terre voit sa situation s'améliorer tandis que celle des artisans libres et des colons s'aggrave (6 36$)$. C'est prócisément à l'époque où les juristes prennent le plus en compte les colons qu'ils se tournent résolument vers les esclaves privilégiás. On observe alors, comme on l'avait supposé au début de ce travail, la substituticn d'une exploitation subtile à l'exploitation exclusivement brutale de la main-d'ceuvre servile. Au tarme de cette évolution, irréversible dès la seconde moitié du 2è̀me sjècle, les contradictions entre esclaves et propriétaires d'esclaves tendront à céder le pas aux contradictions entre pauvres et riches. Surtout, les contrsdictions entre états, entre esclaves et libres, s'effaceront, devant les contradictions économiques (637).

Si le Digeste autorise à dater la crise du système esclavariste dès la seconde moitié du deuxième siòcle (638), permet-il en revanche d'en dégager les causes avec précision ? On a vu que le grouve servile était loin d'être homogène et que les juristes se préoccupaient surtout de la frange des privilégiés, urbains pour la plupart $(6,39)$. Si l'on peut détecter la crise chez ceux-ci par le développement d'une "capacité anormale", il ne s'agit, dans une société essentiellement agricole, que d'une vision fragmentaire de la réalité. Pourtant, le Digeste n'est

636- P. PETIT, L'esclavage antique, in Coll.Hist. Soc. Besançon (1970), p. 19. L'existence d'esclaves installés sur des parcelles est attestée par Scaevola (D 33.7.27.1). Sur le problème, P. DOCKFS, La libération, cit, p. $145 \mathrm{~s}$. Selon J. KOLENDO, La formation du colonat, cit, p. 149, il ne faut pas surestimer l'étendue de ce phênomène.

637 M. RASKOLNIKOFF, L'apport de la recherche, cit, II, p. 359.

638- Cette conclusion vérifie statistiquement celle de E.M. SCHTAERMAN in La schiavitù, cit, p. 336 , et in La chute du régime esclavagiste, Recherches internationales, cahier $n^{\circ} 2, p .137$ (tr. fr. d'un article paru in VDI (1953), 2, p. 51-79). Cependant, selon S. KOVALEV ; Le tournant social du IIle au Ve siècle dans $1^{\prime}$ Empire romain d'Occident. Recherches internationales, cahier $n^{\circ} 2$ (tr. fr. d'un article paru dans VDI (1954) 3, p. 3344) "crise du 3emét étapes de la pe 44): La "crise du zeme siècle" n'aurait été qu' une des étapes de la révolution sociale qui s'opere (p. 162 ), car il n'existait pas alors de clas vraiment révolutionnaire ( $\mathrm{p}$. 173), et il aurait fallu at tendre les invasions barbares pour porter un coup décisif à la société esclavagiste (p. $176 \mathrm{s.})$. On trouve un bref résumé des deux articles précités chez A. DEMAN, in Latomus (1960), p. $788-789$.

639- Cf. supra n304, et conclusions dégagées du Titre II sur 1'esclave travailleur. pas dénué d'intérêt quant aux raisons de la crise envisagáe sous in angle plus global que celui des seuls privilégiés.

Une des explications proposées réside dans la réduction de l'afflux externe d' "esclaves frais", suite à la cessation de la conquête systématique, ce qui aurait provoqué une diminution du nombre d'esclaves et parallèlement une augmentation de leur prix ( 540$)$. On a vu que rien n'stayait dans le Digeste une hausse des prix de la main-d'osuvre servile entre la fin de l'époque républicaine et les deux promiers siècles de l'mpire, mais les prix fournis par les juristes sont il est, rrai serlement, indicatifs. Surtout, rien ne prouve que la rédurtion de l'afflux externe ne soit pas compensée par la natalité (אil). Pourquoi donc les juristes du 3ème siècle attacheraient-ils autant d'importance aux naissances à l'intérieur du groupe servile que leurs pródécesseurs des ler et 2ème siècles si les esclaves ne se reproduisaient plus?

Un deuxième argument, plus solide, fonde le déclin du système esclavagiste sur son manque de rentabilité. Il convient de se tourner tout particulièrement vers les esclaves ruraux : frais de surveillance (642), mauvaise volonté de ces esclaves à utiliser des moyens techniques complexes, mais aussi peur des mâtres d'instruire leurs esclaves (6.43). Sont-ce là des raisons suffisantes ? Les frais de surveillance engloutissent-ils la totalité du profit dominical ou bien la gestion centralisée de la main-d'oeuvre servile permet-elle de grossir la part du maître

640- Par exemple, W.L. WESTERMANn, Slave Systems, cit, p. 101, et RE Suppl. VI, p. 1012 s. ; F. DE MARTINn, Storia, cit, IV, p. 25 s, 339 et $n 6$; J. KOLENDO, Lavoro servile, cit, p. 21 . Contra, E.M. SCHTAERMAN, La schiavitũ, cit, p. 3,25 s, 329.

641- M.I. FINLEY, L'économie antique, cit, p. 113. L'auteur explique la crise par une transformation de structure de la société dans son ensemble, le processus de soumission de la paysannerie rendant l'esclavage inutile (p. 114 s.). Une critique de cette explication chez P. DOCKES, La libération, cit, p. $252 \mathrm{~s}$.

642- P. DOCKES, o.c, p. $160 \mathrm{~s}$; A. CARANDINI, L'ana tomia della scimmia cit, p. 138, 148. On invoque aussi le fait que l'esclave est un capital périssable (mort, maladie), $c f$. M. BLOCH, dont les idées sur le problème sont résumées in P. DOCKES, O.c. p. 147.

643 - E.M. SCHTAERMAN, La schiavitũ, cit, p. 5, 331. Selon 1'auteur (p. 330), il y a crise parce que les rapports de production de la société esclavagiste ne correspondent plus au niveau atteint par les forces productives. C'est également la thèse de Ch. PARAIN, De l'Antiquité au féodalisme, in Quel avenir attend 1 'homme ? (Entretiens de Royaument, 1961), p. 39. 
au-roli de ce qui doit revenir au surveillant ( $6 / 4$ ) ? Ne voit-on nas parfois le progrès technique coexister nvec des formes d'exploitation brutales (645) ? De plus, si le travail servile n'était réellement plus rentable, les ventes d'esclaves occuperaient-elles encore une place aussi importante dans le discours des juristes de l'énoque des Sévères ?

Alors, faut-il chercher ailleurs les causes du déclin de l'esclavage? Quel a étó 1 'impact des idées humanitaires sur la condition de l'esclave? Y-a-t-il eu des réactions serviles sous l'Empire ? Quel a áté leur rôle ? Ce sont là des questions auxquelles il nous faut essayer de rópondre en examinant les pratiques sociales en matière d'esclavage, sur lesquelles le Digeste nous fournit de numbreuses indications.

644- P. DOCKES, La libération, cit, p. $161 \mathrm{~s}$, remarque ailleurs (p. 149) que la rationalité économique des maîtres n'est pas clairement dégag̣ée des autres motivations; parmi celles-ci, il relève la tradition, 1 'honneur, la puissance, 1 'orgueil... M. MAZZA, dans sa préface à La schiavitú, cit., (p. $X X X V)$, note que rien $n^{\prime}$ interdit de penser que le travail des esclaves était aussi rentable que celui des hommes libres ; contra J. KOLENDO et I. BIEZUNSKA-MALONTST, ibid., p. XIIV n 104.

645- M.I. FINLEY, Techical Innovation, Economic History Review, 2e série, $\mathrm{n}^{\circ} 18$ (1965) p. 43 ; ID, L'économie antique, cit, P. 110 ; P.R. LEWIS et G.D.B. JONES, Roman Gold-mining in North-West Spain, in JRS 60 (1970) p. 169-185 ; v. aussi S. KOVALEv, Le tournant social, cit, p. $168 \mathrm{~s}$. J. KOLENDO, Lavoro servile, cit, p. $23 \mathrm{~s}$, explique les changements survenus dans la technique agricole par le manque de main-d'oeuvre et son prix élevé. dans la technique agricole par le manque de main-d'oeuvre et son prix éleve
Pour P. ANDERSON, Les passages, cit, p. 86 , L'esclavage était un obstacle au progrès technique. 
DEUXIEME PARTIE

ESCLAVAGE ET PRATIQUES SOCIAIES 
On examinera tout d'abord l'ensemble tes données par lesquelles les juristes caractérisent l'esclave. Ensuite seront étudiés les aspects plus concrets du rôle de l'esclnue dans les rapports socialux tel qu'il ressort du Digeste.

\section{TITRE I}

LES ENONCES

En premier lieu, il s'agira de recenser les termes spécifiques de l'esclavage et de l'après-esclavage. En second lieu, une étude du statut permettra de différencier les esclaves et affranchis privés de ceux qui sont soumis à un lien de dépendance public. Enfin seront rassemblées les données relatives è la population servile (1).

\section{CHAPITRE I : LA TERMINOLOG IE DE L'ESCLAVAGE ET DE L'APRES-ESCLAVAGE}

La chronologie veut que cette analyse commence par la prise en considération des termes utilisés par les juristes pour désigner l'esclave.

\section{SECTION 1 : LA TERMINOLOGIE DE L'ESCLAVAGE}

L'intérêt sur le plan du droit d'une telle étude est de se demander pour chaque terme s'il intervient en situant l'esclave

1- L'index thématique de Besançon est sur ce point fidèlement suivi. 
comme agent ou comme objet d'acte ou de fait juridique. Il est aussi necessaire d'envisager les rapports qui s'établissent entre le terme désignant l'esclave et ceux avec lesquels il est fréquemment en relation.

\section{Paragraphe 1:La résonance juridique des termes désignant 1'esclave}

La désignation de l'esclave s'opère le plus souvent de manière impersonnelle, mais il arrive parfois que les textes fournissent des noms propres.

\section{I - La dósignation impersonnelle de l'esclave}

Il convient de distinguer la désignation individuelle de la désignation collective.

\section{$\Lambda$ - La désignation individuelle de l'esclave}

Des termes spócifiques servent à désigner l'homne, la femme et l'enfant esclaves, mais il est possible qu'en l'absence de tels termes une autre indication permette de savoir qu'on a affaire à un esclave.

\section{1/ Les termes servant à désigner l'horme esclave}

Ils sont au nombre de trois : servus, homo, et moncipium.

\section{a/ servus (2)}

2- Diverses hypothèses ont êté avancées quant à l'origine du mot servus. L'avesta a un correspondant phonétique de servus au deuxième terme de deux composés qui désignent des chiens : pasus-hourvo (qui garde le troupeau) et vis-hawrvo (qui garde le village), A. ERNout et A. MEILLET, Dictionnaire étymologique de la langue latine, cit., p. 620. Selon E. BENVENISTE, Le nom de 1 'esclave à Rome, in RFL 10 (1932) p. 432, 1 'acception de gardien oul 1 'on a enfermé l'emploi préhistorique de servus est entièrement fictive. La condition-de l'esclave se définit en droit de la manière la plus stricte (cf Gaius I, 9 : Suma divisio de iure personarum haec, quod homines aut liberi sunt aut servi). Or, comment libre aurait-il pu être le contraire de gardien ? D'autre part les Etrusques possèdent un mot d'où 1 'on aurait pu tirer servus. Il s'agit de Servius (Tullius), cf Festus, 510 (343). Servomum dies vulgo existimatur idus Aug quod oo die Ser. Tulliug, natus servor aedem dedicaverit in Avontino, quod aedem dedicaverit in Aventino, E. BENVENISTE, o. c., p. 436. Signalons enfin 1 'étyrologie fantaisiste (cF. J. MACQUERON, Droit romain, cit., p. 310. Florentinus D 1.5 .4 .2 .) selon laquelle les esclaves s'appellent servi parce que les empereurs, en ordonnant de vendre les prisonniers de guerre, leur conservent la vie, servare, au lieu de les tuer. V. sur le thème, L. LANTELLA, Il la-
Procédons à partir de la démarche quantitative qui a été utilisée jusqu'à présent (3) et rangeons les emplois de servus dans les rubriques annoncées : agent d'acte juridique (4),

voro sistematico, cit., p. $51 \mathrm{~s}$.

3- Il s'agit d'un traitement statistique êlémentaire bien moins sophistiqué que ceux qu'expose R ROBIN ans son ouvrage Histoire et Linguistique, Paris, 1973, p. $124 \mathrm{~s}$,, dont on a essayé de suivre les conseils.

4- Un problème se posait avec les textes oũ il est question du pëcule. Bien qu'intervenant dans le sillage du maître, l'esclave est dans ce cas un "instrument d'action juridique" (pour reprendre l'expression utilisée par P.F. GI RARD, Droit romain, cit., p. 101), autrement dit indirectement un agent d'acte juridique. Ont été relevés dans cette rubrique : Alfenus D 11.3.16; D 15.3.16; D 33.8.14; D 40.1.6; D 40.7.14 pr ; Proculus D 46.3.84; Iavolenus D 8.1.12 ; D 15.1.33; D 15.3.2; D 16.3.33 (Labéon) ; D $181.63 \mathrm{pr}$; D 18.6.17; D 19.2.60.7 (Labéon);D 24.1 .20 ; D 28.5 .65 (Labéon); D 28.8 .11 (Trebatiug, Labéon); 29.262 : D 292.64; D1.38; D 31.40 . D 32.29 .4 (Tubero, Labéon); D 32.30 .2 (Trebatius, Labéon)

D 33.5 .15 ; D 33.8.17; D 33.8.22 pr (Labéon)-1 (Trebatius, Labéon);

D 33.5 .15 ; D 33.8 .17 ; D $33.8 .22 \mathrm{pr}$ (Labéon)-1 (Trebatius, Labéon);
D 35.1 .40 .3 ; D $40.7 .28 \mathrm{pr} ; \mathrm{D} 40.7 .39 \mathrm{pr}$ (Q.Mucius, Gallus, Servius, ofilius D 35.1 .40 .3 ; D $40.7 .28 \mathrm{pr} ; \mathrm{D} 40.7 .39 \mathrm{pr}$ (Q.Mucius, Gallus, Servius, Ofili
Labéon)-2 (Trebatius, Labéon)-4 (Trebatius, Ofilius, Labéon); D 41.2 .24 ; D 44.3.4; D 45.1.104; D 45.3.34; D 45.3.36; D 46.1.20; Neratius D 15.3. 18 ; D 28.5.55; D 40.7.17; D 41.10.5.1 ; D 45.3.22 ; Celsus D 15.1.6 (Tubero, Labéon) ; D 28.5.61 ; D 28.7.21 ; D 29.4.25; D 31.20 (Celsus P., Proculus); D 33.8.25; D 46.3.71.2; D 47.2.68.4; Julien D 2.11.13; D 2 . $14.55 ; \mathrm{D} 10.2 .52 .1 ; \mathrm{D} 10.3 .24 \mathrm{pr} ; \mathrm{D} 10.3 .25 ; \mathrm{D} 13.6 .20 ; \mathrm{D} 13.7 .28 .1$;

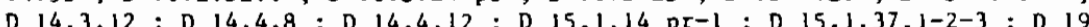
1.24 Pr-1 (Sabinus, Cassius) $D 21.2 .8 ; D 21.2 .39 .1$;D $22.1 .25 .2 ; D 23$. $1.24 \mathrm{pr}-1$ (Sabinus, Cassius); D 21.2 .8 ; D 21.2 .39 .1 ; 22.1 .25 .2 ; D 23 Pr-1; D 28.5.38.1-2-4-5; D 28.5.41; D 28.6 .19 ; D 29.2 .43 ; D $29.2 .45 .1-3$ $\mathrm{Pr}-1$; D $28.5 .38 .1-2-4-5$; D $28.5 .41 ;$ D 28.6 .19 ; D 29.2 .43 ; D $29.2 .45 .1-3$
D $30.81 .1 ;$ D 30.86 .2 ; D $3091.1-3-4-6-7$; D 30.94.1 ; D 30.98; D 30.99; D $30.101 \mathrm{pr}$; D $30.102 ; \mathrm{D} 33.5 .10 ; \mathrm{D} 33.5 .11 ; \mathrm{D} 33.8 .12 ; \mathrm{D} 34.3 .12$; D $36.1 .26 .1-3$; D $36.1 .28 .1-17$; D $36.2 .16 .1-2$; D 36.2 .17 ; D 38.2.13; D 38.13 .1 ; D 39.2.42; D40.4.19; D 40.7.13.1; D 41.1.37 pr-1-2-3-4-5-6; D 41.1 .39 ; D 41.2 .38 .2 ; D $41.4 .7 \mathrm{pr}-2-8$; D 41.4.9. ; D 44.7 .16 ; D 45 . $1.54 .1 ; D 45.1 .62 ; D 45.3 .1 \mathrm{pr}-1-2-3-4-5-6 ; \mathrm{D} 45.3 .14$ (Sabinus) ; D 46 $1.16 \mathrm{pr} ; \mathrm{D} 46.1 .19 ; \mathrm{D} 46.3 .32$; D 47.2.57.2-3; D 49.15.22.1-3 ; Pomponius $D 6.2 .15 ; 07.4 .18 ; D 7.8 .16 .2 ; 012.1 .12 ; 013.6 .13 .2$ (Celsus); D $15.1 .2 ; 015.1 .4 \mathrm{pr}-1-2-3-4-5 ; 015.1 .25 ; 015.149 \mathrm{pr}-1-2 ; 015.2 .3$;

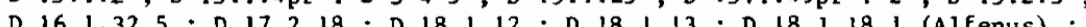
D $23.365 ; D 24.67$; D 18.1 .12 ; D 18.113 ; D 18.1.18.1 (Alfenus); D 23.3 .65 ; D 24.3 .67 ; D 28.1.16pr ; D 28.5.21.1 ; D 28.5.42 ; D 29.2.36 D 30.12 .2 ; D 30.13 (Neratius); D $31.11 \mathrm{pr}$ (Labéon); D 33.8.10; D 34.4 20 ; D 35.1.57; D 38.1.8pr (Labéon); D 40.4.8; D 40.5.44; D 40.7.21p (veteres, Labéon) : D 40.7.29pr-1 (Q. Mucius) ; D 41.1.2Ipr (Proculus) ; D 41.1 .54 .4 ; D 41.2 .25 .1 ; D 41.3.28; D 43.26.13; D 44.2.21.4; D 44. $7.24 \mathrm{pr}-1$; D 45.1 .40 ; D 45.3.17; D 45.3.37; D 45.3.38 (Celsus); D 45 . 3.39 (Gaius) ; D 45.3.40; D 46.1.2 ; D 46.3.19 (Labéon); D 46.3.83 ; AfriCain D 4.4.4; D 7.8.17; D 10.3.9; D 12.1.41; D 14.1.7.2 ; D 15.1.38.23 ; D 15.3.17pr-1; D 19.1.30pr ; D 21.1.51pr;D 29.2.47; D 30.107.1; D 30.108 .1 ; D 34.6.1; D 35.1.32; D 35.1.42; D 39.6.23; D 40.4.22;D 41 . $1.40 ; \mathrm{D} 41.2 .40 \mathrm{pr}-2 ; \mathrm{D} 41.4 .11 ; \mathrm{D} 46.1 .21 .2 ; \mathrm{D} 46.3 .38 \mathrm{pr}-2 ; \mathrm{D} 47.2 .62$. $9 ; \mathrm{D} 48.10 .6 .2 ; \mathrm{T}$. Clemens D $29.2 .28 .2 ; \mathrm{D} 31.59$; Gaius D 2.14.28.2 (Julien); D 5.3.35 (Julien); D 6.1.20; D 7.1.6.3; D 15.1.27.1-2-4 (Julien)lien); D 5.3.35 (Julien) ; D 6.1.20; D 7.1.6.3 ; D 15.1.27.1-2-4 (Julien)-
5 (Julien) -6 (Julien)-7 (Julien)-8 (Julien) ; D 15.1.29pr-1 ; D 15.3.12; 5 (Julien)-6 (Julien)-7 (Julien)-8 (Julien); D 15.1.29pr-1 ; D 15.3.12;
D 20.6.7.1-2 ; D 21.1.18.2 ; D 21.1.24; D 21.2.54.1 ; D 28.5.31pr-1; D $28.5 .32 .1 ; D 28.5 .89 ; D 29.2 .57 .2 ; D 30.68 .1$; 
D $30.69 \mathrm{pr}-4$; D 31.55 .1 ; D $35.2 .73 \mathrm{pr}$; D 36.1.65.4 (Julien)-15; D 38 . 1.22 .1 (Julien) : D 40.7.31 pr:D 40.12.9.2: D 40.12.25.2: D 4i.1.10 pr1-3-4-5; D $41.1 .32 ; 041.1 .43 \mathrm{pr}-2 ; \mathrm{D} 41.1 .45 ; \mathrm{D} 45.1 .141 \mathrm{pr} ; \mathrm{D} 45$. 3.8 ; D $45.3 .28 \mathrm{pr}$ (Julien)-1-2-3-4 (Proculus, Cassius); D 46.1.70.3 D 46.2.34 pr; D 46.4.22; D 46.6.6; D 50.17.107; D 50.17.133; Venuleius D 34.4.32.1 ; D $40.12 .44 \mathrm{pr} ; \mathrm{D} 42.8 .25 .3 ; \mathrm{D} 44.3 .15 .3 ; \mathrm{D} 45.2 .12 .1$ (Ju-
lien): D 45.3 .21 (Julien) ; D 45.3.25 (Cassius) ; Maecianus D 29.4.28 pr: Dien) : D 45.3.21 (Julien) ; D 45.3 .25 (Cassius) ; Maecianus D 29.4.28 pr D $1.7 .18 ; D 7.8 .20$; D 9.2.36.1 (Sabinus); D 15.1.16 (Julien); $D 19$. 1.23 (Julien); D 26.8.12 (veteres, Julien); D 28.5.40 (Julien); D 29. 2.63 (Pomponius) ; D 29.4.5; D 33.3.2 ; D 35.1.47; D 35.2.56 pr-1-4. D 37.15 .3 ; D 38.15.5.2; D 39.6.38;D $46.3 .68 ; D 49.1 .15 ; D 49.15 .1$; Florentinus D 15.1.39; D 28.5.50 pr; D 3n.116.2-3 ; D $34.4 .14 \mathrm{pr}$; D 45.1 .65 .1 ; D 45.3.15;D 46.2.16;Scaevola D 4.3.32; D 15.1.51; D 15.1.54; D 15.1.58; D 15.3.20.1; D 18.5.8;D 21.2.69.3 (Servius) -4 ; D 28.5.84 pr-1; D 28.5.86; D 28.6.48 pr-2;

D 33.7.20.1; D 33.8.23 pr-1-2 ; D 35.2.20 (Maecianus); D 35.2.56.2 (Marcellus) ; D 36.1.80.2; D40.5.19.1; D 40.5.41.7-10-13-14; D 40.7.40.2-3$7-8$; D 45.1.122.1-4; D 45.3.19; D 46.8.89 pr; D 46.3.102.2; D 48.10 24 ; D 49.1.28 pr ; Papinien D 5.1.44.1; $D$ 12.6.58; D 14.3.19.1-3; D $15.1 .50 .2-3 ;$ D $16.1 .27 .1 ;$ D $16.2 .19 ;$ D 16.3 .24 ; D 17.1.54 Pr;D 20. I.1.1 ; D 22.1.4.1 ; D 22.2.4.1; D 26.7.37.1 ; D 26.7.39.18 ; D 28.5.77 (Julien) ; D 30.11; D 31.76.4; D 33.2.2; D 33.3.5; D 33.8.19 pr ; D $35.1 .77 \mathrm{pr}$ (rescrit d'Antonin le pieux); 035.2 .13 ; D 36.1.54.1; D 36. I. 55 ; D 40.3.3; D 40.4.50.1; D 40.4.51.1; D 40.5.23.4; $040.7 .34 \mathrm{pr}$; D 40.7.35; D 40.7.36; D 41.2.44.1-2; D 41.2.45; D 41.2.49 pr;D 41.3. 44.3-7; D 41.3.45.1; D 45.1.118 pr ; D 45.3.18 pr-1-2-3 (Julien) ; D 46.3 .94 .3 ; D 46.3.95.7 ; D 48.19.33 (rescrit de Marc-Aurèle et Verus ; D $49.17 .14 .1-2 ;$ D 49.17 .15 .3 ; Tryphoninus D 12.6.64; D 15.1.57 pr-1(Ju1ien)-2 ; D 15.3.6; D 26.7.58 pr (Scaevola); D 28.5.9i; D 38.2.50 pr-1; D 41.1.63.1-2-3; D 49.15.12.1 ; D 49.17.19.1-2-5; Callistrate D 35.1.82; D $48.10 .15 \mathrm{pr}$ (édit de Claude)-1-2-3-4; Paul D 2.8.8.2; D 2.14.17.7; D 2.14.21.1 ; D 2.14.27.10; D 3.2.14; D 3.3.43.5 (Atilicinus); D 3.4.10; D 3.5.14 (Pomponius) ; D 3.5.16. D 3.5.17 (Proculus, Pegasus, Neratius);D 3.5.18.1 (Sabinus, Scaevola) ; D 3.5.41 ; D 4.3. $20 \mathrm{pr}$ (Labéon); D 4.3.25; D 4.4.24.3; D 4.5.7.2 (Julien); D 4.8.32.8 (Octavenus) ; D 5.1.12.2 ; D 5.3.32 ; D S.3.34.1 (Julien); D 5.3.36 pr (Proculus, Julien, Mauricianus) ; D 6.1.27.4; D 6.2.10; D 7.1 .24 ; D 7.1.31; D 8.3.19; D 9.4.19.2;D 10.2.15;D 10.4.15; D 11.5.4.1; D 12.1 .2 .4 ; D 12.1 .29 (Julien); D 12.1.31.1 (Sabinus, Cassius, Julien) ; D 12.2.20; D 12.2.22; D 12.6.13 pr (Pomponius); D 12.6.36; D 12.6.65.3; D 13.1.3 (Julien) ; D 13.7.18.4; D 14.1.5 pr-1;D 14.1.6 pr-1 ;D 14.3.14; D 14.3.17.1-4 (Proculus) ; D 14.4.10; D 14.5.3; D 15.1.26; D 15.1.43 (Labéon) ; D 15.1.47 pr-1 (Sabinus)-3 (Proculus)-5-6; D 15.1.52 pr; D 15.1. 56 ; D 15.2.2.1 ; D 15.3.8 (Pomponius); D 15.3.11; D 15.3.14 (Julien, Marcellus) : D 15.4.2 pr-2 ; D $15.4 .5 \mathrm{pr}-1 ; \mathrm{D} 16.1 .9 ; \mathrm{D} 16.2 .9 \mathrm{pr}$ D 16.3.21.1 (Trebatius); D 16.3.27; D 17.1.5.4; D 17.1.22.8 (Neratius)-9 (Me1a); D 18.1.40.5; D 20.1.29.3; D $21.1 .43 .10 ; \mathrm{D} 21.1 .44 \mathrm{pr}$ (Pedius) ; D 21.5 pr-1; D 21.2.3; D 21.2.5 (Labêon) ; D 22.1.11.1; D 24.1.28.5 (Julien, Pomponius) ; D $24.1 .38 \mathrm{pr}$ (Alfenus); D 28.5.53; D 28.5.58; D $28.5 .85 \mathrm{pr}^{-1}$ (constitution de Marc-Aurèle) ; D 28.5.9n; $\mathrm{D} 29.1 .40 .1$; D 29.2.22 ; D 29.2.26 (Julien, Pomponius, Marcellus); D 29.2.58; D 29.2. 60 (Labéon, Proculus, Iavolenus) ; D 29.2.65; D 29.2.68; D 29.2.80.2 ; D 29.2.93.1-2; D 29.7.8.5; D 30.7 ; D 30.52 pr; D 31.14.1; D 31.82.2; (Valens, Julien) ; D 31.83 ; D 31.84 ; D 32.4; D 32.8.1; D 33.1.11; D 33. 1.16 ; D 33.8.1; D 33.8.5; D $33.8 .9 \mathrm{pr}^{-1}$ (Marcellus); D 33.8 .15 (Alfenus)

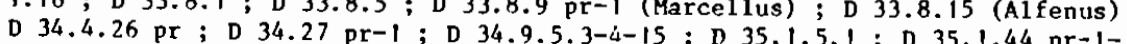
3-8-10 (P1autius) ; D 35.2.1.4; D 35.2.21.1; D 35.2.22.1-2; D 35.2.49 pr (Atilicinus, Nerva, Sabinus, Cassius, Plautius ; rescrit
Antonin le pieux) : D 35.2.63pr (Pedius) ; D 36.1 .42 : D 36.3 .7 ; D 37.11 .10 (Scaevola) ; D 38.2.45; D 39.5:10; D 39.6.44; D 40.4.36; D 40.4 .53 ; D 40 7.4.4 ; D 40.7.20pr-3 (Sabinus, Julien) ; D 40.12 .17 ; D 41.1 .47 ; D 41.1 .57 (Julien) : D 41.2.1.5 (Sabinus, Cassius, Julien)-7-10-13-14 (Nerva filius, Cassius, Julien)-15 (Julien)-16 (veteres)-17-18-19-22 (Nerva filius): 041. 2.3.8 12 ; D 41.2.14pr; D41.2.32.2; D 41.3.4.4-7 (Labéon)-16 (Sabinus, Cas2.3.8 12; $\mathrm{D} 41.2 .14 \mathrm{pr}$; $\mathrm{D} 41.2 .32 .2$; $\mathrm{D} 41.3 .4 .4-7$ (Labéon) 16 (Sabinus, Cas sius) ; D 41.3.8pr (Labéon, Neratius, Julien)-1 (Pedius); D 41.3 .11 : D 11. 3.15pr (Jullen, Marcellus) ; D 41.3.31.2-3 ; $D$ 41.3.34 (Alfenus) ; $D$ 41.4.2. 10-11 (Celsus)-12 (Pomponius)-13 (Celsus)-14 (Celsus) ; D 42.5.4; D 44.5.2. 2 ; D 44.7.43; D 44.7.49; D 45.1.39; D 45.1.75pr ; D 15.1.126.2 ; D 45.3. 16 ; D 45.3.20pr-1 (Labéon) ; D 45.3.26; D 45.3.27; D 45.3.29; D 15.3.30 D 45.3.31 ; D 45.3.32 (Sabinus) ; D 45.3.33pr (J111ien)-1 ; D 46.1.35; D 46.1 56.1 ; D 46.1.66 : D 46.3.35 (Alfenus) ; D 46.3.63 ; D 46.3.98.7 ; D 46.4.11pr -1-2-3 ; D 47.2.42pr ; D 48.10.14pr-1 (Julien, Marcellus) ; D 48.10.22.3-45-9 : D 49.14.13.8 (beneficium de Trajan) : D 49.15.29 (Labéon); D 50.17 .146 ; D $50.17 .175 \mathrm{pr}$; D 50.17.211; Ulpien D 1.7.19pr; D 1.12.1.1-8; D 1.19 .1 .2 D 2.7.3pr (Pedius) ; D 2.11.9pr-1 (Labéon) ; D 2.13.4.3 : D 2.14.7.18 (Vindius Verus. Marcellus) : D 3.3.33pr ; D 3.5.3.6 : D 3.5.5.7-8 (Labéon, Pomponius)-9 (Pomponius) ; D 4.3.7pr-8 (Pomponius); D 4.3.9.4 (Labéon); D 4.3. 11 (Marcellus) ; D 4.4.5; D 4.4.7.5-10 (Papinien, Antonin le pieux, Caracalla) ; D 4.8.7pr(Labéon) -1 (Julien) ; D 4.8.9pr; D 4.9.3.3; D 4.9.7.6; D 5. 1.19 .3 (Labéon) ; D 6.1.41.1 ; D 6.2.7.10-13; D 6.2.9.6 ; D 7.1.12.3 (Julien Pomponius) -4 (Julien) ; D 7.1.21 (Labéon) ; D 7.1.22; D 7.1.25pr-1 (Julien, Mauricianus, Marcellus)-4-5 (Julien) 6 (Scaevola) ; D 7.2.1.1 (Julien)-2 (Julien) ; D 7.3.12 ; D 7.4.5.1 ; D 7.6.5.4 (a) ; D 7.8.12.5 ; D 7.8.14pr ; D 9.2.23pr (Neratius) 1 (Julien) ; D 9.3.1.8; D 9.4.38.3; D 10.2.12.1 ; D 10.4.3.14 (Julien) ; D 11.1.9.2-8; D 11.6.3.6 (Pomponius) ; D 12.1.11.2 ; D 12.1.13.2 (Papinien) ; D 12.2.23; D 12.2.25; D 12.4.3.7 (Celsus p/Celsus); D 13.5.5.10; D 13.6.3.4; D $13.6 .14 ; D 13.7 .11 .5 ; 014.1 .1 .4-16-22$ D 14.1.4.2-3-4; D 14.3.1 (Marcellus); D 14.3.5.3-7 (Labéon)-8 (Labéon)-9 (Labéon) ; D 14.3.7.1 ; D 14.3.11.8; D 14.3.13pr (Julien)-2. (Julien) ; D 14. 4.1pr-1-5 ; D 14.4.3pr (Julien)-1 (Pomponius) ; D 14.4.5.1 (Pomponius)-3-7 (Labéon)-12-13 (Labéon)-14-15; D 14.4.7.2 ; D 14.4.9.2 (Labéon, Pomponius) ; D 15.1.1.4-5-6; D 15.1.3pr-1 (Labéon)-2-3 (Pedius)-4-5 (Celsus)-6 (Julien) -7 (Julien)-8 (Nerva filius)-9 (Sabinus, Cassius)-12 (Labéon) ; D 15.1.5.12-4 (Tubero,Cel sus) ; $D$ 15.1.7.1 (Juilien, Marcellus)-3 (Pedius)-5 (Labéon)-6-7 ; D 15.1 9pm-i (Neratius, Ponponius)-2-4 (Pedius)-5-6-7-8 (JuIien, Marcellus); $15.1 .11 .1-2$ (Julien)-4 (Julien) -5 (Julien) 7 (Julien)-8 (Julien) : lus, Papinien); D 15.1.24; D 15.1.30.2; D 15.1.32pr (Julien)-2 (Neratius) lus, Papinien); D 15.1.24; D 15.1 .36 (Pomponius) : $15.1 .41 ;$ D $15.2 .1 .1-4-6-7$ (Pegasus, Africain) -8 D 15.1.36 (Pomponius) ; D 15.1.41 ; D 15.2.1.1-4-6-7 (Pegasus, Africain)-8
(Scaevola) ; D 15.3.1.1 (Labéon)-2 (Julien, Pomponius); D 15.3.3pr-1 (Pomponius)-2-3 (Labéon)-5 (Labēon, Pomponius)-6 (Labếon)-7-8-9-10; $\mathrm{D} 15.3 .5 \mathrm{pr}$ (Pomponius)-1 (Pomponius)-2-3; D 15.3.7pr-1-2 (Mela)-3 (Labéon)-4 (Labéon) ; D 15.3.10.5 (Marcellus)-6-7 (Pomponius) ; D 15.4.1.1-4-5 (Marcellus)-6-8 (Marcellus)-9 (Labéon) ; D 15.4.3 ; D 15.4.4 (Pomponius) ; D 16.3.1.17-18 (Marcellus)-27-28-29-30-31-32 (Celsus)-33 (Julien)-42 (Julien) ; D 16.3.11 (Sabinus, Pomponius) ; D 17.1.10.7 ; D 17.1.12.2 (Marcellus)-3; D 17.1.19 (Poraponius); D 17.2.23.1 (Marcellus ; Marc-Aurè1e); D 17.2.24; D 17.2.58.3 ; ponius) ; D 17.2.23.1 (Marcellus ; Marc-Aurè 17.2 .63 .9 (Sabinus, Julien, Pomponius) ; D 18.1.29; D 18.4.2.6-12 (JuI ien) -13 (Marcellus) ; D 19.1.13.4-18; D 19.2.13pr; D 21.1.23.4-6-9 (Pedius); D 21.1.31.7 (Marcellus); D 21.1.33pr (Pomponius) ; D 21.2.51.3 ; D 21.3.1.4 ; D 23.3.39pr ; D 24.1.3.8 ; D 24.1.11.6 (Marcellus, Scaevola) ; D 24.1.17.1 ; D 24.1.19pr (Julien); D 24.1.22; D 25.4.1.13 (Aristo); D 26.2.10.4 ; D 26 2.22 ; D 26.7.9pr (Julien) ; D 26.7.11 (rescrit d'Antonin le pieux) ; D 26. 8.1pr (rescrit d'Antonin 1e pieux) 
D 27.3.1.3 (décret de Sévère) ; $D$ 27.5.1.2 (rescrit de Sévère) ; $D$ 27.8.1.1516 ; D 28.1.20.7; D 28.5.3pr-1; D 28.5.6.2-3 (Julien)-4 (Julien) ; D 28.5. 9.16-17-20: D 28.5.30 (rescrit de Sévère); D 28.5.35.3 (Q. Mucius): 028.5 5lpr; D 28.6.8.1; D 28.6.10.1; D 28.6.18pr (Julien)-1; D 28.7.2pr-1 (Ce1 sus) ; D 28.8.1pr ; D 29.1.13.3-4 (rescrit de Sévère); D 29.2.6.7 (Celsus)

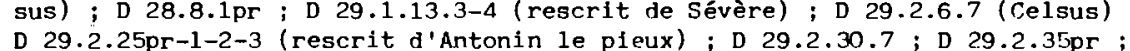
D 29.2.25pr-1-2-3 (rescrit d'Antonin le pieux) ; D 29.2.30.7 ; D 29.2.35pr ;
D 29.2.66; D 29.2.67; D 29.2.71pr ; D 29.4.1.3-4 ; D 29.6.1.1 ; D 30.34.9 ; D $30.50 \mathrm{pr}$; D 30.53 .2 (Julien, Marcellus) ; D 32.1 .1 ; D $32.7 \mathrm{pr}$; D 32.11 .6 D 32.49 .7 ; D 33.6.9.3; D 33.8.6pr (Celsus, Jullen)-1-3-4 (Nerva, Atilicinus Pegasus ; rescrit de Sévère et Caracalla) ; D 33.8.8.1 (Julien)-2-3 (Sabinus) -5 (Labéon)-6-7 (rescrit de Sévère et Caracalla)-8 (Julien) ; D 33.8.24 ; D 35.2.35 (Seaevola); D 36.1.1.10; D 36.1.17.10-13 (Maecianus)-14 (Maecianus) -17 (rescrit d'Antonin le pieux) ; D 36.1.23.1; D 36.2.7.6; D 36.2.9 D 36.2 .12 .2 (Jullen) ; D 36.2.23; D $37.1 .7 \mathrm{pr} ; \mathrm{D} 37.4 .3 .15-16 ; 037.5 .3 .2$;

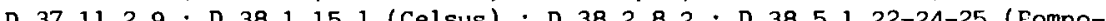
nius) : D 38.9.1.3-14; D $39.1 .5 .1-3 ; 039.5 .13 ; D$ D $39.5 .19 .4 ; 040.1 .4 .1$ (epistula D0.5.24.19: D 40.5 .37 (rescrit de Marc-Aurè1e); D 40.7.2.1 (Cassius, Julien) -3 (Julien)-4 (Celsus) ; D 40.7.3.1-2 (Servius, Labéon, Cassius)-7-814 ; D 40.7.6.6 ; D 41.1.17 ; D 41.1.18; D 41.1.22; D 41.1.23pr-3 (Julien, Scaevola); D 4l.1.33pr (Marcellus, Scaevola)-2 (Julien); D 11.2 .13 .3 ; D 41 2.34 .2 (Celsus) ; D 42.4.3.1; D 42.4.7.15; D 42.6.1.18; D 42.8.6.5-12 (Labéon) ; D 42.8.10.10; D 43.5.3.4; D 43.16.1.22; D 43.19.3.4; D 43.24.3pr: D 43.24.5.6 (Servius)-11; D 43.26.4.2; D 44.4.4.17; D 44.5.1.4; D 44.6.2 (Julien) i $44.7 .14 ; 045.1 .1 \mathrm{pr} ; \mathrm{D} 45.1 .38 .6$ (Julien) $7-8-17$; D 45.3 .2

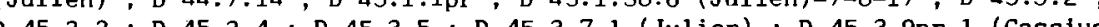
D 45.3 .7 .1 (Julien) ; D $45.3 .9 p r-1$ (Cassius, Jullen) i D 45.3 .13 7.8 .1 (Octavenus) 2 (Labêon) 4 ; D 46.6 .2 ; D $47.2 .52 .9-15-26-28$; D 47.4 .1$. 7 (rescrits de Marc-Aurêle et de sévère et Caracalla ; D 47.10.5.11 ; D 48.5 . 18.5 ; D 49.17.9 (Marcellus) ; D 50.12.2.1 ; D 50.16.17pr ; D 50.16.68; D 50 . 16.99 .3 ; D 50.16.182 ; D 50.17.22pr ; D 50.17.118; L. Rufinus D 31.62 ; D 34.4.21 ; D 40.7.32; Macer D 48.10.10pr; Marcien D 15.1.40.1 (veteres) ; D $20.3 .1 .1 ; \mathrm{D} 20.6 \cdot 8.5$

D 22.1.32.1 ; D 28.5.39 ; D 28.5.49.2 (constitution de Sévère et Caracalla) ; D 28.5.52.1 (Celsus) ; D 28.6.36pr ; D 28.7.18pr (rescrit d'Antonin le pleux); D 30.113pr (Marcellus) ; D 30.114.10-12 (rescrit de Sévère et Caracalla) ; D 30.119 ; D 33.8.18; D 34.8.3.2 ; D 36.1.31.2 ; D 39.4.16.11 (rescrit de Caracalla) ; 0 40.1.5pr-1; 0 40.4.26 (rescrits d'Antonin le pleux et de MarcAurèle et Verus) ; D 48.10.1.8; D 48.10.7 (rescrit de Marc-Aurẻle et Commode) ; D 48.12.1; Modestin D 5.2.12pr; D 16.1.25pr; D 22.5.7; D 24.3.58; D 26.7.32.3 ; D 28.1.19; D 28.1.28; D 28.6.3; D 28.7.25; D 29.2.50; D 31 . 34.2 ; D 34.3.19; D 35.1.53 ; D 40.7.26pr-1; D 45.3.35 ; D 48.4.7.2 ; Hermogénien D 5.1.53 ; D 37.14.21.1-3; D 41.1.61pr-1 ; D 41.2.50.1 ; D 49.14 .46 8 ; I. Aquila D 26.7.34; D 26.10.12 ; Auteur anonyme D 36.4.16. Il faut rapprocher de ces textes ceux où le servus recoit un legs d'aliments, bien qu'il s'agisse d'un legs spécifique (Scaevola D 10.2.39.2 ; D 34.1.20.3; Paul D 26. 7.12 .3 ; 33.10 .12 ; D 37.9.4.1; Ulpien D 27.3.1.8; Marcien (rescrit de Sévère et Caracalla) D 30.113 .1 ) et ceux oì il est question d'une dénonciation par le servus (Marcellus D 29.5.16 i Callistrate (constitution de Sévère) D 49.14.2.6 ; Paul D 29.5.12 ; U1pien D 47.10.5.11 ; Marclen D 48.2 13). Conservus a été intégré dans cette rubrique : ce mot apparait seul chez Ulplen D 15.1.7.7 ;'D 15.1.9.1 (Neratius, Pomponius), aux côtés de servus chez Florentinus D 45.3.15 ; Scaevola D 45.1.122.1 ; Paul D 33.8.9 pr-1 (Marcellus) ; Ulpien D 33.8.6pr (Celsus, Julien), étant bien entendu que dans ces textes conservus apparait en tant qu'agent d'acte juridique. ont également été retenus dans cette rubrique les textes oì objet d'acte juridique (5)

n'apparaît que la formule qui in servitute est (Paul D 3.5.16: D 3.5.17 (Proculus, Pegasus, Neratius) ; D 3.5.18.1 (Sabinus, Scaevola) ; U1pien D 50.16.99.3; D 50.17 .118 ) ou personam servilem (U1pien D 50.17.22pr : In personam servilem nulla cadit obligatio, ; ce dernier texte montre qu'il a également été tenu compte des paragraphes où servus n'apparaissait comme agent que de manière négative (Paul D 4.5.7.2 (Julien) ; D 5.1.12.2; D 50.17.175pr ; Ulpien D 4.8.7pr (Labéon) ; D 11.1.9.2 ; D 28.1.20.7; D 50.16.6.8; Marcien (rescrit de MarcAurèle et Commode) D $48,10.7)$.

5- Alfenus D 10.3.26 ; D 40.1 .7 ; D 44.1.14; Proculus D 33.6.6 ; Iavolenus D 9.2.38; D 15.1.33; D 18.6.17; D 19.2.60.7 (Labéon) ; D 21.2.58) ; D 24.1.20 ; D 24.1.50pr-1 ; D 28.5.65 (Labéon) ; D 28.7.20.1 (Labéon); D 29.2.62.1 ; D 29.2.64 D 31.38 ; D 32.29 .3 (Labéon)-4 (Tubero, Labéon); D 32.10npr (Cascellius, Labéon) ; D 33.4.6.1 (Servius, Namusa, Cascellius, Ofilius, Labéon); D 33.5.14; D 33.5.15 ; D 34.5.28 ; D 40.7.39pr (Q.Mucius, Gallus, Servius, Oft11us, Labéon) ; D 40.12 .42 ; (Labéon) ; D 41.3.16 ; D 44.3.4. ; D 45.1.105 (Sabinus) ; D 45.3.34; D 45.3.36 ; D 49.15 .27 (Ofilius, Trebatius, Labéon); Neratius D 28.5.55; D 45.3.22; D 47.2.6 Celsus D 31.18 ; D 31.20 (Proculus, Celsus p.) ; D 32.79.3 ; D 33.8.13; D 47.2.68. Valens D 4.4.33 (Julien) ; D 35.2.37pr-1 ; Julien D 2.14.55; D 5.1.25; D 7.1.35.1

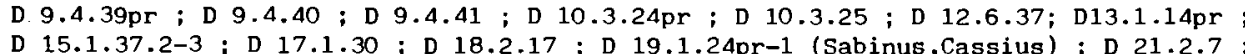
D $15.1 .37 .2-3 ; \mathrm{D} 17.1 .30 ; \mathrm{D} 18.2 .17 ; \mathrm{D} 19.1 .24 \mathrm{pr}-1$ (Sabinus,Cassius) ; D 21.2.7
D $21.2 .8 ; \mathrm{D} 21.2 .39 .3-5 ; \mathrm{D} 22.1 .25 .2 ; \mathrm{D} 23.3 .47 ; \mathrm{D} 24.3 .31 .4 ; \mathrm{D} 28.5 .7 \mathrm{pr}$; D 28.5.8pr-1 ; D 28.5.38.2-4-5; D 28.6.19; D 29.2.43; D 29.2.45.1-3; D 29.7.2.2 D $30.81 .1-4-10$; D 30.84.4-5-12; D 30.86.2; D 30.91.3-4-6 ; D 30.92.1 ; D 30.94.13 ; D 30.96.2 ; D 30.97; D 30.98; D 30.102 ; D 32.62 (Servius) ; D 33.5.9.1-2 (Q. Mucius) ; D 33.5.10 ; D 33.5.11 ; D 33.5.12 ; D 34.5.13.1 ; D 36.1.26.2 ; D 36.1 .28 .1 ; D 36.2.16.1-2; D 36.2.17; D 37.4.13pr ; D 39.2.42 ; D 40.2.6. D $40.5 \cdot 47.1-3 ;$ D 40.9.7.1 (Proculus); D 41.1.37pr-1-2-3-5-6; D 41.1.39;

D 41.2.38pr-2 ; D 41.4.7.7 ; D 41.4.8; d 43.24.14; D 43.26.19.1 ; D 44.7.16 D 45.1.54.1 ; D 45.3.1.1-2-4-5-6; D 46.1.16pr; Pomponius D 7.4.18; D 7.8.16.2 D 9.4.18 : D 11.1.15pr-1 ; D 13.7.8pr ; D 15.1.2 ; D 17.2.60.1 ; D 19.1 .55 (Octavenus) ; D 21.1.48.1-3 ; D 21.1.64pr (Labéon)-1 (Labéon); D 21.2.16.2 (Proculus) ; D 21.2.30; D 21.2.34.1; D 23.3.65; D 24.1.18; D 24.1.29pr (Fulcinius);D 24.3.67 D 26.7.61 ; D 30.8.2 ; D 30.12.1 (Proculus) ; D 30.20 ; D 30.38.1 ; D 30.48 .1 ;

D 30.56 ; D 32.74 ; D 33.2.20 ; D 33.5.6 ; D 33.5.8pr-2; D 35.1.1.3; D 36.1.72.1; D 38.1.8pr (Labéon) ; D 40.1.13 (Octavenus) ; D 40.4.11pr ; D 40.4.40.1 (Ofilius) ; D 40.5.20 (Aristo, Julien, Octavenus) ; D 40.5.34.2 (Sabinus) ; D 40.7.29.1

(Q. Mucius, Labéon, Aristo, Celsus) ; D 41.1.21pr (Proculus) ; D 41.1.54.4; D 41. 4.6.1;D44.7.56;D45.3.17;D45.3.37;D45.3.39;D45.3.40: D 46.3.26:n 46.3 .83

D 46.3.92pr-1; D 47.2.44.2; Africain D 3.5.48;D 7.1.36.2;D $9.4 .28 ; 0$ i 10.3 .9 D 12.1 .23 (julien); D 13.7.31 (Julien); D 15.1.38.3; D 19.1.30pr; D 21.2.24 D 21.2.47; D 30.107.1. ; D 30.108pr-9-11; D 30.110; D 40.4.20; D 40.5.49; D $41.2 .40 .2-3$; D 44.3.6.1; D 46.3.38.3 ; D 47.2.62pr-1-4-5-6; T. Clemens D 7. 7.5 (Julien) ; D 29.2.82 ; D 33.5.17 ; Gaius D 1.6.1.1 ; D 5.3.39pr ; D 6.1.36.1 D 7.1.3.1 ; D 7.1.45; D 9.4.27pr-1; D 10.3.11 : D 13.6.18pr-2 ; D 15.1.27.2-4 (Julien)-5 (Julien)-6 (Julien)-7 (Julien)-8 (Julien) ; D 17.1.27.1 ; D 21.1.18.2 D 21.1.24; D 24.1.8; D 26.7.13pr ; D 28.5.31.1; D 30.65pr; D 30.67pr ; D 30 . 68.2-3 (Julien) ; D 30.69pr-1-4 ; D 30.70pr-2-3; D 31.55.1; D 33.8.4 ; D 35.1 .17 pr-1;D35.2.73pr-3;D36.1.65.4 (Julien) ; D39.4.13.2;D 40.9.3; D 40.9.29 or-1 : 

D 41.1.10pr-3-4-5; D 41.1.43pr-2; D 41.1.45; D 45.3.8; D 45.3.28pr (Julien)
$-1-2-3-4$ (Proculus, Cassius) ; Venuleius D 21.1 .65 .2 (Caelius Sabinus) D 43.29 . -1-2-3-4 (Proculus, Cassius); Venuleius D 21.1 .65 .2 (Caelius Sabinus) D 43.29 .
4.1 (Trebatius); D 45.1.137.4: D 45.3.21 (Julien): D 45.3 .25 (Cassius) D 46. 2.31pr; D 48.8.6 ; Maecianus D 29.4.28pr-1 ; D 35.2.30pr; D 40.4.55pr; D $40.5 .32 \mathrm{pr}-1$; D $49.17 .18 \mathrm{pr}-3$; Marcellus D 7.8.20 ; D 15.1.16 ; D 17.1.49; D D 18.7.4; D 19.1.23 (Ju1ien) ; D 19.2.48.1 ; D 26.8.12 (veteres/Julien) D 28.5.40 (Julien) : D 29.1.31 ; D 29.2.63 (Pomponius) ; D 29.5.16 ; D 31.26 : D 33.3.2. : D 35.2.34 (Julien); D 35.2.56.3; D 38.15.5.2; D 40.5 .9 ; D 40. 5.10pr ; D 40.13.2 ; D 45.1.96; D 46.3.72pr-1-2-3-4-5-6; D 47.6.5 ; Florentinus D 18.1.43pr ; D 30.116.2-3 ; Scaevola D 4.3.32 ; D 15.1.54 ; D 15.1.58 ; D 15.3.20pr; D 15.3.21; D 18.5.8; D 20.1.32; D 20.1.34.1; D 21.2.69.3 (Servius)-4-5 ; D 23.4.29.2 ; D 28.6.48pr-2 ; D 31.88.7 ; D 32.37.3; D 32.101

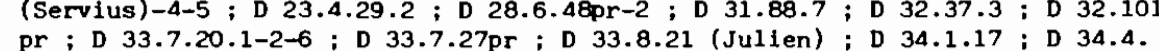
pr ; D 33.7.20.1-2-6; D 33.7.27pr; D 33.8.21 (Julien) ; D 34.1.17 ; D 34.4;
$31 \mathrm{pr}-1$ : D 36.1 .80 .12 ; D 40.7.40.2-7; D 40.9 .6 ; D 44.3.14.2-4 ; D 45.1.135.3; D 45.3.19 ; Papinien D 4.4.31; D 4.8.42 : D 10.2.34; D 12.6.55; D 13.1.17 ; D 15.1.50.3 ; D 17.1.54pr ; D 17.1.57; D 18.7.7; D 18.7.8; D 20.1.1.1 ; D 21.2.64pr; D 21.2.67; D 22.1.4.1; D 24.3.61; D 28.5.77 (Julien) ; D 29.2. 86.2 ; D 31.65pr-3; D 31.76.4; D 32.91 .2 ; D 33.2.2 ; D 33.2.24.1; D 33.7. 3.1 ; D 33.8.19.2 ; D 35.2.11.1-4 ; D 36.1.55; D 40.1.20pr (Litterae de MarC-Aurè1e) ; D 40.1.21 : D 40.4.51pr ; D 40.5.23.2; D 40.7.35; D 40.7.36 ; D 40.12 .36 ; D $41.2 .44 \mathrm{pr} ; \mathrm{D} 41.2 .48 ; \mathrm{D} 41.2 .49 \mathrm{pr}$; D $41.3 .44 \mathrm{pr}-2-3$; D 41.3 . 45.1 ; D 45.1.118pr ; D 45.3.18pr-1-2-3 (Julien) ; D 46.3.95.1 ; D 47.2.81pr-2 D 48.18.6pr-1 ; D 48.18.17.2 (rescrit d'Hadrien) ; D 48.19.33 (rescrit de MarcAurèle et Verus); D 49.17.13 (rescrit d'Hadrien) ; D 49.17.14.1-2 ; D 49.17. 15.3-4; D 50.17.77; Tryphoninus D 9.4.37; D 38.2.50.1; D 41.1.63.1-2-3; D 43.16.19 (Julien) ; D 49.15.12.7-8-9 (Sabinus)-12-13-16 ; D 49.17.19.1-3 (constitution d'Hadrien)-4-5; Callistrate D 48.18.15.2 (rescrit d'Antpnin le pieux) ; Paul D 2.9.2.1 (V. Verus, Julien, Pomponius) ; D 2.11.7 ; D 4.3.18. 1-5; D 5.1.24.2 (Cassius, Julien) ; D 5.3.14; D 5.3.32 ; D 5.3.36.2 (Pomponius) ; D 5.3.40.4; D 6.1.21 (Julien, Pomponius) ; D 6.1.27.5 (Proculus) D 6.1.58; D 6.2.6 ; D 6.2.12pr (rescrit d'Antonin le pieux) ; D 7.1.24; D 7 . 1.26 ; D 7.1.31 ; D 7.1.66 ; D 7.4.26 ; D 7.4.27 ; D 7.8.15pr ; D 7.9.10 ; D 8. 3.19 ; D 9.2 .12 ; D 9.2.18; D 9.2.22pr ; D 9.2.30.1 ; D 9.4.4.1. ; D 9.4.9;

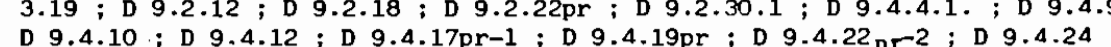
(Labéon,Julien) ; D 9.4.26.6 ; D 9.4.31 (Sabinus, Cassius, Julien, Pomponius) ; D 10.2.15 ; D 10.2.25.15; D 10.3.8.2-3; D 10.3.15; D 10.3.19.3; D 10.3.27 ; D 11.1.20pr; D 11.3.8; D 11.3.14.2 (Sabinus)-3-7; D 12.1.31.1 (Sabinus, Cassius, Ju1ien) ; D 12.2.30.1 ; D 12.6.65.8 ; D 13.1.3 (Jul1en) ; D 13.6.22 ; D 14.1.5pr ; D 14.1.6.1 ; D 14.4.10 ; D 15.1.26; D 15.1.43 (Labéon) ; D 15.1. 47.3 ( Proculus) -5-6 ; D 15.3.14 (Julien, Marcellus); D 15.4.2.2. ; D 15.4.5.1; D 17.1.5.5. ; D 17.1.22.9 (Mela) ; D 17.1.26.8 (Mela) ; D 17.2.56 ; D 18.1.5; D 18.1.34pr (Labéon)-2 ; D 18.2.14.3. D 18.6.8.1 (Julien) ; D 18.7.9 ; D 19.1. 4pr ; D 19.1.43 (Julien, Ulplen) ; D 19.1.45pr (Julien, Africaln)-1 ; D 19.1. 54pr (Labéon); D 19.2.42; D 19.2.43; D 19.2.54.2; D 19.4.2 (Aristo); D 19.

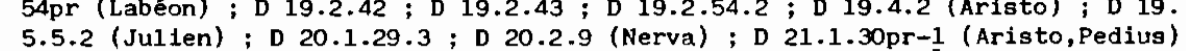
D 21.1.43.3-5-8-10 ; D 21.1.44pr (Pedius) -2 ; D 21.1.57.1;

D 21.1.58pr-1-2 ; D 21.2.3 ; D 21.2.5 (Labéon) ; D 21.2.9 (Sabinus) ; D 21.2. 15.1 ; D 21.2.35; D 21.2.41.1 ; D 21.2.56.3 ; D 22.3.4; D 24.1.28.1-3 (Plautius)-4 (Pomponius)-5 (Jullen, Pomponius); D 24.1.38pr (Alfenus) D 24.1. 67 (Lábéon) ; D 24.3.25.3 ; D 24.3.26 ; D 24.3.63 ; D 25.1.2 ; D 25.1.6 ; D 25.2.21.2 ; D 28.5.53 ; D 28.5.85.1 (const1tution de Marc-Aurèle) ; D 28.5. D 31.5.1; D 31.8pr; D 31.14pr ; D 31.82.2 5Valens, Julien) ; D 32.60 .1

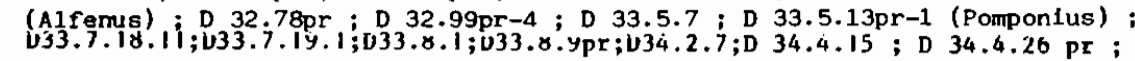

D 34.4.27pr-1 ; D 34.9.5.4; D 35.1.37 (Neratius) : D 35.1.44.8; D 35.1.96 pr-1 ; D 35.2.1.9 (veteres) ; D 35.2.21.1 ; D 35.2.33 ; D 35.2.36pr-1-3-4 : D 35.2.49 pr(Sabinus, Nerva, Atilicinus, Cassius, Plautius : rescrit d'Antonin le pleux) ; D 35.2.63pr(Pedius) ; D 36.1.68.2 ; D 38.2.44.1 ; D 39.3.11.3 (Julien) ; D 39.6 .39 ; D 40.1 .3 ; D 40.1 .9 ; D 40.1 .11 ; D 40.2 .4 .2 (Julien) ;
D 40.4.10.1 D 40.4 .39 ; D 40.5 .6 D 40.5 .25 (Valens) ; D 40.5 .29 ; D 40.5. 31.3-4 (rescrit de Caracalla) ; D 40.5.33.2 ; D 40.5.39pr ; D 40.5.40.1 ; D 40.8 .1 (rescrit de Marc-Aurèle) ; D 40.8 .9 ; D $40.9 .16 \mathrm{pr}-1$; D 40.9 .26 (Scaevola) : D 40.9 .28 ; D 40.12.23.1 ; D 40.12.38.2-3 ; D 40.13.4 (L. Rufinus) ; D $41.1 .47 ;$ D 41.1 .57 (Julien); D 41.2 .1 .5 (Sabinus, Cassius, Julien) $7-15$ (Julien)-16 (veteres)-17-18-22 (Nerva filius): D 41.2.30.4; D 41.3.15.1; D 44.4.5.4. ; D 45.1.49.3; D 45.1.73.1; D 45.1.91pr-1(Julien) -5 (Pomponius) D 44.4.5.4. ; D 45.1.49.3 ; D 45.1.73.1 ; D 45.1.91pr-1(Julien)-5 (Pomponius)
D 45.3.16 ; D 45.3.20.1 (Labéon) ; D 45.3.26; D 45.3.27; D 45.3.29 ; D 45. D 45.3.16 ; D 45.3.20.1 (Labéon) ; D 45.3.26 ; D 45.3.27 ; D 45.3.29 ; D 45.
3.31 ; D 45.3.32 (Sabimus) ; D 45.3.33pr (Julien) -1 ; D 46.3.35 (Alfenus) ; D 46.3.63; D 46.3.98.7-8 (Celsus) ; D 46.4.11pr-2 ; D 46.7.11 ; D 47.2.18 (cassiani) ; D 47.2.86; D 47.10.29; D 48.10.14pr-1 (Julien, Marcellus)-2 (Edit de Claude) ; D 48.10.17 ; D 48.10.22.4-7-10 ; D 48.18.11 ; D 48.18.18. $4-6$; D 49.15.29 (Labéon); $D$ 49.17.20; Ulpien D 2.9.1.1 ; D 2.9.3; D 2.11 . 9.1 (Labéon): D 4.3.7pr (Julien)-3 (Labéon)-4-5; D 4.3.9.1-4 (Labéon) : D 4.4.11.1 (Scaevola ; constitition de Marc-Aurèle) -4 (Julien, Marcellus)-5 (Julien, Marcellus) ; D 5.2.8.9; D 5.2.26; D 6.1.5.5 (Pomponius) ; D 6.1.15.3 D 6.2.9.6 ; D 7.1.12.3 (Julien, Pomponius)-4 (Julien)-5 (Julien) ; D 7.1 .17 .1 (Cassius, Aristo) $-2-3$; $\mathrm{D} 7.1 .21$ (Labéon); $7.1 .22 ; \mathrm{D} 7.1 .25 \mathrm{pr}-1$ (Julien, Mauricianus, Marcellus)-2-4-5 (Julien)-6 (Scaevola); D 7.1.27.2-5; D 7.2.1.1 Mauricianus, Marcellus)-2-4-5 (Julien)-6 (Scaevola) ; D 7.1.27.2-5; D 7.2.1.1
(Julien)-2 (Julien) ; D 7.3.1.2 (Julien) : D 7.4.5.1; D 7.6.5.4 (a)-5 ; D 7.8. $12.5-6$ (Labéon, Sabinus, Octavenus) ; D 7.8.14pr ; D 9.2.13.2 (Celsus) -3 (Julien) ; D 9.2.15pr-1 (Julien) ; D 9.2.17 ; D 9.2.19 (Celsus) ; D 9.2.27.1 (Proculus, U. Ferox)-2 (Celsus)-34 (Mela); D 9.4.2.1 (Celsus, Julien, Marcel1us) ; D 9.4.5-pr-1 ; D 9.4.7.1. (Pomponius); D 9.4.8; D 9.4.11; D 9.4.21.1 D 9.4.36; D 9.4.42.2 ; D 10.2.8pr (Pomponius) ; D 10.2.12.1; D 10.2.16.6 (Ofilius) ; D 10.2.18pr (Julien) -2 (Pomponius) ; D 10.2.49; D 10.3.6.9 (Julien) D 10.4.3.6-7-10 (Julien) ; D 10.4.5.6 ; D 10.4.11pr-1 (Labéon) ; D 11.1.16.1 ; D 11.1 .17 ; D 11.3.1.1; D 11.3.5.4; D 11.3.9pr (Julien)-1 ; D 11.3.13.1 ; D 12.1 .13 .2 (Papinien) ; D 12.4.5.1-2-3-4; D 13.1.7.2; D 13.3.3; D 13.6.5.7 (Namusa, Mela)-13 (Cartilius) ; D 13.7.24.3; D 13.7.25; D 14.1.1.22 ; D 14. 1.4.2-3; D 14.3.13.2 (Julien) : D 14.4.1.5. ; D 14.4.3pr (Julien) ; D 15.1. 3pr ; D 15.1.7.1 (Julien, Marcellus) 6 ; D 15.1.9.5 (Julien) ; D 15.1.11.7 (Jullen)-8 (Julien) ; D 15.1.19.1 (Pomponius, Marcellus, Papinten) ; D 15.1 . 32pr (Jullen)-2 (Neratius) ; D 15.2.1.6-7 (Pegasus, Africain)-8 (Scaevola) D 15.3.3.8; D 15.3.5pr (Pomponius) ; D 15.4.1.8 (Marcellus) ; D 16.1.26 ; D 16.3.1.9-10 (pomponius)-28-29-30-31 ; D 16.3.11 (Sabinus, Pomponius) ; D 17.1.8.4 (Julien)-10; D 17.1.10.1; D 17.1 .19 (Pomponius) ; D 17.2.23.1 (Marcellus, Marc-Aurèle) ; D 17.2.24 ; D 17.2.49 ; D 17.2.58.3 ; D 17.2.63.9 ; (Sabinus, Julien, Pompontus) ; D 18.1.17pr (veteres) ; D 18.1.29; D 18.2.4.2 (Jullen); D 18.4.2.12 (Julien)-13 (Marcellus); D 18.7.1 (Papinien) ; D 19.1. 11.8-12 (Neratius)-15; D 19.1.13.4-13-18-22 (Trebatius, Labéon) ; 0 19.2.9.1. (Marcellus) ; D 19.5.14.1 ; D 20.1.21.1 (Julien) ; D 20.1 .27 (Marcellus) ; D $20.6 .4 \mathrm{pr}$; D 21.1.1.9 (Vivianus) ; D 21.1 .4 .3 (Pompontus) ; D 21.1 .17 .6 (Caellus Sabinus)-7 (Caellus Sabinus)-8 (Caelius Sabinus)-11-15 (Labéon, Caelius Sabinus)-16 (Caellus Sabinus) $-19-20 ; D 21.1 .19 \mathrm{pr}$ (Pedius) ; $D$ 21.1.21.1 (Pompontus)-2 (Pomponius) ; D 21.1.23.3-8 (Julien)-9 (Pedius) ; D $21.1 .25 \mathrm{pr}-4$ (Pedius) $-5-6+7$; D 21.1.29.3 (Julien) ; D 21.1.31pr-1-7 (Marcellus)-9 (Pomponius)-10-16 (Ju1ien)-21 ; D 21.1.33 pr (Pomponius)-1 ; D 21.2.21pr ; D 21.2.25 D 21.2 .33 ; D 21.2 .37 .1 ; D 21.2 .51 .3 ; D 23.3.10.2 ; D 23.3.12.1 (Marcellus,
Scaevola) 
Aurèle) ; D 24.1.17.1 ; D 24.1.19pr (Julien) ; D 24.1.22; D 24.3.7.10-16 (Pomponius); D 24.3.24.4-5; D 24.3.62; D 24.3.61 pr; D 28.5.6.2-3 (Julien); D 28. nius) ; $\mathrm{D} 24.3 .24 .4-5 ; \mathrm{D} 24.3 .62 ; \mathrm{D} 24.3 .51 \mathrm{pr} ; \mathrm{D} 28.5 .6 .2-3$ (Julien) ; $\mathrm{D} 28$.
$5.9 .16-17-20 ; \mathrm{D} 28.5 .30$ (rescrit de Sévère); D 28.5.35.3 (Q. Mucius) ; 28. 5.9.16-17-20 ; D 28.5.30 (rescrit de Sévère) ; D 28.5.35.3 (Q. Mucius) ; D 28.
$5.51 p r ; D 28.6 .18 p r$ (Julien)-1 ; D 28.7.2pr-1 (Celsus) ; D 28.7.4.1 (Julien) D 28.8.1pr ; D 29.2.20.1; D 29.2.25pr-1; D 29.2.42.2-3; D 29.2.66; D 29.2. 67 ; D 29.2.71pr ; D 29.5.1.2 -3-4; D 29.5.3.1-1.3; D 30.39pr (Julien, Africain)-1 ; D 30.44Pr-7 ; D 30.47.2 : D 30.50pr ; D 30.53.8-9 ; D 30.71.1-3 ; D 32.3.1 (Julien) ; D 32.7pr; D 32.68.2 ; D 32.71 ; D 32.73pr-3-5 (Pomponius) ; D 33.5.2pr-2 ; D 33.7.12.3 (Labéon, Pegasus)-8-31 (Celsus)-33-44 (Celsus)-45 (Papinien) ; D 33.8.8.4 (Sabinus); D 33.8.24;D $35.2 .43 ; \mathrm{D} 36.1 .17 .10-14$ (Maecianus)-17 (rescrit d'Antonin le pieux);D 36.1.23.3 (Neratius); D 36.2.7.6 ; D 36.2 .23 ; D 37.11.2.9; D 38.1.15.1 (Celsus); D 38.2.16.1-9; D 38.4.3.3 (Pomponius) ; D 38.5.1.25 (Pomponius); D 38.16.3.2-7; D 39.5.13; D 39.5.18.1 (Aristo, Pomponius)-2 (Aristo, Pomponlus) ; D 39.6.37.1 (Julien) ; D 40.1 .2 ; D 40.1.4.1 (epistula de Marc-Aurèle et Verus)-2-3-4-8-9-11-1.3; D 40.2 .3 D 40.2 .16 .1 ; D 40.2.20pr-1 (constitution de Marc-Aurèle) ; D 40.4.6 ; D 40.5 4.15; D 40.5.7; D 40.5.24.6 (rescrit d'Antonin le pleux)-8 (Celsus)-10-1516-17-21 (rescrits d'Hadrien et d'Antonin le pieux) ; D 40.5 .26 .11 ; D 40.5 28pr(Marcellus)-4 ; D 40.5.30.6 (rescrit d'Antonin le pieux) ; D 40.5.45.2 ; D 40.5.45.5; D 40.5.52; D 40.7.2pr-2 (Julien)-3 (Julien) ; D 40.7.3.7; D 40. 7.16 ; D 40.9 .4 ; D 40.9.12.1-2 (Africain)-7; 0 40.9.30pr (constitution de Marc-Aurèle)-2; D 40.128.1-2; D 40.12 14pr; D 40.12.16pr; D 40.12 .20 .4 D 41.17 ; D $41.1 .18 ; D 41.1 .22 ; D 41.1 .23 p r-3$ (Ju1ien Scaevola) ; D 41.1 . D 41.1 .17 ; Dal.18; ; 41.22 ; D $41.1 .23 p r-3$ (Julien Scaevola) ; D 41. 33pr (Marcellus, Scaevola) $2(J u l i e n) ; D ~ 41.2 .4 ; D 41.2 .13 .3 ; D 41.2 .34 .2$ (Celsus) ; D 41.2.A?pr ; D 42.1.4.8 (Celsus) ; D 42.8.6.5 ; D 43.16.1.46 (Vivianus) ; D 43.24 .7 .1 (Neratius) ; D 44.3 .8 ; D 44.4 .4 .5 (Labéon); D 45. $1.29 \mathrm{pr}$; D 45.1.32; D 45.1.51 ; D 45.1.81pr (Celsus) ; D 45.3.2 ; D 45.3.4; D 45.3.5 ; D 45.3.7.1 (Ju1ien) ; D 45.3.9pr-1 (Cassius, Ju1ien) ; D 46.3.29pr ; D 46.4.8.1 (Octavenus) -2 (Labéon) ; D 46.6.2 ; D 47.2.14.5 (Papinien)-6 (Papinien)-11-15 ; D 47.2.17.2 (Sabinus)-3 (Julien, Pomponius) ; D 47.2.43.12 D 47.2.46.1-3-4-5 ; D 47.2.52.4-23 (Mela) ; D 47.4.1.1-5; D 47.6.3.1-2; D 47. 10.1.6 (Julien)-7 (Lahéon) ; D 47.10.15.36-47-48-49; D 47.10.17.9 ; D 47.10.24 D 48.5.28.3-4-8-9-11-14; D 48.15.2pr-1-2-3; D 48.18.1.6 (rescrit de MarcAurèle et Verus)-8-27 (epistula de Marc-Aurè1e et Verus); D 48.18.2; D 48.18. 3 (constitution de Sévère et Caracalla) ; D 49.17.6 ; D 49.17.9 (Marcellus); D 50.15.4.5; D 50.16.160 (Marcellus); L. Rufinus D 40.7.3?. : Macer D 48.19. 10pr ; D 48.19.14 (Menander); Marcien D 1.8.6.1 (rescrit de Marc-Aurèle et Verus) ; D 15.1.40.1 (veteres) ; D 18.1.42 (rescrit de Marc-Aurèle et Verus) D 18.1.44; D 21.1.52; D 28.5.39; D 28.5.52.1 (Celsus) ; D 29.2.52pr (rescrit d'Antonin le pieux) ; D 30.112 .1 ; D 30.114.19; D 32.65pr-2 ; D 34.1.2pr (rescrit de Sévère et Caracalla) ; $\mathrm{D} 34.5 .18 .1$; D 34.8.3.2 ; D 38.2.29.1 ; $\mathrm{D} 38.4 .6$; $\mathrm{D} 38.4 .16 \mathrm{pr}$ (rescrit de Sévère et Caracalla)-1 (rescrit de Sévère et Caracalla)-4 (rescrit d'Antonin le pieux)-10 (rescrit de Marc-Aurèle et Verus) ; D 40.1.5pr ; D 40.2 .14 .1 ; 40.5 .50 (Scaevola) ; $40.5 .51 .2-8$ D 40.8.6 (constitution de Marc-Aurè̀e) ; $\mathrm{D}$ 48.15.3pr (constitution de Sévère Caracalla) ; Modestin D 3.3.63; D 16.3.23; D 24.3.58; D 31.34pr ; D 31.35 ; D 32.81pr ; D 32.82 ; D 37.14.8.1; D 37.14.13 ; D 40.1.17; D 40.5.12.1; D 40.5 .15 ; D 40.7 .27 (Julien); D 45.3.35; D 48.8.11.1; D 50.16.101.3 Hermogénién D 5.1.5.3; D 32.22.2 ; D 35.2.38.1 ; D 40.9.27.1 ; D 41.1.61pr-1 ; D 41.2.50.1 ; Auteur anonyme D 36.4.16. I1 faut assimiler à ces textes ceux où le seul critère d'activité de l'esclave est operae : l'esclave y ressort comme un agent économique par son travail, mais juridiquement, il n'est qu'un outil, un objet (Alfenus D 40.7.14.1 ; Iavolenus D 40.7.39.3 (Servius, Labéon) -5 ; Julien D 22.1.25.1 ; Gaius D 7.8.13 (Labéon) ; Pau1 D 6.1.31 ; D 9.4.19.1 D 24.1.28.2 ; D 40.5.3.3pr ; D 40.7.41pr (Labéon) ;
U1pien D 5.3.29:D 7.7.2; D 7.7.6pr (Mela) -1:D 7.8.4pr ; D 7.9.5.3; D 33.2.7 ; D 40.7.3.3); idem pour merces chez Ulpien D 42.5.8.2. Par contre, un texte tel que $\mathrm{D} 40.4 .36$, qui contient un autre critère, a été relevé dans la rubrique "agent d'acte juridique" (Paul lib. 7 ad Plautium : Gervum testament, ita manumisi : si iuraverit se Cornelio filio meo decem operamum daturum, liber estol. Si ces textes concernaient simultanément une hypothèse d'operae et de fatt juridique, ils ont été retenus dans les deux rubriques car la problèmatique n'était plus seulement celle de 1 'acte juridique, cf. Alfenus D 40.7.14.1; I avolenus D 40.7.39.3 (Servius, Labéon) ; Pau1 D 9.4.19.1) Notons enfin que conservus a été relevé avec servus (Scaevola D 34.3.31.1. D 33.8.9pr (ressort avec servus) ; Ulpien D 21.1.25.6 (idem), ainsi que in servitutem chez Macer D 48.19.14 (Menander).

6- Cette rubrique a un caractère relativement extensif : ont été pris en considération non seulement les textes où servus apparaît directement comme agent délictuel, mais également ceux où il est question de répression, d'accusation ou de simple suspicion, car ces textes visent, bien que de manière indirecte, l'esclave agent de fait juridique. Ont été relevés Alfenus D 18.6. 12 ; D 40.7.14.1; D 44.7.20; Iavolenus D 9.2.38 ; D 11.1.14pr ; D 40.7.39.3 (Servius, Labéon); Neratius D 47.2 .65 ; D 47.2 .84 .1 ; Celsus D 13.1.15; D 47 $2.68 .3-4$; Julien D 9.4.16;D 9.4.39pr-1-2-3-4; D 9.4.40; D 9.4.41; D 9.4.42 pr-1-2 ; D 12.5.5 (Proculus) ; D 41.3 .33 .6 ; D 41.4 .9 ; D 41.4.10; D 43.24.14 D 47.2.57.3-5; Pomponius D 6.2.15; D 9.4.18; $0.4 .33 ; \mathrm{D} 9.4 .43$; D 10.2. 45.1 ; D 12.4.15 (Labéon, Proculus) ; D 15.1.4.2-3-4 ; D 17.2.60.1 (Labéon) ; D 21.1.48.3 ; D 21.2.16.2 (Proculus) ; D 21.2.30; D 30.38.1; D 30.48pr (Ati1icinus) ; D 30.56 ; D 40.7.29pr ; D 41.1.54.4 ; D 43.24.21.1 ; D 46.3.19 (Labéon) ; D 47.2.35.1 ; D 47.2.44.2. ; Africain D 3.5.48; D 9.4.28; D 13. $6.21 .1 ; \mathrm{D} 13.7 .31$ (Ju1ien) : D 19.1.30pr; D 30.108pr; D 30.110 ; D 40.4 .22 D $47.2 .62 \mathrm{pr}-1-4-5-6-9$; Gaius D $6.1 .36 .1 ; \mathrm{D} 9.2 .4 \mathrm{pr} ; \mathrm{D} 9.4 .1 ; \mathrm{D} 9.4 .15$ (Sabinus, Cassius) ; D 9.4.20 ; D 9.4.23; D 9.4.25; D 9.4.27pr-1 ; D 13.6. $18 \mathrm{pr}-2$; D 15.1.27.1 ; D 29.5.25pr-1 ; D 30.67pr ; D 30.70pr-2-3 ; D 39.4.13.2-3; B 40.12 .25 .2 ; D $44.7 .5 .5 ; D 47.4 .2$; D 47 . 10.34 : Venuleius D48.2.
$12.4 ;$ D $48.19 .16 .3 ;$ Maecianus D $48.1 .11 ;$ Marcellus D $45.1 .96 ; D$
$47.6 .5 ;$ Papinien D 19.5.8 ; D 31.76.4 ; D 43.10.1.2 ; D 48.1.14; D $48.3 .2 \mathrm{pr}-1$ (édit de Domitien) ; D 48.18 .17 .3 ; D 48.19 .33 (rescrit dc Marc-Aurèle et Verus) ;
D 48.19.34pr ; Tryphoninus D 11.4.5 (rescrit d'Antonin le pieux) ; D 49.15 .12 16 ; Menander D 49.16.4.14-15 ; Callistrate D 29.5 .2 (rescrit de Commode) D 479.7 ; D 47.21.3.1 (lex agraria de 1 'empereur Nerva) ; D 48.15.6.2; D 48. 19.28.4-11-16 ; Paul D 2.1.9 (Pomponius, Octavenus) ; D 2.9.2.1 (V. Verus, Julien, Pomponius) ; Paul D 2.10.2 (Ofilius, Sabinus) ; D $3.2 .14 ; \mathrm{D} 3.5 .40 ; \mathrm{D} 4$ 4.24.3 ; D 4.9.6.1 ; D 5.1.24.1; D 5.3.40.4; D 6.1.21 (Julien, Pomponius) ;

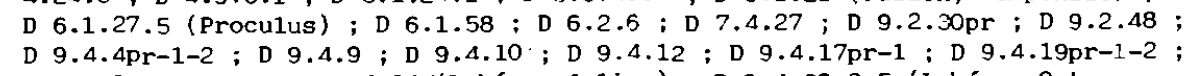
D 9.4.22pr-2-3-4; D 9.4.24 (Labéon, Ju1ien) ; D 9.4.26.3-5 (Labéon, Octavenus)-6; D 9.4.31 (Sabinus, Cassius, Julien, Pomponius) ; D 10.2.25.15; D 10.3.8.3 ; D 10.3.15 ; D 10.3.19.3 ; D 10.4.16 ; D 11.1.8 ; D 11.1.20pr D 11.3.14.2 (Sabinus)-3-6-7 ; D 11.5.4.1 (par a contrario) ; D 12.6.36 ; D 13. 6.22 ; D 14.2 .2 .5 ; D $14.5 .8 ;$ D 17.1.22.9 (Mela) ; D 17.1.26.7 (Neratius) ; D 18.7.9 ; D 19.1.14pr; D 19.2.30.4; D 19.2.45pr; D 19.4.2 (Aristo) ; D 21

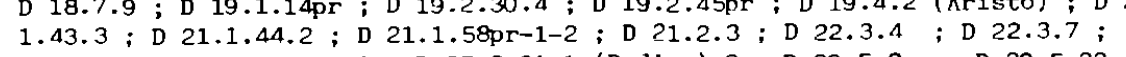
D 24.3.25.3 ; D 25.2.3.1 ; D 25.2.21.1 (Pedius)-2; D 29.5.8pr ; D 29.5.22 ; 
D 31.8pr ; D 33.8.9.1 (Marce11us) ; D 35.2.63pr (Pedlus) ; D 38.2.51 (Labéon) ; D 39.3.11.3 (Jullen) ; D 40.1.12; D 41.2.1.14 (Nerva fillus, Cassius, Jullen) D1.3.4-8-9 (Pomponius)-16 (Sabinus, Cassius) : D 41.3.15.1; D 47.2 .18 (casslani) ; D 47.2.42pr ; D 47.2.54.1 (Sabinus)-2 ; D 47.8.3 (Labéon) : D 47.9.4.1. (rescrit d'Antonin le pieux) ; 0 47.10.18.1 ; D 48.16.16 (rescrit de Damitien) D 49.15.19.5 ; D 50.16.215 (Sabimus, Cassius) ; Ulpien D 1.12.1.5; D 1.14.3 (Pomponius) ; D 2.1.7.1-3 ; D 2.1.12 ; D 2.7.1.1 (Pomponius) ; D 2.9.1.1. ; D 2.9.3; D 2.9.5 D 2.10.1.5; D 2.14.50; D 3.2.4.3 (Pomponius) ; D 4.2.14. 5-11; D 4.2.16.1; D 4.3.7.7 (Scaevola, Labéon) : D 4.3.9.4 (Labén) ; 0 4.4.11 pr (rescrit de Sévère)-5 (Jullen, Marcellus): D 4.9.3.3; D 4.9.7pr-3-4; 0 D.

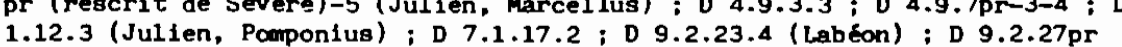

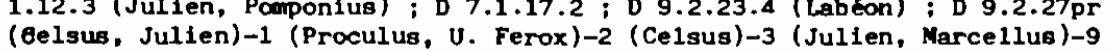
(Neratius)-11 (Proculus)-34 (Mela) ; D 9.2.44.1 ; D 9.3.1pr-8; D 9.3.5.6-10 : D.9.4.2pr-1 (Celsus, Julien, Marcellus): D 9.4.3; D 9.4.5pr-1; D 9.4.6 : D 9.4.7pr-1 (Pomponius) ; D 9.4.8; D 9.4.11; D 9.4.14pr; D 9.4.21.1-2;D 9 . 4.38 .1 (Julien) $-2-3 ; D$ 10.2.16.6 (Ofilius) ; D 10.2.18pr (Jullen) : $D$ 10.4.3.7 ; D 10.4.5.6 ; D 10.4.20; D 11.1.16pr (par a contrario) ; D 11.3.1.4-5; D 11.3. 5.3 ; D 11.3.7 ; D 11.3.11.1; D 11.6.3.6 (Pomponius) ; D 12.1.11.2 ; D 12.4. 5.3 ; D 13.1.4 ; D 13.6.5.13 (Cartilifus) ; D 13.7.24.3; D 14.3.5.8 (Labéon) : D 15.1.3.12 (Labéon) ; D 15.1.9.1 (Neratius, Pcomponius) Gonsererus ) -6-7 D 15.3.3.9; D 16.3.1.18 (Marcellus); D 16.3.11 (Sabtnus, Pomponius) ; D 17.1.

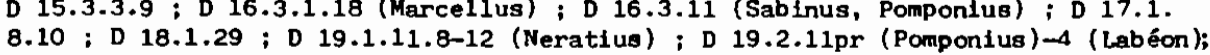
8.10 ; D 18.1.29; D 19.1.11.8-12 (Neratius) ; D 19.2.11pr (Pomponius) -4 (Labé
D $19.5 .15 ; \mathrm{D} 20.1 .27$ (Marcellus) : D 21.1.17.6 (Caelius Sabinus) -7 (Caelius Sabinus) -8 (Caelius Sabinus)-11-15 (Labéon, Caelius Sabinus)-16 (Caelius Sabinus)-19-20 ; D 21.1.21.1 (Pomponius) ; D 21.1.23.3-8 (Julien) ; D 21.1.25.7 ; D 21.1.29.3 (Julien); D 21.1.31pr-1-16 (Julien); D 29.5.1.12 (Scaevola)-14 -18-22-28 (rescrit d'Hadrien)-29 (rescrit d'Hadrien)-30-34-37-38; D 29.5.3.1-2-3 -4-5-12-13-16-17; D 30.39pr (Julien, Africain); D 30.47.2 ; D 30.53.8; D 39 . 12.6 (Celsus) ; D 39.4.1.5-6 ; D 39.4.3pr-3 ; D 39.4.12.2 ; D 40.9.2 ; D 40.9 9.3 ; D 43.16.1.15-16-17-19-19-21; D 43.24.7.1 (Neratius) ; D 43.24.11.7 ; D 44.3 .8 ; D 44.4.4.5 (Labéon)-17; D 44.7.14; D 47.1.2.3 (Pomponius) ; D47. $2.7 \mathrm{pr}$ (quis in servitute); $047.2 .17 \mathrm{pr}$ (veteres)-2 (Sabinus) -3 (Julien, Pomponius) ; D 47.2.36pr (Pomponius)-1-3 (Sabinus) ; D 47.2.43.12 ; D 47.2.52. 3-4-9-15-23 (Mela) ; D 47.4.1.1-2-3-5-6-7 (rescrits de Marc-Aurèle et de sevère et Caracalla)-10 (Scaevola)-11-14-19; D 47.5.1.5; D 47.6.1.pr-2-3 ; D 47.6.3. $1-2$; D 47.7.7.4-5 (Sabirius) ; D 47.8.2pr-5-14-15-19; D 47.8.4.15; D 47.10.9.3; D 47.10.17.3-4-7 (Labéon)-9 ; D 47.12.3.11; D 47.11.5; 0 48.2.5 (rescrít de Marc-Aurèle); D 48.2.6 ; D 48.2.7.4 (rescrit d'Antonin le pleux) ; D 48.5.28pr -3-4-14-16 ; D 48.8.4.2 (rescrit d'Hadrien) ; D 48.10.8; D 48.15.2pr-1-2-3 ; D 48.18.1.5 (rescrits d'Hadrien et d'Antonin le pleux)-19 (rescrit de Trajan) -20-27 (epistula de Marc-Aurèle et Verus) ; D 48.19.1.1 ; D 48.19.6.1; D 48.19. 8.12-13 ; D 48.19.19 ; D 50.17.23 ; D 50.17.157pr ; Macer D 48.5.25pr ; D 48.7 . 3pr ; D 48.10.10pr ; D 48.19.10pr ; Marcien D 18.1.42 (rescrit de Marc-Aurèle et verus ) ; D 21.1.52; D 28.5.49.2 (constitution de Sévère et Caracalla); 39.4.16.1 (rescrit de Sévère et Caracalla)-4 (rescrit d'Antonin le pieux)-11 (rescrit de Caracalla); 0 48.1.9 ; $048.5 .34 \mathrm{pr}$ (rescrit d'Antonín le pieux)-1 ; D 48.6.3pr ; D 48.10.1.13 ; D 48.19.11.1 ; D 49.16.11 ; Modestin D 32.82 ; D 48 . 2.17 ; D 48.8.11.1-2 ; D 48.18.14 ; D 49.1.18; Hermogénien D 41.2.50.1 ; D 47.10 .45 .
7- Ont óté relevés dans cette rubrique les textes où servus est objet délictuel aingi que, plus généralement, ceux où on a affaire à un semus deterior : Alfenus D 9.2.52pr-3-4 ; D 11.3.16; Iavolenus D 9.2.38; D 24.1. 50pr-1 : D 49.15.27 (Ofillus, Trebatius, Labéon) ; Celsus D 47.2.68.4 ; Julien D 9.2.47 - D 9.2.51pr-1 (veteres) ; D 13.1.14pr ; D 19.1.24.1 (Sabimus, Cassius) ; D 41.1.39 ; D 41.4.7.7; D 45.3.14 (Sabinus) ; Pomponius D 17.2.60.1 (Labéon); D 21.2.16.2 (Proculus): D 26.7.61; D $30.48 .1 ; D 36.1 .72 .1$ :

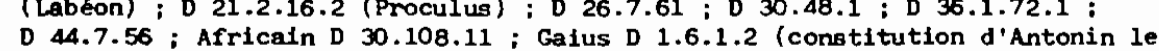
pieux) ; D 9.2.2pr-2 (Labéon) : D 9.2.32.1 ; D 9.2.4pr; D 11.3.15; Venuleius D 48.8.6 ; Marcellus D 9.2.36.1 (Sabinus); D 46.3.72.3; Papinien D 11.3.14. 8-9 ; D 12.1.31.1 (Sablnus, Cassius, Julien); D 13.1.3 (Julien); D 21.2.64pr D 47.2.81pr-2; D 48.5.6pr ; Tryphoninus D 9.4.37; 043.16 .19 (Julien) : Callistrate D 48.15.6pr (rescrit d'Hadrien)-2 ; Paul D 1.18.21; D 4.3.18.5 ; D 5.3.36.2 (Pomponius) ; $07.1 .66 ; \mathrm{D} 9.2 .12$. ; D 9.2.18; 0 9.2.22pr ; 0 9.2. 30.1-4 ; D 9.2.33pr (Pedius) ; D 9.2.45.1-2; D 9.4.10; D 10.3.8.2 ; 011.1 . $20 \mathrm{pr}$; D 11.3.8 ; D 11.3.10 ; D 11.3.14.1-2 (Sabinus) -3-6-7; D 12.1.31.1 (Sabinus, Cassius, Julien); D 12.2.30.1; D 13.1.3 (Julien); D 17.2.56; D 19 $1.45 \mathrm{pr}$ (Julien, Africain); $\mathrm{D} 19.1 .54 \mathrm{pr}$ (Labéon); $\mathrm{D} 19.2 .42 ; \mathrm{D} 19.2 .43$; $\mathrm{D} 21$ 1.43 .5 ; D 33.8.9.1 (Marcellus) ; 36.1 .68 .2 ; D 37.15.6; D 42.2.4; 0 44.7. $34 \mathrm{pr}$ : D 45.1.91.5 (Pomponius) ; D 47.2.86; D 47.2.86; D 47.10.4; D 47.10 . 18.1 ; D 47.10.26; D 47.10.29; D 48.7.4.1 (Labéon); Ulpien D 1.6 .2 (rescrit d'Antonin le pleux) ; D 2.14.50; D 3.5.5.10; D 4.3.7.3; (Labéon)-4-5; D 7. $1.17 .3 ; 09.2 .3 ; D 9.2 .5 .3$. (Julien) ; $D 9.2 .7 .2$ (Pegasus)-4-5 (Labéon) : $D 9.2$ 9.3 (Ofillus)-4; D 9.2.11pr (Mela, Proculus)-2 (Julien); D 9.2.13.2 (Celsus) -3 (Julien) ; D 9.2.15pr-1 (Julien) : D 9.2.17; D 9.2.19 (Celsus) ; 0 9.2.23pr (Neratius)-1 (Julien)-4 (Labéon)-5-6-9; $D$ 9.2.27pr (Celsus, Julien)-1 (Procu1us, U. Ferox) -2 (Celsus) -17 ; D 9.2.46; D 9.4.36; D 11.3.1pr-1-2-3-4-5 ; D 11.3.3.1; D 11.3.5.1-4; D 11.3.7; D 11.3.9pr (Jullen)-1-3 (Neratius) ;

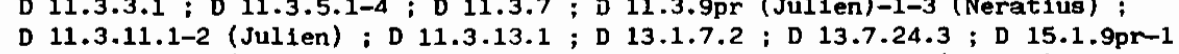

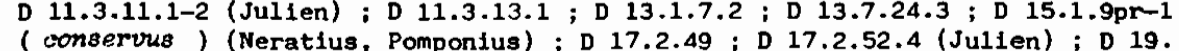
2.13pr; D 21.1.25pr-4 (Pedius)-5-6; D 21.1.31.9 (Pomponius); D 29.5.1.11; D 29.5.3.12; D 30.53.8 et 9 (par a contrar10); D 40.5.24.8 (Ce1sus) ; 043. 16.1.46 (Vivianus) ; D 47.1.2.5-6; D 47.2.14.5 (Papinien)-6 (Papinien)-7 (Pomponius, Papinien)-11-15; D 47.2.36pr (Pomponius)-3 (Sabinus) ; D 47.2.46pr-1-3-4 -5 ; D 47.2.48.2 ; D 47.2.52.23 (Mela)-24-28; D 47.8.4.13-14; D 47.10.1.3-6 (Julien) $\rightarrow 7$ (Labeon); D 47.10.3.4; D 47.10.7.2; D 47.10.9.4; D 47.10.11.9 ; D-47.10.13pr ; D 47.10.15.16 (Labéon)-34-35-36-39 (Labéon)-43-44-45 (Mela)-46 (Labéon) $-47-48-49$; D 47.10 .17 .2 (Trebatius, Mela, Labéon) ; D 47.10.30pr ; D 47.11.5; D 48.8.4.2 (rescrit d'Hadrien); D 48.15.2pr-1-2-3; Marcien D 11. 3.17 ; D 48.15.3pr (constitution de Sévère et Caracalla) ; Modestín D 40.8 .2 (édit de Claude) ; D 48.8.11.1. D 16.3.31.1 (Tryphoninus) n'a pu être retenu car l'esclave n'est pas dans ce texte un objet délictuel, on lui vole quelque chose. 
8- N'ont pu être intégrés dans aucune rubrique en premier lieu les textes dont le seul critère est constitué par le mot quaestio (ou torqueri ou tormenta. , la quaestio pouvant âtre appliquée sans que l'esclave soit nécessairement coupable (donc à écarter de la rubrique "agent de fait juridique"), et n'étant pas ce que $l^{\prime}$ 'on entend communément par acte juridique (donc à écarter également de la rubrique "objet d'acte juridique"). Il s'agit de : Papinien D 3.6.9 ; D 29.5.21pr ; D 48.5.40.8 ; D 48.18.17pr (rescrits de MarcAurèle et de Caracalla)-1 ; Tryphoninus D 18.18.19; Paul D 1.15.3.2 (rescrit, d'Antonin le pieux) ; D 19.2.55pr ; D 29.5.6.1 ; D 48.18.8pr (édit d'Auguste)-1 D 48.18.18.5-7-8; Ulpien D 27.3.1.3 (décret de Sévère) ; D 29.5.1pr-15-16 (Marcellus) -32; D 48.5.28.5 (Africain)-6 (rescrit d'Hadrien)-10; D 18.18.1.1 (rescrit d'Hadrien)-4 (rescrit de Marc-Aurèle et Verus)-7-11 (rescrit de Trajan)-12 (rescrit de Trajan)-13 (rescrit d'Antonin le pieux)-16 (rescrit de jan)-12 (rescrit de Trajan)-13 (rescrit d'Antonin 1e pieux)-16 (rescrit de
Sóvère)-18 (rescrit de Sévère et Caracalla)-22 (rescrit d'Hadrien); D 48.18.4 Sávère)-18 (rescrit de Sévère et Caracalla)-22 (rescrit d'Hadrien) ; D AR.18.4
(Papinien) ; D 48.18.7 ; Marcien D 48.18 .5 ; D 48.18.9pr (rescrits d'Autonin 1e pieux et (le Sévère) -1 ; Modestin $D$ 48.18.13; $\Lambda$. Charisius $D$ 48.18.10.4-2. De même, $n$ 'ont pu être retenus les textes concernant seulement 1 'affranchissement ou plus généralement le status de l'esclave : Proculus D 12.6.53 ; Iavolenus D 28.5.66 ; D 35.1.67 ; D 40.1.26 (Labéon) ; Celsus D 40.2.19 ; Julien D 38.1.25. 2 ; D 40.2.4pr-1 ; D 40.2.5 (Iavolenus) ; D 40.4.18pr (Sabinus) ; D 40.5.47.2 ; D $40.7 .13 .2-4 ; 0$ 40.12.30 (Sabinus, Cassius) ; Pohponius D 1.2.2.24; D 28.5 $23.1 ; 030.54 .2 ; 035.1 .6 .1$ (Neratius) $D 40.43 ; 040.4 .11 .2 ; D 40.4 .41$

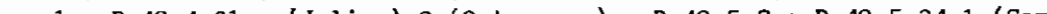
pr-1 ; D 40.4.61pr (Julien) 2 (Dctavenus) i D D 10.9 .22 ; D 40.12 .28 ; D 40.12 .43 (rescrit difadien) ; D 40.13 .3 (servitus); Africain D 30.108 .14 ; T. Clemens D 40.9.2A (Julien) (servitus ) ; Gaius D 1.5 . 3 ; D 1.5 .6 (servitus) ; D 38.2.30 (servitus) ; D 40.2 .7 ; $\mathrm{D} 40.4 .24$; 040 9.10 ; D 40.12 .2 ( servitus ) ; D 40.12 .6 (servitus ) ; D 40.12 .26 ; D 40.16 .1 D 47.10.12 (servitus) ; Venuleius D 43.29 .2 (servientium) ; D 46.8.8.2 (Labéon) ; Maecianus D 35.2.32.5 ; D 40.4.55.1 ; D 40.5.35 (Cassius) ; Marcellus D 28.4.3.1 (rescrit de Marc-Aurèle) ; D 38.2.32 (servitus) ; D 40.1.15 ; D 40.4.42 ; D 40.5.10.2 ( servitus) ; D 40.5 .56 ;Florentinus D 1.5.4.1 ( servitus )-2 ; Scaevola D 34.1.13.2 (servitus) ; D 40.4.29; D 40.4.54pr ; D 40.4. 59pr ; D $40.5 .19 \mathrm{pr}$; D $40.5 .41 \mathrm{pr}$ (servitus)-1 (Servitus )-2-4-6-11; D 44.7. 30 : Papinien D 38.1.42; D 40.1.19; D 40.1.20.3 (Servitus) ; D 40.4.47.1 ; D 40.5 .21 (Servitus; ; D $40.5 .22 \mathrm{pr}-1$; D $40.5 .23 .4 ; \mathrm{D} 40.7 .34 \mathrm{pr} ; \mathrm{D} 40.12 .35$. D $44.2 .29 \mathrm{pr}$ (servitus) ; D 44.2.29pr (servitus) ; Tryphoninus D 1.5 .15 Servitus ; 18.7 .10 (Scaevola ; constitution d'fadrien) ; D 34.3 .28 .7 (Scaevola) ; D $37.14 .23 p r-1$ (res-
crit d'Hadrien); Menander D 40.12.29pr ; D 49.16.4.10; Callistrate D 4.6.11 crit d'Hadrien) ; Menander D $40.12 .29 \mathrm{pr}$; D 49.16 .4 .10 ; Callistrate D 4.
(servitus) ; D 40.12.37; D 48.20.1pr (servilis) ; D 49.14 .12 (rescrit (servitus) ; D 40.12.37 ; D 48.20.1pr (sorvilis) ; D 49.14.12 (rescrit
d'Antonin le pieux) ; Paul D 3.5.18.2 (Labéon) (servili ) D 4.2 .4 (servitus) d'Antonin le pieux) ; Paul D 3.5.18.2 (Labéon) (servili ) ; D 4.2.4 (semitur D 19.5.5.5 ; D 22.6.1.2 ; D 23.2.14.2 (servitus)-3 (servitus) : D 24.2.1 (servitus) ; D 28.6.43.3 (servitus) ; D 35.2.36.2 ; D 37.10..6.3 ; D 38.2.4pr; D 38.2.9 (servitus) ; D 38.10.10.5 (servilis) ; D 40.1.14.1 (Zex, d'Auguste) ; D 40.1 .23 ; D 40.2.15pr-2 ; D 40.2.22; D 40.4.31 ; D 40.4.35 (Servius) ; D 40. 4.36 ; D 40.4 .37 ; D $40.4 .38 ; \mathrm{D} 40.4 .52 ; \mathrm{D} 40.4 .53 ; \mathrm{D} 40.4 .56$ (rescrit de Marc-Aurèle); $\mathrm{D} 40.5 .5 ; \mathrm{D} 40.5 .31 \mathrm{pr}-1$ (rescrit de Marc-Aurèle et Verus) : D 40.5.33.1; D 40.5.39.1 ; D 40.7.20.2-3 (Sabinus, Julien) ; D 40.9.13; D 40. 9.16.2-3 (Aristo) (servitus) ; D 40.12 .32 (servitus); D 40.13 .5 ; D 41.2 .3 . 10 ; D 44.5.2.2 ; D 45.1.83.5; D 45.1.66; D 49.15.19.10; D 49.15.30 (Labéon) D 50.2.9pr (Sévère) (servitus) ; U1pien D $1.1 .4 ; D 1.6 .7 ; D 1.7 .46$ (servitus);
- analyse des paragraphes (par rapport au nombre total de paragraphes où servus apparâ̂t lié à un acte juridique, par périodes).

$\begin{array}{llllllll}\text { I } & - & & - & & \text { I } & - & = \\ \text { II } & 1 / 2 & = & 50 \% & \text { II } & 1 / 2 & = & 50 \% \\ \text { III } 284 / 556 & = & 51 \% & \text { III } 272 / 556 & = & 49 \% \\ \text { IV } & 81 / 160 & = & 51 \% & \text { TV } 79 / 160 & = & 49 \% \\ \text { V } & 738 / 1515 & = & 49 \% & \text { V } 777 / 1515 & = & 51 \% \\ \text { TOTAL } 1104 / 2233 & = & 49 \% & \text { TOTAL } 1129 / 2233 & =51 \%\end{array}$

- analyse des citations (par rapport au nombre total de citations ou servus apparaît 1 ié à un acte juridique, par périodes).

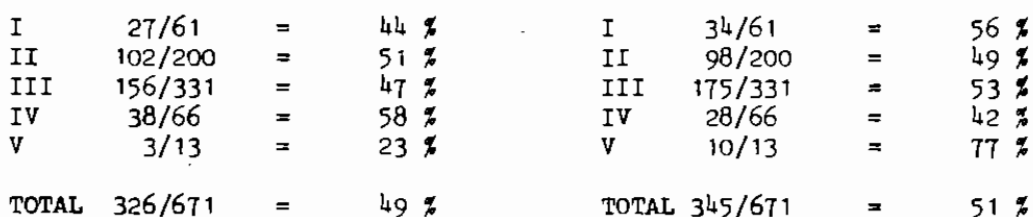

D 1.10.1.2; D 1.18.2; D 2.4.4.3 (Servitus) ; D 2.4.8.1 ; D 2.4.10.11 (Labéon) (Servitus) ; D 3.2.4.3 (Pomponius) (Servitus); D 3.2 .24 (rescrit de Sévẽre) (servitus) ; D 3.3.39.5 (servitus) ; D 4.1.6 (Julien) (servitus) ; D 4.2.9.2 (Pomponius) ; D 4.4.9.4 (Papinien) ; D 4.6.1.1 (servitus) ; D 4.6.12 (servitus); D 4.6.23pr (servitus) ; D 5.3.7.1 (rescrit d'Antonin le pieux) (servitus) ; D 12.4.1pr ; D 12.4.3.7 (Celsus P., Celsus) ; D 22.3.14 ; D 23.2.45.6 (Julien) (servitus) ; D 23.5.2 (servitus) ; D 24.1.32.6; D 24.3.22.13; D 26.1.14.1 (servitus); D 26.4.1.3 (rescrit d'Antonin le pieux); D 26.4.3.5 (servitus) ; D28.3.6.5. (servitus) -6 (rescrit d'Hadrien) $-7 ;$ D 29.1.13.2; D 29.4.29; D $32.1 \mathrm{pr}$; D $34.5 .10 \mathrm{pr}$; D 35.1 .59 .2 (servitus); D 35.1 .92 ; D 36.1 .18 .6 ; D $32.1 \mathrm{pr}$; D $34.5 .10 \mathrm{pr}$; D 35.1 .59 .2 (servitus) ; D 35.1 .92 ; D 36.1.18.6 ;
D 37.4 .1 .9 ; D 37.10 .1 .5 (Julien;rescrit d'Antonin le pieux) -6 ; D 37.12 .1 .2 D 37.4 .1 .9 ; D 37.10 .1 .5 (Julien;rescrit d'Antonin le pieux) 6 ; D 37.12 .1 .2 (servitus); D 38.2 .1pr (Servius) (servitus); D 38.2.3.8 (constituon d'Hadrien); (servitus); D 38.2.1pr (Servius) (servitus) ; D 38.2.3.8 (constituon d'Hadrien
D $38.2 .14 \mathrm{pr}$ (sexvitus); D 38.2.16pr (servitus) -2 (servitus) -3 (servitus) 4 (rescrit de Marc-Aurèle et Verus) (servitus) ; D 38.8.1.2 (servilis) ;

D 38.16.3.1 (servitus)-4-6-12 (rescrit d'Antonin le pieux) (servitus)'; D 38.17.1.3 (servitus) -4 (servitus) ; D 38.17.2.3; D 40.4.1; D 40.4.7 (Neratius) (servitus) ; D 40.4.9.1 ; D 40.4.13pr-2 (Julien) ; D 40.4.14. D 40.4.30; D 40.5.2 (constitution de Marc-Aurèle); D 40.5.4.14; D 40.5.24.1-2$5=4-5$ (rescrit de Caracalla) - 12 (Marcellus) ; i 40.5 .20
pr-3 (rescrit de Sévére et de Caracalla) -7 
Servus come sgent de fait juridique. Servus comme objet de fait juridique

- analyse des parazraphes (par rapport au nombre total de paragraphes ou servus apparaît lié à un fait juriaique, par période

\begin{tabular}{|c|c|c|c|c|c|c|c|}
\hline I & - & $=$ & - & I & - & $=$ & - \\
\hline I I & - & $=$ & - & II & - & $=$ & - \\
\hline III & $86 / 112$ & $=$ & $77 \%$ & III & $26 / 112$ & $=$ & 238 \\
\hline IV & $5 / 8$ & $=$ & $62,5 \%$ & IV & $3 / 8$ & $=$ & $37,5 \%$ \\
\hline v & $357 / 532$ & $=$ & $67 \%$ & $\mathrm{v}$ & $175 / 532$ & $=$ & $33 \%$ \\
\hline TOTAL & $448 / 652$ & $=$ & $69 x$ & TOTAL & $204 / 652$ & $=$ & $31 \%$ \\
\hline
\end{tabular}

- analyse des citations (par rapport au nombre total de citations ou servus ípparaît lié à un fait juridique, par périodes).

\begin{tabular}{|c|c|c|c|c|c|c|c|}
\hline I & $8 / 17$ & $=$ & $47 \%$ & I & $9 / 17$ & $=$ & $53 \%$ \\
\hline II & $50 / 81$ & $=$ & $62 *$ & II & $31 / 81$ & $=$ & $38 x$ \\
\hline III & $57 / 92$ & $=$ & $62 \%$ & II I & $35 / 92$ & $=$ & $38 \%$ \\
\hline IV & $9 / 10$ & $=$ & $90 \%$ & IV & $1 / 10$ & $=$ & $10 \%$ \\
\hline v & $0 / 3$ & $=$ & - & v & $3 / 3$ & $=$ & $(100 \%)$ \\
\hline TOTAL & $124 / 203$ & $=$ & $61 \%$ & TOTAL & $79 / 203$ & $=$ & $39 \%$ \\
\hline
\end{tabular}

(servari) ; D 40.5.30.3 (rescrit de Marc-Aurèle et Verus)-12-17 (rescrit de Sévère et Caracalla); D 40.5.45.1; D 40.7.3.15-16 (Julien); D 40.7.6.1 ; Sévère et Caracal1a) ; D 40.5.45.1 ; D 40.7.3.15-16 (Julien) : D 40.7.6.1
D 40.7.9pr-3; D 40.12.1pr-1; D 40.12.7pr (servitus -5 ; D 40.12.12pr-1 D $40.7 .9 \mathrm{pr}^{-3}$; D 40.12.1pr-1 ; D 40.12.7pr (servitus) -5 ; D $40.12 .12 \mathrm{pr}-1$
(servitus) -2 ; D 40.12.27.1 (rescrit d'Hadrien) ; D 40.12 .31 ; D 40.12 .34 (servitus)-2 ; D 40.12.27.1 (rescrit d Hadrien) ; D 40.12.31 ; D 40.12.34
(constitution de Caracalia) (servitus) ; D 40.13.1.1 (servitus ; D 43.29.3.7 ; D 48.19.2pr (servitus) ; D 48.19.6.2 (servitus); D 48.23.1.1 (servitus); D 49.16.8 (servitus) : D 50.17.209 (servitus); L.Rufinus D 40.5.16; Macer D 42.1.63; Marcien D 1.5.5pr-1-3; D 1.16.2pr; D 34.8.3pr (rescrit d'Antonin le pieux) ; D 37.14.5pr (Claude) ; D 40.1.5.1; D 40.1.8pr-1-2 (rescrit d'Antonin le pieux) ; D 40.2.9pr-1 (rescrit d'Antonin le pieux); D 40.9.9pr; D 40.11.2 ; D 40.15.1.4 (Marcellus) ; Modestin D 37.14.8pr (rescrit d'Hadrien) ; D 38.8.7; D $40.1 .16 ; 040.2 .21$ (constitution d'Auguste) $: 040.4 .44 ; 040.5 .11 ; 040.5 .14$; D 40.7.26pr-1 ; D 40.9.19; D 40.9.20; D 40.11.5.1 (servitus); Hermogénien D 1.5 .13 ; D 37.4 .2 ; D 37.5 .23 (constitution d'Antonin le pieux) ; D 40.15 .3 (servitus); D 48.19.36. N'ont pas non plus été retenus les textes od servus intervient dans une définition (Alfenus D 50.16.203; Pomponius D 50.16.166pr: D 50.16.239.1 ; Paul D 50.16.204pr; Ulpien D 24.1.3.3 ; D 50.16.195.3 (servitutum) ; D 50.17.32 ; Marcien D 50.16.210), ou encore des textes divers qu'il est impossible de ranger dans aucune des rubriques envisagées (Paul D 14.2.2.2 -
On constate tout d'abord que l'utilisation juridique de sermus est polyvalente. Ce wot est en effet abondarment reprósenté dans chacune des rubriques envisagées. L'examen des totaux permet ensuite de remarquer d'une part que serrus est presque autant employé pour désigner l'esclave agent d'acte juridique qu'objet d'acte juridique, et d'autre part que ce terme désigne plus l'esclave agent de fait juridique qu'objet de fait juridique. La première remarque ne doit pas surprendre, il est en effet normal que les juristes emploient rréquement servus comme agent d'acte juridique, le mot átant le plus courant et la fonction posant des problèmes quelquefois difficiles à résoudre : on notera d'ailleurs que la légère prépondérance de cette utilisation porte dans $l$ 'analyse des paragraphes sur les juristes de l'époque des Antonins, époque qui correspond, on l'a vu, à un changement d'utilisation de l'esclave. La deuxième remarque revêt un intérêt particulier quant à l'idéologie véniculée par le vocabulaire juridique : celui-ci se fait plus l'écho d'une peur des maitres vis-à-vis des esclaves, qui ressortent fréquement des textes comme des agents délictuels, que d'une crainte de ces mêmes mâtres de perdre leur propriété ou de la voir se détériorer. Hormis pour la période républicaine, dans l'analyse des citations, ol̀ on observe un équilibre de ces deux emplois de servus, la peur des propriétaires d'esclaves se présume nettement de l'emploi majoritaire de servus comme agent de fait juridique dans les autres périodes prises en considération. Le pourcentage réalisé par la période $V$ dans les citations visant servus objet de fait juridique ne remet pas ce développement en cause, se fondant sur un nombre de textes trop faible pour être pertinent.

Les mêmes observations découlent-elles de l'emploi de homo?

-passagers sur un navire-; D 19.1.21.3 -esclaves mourant de faim-; Ulpien D 4.6.10 -esclaves des magistrats municipaux-; D 11.7.2pr (Aristo) -sépultureD 11.7 .31 .1 -idem- ; D 19.2.13.3 -instruction-; Marcien D 47.22.3.2 in colle$g^{-1} 0^{-)}$. 
Oṕrona te la même manièr que pour servus en classant homo dans les rubriques agent d'acte juridique (10), objet d'acte juridique (11), agent

9- Homo ne désigne pas forcêment un esclave (par exemple, Paul D 4.6.13pr (I.abon): Liheris hominibus; de même ches panl D 1.15.3.2, homines ne vise pas des esclaves : Effracourie fiunt plerumprue in inaulis in horreisque, ubi homines protiosissiman portom fortunctum suarum repenunt...). La signification générale de homo oppose homme a femme, vivant $a$ mort, ou encore créature raiscnnable forc ou bestiz; au pluripl, homines signifie souvent soldats, fantassins (ef A.ERNOUT et A.ME ILLFT, Dictionnaire étymologique, cit. p297). Il est drs textes nù hom peut aussi bien viser un home libre qu'un esclave (of Julien 0 40.12.30 (Sabinus,Cassius) ; Gaius D 1.5.3; D 40.12.25.2 ; D 40.16.1 : Raul D 40.12.24.4 (Mela) : D 42.1.19pr ; D 43.1.2.1; D 49.15.19.10: U1pien D 1.18.13pr: D 47.8.2pr-14: D $48.6 .10 p r ; 050.17 .32$ : Marcien D 15.1 .40 pr (P.Fronto) ; D $40.11 .2 ;$ D $48.8 .3 p r$ ).

10- Calsus D 19.1.38pr; Julien D 21.2 .8 ; Scaevola D 10.2.39.4; 021.2 .69 .2 ; Papinien D 36.1.55: 041.2 .47 (Nerva filius) : Paul D 13.1.13 (Julien); D 21.2.3; IItpien D 46.1 .33

11- Alfenus (chez auteur anonvme) D 44.1 .14 ; tavolenus D 41.3 .19 ; Neratius D 47.2.65: Celsus D 12.4.16: D 19.1.38pr : D 32.79pr : D 46.3.69; Julien D 7.1.15.1 (Sabinus) ; D 10.4.8 : D 12.6.32.3: D 13.1.14pr ; D 13.5.23; D $17.1 .30: 018.5 .5 .2$; 019.1 .26 .1 (Sabitls, Cassius) ; D 21.2.8; D 21.2.39.1; D 30.84.3-5; D 30.86rr: D 30.91.7: D 34.4.11:D 34.5.13.2: D 36.2.11; ; D 45.1 .56 .8 : D $46.3 .33 \mathrm{pr}-1$ : $046.3 .34 \mathrm{pr}-1-2-10 ; 0$ 46.4.17 ; Pomponius D 12.4 15 (Proculus) ; 0 17.1.47.1 (Julien) ; D 21.1 .48 .6 ; 0 30.8.2 : D 30.13 (Neratius D 30.45.2 ; D 30.46: D 33.5.8.2 ; D 40.4.40pr (Julien) ; D $43.3 .30 \mathrm{pr}$; D $41.4 .6 .2 ; 041.10 .3$ (Netatius) : $043.26 .15 .4 ; 045.1 .23 ; 046.4 .10$ : Africain D 17.1.37; D 20.4.9pr; D 21.1.51 pr ; D 30.108.2; D 30.110: D 44.3.6.1 : D $46.3 .38 .3 ; 047.2 .62 .2-5 ; D 50.16 .207$ (Mela) : Gaius D 1.8.1.1.; D 4.7.3.1; D 6.1.18; D 6.1.20; D 6.1.76.1 ; 0 7.7.3; D 7.7.4;

D 12.6 .63 (Neratius) : $D$ 16.3.14.1 (Sabinus, Cassius); D 21.1 .22 ; D 21.1..32 ; D 22.1.28.1 ; D 30.60.5: D 35.1.17pr ; D 40.9.3; D 41.1.43.2; 044.7 .1 .9 ;

D 45.1 .74 : Venuleius D 21.1 .65 .1 (Cassius) : 045.1 .137 .1 ; $046.2 .31 \mathrm{pr}$ :

Marcellus D 19.1 .23 (Julien) ; $D 46.3 .67$; D 46.3.72.1-4-5-6 ; Florentinus $D 18$.

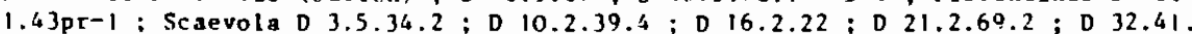
$10: D 33.5 .18$ (Neratius): D 33.7.20.5:D 36.1.80.12:D 38.1 .44045 .1 .122 D 45.1 .127 ; Papinien D 18.7.6.1 (Sabinus) ; 018.7 .7 ; 021.2 .66 .2 ; 031.66 .3 ; D 33.2.2; D 35.2.9.1; D 36.1.55; D 40.1.20.3; D 40.4.47pr: D 41.2.47 (Nerva filius): D 45.1.115.2 (Q.Mucius, Sabirns , Pegasus) ; $045.1 .117 ; 046.3 .95 .1$;

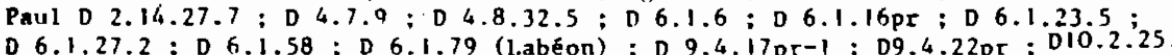

17;D10.4.12.4;D12.1.31pr-1 (Sab.,Cass.,Jul.);D17.1.26.8(Mela);D18.1.1.1(Sabinus); D 18.4.21 ; D 19.1.43 (Julien,Ulpien) ; D 19.2.45.1 ; D 20.6.10.1 ; D 21.1.11; D 21.1.43.9:D 21.1.44pr (Pedius); D 21.1.47pr (Labéon)-1; D 21.1.57.1; ; D 21.2 .3 ; D 21.2.11.1 ; D 21.2.41pr; D 21.2.56.2; D 30.35; D 31.8.1; D 34 5.21pr ; D 35.2.48; D 35.2.63pr (Pedius); D 40.7.42 (Labéon); D 41.2.3.13 (Nerva filius) ; D 42.1.8; D 43.16.6; D 44.7.44.4; D 45.1.2.1-3; D 45.1 .8 (Proculus, Sabinus, Marcellus) ; D 45.1.49.3; D $45.1 .83 .4-5 ; \mathrm{D} 45.1 .91 \mathrm{pr}-1$. (Julien)-6; D 45.1.93; D 46.2.32; D 46.3.60; D 46.3 .98 .8 (Celsus); D 46.7

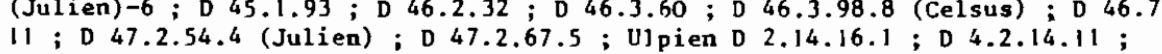

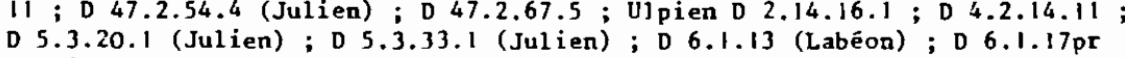
(Julien) -1 (Julien) ; $06.1 .45 ; \mathrm{D} 7.1 .68 \mathrm{pr}$ (Brutus) ; D 9.2.11.7 (Julien) ; D 9.4.36; D 10.4.3.12 (Pomponius); D 10.4.9pr (Julien); D 10.4.17; D 12.6.26. 12 (Celsus) ; D 13.4.2.2; D 13.6.5.6 (veteres)-7 (Namusa,Mela); D 16.3.1.5; D 16.3.7pr ; D 17.1.8.10; D 18.4.2.15; D 21.1.1.7 (Sabinus)-8; D 21.1.10pr (Ofilius) -4 ; D 21.1.14.10 (Caelius Sabinus); D 21.1.21.3 (Pomponius); D 21.1.23pr (Pomponius)-9 ; D 21.1.25.8-9; D 21.1 .27 ; D 21.1.29.3 (Julien) ; D $21.1 .31 \mathrm{pr}-5$ (Pomponius)-6 (Pooponius)-11; D 21.1 .38 .7 (ofilius, Caelius Sabinus)-14; D 21.1.59.1 ; D 21.2.21.2 (Julien)-3 (Julien) ; D 21.2.51.3; D 30.37pr (Cassius, rescrit de Sévère et Caracalla) ; D 30.53.3 ; D 30.71.4 ; D 33.5.2.1 ; D 33.7.8pr (Sabinus); D 33.7.12.2 (Al fenus); D 36.2.12.7; D 41.2.13.2; D 43.31.1pr; D 43.32.1pr; D 44.2.11.1 (Celsus)-4-8 ;D 44.4.4.7 (Labéon); D 45.1.29.1 (Marcellus); D 45.1.69; D 45.1.75.1-8; D 45.1.82.1; D 45.3.9pr ; D 46.1.8.9 (Julient 10; D 46.1.33; D 46.3.9.1; D 46.3.29pr ; D 46.4.13.4 (Julien) ; D 48.5.28.11; Modestin D 45.1.103.

12- Alfenus D 9.2.52.2-3; Julien D 13.6.20; D 39.6.18.3; Africain D 30.110; $D$ 47.2.62.1-2-5; D 47.2.65; Gaius D 2.9.4 (Sabinus); D 21.1 .22 (pris par contexte, cf D 21.1.21.3) : Papinien D 48.3.2.2; Paul D 4.9.6.4; D 6.1.58;

D 9.4.17pr-1; D 9.4.22pr ; D 19.2.45.1; D 21.2.3 ; D 21.2.11.1 ; D 47.2.54.4 (Julien) ; Ulpien D 4.2.14.11; D 9.1.1.14; D 21.1 .21 .3 (Pomponius) ; D 21.2. 21.3 (Julien) ; D 30.53.3; D 50.16.174.

13- Julien D 9.2.47; 0 9.2.51.2 ; D 13.1.14pr ; Gaius D 9.2.8.1 ; Papinien D 13.1.17; Paul D 1.18.21 ; D 9.2.24; D 9.2.31 (Q.Mucius) ; D 11.3.14.9; D 13.1 .3 (Julien) ; D 17.1.22.6 ; D 42.2.4; Ulpien 04.2 .14 .11 ; D 6.1 .13 (Labéon); D 6.1.17pr (Julientl (Julien); D 9.2.9.2 (Neratius)-3 (Ofilius); 0 9.2.11.4 (veteres)-7 (Julien) ; D 9.2.2ipr ; D 9.2.23.3 (Julien)-1l (Julien) D 9.2.27.5-6:D 9.4.36; D 10.4.9Pr (Julien) : D 10.4.17;D 11.3.11.2 (Julien) D21.1.25.8;021.1.31.6 (Pomp.)-11; D 43.16 .1 ; (Julien); 45.39 (Jarcellus) ; 047.1 .2 .1 ; D 47.2.46pr; D 47.10.7.1 ; Marcien D48.8.1.2. Ne rentrent dars aucune de ces catégories D 34.4.27.1 Paul (sur le statiss) et D 47.22.3.2 Marcien (in collegio) 
Far rapport au nombre total de paragraphes ou de citations dans lesquels homo apparait lié à un acte ou à un fait juridique, pour chaque période, on obtient les pourcentages suivants :

homo comme agent d'arte juridique.

\section{homo comme objet a'acte juridique.}

\section{- analyse des paragraphes}

$\begin{array}{llll}\text { I } & - & = & - \\ \text { II } & - & = & - \\ \text { III } & 2 / 83 & = & 2 \% \\ \text { IV } & 2 / 24 & = & 8 \% \\ \text { V } & 5 / 148 & = & \end{array}$

- analyse des citations

$\begin{array}{llll}\text { I } & 0 / 7 & = & - \\ \text { II } & 1 / 32 & = & 3 \% \\ \text { III } & 1 / 33 & =3 \% \\ \text { IV } & 0 / 2 & = & - \\ \text { V } & 0 / 1 & = & \\ \text { TOTAL } & 2 / 75 & =\end{array}$

homo conme agent de fait juridique.

- analyse des paragraphes

$\begin{array}{llll}\text { I } & - & = & - \\ \text { II } & \overrightarrow{9} & = & - \\ \text { III } & \bar{g} / 13 & = & - \\ \text { IV } & \overline{6} & = & - \\ \text { V } & 16 / 50 & = & \\ \text { TOTAL } & 25 / 63 & & 40 \%\end{array}$

- analyse des citations

$\begin{array}{llll}\text { I } & 0 / 3 & = & - \\ \text { II } & 1 / 2 & = & 50 \% \\ \text { III } & 3 / 14 & = & 21 \% \\ \text { IV } & 0 / 1 & = & - \\ \text { V } & - & = & - \\ \text { TOTAL } & 4 / 20 & = & \end{array}$

$\begin{array}{lll}\text { I } & = & - \\ \text { II }- & = & - \\ \text { II } 81 / 83 & = & 98 \% \\ \text { IV } 22 / 24 & = & 92 \% \\ \text { V } 143 / 148 & = & 97 \% \\ \text { TOTAL } 246 / 255 & = & 96 \%\end{array}$

$\begin{array}{lrlr}\text { I } & 7 / 7 & = & 100 \% \\ \text { II } 31 / 32 & = & 97 \% \\ \text { II } 32 / 33 & = & 97 \% \\ \text { IV } 2 / 2 & = & 100 \% \\ \text { V } 1 / 1 & = & 100 \% \\ \text { TOTAL } 73 / 75 & = & 97 \%\end{array}$

homo comme ob.jet de fait juridique.

$\begin{array}{lll}\text { I }- & = & - \\ \text { II } \overline{4} & = & - \\ \text { III } 4 / 13 & = & 31 \% \\ \text { IV } \overline{7} & = & -\overline{8} \% \\ \text { V } 34 / 50 & = & 60 \%\end{array}$

$\begin{array}{llll}\text { I } & 3 / 3 & = & 100 \% \\ \text { II } 1 / 2 & = & 50 \% \\ \text { II } 11 / 14 & = & 79 \% \\ \text { IV } 1 / 1 & = & 100 \% \\ \text { V }- & = & - \\ \text { TOTAL } 16 / 20 & = & 80 \%\end{array}$

Homo est beaucoup moins usité que servus et, bien qu'apparaissant dans toutes les rubriques, désigne principalement l'esclave objet de droit, que ce soit d'un acte ou d'un fait juridique. Seule la période III, dans l'analyse des paragraphes, indique un pourcentage élevé de l'emploi de homo comme agent délictuel, mais il n'est pas appuyé par l'analyse des citations. Du reste, la vocation essentielle de homo à désigner l'esclave objet ressort de deux faits. Tout d'abord, lorsque homo signifie esclave agent d'acte juridique, il ne s'agit jamais d'un emploi isolé en ce sens que le paragraphe concerne chaque fois simultanément une hypothèse d'esclave objet d'acte juridique. Ensuite, il arrive fréquemment que, dans un même texte, homo signifie esclave objet d'acte juridique alors que servus vise au contraire l'esclave agent (14).

On notera donc principalement la vocation essentielle de homo à désigner l'esclave objet de droit. C'est une utilisation a priori surprenante, homo ne signifiant pas forcément, comme on l'a déjà vu, esclave, mais pourant viser un home libre. Il n'est pas exclu qu'il s'agisse là d'un phénomène compensatoire par rapport à l'emploi de servus, fréquement usité pour désigner l'esclave agent de droit. Qu' en est-il de la résonance de mancipium ?

$$
\text { c- mancipiwn (15) }
$$

Mancipizm est moins enployé que homo.L'immense majorité des paragraphes où apparaît mancipiwn concerne l'esclave objet d'acte juridique, et on n'en dénombre que $135(16)$ contre 2 h6 pour homc. On compte d'autre part 10 textes

14- Cf par exemple Julien D 21.2.39.1 : 5i semus tuus emerit hominem; Africain D 21.1.5ipr : ...Sed si servus mandatu domini hominem ememit ; Gaius D 41.1.43.2 : Cwn servus, in quc alterius usus fructus est, hominem emit. 15- De manus, capio ; sur la signification -esclave-, au pluriel cf Cic., Par. 35 ; Att. $8,11,4$; au sing. Pline, Ep, 686 et...; aussi Florentinus D 1.5.4.3 : mancipia vero dicta, quod ab hostibus manu capiantur.

16- Proculus D 31.48pr ; Iavolenus D 24.3.66.3 (Labéon) ; D 38.2.36 : Neratius D 25.1.15; Celsus D 19.1.38.1 (Sextus Aelius, Drusus); Julien D 23.4.21 ; D 30.84 .10 ; Pomponius D 13.7.8.1; D 21.1 .36 ; D 21.1.48.6; D 21.2.34pr;

D 23.3.18 (Labéon) ; D 24.1.31.3; D 30.8.2 ; D 33.5.6; D 41.10.4pr (Trebatius); Africain D 21.1.51pr ; Gaius D 21.1.18pr ; D 21.1.20 (Caelius Sabinus) ; D 21.1 . 26 ; D 21.1.32 ; Maecianus D 32.95 (Aristo); D 36.1.71; Scaevola 0 3.5.34.2 ; D 16.1.28pr; D 24.1.58pr-1-2; D 32.37.3; D 32.41.2-10; D 32.93.2 ; D 32.101. D 33.2 .27 ; D 33.5.21; D 33.7.20.3-5-9; D 33.7.27.1-4; D 34.2.18pr; D 34.4 .

31 pr ; D 41.9.3; Papinien D 6.1.65.1 ; D 21.1.54; C 31.77 .17 ; D 33.4.8; D 34.4.24.1; D 36.1.60.6; D 39.5.31.2 ; D 40.4.48 (Servius) ;D 40.8.8 (constitution de Marc-Aurèle); Tryphoninus D 18.7.10 (Scaevola) ; D 34.1.15.1 (Scaevola) ; Callistrate D 21.2.72 ; Paul D 3.6.7.2 ; D 5.3.40pr (Proculus, Cassius); D 10.3.31; D 14.2.10pr (Labéon) ; D 14.3.17pr ; D 17.2.65.5 (Labéon, Proculus) ; D 18.1.56 ; D 18.7.9; 
visant l'esclave objet de fait juridique (17). Par ailleurs, seuls 2

textes donnent à mancipium la signification d'agent d'acte juridique (18). Encore s'agit-il, conme on l'a vu à propos de homo, de textes oul cette signification n'est pas dissocife de celle d'esclave objet. In outre, on ne possède que 3 textes ou mancipinon est utilise a propos d'un esclave agent delictuel (19). Entin, la rocation de ce terme desigmer 1'eschve objet ressort, comwe pour hamo, du fait qu'il peut apparaftre dans un ame texte que servus, ce mot visant par opposition un esclave agent d'acte juridique $(20)$.

Les termes dfgignant 1 'home esclave ayant Ett examints, il convient de passer l'analyse de ceux qui indiquent la présence d'une femme esclave.

D 19.1.43 (Julien,U1pien); D 20.1.29.1; D 20.2.9; D 21.1.43.6-7; D 21.1. S6 (Marcellus); D 21.1.37pr ; D 25.1.12; D 31.86pr; D 32.78.2-3; D 32.92pr ; 032.99pr;D33.7.18.9-11-13 (Scaev.);D33.7.19pr;D33.7.22.1;D40.12.38.1 (constitucion de Marc-Aurele); D 41.4.2.7 (Trebatius); D 49.14.45.3 ; D 50.16.79.1 (Fulciaius); Ulpien D 5.3.20pr ; D 7.1.15.1-2; D 14.4.5.14; D 17.1.8.4 (Julien) ; D 20.1.6 ; D 21.1.1.1-6; D 21.1.19.6; D 21.1.21.1 (Pomponius) ; D 21.1 .23pr (Pomponius)-9; D 21.1.31.10-11-19-20-21-23; D 21.1.33pr (Pouponius) D 21.1.35;D 21.1.37; D 21.1.38.2-3-10;021.1.59pr-1; D 21.1.63; D 21.2 4pr ; D 23.3.10.5; D 26.7.5.9; D 27.2.3.2; D 27.3.13; D 32.49pr;D 32.68.3 ; D 32.73.4; D $33.4 .1 .6 ;$ D 33.7.12.35 (Neratius) -37 (Papinien) 46 (Fapinien):; D 42.73 .4 ; D 33.4 .1 .6 ; D 33.7.12.35 (Neratius) -37 (Papinien) 46 (Fapinien):; D $40.9 .12 .3-4$; D 42.6 .1 .12 ; D 48.5 .28 .6 (rescrit d'Hadrien) ; Marcien D 39.4.
16.2 (rescrit de SEveare et Caracalla)-3-9 (rescrit d'Antonin le pieux); D 48. 5.3pr (constitution de Sévère et Caracalia); Modestin D 20.1.26.2; D 31.34pr ; D 44.3.3.

17- Pomponius D 21.2.34pr ; Ulpien D 1.12.1.8 (SÉvère) ; D 3.2.4.2-3 (Pomponius); D 19.1.13.5 (Julien); D 21.1.1.1; D 21.1.23pr (Pomponius); D 21.1.31.11; D 47.10.25; Marcien D 48.15.3pr.

18- Gaius D 21.1.18pr ; Marcien D 39.4.16.2 (rescrit de Sêvère et Caracalla), tous deux par allusion au pécule.

19- Papinien D 21.1.54; Vlpien D 21.1.1.1; D 21.1.23pr (Pomponius). Ne rentrent dans aucune rubrique D 1.5.4.3 Florentinus (citê supra n15) et D 50.16.210 Marcien (natus ex mancipiis urbanis).

20- Africain D 21.1.51pr : Cum mancipium morbosum vel vitiosum servus emat ; Paul D 21.1.57pr : Si servru mancipivon emit.

\section{2 - les termes servant à désigner la pemme esclave.}

Le terme le plus courant est ancilla, mais on peut aussi relever serva. On remarque enfin d'autres termes, moins spécifiques, qui visent dans certaine cas la femme esclave.

$$
\text { - ancilla }
$$

Par rapport au nombre total de paragraphes ou de citations dans lesquels ancilla apparait lie à un acte ou à un fait juridique, pour chaque periode, on obtient : gncilla coune asent d'acte juridique (21).

\section{- anelyse des pararraphes}

$$
\begin{aligned}
& \text { I } \\
& \text { II } \\
& \text { III } \\
& \text { IV } \\
& \text { V }
\end{aligned}
$$

II
III
IV
V

$$
\begin{aligned}
& - \\
& 1 / 1 \\
& 4 / 18 \\
& 2 / 5 \\
& 10 / 51
\end{aligned}
$$

TOTAL

$17 / 75$

- analyse des citations

$\begin{array}{llll}\text { I } & 0 / 5 & = & - \\ \text { II } & 1 / 4 & = & 25 \% \\ \text { III } & 1 / 8 & = & 12,5 \% \\ \text { IV } & 0 / 3 & = & - \\ \text { V } & - & = & - \\ \text { TOTAL } & 2 / 20 & = & 10 \%\end{array}$

ancilla comme objet d'acte juridique (22).
21- Proculus D 23.3.67; Julien D 30.84.10 ; Pomponius D 35.1.58(Proculus); Gaius D 15.1.21pr (Julien); D 16.1.13pr; Scaevola D 31.88.13; D 32.38.1; Paul D 15.4.2.1; D 29.1.40.2; D 31.83 ; D 41.2.1.12; U1pien D 3.5.13; D 13.6.3.4; D 14.4 .5 .2 ; D 21.1 .23 .4 ; D 27.6.11.2 ; D 37.10.3.5.

22- Tavolenus D 47.2.75; Celsus D 23.3.58.1; D 30.63 (Servius) ; Julien D 30.84.10; D 41.3.33pr ; D 43.33.1pr; Pomponius D 24.1.31.1 (operae) : D 30.45pr (Ofilius, Aristo, Neratius) ; D 41.10.4pr ; D 43.26.10; Gaius
D6.1.20;D7.8.13(Lab.) (operae);v2U.4.il.3;041.3.36.i;Venul.D42.8.25.4;Scaevola 


\section{- analyse des paragraphez}

\begin{tabular}{|c|c|c|c|c|c|c|c|}
\hline I & - & $=$ & - & I & - & $=$ & - \\
\hline II & - & $=$ & - & II & - & $=$ & - \\
\hline III & $1 / 8$ & $=$ & $12,5 \%$ & $\operatorname{trI}$ & $7 / 8$ & $=$ & $87,5 x$ \\
\hline IV & - & $=$ & - & $\Gamma V$ & - & $=$ & - \\
\hline $\mathrm{v}$ & $2 / 18$ & $=$ & $11 \%$ & $v$ & $16 / 18$ & $=$ & $89 x$ \\
\hline TOTAL & $3 / 26$ & $=$ & $12 \%$ & TOTAL & $23 / 26$ & $=$ & $88 \%$ \\
\hline \multicolumn{8}{|c|}{ - analyse des citations } \\
\hline I & $0 / 1$ & $=$ & - & I & $1 / 1$ & $=$ & $100 \%$ \\
\hline II & $0 / 2$ & $=$ & - & II & $2 / 2$ & $=$ & $100 x$ \\
\hline III & $0 / 1$ & $=$ & - & III & $1 / 1$ & $=$ & $100 \%$ \\
\hline IV & $0 / 2$ & $=$ & - & IV & $2 / 2$ & $=$ & $100 \%$ \\
\hline v & - & $=$ & - & $\mathrm{v}$ & - & $=$ & - \\
\hline TOTAL & $0 / 6$ & $=$ & - & TOTAL & $6 / 6$ & $=$ & $100 \%$ \\
\hline
\end{tabular}

D 33.5.21; D 40.5.41.5 ; Papinien D 6.1.65.1; D 23.3.69.9; D 35.2.9.1 ; Paul D 4.4.48.1; D 18.1.56; D 21.2.42; D 22.1.14.1 (Neratius); D 24.1.28.1; D 30.62 ; D 32.60 .1 (Alfenus) ; D 33.7 .16 .2 (Alfenus) ; D 33.8.3 ; D 33.8 .15 (Alfenus) ; D 35.1.28.1 ; D 40.1.10 (constitution de Marc-Aurèle) ; D 40.1.23 ; D 40.5.40pr ; D 41.3.4.17; D 41.10.2 ; D 45.1.83.8 ; Ulpien D 5.3.20.3 ;

D 5.3.27pr ; D 7.8.12.6 (Labêon, Sabinus,octavenus) ; D 13.7.24.3 ; D 18.2.4.1 (Julien) ; D 21.1.31.2 ; D 23.2.45.3 ; D 24.1.19pr (Julien)-1 (Jul ien, Marcellus) D32.71;D32.73pr;D40.5.26.2 (rescrits d'Antonin et de Sévère et Caracalla); D 40.5.45pr-2 ; D 41.3.10.2 (Marcellus;Scaevola); D 44.2.7.1; D 47.2.48.5; Marcien D $37.14: 3$ (rescrit de Sêvère et Caracalla) ; Modestin D 20.1.26.2 ; D. 32.81pr ; D 50.16.101.3.

23- Africain D 47.2.61 ; Ulpien D 29.5.1.28 (rescrit d'Hadrien)-32.

24- Iavolenus D 47:2.75; Julien D 1.5.26; D 41.3.33pr ; D 41.4.9; D 41.4.10 ; Pomponius D 41.10.4pr (Trebatius) ; Africain D 47.2.61 ; Paul D 1.18 .21 ; D 18.1.56 ; D 40.8.7 (constitution de Sévère et Caracalia) ; D 41.3.4.15-16 (Sabinus, Cassius)-17; D 47.2.83.2 ; Ulpien D 1.6.2 (Hadrien); D6.2.11.2-5 (Pomponius);D13.7.24.3;D41.3.10.2(Marcel lus, Scaevola);D47.1.2.5; D 47.2.39; D 47.2.48.5; D 47.10.15.15. Ne rentrent dans aucune de ces catégories des textes concernant seulement la quaestio (Paul D 48.18.20 ; Ulpien (rescrit d'Hadrien) D 48.5.28.6) ; l'affranchissement ou le status de l'ancilLa (Julien D 40.5.47.4 ; Scaevola D 40.4.59pr ; D 40.5.41 pr ; Paul D 24.3.56; D 40.8.9; D 40.12.38:1; D 41.7.8 ; Ulpien D 4.1.6 (Julien) ; D 25.4.1.10 ; D 34.5.10.1; D 40.5.26.5 ; L.Rufinus D 23.2.51.1 ; Marcien D 40.5.53pr-1; D 40.5.55pr-1 (Marcellus) ; Modestin D 25.3.7; D 40.4.44;D 40.5.13; D 40. 9.21 (Julien); un problème de-naissance (Ulpien D 37.10.1.5 (Julien; rescrit d'Antonin le pieux) ; D 40.4.13.3 ; D 40.11.1 ; Marcien D 1.5.5.1-2-3;

D 40.15.1.4 (Marcellus); Modestin D 1.5.22);
Ce tableau montre que la résonance juridique d'anciiliz est, l...lyvalente. Néanmoins, elle n'est pas équivalente à celle de serius pour I'homme esclave. Dans la plupart des cas, ancilla désigne en eifet la femme ohjet de droit. Toujours d'un point de vue terminologique, il est intéressant de relever qu'ancilla intervient le plus souvent en compagnie d'un terme marquant la fonction reproductrice de la feme tel que partus, natus ou mater (25), ce qui ne laisse planer aucun dopte sur le rôle qui lui est dévolu. Il faut aussi remarquer que le terme mêmed'ancilla dénote une conception différente de l'esclave suivant son sexe : la fenme esclave est une servante (26). Ancilla vise la fonction de la feme esclave, serva sa condition juridique (2T).

\section{b - serva}

Peu de textes contiennent une référence au mot serva. Ce terme est cependant utilisé par les juristes de toutes les périodes prises en considération (28). Sa résonance juridique est particulièrement équilibrée. Serva est agent d'acte juridique dans quatre paragraphes (29), objet dans trois $(30)$ et

de même que les textes traitant au premier plan du partus et où ancilla $n$ 'intervient en quelque sorte que comme appoint terminologique par la forme partus ancillae (Julien D 21.2.8; D 24.3.31.4; D 30.91.7; Pomponius D 15. 2.3 ; D 30.24pr ;Gaius D 20.1.15pr ; D 35.2.73pr ; Maecianus D 35.2.30pr ; Papinien D 22.1.4pr ; D 36.1.60.4; Tryphoninus D 15.1.57.2 ; D 28.2.28.3; Paul D 13.7.18.2 ; D 19.1.21pr; D 22.1.14pr; D 41.3.4.5; Ulpien D 33.8.; 8.8 (Julien) ; D 45.1.75.4; D 47.2.48.6); enfin des textes divers (Scaevola D 34.2 .13 -vêtements-; Ulpien D 11.7.31.1'-sépulture- ; D 50.16.40.1 -dêfinition-).

25- Par exemple, sur un total de 58 textes traitant d'une ancilla objet d'acte juridique, 32 concernent directement ou indirectement la maternité. 26- WACKERNAGEL, GLOTTA II (1909) p7.

27- A.ERNOUT et A.MEILLET, Dictionnaire étymologique. cit. p620.

28- V.n29 à 32.

29- Paul D 29.1.40.2; Ulpien D 14.1.1.21; D 23.3.39pr ; D 32.49.7.

30- Julien D 32.62 (Servius) ; Scaevola D 33.7.27.1 (conservae); Papinien D 18.7.6pr (Sabinus). 
2port de ait juridique dans cinq (31), objet dans huit (32).

Mais ancilla et serva ne sont pas les seuls termes servant à

désigner la remme esclave.

c - autres termes servent à désigner la femme esclave.

Excepté contubernalis qui réfère à l'union servile, aucun de ces termes n'est propre à l'esclavage. Ainsi trouve-t-on uxor, normalement réservé à l'épouse libre, mais dont l'emploi à propos d'une esclave cadre avec une certaine reconnaissance des liens. familiaux serviles (33). On remarque aussi mulier, qui est à ancilla ce qu'homo est à servus, puis mater, concubina. La valeur juridique de ces dénominations est dans la ligaée de celle des termes spécifiques à la ferme esclave (34).

Ces termes sont, comme ancilla, fréquemment en relation avec ceux qui désignent l'enfant esclave.

31- Callistrate D 47.21.3.1 (empereur Nerva); D 48.15.6.2; U1pien D 13.3 .5 .3 ; D 29.5.3.12; D 40.7.6pr.

32- Gaius D 9.2.22pr ; Papinien D 18.7.6pr (Sabinus); D 48.5.6pr ; Callistrate D 48.15.6.2; U1pien D 9.2.3; D 11.3.1pr; D 29.5.3.12; D 47.10.25. Ne sont intégrables dans aucune rubrique les textes concernant seulement le status (Papinien D 24.3.42.1; Paul D 41.7.8; U1pien D 38.16.1.1 (rescrits de MarcAuréle et Verus et de Caracalla); Modestin $D$ 25.3.7) ainsi que les textes ou il Auréle et verus et de Caracalla) ; Modestin $D$ 25.3.7) ainsi que les textes ou il est question d'une serva pcenae sans que 1 on sache si celle-ci est au départ une
esclave (Ulpien D $48.19 .8 .8 ; \mathrm{D} 48.20 .5 \mathrm{pr} ; \mathrm{D} 38.17 .23$ ), contrairement a D 40.7 . $6 \mathrm{pr}$ ( $S i$ statulibera serva poenae facta sit).

33- Des inscriptions mentionnent à propos des unions serviles non seulement uxor, mais aussi coniux, maritus, Cf CIL, VI, $8409 \mathrm{~A}, 4847,8819,8827$, 22425; XI, 4294 .

34- Si on classe ces termes par rubriques, on obtient : objet d'acte juridique, Pomponius D 33.7.15.2 (muiier) ; D 40.13.3 (melier); Venuleius D 41.1.66 (mulier) ; D 42.8.25.5 (Proculus) (milier); Scaevola D 32.41.2 (contubernalis) -5 (contubernalis) ; D 33.7.20.1 (contubernalis) 4 (uxor); D 34.1.20pr (contubernalis) ; Papinien D 31.77 .17 (mater) ; D 31.73 (mater) ; D 41.3.44.2 (mulier) ; Tryphoninus D 49.15.12.18 (mater); Paul D 42.5.38pr (concubina) (par a contrario); Ulpien D 6.2.11.3 (Julien) (mater)-4 (Julien) (mater) ; D 10.4.9.7 (Sabinus, Pomponius) (mulier) ; D 18.1.11.1 (mulier) ; D 19.1.11.5 (mulier) ; D 20.1.8 (concubina) (par a contrario); D 21.1.14pr (Sabinus) (mulier)-1 (mulier)-2 (puerpera)-7 (mulier) ; 021.1 .31 .6

(Pomponius) (mater); B 33.7.12.5 (Trebatius) (mulier)-7 (uxor)-33 ruxor

\section{3 - Les termes servant à désigner l'enfant esclave}

Ces termes ont été présentés lors de l'étude consacrée à la reproduction naturelle du groupe servile (35). Partus est toujours employé dans le sens d'objet de droit, qu'il s'agisse d'un acte (36)ou d'un fait juridique(37):

contubernalis) ; Modestin D 32.81.1 (mulier) ; agent d'acte juridique, Africain D 35.1 .31 (uxor, mulier) ; Marcellus D 23.3.59.2 (mulier); Scaevola D 34.1.20.1 (contubermalis) (legs d'aliments); Paul D 35.1.81pr (contubernalis); objet de fait juridique, Ulpien D 3.2 .24 (mulier) ; D 6.2. 11.3-(Julien) (mater)-4 (Julien) (mater); $\mathrm{D}$ 9.2.9pr (Labéon) (mulier); D 9.2.27.22 (Brutus) (mulier) ; aucun texte sur agent de fait juridique. He D 9.2 .27 .22 (Brutus) (mulier) ; aucun texte sur agent de fait juridique
rentrent dans aucune de ces rubriques les textes concernant la quaestio (Ulpien D 48.19.13 (mulier) ) ; I'affranchissement ou le status de la mère ou de L'enfant (Celsus D 40.2.19 (praegnas); Gaius D 38.8.2 (mater); D 40.7.31.1 (Julien) (contubermatis) ; Maecianus D 40.5.54 (mater); Scaevola D 40.4.60 (mater); D 40.5.41.15 (contubermalis) ; Paul D 23.2.14.2 (mater)3 (mater,uxor) ; Ulpien D 38.16.3.12(rescrit d'Antonin le pieux) (mater) ; D 38.17.1.3 (mater) ; D 40.2.13 (mulier, uxor) ; D 40.2.20.2 (uxor) ;

D 40.5.24.6 (rescrit d'Antonin le pieux) (damnate in metallum) ; D 40.5.26.1 (mater)-3 (rescrit de Sévère et Caracalla) (mater) ; D 40.7.6.1 (mater) ;

Marcien 0 40.5.51.12 (uror) ) ; les textes qui traitent de 1 'enfant au premier plan, le terme désignant la mère n'apparaissant qu'accessoirement (Papinien D 22.1.8 (mulier); Paul D 22.1.10 (mater) ; Ulpien D 21.1.17.5 (Vivianus) (mater) ). Il faut enfin signaler deux textes dans lesquels il y a doute sur le status de la mère (Julien D 37.10 .7 .3 (mater) et Paul (décret d'Hadrien) D 40.12 .23 .2 (mater)).

35- V. supra n158 et s., $1^{\circ}$ partie.

36- Proculus D 31.48pr ; Iavolenus D 24.3.66.3 (Labéon) ; Celsus D 23.3.58.1 ; D 30.63 (Servius); Julien D 12.4.7.1; D 21.2.8; D 24.3.31.4; D 30.82.4; D 30.84 .10 ; D 30.91.7; D 41.3.33pr ; D 41.4.9; D 41.4.10; Pomponius D $15.2 .3 ;$ D $18.1 .8 \mathrm{pr} ; \mathrm{D} 18.1 .31 ; 023.3 .18$ (Labéón): Gaius $\mathrm{D} 6.1 .20 ; \mathrm{D} 20$. 1.15pr (Julien); D 20.4.11.3; D 22.1.28.1; D 35.2.73pr; D $39.5 .11 ; \mathrm{D} 41.3$.

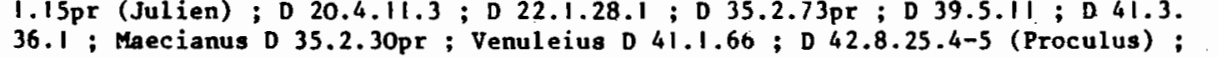

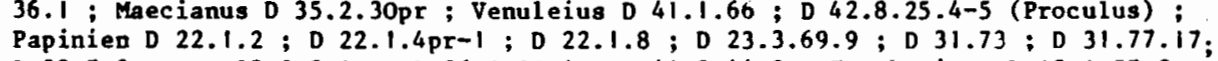
D 33.7.3pr : D 35.2.9.1; D 36.1.60.4; D 41.3.44.2 ; Tryphoninus D 15.1.57.2 : D 49.15.12.18; Paul D 6.1.16pr; D 10.2.11; D 12.4.12 ; D 12.6.65.5; D 13.7. 18.2 ; D 19.1.21 pr ; D 21.2.42; D 22.1.10; D 22.1.14pr-1 (Neratius);

D 24.1.28.5 (Julien, Pomponius) ; D 35.2.24.1; D 41.3.4.5-16 (Sabinus,Cassius) ; D 41.10.2 ; D 45.1.83.8; Ulpien D 3.5.3.6; D 4.2.12pr; D 5.3.20.3; D 5.3.27pr D 6.1.17.1 (Julien); D 6.2.11.2-3 (Julien) -4 (Julien)-5 (Pomponius);

D 7.1.68pr (Brutus); D 10.3.6.4 (Sabinus, Atilicinus); D 10.4.9.7 (Sabinus, Porponius) ; D 12.2.11.1; D 16.3.1.24; D 18.2.4.1 (Julien)-2 (Juliea); D 21.1.31.2-6 (Pomponius) $-24 ; D$ 33.8.8.8 (Julien); D 36.1.23.3 (Neratius) D 36.4 .58 ; D 41.3.10.2 (Marcellus,Scaevola); D 43.8.10.21 ; D 44.2.7.1-3; D $47.2 .48 .5-6$.

37- Africain D 47.2.61; U1pien D 47.2.48.5-6; D 47.4.1.11: D 50.16.26 (Scaevola) (au deuxième degré : vol de la mère). Ne sont intégrables dans aucune 
de même qu'infars(38). Filius (39), impubes (140), natus(41),

de ces rubriques, Tryphoninus D 1.5.15; Ulpien D 40.5.26.3 (rescrit de Sévère et Caracalla) -5 ; D 40.7.6pr ; Marcien D 40.5.53pr.

38- Infare signifie objet d'acte juridique chez Pomponius D 7.1 .55 ; Paul D 6.2.12.5; D 13.1.13; U1pien D 7.1.12.3 (Pomponius) ; D 33.7.12.7; D 40.5.26.2; D 44.4.4.9. Il signifie objet de fait juridique chez Celsus D 47.2.68.2; Paul D 13.1.13 ; Ulpien D 9.2.23.7. N'est pas intégrable D 40.1 .25 de Gaius qui ne concerne que 1 'affranchissement.

39- Filius employé pour l'enfant esclave signifie agent d'acte juridique chez Scaevola 0 36.1.80.2 ; objet d'acte juridique chez Scaevola D 32.37.7; D 32.41.2-5; D 33.7.20.1-4; D 40.5.41.5 ; Papinien D 17.1.54pr; Paul D 35.2.63pr ; Ulpien D 20.1.8; D 21.1.35; D 32.68.1; D 36.1.11.2 (rescrit d'Antonin le pieux) (filia); objet de fait juridique chez Paul D 9.2.33pr (Pedius). Ne rentrent pas dans ces rubriques Julien $D$ 42.8.17.1; Gaius $D 40.4$. 24 ; Maecianus D 40.5.54; Tryphoninus D 31.88 .12 (Scaevola) (filia); Paul D 19.5.5pr ; D 23.2.14.2 (filia) ; D 40.5.39.1; U1pien D 40.2.13 (filius,filia) ; D 40.2.20.3 (Marcellus) ; D 40.4.13.3; D 40.12.3pr, qui concernent af franchissemt ou statut, ni les textes suivants : Iavolenus D 40.13.23.2; Ulpien D 1.7.46; D 34.5.10.1 (filia); D 36.1.18.4 (Papinien); D $37.15 .1 .1 ; 040.2 .11$ (filius, filia).

40- Impubes est employê dans le sens d'agent d'acte juridique chez Gaius D 29.2.57.2 (on a servus); Venuleius D 40.12.44.2; Papinien D 26.5.13pr (rescrit d'Hadrien); Paul D 41.2.1.13 (associé à serrus); dans le sens (rescrit d'Hadrien); Paul D 4l.2. Pomponius). Ne sont pas intêgrables Maecianus D 29.5.14 (/servus ; (puer); U1pien D 29.5.1.32 (/ancilla)-33, qui concernent la quaestio, ainsi que Papinien D 26.5.13.1 -affranchissement-.

41- Natus est usitê pour agent d'acte juridique chez Tryphoninus D 28.2.28.3; pour objet d'acte juridique chez Julien $D 23.4 .21$; $D$ 36.2.16pr ; D 43.33.1pr ; Pomponius D 35.1.1.3; D 41.10.4pr (Trebatius); 043.26 .10 ; Gaius D 20.4.11.2 ; Scaevola D 32.37 .3 (adgnata) ; D 32.41.10 (adgnata); D 33.5.21 (adgnata) ; D 33.7.28 (adgnata) ; Papinien D 36.2.25.1 ; Paul D 7.7.1; D 12.6.15pr ; D 20.1.29.1; D 32.99.3; D 33.8.3; Ulpien D 21.1.1.1; D 33.7.12.33; D 40.5.24.6 (rescrit d'Antonin le pieux); D 43.32.1pr; 045. 1.75.4; $D$ 50.15.4.9; Modestin D 20.1.26.2. N'entrent pas dans ces rubriques Pomponius D 40.13.3 (nascuntur) ; Scaevola D 40.4.60; Paul D 41.7.8; D 50.2.9pr (Sêvère): Ulpiend 38.16.1.1 (rescrits de Marc-Aurèle et Verus et de Caracalla); D 38.16.3.12 (rescrit d'Antonin le pieux); D 37.10.1.5 (editus); D 38.17.1.3; ; D 40.11 .1 ; Marcien D 40.5.53.1 ; D 40.11.2; 040.15 .1 .4 (Marcelius); Modestin D 40.5.13, qui visent affranchissement ou status; on ne peut pas non plus intégrer Paul D 45.1.75.4; Ulpien D 11.4.1.5 (Labéon); Marcien D 50.16. 210 puer (42) ont par contre une résonance jurisique diversifiée : ils peuvent signifier esclave agent ou objet de droit. Quant aux autres termes, ils n'apparaissent que très peu et visent essentiellement l'esclave objet de droit (43).

A côté de ces termes qui désignent l'homme, l'enfant ou la femne esclave, il en est qui ne leur sont nullement spécifiques, mais le contexte permet chaque fois de savoir qu'on a affaire à un esclave.

\section{4 - autres indications terminologiques de la présence} d'un esclave

Il est possible que le seul indicateur pouvant. être relevé dans un texte soit un nom de métier (44) ou que la présence d'un esclave soit signalée

42- Puer est employé dans le sens d'objet d'acte juridique chez Marcellus D 37.15.3; Scaevola D 32.41.4; Paul D 6.1.6; D 24.1.28.1; D 24.1.38pr (Alfenus) ; D 35.1.38.1 ; D 6.1.27.5 (Proculus) ; D 50.16.79.1 (Fulcinius) ; Ulpien D 18.1.11.1; D 19.5.13.1 (Julien) ; Modestin D 32.81pr-1 ; dans le sens d'agent d'acte juridique chez Marcellus D 37.15 .3 ; dans le sens d'objet de fait juridique chez Alfenus (chez auteur anonyme) D 9.2.52.2 et chez Ulpien D 9.2.7.7 (Celsus) ; D 9.2.27.28 (Vivianus); dans le sens d'agent de fait juridique chez U1pien D 21.1.17.5 (Vivianus). Un seu1 texte ne rentre pas dans ces rubriques, il s'agit de D 29.5 .14 Maecianus qui concerne 1a quaestio. Quant au terme puella, il intervient trois. fois paur viser une esclave objet d'acte juridique : Papinien D 40.1.20.2 ; Ulpien D 32.49pr ; Modestin D 32. $81 \mathrm{pr}$.

43- Ainsi servulus dans le sens d'objet de fait juridique chez Al fenus (chez auteur anonyme) D 9.2.52.4; D 40.1.7. Suboles dans le sens d'objet d'acte juridique chez Ulpien $D$ 23.3.10.2 ; iuvenes, idem chez Marcellus D 32.69.1 ; novitius, idem chez Venuleius D 21.1.65.2 (Caelius Sabinus) U1pien D 21.1.37; Pomponius D 3.5.10 (Proculus).

44- Un nom de métier désigne un esclave objet d'acte juridique chez Pomponius D 33.7.15pr (Servius) (pistores, institores, focaria); Africain D 21 . $1.34 \mathrm{pr}$ (comoedi, chorus)-1 (comoedos) ; Gaius 06.1 .28 (pictorem, librarium); D 21.1.18.1 (cocum) ; Scaevola D 33.7.3 (institores); D 33.7. 20pr (vilicis) 4 (actor) ; Papinien D 32.91pr (actor) ; Paul D 32.60 .3 (Alfenus) (topiarium, saltuarium, opilionem) ; D 32.61' (textoribus, ostiarium) ; 0 32.66 (phasianarii, pastores) ; D 32.99.1 (venatores, aucupes)-2 (muliones); 0 33.7.13pr (Neratius) (institor)-1 (Neratius) (balneatorem) ; 033.7 .14 (fornacator) ; D 33.7.18.1 (pistores) -4 (Scaevola) (vilicus)-5 (rusticis operariis)-6 (bubulco)-7 (putatoribus)-8 (pastores,foseores)-10 (Cassius) (medicus); U1pien D 7.4.12.1 (histrio); o 33.7.8.1 (atrienses, scoparii, topiarii, saltuarii, pastores) ; D 33.7.12pr (Servius) (par cultorum)-4 (Labéon, Neratius) (saltuarium) -6 (Servius, Namusa) (lanificae, tonscres, fullones, focariae)-9 (cellarium, ostiarium, mulionem)-12 (venatores, vestigatcres)-13 (aucupes) 
Dar un terme comme venalis ou venaticius (i5). Mais beaucoup plus intéressante est la pratique des juristes qui consiste à désigner l'esclave par un terme à vocation délictuelle tel que fur ou fugitivus (46). Dans ce dernier

-38 (Papinien) (actorem) ; Marcien D 32.65.1 (Lecticarius, cocus)-3 (omatricibus) (Celsus); D 33.7.17.2 (balneator, topiarii, saltuarius, institor); Modestin D 40.5.12pr (rescrit de Caracalla) (tragoedos). Esclave agent d'acte juridique chez Pomponius D 33.7.15pr (Servius) (institores); Scaevola D 33.7.7 (institores) ; D 33.7.20.4 (actor); D 34.1.18.3 (actcres) ; D 40.7.40.5 (vilicis) ; Papinien D 32.91 pr (actor); D 33.1 .103 (actor) Paul D 14.3.17.3 (Pomponius) (institor) ; D 26.7.24pr (actor); D 32.97 (Caracalla) (actori) ; D 33.7.13pr (Neratius) (institor); D 33.7.18.4 (Scaevola) (vilicus) ; D 46.3.62 (dispensator); Ulpien D 14.1.1.20 (Pomponius) (institor, exercitor)-23 (Julien) (exercitor); D 33.7.12.38 (Papinien) (actor); Marcien D 33.7.17.2 (institor). Esclave objet de fait juridique chez Paul D 9.2.22.1 (comoedis, symphoniacis). Esclave agent de fait juridique chez Paul D 1.15.3.5 (capsarios).

45- Ces termes visent 1 'esclave objet de vente : Pomponius D 6.1.29 (venalem) ; J1pien D 21.1.17.2 (Labéon, Caelius Sabinus) (venales) ; D 28.8.5.1 (venalicia); de même que venaliciarius, marchand d'esclaves : Paul D 21.1 .44 1; U1pien D 9.2.27.24 (Vivianus).

46- Le terme à dénotation délictuelle peut ne désigner que l'esclave agent de fait juridique : Gaius D 40.12.11 (par fugae) ; Tryphoninus D 50.16.225 (fugitivus, fur) ; Callistrate D \$1.4.2 (fugitiorus) ; Paul D 11.4.4 (fugitivus) ; D 33.8.9.2 (par fugerit); 0 40.7.4.8 (Cassius) (in fuga); Ulpien D 1.15 .5 (rescrit de Sévère et Caracal1a) (fugitiorus) ; D 9.4.21.3 (in fuga); D 11.4 .1 .1 (fugitivus)-2 (epistula de Marc-Aurèle et Commode) (fugitivus)-3 (fugitivus) -5 (Labéon) (fugitivus, fugitiva)-8 (fugitivus) ; D 11.4 .3

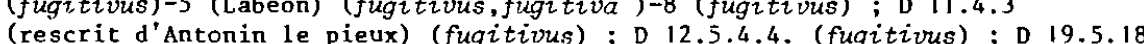
(rescrit d Antonin le pieux) (fugitivus); D 12.5.4.4. (fugitivus) ; D 19.5.1 (fugitivus) ; D 47.2.36.2 (Sabinus, Pomponius) (fugitivus); D 47.10.22 (fugitivus); Modestin D 48.3.14.7 (Venuleius) (fugitivus). Mais il peut avoir une résonance supplêmentaire : objet d'acte juridique chez Pomponius D 21.1.48.4 (par fuga) ; D 21.1.64.2 (Labéon) (fugitivrus); D 24.1.31.5 (fugitivus) ; Africain D 47.2.62.3 (fur) ; Gaius D 18.1.35.3 (fugitivus); D 41.2.15 (fugitivus) ; Papinien D 21.1.55 (fugitivus) ; Paul D 21.1.43.1 (fugitivus)-2 (fugitivus) ; Ulpien D 19.1.11.7 (Neratius) ; D 19.1.13.1 (fur, fugitivus) -2 (par fugerent) $\rightarrow 3$ (fur) ; D 21.1.17pr (0filius) (fugitivus)-1 (Caelius Sabi nus) (fugitivus)-2 (Cassius) (fugitivus)-3 (vivianus) (fugitivus) - 4 (Proculus) (fugitivus)-5 (Vivianus) (fugitivus)-9 (Caelius Sabinus) (fugitivus)-10 (Caelius Sabinus) (par fugisse)-12 (Labéon,Caelius Sabinus) tivus)-10 (Caelius Sabinus) (par fugisse) -12 (Labéon,Caelius Sabinus)
(fugitiorus)-13 (Caelius Sabinus) (fugitivus)-14 (Labéon) (fugitivus) : (fugitivus)-13 (Caelius Sabinus) (fugitivus)-14 (Labéon) (fugitivus) ;
D 21.1.19.1 (fur) ; D 21.2.31 (fur) ; D 21.2.32pr (fugitivus)-1 (Julien) (fugitivus) ; D 41.2.13pr (fugitivus) ; D 47.2.17.1 (fur) ; D 47.8.2.18 (fugitivus) ; agent d'acte juridique chez Julien D 46.3.34.5 (fugitions) ; Gaius D 41.2.15 (fugitivus) ; Paul D 15.1.48pr (fugitivus) ; Ulpien D 47.8.2.25 (fugitivus) ; objet de fait juridique chez Gaius D 9.2.4.1 (fur) ; Callistrate D 48.15.6.1 (rescrit d'Hadrien) (fugitivus) ; Paul D 15.1.48pr (fugitious) Ulpien D 9.2.5pr (fur) ; D 9.2.13pr (fugitivus); D 11.4.1pr (fugitivus); D 47.2.52.15 (fugitivus) ; D 47.8.2.18 (fugitivus); Marcien D 47.2.63 (fugitivus) (pris par extension); Modestin D 48.15.5.

cas, I'esclave est bien entendi envisagé comme un agent de rait jüidizue mais le terme peut aussi avoir une résonance suppiémentaire. Quoi qu'il en soit, la désignation de l'esclave par fur ou fugitivus dénote de la mentalité esclavagiste du langage juridique.

Si les termes désignant l'esclave individueilement sont variés, un seul vocable permet $d$ 'englober un groupe d'esclaves. Il s'agit de familia.

\section{B - La désignation collective par familia ( 46 bis)}

Par rapport au nombre total de paragraphes ou de citations dans lesquels familia apparaît lié à un acte ou à un fait juridique, pour chaque période, on obtient :

familia comme agent d'acte juridique (47).

- analyse des paranraphes

$\begin{array}{lllr}\text { I } & - & = & - \\ \text { II } & 1 / 1 & = & 100 \\ \text { III } & 1 / 5 & = & 20 \\ \text { IV } & 0 / 2 & = & - \\ \text { V } & 0 / 17= & \\ \text { TOTAL } & 2 / 25= & \end{array}$

TOTAL

- analyse des citations

$\begin{array}{lllllllr}\text { I } & 0 / 1 & = & - & \text { I } & 1 / 1 & = & 100 \% \\ \text { II } & 0 / 2 & = & - & \text { II } & 2 / 2 & = & 100 \% \\ \text { III } & - & = & - & \text { III } & - & = & - \\ \text { IV } & - & = & - & \text { IV } & - & = & - \\ \text { V } & 0 / 1 & = & - & \text { V } & 1 / 1 & = & 100 \% \\ \text { TOTAL } & \text { O/L } & = & - & \text { TOTAL } & 4 / 4 & = & 100 \%\end{array}$

46 bis- Familia, rappelons-le, peut avoir une signification plus générale qu'ensemble des esclaves d'un même maître (cf. supra lère partie n. 240).

47- Proculus D 4.3.31 ; Gaius D 45.3.28.4

48- Iavolenus D 33.5.14; Celsus D 32.79pr ; Pomponius D 24.1.31.10; Gaius D 3.5.21; Marcellus D 47.6.5 ; Scaevola D 33.7.27.3 ; Papinien D 31.65pr ; Paul D 9.4 .9 ; D 32.78.1 ; D 33.5.7; D 33.5.13pr ; D 32.99pr (intégré en fonction du contexte) ; Ulpien D 4.3.9.1 (au deuxième degré : choix de l'esclave le meilleur de la familia) ; D 5.3.5pr ; D 29.5.1.15; D 33.7.12.3 (Labéon,Pegasus)-5 (Trebatius) -10 (intégré en fonction du contexte) -42 (Papinien); D 42.5.9.5; D 45.1. $29 \mathrm{pr} ; \mathrm{D}$ 47.6.3pr (au deuxième degré). 
- analyse des paragraphes

$\begin{array}{llll}\text { I } & - & = & - \\ \text { II } & - & = & - \\ \text { III } & 6 / 6 & = & 100 x \\ \text { IV } & 4 / 4 & = & 100 x \\ \text { V } & 44 / 47 & = & 04 \% \\ \text { TOTAL } & 54 / 57 & = & 95 \%\end{array}$

$\begin{array}{llll}\text { I } & - & = & - \\ \text { II } & - & = & - \\ \text { III } & 0 / 6 & = & - \\ \text { IV } & 0 / 4 & = & - \\ \text { V } & 3 / 47 & = & 6 x \\ \text { TOTAL } 3 / 57 & = & 5 \%\end{array}$

- analyse des citations

\begin{tabular}{|c|c|c|c|c|c|}
\hline & & $=\quad \bar{m}$. & I & & $=$ \\
\hline $\begin{array}{l}11 \\
\text { III }\end{array}$ & $\begin{array}{l}4 / 4 \\
5 / 5\end{array}$ & $\begin{array}{l}=100 \% \\
=100\end{array}$ & $\begin{array}{l}\text { II } \\
\text { III }\end{array}$ & $\begin{array}{l}0 / 4 \\
0 / 5\end{array}$ & $\begin{array}{l}= \\
=\end{array}$ \\
\hline IV & - & - & IV & - & $=$ \\
\hline $\mathbf{v}$ & - & $=$ & $\mathrm{v}$ & - & $=$ \\
\hline TOTAL & 9/9 & $100 \%$ & TOTAL & $0 / 9$ & $=$ \\
\hline
\end{tabular}

49- Julien D 8.5.18; D 47.6.4 ; Pomponius D 21.1.48pr ; Gaius D 9.2.32pr ; D 29.5.25pr ; D 39.4.13.2; Venuleius D 29.5.13; Marcellus D 29.5.16; D 47.6.5; Scaevola D 47.6.6 (Labéon); Papinien D 1.21.1pr; D 29.5.4 ; D 29.5.21pr ;

D 35.2.11.4; Paul D 2.1.9; D 9.4.9; D 9.4 .31 (Sabinus,Cassius, Jul ien, Pomponius); D 29.5.6.3 ; Ulpien D 21.1.1.1; D 21.1.25.1-4 (Pedius)-2 (intégrable par le contexte); D 21.1.31.9. (Pomponius)-11-15 (intëgrable par le contexte); D 29.5 .3$. 12-18-29; D 29.5.5.2 ; D 39.4.1pr-5 (intégrable par le contexte) ; D 39.4.12. 1-2 (intégrable par le contexte) ; 040.12 .7 .4 ; D 43.16.1 pr-11-12-15-16 (intégrable par le contexte)-17 (idem)-18 (idem)-19; D 43,16.3.11; D 47.6.1pr ; D 47.6.3pr ; D 47.8.2.14-16-19; D 47.8.4.15; D 47.9.3.7 (Labéon, Celsus) ; D 50.16.195.3 (intégrable par le contexte); Marcien D 48.8.3.4 ; Modestin D 29. 5.17; D 29.5.19.

50- Papinien D 47.2.81.2 (au deuxième degré : servus ex familia subreptus); Ulpien D 29.5.3.12 (idem) ; D 42.5.9.5. Ne sont intégrables dans aucune de ces rubriques les textes concernant seulement la quaestio (Ulpien D 29.5.1pr-24 ; D $30.50 .2 ; D 36.1 .3 .5 ; D 38.2 .37 .1$ (Julien) ) ou donnant simplement une définition (Pomponius D 50.16.166pr ; Ulpien D 50.16.40.2-3) ou enfin concernant des oroblèmes divers, en particulier nourriture et habillement (Iavolenus D 18,1.78.1 (Labêon) ; Neratius D 15.3.18; Africain D 40.7.15pr ; Papinien D 21.1.54; D $33.1 \cdot 10.3$; Paul D $11.5 .4 \mathrm{pr}$; D 19.1.21.3 ; D 33.9.4.2 ; Ulpien D 7.8.2.1 ; D 7.8.10.4 (Labéon); D 15.3.3.1 (Pomponius)-7-10; D 33.9.3pr (Sabinus)-6 (Q.Mucius, Servius)-8 (Ofilius) ;. D 34.2.23.2; D 42.5.9.3; Hermogénien D 35.2.38pr). Papinien parle d'une familia libertorum (D 31.77 .27 )
La quasi-absence de textes visant familia en tant qu'agent d'acte juridique eut nomale, la collectivitá des esclaves n'agissant pas. Le pourcentage ráalisé par la nériode II dans l'analyse des paragraphes a'est pas pertinent, ne reposant que sur un seul texte.

Mais la constatation la plus importante découle de ce que, dans la plupart des tèxtes, familia a le sens d'agent délictuel. Bien que les chiffres soient en l'espèce assez faibles, cet usage semble refléter une certaine inquiétude des maîtres dès qu'intervient une prise en considération numérique des esclaves. Cette inquiétude peut revêtir un aspect simplement patrimonial, le mantre devant payer la poena ou procéder à l'abandon noxal des esclaves coupables du délit(50 bis), mais elle peut même aller jusqu'à se muer en véritable peur lorsqu'il est question de violence et surtout $d$ 'assassinat du maître (51).

Certains des termes désignaıt l'esclave de manière impersonnelle ont une résonance juridique relativement équilibrée; c'est le cas de servus et dans une moindre mesure, ce deuxième terme $n$ ' apparaissant que très rarement, de serva. La résonance de la plupart est diversifiée, hormis pour partus qui ne signifiequ'objet de droit et pour mancipium dont la vocation essentielle est d'avoir la même signification. Quant au terme familia, il se distingue des autres par la crainte qu'il évoque. ( 52 ).

De nombreux textes fournissent aussi des noins propres d'esclaves.

50 bis- Très explicite sur ce point, Ulpien D 47.6.1 pr.

51- Violence : Ulpien D 39.4.1 pr-(5); D 43.16.1 pr-11-12-15-(17-18)-i9 ; D 43.16.3.11; D 47.8.2.14-16-19; D 47.8.4.15; D 50.16.195.3; Marcien D 48.8.3.4. Meurtre du maître : Venuleius D 29.5.13; Marcellus D 29.5.16; Papinien D 1.21.1 pr ; D 29.5.4; D 29.5.21 pr ; D 35.2.11.4; Ulpien D 29.5. 3.12-18-29; D 29.5.5.2; D 40.12.7.4; Modestin D 29.5.17. A propos du s.c. silanien, il faut aussi signaler Paul D 29.5.6.3 et Modestin D 29.5.19. Par ailleurs, Gaius (D 39.4.13.2) parle d'une tam factiosam familiam. 52- Signalons encore pour en terminer avec cette etude que 1 'esclave est parfois dégigné par persona (cf Gaius D 9.4.29 -peut concerner le fils de famille ou l'esclave, le contexte étant celui des actions noxales-; Paul D 30.5 (Trebatius, Labéon, Cassius) ; Ulpien D 21.1.35; D 24.3.21; D 40.9. 14pr). On trouve aussi deux fois vir (Ulpien D 33.7.12.5: Modestin D 32.81.1). 


\section{II - L'onomastique de l'esclavage}

Une simpléénumération des noms d'esclaves est insuffisante. Il paraît en revanche intéressant, conformément à l'analyse entreprise jusqu'à présent, de tenter de voir si certains noms semblent destinés à placer l'esclave à la tête d'un rapport juridique ou bien en font plutôt l'ob.jet.

Pour cela, il convient de présenter ces différents noms par rubriques juridiques .

- I'esclave objet d'acte juridique -

\begin{tabular}{|c|c|c|c|}
\hline $\begin{array}{l}\text { juriste auteur } \\
\text { du passage }\end{array}$ & juriste cité & nom & source \\
\hline Iavolenus & $\begin{array}{l}\text { Labéon } \\
\text { Tubero, Labéon } \\
\text { Cascellius, Labéon } \\
\text { Q.Mucius, Gallus, } \\
\text { Servius, Ofilius, } \\
\text { Labéon. } \\
\text { Sabinus }\end{array}$ & $\begin{array}{c}\text { Stichus } \\
\text { Stichus/Dama } \\
\text { Stichus } \\
\text { Flaccus/Philonicus } \\
\text { Stichus } \\
\text { Dama/Fros }\end{array}$ & $\begin{array}{l}\text { D } 32.29 .3 \\
\text { D } 32.29 .4 \\
\text { D } 32.100 \mathrm{pr} \\
\text { D } 34.5 .28 \\
\text { D } 40.7 .39 \mathrm{pr}\end{array}$ \\
\hline Celsus & & $\begin{array}{c}\text { Stichus } \\
\text { Stichus } \\
\text { Pamphila } \\
\text { Stichus } \\
\text { Stichus } \\
\text { Stichus/Pamphilus } \\
\text { Stichus }\end{array}$ & $\begin{array}{ll}\text { D } & 12 \cdot 4 \cdot 16 \\
\text { D } & 17 \cdot 1 \cdot 50.1 \\
\text { D } & 23 \cdot 3 \cdot 58.1 \\
\text { D } & 31.15 \\
\text { D } & 31.18 \\
\text { D } & 31.19 \\
\text { D } & 40.7 .23 \mathrm{pr}\end{array}$ \\
\hline Valens & & Stichus & D 35.1 .89 \\
\hline Julien & & $\begin{array}{c}\text { Stichus/Famphilus } \\
\text { Stichus/Pamphilus } \\
\text { Stichus } \\
\text { Pamphilus } \\
\text { Stichus } \\
\text { Stichus/Pamphilus } \\
\text { Stichus } \\
\text { Stichus } \\
\text { Stichus } \\
\text { Famphila } \\
\text { Stichus/Pamphilus } \\
\text { Stichus } \\
\text { Stichus } \\
\text { Stichus/Pamphilus } \\
\text { Stichus/Pamphilus }\end{array}$ & $\begin{array}{ll}\text { D } & 10.3 .25 \\
\text { D } & 12.6 .32 \mathrm{pr} \\
\text { D } & 12.6 .32 .3 \\
\text { D } 28.5 .38 \mathrm{pr} \\
\text { D } 30.6 \\
\text { D } 30.76 \\
\text { D } 30.81 .2 \\
\text { D } 30.81 .6 \\
\text { D } 30.82 \mathrm{pr} \\
\text { D } 30.82 .4 \\
\text { D } 30.82 .6 \\
\text { D } 30.84 .3 \\
\text { D } 30.84 .8 \\
\text { D } 30.84 .9 \\
\text { D } 30.84 .11\end{array}$ \\
\hline
\end{tabular}

\begin{tabular}{|c|c|c|c|}
\hline (Juzien suite) & Q.Mucius & $\begin{array}{c}\text { Stichus } \\
\text { Stichus } \\
\text { Stichus } \\
\text { Stichus/Dama } \\
\text { Stichus/Pamphilus } \\
\text { Stichus } \\
\text { Stichus } \\
\text { Stichus/Pamphilus } \\
\text { Stichus } \\
\text { Stichus } \\
\text { Eros } \\
\text { gtichus } \\
\text { Dion } \\
\text { Stichus/Dama/Eros } \\
\text { Stichus } \\
\text { Stichus/Pamphila } \\
\text { Stichus } \\
\text { Stichus } \\
\text { Stichus } \\
\text { Stichus/Pamphilus } \\
\text { Stichrs } \\
\text { Stichus/Pamphilus } \\
\text { Stichus/Pamphilus }\end{array}$ & $\begin{array}{ll}\text { D } & 30.85 .1 \\
\text { D } & 30.91 .2 \\
D & 30.01 .5 \\
\text { D } & 30.94 .2 \\
\text { D } & 30.97 \\
\text { D } & 30.99 \\
D & 30.101 \mathrm{pr} \\
\text { D } 33.5 .9 \mathrm{pr} \\
\text { D } 33.5 .9 .1 \\
\text { D } 33.5 .9 .2 \\
\text { D } 33.5 .11 \\
\text { D } 34.14 .11 \\
\text { D } 34.5 .13 .1 \\
\text { D } 34.5 .13 .2 \\
\text { D } 36.1 .26 .2 \\
\text { D } 36.2 .16 \mathrm{pr} \\
\text { D } 39.5 .2 .7 \\
\text { D } 40.4 .15 \\
\text { D } 40.5 .48 \\
\text { D } 40.9 .5 .2 \\
\text { D } 44.7 .18 \\
\text { D } 45.1 .54 \mathrm{pr} \\
\text { D } 46.3 .33 .1\end{array}$ \\
\hline Pomponius & $\begin{array}{l}\text { Neratius } \\
\text { Juzien } \\
\text { Labéon }\end{array}$ & $\begin{array}{l}\text { Stichus/Pamphilus } \\
\text { Stichus } \\
\text { Stichus } \\
\text { Stichus } \\
\text { Stichus/Pamphilus } \\
\text { Stichus } \\
\text { Stichus } \\
\text { Stichus } \\
\text { Arescusa (53) } \\
\text { Stichus } \\
\text { Stichus } \\
\text { Stichus/Pamphilus } \\
\text { Stichus/Pamphilus } \\
\text { Stichus } \\
\text { Stichus/Pamphilus } \\
\text { Stichus } \\
\text { Stichus/Pamphilus }\end{array}$ & $\begin{array}{ll}\text { D } & 12.6 .19 .3 \\
\text { D } & 30.13 \\
\text { D } & 30.36 .3 \\
\text { D } & 30.16 \\
\text { D } & 31.11 .1 \\
\text { D } & 32.85 \\
\text { D } & 33.1 .2 \\
\text { D } & 34.2 .10 \\
\text { D } & 35.1 .1 .3 \\
\text { D } & 36.1 .72 .1 \\
\text { D } 40.7 .30 \\
\text { D } 44.2 .21 .2 \\
\text { D } 45.1 .16 \mathrm{pr} \\
\text { D } 45.1 .33 \\
\text { D } 45.1 .112 \mathrm{pr} \\
\text { D } 45.2 .18 \\
\text { D } 46.4 .15\end{array}$ \\
\hline Africain & & $\begin{array}{l}\text { Pamphilus } \\
\text { Stichus } \\
\text { Eros } \\
\text { Stichus } \\
\text { Stichus } \\
\text { Stichus } \\
\text { Pamphilus } \\
\text { Pamphilus } \\
\text { Stichus }\end{array}$ & $\begin{array}{ll}\text { D } & 15.1 .38 .2 \\
\text { D } & 15.3 .17 .1 \\
\text { D } & 20.4 .9 \mathrm{pr} \\
\text { D } & 21.2 .45 .2 \\
\text { D } & 30.108 .4 \\
\text { D } & 30.108 .5 \\
\text { D } & 33.8 \cdot 16 \mathrm{pr} \\
\text { D } & 33.8 .15 .1 \\
\text { D } & 46.3 .38 .3\end{array}$ \\
\hline
\end{tabular}

53- Objet indirectement par natus. 


\begin{tabular}{|c|c|c|c|}
\hline Gaius & Julien & $\begin{array}{l}\text { Stichus } \\
\text { Stichus } \\
\text { Stichus } \\
\text { Stichus } \\
\text { Stichus } \\
\text { Stichus } \\
\text { Stichus }\end{array}$ & $\begin{array}{l}\text { D } 21.1 .32 \\
\text { D } 30.65 .1 \\
\text { D } 35.1 .17 \mathrm{pr} \\
\text { D } 35.1 .17 .1 \\
\text { D } 40.7 .37 \\
\text { D } 45.1 .74 \\
\text { D } 45.2 .15\end{array}$ \\
\hline Venule ius & & Stichus & D 45.1 .137 .4 \\
\hline Marcellus & & $\begin{array}{c}\text { Stichus } \\
\text { Stichus } \\
\text { Stichus } \\
\text { Stichus } \\
\text { Stichiss } \\
\\
\text { Stichus/ramphilus } \\
\text { Stichus } \\
\text { Stichus } \\
\text { Stichus/Pamphilus } \\
\text { Stichus } \\
\text { Stichus }\end{array}$ & $\begin{array}{l}\text { D } 9.2 .34 \\
\text { D } 30.83 \\
\text { D } 31.50 .1 \\
\text { D } 35.2 .56 .4 \\
\text { D } 40.7 .24 \\
\text { D } 46.1 .38 \mathrm{pr} \\
\text { D } 46.3 .67 \\
\text { D } 46.3 .72 .1 \\
\text { D } 46.3 .72 .4 \\
\text { D } 46.3 .72 .5 \\
\text { D } 46.8 .17\end{array}$ \\
\hline Scaevola & $\begin{array}{l}\text { Julien } \\
\text { Julien } \\
\text { Neratius }\end{array}$ & $\begin{array}{c}\text { Stichus } \\
\text { Stephanus } \\
\text { Stichus } \\
\text { Stichus } \\
\text { Stichus } \\
\text { Stichus } \\
\text { Stichus/Fros } \\
\text { Pamphilus } \\
\text { Stichus } \\
\text { Stichus } \\
\text { Stichus/Dama } \\
\text { Pamphila } \\
\text { Stichus } \\
\text { Stichus } \\
\text { Stichus }\end{array}$ & $\begin{array}{ll}\text { D } & 2.14 .54 \\
\text { D } & 10.2 .39 .4 \\
\text { D } & 20.1 .32 \\
\text { D } & 21.2 .69 .1 \\
\text { D } & 21.2 .69 .2 \\
\text { D } & 28.6 .48 .1 \\
\text { D } & 32.41 .2 \\
\text { D } & 33.5 .18 \\
\text { D } & 33.7 .20 .1 \\
\text { D } & 33.7 .20 .6 \\
\text { D } & 34.4 .31 \mathrm{pr} \\
\text { D } & 40.5 .41 .5 \\
\text { D } & 40.7 .40 .2 \\
\text { D } & 45.1 .122 .2 \\
\text { D } & 45.1 .127\end{array}$ \\
\hline Papinien & $\begin{array}{l}\text { Servius } \\
\text { Q.Mucius, Sabinus, } \\
\text { Pegasus. }\end{array}$ & \begin{tabular}{|} 
Stichus/Dama \\
Stichus \\
Damphila \\
Scorpus \\
Arethusa (5/4) \\
Pamphilus \\
Stichus \\
Pamphilus \\
\\
Stichus/Pamphilus
\end{tabular} & $\begin{array}{l}\text { D } 3 \cdot 3.66 \\
\text { D } 31.65 .2 \\
\text { D } 31.73 \\
\text { D } 33.2 .24 .1 \\
\text { D } 36.2 .25 .1 \\
\text { D } 40.4 .48 \\
\text { D } 40.5 .21 \\
\text { D } 45.1 .115 .2 \\
\text { D } 46.3 .95 \mathrm{pr}\end{array}$ \\
\hline
\end{tabular}

54- objet indirectement par partus.

\begin{tabular}{|c|c|c|c|}
\hline Tryphoninus & $\begin{array}{l}\text { Scaevola, const. } \\
\text { Hadrien et Marc- } \\
\text { Aurèle }\end{array}$ & Stichus/Parphila & D 18.7 .10 \\
\hline Paul & $\begin{array}{l}\text { Labéon } \\
\text { Julien, } \\
\text { Ulpien } \\
\text { rescrit d'Antonin } \\
\text { le pieux. } \\
\qquad \\
\text { Neratius } \\
\text { Sabinus, Proculus, } \\
\text { Marcellus }\end{array}$ & $\begin{array}{c}\text { Pamphila } \\
\text { Stichus } \\
\text { Stichus } \\
\text { Arethusa (55) } \\
\text { Stichus/Pamphilus } \\
\text { Stichus } \\
\text { Stichus/Pamphilus } \\
\text { Stichus } \\
\text { Stichus } \\
\text { Stichus } \\
\text { Stichus } \\
\text { Stichus } \\
\text { Stichus/Pamphilus } \\
\text { Stichus } \\
\text { Stichus/Pamphilus/Ares- } \\
\text { cusa } \\
\text { Stichus } \\
\text { Stichus } \\
\text { Stichus } \\
\text { Stichus } \\
\text { Stichus } \\
\text { Stichus } \\
\text { Stichus } \\
\text { Stichus } \\
\\
\text { Stichus } \\
\text { Phylargyrus/Agathea } \\
\text { Stichus } \\
\text { Evemeria } \\
\text { Pamphila } \\
\text { Stichus } \\
\text { Stichus } \\
\text { Concordia } \\
\text { Stichus } \\
\text { Stichus } \\
\text { Stichus } \\
\text { Stichus/Dama } \\
\text { Stichus } \\
\text { Stichus/Pamphilus } \\
\text { Stichus } \\
\text { Stichus/Pamphilus } \\
\text { Stichus/Pamphilus } \\
\text { Stichus } \\
\text { Stichus } \\
\text { Stichus } \\
\text { Stichus } \\
\text { Stichus } \\
\text { Stich }\end{array}$ & $\begin{array}{l}\text { D } 49.15 .12 .1 \mathrm{C} \\
\text { D } 2.14 .27 .6 \\
\text { D } 2.14 .27 .7 \\
\text { D } 7.7 .1 \\
\text { D } 9.2 .55 \\
\text { D } 12.2 .30 .1 \\
\text { D } 12.6 .21 \\
\text { D } 13.5 .12 \\
\text { D } 13.5 .21 \mathrm{pr} \\
\text { D } 17.1 .5 .5 \\
\text { D } 17.1 .46 \\
\text { D } 18.1 .34 \mathrm{pr} \\
\text { D } 18.1 .34 .6 \\
\text { D } 18.4 .21 \\
\text { D } 19.1 .43 \\
\text { D } 19.5 .5 .1 \\
\text { D } 23.3 .25 \\
\text { D } 23.3 .56 \mathrm{pr} \\
\text { D } 31.5 .1 \\
\text { D } 31.8 .1 \\
\text { D } 32.78 \mathrm{pr} \\
\text { D } 33.2 .21 \\
\text { D } 34.3 .25 \\
\\
\text { D } 34.5 .21 \mathrm{pr} \\
\text { D } 35.1 .28 .1 \\
\text { D } 35.2 .21 \mathrm{pr} \\
\text { D } 40.1 .10 \\
\text { D } 40.1 .22 \\
\text { D } 40.4 .39 \\
\text { D } 40.5 .6 \\
\text { D } 40.5 .40 \mathrm{pr} \\
\text { D } 40.5 .40 .1 \\
\text { D } 40.12 .38 .3 \\
\text { D } 41.2 .5 \\
\text { D } 41.4 .2 .6 \\
\text { D } 44.7 .45 \\
\text { D } 45.1 .2 .1 \\
\text { D } 45.1 .8 \\
\\
\text { D } 45.1 .24 \\
\text { D } 45.1 .49 \mathrm{pr} \\
\text { D } 45.1 .73 .2 \\
\text { D } 45.1 .83 .1 \\
\text { D } 45.1 .83 .2 \\
\text { D } 45.1 .83 .4 \\
\text { D } 45.1 .83 .5\end{array}$ \\
\hline
\end{tabular}

55- Objet indirectement par natum. 


\begin{tabular}{|c|c|c|c|}
\hline (Paul suite) & $\begin{array}{l}\text { Sabinus } \\
\text { Celsus }\end{array}$ & $\begin{array}{c}\text { Stichus } \\
\text { Stichus } \\
\text { Stichus/Panphilus } \\
\text { Stichus } \\
\text { Stichus/Pamphilus } \\
\text { Stichus } \\
\text { Stichus / Pamphilus } \\
\text { Eros }\end{array}$ & $\begin{array}{l}\text { D } 45 \cdot 1.83 .7 \\
\text { D } 45.1 .91 .3 \\
\text { D } 45.1 .128 \\
\text { D } 45.3 .20 p r \\
\text { D } 46.1 .34 \\
\text { D } 46.1 .58 .1 \\
\text { D } 46.3 .98 .6 \\
\text { D } 47.2 .67 .5\end{array}$ \\
\hline Ulpien & $\begin{array}{l}\text { Julien, Mauricianus } \\
\text { Pomponius } \\
\text { Julien } \\
\text { Marcellus } \\
\text { Celsus, Marcellus } \\
\text { Celsus } \\
\text { Scaevola } \\
\text { Julien } \\
\text { Julien } \\
\text { Antonin le pieux } \\
\text { Antonin le pieux } \\
\text { Octavenus } \\
\text { Julien }\end{array}$ & $\begin{array}{c}\text { Stichus } \\
\text { Eros } \\
\text { Stichus } \\
\text { Stichus } \\
\text { Stichus } \\
\text { Stichus } \\
\text { Stichus } \\
\text { Stichus } \\
\text { Stichus/Pamphilus } \\
\text { Stichus/Pamphilus } \\
\text { Stichus } \\
\text { Stichus } \\
\text { Stichus } \\
\text { Stichus } \\
\text { Stichus/Panphilus } \\
\text { Stichus } \\
\text { Stichus } \\
\text { Stichus } \\
\text { Stichus } \\
\text { Eppphroditus } \\
\text { Hermias } \\
\text { Stichus } \\
\text { Stichus } \\
\text { Stichus } \\
\text { Stichus } \\
\text { Stichus/Pamphilus } \\
\text { Stichus } \\
\text { Arethusa (56) } \\
\text { Stichus } \\
\text { Stichus } \\
\text { Stichus } \\
\text { Stichus/Pamphilus } \\
\text { Stichus } \\
\text { Stichus } \\
\text { Stichus } \\
\text { Stichus/Panphilus } \\
\text { Stichus } \\
\text { Stichus } \\
\text { tch }\end{array}$ & 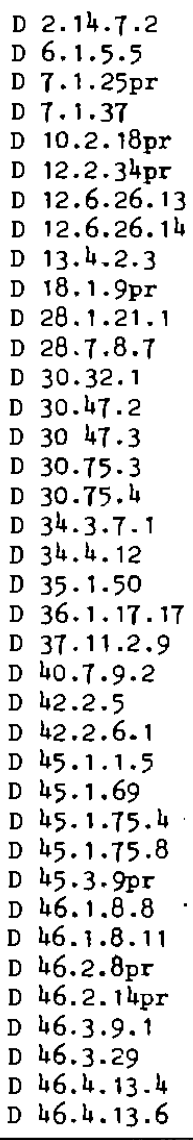 \\
\hline Marcien & Celsus & $\begin{array}{c}\text { Stichus } \\
\text { Stichus } \\
\text { Stichus } \\
\text { Stichus/Pamphilus }\end{array}$ & $\begin{array}{l}\text { D } 12 \cdot 3 \cdot 5 \cdot 4 \\
\text { D } 22.1 \cdot 35.2 . \\
\text { D } 28.5 \cdot 52.1 \\
\text { D } 35 \cdot 1.33 .4\end{array}$ \\
\hline
\end{tabular}

\begin{tabular}{|c|c|c|c|}
\hline $\begin{array}{l}\text { Juriste auteur } \\
\text { du passage }\end{array}$ & Juriste cité & Nom & Source \\
\hline $\begin{array}{l}\text { Alfenus (chez } \\
\text { auteur anonyme) }\end{array}$ & & Pamphilus & D 33.8 .14 \\
\hline Iavolenus & $\begin{array}{l}\text { Trebatius, Labéon } \\
\text { Tubero, Labéon } \\
\text { Trebatius, Labéon } \\
\text { Namusa, Servius } \\
\text { Trebatius, Ofilius, } \\
\text { Labéon }\end{array}$ & $\begin{array}{c}\text { Dama } \\
\text { Stichus/Dama } \\
\text { Stichus } \\
\text { Stichus } \\
\text { Stichus }\end{array}$ & $\begin{array}{l}\text { D } 28.8 .11 \\
\text { D } 32.29 .4 \\
\text { D } 32.30 .2 \\
\text { D } 35.1 .40 .3 \\
\text { D } 40.7 \cdot 39.4\end{array}$ \\
\hline Celsus & & Stichus & D $40.7 .23 \mathrm{px}$ \\
\hline Valeng & Pegasus & Stichus & D 32.12 \\
\hline Julien & & $\begin{array}{l}\text { Stichus } \\
\text { Pemphilus } \\
\text { Stichus } \\
\text { Apollonius } \\
\text { Stichus } \\
\text { Stichus } \\
\text { Stichus } \\
\text { Stichus } \\
\text { Pamphila } \\
\text { Pamphilus } \\
\text { Eros } \\
\text { Eros } \\
\text { Stichus } \\
\text { Pamphilus } \\
\text { Stichus }\end{array}$ & 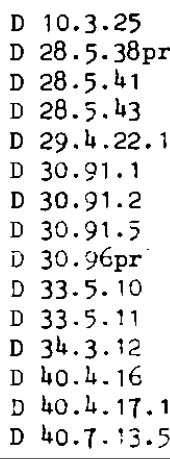 \\
\hline Pomponiug & $\begin{array}{l}\text { Trebatius, Labéon. } \\
\text { Constitution de } \\
\text { Tibère. } \\
\text { Q. Mucius }\end{array}$ & $\begin{array}{c}\text { Stichus } \\
\text { Parthenius } \\
\text { Stichus } \\
\text { Stichus } \\
\text { Stichus } \\
\text { Stichus / Pamphilus } \\
\text { Stichus } \\
\text { Andronicus }\end{array}$ & $\begin{array}{l}\text { D } 28 \cdot 5 \cdot 21 \mathrm{pr} \\
\text { D } 28.5 .42 \\
\text { D } 40.4 \cdot 4 \mathrm{pr} \\
\text { D } 40.4 .4 .1 \\
\text { D } 40.4 .8 \\
\text { D } 40.4 \cdot 11.1 \\
\text { D } 40.4 .41 .2 \\
\text { D } 40.7 .29 .1\end{array}$ \\
\hline Africain & & \begin{tabular}{l}
\multicolumn{1}{c}{ Stichus } \\
Stichus \\
Stichus/Pamphilus \\
Stichus/Pamphilus \\
Stichus/Pamphilus \\
Stichus /Pamphilus \\
Stichus/Pamphila \\
$\quad$ Stichus
\end{tabular} & $\begin{array}{l}\text { D } 12 \cdot 1.41 \\
\text { D } 14.1 .7 \mathrm{pr} \\
\text { D } 15 \cdot 1 \cdot 38.2 \\
\text { D } 15 \cdot 3 \cdot 17.1 \\
\text { D } 33.8 \cdot 16 \mathrm{pr} \\
\text { D } 33.8 .16 .1 \\
\text { D } 35 \cdot 1.31 \\
\text { D } 40.6 .2 ?\end{array}$ \\
\hline
\end{tabular}

56- Objet indirectement par parís. 


\begin{tabular}{|c|c|c|c|}
\hline Gaius & Julien & $\begin{array}{c}\text { Stichus/Pamphilus } \\
\text { Stichus } \\
\text { Stichus }\end{array}$ & $\begin{array}{l}\text { D } 7.1 .74 \\
\text { D } 28.5 .89 \\
\text { D } 40.7 .31 .1\end{array}$ \\
\hline Marcellus & & $\begin{array}{l}\text { Stichus } \\
\text { Pamphilus } \\
\text { Stichus }\end{array}$ & $\begin{array}{l}\text { D } 36.1 .46 \mathrm{pr} \\
\text { D } 39.6 .38 \\
\text { D } 40.7 .24\end{array}$ \\
\hline Scaevola & Julien & 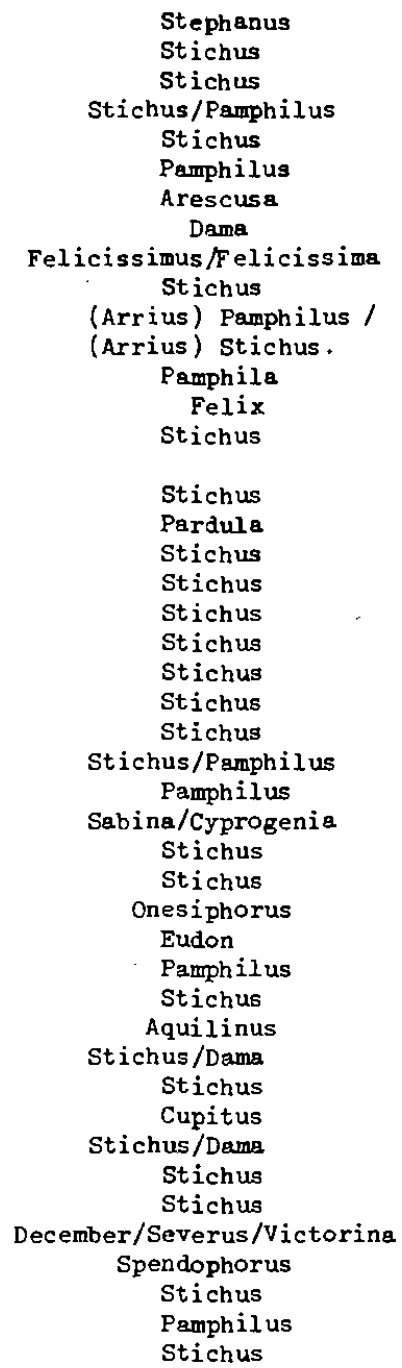 & 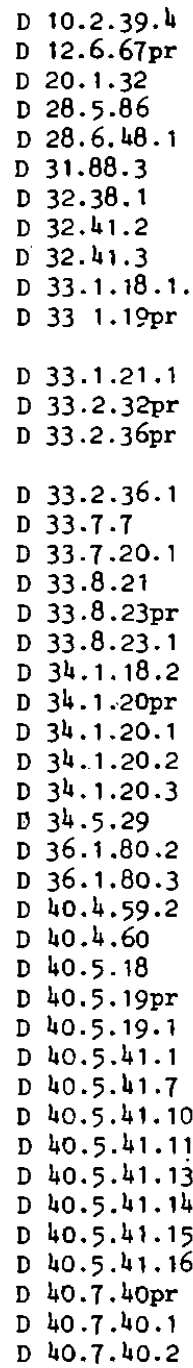 \\
\hline
\end{tabular}

\begin{tabular}{|c|c|c|c|}
\hline (Scaevola suite) & & $\begin{array}{c}\text { Stichus } \\
\text { Pamphilus/Dama } \\
\text { Stichus/Eros } \\
\text { Stichus } \\
\text { Valerius } \\
\text { Aithales }\end{array}$ & $\begin{array}{l}\text { D } 40.7 .40 .3 \\
\text { D } 40.7 .40 .8 \\
\text { D } 45.1 .122 .11 \\
\text { D } 46.3 .89 \mathrm{pr} \\
\text { D } 46.3 .102 .2 \\
\text { D } 48.10 .24\end{array}$ \\
\hline Papinien & Cassius, Caelius Sabi & $\begin{array}{l}\text { Stichus } \\
\text { Stichus } \\
\text { Pamphilus } \\
\text { Stichus }\end{array}$ & $\begin{array}{l}\text { D } 16.3 .24 \\
\text { D } 31.65 .2 \\
\text { D } 35.1 .72 .7 \\
\text { D } 46.3 .95 .7\end{array}$ \\
\hline Tryphonius & $\begin{array}{l}\text { Scaevola } \\
\text { Scaevola }\end{array}$ & $\begin{array}{c}\text { Arescusa } \\
\text { Pamphilus/Diphilus } \\
\text { Titia } \\
\text { Dama/Pamphilus/Pamphila } \\
\text { Stichus }\end{array}$ & $\begin{array}{l}\text { D } 1.5 .15 \\
\text { D } 26.7 .58 \mathrm{pr} \\
\text { D } 28.2 .28 .3 \\
\text { D } 31.88 .12 \\
\text { D } 34.3 .28 .7\end{array}$ \\
\hline Paul & Neratius & $\begin{array}{c}\text { Stichus } \\
\text { Ianuarius } \\
\text { Stichus } \\
\text { Pamphilus } \\
\text { (Lucius) Eros } \\
\text { Stichus } \\
\text { Pamphila } \\
\text { Stichus } \\
\text { Stichus } \\
\text { Antiochus } \\
\text { Stichus } \\
\text { Stichus } \\
\text { Stichus } \\
\text { Chrysogonus }\end{array}$ & $\begin{array}{ll}\text { D } & 15.1 .18 \\
\text { D } & 15.1 .47 \mathrm{pr} \\
\text { D } & 15.1 .53 \\
\text { D } & 16.3 .27 \\
\text { D } 26.2 .32 .2 \\
\text { D } 28.5 .56 \\
\text { D } 29.1 .40 .2 \\
\text { D } 29.2 .58 \\
\text { D } 32.6 .1 \\
\text { D } 32.97 \\
\text { D } 33.5 .13 \mathrm{pr} \\
\text { D } 35.1 .31 \mathrm{pr} \\
\text { D } 40.7 .4 .4 \\
\text { D } 45.1 .126 .2\end{array}$ \\
\hline Ulpien & $\begin{array}{l}\text { Labéon, Aristo, } \\
\text { Neratius } \\
\text { Antonin le pieux } \\
\text { Antonin le pieux } \\
\text { Antonin le pieux } \\
\text { Aristo } \\
\text { Pomponius }\end{array}$ & $\begin{array}{c}\text { Stichus } \\
\text { Stichus } \\
\text { Stichus } \\
\text { Stichus } \\
\text { Stichus/Pamphilus } \\
\text { Eros } \\
\text { Epaphroditus } \\
\text { Albins } \\
\text { Stichus } \\
\text { Hermias } \\
\text { Stichus } \\
\text { Stichus } \\
\text { Stichus } \\
\text { Stichus }\end{array}$ & $\begin{array}{l}\text { D } 7.1 .25 \mathrm{pr} \\
\text { D } 15.4 \cdot 1.1 \\
\text { D } 28.5 \cdot 9.14 \\
\text { D } 28.6 \cdot 10.7 \\
\text { D } 33.8 .8 .2 \\
\text { D } 34.3 \cdot 12 \\
\text { D } 35.1 .50 \\
\text { D } 36.1 .11 .2 \\
\text { D } 36.1 .17 .16 \\
\text { D } 36.1 .17 .17 \\
\text { D } 37.11 .2 .9 \\
\text { D } 40.4 .2 \\
\text { D } 40.7 .3 .13 \\
\text { D } 46.3 .9 \mathrm{pr}\end{array}$ \\
\hline Marcien & $\begin{array}{l}\text { rescrit de Marc-Aurèle } \\
\text { Celsus }\end{array}$ & $\begin{array}{l}\text { Stichus } \\
\text { Stichus }\end{array}$ & $\begin{array}{l}\text { D } 28 \cdot 5 \cdot 52 \mathrm{pr} \\
\text { D } 28.5 \cdot 52.1\end{array}$ \\
\hline
\end{tabular}


- L'escleve objet de fait juridique

\begin{tabular}{|l|c|c|c|}
\hline $\begin{array}{c}\text { Juriste auteur } \\
\text { du passge }\end{array}$ & Juriste cité & Nom & Source \\
\hline Pomponius & & Stichus & D 36.1.72.1 \\
\hline Marcellus & & Stichus & D 9.2.34 \\
\hline Paul & & Stichus/Pamphilus & D 9.2.55 \\
\hline Ulpien & Julien & Stichus & D 9.2.23.2 \\
\hline
\end{tabular}

- L'esclave agent de fait juridique

\begin{tabular}{|l|c|c|c|}
\hline Iavolenus & Servius, Labéon & Dams & D 40.7.39.3 \\
\hline Africain & & $\begin{array}{c}\text { Pamphilus } \\
\text { Pamphilus }\end{array}$ & $\begin{array}{l}\text { D 33.8.16pr } \\
\text { D 33.8.16.1 }\end{array}$ \\
\hline Screvola & & Stichus & D 40.7.40pr \\
\hline Tryphoninus & Scaevola & Stichus & D 40.4.59.1 \\
\hline Peul & & Stichus & D 40.12.38.3 \\
\hline Ulpien & Julien & $\begin{array}{c}\text { Barbarius Philippus } \\
\text { Stichus } \\
\text { Stichus /Pasphilus }\end{array}$ & $\begin{array}{l}\text { D } 1.14 .3 \\
\text { D } 10.2 .18 \mathrm{pr} \\
\mathrm{D} \mathrm{30.47.3}\end{array}$ \\
\hline
\end{tabular}

Une première remarque s'impose : elors que trente-cinq noms d'esclaves apparaissent dans $1_{a}$ rubrique agent de droit, on n'en relève que dix-huit dans celle qui concerne 1 'esclave objet de droit. La plus grande diversité dans le premier cas s'explique per le fait que l'escleve intervient beaucoup moins comme exemple que dans 1 'hypothèse où il est simplement objet.

D'autre part, deux noms ressortent très souvent des textes. Il s'agit de Pomphilus et surtout de Stishus. Une lecture superficielle du Digeste semblerait indiquer que ces noms sont symboliques de 1 'esclave objet. L'impression n'est pas fausse mais elle est incomplète. En effet, on relève aussi fréquement ces noms à propos de l'esclave agent. Ainsi, Stichue apparaît dans $86 \%$ des textes traitant de l'esclage objet, mais également dans $64 \%$ de ceux qui visent l'esclave agent (57). Pamphilue ressort de $21 \%$ des textes concernant l'esclave objet, mais eussi de $18 \%$ de ceux qui parlent de l'esclave agent $(58)$.

57- L'analyse te la fréquence de Stichus (analyse des paragraphes) montre que l'emploi de co nom est sensiblement le même de l'époque des Antonins a celle deg Sévères. Les pourcentages sont calculés par rapport aux textes concernant objet et agent séparément.

$$
\text { Stichue objet }
$$

- d'acte juridique

III $\quad 73 / 85 \quad 86 z$

$\begin{array}{lll}\text { IV } & 24 / 27 & -89 z \\ V & 89 / 105 & 85 \%\end{array}$

Total rubrique $186 / 105=$

$86 z$

- de fait juridique

$\begin{array}{llll}\text { III } & 1 / 1 & & 100 z \\ \text { IV } & 1 / 1 & =100 z \\ \text { V } & 2 / 2 & = & 100 z \\ \text { Total rubrique } & 4 / 4 & =100 \% \\ \text { TOTAL } & 190 / 221 & = & 86 z\end{array}$

Stichus agent

- d'acte juridique

$\begin{array}{ll}\text { III } & 31 / 41 \\ \text { IV } & 31 / 53 \\ \text { V } & 24 / 39\end{array}$

Total rubrique $86 / 133$

- de fait juridique

$\begin{array}{ll}\text { III } & 0 / 3 \\ \text { IV } & 1 / 1 \\ \text { V } & 4 / 5\end{array}$

Total rubrique $5 / 9$

TOTAL $\quad 91 / 142$

58- Même procédé et même conclusion pour Pamphilus.

Ponghilus objet

- d'acte juridique

\begin{tabular}{|c|c|c|}
\hline III & $22 / 85$ & $=$ \\
\hline IV & $3 / 27$ & - \\
\hline v & $20 / 105$ & - \\
\hline
\end{tabular}

- de fait juridique

$\begin{array}{llll}\text { III } & 0 / 1 & = & - \\ \text { IV } & 0 / 1 & = & - \\ \text { V } & 1 / 2 & = & 50 \% \\ \text { Total rubrique } 1 / 4 & = & 25 \% \\ \text { TOTAL } & 46 / 221 & = & 21 \%\end{array}$

\section{Pomphilus agent}

- d'acte juridique

$\begin{array}{ll}\text { III } & 9 / 41 \\ \text { IV } & 9 / 53 \\ \text { V } & 5 / 39\end{array}$

Total rubrique $23 / 133$

- de fait juridique

$\begin{array}{ll}\text { III } & 2 / 3 \\ \text { IV } & 0 / 1 \\ \text { V } & 1 / 5\end{array}$

Total rubrique $3 / 9$

TOTAL

$26 / 142$

$=\quad-\bar{z}$
$=\quad 80 z$
$=\quad 56 z$
$=\quad 64 z$


Si l'on sssocie aux textes que l'on vient d'examiner ceux qui ne rentrent pas dans ces rubriques juridiquas (59), on dénombre quatre-vingt neuf noms desclaves et affranchis, hommes et femmes, dont voici la liste alphabétique. En ce qui concerme les noms d'homes, on relève Agricola, Aitha-

59- Agricola dang un texte d'Ulpien concernant la quasetio (D 48.18.1.22 rescrit d'Hadrien); Vindex, esclave d'un certain Vitellius chez Pomponius D 1.2.2.24; Menonder, interprète capturé par les $R$ omains, chez Pouponius D 49.15.5.3. Les autres textes concernent 1 'affranchissement ou des af $\mathrm{fran}^{-}$ chis : on relève Iavolenus D 40:7.39.1 (Ofilius, Labéon) (Stichus); Julien D 12.1.19pr (Stichus); D 30.91 .1 (Stichus) (daas ce texte, serrus intervient come gent); D 40.2.4.1 (Stichus); D 40.4.17pr (Stichus); D 40.

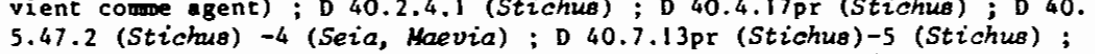
5.47 .2 (Stichus) -4 (Seia, Maevia) : D 40.7.13pr (Stichus)-5 (Stichus) :
D 40.9.5.1 (Stichus) Pomponius D 40.4.41pr (Stichus)-1 (Stichus) ; D 40.4. 6lpr (Julien) (Stichus)-1 (Stichus); D 50.16.162.1 (Dama); Africain D 40. 4.21 (Stichus, Pomphilus); Gaius D 40.4 .24 (Pamphilus); D 40.4 .57 (Sabinus, Cassius, Julien) (Stichus): Marcallus D 40.5.56 (Stiohus. Pomphilus, Eros, Diphilus) ; Scaevola D 10.2.39.2 (Stichus); D 14.3.20 (Terminalis); D 31.88.11 (Dama, Pamphilus); D 32.37.3 (Lucrio)-2 (Seia, Maevius) ; D 32.38.1 (Stichus, Erss) ; D 32.41.1 (Fortunius) ; D 32.102.3 (Seius) ; D 33.1.21pr (Philo)-4 (Titius) ; D 33.2.33pr (Sempronius)-2 (Nigidius, Titius, Dion); D 33.2.34pr (Pactia, Trophima) ; D 33.7.20.6 (Panphila) (dans le même texte, Stichus intervient comale objet) ; 033.7 .27 .3 (Pam philus); D 34.1.16.1 (Rufinas); D 32.39pr (constitution de Marc-Aurelle) (Pamphilus) ; D 34.4.30.1 (Seia, Pamphilus) : D 35.2 .94 (Titius) : D 36. 1.80 .13 (Otacilius) ; D 36.3.18pr (Seius); D 40.4.54pr (Cratistus, Cratinus); D 40.4.59pr (Eutychia) ; D 39.5.35pr (Stichus) : D 40.5.41pr (Thaie)-1 (Stichus, Dama, Pamphilus)-3 (Stichus) ; D 40.7.40.2 (Pcomphilus) (dans le même texte, Stichus est objet et agent) ; Papinien D 19.5 .7 (Stichus) ; D 34.1.9pr (Numerius); D 40.4.49 (Samia) ; D 40.7.34.1 (Doma). Tryphoninus D 34.1 .16 .2 (Scaevola) (Basilica, Epictetus, Callistus); Paul D 19 1.45 .2 (Arescusa) ; D 31.87 .2 (Verus, Sapidus, Symphorus, Beryilus) ;

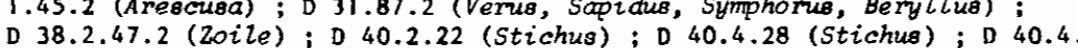
D 38.2 .47 .2 (Zoile) ; D 40.2 .22 (Stichus) ; D 40.4 .28 (Stichus) ; D 40.4 .
34 (Stichus) ; D 40.4.28 (Stichus) ; D 40.4 .34 (Stichus) ; D 40.4 .52 (Sévère et Caracalla) (Stephanus) ; D 40.5.39.1 (Zoile); D 40.7.4.5 (Pomponius) (strchus) ; D 40.7.20.6 (Stichus); D 41.7 .8 (Thetis); D 45.1.126.2 (20sas) (dans le même texte. Chrysogonus intervient comme agent); Ulpien D 1.5.16 (Arescusa) ; D 5.2.26 (Stichus); D 5.3.7.1 (regcrit d'Antonin le pieux) (Licinnicans ; D 12.4.3.1 (Stichus)-2 (Stichus)-3 (Proculus) (Stichus)-5 (Neratius, Julien) (Paris) ; D 35.1.7pr (Stichus) ; D 36.1.17. 17 (Antonin le pieux) (Pamphilus) (dans ce texte, Bermias est objet et agent) ; D 40.4.7 (Neratius) (Stichus) ; D 40.5.24.7 (Stichus); D 40.5 .37 (rescrit de Marc-Aurèle) (Trophimus); D $40.5 .46 \mathrm{pr}$ (Stichus)-1 (Stichus)-2
(Stichos) (Stichus) : D 40.7.3.16 (Julien) (Aretusa) ; Marcien D 23.2.57 (rescrit de Marc-Aurèle et Verus adressé par l'affranchi Mensor); Modestin D $31.34 \mathrm{pr}$ (Stichus) ; D 34.1 .5 (Prothymus, Polychronius, Hypathius) ; D 37.14 .12 (Iulius) ; D 40.4 .44 (Saccus, Eutychia, Hirena); D 40.5.14 (Ëros, Psyilus). les, Andronious, Antiochus, Apollonius, Aquilinus, Barbarius Philippus, Beryllus, Callistus, Chrysogonus, Cratinus, Cipitus, Dma, December, Dion, Diphilus, Epqphroditus, Epictetus, Eros, Eudon, Folicissimus, Felir, Flaccus, Fortunius, Hermias, Bypathius, Ionuarius, Iulius, Licinnionus, Lucrio, Maevius, Menander, Mensor, Nigidius, Numerius, Onesiphorus, Otacilius, Pamphilus, Páris, Parthenius, Philo, Philonicus, Phylargyrus, Polychronius, Prothymus, Psyllus, Rufinas, Saccus, Sapidus, Scorpus, Seius, Sempronius, Severus, Spendophorus, Stephanus, Stichus, Symphorus, Terminalis, Titius, Trophimus, Valerius, Verus, Vindex, Zoile, Zosas. Les noms de femmes sont moins nombreux : Agathea, Albina, Arescusa, Arethusa, Basilica, Concordia, Cyprogenia, Eutychia, Evemeria, Felicissima, Hirena, Maevia, Pactia, Pamphila, Pariula, Sabina, Samia, Seia, Thais, Thetis, Titia, Trophima, Victorina.

Il faut noter que certains de ces noms, comme Iulius, Titius (-a), Seius (-a), Maevius (-a), Sempronius désignent habituellement, dans le Digeste, des personnes libres. Hormis un texte ou Titia désigne une ancilla (60), ces noms sont employés à propos d'affranchis. Il s'agit probablement alor: du nomen de celui qui les a affranchis. Un passage de Paul va clairement dans ce sens, il y est question d'un certain Iulius Zosas, affranchi de Iulius Quintilicanus (61).

Nombreux sont les noms d'origine grecque mais il n'est pas pour autant possible de déduire qu'ils sont portés par des esclaves de la même origine. En effet, il était certainement de bon ton de faire porter à ses esclaves des noms grecs.

60- Tryphoninus D 28.2.28.3.

61- Paul D 45.1.126.2 ; dans le mêre sens, Scaevola D 14.3.20 où il est question d'Oatavius Terminalis, commis d' Octavius Felix. 
Les texmes désignant l'esclave ayant été examinés, il est possible de passer 1 'analyge des relations terminologiques.

\section{Paragraphe 2 - Les relations terminologiques}

Elles sont de trois ordres : la première est une relation de domination (servus/dominus); la seconde est soit une relation d'assimilation, soit de domination (serrug/filizs) ; la demière est une relation d'opposition (servro/liber).

Serrus étant le terme qui désigne le plus fréquement l'esclave, on fera exclusivement porter 1 'analyge chiffrée sur lui. Avant de déterminer laquelle de ces troig relations est dominante, il convient de les envisager sêparément.

\section{$I-$ Servus/dominus}

Le relevé des textes où servus s'oppose à dominus (62) nous conduit a la répartition par périoder suivante :
Analyse des paragraphes

$\begin{array}{lr}\text { I } & - \\ \text { II } & 2 \\ \text { III } & 144 \\ \text { IV } & 38 \\ V & 471\end{array}$

Analyse des citations

$\begin{array}{lr}\text { I } & 24 \\ \text { II } & 56 \\ \text { II I } & 90 \\ \text { IV } & 10 \\ V & 1\end{array}$

62- Serrus s'oppose a dominus chez $A 1$ fenus (chez auteur anonyme)

D $9.2 .52 \mathrm{pr}-4 ; \mathrm{D} 10.3 .26 ; \mathrm{D} 11.3 .16 ; \mathrm{D} 15.3 .16 ; \mathrm{D} 18.6 .12 ; \mathrm{D} 40.1 .6$; D $40.7 .14 \mathrm{pr}-1$; Proculus D 12.6.53 ; D 46.3 .84 ; Iavolenus D 18.1.63pr; D 29.2.62.1; D 29.2.64; D 32.29.4 (Tubero, Labêon) ; D 33.5.15 ; D 33. 8.22pr (Labéon)-l (Trebatius, Labéon); D $34.5 .28 ; 035.1 .40 .3 ; \mathrm{D} 40.12$. 42 (Labéon); D 41.2 .24 ; D 45.3 .36 ; D 46.1.20; Neratius D 15.3 .18 ; D 40.7.17; D 45.3.22; Celsus D 15.1.6 (Tubero, Labéon); D 31.20 (Célsus P., Proculus) ; D 47.2.68.4; Julien D 9.4.39.2-3-4; D 9.4.4.1; D 10.3 . 240r ; D 13.7.28.1; D 14.4.8; D 15.1 .14 .1 ; 15.1 .37 .3 ; D 17.1 .30 ; D 23.2 .39 .1 ; D 23.3.46pr ; D 28.5.41; D 30.81.1 ; D 30.92.1 ; D 30.94.1 ; D $30.99 ; D 30.102$; D 33.5.10;D 36.1.26.1;D 38.13.1; D 39.2 .42 ;
D $41.2 .4 \mathrm{pr}-1 ; \mathrm{D} 41,1.37 .1-2-3-4-5-6 ; \mathrm{D} 41.3 .33 .6 ; \mathrm{D} 41.4 .8$; D 41.4 .10 ; D 45.1 .54 .1 ; D 45.1.62; D 45.3.1pr-4-6; D 46.1.19; D 46 . 3.32 ; D 47.2.57.2-3; D 49.15.22.1-3; Pomponius D 9.4.18;D 9.4.43; D 10.2.45.1 ; D 11.1.15pr ; D I2.1.12; D 12.4.15 (Labêon, Proculus); D 15.1.2; D 15.1.4pr-1-2-3-5; D 15.1.25; D 15.1.49pr;D 17.2.18; D 18.1.18.1 (Alfenus); D 29.2.36; D 30.13 (Neratius); D 30.48pr (Atilicinus) ; D 40.4.8; D 40.5.44; D 40.7.21 pr (veteres, Labéon) ; D 40. 12.28 ; D 41.1 .54 .4 ; D 44.7.24.1; D 45.3.37; D 45.3.39 (Gaius) ; D 46. $3.92 \mathrm{pr}-1$; D 47.2.44.2 ; D 50.16.166pr ; Africain D 4.4.4 ; D 7.8.17; D 9.4.28; D 10.3.9; D 15.1.38.2; D 15.3.17pr; D 21.1.51pr ; D 21.2. 24 ; D 29.2 .47 ; D 34.6 .1 ; D 35.1.32; D 35.1.32; D 35.1.42; D 41.2 40.2-3; D 46.3.38.2;D 47.2.62pr; T. Clemens D 29.2.82; D 31.59;

Gaius D 1.6.1.1-2 (congtitution d'Antonin le pieux); D 2.14.28.2 (Julien) D 2.14.30.1 (Julien) ; D 5.3.35 (Julien) ; D 9.4.20; D 9.4.25;D 11.3.15; D 15.1.27.1-2-8 (Julien); D 15.1.29.1; D 15.3.12; D 28.5.31pr-1 (domina) : D 30.68.1 ; D 31.55.1; D 40.12.25.2; D 40.16.1 ; D 41.1.10.3-4-5; D 45.1.4I pr ; D 45.3.8; D 45.3.28pr (Julien)-1-2-3; D 46.1.70.3;

D 46.4.22 ; D 47.4.2 ; Maecianus D 29.4.28pr; D 29.5.14; D 36.1.67pr ;

D 40.5.32.1 ; D 48.1.11 ; Venuleius D 44.3.15.3; D 45.1.137.4; D 45.3.21 (Julien); $D$ 45.3.25 (Cassius) ; $D 46.8 .8 .2$ (Labéon) ; D 48.19.6.3 : Marcellug D 9.2 .36 .1 (Sabinus) : D 15.16 (Julien); D 29.2.63 (Pomponius) Cellus D D 29.4 .5 ; D JS.H.47, D J8.15.5.2; D 40.1.15; D 46.3.72.3; D 49.1.15 Florentinus D 34.4.14pr; D 45.1.65.1; D 46.2.16; Scaevola D 15.1 .51 D 15.1 .54 ; D 15.1 .58 ; D 20.1 .32 ; D 28.6.48 pr ; D 33.7.20.1 (par fide dominica) ; D 33.8.23pr : D 34.3.31.1 ; D 40.5.19pr ; D 40.5.41.7; D 40 7.40 .7 ; D 45.1.122.1; D 45.3.19; D 48.10.24; D 49.1.28pr ; Papinien D 3.6.9; D 5.1.44.1; D 14.3.19.1-3; D 15.1.50.2 ; D 16.1.27.1; D 17.1. 54pr ; D 17.1.57 ; D 19.5.8; D 20.1.1.1; D 21.2.67; D 26.7.37.1 (dominicas mexcis) ; D 29.5.21 pr ; D 30.11 ; D 33.3.5; D 33.8.19pr ; D 35.2 .11 . 4 ; D 35.2.13; D 40.5.23.2 ; D 40.7.34pr ; D 40.12.36; D 41.2.44.1 ; D 41 . 3.44 .7 ; D 41.3.45.1; D 45.1.118pr ; D 45.3.18pr-2-3 (Julien); D 46.3. $94.3 ;$ D $46.3 .95 .7 ;$ D 48.3 .2 .I (édit de Domitien); D 48.5.40.8; D 48 .

18.17pr (rescrits de Marc-Aurêle et de Caracalla) $-1-2$ (rescrit d'Hadrien) -3 ; $18.17 \mathrm{pr}$ (rescrics de Marc-Aurèle et de Caracalla)-i-2 (rescrit d'Hadrien)-3 ; D $48.19 .34 \mathrm{pr}$; Tryphoninus D 11.4 .5 (rescrit d'Antonin le pieux) ; D.16.3.31 1 ; D 28.5.91 ; D 41.1.63.1-2-3 ; D 49.15.2.8-9 (Sabinus) ; Callistrate D 29.5.2 (rescrit de Commode); D 47.21.3.1 (1'Empereur Nerva) ; D 48.10.15. $1-2-4$; D 48.15.6.2 ; D 48.18.15.2 (rescrit d'Antonin le pieux); D 48.19. 28.4-II ; D 49.14.2.6 (constitution de Sévère); Paul D 2.9.2.1 (Julien, V. Verus, Pomponius); D 2.10.2 (Ofilius, Sabinus); D 2.14.17.7; D 2.14.21. I ; D 2.14.27.10 ; D 3.2.14 ; D 4.3.20pr (Labéon) ; D 4.4.24.3 ; D 4.5.7.2 (Julien) ; D 5.3.34.1 (Julien); D 5.3.36pr (Proculus, Julien, Mauricianus): D $6.1 .27 .4 ; 07.4 .27 ; 09.2 .12 ; 0.2 .30$. I D D $9.4 .4 \mathrm{prl}-2 ; \mathrm{D} 9.4 .9$; $39.4 .17 \mathrm{pr}^{-1} ; \mathrm{D} 9.4 .19 \mathrm{pr}-1 ; \mathrm{D} 9.4 .22 \mathrm{pr}-3-4 ; \mathrm{D} 9.4 .26 .6 ; \mathrm{D} 9.4 .31$ (Sabinus, Cassius, Julien, Pomponius); D 10.4 .16 ; D 11.1 .8 ; D $11.1 .20 \mathrm{pr}$; D $11.3 .14 .9 ; \mathrm{D} 11$. 5.4 .1 ; D 12.1.2.4; D 12.2.22 ; D 14.1.6pr-1 ; D 15.1.26 ; D 15.1.47pr-1 (Sabinus)-5 ; D 15.1.52pr ; D 15.2.2.1 ; D 15.3.8 (Pomponius) ; D 15.3.11; D 15.4.5pr-1; D 16.1.9; D 16.3.21.1 (Trebatius) D 16.3.27; D 17.1 .5 .4 ; D 18.1.40.5; D 18.2.14.3; D 19.1.45.1; D 19.2.55Pr ; D 201.29.3; D 21 . 1.57pr-1 ; D 21.2.41.1; D 22.3.7; D 25.2.21.1 (Pedius) (domina) ; 028 . 5.90 ; D 29.2.22 (Pomponius) ; D 29.2.68; D 29.2.93.1-2 ;

D 29.5.6.1-2;D $31.14 .1 ; 031.82 .2$ (Valeng, Ju-

lien); D 31.84 ; D 32.4; D 33.1.11; D 33.3.9.1 (Marcellus); D 34.9.5.3$4 ; \mathrm{D} 35.1 .5 .1 ; \mathrm{D} 35.1 .44 \mathrm{pr}-1-3 ; \mathrm{D} 35.2 .22 .1-2 ; \mathrm{D} 36.3 .7 ; \mathrm{D} 38.2 .4 \mathrm{pr}$; D $39.5 .10 ; D 39.6 .44 ; D 40.1 .12 ; 040.2 .4 .2$ (Julien); D 40.5.31.4 (resCrit de Caracalla); D $40.12 .23 \mathrm{pr} ; \mathrm{D} 41.2 .3 .10$; D 41.3 .4 .16 (Sabinus, Cassius); D $41.3 .8 \mathrm{pr}$ (Labéon, Neratius, Julien); $41.3 .11: 041.3 .15 \mathrm{pr}$ (Julien, Marcellus) -1 ; D 41.3 .34 (Alfenus); D 41.4.2.12 (Pomponius); D 44.4 . $5.4 ; D 44.5 .2 .2 ; D 45.1 .39 ; D 45.1 .126 .2 ; D 45.3 .16 ; 045.3 .27 ; D 45.3$.

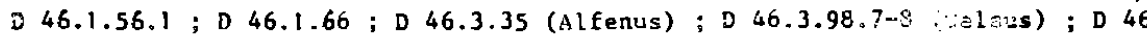
$4.11 \mathrm{pr}-1-3 ; \mathrm{D} 47.2 .42 \mathrm{pr}$; D 47.2.54.1 (Sabinus); D 47.8.3 (La)on); 047. 20.18 .1 ; $D 47.10 .26$; D 48.10.22.5-9; D 48.18.18.5-6-7-8; 
D 49.15 .30 (Labéon) : v1pien D 1.12.1.1-8; D 2.1.7.3; D 2.7.1.1 (P omponius); D 2.13.4.3: D 3.5.5.8 (Labéon, Pomponius) ; 3.5.9.1 (Labéon, Proculus, Celsus, Julien) : D 4.2.14.11; D 4.2.16.1; D 4.3.7.8 (Pomponius): D 4.4.3.11 ; D 4.4.11 Pr (rescrit de Sévère) : D 4.9.3.3 ; D 5.1.19.3 (Labeon) : D 6.1.41. I ; D 7.1.17:1 (Cassius, Aristo) ; D 7.1.25.4-5 (Julien)-6 (Scaevola) ;

D 7.2.1.1 (Julien) ; D 7.3.1.2 (Julien) ; D 7.7.6.1; D 7.8.12.5; D 9.2.13. 2 (Celsus) ; D 9.2.17; D 9.2.27.2 (Celsus)-3 (Julien,Marcellus)-34 (Mela) ; 2 (Celsus): D $2.37: D$ D 2.27 .2 (Celsus) 3 (Julen, Marcellus) 34 (Mela) D 9.2 .44 .1 ; $9.3 .1 p r ; D$,

lus) ; 0 9.4.3; D 9.4.5.1; 0.4 .8 (Pomponius) ; 0 9.4.11; 0 9.4.38.1

(Julien); D 9.4.42.2; D 10.4.3.7; D 11.9.8; D 11.7.31.1 ; D 12.1.11.2 ; D 12.2.23;D 12.2.25;D 13.1.4; D 13.5.5.10;D 14.1.4.3-4; D 14.3.11.8 D 14.4:1pr; D 14.4.3pr (Julien) ; D 14.4.7.2; D 15.1.1.4; D 15.1.3.3-5 (Celsus)-6 (Julien)-12 (Labeon); D 15.1.5.1-4 (Tubero,Celsus) ; D 15.1.7.1 (Julien, Harcellus)-3 (Pedius)-6 ; D 15.1.9pr-1 (Neratius, Pomponius) (conservus ) $\rightarrow$ (Pedius) -5 (Julien)-6-7-8 (Julien, Marcellus) ; D 15.1.11.1-2 (Julien) -4 (Julien)-5 (Julien)-7 (Julien) ; D 15.1.17 (Proculus, Atilicinus, Servius) ; D 15.1.36 (Pomponius) ; D 15.1.41; D 15.3.1.1-2 (Julien, Pomponius); D 15. 3. 3pr-1 (Pomponius) -2-3 (Labeon)-5 (Labeon, Pomponius)-6 (Labeon)-7-8-9-10; D 15.3.5pr (Pomponius) -1 (Pomponius)-2-3; D 15.3.7pr-3 (Labeon)-4 (Labeon). D $15.3 .5 \mathrm{pr}$ (Pomponius) -1 (Pomponius) $-2-3 ; \mathrm{D} 15.3 .7 \mathrm{pr}-3$ (Labeon) -4 (Labeon)

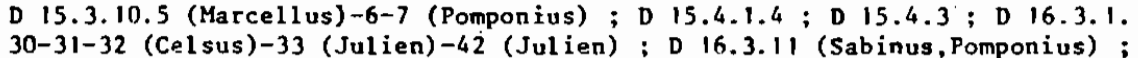
30-31-32 (Celsus)-33 (Julien) 42 (Jul ien) : D 16.3.11 (Sabinus, Pomponius) ; $7 \mathrm{pr}$ (veteres) ; D 18.7.1 (Papinien); D 19.5.15; D 20.1.21.1 (Julien) ; D 21.1.17.6 (Caelius Sabinus)-11-19; D 21.1.23.4; D 21.1.31.1; D 21.3.1. 4 ; D 24.1.22; D 27.5.1.2 (rescrit de Sévère); 028.5 .6 .3 (Julien); D 28.

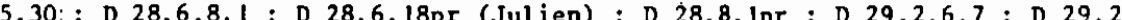
30.7 D 29.2.35pr; D 29.2 .66 ; D 29.2 .67 (par domica) ( D D 29.4.1.3-4; D 29.5. lpr-2-18-28 (rescrit d'Hadrien)-29 (rescrit d'Hadrien) -37-38; D 29.5.3.1-3-4-5 (domina) -13; D 29.6.1.1 ; D 30.50pr ; D 32.11 16 ; D 33.8.6pr (Celsus, Julien); D 33.8.8. I (Julien) -5 (Labéon); D 36.1.1. 10 : D 36.1.17.14 (Maecianus) ; D 36.2.9; D 36.2.23; D 38.9.1.3; D 38.16 $3.4 ; 039.1 .5 .3 ; D 39.4 .1 .6 ; 040.4 .6 ; 040.5 .7 ; D 40.5 .24 .5$ (rescrit de Caracalla); -8 (Celsus); D 40.7.3.2 (Servius, Labéon, Cassius)-8; D 40.12.7.5; D 40.12 12.2 ; D 41.1.17; D 41.2.13.3; D 4I.2.42pr; D 42.4.3.1; D 42.8.6.12 (Labêon) ; D 43.5.3.4; D 43.16.1.15-22 ; D 43.24.7.1 (Neratius); $D 43.24$. I1.7; D 44.3.8;D 44.4.4.17;D 44.6.2 (Julien); D 45.1.38.17; D 45.3.2 ; $D 45.3 .4 ; D 45.3 .5$; D 45.3.7.1 (Julien) ; D 45.3.9pr-1 (Cassius,Julien); $D$ 45.3.13;D 46.3.9pr; D 46.3.18; D 46.4.8.1 (Octavenus) -2 (Labéon) -4 ; D 47.2 .17 .3 (Pomponius); D 47.2.43.12; D 47.2.46pr-1-5; D 47.2.48.2 ;

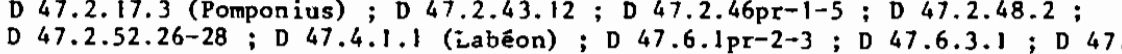
7.7 .5 (Sabinus) ; D 47.8.2pr-15;D 47.10.9.3; D 47.10.11.9; D 47.10.15. 34-35-36-45 (Mela) -48 ; D 47.10.17.2 (Trebatius, Labéon, Mela)-4-7 (Labéon)9 ; D 47.11.5 ; D 48.2.5 (rescrit de Marc-Aurèle) ; D 48.2.7.4 (rescrit d'Antonin le pieux) ; D 48.5.18.5; D 48.5.28.3-16; D 48.15.2.3; D 48.18. 1.5 (rescrit d'Hadrien et d'Antonin le pieux)-13 (rescrit d'Antonin le pieux) -16 (rescrit de Sévère)-18 (rescrit de Sévère et Caracalla)-19 (rescrit de Trajan)-27 (epistula de Marc-Aurèle et Verus) ; D 48.18.2; D 48.19.19; D $50.12 .2 .1 ;$ D $50.17 .157 \mathrm{pr} ; \mathrm{L}$. Rufinus D $31.62 ; \mathrm{D} 34.4 .21$; Macer D 48 $10.10 \mathrm{pr}$; D 48.19.jOpr; Marcien D 15.1.40.1 (vereres); D 18.1.42 (rescrit de Marc-Aurèle et Verus) ; D 21.1.52;D22.1.32.1;D22.5.49.2 (Sévère et et Caracalla) (domina) : D 30.114 .12 (rescrit de Sévère et Caracalla); D 34.5.18.1; D 36.1.31.2; D 39.4.16pr (rescrit de Sévère et Caracalla)-1
Mais servrua n'est pas le seul terae désignant l'esclave qui s'oppose a dominus. On relève égalerent homo (63), nancipivon (64), vicarius (65). Dominus peut aussi être en relation avec un terme concernant un esclave en voie d'affranchissement (66) ou affranchi (67). On peut le trouver aux côtés d'ancilla (68), de serva (69), de mulier (70), de partus ( 71 ), de

(rescrit des mêmeg)-11 (rescrit de Caracalla): 0 40.1.5pr-1; D 40.2.9pr; D 40.5.51.2; D 40.9.9pr; D 47.22.3.2; D 48.1.9; D 48.10.7 (rescrit de Marc-Aurêle et Comode) ; 48.12 .1 ; D 48.15.3pr (constitution de Sévęre et Caracalla) ; D 48.18.5; D 48.18.9.1 ; D 48.19.11.1 : Modestin D 3.3.63 ; D 16.1.25pr (domina) ; D 28.6.3; D $28.7 .25 ; \mathrm{D} 31.34 .2 ; \mathrm{D} 40.8 .2$ (dit de Claude ) $D$ 40.9.20; D 48.2.17; D 48.4.7.2; $D 48.8 .11 .2$; Hermogênien D 1.5 .13 : D 5.1 .53 ; D $41.1 .61 \mathrm{pr}$; D 47.10 .45 .

63- En rie recenant que les textes où homo est la seul indicateur de la présence d'un esclave (dans le même cexte, on peut en effec aussi ralever cexrus, par ex. chez Paul D 9.4.17pr-1) : Pomponius D 43.26.15.4; Gaius D 2.9.4 (Sabinus); Papinien D 31.66.3; Paul D 30.35; Ulpien D 2.14.16.1; D 4.2 .14 .11 ; D 47.10 .7 .1

64- Moncipium s'oppose a dominus chez Paul D 21.1.43.6; D 40.12.38.1 (Constitution de Marc-Aurèle). Ce terme 'oppose à pater familias chez Scaevola D 32.93 .2 ; D 41.9.3; Ulpien D 33.7.12.37 (Papinien); D 47 10.25 .

65- Vicarius/dominus chez Pomponius D 15.1.4.6; Africain D 33.3.16.1.

66- Statuliber/dominus chez Paul D 35.1.44.2; statuliber/pater fomilias chez Pomponius D 35.1 .112 .3 (Neratius)

67- Manumissus, seul indicateur de la présence d'un esclave, s'oppose à dominus chez Pomponius D 40.14.3.1; Paul D 28.1.14; D 40.9.15pr (rescrit de Caracalla); D 40.9.17.1; D 47.2.42.1 (Sabinus); Ulpien D 29.2.71.2 $D 41.2 .13 .8$; Hermogénien $D 40.2 .23$. Liber ou libertas dans le sens d'afD 41.2 .13 .8 ; Hermogénien $D$ 40.2.23. Liber ou libertas dans le sens d'af franchi ou d'affranchissement, seul indicateur, s'oppose à dominus chez Fomponius D 35.1.111 ; Paul D 40.5.43; D 48.19.38.4; Ulpien D 3.5.44.1; $D$ 36.2.5.7; D 40.5.26.11; D 47.4.1Pr ; Marcien D 40.8.5; D 40.9.9.1; ; liber ou libertas, dans le même sens, s'oppose à pater farmilias chez Julien D40.4.17.2; Ulpien D 40.5.24.11.

68- Ancilla/dominus chez Lavolenus D 47.2.75; Gaius D 15.1.27pr (Julien); Paul D 15.4.2.1; D $31.83 ; 040.1 .23 ; 0$ 40.12.38. (constitution de MarcAurèle); D 41.7 .8 ; Ulpien D 1.6.2 (Hadrien, rescrit d'Antonin le pieux) (matrona/ancilla) ; D 4.1.6 (Julien) ; D 11.7.31.1; D 29.5.1.28 (rescrit d'Hadrien) (domina/ancilla).

69- Serva/dominus chez Callistrate D 47.21.3.1 (Empereur Nerva); D 48.15. 6.2 (dominus, domina); Ulpien D 47.10.25.

70- Mulier/dominus chez Marcellus D 23.3.59.2

71- Partus ancillae/dominus chez Gaius D 22.1.28.1. 
puer (72) ou de familia (73), et même de fugitivus (74), ou encore d'un mot qui $n$ 'indique que l'activité professionnelle de l'esclave (75), ou enfín d'un nom propre (76).

En l'absence de dominus, on a parfois affaire à un rapport de même nature. C'est notanment le cas lorsque serrus est opposé à pater famiiias (77).

Opérons de mêne pour la relation servus/filius.

\section{II - Servus/filiug}

Comue servus/dominus, servus/filius peut signifier un rapport

72- Puer/dominus chez Alfenus D 9.2.52.2 (chez auteur anonyme).

73- Fomilia/dominus chez Gaius D 9.2.32pr ; Papinien D 1.21.1pr ; Paul D 2.1.9 ; (Pomponius, Octavenus) ; D 29.5.6.3 ; Ulpien D 7.8.10.4 (Labēon); D $39.4 .1 \mathrm{Pr}$; D $47.6 .3 \mathrm{pr}$; Modestin D 29.5.19. I1 s'agit $1 \mathrm{a}$ deg textes ou familia est le seul indicateur, mais on relève ausai l'opposition familial domimus dans des textes où on a servus : Neratius D 15.3.18; Papinien D 29.5.21 pr ; D 35.2.11.4; Paul D 9.4.9; D 9.4.31 (Sabinus, Cassius, Julien, Pomponius) ; Dlpien D 15.3.3.1 (Pomponius)-7-10.

74- Fugitivres (seul indicateur)/dominus chez Julien D 46.3.34.5 ; Tryphoninus D S0.16.225; Callistrate D 11.4.2; Paul D 21.1.43.1-2; Ulpien D 1.15 .4 (rescrit de Severe et Caracalla); D 9.2.13pr; D 11.4 .1 .1 ; D 21 . 1.17pr (Ofilius)-1 (Caelius Sabinus)-2 (Cassius)-3 (Vivianus) -4 (Proculus)5 (Vivianus) -9 (Caelius Sabinus)-13 (Caelius Sabinus) ; D 47.2.52.12 ; Modestin D 48.3.14.7 (Venuleius).

75- Papinien D 33.1.10.3 (actor/domina); Paul D 14.3.17.3 (Pomponius) (institor/dominus) ; D 33.7.18.4 (vilicus/fide dominica) (Scaevola) ; U1pien D 14.1.1.20 (Pomponius) (institor/dominus)-23 (Julien) (exercitor/ dominus). Le nom de métier peut aussi s'opposer à pater familias : Paul D 32.99 .1 (venatores, aucupes); D 33.7.18.1 (pistores).

76- Nom propre (seul indicateur)/dominus chez Africain D 14.1.7pr; Mar cellus D 46.8.17; Scaevola D 12.6.67pr; D 40.5.41.17 (on a eussi arcazrius) ; D 40.7.40.1 ; Tryphoninus D 40.4.59.1 (Scaevola); Paul D 26.2. 32.2 : Ulpien D 35.1 .50 (Antonin le pieux). On relève un nom d'esclave opposé a pater familias chez Julien D 30.96pr : Pomponius D 40.4.4pr Scaevola D 40.5 .41 .16 .

77- On trouve servus aux côtés de pater farrilias chez Alfenus (chez auteur anonyee) $D$ 50.16.203; Julien D 10.2.52.1; D 36.2.17; Paul D 11.1 . 13.1 ; D 32.60.1 (Alfenus) (on a aussi anciliza); U1pien D 14.1.1.16: D 32.71 (on a aussi ancilla); D 47.10.1.3 (on a aussi mater familias); D 47.10.15.15 (ancilla/mater fomilias). Servus ressort quelquefois dans 1 e mêne texte que le mot patronus, il $s^{\prime}$ agit dans chaque cas de $1^{\prime}$ esclave du patron : Tryphoninus D $37.14 .23 \mathrm{pr}$; D $38.2 .50 \mathrm{pr}-1$; Paul D 38.2.44.1; D 38.2.45;D 40.5.33 Pr ; Ulpien D 38.2.8.2; D 38.16.3.2. de domination ( 78 ), mais ce n'est pas 1 'hypothèse la plus intéressante. L'originalité de la relation semra/filizus consiste dans le fait qu'elle vise parfois un rapport d'assimilation.' Celle-ci, compte tenu du nombre de textes ou elle se produit, semble être la manifestatıon arun certain "paternalisme" a I'Egard de l'esclave. En effet, serrus désigne alora un

78- Il s'agit de l'esclave du fils. Le texte indique qu'il est dens son pécule, ou qu'il lui a été légué ou donné, ou qu'il a été affranchi par lui. On relève semrs/filius dans ce sens chez Alfenus (chez auteur anongme) D 40.1.7; D 44.1.14; Julien D 30.81.10; D 36.1.26.2 ; D 38 . 2.13 ; D 40.2.4pr ; Gaius D 38.1.22.1 (Julien); Maecianus D 49.17.18pr; Marcellus D 37.15 .3 ; Scaevola D $4.3 .32 ; \mathrm{D} 15.1 .54 ; 00.5 .41 .1$ : Papinien D 29.2.86.2; D 33.8.19.2; D 40.5.23.2-4; D 45.3.18pr; D 49.17. 13 (rescrit d'Hadrien); D 49.17.15.3; Tryphoninus D 37.14.23pr ; D 49. 17.19.2-3-4-5 : Paul D 40.2.22 ; D 40.5.31.1 (rescrit de Marc-Auràle et Verus); D 40.5.33pr-1; D 40.5.39.1; D 40.9.15.1 (Julien) ; D 45.1.91. 5 (Pouponius) ; D 48.10.14pr ; D 49.17.20 ; Ulpien D 24.1.19pr (Julien) ; D 29.2.42.2-3; D 30.44pr; D 38.2.3.8 (constitution d'Badrien) : D 38 4.3.3. (Pomponius); $\mathrm{D} 40.9 .12 .7$ (filia); $\mathrm{D} 40.9 .30 .1-2 ; \mathrm{D} 41.1 .33 \mathrm{pr}$ (Marcellue Scaevola) D 47.2 .52 .4 : 49.17.6:D 49.17.9 (Marcellus)

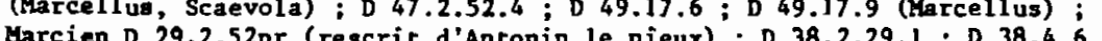
Marcien D 29.2.52pr (rescrie diAnconin le pleux); D $38.2 .29 .1 ; D 38.4 .6$ Modestin D 5.2.12pr ; D 37.14.8pr (rescrit d'Badrien) ; D 37.14.13 ; D 40 1.16 ; D 40.1.17; A. Charisius D 48.18.10:2. Il faut de même signaler oncilla/filia chez Papinien D 6.1.65.1 : Paul D 40.5.40pr, et ancilla/filius chez Ulpien D 23.2.45.3; L. Rufinus D 23.2.51.1.

Il est par ailleurs des textes où servus et fílius (ou filita) apparaissent mais ne sont reliables ni a un rapport de domination ni d'assimilation. C'est par exemple le cas lorsqu'il est traité d'un esclave qui parviendra a la liberté lorsque le fils aura tel âge. On rencontre ve qui parviendra a la liberté lorsque le fils aura tel age. On rencontre
ce problème chez Pouponius D 35.1.6.1 (Servius, Labeon, Sabinus, Cassius, Ce probleme chez Pomponius D 35.1 .6 .1 (Servius, Labeon, Sabinus, Cas Scaevola D 40.4.29; 0 40.5.41.1C-13-14 (filia) $-15-16$ (filia) ;

Papinien D 40.4.47.1 ; Callistrate D 48.10.15.4 : Paul D 16.3.27 (filia); D 40.4.53; D 45.3.20.1 (Labēon) (fizius, filia); Ulpien D 5.2.8.9;

D 40.7.19: 40.12 .1 .1 ; 40.12 .3 .1 : Modestin D 40.5 .14 (filia). 
La répartition par périodeg des textes où aerrus/filius vise un rapport de donination ou d'assinilation s'opère de la manière suivante;

79- Servus et filius sont agents d'acte juridique chez Neratius $D 28$. 5.55 ; Celsus D 46.3.71.2 ; Julien D 23.3.46pr ;Pomp.D28.1.16pr;D29.2.36; 6.1 D ; 43.26 .13 ; D 45.3.40; D 45.1 .40 ; Africain D 7.8 .17 ; D D.1 D J5.1.42 D 39.6 .23 ; Gaius D 2.14 .28 .2 (Julien) ; D is.3.12 D 122.4 ; Papinien D 30.11 ; D 35.2 .13 ; D 41.3.44.7; D 45.3.18.2 ; Tryphoninus D 15.1.57pr; D 49.15.12.1; Callistrate D 48. 10.15pr (édit de

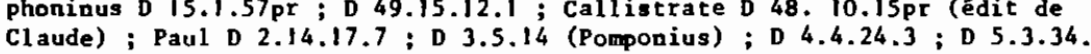
1 (Julien) ; D 5.3.36pr (Proculus, Julien, Mauricianus) ; D 6.1.27.4 : D 11.5 .4 .1 ; D 12.1.2.4; D 12.2.22 ; D 15.3.8 (Pomponius) ; D 15.4.5pr ; D 16.2.9pr ; D 18.2.14.3; D 21.1.57.1; D 29.2.26 (Julien, Pomponius, Harcellus) ; D 29.2.93.1; D 32.4; D 34.9.5.3; D 35.1.5.1; D 36.3.7 ; D 41.2 .1 .5 (Sabinus, Cassius, Julien); D41.2.14pr ; D 41.3 .31 .3 ; D 44. $7.49 ; D 45.1 .39 ; D 45.1 .76 \mathrm{pr} ; \mathrm{D} 46.1 .56 .1 ; \mathrm{D} 48 \cdot 10.22 .5$; Ulpien D 2.14.7.18; (v. Verus, Marcellus) ;D3.3.33pr (Labéon) ;D3.5.5.8 (Labéon, Pomponius); D 4.4.7.5; D 4.9.3.3; D 4.9.7.6; D 6.1.41.1; D 7.8.12.5; D 11.1.9. 8 ; D 13.6.3.4; D 14.1.1.16-21; D 15.1.1.4-5; D 15.1 .3 .5 (Celsus) -7 (Julien)-9 (Sabinus, Cassius) -12 (Labéon); D 15.1.5.1 -2;D15.1.7.3(Pedius); D 15.1.11.2 (Julien) ; D 15.1.24; D 15.1.36 (Pomponius); D 15.2.1.1-4; D 15.3.3.3 (Labéon) ; D 15.3.10.6; D 15.4.1.6; D 16.3.1.42 (Julien) ; D 17.1.12.2 (Marcellus); D 18.4.2.6; D 21.1.23.4; D 26.8.1pr (rescrit d'Antonin le pieux); D 28.6.8.1; D 29.2.30.7; D 29.2.35pr ; D 30.53.2 (Julien. Marcellus) ; D 32.1.1; D 32.49 .7 ; D 33.8.6.4 (Nerva, Atilicinus, Pegasus; rescrit de Sévère et Caracalla) (filia) ; $\mathrm{D} \mathrm{36.1.1.10;} \mathrm{D} 36.2 .9$; D 38.2 .8 .2 ; D 38.5.1.22; D 42.4.7.15; D 42.8.6.12 (Labéon) ; D 42.8.10.10; D 43.5.1.7; D 44.4.4.17; D 45.1.38.6 (Julien) -17 ; D 46.4.8.4; D 47.2. 52.9-15; D 50.12.2.1; L. Rufinus D 34.4.21; Macer D 48.10.10pr ; Marcien D 20.3.1.1; D 20.6.8.5; D 30.114.12 (rescrit de Sévère et Caracalla); D $36.1 .31 .2 ;$ D 48.10 .1 .8 ; Modestin D 28.1.19. On trouve ancilla/filia dans le même sens chez Gaius D 15.1.27pr (Julien) ; Paul D 15.4.2.1; dans le même sens chez Gaius D 15.1.27pr (Julien) ; Paul D 15.4.2.1 ;
U1pien D 13.6.3.4; D 14.3 .7 .1 ; D 21.1 .23 .4 ; D 27.6.11.2 ; ancilla/filius chez Ulpien D 3.5.13; serva/filia chez Ulpien D 14.1.1.21; D 32.49 .7 . Servus et filius sont employés dans le sens d'agents de fait juridique chez Pomponius D 47.2.36.1 ; Paul D 11.5.4.1 (par a contrario) ; Ulpien D 2.1.7.1; D 13.1.4; D 15.1.3.12 (Labéon); D 44.4.4.17; D 47.2.17pr (veteres) ; D 47.2.52.9-15; Macer D 48.10.10pr ; l'hypothèse oi filius... servion (poenae) efficit (Ulpien D 37.4 .1 .9 ), de par sa spécificité, n'est pas intégrable.

80- Serrus et filius objets d'acte juridique chez Julien D 21.2.39.3 (il $s^{\prime}$ agit de la vente d'un fills conme esclave); objets de fait juridique chez Paul D 11.3.14.1 (filius, filia); D 47.10.26 ; Ulpien D 9.2.5.3 (Julien);

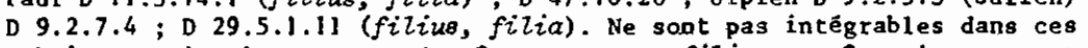
rubriques mais situent tout de même servus et fílius au même niveau un texte d'Ulpien (D 50,16.195.3) qui traite de la composition de la familia, et d'autres qui rapprochent semrus manwissus de filius emoncipatus (Jul ien

\section{Rapport de domination}

Rapport d'asgimilation

\section{- enalyse des paragrapbes}

$\begin{array}{llll}\text { I } & - & = & - \\ \text { II } & - & * & 17 \\ \text { III } & 5 / 29 & = & 71 \\ \text { IV } & 5 / 7 & = & 25\end{array}$

TOTAL $57 / 206$

$\begin{array}{ll}- \\ - \\ 17 & \% \\ 71 & 8 \\ 25 & \%\end{array}$

$28 \%$

- analyse des citations

$\begin{array}{lllllll}\text { I } & 0 / 1 & = & - & \text { I } & 1 / 1 & =100 \% \\ \text { II } & 0 / 15 & * & - & \text { II } & 15 / 15 & =100 \% \\ \text { III } & 5 / 27 & \Rightarrow & 19 \% & \text { III } & 22 / 27 & =81 \% \\ \text { IV } & 3 / 7 & = & 43 \% & \text { IV } & 4 / 7 & =57 \% \\ \text { V } & - & * & - & \text { V } & - & = \\ \text { TOTAL } & 8 / 50 & = & 16 \% & \text { TOTAC } 42 / 50 & =84 \%\end{array}$

Le rapport d'assimilation ressort de ce tableau conme étant nettement prépondérant. La période IV, dans l'analyse des paragraphes, dément cette prépondérance, mais elle ne s'appuie que sur peu de textes et le pourcentage qu'elle réalise a'est pas confirmé par l'analyge des citations.que le rapport d'assimilation soit le plus important tient au srand nombre de textes où servus et filius apparaissent come asents juridiques. Ce nombre est élevé car déterminer les effets des opérations accomplies par l'alieni juris dans l'intérêt du maître ou du père constitue un problème souvent épineux pour les juriștes.

D 38.13.1 ; Pomponius D 45.3.40; Paul D 4.5.3.1; D 5.3.36pr (Proculus, Julien, Mauricianus) ; D 32.4 ; D 36.3 .7 ; Ulpien D 1.18 .2 ; $\mathrm{D} 12.4 .1 \mathrm{pr}$; D 15.2.1.4; D 29.2.35pr; de même qu'un texte de Tryphoninus (D 16.3.31. 1) ol il est quegtion d'un vol commis au passif d'un esclave ou d'un fils. 
On remarquera également, lorsque fils et esclave ressortent comme agents d'actes juridiques, que les juristes ne rapportent que très peu de solutions différentes ( $8 \cap$ bis). Esclave et fils interviennent par ailleurs le plus souvent dans les textes de manière simultanée. On trouve alors aussi bien servus vel filius que filius vel servus. Quelquefois, filius apparait dans le passage avant servus, le juriste indiquant simplement que la solution est identique pour l'esclave, mais l'inverse se produit aussi et non moins fréquemment : rien ne permet donc d'avancer que le développement de la "capacité servile" ne serait que la répercussion de l'évolution concernant le filiusfamilias ( 80 ter). Il faut chercher ailleurs les causes de ce développement.

Outre cette constatation, qui est la plus importante, on peut ajouter que servus apparait parfois aux côtés d'un autre terme désignant le fils que filius : notamment minor (81), pupillus (82), adulescens (83), liber (84). Inversement, d'autres termes que servus peuvent entrer en relation avec filius : c'est le cas de homo (85), de mancipizon (86), de

80 bis- v. Tryphoninus D 49.15.12.1; Ulpien D 3.3.33 pr ; D 15.1.3.9; D 15.1.5.2.

80 ter- Filius précède servus chez Africain D $35.1 .42 ; 039.6 .23$; U1pien D 4.4.7.5; D 16.3.1.42; D 42.8.10.10; D 46.4.8.4 . Serrus précède filius chez Pomponius D 45.3.40; Papinien D 35.2 .13 ; Paul D 12.2 .22 ; D 48.10.22.5; Olpien D 2.14.7.18; D 15.3.3.3; D 33.8.6.4; D 42.8.6.12 D 48.10 .22 .5 ; OlPien D 2.14.7.18; D 15.3 .3 .3 ; D 33.8 .6 .4 ; D 42.8 .6 .12 ;
D 47.2.52.15. M. RASER, RPR I2, p. 343 , 605 ; v. Supra $n .544$ et 569 ( $1^{\circ}$ part.). 81- Servus/minor chez Valens D 4.4 .33 (Julien); Julien D 30.102 ; Papiaien D 33.7.3.1 ; Ulpien D 4.3.7pr (Juliea) ; D 4.4.11.1 (Scaevola ; constitution de Marc-Aurèle)-4 (Julien, Marcellus)-5 (les mêmes). Ancilla/minor chez Paul D 4.4.48.1.

82- Servus/pupillus chez Gaius D 26.7.13pr ; Julien D 40.2.6 ; Maecianus D 36.1.67.3 ; Scaevola D 15.3.20.1 ; D 15.3.21 (filia) ; D 28.6.48.2 Papinien D 40.1.20pr-1 ; Paul D 15.4.2pr ; D 29.2.60 (Labéon, Proculus, Iavolenus); D 40.2.15pr; Ulpien D 15.1.7.1 (Julien, Marcellus); D 26.7.9pr (Julien) ; D 27.8.1.15 ; D 28.6.10.1; D 40.2.16.1 ; Modestin D 26.7.32.3 (pupilla) ; D 29.2.50; I. Aquila D 26.7.34.

83- Servus/adulescens chez Papinien D 26.7.39.18; Ulpien D 15.4.1.9 (Labéon).

84- Liber dans le sens d'enfant, bien entendu, (sur ce sens, A. ERNOUT et A. MEILLET; Dictionnaire étymologique cit. p. 355) : Ulpien D 29.2 20.1 ; D 35.1.92; D 37.4.3.15; D 38.9.1.14.

85- Sur ancilla et serva, ef supra n 78 et 79 . On trouve hums/filius chez Scaevola D 10.2.39.4; Ulpien D 44.2.11.8.

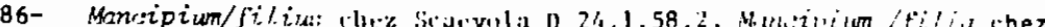
Scaevola 041.9 .3 : Yapillien 1) 11.77 .17 : 0 39.5.31.2: 010.8 .8 (cunsti-

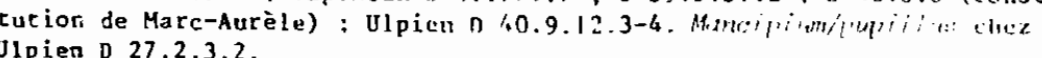

statuliber (87). Filius peut aussi s'opposer à un nom propre d'esclave (88). Chaque fois que ces termes sont en relation avec filius, on a affaire à un rapport de domination.

Il nous reste à examiner la relation gervus/liber.

\section{III -" Servus/liber}

La relation servus/liber marque une opposition statuaire. Liber peut désigner $1^{\prime}$ homme libre, l'ingénu, et c'est là que l'opposition prend toute sa valeur, On trouve par exemple cette signification de liber chez Gaius :

D 1.5.3 lib. 1 Institutionum : Summa itaque de iure personarm divisio haec est, quod ommes homines autliberi sunt aut servi.

Liber peut aussi indiquer l'affranchissement de l'esclave ; c'est le sens de cun testamento servus liber esse iussus est (89). L'opposition servus/liber est alors toujours de nature statutaire, l'emploi de liber dans ce sens étant plus proche de manumissus que de libertus qui désigne véritablement l'état d'affranchi.

La répartition des textes où cette opposition apparaît s'opère aingi :

\section{- Servus/liber dans le sens esclave/ingénu $(90)$}

- Anelyse des paragraphes

\begin{tabular}{|c|c|c|c|c|c|c|c|}
\hline I & - & $=$ & - & $I$ & - & $=$ & - \\
\hline II & $0 / 1$ & $=$ & - & II & $1 / 1$ & $=$ & $100 \%$ \\
\hline III & $27 / 228$ & $=$ & $21 \%$ & III & $101 / 128$ & $=$ & $79 \%$ \\
\hline IV & $7 / 66$ & $=$ & $11 x$ & IV & $59 / 66$ & $=$ & $89 \%$ \\
\hline $\mathrm{v}$ & $132 / 437$ & $=$ & $30 \pi$ & $\mathrm{v}$ & $305 / 437$ & $=$ & $70 \%$ \\
\hline TOTA & $166 / 632$ & $=$ & $26 \%$ & $\mathrm{v}$ & $466 / 632$ & $=$ & \\
\hline
\end{tabular}

87- Statuliber/filia chez Paul D 46.3.98pr. Statuliber et filia apparaissent dans le même texte, mais ne sont pas liés, chez Ulpien D 5.2.8.10 ; D 39.6.8pr (Julien); D 40.7.6.4.

88- Stichus/filius chez Paul D 45.1.49pr.

89- Pomponius D 40.4.11.2

90- On relève 1 'opposition servus (ou servitus)/2iber (ou libertas)
Semus/liber dens le sens esclave/affranchi ( 91 ) 


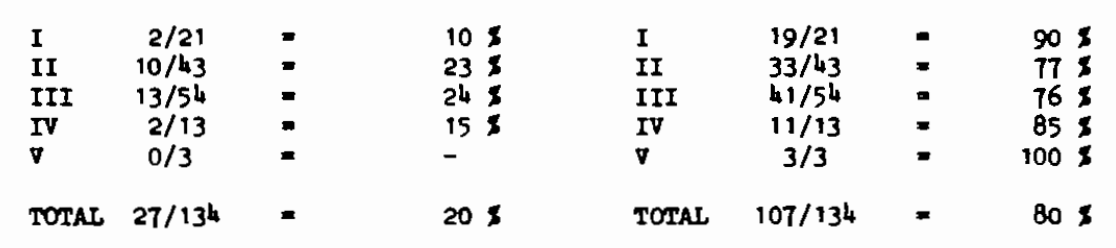

Il ressort clairement de ce tableau que l'opposition servus/liber dans le sens esclave/affranchi est preponderante, quelle que soit la ptriode prise en consideration. C'est dire la place importante que tient l'affranchissement, sur lequel on reviendra, dans les écrits des juristes romains.

dans ce sens chez Iavolenus D 11.1.14pr; D 18.1.63pr; D 40.12 .42 (Labéon) ; D 45.3.34; Celsus D 40.2.19; Julien D 21.2.39.3; D 40.12.30 (Sabinus, Cassius); :D 41.2.38pr; D 41.4.7.8; Pomponius D 9.4.33; D 13.

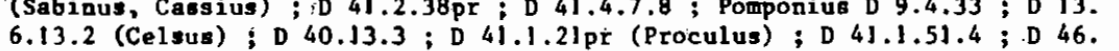

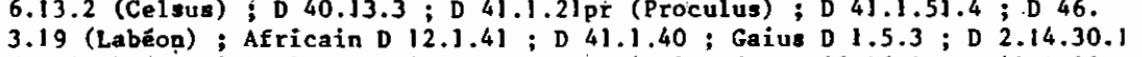
(Julien); D $28.5 .31 \mathrm{pr}$; D $40.12 .25 .2 ; \mathrm{D} 40.12 .26$; D 40.16 .1 ; D $41.1 .10 \mathrm{pr}-$ 4 ; D 44.7.5.5; D 47.10.12 ; Venuleius D 43.29.4.1 (Trebatius) ; D 45.2. 12.1 (Julien) D 46.8.8.2 (Labéon); D. 48,19.6.3; Marcellus D 40.5.10.2 ; Scaevola D 21.2.69pr:; D 44.7.30.; Papinien D 16.1.27.1; D 33.3.5 ; D 41 .' $3.44 \mathrm{pr}$; D 43.10.1.2 ; D 45.1.118pr ; Tryphoninus D J.5.15 ; D 49.15.1.2.9 (Sabinus) ; Menaider D. 40.12.29pr ; D 49.16.4.10; Callistrate D 48.10.15. 3 ; D 48.15.6.2; D 48.19.28.1.1-16; D 49.14.12 (rescrit d'Antonin 1e pieux) ; Paul D 2.1.9 (Pomponius, Octavenus); D 2.1. pieux) (Jaul D 2.19 (Pomponius, Octavenus) ; D 2.1.7 ; D 4.2.8.1 ; D 4 . $13.1 ; \mathrm{D} 14.1 .5 \mathrm{pr} ; \mathrm{D} 14.2 .2 .2 ; \mathrm{D} 15.1 .47 .6 ; \mathrm{D} 15.1 .52 \mathrm{pr} ; \mathrm{D} 18.1 .5 ; \mathrm{D} 18$. I.34.2; D 18.2.14.3 ; D 22.3.8; D 22.6.1.2; D 28.5.58; D 28.6.43.3; D 40.7.4.4; D 40.12.17.; D 40.12.23pr-1; D 40.12.24.4; D 40.13.4; D $40.13 .5 ; D 41.2 .3 .10 ; D 41.3 .15 .1 ; D 41.3 .31 .2 ; 045.1 .83 .5 ; D 45$. J.126.2 ; D 45.3.20pr; D 47.9.4.1 (rescrit d'Aneonin le pieux); D 49.15.19. 10 ; D S0.2.9pr ;.D S0.17.175pr ; Ulpien D 1.1.4;D 3.2.4.3 (Pomponius); D 3.3.39.5; D 3.5.5.9 (Pomponius) ; D 4.9.7pr ; D 7.8.4pr : D 9.3.1pr ; D 11.3.1.1; D 11.3.5.1 ; D 14.1.1.4 ; D 14.3.1 (Marcellus); D 14.3.7.1.; D 14.4.1.5;D15.1 1.6;D15.1.3.8 (Nerva f.);D21 2.23.6;D24.1.3.3-8; D 24 .

3.22 .13 ; D 26.2.22; D 27.3.1.8; D 29.1.13.3; D 29.2.6.7; D 33.5.2. 2 ; D 38.2.16pr-2; D 39.4.1.5; D 39.4.3.3; D 40.5.24.3; D 40.7.6.1; D $40.12 .3 \mathrm{pr} ; \mathrm{D} 40.12 .7 \mathrm{pr}-5$; D 40.12 .10 (Alfenus, Julien) ; D 40.12.12pr-12 ; D 40.12.14pr ; D 40.12.27.1 (rescrit d'Hadrien); D 40:12.34 (constitution de Caracalla).:D 41.1.23pr3 (Julien, Scaevola) ; D 41:2.13.3;
D 43.16.1.21;D 43.24.3 pr ; D 43.29.3.7; D 45.1.81 $\mathrm{nr}$ (Ce1sus) ; D 47. $2.14 .17 ;$ D $47.2 .52 .15 ; D 47.4 .1 .1-2 ;$ D $47.5 .1 .5 ; D 47.5 .1$ pr-3; D 47 $6.3 .2 ; \mathrm{D} 47.7 .7 .4 ; \mathrm{D} 47.8 .2 .5-14 ; \mathrm{D} 47.10 .3 .4 ; \mathrm{D} 47.10 .5 .11 ; \mathrm{D} 47.10$ 11.9 ; D 47.10.15.16 (Labéon) 45 (Mela) 48 ; D 47.10.17.3 ; D 48.2.5 (rescrit de Marc-Aurèle); D 48.5.28.5 (Africain) ; D 48.8.4.2 (rescrit d'Radrien); D 48.10.8; D 48.18.1.8; D 48.19.8.11; D 49.16.8; Macer D 48 . 7.3 ; D 48.19: $10 \mathrm{pr}$; Marcien D 1.5.5 pr-3; D 1.16.2 pr; D 48.6.3 pr; Hermogeñen D 1.5.13; D 40.15.3 ; D 41.2.50.1 ; D 47.10.45.

On trouve quelquefois liber-tas dans le même sens, sans autre indicateur : Yavolenus D 41.2.23.2 ; Julien D 22.3.20; D 37.10.7.3 ; Pomponius D 18.1.4; D 18.1.6 pr (Ce1 sus) ; D 50.17.20; Gaius D 10.4.13; D 28.1.8.4; D 40.12.9 pr ; D 40.12.25 pr-1;D 48.15.4; D 48.19.29; D 50.17.122; Venuleius D 40.14.2 pr ; D 43.29 .4 pr-2 ; Callistrate D 48 . 19.28.6 (rescrit d'Hadrien) ; D 49.14.3.9 (rescrit d'Hadrien) ; D 50.13.5. 3 ; Paul D 1.5.14; D 4.6.13 pr ; D 4.8.32.7; D 10.4.12 pr ; D 40.12.24 $\mathrm{pr}-2-3$; D 40.12.33; D 40.12.41 pr-1; D 41.2.30.1; D 42.1.38 pr (const $\mathrm{i}$ tution d'Antonin 1e pieux) ; D 49.1.25 (Alexandre-Sévère) ; D 50.17.106 : ulpien D 4.3.24; D 21.1.42; D 40.5.24.9 (Marcellus; rescrit de Sévère et Caracalla); D 40.7.6.2; D 40.12.3.1; D 40.12.7.1-2; D 40.12.8 pr ; D $40.12 .12 .3-4-5-6 ; D 40.12 .14 .1 ; 040.12 .16 .2 ; D 40.12 .22 .3 ; D$ D 40.12 . 27.2 ; D $40.13 .1 \mathrm{pr}$; D 41.1 .23 .1 ; D $43.29 .1 \mathrm{pr}-1$; D $43.29 .3 .1-6-9$; D 47 . 6.1 .1 ; $D 48.1 .5 .1$ (constitution de Sévère et Caracal1a); $\mathrm{D} 48.15 .1$; $\mathrm{D} 48$. 18.12 (rescrit d'Hadrien) ; L. Rufinus D 18.1.70; Macer D 49.1.9; Marcien D 48.22.15 pr ; A. Charisius D 48.18.10.6.

Dans certains textes, liber, -tas peut avoir les deux significations (affranchi, ingénu). C'est par exemple le cas chez Iavolenús D 45.3 .34 : Si servus testamento manumissus, cion se liberum (af franchi) esse ignoraret... Distat ista cousa eius, qui liber (né libre) emptus bona fide servit. Ceci explique que des textes relevés dans cette rubrique le soient aussi dans celle qui suit.

91- Servus (ou servitus)/Ziber (ou libertas) dans ce sens chez Alfenus (chez auteur anonyme) D $11.3 .16 ; \mathrm{D} 33.8 .14 ; \mathrm{D} 40.1 .6 ; \mathrm{D} 40.1 .7$; D 40 . $7.14 \mathrm{pr}^{-1}$; D 44.7.20 ; Proculus D 12.6.53; Iavolenus D 15.3.2 ; D 28.8.11 (Trebatius, Labéon); D 32:29.4. (Tubero, Labéon); D 32.30.2 (Trébatius, Labéon) ; D 33.5.14 ; D 33.5.15 ; D 35.1.40.3 ; D 40.1.26 ; D 40.7.28 pr ;; D 40.7.39 pr (Q. Mucius, Gallus, Servius, ofilius, Labêon)-3 (Servius,

Labéon) -4 (Trebatius, ofilius, Labéon)-5 (2iberare servum) ; D 45.1 .104 ; D 45.3.34; Neratius D 28.5.55; D 40.7.17; Celsus D 13.1.15; D 28.7.21; D 33.8.25; Julien D $9.4 .16 ; \mathrm{D} 10.2 .52 .1 ; \mathrm{D} 13.1 .14 \mathrm{pr} ; \mathrm{D} 15.1 .14 \mathrm{pr}$ : D 28.1.12; D 28.5.7 pr; D 28.5.8 pr-1; D 28.5.38.1-2-4; D 29.7.2.2 ; D 30.81 .10 ; D $30.91 .1-4$; D 30.102 ; D 33.5.9.1-2 (Q. Mucius) ; D 34.3. 12 ; D 36.1.26.1-2-3; D $40.2 .4 \mathrm{pr}-1$; D $40.4 .18 \mathrm{pr}$ (Sabinus); $\mathrm{D} 40.4 .19$; D 40.5.47.1-2-3; D 40.7.13.1-2-4; D 40.9.7.1 (Proculus); D 41.4.9; Pomponius D 28.5.21.1 ; D 30.54.2 ; D 31.1.1 pr (Labéon) ; D 33.2.20 ; D 33.9 .6 ; D 34.4 .20 ; D 35.1.57; D 40.1.13 (Octavenus) ; D 40.4 .8 ; D 40.4.11 $\mathrm{pr}^{-2}$; D 40.4.40.1 (Ofilius) ; D 40.4.41 pr-1 ; 40.4 .61 pr (Julien)-2 (Octavenus) ; D 40.5.8; D 40.5.34.1.(Campanus) -2 (Sabinus) ; D 40.5.44:D 40.7.21 pr (veteres Laben) : 40.7 .29 .1 (Q Mucius Lat béon, Aristo, Celsus); $\mathrm{D} 40.12 .28$ (pris par ex voluntate domini); $\mathrm{D} 40.12$ 43 (rescrit d'Hadrien); Africain D 12.1.41; D 30.107.1;D 30.108.9-14; 43 (rescrit d'Hadrien); Africain D 12.1.41; D 30.107.1 ; D 30.108.9-14;

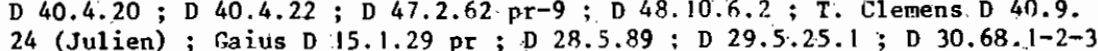
24 (Julien); Gaius D 15.1.29 pr;D $28.5 .89 ;$ D 29.5 .25 .1 ; D 30.68.1-2-3
(Julien); D35.2.73.3; D 36.1.65.15; D 40.4.24; D 40.9.10; D 40.9.29 pr-1; D 47.4.2 ; Maecianus D 29.4.28.i ; D 35.2.30.8 ; D 40.4.55 pr ; D 40.5.32.1; Venuleius D 34.4.32.1; D 40.12.44 pr ; Marcel1us D 9.2.36.1 (Sabinus) ; D 18.7.4 ; D 28.4.3.1 (rescrit de Marc-Aurèle) ; D 29.1.31 ; 
D $29.5 .16 ;$ D $35.1 .47 ;$ D 35.2.56.3-4;D 37.15.3; D 39.6.38; D 40.1.15; D 40.4 .42 ; D 40.5 .9 ; D $40.5 .10 \mathrm{pr}^{-2} ; \mathrm{D} 40.5 .56 ; \mathrm{D} 40.13 .2 ; \mathrm{D} 46.3$. 68 ; D 46.3.72.5-6; Florentinus D $28.5 .50 \mathrm{pr}$; Scaevola D 4.3.32 ; D 10. 2.39 .2 ; D 21.2.69 pr-4-5; D 28.5.84 pr-1; D 28.5.86; D 28.6.48.2; D 32.37.3; D 33.8.23 pr-1-2 ; D 34.1.20.3; D 40.4.29; D 40.4.54 pr D 40.4.59 $\mathrm{pr}$; D 40.5.19 $\mathrm{pr}-1$; D 40.5.41 pr-1-2-4-6-7-10-11; D 40.7. 40.2-3-7-8 ; D 48.10.24 ; Papinien D 4.4.31 ; D 14.3.19.1; D 26.7.37.1 ; D 29.5.21 pr ; D 31.65.3; D 31.76.4; D 33.7.3.1 ; D 33.8.19 pr ; D 35.1 $77 \mathrm{pr}$ (rescrit d'Antonin le pieux) ; D 35.2.11.1; D 36.1.54.1 ; D 36.1. 55 ; D 38.1.42.; D 38.2.41; D 40.1.19; D 10.1 .20 pr (iitterae de MarcAurēle)-3 ; D 40.1.21; D 40.4.51 pr-1; D 40.5.21; D 40.5.22 pr-1; D 40.7.34 Pr; D 40.7.35; D 40.7.36;D 44.2.29 pr; D 48.19.33 (rescrit de Marc-Aurèle et Verus) ; Tryphoninus D 1.5.15; D 12.6.64; D 15.1.57.1 (Julien) ; D 28.5.9.1 ; D 34.3.28.7 (Scaevo1a); D37.14.23 pr-1 (rescrit d'Hadrien) ; D 48.18.19; D 49.15.12.16; D 49.17.19.3 (constitution d'Hadrien)-4-5; Callistrate D 35.1 .82 ; D 48.10.15.2-3; Paul D 3.2.14; D 3.5.14 (Pomponius); D 3.5.18.1 (Sabinus, Scaevo1a) ; D 9.2.48; D 14.3.14; D 19.1 .43 (Julien, Ulpien) ; D 19.1.45.1 ; D 20.2.9 (servos liberari); D 28.5.58; D 28.5.85 $\mathrm{pr}^{-1}$ (constitution de Marc-Aurèle) ; D 28.5.90; D 29.1.40.1 ; D 29.2.58 ; D 29.2.60 (Labéon, Proculus, Tavolenus) ; D 29. $5.12 ; \mathrm{D} 29.7 .8 .5 ; \mathrm{D} 31.14 \mathrm{pr}^{-1} ; \mathrm{D} 31.82 .2$ (Valens, Julien); D 31.83 ; D $31.84 ; D 33.1 .16 ; 0$ D $3.5 .13 \mathrm{pr} ; \mathrm{D} 34.4 .26 \mathrm{pr} ; 34.9 .5 .15$ (Antonin ; D $31.84 ; D$ D 35 ; D 33.5 .13 pr ; D 34.4.26 pr ; D 34.9.5. I5 (Antonin le pieux) ; D 35.1.44.10; D 35.1.96 pr-1; D 35.2.1.4 ; D 35.2.36.3-4 (Africain) ; D $38.2 .4 \mathrm{pr} ; \mathrm{D} 40.1 .9$; D 40.1.11 ; D 40.1.14.1 (Lex d'Auguste) ; D 40.2.15 pr ; D 40.2.22; D 40.4.10.1; D 40.4.31; D 40.4.35 (Servius) ; D 40.4.36; D 40.4.38; D 40.4.39;D 40.4.52;D 40.4.53; D 40.4.56 (rescrit de Marc-Aurèle) ; D 40.5.5; D 40.5.29; D 40.5.31 pr-4 (rescrit de Caracalla) ; D 40.5.33 pr-1-2 ; D 40.5.39 pr-1 ; D 40.5.40.1 ; D 40.7.4.4; D 40.7.20 pr-2-3 (Sakinus, Julien) ; D 40.7.41 pr (Labéon) ; D 40.8.1 (rescrit de Marc-Aurèle) ; D 40.8.9; D 40.9.16 pr-3 (Aristo); D 40.9 .26 (Scaevola) ; D $40.12 .24 .3 ;$ D $40.12 .38 .2-3 ; 040.13 .4 ; D 41$. 3.4.16 (Sabinus, Cassius) ; D 41.4.2.14 (Ce1sus); D 42.5.4; D 44.5.2.2 D 45.1.83.5; D 47.8.3 (Labéon); D 48.10.22.3-5-7-9-10;D 49.17.20; D 50.17.146; Ulpien D 1.1.4; D 1.14.3 (Pomponius); D 2.9.5; D 2.14.7.18 (v, Verus, Marcellus) ; D 3.5.16; D 4.3.7.8 (Pomponius); D 4.4.5; D 4. 4.7.5-10 (Papinien; Antonin le pieux, Caracalla); D 4.4.11.1 (Scaevola; constitution de Marc-Aurèle) ; 04.8 .9 pr ; D 5.3.7.1 (rescrit d'Antonin le pieux); D 92.15.1 (Julien) ; D 9.2.23.1 (Julien) ; $09.4 .42 \mathrm{pr}$; D 10.2.49; D 11.3.7; D 12.4.3.7 (Ce1sus p., Ce1sus); D 12.4.5.1; D 15.1.3.1 (Labéon) ; D 15.1.9.5 (Julien) ; D 15.1.11.1; D 15.1.32 pr (Julien); D 15.2.1.7 (Pegasus, Africain); D 16.3.1.33 (Julien); D 21 . I. 17.16 (Caelius Sabinus) ; D 21.3.1.4; D 24.1.7.8 (Sabinus, Papinien constitution de Marc-Aurèle) ; D24.1.16 (Marcellus, Scaevola) ; D 24.1.22; D 24.3.24.4; D 26.2.10.4; D 26.2.22; D 26.4.1.3 (rescrit d'Antonin le pieux); D 28.5.3 pr-1; D 28.5.6.3 (Julien)-4 (Julien); D 28.5.9.16-17;

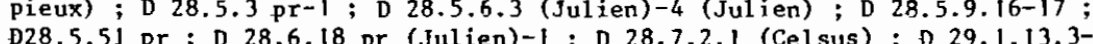

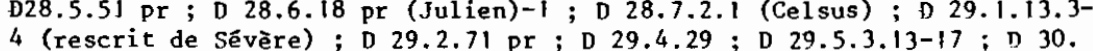
4 (rescrit de Sévère) ; D $29.2 .71 \mathrm{pr}$; D 29.4.29; D 29.5.3.13-17; D 30 34.9 ; D 30.44 .7 ; D 33.7.12.31 (Celsus) ; D 33.8.6.3 ; D 33.8.8.1 (Julien)-3 (Sabinus)-5 (Labénn)-7 (rescrit de Sévère et Caracalla); D 34.5 $10 \mathrm{pr}$; D 35.1 .92 ; D 36.1.17.13 (Maecianus) -17 (rescrit d'Antonin le pieux) ; D 36.1.23.1.; D 36.2.12.2 (Julien) ; D 38.2.16.9; D 38.16.3.4; D 38.17 .1 .4 ; D 38.17.2.3 ; D 39.5.19.4; D 40.1.2; D 40.1.4.3-9; D 40 . 2.3 ; D 40.2.16.1; D 40.4.I ; D 40.4.6; D 40.4.7; D 40.4.9.1; D 40.4. $13 \mathrm{pr}-2$ (Julien) ; D 40.4.14; D 40.4.30; D 40.5.2 (constitution de Ma Aurèle) ; D 40.5.4.14-15; D 40.5.24.1-2-3-4-5 (rescrit de Caracalla)-6 (rescrit d'Antonin le pieux)-8 (Ce1sus)-10-19-21 (rescritsd'Hadrien et
D 40.5.30.3 (rescrit de Marc-Aurèle et de Verus)-6 (rescrit d'Antonin le pieuxt12-17 (rescrit de Sévẽre et Caracalla); D 40.5.37 (rescrit de Marc-Aurèle) ; D 40.5.45.1-2 ; D 40.5.46.5; D 40.5.52; D 40.7.2 pr-1 (Cassius, Julien)-2 (Julien)-3 (Julien)-4 (Ce1sus); D 40.7.3.1-2 (Servius, Labéon, Cassius)-3-7-8-14-15 ; D 40.7.9 pr-3 : D 40.7.19; D 40.9.2 ; D 40.9.30 pr (constitution de Marc-Aurèle) ; D 40.12.7.5; D 40.12.12.2; D 40.13.1.1; D 42.6.1.18; D 44.5.1.4; D 45.1.51; D 47.4.1.1-2-3-6-7 (rescrits de Marc-Aurèle et de Sévère et Caracalla)-11-19; D 47.10.1.7 (Labéon); D 48.5.28.10; D 48.19.1.1 D 49.17.6; D 49.17.9 (Marcel1us) L. Rufinus D 40.5.16; D 40.7.32; Marcien D 28.5.49.2 (constitution de

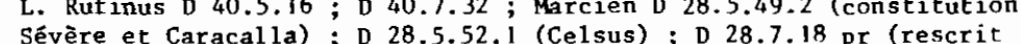
Sévère et Caracalla); D 28.5.52.1 (Celsus) ; D 28.7.18 pr (rescrit
d'Antonin le pieux); D 30.113 .1 (rescrit de Sévère et Caracalla) ; D 30. d'Antonin le pieux); D 30.113 .1 (rescrit de Sévère et Caracalla) ; D 30 . 114.10 ; D 38.4.6; D 40.1.5.1; D 40.1.8.2 (rescrit d'Antonin le pieux);

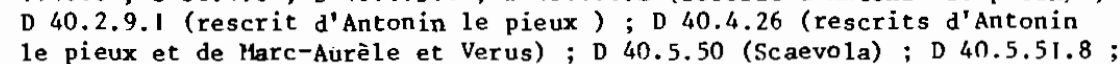
le pieux et de Marc-Aurèle et Verus) ; D 40.5 .50 (Scaevola) ; D 40.5 .51 .
D 40.8.6 (constitution de Marc-Aurèle) : D 40.9.9 pr ; D 48.1.9; D 48 . 10.7 (rescrit de Marc-Aurèle et de Conmode); Modestin D 28.1.19; D 31 . $34 \mathrm{pr}$; D 35.1.53 ; D 37.14.8.1 ; D 40.4.44; D 40.5.11 ; D 40.5.14; D 40.7.26 pr-1; D 40.8.2 (édit de Claude) ; D 40.9.19; D 40.9.20;D 48 . 18.14 ; Hermogénien D 5.1 .53 ; D 37.5 .23 (constitution d'Antonin le pieux) : D 40.9 .27 .1 .

On relève liber-tas dans ce sens sans autre indicateur chez Tavolenus $D 32.77$; Celsus D 34.5 .25 ; D 35.1 .49 ; Valens D 35.1 88 ; Julien D 28.2.13.1; D 28.5.22; D 29.7.4; D 35.2.87.3 ; D 40.4.17. 2 ; D 40.4.18.1-2;D 40.5.47 pr;D 40.7.12;D 40.7.13.3; D 40.9.5 pr ;

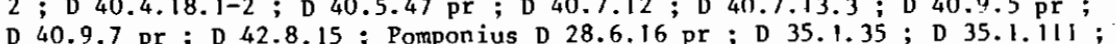
D 40.4 .46 (Aristo) ; D 40.7.8 pr ; D 40.9.23; D 50.16.120; Africain D 21.46 .3 ; 40.9 , T C D 28.7.22; D 29.4.17; D 29.5.9; D 35.1.90 (rescrit d'Antonin le pieux); D 49.14.14; Maecianus D 40.4.58; D 40.5.36.2 ; D 40.5.42 (Antonin le pieux) ; Marcellus D 28.5.54; D 40.7.24 ; F1orentinus D 29.1.24 (rescrit de Trajan) ; D 34.4.14.1 ; Scaevola D 40.4.54.1 ; D 40.5.41.12 ; Papinien D 20.1.3 pr ; D 28.3.17; D 29.7.11 ; D $31.76 \mathrm{pr}$; D 31.77.11-29; D 35.1. $78 \mathrm{pr}$; D 36.1.57.1 (rescrit d'Antonin le pieux)-2 ; D 37.7.6 ; D 40.4.50 pr (constitution de Marc-Aurèle) ; D 40.5.22.2 ; D 40.5.23 pr-3 ; D 40.9 . 25 ; D 40.15. 2 pr; Tryphoninus D 49.15.12.14; Paul D 4.6.13 pr ; D 4.8. 32.7 ; D 28.5.85.2; D 28.6.38.3 (Scaevola); D 29.2.74.4; D 35.2.39; D $38.1 .39 \mathrm{pr}: \mathrm{D} 40.2 .15 .1 ; \mathrm{D} 40.4 .33 ; \mathrm{D} 40.5 .27 ; \mathrm{D} 40.7 .4 .2-3-6-7$; D 40.7.10; D 40.7.18; D 40.7.41.1 (Labéon) : D 40.9.18 pr-1 ; D 40.12.24. 3 (voluntate domini) ; D 48.19 .38 .4 ; Vlpien $\mathrm{D} 3.5 .44 .1$; D 4.4 .9 .6 ; D 4.4 .22 ; D 5.1.67;D 5.2.8.16-17; D 5.3.7 nr (rescrit de Trajan); ${ }^{2}$ D $24.1 .9 \mathrm{pr}$; D 24.3 .64 .1 ; D 25.6 .1 .11 ; D 28.3.12.1; D 28.5.3.3 ; D 28 . $5.9 .18-19$; D 29.1 .19 .1 ; D 29.4 .10 ; D $29.4 .12 \mathrm{pr}$; D 29.5.1.5-13 (Scaevola); D 35.3.1.11-12; D 36.1.6.5; D 36.1.15.3; D 36.1.18.3; D 36.2.5.7 drien); D 40.1.4 pr (epistula de Marc-Aurèle et Verus)-5-10; D 40.2.16 $\mathrm{pr}$; D 40.4.12 $\mathrm{pr}-1-2$ (Julien) ; D 40.4.32; D 40.5.1; D 40.5.4.-2-3-4-5-6Pr-19-20; 40.5 .24 pr-9 (Marcellus; recrit de Sévère et Caracalla)-11-1418-20; 40.56 (rescrits d'Antonin le niemx et de Sévère et Caracalla) 8 (rescrit de Sévère et Caracalla)-9-10;040.5.28.1-2; D 40.5.30.4-7-910-11-13 (rescrit de Marc-Aurèle et Verus)-14-15(rescrít de Caracalla)-16 (rescrit de Marc-Aurèle); 0 40.7.3.5-9-11 (Trebatius, Labéon)-12 (Treba-

tius);D40.7.6.7;D40.12.7.4;D44.2.1;D44.5.1.9;D47.4.1 pr-4-8-9; D 48 .
$19.8 .4 ; \mathrm{D} 49.1 .14 \mathrm{pr}$ (Antonin le pieux)-1 (spistufa de Marc-Aurèle et verus); D 50.17.161; Marcien D 36.1.32 $\mathrm{pr}^{-1}$ (rescrit d'Antonin 1e pieux) : D40.4. 23.1 ; D 40.9.9.1 ; D 49.1.5.1 ; Modestin D 5.2.9 (Pau1) : D 31.34.6; D $35.1 .5 \mathrm{pr}-1$; D 38.1.32;D 40.4.43;D 40.4.45;D 40.5.12.2 (Fertinax) ; Hermogénien D 35.1.94.1; D $40.1 .24 \mathrm{pr}-1$. 
Pour mémolre, on peut aussi remarquer que liber ressort quelquefois accompagné de libertus, statuliber, manimissus. Le rapprochement n'est alors intéressant que si líber signifie ingénu. En ne tenant compte que des textes non relevés auparavant dans la rubrique serrus/liber, on trouve : sur libertus/liber, Paul D 43.1.2.1; Ulpien D 43.29.3.4; sur statuliber/liber, Ulpien D 30.44 .8 ; D 35.2.44; sur mantonissug/liher,

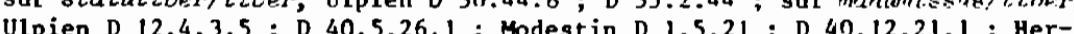

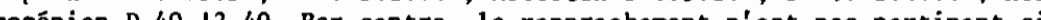
mogenien $D$ in iber signifie affranchi. On peut neanmoins signales les textes qui $n$ ' ont pas été intégrés dans la rubrique servus/liber : liber signifie affranchi (dans un texte od intervient Libertus) chez Pomponius D 38.1.12; D 40.5 . $34 \mathrm{pr}$; Gaius D 40.12.4 ; Maecianus D 40.5.36 pr; Marcellus D 23.2.50 (Liberta) ; Papinien D 33.8.19.1 ; Paul D 37.14.20; D 39.5.8; D 50.1. $22 \mathrm{pr}$; Ulpien D 38.1.7 pr ; D 38.1.13.1 (constitution de Marc-Aurèle) ; D 38.1 .36 (Labéon) ; D 38.2.37 pr (Julien); D 40.5.4.7-12-13; $\mathrm{D} 40.5 .28$. 3-5; D 44.5.1.5-6-7-8-10 (Cassius, U. Ferox) ; Modestin D 25.3.6 pr ; D 37.14 .7 pr (7.iberta) (décret de Vespasien); D 38.2.33: D 46.3.77; liber signifie affranchi (dans un texte ou intervient statuliber) chez Iavolenus D 40.7.28.1 (Cassius) ; Julien D 30.81.9; D 30.76.4 : Pommonius D $35.1 .110 ; D 40.7 .5$ (Neratius) : D 40.7 .11 (Aristo) ; Africain D 35.1.32; D 40.7.15 pr-1 ; Gaius D 9.4.15 (Sabinus, Cassius) ; D 21.2 . 54.1 ; Scaevola D 21.2.69.1 ; Tryphoninus D 49.15.12.10 (Sabinus)-11; Paul D 2.9.6 ; D 29.2.74.3 ; D 40.7.1 pr ; D 40.7.4 pr-1 ; D 40.7.20.5; D 40.7.38; D 40.9.16.4; D 49.15.19.6; Ulpien D 9.4.14.1; D 12.4.3.8 (Ce1sus)-9 ; D 29.2.20.4 (Julien, Papinien) : D 40.7.3.4-6-10-17; 0 40.7.6. 3-4-5-6 ; D 40.7.9.1-2 ; D 44.4.2.7 (Julien) ; D 48.19.9.16 (rescrit d'Antonin le pieux) ; Marcien D 40.5.51.3 ; Hermogénien D $35.1 .94 \mathrm{pr}$; D 36 . $4.11 \mathrm{pr}$; liber signifie affranchi (dans un texte oì intervient manumissus) Chez Iavolenus D 31.37 ; Pomponius D 40.4.4.2 ; D 40.4.5; D 40.5.34 pr ; Africain D 21.2.46.2 ; D 35.1.32 ; Maecianus D $35.1 .86 \mathrm{pr}$ (Julien)-1; Marcellus D 40.5.10.1; Scaevola D 34.1 .18 .2 ; D 36.2.27.1 ; D 42.8.23; Papinien D 12.6.58; D 26.2.28.1 ; D 27.1.24; D 33.8.19.1; D 34.1.10.1 ; D 40.1.20.1 ; D 40.5.23.1 ; D 50.1:17.8 ; Tryphoninus D 40.5.17 (Scaevola) Callistrate D 40.8.3 (rescrit de Marc-Aurèle et Commode) ; Paul D 18.7.3 ; D 34.4.26.1 ; D 40.4.27; D 40.5.31.2 ; D 40.9.15 pr (rescrit de Caracalla); D 40.9.16.4-5 ; D 40.9.17 pr (Marc-Aurèle) 1-2 ; D 50.17.179; Ulpien D 2.4.10 pr (constitution de Marc-Aurèle) ; D 24.1.7.9 ; D 26.4.3.2 (constitution de Marc-Aurèle)-3; D 29.2.71.1 ; D 38.1.13 pr et I (constitution de Marc-Aurèle) ; D 38.16.3.3 (idem) ; D 40.1.4.6-7; D 40.4.9 pr i D 40. 4.12 3 : D 40.4.25; D 40.5.4.7-13-16-18; D 40.5.26-2 (cescrits d'Anto4.12.3 0 (rescrits d'Anto(rect le pieux et de severe et Caracalla) 7 ; 0 40.5.30.1 ; D 40.5.4h.3 (rescrit de Sévère) ; D40.8.4 ; Marcien D 40.1.8.3 (rescrit d'Hadrien) : D $40.4 .23 \mathrm{pr}$; D 40.5.51 pr-4-6 ; D 40.9.9.2; D 40.9.11 pr-1 (rescrit de Marc-Aurèle et de Verus) ; Modestin D 31.31 ; D 34.5 .27 ; Hermogénien D $40.9 .27 \mathrm{pr}$.
Comme on l'a remarqué ì pronos des relations servus/dominus ou servus/filius, servus n'est pas le seul terme désignant l'esclave qui s'oppose à liber. On relève ainsi ancilla (92), serva (93) et mancipium (94), homo (95) ou fugitivus (96), s'odposant ذ̀ liber dans les deux sens de ce terme. Par contre, les mots risant l'enfant esclave (07), les noms de mstiers (98), et les noms propres ( $(00)$, lorsqu'ils sont les

92- Ancilla/libera dans le sens esclave/1ibre chez Proculus D 23.3.67; Ulpien D 25.4.1.10 ; Marcien D 1.5.5.2 ; dans le sens esclave/affranchie chez Proculus D 23.3.67 : chez Julien D 40.5.47.4 : faius D 16.1.13 pr : Scaevola D 32.38.1 ; D 40.4.59 pr ; D 40.5.41 pr-5; Paul D 29.1.40.2 ; D 40.1 .23 ; D 40.8.7 (constitution de Sêvère et Caracalla) ; D 40.8.9; D 41.7.8; U1pien D 40.4.13.3; D 40.5.26.5; D 40.5.45 pr-2 ; Marcien D 40.5.53 pr ; D 40.5.55.1 (Marcellus) ; D 40.15.1.4 (Marcellus) ; Modestin D 40.4.44; D 40.9.21 (Julien).

93- Serva/libera dans le sens esclave/ingénue chez Papinien D 24.3.42. 1 ; D 48.5.6 pr ; Callistrate D 48.15.6.2 ; dans le sens esclave/aff ranchie chez Paul D 29.1.40.2; D 41.7.8. Dans ce deuxième sens, on trouve aussi contubernalis chez Caius $D$ 40.7.31.1: Paul D 35.1.81 pr ; mater chez Scaevola D 40.4.60; uxor chez Marcien D 40.5.51.12; prostituta chez Paul D 18.7.9; Ulpien D 2.4.10.1. Dans le premier sens, on a mulier chez Pomponius D 40.13.3; Marcellus D 23.3.59.2 ; Paul D 50.2.9 pr. 94- Mancipizm/liber dans le sens esclave/ingênu chez ulpien D 3.2.4.2 ; dans le sens esclave/affranchi chez Pomponius D 21.2.34 pr ; Papinien D 40.4.48; D 40.8.8; Tryphoninus D 18.7.10 (Scaevola) ; Paul D 40.12.38. I ; D 49.14.45.3; dans le même sens, vicarius chez Paul D 40.4.10 pr. 95- Dans deux textes, homo vise simultanément un homne libre et un esclave (Hodestin D 45.1.103 : homo liber/mortuum hominem; Gaius D 44.7.1. 9 : homine libero/vel ian mortuo). Sinon, homo (esclave) s'oppose à liber (affranchi), chez Julien D 46.3.33 pr ; Pomponius D 40.4.40 pr ; Scaevola D 21.2.69.2 ; D 45.1.122.2 ; Papinien D 40.4.47 pr; Paul D 40.7.42 (Labéon) ; Ulpien D 46.1 .33 .

96- Fugitivus/liber dans le sens esclave/Libre chez Julien D 46.3.34.5; Gaius D 40.12.11 (par fugae) ; Callistrate D 11.4 .2 ; Ulpien D 47.10.22 ; D 9.2.13 pr; dans le sens esclave/affranchi, Paul D 40.7.4.8 (par in fuga).

97- Partus chez Scaevola D 40.5.41.5; Ulpien D 40.5.26.3 (rescrit de Sévère et Caracalla) ; Marcien D 40.5 .53 pr ; natus chez tlpien D 38,16 . 1.1 ; D 38.17.1.3; Marcien D 40.5.53.1; Modestin D 40.5.13; infans chez Gaius D 40.1.25; impubes chez Papinien D 26.5.13 pr (rescrit d'Hadrien) ; alumna chez Paul D 40.5.38 ; filius chez Gaius D 40.4.24; Maecianus D 40.5.54. Les termes envisagés dans cette note ainsi que dans les deux suivantes sont les seuls indicateurs de la présence d'un esclave. 98- Actor chez Scaevola D 34.1.18.3 ; Paul D 32.97 ; dispensator chez Paul D 46.3.62.

99- Iavolenus D 40.7.39.1 (Stichus) ; Celsus D $40.7 .23 \mathrm{pr}$ (Stichus); Valens D 32.12 (Pegasus) (Stichus) ; Julien D 28.5 .43 (Apollonius) ; D 29. 4.22.1 (Stichus); D 30.81.2 (Stichus); D 30.91.2 (Stichus) ; D 40.4.15 (Stichus) ; D 40.4.16 (Stichus) ; D 40.4.17 pr (Stichus)-1 (Pcomphizus) ; D 40.5 .48 (Stichus); D $40.7 .13 \mathrm{pr}$ (Stichus)-5 (Stichus); D 40.9.5.1 (Stichus)-2 (Stichus, Pamphizus) ; Pomponius D 40.4.4 pr (Stichus)-1 (Stichus); D 40.4.11.1 (Stichus, Fomphizizus) ; D 40.4.41.2 (Labéon) (Stichus) ; D 40.4.61.1 (Stichus); D 40.7.30 (Stichus); D 50.16.162.1 
seuls indirnt,eurs de J prózence d'un escjave, ne 3 'opposent ì liber que dants le sens esclaye/arrranchi.

Pour dégager de ces relations celle qui est dominante, il faut faire porter la comparaison sur des élfments pertinents. Ainsi ne convient-il de prendre en considération, à propos de servus/filiug que le rapport d'sssinilation, le rapport de domination n'ftant pas original vis-â-vis de servus/dominus. De même est-il seulement possible de terir compte de la relation sorvus/liber dans le sens esclave/ingenu, cette opposition dans le sens esclave/affranchi recoupant, pour des raisons de.jà exposéos, une réqlité différente

Par rapport au nombre total de textes où ces relations apparaissent par périodes en leurs éléments pertinents, on obtient :

analyse des paragraphes

- servus/dominus

$\begin{array}{lll}\text { I } & - & = \\ \text { II } & 2 / 2 & = \\ \text { III } & 144 / 196 & = \\ \text { IV } & 38 / 47 & = \\ \text { V } & 471 / 726 & =\end{array}$

$47=$

- gervus/filiue (assimilation)

$\begin{array}{llllllll}\text { I } & - & = & - & \text { I } 1 / 27 & = & 4 \% \\ \text { II } & 0 / 2 & = & - & \text { II } 15 / 81 & = & 19 \% \\ \text { III } 24 / 196 & = & 12 \% & \text { III } 22 / 125 & = & 18 \% \\ \text { IV } & 2 / 47 & = & 4 \% & \text { IV } 4 / 16 & = & 25 \% \\ \text { V } & 123 / 726 & = & 17 \% & \text { V } 0 / 1 & = & -\end{array}$

- gervus/liber (esclave/ingénu)

$\begin{array}{llllllll}\text { I } & - & = & - & \text { I } & 2 / 27 & = & 7 \% \\ \text { II } & 0 / 2 & = & - & \text { II } & 10 / 81 & = & 12 \% \\ \text { III } 27 / 196 & = & 14 \% & \text { III } 13 / 125 & = & 10 \% \\ \text { IV } & 7 / 47 & = & 15 \% & \text { IV } 2 / 16 & = & 12,5 \% \\ \text { V } & 132 / 726 & = & 18 \% & \text { V } & 0 / 1 & = & -\end{array}$

La relation la plus fréquente est oervus/dominus, quelle que soit la période. Quant à la relation servus/filius en tant que rapport d'assimilation, elle est légèrement moins importante, dans l'analyse des paragraphes, que servus/liber, mais les citations rétablissent un certain équilibre entre ces deux rubriques.

Du point de vue des relations terminologiques qu'ils utilisent, les juristes se préoccupent donc davantage du rapport de domination/subordination existant entre le maitre et l'esclave, symbolisant une intervention étatique importante, que de l'opposition statutaire entre esclave et libre. Par contre, on l'a vu à travers l'étude de serrus/liber dans le sens esclave/affranchi, une relation est loin d'être négligeable chez les juristes, celle qui distingue l'esclave de l'affranchi.

100- Pourcentage exact $73,46: 74$ \% pour arrondir total de la période a $100 \%$. 


\section{SECTION 2 - LA TERMINOLOGIE DE L'APRES-ESCLAVAGE}

Il s'agit de suivre la même démarche que celle adoptée à l'ágard de la terminologie de l'esclavage, c'ast-à-dire examiner dans un premier temps les termes désignant l'affranchi pour envisager par la suite les rapports s'établissant entre ces termes et ceux avec lesquels ils sont fréquenment en relation.

\section{Paragraphe 1 - Les termes désignant l'affranchi.}

Il existe à cet égard une gradation. Statuliber vise l'esclave affranchi par testament sous condition. Manumissus concerne l'opération de l'affranchissement. Enfin, libertus, $-a$ et libertinus, $-a$ désignent $l$ 'affranchi(e) proprement dit.

\section{I - Statuliber : une situation transitoire.}

Bien qu'il jouisse d'une situation assez avantageuse dès le décès du testateur, le statuliber est, esclave tant que la condition sous laquelle la liberté lui a été laissée n'est pas réalisée (101). C'est ce qui ressort de la résonance juridique de ce terme. Statuliber désigns le plus souvent un agent de droit (102) mais le nombre de textes qui en

101- Sur la situation avantageuse : l'hêritier ne doit rien faire qui rende impossible ou plus difficile la réalisation de la condition ; Celsus (D 40.7.23.1) admet que l'esclave affranchi sous la condition de reddere rationes utilise le pécule (ou plus prếcisément, vende les res peculiares) pour solvere reliqua. Sur le statuliber, f. DONATUTI, Lo statulibero, Milan (1940) ; M. BRETONE, Statuliber, in NNDI 18 (1971) p. $380 \mathrm{~s}$.

102- Statuliber est employé dans le sens d'agent d'acte juridique chez Iavolenus. D 40.7.28.1 (Cassius) ; D 40.7.39.2 (Trebatius, Labéon); Neratius D 40.7.17; Julien D 40.7.13.1 ; Pomponius D $31.11 \mathrm{pr}$ (Labêon); D 35.1 .110 ; D 35.1.112.3 (Neratius); D 40.7.5 pr (Artisto, Neratius)-1 ; D 40.7.8.1 (Labéon) ; D 40.7.11 (Aristo) ; D 40.7.29 pr ; Africaín 35. 1.32 ; D 40.4.22 ;D 40.7.15.1 ; Gaius D 21.2.54.1 ; D 35.2.76 pr ; traitent come un objet est loin d'âtre négligeable (103) puisqu'on en compte trente-cinq contre soixante-cinq dans le premier cas.

Cependant, la statuta libertas ne constitue qu'un des aspects de l'affranchissement.
D 39.6.31.2 ; Papinien D 39.6.41; D 40.7.34 pr-1 (rescrit de Caracalla); D 40.7.35; D 40.7.36; Tryphoninus D 49.15.12.11; Paul D 19.1.42; D 29. 2.74 .3 ; D 35.1.44.2 ; D 35.1.46; D 40.7.4 pr-1 (Julien) ; D 40.7.20.1 (Julien)-5; Ulpien D 5.2.8.10; D 5.3.13.6 (Sabinus); D 10.2.20.9 (Papinien); D 12.4.3.6-8 (Celsus) -9 ; D 29.2.20.4 (Julien, Papínien); D 35. 2.44 ; D 33.8.8 pr (Pegasus) 4 (Sabinus); D 39.6.8 pr (Julien) ; D 40.7 3.2 (Servius, Labéon, Cassius)-3-4-6; D 40.7.6.4-5; D 44.4.2.7 (Julien); Modestin D 5.2.12.1; D 35.1.66; D 40.7.27 (Ju1ien); Hermogénien D 35. $1.94 \mathrm{pr}$. Dans le sens d'agent de fait juridique, Julien $\mathrm{D} 9.4 .16$; Pomponius D 40.7.29 pr; Africain D 47.2.62.9; Gaius D 9.4.27.1; Paul D 2.9. 6 ; D 49.15.19.6; Ulpien D 9.4.14.1; D 40.7.6 pr (statulibera); D 40 .

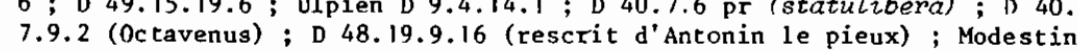
7.9 .2 (Octave
D 48.18 .14 .

103- Statuliber vise un esclave objet d'acte juridique chez Julien D 21. 2.39.4; D 30.81.9; Pomponius D 40.7.8.1 (Labéon); D 40.7.30; Africain D 21.2.46.2 ; D 40.7.15.1 ; Gaius D 12.6.63 (Neratius) ; D 21.2.54.1; Scaevola D 21.2.69.1-2-4-5: Papinien D 40.7.35; D 40.7.36: Tryphoninus D 49.15.12.10-11; Paul D 19.1.42; D 40.7.7; D 46.3.98 pr ; Ulpien D 10.2.12.2 ; D 30.44.8; D 40.7.2 pr ; D 40.7.3.17; D 40.7.6.3-5-6; D 40.7.9.1; D 44.4.2.7 (Julien) ; Marcien D 20.1.13.1; D 40.5.51.3; Modestin D 40.7.25; D 40.7.27 (Julien); Hermogénien D 36.4.11 pr. Objet de fait juridique chez Papinien D 47.2.81.1 ; Ulpien D 47.2.52.29. Ne sont pas intégrables dans ces rubriques trois textes concernant seulement la quaestio (Paul D 48.18.8.1 ; Ulpien D 29.5.1.4 : Marcien D 48.18.9.3) et d'autres dans lesque1s, bien que statuliber apparaisse, c'est un autre terme désignant 1 'esclave qui est porteur du rapport juridique : ainsí D 40.7.2.3 Ulpien (Julien) (servus agent et objet d'acte juridique); D 40.7.2.4 Ulpien (Celsus) (servus agent d'acte juridique) ;

D 40.7.39 pr Iavolenus ( $Q$. Mucius, Gallus, Servius, nfilius, Labéon) (servus objet d'acte juridique); 0 45.1.91.1 Paul (Julien) (homo 


\section{II- Mamumissus}

Manumissus a au contraire une vocation générale. Ce terme, qui signifie littéralement renvoyé de la main, geste représentant une des formalités de earactère symbolique de l'affranchissement, désigne le changement d'état entre l'esclave et l'affranchi, l'opération d'affranchissement. Compte tenu de 1a réalité plus large qu'il recoupe, il est évidemuent plus représenté que statuliber (104).

Contrairement à manumisous, libertus a'indique plus 1 'acte d'affranchissement mais 1 'esclave qui a déjà obtenu sa liberté.

objet d'acte juridique); D 46.3.33pr Julien (homo objet d'acte juridique) ; D 46.3.38.3 Africain (homo objet d'acte juridique) ; D 46.3.92.1 Pomponius (servus objet d'acte juridique).

104- Il est nécessaire de tenir compte également de manumissio, manumissor et monumittere qui recoupent la même réalité. On relève ainsi : Alfenus (chez auteur anonyme) D 11.3 .16 ; D 40.1.6; D 40.1.7; D 44.7.20; Iavolenus D 15.3.2; D 28.5.66 ; D 28.7.20.1 (Labéon); D 29.2.64; D 31.37 ; D 32.29 .4 (Tubero, Labéon); D 33.5.14; D 33.8.22pr (Labéon)-I (Trebatius, Labéon) ; D 40. 1.26 (Labéon) D 40.7.28pr ; D 45.1.104; D 45.3.34; Celsus D 1.18.17; D 32.79. D 40.2.19; Valens D 4.4.33 (Julien); D 35.2.37.1; D 36.4.15; D 38.1.47 (Campanus) ; Julien D 12.1.19pr ; D 17.1.30; D 28.5.38.4; D 30.81.2; D 30.91.5 ; D 30.92.1; D 30.94.3; D 30.99; D 30.102; D 33.5.10; D 36.1.26.3; D 36 . 1.28 .17 ; D 36.2.17; D 38.13.1; D 40.2.4pr-1 ; D 40.2.5 (Tavolenus) ; D 40.2 .6 D 40.4.15;D 40.4.19;D 40.5.47.1; D 40.5.48;D 40.7.13pr-4;D 40.9.5.2 ; D 40.9.7.1 (Proculus); D 41.4.7.2;D 43.24.14; D 46.3.32; Pomponius D 15.2.3; D 21.2.16.2 (Proculus); D 21.2.34pr; D 28.5.23.1; D 30.54 .2 ; D 33.5.6; D 35.1.6.1 (Neratius); D 35.1.58 (Proculus) ; D 38.1.4; D 38.4.13pr D 40.1.13 (Octavenus) ; D 40.2.1; D 40.4.3; D 40.4.4pr-2; D 40.4.5; 40.4 . Ilpr ; D 40.5.8; D 40.5.20 (Aristo, Julien, Octavenus) ; D 40.5.34pr-1 (Campanus) -2 (Sabinus) ; D 40.9.22 ; D 40.12.43 (rescrit d'Hadrien) ; D 40.14.3.1 ; D 44.7.56; D 45.3.40; D 46.3.83; D 46.3.92pr; D 47.2.44.2 ; Africain; D 19.1.30.1 ; D 21.2.46.2 ; D 30.108.14; D 30.109pr ; D 33.8.16pr ; D 35.1.32; D $40.4 .20 ; D 40.5 .49 ; \mathrm{D} 40.9 .8 \mathrm{pr}$; D $4 \mathrm{~T} .1 .40 ; \mathrm{D} 46.1 .21 .2$; T.Clemens D 29.2.82; D 40.9.24 (Julien) ; Gaius D 1.5 .6 ; D 4.7.3.1; D 5.3.3; D.6.1. 18 ; D 16.1.13pr; D 17.1.27.1; D 24.1 .8 ; D 26.8.9.1 ; D 33.8.4; D 38.8.2 ; D 39.4.13.2 ; D 40.1.18; D 40.2.7; D 40.2.25 (Fufidius, Nerva filius); D 40.4 . 57 (Sabinus, Cassius, Julien) ; D 40.7.37; D 40.9.3; D 40.9.10; D 40.12.9.2; Maecianus D 29.4.28.1 ; D 35.1.86pr (Julien)-1; D 35.2.32.5; D 40.5.32pr-1; D 40.5.35 (Cassius); Venuleius $D 40.12 .44 \mathrm{pr}$; D 40.14.2.1 ; Marcellus $D 18$. 7.4 ; D 19.1.23 (Julien) ; D 23.2.50; D 23.3.59.2; D 29.1.29.1 ; D 29.1.31 ; D 35.2 .34 (Julien) ; D 35.2.56.3; D 36.1.46pr; D 37.15.3; D 38.2.32; D 40.1. 15 ; D 40.5.10.1-2 ; D 40.5.56 ; Florentinus D 38.2.28pr ; Scaevola D 2.15. $3 \mathrm{pr}$; D 4.3.32; D 10.2.39.4; D 22.2.5pr ; D 23.4.29.2; D 31.88.3-6-11; D 32.37 .7 ; D 33.2.34 pr ; D 33.2.36pr ; D 33.7.7; D 33.7.27.3; D 33.8.21 (Julien); D 33.8.23.1; D 34.1.13.2; D 34.1.16.1-3; D 34.1.18pr-1-2-3; D 34.1.19; D 34.5.29; D 36.1.80.2; D 36.2.27.1; D 39.5.35pr; D 40.4.29;
D 40.4 .60 ; D 40.5 .19 .1 ; D 40.5.41.2-3-5-6-13-14-17; D 40.7.40pr-2-3-7 ; D 40.9 .6 ; D 41.6.5; D 42.8.23; D 45.1.122.2; D 50.16.243; Papinien D 4.4 31 ; D12.6.58; D 15.1.50.3; D 17.1.54pr-1 (à propos d'un iiber homo bona fide serviens) : D 18.7.6pr (Sabinus) ; D 18.7.8 ; D 24.3.61; D 26.2.28.1 D 26.5.13.1; D 26.7.39.18; D 27.1.24; D 29.2.86.2 ; D 29.7.13pr ; D 31.76.4 D 33.7.3.1; D 33.8.19pr-1-2 ; D 34.1.10.1; D 35.2.11.4; D 36.1 .55 ; D 38.1 . 42 ; D 38.2.41 ; D 40.1.19 ; D $40.1 .20 \mathrm{pr}$ ( Litterae de Marc-Aurèle)-1-2-3 ; D 40.1 .21 ; D 40.1 .22 ; D 40.3.3; D 40.4.47.1; D 40.4 .48 (Servius) ; D 40. 4.49 ; D 40.4.50.1 ; D 40.4.51pr ; D 40.5.22pr-1 ; D 40.5.23.1-2-4 ; D 40.7.34 pr ; D 40.12.35; D 40.14.4 ; D 44.2.29pr ; D 48.19.33 (rescrit de Marc-Aurèle et Verus) ; D 49.17.13 (rescrit d'Hadrien) ; D 50.1.17.8 ; Tryphoninus D 1.5 .15 D 12.6.64; D 12.6.64; D 15.1.57.1 (Julien) ; D 18.7.10 (Scaevola ; Hadrien, Marc-Aurèle); D 31.88.12 (Scaevola) ; D 34.3.28.7 (Scaevola) ; D 37.14.23.1 (rescrit d'Hadrien) ; D 38.2.50.1 ; D 40.5.17 (Scaevola) ; D 49.15.12.9 (Sabinus) -16 ; D 49.17.19.3 (constitution d'Hadrien) 4 ; Callistrate D $38.1 .38 \mathrm{pr}-1$; D 40.8 .3 (rescrit de Marc-Aurèle et Commode) : Paul D 1.7.36.1 ; D 2.4.9; D 4.4.48.1 ; D 4.5.3.1 ; D 5.3.36pr (Proculus, Julien, Mauricianus); D 9.4.24 (Labéon, Julien) ; D 11.3.14.9 ; D 11.7.25 ; D 12.6.13pr (Pomponius) ; D 12.6 . 65.3-8; D 15.1.26; D 15.1.53 ; D 16.3.21.1 (Trebatius) ; D 18.7.3; D 19.1. 43 (Jullen, Ulpien) ; D 19.5.5pr-2 (Julien)-5 ; D 20.2.9 (Nerva p.) ; D 21.1.47 pr (Labéon) ; D 21.2.26; D 23.2.14.2 ; D 24.2.9; D 24.3.63; D 28.1.14; D 28. 5.56 (Neratius) ; D 28.5.85.1 (constitution de Marc-Aurèle) ; D 29.1.40.2 ; D 29.2.80.2; D 29.5.10.1 (constitution de Trajan) ; D 30.35 ; D 31.8 .5 (rescrit d'Hadrien) ; D 31.83 ; D $31.84 ; \mathrm{D} 32.4$; D 32.8 .1 ; D 33.8 .1 ; D 33 . 8.3; D 33.8.15 (Alfenus) ; D 34.4.26.1;

D 34.4.27.1; D 34.9.5.4; D 35.1.37 (Neratius) ; D 35.1.81pr; D 35.2.33; D 35.2.36pr-2 ; D 36.3.7; D 38.1.16pr; D 38.2.51 (Labéon) ; D 39.6.39; D 39. 6.44 ; $\mathrm{D} 40.1 .3$; $\mathrm{D} 40.1 .9$; D 40.1 .10 (const1tution de Marc-Aurèle) ; $\mathrm{D} 40.1$. 11 ; D 40.1 .12 ; D 40.1.14pr-1 ( lex d'Auguste) ; D 40.1 .23 ; D 40.2 .4 .2 (Julien)
D 40.2.15pr-2-4-5 ; D 40.2.17; D $40.2 .18 \mathrm{pr}-1-2$; D $40.2 .22 ; \mathrm{D} 40.2 .24$; D 40 . $1.10 \mathrm{pr}$; D 40.4 .27 ; D 40.4 .36 ; D 40.4 .37 ; D 40.5 .6 ; D 40.5 .29 ; D 40.5 .31 .1 (rescrit de Marc-Aurèle et Verus)-2-3-4 (rescrit de Caracalla) ; D 40.5.33pr-2 ; D 40.5 .38 ; D $40.5 .39 \mathrm{pr}$; D $40.5 .40 \mathrm{pr}-1$; D 40.7.1.1; D 40.7.4.1 (Julien) D $40.7 .20 \mathrm{pr}-6$; D 40.8 .1 (rescrit de Marc-Aurele) ; D 40.8 .9 ; D 40.9 .13 ; D 40. 9.15pr (rescrit de Caraca1la)-1 (Julien) ; D 40.9.16pr-1-2-3 (Aristo)-4-5 ; D 40.9.17pr (Marc-Aurèle)-i-2; D 40.9 .26 (Scaevoiai i $40.9 .25 ; 2$ i $0 . j 2.32$ D $40.12 .38 \mathrm{pr}$ (constitution de Marc-Aurè1e) - 3; D 41.2.30.4; D 41.3.4.17;

D 40.12 .38 pr (const1tution de Marc-Aurè1e) -3 ; D 41.2.30.4; (Julien) -2 (Ju1ien) D 41.7 .8 ; D $44.5 .2 .2 ;$ D 44.7 .4 .5 ; D 45.1 .66 ; D 45.1 .91 .1 (Julien) -2 (Julien)
D 46.3 .35 (Alfenus); D 46.3 .98 .8 (Ce1sus) D $47.2 .42 \mathrm{pr}-1$ (Sabinus) ; D 47.2 .67 .3 (Julien) : D 47.8.3. (Labéon); $\mathrm{D} 47.10 .29$; $\mathrm{D} 48.10 .14 .2$ (édit de Claude) ; D 48.10 .17 ; D 48.10.22.3-7-10 ; D 48.20.7.1 ; D 48.22.13 ; D 49.14.45.3 ; D 49.17 .20 ; D 50.17 .179 : U1pien D 1.1 .4 ; D $1.10 .1 \mathrm{pr}-1-2$; D 1.12 .1 .1 ; D 1.14.1 ; D 1.18.2 ; D 2.4.8.1 ; D 2.4.10pr (constitution de Marc-Aurè1e)-4 ; D 3.5 .16 ; D 4.2.9.2 (Pomponius) ; D 4.3.7pr (Ju1ien)-8 (Pomponius) ; D 4.4. 11pr (rescrit de Sévère)-1 (Scaevola ; constitution de Marc-Aurèle) ; D 5.2.26 D 5.3.7.2 (rescrit d'Antonin le pieux) : D 7.4.15; D 9.2.15.1 (Julien) : D 9. 4.2.1 (Celsus, Julien, Marcellus) ; D 9.4.5.1 ; D 9.4.6 ; D 11.3.5.4; D 11.3.7 ; D 12.4.1pr ; D 12.4.3.4-5 (Neratius, Julien) ; D 12.4.5.1-2-3-4 ; D 14.4.9.2 (Labéon, Pomponius) ; D 15.1.9.5 (Julien) ; D 15.1.11.1 ; D 15.1.32pr(Julien) D 15.2.1pr-4-7 (Pegasus, Africain) ; D 15.3.3pr ; D 16.3.1.18 (Marcellus)-30 ; D 16.3.11 (Sabinus, Pomponius); D 17.1.8.5 (Julien) (à propos d'un liber homo bone fide serviens) ; D 20.2.6 (Pomponius) ; D 21.1.17.19; D 21.2.25 ; D 23.2.29; D 23.2.45pr (rescrit de Sévère et Caracal1a)-3; D 24.1.7.8 (Sabinus, Papinien; constitution de Marc-Aurè1e)-9; $\mathrm{D} 24.1 .9 .1$ (Ju1ien); $\mathrm{D} 24.3 .24$ 
(constitution de Marc-Aurè1e)-3-4 ; D 28.5.9.17.19 ; D 28.7.8.7 ; D 29.2.25.1; D 29.2.35pr; D 29.2.42.2-3; D 29.2.71.1-2; D 29.5.1.13 (Scaevola) ; D 29.5 . 3.15-16 : D 29.6.1.1; D 32.1.1; D 32.3.1 (Julien); D 33.8.8.2-8 (Julien): D 35.1 .92 ; D 36.1 .11 .2 (Antonin le pleux) ; D $36.1 .17 .16 ; \mathrm{D} 36.2 .7 .6$; D 37.12 . 1.2 ; D 38.1 .7 .2 ; D 38.1.13pr (constitution de Marc-Aurèle)-1 (idem); D 38 . 1.2 ; D 38.1 .7 .2 ; D 38.1.13pr (constitution de Marc-Aurèle)-1 (idem); $\mathrm{D} 38$.
$2.3 .3-4-8$ (constitution d'Hadrien) ; $\mathrm{D} 38.4 .1 \mathrm{pr}: \mathrm{D} 38.4 .3 .3$ (Pomponius) : $\mathrm{D} 38$. 5.1.24-25 (Pomponius) : D 38.16.1.1 (rescrits de Marc-Aurèle et Verus et de Caracalla) ; D 38.16.3.1-2-3 (constitution de Marc-Aurèle)-6-7 ; D 38.17.1.3 ; D 38.17.2.3 ; D 39.5.18.1 (Aristo: Pomponfus)-2 (Aristo, Pomponius) : D 40.1 .1 ; D $40.1 .2 ; \mathrm{D} 40.1 .4 .6-7-9-13 ; \mathrm{D} 40.2 .3 ; \mathrm{D} 40.2 .8 ; \mathrm{D} 40.2 .11 ; \mathrm{D} 40.2 .13$; D $40.2 .16 .1 ; \mathrm{D} 40.2 .20 \mathrm{pr}-1$ (constitution de Marc-Aurèle)-2-3 (Marcellus)-4 : D 40.3.1 (Marc-Aurèle) ; D 40.4.9pr-1; D 40.4.12.3; D 40.4.25; D 40.5.4.71.3-15-16-18; D 40.5.7 ; D 40.5.24.12 (Marcellus)-13 (Marcellus)-16-17-19-21 D $40.5 .26 \mathrm{pr}-1$ (constitution de Sévère)-2 (rescrits d'Antonin le pieux et de Sévère et Caracalla)6-7 ; D 40.5.28pr (Marcellus)- A; D $0.5 .30 .1-3$ (rescrit de Marc-Aurèle et Verus) -6 (rescrit d'Antonin le pleux) ; D $40.5 .45 \mathrm{pr}-1-2$; D 40.5.46pr-3 (rescrit de Sévère) -5 ; D 40.7.2pr ; D 40.7.3.15 ; D 40.8.4; D 40.9 .1 (Celsus) ; D 40.9.4; D 40.9.12pr-1-2 (Africain)-3-4-5-6 (Arricain)-7 D 40.9.14pr-1-2-3-4-5; D 40.9.30pr (constitution de Marc-Aurèle)-1-2; D 40 . 12.3pr ; D 40.12.31; D 41.2 .13 .8 ; D 42.8 .6 .5 ; D 44.5.1.4 ; D 44.7.14 ; D 46. 3.18; D 47.2.7pr; D 47.2.17.1 ; D 47.4.1.7 (rescríts de Marc-Aurèle et de Sévère et Caracalla) ; D 47.4.3 (Labéon) ; D 47.6.3pr ; D 47.10.1.7 (Labéon) ; D 47.10.17.7 (Labéon) : D 47.10.30pr ; D 48.18.1.13 (rescrit d'Antonin le pleux) ; D 49.17.6 ; D 50.1.1pr ; D 50.1.7; D 50.1.27pr ; L. Rufinus D 23.2 .51 pr-1; D 31.62 ; Marcien D 1.5.5.3 ; D 1.16.2pr-1; D 28.5.19.2 (constitution de Sévère et Caracalla) ; D 28.5.52.1 (Celsus) ; D 29.2.52pr (rescrit d'Antonin le pieux) ; D 30.112.1; D 33.8.18; D 33.8.20; D 34.1.2pr (rescrit de Sévère et Caracalla) ; D 37.14.3 (rescrit des mêmes) ; D 38.2 .22 (Julien ; rescrit d'Hadrien) ; D 38.2.29pr-1 ; D 38.4.6; D 40.1.5pr ; D 40.1.8pr-1-3 (rescrit d'Hadrien); D 40.2.9pr-1 (rescrit d'Antonin le pleux) ; D 40.2 .10 ; D 40.2 . $14 \mathrm{pr}-1$; D 40.4.23pr ; D 40.5 .50 (Scaevola) ; D 40.5.51pr-1-2-4-6-8-9 (rescrit d'Antonin le pieux)-10-11 (Antonin le pieux)-12; D 40.5.53.1; D 40.5.55pr-1 (Marcellus) ; D 40.8.6. (constitution de Marc-Aurèle) ; D 40.9.9pr-2 ; D 40.9 . $11 \mathrm{pr}-1$ (rescrit de Marc-Aurèle et Verus) ; D 48.22.2 (rescrit d'Antonin le pieux) ; D 49.14 .30 (rescrit de Sévère et. Caracalla) : Modestin D 1.5 .21 (a propos d'un homme libre qui s'est laissé vendre comme esclave) ; D 1.5 .22 ; D5.2.12 pr ; D 24.2.10; D 27.1.14.3; D 31.9; D $31.31 ; D 31.34 \mathrm{pr} ;$ D 34.5 . 27 ; D 35.1.66 ; D 37.14.8pr (rescrit d'Hadrien)-1 ; D 37.14.13; D 38.1.31; D 38.4.8 (Julien, Marcellus) ; D 38.8.7; D 40.1 .16 ; D 40.1 .17 ; D 40.2.21 (constitution d'Auguste) ; D 40.5.12pr (rescrit de Caracalla)-1 : D 40.5.13 ; D 40.5 .15 ; D 40.7.25; D 40.9.20 ; D 40.9.21 (Julien) ; D 40.12.21.1 ; Hermogénien D 5.1.53 ; D 40.2.23 ; D 40.9.27pr ; D 40.12 .40 ; $\mathrm{D} 50.1 .23 \mathrm{pr}$. Il arríve que manumittere soit employé comme équivalent d'emancipare, par exemple chez Ulpien D 35.1.92 ( $\mathrm{Si}$ cui legatum fuerit relictum isque rogatus sit liberos. suos emancipare, an cogi debeat manumittere? ; cette acception rare de manumittere a été écartée car elle ne concerne pas 1 'esclavage.

\section{III - Libertus et libertinus.}

Ces deux termes sont des dérivés de liber (105). Libertus vise essentiellement les rapports existant entre l'affranchi et son patron, tandis que libertinus distingue 1 'affranchi de 1 'ingénu (106). Libertus(107)

105- A. ERNOUt et A. MEILLET, Dictionnaire étymologique, cit., p. 355. 106- Gaius I,10 : Liberorum hominum alii ingenui sunt, alii libertini.

107- Libertus apparaît chez Alfenus (chez auteur anonyme) D $38.1 .26 \mathrm{pr}^{-1}$; D 40.1.7; D 48.22.3; Iavolenus D 28.5.66; D 33.8.22.1 (Trebatius, Labéon); D 38.1 .33 ; D 38.2.34; D 38.2.35; D 38.2.36; D 38.5.12 ; Neratius D 38.1.50. 1: Celsus D 31.29 pr; D 38.1.30 pr; Valens D 32.94 (Julien); D 34.1.22.1 ; Julien D $1.5 .26 ; \mathrm{D} 12.4 .11 ; \mathrm{D} 28.2 .13 .2 ; \mathrm{Dr} 32.94$ (Julien) ; D 34.1 .22 .1 ; 7 ; D 38.1.23 pr-1; D 38.1.24; D $38.1 .25 \mathrm{pr}-1-2-3-4 ; \mathrm{D} 38.1 .27$; D 38.2 .11 ;

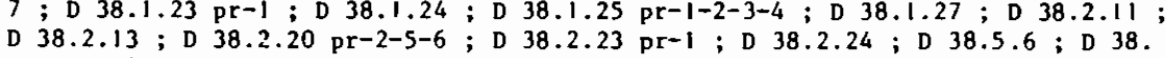
5.9; D 42.8.17.1; D 45.1.54.1; Pomponius D 38.1.3.1; D 38.1.4 (Aristo) ; D $38.1 .8 \mathrm{pr}$ (Labéon) -1 ; D $38.1 .10 \mathrm{pr}^{-1}$; D 38.1.12; D 38.1.34; D $38.2 .2 \mathrm{pr}-$ $1-2$; D 38.4.2 ; D 38.4.4; D 38.4.12; D 38.4.13 pr ; D 40.5.34 pr ; D 45.3. 38 (Ce1sus) ; D 50.16.171; Africain D 30.108.3-15; D 38.2.26; D 38.2.27 ; D 40.5.49; D 48.10.6.3; T. Clemens D 23.2.48.2; D 37.14 .10 ; D 38.2 .38 pr1 ; D 38.2.39; D 38.2.40; D 38.4.10; D 40.9.32.1-2 (Octavenus) ; Gaius D 38.1.22 pr-1 (Julien)-2 ; D 38.1.49 (Neratius); D $38.2 .5 \mathrm{pr}-1$; D 38.2.30 ; D 40.9.10; D 40.12.4; D 40.12.9.2; D 50.16.58.1 ; Maecianus D 36.1.66.3; D 40.5.36 pr-1; Venuleius D 40.12.44 pr; Marcellus D 19.1.23 (Julien); D 29. D $40.5 .36 \mathrm{pr}-1$; Venuleius D 40.12.44 $\mathrm{pr}$; Marcellus D 19.1.23 (Julien) ; D 29.
4.5 ; D 31.17.1; D 35.2.52 pr; D 36.1.46 pr; D 38.2.31; D 38.2.32; D 39 .

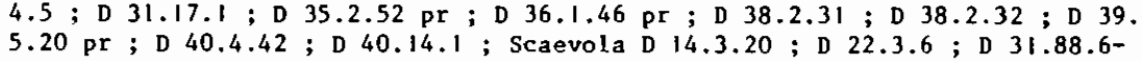
9-11-14; D 31.89 .6 ; D 32.35 pr-2 ; D 32.37.2; D 32.38.1-2; D 32.39 pr (constitution de Marc-Aurèle) ; D 32.41.1-2; D 32.93.2; D 32.102.3; D 33. 1.13 .1 ; D 33.1.18 pr ; D 33.1.20.1; D 33.1.21 pr-1-4;D 33.2.33.1-2; D 33. $2.34 \mathrm{pr}$; D 33.2.35; D 33.7.20.1 ; D 33.7.27.3; D $33.8 .23 \mathrm{pr}$; D 34.1.13 pr1 (rescrit d'Antonin le pieux) ; D 34.1.15.2; D $34.1 .16 \mathrm{pr}-1-3 ; \mathrm{D} 34.1 .18$ pr-3-5; D 34.1.19;D 34.1.20 pr-1 ; D 34.3.31.1 ; D 34.4.30.1; D 35.1.108; D $35.2 .25 .1 ; D 35.2 .94 ; D$ D $36.1 .80 .13 ; D 36.3 .18 \mathrm{pr} ; \mathrm{D} 37.14 .18 ; \mathrm{D} 38.1$. 44 ; D 38.1.45;D $38.2 .48 ; \mathrm{D} 38.4 .7 ; \mathrm{D} 39.5 .35 \mathrm{pr} \mathrm{D} 40.5 .41 .6-15 ; \mathrm{D} 40.7$. 40.2 ; D 50.16.243; Papinien D $1.9 .9 ;$ D $2.4 .14 ; D 3.5 .30$ pr-4; D 7.1 .57 . 1 ; D 18.6.19 pr; D 23.2.62.1; D 24.3.61;D 26.2.28.2; D 26.5.13.1; D 26. 5.14 ; D 27.1 .24 ; D 27.1 .30 .3 ; D $28.5 .79 \mathrm{pr}$; D 31.67.7; D 31.77.13-15-21$27-28-32$; D 31.79 ; D 33.1.10.3 ; D 33.7 .3 pr ; D 33.8.19.1 ; D 34.1 .8 ; D 34.1.9 $\mathrm{pr}-1$; D 34.1.10 pr ; D 35.1.71.2; D 35.1.72.1; D 35.1.101.4; D 37.10 .11 ; D 38.1 .41 ; D 38.2.41; D 38.2.42.1-2-3 (Marc-Aurèle) ; D 38.4 . 11 ; D 40.1.22 ; D 40.4.48 (Servius); D 40.4.50.1; D 40.10.1 pr ; D 48.2.2 pr; D 48.5.39.9; D 49.17.13 (rescrit d'Hadrien); D 50.1.17 pr-1; Tryphoninus (Scaevola); D $34.5 .9 .2 ;$ D $34.9 .22 ;$ D 37.14 .23 .1 (rescrit dithadrien); D 37. (Scalo; D 38.2.15; D 38.2.50.1-2-3-4-5-6; D 48.5.43; Callistrate D 23.2. $15.10 ;$ D 38.2 .15 ; D $38.2 .50 .1-2-3-4-5-6$; D 48.5 .43 ; Callistrate D 23.2 .
$64 \mathrm{pr} ;$ D 38.1 .38 .1 ; D 40.12 .37 ; D 47.9.7; D 49.14 .2 .6 (constitution de Sévêre) ; D 50.1 .37 .1 ; D 50.6 .6 .4 ; Paul D 2.4 .11 (Labéon) ; D $2.4 .15 ; \mathrm{D} 2.7$. $2 \mathrm{pr} ; \mathrm{D} 5.3 .32$; D 10.2.41; D II.3.14.9; D 12.2.30.4; D 12.6.65.8; D 17.2. 10 ; D 17.2.71.1; D 19.5.5.5; D 21.2.26; D 22.5.4; D 23.2.37; D 23.2.66.1; D24.2.9;D25.3.9:D26.7.12.3;D29.1.37;D29.2.73(Labéon);D29.5.7;D29.5.10.1 
(constitution de Trajan) ; D 31.87.2 ; D 33.1.9 (Papinien) ; D 34.1 .12 ; D 34. 2.4 (Servius) ; D 34.4.29 ; D 34.9.21 ; D 35.1.43pr (Proculus, Cassius, Plautius) ; D 35.1.84 ; D37.14.6pr-1-2-4; D 37.14.15 ; D 37.14.19 ; D 37.14.20 ; D 37.14.24 ; D 37.15.8 ; D 38.1.16pr; D 38.1.18 (Sabinus) ; D 38.1.20.1 (Proculus) : D 38.1.37.4-7-8 ; D 38.1.39.1 ; D 38.1.51; D 38.2.4pr-1-2-3; D 38. $2.9 ; 038.2 .18 ; 038.2 .42 \mathrm{pr}$ (Papinien); D $38.2 .44 .2 ; 038.2 .46$; D 38.2 $47 \mathrm{pr}-1-2-3-4 ; \mathrm{D} 38.2 .49 ; \mathrm{D} 38.2 .51$ (Labéon) $\mathrm{D} 38.5$ i1 38.45 .8 ; D 40.5 25 (Valens); D 40.5 .31 .3 ; D $40.5 .33 .1 ; \mathrm{D} 40.7 .20 .6 ; \mathrm{D} 40.11 .4 ; \mathrm{D} 42.1 .19 \mathrm{pr}$ D 43.1 .2 .1 ; D 44.5 .2 .2 ; D $45.1 .73 \mathrm{pr} ; \mathrm{D} 46.1 .56 \mathrm{pr} ; \mathrm{D} 46.3 .98 .1$; D 47.2 .90 ; D 43.1 .2 .1 ; D 44.5 .2 .2 ; D $45.1 .73 \mathrm{pr}$; D $46.1 .56 \mathrm{pr}$; D 46.3 .98 .1 ; D 47.2 .90 D 47.2.92 (Labéon) ; D 48.10.22.8 ; D 48.18.20 ; D 50.1.22pr-2 ; D 50.16 .70 ; Ulpien D 1.1.4; D 1.7.15.3; D 1.12.1.2-10; D 1.16.9.3 ; D 2.1.10 ; D 2.4.8.12 (Celsus, Julien) ; $\mathrm{D} 2.4 .10 .2-4-8-10-11$ (Labéon)-12 (Pedius) ; D 2.4.12 ; D3.3.35pr-1 ; D 3.5.5.7 ; D 4.3.7.8 (Pomponius); D 4.3.11.1 (Labéon) ; D 5.1 . 52.1 ; D 5.3.16.6 ; D 7.8.2.1 (Tubero, Labéon, Celsus) ; D 9.3.5.1 (Trebatius) D 11.7.6pr (Papinien) ; D 12.2.1.3pr ; D 12.6.26.12 (Celsus, Julien, Marcellus) D 16.3.1.14 (Labéon, Pomponius) ; D 17.1.12.8 (Papinien) ; D 21.1.17.15 (Labéon, Caelius Sabinus) ; D 22.3.14 ; D 22.3.18pr (Julien) ; D 23.2.13 ; D 24.1.9.1 (Julien) ; D 24.3.24.4; D 24.3.64.3-5-8-10; D 25.3.5.18-21-22 (Marcellus) -23 (Marcellus)-25-26 ; D 26.1.8; D 26.4.1.3 (Marcellus ; rescrit d'Antonin le pieux) ; D 26.4.3.3 ; D 26.7.3.4 (rescrit de Sévère et Caraca11a) ; D 26.10.2 ; D 26.10.3.1; D 27.2.1.2 ; D 27.3.1.6 (Mela)-8; D 27.9.5.11; D 29.2.20.2 ; D 29.4.29; D 30.95 ; D 31.24 (Marcellus, Scaevola); D 33.4.1.10 (Julien); D 34.1.3 (rescrit d'Antonin le pleux) ; D 34.1.14.3; D 35.3.3.4 (rescrit de Marc-Aurèle et Verus) ; D 36.1.18.4 (Papinien) ; D 37.12.1pr-2:37.14.1;37.14.2: D $37.14 .17 \mathrm{pr}$ (Proculus, Julien, Maecianus ; rescrit de Marc-Aurèle et Verus) -1 (Proculus, Julien) ; D 37.15.1.1 ; D 37.15.7.4 ; D 37.15.9 ; D 38.1.2.1 D 38.1.7pr-1 (Celsus) ;D 38.1.13.1 (constitution de Marc-Aurè1e)-3 ; D 38.1. 15pr-1 (Ce1sus, Papinien); D 38.1.29; D 38.1.36 (Labéon) ; D 38.2.1pr (Servius) -1 ; $038.2 .3 p r-6-7-9-14-18-19-20 ; 0$ 38.2.6pr (Marcellus)-1 (Marc-Aurè1e) vius) -1 ; D 38.2.3pr-6-7-9-14-18-19-20; D 38.2.6pr (Marcellus)-1 (Marc-Aurèle) $-2-4$; D 38.2.8.3-4-5 ; D 38.2.10pr-1 (Jialien) ; D 38.2.12.1.6-7 ; D 38.2.14pr 4-5-6-7-8-9-10-11 ; D 38.2.16.4-5 (Ju1len) ; D 38.2.17; D 38.2.37pr (Julien) -1 (Julien) ; D 38.3.1pr-1 ; D 38.4.1pr-1-2-5-8 (Julien) ; D 38.4.3pr-3 (Pom ponius)-4-5-7 ; D 38.4.5.1 (Julien) ; D 38.5.1pr-6 (Julien)-10-11-12-13-14 (Pomponius) -16-17-19-20-22-24-26-27 (Pomponius, Papinien) ; D 38.5.3pr (Papinien)-3-5 (Papinien) ; D 38.5.4pr-1 ; D 38.16.3pr-1-3 (constitution de MarcAurèle) $-4-6-7-8$; D 38.17.2.24-27; $\mathrm{D} 39.5 .16$; $\mathrm{D} 40.3 .2 ; \mathrm{D} 40.4 .7$ (Neratius) ; D 40.5.4.7-12-13-23 ; D 40.5.24.21 (rescrits d'Hadrien et d'Antonin le pieux) ; D 40.5.28.3-5; D 40.5.30.3 (rescrit de Marc-Aurèle et Verus)-5-12 ; D 40.7 . 2pr ; D 40.9.30.4-5 (Ju1ien) ; D 40.12.3.3 ; D 40.12.5pr ; D 40.14.6 ; D 12. 4.5.1 ; D 43.16.1.43; D 43.29.3.4; D A4.4.4.16; D 44.5.1.4-5-6-7-8-10 (Cassius, U. Ferox)-11-12; D 44.7.25.1 ; D $46.4 .13 \mathrm{pr} ; \mathrm{D} 47.4 .1 .2$; D 47.10 7.2 ; D 47.10 .11 .7 (Marcel1us) -8 ; D 48.18 .1 .9 ; D $48.23 .1 \mathrm{pr} ; \mathrm{D} 49.17 .6$; D 50.4.3.8 ; D 50.16.195.1; D 50.17.126.1; Macer D 42.1.5.3; D 48.2.11.1; D $48.5 .25 \mathrm{pr}$; D $48.20 .8 \mathrm{pr}-1-2-3$; Marcien D 1.8 .6 .1 ; D 1.22.2 ; D 2.4.23;

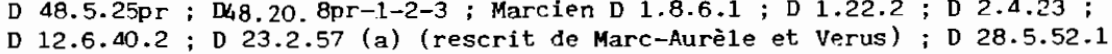
(Celsus) ; $D$ 30.114.1-14 (rescrit de Sévère et Caracalla) ; $D$ 34.1.2pr (rescrit đes mêmes) ; D 34.4.13 (idem) ; D 34.9.1 (idem) ; D 35.3.33.2 (idem) ; D 37.14.4 (idem) ; D 37.14.5pr (C1aude)-1; D 37.15.4 (rescrit de Sévè re et Caracalla) ; D 38.2.22 (Julien ; rescrit d'Hadrier) ; D 38.2.29pr ; D 38.5.2 : D 40.5 .53 .1 ; D 40.8 .5 ; D 40.15.1.4 (Marcellus) ; D 48.5.34.1 ; D 48.19.11.1; D 48.22.16 ; Modestin D 2.4.25; D 25.3.6pr-1 (constitution de Commode) ; D 26. 6.21 ; D 26.10.9 ; D 27.1.10.7; D 27.1.14pr-2 (rescrit de Caracalla)-3-4;

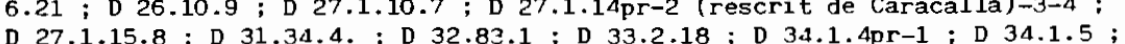
D 37.14 .7 .13 ; ${ }^{37}{ }^{14.8 p r}$ (rescrit d Hadrien)-1; D $37.14 .9 p r-1 ;$ D 37.14 .12 ; est beaucoup plus fréquent que libertinus (108). D'alutre part, les terines désignant l'affranchi apparaissent plus souvent que ceux indiquant la présence d'une affranchie, liberta et libertina (109) : cela ne tient probablement pas au fait que les affranchis sont plus nombreux, mais plutôt à ce que libertus a une vocation terminologique plus globale que $i_{i}$ berta, de la nême manière que servus par rapport à serva ou à ancizla.

D 38.4 .9 ; D 40.1.16; D 40.11.5pr; D 46.3.77; D 48.4.7.2; D 50.16.105 ; D 50.16.107 ; Hermogénien D 26.5.27.1 ; D 27.1.43 ; D 37.14 21 pr-1-3-4; D 48.4.9 (décret de Sévère) ; A. Charisius D 48.18.10.4. Les textes où on trouve collibertus ou conlibertus, affranchi d'une même personne, ont été intégrếs dans la liste, que ccs termes apparaissent seuls (Scaene, ont été intégrês dans la liste, que ccs termes apparaissent seuls (Scae18.4 (Papinien); D 37.15 .1 .1 ) ou en compagnie de libertus (Valens D 32.94 18.4 (Papinien) ; D 37.15 .1 .1 ) ou en compagnie de libertus (Valens D 32.94
(Julien); Tryphoninus D 32.38 .5 (Scaevola); Ulpien (Marcellus) D 47.10.11.7) 108- Libertinus apparaît chez Iavolenus D 28.8.11 (Trebatius, Labéon) Julien D 38.2.20.4; Pomponius D 1.2.2.7; D 23.2.8; Gaius D 1.5.6; D 38. $2.5 \mathrm{pr}$; Marcellus D 23.2.32 ; Florentinus D 38.2.28 pr ; Papinien D 23.2. 34.3 ; Tryphoninus D 27.1.44 pr (rescrit de Sévère et Caracalla)-1-3; D 48. 5.43 ; Callistrate D 48.15.6.2 ; Paul D 23.2.16; D 23.2.44.1; D 23.2.47; D 29.2.22 (Pomponius); D 31.83 ; D 38.1.37 pr ; D 48.20.7.1; U1pien D 1.5. 25 ; D 1.5.27; D 1.7.46; D 2.15.8.7; D 22.4.6 ; D 24.1.3.1; D 26.4.1.1; D 36.1.18.4 (Papinien) ; D 38.1.2 pr; D 38.5.1.4; D 38.7.2.1; D 38.17.2.2 (Julien ; constitution de Caracalla) ; D 40.10.6 (rescrit d'Hadrien) ; D 40. $14.6 ; 040.16 .4 ;$ D 47.10.9.4; D 50.1.6.3; Macer D 1.18.16; D 49.4.2.2; Marcien D 1.5.5 Pr ; D 40.11.2; Modestin D 1.5.21; D 23.2.42.1; D 27.1.1. 4 (rescrit de Sévère) : D 38.10.4.9; D 40.11.5.1; Hermogénien D 26.5.27.1. Il faut également tenir compte des textes où on a iibertinitas (Venuleius D 40.14.2.1; Ulpien D 22.3.14 et D 40.16.2.3).

109- On trouve liberta chez Celsus D 38.1.30.1; Valens D 38.1.46; Julien D 42.6.6.1 ; Pomponius D 38.1.34; T. Clemens D 23.2.48 pr-1; D 40.9.31 (Julien) ; D 40.9.32 pr ; Gaius D 23.2.46 (Lavolenus) ; Marcellus D 23.2.50; Scaevola D 31.88 .6 ; D 33.2.34 pr ; D 33.7.20.6; D 34.1.15.2; D 34.1.16.1; D 34.4 .30 .1 ; D 40.5.41 F ; Papinien D 23.3.69.6 ; D 31.77.28; D 37.15 .11 ; Tryphoninus D 34.1.16.2 (Scaevola) ; Paul D 19.1.45.2; D 25.7.2; D 37.14 .6 ; pr-3 (Julien)- 4 ; D 38.1.28; D 38.1.35; D 40.12.39.3; D $50.1 .22 \mathrm{pr}$; U1pien D 12.2.16 ; D 23.2.45 pr (rescrit de Sévère et Caracal1a)-4-6 (Julien) ; D 24.1 .3 .1 (rescrit de Sévère) ; $D 24.2 .11$ pr (Julien)-2 (rescrit de Sévère et Caracalla) ; D 25.3.5.24 ; D 25.7.1 pr ; D 38.1.2.1 ; D 38.1.13.4-5; D 38. $3.1 \mathrm{pr} ; \mathrm{D} 38.4 .1 \mathrm{pr}-1$; D 38.11.1.1 ; D 38.16.3.5 (dans ce texte, on relève. aussi conliberta)-6 ; D 40.12.3.3 ; D 40.12.5 pr ; D 40.10.11.7 (Marcellus); Marcien D 23.2.28 ; D 24.3.35 ; D 25.7.3 pr ; Modestin D 24.2.10 ; D 34.1 .4 pr-1 ; D 37.14.7 pr (décret de Vespasien) : D 50.16.105; Hernogénien D 24. 1.62 .1 ; D 38.1 .48 pr-2. On relève libertina chez Celsus D 23.2.23 ; Pomponius D 23.2.8; Paul D 23.2.44 pr-1; Ulpien D 23.2.27; D 23.2.31; D 23.2. 56 ; D 24.1.32.8; D 38.17.1 pr ; D 50.16.46.1 ; Marcien D 23.2.58.2 (rescrit d'Antonin le pieux); Modestin D 38.10.4.9. On peut savoir, en 1'absence de ces termes, qu'on a affaire à une affranchie, par exemple Paul D $4.2 .21 \mathrm{pr}$
(mulier ingrata/patronus) ou Scaevola D 31.88 .14 (Iibertis utriusque sexus). 
Bien que mimumissus soit le terme le plus employé, il convient de faire porter l'étude des relations terminologiques sur iibertus et libertinus car celux-ci marquent véritablement l'état d'affranchi (110).

\section{Paragraphe 2 . Les relations terminologiques}

Comme en matière d'esclavage, elles sont multiples, c'est pourquoi il est nécessaire de ne comptabiliser que celles qui sont pertinentes : la relation de domination libertus / patronus et les relaticns d'opposition statutaire libertus / servus et libertinus / ingemus.

\section{I - Libertus/patronus : la relation de domination.}

Il est permis de tenir également compte de l'opposition liberta / patronus, $-a$ car, à 1 'exception de libertina, pendant de libertinus, on ne rencontre pas, contrairement à ce qui se produisait pour la femme esclave, une terminologie diversifiée.

Les textes ou on relève le rapport libertus,-a/patronus, -a se répartissent ainsi (111) : analyse des paragraphes

$\begin{array}{ll}\text { I } & \\ \text { II } & \\ \text { III } & 56 \\ \text { IV } & 16\end{array}$

analyse des citations

$\begin{array}{lr}\text { I } & 2 \\ \text { II } & 16 \\ \text { III } & 26 \\ \text { IV } & 6 \\ \text { V } & 7\end{array}$

110- Manumissus, - $a$ apparaît dans 662 textes (supra n. 104), libertus, $-a$ dans 648 (supra n. 107 et 109). Libertus et libertinus désignent l'état d'affranchi, alors que manumissus et même liber visent l'affranchissement. 111- L'opposition libertus/patronus ressort de : Alfenus (chez auteur anonyme) D 38.1.26.1; Lavolenus D 38.2.34; D 38.2.36; D 38.5.12 ; Celsus D 38.1.30 pr-1; Valens D 38.1.46 (Ziberta) ; Julien D 1.5.26 ; D 30. 81.4 ; D 37.10.7.7; D $38.1 .23 \mathrm{pr}-1$; D $38.1 .24 ; \mathrm{D} 38.1 .25 \mathrm{pr}-4 ; \mathrm{D} 38.1$ 27 ; D 38.2.13 ; D 38.2.20 pr-2-5-6; D 38.2.23 pr-1 ; D 38.2.24; D 38.5 6 ; D 38.5.9 ; D 42.E.6.1 (Ziberta); D 45.1.54.1; Pomponius D 38.1 .4 (Aristo): D38.1.8pr(Labếon);D38,1;10pr-1;D38.1.12;038.1.34(2ibertus, -a);D $38.2 .2 \mathrm{pr}-1$ :
D 38.4.2; D 38.4.12; D 50.16.171; Africain D 38.2.26; D 38.5.10; D 48 10.6 .3 : T. Clemens D 23.2.48n (liberta) -1 (liberta)-2 : D 37.14.10; D 38.2.38.1 ; D 38.2.39; D 40.9.31 (Julien) (liberto) ; D 40.9.32pr (libertr )-1 ; Gaius D 38.1.22pr-1 (Julien) ; D 38.1.49 (Neratius) ; D 38. 2.5pr-1 ; D 40.12 .4 ; D 40.12.9; Maecianus D 36.1.66.3 ; Venuleius D 40.12 . 44pr ; Marcellus D 29.4.5 ; D 35.2.52pr ; D 38.2.31 ; D 39.5.20pr ; D 40.14 . 1: Scaevola D 22.3.6; D 31.88.11;D 32.35pr ; D 33.2.33.1 : D 33.8.23pr

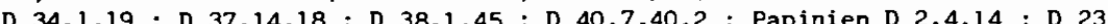

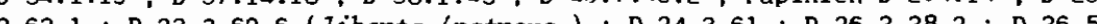
.62.1 : D 23.3 .69 .6 ( Liberta /patrona ) : D $24.3 .61 ; 0$ Da. D 13.1 ; D 26.5.14 ; D 27.1.24 ; D 27.1.30.3 ; D 31.77.28 (libertus, liberta) ; D 33.1.10.3 (patrona) ; D 33.7.3pr ; D 34.1.9.1; D 35.1.72.1 ; D 35.1.101.4 : D 37.15.11 (Liberta /patrona) ; D 38.1.41; D 38.2.41; D 38.2.42.1-2-3 (MarcAurèle ) ; D 38.4.11 ; D 48.5.39.9; D 50.1.17pr-1 ; Tryphoninus D 26.7.58pr (Scaevola) ; D 34.5.9.2 ; D 37.14.23.1 (rescrit d'Hadrien) : D 37.15.10 ; D 38.2.50.1-2-3-4-5-6; D 48.5 .43 ; Callistrate D 38.1.38.1 ; D 49.14.2.6 (constitution de Sévère) ; D 50.1.37.1 ; Paul D 2.4.11 (Labéon) ; D 2.1.15 ; D 2.7.2pr ; D 12.2.30.4; D 17.2.71.1 (collibertus) ; D 19.1.45.2 ; D 22.5.4 D 23.2.66.1 ; D 25.3.9 ; D 25.7.2 (liberta) ; D 29.1.37 ; D 29.2.73 (Labéon) D 35.1.43pr (Proculus, Cassius, Plautius) ; D 37.14.6pr-2-3 (Julien) (Liberta) D $37.14 .19 ; \mathrm{D} 37.14 .20 ; \mathrm{D} 37.15 .8 ; \mathrm{D} 38.1 .18$ (Sabinus); D 38.1 .20 .1 (Proculus); D 38.1.28 (liberta); D 38.1.35 (2iberta); D 38.1.37.4; D 38.1 . 39.1: D 38.1.51: D 38.2.4.1-2-3:D 38.2.18 (patrona) ; D 38.2.42pr (Pap 1nien) (patronus, patrona) ; D 38.2.44.2; D 38.2.46;D 38.2.47.1-2; D 38.2 . 49 ; D 38.5.11 ; D 40.5.31.3; D 40.11.4 : D 42.1.19pr; D 44.5.2.2 ; D 46.3. 98.1 : D 47.2.90 ; D 47.2.92 (Labéon) : D 50.1.22pr-2 ; U1pien D 1.12.1.2-10 D 1.16.9.3; D 2.4.8.1-2 (Celsus, Julien) ; D 2.4.10.2-10 (2iberta) -11 (Labéon)-12 (Pedius) ; D 2.4.12; D 3.3.35.1 ; D 4.3.11.1 (Labéon) ; D 5.3. 16.6 ; D 11.7.6pr (Papinten) ; D 12.2.13pr ; D 12.2.16 (Ziberta) ; D 12.6. 26.12 (Celsus, Julien, Marcellus) ; D 17.1.12.8 (Papinien); D 21.1.17.15 (Labéon, Caelius, Sabinus) ; D 22.3.18pr (Julien) ; D 23.2.13 (patrona) : D 23.2.45pr (rescrit de Sévè̀re et Caracalla) (2iberta )-4 (2iberta) -6 (Julien) (Liberta) ; D 24.2.11pr (Julien) (Liberta) -2 (rescrit de Sévè re Caracalla) (liberta); D 24.3.64.5-8; D 25.3.5.18-23 (Marcellus)-24 (iiberta)-25-26; D 25.7.1pr (2iberta); D 26.1.8; D 26.4.1.3 (Marcellus ;

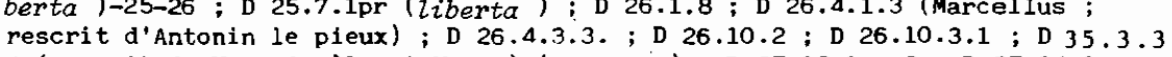
4 (rescrit de Marc-Aurèle et Verus) (patrona); $\mathrm{D} 37.12 .1 \mathrm{pr}-2$; $\mathrm{D} 37.14 .1$ (patronus, patrona) : D 37.14.2; D 37.14.16pr (Julien) D 37.14.17.1 (Proculus, Julien) ; D 37.15.5.4 (patrona ) : D 37.15.9 ; D 38.1.13.3-4 (2iberta) -5 (2iberta) ; D 38.1.15.1 (Celsus, Papinien) ; D 38.1 .29 ; D 38.1 .36 (Labéon) D 38.2.1pr (Servius)-1 ; D 38.2.3pr-6-7-14-18-19-20 ; D 38.2.6pr (Marcellus) -1 (Marc -Aurèle)-2-4 ; D 38.2.8.4-5 ; D 38.2.10pr ; D 38.2.12.6-7 ; D 38.2 14.4-6-8-9-11; D 38.2.16.4 (rescrit de Marc-Aurèle et Verus)-5 (Julien) : D 38.2 .17 (patronus, patrona); D 38.2.37pr (Julien)-1 (Julien) ; D 38.3.1pr (Libertus, Liberta), ; D 38.4.1pr (2ibertus, liberta)-5 (ius patronatus) (Libertus, liberta ) : D 38.4.1pr (Zibertus, liberta)-5 (ius patronatus
-8 (Julien) ; D 38.4.3pr ; D 38.5.1.6 (Julien)-10-11-12-13-14 (Pomponius)-17 -8 (Julien) ; D 38.4.3pr ; D 38.5.1.6 (Julien)-10-11-12-13-14 (Pomponius)-17
$-19-22-24-26-27$ (Pomponius, Papinien) ; D 38.5.3pr (Papinien)-3-5 (Papinien) -19-22-24-26 27 (Pomponius, Papinien); D 38.5.3pr (Papinien)-3-5 (Papinien) D 38.5 .4 .1 ; D 38.11 .1 .1 (Ziberta) ; D 38.16.3pr-5 (Ziberta ) ; D 40.9 .30 .
$4-5$ (Julien) ; D 40.14 .6 ; D 43.16.1.43 ; D 44.4 .4 .16 ; D 44.5.1.4-5-6-7-8-10 (Cassius, U. Ferox) $-11-12$; D 44.7.25.1 ; D 47.4 .1 .2 ; D 47.10.7.2; D 47.10. 11.7 ; D 48.18.1.9 ; D 48.23.1pr ; D 50.4.3.8; D 5C.16.195.1 ; Macer D 48.2. 11.1 ; D 48.20.8pr-1-2 ; Marcien D 2.4.23; 012.6 .40 .2 ; D 23.2.28 (Ziberta); D 24.3.35 ; D 30.114.1 ; D 34.9.1 (rescrit de Sévère et Caracalla) ; D 37.14. 4 (rescrit de Sévère et Caracalla); $D$ 37.14.5pr (Claude) $-1 ; D 38.2 .29 \mathrm{pr}$; D 48.19.11.1: Modestin D 2.4.25: D 24.2.10 ( liberta) ; D 25.3.6pr-1 (constitution de Commode); D 26.10.9; D 27.1 .10 .7 ; D 33.2.18; D 34.1.4pr

(libertus liberta/patronus, patrona)-1 (libertus, liberta) ; D 37.14.7.1 ; (libertus liberta /patronus, patrona)-1 (Jibertus, liberta) ; D 37.14.7.1 ;
D 38.2.33 ; D 38.4.8 (Julien, Marcellus) ; D 38.4.9; D 40.11.5pr ; D 48.4 .72 tiermogénien D 24.1.62.1 (2iberta) ; D 37.14.21pr-1-3-4; D 38.1.48pr (Ziberta )-2 (liberta/patrona) 
Il est possible par ailleurs que patronus soit en relation non pas avec libertus, mais avec libertinus (112), ou bien avec liber, dans le sens d'affranchi (113), manumissus (114), ou que la présence de l'affranchi soit simplement sous-entendue par ius aureorwm anulorum (115).

Cette relation est-elle prépondérante par rapport aux relations statutaires ?

II - Libertus/servus et libertinus/ingenuus : les relations statutaires.

Il faut distinguer à propos de libertus/servus deux sortes de textes : dans les uns, servus et libertus visent le même individu qui passe donc de l'ótat d'esclave à celui d'affranchi (116), ainsi un passage de Julien (D 38.1.25.2) :

Item plerumque medici servos eiusdem artis libertos perducunt;

112- A 1 'exception des textes où on relève également líbertus, il s'agit de Julien D 38.2.20.4; Florentinus D 38.2.28 pr ; Tryphoninus D 27.1.44.3; Paul D 31.83 ; D 38.1 .37 pr (patronus, $-\alpha$ ) ; D 48.20.7.1 ; v1pien D 1.5.27; D 26.4.1.1 (patronus,-a) ; D 38.5.1.4; D 38.7.2.1 (patronus,-a) ; D 40.10. 6 (rescrit d'Hadrien) ; D 50.1.6.3 ; Marcien D 40.11.2.

113- Liber,-tas/patronus est le seul rapport décelable chez Tryphoninus D 37.14.23 pr ; Paul D 38.1.39 pr ; D 40.5.33 pr ; U1pien D 2.14.10.1 (Marcellus) ; D 2.12.2 ; D 38.2.16.9; D 44.5.1.9; Marcien D 40.5.51.3 ; Modes tin D 38.1.32.

114- A 1 'exception des textes où on trouve libertus ou libertinus, on a Alfenus (chez auteur anonyme) D 40.1.6; Callistrate D $38.1 .38 \mathrm{pr}$; Paul D 19.1 .43 ; D 48.10 .14 .2 (ëdit de Claude); D 48.22.13; v1pien D 2.4.10 pr (constitution de Marc-Aurèle); D 4.3.7.8 (Pomponius); D 23.2.29; D 23.2. 45.3 ; D 38.2.3.8 (constitution d'Hadrien) ; D 38.16.3.2; D 50.1 .7 ; D 50. $1.27 \mathrm{pr}$; L. Rufinus D 23.2.51 pr ; Marcien D 38.2.29.1.

115- Papinien D 40.10.1.1 ; Paul D 40.10.5; U1pien D 2.4.10.3; Marcien D 40.10 .3 (Commode).

116- On relève servras (ou servitus) / libertus dans ce sens chez Iavolenus D 28.5.66 ; Julien D 38.1.25.2 ; D 45.1.54.1 ; Pomponius D 38.4.13 pr ; Africain D 40.5.49 ; Gaius D 38.2.30 ; Venuleius D 40.12.44 pr ; Marcellus D 19.1.23 (Julien); D 38.2.32; D 40.4.42; D 40.5.41.6; Papinien D 14.3. 19.1; D 40.4.50.1; D 49.17.13 (rescrit d'Hadrien); Tryphoninus D 26.7.58 pr (Scaevola); Callistrate D 40.12.37; D 47.9.7; D 49.14.2.6 (constitution de Sévère) ; Paul D 15.3.32 ; D 12.6.65.8; D 19.5.5.5; D 38.2.4 pr ; D 38. 2.9 ; D 40.5.25 (Valens) ; D 40.5.33.1; D 44.5.2.2; vipien D $1.1 .4 ; 02.4$. dans d'autres, servus et libertus sont dissociés (117), que le passage contienne deux hypothèses distinctes (118) ou qu' il s'agisse de l'esclave d'un affranchi, comme c'est par exemple le cas chez Paul (D 38.2.44.2):

$S i$ ex bonis, quae mortis tempore fuerunt, debitam partem dedit $l_{i}$ bertus in hereditate vel legato, servus tamen post mortem liberti reversus $a b$ hostibus alugeat patrimonium.

Dans ce dernier cas, la relation statutaire ne joue pour ainsi dire qu'au deuxième degré.

Si l'on tient compte de ces deux sortes de textes, la répartition par périodes s'opère de la façon suivante : analyse des paragraphes

I

II

IV

$$
\begin{array}{r}
- \\
12 \\
8
\end{array}
$$

analyse des citations

$\begin{array}{ll}\text { I } & - \\ \text { II } & 5 \\ \text { III } & 5 \\ \text { IV } & 2 \\ \text { V } & -\end{array}$

10.11 (Labéon); D 3.5.5.7; D 22.3.14; D 24.3.24.4; D 29.4.29; D 37.12 . 1.2 ; D $38.2 .14 \mathrm{pr}$; D 38.2.16.4 (rescrit de Marc-Aurèle et Verus) ; D 38. 16.3.4-6 (Zibertus, $-a)-7$; D 40.4.7 (Neratius); D 40.5.24.2I (rescrits d'Hadrien et d'Antonin le pieux) ; D 40.5.30.3 (rescrit de Marc-Aurèle et Verus) -12 ; D 40.7.2 pr ; D 44.5.1.4; D 47.10.7.2 ; D 49.17.6 ; Macer D 42.1 .63 ; D 48.5.25 pr ; Marcien D 1.8.6.1 (rescrit de Marc-Aurèle et D 42.1.63 ; D 48.5.25 pr; Marcien D 1.8.6.1 (rescrit de Marc-Aurèle et Verus) ; D 28.5.52.1 (Celsus) ; D $37.44 .5 \mathrm{pr}$ (Claude) ; D 38.4.6 ; D 40 . D $37.14 .8 \mathrm{pr}$ (rescrit d'Hadrien)-1 ; D 37.14 .13 ; D 40.1.16 ; D 48.4.7.2; A. Charisius D 48.18.10.4. Il faut aussi signaler dans ce sens ancilla/li-berta chez Scaevola D 40.5.41 pr et mancipium/libertus chez Papinien D 40. 4.48 (Servius).

117 - Julien D 38.2.13 ; Pomponius D $38.1 .8 \mathrm{pr}$ (Labéon) : D $38.1 .10 \mathrm{pr}$; D 45.3.38 (Ce1sus); Gaius D 40.9.10; D 40.12.9.2 ; Scaevola D 33.8.23 pr ; D 34.3.31.1 (conservus) ; D 40.7.40.2 ; Papinien D 38.2.41 ; Tryphoninus D 38.2.50.1; Paul D 26.7.12.3; D 38.2.44.2; D 38.2.51 (Labéon); D 40.5.31.3; Ulpien D 21.1.17.5 (Labéon, Caelius Sabinus); D 38.5.1.2224 ; Marcien D 34.1 .2 pr (rescrit de Sévère et Caracalla) ; Hermogénien D 37.14.21.1-3. Dans ce sens, on relève aussi ancilla chez Paul D 48.18 . 20 et mancipium chez Iavolenus D 38.2.36.

118- Par exemple Julien D 38.2.13: Filius patrori exheredatus, quamis nepos ex eo heres scriptus fuerit, bonomum possessionem contra tabulas paternorum libertorum accipere non potest : licet enim necessarius existat patri suo, non per semetipsum, sed per alium ad hereditatem admittitur. Et certe constat : si emancipatus filius exheredatus fuerit et servus eius heres scriptus, etsi iusserit semo hereditatem adire et ita patri suo heres extiterit, non habebit contra tabulas paternorum libertorym

bonorum possessionem. La dissociation peut se produire a un moindre niveau, 
On remarque que servus/libertus, qui désigne la condition servile et celle de l'affranchi. est beaucoup moins fréquent que semus/liber (110) ou semus/manumissus, qui vise le passage de l'esclavage à l'après-esclavage $(120)$.

s'il s'agit par exemple d'un commerce exercé par des esclaves et des affranchis come c'est le cas chez Gaius D 40.9.10 : ...Qucd frequenter accidit his, qui transmarinas negotiationes et aliis regionibus, quan in quibus ipsi morantur, per servos atque libertos exercent.

119- cf. supra n. 91 .

120- Sur un total de 662 textes où on trouve manumissus (ou manumittere, etc.), servus (ou servitus) apparaît dans 373 . Manumissus ressort seul chez Iavolenus D 31.37 ; Celsus D 1.18.17; Valens D 36.4 .15 ; D 38.1 .47 (Campanus) ; Pomponius D $38.1 .4 ; D 40.2 .1 ; D 40.4 .4 .2 ; 040.4 .5 ; D 40.5$. $34 \mathrm{pr}$; D 40.14.3.1; Africain D 19.1.30.1 ; D $30.109 \mathrm{pr}$; D 40.9.8 pr; Gaius D 5.3.3 ; D 26.8.9.1 ; D 40.1.18; D 40.2.25 (Nerva filius, Fufidius) ; Maecianus D $35.1 .86 \mathrm{pr}$ (Julien)-1 ; Venuleius D 40.14.2.1 ; Marcellus D 23.2.50; D 29.1.29.1 ; D 40.5.10.1; Florentinus D 38.2.28 pr ; Scaevola D 2.15.3 pr ; D 22.2.5 pr; D 31.88.6-11; D 32.37.7; D 33.2. $34 \mathrm{pr}$; D 33.7.27.3; D 34.1.16.1-3; D 34.1.18 pr-1 ; D 34.1.19; D 36. 2.27.1 ; D 41.6.5; D 42.8.23; D 50.16.243; Papinien D 17.1.54.1 (Ziber homo bona fide serviens) ; D 26.2.28.1; 026.5 .13 .1 ; D 27.1 .24 ; D 29. $7.13 \mathrm{pr}$; D 33.8.19.1; D 34.1.10.1; D 40.1.20.1; D 40.1.22; D 40.5.23. I ; D 40.14.4; D 50.1.17.8; Tryphoninus D 40.5.17 (Scaevola) ; Callistrate D 38.1.38 pr-1 ; D 40.8.3 (rescrit de Marc-Aurè le et Commode) ; D 28.1.14; D 29.5.10.1 (constitution de Trajan); D 31.8.5 (rescrit d'Hadrien) ; D 34.4.26.1 ; D 38.1.16 pr ; D 40.1.14 pr ; D 40.2.15.4-5 ; D 40.2.17; D 40.2.18 pr-1-2; D 40.2.24; D 40.4.27; D 40.5.31.2; 40. $9.15 \mathrm{pr}$ (rescrit de Caracalla)-1 (Julien); D 40.9.16.5; D 40.9.17 pr 9.15 pr (rescrit de Caracalla)-1 (Julien); D 40.9.16.5; D 40.9.17 pr (Marc-Aurèle)-1-2 ; D 40.9.26 (Scaevola); D 40.12.38 pr (constitution
de Marc-Aurêle) ; D 45.1.91.2 (Julien); D 47.2.42.1 (Sabinus) ; D 47.2. 67.3 (Julien) ; D 48.20.7.1 ; D 48.22.13; D 50.17.179; Ulpien D 1.10.1 $\mathrm{pr}-1$; D 2.4.10 pr (constitution de Marc-Aurèle) 4 ; D 5.3.7.2 (rescrit d'Antonin le pieux) ; D 7.4.15; D 11.3.7; D 12.4.3.4-5 (Neratius, Julien) ; D 15.2.1 pr; D 17.1.8.5 (Julien) (Ziber homo bona fide serviens) ; D 20. 2.6 (Pomponius) ; D 23.2.29; D 23.2 .45 pr (rescrit de Sévère et Caracalla) ; D 24.1.7.9; D 24.1.9.1 (Julien) ; D 24.3.64.3; D 25.3.5.22 (Marcellus); D 26.4.3.1-2 (constitution de Marc-Aurèle)-3-4; D 28.5.9.19; D 29.2. 71.1-2; D 29.5.1.13 (Scaevola) ; D 29.5.3.15; D 38.1.7.2; D 38.1.13 pr constitution de Marc-Aurêle)-1 (idem); D 38.2.3.3-4; D $38.4 .1 \mathrm{pr} ; \mathrm{D} 38$. 16.3.3 (constitution de Marc-Aurèle); D 40.1.1; D 40.1.4.6-7; D 40.2. 8 ; D 40.2.20.4; D 40.3.1 (Marc-Aurèle); D 40.4.9 $\mathrm{pr}^{-1} ; \mathrm{D} 40.4 .12 .3$; D 40.4.25;D 40.5.4.7-13-16-18; D 40.5.24.13 (Marcellus) ; D 40.5.30.1; $\mathrm{D} 40.5 .46 .3$ (rescrit de Sévère) ; D 40.8.4; D 40.9.1 (Celsus) ; D 40.9 . 14.1-2-3-4-5; D 40.9.30.1; D 41.2.13.8; D 47.4.3 (Labéon); D 47.6.3 pr ; D 50.1.1 pr ; D 50.1.7 ; D 50.1.27 pr ; Marcien D I.16.2.1; D 33.8.20; D 38.2.22 (Julien; rescrit d'Hadrien); D 38.2.29 pr; D 40.1.8.3 (rescrit d'Hadrien) ; D 40.2.10 ; D 40.4.23 pr ; D 40.5.51 pr-1-4-6-9 (rescrit d'antonin le pieux)-10-11 (Antonin le pieux); D 40.9.9.2; D 40.9.11 pr-1

(rescrit de Marc-Aurèle et Verus) ; D 48.22.2 (rescrit d'Antonin le pieux) ;

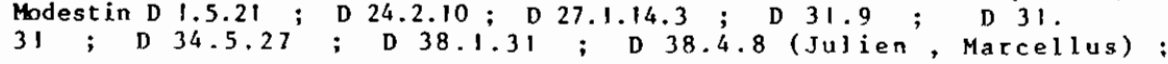

D 40.12.21.1 ; Hermogénien D 40.2.23; D 40.9.27pr : D 40.12 .40 ; D 50.1.23pr. Manumissus peut apparattre accompagné d'un autre terme désignant l'esclave que servrs : homo chez Gaius D 4.7.3.1 ; D 6.1.18 : Marcellus D 19.1.23 (Julien) : Scaevola D 10.2.39.4; D 45.1.122.2; Papinien D 40.1 .20 .3 ; Paul D 21.1.47pr (Labéon); 0 30.35; mancipium chez, Pomponius D 21.2.34pr : Papinien D 40. 4.48 (Servius): Tryphoninus D 18.7.10: Paul D 49.14.45.3: U1pien D 40.9 .12 pr-3-4-5; statuliber chez Paul D 40.7 .1 .1 ; D 40.7 .4 .1 (Julien); D 40.9 . pr-3-4-5 ; strtuliber chez Paul D 40.7 .1 .1 ; D 40.7 .4 .1 (Julien) ; D 40.9 .
16.4 ; Modestin D 35.1.66; D 40.7.25; vicarius chez Paul D 40.4.10pr ; persona chez Ulpien $D$ 40.9.14pr ; un terme désignant la femme esclave, chez Celsus D 40.2.19 (praegnas) ; Gaius D 16.1.13pr (ancilla) ; D 38.8.2 (mulier) : MarcellusD 23.3.59.2 (milier) ; Scaevola D 40.4.60 (mater) ; D 40.5.41.5 (ancilla) ; Pap1nien D 18.7.6pr (Sabinus) (serva) ; Callistrate D 38.1.38pr (par meretrix) : Paul D 4.4.48.1 (ancilla) : D 29.1.40.2 (ancilla serva ) ; D 31.83 (ancilla) ; D 33.8 .3 (ancilla) ; D 40.1 .10 (ancilla) D 40.5.40pr (ancilla) ; D 40.8.9 (ancilla); Ulpten D 38.16.1.1 (rescrits de Marc-Aurèle et Verus et de Caracalla) (serva) : D 38.17.2.3 (serva) ; D 40.2 .20 .2 (uxor) : $D$ 40.5.26.1 (constitution de Sévère) (mater ) -2 (rescrits d'Antonin le pleux et de Sévère et Caracal la) (mater) ; $D$ 40.5.45pr (ancilla) ; L. Rufinus D 23.2.51pr (ancilla) -1 (ancilla) ; Marcien D 1.

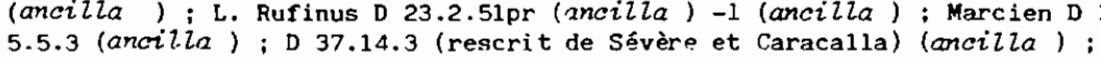
D 40.5.51.12 (uxor) ; D 40.5.53.1 (ancilla) ; D 40.5.55pr (ancilla) -1 (Marcellus) (ancilla) ; Modestin D 1.5 .22 (ancilla) ; D 40.5 .13 (ancilla); D 40.9.21 (Julien) (ancilla) ; un terme désignant l'enfant esclave : puella chez Papinten D 40.1.20.2 ; Paul D 41.7.8; filius chez Paul D 19.5.5pr ; Ulpien D 40.2.20.3 (Marcellus) ; filia chez Ulpien D 36.1.11.2 ; D 40.2.11 ; alummus chez Marcien D 40.2.14pr ; alumna chez Paul D 40.5.38; un terme à dénotation délictuelle, chez Ulpien $D 47.2 .17 .1$ (fur) ; un nom de métier, chez Scaevola D 34.1.18.3 (actor); Modestin D 40.5.12pr (rescrit de Caracalla) (tragoedi); ou enfin un nom propre, chez Julien D 12.1.19pr; D 30 .

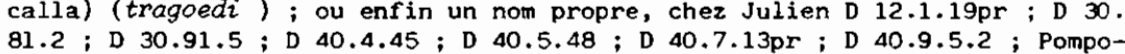
nius D 40.4.4pr ; Africaln D 33.8.16pr ; D 21.2.46.2 ; Gaius D 40.4.57 (Sabinus, Cassius, Jullen) ; D 40.7.37 ; Marcellus D $36.1 .46 \mathrm{pr}$; Ścaevola D 31. 88.3; D 33.2.36pr; D 33.8.21 (Julien) ; D 33.7.7; D 34.1.18.2 ; D 34.5 .29 ; D 36.1.80.2 ; D 39.5.35pr ; D 40.5.41.3-17 ; D 40.7.40pr ; Papinien D 40.4 . 49 ; Tryphontnus D 31.88 .12 (Scaevola) ; D 34.3.28.7 (Scaevola); Paul D 15.1. 53 ; D 28.5.56 (Neratius) ; D 35.1.81pr ; D 40.1 .23 ; D 44.7 .45 ; Ulpien D 28 7.8 .7 ; D 36.1.17.16; D 40.5.46pr; Modestin D $31.34 \mathrm{pr}$. 
Il faut aussi noter que parfois, au lieu de s'opposer à libertus, servus s'oppose à libertinus (121).

Quant à la relation libertinus/ingenuus, elle ressort ainsi (122) :

\section{analyse des paragraphes}

-
-
28

En outre, on peut trouver ingenuus en rapport non avec libertinus, mais avec libertus (123), manumissus (124), ou bien avec ius oureorwm anulorum ou restitutio natalium qui sous-entendent la présence $d$ 'un affranchi (125).

121- Iavolenus D 28.8.11 (Trebatius, Labêon) ; Gaiús D 1.5.6 ; Paul D 40. 12.32 ; Ul pien D 1.7.46; D 4.8.7 pr (Labéon, Pedius, Pomponius) ; D 47.10. 9.4 ; Marcien D 40.11.2. Ont été intégrés dans cette 1 iste les textes oũ 1 'on trouve servitus (D 1.5 .6 et D 1.7 .46 ).

122- En tenant compte de libertina/ingenua, on remarque Celsus D 23.2.23; Venuleius D 40.14.2.1 ; Marcellus D 23.2.32 ; Tryphoninus D $27.1 .44 \mathrm{pr}$ (rescrit de Sévère et Caracalla)-1-3 ; Callistrate D 48.15.6.2 ; Paul D 4. 8.32 .7 ; D 29.2.22 (Pomponius); D 40.12.32 ; Ulpien D 1.5.25; D 1.5.27: D 2.15.8.7 ; D 4.8.7 pr (Pedius, Pomponius); D 22.3.14; D 22.4.6; D 38 5.1.4; D 38.7.2.1; D 38.17.1 pr (Zibertina/ingenua); D 40.10.6 (rescrit d'kadrien); D 40.16.2.3; D 40.16.4; D 47.10.9.4; D 50.16.46.1 (libertina/ingenua) ; Macer D 49.4.2.2 ; Marcien D 1.5.5 pr; D 23.2.58.2 (rescrit d'Antonin le pieux) ; D 25.7 .3 pr (libertina/ingenua) ; Modestin D 27.1.1. 4 (rescrit de Sêvère) ; D 40.11.5.1 ; Hermogénien D 26.5.27.1. On peut rapprocher de cette relation D 1.5 .21 de Modestin (homo Liber/Libertinus).

123- Marcellus D 40.14.1; U1pien D 2.4.10.2; D 3.5.5.7; D 38.2.3 pr.

124- Papinien D 40.14.4; Ulpien D 40.5.26.1 (constitution de Sévère)-2 (rescrits d'Antonin le pieux et de Sévère et Caracalla).

125- Scaevola D 40.11.3 ; Papinien D 40.10.1.1; D 40.10.2 ; Tryphoninus D 27.1.44.2; Paul D 40.10.5; Ulpien D 2.4.10.3; D 40.10.4.

Reprenons ces différentes données. En comparant les chiffres obtenus au nombre total de textes où ces relations apparaissent par périodes, on parvient à :

analyse des paragraphes

- Domination : Zibertus/patronus.

\begin{tabular}{|c|c|c|c|c|c|c|}
\hline I & - & $=$ & I & $2 / 2$ & $=$ & $100 \%$ \\
\hline II & - & $=$ & II & $16 / 21$ & $=$ & 76 \\
\hline III & $56 / 69$ & $81 \%$ & III & $26 / 34$ & $=$ & 76 \\
\hline IV & $16 / 26$ & $62 \%$ & IV & $6 / 8$ & $=$ & 75 \\
\hline & $249 / 340$ & $73 \%$ & $\mathrm{v}$ & $7 / 7$ & $=$ & 100 \\
\hline
\end{tabular}

\section{-Oppositions statutaires.}

-Libertus/servus.

\begin{tabular}{|c|c|c|c|c|c|c|c|}
\hline I & - & $=$ & - & I & $0 / 2$ & $=$ & - \\
\hline II & - & $=$ & - & II & $5 / 21$ & $=$ & $24 \%$ \\
\hline III & $12 / 69$ & $=$ & $17 \%$ & III & $5 / 34$ & $=$ & \\
\hline IV & $8 / 26$ & $=$ & $31 \%$ & IV & $2 / 8$ & $=$ & $25 \underset{\oplus}{\mathbb{\phi}}$ \\
\hline $\mathrm{v}$ & $63 / 340$ & $=$ & $19 \%$ & $\mathrm{v}$ & $0 / 7$ & $=$ & - \\
\hline \multicolumn{8}{|c|}{-Libertinus/ingenuus. } \\
\hline I & - & $=$ & - & I & $0 / 2$ & $=$ & - \\
\hline II & - & $=$ & - & II & $0 / 21$ & $=$ & - \\
\hline III & $1 / 69$ & $=$ & $2 \%(126)$ & III & $3 / 34$ & $=$ & $9 \%$ \\
\hline IV & $2 / 26$ & $=$ & $7 \%(127)$ & IV & $0 / 8$ & $=$ & - \\
\hline $\mathrm{v}$ & $28 / 340$ & $=$ & $8 \%$ & v & $0 / 7$ & $=$ & - \\
\hline
\end{tabular}

D'une part, en ce qui concerne les relations statutaires, on constate que libertus/servus est plus fréquent que iibertinus/ingenuus. Cela va dans le sens de ce qui a précédemment été dégagé à propos de l'esclave, serius/liber visant plus l'esclave par opposition à un affranchi que par opposition à un ingénu. Toutefois, cette dernière interprétation de servus/liber ressort beaucoup plus des textes que libertinus/ingenuus. Autrement dit, les juristes se préoccupent davantage de distinguer l'esclave que l'affranchi d'un ingénu. Il est vrai que l'affranchi, malgré certaines infériorités, est juridiquement libre.

126- Pourcentage exact de $1,44 \%: 2 \%$ pour arrondir le total de 1 a période à $100 \mathrm{z}$.

127- Pourcentage exact de 7,59 $\%: 7 \%$ pour arrondir le total de 1 a période à $100 \%$. 
D'autre part, 1s relation de domination est prépondérante par rapport aux relations atatutaires quelle que soit la période envisagfe. Cela montre que le problème central de l'après-esclavage reste le fait de maintenir l'ancien esclove dans une situation de dépendance vis-à-vis de son ancien maitre.

$$
-0^{-}
$$

Reprenons rapidement les résultats auxquels cette analyse terminologique nous a permis de parvenir. La plupart des termes qui désignent l'esclave ont une résonance juridique diversifiée, à l'exception de partuo qui ne vise que l'esclave objet, aingi que hamo et surtout mancipium dont c'est aussi la vocation essentielle. Sorvus apparait come le terme aysnt la résonance ls plus Equilibrée. L'emploi du mot fanilia dénote une peur latente des propriftaires d'esclaves. De même est intéressante, du point de vue idfologique, la pratique qui consiste à désigner l'esclave par un terme à signification délictuelle. Le problème de la résonance juridique ne se pose pas pour libertus et libertinus, l'affranchi apparaissant comme un sujet de droit (128).

Quant aux relations terminologiques prises en leurs éléments pertinents. elles consacrent une prépondérance du rapport de domination/subordination aussi bien en ce qui concerne l'esclavage que l'après-esclavage. Il est utile à ce sujet d'examiner les différents modes d'affranchissement tels qu'ils ressortent du Digeste.

$-0$

128- Il est cependant vrai qu'un affranchi peut être commun, en d'autres termes dépendre de plusieurs patrons. C'est un point sur lequel il sera revenu plus loin.

\section{CHAPITRE II : LE STATUT DU DEPENDANT}

Deux problèmes se posent : celui du passage de l'état d'esclave à l'état d'affranchi et celui de la nature du lien de dépendance.

\section{SECTION 1. - LE PASSAGE DE L'ETAT D'ESCLAVE A CELUI D'AFFRANCH}

Des trois procédés civils d'affranchissement, le cens tombe en désuétude (129). La vindicts demeure sous l'Empire le procédé par excellence d'sffranchissement exitre vifs (130). Quent au testament, il a cessé de se faire devant les comices et devient un acte purement privé (131). Des modes non formalistes vont être utilisés pour les affranchissements entre vifs, le maittre se contentant d'exprimer sa volonté hors des formes usuelles, por epistulam, par lettre ou inter

129- J. GaUdEmET, Institutions de 1'antiquité, cit. p. 557. L'affranchissement par le cens est cependant encore signalé théoriquement par les jurisconsultes classiques, R. MONIER, Droit romain, cit. I, p. 220 n.2 ; aussi R. DANIELI, In margine a un recente studio sulla manumissio censu SDHI 15 (1949) 198-202 M. LEHOSSE, L'af franchissement par censu, SDHT 15 (1949) $P$. T $161-203$. Rien ne ressort dans sur ce procédé d'affranchissement.

130- A. GUARINO, Diritto privato romano, cit. p. 61। ; APPLETON, L'affranchissement vindicto ne s'appliquait anciennement qu'aux esclaves pour dettes, in Mél. FOURNIER (1929)p.1s; ;. CUQ, Une scène d'affranchissement par la vindicte au $1^{\circ}$ siècle de notre ère, in CRAI (1915) p. 537-551; H. LeVY-BRUHL, L af franchissement par la vindicte, in St.

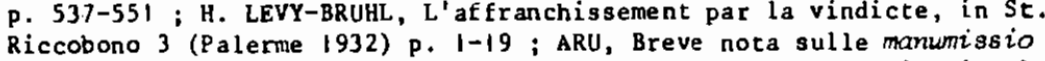
vindicta, in St. Solmi 2 (1940) p. 301 s.; R. MONIER, Contribution a l'étude des rites de la manumissio vindicta, in St. Albertario I (1952) p. 195 s. ; M. KASER, ther Verfügungsakte Gewal tunterworfener, mit Studien zur Natur der manumissio vindicta, in SDHI 16 (1950) p. $59 \mathrm{~s}$.; Ph. MEYLAN Lindividualité de la manumissio vindicta in St. Arangio, Ph. ME (1953) p. 469 o. CHARET Alapa, in SDHI 19 (1953) po 334 , Ruiz 4 (1953) p. $469 \mathrm{~s}$. ; CHARVET, Alapa, in SOHI TONDO, Aspetti simbolici e magici nella struttura della manumissio
dicta (1966); $\mathrm{M}$. DE DOMINICIS, La manumissio vindicta nel diritto giustinianeo, in St. De Francisci 2 (Milan 1956) p. 275-294; sur la manumisoio vindicta à 1 'époque républicaine, S. TREGGIARI, Roman freedmen during the Late Republic,0xford (1969), p. $21 \mathrm{~s}$

131- Sur la manumis8io testamento, G. IMPALLOMENI, Le manomissioni mortis causa, Studi sulle fonti autoritative romane, Padoue (1961) $\mathbf{x}+$ $276 \mathrm{p}$. ; sur ls manumissio testamento ạ l'époque républicaine, S. TREGGIARI, Roman feedmen, cit., p. $27 \mathrm{~s}$. 
amicos, devant quelques amis (132). Ces affranchissements placent l'esclave dans une situation de liberté de fait (133) mais ils vont émerger dans le domaine du droit lorsque le préteur s'opposera à ce que l'ancien maître fasse retomber l'affranchi en servitude (134). La lex Iunia ira plus loin en reconnaissant le statut, inférieur il est vrai, de latin junien à de tels affranchis (135).

A côté des legs va aussi se développer une forme simplifiée, le fidéicommis, disposition de dernière volonté par laquelle on charge une personne, légataire ou héritier, de remettre quelque chose à un tiers

132- J. GAUDEMET, Institutions de l'Antiquité, cit. p. 558 ; B. ALBANESE, La struttura della manumissio inter amicos, in Annali del seminario giur. Univ. Palermo 29 (1962) p. 5-103. Une autre forme d'affranchissement consistait inviter 1 'esclave à la table du maitre (affranchissement per mensam). On croit trouver cette forme dans le Satiricon (Ch. LXX) $L X X)$, mais 1 allusion est imprecise; la pratique est par contre certaine au bas Empire. Sur ce probleme, A. BISCARD, Petronio LXX, 10-11, in SIFC (1938) p. 7 , , , manumissio per menson $e$ affrancazioni pretorie, Florence (1939), 92 p.; G. FUNAIOLI, Ancora sull'età di Petronio, A proposito della mamorissio per mensam, in BIDR 44 (1936-37) p. 385-395 ; R. HENRION, Satiricon et mamumissio per mensam in RBPH 22 (1943) p. 198-204; U.E. PAOLI, De manumissione per mensam, in SDHI 2 (1936) p. 369-372 ; id., L'età del Satiricon, in SIFC (1937) p. 1346. I1 faut encore remarquer que Paul critique une opinion de Nerva selon qui on peut affranchir des esclaves retenus en gage en les présentant par sa fenêtre, cf. D 20.2.9: ". . et derisus Nerva iumis consuitus, qui pr (D) pensionem liberari posse".

133- Ces affranchissements naissent hors du cadre juridique. I1 ae saurait être question de droit de cité pour ces esclaves, E. VOLTERRA, Maromissioni di schiavi compiute da peregrini, in St. De Francisci 4 (Milan 1956), p. 89-90. Une loi rapportée par Paul (D 40.1.14.1 : "Imperator cum servum manumittit, non vinfictam imponit, sed cum voluit, perator cum servum manumitit, non vindictam imponit, sed cum voluit, fit liber is qui manomittitur ex lege Augusti") permet à Augus te d'af-
franchir par simple effet de sa volontê ; on entre ici dans le domaine du droit civi1, G. BOULVERT, Domestique et fonctionnaire, cit. p. 96.

134- L'esclave est in libertate tuitione Praetoris, .. Roman law, cit., p. 445 ; M. DE DOMINICIS, Les Latins Juniens dans la pensée du législateur romain, RIDA 20 (1973) p. 311.

135- Gaius I, 22 et III, 56 ; fragment de Dosithée, 6-7. Selon les Institutes de Justinien (1.5.3.), il s'agirait d'une lex Iunia Norbana, ce qui la daterait de 18 ap. J-C, date douteuse si la lex Aelia Sentia ( 4 ap. J-C.) se réfère déjà au statut des latins juniens. J. GAUnEMET, Int. Institutions de 1 Aritiquité, cit. p. $558 \mathrm{n.7}$, admet que lá lex Aelia Sentia a pu prévoir la condition de latin (non junien) pour ceux qui étaient libérés en dehors des conditions légales en leur refusant la civitas. M. DE DOMINICIS, La latins Juniens, cit., p. 313, fait remonter la lex Irnia à 17 av. J-C ; sur le problème, aussi A. WILINSKI, Zur frage von Latinern ex lege Aelia Sentia, in ZSS 80 (1963) p. 378-392 ; A.M. DUFF, Feedmen in the early Roman Empire, (éd. Cambridge 1958), p. $210 \mathrm{~s}$. ou de faire quelque chose. Aruguste a donné par la procédure extraordinaire force juridique à cette disposition. On va pouvoir utiliser cette forme pour charger quelqu'un de donner la liberté à un esclave (136). Divers sénatus-consultes vont reprendre cette disposition, et spécialement en 101 ou 103, le s. c. Rubrien par lequel le préteur peut déclarer lui-même la liberté à défaut d'exécution par l'héritier ou le légataire (137).

Ces divers modes d'affranchissement étant énumérés, il s'agit de voir quelle est leur place dans le Digeste d'une part, et d'autre part quelles sont les formes qu'ils revêtent : autrement dit, l'affranchissement est-il onéreux ou gratuit, pur ou conditionnel ?

\section{Paragraphe 1: Affranchissement entre vifs et affranchissement à cause} de mort.

Il est des textes qui renvoient clairement à l'affranchissement entre vifs, soit par la mention de la vindicta (138), soit par les paroles vivus manumisit (139). Dans d'autres, la référence à ce mode

136- WALLON H., Histoire de 1 'esclavage, cit. III, p. 67 ; M. KASER, mortis causa, cit. P. 259 , cr. de G. FRANCIOSI, in Labeo 13 (1967) p. $273 \mathrm{~s}$.

137- E. VOLTERRA, in NNDI 16 (1969) p. 1072 ; C. CASTELLO, D 40.5.26.7, in tema di senatoconsul to Rubriano, Studi Scheri1lo I (1972) p. $201 \mathrm{~s}$. S.C. Dasumianum (sous Hadrien) : procédure de défaut simplifiée en cas d'absence ex iusta causa; 5.C. Articuleianum (123) : procédure en province; S.C. Iuncianum (127) : contrainte contre celui qui doit affranchir 1 esclave ex quacromque causa; S.C. Vitrasianom (Hadrien) : en chis de pluralité d'héritiers, contrainte excercée aussi contre ceux qui ne sont pas chargés du fidéicommis (ếlargi par un rescrit d'Antonin le ne sont pas chargés du fidéi comais
pieux, ef. Ulpien D 40.5 .30 .6 ).

138- Cf. D 40.2, De manwissis vindicta.

139- Par exemple D 33.7.3.1 Papinien : Minor viginti annis instructa praedia consobrinae suae dari voluit et quosdam semos praediomm vivus manumisit. L'affranchissement entre vifs ressort explicitement de Celsus D 40.2.19; Julien D 28.5.7 pr ; D 30.91.2 ; D 33.5.10 ; D 34.3.12; D 40.2.4.pr-1; D 40.2.5 (Iavolenus) ; D 40.2.6; Pomponius D 40.2.1.; D 40.4.4 pr-2; Africain D 40.4.20; Gaius D 40.2.7; D 40.2.25 (Nerva filius, Fufidius); Maecianus D $35.1 .86 \mathrm{pr}$ (Ju1ien); Marcellus D 40.1 . 15 ; Scaevola D 10.2.39.4; D 32.37.7; D 32.41.2 ; D 34.1.19; Papinien D 33.7.3.1 ; D 33.8.19.1-2; Tryphoninus D 49.17.19.4 ; Pau1 D 29.5.10. 1 (constitution de Trajan); D 34.4.26.1 ; D 34.4.27.1; D 40.1.14 pr-1 (lex Augusti) ; D 40.2.4.2 (Julien) ; D 40.2 .15 pr-1-2-3-4-5; D 40.2. 17 ; D 40.2.18 pr-1-2 ; D 40.2.22; D 40.2.24; D 40.4.27; D 41.7.8; U1pien D 5.3.7.2 (rescrit d'Antonin le pieux) ; D 34.3.12 ; D 40.2.2.; 
d'affranchissement est. seulement implicite.

Pour illustrer cette deuxième hypothèse, prenons un passage de L. Rufinus (D 31.62 ) :

Si aliems servus heres institutus fuerit, a domino eius fideicommissum relinqui potest. Sed ita hoc fideicommissum domims praestare debet, si per serrrm factus sit heres : quod si ante, quan iussu eius adiretur hereditas, servus manumissus fuerit et suo arbitrio adierit hereditatem, dominus id debitums non est, quia heres factus non e6t...

Il s'agit en l'espèce d'un esclave institué héritier par un tiers qui charge son maître d'un fidéicomuis. Si, avant de donner l'ordre à son esclave d'accepter la succession, le mâtre l'affranchit, il ne sera pas tenu du fidéicomais car l'esclave aura acquis la succession de son propre arbitre. Il est clair qu'on a affaire à un affranchissement entre vifs $(140)$.

D 40.2.3.; D 40.2.8; D 40.2.11; D 40.2.12:D 40.2.13; D 40. $2.16 \mathrm{pr}-1$; $\mathrm{D} 40.2 .20 \mathrm{pr}-1$ (constitution de Marc-Aurèle)-2-3 (Marcellus) -4 ; D 40.4 .12 .3 ; D 40.8.4; D 40.12.12.2 ; Marcien D 33.8.20; D 38.4 .6 ; D $40.2 .9 \mathrm{pr}-1$; D 40.2.10; D 40.2.14 pr-1; Modestin D 40. 2.21 (constitution d'Auguste); Hermogénien $D$ 40.2.23.'

140- A 1'exception des cas signalês infra (n. $141 \mathrm{a} 143$ ), on relève Alfenus (chez auteur anonyme) D 11.3 .16 ; D 40.1 .7 ; tavolenus $D 33.5$. 14 ; Celsus D 32.79 .3 ; Valens D 36.4 .15 ; Julien D 30.99 ; D 43.24 .14 ; D.45.1.54.1 ; Pomponius D 21.2.16.2 (Proculus); D 33.5.6; D 36.1.72.1;; D 44.7.56; D 46.3.83; Africain D 46.1.21.2; T. Clemens D 29.2.82; Gaius D 28.7.22; D 39.4.13.2 ; D 40.9.3; Marcellus D 19.1.23 (Julien); D 29.1.31; Scaevola D 34.1.16.3; D 40.4.60; Papinien D 14.3. 19.1 ; D 19.5.7 (par a contrario); D 24.3.61; D 26.7.39.18; D 40.3.3; D 49.17.13 (rescrit d'Hadrien); Tryphoninus D 12.6.64; D 38.2.50.1; Paul D 4.4.48.1; D 5.3.36 pr ; D 9.4.24 (Labéon, Julien); D 11.3 .14 . 9 ; D 12.6.65.3-8 ; D 15.1 .26 ; D 15.1.53; D 16.3.21.1 (Trebatius); D 19.5.5 pr-2 (Julien) (par a contrario)-5; D 20.2 .9 (Nerva); D 21.1. $47 \mathrm{pr}$ (Labéon) ; D 21.2.26; D 24.3.63; D 29.2.80.2; D.31.83; D 36 . 3.7 ; D 39.6.39; D 40.1.11; D 40.9.17.1 (Marc-Aurẽ le) ; D 40.12.38 pr (constitution de Marc-Aurèle)-3 ; D 44.7.45; D 45.1.91.1 (Julien)-2 (Julien) ; D $47.2 .42 \mathrm{pr}-1$ (Sabinus) ; D 47.8.3 (Labéon); D 47.10.29; D 48.10.14.2 (édit de Claude); U1pien D 4.3.7 pr (Julien) ; D 4.4.9.6; D 9.4.2.1 (Celsus) ; D 9.4.5.1; D 11.3.5.4; D 15.1.32 pr ; D 15.2.1. 7 (Pegasus, Africain); D 21.2.25; D 23.2.45.2-3; D $24.3 .24 .4 ; D 24$.

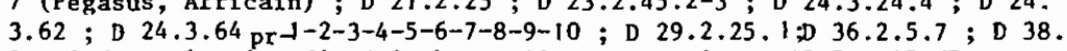
2.3.8 (constitution d'Hadrien); D 38.3.1 pr-1-2; D 38.5.1.25 (Pomponius) ; D 38.16.3..2-6-7-8; D 40.1.2; D 40.3.1 (Marc-Aurèle) ; D 40 . $3.2 ;$ D.40.9.30.1; D $41.2 .13 .8 ; \mathrm{D} \mathrm{44.5.1.4;D} \mathrm{47.6.3} \mathrm{pr;} \mathrm{D} \mathrm{47.10.7.}$ 2 ; D 48.18 .1.13 (rescrit d'Antonin le pieux); D 50.1.7; L. Rufinus D 31.62 ; Marcien D 30.112 .1 ; D 38.2 .22 (Julien ; rescrit d'Hadrien); Modestin D 5.2.12 pr ; D 37.14.8 pr (rescrit d'Hadrien) ; D 37.14.9.1; D 40.9.20; D 40.9.21 (Julien). Ont été intégrês dans cette liste les textes concernant les esclaves affranchis par les municipes ou les universitates et par 1 'empereur.
En dehors de toute indication sur l'affranchissement, il peut néanmoins être possible de présumer qu'il a eu lieu du vivant du maître, c'est le cas lorsque l'esclave paye le prix de sa liberté à son mâtre (141). Il existe par ailleurs deux hypothèses qui ne peuvent concerner en tout état de cause qu'un esclave affranchi entre vifs : les alimenta $(142)$ et surtout les operae (143) que l'affranchi doit fourair à son patron. En effet, l'esclave affranchi par testament n'a pas de patron, ou plutôt il n'a pour patron que le défunt, patron peu gênant (144). Quant à celui qui a étê affranchi par fideicommis, il ne doit à son patron, le fiduciaire, ni les alimenta (145), ní les operae

141- Ainsi Alfenus (chez auteur anonyme) D 40.1.6; Iavolenus D 15.3 . 2; D 45.1.104; Julien D 41.4.9; Gaius D 16.1.13 pr; Marcellus D 37 15.3 ; Papinien D 15.1.50.3; D 40.1.19; Paul D 41.3.4.16 (Sabinus, Cassius) ; D 41.4.2.14 (Celsus) ; D 44.5.2.2 ; D 48.19.38.4 ; Ulpien D 15.1.11.1 ; D 15.3.3 pr ; D 4.3.7.8 (Pomponius); D 16.3.1.33 (Julien); D 25.3.5.22 (Marcellus) ; D 29.2.71.2; D 33.8.8.5 (Labéon); D 40.1.4 pri-1-2-3-4-5-6-7-8-10-11-12-13-14 (epistula de Marc-Aurèle et Verus); Marcien D 40.1.5 pr (constitution de Marc-Aurèle et Verus) ; Modestin D 27.1 .14 .3 .

142- Il faut ainsi tenir compte de Ulpien D 25.3.5.18-19-20-21-23 (Marcellus) $-24-25-26$.

143- Il faut donc aussi relever Alfenus (chez auteur anonyme) D 38.1. $26 \mathrm{pr}-1$; Iavolenus D 38.1.21 ; D 38.1 .33 ; Neratius D $38.1 .50 \mathrm{pr}-1$.; Celsus D $38.1 .30 \mathrm{pr}-1$; Valens D 38.1 .46 (par a contrario); Julien D 38 . 1.11 ; D 38.1.23 pr-1; D 38.1.24; D $38.1 .25 \mathrm{pr}-1-2-3-4$; D 38.1 .27 ; Pomponius D $38.1 .3 \mathrm{pr}-1$; D 38.1 .4 (Aristo) ; D $38.1 .8 \mathrm{pr}$ (Labéon)-1; D 38.1.10 pr-1; D 38.1.12; D. 38.1.34; Gaius D 38.1.19; D 38.1.22 pr-1 (Julien)-2; D 38.1 .49 (Neratius); T. Clemens D 38.1 .1 .4 ; Scaevola D 38.1.44; D 38.1.45; Papinien D 38.1.40; D 38.1.41; D 38.1.43; Callistrate D $38.1 .38 \mathrm{pr}-1$; Paul D 38.1.1. ; D $38.1 .16 \mathrm{pr}-1$; D 38.1 .17 ; D 38.1.18 (Sabinus); D 38.1.20 pr-1 (Proculus); D 38.1.28; D 38.1.35 (par a contrario); $38.1 .37 \mathrm{pr}$ (idem) -1 (idem) -2 (Pomponius) $-3-4-5-6$ (Julien) $-7-8$; D 38.1.39 pr-1 ; D 38.1.5 ; Ulpien D $38.1 .2 \mathrm{pr}-1$; D 38 . (Julien) -7.8 ; D $38.1 .39 \mathrm{pr}-1$; D 38.1 .51 ; Ulpien D 38.1.2 $\mathrm{pr}^{-1}$; $\mathrm{D} 38$. 1.5; D 38.1.6 ; D 38.1.7 $\mathrm{pr}^{-1}$ (Celsus) $-2-3-5-6-7-8-9 ; \mathrm{D} 38.1 .9 \mathrm{pr}-1$
D $38.1 .13 .2-3-4-5$; D $38.1 .15 \mathrm{pr}^{-1}$ (Celsus) ; D 38.1 .29 ; D 38.1 .36 (Labéon); Modestin D 38.1.31 (par a contrario); Herrogénien D $38.1 .48 \mathrm{pr}-1-2$.

144- Cet affranchi est appelé libertus orcinus ; sur le thème, LORETI LORINI, La condizione del liberto orcino nella compilazione giustinianea, in BIDR 34 (1925) p. $29 \mathrm{~s}$. ; HARADA, Textkritische Studien zur adsignatio i iberti orcini, in ZSS 59 (1939) p. $498 \mathrm{~s}$.

145- Ulpien D 25.3.5.22 : Si quis a liberti ali se desiderot vel $a b$ eo, quem ex causa fideicommissi manumisit quemve suis numis redemit, non debet audiri, ut et Marcellus seribit, exaequatque ewm, qui mercedes exigendo ius libertomum amisit. 
(146). Il faut enfin signaler deux textes qui semblent correspondre à un affranchissement entre vifs par un mode non formaliste, par la formule domini voluntate in libertate morari (147).

En ce qui concerne 1'affranchissement à cause de rort, il est parfois difficile de distinguer la liberté testamentaire directe de la liberté fidéicommissaire, le second procédé relevant aussi d'une disposition mortis causa (148). Une première nuance entre ces deux procédés consiste dans le fait que le fidéiconnis de liberté donne lieu à un affranchissement entre vifs par le fiduciarius (149). La seconde et la 146- Valens D 38.1.47: Campanus scribit non debere praetorem pati
domum mumus operas imponi ei, qui ex fideicommissi causa manumittatur ; donum munus operas imponi ei, qui ex fideicommissi causa manumitatur Ulpien D 38.1.7.4 : Rescriptum est a divo Hadriano e deinceps cessare operamom persecutionem adversus erm, qui ex causa fideiconmissi ad $l_{i}$ bertatem perductus est ; dans le même sens, Papinien D 38.1 .42 et une constitution de Marc-Aurèle chez Ulpien D 38.1.13.1. Cependant, Valens admet une réserve (D $38.1 .47: \ldots$ Sed si, com sciret posse se id recusare, obligari se passus sit, non inkibendam operamon petitionem, quia donasse videtur).

147- Il s'agit de Pomponius D 40.12.28: Non videtur domini voluntate servus in libertate esse, quem dominus ignorasset surm esse : et est hoc verrom : is enim demon voluntate domini in libertate est, qui posses-

sionem libertatis ex voluntate domini consequitur, et de Paul D 40.12.

$24.3: \ldots$ voluntate domini in libertate eum morari, cf. M. DE DOMINICIS, Les Latins Juniens, cit., p. 311 ; W.W. BUCKLAND, Roman 1aw, cit., p. 446 n. 12 .

Il faut enfin remarquer que certains textes signalent un affranchissement sans en indiquer le mode ; il est alors évidemment impossible de trancher. Le problème se pose notament avec Iavolenus D 29.2. 64 ; Julien D 30.91.4 ; D 30.102 ; D 36.1.26.1 ; Pomponius D 20.2.6 ; D 40.1.13; D 45.3.40; D 46.3.92 pr ; Africain D 19.1.30.1 ; Gaius D 27.10 .17 ; D 38.8.2 ; D 40.1 .18 ; Paul D 30.35 ; D 31.82 .2 (Valens, Julien) ; D 38.2.51 (Labéon) ; D 40.1.3 ; D 40.9.26 (Scaevola) ; Ulpien D 2.9.5; D 24.1.11.6 (Marcellus, Scaevola) ; D 29.2.35 pr ; D 38.5.1. 24 ; D 38.16.3.1-12 (rescrit d'Antonin le pieux); 038.17 .2 .2 ( Julien) $-3 ; 040.1$; D 40.9.12.1-2 (Africain); $40.9 .14 .5 ; 046.3$ 18 ; D 47.2.46 pr ; Marcien D 29.2.52 pr (rescrit d'Antonin le pieux) D 30.114 .10 ; Modestin D 37.14 .13 ; D 38.8.7; D 40.1.16.

148- Paul D 40.4.56 : Si quis servo testamento dederit libertatem et directo et per fideiconmissum, in potestate semi est, utrum velit ex directo an ex fideiconmisso ad libertatem pervenire et ita Marcus imperator rescripsit. L'opposition liberté testamentaire directe/fidéicomrator rescripsit. L opposition liberté filius); Maecianus D 29.4.28.1 ; Scaevola D 40.5.41 pr ; Papinien D 26. $5.13 \mathrm{pr}$ (rescrit d'Hadrien); Paul D 40.5.33 pr ; Ulpien D 25.6.1.11 ; D 26.4.3.3; D 29.4.12 pr ; D 35.3.1.12; D 36.1.11.2 (Antonin le pieux) ; D 36.1.17.3 (Maecianua) ; D 36.1.23.1 ; D 38.1.13.1 (cons ti tution de Marc-Aurèle) ; 0 40.5.4.19 (constitution de Marc-Aurèle) ; D 40 . 5.24.10-11 ; D 40.5.26.7 ; L. Rufinus D 40.5.16 ; Marcien D 40.5.55.1 (Marcellus) ; Modestin D 40.4.43 ; D 40.5.12.2 (rescrit de Pertinax).

149- W.W. BUCKLAND, Roman law, cit., p. 513. plus importante quant à son utilité pratique est que; par l'affranchissement fidéicommissaire le patron a droit aux bona de l'affranchi, c'est-à-dire a un droit successoral sur son patrimoine (150), alors que le libertus orcinus n'est pas tenu à ce devoir et pour cause.

Cependant, certains textes manquent de clarté. Par exemple, dans le livre D 40.5 (De fideicomissariis libertatibus), il arrive que $1 \mathrm{a}$ forme de l'affrachissement rappelle plutôt une liberte testamentaire directe. Il en est ainsi dans ce passage de Scaerole :

Titius Sticho servo suo libertatem dedit, si rationes sic dederit (151).

Il n'est nullement question du fiduciaire.

Inversement, dans le livre D 40.4 (De manumissis testamento), il semble que ce texte de Papinien concerne une liberté fidéicommissaire :

Testamento centurio servos ouos venire prohibuit ac petit, prout quisque memisset, eos manumitti. Libertates utiliter datas respondit, cum, si nemo servorum offenderit, omnes ad libertatem pervemire possunt; quibusdam autem per offensam exclusis residui in libertatem perveniunt (152).

En effet, cette disposition testamentaire qui vise à affranchir les esclaves qui le "méritent" nécessite l'intervention d'un tiers.

Que la confusion soit le fait des compilateurs ou des juristes eux-mêmes, au fond peu importe. L'attitude la plus objective consiste, à notre sens, à relever ces textes deux fois, dans la rubrique concernant l'affranchissement testamentaire et celle concernant l'affranchissement fidéicommissaire (153).

150- On relève une autre différence dans le fait que la liberté fidéicoumissaire peut être donnée à un sexrus alienus, W.W. BUCKL.AND, Op. cit., p. 513.

151- D 40.5.41.7.

152- D 40.4.51 pr.

153- Il n'y a bien entendu pas lieu à confusion lorsque, malgré le contexte, le contenu du paragraphe est clair ; par exemple, à propos du livre D 40.4 (De manumissis testanento), D 40.4.11 pr de Pomponius (Si legato semo fideicommissa libertas relicta est, vel heres vel legatarius eum cogitur manumittere) traite sans équivoque d'une liberté fidéicommissaire. 
En outre, d'autres textes parlent seulement de liberté conditionnelle. Or, ces deux procédess pourant $s$ 'en accommoder,il était difficile d'opérer une distinction (154), c'est pourquoi ces textes ont aussi été relevés dans les deux rubriques (155).

Une comparaison chronologique de la fréquence de la liberté testamentaire directe (156) et fiđéicomnissaire (157) nous conduit au tableau suivant :

154- W.W. BUCKIAND, Roman 1aw, cit., p. 517 s., cf. Paul D 40.5.33.1 ; Julien D 40.5.47.2 (Si Sticho libertas per fideicarmisoum data fuerit sub condicione).

155- Psr exemple, le texte indique seulement : si servus sub condicione liber $88 s 8$ iussus fuisset (Julien D 13.1.14 pr). Le problème apparaît, outre chez Julien D 13.1.14 pr, chez le même auteur D 30.91.1; D 40.9.5; Pomponius D 33.2.20; D 35.1.35; D 35.1.57; D 35.1.111 ; Africain D 21.2.46.3; Gaius D 28.7.22; D 30.68.2; Maecisnus D 35.í. A6.1; Marcellus D 39.6.38; Paul D 29.2.74.4; Ulpien D 15.1.9.5 (Julien); D 33.8.8.3 (Sabinus) ; D 34.5.10.1; D 50.17.161.

156- Ont Evidenment êté intégrîs dans cette rubrique les hypothèses concernant 1 'institution coume héritier de 1 'esclave par son maître et le legs fait a l'esclave par son maître, car elles requièrent pour leur validité l'affranchissement de l'esclave, w.W. BUCKLAND, Roman law, p. 505 , p. 144. Un problème se posait avec 1 'appellation statuliber. Selon la definition de Gaius (Inst. II, 200 : qui testamento aub aliqua condicione liber esse iussus est), le terme statuliber designe normalement 1 'esclave affranchi par testament sous condition. Paul donne en revanche une définition beaucoup plus large (D $40.7 .1 \mathrm{pr}$ : Statuliber est qui statutam et destinatom in tempus vel condicionem libertatem habet). Selon ce juriste, la statulibertas peut donc concerner un affranchissement non testamentaire; d'autre part, le dies est rajouté a la condicio non testamentaire ; d'autre part, le dies est rajouté a la condicio
(M. BRETONE, Statuliber, NNDI 18 (1971) p. 380). L'extension est surprenante et $n$ 'est probablement pas due a Paul mais aux compilateurs. La critique porte aussi sur D 40.7 .1 .1 où sont qualifiés de statuliber les esclaves affranchis en fraude des créanciers, (sur ce point, $F$. SCHULZ, Die fraudatorische Freilassung im Klassichen und justinianischen Recht, in ZSS Rom.Abt. (1928) 48, p. 234 s. ; A. METRO, La lex Ablia Sentia e le manomissioni fraudolente, in Labeo 7 (1961) p. 191 s.). On a néanmoins temu compte de ces deux textes car leur portée est relativement générale, notament dans $D$ 40.7 .1 .1 , oü 1 'affranchissement en fraude des créanciers n'intervient qu'accessoirement. En ce qui concerne D 40 . 9.16.4 qui traite au contraire de maniêre principale de l'affranchisse9.16.4 qui traite au contraire de manière principale de l'affranchiss
ment en fraude des crêanciers, ce texte est intégrable par la formule ment en fraude des créanciers, ce texte est intégrable par la formule
quasi statuliber. Ont ainsi été relevés Alfenus (chez auteur anonytre) quasi statuliber. Ont ainsi été relevés Alfenus (chez auteur anonyt the)
D 33.8 .14 ; D 40.1 .6 ; D 40.1 .7 ; D $40.7 .14 \mathrm{pr}^{-1}$; Proculus D 12.6.53 Iavolenus D 28.5 .66 ; D 31.37 ; D 32.29 .4 (Tubero, Labéon) ; D 32.30 . 2 (Trebatius, Labéon); D 32.77 ; D $33.8 .22 \mathrm{pr}$ (Labéon)-I (Trebatius, Labéon) ; D 35.1 .40 .3 ; D 40.7.28 pr-1 (Cassius) ; D 40.7.39 pr (Q. Mucius, Gallus, Servius, ofilius, Labéon)-1 (ofilius, Labéon) -2 (Trebatius, Labéon) -3 (Servius, Labéon) -4 (Trebatius, ofilius, Labéon) -5 ; $D$ 45.3.34; Neratius D 28.5.55; D 40.7.17; Celsus D 33.8 .25 ; D 34.5 .25 ; D 35 . 1.49 ; D $40.7 .23 \mathrm{pr}-1$; Valens $\mathrm{D} 32.12$ (Pegasus); D 35.1 .87 ; D 35.2 .
37 pr : Julien D 9.4.16; D 10.2.52.1; D 13.1.14 pr ; D 15.1.14 pr ; D 21.2 .39 .4 ; D 28.1.12; D 28.2.13.1; D $28.5 .7 \mathrm{pr}$; D $28.5 .8 \mathrm{pr}-1$; D 28.5.22; D 28.5.38.1-2-3; D 28.5.43; D 28.6.19; D 29.4.22.1; D 29.7 .2 .2 ; D 29.7 .4 ; D 30.81 .9 ; D $30.91 .1-5$; D 30.94 .3 ; D 30.96 4 ; D 33.5.9.1-2 (Q.Hucius) ; D 33.8.12; D 34.3.12; D 35.2.87.3 ; D $36.1 .26 .2-3$; D 40.4.15; D 40.4.16; D 40.4.17 pr-1-2; D 40.4. $18 \mathrm{pr}$ (Sabinus)-1-2 ; D 40.4.19.1; D 40.7.12; D $40.7 .13 \mathrm{pr}-1-2-3-4-5$; D 40.9 .5 pr (par a contrario)-1 ; D 40.9.7 pr ; D 42.8.15; D 46.3.33 Pr ; Pomponius D 28.5.21 Pr (Trebatius, Labéon)-1 ; D $31.11 \mathrm{pr}$ (LaPr ; Pomponius D 28.5.21 Pr (Trebatius, Labéon)-1; D 31 . W Pr (La-

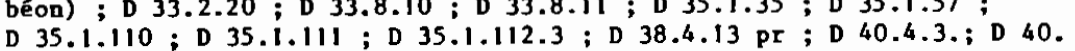

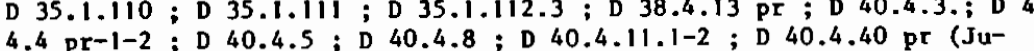

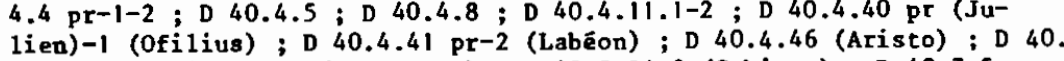
$4.61 \mathrm{pr}$ (Julien)-1-2 (Octavemus); D 40.5.34.2 (Sabinus); D 40.7.5 pr (Aristo, Neratius)-1 ; D $40.7 .8 \mathrm{pr}-1$ (Labéon); D 40.7 .11 (Aristo); D 40.7.21 pr (veteres, LabÉon) ; D 40.7.29 pr-1 (Q. Mucius, Labéon, Aristo, Celsus) ; D 40.7.30 ; D 46.3.92.1 ; D 47.2.44.2 ; D 50.16.120 D 50.16 .162 .1 ; Africain D 12.1.41; D 21.2.46.2-3; D 30.108.9; D 33. $8.16 \mathrm{pr}^{-1}$; D $35.1 .31 ;$ D $35.1 .32 ; \mathrm{D} 40.4 .20 ; \mathrm{D} 40.4 .21 ; \mathrm{D} 40.4 .22$; D $40.7 .15 \mathrm{pr}^{-1}$; D 40.9.8.1; D 46.3.38.3; D 47.2.62.9;D 48.10.6. 2 ; T. Clemens D 40.6.1 ; Gaius D 9.4.15 (Sabinus, Cassius) ; D 9.4 27.1 ; D 12.6.63 (Neratius); D 21.2.54.1; D 28.5.89; D.28.7.22; D 29.4 .17 ; D 29.5 .9 ; D 29.5 .25 .1 ; D $30.68 .1-2-3$; D 35.1 .88 ; D 35 . 2.73 .3 ; D 35.2.76 pr; D 36.1.65.15; D 39.6.31.2; D 40.4.24; D 40 . 4.57 (Sabinus, Cassius, Julien); D 40.7.31 pr-1 (Julien); D 40.7.37; D 40.12 .25 .1 ; D 47.4.2 ; D 49.14.14; Maecianus D 29.4.28.1 ; D 35 . $1.86 \mathrm{pr}$ (Julien) -1 ; D $40.4 .55 \mathrm{pr}-1$; D 40.4 .58 ; Venuleius D 34.4 .32 . 1 ; Marcellus D 9.2.36.1 (Sabinus) ; D 28.4.3 pr et 1 (rescrit de MarcAurèle) ; D 28.5.54; D 29.1.29.1; D 35.1.47; D 36.1 .46 pr ; D 39. $6.38 ; \mathrm{D} 40.1 .15 ; \mathrm{D} 40.4 .42 ; \mathrm{D} 40.5 \cdot 10.1-2 ; \mathrm{D} 40.7 .24 ; \mathrm{D} 40.13 .2$ D 46.3.68; Florentinus D 28.5.50 pr; D 29.1.24 (rescrit de Trajan); D 46.3.68; Florentinus D 28.5.50 pr ; D 29.67 pr ; D 21.2.69 pr-1-23 (Servius) $-4-5$; D 23.4.29.2 ; D 28.5 .84 pr (par a contrario)-1 ; D 28.5.86 (par a contrario); D 28.6.48.1 (Julien) 2 ; D 31.88.3-6; D 32.37 .7 ; D 32.38 .1 ; D $32.41 .2-3$; D 33.7 .7 ; D 33.8 .21 ; D 33.8 . $23 \mathrm{pr}^{-1-2-3}$; D 34.1.16.1 ; D 34.1.18 pr-1-3; D 34.1.20 pr-1 ; D 34.2 15 ; D 34.5.29; D 39.5.35 pr ; D 40.4.29; D 40.4.54 pr-1; D 40.4. $59 \mathrm{pr}-2$; D 40.4.60; D 40.5.41 pr-7-8-9-10-11-12-15-16-17; D 40.7.40. $1-3-4-5-6-7-8 ;$ D $42.8 .23 ; D 50.16 .243$; Papinien D 12.6.58; D 20.1. $3 \mathrm{pr}$; D 26.5.13 pr (rescrit d'Hadrien); D 28.3.17; D 28.5 .77 (Julien); D 29.5. $21 \mathrm{pr}$; D 29.7.11; D $31.65 .2-3$; D $31.76 \mathrm{pr}-4$; D 33. $8.19 \mathrm{pr}-1$; D 35.1 .72 .7 (Cassius, Caelius Sabinus) ; D $35.1 .77 \mathrm{pr}$ (rescrit d'Antonin le pieux) ; D 35.1 .78 pr ; D 35.2.11.1-4; D 36.1.57. I (rescrit d'Antonin le pieux) ; D 37.7.6; D 39.6.41; D 40.1.20.3; D $40.4 .47 \mathrm{pr}$; D 40.4 .48 (Servius) ; D 40.4 .49 ; D $40.4 .50 \mathrm{pr}$ (constitution de Marc-Aurèle) -1 ; D $40.4 .51 \mathrm{pr}-1$; D.40.5.21; D 40.7.33 D 40.7.34 pr-1 (rescrit de Caracalla) ; D 40.7.35 ; D 40.7.36 (prudentes) ; $D 47.2 .81 .1$; $D$ 48.19.33 (rescrit de Marc-Aurèle et Verus) ; Tryphoninus D 1.5.15;D $15.1 .57 \mathrm{pr}$; D 28.5 .91 ; D 31.88 .12 (Scaevola) ; D 34.3.28.7 ; D 40.4.59.1 (Scaevola); D 49.15.12.10-11 ; D 49.17.19.3 (constitution d'Hadrien)-5; Callistrate D 35.1 .82 ; Paul D 2.9.6; (constitution d'Hadrien)-5 ; Callistrate D 35.1 .82 ; Paul D 2.9.6 ;

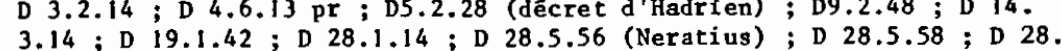
5.85 pr-1 ; D 28.6.38.3 (Scaevola) ; D 29.1.40.1-2 ; D 29.2.58; D 29. 2.60 (Labéon, Proculus, Iavolenus) ; D 29.2.74.3-4; D 29.7.8.5; 
D 31.8 .5 (Rescrit d'Hadrien) ; D 31.14 pr ; D 32.97 ; D 33.1 .16 ; D 33 8.15 (Alfenus) ; D $34.4 .26 \mathrm{pr}-1$; D 34.9 .5 .15 (Antonin le pieux)-18; D 35.1.44.2-10 (Plautius) ; D 35.1.46; D 35.1.81 pr ; D $35.1 .96 \mathrm{pr}-\mathrm{i}$; D 35.2.1.4; D 35.2.39; D 40.1.12; D 40.4.10 pr-1; D 40.4.27; D 40. $4: 28$; D 40.4.31; D 40.4.33;D 40.4.34; D 40.4.35 (Servius) ; D 40. 4.36 ; D 40.4.37;D 40.4.38; D 40.4.39; D 40.4.52; D 40.4.53. D 40.4 .56 (rescrit de Marc-Aurèle) ; D 40.5.33 pr ; D 40.5.38; D 40 . 5.40 .1 ; D 40.7.1 pr-1 ; D 40.7.4 pr-1 (Julien)-2-3-4-5 (Pomponius) 6 $-7-\mathrm{A}$ (Cassius); D 40.7.7; D 40.7.10; D $40.7 .18 ; \mathrm{D} 40.7 .20 \mathrm{pr} \rightarrow 1$ (Julien) $-2-3$ (Sabinus, Julien) 4 (rescrit d'Hadrien)-5-6; D 40.7 .22 pr-1-2; D 40.7.38; D 40.7.41 pr (Labéon)-1 (Labéon); D 40.7.42 (Labéon) ; D 40.9.13 ; D 40.9.16.4; D 40.9.17.2 ; D 40.9.18 pr-1 ; D 40.13. 4 (L. Rufinus) ; D 42.8 .16 ; D 44.4.3; D 45.1.91.1 (Julien); D 46.3 . 62 ; D 46.3.98 Pr ; D 48.10.22.7; D 48.18.8.1;D 49.15.19.6; D 49. 17.20 ; Ulpien D 1.5.16 ; D 3.5.44.1 ; D 4.4.7.5-10 (Papinien ; Antonin le pieux, Caracalla) ; D 4.4.22 ; D 5.2.8.9-10-16-17 ; D 5.2.29.2 D 5.3.7 pr (rescrit de Trajan)-1 (rescrit d'Antonin le pieux)-2 (idem); D 5.3.13.6 (Sabinus); D 9.2.15.1 (Julien); D 9.2.23.1 (Julien); D 9. $4.14 .1 ; D 10.2 .12 .2 ;$ D 10.2 .20 .9 (Papinien); D $10.2 .49 ; \mathrm{D} 12.4 .3 .6$ (Celsus) -7 (Celsus p., Celsus)-8 (Celsus)-9; D 14.4.9.2 (Labéon, Pomponius) ; D 15.1.3.1 (Labéon) ; D 15.1.9.5. (Julien) ; D 15.1.32 pt (Julien); D 21.1.17.16 (Caelius Sabinus); D 21.2.51.! (Julien); D 21 . 3.1 .4 ; D 24.1 .22 ; D 25.6.1.11 ; D 26.4.3.3; D 28.3.12.1 ; D 28.5.3.1-3; D 28.5.6.3 (Julien) 4 (Julien); D 28.5.9.14 (Labêon, Aristo, Neratius)-ló17-18-19: D 28.5.51 pr; D 28.6.10.7; D 28.6 .18 pr (Julien)-1 ; D 28 . 7.2.I. (Celsus) : D 29.1.13.3 (par a contrario)-4 (rescrit de Sévère) : D 29.1.19.1; D 29.2.20.4 (Julien, Paninien) ; D 29.2.71 pr-i-2 ; D 29.4.6.10; D 29.4.12 or ; D 29.4.29; D 29.5.1.4-13 (Scaevola) D $30.44 .7-8$; D 30.95; D 33.4.1.10; D 33.7.12.31 (Celsus); D 33.' 8.6.2-3-5; D 33.8 .8 pr (Pegasus) -1 (Julien) $-2-3$ (Sabinus) 4 ' (Sabinus)-5 (Labéon)-6-7 (rescrit de Sévère et Caracalla)-8 (Julien) ; D 34.3 .12 ; D 34.4.3.9 (Julien, Papinien) ; D $34.5 .10 \mathrm{pr}^{-1}$; D35.1.50 (Antonin le pieux) ; D 35.2.44 ; D 35.3.1.II-12 ; D 36.1.11.2 (Antonin le pieux); D $36.1 .15 .3 ; \mathrm{D} 36.1 .17 .3$ (Haecianus) ; D 36.1.23.1; D 36.2.7.6 ; D $36.2 .8 ; \mathrm{D} 36.2 .17$; D 37.5.8.2; D 37.10.3.11 (Julien); D 3E.1.13. 1 (constitution de Marc-Aurèle) ; D 39.6.8 pr (Julien); D 39.6.36; D 40.4.1; D 40.4.2 (Aristo); D 40.4.6; D 40.4.7 (Neratius); D 40.4. $9 \mathrm{pr}-1$ (par a contrario); D $40.4 .12 \mathrm{pr}-1-2$ (Julien)-3; D $40.4 .13 \mathrm{pr}-1$ -2 (Julien) -3 ; D 40.4.14; D 40.4.25;D 40.4.30;D 40.4.32; D 40 . 5.2 ; D 40.5 .3 ; D 40.5 .4 pr-1-2-3-4-5-6-12-13-14-17-18-19-20-21-22-23 (constitution de Marc-Aurèle) ; D 40.5.24.10-11; D 40.5.26.7 ; D 40 . 5.30 .12 ; D 40.7.2 pr-1 (Cassius, Julien)-2 (Julien) $-3-4$ (Celsus); D $40.73 \mathrm{pr}-1-2$ (Servius Laben, Cassius) $-3-4-5-6-7-8-9-10-11-12-13$ (Pomponius) $-14-15-16$ (Julien) $-17 ; \mathrm{D} 40.7 .6 \mathrm{pr}-1-2-3-4-5-6-7 ; 040$ 7.9 pr-1-2 (Octavenus)-3; D 40.7.16; D $40.7 .19 ; 040.12 .7 .4 ; 040$ $7.9 \mathrm{pr}-1-2$ (Octavenus) -3 ; D $40.7 .16 ; \mathrm{D} 40.7 .19 ; \mathrm{D} 40.12 .7 .4 ; \mathrm{D} 40$.
$12.12 .2 ; \mathrm{D} 40.12 .31 ; \mathrm{D} 44.2 .1 ; \mathrm{D} 44.4 .2 .7 ; \mathrm{D} 47.2 .52 .29 ; \mathrm{D} 47.4 .1$ pr-1 (Labéon) $-2-3-4-5$ (intégré en fonction du contexte)-6-8-9-10 (Scaevo1a) $-11-12-13-14-15$ (Scaevcla)-16-17-18-19; 0 47.4.3 (Labéon); 47 . 10.1.7 (Labéon); D 48.5.28.10; D 4́s.19.9.1ó (rescrit d'Antonin ie pieux) ; D 49.1.14 pr (Antonin le pieux)-1 (ezistǘa de Narc-Aurèle et Verus) ; D 49.17 .9 (ITarcellus) ; D 50.17.161 ; L. Rufinus D 40. 5.16; D 40.7.32 ; Marcien D 20.1.13.1; D 25.5.49.2 (par a contrario) (constitution de Sévère et Caracalla); 28.5 .52 pr (rescrit de MarcAurèle)-I (Celsus) 113.1 (rescrit de Sévère et Caracalla); $30.119 ; \mathrm{D} 33.8 .18 ; \mathrm{D} 36$.
1. $32 \mathrm{pr}-1$ (rescrit d'Antonin le pieux);D $38.4 .6 ; 040.1 .5 .1 ; \mathrm{D} 40$. $4.23 \mathrm{pr}-1$; $\mathrm{D}$ 40.4.26 (rescrits d'Antonin le pieux et de Marc-Aurèle Verus); D 40.5.51.3; D 40.5.55.1 (Harcellus); D 48.10.7; D 48.18. 9.3 ; D 49.1.5.1 (rescrit d'Antonin le pieux); Modestin D 5.2.9 (Pau1); D 5.2.12.1; D 31.9 ; D 31.34 .6 ; D 34.1 .4 pr ; D $35.1 .51 \mathrm{pr}^{-1}$; D $35.1 .53 ; \mathrm{D} 35.1 .66$; D $40.4 .43 ; \mathrm{D} 40.4 .44 ; \mathrm{D} 40.4 .45 ; \mathrm{D} 40.5 .12$. 2 (rescrit de Pertinax); D 40.5.14; D 40.7.25;D 40.7.26 pr-1; D 40 . 7.27 (Julien) ; D 48.18.14 ; Hermogénien D 5.1 .53 ; D $35.1 .94 \mathrm{pr}-1$; D $35.2 .38 \mathrm{pr} ; \mathrm{D} 36.4 .11 \mathrm{pr}$; D 37.5 .23 (constitution d'Antonin le pieux).

157- Iavolenus D 28.7.20.1 ; Valens D 4.4.33 (Julien) ; D 35.2.37 pr-1 ; D 38.1 .47 (Campanus) ; Julien D 13.1.14 pr ; D 29.7.2.2 ; D 30. $81.2-10$; D 30.91.1 ; D 30.92.1 ; D 30.94.3; D 36.1 28.17; D 40.4 . 15 ; D 40.4.19; D 40.5.47 pr-1-2-3-4 ; D 40.5.48; D 40.9.5 pr ; Powponius D 28.5.23.1; D 28.6.16 pr; D 30.54.2; D 33.2.20; D 35.1.6. 1 (Neratius); D $35.1 .35 ;$ D 35.1 .57 ; D 35.1 .111 ; D 40.1.13 (Octavenus) ; D 40.4 .5 ; D $40.4 .11 \mathrm{pr}$; D $40.4 .40 \mathrm{pr}$ (Julien)-I (Ofilius) : D 40.5.8 ; D 40.5.20 (Aristo, Octavenus, Julien); D $40.5 .34 \mathrm{pr}-1$ (Campanus) -2 (Sabimus); D 40.5.44; D 40.7.21.1 (P. Clemens) ; D 40 . 12.43 (rescrit d'Hadrien); Africain D 21.2.46.3; D 30.108 .14 ; D 30 . $109 \mathrm{pr}$; D 40.4.20 ; D 40.5.49 ; Gaius D 28.7.22 ; D 30.68.2 ; D 40 . 7.37 ; D 35.1 .90 (rescrit d'Antonin le pieux) ; Maecianus D 29.4.28.1 D 35.1 .86 .1 ; D 35.2.32.5; D $40.4 .55 \mathrm{pr}-2$; D $40.5 .32 \mathrm{pr}-1-2$; D 40 . 5.35 (Cassius) ; D 40.5.36 pr-I-2 ; D 40.5.42 (Antonin le pieux) ; D 40 5.54 ; Marcellus D 35.2.34 (Julien) ; D 35.2.56.3-4; D 39.6.38; D 40 . 5.9 ; D 40.5.10 pr-1-2; D 40.5.56;D 46.3.72.5 : Scaevola D 4.3.32 D 28.5.84.1; D 32.37.3 (constitution d'Antonin le pieux); D 34.1.18.2; D 34.1 .20 .3 ; D $36.1 .80 .2-3$; D 36.2.27.1 ; D 40.5.18; D 40.5.19 pr-1; D $40.5 .41 \mathrm{pr}-1-2-3-4-5-6-7-8-9-10-11-12-13-14-15-16-17$; D 40.7 .40 pr-2 ; D 48.10.24; Papinien D4.4.31; D 26.2.28.1 ; D 26.5.13 pr-1-2 ; D 27.1.24; D 29.2.86.2 ; D 29.7.11; D 29.7.12; D 29.7.13 pr ; D 31 . $77.11-29$; D 34.1.10.1 ; D 36.1.54.1 ; D 36.1 .55 ; D 38.1.42; D 38.2 . 41 ; D 40.1.20.1; D 40.4.47.1; D 40.4.51 pr;D 40.5.21; D 40.5.22 pr-1-2 ; D 40.5.23 pr-1-2-3-4; D 40.8.8 (constitution de Marc-Aurèle) : D 44.2.29 pr; D 50.1.17.8; Tryphonimus D 37.14.23.1 (rescrit d'Hadrien) ; D 40.5.17 (Scaevola) ; D 48.18.19; D 49.15.12.14; Callistrate D 48.10.15.2-3 (rescrit d'Antonin le pieux); Paul D2.4.9; D 4.8. 32.7 ; D 19.1.43; D 19.1.45.2 ; D 21.2.26 ; D 28.5 .56 (Neratius) D 28.5.85 pr ; D 29.1.40.2; D 29.2.74.4; D 31.5.1; D 31.14.1; D 31 . 84 ; D 32.8.1; D 34.5.9.4; D 35.1.37 (Neratius) ; D 35.2.33; D 35.2. $36 \mathrm{pr}-1-2-3-4$ (Africain); D $40.2 .15 \mathrm{pr} ; \mathrm{D} 40.4 .56$ (rescrit de MarcAurèle) ; D 40.5.5 ; D 40.5.6 ; D 40.5.25 (Valens) ; D 40.5.27 ; D 40. 5.29 ; D 40.5.31 pr-1 (rescrit de Marc-Aurèle et Verus)-2-3-4 (rescrit de Caracalla) ; D 40.5 .33 pr-1-2; 40.538 ; $40.539 \mathrm{pr}^{-1}$; 40.5 $40 \mathrm{pr}-1$ (par a contrario); $40.5 .43 ; \mathrm{D} 40.9 .16 \mathrm{pr} ; \mathrm{D} 40.13 .4$ (L. Rufinus) ; D 48.10.17; D 48.10.22.3-9-10; U1pien D 2.12.2; D 4.4.5; D 5.1 .67 ; D $5.2 .26 ;$ D 9.2 .23 .2 (Julien) D $9.4 .14 .1 ; 0$, 15.1 .9 .5 (Julien); D 25.3.5.22 (Marcellus); D 25.6.1.11; D 26.2.10.4; D 26 4.1.3 (rescrit d'Antonin le pieux); D 26.4.3.1-3; D 28.5.3.3; D 28 . 7.8 .7 ; D 29.2.42.3; D 29.2.71.1; D 29.4.12 pr ; D 29.5.1.5 (rescrit d'Antonin le pieux) ; D 32.3.1 (Julien) ; D 33.8.8.3 (Sabinus) ; D 34 5.10 .1 ; D 35.1.92; D 35.2.35 (Scaevola) ; D 35.3.1.12; D 36.1.6. 5 ; D 36.1.11.2 (Antonin le pieux) ; D 36.1.17.3 (Maecianus)-17 (rescrit d'Antonin le pieux); D 36.1.18.3; D 36.1.23.1; D 38.1.7.4 (rescrit d'Hadrien); D 38.1.13.I (constitution de Marc-Aurèle); D 38.2.16.9; 
D.38.16.1.1 (rescrits de Marc-Auréle et Verus et de Caracal1a) ; D 38. 16.3.1; D 38.17.1.3; D 38.17.2.3; D $40.2 .20 \mathrm{pr}$; D $40.4 .9 \mathrm{pr}$; D 40.5.1; D 40.5.2; D 40.5.3; D 40.5.4 pr-1-2-3-4-5-6-7-8-9-10-1112-13-14-15-16-17-18-19-20-21-22-23 (constitution de Marc-Auréle) D 40.5.7; D 40.5.24 pr-1-2-3-4-5 (rescrit de Caracalla)-6 (regcrit d'Antonin le pieux)-7-8 (Celsus)-9 (Marcellus)-10-11-12-13 (Marceld'Antonin le pieux) -7-8 (Celsus)-9 (Marcellus)-10-11-12-13 (Marce pieux); D $40.5 .26 \mathrm{pr}-1$ (constitution de Sévère)-2 (Antonin le pieux, rescrit de Sêvère et Caracalla)-3 (rescrit de Sévère et Caracalla) -4 (rescrits d'Antonin le pieux et de Sévère et Caracalla)-5-6-7-8 (rescrit de Sévère et Caracalla)-9-10-11; D 40.5.28 pr (Marcellus)-1-23-4-5 ; D 40.5.30 pr (rescrit de Caracalla)-1-2-3 (rescrit de MarcAurêle et Verus)-4-5 (rescrit d'Antonin le pieux) -6 (idem) 7 (idem) -8 -9-10-11-12-13 (rescrit de Marc-Aurèle et Verus)-14-15 (rescrit de Caracalla)-16 (rescrit de Marc-Aurèle)-17 (reacrit de Sévère et Caracalla) ; D 40.5.37 (rescrit de Marc-Aurèle) ; D 40.5.45 pr-1-2 ; D 40 $5.46 \mathrm{pr}^{-1-2-3}$ (rescrit de Sévère) $-4-5 ; \mathrm{D} 40.5 .52 ; \mathrm{D} 47.4 .1 .7$ (rescrits de Marc-Aurèle et de Sévère et Caracalla); D 48.5.28.10; D 50 crits de Marc-Aurèle et de Sévère et Caracalla) ; D 48.5.28.10 ; D 50 17.161 ; L. Rufinus D 40.5 .16 ; Marcien D $34.1 .2 \mathrm{pr} ; \mathrm{D} 37.14 .3$ (res-
crit de Sévère et Caracalla) ; D $38.2 .29 \mathrm{pr}-1$; D $40.2 .14 .1 ; \mathrm{D} 40.5$. 50 (Scaevola); $040.5 .51 \mathrm{pr}-1-2-3-4-5-6-7-8-9$ (rescrit d'Antonin le pieux)-10-11-12 ; D 40.5.53 pr-1 ; D 40.5.55 pr-1 (Marcellus) ; Modestin D 5.2.9 (Pau1) ; D 31.31; D 31.34 pr ; D 34.5.27; D 40.4.43; D 40.5.11; D 40.5.12 pr (Caracal1a)-1-2 (rescrit de Pertinax); $D 40$. 5.13 ; D 40.5.14; D 40.5.15; Hermogénien D 5.1.53.

Le fidếicoumis peut correspondre à trois hypothèses : en premier lieu, l'hêritier peut être chargé d'affranchir l'esclave du testateur, de mềme qu'une personne qui 1 'a reçu en legs ou fidéicommis (W.W. BUCKLAND, Roman law, cit., p. 527) ; en second lieu, l'héritier, le légataire ou celui qui reçoit le fiđêicormis peuvent être chargés d'affranchir leur propre esclave (ib., p. 529) ; enfin, le bênéficiaire doit parfois acheter et affranchir un esclave (ib., p. 530). Ces trois hypothèses sont distinctes de celle qui concerne le transfert d'un esclave ut marumittatur. Ce cas n'entrant pas directement dans la problématique du texte, il convient de 1 'exposer rapidement dans cette note. On esclave peut être vendu ou donné ( $p a r$ ex. D.38.1.13 pr; $\mathrm{D} \mathrm{40.9.30} \mathrm{pr)} \mathrm{pour} \mathrm{ê-}$ tre affranchi immédiatement (par ex. D 40.1.20 pr ; D 24.1 .7 .9 ; D 40 . tre affranchi izmêdiatement (par ex. D 40.1 .20 pr ; D 24.1 .7 .9 ; D 40. 8.9), ou durant une certaine période (par ex. D 37.14.8.1 ; $D 40.8 .1$ D 29.2.71.1), ou après un certain délai (par ex. D 18.7.10; D 24.1. 7.9 ; D 40.1.20.2 ; D 40.12.38.1-3). Une constitution de Marc-Aurè1e prévoit que s'il n'est pas dûment af franchi, il sera libre en vertu de la transaction (par ex. D 38.1 .13 pr : Si quis hac lege emptus sit, ut manwittatur, et ex constitutione divi Marci pervenerit ad libertatem...), w.w. BUCKLAND, op. cit., p. 628, On relêve cette hypothẻse dans le Digeste chez Julien D 17.1.30; D 40.9.7.1 (Proculus); Gaius D 17. 1.27.1; D 24.1.8; Marcellus D 18.7.4; Scaevola D 45.1.122.2 ; Papinien D 18.7.8; D 40.1.20 pr (Zitterae de Marc-Aurèle)-1-2-3; D 40 . 8.8 (constitution de Marc-Aurèle) : Tryphoninus D 18.7.10 (Scaevola ; Hadrien, Marc-Aurèle) ; Callistrate D 40.8.3 (rescrit de Maśc-Aurèle et Commode) ; Paul D 18.7.3; D 24.3.63; D 28.5.85.1 (constitution de Marc-Aurèle) ; D 40.1.10 (constitution de Marc-Aurèle) ; D 40.1.23 ; D 40.8.1 (rescrit de Marc-Aurèle) ; D 40.8.9 ; D 40.9.16 pr-1; D 40. 12 $38 \mathrm{pr}$ (constitution de Marc-Aurèle)-1 (idem)-3; D 49.14.45.3; Ulpien D 2.4.10 pr (constitution de Marc-Aurèle); D 2.14.7.2(Julien, Mauricianus); D 4.4.11.1; D 12.4.5.1; D 23.2.45 pr; D 24.1.7.8-9; D 24.1.9 pr-1(Julien); D 26.4.3.2(constitution de Marc-Aurẽle); D 29.2.71.1; D 38.1.13 pr(constitution de Marc-Aurèle); D38.2.3.3; D38.16.3.3(constitution de Marc-Aurèle);
- analyse des paragraphes

\begin{tabular}{|c|c|c|c|c|c|}
\hline I & - & $=\quad-$ & I & - & $=$ \\
\hline II & $1 / 1$ & $=100 \%$ & II & $0 / 1$ & $=$ \\
\hline III & $163 / 215$ & $76 \%$ & III & $52 / 215$ & $=$ \\
\hline IV & $85 / 14 !$ & $60 \%$ & IV & $56 / 141$ & 40 \\
\hline v & $403 / 666^{\circ}$ & $61 \%$ & $v$ & $263 / 666$ & 39 \\
\hline TOTAL & $652 / 1023$ & $64 x$ & TOTAL & $370 / 1023$ & $=$ \\
\hline
\end{tabular}

- analyse des citations

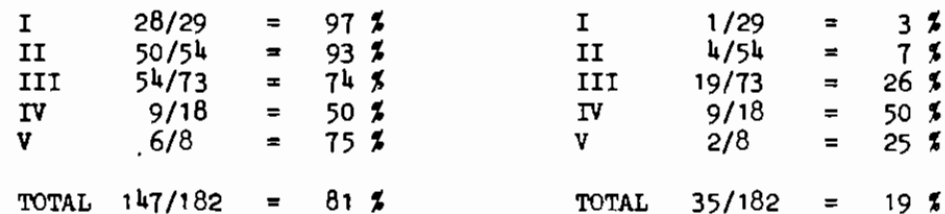

Les références à 1 'affranchissement testamentaire direct sont nettement plus nombreuses que les références à l'affranchissement fidéiconmissaire. Cette suprématie est vraisemblablement due à la faculté qu'a le maître d'instituer son esclave heres necessarius, celuici ne pouvant refuser la succession et devant en supporter les dettes (158).

Le fidéicommis de liberté est un développement du droit impérial (159). On remarque surtout une augmentation du pourcentage de textes concernant la liberté fidéicormissaire à partir de la période IV, correspondant aux règnes des derniers Antonins. Notament, les pourcentages issus de l'analyse des citations pour cette période indiquent un équilibre parfait entre les deux procédés d'affranchissement. Cette

D 39.5.18.1 (Aristo, Pomponius)-2 (les mênes) ; D 40.1.4.6; D 40.2 16.1 ; D 40.2.20.1 (constitution de Marc-Aurèle); D 40.8.4; D 40.9 . $30 \mathrm{pr}$ (constitution de Marc-Aurèle) ; D 49.17.6; Modestin D 1.5.22 (constitution de Marc-Aurêle); D 37.14.8.1. Sur les problèmes juridi(constitution de Marc-Aurèle) ; D 37.14 .8 .1 . Sur les problèmes juridi-
ques posés par cette forme originale d'acquisition de la liberté, W.W. BUCKLAND, op. cit., p. $628 \mathrm{~s}$.

158- M. KASER, RPR. I 2 , p. 684 n 33, p. 714 .

159- R. MONIER, Droit romain, cit., I, p. 220 . Le renvoi unique a 1a pêriode I s'explique par le fait qu'Ofilius (chez Pomponius D 40.4.40.1) connaît le début de 1 'époque impériale. 
progressicn correspond sans doute à la pénétration dans la pratique des sénatus-consultes de l'époque d'Hadrien assurant aux fidéicommis de liberté une pleine validité juridique $(160)$.

On peut maintenant se demander quelle est la place de l'affranchissement testamentaire, direct et fidéicommissaire, par rapport à l'affranchissement entre vifs. En tenant compte pour ce dernier procédé des textes où l'indication est seulement implicite, on obtient :

\section{Afrranchissement testamentaire}

Affranchissement entre vifs (16i)

\section{- analyse des paragraphes}

$\begin{array}{lcll}\text { I } & - & = & - \\ \text { II } & 1 / 1 & = & 100 \% \\ \text { III } & 215 / 283 & = & 76 \% \\ \text { IV } & 141 / 154 & = & 92 \% \\ \text { V } & 666 / 902 & = & 74 \%\end{array}$

TOTAL $1023 / 1340=76 \%$

- analyse des citations

\begin{tabular}{|c|c|c|c|c|c|c|}
\hline I & $29 / 35$ & $83 \%$ & I & $6 / 35$ & $=$ & $17 \%$ \\
\hline II & $54 / 70$ & $77 \%$ & II & $16 / 70$ & $=$ & $23 \%$ \\
\hline III & $73 / 97$ & $75:$ & III & $24 / 97$ & $=$ & $25 \%$ \\
\hline IV & $18 / 21$ & $86 \%$ & $\Gamma v$ & $3 / 21$ & $=$ & $14 \%$ \\
\hline $\mathrm{V}$ & $8 / 8$ & $100 \%$ & $v$ & $0 / 8$ & $=$ & - \\
\hline TOTAL & $182 / 231$ & $79 \%$ & TOTAL & $40 / 231$ & $=$ & 21 \\
\hline
\end{tabular}

La prépondérance de textes traitant d'affranchissement à cause de mort est écrasante quelle que soit la période envisagée. Ces chiffres doivent il est vrai être nuancés du fait que les textes concernant 1 'affranchissement entre vifs manquent souvent de clarté et surtout que ce procédé pose apparement beaucoup moins de problèmes aux juristes que l'affranchissement à cause de mort.

Il $n$ 'en demeure pas moins intéressant de constater que la Lex Fufia Caninia (2 ap. J-C.), qui limitait dès Auguste le nombre maximum

160- Cf. supra n 137.

161- Cf. supra n 139, 140, 141, 142, 143, 147. des esclaves pouvant être affranchis par testament (162), ne semble pas, si l'on s'en tient aux pourcentages extraits du Digeste et même compte tenu de la réserve précédemment formulée, avoir mis un frein à la pratique des affranchissements testamentaires. Il est vrai que ce procédé n'engageait en rien le testateur qui profitait, durant son existence, du travail de l'esclave, et qui ne faisait preuve de générosité qu'aux dépens de ses héritiers (163).

Le fait de se demander si l'affranchissement a lieu entre vifs ou à cause de mort entraîne une vision de l'affranchissement côté dominant. Or, il est fondamental, toujours d'un point de vue juridique, de tenter de déterminer la manière dont 1 'affranchissement est vécu par l'esclave. Pour cela, il faut poser aux textes d'autres questions : 1'affranchissement est-il onéreux ou gratuit ? Est-il simple ou conditionnel

Paragraphe 2: Affranchissement gratuit ou onéreux et affranchissenent simple ou conditionnel

\section{I - Affranchissement gratuit ou onéreux}

Les textes traitant d'un affranchissement onéreux sont nombreux et il convient de distinguer plusieurs hypothèses.

La suis nummis redemptio (164) est une variété du pacte de liberté (165). L'esclave, qui a l'initiative de la procédure (166), au

162- Ce nombre variant selon celui d'esclaves appartenant au testateur mais ne pouvant jamais dêpasser cent, cf. Gaius I, 42-46; critique du paragraphe 43 par S. SOLAZZI, Un ingenuo commento della legge Fufia Caninia in Gai.. I, 43, in SDHI 20 (1954) p. $303 \mathrm{s.}$ J. GAUDEMET, Institutions de 1'Antiquité, cit., p. 559 n. 1.

163- J, MACQUERON, Droit romain, cit., p. 342.

164- Concernent la suis nummis redemptio, dans le Digeste, les textes suivants : Papinien D 40.1.19; Paul D 28.5.85.2 ; D 48.19.38.4 ; Ulpien D 1.12.1.1 ; D 2.4.10 pr (constitution de Marc-Aurèle) ; D 5.1.67 ; D 23.2.45.2 ; D 25.3.5.22 (Marcellus) ; D 40.1.4 pr à 14 (epistula de Marc-Aurèle et Verus) ; Marcien D 40.1 .5 pr (constitution de Marc-Aurèle et Verus) ; Modestin D 27.1.14.3.

I65- M. JACOTA, Les pactes de l'esclave en son nom propre, RIDA 13 (1966), P. 219

166- W.W. BUCKL.AND, Roman law, cit., p. 645 
lieu de traiter directement avec son mâtre, agit par l'intermédiaire d'un tiers (167). Celui-ci,avec l'argent procure en principe par l'esclave (168), le rachète à son mâtre pour l'affranchir ensuite (169). Ce pacte est d'un usage courant à l'époque classique. Il est sanctionné par une constitution de Marc-Aurèle et Verus selon laquelle si le redemptor, c'est-à-dire le tiers, refuse d'affranchir l'esclave racheté, celui-ci pourra porter plainte devant le préfet de la ville à Rome et devant le gouverneur en province (170).

La deuxième hypothèse concerne le cas oul le maître reçoit de l'argent pour affranchir son esclave. Blle est distincte de la première à deux titres : premièrement, on a affaire ici au véritable mafitre de l'esclave alors que, dans le cas précédent, le manumissor ne se trouvait dans la position de maitre qu'aux frais d'un autre et dans l'unique but d'affranchir (171); deuxièmement, l'esclave ne joue plus forcément un rôle actif. En effet, l'argent peut être versé par l'esclave lui-même

167- Le choix de ce tiers n'est soumis aucune condition restrictive. Ainsi, il peut $s^{\prime}$ agir d'un esclave ou d'un pupille, W.W. BUCRLAND, op. cit.; p. 637. Aucune importance non plus quant a 1 'âge du vendeur, cf. Ulpien D 40.1.4.8.

168- L. VON SEUFFERT, Das Loskauf von Sklaven mit ihrem Geld, in $2 S S$, Rom. Abt. 29 (1908) p. 516 s. Ph. LOTMAR, Marc Aurels Erlass uber die Freilassungsauf lage, in ZSS, Rom. Abt. 33 (1912) p. $304 \mathrm{~s}$. ; M. JACOTA, Les pactes de 1 'esclave, cit., p. 219 n 23. L'argent peut provenir du pécule ; il peut être prêté ou donné par un ami ; il peut provenir de ce que l'esclave a gagné hors de la domus; l'esclave peut aussi donner ce qu'il a acquis par ses travaux ou payer une partie du prix par ces ce qu'il a acquis par ses travaux ou payer une partie du prix par ces
travaux, cf. Ulpien D 40.1 .4 .10 . W.W. BUCKI.AND, Roman law, cit., p. 637, relève que 1 'expression suis numis emptus est incorrecte puisque 1 'es-' clave ne peut pas avoir d'argent. Le raisonnement juridique fait en l'espece appel $a$ une fiction pour mettre le droit en accord avec les faits, M. JACOTA, Les pactes de l'esclave, cit., p. 223, cf. Ulpien D 40.1.4.1 : Et primo quidem numris suis non proprie videtur emptus dici, cum suos numos servus habere non possit : verum coniventibus oculis credendun est suis numis evon redemptum, owm non momis eius, qui eun redimit, comparatur.

169- L'esclave peut choisir ce mơyen pour parvenir à la liberté par exemple parce qu'il ne veut pas que son maître connaisse ses capacités financières ou parce que celui-ci, n'étant pas citoyen romain, ne pourrait pas lui donner le droit de cité, M. JACOTA, Les pactes de l'esclave, cit., p. 219.

170- Marcien D $40.1 .5 \mathrm{pr}$.

171- W.w. BUCKLAND, Roman law, cit., p. 642 ; Marcellus D 37.15.3. ou en son nom mais il peut aussi l'être par un tiers (172). Seuls deux textes du Digeste réfèrent à ce mode d'acquisition de la liberté comme devant produire un effet obligatoire. Selon Paul, la constitution de Marc-Aurèle concernant l'esclave vendu ut mornumittatur doit s'appliquer à cette espèce (173) ; autrement dit, la liberté doit prendre effet automatiquement (174). Papinien assimile ce cas à celui du semns suis nommis enptus, estimant que le magistrat peut contraindre le maitre à affranchir en cas de refus de celui-ci (175). Mais il semble que ces deux textes soient interpolés et qu'il ne s'agisse donc pas de solutions classiques (176).

Cette deuxième hypothèse ne doit pas être confondue avec celle de la liberté fidéicommissaire laissée à l'esclave du fiduciaire (177). Le droit est alors fondé sur l'idée de fides et sur le caractère inviolable de la volonté du testateur, deux considérations étrangères au

172- L'argent est fourni par' l'esclave : Alfenus (chez auteur anonyme) D 40.1.6 ; tavolenus D 15.3.2 ; Julien D 41.4 .9 (il fournit une esclave volée pour payer le prix de sa liberté) ; Gaịs D $16.1 .13 \mathrm{pr}$; Marcellus D 37.15.3 ; Pspinien D 15.1.50.3 (i1 emprunte l'argent à un tiers); Paul D 41.3.4.16 (Sabinus, Cassius)(il vole une esclave et la remet a son mâ̂tre comme prix de sa libertê); D 41.4.2.14 (Celsus) (il remet au maître une chose qu'il a achetée de mauvaise foi); D 44.5.2.2 ; Ulpien

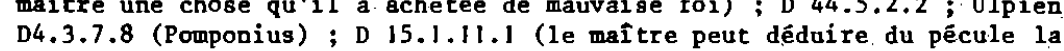
sorme que 1 'esclave doit lui verser pour l'obtention de sa liberté) D $15.3 .3 \mathrm{pr}$ (il emprunte 1 'argent $\mathrm{a}$ un tiers); D 16.3.1.33 (Jutien) (indirectement : il dépose la somme chez un tiers afin qu'il la remette a son maître) ; D 33.8.8.5 (Labéon); Modestin D 38.1.32. L'argent est fourni par un tiers chez Julien D $12.1 .19 \mathrm{pr}$; Marcellus D 37.15.3 ; Scaevola D 22.2.5 pr ; Papinien D 19.5.7; D 40.1.19; Paul D 19.5.5.2 (Julien); D 40.12.38.1 (constitution de Marc-Aurèle); 04 ! 3.4 .17 ; Ulpien D 12.4.1 pr ; D $12.4 .3 .2-3$ (Proculus) $-4 ; D$ 12.4.5.2-3-4;D 38.2 . 34 .

173- Cf. supra n. 157 in fine.

174- Paul D 40.12.38.1.

175- Papinien D 40.1.19: Si quis ab alio numos acceperit, ut servum sunon manumittat, etion ab invito libertas extorqueri potest, licet 'plerumque' pecunia eius numerata sit, maxime si frater vel pater naturalis pecunion dedit : videbitur enim similis ei qui suis numis redemptus est.

176- W.W. BUCKLAND, Roman law, cit., p. 642-643 ; M. JACOTA, Les pactes de 1 'esclave, cit., p. 222-223, ne soulève pas le problème a propos de D 40.1.19.

177- Renforcée par le s.c. Iuncianum, cf. supra n. 137. 
cas précédemment envisagé (178). Néanmoins, cette forme d'affranchissement apparaît aussi come étant onéreuse par le fait que le fiduciaire reçoit quelque chose en échenge de son acte (179). Plus généralement, il en va de même lorsque l'héritier ou le légataire est prié de racheter un esclave et de l'affranchir (180) ou lorsque, pour affranchir son esclave, le testateur lui remet quelque chose (181). Si l'esclave apparaît le plus souvent cantonné dans un rôle passif, des textes de Scaevola et Papinien nous apprennent qu'il n'en est pas toujours ainsi (182).

Tout comme l'affranchissement par fidéicommis (183), la manumissio testamento peut avoir un caractère onéreux. L'esclave affranchi par testament peut voir l'obtention de sa liberté conditionnée par la remise d'une somme à l'héritier (184) ou à un tiers (185). Dans un cas comme dans l'autre, il est perris au statuliber d'utiliser son pécuIe (186).

178- W.w. BuCKLAND, Romen law, cit., p. 646 n.. 2.

179- Marcelius lib. 42 digest. Iuliani notat : In testatoris servo non erit Falcidiae locus : ai vero pecumian aliudve quid legaverit fideique legatarii commiserit, ut aliemon servu vel legatarii manumitteret, locus erit, (D 35.2.34). 180- Par exemple, Maecianus D 29.4.28.1 : ... $8 i$ alienos servos roga-
tus sit marwittere, utique redimere eos debebit.

181- Par exemple, Scaevola D 40.5.41:3 : Seio auri libras tres et Stichum notarium, quem peto manumittas.

182-. Scaevola D 40.7.40.2 ; Papinien D 40.5.23.4.

183- L'affranchissement fidéiconmissaire ressort coume étant onéreux chez Valens $D$ 35.2.37.1 ; Pomponius D 40.5.8; $\mathrm{D} 40.5 .20$; Africain D 30.108.14; Maecianus D 29.4.28.1; D 40.4.55.2 ; D 40.5.32 pr-1; Mercellus D 35.2.34 (Julien); Scaevola D 40.5.41.3-5; Papinien D 40. $5.22 \mathrm{pr}$; Paul D 31.5 .1 ; D 35.2.36 pr-1 ; D 40.5 .6 ; D $40.5 .39 \mathrm{pr}$; Ulpien D 28.7.8.7 ; D 38.2.16.9 ; D 40.5.4.15 (constitution de MarcAurèle); D 40.5.7; D 40.5.24.12 (Marcellus)-13 (Marcellus) -14-15-17 -18 ; D 40.5.26.6 ; D 40.5.45.1 ; Marcien D 40.5.51.2.

184- Par exemple, Neratius $D$ 40.7.17 : si decem heredi dedisset, iussus eat liber esse.

185- Par exemple, PauI D 40.7.20 pr: Si peculium servo legatum sit, qui iussus est alii dare decem et sic liber esse...

186- Ce principe $n$ 'est sûrement pas inconnu des juristes' les plus anciens (cf. M. BRETONE, Statuliber, cit., p. 381). Il est appliqué par Sa dans la jurisprudence postérieure (cF. Lavolenus D40.7.39.2; Africain D47. 2.62.9; Gaius D 35.2.76 pr ; Papinien D 39.6 .41 ; D 40.7.35; Tryphoninus D 49.15.12.11; Paul D 40.9.18.1 ; Ulpien D 12.4.3.9; D 29.2.20. 4 (Papinien); D 35.2.44;D $33.8 .8 .7 ; \mathrm{D} 39.6 .36$; D 40.7.3.1-2-8;
Le paiement du prix de la liberté s'effectue fréquemment en argent (187). On trouve une liste des sommes que doit verser l'esclave dans l'étude sur le prix de l'esclave (188). Les sommes signalées à propos du paiement réalisé par un tiers sont du même ordre (189). Certains textes parlent de la remise d'une ancilla volée en échange de

D 44.4.2.7). La validité de 1 'alienatio numomon, invito herede, lorsque la condition consiste en une datio pecuriale en faveur d'un tiers, est discutée (cf. Paul D 40.7.20.1). Le statuliber peut aussi se libérer de la condition par ses operae (cf. Pomponius D 35.1.57).

187- Le statuliber doit fournir une some pour l'obtention de sa liberté chez Alfenus (chez auteur anonyme) D 40.7.14 pr ; Proculus D 12.6.53; Iavolenus D 40.7.39 pr (Q. Mucius, Gallus, Servius, Ofilius, Labéon) -2 (Trebatius, Labéon) -4 (Trebatius, ofilius, Labéon) -5 ; Neratius D 40 . 7.17 ; Celsus D 40.7.23 pr; Julien D 28.5.38.1; D 30.91.1; D 40.7 . 7.17 ; Celsus D 40.7.23 pr ; Julien D 28.5.38.1 ; D 30.91 .1 ; D 40.7. 13.1-5 ; Pomponius D 35.1.57 ; D 35.1.110; D 40.4.11.1 ; D 40.4.41.2 (Labéon) ; D 40.7.5.1 ; D $40.7 .8 \mathrm{pr}-1$ (Labéon) ; D 40.7.11 (Aristo); D 40.7.21 pr ; D 40.7.29.1 (Q. Mucius, Labéon, Aristo, Celsus) ; Africain D 21.2.46.3 ; D 40.7.15 pr-1 ; D 47.2.62.9 ; Gaiue D 21.2.54.1 ; D $35.2 .76 \mathrm{pr}$; D 39.6.31.2 ; Maecianus D 35.1 .86 pr (Julien) ; Marcellus D 39.6 .38 ; D 40.7 .24 ; D 46.3 .68 ; Scaevola D 12.6.67 pr ; D 21. 2.69.2-3 (Servius) -4 ; D 40.5.41.8-9-14; D 40.7.40.1 ; Papinien D 35. 1.72 .7 (Cassius, Caelius Sabinus) ; D $39.6 .41 ; \mathrm{D} 40.7 .34 \mathrm{pr} ; \mathrm{D} 40.7$ 35 ; Tryphoninus D 1.5.15; D 49.15.12.11; Paul D 19.1.42; D 35.1.44. 2-10 (Plautius); D 35.1 .46 ; D 40.4 .36 ; D $40.7 .4 \mathrm{pr}-1$ (Julien) $-3-4$ -6 ; D 40.7.10; D 40.7.18; D 40.7.20 pr-1 (Julien) $-2-3$ (Sabinus, Ju-6 ; D 40.7 .10 ; D 40.7 .18 ; D $40.7 .20 \mathrm{pr}-1$ (Julien) $-2-3$ (Sabinus, Ju-
lien) 4 (rescrit d'Hadrien)-5; D $40.7 .22 \mathrm{pr}-1-2$; D 40.7 .41 . I (Labéon) ; D 40.9.18.1 ; D 44.4.3' ; Ulpien D 5.2.8.10 ; D 5.3.13.6 (Sabinus); D 10.2.20.9 (Papinien); D 12.4 .3 .6 (Celsus)-7 (Celsus p., Celsus) -8 (Celsus) -9 ; D 29.2.20.4 (Julien, Papinien) ; D 29.4.6.10; D 33.8.8.3 (Sabimus)-7 (rescrít de Sévère et Caracalla) ; D 35.2 .44 ; D 39.6.8 pr (Julien) ; D 39.6.36 ; D 40.5.4.6 (constitution de MarcAurèle) ; D 40.7.3.1-2 (Servius, Labéon, Cassius)-3-4-5-6-7-8-9-11-12-13 -14 ; D 40.7.6.4-5-6-7; D 44.4.2.7; L. Rufinus D 40.7.32; Modestin D 5.2.12.1; D 35.1.51.1 ; D 40.7.27 (Julien) ; Hermogénien D 35.1 . $94 \mathrm{pr}-1$.

188 - Intitulée "Le prix de l'esclave ressort de la sonme qu'il doit verser pour l'obtention de sa liberté" (cf. supra première partie).

189- Par exemple, Julien D 12.I.19 pr:... si Titio decem dedero, ut Stichum intra calendas monumitteret; Papinien D 19.5.7 : Si tibi decem dedero, ut Stichum maramittas. A propos de la liberté fidéicomnissaire, Paul D 40.5.6 : Decem legata sunt et rogatus est legatarius Stichum emere et manumittere; vipien D 40.5.7 : Si cui legata sint centum ita, ut servw alienum redimat et manumittat; mais Pomponius rappelle que la some remise n'est pas forcément égale à la valeur de l'esclave, D 40.5.8 : Eun cui mille nami legati fuissent, si rogatus fuisset viginti semum manomittere... 
1a liberté de l'esclave (190). Le fait que celle-ci puisse être fournie par l'esclave lui-même est édifiant sur les rapports existant à l'intérieur du groupe servile, rapports dus à la diversité de condition économique de ses membres. Les esclaves qui se rachètent à leurs propres frais appartiennent principalement à de gros propriétaires, ce qui leur permet d'acquérir les moyens financiers nécessaires au rachat (191).

Quant à l'expression gratuita libertas, elle n'apparaît qu'une seule fois et par a contrario dans un contexte d'affranchissement onéreux (192). Il doit s'agir d'une pratique rare mais son existence est tout de même attestée par les textes sur les operae liberti. Reprenons en effet les différentes hypothèses d'affranchissement, onéreux et penchons-nous sur leurs conskquences juridiques. On a vu que l'esclave affranchi par fidéicomnis n'était pas tenu aux operae (193). Le problène ne se pose pas pour celui qui a directement été affranchi par testament. Quant à celui qui obtient sa liberté contre paiement à son maître, il n'est pas non plus soumis à cette obligation (194). Il

190- Celle-ci est remise par 1'esclave (Julien D 41.4.9: Qui ob pactionem libertatis ancilion furtivan a servo accepit, potest partion pactionem libertatis ancillan furtivan a servo accepit, potest partion eius quasi emptor usucapere; Paul D 41.3.4.16 : De illo quaeritur,
si servus meus ancillam, quam subripuit, pro libertate sua mihi dederit, an partum apud me conceptiom usucapere possim) ou par un tiers (Paul D. 41.3.4:17 : Sed et si, ut servon meron manumitterem, alius mihi furtivan ancilizon dederit).

191- De même, le vilicus ou l'institor du moyen ou du petit proprirtaire peuvent acquérir ces moyens, mais leurs possibilités sont probablement plus limitées, E.M. SCHTAERMAN, La schiavitũ, cit., p. 114 . 192- Marcellus D 37.15.3. Sur la fréquence de 1 affranchissenent onéreux, v. K. HOPKINS, Conquerors and Slaves, Cambridge(1978), p. $115 \mathrm{~s}$. 193- Cf. supra n 146 .

194- Le servus suis nummis redemptus $n$ 'est pas tenu aux operae (cf. D 37.15.3 Marcellus ; C 6.3.8. W.W. BUCRLAND, Roman law, cit., p. 640), de mêne que 1 'esclave transféré uí marumittatur (cf. Ulpien D 38.1 . $13 \mathrm{pr}$. W.W. BUCKIAND, op. cit., p. 631). Les droits du patronat sont plus complets dans le cas du maître qui reçoit une somme pour affranchir son esclave : ainsi, ce maître peut intenter contre son affranchi une accusation d'ingratitude (cf. D 37.15.3). C 6.4.1 pr (210) nous apprend qu'il détient ormia iura patronatus. Toutefois, c 6.3 .3 (206) ne lui reconnaît pas le droit d'exiger des operae. W.W. BUCKLAND, op. cit., p. 642, justifie cette solution par le fait que 1 'affranchissement cit., p. 642 , justifie cette solution par le fait que l'affranchissement
n'apas été gratuit en 1 'espèce. Sur le thème, voir aussi E.M. SCHIAERMAN, La schiavitu, cit., p. 108, 109, 112. On notera cependant qu'accepter de $1^{\prime}$ argent ne détruit pas le droit du patron aux bona de $1^{\prime}$ affranchi, w.W. BUCKIAND, op. cit., p. 641, n. 14. découle de cela que seuls les esclaves afranchis gratuitement entre vifs sont concernes par ce devoir, ce qui restreint certes grandement sa portée.

Il ressort donc du Digeste une prépondérance de l'affranchissement onéreux. Qu'en est-il de l'affranchissement conditionnel par rapport à l'affranchissement simple?

\section{II - Affranchissement simple ou conditionnel}

La concession testamentaire de la liberté peut être soumise à un délai ou à une condition (195) ainsi que le transfert d'un esclave en vue de son affranchissement (196), mais cette seconde modalité n'apparaît qu'assez rarement (197).

Le délai peut constituer le seul obstacle à l'obtention de la liberté testamentaire (198) : il en est ainsi de la forme "j'affranchis

195- W.W. BUCRLAND, Román law, cit., p. 479. La diotinction entre délai et condition est faite par Gàus D 28.7.22 ; Paul D 35.2.36.4 (Africain); D 40.7.1 pr; Ulpien D 32.3 .1 (Julien); D 40.5 .4 .5 (cotstitution de Marc-Aurềle) ; D 40.5.26.5. Le délai peut être connu (dies certus) ou non (dies incertus) ; dans le deuxième cas, il produit le. même effet qu'une condition, si l'on en croit Papinien (D 35.1.75:Dies incertus condicionem in testomento facit).

196- Il s'agit par exemple d'une esclave vendue a condition d'être affranchie après un an ( $c f$. Papinien $D$ 40.1.20.2) ou d'un esclave livré pour être affranchi après 5 ans (cf. Papinien D 40.1.20.3).

197- Il ne faut pas confondre un esclave livré pour être affranchi avec un statuliber (cf. Papinien D 40.1.20.3 : ... neque enim in omnibus libertatis causa 'traditun' comparari statuliberwon). Les textes où il est question d'un delai a propos du transfert d'un esclave en vue de son affranchissement sont les suivants : Papinien D 18.7.8; D 40.1 .20 2-3 ; Tryphoninus D 18.7.10 (Scaevola ; Hadrien, Marc-Aurèle); Callistrate D 40.8.3 (rescrit de Marc-Aurèle et Cormode) ; Paul D 18.7.3 ; D 40. 1.23 ; D 40.8.1 (rescrit de Marc-Aurèle) ; D 40.12.38 pr-3; Ulpien D 29.2.71.1; D 39.5.18.1 et 2 (Aristo, Pomponius); D 40.8.4; Modestin D 37.14 .8 .1 . On trouve une condition assortie d'un délai à propos de la même hypothèse chez Paul $\mathrm{D} 40.12 .38 .3$ : Gaius Seius Stichum servum Lucio Titio vendidit ita, ut Titius Stichrom post triennirom manumitteret, si continuo triennio servisset. On peut aussi relever la mention d'un délai à propos de la remise d'une somme au maître pour qu'il affranchisse son esclave (cf. Ulpien D 12.4.3.3 (Proculus) : Quid si ita dedi, ut intra certum tempus manwrittas?l.

198- Celsus D 35.1.49; Julien D 30.81 .2 (legs pour être affranchi); D 40.4.15 (idem) ; D.40.4.16 ; D 40.4.17.2 ; D 40.4.18 pr (Sabinus) -1-2 ; Pomponius D 40.4.4.1 ; D 40.4.41 pr-2 (Labéon) ; D 40.4.46 (Aristo) ; 
Stichus lorsque Titius aura 30 ans" (199) ou encore " $j$ 'affranchis tel esclave pour qu'il jouisse de la liberté dans dix ans" (200). Si on laisse la liberté à un esclave pour qu'il l'acquière effectivement après plusieurs années, sans fixer de terme, Julien estime par favor libertatis qu'il sera libre au bout de deux ans (201).

Le délai peut aussi se greffer à la condition sous laquelle la liberté testamentaire a été laissée (202) : par exemple, l'esclave est affranchi s'il parvient à fournir $l_{a}$ somme de cent en cinq ans (203) ou s'il donne dix avant telle date (204).

Les délais imposés sont très variables. L'esclave peut être tenu de faire quelque chose dans un intervalle réduit, six mois par exemple (205). Quelquefois, il ne peut être affranchi avant que s'écoulent sept, dix, voire même douze ans (206). Cet obstacle temporel à

56.3 (legs pour être affranchi) ; D 40.5 .56 ; Scaevola D 10.2.39.2 ; D 21.2.69.2; D 34.5 .29 ; Papinien D 31.65.2-3; Paul D 19.1.43; D 33.1 .16 ; D 35.2 .36 .4 (Africain) ; D 40.7 .1 pr ; Ulpien D 12.4 .5 .1 ; D 33.1 .16 ; D 35.2 .36 .4 (Africain) ; D 40.7 .1 pr ; Ulpien D 2.4 .5 (Julien); D 28.5.9.17-18-19; D 32.3.1 (Julien); D 40 . 5.4.5 (constitution de Marc-Aurèle) ; D 40.5.26.5; D 40.7.19; Marcien D 30.113.1 (rescrit de Sévère et Caracalla) ; D 36.1.32.1 (rescrit d' Antonin le pieux) ; D 40.4.23.1 ; Modestin D 1.5.22. Le délai peut aussi bien affecter la liberté testamentaire directe que la liberté fidéicommissaire (cf. pour cette dernière Julien D 30.81.2 ; D 40.4.15; Marcellus D 35.2.56.3; Paul D 19.1.43; Ulpien D 32.3.1 (Julien) ; D 40.5.4.5; D 40.5.26.5). La concession de la liberté $\mathrm{a}$ un egclave ior 40.5 .4 .5 ; 0 40.5. esclave lorsqu li mourca est évidement $1 \mathrm{aw}$, cit., p. 484 , on renconcre 1 hypothe chez Julien D 40.4.17 p (Libertas, quae in ultimon vitae tempus confertur, veluti 'Stichus oum morietur, liber esto', nullius momenti existinanda est) et chez Pomponius D 40.4.61 pr, et Paul D 40.7.4.1., ces deux juristes citant Julien.

199- Ju1ien D 40.4.16.

200- Paul D 33.1.16.

201- Julien D 40.4.17.2.

202- A1fenus (chez auteur anonyme) D 40.7.14.1; Iavolenus $D$ 32.30.2 (Trebatius, Labéon) ; D 40.7.28 pr; D 40.7.39.3; Celsus D 40.7.23 pr ; Julien D 28.5.38.1 ; D 40.7.13.5 ; Scaevola D 12.6.67 pr ; D 40.5.41 pr-10-12-13-15; D 40.7.40.2-7-8; Papinien D 40.5.23.4; D 40.7.34.1; Paul D 35.1.46; D 40.7.4.2.-4-5 (Pomponius)-8 (Cassius) ; D 40.7.18; D 40.7.41 pr (Labéont); vlpien D 40.7.3.11-13 (Pomponius)-14-15.

203- Ce1sus D 40.7.23 pr.

204- Julien D 28.5.38.1.

205- Scaevo1a D 40.7.40.8.

206 - Lavolenus D 40.7.39.3 : Dama semus cum heredi meo anomum septem oreras solverit, liber esto; Scaevola D 12.6 .67 pr : Stichus testcmento l'obtention de la liberté se caractérise par ce qu'il est extérieur à l'esclave. Cela est vrai même lorsqu'il est accouplé à une condition, celle-ci étant alors déterminée par le délai (207).

La condition peut elle aussi être extérieure à l'esclave qui ne joue dans ce cas aucun rôle dans sa réalisation (208). On peut ainsi laisser la liberté à l'esclave si l'héritier ne l'affranchit pas dans son testament (209) ou s'il ne l'aliène pas (210), si le testateur meurt sans enfant (211) ou si son fils est décédé (212), si la ferme à qui l'usufruit de l'esclave a été légué se marie (213), ou bien lorsque les dettes seront payées (214), ou encore, exemples types de conditions ne tenant pas à l'esclave, oi novio ex Asia venit ou si Titius consul factus sit (215). Bien que toujours extérieure à l'esclave, il est possible que la condition nécessite de sa part une certaine "activité" :

eius, quem dominum surm arbitrabatur, libertate accepta, si decem annis ex die mortis annuos decem heredibus praestitisset; Pomponius D 40. $4.41 \mathrm{pr}:$ :Stichus semrus meus anno duodecimo, postquam ego martus ero, liber esto.

207- Ainsi, dans ce passage de Scaevola (D 40.5.41.10: Cupitum servrom meum, cum Marcianus filius meus sedecim annos impleverit, rationibus redditis liberum egse volol, il est clair que l'obtentinn de la liberté ne dépend pas seulement du fait que l'esclave rende ses comptes mais aussi de ce que le fils atteigne l'âge de seize ans. De même, dens ce texte d'Alfenus (D 40.7 .14 .1 . Sermus cum heredi annorm septem opepas dediooets liber eooe iusous erat), l'esclave ment fournir ses operae à l'héritier, encore faut-il qu'il le fasse pendant sept ans.

208- Les textes où on relève des conditions ne tenant pas à 1'esclave sont : Iavolenus D 40.7.39.1 (Ofilius, Labéon) ; Julien D 40.7.13 pr ; D 40.9.5.1; Pomponius D 40.7.30; D 50.16.162.1; Africain D 21.2.46.2 ; Gaius 40.4 .57 (Sabinus Cassius ; Du1ien) : Gaius D 40.4.57 (Sabinus, Cassius, Julien) ; Maecianus D 40.4.55 pr ; Marcellus D 35.1 .47 ; Scaevola D 40.5 .41 .16 (la condition extérieure à 1 'esclave est associée à une condition tenant à 1ui : Spendophorus, cum filia mea in familia rupoerit, si rationes idonee filiae meae administratas reddiderit, liber esto); Papinien D 40.4.48 (Servius) ; Paul D 35.1.96 $\mathrm{pr}^{-1}$; D 40.4.28;D 40.4.38; D 40.7.20.6; D 40.7.42 (Labéon) ; D 40.9.16.4; Ulpien D 40.4.7 (Neratius)

209- Julien D 40.7 .13 pr.

210- Pomponius D 40.7.30.

211 - Vlpien D 40.4.7.

212- Pomponius D 50.16.162.1.

213- Pau1 D 35.1.96.1.

214- Julien $\mathrm{D} 40.9 .5 .1$.

215- Africain D 21.2.46.2. 
c'est ce qui ressort des fomules "mon fils, je te demande d'afrranchir mes trcis esclaves si tu es content de leurs services" ou "je veux que tu affranchisses mes esclaves s'ils le méritent" (216).

Si les conditions ne tenant pas à l'esclave ne soulèvent pas de problèmes juridiques particuliers (217), on ne peut en dire autant de celles dont 1 'accomplissement dépend directement de son activité (218). En

216- Africain D 40.4.20 : Servos legavit et cavit ita: rogo, si te promeruerunt, dignos eos libertate existimes ; Scaerola D. 40.5.41.1 : Lucius Titius ita cavit : Maevi fili camissime, te rogo, ut, si Stichus et Damas et Parmhilus te promeruerint, aere alieno liberato ne alterius quam tham servitutem experiantur ; D 40.5.41.4 : ... Ohasro, Bi paratis actoribus rationes reddere heres libertatem non praestet, paratis actoribus rationes reddere heres libertatem non praestet,
dicendo eos non placere sibi, an audienda esse. Respondit non spectondicendo eos non placere sibi, an audienda esse. Respondit nom specton-
dum, quod heredibus displiceret, sed id quad viro bono posset placere, ut libertatem consequantur; Papinien D $40.4 .51 \mathrm{pr}$ : Testamento centurio servos suos venire prohibuit ac petit, prout quisque meruisset, eos manumitti; Modestin D 40.5.14 : Erotem servion meum, qui et Fsytlus vocatur, libemon esse volo, si uxori meae placsat.

217- M. BRETONE, Statuliber, cit., p. 381 .

218- Traitent de conditions tenant 1 'esclave : Alfenus (chez auteur anonyme) D 40.7.14 pr-1; Proculus B 12.6.53; Iavolenus D 32.30.2 (Trebatius, Labéon) ; D $40.7 .28 \mathrm{pr}-1$ (Cassius).; D $40.7 .39 \mathrm{pr}$ (Q. Mucius, Gallus, Servius, Ofilius, Labéon)-2 (Trebatius, Labéon)-3 (Servius, Labéon) -4 (Trebatius, ofilius, Labêon) -5 ; Neratius $\mathrm{D} 40.7$. 17 ; Celsus D 40.7.23 pr-1; Valens D 35.1.87; D $35.2 .37 \mathrm{pr}$; Julien D 9.4.16; D 13.1.14 pr ; D 21.2.39.4; D 28.5.7 pr ; D 28.5.8.1; D 28.5 .22 ; D 28.5.38.1-2-3; D 30.81.9; D 30.91.1; D 30.96.4; D 33 . 5.9.1-2 (Q. Mucius) ; D 38.2.20 pr ; D 40.4.17 pr ; D 40.5.47.2-3. D 40.7 .12 ; D $40.7,13 \cdot 1-2-3-4-5$; D 46.3 .33 pr ; Pomponius D $31.11 \mathrm{pr}$ (Labếon) ; D 33.2 .20 ; D 35.1 .35 ; D 35.1 .57 ; D 35.11110 ; D 35.1 .111 ; D $35.1 .112 .3 ; \mathrm{D} 40.4 .8 ; \mathrm{D} 40.4 .11 .1 ; \mathrm{D} 40.4 .41 .2$ (Labéon); $\mathrm{D} 40.4 .61$ pr (Julien) $-1-2$ (Octavenus); D 40.7 .5 pr (Aristo, Neratius) -1 ; D 40 .

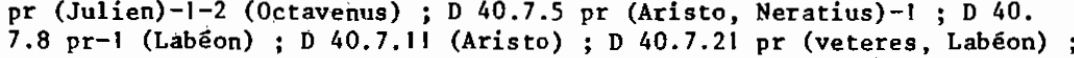
$7.8 \mathrm{pr}-1$ (Labéon) ; D 40.7.11 (Aristo); D 40.7.21) pr (veteres, Laber
D $40.7 .29 \mathrm{pr}-1$ (Q. Mucius, Labéon, Aristo, Celsus) ; D 46.3.92.1; Africain D 21.2.46.3 ; D 35.1.32; D 40.4.22; D 40.7.15 pr-1 ; D 46 . 3.38.3 ; D 47.2.62.9; Gaius D 9.4.15 (Sabinus, Cassius); D 9.4.27. 1 ; D 12.6.63 (Neratius) ; D 21.2.54.1; D 28.7.22; D 30.68 .2 ; D 35 1.88 ; D 35.2.73.3 ; D 35.2.76 pr ; D 39.6.31.2 ; D 40.7.31 pr (pris en fonction du contexte)-1 (Julien) ; D 40.7.37 (pris en fonction du contexte) ; D 40.12.25.1; D 47.4.2 ; Maecianus D $35.1 .86 \mathrm{pr}$ (Julien)-1'; Marcellus D 39.6 .38 ; D $40.5: 10.2 ;$ D 40.7 .24 ; D 46.3 .68 ; Scaevola D4.3.32; D 12.6.67 pr ; D 21.2.69 pr-1-2-3 (Servius) $-4-5 ; \mathrm{D} 33.8 .23 \mathrm{pr}$

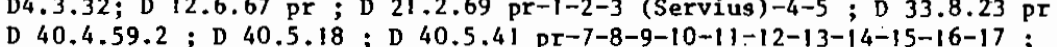

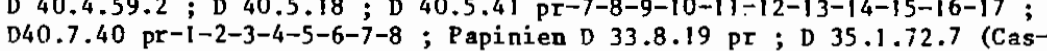
sius, Caelius Sabinus) ; D $35.1 .78 \mathrm{pr}$; D 35.2.11.1; D 39.6.41; D 40 . 4.51 .1 ; D 40.5.21; D 40.5.23.4; D 40.7.33; D 40.7.34 pr-1; D 40.7. 35 ; D 40.7.36; D 47.2.81.1 ; Tryphoninus D 1.5.15; D 49.15.12.10-11; Callistrate D 35.1.82 ; Paul D 2.9.6; D 19.1.42; D 29.2.74.3-4; D 34 . 9.5.18; D 35.1.44.2-10 (P1autius) ; D 35.1.46;D $35.1 .81 \mathrm{pr} ; \mathrm{D} 35$ 2.36.4 (Africain) ; D 40.4.28; D 40.4.36; D 40.4.39; D 40.4.53; D 40. 5.33 .1 ; D 40.7.1 pr-1 ; D $40.7 .4 \mathrm{pr}-1$ (Julien) $-2-3-4-5$ (Ponponi -8 (Cassius) ; D $40.7 .7 ; \mathrm{D} 40.7 .10 ; \mathrm{D} 40.7 .18 ; \mathrm{D} 40.7 .20$ pr-1-2-3 effet, celles-ci présentent deux difficultés : 1 a première quant, à $1 \mathrm{a}$ sphère d'autonomie dont jouit l'esclave afrranchi sous condition pour la réalisation de celle-ci (219); la seconde quant aux conséquences d'un obstacle mis par l'héritier à cette réalisation (2乞0). Mais ce

(Sabinus, Julien) 4 (rescrit d'Hadrien)-5 ; D $40.7 .22 \mathrm{pr}-1-2 ; D$ 40.7.38; D $40.7 .41 \mathrm{pr}$ (Labéon)-1 (Labéon) ; D 40.9.13; D 40.9.18.1 ; D 40.12 . 38.3 (il s'agit d'un transfert ut monomittatur); D 40.13.4 (L. Rufinus); D 44.4.3; D 45.1.91.1 (Julien); D 46.3.98 pr; D 48.18.8.1; D 49.15.19.6; Uipien D 1.5.16; D 5.2.8.10; D 5.3.13.6 (Sabinus): D 49.15 .19 .6 ; Ulpien D 1.5.16 ; D 5.2.8.10 ; D 5.3.13.6 (Sabinus) : D 9.4.14.1; D 10.2.12.2; D 10.2.20.9 (Papinien); D 12.4.3.6 (Celsus) -7 (Celsus P.. Celsus) -8 (Celsus) -9 ; D 15.1.9.5 (Julien); D 28 5.3.1-3 ; D 29.2.20.4 (Julien, Papinien); D 29.4.6.10; D 29.5.1.4 D 30.44 .8 ; D 32.3.1 (Julien); D 33.8 .8 pr (Pegasus) -3 (Sabinus) 4 (Sabinus)-7 (rescrit de Sévère et Caracalla); D 34.4 .3 .9 (Julien, Papinien): D 34.5.10.1; D 35.1.50 (Antonin le pieux); D 35.2.44; D 35.3.1.11; D 36.2.8; D 39.6.8 pr (Julien); D 39.6.36; D 40.4.6; D 40.4.12 pr-1-3; D 40.4.13 pr-1-2 (Ju1ien); 140.5 .4 .5 (constitution de Marc-Aurèle)-6 (idem); D 40.5.24.3-21 (rescrits d'lladrien et d'Ande Marchurele)-6 (idem) ; D 40.5.24.3-21 (rescrits d hladrien et d'Antonin le pieux) ; D 40.5.26.5; D 40.7.2 pr; D 40.7.2.1 et 2 (pris en fonction du contexte) ; D 40.7.2.3-4 (Ce1sus); D 40.7.3 pr-1-2 (Servius, Labéon, Cassius)-3-4-5-6-7-8-9-10-11-12-13 (Pomponius)-14-15-16 (Julien)-17; D 40.7.6 $\mathrm{pr}-1-2-3-4-5-6-7$; D $40.7 .9 \mathrm{pr}-1-2$ (Octavenus) -3 ; D 40.7.16; D 44.4.2.7; D 47.2.52.29; D 47.4.1.3; D 47.4.3 (Labéon); D 48.5.28.10; D 48.19.9.16 (rescrit d'Antonin le pieux) ; D 50.17.161 ; L. Rufinus D 40.7.32 ; Marcien D 20.1.13.1 ; D 40.1.5.1; D 40.4.23. 1 ; D 40.5.51.3 ; D 48.18.9.3; Modestin D 5.2.12.1; D 35.1.51 Pr-1; D 35.1 .53 ; D $35.1 .66 ; \mathrm{D} 40.4 .45 ; \mathrm{D} 40.7 .25 ; \mathrm{D} 40.7 .26 \mathrm{pr}-1$; D 40 . 7.27 (Julien) ; D 48.18 .14 ; Hermogénien D 5.1 .53 ; D 35.1 .94 pr-1 ; D $35.2 .38 \mathrm{pr} ; \mathrm{D} 36.4 .11 \mathrm{pr}$

219- On a envisagé ce problème a propos de la datio pecuniae du statuliber (cf. supra n. 184 a 186).

220- Pour permettre la réalisation de la condition de manière fictive, les juristes semblent requérir que l'empêchement dû à 1 'hëritier soi empreint de mauvaise foi ou bien que celui-ci ait simplement ignoré que son action nuisait à la liberté (cf. Ulpien D 40.7.3.11 : Si quis heredi in diebus triginta proximis mortis testatoris dare iussus fuemit, deinde heres tardius adierit. Trebatius et Labeo, si sine dolo malo tardius adierit, dontem eum intra dies tringinta aditae hereditatis ad libertatem pervenire : quae sententia vera est; Iavolenus D $40.7 .28 \mathrm{pr}$ Si. hereditas eius, qui servum, intra dies triginta mortis suae si rationes reddidisset, liberun esse iusserat, post dies tringinta adita est, iure quidem stricto ita manomissus liber esse non potest, quoriam condicione deficitur : sed favor libertatis eo rem perduxit, it respondeatur expletam condicionem, si per eum, cui data est, non staret quo minus expleretur). Mais la matière a été discutée (cf. Paul D 40.7. 38, lib. 1 ad Neratium, qui selon M. BRETONE, Statuliber, cit., p. 382 . répète peut-être des paroles de Neratius : Non onne ab heredis persona interveniens impedimentum statulibero pro expleta condicione cedit, sed id drontaxat, quod impediendae libertatis factum est). 
sont là deo problènes que la romanistique a dejà pris en considération et qu'il convient de délaisser pour examiner l'aspect pratique de ces conditions tenant à l'esclave (221).

D'un point de vue général, il faut tout d'abord remarquer que l'accomplissement de la condition peut être impossible. C'est le cas si on demande à l'esclave de fournir une some considérable qu'il ne pourra se procurer. La coneession de la liberté est alors nulle (222). Bosuite, la condition peut être négative et consister dans la nonréalisation d'un événement : par exemple, tel esclave sera affranchi s'il ne monte pas au Capitole (223). La validité de la concession dépend alors de l'intention du testateur. Si, par les paroles si Capitolium non ascenderit, celui-ci a voulu que la liberté ne produise aucun effet jusqu'à la mort de l'esclave, Julien écrit que la concession est nulle (224). Si par contre le testateur a employé ces paroles dans l'intention que l'esclave ne monte pas au Capitole dès qu'il en aurait l'occasion, ce dernier devient alors libre par son abstention. Telle est la solution qu'inspire le favor libertatis au même juriste (225). Cependant, la condition d'obtention de la liberté est le plus sourvent positive.

221- Il ne faut pas confondre ordre et condition : un esclave affranchi ita ut rationes reddat, de manière ce qu'il rende les comptes, n'est pas affranchi sous condition, W.W. BUCKLAND, Roman law, cit., p. 483, (cf. Julien D 40.4.17.1 : Haec scriptura testamenti 'Panphilus liber esto, ita ut filiis meis rationem reddat' an sub condicione libertas data videretur', quaesitum est. Respondi pure 'quidem' datam libertatem et illam adiectionem 'ita ut rationes reddat' condi-

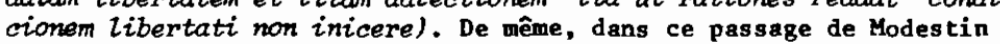
(D 40.4.44: Maevia decedens servis suis nomine Sacci et Eutychiae et Irenae sub condicione libertatem reliquit his verbis : 'Saccus servus sunto, ut monumento meo alternis mensibus lucernam accendant et sollemria mortis peragant' : quaero, cum adsiduo momonento Maeviae Saccus et Eutychia et Irene non adsint, an liberi esse possunt. Modestinus respondit neque contextum verborm totius scripturae neque mentem testatricis eam esse, ut libertas sub condicione suspensa sit, cum $l_{i}$ beros eos mommento adesse voluit : officio tamen iudicis eos esse compellendos testatricia iussioni parere), il s'agit encore d'un ordre et non d'une condition.

222- Paul D 40.7.4.1.

223- CF. Julien D 40.4.17 pr ; Pomponius (Julien) D 40.4:61 pr ; Paul D 40:4.28.

224- Chez Pomponius D $40.461 \mathrm{pr}$.

225- Julien D 40.4.17 pr.
Les conditions positives présentées par le Digeste sont nombreuses. L'esclave doit fournir ses operae, servir l'héritier (226), participer plus précisément à la construction d'un navire (227), d'une maison ou à l'érection d'une statue (228). Quelquefois, il doit seulement aller à Capoue (229) ou monter au Capitole (230). Ailleurs, une fenme esclave obtient la liberté si elle a trois enfants (231), on retrouve ici 1' "utilité" de la feme, ou si le premier enfant dont elle accouche est de sexe masculin (232). Mais les deux conditions les plus courantes sont la redditio des comptes (233) et la remise d'une some d'argent (234), des conditions à caractère financier. 226- Servire et operas dare sont équivalents ; les services doivent
être rendus personnellement, cf. Iavolenus D 40.7.39.5: Si serrus eperas extroneo dare iussus esset, extroneus nuilus nomine servi suas operas dando liberare servin potest. Quod in pecunia aliter observatur, utpote cum extraneus pro eo servo dando pecuniam servion liberaret. Fourair cent operae signifie fournir cent journées de travail, W.W. BUCRIAND, Roman law, cit., p. 487, cf. Paul D 40.7.4.4.

227- U1pien D 40.4.13.1.

228- Ulpien D 40.4.13 pr.

229- Pomponius D 40.4.61.1.

230- Julien D 30.91 .1 .

231- Tryphoninus D1.5.15: Arescusa, si tres pepererit libera esse testamento iussa... ; Ulpien D 40.7.3.16 : Item Iulianus libro sexto decimo digestorm scripsit, oi Arethusae libertas ita sit data, si tres servos pepererit...

232- Ulpien D 34.5.10.1 : Plane si ita libertatem acceperit ancilla 'si primum narem pepererit, libera esto'...

233- Cette condition consiste pratiquement à déclarer et à rendre compte des effets que 1 'esclave a eu a administrer, W.. . BUCR.AND, Roman law, cit., p. 494 ; si l'esclave n'a jamais eu à administrer, la condition est nulle, cf. D 40.7.26.1 ; Modestin : Servus iussus reddito ratione ad libertatem perverire, licet nullan administroverit rationem, nihilo mimus erit liber. nihito arms erit tiber. set implique rationes reddere, c'est-a-dire que la gestion a été exercée dans 1 intêret du womponius D 40.7.21 pr : Labeo libro posteriomm ita refert : 'Calenus dispensator meus, si rationse diligenter tmetasse videbitur, liber es to suaque omnia et centum habeto'. Diligention desiderare eam debemus, quae domino, non quae servo fuerit utilis). La condition reliqua reddere consiste simplement pour l'esclave à payer ce dont il est reliquataire sans avoir à fournir les pièces justificatives de sa gestion (cf. Callistrate D 35.1 .82 ).

234- Cf. supra n 184 a 186 . 
Envisageons đésormais ces differents textes de manière à établir 1a comparaison : la liberte depend-elle le plus sourent de conditions tenant à l'esclave ou d'un événement qui lui est extérieur ? Bn ce qui concerne le deuxième élément de la question, il convient de tenir compte des textes mentionnant un délai car, bien que ne concernant pas à proprement parler l'affranchissement conditionnel, ils visent l'acquisition de la liberté par un événement indépendant de l'activité ou de la volonté de l'esclave. Toutefois, il s'agit d'écarter les textes où le délai apparait greffé d̀ une condition tenant à l'esclave, les deux problématiques se trouvant alors confondues.

\section{L'acquisition de la liberté dépend de l'esclave (235)}

- analyse des paragraphes

$\begin{array}{lcll}\text { I } & - & = & \\ \text { II } & 1 / 1 & = & 100 \% \\ \text { III } & 82 / 107 & = & 77 \% \\ \text { IV } & 38 / 48 & = & 79 \% \\ \text { V } & 174 / 220 & = & 79 \% \\ \text { TOTAL } & 295 / 376 & = & 79 \%\end{array}$

- analyse des citations

$\begin{array}{lclllrl}\text { I } & 17 / 20 & & 85 \% & \text { I } & 3 / 20 & =15 \% \\ \text { II } & 29 / 36 & & 81 \% & \text { II } & 7 / 36 & =19 \% \\ \text { III } & 29 / 41 & = & 71 \% & \text { III } & 12 / 4 \% & =29 \% \\ \text { IV } & 0 / 1 & = & - & \text { IV } & 1 / 1 & =100 \% \\ \text { V } & 4 / 4 & = & & & \end{array}$

A l'exception de la période IV dens l'analyse des citations, qui ne compte qu'un seul texte et dont le pourcentage est d'ailleurs démenti par l'analyse des paragraphes, il est invariablement plus question d'un rôle actif de l'esclave dans l'acquisition de sa liberté que d'un rôle purement attentiste de celui-ci. Cela recoupe les données issues de l'analyse de l'affranchissement onéreux à travers le Digeste.

235- Cf. supra n. 218.

236- Cf. supra n. 197, 198, 208, 216.
Cependant, il est un aspect qui ne ressort pas de la prise en consideration quantitative des textes et qu'il est important de souligner. Il est possible que l'esclave ignore la condition sous laquelle la liberté lui a été laissée. Il ne semble pas pour autant que le cas soit fréquent, on le relève dans deux textes seulement (237). Cette ignorance est-elle le fait d'une négligence de sa part ? Lui a-t-on caché la nature de la condition pour l'empêcher de parvenir à la liberté ? Les textes ne répondent pas à ces questions. Méanmoins, un passage d'Ulpien, qui s'éloigne de notre problématique en ce qu'il ne vise pas l'affranchissement conditionnel, est tout de même intéressant. Ce juriste écrit que la liberté fidéicommissaire n'est quelquefois pas réclamée par ceux à qui elle est due en raison de leur négligence ou de leur crainte, timiditas, ou bien à cause du rang social élevé de ceux qui sont chargés de la délivrer (238).

Marcien nous apprend que l'affranchissement est simple lorsque la liberté n'est soumise à aucun terme ni à aucune condition (239). On troure alors les paroles pura libertas (240). Une comparsison quantitative des textes concernant l'affranchissement simple et de ceux traitant de l'affranchissement conditionnel ou grevé d'un délai montre une

237- Paul D 29.2.74.3-4 (Julien).

238- U1pien D 40.5.26.1.

239- Marcien D 40.4.23.1 : Testamento data libertas competit pure quidem data statim, quam adita fuerit hereditas vel ab uno ex hereiibus : si in diem autem libertas dato est vel sub condicione, tunc competit libertas, cun dies venerit vel condicio extiterit.

240- La terminologie pure ou pura libertas ressort de Julien $D 30$. 91.1 ; D 33.5.9.2 (Q. Mucius) ; D 40.4.17.1 ; Pomponius D 28.5.21.1 ; Scaevola D 40.5.41 pr ; Papinien D 31.76 .4 ; D34.1.10.1 ; D 35.1.

$77 \mathrm{pr}$ (rescrit d'Antonin le pieux) ; Tryphoninus D 40.4.59.1 (Scaevola); Paul D $35.1 .81 \mathrm{pr}$; Ulpien D 3.5.44.1; D 28.5.3.3; D 29.5.1.5 (rescrit d'Antonin le pieux)-13 (Scaevola); D 33.8.8.1 (Julien); D 40.4.12.2 (Julien); D 40.4.14; D 40.4.25; D 40.5.4.5 (constitution de Marc-Aurẽle) ; D 40.5.37 (rescrit de Marc-Aurèle) ; D 47.4.1.7 (rescrits de Marc-Aurèle et de Sévère et Caracalla); Marcien $D 36.1$. $32 \mathrm{pr}$; D 40:4.23.1. Si on suit ce dernier texte (cité supra n. 239), il faut aussi tenir compte des paragraphes où il est question d'une liberté accordée sans dêlai (Julien D 33.5.9.1; Pomponius D 40.4. 4.1 ; Ulpien D 39.5.18.1 (Aristo, Pomponius). 
- analyse des paragraphes

$\begin{array}{llll}\text { I } & - & = & - \\ \text { II } & 0 / 1 & = & - \\ \text { III } & 7 / 114 & = & 6 \% \\ \text { IV } & 1 / 49 & = & 2 \% \\ \text { V } & 19 / 239 & = & 8 \% \\ \text { TOTAL } & 27 / 403 & = & 7 \%\end{array}$

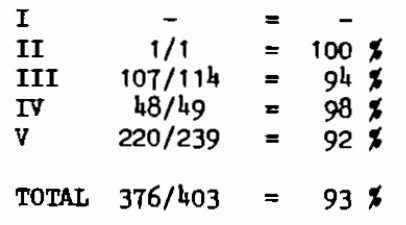

- analyse des citations

$\begin{array}{lllllll}\text { I } & 1 / 21 & = & 5 * & \text { I } & 20 / 21 & =95 \% \\ \text { II } & 0 / 36 & = & - & \text { II } & 36 / 36 & =100 \% \\ \text { III } & 4 / 45 & = & 9 * & \text { III } & 41 / 45 & =91 \% \\ \text { IV } & 2 / 3 & = & 67 \% & \text { IV } & 1 / 3 & =33 \% \\ \text { V } & 0 / 4 & = & - & \text { V } & 4 / 4 & =100 \% \\ \text { TOTAL } & 7 / 109 & & 6 \% & \text { TOTAL } 102 / 109 & =94 \%\end{array}$

supériorité très nette de ces derniers (241). La liberté, lorsqu' elle est assortie d'un délai ou d'une condition, pose évidement plus de problèmes aux juristes. Ainsi s'explique le caractère écrasant de cette prépondérance. En matière d'affranchissement, on peut sans doute parler de générosité des maîtres, mais cette générosité est-elle toujours désintéressée ? Les maîtres n'ont-ils pas tenđance à profiter au maximum de leurs esclaves jusqu'à l'obtention par ceux-ci de la liberté ? D'ailleurs, l'exploitation ne prend pas pour autant fin, on l'a vu, à l'affranchissement de l'esclave. C'est dans un tel sens que vont_ces édifiantes paroles de Modestin :

Interest enim nonnumquan a sene potius manwitti quan a iuvene (242),

c'est-à-dire : mieux vaut être affranchi par un honme âgé que jeune.

$$
-0^{-}-
$$

241- Il faut êmettre la même réserve qu'à propos du tableân précédent en ce qui concerne la période IV dans 1 'analyse des citations, mais le pourcentage de cette période est démenti par l'analyse des paragra-
phes.

$242-\quad D \quad 40.5 .15$.
On a dégagé de cette étude sur le passage de l'état d'esclave à celui d'affranchi les traits suivants : l'affranchissement à cause de mort ressort plus souvent des textes que l'affrenchissement entre vifs, malgré les limites posées par la lex Fufia Caninia au début de notre ère ; l'affranchissement onéreux apparâ̂t beaucoup plus fréquemment que l'affranchissement gratuit, tout comme l'affranchissement assorti d'un terme ou d'une condition par rapport à I'affranchissement simple. Autant d'appects qui vont dans l'intérêt bien senti des mâ̂tres.

De même que le groupe servile est composé d'éléments hétérogènes, la conđition des affranchis n'est pas monolithique. La première division qui vient à l'esprit est celle qui oppose les affranchis citoyens romains aux affranchis latins juniens (243) et pérégrins dêaitices (244), mais notre propos n'est pas de s'étendre sur ces différences juridiques qui n'epparaissent que peu dans le Digeste (245).

Par contre, les textes juridiques sont porteurs de distinctions au sein-même de le catégorie des affranchis citoyens romains. En premier lieu, la forme de l'affranchissement determine le contenu des droits du patron. Ainsi, on a vu que I'esclave affranchi par fidéicommis et celui qui rachetait sa liberté n'étaient pas tenus aux operae.

243- Sont latins juniens : en vertu de la lex Iunia, les affranchis qui tiennent leur liberté d'un acte non solennel, ou d'un maître qui n'avait sur eux que la propriété bonitaire; en vertu de la lex Aelia Sentia, ceux qui ont obtenu leur liberté avant l'âge de trente ans sans autorisation du conseil des affranchissements; 1 'esclave infirme abandonné, déclarế libre par un édit de Claude ; les enfants nés de parents latins juniens. Le latin junien vit libre, mais meurt esclave : à son dêcès, son patrimoine est considerré cơme un pécule d'esclave et retourne au maître, J. MACQUERON, Droit romain, cit., p. $354 \mathrm{~s}$.

244- Ils ont la pessima libertas et sont traités comme les pérégrins déditices. Ils n'ont aucun droit politique, ni familial, ni patrimonial, J. MACQUERON, Droit Romain, cit., p. $356 \mathrm{~s}$.

245- On peut présumer la présence d'un latin junien lorsqu'il s'agit par exemple de l'affranchissement d'un esclave de moins de trente ans sans 1 'autorisation du conseil des affranchissements. On peut également raisonner de même à partir de la formule in libertate (par ex. Pomponius D 40.12.28; Paul D 40.12.24.3, cf. supra $n$, 147). En revanche, rien n'apparaît sur des affranchis pērégrins déditices. 
Ils sont en revanche soumis au devoir d' obsequiwn envers leur patron (246) et celui-ci a droit à une part de leur succession (247). En second lieu, l'empereur peut de ga seule volonté mettre l'affranchi dans une situation privilégiée. Les inferiorités politiques et sociales qui sont le lot des affranchis citoyens romains ne pèsent pas sur

246- Ce devoir est envisagé chez Julien D $37.15 .2 \mathrm{pr}-1$; Marcellus D 37.15.3 ; Papinien D 37.15.11; D 38.1.41 (verecundia); Tryphoninus

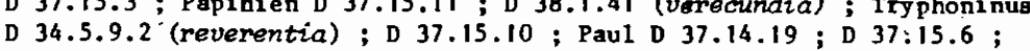
D 37.15 .8 ; D 47.2 .92 (Labéon) (verecundia) ; U1pien D 1.16 .9 .3 ; D 25 . 3.5 .20 ; D 37.15.1.1; D $37.15 .5 \mathrm{pr}-1$; D $37.15 .7 \mathrm{pr}-1-2-3-4-5$; D 37.15. 9 ; D 40.14.6 ; Marcien D 37.15 .4 (rescrit de Severre et Caracalla). Particulièrement êloquent ce passage d'Ulpien (D 37.15.9) : Liberto et filio aemper honesta et ancta persona patris ac patroni videri debet. L'obsequizm consiste notamment a ne pas intenter contre le patron d'action infamante (cf. Ulpien D 37.15.5.1 : Sed nec fomosae actiones adversus eos dontur, nec hae quidem, quae doli vel fraudis habent mentionem); sur les autres actions of 17.15 : De obsequi pant mentionem); sur les autres actions, Cr. D 37.15 . De ohsequits parentibus et patronis praestandis. L' obsequizon a un caractere personnel, il n'est dû qu'au patron et non a ceux qui interviennent pour lui (cf. Ulpien D 37 15.1.5 : Honor autem his personis habebitur ipsis, non etiam interventoribus eornon) ; de même, I'héritier de $I^{\prime}$ affranchi $n^{\prime \prime} y$ est pas tenu (cf. Paul D 37.15.8 : Heres liberti omnia iura integra extronei hominis adversus patronim defuncti habet) ; sur 1 'obsequizon a 1 'époque rêpublicaine, s. TREGGIARI, Roman freedmen, cit., p. 69 s. ; pour certains, l'obsequizon n'aurait pas été imposé par la loi à l'époque républicaine et $n$ 'aurait été sanctionné que lors de l'apparition de l'accusatio in grati liberti avec la lex Aelia Sentia, A.M. DUFF, Freedmen in the early Roman Empire, Oxford (1928), nouvelle édition Cambridge (1958),

37 ; C. COSENTINI adopte une attitude extrême en soutenant que 1 'obse37 ; C. COSENTINI adopte une attitude extrême en soutenant que l'obse-
quium, dans le sens technique de devoir dû par l'affranchi, n'apparaît pas en droit avant 1 'époque post-classique (Studi sui liberti, Catania (1948) p. 72 s.).

247- Sur 1'esclave affranchi par fj.déicoumis, cf. Marcien D 38.2. $29 \mathrm{pr}$. ; Gui ex causa fideiconorissi manumittitur, est quidem libertus manumissoris et tam contra tabulas quam ab intestato ad bona eius venire potest quasi patronus : sed operas ei imponere non potest nec impositas ab eo petere. Sur celui qui rachète sa liberté, cf. Ulpien D 38.2.3.4 (W.W. BUCKLAND, Roman law, cit., p. 641 n. 14). Sur le contenu et I'évolution de ce droit patronal aux bona de 1 'affranchi, cf. J. GAUDEMET,Institutions de 1'Antiquité, cit., p. $563 \mathrm{~s}$. ; J. MACQUERON, Droit Romain, cit., p. 352 s. : plus précisément, VAN WARMELO, Bona liberti, bona Latini Iuniani. Butterworth South Afr. Law Review III in memory of Jolowicz (1956), p. 27-38. celui qui a obtenu le ius aureorum conulomom (248). Il n'est pas tenu non plus aux operae mais son patron conserve le droit aux bona (249). L'individu qui a obtenu ce privilège vit comme un ingénu mais meurt comme un affranchi, écrit Ulpien (250). Seuls ceux à qui l'empereur confère la reatitutio natalium sont ingénus à part entière. Selon Modestin, ils sont ainsi lavés de toute macule servile, à tel point qu'ils sont considérés comme n'ayant jamais été esclaves (251). Il g'agit certes d'un discours juridique théorisant ; dans la pratique, on peut penser qu'il n'en allait pas forcément ainsi. Les textes concernant ces privilèges accordés par l'empereur ne sont pas nombreux,

248- Sur ius oureomom onulomon et restitutio natalizon, M. BARTOSEK, De 1 'ingénuité en droit romain, Czasopismo Prawno-Historyczne III (1951) p. 12-40. Concernant le ius aureorum anulomon dans le Digeste, Papinien D 40.10.1 pr-1 ; D 40.10.2 ; Tryphoninus D 27.1. $44 \mathrm{pr}$ (rescrit de Sévère et Caracalla)-1-2-3 ; D 29.5.11 ; D 48.5 .43 ; Paul D 40.10.5; U1pien D 2.4.10.3; D 38.2.3 pr ; D 40.10.4; D 40 . 10.6 (rescrit d'Hadrien) ; Marcien D 35.1 .33 .2 (rescrit de Sévère et de Caraca11a) ; D 40.10.3 (Commode); Modestin D 27.1.14.2 (rescrit de Caracalla). Sur les infériorités politiques et sociales des affranchis. cf. 1. MACQUERON, Droit romain, cit., p. 349 ; J. GAUDEMET, Insti tucf. 1. MACQUERON, Droit romain, cit., p. 349 ; J. GAUDERET, Ins ti tu-
tions de l'Antiquité, cit., p. $565 \mathrm{~s}$.; F. DE MARTINO, Storia, TV, cit. p. 352 (infériorités politiques : ius suffragii limité ; exclusion du ius honorion à Rome ; infériorités sociales : interdiction du mariage entre affranchis et personnes appartenant à L'aristocratie sénatoriale (lex Iulia de maritandis ordinibus) ; exclusion des droits d' agnation) ; sur l'évolution de ces inférioritês, cf. les ouvrages cités.

249- Paul D 40.10.5: Is, qui ius anulorrm impetravit, ut ingenus habetur, quamvis in hereditate eius patronus non excludatur.

250- Ulpien D 38.2.3 pr : Etiansi ius anulomon consecutus sit libertus a principe, adversus huius tabulas venit patronus, ut multis rescriptis continetur : hic enim vivit quasi ingenuus, moritur quasi libertus.

251- Modestin D 40.11.5.1 : Libertinus, qui natalibus restitutus est, perinde habetur, atque si ingenuus natus medio tempore maculam servitutis non sustimisset. Concernant la restitutio natalizm dans le $\mathrm{Di}$ geste : Scaevola D 40.11.3; Paul D 40.11.4; Ulpien D 2.4.10.3; D 38. 2.3 .1 ; D 40.10 .4 ; D 40.11 .4 ; Marcien D 40.11.2 ; Modestin D 40.11 . $5 \mathrm{pr}-1$. Le patron dont 1 'affranchi (ou l'affranchie, cf. D 40.10.

4) a obtenu la restitutio natalizm perd son droit aux bona, cf. Ulpieo D 38.2.3.1 : Plane si natalibus redditus sit, cessat contra tabulas bonomon posses8io; Marcien D 40.11.2 : ... Hic enim, quantiom ad totum ius pertinet, perinde habetur, atque si ingenuus natus esset, nec patronas eius potest ad successionem venire. Ne doit pas non plus les bona celui à qui l'empereur a concédé la liberté de tester, cf. U1pien D 38.2.3.2 : Idem et si a principe liberam testamenti factionem impetravit. 
mais le fait que deux titres du Digeste leur soient consacrés montre qu'on a probablement affaire ̀̀ une pratique non négligeable (252). Il faut cependant opérer une distinction à cet égard entre ius aureorum anuloron et restitutio natalizon. La première faveur n'entraînant plus à partir du règne de Comode l'accès à $l$ 'ordre équestre et n'étant donc plus subordonnée à ume exigence censitaire, on peut penser que sa concession fut assez fréquente (253). Si l'on suit Marcien, la seconde, décision impériale rendue avec l'assentiment du patran, n'était accordée que rarement (254).

$$
-0
$$

\section{SECTION 2. FORMES PARTICULIRRES DE LA DEPENDANCE}

Peu de textes traitent des esclaves ou affranchis publics et impériaux dans le Digeste. Le but de cette section sera donc essentiellement de rappeler brièvement l'état des connaissances sur la question.

\section{Paragraphe-1: Les esclaves et affranchis publics.}

Il faut distinguer les esclaves des villes de ceux de l'Etat.

Le servus publicus municipii (255) n'est qu'un exemple de l'ensemble plus vaste des servi universitatis (256). Il appartient à la

252- Cf. D 40.10 : De iure aureomon amilorum ; D 40.11: De natalibus restituendis.

253- J. GAUDEMET, Institutions de I'Antiquitê, cit., p. 567.

254- Marcien D 40.11.2 : ... Ideoque imperatores non facile solent quemquam natalibus restituere nisi consentiente patrono.

255- Il est question de servi publici municipii chez Iavolenus D 8 . 1.12 ; Paul D 41.2.1.22 (Nerva filius); U1pien D 6.2 .9 .6 ; D 29.2. 25.1 ; D 38.16 .3 .6 ; D 40.5 .24 .1 ; D 41.2 .2 ; D 48.18 .1 .7 ; D 45.3 .3 On trouve la terminologie ssrvus civitatis chez Papinien $D$ 40.3.3. Paul D 3.4.10; Ulpien D 2.4.10.4; D 15.4.4 (Pomponius) ; D 50.16 .17 pr Marcien $D$ 1.8.6.1. On relève 1a présence d'esclaves af franchis par des municipes chez Ulpien $D$ 38.3.1 pr-1-2 (Papinien).

256- W.w. BUCKJ.AND, Koman 1 aw, cit., p. 327 . On trouve servus corporis chez U1pien D 2.4.10.4; serrus decuriae chez U1pien D 29.2.25.1; serrus collegii chez U1pien D 2.4.10.4; D 29.2.25.1; D 40.3.1 (MarcAurè̀le); 040.3 .2 (il s'agit ici d'un affranchi). communauté (257) qui a sur lui les mêmes droits qu'un propriétaire ordinaire (258). Ses activités sont diverses (259) mais il semble qu'il soit souvent employé dans 1 'administration financière (260). On relève dans cette catégorie la présence de femmes (261), ce qui permet aux eervi publici civitatis de se reproduire par la naissance (262), contrairement aux servi publici populi romani (263).

Le peuple romain (264) possède aussi des esclaves publics (265).

257- Gaius II, 11. Ces esclaves peuvent être soumis a la quaestio contre un membre de la collectivité, cf. Ulpien D 48.18.1.7 : Servum manicipum posse in caput civizm torqueri saepissime rescriptran est, quia non sit illorm servus, sed rei publicae idemque in ceteris servis corpornon dicendum est; nec onim plurivm servus videtur, sed corporis. Ceux qui utilisent de tels esclaves pour leur usage personnel encourent de lourdes peines, cf. C I.6.1.5.

258- W.W. BUCRJAND, Roman law, cit., p. 327 et n.8

259- Il arrive même qu'ils soient utilisés comme gardes militaires, cf. Plin, Litt. Trai., 19, 20.

260- C I. 11.40 .

26I- L. HALRIN, Les esclaves publics chez les Rowains, Bruxelles (1897), Bib1, de la Fac. de Philosophie et Lettres de I'Université de Liège, fasc. 1, p. 120. Les textes cités par cet auteur (UIpien D 38.3 . $1 \mathrm{pr}$ : Municipibus plemon ius in bonis libertorwn libertamon defertur, hoc est id ius quod plenom ius in bonis libertorwn libertarm defertur servion mamomiserint, admittentur ad legitimam hereditatem in ban is libert servim mamomiserint, admittentur ad legitimam hereditatem in ban is libertivel libertae intestatoron) font allusinn a d'anciennes ancillae muni-
cipales. On cherche en vain des ancillae de I'Etat, cf. N. ROULAND A cipales. On cherche en vain des ancillae de I'Etat, cf. N. ROULAND、A
propos des eervi publici populi ramani, Aix-en-Provence (i975), p. 9 n. 1 .

262- Les enfants naissant dans ce groupe sont eux-têmes publics, cf. CIL, $11,2656$.

263- Sur la reproduction naturelle, cf. supra n. 262. L'appartenance au peuple romain entraîne une modification de certaines règles régissant 1 'esclavage : L'Etat peut acheter des esclaves pour en faire des servi publici, il peut aussi en acquérir par donation, succession (Th. MOMMSEN, Le droit public romain ( $=$ D.P.), trad. en 7 vol. par P.F. GIRARD, Paris (1889-1896), I, p. 363 ; L. HALRIN, Les esclaves publics, cit., p. 18 s.), mais il dispose aussi de modes d'acquisition inaccessibles aux particuliers, capture $\mathbf{z}$ la guerre et confiscation (Th. MOMMSEN, D.P., I, p. 363 ; L. HALKIN, op. cit., p. 16-18). La procêdure d'affranchissement de ces esclaves diffère également de celle du droit commun (cf. G. BOULVERT, Esclaves et affranchis impériaux, cit. P. 11) : Ie sénat décide de 1 'affranchissenent (I. HAtrin, op. une déclaration non é une déclaration non équivoque du magistrat cotopétert produit les effet de 1 'affranchissement solennel (Th. MOMMSEN, op. cit., p. 364 ; L HALRIN, op. cit., p. 30).

264- Th.MOMMSEN, D.P. I,p.362 s.: E.DE RUGGIERO, D.E.II, snR su , D.750 s. W.L. WESTERMANN, Slave systems, cit., p. 70-71. Sur 1'identification 
Ces esclaves sont d'une part chargés de représenter les citoyens dans la conclusion d'actes juridiques (266), et sont d'autre part nécessaires au fonctionnement des institutions (267). Le rôle qu'ils jouent dans la vie de l'Etat les place dans une situation privilégiée (268). Le salaire qu'ils perçoivent leur permet de mener une vie autonome (269). Preuve en est que des ingénues vont même jusqu'à s'unir à eux (270). Ils ont de plus la possibilité, par rapport aux esclaves "normaux", de disposer par testament de la moitié de leur pécule (271), et s'en distinguent donc par ce qu'ils sont investis d'une certaine capacité à

entre l'Etat et le populus romarus, M. RASER, RPR $I_{2}$, P. 304; sur les rapports entre personnalite juridique, Etat et populus romanus, B. ELIACHEVITCB, La personnalité juriđique en droit romain, Paris (1942). p. $3 i-32$.

265- Comme les autres institutions du régine républicain, ces esclaves continuent d'exister oous 1 'Empire, G. BOULVERT, Esclaves et affranchis impériaux (- EAI) p. $10 \mathrm{n}$ il ; L. HALRIN, Les esclaves publics, cit., p. 21-22 ; E. DE RUGGIERO, op. cit., p. 750 . Traitent de ces esclaves dans le Digeste: Marcellus D 1.7.18; Papinien D 16.2.19; Ulpien D 27. 8.1.15-16; D 45.3.3; D 46.6.2. Quant a D 22.1.11.1 de Paul (Quid si $8.1 .15-16$; D 45.3 .3 ; D 46.6 .2 . Quant a $\mathrm{D} 22.1 .11 .1$ de Paul (Quid $8 i$
servus publicus obligationem usuramon rei publicae adquisiit ? Aequrm est, quarvis ipso iurs usurae rai publicae debeantur, tamen pro defectis nominibus compensationem maiomon usuramon fieri, si non sit parata res publica universomom debitomu fortunam suscipere. Eadem fere in tutoribus Marcellus refert), W.H. BUCKLAND en traîte a propos des servi municipii (Roman law, cit., p. 329 , n 3).

266- Les citoyens pris tant collectivement qu'individuellement, $L$. hALRIN, Les esclaves publics, cit., p. 41 .

267- G. BOULVERT, EAI, p. 10 : cêlêbration ou aide des prêtres chargés de cêlébrer les cultes officiels (Th. MOMMSEN, D.P.,I, P. 368 ; L. HALKIN, Les esclaves publics, cit., p. 48 s. ; E. DE RUGGIERO, op. cit., p. 756-757) ; auxiliaire auprès des magistrats ; fonctionnement de grands services publics (ainsi une familia aquaria au service des eaux, cf. Th. MOMMEN, D.P. I, P. 370-374; L. HALRIN, Les esclaves publics, cit., p. 77-106).

268- Appartenant $a$ un être collectif, donc à tous, ces esclaves n'appartiennent en fait a personne, G. BOULVERT, EAI,p. 10.

269- Th. MOMMSEN, D.P. I, P. 365 ; L. HALRIN, Les esclaves publics, cit., p. I31. I1 semble qu'ils recevaient un salaire annuel (W.W. BUCRLAND, Roman law, cit., p. 320) ou au moins des cibaria anmua provenant de 1 ' aerarizon (L. HALKIN, op. cit., p. 115 ; cf. Plin., Litt. Trai, 31). Sur le mode de vie autonome les favorisant, N. ROULAND, Les servi publici dans le monde occidental romain, mêmoire D.E.S. dactyl. Aixen-Provence (1970) p. $281 \mathrm{~s}$. Un emploi simplement temporaire aux travaux publics ne leur donnait pas le titre de servus publicus (Tite-Live, 26, 47 ; Polybe 10, 17).

270- W.W.' BUCRLAND, Roman law, cit., p. 319 ; l. BALRIN, Les esclaves publics, cit., p. 135. Ils utilisent les termes employés par des époux unis en justes noces, ef. G. BOULVERT, EAI, p. 11 ; L. HALKIN, op. cit., p. $116 \mathrm{~s}$., mais ils n'ont pas le conubium, cf. J. PLASSARD, Le concubinat titre personnel reconnue par le droit (272). Ces esclaves étant principalement utilisés dans les affaires relevant du département sénatorial, ils vont diminuer avec l'absorption graduelle du pouvoir sénatorial par les empereurs (273).

\section{Paragraphe 2: Los esclaves et affranchis impériaux (274).}

Les esclaves et affrenchis impériaux n'appartiennent pas au popuLus mais personnellement à l'empereur (275). Le lien qui les unit à $l$ 'empereur n'est pas de nature publique, il est régi par les seules normes du droit privé (276). Combe un maître ordinaire, l'empereur exerce sur ses esclaves la dominica potestas (277). Il leur concòde un pécule a propos duquel certains textes semblent admettre une capacité

romain sous le Baut-Empire (1921) p. $120 \mathrm{~s}$. Les enfants issus de ces unions suivent la condition de la mère, cf. L. HALKIN, op. cit., p. 119. 271- Règles d'Ulpien, 20, 16 ; N. ROULAND, les servi publici, cit., p. 244. A 1 'appui de CIL, 10, 4687, L. HALKIN, op. cit., p. 197, pense que les servi moricipii ont la même facul té; contra, W.W. BUCKLAND, Roman law, cit., p. 328.

272- Cette facultê de disposer par testament de la moitiê de son pécule n'est pas une simple tolêrance de la part du maître dans le cadre de la fomilia (come par ex. chez P1ine, Lettres, VIII, 16) mais un véritable droit, G. BOULVERT, EAI, p. 12.

273- W.W. BUCRLAND, Roman law, cit., p. 319, 322.

274- W.w. BUCKIAND, op. cit., p. 323 s., distingue trois sortes d'esclaves impériaux : les servi (patrimoniales) Caesaris ou Augusti; les servi fiscales et les servi privatae rei Caesaris ; renvoi a 1 'ouvrage sur le contenu de la distinction. Dans le Digeste, on relève la terminologie servus Caesaris chez Pomponius D 28.5.42 (constitution de Tibère); U1pien D 1.19.1.2 ; Marcien D 48.19.17 pr ; Hermogénien D 49.14.46.8 (W.W. BUCKIAND, op. cit., p. 326, voit là un servus privatae rei); servus principis chez Callistrate D 47.9 .7 ; Ulpien D 40.5.24.1; Zibertus principis chez Callistrate D 47.9 .7 ; Ulpien D 38.16.3.8; fisci servus chez Callistrate D 49.14.12; Ulpien D 29.2.25.2 (selon W.W. BUCKLAND, Roman law, p. 325, le terme exclut les esclaves qui sont devenus 1a propriété du fisc par condamation (fisci mancipia), cf. C I.9.51.8). I1 faut aussi signaler des textes de Paul $D 1.15 .3 .2$ et $D$ 40.1.14.1. 275- G. BOULVERT, EAI, p. 12.

276- M. HAMMOND, The Antonine Monarchy, Rome (1957), p. 451 ; G. BOULVERT, Domestique et fonctionnaire (= DEF), cit., p. 10-43.

277- Comme tout propriétaire, 1 'empereur a sur son esclave la facultê de jouir de son activité a sa guise, ainsi qu'en témoignent toutes les inscriptions faisant état de la fonctinn exercée par l'esclave au service de l'empereur, que ce soit au palais dans le service domestique proprement dit, ou que ce soit dans un secrétariat ou un service financier impérial, G. BOULVERT, DEF, p. 85 s. 
jurldique $(238)$. Ii peut les aiiéner ou les affranchir, il a elors sur eux les droits de patronat (279). A 1a différence des esclaves publics, I'affranchissement ne marque pas pour les esclaves impériaux la fin de leur activité au service de l'Etat (2BO).

Du point de vue familial, les esclaves et affranchis impériaux contractent généralement des unions à 1 'intérieur de leur groupe (281). Quelle que soit la nature juridique de $I^{\prime}$ union, une famille est fondée (282) qui permet au groupe de se reproduire lorsque la mère est esclave (283).

Les esclaves et affranchis impériaux dispantront de 1 'administration avec le Dominat (284).

$-\circ$

278- Sous réserve de ne pas porter atteinte à la portion destinée a 1 'empereur, ef. Ulpien, Fr. de iure fisci, 6 ; w.W. BUCKLAND, Roman 1 aw, cit., p. 325 ; E.M. SCHTAERMAN, La schiavitu, cit., p. 149.

279- Obsequizm, operae, bona, ef. G. BOULVERT, DEF, p. 101-107.

280- G. BOULVERT, EAI, p. 13.

$28 \mathrm{I}$ - G. BOULVERT, EAI, p. 452 ; Id., DEF, p. 261 s. Des unions peuvent aussi.se nouer entre personnes de condition inégale : homane affranchi impérial, femme esclave du prince ou inversement (DEF, p. 274 s.) ; il n'est pas rare non plus que des esclaves impériaux, de sexe masculin ou féminin, de même que des affranchis (ou affranchies) impériaux s'unissent à des ingénus ou ingénues (DEF, p. $279 \mathrm{s.}$ ).

282- L'affranchissement permet à 1 'esclave d'acquêrir le comubium, la capacité matrimoniale (G. BOULVERT, DEF, p. 283), mais parmi les affranchis impériaux, il en est certains qui préfèrent au mariage une union licite, mais non juridique, le concubinat (ib., p. 284). Quant aux esclaves impériaux, ils sont dans $1^{\prime}$ impossibilitê de contracter un iustum matrimonizm (ib., p. $300 \mathrm{s.}$ ).

283- Ce mode d'acquisition d'esclaves impériaux est le meilleur car l'éducation dans une famille de fonctionnaires est de nature à préparer de bons fonctionnaires (G. BOULVERT, EAI, p. 452). L'encouragement à 1a reproduction naturelle apparaît clairement dans une mesure qui prévoit qu'une esclave impériale qui aura eu quatre enfants obtiendra automatiquement ia liberté (cf. Ulpien, Fr. de iure fisci, 13).

284- Le terme "Dominat" est traditionnellement utilisé pour désigner ie régire politique faisant suite au Principat (Th. MOMMSEN, DP, V, p. 19-20; P. DE FRANCISCI, Arcana Inperii, vol. 3, 1, Milan (1948), p. 269 s., $339 \mathrm{s.}$ ). Cependant, G. BOULVERT, EAI, p. 453, n. 1, souligne qu'il est inexactement opposê à Principat, Dominus étant déjà employé à propos de Caligula et se diffusant au 2 e siècle (3. BERANGER, Recherales sur íaspect idéologique du ?aincidat (1953), 0. 6l-63 ; J. GAI-

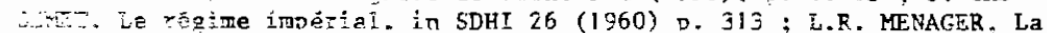

servile. chute de 1 'Empire romain, Aix-en-Provence (1966) p. 33). Il convient, avant d'en terminer avec cette rapide évocation des esclaves et affranchís impériaux, de citer les ouvrages de P.R.C. WEAVER, E(anilia Caesamis, Cambridge (1972), 330 p. ; H. CHANTRATNE, Feigelassene und Sklaven im Dienst der römischen Kaiser, Studien zu ihrer Nomenklatur, Wiesbaden (1967), $419 \mathrm{p}$. et le livre plus ancien de 0 . HIRSCHFELD, Die Raiserlichen Verwaltungsbeamten bis auf Diocletian (2e êd. 1905). Cependant, malgré leur élimination de I'administration impériale, des esclaves et affranchis impériaux continuent à exister, G. BOtrVERT, EAI, P. 456 : ainsi, un auteur de la fin du $3 e$ siècle, Hermogênien, traite des questions relatives aux esclaves ippériaux (cf. D 49.14 .46$. traite des questions relatives aux esclaves impériaux (cf. D 49.14 .46 voienti adquirit). 
CHAPITRE III : LES DOHHEES DES JURISTES SUR LA POPULATION SERVILE.

Le problème de la natalité a été eraminé propos de la reproduction naturelle du groupe servile, il n'est donc pas utile d'y revenir. Celui de la nuptialité sera envisagé plus loin. Bn ce qui concerne l'appartenance sexuelle des esclaves, il surfit de se reporter à l'étude terminologique pour constater que serva, ancilla et les autres termes désignant la femme esclave sont peu représentés par rapport à servus, homo et moncipirm. Cela ne signifie pas qu'il y ait moins d'esclaves du sexe féminin que mascuin. En effet, les termes désignant $l^{\prime}$ 'homme esclave ont une vocation plus générale, de la même manière que le mot homme englobe souvent le not femme. On possède aussi quelques indications sur l'âge de l'esclave, sans qu'il soit toutefois possible d'en tirer des conclusions sur sa duré de vie moyenne. Les tertes relevés sur la naissance font évidemment allusion à des enfants esclaves mais leur âge apparâ̂t rarement de manière précise. Paul parle d'une ancilla de cinquante ans (285). A propos de l'affranchissement, on note les âges de douze ans (286), quinze (287), vingt-cinq (288), trente (289), plus de cinquante (290). Ce dernier cas doit être exceptionnel puisqu'on prévoit une dispense des operae pour l'affranchie ayant passé ce cap. Un rescrit d'Antonin le pieux ordonnent la libération des vieux travailleurs des mines laisse à penser qu'il devait être difficile de "se faire vieux" lorsque les travaux exigés étaient durs (291).

\section{5- D. 19.1.21 pr.}

286- Papinien D 26.5.13.2.

287- Scaevola D 10.2.39.2.

288- Pau1 D 26.2.32.2.

289- Julien D 40.7.13.5 ; Pomponius D 40.4.46 (Aristo); Scaevola D $10.2 .39 .2 ; 034.5 .29$.

290- Paul D 38.1.35.

291- Chez Modestin D 48.19:22.. Selon J. SzILAGYI, Beiträge zur Statistik der Sterblichkeit in der illyrischen Provinzgruppe und in Norditalien (Gallia padana), in Acta arch. Acad. Sc. Hung. (1962), p. 297396 (tableau, 347), la longévité moyenne d'un esclave était d'une vingtaine d'années.
Les tertes juridiques sont un peu plus loquaces sur la situation geographique des esclaves. C'est sur la maladie et la mortalité que leurs renseignements sont les plus précieux.

\section{SECTION I. IA STTUATION GEOGRAPHIQUE DE L'ESCLAVE.}

Il est question dans trois textés seulement de l'origine geographique de l'esclave. Pomponius traite d'un interprète carthaginois capturé par les Romains (292). Ulpien écrit qu'on doit déclarer au censeur la natio de l'esclave (293), mais le passage le plus intéressant, du même auteur est celui-ci (294) :

Qui mancipia vendunt, nationem cuiusque in venditione pronunticare debent : plermaue enim natio servi aut provocat aut deterret emptorem : idcirco interest nostra scire nationem.

c'est-@̀-dire : les vendeurs doivent déclarer la natio des esclaves car c'est souvent le lieu de naissance qui détermine le choix de l'acheteur. Puis Ulpien poursuit : si le vendeur n'a pas fait cette déclaration, l'acheteur aura contre lui l'action redhibitoire. Un certain racisme ressort de ces lignes (295).

Plus nombreux sont les textes indiquant le lieu d'activité de l'esclave. Certains sont peu précis : l'esclave est simplement situé hors d'Italie (296), en province (297). D'autres montrent la présence

\section{2- D 49.15.5.3. \\ 293- D 50.15 .4 .5 .}

294- D 21.1.31.21.

295- J. GAUDEMET, Institutions de 1 'Antiquité, cit., p. 368 n. 4 : I'assimilation de l'étranger à un esclave au moins virtuel (H. LEVYBRUHII, Esquisse d'une thêorie sociologique de I'esclavage, in Problèmes du très ancien droit romain (1934), p. 15-34) est écartée par J. VOGT, Röm. Geschichte (1932) ; A. HEuss, völkerrecht, Grundlagen der rö̀m. Aussenpolitik, KIio 31 (1933) ; E. MEYER, Röm. Staat (1948), p. 220 Fus senpolitik, Klio 31 (1933) ; E. MEYER, Rö. Staat (1948), P. 220 ; tion Antonine de I'an 212 , in Rec. Soc. J. Bodin IX, I. L'étranger, Bruxelles (1958), p. 195-208; BONFANTE, in St. Betti 2 (1962), p. 8788 ; P. CATALANO, Sistema Sovranazionale romano, Turin (1965), p. 51-61. 296- Par exemple Papinien D 18.7.7.

297- Ainsi Paul D.41.2.1.14; Ulpien D 21.1.17.16; D 33.7.12.38; D 40.9.12.1. 
d'esclaves à Rome (298), à Capoue (299), en Ombrie (300), en Sicile (301) ; en Gaule (302), à Arles (303) ; en Hispanie (304), à Gades (305) ; en Afrique (306); en Syrie (307). Il est intéressant de noter qu'à propos de l'Afrique et de la Syrie, Julien parle de Iavolenus comme propriétaire d'esclaves (308). Il est ailleurs question d'un esclave capturé par les Germains ; il s'agissait d'un esclave volé (309). Les données relatives aux affranchis sont de la même nature : soit l'affranchi est simplement situé en province (310), soit il apparaît plus précisêment à Rome (311), en Italie (312), à Arles (313), en Afrique (314). Papinien parle d'um affranchi institué héritier par son patron de biens situés en Pannonie (315). L'esclave jouit dans certains textes d'une autonomie de fait, c'est le cas lorsque le lieu d'exercice de son activité est distinct du lieu de résidence de son maitre (316).

298- Paul D 18.7.9; Ulpien D 5.1.19.3.

299- Ulpien D 40.7.3.3.

300- Scaevola D 32.41.2.

301- Alfenus D 50.16.203.

302- Ulpien D 28.5.35.3.

303- Ulpien D 14.3.13 pr.

304- Papinien D 40.7.34.1.

305- Pomponius D 30.12.1 (Proculus).

306- Scaevola D 33.7.27.1.

307- Scaevola D 45.1.122.1.

308- Julien D 40.2.5 : An apud se manumittere possit is qui consilizm praebeat, saepe quaesitum est. Ego, qui meminissem Iavolenum praeceptorem meum et in Africa et in Symia servos suos mamomisisse...

309- Iavolexus D 49.15.27.

310- Paul D 38.1.20.1 (Proculus).

311- Paul D 35.1.84.

312- Dlpien D 26.7.3.4.

313- Scaevola D 33.2.34 pr.

314- vlpien D 26.7.3.4.

315- Papinien 528.5 .79 pr.

316- Par exeuple, Paul D 41.2.1.14 (Nerva filius); vlpien D 5.1.19.3 (Labéon) ; D 21.1.17.16 (Caelius Sabimus); D 28.5.35.3; D 33.7.12.38 (Papinien). Intéressant à un autre titre, Alfenus (chez auteur anonyme) D 50.16.203 : ... Quaerebatur, si quis a Sicilia servos Roman mitteret fundi instruendi causa...
Il est certes important de savoir avec précision où se trouve un esclave, mais le discours des juristes relatif aux maladies contractées par l'esclave ou à sa mort est plus significatif quant à la valorisation sociale de l'exploité.

SECTION 2. MALADIE ET MORTALITE : I'ESCLAVE, CAPITAL PERISSABLE.

\section{Paragraphe 1: L'esclave malade}

La définition de la maladie qu'il convient d'adopter est une définition extensive. I'esclave malade est pour le juriste un esclave que ses "défauts" physiques ou psychiques rendent inutilisable. C'est ce qu'écrit Paul (317). C'est aussi ce qui ressort de ces lignes qu'Ulpien emprunte à Sebinus :

"Sed sciendum est morbum opud Sabinum sic definitum esse habitum cuiusque corporis contra naturam, qui usum eius ad id facit deteriorem, cuius causa natura nobis eius corporis sonitatem dedit (318).

La maladie est l'affection du corps qui le rend moins apte à remplir les fonctions auxquelles la nature l'avait destiné.

Cependant, il apparâ̂t dans le cormentaire d'Ulpien sur l'édit des édiles, source principale du thème envisagé, que tout défaut physique n'est pas ume cause de redhibitio. Ainsi, si un esclave est doté d'un nombre de doigts supérieur à la normale, il est considéré comme sain pourvu que cela ne l'empêche pas de les utiliser (319). De même est sain celui dont I'oeil ou la joue est frappé de proéminence s'il parvient néanmoins à remplir sa fonction (320). Le fait que l'esclave ait une main palmée n'est pas une cause de redhibitio s'il en a $I^{\prime} u-$ sage (321). La maladie de l'esclave est donc clairement conçue par le juriste comme l'impossibilité de rentabiliser son travail.

317- Paul D 21.1.5: Et quantum interest inter haec vitia quae Graeci morbi malignam speciem dicunt interque malion aut morbum aut aegrotationem tantrom inter talia vitia et ewm morbum, ex quo quis minus optus usui sit, differt.

318- Jlpien D 21.1.1.7.

319- Ulpien D 21.1 .10 .2 .

320- Ulpien D 21.1.12.1 (Pedius).

321- U1pien D 21.1 .14 .6 . 
Les textes traitant de ce problème sont-ils nombreux ? On n'en relève pas moins de cent deux qui, si on les met en relation avec le nombre total de textes concernant 1 'esclavage par périodes, se répar tissent de la manière suivante (322) :

\section{- snalyse des paragraphes}

$\begin{array}{llll}\text { I } & - & = & - \\ \text { II } & -16 & = & - \\ \text { III } 23 / 985 & = & 2 \pi \\ \text { IV } 1 / 354 & = & 0,28 \\ \text { V } & 78 / 3840 & =\end{array}$

Réserve faite de la période IV qui manque de représentativité en l'espèce, on constate en général une continuité du thème de la maladie. La seule référence au thème consiste parfois dans l'emploi du mot morbus ou aeger (323), mais souvent la précision du texte est plus grande.

Parmi les maladies dont les juristes font état, il faut distinguer les maladies physiques des maladies psychiques. Le distinction est il est rrai fragile du fait que, comme le souligne ulpien, une

322- Ces textes sont les suivants : Iavolenus D 21.1.53; D 40.1 .26 (Labéon) ; Celsus D 17.1.50.1 ; Julien D 21.1.50; D 30.84.4 ; Pomponius D 12.1 .12 ; D 13.7.8 pr ; D 21.1.16; D 21.1.48.1 ; D 21.1.64.1 (Labéon); D 21.2.16.2 (Proculus) ; D 30.45.1; D 41.2.25.1; Airricain D 21.1 .34 pr ; D $21.1 .51 \mathrm{pr}-1$; D 30.108.11 ; Gaius D 7.1.45; D 9.2.8 pr ; D 13. 6.18.2; D 21.1.3; D 21.1.13; D 21.1.32; Venuleius D 21.1.65.j (Cassius); Paul D 6.1.79; D 13.6.22; D 14.2.2.5; D $21.1 .5 ;$ D 21 . l.11; D 21.1.15 ; D 21.1.30.1 (Pedius) ; D 21.1.43.4-6; D 21.1.44.2; D 25.1.2 ; D 25.1.12; D 29.2.93.2; D 40.7.4.5; D 41.2.1.10; D 41 . 3.31.3; D 45.1.91 pr; D 47.2.54.4 (Julien); Ulpien D 7.1.12.3 (Pomponius ; D 9.2.7.5 (Labeon)-6 (Celsus)-8 (Proculus); D 9.2.27.6 ; D9.2.9.1 ; D 10.4.17; D 19.1.13.22 (Labéon); D 21.1.1.1-6-7 (Sabinus) -8-9 (Vivianus) -10 (Vívianus) ; D 21.1.4.1-5 (Pomponius)-6 (Pomponius); D $21.1 .6 \mathrm{pr}$ (Pomponius)-1 (Trebatiug)-2; D 21.1 .7 ; D 21.1 .8 ; D 21.1 . 9 (Sabinus) ; D 21.1.10 pr (Ofilius) -1 (Cato) $-2-3-4$; D 21.1.12 $\mathrm{pr}^{-1}$ (Pedius) -2-3-4 (Trebatius) ; D 21.1.14 pr (Sabinus)-2-3 (Trebatius, Caelius Sabinus) -4 (Pedius) -5 (Pedius) $-6-7-8-9-10$ (Caelius Sabinus), Caelius Sabinus) 4 (Pedius) (Pedius) $6-7-8-9-10$ (Caelius Sabinus); D 21.1 .23 .2 (Pomponiug); D 21.1.31.12-16 (Julien); D 21.1.35; D 21 1.38.3-7-10; D 21.2.32.1 (Julien); D 24.3.7.16 (Pomponius) ; D 29.5. 3 pr-8-9-10-11; D 33.2.7 ; Modestin D 40.8.2 (édit de Claude) ; D 48 . 19.22 (rescrit d'Antonin le pieux).

323- Par exemple Ulpien D 21.1.38.3: Et fere eadem, sunt in his, quae in mancipiis, quod ad morbon vitiwne attinet; $\mathrm{D} 33.2 .7 ; \ldots$ aeger semus fuit. affection physique peut avoir des conséquences sur le psychisme de l'esclave (324). Autre distinction, qu'Ulpien attribue à Pomponius, celle qui oppose les maladies incurables aux maladies seulement temporaires (325).

Le titre du Digeste consacré à l'édit des édiles (D 21.1) donne une Véritable panoplie de ces maladies t, plus généralement, des défauts physiques de l'esclave. On trouve des esclaves frappés d'infirmité : un aveugle (326), un sourd (327), un muet (328), un bossu (329), un manchot (330), un esclave dont une jambe est trop courte (331). Ailleurs, l'esclave est atteint de fièvre tierce ou quarte (332), d'épilepsie (333), de phtisie (334), d'une affection du foie (335), d'une tumeur (336), d'une dartre (337), d'un polype (338). Mais le mal peut être plus léger : il s'agit de myopie (339), d'ophtalmie (340), d'un goître (341), d'un simple mal aux dents ou à

\section{4- Ulpien D 21.1.1.9 (Vivianus).}

325- Ulpien D 21.1.6 pr (Pomponius).

326- Ulpien D 21.1.1.7 (Sabinus); D 21.1.14.10 (Caelius Sabinus); D 29.5.3.9.

327- Gaius D 21.1.3; Ulpien D 29.5.3.8.

328- Gaius D 21.1.3; Paul D 29.2.93.2; Ulpien D 21.1 .9 (Sabinus); D 29.5.3.10.

329- Gaius D 21.1.3.

330- Ulpien D 21.1.12.3.

331- Ulpien D 21.1.12.1 (Pedius).

332- Iavolenus D 21.1.53; U1pien D 21.1.1.8.

333- Venuleius D 21.1.65.1 (Cassius); Ulpien D 21.1.4.5 (Pomponius).

334- Ulpien D 21.1.1.7 (Sabinus).

335- Ulpien D 21.1.12.4 (Trebatius).

336- Par exemple, une tumeur incurable dans la gorge, cf. Ulpien D21.1.14.8.

337- Ulpien D 21.1.6.1 (Trebatius).

338- vlpien D 21.1.12 pr.

339- Ulpien D 21,1,10.3.

340- Ulpien D 21.1.4.6 (Pomponius).

341 - Ulpien D $21,1 \cdot 12.2$. On peut relever par ailleurs un texte où 1 'esclave est atteint d'une incontinence de la vessie, cf. Ulpien D 21 . 1.14 .4 : Item de eo qui urinam facit quaeritur. Et Pedius ait non $o b$ ean rem sanum non esse, quod in lecto somno vinoque pressus aut etiom pigritia surgendi urinam faciat : sin outem vitio vesicae collectum unorem continere non potest, non quia uninam in lecto facit, sed quia vitiosan vesican habet, redhiberi posse : et verius est quad Pedius. 
l'oreille (342), de varices (343), de démangeaisons (344) ou encore d'une fièrre sans gravité (345).

Il est aussi important de remarquer, dans le sens développé plus haut de la rentabilisation de l'activité servile, que la feme dont la fréquence des règles est anormale (346), la femme stérile (347), celle qui souffre d'un vice de la matrice ( 348 ), ou qui est trop etroite pour enfanter n'est pas considérée comme saine (349). Il est tout à fait remarquable qu'Ulpien croit utile de préciser qu'une femme en couche est saine, pournu que ne survienne aucun accident (350).

Cette éventualité nous amène à envisager le pire: comment la mort de l'esclave apparaît-elle ?

\section{Paragraphe 2 : La mort de l'esclave}

Voyons d'abord dans quelles proportions ce thème ressort du Digeste. En adoptant la même démarche que précédemment, on parvient au tableau :

- analyse des paragraphes

$\begin{array}{lllllll}\text { I } & - & = & & \text { I } & 12 / 128 & =9 \% \\ \text { II } & -/ 6 & = & \text { II } & 33 / 347 & =10 \% \\ \text { III } & 63 / 985 & = & 6 \% & \text { III } & 52 / 519 & =10 \% \\ \text { IV } & 18 / 354 & =5 \% & \text { IV } & 10 / 105 & =10 \% \\ \text { V } & 256 / 3840 & =7 \% & & \text { V } & -135 & =\end{array}$

On ne relève pas moins de 332 textes concernant une mort d'esclave (351), ce qui constitue, par rapport au nombre total de textes

351- Il s'agit de Alfenus (chez auteur anonyme) D $9.2 .52 \mathrm{pr}-2$; Iavolemus D 24.1.50 pr-1 ; D 33.4.6.1 (Servius, Cascellius, Namusa, Ofilius, Labéon) : D 45.1.105 (Sabinus) ; Celsus D 17.1.50.1; D 30.63 (Servius); Julien D 9.2.47 ; D 9.2.51 pr-1 (veteres)-2 ; D 9.4.16 ; D 9.4.39.4; D 10.3 .25 ; D 12.6.32 pr ; D 13.5.23; D 14.4.8; D 15.1.14.1; D 18 . 5.5 .2 ; D 21.2.39.1; D 30.84.3-9; D 30.92 .1 ; D 30.96 .2 ; D 39.5.2.7 ; D 40.4.17 pr; D 45.1.56.8; Pomponius D 11.1.15.1; D 12.4.15 (Labéon, Proculus) ; D 13.7.8 pr; D 15.2.3; D 21.1.48 pr; $\mathrm{D} 30.36 .3 ; \mathrm{D} 30$. 48.1 ; D 30.54 .2 ; D 31.11 .1 (Julien) ; D 36.1.72. I ; D 40.4.6i pr (Julien); D 41.2.25.1; D 44.7.56; D 45.1.23; D 45.1.33; D 46.3.92 pr ; Africain D 7.1.36.2 ; D 12.1.23 (Julien); D 15.3.17.1; D 23.5.9.2 ; D 35.1 .31 ; D 46.3.38.3 ; D 47.2.62 pr ; Gaius D 6.1.36.I ; D 9.2.2 pr ; D 9.2.4 pr-1 ; D 9.2.8.1; D 9.2.32.1 ; D 13.6.18 pr; D 16.3.14.1 (Sabinus, Cassius) ; D 30.69.5 ; D 33.8.4 ; D 35.2 .73 pr ; D 39.4.13.3 ; D 40.7.31.1 (Ju1ien) ; D 44.7.1.9; Maecianus D 29.5.14 ; D $35.2 .30 \mathrm{pr}$; Venuleius D 46.2.31 pr ; Marcellus D 9.2.34; D 9.2.36 pr-l (Sabinus); D $36.1 .46 \mathrm{pr} ; \mathrm{D} 45.1 .96 ; \mathrm{D} \mathrm{46.3.72} \mathrm{pr}-1-3-4$; Scaevola D 2.14.54; D $10.2 .39 .2-4$; D 32.41 .4 ; D 40.9 .6 (Julien); D 45.1.127; Papinien D $10.2 .39 .2-4 ; D 32.41 .4 ;$ D 40.9 .6 (Julien) ; D 45.1 .127 ; Papinien S5; D 36.1.60.6; D 40.5.22.1; D 46.3.95 pr-1 ; D 48.3.2 pr; D 48.18 . $6 \mathrm{pr}$; Tryphoninus D $15.1 .57 \mathrm{pr}$; D 43.16.19 (Julien) ; Callistrate D 47.21.3.1 (Nerva); D 48.19.28.11-16; Paul D 4.3.18.1 ; D 4.3.18.5; D 4.8.32.5; D 5.3.36 pr (Proculus, Julien, Mauricianus)-2 (Pomponius); D 5.3.40 pr (Proculus, Cassius) ; D 6.1.27.2 ; D 6.1.79 (Labéon) ; D 9. 2.12 ; D 9.2.14; $\mathrm{D} 9.2 .18$; D $9.2 .22 \mathrm{pr}^{-1}$; D 9.2.31 (Q. Mucius); D 9. $2.45 \mathrm{pr}-2-3: \mathrm{D} 9.2 .30 \mathrm{pr}-1-4: \mathrm{D} 9.2 .33 \mathrm{pr}$ (Pedius) $: \mathrm{D} 9.2 .55 ; \mathrm{D} 9.4$ $26.4 ; D 10.4 .12 .4 ; D 11.1 .20 \mathrm{pr} ; \mathrm{D} 12.2 .30 .1 ; \mathrm{DI} 3.1 .3$ (Julien); D 13.5.21 pr;
D 14.2.2.5;D14.2.10 pr(Labéon);D15.1.48 pr;D17.1.22.6;D18.1.34.6;D18.4.21; D $14.2 .2 .5 ; D 14.2 .10 \mathrm{pr}$ (Labéon);D15.1.48 pr;D17.1 $22.6 ; \mathrm{D} 18.1 .34 .6 ; \mathrm{D} 18.4 .21$; D $21.1 .43 .4 ; D 21.1 .47 .1 ; D 23.3 .56$ Pr;D24.1.28.3 (PLautius) -4 (Pomponius);D 24

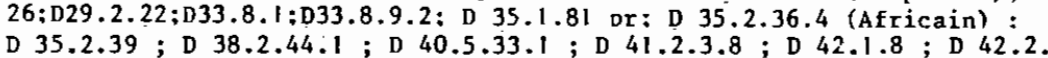
4 ; D 44.7.45 ; D 45.1.8 (Sabinus, Proculus, Marcellus) ; D 45.1.24 ; D $45.1 .49 \mathrm{pr}-3$; D 45.1.83.7 (Sabinus) ; D 45.1.88; D 45.1.91 pr-5 (Pomponius) -6 (Julien) ; D 46.1 .34 ; D 46.1.58.1 ; D 46.7.11 ; Ulpien D 2.1.12 ; D 3.5.5.10 (Pomponius); D 4.2.14.1I ; D 4.3.7.3 (Labéon) -4 -5; D 4.4.11.4 (Julien, Marcellus) -5 (Julien, Marcellus) ; D 4.7.4.5; D 4.9.7.4-6; D 5.3.33.1 (Julien); D 6.1.15.3; D 6.1.17 pr (Julien)-i (Julien); D 7.1.17.3; D 9.2.3; D 9.2.5 pr-3; D 9.2.7.1-2 (Pegasus) -3 (Proculus) $-4-5$ (Labéon) -6 (Celsus) -7 (Celsus) -8 (Proculus) ; D 9.2.9 -3 (Proculus) $-4-5$ (Labéon) -6 (Celsus) -7 (Celsus) -8 (Proculus) ; D 9.2 .9 pr-1-2 (Neratius) -3 (Ofilius) -4 ; D 9.2.11 pr (Mela, Proculus) $-\mathrm{I}-2$ (Julien) -3 (Celsus, Marcellus) 4 (veteres) ; $D$ 9.2.13.2 (Celsus) -3 (Julien); D 9.2.15 pr-1 (Julien); D 9.2.17;D 9.2.19; D 9.2.20; D 9.2. $21 \mathrm{pr}-1$ (Celsus, Julien) -2 ; $\mathrm{D} 9.2 .23 \mathrm{pr}$ (Neratius)-1 (Julien) -2 (Ju1ien) -3 (Julien) -4 (Labéon)-5-6-7-8-9-10-11 (Julien) ; D 9.2.25 pr ; D 9.2.27 pr (Celsus, Julien)-1 (Proculus, U, Ferox)-2 (Celsus) -5 ; D 9.2.35; D 9.2.44.1; D 9.2.46; D 9.4.5.1 ; D 9.4.42.1; D $10.4 .9 \mathrm{pr}$ (Julien):D 11.3.5.4:D 12.2.34 Dr (Marcellus); D $12.3 .5 .4 ; D 12.4$. 3.3 (Proculus) $4 ; \mathrm{D} 12.4 .5 .2-4 ; \mathrm{D} 13.1 .7 .2 ; \mathrm{D} \mathrm{13.3.3.;D} 13.6 .5 .7$ (Namusa, Mela) ; D 14.1.4.3-4; D 15.1.9.5 (Julien) -7 ; D 15.1 .11 .4 (Ju-

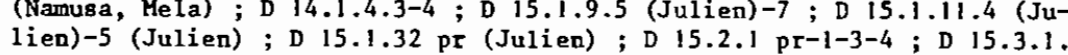
lien)-5 (Julien) ; D $15.1 .32 \mathrm{pr}$ (Julien) ; D 15.2.1 pr-1-3-4; D 15.3.
1 (Labéon) ; D 15.3.3.8; D 15.3 .7 .2 (Mela) ; D 16.3.1.30.; D 17.2 . 49 ; D 18.2.4.1 (Julien)-2 (Julien); D 19.1.13.22 (Labéon); D 19.2. 11.4 (Labéon) ; D 19.2.13 pr ; D 19.5.14.1 ; D 20.1.27 (Marcellus) ; D 21.1.17.4 (Proculus)-6 (Caelius Sabinus); D 21.1.23.3; D 21.1.31.6 (Pomponius)-11-12-13 (Pedius); D 21.2.21 pr ; D 23.3.10.5 ; D 23.3.12.1 
concernant l'eaclavage un pourcentage relativement important qui s'exprime de manièra sensiblement identique aux différentes périodes.

Dans certains paragraphes, la mort n'intervient que conme hypothèse de travail du juriste. Il en est ainsi lorsque celui-ci sippose, pour le développement $d$ 'un problème juridique, que l'escleve est mort ou afrranchi (352), assimilation qui, par ailleurs, ne manque pas d'intérêt. Par contre, il s'agit dans d'autres paragraphes de cas cancrets. On apprend par exemple que l'esclave est mort de maladie (353) ou accidentellement (354) qu'il s'est suicidé (355), ou bien que sa mort se situe au terme d'une procédure répressive (356), ou enfin qu'il a

(Marcellus, Scaevola) ; D 29.5.1.12 (Scaevola)-13 (Scaevola)-14-21-28 (Hadrien)-30-31-33; D 29.5.3.4-13-16-17-18; D 29.5.5.2; D 30.47.3. D $30.53 .3-8$; D 33.4.1.6; D 36.1 .11 .2 (Antonin le pieux) ; D 36.1 .23 . 3 (Neratius); D 38.5.1.25 (Pomponius); D 40.12 .7 .4 ; D 42.2.5; D 43 . 16.1.34; D 43.24.7.1 (Neratius); D 45.1.29.1 (Marcellus); D 45.1. 69 ; D 45.1 .82 .1 ; D 46.1.8.8 (Julien) ; D 47.1.2.1-2-3 (Pomponius)-4; D 47.2.36 pr;D 47.2.46 pr ; D 47.2.52.28; D 47.10.7.1; D 48.5.28. 15 ; D 48.8.4.2 (Hadrien) ; D 48.10.8; D 48.18.1.20; D 48.18.7; D 48.19.8.3; D 48.19.19; Marcien D 18.1.44; D 30.114.19; D 34.5 . 18.1; D 40.9.9.1; D 48.1.9; D 48.10.1.13; D 48.15.3.1 (rescrit de Sévère et Caracalla) (pris par extension, la victime du plagiariuo pouvant être un esclave); Modestin D 28.1.19; D 29.5.19; D 45.1.103 D 48.2.17; Hermogénien D 1.5.13.

352- Pomponius D 15.2.3; D 21.2.16.2 (Proculus); D 36.1.72.1; D 44. 7.56 ; D $46.3 .92 \mathrm{pr}$ (manumissio morti similis est) ; Africain D 47. $2.62 \mathrm{pr}$; Gaius D 33.8.4 ; Paul D 5.3.36 pr (Proculus, Julien, Mauricianus) ; D 33.8.1; D 33.8.3; Ulpien D 9.4.5.1; D il.3.5.4; D 15 . I.32 pr (Julien); D 15.2.1 pr-1-4; D 16.3.1.30; D 28.7.8.7; D 38 . I.32 $\mathrm{pr}$ (Julien); D $15.2 .1 \mathrm{pr}^{-1-4}$
5.1 .25 (Pomponius) ; D $47.2 .46 \mathrm{pr}$.

353- Pomponius D 13.7.8 pr ; Paul D 6.1.79; D 14.2.2.5; Ulpien D21. 1.31 .12 .

354- Alfenus (chez auteur anonytue) D 9.2.52.2; Gaius D6.1.35.1;D 9. 2.8.1 ; Paul D 9.2.31 (Q. Mucius) ; Ulpien D 4.4:11.5 (Julien, Marce1lus) ; D 9.2.7.2 (Pegasus) -8 (Proculus) ; D $9.2 .9 \mathrm{pr}-3$ (Ofilius) -4 ; D 9.2.1I pr (Mela, Proculus) ; D 9.2.15.1 (Julien) ; D 12.4.5.4 ; D 13. 6.5.7 (Me1a, Namusa) ; D 19.2.13 pr.

355- Paul D 14.2.2.5; D 21.1.43.4 ; D 33.8.9.2 ; U1pien D 15.1.9.7; D 21.1.1.1 ; D 21.1.17.4 ; (Proculus) -6 (Caelius Sabinus) ; D 21.1 . 23.3 ; D 47.2.36 pr.

356- Pomponius D 12.4.15 (Labểon, Proculus) ; D 30.48 .1 ; D 36.1 .72 1 ; D 45.1.23; Gaius D 9.2.4 pr-1; Maecianus D 29.5.14 ; Marcellus D 45.1.96; Papínién D 46.3.95.1 ; D 48.3.2 pr ; D 48.18.6 pr ; Callistrate D 47.21.3.1 (Nerva) ; D 48.19.28.11-16 ; Paul D 5.3.36.2 (Pomponius) ; D 9.2.12; D 9.2.14; D 9.2.18; D 9.2.30 pr ; D 35.2 .

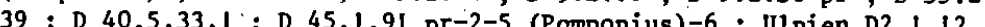
D 3.5.5.10 (Pomponius); D 4.3.7.3 (Labéon) $-4-5$; D 6.1.17 pr (Julien) D $9.2 .5 \mathrm{pr}-3$ (Julien) ; $\mathrm{D} 9.2 .17$; D $10.4 .9 \mathrm{pr} ; \mathrm{D} 17.2 .49$; D 20. 1.27 (Marcellus); D 29.5.1.12 (Scaevola)-13 (Scaevola)-14-21-28 (Hadrien) $-30-31-33$; D 29.5.3.4-13-16-17-18; D 29.5.5.2; D 30.53 . été assassiné ( 357 ). La barrière est fragile entre ces deux derniers cas. La limitation du drcit de vie et de mort du mâttre sur l'esclave n'eupêche apparemment pas le premier nommé d'avoir la main leste dans la pratique, de nombreux textes traitant de ce problème à une époque postérieure à celle d'Antonin le pieux (358). Si un rapport de domination se dégage du texte, on pense à une pratique répressive, mais la disproportion entre acte répressif et acte délictuel initial incite à se voir en présence d'une véritable agression. Ainsi, l'esclave peut être tué pour un délit qui méritait la mort (359) mais il peut l'être aussi pour une faute très légère (360). Dans ce deuxième sens, un texte est particulièrement remarquable, bien qu'il ne s'agisse pas d'un rapport de domination directe :

Si magister in disciplina vulneraverit servun vel occiderit, an Aquilia teneatur, quasi domnum iniuria dedsrit ? Et Iulionus scribit Aquilia teneri evm, qui eluscaverat discipulum in disciplina : malto $3-8 ;$ D 40.12 .7 .4 ; D 48.8.4.2 (Hadrien) ; D 48.10.8; D 48.18.1.20;
D 48.18.7; D 48.19.8.3; D 48.19.19; Marcien D 40.9.9.1 ; D 48.1.9; D 48.10.1.13; Modestin D 29.5.19; D 48.2.17; Hermogënien D 1.5.13.' 357- Alfenus (chez auteur anonyme) D 9.2.52 pr ; Julien D 9.2.47; D 9.2.51 $\mathrm{pr}^{-1}$ (veteres)-2 ; Gaius D 6.1.36.1; $\mathrm{D} 9.2 .2 \mathrm{pr}$; D 9.2.32.1; Dar. Marcellus D 9.2.34 ; D 9.2.36 pr-1 (Sabinus) ; Papinien D 46.3.95.1 ; Paul D 4.3.18.5; D 9.2.22 pr-1 ; D 9.2.26; $9.2 .30 .1-4 ; \mathrm{D} 9.2 .33 \mathrm{pr}$
(Pedius) ; $\mathrm{D} \mathrm{9.2.45} \mathrm{pr}^{-2} ; \mathrm{D} 9.2 .55 ; \mathrm{D} 11.1 .20 \mathrm{pr} ; \mathrm{D} 17.1 .22 .6 ; \mathrm{D} 42$. 2.4 ; D 45.1.88; Ulpien D 6.1 .17 .1 (Julien) ; D 7.1.17.3; D 9.2.3; D 9.2.7.1-3 (Proculus) -4-5 (Labéon) -6 (Celsus) -7 (Celsus) ; D 9.2.9.2 (Neratius)-3 (Ofilius) -4 ; D 9.2.11.1-2 (Julien)-3 (Celsus, Marcellus) ; D 9.2.13.2 (Celsus)-3 (Julien) ; D 9.2.15 pr-1 (Julien); D 9.2.19; D 9.2.21.1 (Celsus, Julien) -2 ; D 9.2.23 pr (Neratius)-1-2-3 (Julien) -4 (Labéon) -5-6-7-8-9-10-11 (Julien) ; D 9.2.25 pr; D $9.2 .27 \mathrm{pr}$ (Celsus, Julien)-1 (Proculus, U, Ferox)-2 (Ce1sus)-5-6;D $9.2 .35 ; D 9.2 .44$. D $9.2 .46 ; D$ 12.4.5.4; D 13.3.3; D 19.5.14.1; D 47.1.2.1-2-3 (Pomponius) -4 ; D 47.10.7.1; Marcien D 22.1.32.5; D 48.8.1.2.

nius) -4 ; D 47. H. WALLON, Histoire de l'esclavage, cit. Irr, p. 56 ; v. A. GUARINO, Diritto privato romano, cit., D. $493 \mathrm{~s}$. ; B. ALBANESE, Note sull' voluzione storica del ius vitae ac necis, in Scr. Ferrini, Milan 3 (1948) p. $343 \mathrm{~s}$.

359- Ulpien D 30.53.3 ; Si heres hominem legatron occidit ob facinus, 'hoc est merentem', sine dubio dicendwon erit eum ex testcomento non teneri.

360- Marcellus D 20.1.27 : Semwn, quem quis pignori dederat, ex levissima offensa vinxit, mox solvit, et quia debito non satisfaciebat, creditor minoris servun vendidit : an aliqua actio creditori in debitorem constituenda sit, quia crediti ipsius actio non sufficit ad id quod deest persequendun? Quid si eun interfecisset aut eluscasset ?... 
magis igitur in occiso idem erit dicendum (361).

Si un magister blesse ou, à plus forte raison, tue un esclave à la formation duquel il procédait, il sera tenu de la lex Aquilia. Le seul motif de la mort est la correction... en l'espèce radicale.

Est-il possible de dégager une fréquence de ces différentes morts ? Si on opère pour chaque période par rapport au nombre total de textes où ces divers types de mort ressortent de manière explicite, on obtient :

- analyse des paragraphes

- analyse des citations

\section{- la maladie}

$\begin{array}{llll}\text { I } & - & = & - \\ \text { II } & \overline{1} & = & - \\ \text { III } & 1 / 16 & = & 6 \% \\ \text { IV } & 0 / 5 & = & - \\ \text { V } & 3 / 150 & = & 2 \%\end{array}$

- l'accident

$\begin{array}{llll}\text { I } & - & = & - \\ \text { II } & - & = & - \\ \text { III } \quad 2 / 16 & = & 12,5 \% \\ \text { IV } & 0 / 5 & = & \overrightarrow{8} \% \\ \text { V } & 12 / 150 & = & 8 \%\end{array}$

$\begin{array}{lllr}\text { I } & 3 / 6 & = & 50 \% \\ \text { II } & 5 / 16 & = & 31 \% \\ \text { III } & 2 / 27 & = & 7 \% \\ \text { IV } & 1 / 5 & = & 20 \% \\ \text { V } & - & = & -\end{array}$

- le suicide

$\begin{array}{llllllll}\text { I } & - & & - & \text { I } & 0 / 6 & = & - \\ \text { II } & - & = & - & \text { II } & 3 / 16 & = & 19 \% \\ \text { III } & 0 / 16 & = & - & \text { III } 0 / 25 & = & - \\ \text { IV } & 0 / 5 & = & - & \text { IV } & 0 / 5 & = & - \\ \text { V } & 9 / 150 & = & 6 \% & \text { V } & - & = & -\end{array}$

\section{- la répression}

\begin{tabular}{|c|c|c|c|c|c|c|}
\hline I & - & $=$ & I & $0 / 6$ & $=$ & - \\
\hline II & - & $=$ & II & $3 / 16$ & $=$ & $19 \%$ \\
\hline III & $6 / 16$ & $=37,5 \%$ & III & $5 / 27$ & $=$ & $19 \%$ \\
\hline IV & $2 / 5$ & $40 \%$ & IV & $3 / 5$ & $=$ & $60 \%$ \\
\hline$v$ & $57 / 150$ & $=38 \%$ & v & - & $=$ & - \\
\hline
\end{tabular}

361- Ulpien D 9.2.5.3. Quelquefois, le magister n'est guère plus tendre a l'égard du fils de famille. Le texte poursuit en effet : ... Sutor, inquit (Iulianus), puero discenti ingenuo filio familias, pamum bene facienti quod demonstraverit, forma calcei cervicem percussit, ut oculus puero perfunderetur.

\section{- le meurtre}

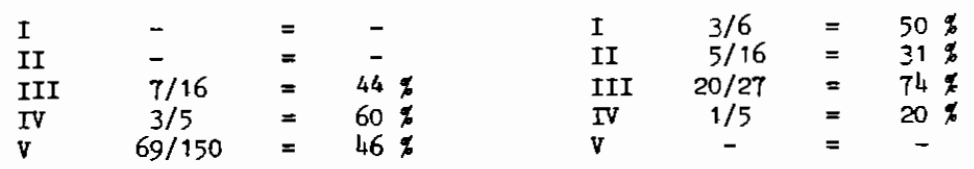

Si l'on en croit ces pourcentages, c'est le meurtre qui est la cause la plus fréquente des morts d'esclaves (362). Vient ensuite le. répression. La mort qu'on pourrait qualifier de "normale" (maladie, accident et suicide) n'est que faiblement représentée.

Ces chiffres ne sont certainement pas le reflet de la réalité quotidienne. Il faut en effet être consciert, une fois encore, de ce qu'on a affaire à des textes juridiques, lonc des textes privilégiant les thèmes qui soulèvent le plus de problèmes juridiques, ainsi le meurtre et la répression : le meurtre parce qu'il s'agit d'assurer une protection du maître quant à la valeur de I'esclave ; la répression parce qu'elle tend de plus en plus à être le fait de l'Etat. C'est in point sur lequel on reviendra. On remarquera cependant qu'à égale "intensité juridique", le meurtre l'emporte sur la répression. Cela montre que, malgré l'intervention étatique grandissante, la protection des intérêts des maîtres reste un problème important.

On s'est contenté jusqu'à présent de conduire l'analyse sur un plan purement quantitatif. Il convient désormais de s'arrêter sur les réalités rapportées par ces textes. Si rien d'intéressant ne ressort sur la maladie, il n'en va pas de même des autres thèmes.

En ce qui concerne tout d'abord la mort accidentelle, l'accident de la circulation ne semble pas être chose rare (363) et ce, quelle que soit la période envisagée. Alfenus évoque un enfant écrasé par deux chariots tirés par des mules (364). Gaius parle d'un esclave piétiné par des mules emballées (365), Ulpien d'un autre qui, ayant loué un char, périt par faute de la témérité du cocher (366). Chez Ulpien toujours, il

362- Le pourcentage faible de la période IV dans I'analyse des citations n'est pas étayé sur ce problème par l'analyse des paragraphes.

363- Un aperçu des problèmes de la circulation dans Rome se trouve dans 1 'ouvrage de J. CARCOPINO, La vie quotidienne a Rome $\lambda$ I'apogée de I'Empire, cit., p. $67 \mathrm{~s}$.

364- D 9.2.52.2.

365- D 9.2.8.1.

366- D 19.2.13 pr. 
est question d'un esclave écrasé par un char (367). L'esclave peut aussi périr dans un incendie (368), un tremblement de terre (369), l'effondrement d'une maison (370), un naufrage (371). Ofilius rapporte un cas de noyade, la monture de l'esclave ayant été efrrayée par un passant et l'ayant fait chuter dans un fleuve (372). Ailleurs, il est tué par un objet tombant d'une saison en construction ou par une branche (373), il est écrasé par le fardeau trop lourd qu'un passant a malencontreusement laisse glisser sur lui ( 374$)$. Mela et Proculus retracent une nort stupide : des gens jouent à la balle à proximité d'un barbier, la halle heurte la main de celui-ci qui tranche la gorge de l'esclave qu'il était en train de raser (375). On peut en dire autant de cette espèce que 1 'on trouve chez Ulpien : l'esclave, passant à proximite d'un lieu d'entrasnement, est tué par un javelot (376), mais le texte précise que, si le jet a éte volontaire, l'action de la lex Aquitia aura lieu. On passe ainsi à un autre thème, le meurtre.

Le Digeste fournit une énumération qui, si elle n'est pas exhaustive, n'en est pas moins intéréssante. L'esclave périt empoisonné (377), étranglé, poignardé, roué de coups de pieds (378). Il est précipité d'un pont (379). On lui fait volontairement emprunter des passages dangereux (380). On le fait mourir de faim (381), de froid (382). Relevons à titre

\section{7- D 12.4.5.4.}

368- Ulpien (Julien, Marcellus) D 4.4.11.5.

369- Ulpien (Julien, Marcellus) D 4.4.11.5.

370- Ulpien (Julien) D 9.2.15.1; D 12.4.5.4

371- Ulpien (Julien) D 9.2.15.1.

372- Chez Ulpien D 9.2.9.3.

373- Paul D 9.2.31 (Q. Mucius).

374- Ulpien D 9.2.7.2 (Pegasus).

375- Chez U1pien D 9.2.11 pr.

376- Ulpien $D$ 9.2.9.4.

377- U1pien D 4.3.7.3 (Labêon); D 9.2.7.6 (Celsus); D 47.10.7.1.

378- Ulpien D 9.2.7.1.

379- Ulpien D 9.2.7.7 (Celsus). Il est également question dans le passage d'un enfant frappé contre un rocher.

380- Gaius D 6.1.36.1.

381- Ulpien D 9.2.9.2 (Neratius).

382- Ulpien D 19.5.14.1. documentaire, à proposs du manque de solidarité à l'intérieur du groupe servile, dejà évoqué, que le meurtrier est parfois lui-même esclave $(383)$.

En ce qui concerne le suicide, le moyen le plus courant semble être la chute dans le vide ( 384 ). Méanoins, on trouve aussi mentionnés le poison et la pendaison (385). Il paut citer ce passage de Paul :

Bortis consciscendas cousa sibi facit, qui propter nequition malosque morss flagitizome aliquod admissum mortem sibi consciscere voluit, non si dolorem corporis non sustinendo id fecerit (386).

Paul estime qu'un esclave est censé avoir attenté à sa vie lorsqu'il a voulu se donner la mort à cause de ses mauvais penchants, le texte dit littéralement "ses mauvaises moeurs", ou pour échapper aux poursuites consécutịves à un délit qu'il avait cormis, non $s^{\prime}$ il a voulu mettre fin par ce moyen aux douleurs insupportables que lui infligeait son corps. Ce texte nous intéresse à un double titre : tout d'abord, il donne des motifs du suicide d'un esclave ; ensuite, les mots nequitian malosque mores sont typiques du point de vue du dominant et montrent que le suicide peut constituer une forme d'opposition de la part du dominé.

Ces lignes nous conduisent déjà à une problématique différente, qui n'est plus celle de la prise en considération des seuls énóncés relevés dans le Digeste, mais de la situation de l'esclave dans la société.

383- Ulpien D 9.2.27 pr (Celsus, Julien)-1 (Proculus, Urseius Ferox)-2 (Celsus).

384- Ulpien D 15.1.9.7; D 21.1.17.4. On peut assimiler a cette forme de mort la noyade : 1'esclave se jette a la mer (Paul D 14.2.2.5) ;

il se précipite du haut d'un pont (Olpien (Caelius Sabinus) D 21.1.

$17.6)$.

385- Ulpien D 21.1.23.3. Y. GRISE, De la fréquence du suicide chez les Romains, Latomus (1980) p. 17-46, signale aussi le glaive(p. 23 s.).

386- D 21.1.43.4. M. MESLIN, L'homme romain, Paris (1973), p. 240, estime que "la variété des jugements émis sur le suicide renvoie à la diversit tê le qualitế des personnes qui $z$ ont recours". A partîr d'un recensement des cas de suicide rapportés par des sources de nature principalement litt ment littêraire, $Y$ chise, o.ch pemble avoir atteint-sa fréquence maximale à l'époque des guerres civiles et de la mise en place du rónes dus à la défaite de la révolte de Sicile- le motif suicides desclaves dus à la défaite de la révolte de Sicile- le motif de suicides d'esclaves que rapporte essentiellement l'auteur est l'attachement de l'esclave à son maître. C'est là une vision beaucoup trop idéaliste de la réalité, réalité qui n'échappe pas au juriste Paul, et Y. GRISE n'aurait pas dû manquer de le souligner(cf. F.DE MARTINO, Storia III,p.119) 
TITRE II

\section{L'ESCLAVE DANS LA SOCIETE}

On examinera en premier lieu les signes sociaux distinctifs de 1 'esclave. On étudiera ensuite sa situation au sein des rapports politiques et juridiques pour en terminer par une analyse de ses comportements.

\section{CHAPITRE I : DES SIGNES SOCIAUX}

Outre ce qui a été dit sur la position économique de l'esclave, la pratique sociale lui confère des signes distinctifs tant au niveau de l'individu qu'au niveau de la société.

\section{SECTION 1. LA. PRATIQUE SOCLILE AU NIVEAU DE L'INDIVIDU}

On envisagera d'une part les thèmes de l'alimentation, de l'habillement et du Iogement, d'autre part, ceux des pratiques sexuelles et formes d'unions.

\section{Paragraphe 1: Alimentation, habillement et logement.}

Ces trois thèmes font l'objet d'une analyse commune car, du point de vue juridique, ils forment un tout.

L'obligation de fournir les alimenta peut dériver d'une convention ou d'un legs, mais aussi de l'ordonnancement juridique (387).

387- A. GUARINO, Diritto privato romano, cit., p. $854 \mathrm{s.}$; R. ORESTAN0, Alimenti (dir. rom.) in NNDI. Sur l'origine et le développement du droit aux alimenta, A. GUARINO, op. cit., p. 854 n. 92.6 .
Indépendamment de ses obligations à l'obsequium, aux overae et aux hona, l'affranchi doit fournir des aliments au patron ou à ses enfants dans le besoin (388) sous peine de se rendre coupable d'ingratitude (389). Inversement, et c'est le sens qui nous intéresse, le petron est tenu au même devoir envers son affranchi sous peine de perdre son droit aux bona. (390), voire tous ses droits de patronat (391).

Les alimenta recourrent la nourriture, l'habillement et le logement. C'est ce que 1 'on peut lire chez Iavolenus

Legatis alimentis cibaria et vestitus et habitatio debebitur (392).

Par contre, sous le vocable cibaria ne sont pas compris l'habitatio, le vestiarivm et le calciarivm (393). Il faut aussi distinguer le legs de l'habitatio de celui d'une domus, le second pouvant représenter pour 1'affranchi un placement et non pas un logement (3gh)

388- H. WALLON, Histoire de 1'esclavage, cit. III, p. 79. La jurisprudence se bornait au cas de nécessité, cf. Paul D 25.3.9 : In bonis superstitum libertorwn nullum omnino ius patroni liberivs patronorm habent, nisi si tom esse infirmos tanque pauperes praesidibus probavemint, ut memito menstmis alimentis a libertis suis adiuvam debecnt. Idque ius ita plumis principum constitutionibus manifestatur. Ique ius ita plurisis principun constitutionibus manifestatur. L'escla (Marcellus) D 25.3.5.22 (cité supra n. 145).

389- L'action tendant a la constatation de l'ingratitude de l'affranchi n'appartient qu'au patron ou à ses fils. La peine a varie d'Hadrien a Justinien, $H$. WAL ' l' Juffranieni tuice du patron ; est vendu corme esclave, cf. Modestin D 25.3.6.1

390- Modestin D 38.2.33.

391- Marcien D 37.14.5.1

392- D 34.1.6. Il arrive fréquemnent que 1 'on trouve, a côtê des alimenta, la mention des cibaria, du vestiarius ou de l'habitatio : il $s$ 'agit de simples redondances de la langue latine, (par exemple, Ulpien D 35.3.3.4 : ... alimentorm et vestiarii libertis relictomom). Pour $\mathbf{F}$. WYCISK, Alimenta et victus dans le droit romain classique, RHD, 50 (1972), p. 205-228 (cr in Bull. an. Hist, rom. XI (1972) p. 633), 引 la fin de la République, alere courre tout ce qui est nécessaire à la vie, mais alimenta correspond souvent à la seule nourriture ; c'est seulement

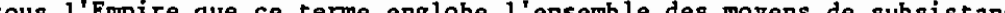
393- U1pien D 34.H.21 : Diariis vei cibariis relictis neque habitationem neque vestiarium neque calciarium deberi palam est, quoniam de cibo tantum testator sensit.

394- Un exemple de maison destinée a procurer des revenus aux affranchis à qui elle a été léguée est fourni par Scaevola D 31.88 .14 : Insulam $t_{i}$ bertis utriusque serus legavit ita, ut ex reditu eius mascuti duplion, feminae simplum percipiant, eamoue alienari vetuit. 
De nombreux textes traitent de legs d'alimenta (395). Is prestation peut avoir lieu en nature ou en argent (396). On relève à ce sujet plusieurs rapports : soit le legs semble adressé à un esclave par son maître ou par un tiers, sans obtention de la liberté ; soit il est rait par le maître à un esclave qu'il affranchit dans son testament ou par le patron à son affranchi. Seuls les deux derniers cas sont pertinents en ce qui nous concerne. On sait en effet qu'un legs fait par un testateur à son propre esclave sine libertate est nul (397). Ainsi, si un individu lègue à sa concubine des esclaves et ordonne qu'on leur fournisse la nourriture qu'il leur donnait de son vivant, le legs de cibaria n'est pas fait à l'esclave mais à la concubine (398). D'autre part, le legs adressé à un servus aliemus est assimilable à un legs fait à son mâttre (399). Si on fait un legs de cibaria aux esclaves de Titius, c'est donc Titius qui est légataire (400). Par contre, par l'acquisition de la liberté, l'esclave devient le véritable légataire. La distinction opérée entre le legs fait par le mâ̂tre à l'esclave affranchi par testament et celui fait par le patron à l'affranchi n'a qu'une valeur chronologique. En effet, dans le premier cas, l'obtention de la liberté et le legs des alimenta sont simultanés (401). Dans le

395- Mais il est Également question d' alimenta dans le Digeste sans qu'il s'agisse de legs. Il en est ainsi a propos d'esclaves, chez Paul D 24.1.28.1 ; D 26.7.12.3; D 41.7.8; U1pien D 44.4.4.9; a propos d'affranchis, chez Iavolenus D 38.1.33 ; Neratius D 38.1.50.1 ; Papinien D 40.10.1.1 ; Paul D 26.7.12.3 ; D 38.1.18 (Sabinus); Marcien D 37.14.5.1; Modestin D 25.3.6 pr ; D 38.2.33. Dans le sens de 1'obligation aux alimenta de 1 'affranchi envers son patron, on relève Papinien D 38.1.41 (un patron simule 1 'indigence pour obtenir des alimenta: ... Alimentomon diversa causa est, cum inopia patroni per invidiam $l_{i-}$ bertum convenit) ; Paul D 25.3.9; D 29.2.73 (Labéon) ; U1pien D 25.3. 5.18 a 26 .

396- Un exemple de prestation en argent chez Tryphoninus D 34.1.16.2. Une même prestation peut consigter en partie en une quantité mensuelle de nourriture, en partie en une somme, cf. Papinien D 34.1.9.1.

397- Ulpien D 30.34.9.

398- Tryphoninus (Scaevola) D 34.1.15.1.

399- Pomponius D 30.12.2.

400 - Africain D 35.1.42. Nếanmoins, le legs fait à 1 'esclave par un tiers est lié a la personne de 1 'esclave; c'est ce que souligne v1pien dans un legs d'habitatio, cf. D 36.2.9.

401- Dans cette note et la suivante, les legs gênéraux d'alimenta sont associés aux legs particuliers de cibaria, vestiania et habitatio, la problématique étant la même. On relève ici Scaevola D 10.2.39.2 (Cibaria, vestiaria) ; 34.1 .18 pr (alimenta... vestiaria)-1 (alimenta)-2 second, on a sfaire un esclave auparavant affranchi, donc envers lequel le patron était dejà tenu aux alimenta (402). Dans un cas corme dans l'autre, le legs peut être conditionnel, la condition étant toujours de même nature. On impose à l'affranchi de rester dans un endroit ou plus généralement chez une personne déterminée (403). On sent

(cibaria, vestiaria) ; D 34.1 .20 .3 (cibaria, vestiaria... alimenta) ; D 34.5.29 (alimenta); D 36.2.27.1 (cibaria); Papinien D 34.110.1 (alimenta) ; Marcien (rescrit de Sévère et Caracalla) D 30.113 .1 (cibaria) ; Hodestin D 34.1.4 pr (cibaria, vestiaria). Une hypothèse originale est développée par Paul (D 33.1.16 : Servus post decem annos liber esse iussus est legatromque ei ex die mortis domini in annos singulos relictum est. Eomon quidem anomon, quibus iom liber erit, legatum debebitur: interin autem heres ei alimenta prasstare compelititur). par testament, un esclave reçoit la liberté pour en jouir dans dix ans et un legs; le legs ne lui sera dû que lorsqu'il aura effectivement et un legs ; le legs ne lui sera dû que lorsqu'il aura effectivement
obtenu la liberté, mais, en attendant, l'heritier doit lui fournłr les alimenta; sur ce texte, W.W. BuCKIAND, Roman $1 \mathrm{aw}$, cit., p. 472 s.

402- Valens D 34.1.22.1 (alimenta); Scaevola D 31.88 .11 (alimenta); D 33.1.13.1 (alimenta); D 33.2 .33 pr (habitatio)-1 (habitatio) ; D 34. $1.13 \mathrm{pr}$ (cibaria, vestiaria)-1 (rescrit d'Antonin le pieux) (alimenta ... vestiaria)-2 (alimenta); D 34.1.15. Acibaria, vestiaria) ; D 34.1 . 16 pr (alimenta... vestioria)-1 (alimenta)-3; D 34.1 .18 .3 (cibaria, vestiaria) $4-5$ (cibaria, vestiaria); D 34.1 . I9 (cibaria); D 34.1 .20 pr (alimenta)-1 (alimenta)-2 (alimenta); Papinien D 7.1.57.1 (alimenta); D 33.1.10.3 (habitatio) ; D 34.1 .8 (alimenta) ; D 34.1.9.1 (cibaria, vestiaria); D 34.1.10 pr (alimenta)-1 (alimenta) ; D 38.4.11 (alimenta); D 40.10.1 pr (alimenta); Tryphoninus (Scaevola) D 34.1.16.2 (alimenta ... cibaria, vestiaria, habitatio) ; Paul D 10.2 .41 (alimenta) ; D 34 . 1.12 (cibaria, vestiaria) ; D 35.1 .84 (alimenta) ; D 37.14.24 (alimenta); U1pien D 2.15.8.7 (alimenta) ; D 34.1.3 (rescrit d'Antonin le pieux) (alimenta) ; D 34.1.14.3 (alimenta... aqua) ; D 35.3.3.4 (rescrit de Marc-Aurèle et Verus) (alimenta...vestiaria) ; D 39.5 .16 (vestem) ; Modestin D 34.1.4 pr (cibaria, vestiaria)-1 (cibaria, vestiaria); D 33.2.18 (habitatio) ; D 34.1 .5 (cibaria). A propos du legs de nourriture, signalons encore le legs de penus, provisions de bouche, qui apparaît à propos d'un esclave chez Paul D 33.9.4.2-5 (Labeon) ; vlpien D 33.9.3 pr (Sabinus)-6 (Q. Mucius, Servius)-8 (Ofilius); à propos d'un affranchi chez Papinien D 34.1 .9 pr.

403- Les affranchis sont tenus de rester dans le fonds où est inhumé le testateur, (cf. Scaevola D 34.1.18.5). Ailleurs, les affranchis doivent demeurer chez une personne indiquée par le testateur : ainsi, un testateur laisse aux affranchis testamentaires la somme de dix par an 'ils restent auprès de sa mère (cf. Scaevola D 34.1.18.1); il charge ses héritiers d'affranchir Stichus a qui il lègue cibaria et vestiaria s'il demeure chez un d'entre eux (Scaevola $\mathrm{D} 34.1 .18 .2$ ); une mère ing$\mathrm{s}$ il demeure chez un d'entre eux (Scaevola $\mathrm{D} 34.1 .18 .2$ ) ; une mère ins
titue son fils et laisse la liberté fidéicoumissaire a un esclave, a qui elle lègue ciboria et vestiaria s'il demeure auprès du fils (Scaevola D 34.1.20.3); ailleurs, une testatrice veut qu'on donne alimenta et vestioria a ses affranchis tant qu'ils resteront chez un tel (Scaevola D 34.1.13.1) ; un testateur cherge sa feme de fournir douze deniers par an, alimentorum nomine, à ses affranchis s'ils restent auprès d'elle (Scaevola D 33.1.13.1) ; un patron lègue les alimenta à ses affranchis s'ils restent avec son fils (Paul D 35.1.84). 
évidement là la contrepartie des alimenta qui lui sont dus. Toutefois, exceptionnellement, la condition peut être levée s'il ne tient pas à l'affranchi qu'elle ne soit pas remplie. L'hypothège est prérue par Scaevola : si l'héritier, auprès duquel le testateur voulait que les affranchis demeurent, a exercé sur eux son pouvoir de manière si dure qu'ils l'aient quitté, il restera néanmoins tenu de leur fournir les alimenta (404).

La communauté d'intérêt juridique entre alimentation, habillement et logement étant précisée, penchons-nous successivement sur chacun de ces thèmes.

Le thème le mieux représenté est celui de la nourriture. Il intervient à propos d'une grande variété de problèmes juridiques, la question étant de savoir à qui incombent les dépenses occasionnées par l'alimentation des esclaves : vente (405), dcnation entre mari et femme (406), dot (407), usufruit (408), dépôt (409), commodat (410), succession (411), ainsi qu'en matière de mandat (412), de tutelle (413), de legs de l'instrumention fundi (414) et à propos de l'actio ad exhibendum (415). Il ressort également en matière de délits (416).

Les denrées mentionnées par les textes sont le blé (417),

\section{4- D 34.1.13.2.}

405- Celsus D 19.1.38.1 (Sextus Aelius, Drusus); Paul D 21.1.30.1

(Aristo, Pedius).

406- Scaevola D 24.1.58.1; Pomponius D 24.1.31.10.

407- Neratius D 25.1.15.

408- Gaius D 7.1.45 ; Ulpien D 7.1.15.2.

409- Ulpien D 16.3.1.9-10 (Pomponius); Modestin D 16.3.23.

410- Gaius D 13.6.18.2.

411- U1pien D 5.3.5 pr (rescrit d'Antonin le pieux); D 29.2.20.1.

412- Paul D 17.1.26.8 (Mela).

413- U1pien D 27.3.1.8.

414- Paul D 33.7.18.5 (Scaevola)-9; Ulpien D 33.7.12 pr (Servius)-1.

415- Ulpien D 10.4.11.1 (Labéon).

416- Pomponius D 47.2.35.1 ; Callistrate D 48.15.6.1 (rescrit d'Hadrien'.

417- Neratius D 15.3.18; Gaius D 3.5.21; Paul D 19.1.21.3; D 33. 7.18.5-9 ; Ulpien D 5.1.52.1 ; D 15.3.3.1 (Pomponius) -7 ; D 33.7.12 or \{5ervins? l'eau (418) et le vin (419) au sujet duquel une distinction discriminatoire est faite par Ulpien qui dissocie le vin destiné à l'usage du mâ̂tre de celui des esclaves, qui peut se trouver dans leur pécule (420).

La nourriture de la familia peut être achetée par un esclave. Le cas n'est pas rare puisqu'on le trouve signalé par Neratius (421), Pomponius (422), Africain (423) et Ulpien (424). Labéon parle d'un esclave qui reçoit personnellement de l'argent pour pourvoir à sa nourriture, selon une consuetudo domini (425). C'est le signe incontestable d'une certaine autonomie. Ulpien évoque un testateur chargeant par fidéicomnis son héritier d'acheter des tessères de blé pour ses affranchis (426).

La nourriture destinée à la fomilia semble être stockée sur le domaine si l'on en croit Gaius qui écrit que le blé et le vin ont péri pour cause d'incendie ou d'effondrement (427). Ulpien parle de la détérioration du blé rangé dans l'horreum dominical (428). Qu'advient-il alors de la farrilia? Paul parle d'une farrilia morte de faim par défaut de livraison du blé (429), mais il ne s'agit certainement là que d'une hypothèse d'école; les maitres les premiers ont du reste intérêt à ce qu'il en soit ainsi.

La nourriture est-elle la même pour tous les esclaves de la $f a-$ milia ? Paul répond par la négative en écrivant qu'elle constitue un

418- Ulpien D 33.7.12.10;D 34.1.14.3.

419- Gaius D 3.5.21 ; U1pien D 21.1.4.2 (Pomponius) ; D 21.1.14.4 (Pedius) ; D 33.6.9.3 ; D 33.7.12 pr (Servius).

420- Ulpien D 33.6.9.3.

421- Neratius D 15.3.18.

422- Pomponius chez Ulpien D 15.3.3.1.

423- Africain D 40.7.15 pr.

424- U1pien D 15.3.3.7.

425- Ulpien D 15.3.3.3 (Labêon).

426- Ulpien D 5.1.52.1.

427- Gaius D 3.5.21.

428- v1pien D 15.3.3.3.

429- Paul D 19.1.21.3. 
critère de distinction entre les esclaves urbains et ruraux (430).

Ulpien ajoute gue l'usufruitier doit alinenter les esclaves on fonction de leur rang et de leur dignité, secunrium ordinem et dignitatem (431). Par rapport aux conclusions dégapées de l'átude sur ln main-d'oeuvro servile, on ne peut que constater une fois de plus le manque d'homogónéité du groupe servile. Qu'une discrimination s'opère en son sein par l'alimentation est en un sens remarquable.

Ce thème apparaît enfin à propos de ce qu'il est convenu d'appeler les défauts de l'esclave. Pompcnius évoque ainsi un esclave enclin à la boisson, vinarius ou encore, défaut moindre sans doute bien qu'il se trouve dans la même énumération, un esclave gourmand, gulosus. Ces défauts n'entraînent toutefois pas, selon le juriste, la possibilité d'une redhibitio (432). Paul est l'auteur d'un passage intéressant :

Guod in convivio vescendi causa poritur, in eam rem familia ludere permittitur (433).

Les esclaves d'une même familia peuvent jouer lour repas. Il est vrai que le jeu n'est pas considéré comme un défaut pouvant donner lieu à redhibitio (434), mais il est permis de se demander si le jeu en question a véritablement lieu par vice ou parce que la quantité de nourriture est insuffisante...

Quel est le coût de la nourriture d'un esclave ? Les textes ne nous donnent pas des indications très précises. Il est néanmoins visible qu'il s'egit d'un coût modique, contrairement aux dépenses occesionnées par la maladie ou la fuite d'un esclave (435). Par ailleurs, si l'on se fie à ce passage de Scaevola, il semble qu'il soit plus onéreux de vêtir un esclave que de le nourrir :

Servo libertatem dedit qui erat annomum quindecim, "cron erit annorum triginta", eidem ex die mortis suae quoad viveret cibarioron nomine denarios denos, vestiarii denarios viginti quinque praestari se velle signifigavit (436).

430- Paul D $32.99 \mathrm{pr}-1-3$

431- Ulpien D 7.1.15.2.

432- Ulpien D 21.1.4.2 (Pomponius).

433- Paul D 11.5 .4 pr.

434- Ulpien D 21.1 .4 .2 (Pomponius).

435- Gaius D 13.6.18.2.

436- D 10.2.39.2.
Un tegtateur laisse à un esclave de quinze ans la liberté mux qu'il en jouisse à l'âge de trente ans et lui lègue durant toit.e sa vie dix deniers devant lui assırer la nourriture et vingt-cinq le rêtement.

Come on l'a remarqué pour l'nlimentation, l'habillement de l'esclave intervient à propos de problèmes juridiques variés : legs (437), usufruit (438), dot (439), dépôt (440), ccommodat (441), actio ad exhibendzon (442) et délits (443). De même, l'esclave peut recevoir de l'argent pour se vêtir lui-même ( 444$)$. Celui qui appartient à la ferme peut à cet usage se voir attribuer une somme par le mari (445). Quoi qu'il en soit, le maître est tenu de lui fournir le vêtement (446). L'esclave peut, aussi être chargé d'acheter les habits utiles à la fomilia (447). Ces habits ne font pas forcément partie du pécule; il est utile à ce sujet de citer un texte de Pomponius :

Id vestimentum peculii esse incipit, quod ita dederit dominus, ut eo vestitu servon perpetuo uti vellet eoque nomine ei traderet, ne quis alius eo uteretur idque $a b$ eo eius usus gratia custodiretur. Sed quod vestimentron servo dominus ita dedit utendum, ut non semper, sed ad certum usum certis temporibus eo uteretur, veluti cum sequeretur eum sive cenanti ministravit, id vestimentum non esse peculii (448).

437- Scaevola D 34.2 .13

438- Ulpien D 7.1.15.2.

439- Iavolenus D 24.3.66.1 (Trebatius, Labëon).

440- Ulpien D 16.3.1.5.

441- Ulpien D 47.2.14.15.

442- U1pien D 10.4.11.1 (Labéon).

443- Actio furti chez vlpien D 47.2.14.15, et iniuriarum chez le même D 47.10 .15 .15 .

444- Ulpien D 15.3.3.3 (Labêon).

445- Iavolenus D 24.3.66.1 (Trebatius, Labéon).

446- Marcien D 15.1.40.1 : Quomodo autem peculium nnscitur, quaesitum est. Et ita veteres distingumt, si id adquisiit servus quod dominus necesse non habet praestare, id esse peculiwn, si vero tunicas aut aliquid simile quod ei dominus necesse habet proestare, non esse peculium.

447- Ulpien D 15.3.3.10.

448. - Cette définition de Pomponius (cf. D 15.1.25) est plus subtile, plus êlaborée que celle donnée par les veteres (chez Marcien D 15.1.40. 1 , cité supra $n$. 446) pour lesquels les vêtements ne font pas partie du pécule quel que soit leur usage. 
Un vêtement fait partie du pécule lorsque le maître l'a remis à l'esclave dans l'intention qu'il ne serve à aucun autre et qu'il le conserve ainsi pour son usage. Si, en revanche, le mâtre ne le lui a remis que temporairement, par exemple pour qu'il le mette quand il 1'accompagne ou le sert à table, le vêtement n'est pas compris dans le pecule.

Comme en matière d'alimentation, l'esclave doit être vêtu secundum ordinem et dignitatem (449), mais cette discrimination vestimentaire ne joue évidenment pas qu'entre esclaves ; on le voit clairement ressortir a'm passage d'Ulpien :

Vestimenta ommia aut virilia sunt aut puerilia aut muliebria out commonia aut familiarica... Familiarica sunt, quae ad familian vestiendam paratu sunt, sicuti saga turicae paenulae lintea vestimenta stragula et conoimilia (450).

On distingue les vêtements d'hommes, d'enfants, de femmes, ou ceux qui leur sont communs et les habits d'esclaves. Ceux-ci portent des sayons, des tuniques, des manteaux, des vêtements de toile, d'étoffe et autres. Cependant, la discrimination n'est pas totale. On relève en effet parmi les vestimenta virilia, vêtements d'howmes libres, des tuniques et des sayons. ; semblablement, on remarque des tuniques dans l'énumération des vestimenta muliebria (451). Il est nécessaire de rappeler à cet égard la proposition sénatoriale, mentionnée par Sénèque, tendant à distinguer les esclaves des citcyens libres par le port d'un habit différent, et le rejet de cette proposition pour le motif que les esclaves auraient alors pu compter les libres : deinde apparuit quantum periculum immineret, si servi nostri numerare nos coepissent (452).

Toujours du point de vue discriminatoire, il faut citer ces lignes fort édifiantes d'Ulpien :

449- Ulpien D 7.1.15.2.

450- Ulpien D 34.2.23.2.

451- Ibid : Virilia sunt, quae ipsius patris familiae causa parata sunt; et plus loin : Muliebria sunt, quae matris fomiliae causa sunt comparata.

452- Sen., De Clem., I, 24,1.
Si quis virgines appellasset, si tamen ancillari veste vestitas, minus peccore videtur... (453).

Si quelqu'un interpelle des jeunes filles vêtues d'habits d'esclaves, il comet une moindre faute que si matrum familiam vestitae fuissent.

Les textes sont assez paurres en ce qui concerne le logement de l'esclave. Plusieurs le situent sous le même toit que le maitre, sub eodem tecto (454). Ces termes ont toutefois une signification assez extensive. Ainsi, Ulpien écrit à propos du sénatus-consulte silanien :

Eodem autem tecto qualiter accipiatur, videamus, utrum intra eosdem parietes an et ultra intra eandem diaetam vel cubiculum vel eandem domum vel eosdem hortos vel totam villam. Et ait Sextus sic esse saepe indicatzon, ut quicromque eo loci fuerunt, unde vocem exaudire potuerunt, hi puniantur, quasi sub eodem tecto fuerunt, licet alii validioris vocis, alii exiguioris ount nec omes undique exaudiri possunt (455).

Eodem tecto signifie-t-il dans l'enceinte de la maison ou en dehors, dans le même pavillon, dans la même chambre, dans le même jardin ou dans la même villa ? La pratique judiciaire montre, selon Sextus (456), qu'il s'agit d'un endroit d'où l'esclave puisse entendre les cris de son maître, d'un endroit donc relativement rapproché, à l'intérieur du domaine.

Il est aussi question du logement de l'esclave à propos du legs d'usage. Celui à qui on lègue l'usage d'ume maison peut y loger ceux qui remplissent les travaux serviles, quos loco servorm in operis habet, qu'ils soient libres ou esclaves d'autrui (457). Il peut également y loger ses affranchis (458). Quant à l'usager d'un fonds, il ne peut pas interdire aux esclaves arriculteurs $d$ 'y habiter, mais rien ne l'empêche par contre de s'opposer à l'hébergement des esclares urbains du propriétaire (459).

453- D1pien D 47.10.15.15.

454- Maecianus D 29.5.14; U1pien D 29.5.1.5 (rescrit d'Antonin le pieux)-26-27-38.

455- D 29.5.1.27.

456- Sur Sextus et 1 'incertitude qui pèse sur l'appellation, cf. 0 . LENEL, Pal. II, P. 1229 n. 2 et note 48 in Chapitre préliminaire.

457- U1pien D 7.8.4 pr. Les personae loco servorum sont des salariés libres assimilés aux esclaves (F.M. DE ROBERTIS, Lavoro e lavoratori nel mondo romano, (Bari), 1963, p. $102 \mathrm{~s}$ ). L'assimilation est d'autant plus fréquente que souvent 1 'engagement de ces travailleurs se fait en 1 'absence d'accords bien definis (E.M. SCHTAERHAN, La Schiavitū, cit., p. 19). 458- Jlpien D 7.8.2.1 (Tubero, Labéon, Celsus).

459- Ulpien D7.8.10.4 (Labéon). 
Ulpien nous dit ailleurs que des esclaves appartenant au mari et à la ferme vivent dans 1 a même maison $(460)$. Cependant, c'est un trait qui a déjà été souligné à propos de l'étude sur la situation géographique, l'esclave ne réside pas obligatoirement chez son maître, en particulier lorsqu'il exerce une fonction relativement autonome. En outre, le lieu de travail habituel de l'esclave peut ne pas coincider avec celui de sa résidence : Scaevola éroque par exemple l'actor d'un fonds demeurant en ville (461).

Une discrimination sociale apparait dans les thèmes qui viennent d'être envisagés. Kessort-elle aussi des textes pour ce qui touche à la vie sexuelle et à la vie fawiliale des esclaves ?

\section{Paragraphe 2: Pratiques sexuelles et formes d'unions}

Ces deux thèmes étant difficilement dissociables, il convient de les envisager dans la même rubrique.

\section{I - Les pratigues sexuelles}

L'esclave apparaît fréquement en tant qu'objet, plus rarement en tant qu'agent, d'actes sexuels répréhensibles. Par ailleurs, certains passages apportent des renseignements intéressants quant à la situation particulière de la femme esclave dans la société, quant à son rôle de reproductrice, rôle inséparable de sa vie sexuelle dans la mesure où il contribue à la déterminer.

C'est de la prostitution dont il est le plus question dans les textes. Dans deux cas seulement, la profession semble être volontaire (462). Ailleurs, elle est imposée par le dominant, qu'il s'agisse du mâ̂tre, c'est le cas le plus fréquent (463), ou du titulaire d'un droit sur l'esclave, par exemple le créancier gagiste (464). Pomponius évoque

\section{0- U1pien D 29.5.1.15.}

461- Scaevola D 33.7.20.4.

462- Heretrix, cf. Callistrate D 38.1 .38 pr. ; U1pien D 47.2.39.

463- Il faut également tenir compte des cas où 1 'ancilla est vendue sous la condition ne prostituatur, cf. Pomponius D 21.2.34 pr. ; Papinien D 18.7.6 pr (Sabinus) ; Paul D 18.1.56; D 18.7.9; D 40.8.7 (constitution de SÉvère et Caracalla); U1pien D 1.12.1.8 (Sévère) ; D 2. 4.10 .1 ; D 3.2.4.2 ; Marcien D 40.8.6 ; Modestin D 37.14.7 pr (décret de Vespasien).

464- U1pien D 13.7 .24 .3 . même un servus ordinarius qui prostitue les esclaves qui se trouvent dans son pécule (465) : mimétisme social de l'esclave privilégié et manque de solidarité au sein du groupe servile sont deux tendances qui ont déjà été dénoncées. Un texte d'Ulpien nous apprend que 1a prostitution des esclaves peut avoir lieu sous le couvert d'un cabaret, d'un hôtel, ou dans les bains, cơme cela s'est produit dans certaines provinces (466). Le droit ne va pourtant pas rester indifferent à cette pratique.

L'esclave vendue sous la condition ne prostituatur obtiendra la liberté en cas de manquement à la condition (467). In ce qui concerne la femme vendue à condition de ne pas être prostitué, dont la vente prévoit expressément qu'elle sera libre dans le cas contraire, Vespasien décrète que si elle passe à un nouvel acheteur et que celui-ci, ignorant la condition, la prostitue, elle obtiendra tout de même la liberté et deviendra l'affranchie du premier vendeur (468). On ignore 1 'origine de cette mesure, visiblement antérieure à vespasien qui en traite comme faisant déjà partie des institutions (469). La dernière hypothèse d'obtention de la liberté par punition du maître concerne toujours une condition ne prostituatur, mais accompagnée d'une possibilité de manus iniectio en cas de non-respect de celle-ci. A ce sujet, Hadrien semble avoir établi que si le titulaire de la manu iniectio y renonce et permet la prostitution, la feme sera déclarée libre par l'autorité du préteur (470). Flus tard, Sévère et Caracalla décideront dans le même sens que l'esclave sera affranchie si celui qui pouvait pratiquer la manus iniectio y a renoncé pour de l'argent, car nihil enim interesse, ipse abducas et prostituas, an patiaris prostitutam esse pretio accepto, cum possis eximere, c'est-à-hire : il n'y a aucune difference entre le fait de prostituer soi-même un esclave et celui d'accepter de l'argent pour "fermer les yeux" (471). Sévère chargera

465- Ulpien D 3.2.4.3 (Pomponius).

466- U1pien D 3.2.4.2.

467- Dans ce sens, une constitution de Marc-Aurèle, chez Marcien D 40.

8.6.

468- Modestin D $37.14 .7 \mathrm{pr}$ : Divrs Vespasianus decrevit, ut, si qua hac lege venierit, ne prostitueretur et, si prostituta esset, ut esset libera, si postea ab emptore alii sire condicione venit, ex lege venditionis liberam esse et libertam prioris venditoris.

469- Cf. W.W. BUCRLAND, Roman 1aw, cit., p. 603.

470- C I.4.56.1; W.W. BUCKLAND, op. cit., p. 604.

471- Paul D 40.8.7. : Imperator noster cum patre suo constituit in eo, 
même le préfet de la ville de défendre les esclaves contre les maîtres qui voudraient les prostituer (472).

Ces mesures, par la distance temporelle qui les sépare, plus d'un siècle s'écoule entre la constitution de Vespasien et celle de Sévère et Caracalla, sont l'aveu à peine voilé de l'impuissance de I'Etat face à des pratiques ancrées dans le quotidien. Que Callistrate se voit contraint de préciser que $I$ 'individu qui a affranchi une meretrix ne peut Iui demander ses "services" en guise d' operae, car cela est contraire à la bienséance, est tout-à-fait éloquent (473).

Ceux qui tirent un gain de la prostitution de leurs esclaves tombent sous le coup du délit de lenocinium, proxénétisme (474). Le Digeste fait état a'un autre délit sexuel, I'cudulterium (475).

La lex Iulia de adulteriis ne s'spplique pas aux femmes esclaves qui sont, sur ce plan, assimilées aux fermes de mauvaise vie (476). La condition de $I$ 'home coupable n'a, par contre, aucune importance. Les conséquences de l'adultère commis avec un esclave (477), hormis le droit

qui, cum possit abducere prostitutom ancillam, pecuria accepta manus iniectionem vendidit, ut libera esset.

472- Ulpien D 1.12.1.8: Hoc quoque officiom praefecto urbi a aivo Severo datum est, ut mancipia tueatur ne prostituantur. Le même empereur défendra que la femme qui a été prostituée alors qu'elle était esclave soit notée d'infamie, cf. Ulpien D 3.2.24 : Imperator Severus rescripsit non offuisse mulieris famae quaestion eius in servitute factum.

473- Callistrate D 38.1.38 pr.

474- Sur le thème, Th. MOMMSEN, Droit pénal romain (tr. fr. J. DUQUESNE, 3 vol. Paris 1907), II, P. $427 \mathrm{~s}$; ; Ulpien D 3.2.4.2 : ... Lenocinium facit qui quaestuaria mancipia habuerit.

475- Sur 1'adulterizon, Th. MOMMSEN, Droit pénal, II, cit., p. 414 s.; FERRINI, Diritto penale romano (1909), p. $306 \mathrm{~s}$.; E. VOLTERRA, Per la storia dell'accusatio adulterii iure mariti vel patris, in SUC (1928); CORSANEGO, La repressione romana del1'adulterio (1936) ; M.A. DE DOMINICIS, Sulle origini romanocristiane del diritto del marito ad accusare constante matrimonio la moglie adultera, in SDHI 16 (1950), p. 211 s. ; CHIAZZESE, Adulterizon (dir. rom.), in NNDI ; G. LONGO, Lex Iulia de achulteriis coercendis, in NNDI ; J.A.C. THOMAS, Lex Iulia de adulteriis coercendis, in Et. Macqueron (1970), p. $637 \mathrm{~s}$.

476- Th. MOMMSEN, Droit Pénal, II, cit., p. 418.

477- On relève un adultère commis avec un esclave chez Tryphoninus D 50.16.225; Paul D 9.2.30 pr ; Ulpien D 1.12.1.5; D 40.9.12.6 (Africain); D 48.2.5 (rescrit de Marc-Aurèle) ; D 48.5.28 pr-1-2-3-4-16 ; Macer D 48.5.25 Pr ; Marcien D 28.5.49.2 (constitution de Sévère et Caracalla) ; D 48.5.34 pr (rescrit d'Antonin le pieux)-1. Le Digeste fournit également des cas d'adultère auxquels sont mêles des affranchis ou des affranchies. L'affranchi est coupable chez Tryphoninus D 48.5 .43 (adultère de mise à mort, ne font l'objet que de mentions rares (478). La situation est il est vrai problématique, mais combien intéressante du point de vue des rapports sociaux, lorsque l'esclave appartient à la fembe coupable $(479)$ ou à I'accusateur (480). L'esclave intervient ici comme

avec la femme du patron); Macer D 48.5.25 pr (adultère avec la femme du patron; avec la patronne) ; Marcien D 48.5.34.1 (adultère avec la femme du patron). C'est une affranchie qui endosse la culpabilité chez Marcellus D 23.2.41.i et chez U1pien D 24.2.11.2 (rescrit de Sévère et Caracalla). Un texte cependant traite de l'affranchi en tant que victime de ce délit, cf. Papinien D 48.5.39.9 : Liberto patroni famam lacessere non facile conceditur : sed si iure mariti velit adulterii accuscre, non facile conceditur: oed oi iure mariti velit adulteril accuscre, patronw, qui sit ex eo momero, qui deprehensus ab alio interfici potest, patronion, qui sit ex eo momero, qui deprehensus ab alio interfici potect, occidere. Quod dumon nobis esse videtur : nom cuius famae, multo magis vitae parcendum est. On nctera essentiellement que 1 'affranchi qui surprend son patron en flagrant délit d'adultère avec sa femme n'a pas le droit de se faire justice. Le motif invoqué est que, si on doit respecter la réputation de quelqu'un, il faut encore davantage respecter sa vie. Motif compréhensible compte tenu du rapport de subordination qui lie l'affranchi a qui lie l'aferanchis mulation, car la réciproque n'est pas vraie, cf. Macer D 48.5.25 pr : Marito quoque adulterm uxoris suce oacidere permittitur, sed non quem libet, ut patri : nam hac lege cavetur, ut liceat viro deprehensw dom suae (non eticm soceri) in adulterio uxoris occidere eum, qui leno fuerit quive artem ludicram ante fecerit in scaenom saltandi cantandive causa prodierit iudiciove publico damatus neque in integrem rostitutus erit, quive libertus eius mariti uxorisve, patris matris, filii filiae utrius eomom fuerit (nec interest, proprius cuius eormon an cum alio conmunis fuerit) quive servus erit.

478- Sur le droit pour le mari de tuer I'esclave surpris en flagrant délit d'adultère avec sa femte, cf. Hacer $D$ 48.5.25 pr (cité supra n. 477). Par ailleurs, il faut signaler ce passage de Marcien, D 48.5.34 pr : Si quis adulterivon a servo suo commisstom dicat in ecm, quam uxorem habuit, divus Pius rescripsit accusare potius mulierem eum debere, quam in praeiudicium eius servum surm torquere; si quelqu'un soupçonne son esclave d'avoir commis un adultère avec sa femme, un rescrit d'Antonin le pieux lui intime d'accuser sa femme avant d'appliquer $1^{\prime}$ esclave à la quaestio.

479- Marcien D 28.5.49.2. Interdum nec mon libertate utiliter servus a domina heres instituitur, ut constitutione divorion Severi et Antonini significatur, cuius verba haec sunt : 'servn adulterii accusation non iure testamento manumissum ante sententiam ab ea muliere videri, quae rea fuerit eiusdem criminis postulata, rationis est'. Quare sequitur, ut in eundem a domina collata institutio nihil momenti habeat.

480- Ulpien D 1.12.1.5 ; D 40.9.12.6 (Africain) ; Marcien D 48.5.34 pr (rescrit d'Antonin le pieux)-1. Il peut aussi s'agir d'un serrus alienus, cf. Paul D 9.2.30 pr : Qui occidit adultemm deprenensum servom alienum, hac lege non tenebitior. 
agent dálictuel

pudeur dont il

- Il n'en est pas ainsi de l'attentat à la prévoient bien une protection de la pudicitia servi (482), mais il est plus juste de parler d'une protection des intérêts du maitre. En effet, le maître a l'actio iniumiamon, l'actio Legis Aquiliae et l'actio de servo corrupto (483). En d'autres termes, L'attentat à la pudeur sur un esclave est clairement considéré par les juristes comme une atteinte au patrimoine de son maître. Cet attentat peut aussi bien être perpétré sur une femme (484) que sur un homme (485). Inversement un esclave peut prêter son assistance à l'abus d'une feme ou d'un enfant (486). D'un point de vue plus général, on relève un rapport homosexuel entre un

481- Il s'agit d'un stuprom au sens philologique du terme (il apparaît sous cette forme ou une forme dérivée chez Papinien $D 48.5 .6 \mathrm{pr}$; Paul D 1.18.21; D 11.3.2 ; Ulpien D 21.1.23 pr (Pomponius) ; D 47. 10.25) (cf. Th. MOMMSEN, Droit pénal, cit. II, p. 421 n.1), mais non dans le sens juridique tel que le donne A. GUARINo, Diritto privato, dans le sens juridique tel que le donne A. GUARINO, Diritto privato cit., P. 525, c'est-a-dire l union avec une femme virgo vel vidua, 1 'exception d'une Zibertina, d'une femme "facile" ou d'une femme obscuro loco nata. On relève encore une atteinte a la pudicitia servi chez Paul D 47.2.83.2 ; Ulpien D 1.12.1.8; D 47.1.2.5; D 47.10.9.4.

482- Les termes pudicitia servi apparaissent chez Ulpien, D 47.10.9.4 : $S i$ quis tan feminam quam masculum, oive ingenuos sive libertinos, impudicos facere adtemptavit, iniuriamon tenebitur. Sed et si servi pudicitia adtemptato sit, ini.uriamon locrm habet.

483- Outre D 47.10.9.4 sur I'actio iniuriamon (cite supra n. 482), il faut mentionner Papinien D 48.5.6 pr : Inter liberas tantwo personas adulterium stupmomve passas lex. Iulia locw habet. Quod autem ad servas pertinet, et legis Aquiliae actio facile tenebit et iniuriamon quoque competit nec erit deneganda praetoria quoque actio de servo cormpto : nec propter plures actiones parcendum erit in huiusmodi crimine reo ; et Ulpien D 47.10.25 : Si stiprum serva passa sit, iniuriarm actio dabitur: aut, $3 i$ celavit mancipium vel quid aliud furandi animo fecit, etion furti : vel, si virginem irmaturam stupraverit, etiam legis Aquiliae actionem competere quidam putant.

484- Paul D 1.18 .21 ; D 47.2.83.2 ; Papinien D $48.5 .6 \mathrm{pr}$; Ulpien D 47.1.2.5;D 47.10.9.4; D 47.10.25.

485- Cela ressort clairement de $D$ 47.10.9.4 Ulpien, et découle de 1 'emploi du masculin chez Paul D 1.18 .21 ; D 11.3 .2 ; U1pien D 1.12.1.8; D 21.1 .23 pr (Pomponius).

486- Paul D 47.11.1.2. Th. MOMMSEN, Droit pénal, cit. II, p. 432 n. 6, III, P. 407. maître et son esclave chez Ulpien (487).

Quant à la castration (488), seuls quatre textes du Digeste y font allusion à propos d'un esclave (489). On sait que Domitien la défendit tant pour les esclaves que pour les homes libres (490). Cette interdiction fut renouvelée par Nerva (401) et Hadrien (402). Salon le rescrit de ce dernier, la peine de mort frappe tout médecin et tout esclave qui pratique la castration, de même que celui qui se laisse châtrer volontairement. Venuleius signale qu'en vertu d'un sénatusconsulte de l'époque du consulat de Neratius Priscus et Annius Verus, celui qui aura livré son esclave pour être châtré verra la moitié de son patrimoine confisquée (493). Quant à la castration d'un servus alienus sans le consentement de son maitre, elle n'est pas considérée comme un dormage causé à la chose d'autrui puisque la valeur de l'esclave s'en trouve augmentée, mais c'est une iniuria (494).

En ce qui concerne les textes traitant de la feme esclave comme reproductrice, la plupart ont déjà été mentionnés à propos de la naissance à l'intérieur du groupe servile (495) ou de l'étude sur la maladie (496). Méritent d'être signalés ici certains textes sur la maternité. Il est ainsi question chez Paul de jumeaux (497), chez Ulpien d'une femme qui accouche simultanément d'un garçon et d'une fille (498).

487- Ulpien D 7.7.6.2 : Item voluptatis vel affectionis aestimatio non habebitur, veluti si dilexerit eum dominus aut in deliciis habuerit. Contrairement $a$ un autre texte d'Ulpien (D 1.12.1.8) od des esclaves sont contraints par leur maître a l'obscénité, le rapport semble ici volontaire. V.aussi D 38.1.7.5 (voluptatis artifex), cf. supra n.506 $1^{\circ}$ part 488- Th. MOMMSEN, Droit pénal, II, cit., p. $354 \mathrm{~s}$.

489- Venuleius D 48.8.6; Ulpien D 9.2.27.29 (Vivianus) ; D 48.8 . 4.2 (rescrit d'Hadrien). D 37.14.6.2 de Paul concerne plus exactement un affranchi.

490- Suétone, Dom., 7.

$491-$ Dion, 68.1.

492- Ulpien D 48.8.4.2.

493- D 48.8.6. Sur le thème, v. DALLA, L'incapacità sessuale in diritto romano, (1978), p. $85 \mathrm{~s}$. Ce s.c. da.terait de 97 ap. J-C., V. SCARANO USSANI, Valori e storia nella cultura giuridica fra Nerva e Adriano, Studi su Nerazio e Celso, Naples (1979), p. 96 s. et n. 43.

494- U1pien D 9.2.27.28 : Et si puerum quis castraverit et pretiosiorem fecerit, Viviams scribit cessare Aquilian, sed iniuriamon erit agendrom aut ex edicto aedilizm aut in quadruplum.

495- Sur le terme virgo, cf. supra n. 171 première partie ; ancilla nondion viripotens, cf. supra $n .172$ première partie; sur l'esclave devant mettre au monde plusieurs enfants pour obtenir la liberté, cf. supra n. 200 première partie. 
Sur l'accouchement proprement dit, on relèvera une césarienne pratiquée sur une ancilla (409), une sage-femne, obstetrix, présente à ce moment-1à (500).

Ces indications sur la maternité de la femme esclave nous éloignent dé la groblématique de la vie sexuelle dófinie au sens strict. Elles nous amènent à envisager plus globalement la vie familiale des escleves.

\section{II - Les formes d'unions}

Les unions dans lesquelles on relève la présence d'esclaves ou d'affranchis peuvent ètre nouées soit à l'intérieur, soit en dehors de leur groupe.

\section{A/ - Les unions nouées à l'intérieur du groupe des es- claves et affranchis}

Esclaves et affrpachis peuvent s'unir à des personnes ayant la même condition qu'eux, mais des esclaves peuvent aussi conclure des unions avec ces affranchis.

\section{1) - Unions entre personnes de même condition}

\section{a - entre esclaves}

Le Digeste fournit vingt-cing cas d'esclaves menent, une vie conjugale (501). Tout en refusant aux unions serviles une existonce

497- Paul D 9.2.22.1 gemelti.

498- U1pien D 34.5.10.1.

499- U1pien D 6.2.11.5 (Pomponius).

500- Ulpien (Labêon) D 9.2 .9 pr.

501- Gaius D 40.7.31.1 ; Scaevola D 32.41.2-5; D 33.7.20.1-4; D 33. 7.27.1 ; D 34.I.20 pr-1 ; D 40.4.59 pr ; D 40.5.41.15 ; Paul D 23.2.

14.3 ; D $35.1 .81 \mathrm{pr}$; D 38.10 .10 .5 (par serviles cognationes) ; U1pien D 21.1 .35 ; D $21.1 .40 \mathrm{pr}$; D 23.3.39 pr ; D 33.7.12.5 (Trebatius)-7-33; D 38.8.1.2 (par servilis cognatio); D 38.16 .1 .1 (rescrits de MarcAurèle et Verus et de Caracalla); D 40.2.12 (par ratio cognationis). On peut intégrer par extension D 2.4.4.3 Ulpien (Labéon), D 38.10.10.5 Paul, où le terme parentes semble désigner un père et une mère esclaves, et $D$ 48.2.12.4 de Venuleius, où le juriste affirme que la lex Pompeia juridique, les juristes vont admettre assez tôt la validité des termes employés par les esclaves quant à ces unions : ainsi trouve-t-on, malgré l'illégitimité de la relation, vir (502) et uxor (503) désignant des esclaves. On relève pareillement des vocables faisant allusion à la parenté par alliance, tel le terme assez général cognatus (504), ou à une parenté plus proche, frater (505) et soror (506). Parens (507), pater (508), mater (509) désiguent les ascendents. Les descendants sont visés par nepos (510) et, plus fráquemment par filius et filia (511).

S'il découle de 1 'emploi de frater ou de soror que plusieurs enfants, en tout état de cause au moins deux, sont issus de l'union, on ne peut tirer les mêmes conclusions à partir de filius et" de filia que lorsque ces termes, employés au pluriel, sont en relation avec un vocable désignant I'ascendant au singulier (512), c'est-g̀-dire rarement.

de parricidiia s'applique aux esclaves : a la fin de la République, le terme parriciąizon semble avoir dêsigné le meurtre d'un proche parent, cf. H. KUPISZEWSRI, Quelques remarques sur le parricidizon dans le droit romain classique et post-classique, in St. Volterra, IV, p. 604, (plus tard, voir la définition de Marcien $D$ 48.9.1) ; s'il s'agit du meurtre du père, on a toutes les raisons de penser que Venuleius sous-entend

qu'il a étê assassiné par son enfant ; or, l'intérêt du passage est que père et enfant soient esclaves; et, si 1 'enfant est esclave, cela père et enfant soient esclaves : et, si l'enfant est esclave, cela
signifie que la mère l'est aussi : on a donc indirectement une possi-

502- Ulpien D 33.7.12.5 (Trebatius).

503- Scaevola D 33.7.20.4 ; Paul D 23.2.14.3; U1pien D 33.7.12.7-33. Concernent une hypothèse d'affranchissement Africain D 35.1.31; Paul D 23.2.14.2-3 ; Ulpien D 40.2.13 ; Modestin D 40.9.21 (Julien). A part, un texte de Modestin sur 1'ignorance par la femme de son status, cf.

D 25.3.7 : uxorem ancillam esse.

504- Paul D 38.10.10.5: Non parcimus his nominibus, id est cognatomon, eticon in servis.

505- Papinien D 17.1.54 pr ; D 40.1.19; Paul D 21.1.39; Ulpien D 21. 1.35 ; $\mathrm{D} 30.71 .3$; $\mathrm{D} 40.2 .11$; $\mathrm{D} 48.18 .1 .10$ (rescrit de Sévère et Caraca11a).

506- Paul D 23.2.14.2; U1pien D 40.2.11.

507- Scaevola D 33.7.20.5 ; Paul D 38.10.10.5 ; U1pien D 2.4.4.3

(Labéon) ; D 21.1.35; D 40.12.3 pr.

508- Scaevola D 33.7.27.1 ; Paul D 23.2.14.2-3; D 50.2.9 pr (Sévẽre); Ulpien D 30.71 .3 ; D 38.16.1.1 (rescrits de Marc-Aurèle et Verus et de Caracalla).

509- Cf. supra n. 34 .

$510-$ Scaevola D $32.41 .5 ;$ D $34.1 .20 \mathrm{pr}$. Dans D $33.2 .36 \mathrm{pr}-1$, les nepotes sont ingénus, le passage évoquant 1'affranchissement de leur aỉeul potes sont

$511-$ Cf. supra n. 159 première partie. 
On ne peut toutefois s'appuyer sur ce fait pour souterir que, de manière plus gécérale, les unions serviles ne sont pas prolifiques (513). Les encouragements $d$ 'un Columelle ou d'un Pline le jeune ne signifient pas nécessairement en effet que les unions serviles n'ont pas la prolificité souhaitée, l'importance des textes traitant de la reproduction naturelle est là pour nous le rappeler, mais plutôt que, l'apport externe d' "esclaves frais" se tarissant, il s'agit de pallier par d'autres moyens cette carence ( 514 ).

Si le Digeste incite donc à la prudence en la matière, il est par contre une source précieuse d!information quant aux effets que les juristes vont permettre à la servilis cognatio de produire dans le domaine du droit.

Déjà Labécn admet que la règle interdisant une in ius vocatio du père, sans autorisation du préteur, $s$ 'applique aux pères qui étaient esclaves au moment de la naissance de l'enfant (515). Pour Pomponius,

512- Scaevola D 32.41.5; D 40.5.41.5; Paul D 40.5.39.1; Ulpien D 40 4.13.3. Rappelons ếgalement Paul D 9.2.22.1, ou il est question de.gemelli.

513- On ne peut être d'accord avec G. SUFFERT, La peste blanche : coir ment éviter le suicide de $1^{\prime}$ Occident, Paris (1976), p. 116-119, selon qui les esclaves n'ont pas d'enfants. Comment serait-il possible d'expliquer que $5 \%$ du nombre total de textes concernant l'esclavage, pour notre période $v$, c'est-à-dire à partir des Sévères, traitent encore de la reproduction naturelle des esclaves?

514- Colum.I,8,19; Pline, Lettres, VIII, 16 ; v. R.H. BARROW, Slavery, cit., p. 87 ; G. BoULVERT, DEF., cit., p. 321 . Si les riches maîtres de 1 'époque républicaine n'étaient que médiocrement intéressés par l'élevage des enfants-esclaves, compte tenu de l'afflux des prisonniers de guerre (cf. R. MARTIN, La vie sexuelle des esclaves d'après les dialogues rustiques de Varron, in $J$. Collart, Varron, Gramaire antique et stylistique latine, Paris (1978), p. 121), il n'en va plus de même lorsque la razzia connaît une baisse ne permettant plus le gaspillage (cf. P. DOCKES, La libération, cit.,p. 165). Sur la rentabilité de l'êlevage, v. aussi I. BIEZUNSKA-MALOWIST, Les enfants esclaves à la lumière des papyrus, Latomus (1969), p. 91-96. Fournir des compagnes aux esclaves, c'était les attacher au domaine, R. MARTIN, Recherche sur les agronomes latins les attacher au domaine, R. MARTIN, Recherche sur les agronomes latins et leurs conceptions était aussi limiter les risques de fuite, ibid., p. 173; peu importait c'était aussi limiter les risques de fuite, ibid., p. 173; peu importait
enfin la beauté de la femme-esclave, sa qualité essentielle étant selon Varron (R.R.L., II, 10,7) la robustesse, l'agronome vantant la résistance et $I^{\prime}$ aptitude à cumuler les tâches de la maternité et les travaux des champs des Illyriennes, ibid., p. 174.

515- Chez U1pien D 2.4.4.3 : Parentes etian eos accipi Labeo existimat, qui in servitute susceperunt. les liens familiaux serviles constituent un empêchement au mariage après affranchissement (516). Selon Venuleius, qui écrit sous les Antonins, la lex Pompeia de parricidiis est applicable aux esclaves (517). A l'époque des Sévères, la reconnaissance des liens ramiliaux des esclaves s'étoffe. Le lien de parenté est une juste cause d'affranchissement dans $l^{\prime}$ hypothèse ol cette cause est requise (518).

Les esclaves unis par des liens familiaux ne peurent être séparés à l'occasion d'une redhibitio (519). Ulpien reconnaît même la validité d'une quasi-dot apportée par une esclave (520).

Cette tendance à admettre une certaine validité des unions serviles ne va pas dans le seul intérêt des esclaves : les juristes $s$ 'efforcent d'attacher au fonds les instruments de production, dont les esclaves font partie (521). C'est ainsi qu'à propos du legs d'un fonds cum instmonto, Paul inclut les uxores des esclaves travaillant sur le fonds (522), Ulpien y ajoute leurs enfants (523). C'est dire qu'on encourage les esclaves à prendre pour compagnes des ancillae de la même familia. D'ailleurs, parni les cas que nous soumet le Digeste, aucun ne traite d'une union entre deux esclaves de familiae différentes.

Si esclaves et affranchis forment un tout du point de vue sociologique, il faut être plus nuancé sur le plan juridique : on vient de voir que les premiers ne pouvaient que contracter une union de fait,

516- Pomponius D 23.2.8; Paul D 23.2.14.2.

517- Venuleius D 48.2.12.4. W.W. BUCKLAND, Roman law, cit., p. 78 ; E.M. SCHTAERMAN, La schiavitò, cit., p. 231 .

518- Ulpien D 40.2.11.

519- Paul D 21.1.39; U1pien D 21.1.35

520- Ulpien D 23.3.39 pr. Proculus refusait cette possitilite (cf. D 23.3.67). On ne sait si I'innovation, dont Ulpien fait état, date de 1 'époque des Antonins ou de celle des Sévères, cf. E.M. SCHTAERMAN, La schiavitù, cit., p. 234.

521- Cf. sur le thème, M. HoRvar, Legation fundi e servi, cit., p. $89-97$.

522- Paul, Sent.III, 6, 38 : Uxores eorm qui operantur magis est ut instrumento cedant.

523- U1pien D 33.7.12.7 : Uxores quoque et infantes eorm, qui supra emonerati sunt, credendwm est in eadem villa agentes voluisse testatorem legato contineri : neque enim duran separationem iniunxisse credendus est ; et D 33.7.12.33 : Contubernales quoque servorm, et natos, instructo fundo contineri verrom est, a propos de legs d'un fonds instructus. 
le contubemizon ; les seconds ont en revanche le conubion, la capacité matrimoniale $(524)$.

\section{$b$ - entre affrenchis}

On trouve dans le Digeste onze cas d'affranchis unis par des liens corjugaux (525). Ces liens sont le plus souvent constitutifs d'une véritable union juridique, le matrimonizon (526), mais il est possible que des affranchis préfèrent au mariage une simple union de fait, le concubinat (527).

Alors que la macule de la naissance servile est indélébile, le fils de l'affrenchi qui naît libre est un ingénu, et n'est pas affecté par les diverses incapacités frappant son père (528).

\section{4- G. BOULVERT, DEF, cit., P. 257.}

525- Africain D 35.1.31 ; Pomponius D 23.2.8; Paul D 23.2.14.2-3 ; D $35.1 .81 \mathrm{pr}$; D 50.1.22 pr ; Ulpien D 23.2 .56 ; D 38.16.1.1 ; D 38 . 16.3.5; D 47.10.11.7; Marcien D 40.2.14.1.

526- Tous les textes citếs (supra n. 525) à l'exception de D 23.2.56 de U1pien.

527- Ulpien D 23.2.56 : Etian si concubinam quis habuerit sororis filiom, licet libertinam, incestrom conmittitur. Sur 1 'inceste, Th. MOMSSEN, Droit péna1, cit., II, p. $406 \mathrm{~s}$. Le concubinat est une union de pur fait et non un mariage d'ordre inférieur; il ne produit donc pas a'effet en droit privé, cf. J. PLASSARD, Le concubinat romain sous le Haut-Empire, (1921), p. 25 ; P. MEYER, Der röm. Konkubinat (1895) ; A. BERGER, Encrcl. Dict., cit., p. 402

528- G. BOULVERT, DEF, cit., p. 322. Néanmoins, les fils d'affiarctis s sont écartés par la tradition et les moeurs du sénat et des magistratures, cf. Cicéron, Pro C1uentio, XlviI, 132 ; Horace, Satires, 1, 6, 20 ; Suétone, Néron, XV ; sous 1a Rëpublique, S. TREGGIARI, Roman freedmen, cit. P. 229 s. Il est question, dans le Digeste, de descendance des, cit., $p$. affranchis chez Celsu $D$ (J0.2.19; Julien $D 38.220 .4-5-6$; T. Clemen D 35.2 .38 .1 ; D 40.9 .31 (Julien) ; Maecianus D 36.1 .66 .3 ; Marcellus D 35.2.52 pr ; Scaevola D 33.1.18 pr ; D 33.2.36 pr-1 ; D 34.1.16.1 ; 36.3.18 pr-1 ; D 40.4.60; D 40.7.40.2 ; Papinien D 13.7.40.1; D $3 !$ 77.13-27-28; D 38.2.42.2; Tryphoninus D 28.2.28.3; D 34.5.9.2 ; Paul D 31.83 ; D $37.14 .6 \mathrm{pr}-2$; D $38.1 .37 \mathrm{pr}-1$ (Ju1ien) -2 (Pomponius) -3 -4-5-6 (Ju1ien)-7-8; D 38.2.4.3; D 48.18.20; D 50.1.22 pr ; U1pien D 2.4.10.10; D 36.1.18.4 (Papinien) ; D 38.2.3.14; D 38.2.6 pr-2-3 D 38.2 .16 .5 (Julien) ; $D$ 38.2.17 (par a contrario); D $38.5 .1 .2-10$. D 38.16.3.12 (rescrit d'Anconin le pieux) ; D 38.17 .1 pr-3.; D 38.17.2. 3 ; D 40.5.26.1-2 (Antonin le pieux, rescrit de Sévère et Caraca11a) -4 (rescrits d'Antonin le pieux et de Sévère et Caracalla) ; D $40.7 .6 \mathrm{pr}-1$ -2 ; D 50.1.6.3; Macer D 48.18.20.1; Marcien D 50.5.53 pr ; Hermogénien D $37.14 .21 .3-4 ;$; 48.4 .9 (décret de Sévère).
L'union conjugale entre affranchis peut être le prolongement de relations contractées durant l'esclavage : soit l'bomme et la ferme ont été affranchis simultanément (529) ; soit l'un d'eux a obtenu la liberté avant son conjoint, il s'agit fréquemment de 1 'homme (530).

\section{2) - Unions entre personnes de conditions inégales}

Les trois textes, tous de Scaevola, qui visent ce problème, font d'ailleurs allusion à une antériorité de l'affranchissement de l'borme (531). Le soin de celui-ci est alors d'obtenir le plus rapidement possible la liberté de sa compagne (532).

Bien que les textes du Digeste ne traitent sur ce point que d'unions conclues alors que les deux conjoints avaient la même condition, l'épigraphie montre que la relation peut être originairement nouée à une époque où les parties svaient déjè un status différent (533). Du reste, des esclaves peuvent même s'unir à des ingénues et des ingénus à des esclaves (534).

\section{B/ - Les unions nouées en dehors du groupe des esclaves et affranchis.}

Il faut à nouveau distinguer les esclaves des affranchis, compte tenu ae la nature différente des unions qu'ils peuvent nover.

\section{1) - Unions ayant trait ̀̀ des esclaves}

Parmi les ingénus, le maître mérite une place particulière.

a) - le mâtre a pour concubine une de ses esclaves

Une telle union peut ressortir de l'emploi des termes filius naturalis (535). Cette expression peut en effet non seulement, viser

529- Par exemple, Paul D 35.1 .81 pr : ... Stichus si rationes reddiderit, cum contubernali sua liber esto...

530- G. BOULVERT, DEF, cit., p. 262.

531- Scaevola D $32.41 .2 ;$ D $34.1 .20 \mathrm{pr}-1$.

532- Petrone, Satiricon, LXVII, 6 ; P. VE YNE, Vie de Trimalcion, AESC. $16(1961)$, p. 222 .

533- G. BOULVERT, DEF, cit., p. $274 \mathrm{~s}$

534- On a déjà évoqué ce problème à propos des esclaves publics, (cf. supra n. 270) et des esc1 aves imperiaux (cf. supra n, 281).

535- On peut relever sur les unions maître/esclave Julien D 42.8.17. 
l'enfant qu'un père a eu alors qu'il était esclave, mais aussi celui que le maître a obtenu de sa concubine esclave (536). L'enfant suivant la condition de la mère, le maître peut agrandir de cette manière son réservoir de main-d'oeuvre (537). Il convient toutefois d'être nuancé sur ce point. Gaius rapporte en effet une disposition de la lex Aelia Sentia qui tolère des affranchissements avant l'âge minimum de 30 ans lorsqu'il s'agit de libérer son propre fils ou fille. C'est la preuve de l'existence d'unions civis/serva fondées sur l'affection, débouchant sur un affranchissement que l'on facilite ( 537 bis). Il est ailleurs spécifié que plusieurs enfants sont issus de l'union (538).

Dans un souci de respect pour la famille de l'esclave, les juristes vont également prendre en consideration cette hypothèse. C'est ainsi que la concubine esclave et son enfant seront exclus de l'obligation générale pesant sur les biens du débiteur (539).

1: Scaevola D 36.1.80.2 ; Papinien D 17.1.54 pr ; Paul D 9.2.33 pr (Pedius) ; D 35.2.63 pr (Pedius); D 42.5.38 pr ; Ulpien D 20.1.8.

536- Compte tenu de son double sens, 1 'expression filius naturalis (cf. supra n. 159 première partie) peut soulever des difficultés d'interprétation. Prenons un exemple, Ulpien D 36.1.18.4 : Si quis rogatus fuerit, ut, si sine liberis decesserit, restituat hereditatem, Papinianus libro octavo responsorom scribit etian naturalem filizon efficere, ut deficiat condicio : et in libertino eodem colliberto hoc scribit. Si le fils naturel de l'affranchi est probablement un enfant qu'il a étant esclave, le premier cas est moins clair : il peut s'agir d'un enfant que 1'héritier a eu d'une de ses esclaves, mais il se peut aussi qu'il ait eu cet enfant alors qu'il était esclave et qu'il ait été affranchi par la suite, ou encore que cet enfant soit simplement le fruit d'une union de fait entre l'hêritier et une ingénue. L'analyse de $D$ 30.71.3 U1pien, D 37.14.21.3 Hermogénien ; D 40.1.19 Papinien ; D 40.2.11 Ulpien, est aussi délicate.

537- La condition de la mère $s$ 'apprécie au jour de la naissance, E. ALBERTARIO, Conceptus pro iom nato habetur, in Scritti I, (1933), p. 8 ; C. CASTELLo, la condizione del concepito da libero e schiava e da libera e schiavo, Studi Solazzi (1948), p. 233, et Sulla condizione del figlio concepito legittimamente e illegittimamente nel dir. rom., RIDA IV (1950) p. $267 \mathrm{~s}$. Sous les Sêvères, il suffit que la mère ait été libre a un moment de la grossesse pour que l'enfant naigse libre, libre a un moment de la grossesse pour que l'enfant naigse libre,
V. ARANGIO-RUIZ, Arch. Giuridico CXIII (1935), p. $78 \mathrm{~s}$.; C. CASTElloRIDA IV (1950), p. $288 \mathrm{s.;}$; contra, E. ALBERTARIO, op. cit., p. $35 \mathrm{s.}$ 537 bis-Gaius, I, 19.

538- Julien D 42.8.17.1; Paul D 42.5.38 pr ; U1pien D 20.1.8. 539- Paul D $42.5 .38 \mathrm{pr}$ : Bonis venditis excipiontur concubina et liberi naturales. b) - sutres cag.

En dehors de 1 'hypothèse où une esclave est la concubine de son maître, le Digeste offre onze exemples d'unions entre esclaves et ingénus ou ingénues (540). Si le père est esclave et la mère libre, l'enfant, suivant la condition de sa mère, sera libre (541). Si, par contre, le père est libre et la mère esclave, l'enfant naîtra esclave (542). Dans les deux cas, il s'agit d'un contubernizon, tout comme dans les ménages de deux esclaves (543). Cependant il arrive que, dans $1 a$ pratique, les choses ne soient pas aussi claires.

On a vu, à propos de l'étude sur le nombre des esclaves, que la confusion s'instaurait fréquement entre esclaves et libres (544). Aussi n'est-on pas surpris de voir un homme on une femme contracter un matrimonium avec une ou un esclave qu'il croyait libre (545).

Ces unions vont être partiellement reconnues par le droit. Paul examine 1 'hypothèse suivante : un horme libre donne sa fille pour épouse à un servus alienus. Il lui remet sa dot. Puis il meurt, ainsi que son "gendre". Le juriste décide que la veuve pourra réclamer que la dot lui soit restituée sur le pécule de son "mari" (546).

Faire allusion au pécule, c'est nécessai rement sous-entendre qu'il s'agit d'un esclave privilégié. Au demeurant, une telle union n'est concevable que si l'esclave occupe une position sociale relativement Élevée (547). En est-il de même pour les affranchis?

540- Proculus D 23.3.67; Marcellus D 23.3.59.2; Papinien D 24.3.42. 1 ; Tryphoninus D 28.2.28.3 ; Paul D 16.3.27; D 24.3.23; D 41.7.8; D $50.2 .9 \mathrm{pr}$ (Sévère) ; Ulpien D 24.3.22.13; D 38.16.1.1 (rescrits de Marc-Aurèle et Verus et de Caracalla) ; Modestin D 25.3.7.

541- Paul D 16.3.27; D 24.3.23; D 50.2.9 pr (Sévère); U1pien D 24. 3.22 .13 .

542- Proculus D 23.3.67; Marcellus D 23.3.59.2 ; Papinien D 24.3.42. 1 ; Tryphoninus D 28.2.28.3; Paul D 41.7.8; Ulpien D 38.16.1.1 (rescrits de Marc-Aurẽle et Verus et de Caracalla); Modestin D 25.3.7.

543- G. BOULVERT, DEF, cit., p. 302.

544- Cf. supra notes 67 a 78 première partie.

545- Ignorance par la femme de son status d'esclave, Proculus D 23.3. 67 ; confusinn, Papinien $D 24.3 .42 .1$, gur la feme ; confusion sur 1 ' homane, U1pien D 24.3.22.13.

546- Paul D 16.3.27.

547- E.M. SCHTAERMAN, La schiavitũ, cit., p. 241. 


\section{2) - Unions ayant trait à des affranchis}

Si rien de précis ne ressort sur la position sociale des ingénus et ingénues unis à des esclaves, les textes juridiques sont plus loquaces sur le problème à propos d'unions nouées avec des affranchis.

$D^{\prime}$ une part, on sait que l'affranchissement, tout en permettant à l'esclave d'acquérir le conubizon, ne l'autorise pas à contracter une union légitime avec une personne de l'ordre sénatorial (548). Ainsi, la fille, la petite-fille et même l'arrière-petite fille d'un sénateur ne peuvent se marier ì un affranchi (549). Ce dernier reste sous le coup de l'interdiction même s'il s'est fait adroger par un ingénu et bénéficie des droits de l'ingénuité (550), ou même si le père de $1 \mathrm{a}$ fille est déchu de sa dignité (551). Il y a deux sortes d'exceptions ì cette rògle, elles sont il est vrai tout-à-frit, limitatives. Fn premier lieu,si ln fille d'un sñnteur s'ert livrée ì la prostitution, a pratiqué un ars ludicra, ou enfin a été condamnée par un iudicium publicom, elle pourra se marier à un affranchi, car, souligne Paul, nec enim honos ei servatur, quae se in tintum foedus dedusit, c'est-à-dire : il n'y a pas lieu d'avoir de la considération pour une personne qui s'est ningi dóshonorée (55?). En scoond lieu, senl l'empereur peut, accorder $\grave{n}$ un sénateur une dérogation lui permettant d'épouser une affrnnchie (553). Crmendant, la pratique peut parfois ]'emporter sur

548- L'interdiction est prévue par les Iois Iulia et Papia Fopproa votées sous Auguste : Paul D $23.2 .44 \mathrm{pr}$ (Lege Iulia ita canetur: Qui senator est quive filius nenosve ex filio proneposve ex filio nato cuius eormom est erit, ne quis eomum oponsam uxoremve sciens dolo malo habeto libertinam aut eam, quae ipsa cuiusve pater materve artem ludicram facit fecerit. Neve senatoris filia neptisve ex filio proneptisve ex nepote filio nato nats libertino eive qui ipse cuiusus pater materve nepote filio nato nata libertino eive qui ipse cuiusve pater materne quis eorum dolo malo eciens sponsan uxoremve eam habeto; Celsus D 23. 2.23 : Lege Fapia cavetur ommibus ingenuis praeter senatores eorumque
liberos libertinam uxorem habere licere; G. BOULVERT, DEF, cit., p. 283, liberos libertinan uxorem habere licere; G. BOUL
n. 138 ; M. MESLIN, L'horme romain, cit., p. 154 .

549- Modestin D 23.2.42.1 : Si senatoris filia neptis proneptis libertino vel qui artem ludicron exercuit cuiusve pater materve id fecerit moserit, nuptiae non erunt.

550- Marcellus D 23.2.32 : Sciendum est libertirnm, qui se ingenuo dedit adrogandum, quamvis in eius fomilia ingenui iura sit consecutus, ut libertinum tanen a senatoriis nuptiis repellendum ssse.

551- Papinien D 1.9.9: Filiam senatoris nuptias liberti secutan patris casus non facit usorem : nom quaesita dignitas liberis propter casum patris remoti a senatu auferenda non est ; (- D 23.2.34.3). le droit : dans ce sens, un rescrit d'Antonin le pieux, rapporté par Marcien, éroque une affranchie qui épcuse un sénateur en lui cachant son véritable status (554).

D'autre part, si l'union légitime d'un sénateur et d'une affranchie est interdite, le concubinat, lui, est autorisé. Ulpien indique à ce sujet un sénateur vivant avec une affranchie sans que celle-ci soit sa ferme (555), et Sévère rappelle qu'un sénateur du nom de Pontius Paulinus avait pour concubine une affranchie (556).

On a distingué, lors de l'étude des unions ayant trait à des esclaves, le maître des autres hommes libres ; il convient d'en faire autant pour le patron.

\section{a) - L'union est nouée avec le patron}

Alors que seul le contubernium est possible pour les esclaves, les conjoints ont ici le choix entre une union juridique ou une simple union de fait.

Dans le premier cas, l'observateur moderne ne peut pas rester indifférent aux prérogatives exorbitantes dont jouit le patron. Bien que Marcien écrive qu'il ne puisse contraindre son affranchie à l'épouser (557), Ulpien admet cette possibilité s'il lui a précisément fait don de la liberté dans ce but (558). Paul estime même que celui qui oblige son affranchie à l'épouser par prestation de serment ne contrevient pas à 1a lex Aelia Sentia (559). Si les rapports sont clairs à

\section{2- Paul D 23.2.47.}

553- Ulpien D 23.2.31 : Si senatori indulgentia principis fuerit permissum libertinam iustam uxorem habere, potest iusta uxor esse.

554- Marcien D 23.2.58: A divo Pio rescriptw est, si libertina senatorem deceperit quasi ingenua et ei nipta est, ad exemplwm praetoris edicti daciam in eam actionem, quia ex dote nullum lucrom habet quas nulla est.

555- U1pien D 23.2.27 : Si quis in senatorio ordine agens libertinam habuerit uxorem, quamvis interim uxor non sit, attomen in ea condicione est, ut, si amiserit dignitatem, uzor esse incipiat.

556- U1pien D 24.1.3.1 : ... Divrus tamen Severus in Liberta Pontii Paulini senatoris contra statuit, quia non erat affectione uroris habita, sed magis concubinae.

557- Marcien D 23.2.28: Invitam libertan uxorem ducere patronus non potest.

558- U1pien D 23.2.29: ... quod et Ateius Capito consulatu suc fertur decrevisse. Hoc tomen ita observandum est, nisi patronus ideo eam manomisit, ut uxorem sam ducat.

559- Paul D 37.14.6.3 : Si patronus likertam iureiuranto alegerit, ut 
propos du mariny?, ils ne le sont pas moins ì propos lu divorce.

Ainsi, 1r patron doit conantir à ce que son affranchie le quit,te (560).

S'il y a rupture de fait, sans le consentement du natron, la femme ne pourra pas róclamer la dot; en outre, elle ne pourra pas contracter un nouveau mariage tent que le patron n'aura pas consenti au divorce (561). Il existe cependant, une exception à cette règle, celle de l'osclave arfranchie par fidéicommis (562).

Trente textes concrinent ce problème (563). Seuls quatre font. allusion à une affranchie concubine de son patron (564), cette seconde

sibi nuberet, si quidem ducturus eam adegit, nihil contra legem fecisse videbitur. Cependant, si le patron obtient de son affranchie ce serment dans le seul but de l'empêcher de se marier a un autre, il contrevient a la lex Aelia Sentia de la même manière que $s^{\prime}$ il lui avait fait promettre de ne pas se marier; c'est ce que nous apprend la suite du passage : si vero non ducturus propter hoc solum adegit, ne ali $i$ nuberet, fraudem legi factom Iulianus ait et perinde patronum teneri, ac bi co jisset iurege facton Iulianus ait et pexinde patronm teneri, ac oi coegibset iurare libertas non naptur et marier, Julien D 38.2.24; T. Clemens D 40.9.31 (Julien) (le patron qui oblige son affranchie promettre de ne pas se marier tant que ses enfants seront impubères ne contrevient pas à la lex Aelia Sentia car il ne lui impose pas une viduité perpétuelle); $D 40.9 .32 \mathrm{pr}$; Paul D $37.14 .6 \mathrm{pr}-2-3$ (Julien) -4 ; D 37.14.15; Ulpien D 2.4.8.2; D 38.16. 3.5.

560- U1pien D 38.11.1.1 : Liberta ab invito patrono divortit : lex Iulia de maritondis ordinibus retinet istam in matrimonio; dans le même sens, D 23.2.45 pr-5.

561- Julien chez Ulpien D 24.2.11 pr. Elle ne pourra même pas être la concubine d'un autre :... Iulianus quidem amplius putat nec in concubinatu eam alterius patroni esse posse. Si, par contre, elle vivait seulement en concubinage avec son patron, elle pourra le quitter pour avoir le même type de relation avec un autre, mais elle, ne pourra néanmoins pas se marier avec celui qu'elle aura choisi, cf. Ulpien D $25.7 .1 \mathrm{pr}$.

562- Marcellus D 23.2.50 : Proxime constitutum dicitur, ut, cum quis libertaon suan duxerit uxorem, quam ex fideicommissi causa manumiserit, liceat libertce invito eo mptias contrahere : puto, quia non erat ferendus is qui ex necessitate manumisit, non suo arbitrio: magis enim debitam libertatem prasstitit quam ullum beneficium in mulierem contulit ; dans le même sens, Modestin D 24.2.10.

563- Celsus D 40.2.19; Valens D 38.1.46; T. Clemens D 23.2.48.1; Gaius D 23.2.46 (Iavolerius); Marcellus D 23.2.50; Paul D 37.14 .6 .3 (Julien); D 40.2.15.4; D 40.12.39.3; U1pien D 12.2.16 ; D 23.2.29; D 23.2.45 pr (rescrit de Sévère et Caracalla)-1-2-3-4-5-6 (Julien) ; D 24.2.11 pr (Julien)-1-2 (rescrit de Sévère et Caracalla) ; D 38.11.1. 1 ; D 40.2.20.2 ; L. Rufinus D 23.2 .51 pr ; Marcien D 23.2.28 ; D 24.3. 35 ; D 40.5.5i.12 ; Modestin D 24.2.10 ; D 40.9.21 (Julien); Hermogénien D 24.1.62.1. Dans D 23.2.13 de Ulpien, c'est une patronne qui épouse son affranchi.

564- Valens D 38.1.46; Paul D 25.7.2; Ulpien D 25.7.1 pr-3. hypothèse soulevant woins de fifficultss juridiques. Devx f'entre elzx móritent nóanmoins d'être signalás. Dans le fremier, teull ácrit, que l'affranchie doit demeurer la concubine de son patrcn, wême si celui-ci est atteint de démence (565). Dans le second, Jlpien remarque que l'arfranchie, qui a été la concubine de son patron, ne peut, sous peine d'inceste, avoir des relations sexuelles arec le fils ou le petit-fils de celui-ci (566). La solution est intéressante en ce que l'affranchie, lors des relations punissables, n'est plus unie au patron.

\section{b) - autres cas}

Une afrranehie peut $s$ 'unir légitimement ì un parent de son patron, son fils par exemple (567). Inversement, un affrenehi peut semblablement épouser une patroni cognata (568). Papinien estime tartefois qu'il y a de l'indécence pour une femme à épouser l'affranchi de son défunt mari (569).

Des unions sont aussi contractées en dehors de la famille du patron. On remarque toujours la même dualité, mariage (570) ou concubinat (571). Le mariage entre ingénu et affranchie peut avoir une origine antérieure. Il peut, par exemple, avoir été contracté alors que la femme était encore esclave et ignorait son status (572). De nombreux textes traitant d'unions matrimoniales ne spécirient pas si le partenaire est affranchi ou bien ingénu (573). Il n'a donc pas été possible

\section{5- Paul D 25.7.2.}

566- U1pien D 25.7.1.3.

567- T. Clemens D 23.2.48 pr-2 ; L. Rufinus D 23.2.51.1 (Julien).

568- Ulpien D 38.16.3.5.

569- Papinien D 23.2.62.1.

570- Proculus D 23.3.67; Celsus D 23.2.23; D 23.2.64 pr Callistrate ; Paul D 23.2.37; D 23.2.66.1; U1pien D 47.10.11.7 (Parcellus)-8.

571- Marcellus D 23.2.41.1; v1pien D 24.2.11 pr (Julien) ; D 25.7. 1 pr ; Marcien D 25.7.3 pr.

572- Proculus D 23.3.67.

573- Celsus D 38.1.30.1 ; T. Clemens D 38.1 .14 ; Marcellus D 23.2. 50 ; D 23.2.59.2 ; Papinien D 23.3.69.6; D 48.5.39.9; Paul D 37.14. 6.3 (Julien) 4 ; D 38.1 .28 ; U1pien D 23.2 .45 pr (rescrit de Sévère et Caracalla)-4-6 (Julien) ; D 24.2.11 pr (Julien); D 25.7.1 pr ; D 38. 1.13.3-4-5; Hermogénien D $38.1 .48 \mathrm{pr}-1-2$. Les textes codés dans la relation patron/affranchi concernent la faculté, ou son refus, pour relation patron/affranchi concernent la faculté, ou son refus, pour c'est pourquoi la référence a été reprise ici. 
de les intégrer dans l'analyse quantitative qui va suivre. A propos du concubinat, il faut relever un texte de Marcien pour la mentalité qui s'en dégage :

In concubinatu potest esse et aliena liberta et ingenua et maxime ea quae obscuro loco nata est vel quaestum corpore fecit. Alioquin si honestas vitae et ingenuan mulierem in concubinatum habere maluerit, sine testatione hoc manifestum faciente non conceditur (574),

ce qui signifie : on peut avoir pour concubine l'affranchie d'autrui, et même une ingénue si elle est de basse naissance ou prostituée. Si, par contre, on veut faire d'une femme de bonnes moeurs, honestae vitae, sa concubine, il est nécessaire de le déclarer devant témoins, car, poursuit le juriste, on ne peut qu'épouser une telle femne ou la déshonorer. Mais peu importent ces derniers motifs. $L^{\prime}$ assimilation de I'affranchie à une femme de mauvaise vie ne manque pas d'intérêt.

$$
-
$$

Conscient désorrais des différents types d'unions qu'esclaves et affranchis peuvent nouer, on peut tenter d'en faire une analyse quantitative à travers les cas que nous livre le Digeste.

II n'est pas possible de poser la question : esclaves ou affranchis s'unissent-ils plus fréquemnent à I'intérieur de leur groupe qu'en dehors $?$ Tout du moins, pas dans ces termes, le Digeste

n'étant pas en ce domaine un reflet obligatoirement fidèle de la réalité sociale. On peut simplement se demander si le droit prend flus en considération, en fonction de l'époque, la première hypothèse ou la seconde. Les citations par renvoi, trop peu nombreuses pour être représentatives, ont été écartées. On n'a pas tenu compte non plus des textes traitant de I'interdiction du mariage entre affranchis' et membres de I'aristocratie sénatoriale, étant donné leur aspect strictement négatif. Les chiffres correspondent bien entendu aux réferences précédemment données en note.

$574-025.7 .3 \mathrm{pr}$ (suite) : ... Sed necesse est ei vel uxorem ean habere vel hoc recusantem stuprum cum ea comittere.
Par rapport au nombre total de paragrapbes traitant d'unions d'esclaves ou d'affranchis, par périodes, on obtient :

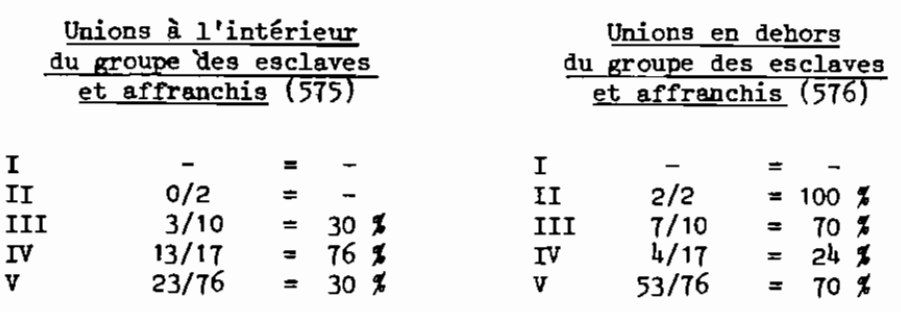

On relève une prépondérance des unions nouées en dehors du groupe des esclaves et affranchis, à l'exception toutefois de la période IV. L'intérêt de cette période pour les unions contractées à I'intérieur du groupe tient moins, semble-t-il, à l'importance croissante de la reconnaissance des liens familiaux serviles par le droit qu'au fait que les paragraphes la représentant sont pratiquement tous de Scaevola, juriste s'intéressant particulièrement à de telles unions. On remarquera par ailleurs que les pourcentages obtenus par les périodes III, correspondant aux règnes des premiers Antonins, et V, époque des Sévères, sont identiques.

La suprématie, dans le Digeste, des unions extérieures au groupe s'explique aisément. Si la reconnaissance des liens conjugaux serviles est en soi problématique, celle des unions noués par des dépendants à l'extérieur de leur groupe l'est encore davantage.

Une analyse plus détaillée montre que ce sont les affranchis qui ont le plus tendance à s'unir en dehors du groupe. Les seuls pourcentages qui démentent cette tendance reposent sur un nombre de textes trop faible pour ètre fiable. Ce besoin de nouer des relations avec des ingénus, ou ingénues, est compréhensible. Dans la mesure où il n'existe pas d'union contractée avec un ou une esclave antérieurement à I'affranchissement, I'affranchi (e) doit ressentir la nécessité de se débarrasser, au moins đans les apparences, de ses antécédents serviles.

575- C'est-a-dire : entre esclaves ; entre affranchis ; entre esclaves et affranchis.

576- C'est-à-dire : entre esclaves et ingênus ou ingénues ; entre affranchis ou affranchies et ingénues ou ingenus. 
Unions ayant trait ì des esclaves

\begin{tabular}{|c|c|c|}
\hline- & & \\
\hline$\overline{1 / 3}$ & & \\
\hline $\begin{array}{l}13 / 16 \\
14 / 23\end{array}$ & (5T7) & \\
\hline
\end{tabular}

\section{- en dehors}

$\begin{array}{llll}\text { I } & - & & - \\ \text { II } & 1 / 2 & & 50 \% \\ \text { III } & 1 / 7 & & =14 \% \\ \text { IV } & 2 / 4 & & =50 \% \\ \text { V } & 14 / 53 & & =26 \%\end{array}$

\section{- à l'intérieur du groupe}

Unions ayent trait a des afrranchis

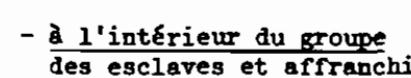

des esclaves et affranchis

$\begin{array}{llll}\text { I } & - & = & - \\ \text { II } & - & = & - \\ \text { III } & 2 / 3 & = & 7 \% \\ \text { IV } & 3 / 16 & =19 \% \\ \text { V } & 9 / 23 & = & \end{array}$

- en dehors

$\begin{array}{lcl}\text { I } & - & =- \\ \text { II } & 1 / 2 & =50 \% \\ \text { III } & 6 / 7 & =86 \% \\ \text { IV } & 2 / 4 & =50 \% \\ \text { V } & 39 / 53 & =74 \%\end{array}$

Si le Digeste est une source importante de renseignements en ce qui concerne la pratique sociale au niveau de l'individu (578), il est en revanche paurre si l'on passe à l'étude de cette pratique au niveau de la société.

\section{SECTION 2 - LA PRATIQUE SOCLALE AU NTVEAU DE LA SOCIETE}

Les usages funéraires sont en relation avec la vie religieuse, et, sur ce point, le droit admet une capacité de l'esclave (579). L'endroit orl est enterré un esclave est ainsi religieux (580). Il n'est donc pas étonnant de rencontrer des esclaves parmi les membres des

577- La relation conjugale entre esclaves et affranchis concernant les deux rubriques, il allu la dédoubler, ce qui fait passer le total de la période IV de 13 a 16 (cf. Scaevola D 32.41 .2 ; D $34.1 .20 \mathrm{pr}-1$ ).

578- D'autres thèmes n'ont pas été envisagés dans cette rubrique car ils le seront plus loin : ainsi. I'héritage, les marques et objets de répression.

579- H.H. BUCKLAND, Roman law, cit., p. 74.

580- UIpien D $11.7 .2 \mathrm{pr}$ : Locwon in quo serrus sepultus est religiogion esse Aristo ait. On trouve par ailleurs des esclaves associés a des pratiques religieuses, tels ces esclaves préposés à la custodi (Papinien D 40.12.35) ou a la custodia de môme, des femple; d'autres des affranchis servent dans un temple (Scaevola D 33.1.20.1), d'autres doivent célébrer chaque année I'anniversaire de la mort de
celui qui leur a donné la liberté (Scaevola D 34.1.18.5). collegia, orgenisues se présentant principalement'sous l'aspect d'associations funéraires et correspondant au besoin de l'individu d'échapper a l'isolement (581).

Servos quoque licet in collegio tenuionum recipi volentibus dominis, ut curatores horem corporwon sciant, ne invito aut ignorante domino in collegion teruiomm reciperent, et in futumm poena teneantur in singulos homines aursorm centum (582).

Les esclaves ne peurent cependant entrer que dans les collèges de personnes paurres, temriorss, et seulement avec le consentement de leur maitre. Marcien, qui est l'auteur du texte, poursuit : les responsables de ces organismes, curatores horm corporm, encourent une amende de cent ourei par esclave admis à l'insu du maître ou malgré lui. Il s'agit d'une amende très lourde si on considère que le prix d'un esclave est, en moyenne, de l'ordre de vingt aurei (583). Ce taux élevé s'ex-plique par un besoin de protection de la puissance dominicale sur l'esclave. Les maîtres hésitent à laisser aller leurs esclaves aux réunions du collège, ils prérèrent les garder chez eux sous leur contrôle. On retrouve là une crainte du même ordre que celle qui ressort de l'emploi du vocable familia (584). Reconnaître à l'esclave la possibilité d'avoir une vie religieuse est une chose, mais il n'est pas question que cette vie religieuse engendre une quelconque pratique politique, surtout lorsque celle-ci risque d'être subversive. En d'autres termes, la religion ne semble acceptable que dans la mesure où elle détourne du politique.

Cette crainte est une des raisons du faible nombre d'esclaves faisant partie de collèges, mais il en existe d'autres. Certains collèges n'acceptent pas d'esclaves parni leurs membres (585). Il faut en outre écarter les esclaves ne disposant pas des moyens financiers nécessaires,

581- P. PETIT, La paix romaine (1971), p. 182. Alors que sous Ia République, les collegia représentaient une force politique active, ils deviennent sous 1 'Empire une arme de pression morale de la part de I' $\mathrm{B}$ tat, cf. E.M. SCHTAERMAN, La schiavitu, cit., p. 104.

582- Marcien D 47.22.3.2.

583- Papinien D 40.4.47 et Ulpien D 5.2.8.17 (citếs supra n. 141 première partie).

584- Cf. Supra analyse terminologique.

585- Par exemple, CIL, VI, 10234. 
le versement initial et les dépenses liées à la fonction de magistrat étant très lourdes (586).

$$
-0_{-}^{-}
$$

Il résulte de cette analyse de la pratique sociale, tant au niveau de l'individu que de la société, que l'idée centrale est celle d'une discrimination opérée au sein du groupe servile : discrimination alimentaire, vestimentaire, conjugale, familiale et enfin religieuse. Il s'agit là d'une confirmation de l'analyse économique : seuls peuvent mener une vie "correcte", à tel point qu'on les confond parfois avec des ingénus, les esclaves privilégiés. Ce sont surtout ces derniers que $l$ es juristes prennent en considération.

$$
-0_{-}
$$

\footnotetext{
586- E.M. SCHTAERMAN, La schiavitù, cit., p. 103.
}

\section{CHAPITRE II - ESCLAVAGE ET DROIT}

Quelle est la place de l'esclave dans les opérations juridiques ? Quelles sont les sanctions du droit à son égard ? Voilà les questions auxquelles on doit maintenant tenter d'apporter une réponse.

\section{SECTION 1. LA PLACE DE L'ESCLAVE DANS LES OPERATIONS JURIDIQUES}

L'esclave apparaît-il plus souvent comme objet ou bien comme agent de droit ? La réponse, quelle qu'elle soit, implique une analyse des actes et des faits juridiques.

\section{Paragraphe 1 : Esclave et actes juridiques.}

L'esclave peut aussi bien être objet qu'agent d'actes juridiques.

\section{I - L'esclave objet d'acte juridique}

De nombreux textes concernant ce problème ont été cités à propos des formes d'acquisition et d'exploitation de la main-d'oeuvre servile. Il s'agit des textes traitant de la vente (587), de l'échange (588), de la stipulation (589), des legs et fidéicommis (590), de la donation (591), de la dot (592), du commodat (593), du louage (594), de l'usufruit (595) et de l'usage (596).

587- Cf. supra n. 3, première partie.

588- Cf. supra n. 5, première partie

589- Cf. supra n. 6, pretrière partie.

590- Cf. supra n. 7, première partie

591- Cf. supra n. 8, première partie.

592- Cf. supra n. 9, première partie.

593- Cf. supra n. 19, première partie.

594- Cf. supra n. 22 et 23 , première partie.

595- Cf. supra a. 24, première partie. 
Outre ces opérations, l'esclave peut encore être l'objet de droits ou d'actions juridiques : copropriété (597), action en

\section{6- Cf. supra n. 25, premiare partie.}

597- On relève un eaclave commun chez Alfenus (chez auteur anonyme) D 10.3.26 ; Iavolenus D 29.2.64; D 33.8.22.1 (Labéon. Trebatius); Celaus D $3 i .20$ (Celsus p., Proculus) ; Julien D 9.4.39 pr ; D 9.4.41;

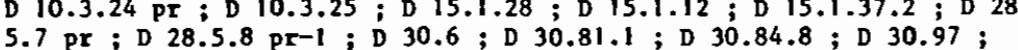

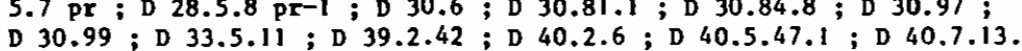
I ; D 40.12.30 (Sabinus, Cassius) ; D 41.1.37.1-2-3-6; D $41.1 .54 \mathrm{pr}-1$; D 45.3.1.2-4-6; D 45.3.10; D 46.1.16 pr ; D 46.3.34.1-10 ; Pomponius D 17.2.60.1 (Labéon); D 21.2.34.1; D 24.1.31.3; D 32.74 ; D 33.5.8.2 ; D 38.1 .4 (Aristo); D $38.1 .8 \mathrm{pr}$ (Labeon); D 40.7.8.1 (Labéon) ; D 45.3.6 (Ofilius, Sabinus, Cassius) ; D 45.3 .17 ; D 45.3.37 ; Africain D 10.3.9; D 40.5.49; D 47.2.62 pr ; Gaius D 6.1.76.1; D 10 . 3.11; D 15.1.27.8 (Julien); D 41.1.15; D 45.3.8; D 45.3.28.1-2-3; Venuleius D 45.3.21 (Julien); Marcellus D 15.1.16 (Julien); D 26.8.12 Venuleius D 45.3.21 (Julien); Marcellus D 15.1.16 (Julien) ; D 26.8.12

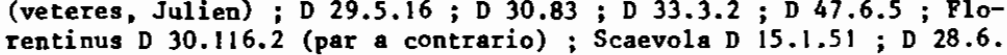
$48 \mathrm{pr}$; D 41.9.3 ; D 45.3.19; Papinien D 23.3.69.9; D 29.7.11; D 29.7.12 ; D 33.8.19.2 ; D 40.4.48 (Servius); D 45.3.18 pr-1-2 (resCrit d'Hadrien) ; Tryphoninus D 41.1.63.1-2 ; D 49.15.12.13; Paul D 4.9.6.1; D 7.9.10; D 8.3.19; D 9.4.9; D 9.4.10; D 9.4.17 pr ; D 9.4.26.1-2-6; D 9.4.31 (Julien) ; D 10.3.8.2-3-4; D 10.3.15 ; D 10. 3.19 .3 ; D 10.3.27; D 10.3.31; D II.1.20 pr; D 11.3 .14 .2 ; D 14.1 . $5 \mathrm{pr}$; D 14.1.6.1; D 15.1.20;D 15.1.47.2 ; D 15.3.14 (Julien, Marcellus) ; D 15.4.5.1 ; D 17.2.56; D 21.1.44.1 ; D 24.1.38 pr (Alfenus) ; D 28.5.90; D 29.2.65 (suite de Iavolenus D 29.2.64) ; D 29.2 68 ; D 29.5.6.2 ; D 30.5.I (Trebatius, Labéon, Cassius) ; D 32.99.5; D 35.1 .44 .8 ; D 35.2.49 pr (Cassius, rescrit d'Antonin le pieux) ; D 39. 3.11.3 (Julien); D 40.2.4.2 (Julien) ; D 40.2.15.4; D 40.7.22 pr-1; D 40.9.16.1; D 41.2.1.7-18; D 45.3.12; D 45.3.27; D 45.3.29 ; D 47 . 10.16 ; D $48.10 .14 \mathrm{pr}-1$; Ulpien D 7.1.25.4; D 7.2.1.1. (Julien) -2 (Julien); D 9.1.1.14; D 9.2.19 (Celsus); D 9.2.20; D 9.2. 27.1 (Proculus, U. Ferox) -2 (Celsus) ; D9.4.2.1 (Celsus, Julien, Marcellus); D 9.4.5 pr ; D9.4.8; D 10.3.6.4 (Sabinus, Atilicinus)-9 (Julien) ; D $11.1 .17 ;$ D 11.3 .9 (Julien) ; D 12.1 .13 .2 (Papinien); D 12.2.26. 14 (Celsus); D 14.1.4.2; D 14.3.13.2 (Julien); D 14.4.3 pr ; D 14.4 . 14 (Celsus) ; D 14.1.4.2 ; D 14.3.13.2 (Julien) ; D 14.4.3 pr ; D 14.4 .
5.10 ; D 15.1.7.1 (Julien, Marcellus); D 15.1.11.9 (Julien); D 15.1.

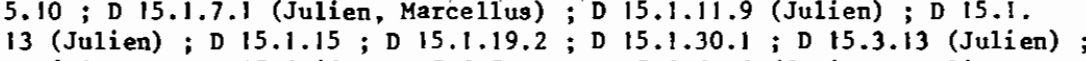
D 16.3.1.31; D 17.2.49; D 17.2.51.1; D 17.2.63.9 (Sabinus, Julien, Pomponius) ; D 21.1.31.5 (Pomponius)-6 (Pomponius)-7 (Marcellus) -8 (Marcellus) -10 ; D 28.5.6.3 ; D 28.6.18 pr (Julien) ; D 28.7.2 pr D 28.8.1 pr ; D 29.2.66; D 29.2.67; D 29.5.1.6 ; D 29.5.3.4; D 30.50 pr ; D 32.68.2 ; D 33.8.6.3 ; D 35.1.50 (Antonin le pieux) ; D 36.1. $17.10 ; \mathrm{D} 36.2 .23$; D 38.1 .15 .1 (Celsus) ; D 38.2.16.1 ; D 39.5.13;D 40.2.3; D 40.5.30.6 (rescrit d'Antonin le pieux); D 40.12.8.1; D 41 1.17 ; D 41.1.23.3 (Julien, Scaevola) ; D 41.2.42 pr ; D 45.3.2 ; D 45. 3.4 ; D 45.3.5 ; D 45.3.7 $\mathrm{pr}^{-1}$ (Julien) ; D 45.3.9 $\mathrm{pr}^{-1}$ (Cassius, Julien) ; D 45.3.11 (Julien); D 45.3.13; D 46.3.29; D 46.4.8.1-2 (Labéon) : D 47.2.46.5; D 4.7.10.15.36-49;D 47.10.17 pr-9 ; D 48.5.28. 4-11; D 48.18.1.7 (par a contrario); D 48.18 .3 (constitution de Sévèré et Caraca11a); Marcien D 1.8.6.1 ; Hermogênien D 35.2.38.1. Sur le thème, voir notamment M. BRETONE, Serrus communis, Contributo revendication (598), restitution (599), confiscation (600), possession (601), traditio (602). Il peut aussi faire l'objet d'une actio ad

alla storia della couproprietă romana in etă classica, Naples (1958) un płoblème particulier est posé par l'affranchissement d'un esclave commun ; cet affranchissement n'est valable a l'epoque classique que s'il est effectué par tous les copropriétaires de 1 'esclave (cf. M. BRETONE, op. cit., p. 140 s. ; A. BERGER, Encycl. Diction., cit., p. 577), sans quoi est applique le ius adcrescendi, c'est-a-dire que la part, dans l'esclave, de ceux qui n'ont pas consenti a sa biberts se trouve accrue (cf. A. GUARINO, Diritto privato romano, cit., p. 487 ; A BERGER, Encycl. Diction., cit., p. 526 ; plus généralement, $\nabla$. LEON-' HARD, RE 10 ; HUNBERT, DSDA 3 ; P. BONFANTE, Scritti giuridici, 3 (1926),
P. 434 ; J. MACQUERON, RHD 8 (1929), p. 580 ; VACCARO-DELOGU, L'accresciP. 434 ; J. MACQUERON, RHD 8 (1929), P. 580 ; VACCARO-DELOGU, L' accre
mento nel diritto ereditario (1941) ; U. ROBBE, IUs adcrescendi e la sostituzione volgare, (1947) ) C'est seulement à l'Epoque post-classique, par une poussee importante du favor libertatis, que le consentement d'un seul des copropriétaires suffira a $l^{\prime}$ affranchissement d'un esclave commun (cf. M. DE DOMINICIS, Mammissio (dir. rom.), NNDI, $X$, (1964) p. 195 ; Paul sent. 4,125: Cormmem sererm unus ex soci is vinciendo futurae libertati' non nocebit).

Bien qu'il ne s'agisse plus d'un rapport de propriété, un affranchi peut egalement être comern : ansi s'il est affropriete, un affranmaîtres ou assigne plusieurs enfants du patron, cf. Iavolenus $D 38.2$. 34 ; Julien D 38.1.23.1 ; D 38.2.21; D 38.2.23.2; D 38.2.24; D 45.1 54.1 ; Pomponius D 38.1 .4 (Aristo); D $38.1 .8 \mathrm{pr}$ (Labéon); D 38.2: $2 \mathrm{pr}$; D 38.4.2; D 38.4.4; D 38.4.12 ; Gaius D 23.2.46 (Iavolenus); D 38.1 .49 (Neratius); T. Clemens D 23.2.48 pr; D 38.4.10 pr-1; Scaevola D 34.1.16.3 ; Papinien D 40.4.48 (Servius); Paul D 38.1 . 28 ; D 38.1.51 ; D 40.2.15.4; Ulpien D 26.4.3.4-5-6-7; D 26.4.5.4; D 38.1.15.1 (Celsus) ; D 38.2.10 pr ; D 38.4.1.1-7 (Marcellus) -8 (Julien); D $38.4 .3 \mathrm{pr}-7$; D $38.4 .5 \mathrm{pr}$ (Julien) -1 (Julien) 2 ; D 38.5 . lien); D 38.4.3 pr-7; D 38.4 .5 pr (Julien)-1 (Julien) $-2 ;$ D 38.5 . 30.4 ; D 40.12 .5 .1 ; Macer D 48.5.25 pr ; Marcien D 2.4.23; Hermogénien D 37.14 .21 .1 .

598- Pomponius D $6.1 .29 ; D$ 44.2.21.2; Gaius D 4.7.3.1; D 6.1 .18 ; D $6.1 .20 ;$ D $6,1.28 ; 06,1.30 ;$ Callistrate D 48.18 .15 .2 (rescrit d'Antonin le pieux); Paul D 6.1.6; D 6.1.16 pr ; D 6.1.21 (Julien, Pomponius) ; D 6.1.27.2-4-5; D 6.1.31; D 6.1.36.1; D 6.2.12.5; D 12 . $1.31 \mathrm{pr}$; D 46.7 .11 ; Ulpien D 6.1.5.5 (Pomponius) ; D 6.1 .13 (Labéon) ; D 6.1 .15 pr (Labéon) -3 ; D 6.1 .17 pr (Julien)-1 (Julien); D 6.1.22 ; D 44.2.7.1-3; D 44.2.11.1 (Celsus) $-4-8$; D 44.4.4.7; Modestin D 6.1. 32. Alors que la vindicatio est une action in rem (A. BERGER, Encycl. Diction., p. 766 ; Gaius, Inst. 4, 5), 1a condictio (cf. Julien D 13. $1.14 \mathrm{pr}$; Papinien D 13.1.17 ; Paul D 13.1.3 (Julien) ; D 13.1.13 ; Ulpien D 13.1.7.2; D 13.3.3) est une action in personan (A. BERGER, Encycl. Diction., p. 405; Gaius, Inst., 4, 5; 17); sur cette distinction, voir anssi A. GUARINO, Diritto privato romano, sur cete distinc tion, voir aussi A. GUARINo, Diritto privato romano, cit., p. $211 \mathrm{~s}$.; cf. SEGRE, Sulla distinzione delle actiones in rem e in personam estranee al ius civile nel diritto romano classico, in RAL (1929)

599- Papinien D 4.8.42 ; D 22.1.2 ; Tryphoninus D 43.16.19 (Julien); Ulpien D 4.2.12 pr; D 10.4.9.7 (Sabinus, Pomponius); D 12.2.11.1.

600- Papinien D 48.18.6 pr ; Ulpien D 48.5.28.11-12-13-14; Marcien D 39.4 .16 pr-1 et 2 (rescrit de Sévère et Caracalla)-3; D 39.4.16.4 
et 9 (rescrit d'Antonin le pieux) ; D 39.4.16.10 (rescrit de MarcAurēle et Verus).

601- La propriêté peut être acquise par une possession prolongée, cf. R. HONIER, Droit romain, I, cit., p. $421 \mathrm{~s}$, Les textes parlent le plus souvent d'usucopio, mais on trouve aussi praescriptio chez Modestin D 44.3.3 (Longae possessionis praescriptionem tam in praediis quam in mancipiis Locwn habere manifestum est), bien que ce deuxiême terme concerne généralement les res inmobiles, cf. A. GUARnNo, Diritto privato romano, cit., p. 625 ; sur $1^{2}$ accessio possessionis, cf. D.44.3.14. $2-4$; A. GUARINO, op. cit., p. 627. Il est question de possession d'un esclave, dans le Digeste, chez Iavolenus D 41.3 .19 ; D 47.2.75; D 49 . 15.27 (Trebatius, Ofilius, Labẻon); Valens D 36.4 .15 ; Julien D 1.5 . 26 ; D 10.4.8; D 41.1.37 pr ; D 41.2.38 pr; D 41.3.33 pr ; D 41.3. 35 ; D 41.4.7.7; D 41.4.8; D 41.4.9; D 41.4.10; D 43.33.1 pr; D 45.3.1.1; Pomponius D 15.1.23; D 17.1.47.1 (Julien); D $41.1 .21 \mathrm{pr}$ (Proculus) ; D 41.1.54.4;D 41.3.30 pr;D 41.4.6.1-2; D 41.10.3 (Neratius) ; D 41.10.4 pr; D 43.26.15.4; D 44.7.56; D 45.3.39; AfriCain D 9.4.28; D 12.1.23 (Julien); D 12.1.41; D 41.1.40; D 41.2. 40.2-3; D 44.3.6.1; Gaius D 9.4.13; D 41.1 .10 pr-4-5; D 41.1 .43 pr ; D 41.2.15; Venuleius D 41.1.66; Marcellus D 17.1.49; Scaevola D 41. 6.5 ; D 41.9.3; D 44.3.14.2-4; D 45.3.19; Papinien D 15.1.50.3; D 17.1.57; D 41.2.44 pr ; D 41.2.47 (Nerva f.) ; D 41.2 .48 ; D 41.2 .49 pr ; D 41.3.44.2 ; D 45.1.118 pr ; Tryphoninus D 49.15.12.8-9 (veteres) ; Paul D 6.1.21 ; D 6.1.22 ; D 9.4.12; D 12.1.31.1 ; D 21.1.43. 3 ; D 21.1.44.2; D 21.2.56.3; D 22.1.10; D 35.2.63 pr (Pedius) ; $\mathrm{D} 40.7 .4 .4 ; \mathrm{D} 4 \mathrm{I}, 1.57$ (Julien); $\mathrm{D} 41.2 .1 .6-16$ (veteres) ; D 41.2 ; $3.10-13$ (Nerva f.) ; D 41.2 .5 ; D 41.2 .30 .4 ; D $41.3 .4 .5-15-16$ (Sabinus, Cassius) $-17-18$; D 41.3 .15 .1 ; D 41.4 .2 .6 (Neratius) -7 (Trebatius) ; D 41.10.2 ; D 43.16.6; D 45.3.20.1; D 45.3.31; D 45.3.33 pr (Julien)-1 ; D 46.3.60; D 46.4.11 pr ; D 46.7.11; D 47.2.54.4; D 48.10.22.4 ; Ulpien D 4.2.14.11; D 6.2.11.4 (Julien)-5 (Pomponius) ; D 7.1.25.6 ; D 9.2.17; D 9.4.11; D 9.4.21 pr-1 ; D 10.4.3.12 (Pomponius) ; D 10.4.5.6; D 10.4.9 pr (Julien); D 15.1.15; D 15.1.32 pr (Julien) ; D 15.4.1.8; D 24.1.19 pr (Julien) ; D 28.5.6.2 ; D 29.2.25 Pr ; D 29.5.1.2 ; D 32.73.1 ; D 39.4.12.2 ; D 39.5.18.2 (Pomponius) ; D 40.7.2 pr-3 (Julien); D 40.7.6.3; D 40.7.9.3; D 41.1.22; D 41.i. $23 \mathrm{pr}-1-2-3$ (Julien, Scaevola) ; D 41.2 .4 ; D $41.2 .13 \mathrm{pr}-2-3$; D 41.2 . 34.2 ; D 41.2.42 pr; D 41.3.10.2 (Marcellus, Scaevola); D 43.16. 1.46 (Vivianus) ; D 43.31.1.1; D 44.3.8; D 47.2.17.3 (Julien, Pomponius) ; D 47.2.48.5; D 47.8.2.14-18; D 47.10.15.47-48; D 48.5.28.9; D 48.18.1.8 ; Marcien D 40.5.55.1 (Marce1lus); D 48.15.3 pr (constitution de Sévère et Caracalla) ; Modestin $D$ 44.3.3 ; Hermogénien $D$ 36.4. $11 \mathrm{pr}$; D 41.2.50.1. Sur 1a distinction entre possessio civilis et possessio naturalis ou comporalis, et, au sein de cette dernière, la sous distinction entre possessio pro suo et posgessio pro alieno, $v$. A. GUARnNo op. cit, 467 s. La possession du précariste est une possescio pro atieno, pro alieno, cf. ibid., p. 469 n. 44.2 . esclave apparalt comme objet de precarivm chez Julien D 43.26.19.1; Pomponius D 43.26.10; D 43.26.15.4; Africain D 41.2.40.3 ; Paul D 9.4.22.1 ; Ulpien D 41.1 .22 ; D 43.31.1.1; D 47.2.14.11. Sur le thème, cf. A. GUARINo, op. cit., p. $850 \mathrm{~s}$.; ZAMORANI, Precorio habere (1969).

602- Ne sont relevés ici que les textes oũ tradere ou traditus constitue le seul critère d'intégration dans la rubrique "esclave objet d'acte juridique" : Pomponius D 40.5.34 pr ; Gaius D 17.1.27.1 ; Venuleius D 48. 8.6 ; Paul D 4.3.18.1 ; D 40.9.16.1 ; Ulpien D 12.4.5.1; D 28.5.6.2 ; D 40.7.6.3 ; D 44.2.11.1 (Celsus) ; D 44.4.4.7 ; Marcien D 40.5.53.1; exhibendion (603). On le troure quelquefois dans le pécule d'un fils de famille, prenant l'appellation de castrensis lorsque celui-ci est militaire (604), ou bien dans le pécule d'un servos ordinarius (605).

Il est question d'esclaves aliénés. La vocation juridique du terme aliénation étant anbivalente et pouvant aussi bien viser une vente (606) qu'une donation (607), il convenait de tenir également compte des textes ne rentrant dans aucune de ces deux rubriques (608). On

D 40.5 .54 ; D $40.5 .55 \mathrm{pr}$. On trouve chez ce dernier juriste transferendus, cf $\mathrm{D}$ 40.5.51.3. Sur la traditio comme mode de transfert de la propriété, cf. A. GUARINO, Diritto privato romano, cit., p. $624 \mathrm{~s}$. biblio., p. $593, \mathrm{n} .58 .12$ et p. 594 n. 58.12 .1 .

603- Paul D 2.9.2.1 ( $\nabla$. Verus, Julien, Pomponius) ; U1pien D 2.9 .5 ; D 10.4.3.7; D 10.4.5.6; D 10.4.11 pr-1 (Labéon). Sur 1'actio ad exhibendum, v. A. GUARINO, Diritto privato romano, cit., p. 600 ; MARRONE, Actio ad exhibendion, in AUPA (1957) ; BURILL0, Contribuciones al estudio de la actio ad exhibendum en derecho clasico, in SDHI 26 (i960) al estudio de la actio ad exhibentron en derecho clasico, in silleurs, il p. $190 \mathrm{~s}$. ; A. BERGER, Encycl. Diction., cit., p. 463. Par ailleurs,
est objet dans deux textes de la promesse faite par quelqu'un de le présenter en justice, cf. Paul D 2.11.7 ; Ulpien D 2.11.9.1 (Labéon); A. BERGER, op. cit., p. 708 .

604- On relève la présence d'un esclave dans le pécule de la fille chez Papinien D 6.1.65.1, ou du fils chez Alfenus (chez auteur anonywe) D 44.1 .14 ; chez Papinien D 33.8 .19 .2 ; Ulpien D 14.1 .1 .22 . Le pécule
de celui-ci est castrense chez Maecianus D $49.17 .18 \mathrm{pr}-3$; Papinien D 49. 17.14.1-2 ; D 49.17.15.3-4; Tryphoninus D 49.17.19.1-3-4-5; 01pien D 30.44 Pr ; D 40.9.30.2; D 47.2.52.4; D 49.17.6; D 49.17.9 (Marcellus). Sur le peculizm castrense, A. GUARINo, Diritto privato romano, cit., p. 501 s. ; FITTING, Das castrense peculiwn (1871) ; GUARINO, L'oggetto del castrense pecutium, in BIDR 48 (1941), p. $41 \mathrm{~s}$. ; LA ROSA, I peculi speciali in diritto romano (1953), et Ancora in tema di peculizom castrense, in St. De Francisci 2 (1956) p. $393 \mathrm{~s}$.; G. LONGO Sulla capacita patrimoniale e processuale del filiusfomilias fornito Sulla capacita patrimoniale e process
di peculio castrense, in AUMA (1957).

605- Cf. supra n. 622, première partie.

606- Par exemple D 30.91.3-5

607- Par exemple D 40.1.15.

608- Iavolenus D 29.2.62.1; D 32.29 .4 (Tubero, Labéon) ; Julien D 28. 5.7.1 ; D 28.5.38.4 ; D 29.7.2.2 ; D 30.6 ; D 30.102 ; Pomponius D 40 . 7.30 ; D 44.7.56; D 45.3.40 ; Africain D 15.3.17.1; Gaius D 15.1.27 2-3 (Julien); D 30.69.1; D 41.3.36.1; T. Clemens D 29.2 .82 ; $\mathrm{Mae}^{-}$ cianus D 40.4.58; D 40.5.32 pr ; Venuleius D 42.8.25.4-5 (Proculus); Marcellus D 35.1.47; D 40.5.9 ; Papinien D 31.66.3; D 40.1.20.3 ; D 40.7.36; Paul D 9.4.24; D 15.1 .26 ; D 33.8.1; D 33.8.3; D 33.8 . 4 ; D 40.4.39; D 40.5.29; D 40.5.31.2; D 47.10.29; Ulpien D 4.2.7.1; D 4.7.4.5; D9.2.15.1 (Julien) ; D 9.4.2.1 (Celsus); D 9.4.5.1; D 11 . 3.5 .4 ; D 15.1.32 pr (Julien) ; D 15.2.1 pr-1-3-4-5; D 16.3.1.30; D 16.3.11 (Sabinus) ; D 28.5.9.17-19-20; D 28.7.2 pr-I (Celsus) ; D 28. 7.4.1 (Julien) ; D 29.2.25.1 ; D 32.7 pr ; D 37.11.2.9 ; D 40.5.24.21 (rescrits d'lladrien et d'Antonin le pieux) ; D 40.7 .2 pr ; D 40.9.12. 
remarque encore des esclaves dans des successions (609). Il s'agit plus précisément de servi hereditarii en cas d'hersditas iacens, svant qu'ait en lieu $l^{\prime}$ adition d'hérédité $(610)$. On roit aussi des esclaves être objets de dépôt (611), de gage ou d'bypothèque (612). On relève

1-3-4-6 (Africain)-7; D 40.9.14 pr-4-5-6; D 47.2.17.1; D 47.4.1.1 (Labeon); Marcien D 35.1.33.4; D 40.5.51.1; D 49.14.30 (rescrit de Severre et Caracal la).

609- Il convient d'ecarter les esclaves affranchis par testament car ils n'apparaissent pas a proprement parler en tant qu'objets de droit. Trois textes correspondant a cette hypothèse peuvent néanmoins être integres: Iavolenus D 45.3.34 (un esclave est affranchi par testament, mais il 1 'ignore : in causa hereditaria maneret) ; Papinien D 35.2.11. $1-4$ (un esclave affranchi conditionnel lement par testament meurt avant 1a réalisation de la condition : il est toujours censé faire partie de 1a réalisation de la condition : il est toujours censé faire partie de la succession). Doivent par ailleurs être relevés dans cette rubrique :
Iavolenus D 38.2.36; Valens D 36.4.15; Julien D 19.1.13.5; D 44.7. 18 ; D 45.1.54 pr ; Pomponius D 21.1.48.5; D 21.2.30; D 40.7.8.1 (Labéon); Africain D 40.5.49; Gaius D 5.3.39 pr; D 35.2.73.3; Maecianus D 35.2.30 pr ; Scaevola D 34.3.31.1 ; Papinien D 10.2.31; D 10. 2.34 ; D 22.1 .6 pr (Caracal1a) ; D 29.2.86.2 ; D 35.2.9.1; D 45.1.117; Paul D 5.3.32; D 5.3.40 pr (Proculus, Cassius) -4 ; D 6.1 .6 ; D 10.2.11; D 10.3.31; D i1.3.8; D 18.4.21;D 21.2.41 pr; D 22.1.14.1 (Neratius); D $31.86 \mathrm{pr}$; D 35.2 .24 .1 ; D $38.2 .44 .2 ; \mathrm{D} 47.2 .86$; Ulpien D5. tius) ; D $31.86 \mathrm{pr}$; D $35.2 .24 .1 ; \mathrm{D} 38.2 .44 .2$; D 47.2 .86 ; Ulpien DS.

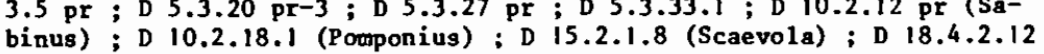
(Julien)-13 (Marcellus); D 21.1.31.5 (Pomponius)-6 (idem)-7 (Marcellus)9 (Pomponius); D 29,2.42.2-3; D 35.2.43; D 44.2.11.1: Marcien D 29.2.52 pr:

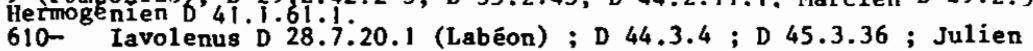
D 29.2.43; D 36.1.28.1; D 37.4.13 pr; D 44.7.16 ; Pomponius D 7.4.18; D $11.1 .15 \mathrm{pr}$; D 26.7.61; D 36.1.72.1; D 47.2.44.2 ; Gaius D 3.5.21 ; D 28.5.31.1; D 31.55.1; D 35.2.73 pr ; D 36.1.65.4 (Julien) ; D 45.2. 38.4 (Proculus, Cassius); Maecianus D $40.4 .55 \mathrm{pr}$; D 40.4 .58 ; Venuleius (Cassius) D 45.3.25 ; Florentinus D 30.116 .3 ; Scaevola D 18.5.8 ; Papinien D 4.4 .31 ; D 6.1.65.1; D 41.3.44.3; D 41.3.45.1; D 45.3.18.2; D 48 . 18.6.1 ; D 48.18.17.2 ; D 49.17.13 (rescrit d'Hadrien) ; D 49.17.14.1; Paul D 2.14.27.10; D 5.3.14; D 5.3.36.2 (Pomponius); D 10.2.15; D $10.2 .25 .15 ; \mathrm{D} 11.3 .14 \mathrm{pr} ; \mathrm{D} 28.5 .53 ; \mathrm{D} 31.82 .2 ; \mathrm{D} 36.1 .68 .2 ; \mathrm{D} 41$. $D 10.2 .25 .15 ; D 11.3 .14 \mathrm{Pr} ; \mathrm{D} 28.5 .53 ; \mathrm{D} 31.82 .2 ; \mathrm{D} 36.1 .68 .2 ; \mathrm{D} 41$
$2.1 .5-16$ (veteres) ; D $41.10 .2 ; \mathrm{D} 45.1 .73 .1 ; \mathrm{D} 45.3 .16 ; \mathrm{D} 45.3 .26$; D 46.4.11.2; D 48.18.18.4; D 49.15.29 (Labéon); U1pien D 6.2.9.6; D 46.4 .11 .2 ; D 48.18.18.4 ; D 49.15.29 (Labéon) ; U1pien D 6.2.9.6 ;
D7.3.1.2 (Julien) ; D 9.2.13.2 (Celsus) ; D 10.2.12.1; D 10.2.16.6 (Ofilius) ; D 11.3.13.1; D 15.1.3 pr ; D 16.3.1.29; D 28.5.6.2 ; D 28.7.4.1 (Julien); D 29.2.20.1; D 30.71.3; D 41.1.18; D $41.1 .33 \mathrm{pr}$ (Marcellus, Scaevola) -2 (Julien) ; D 42.6.1.12; D 47 . 10.1 .6 (Julien) 7 (Labéon) ; D 48.18.2 ; Marcien D 40.5.51.8; Modestin D $31.34 \mathrm{pr}$; D 45.3 .35 ; Hermogénien D $41.1 .16 \mathrm{pr}$. Sur le thème, W.W. BUCKIAND, Roman law, cit., p. $252 \mathrm{~s}$. ; sur le concept d'hereditas iacens, A. BERGER, Encycl. diction., cit., p. 486 ; A. D'AMIA, L'eredita giacente (1937) ; A. GUARINO, Diritto privato romano, cit.', p. dita giacente (1937); A. GUARINO, Diritto privato romano, cit.'P.

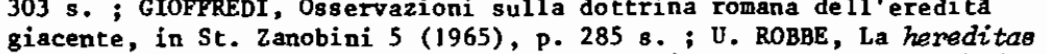
iacet e il significato della hereditas in diritto romano, 1 (1975) (XX + 616 p.), cr de P. LEUREGANS, in RHD (1978), p. 97-100. enfin quelques tertes, peu nombreux, ou un esclare est donné en soIutio (613), remis come caution (614), objet d'un constitut (615),

6I1- Africain D 13.7.31 (Julien) ; D 47.2.62.5-7; Gaius D 16.3.14.1 (Sabinus, Cassius) ; Paul D 9.4.22 pr ; D 41.4.2.7 (Trebatius); Vlpien D 2.14.50; D .16.3.1.5-9-10 (Pooponius)-24; D 16.3.7 pr ; Modestin D 16.3.23. Sur le servus depositus, W.H. BOCKLAND, Roman law, cit., p. 265 s. Sur le depositum, v. A. GUAR nNo, Diritto privato romano, p. 265 s. Sur le depositum, v, A. GUAR No, Diritto privato rowano, Deposito (storia), in ED.

612- Iavolenus D 41.3 .16 ; Celsus D 46.3 .69 ; Julien D 30.86 pr ; D 40.9.5.2 ; D 41.1.37 pr ; D 41.4.7.7; D 43.33.1 pr ; D 44.7.16 ; Pomponius D 13.7.8 pr-1 ; D 15.1.23; D 33.5.6; D 46.3.26; Africain D13.7.31;D 20.4.9 pr ; D 47.2.62.1-3-5; Gaius D 6.1 .18 ; D 9.4 .27 . pr-1 ; D 20.1.15 pr (Julien); D 20.4 .11 .3 ; D 40.9.29 pr ; Scaevola D 20.1.32 ; D 20.1.34.1 ; Papinien D 10.2.31; D 20.1.1.1 ; D 48.19.33; Tryphoninus D 49.15.12.12 ; Paul D 9.2.18; D 9.2.30.1; D 9.4.22.1-2; D 9.4.26.6; D $11.3 .14 .4 ; D 13.7 .25 ;$ D 20.1.29.1-3; D 20.2.9 (Nerva) D 20.6.10.1; D 21.1.43.8; D 21.2.35; D 40.1.3; D 40.2.4.2; D 40.9: 26 (Scaevola); D 41.2.1.15 (Julien); Ulpien D 4.3.9.4 (Labéon) ; D 9.2.17; D 9.4.36; D 10.3.6.9 (Julien); D 10.4.3.12 (Pomponius) ; D 13.7.24.3; D 20.1.6; D 20.1.8; D 20.1.21.1 (Julien) ;D 20.1.27(Marce11us);D20.2.6(Pomponius);D20.4.11.2;D20.6.4 pr;D21.1.17.11;D21.1.31.8(Marrollua):D28.5 . 30(rescrit de Sévère):D29.5.1.3:D30.44.7:D32.7.3.2:D40.5 .24.1n; D40.5.26.2(rescrits.d Antonin le pieux et de Sévère et Caracal1a):D40.5.45pr D 40.9 .4 ; D 40.12 .8 .2 ; D $43.32 .1 \mathrm{pr}$ (par a contrario) ; D.47.2.14 5 (Papinien)-6 (Pomponius, Papinien)-7 (les mêmes); D 47.2.46.4 (Africain); D 48.5.28.2; Marcien D 20.1.13.1; D 40.8.6 (constitution de Marc-Aurè1e) ; Modestin D 20.1.26.2 ; Hermogénien D 35.2.38.2 ; D 40.9.27.1. Sur le servus pigneraticius, W.W. BUCKLAND, Roman law, cit., p. 281 s. Sur pignus et hypotheca, cf. A. GUARINo, Diritto privato romano, cit., p. 680 s. ; PALMIERI, Ipoteca, (dir. rom.) in NNDI ; BOVE, Pegno (dir. rom.) in WNDI ; KUNKEL, Hypothesen zur Geschichte des rồmisPegno (dir. rom.) in chen pfandorech, in zss sidérés de manière unitaire, le pignus semble plus ancien que 1 'hypotheca, cf. A. GUARINO, op. cit.,p. 680, n. 70.1; A. BISCARDI, Die mehrfache Verpfändung einer Sache vom attische bis zum spätrömischen Recht, in zSS $86(1969)$, p. $146 \mathrm{~s}$.Sur $1^{\prime}$ attribution, par les compilateurs, au pignus de. textes concernant la fiducie, J. MACQUERON, Obligations, cit., p. 91. 613- Dans le sens large de libération du débiteur, A. BERGER, Encyci. Diction., cit., p. 710 ; cf. Paul D 41.3 .4 .17 ; D 46.1.58.1 ; D 46.3. 60 .

614- Africain D 17.1.37 : Hominem certum pro te dari fideiussi; sur la fideiussio, v. A. GUARINo, Diritto privato romano, cit., p. $758 \mathrm{~s}$.

615- Paul D 13.5.12; D 13.6.21. Le constitut est 1a convention par laquelle une personne, appelée constituant, s'engage, sans 1'emploí de fnrmes déterminées, à payer une dette préexistante, soit sa propre dette, soit celle d'autrui, R. MONIER, Droit romain, cit II, p. 200 sur le theme, A. GUARINo, Diritto privato romano, cit., p. 839 ; ASTUTI sur le theme, A. GUARnNo, Diritto privato romano, cit., p. 839 ; ASTU et Studi intorno alla promessa di pagamento, Il costituto di debito (1941) ; ROUSSIER, Le constitut (1958); GUIZZI, Constitutum debiti in NNDI ; FREZZA, Questioni esegetiche e sistematiche in materia di constitutrom debiti, in St. Funaioli (1961), p. $699 \mathrm{~s}$. ; RARADENIZ, Rlassik Roma Hukukunda constitutum debiti (1968). 
d'un compromis (616).

L'esclave apparaît aussi corme agent à propos d'actes très divers.

$$
\text { II - L'esclave agent d'acte juridique (617). }
$$

On voit intervenir l'esclave en matière de vente $(618)$, de lousge $(619)$, de société $(620)$, de mandat (621), de dépôt (622), de gage (623), de commodat (624), de donation (625), et surtout de stipulation

616- Paul D 4.8.32.5. Le compromis n'est pas un pacte à l'époque classique, c'est 1 'ensemble des stipulationes en vertu desquelles les parties $s^{\prime}$ engagent à payer une amende si elles ne se soumettent pas a l'éventuelle condamnation de l'arbitre. Il devient un pacte légitiue avec Justinien, cf. A. GUARINO, Diritto privato romano, cit., P. 841 sur le thème, IA PIRA Compromissum e litis contestatio, in St. Ricsur le theng, IA PIRA, Compro isuste litis contestatio, in st. Ricromain classique, in RHD. 18 (1939) p. $167 \mathrm{s.}$; BONIFACIO, Compromes so (dir. rom.) in NNDI ; TALAMANCA, Ricerche in tema di compromissum (1958).

617- La liste exhaustive des paragraphes où 1 'esclave apparaît contme agent d'acte juridique sera donnée plus loin. Pour le moment, on se contentera d'apporter des exemples, la question êtant pour une large contentera d'apporter des exemples, la question étant pour une large
part traitée dans 1 'ouvrage de I. BUTI, Studi sulla capacità patrimopart traitée dans l'ouvrage de I. BUTI, Studi sulla capacità patrimo-
niale dei servi, cit., Ch. II, L'agire del servo nelle varie fattispecie, p. 73-143.

618- Par exemple, Alfenus (chez auteur anonyme) D 15.3.16 (achat de boeufs) ; Julien D 10.3.25 (achat d'un vicarius); D 19.1 .24 pr (achat d'un fonds) ; D 21.2.39.1 (achat et vente d'un esclave).

619- Ainsi, Alfenus (chez auteur anonyme) D 15.3.16 (le maître loue un fonds $\bar{a} l^{\prime}$ 'esclave) ; D 40.7.14 pr ( 1 'esclave loue un fonds de son maître).

620- Par exemple, Pomponius D 17.2.18.

621- Par exemple, Julien D 41.4.7.2 (un esclave charge par mandat un tiers d'acheter un fonds); cependant, le mandat par lequel un esclave chargerait quelqu' un de $1^{\prime}$ 'acheter serait nul, cf. Papinien D $17.1 .54 \mathrm{pr}$

622- Dêpôt fait par un esclave, cf. Ulpien D 16.3.1.17-27-28-29-30-3132 (Celsus)-33 (Julien); dépôt fait chez un esclave, cf. Ulpien D 16. $3.1 \cdot 18-42$ (Julien).

623- L'esclave reçoit un gage, cf. Julien D 13.7.28.1; il remet en gage une res peculiares, cf. Paul D 13.7.18.4.

624- Esclave en tant que commodant, cf. Paul D 12.6.36 ; en tant que conmodataire, cf. Ulpien D 13.6.3.4.

625- Alfenus chez Paul D 24.1.38 pr. Cependant, les esclaves qui sont sous la puissance du mari ne peurent faire de donation a la femme, cf. v1pien $D$ 24.1.3.3. De même, les esclaves ne peuvent aliêner leur pécule par donation, cf. Ulpien D 24.1.3.8.
(626). Ailleurs, il reçoit une caution en garantie d'un paiement (627). Il prête (628) ou emprunte (629) une somne d'argent. Il est vrai que ces opérations sont effectuées dans le sillage du maître. Il en va plus généralement de même dès qu'on est en présence d'une action exercitoria, institoria ou quod iussu (630). Il faut être plus nuancé avec les actions de peculio, tributoria et de in rem verso car il agit alors de sa prcpre initiative (631). Ce $n$ 'est toutefois qu'exceptionnellement que 1 'esclave semble agir en nom propre. (632).

D'autres cas sont liés à la libertê : ainsi, en matière de legs (633) et d'institution d'héritier (634). A un autre niveau, 1'esclave

626- Le fait qu'un titre du Digeste (D 45.3 De stipulatione servoram) soit consacré a ce problème montre son importance. Cependant, les stipulations accouplies par l'esclave en son nom propre sont considerrées coume inutiles par le ius civile, cf. Gaius 3, 104 ; elles ne sont reconnues que de manière limitée par le ius honorariun et le ius novrom impérial, cf. I. BUTI, op. cit., p. 113.

627- Par exemple, Iavolenus $D$ 44.3.4. On relève chez U1pien D 17.1.12. 2 un servus fideiussor.

628- Par exemple, U1pien D 12.1.11.2; D 39.5.19.4.

629- Par exemple, Paul D 4.3.20 pr ; D 17.1.22.8.

630- Cf. supra Sect.2 Ch.I Titre II prem. partie : 1'indépendance de fait de 1 'esclave (action exercitoria, inatitoria, quod iussu.) Au même titre que l'institor et 1'exercitor, le vilicus, 1'actor et le dispensator sont disposés par leurs fonctions a intervenir en tant qu'agents $d^{\prime}$ actes juridiques, et les textes ou il est question de tels esclaves seront donc pris en considerration dans les totaux.

631- I. BUTI, op. cit., p. 152 ; cf. supra paragraphe 2 , section 2 Ch.I titre II, première partie : 1'autonomie relative de 1 'esclave (actions de peculio, tributoria, de in rem verso).

632- Cf. supra paragraphe 2 , même section, et les articles cités de M. JACOTA, L'esclave créancier, et les pactes de l'esclave en son non propre.

633- Legs fait par le maître, cf. par exemple, Iavolenus D 32.30.2 (Trebatius, Labéon); Celsus D 34.5.25; Julien D 28.5.38.4; Gaius D $30.69 \mathrm{pr}$; Scaevola D 31.88.3 ; Paul D 28.5.90; Ulpien D 30.34.9; Modestin D 35.1 .66 ; Legs fait par un tiers, ef. par exemple, Iavolenus D 31.40 ; Pomponius D 7.4.18; Marce11us D 35.2.56.4; Paul D 10.2.15; Ulpien D 30.53 .2 (Julien, Marcellus). Il faudra également tenir compte dans les totaux des textes concernant les legs d'alimenta faits a un esclave au moment de son affranchissement, cf. supra n. 40I. On peut aussi léguer à l'esclave un droit d'usufruit, cf. par exemple Julien aussi léguer

634- L'esclave peut être institué héritier par son maître, cf. par exemple Celsus D 28.5.61 ; Pomponius D 28.5.21 pr (Trebatius, Labéon) ; Gaius D 28.5.89 ; Papinien D 28.5.77 (Julien) ; Paul D 35.1.44.10 (Plautius); Ulpien D $28.7 .2 \mathrm{pr}$; ou par un tiers, cf. par exemple Ce1sus D 29.4.25; Julien D 38.2.13; Africain 29.2.47; Scaevola D 28. 
apparaît toujours comme agent lorsqu'il est afranchi à condition de rendre les comptes (635) ou de remettre une somme (636), ou lorsqu'il fait un pacte de liberté avec son maître (637).

Enfin, il se voit reconnaître, dans des hypothèses déterminées, la possibilité de se pourvoir en justice (638).

$$
-\circ-
$$

Si on opère une comparaison entre esclave objet (639) et esclave agent (640) d'acte juridique, par rapport au nombre total de textes

$6.48 \mathrm{pr}$; Paul D 35.1.44.1; Ulpien D 30.39.1 ; Marcien D 28.5.39; Hermogénien $D 37.14 .21 .3$. La liste complète des références concernant les legs et institutions d'héritiers intéressant des esclaves sera donnée infra a propos des rapports sociaux. Signalons que l'esclave ne donnée infra à propos des rapports sociaux. Signalons
peut être possessor pro herede, cf. Paul D 41.3 .4 .4 .

635- Cf. supra n. 492, première partie.

636- Cf. par exemple D 40.7.17 Neratius (remise de la somme à 1 'hêritier) ; $D 40.7 .20 \mathrm{pr}$ Paul (a un tiers).

637- Cf. par exemple Ulpien D 4.3.7.8; v. M. JACOTA, Les pactes de 1 'esclave, cit., p. 209 s.

638- Hermogénien D 5.1.53 : Vix certis.ex causis adversus dominos servis consistere permissum est : id est si qui suppressas tabulas testamenti dicant, in quibus libertatem sibi relictam adseverant. Item articris annonae populi Romani, census etiam et falsae monetae criminis reos dominos detegere servis permissum est, praeterea fideiconnissam libertatem ab his petent : sed et si qui suis numis redermptos se et non manumissos contra placiti fidem adseverent. Liber eticm esse iussus si rationes reddiderit, orbitron contra dominom rationibus excutiendis recte petet. Sed et si quis fidem alicuius elegerit, ut numis eius redimatur atque his solutis monomittatur, nec ille oblatom pecumion suscipere velle dicat, contractus fidem detegendi servo potestas tributa susciper.

639- Comme on l'a signalé à propos de 1 'analyse terminologique, il faut tenir compte dans cette rubrique des textes concernant les operae de $l^{\prime}$ esclave, cf. supra n. 5. A un niveau plus gênéral, il convient d'intégrer aussi les textes où il est question du prix de l'esclave, celui-ci apparaissant indubitablement alors comme objet, (par ex., Valens D 35.1 .37 .1 ). L'esclave ressort come objet d'acte juridique chez lens D 35.1 .37 .1 ). L'esclave ressort comme objet d acte juridique che Proculus D 12.6.53; D 31.48 pr ; D 33.6.6 ; Iavolenus D 9.2.38; D 15 1.33 ; D 18.6.17; D 19.2.60.7 (Ĺabéon); D 21.1 .53 ; D 21.2.58; D 24 . 1.20 ; D 24.1.50 pr-1 ; D 24.3.66.3 (Labéon) ; D 28.7.20.1 (Labéon) D 29.2.62.1; D 29.2.64; D 31.37 ; D 31.38 ; D 32.29.4 (Tubero, Labéon) ; D 32.30.2 (Trebatius, Labéon) ; D 32.39.3 (Labéon) ; D 32:100 pr (Cącellius, Labéon) ; D 33.4.6.1 (Servius, Cascellius, Namusa, Labéon); D 33.5.14; D 33.5.15; D 33.8.22.1 (Trebatius, Labéon); D 34.5 .28 ; D 38.2 .36 ; D 40.7 .39 pr (Q. Mucius, Servius, ofilius, Labéon) -3 (Servius, Labéon) -4 (Trebatius, ofilius, Labéon) -5 ; D 40.
12.42 ; D 41.3.16; D 41.3.19; D 44.3.4; D 45.1.105 (Sabinus) ; D 45 . 3.34 ; D 45.3.36 ; D 47.2.75 ; D 49.15.27 (Trebatius, Ofilius, Labéon) Neratius D 19.1.31.1; D 25.1.15;D 28.5.55; D 40.7.17; D 45.3.22. D $45.3 .24 ; D 47.2 .65$; Celsus D 12.4.16; D 12.6.26.14;D 15.1.6 (Tu-

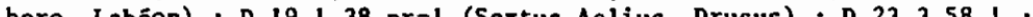
bero, Labeon) ; D I D 30.63 (Servius) ; D 31.15 ; D 31.18 ; D 31.19 ; D 31.20 (Celsus P. Proculus) ; D $32.79 \mathrm{pr}-3$; D 33.8 .13 ; D 33.8 .25 ; D $40.7 .23 \mathrm{pr}$; D 46 3.69 ; D 47.2 .68 .3 ; Valens D 35.1 .89 ; D $35.2 .37 \mathrm{pr}-1$; D 36.4.15 ; Julien D 1.5.26; D 2.14.55; D 5.1.25; D 7.1.35.1 (Sabinus) : D 9.4 $39 \mathrm{pr} ; \mathrm{D} 9.4 .40 ; \mathrm{D} 9.4 .41 ; \mathrm{D} 10.3 .24 \mathrm{pr}$; D $10.3 .25 ; \mathrm{D} 10.4 .8$; D 12.4.7.1; D 12.6.32 pr-2-3; D 12.6.37; D 13.1.14 pr ; D 13.5.23; D $14.3 .12 ;$ D 15.1.12;D 15.1.28;D 15.1.37.1-2-3; D 17.1.30;D 18. 2.17 ; D 18.5.5.2; D 19.1.13.5; D 19.1.24 pr-1 (Sabinus, Cassius) ; D 21.1 .50 ; D 21.2 .7 ; D 21.2 .8 ; D 21.2.39.1-3-4-5; D 22.1.25.1-2 D 23.3 .47 ; D 23.4.21; D 24.3.31.4; D $28.5 .7 \mathrm{pr}^{-1}$; D 28.5.8 $\mathrm{pr}^{-1}$; D 28.5.38 pr-1-2-3-4-5; D 28.6.19; D 29.2.43; D 29.2.45.1 ; D 29 .

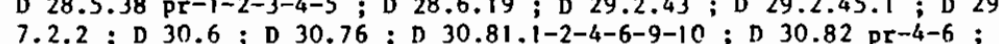
D $30.84 .3-4-5-8-9-10-11-12$; D $30.86 \mathrm{pr}-1-2 ; \mathrm{D} 30.91 .1-2-3-4-5-6-7$; D $30.84 .3-4-5-8-9-10-11-12$; D $30.86 \mathrm{pr}-1-2$; D $30.91 .1-2-3-4-5-6-7 ;$; D 30.92 .1 ; D $30.94 .1-2-3$; D 30.96 .2 ; D 30.97 ; D $30.98 ; \mathrm{D} 30.99 ; 8$; 5.11 ; D 33.5.12; D 34.4 .11 ; D $34.5 .13 .1-2$; D 36.1.28.1 ; D 36.2 . $16 \mathrm{pr}^{-1} ; \mathrm{D} 37.4 .13 \mathrm{pr}$; D 39.2.42; D 39.5.2.7; D 39.6.18.3; D 40. 2.6 ; D 40.4.15; D 40.5.47.1-3; D 40.5.48; D 40.7.6.7; D 40.7.13.1-5; D 40.9.5.2 ; D 40.9.7.1 (Proculus); D 40.12.30 (Sabinus, Cassius) ; D $41.1 .37 \mathrm{pr}-1-2-3-5-6$; D 41.1 .39 ; D $41.1 .54 \mathrm{pr}-1$; D $41.2 .38 \mathrm{pr}-2$; D $41.3 .33 \mathrm{pr} ; \mathrm{D} 41.3 .35 ; \mathrm{D} 41.4 .7 .7 ; \mathrm{D} 41.4 .8 ; \mathrm{D} 41.4 .9 ; \mathrm{D} 41.4 .10$; D $43.24 .14 ;$ D $43.26 .19 .1 ;$ D 43.33 .1 pr ; D 44.2.25.1; D 44.7 .16 ; D $44.7 .18 ; \mathrm{D} 45.1 .54 \mathrm{pr}^{-1} ; \mathrm{D} 45.1 .56 .8 ; \mathrm{D} 45.3 .1 .1-2-4-5-6 ; \mathrm{D} 45.3$ 10 ; D 46.1.16 pr ; D 46.3.33 $\mathrm{pr}^{-1}$; D 46.3.34 pr-1-2-10; D 46.4.17; Pomponius D 3.5.10 (Proculus) ; D 6.1 .29 ; D 7.1 .55 ; D 7.4.18;D 7.8 . $16.2 ; \mathrm{D} 9.4 .18$; D 11.1.15 pr ; D 12.6.19.3; D 13.7.8 $\mathrm{pr}-1$; D 15. 1.2 ; D 15.1.4.6; D 15.1.23; D 15.1.34; D 15.2.3; D 17.1.47.1 (Julien) ; D 17.2.60.1 (Labéon); D 18.1.8 pr; D 18.1.13; D 18.1.31; D 19.1.55 (0ctavenus) ; D 21.1.16; D 21.1.36; D 21.1.46; D 21.1. $48 \mathrm{pr}-1-2-3-4-5-6-7-8$; D $21.1 .64 \mathrm{pr}$ (Labéon)-1 (Labéon) -2 (Labéon) D 21.216 .2 (Proculus); D $21.2 .30 ;$ D 21.2.34 pr-1; D 23.3.18 (Labéon); D 23.3.65;D 24.1.18; D 24.1.28.4;D 24.1.29 pr (Fulcinius) D $24.1 .31 .3-5-10$; D 24.3 .67 ; D 26.7.6.1; D 28.6.16 pr; D 30.8.2; D 30.12 .1 (Proculus) ; D 30.13 (Neratius) ; D 30.20 ; D $30.24 \mathrm{pr}$; D $30.36 \mathrm{pr}$ (Labéon) $-1-3$; D 30.38 .1 ; D $30.45 \mathrm{pr}$ (Ofilius, Aristo, Neratius) $-1-2$; D 30.46 ; D 30.48 .1 ; D 30.56 ; D 31.11 .1 (Julien) D 32.74 ; D 32.85 (Labéon) ; D 33.1.2 ; D 33.2.20; D 33.5 .6 ; D 33.5 .8 pr-2 ; D 33.7.15 pr (Servius) -2 ; D 34.2.10; D 35.1.1.3; D 36.1.72.1 ; D 38.1 .4 (Aristo) ; D 38.1.8 pr (Labéon); D 40.1 .13 (Octavenus); D 40.4.11 pr ; D 40.4.40 pr (Julien)-1 (Ofilius); D 40.4.41.1 ; D 40. 5.8; D 40.5.20 (Julien) ; D $40.5 .34 \mathrm{pr}-2$ (Sabinus); D 40.7.8.1 (Labéon); D 40.7.29.1 (Q. Mucius, Labéon, Aristo, Celsus) ; D 40.7.30. D $41.1 .21 \mathrm{pr}$ (Proculus); D $41.1 .54 .4 ; \mathrm{D} 41.3 .30 \mathrm{pr} ; \mathrm{D} 41.4 .6 .1-2$; D 41.1 .21 pr (Proculus) ; D 41.1 .54 .4 ; D 41.3.30 pr ; D 41.4.6.1-2 ; 26.10 ; D 43.26.15.4; D 44.2.21.2; D 44.7.56;D 45.1.16 pr;D 45 . 1.23 ; D 45.1.33;D 45.1.112 pr; D 45.2.18;D 45.3.6 (Ofilius, Sabinus, Cassius) ; $D 45.3 .17$; D 45.3.37 ; D 45.3.39 (Gaius) ; D 45.3. 40 ; D 46.3.26; D 46.3.83; D 46.3.92 $\mathrm{pr}-1 ; \mathrm{D} 46.4 .10 ; \mathrm{D} 46.4 .15$; D 47.2.44.2; D 50.16.166 pr ; Africain D 3.5.48; D 7.1.36.2; D 7.1. 37 ; D9.4.28; D 10.3.9; D 12.1.23 (Julien) ; D 12.1.41; D 13.7.31 
(Julien) ; D 15.1.38.2-3; D 15.3.17.1; D 17.1.37; D 19.1.30 pr-1; D 19.1.44; D 20.4.9 pr ; D $21.1 .34 \mathrm{pr}-1$; D 21.1.51 pr-1; D 21.2.24; D 21.2.46.2-3; D 21.2.47; D 23.5.9.2 ; D 30.107.1 ; D 30.108 pr-2-4 $-5-9-11$; D 30.110; D 33.8.16 pr; D 40.4.20; D 40.5.49; D 40.7.15. I ; D 41.1.40; D 41.2.40.2-3; D 44.3.6.1; D 47.2.62 pr-1-2-3-4-5-6-7 -9; D 50.16.207 (Mela); T. Clemens D 7.7.5; D 29.2.82; D 33.5.17; Gaius D 3.5.21;D 4.7.3.1; D 5.3.39 pr ; D 6.1.18; D 6.1.20; D 6.1. 28 ; D $6.1 .30 ; \mathrm{D} 7.1 .31 ; \mathrm{D} 7.1 .45 ; \mathrm{D} 7.1 .76 .1 ; \mathrm{D} 7.7 .3 ; \mathrm{D} 7.7 .4$; D ; 8.13 (Labéon); $9.4 .13 ; 0.4$; $27 \mathrm{pr}-1 ; \mathrm{D} ; .4 .30 ; \mathrm{D} 10.3 .1 \mathrm{I}$; D 7.8.13 (Labéon) ; D 9.4.13 ; D 9.4.27 pr-1 ; D 9.4.30; D 10.3.1I ; D 12.6.63; D 13.6.18 pr-2; D 15.1.27.2-3 (Julien)-4 (Julien)-5 (Julien)-6 (Julien)-7 (Julien)-8 (Julien) ; D 16.3.14.1 (Sabinus, Cassius) ; D 17.1.27.1; D 18.1.35.3; D 20.1.15 pr (Julien); D 20.4.11.3; D 21 . 1.3 ; D 21.1.13 ; D 21.1.18 pr-1-2 ; D 21.1.20 (Caelius Sabinus) ; D 2! 1.22 ; D 21.1.24; D 21.1.26; D 21.1.28.; D 21.1.32; D 21.1 .45 ; D 21 . 2.54 .1 ; D 24.1.8; D 28.5.31.1 ; D 30.65 pr-1 ; D $30.67 \mathrm{pr}$; D 30.68.23 (Julien) ; D $30.69 \mathrm{pr}-1-4-5$; D $30.70 \mathrm{pr}-2-3$; D 31.55 .1 ; D 33.8 .4 ; D 35.1.17 pr-1; D 35.2.73 pr-3; D 36.1.65.4 (Julien); D 39.4.13.2. D $35.1 .17 \mathrm{pr}^{-1} ; \mathrm{D} 35.2 .73 \mathrm{pr}^{-3} ; \mathrm{D} 36.1 .65 .4$ (Julien) ; D 39.4 .13 .2 ; $12.9 \mathrm{pr} ; \mathrm{D} 41.1 .10 \mathrm{pr}-3-4-5 ; \mathrm{D} 41.1 .15 ; \mathrm{D} 41.1 .43 \mathrm{pr}-2 ; \mathrm{D} 41.2$. $12.9 \mathrm{pr}$; D $41.1 .10 \mathrm{pr}-3-4-5$; D $41.1 .15 ; \mathrm{D} 41.1 .43 \mathrm{pr}-2$; D 41.2 .
15 ; D 41.3.36.1 ; D 44.7.1.9; D $45.1 .74 ; \mathrm{D} 45.2 .15$ (Julien) ; D 45 . 3.8 ; D 45.3 .28 pr-1-2-3-4 (Próculus, Cassius); Maecianus D 29.4.28 pr-1; D 32.95 (Aristo) ; D 35.1.86 pr (Julien) ; D 35.2.30 pr ; D 36.1.71 ; D 40.4.55 pr-2; D 40.4.58; D 40.5.32 pr-1 ; D 49.17.18 pr-3; Venuleius $\mathrm{D} 21.1 .65 \mathrm{pr}-1$ (Cassius) -2 (Caelius Sabinus) ; D 41.1 .66 ;

D 42.8.25.4-5 (Proculus) ; D 43.29.4.1 (Trebatius) ; D 45.1.137.1-4 ; D 45.3.21 (Julien) ; D 45.3.25 (Cagsius) ; D 48.8.6; Marcellus D 7.8 . $20 ; \mathrm{D} 9.2 .34 ; \mathrm{D} 9.2 .36 \mathrm{pr}$; D 15.1 .16 (Julien); D $17.1 .49 ; \mathrm{D} 18.7$ $4 ; \mathrm{D} 19.1 .23$ (Julien); $\mathrm{D} 19.2 .48 .1 ; \mathrm{D} 26.8 .12$ (veteres, Julien) 4 ; D 19.1.23 (Julien); D 19.2.48.1; D 26.8.12 (veteres, Julien) ; D 32.69 .1 ; D $33.3 .2 ;$ D $35.1 .47 ;$ D 35.2 .34 (Julien).; D 35.2 .56 . D 32.69 .1 ; D 33.3 .2 ; D 35.1.47; D 35.2.34 (Julien); D 35.2 .56 . $3-4 ; \mathrm{D} 37.15 .3 ; \mathrm{D} 38.15 .5 .2 ; \mathrm{D} 39.6 .38 ; \mathrm{D} 40.1 .15 ; \mathrm{D} 40.5 .9 ;$
$\mathrm{D} 40.5 .10 \mathrm{pr}-1-2 ; \mathrm{D} 40.7 .24 ; \mathrm{D} 40.13 .2 ; \mathrm{D} 45.1 .96 ; \mathrm{D} 46.1 .38 \mathrm{pr}$ D 46.3.67; D 4ó.3.68; D 46.3.72 pr-1-2-4-5-6; D 46.8.17; D 47.6.5; Florentinus D $18.1 .43 \mathrm{pr}^{-1}$; D $30.116 .2-3$; Scaevola D 2.14.54 ; D 3 . $5.34 .2 ; \mathrm{D} 4.3 .32$; D 10.2.34.9; D 12.6.67 pr; D 15.1.51; D 15.1. $54 ; \mathrm{D} 16.1 .28 \mathrm{Pr} ; \mathrm{D} 16.2 .22 ; \mathrm{D} 18.5 .8 ; \mathrm{D} 20.1 .32 ; \mathrm{D} 20.1 .34 .1$ D 21.69 pr-1-2-3 (Servius) $4-5 ; 024,1.58$ Pr-1-2;D 28.6 .48 Pr-1 (Julien)-2; D 31.88.7; D 32.37.3-7; D 32.41.2-4-5-10; D 32.93 .2 ; D $32.101 \mathrm{pr}-1 ; \mathrm{D} 33.2 .37 ; \mathrm{D} 33.5 .18$ (Neratius); D $33.5 .21 ; \mathrm{D} 33$. D 32.101 $\mathrm{pr}^{-1}$; D 33.2.37; D 33.5.18 (Neratius) ; D 33.5.21 ; D 33 ;

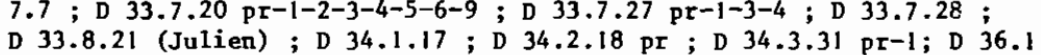
$80.12 ; \mathrm{D} 38.1 .44 ; \mathrm{D} 40.5 .41 .3-5 ; \mathrm{D} 40.7 .40 .2-7$ : D 40.9.6; 41.6 5 ; D 41.9.3; D 44.3.14.2-4; D 45.1.122.2; D 45.1.127; D 45.1.135 3 ; D 45.3.19; Papinien D 3.3.66 ; D 4.4.31; D 4.8.42; D 6.1.65. I ; D 10.2.31; D 10.2.34;D 12.6.55;D 13.1.17; D 15.1.50.3; D $17.1 .54 \mathrm{pr}$; D 17.1.57; D 18.7.5 ; D 18.7.6 pr (Sabiaus)-1 (Sabinus) ; D 18.7.7; D 18.7.8; D 19.5.8 ; D 20.1.1.1 ; D 21.1.54 ; D 21 1.55 ; D $21.2 .64 \mathrm{pr}$; D 21.2 .66 .2 ; D 21.2 .67 ; D 22.1 .2 ; D 22.1 .4 $\mathrm{pr}-1$; D 22.1.6 pr (Caracalla) ; D 22.1.8; D 22.2.4.1; D 23.3.69.9 D 24.3 .61 ; D 28.5 .77 ; D 29.2.86.2; D 29.7.11; D 29.7.12; D 31 . $65 \mathrm{pr}-3$; D 31.66 .3 ; D 31.73 ; D 31.77 .17 ; D $32.91 \mathrm{pr}-2$; D 33 . 2.2 ; D 33.2.24.1 ; D 33.4.8; D 33.7.3 pr-1 ; D 33.8.19.2; D 34.4 . 24.1 ; D 35.2 .9 .1 ; D $35.211 .1-4$; D 36.1 .55 ; D 36.1.60.4-6; D 36 . 2.25 .1 ; D 38.2.41; D 39.5.31.2; D 40.1.19; D 40.1.20 pr (Litterae de Marc-Aurèle)-2-3 ; D 40.1.21 ; D 40.4.47 pr ; D 40.4.48 (Servius);
D 40.5.21; D 40.7.34 pr; D 40.7.35; D 40.7.36; D 40.8.8 (constitution de Marc-Aurèle); 41.2 .47 (Nerva f.); D $41.2 .48 ; \mathrm{D} 41.2$ $49 \mathrm{pr} ; \mathrm{D} 41.3 .44 \mathrm{pr}-2-3 ; \mathrm{D} 41.3 .45 .1 ; \quad \mathrm{D} 45.1 .115 .2$ (Sabinus, Pegasus) ; D 45.1.117; D 45.1.118 pr ; D 45.3.18 pr-1-2-3 (Julien) ; D 46.3.95 pr-1 ; D 47.2.81 $\mathrm{pr}^{-2}$; D 48.18.6 $\mathrm{pr}^{-1}$; $\mathrm{D} 48.18$ 17.2 (regcrit d'Hadrien) ; D 48.19.33 ; D 49.17.13; D 49.17.14.1-2 ; D 49.17.15.3-4; D 50.17.77; Tryphoninus D $1.5 .15 ; 0$ D $9.4 .37 ; \mathrm{D} 15$; $1.57 .2 ; \mathrm{D} 18.7 .10$ (Scaevola); D 34.1.15.1 (Scaevola); D 38.2.50.1-2 1.57.2 ; D 18.7.10 (Scaevola) ; D 34.1 15.1 (Scaevola) ; D 38.2.50.1-2;
D 41.1.63.1-2-3; D 43.16.19 (Julien); D 49.15.12.7-8-9 (veteres)-10-11 -12-13-14-16-18; Callistrate D 21.2.7.2 ; D 40.8.3 (rescrit de MarcAurèle et Commode) ; D 48.15.6.1 (rescrit d'Hadrien) ; D 48.18.15.2 (rescrit d'Antonin le pieux) ; Paul D 2.9.2.1 (V. Verus, Julien, Pomponius) ; D 2.11.7; D 2.14.27.6-7-10; D 3.6.7.2; D 4.3.18.1-5 D 4.4.48.1; D 4.7.9; D 4.8.32.5; D 4.9.6.1-2 (Pomponius) ; D 5.3.14; D 5.3 .32 ; D 5.3.36.2 (Pomponius) ; D 5.3 .40 pr (Proculus, Cassius) 4 ; D $6.1 .6 ; 06.1 .16$ Pr ; D 6.1.21 (Julien, Pomponius); D 6.1.27.2-4-5 D 6.1.31; D 6.1.36.1; D 6.2.6;0 6.2.12 Pr-5; D 7.1.24;D 7.1 .26 D $7.1 .31 ; \mathrm{D} 7.1 .66 ; \mathrm{D} 7.4 .26 ; \mathrm{D} 7.4 .27$; D $7.7 .1 ; \mathrm{D} 7.8 .15 \mathrm{pr} ; \mathrm{D} ;$.

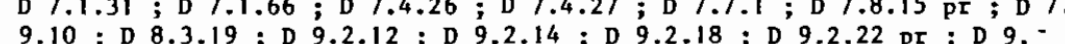

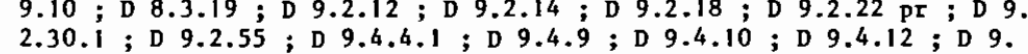
4.17 $\mathrm{pr}^{-1}$; D 9.4.19.1-2 ; D 9.4.22 $\mathrm{pr}^{-1-2}$; D 9.4.24; D 9.4.26.1-2-6; D 9.4.31 (Julien) ; D 10.2.11; D 10.2.15; D 10.2.25.15-17; D 10.3.8. $2-3-4 ; D$ 10.3.15; D 10.3.19.3; D 10.3.27; D 10.3.31; D 11.1.20 $\mathrm{Pr}$; D 11.3 .8 ; D 11.3.14 pr-2-3-4-7 ; D 12.1.31 pr-1 (Sabinus, Cassius, Julien) ; D 12.2.30.1; D 12.4.12; D 12.6.65.5-8; D 13.1.3 (Julien) ; D 13.1.13; D 13.5.12 ; D 13.5.21 pr; D 13.6.21_; D 13.6. 22 ; D 13.7.18.2; D 13.7.25; D I4.1.5 pr; D 14.1.6.1; D 14.3.17 Pr ; D 14.4 .10 ; D 15.1.18; D 15.1.20; D 15.1.26; D 15.1.43 (Labéon); D 15.1.47.2-3 (Proculus)-4 (Julien)-5-6; D 15.3.14. (Julien, Marceliug) ; D 15.4.2.2 ; D 15.4.5.1; D 17.1.5.5; D 17.1.22.9; D 17. 1.26 .7 (Neratius)-8 (Mela); D $17.1 .46 ; 017.2 .56 ; D 17.2 .65 .5$ (Labéon, Proculus) ; D 18.1.1.1 (Sabinus) ; D 18.1 .34 pr (Labéon)-6 ; D 18.1.56; D 18.4.21; D 18.6.8.1 (Julien); D 18.7.3; D 18.7.9; D $19.1 .4 \mathrm{pr}$; D 19.1.21 pr ; D 19.1.42; D 19.1 .43 (Julien, Ulpien) ; D $19.1 .45 \mathrm{pr}$ (Julien, Africain) $-1-2$; D $19.1 .54 \mathrm{pr}$ (Labẻon) ; D 19.2. 42 ; D 19.2.43; D 19.2.45.1-2 ; D 19.4.2 (Aristo); D 19.5.5.1-2 (Julien); D 20.1.29.1-3; D 20.2.9 (Nerva); D 20.6.10.1; D 21.1.2. D 21.1 .5 ; D 21.1 .11 ; D 21.1.15; D 21.1.30 pr-1 (Aristo, Pedius) ; D 21.1 .39 ; D $21.1 .43 .1-2-3-4-5-6-7-8-9-10 ;$ D $21.1 .44 \mathrm{pr}^{-1-2}$; D 21.1 $47 \mathrm{pr}$ (Labéon) -1 ; D 21.1 .56 (Marcellus); D $21.1 .57 \mathrm{pr}-1$; D 21.1 $58 \mathrm{pr}-1-2 ; \mathrm{D} 21.1 .60 ; \mathrm{D} 21.2 .3$; D 21.2.5 (Labéon) ; D 21.2 .9 (Sabinus) ; D 21.2.11.1; D 21.2.26; D 21.2.35; D 21.2.41 pr-1; D 21.2.42; D 21.2.56.2-3 ; D 22.1.10 ; D 22.1.14 pr-1 (Neratius) ; D 22.3.4 ; D 23.3.25; D 23.5.50 pr ; D 24.1.28.1-2-3 (Plautius) -4 (Pmponius) -5 (Julien. Pomponius) ; D 24.1.38 pr (Alfenus) ; D 24.1.67 (Labéon) ; D24.3.
$25.3 ;$ D24.3.26;D24.3.63;D25.1.2; D 25.1.6; D 25.1.12; D 25.2.21.2; D 28.5.53; D 28.5.85.1-2; D 28.5.90; D 29.1.40.2; D 29.2.65 D 29.2.68; D 29.5.6.2 ; D 29.5.8 pr ; D 29.5.12 ; D 30.5 pr-1 (Trebatius, Labéon, Cassius) ; D 30.35 ; D 30.52 pr; D 30.62; D 31.5 .1 ; D (Alf ; D 31.14 pr ; D 31.82 .2 ; D 31.86 pr ; D 32.60.1 (Alfenus) -3 (Alfenus) ; D 32.61 ; D 32.66 ; D $32.78 \mathrm{pr}-1-2-3$; D $32.92 \mathrm{pr}$; D 32 . $99 \mathrm{pr}-1-2-3-4-5$; D 33.2.21 ; D 33.5 .7 ; D $33.5 .13 \mathrm{pr}-1$ (Pomponius) D $33.7 .13 \mathrm{pr}$ (Neratius)-J (Neratius); D 33.7.14; D 33.7.16.2 (Alfenus) ; D 33.7.18.1-4 (Scaevola) -5-6-7-8-9-10 (Cassius)-11-13 (Scaevola); 
9 pr ; D 33.8.15 (Alfenus); D 34.2.7; D 34.3.25 (rescrit d'Antonin le pieux) ; D 34.4.15; D 34.4.26 pr ; D $34.4 .27 \mathrm{pr}-1$; D 34.5.2I pr ; D 34.9.5.4; D 35.1.28.1; D 35.1.37 (Neratius); D 35.1.44.8-10 (Plautius) ; D $35.1 .96 \mathrm{pr}-1$; D 35.2 .1 .9 (veteres, Aristo) ; D 35.2 . $21 \mathrm{pr}-1$; D 35.2.24.1; D 35.2.33; D 35.2.36 pr-1-3-4 (Africain) ; D 35.2.48; D 35.2.49 pr (Sabinus, Cassius, Nerva, Atilicinus, Plautius ; rescrit d'Antonin le pieux); D $35.2 .63 \mathrm{pr}$ (Pedius); D 36.1.68.2 ; D 38.2.44.1-2; D 39.3.11.3 (Julien) ; D 39.6.39; D 40.1.3; D 40.1. 9 ; D 40.1.10; D 40.1.11; D 40.1.23; D 40.2.4.2 (Julien); D 40.2.15 4 ; D $40.4 .10 \mathrm{pr}-1$; D 40.4.39; D 40.5 .6 ; D 40.5.25 (Valeos) ; D 40 5.29 ; D 40.5.31-2-3-4;D 40.5.33.2; D $40.5 .39 \mathrm{pr} ; \mathrm{D} 40.5 .40 \mathrm{pr}-1$ D 40.7.4.3-4-6; D 40.7.7; D 40.7.10; D 40.7.18; D $40.7 .20 \mathrm{pr}-2-3$ (Sabinus, Julien)-5; D 40.7.22 pr-1-2 ; D 40.7.41 pr (Labéon)-1 (La(Labéon) ; D 40.8.1 (rescrit de Marc-Aurèle) ; D 40. $.16 \mathrm{pr}-1$; D 40.9.18.1 ; D 40.9.26 (Scaevola); D 40.9.28 D 40.12 .23 .1 ; D 40.12.38 F (constitution de Marc-Aurèle)-1-2-3 ; D 40.13.4 (L. Rufinus); D 41.1.47; D 41.1.49;D 41.1 .57 (Julien); D 41.2.1.5-6-7-8-15 (Julien)-16 (veteres)-17-18; D 41.2.3.10-13 (Nerva f.) ; D 41.2.5; D 41.2.30.4; D 41.3.4.5-8-16 (Sabinus, Cassius) -17 ; D 41.3.15.1; D 41.4.2.6 (Neratius)-7 (Trebatius) ; 041 . 10.2 ; D 42.5.38 pr;D 43.16 .6 ; D 44.4.3;D 44.4.5.4; D 44.7.44.4; D 44.7 .45 ; D 45.1.2.1-3; D 45.1.8 (Sabinus, Proculus, Marce1lus): D 45.1.24; D 45.1.49 pr-3; D 45.1.73 pr-1-2 ; D 45.1.83.1-2-4-5-7 (Sabinus)-8 ; D 45.1.85.4; D 45.1.88; D 45.1.91 pr-1 (Julien)-2-3 (Celsus, Julien) -5 (Pomponius) 6 (Julien) ; D 45.1.93 ; D 45.1.128; D 45.3.12;D 45.3.16;D 45.3.20 pr-1;D 45.3.23; D 45.3.26; D 45.3.27;D 45.3.29;D 45.3.31; D 45.3.32; D 45.3.33 pr (Julien) -1; D 45.3 .27 ; D 45.3 .29 ; D 45.3 .31 ; D 45.3 .32 ; D 45.3 .33 Pr (Julien) 60 ; D 46.3.63; D 46.3.98 pr-6-7-8 (Celsus) ; D $46.4 .11 \mathrm{pr}-2$;

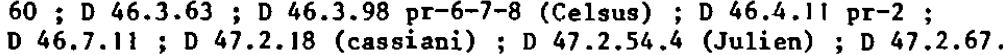
5 ; D 47.2.86;D 47.10.16; D 47.10.29; D 48.10.14 pr-1-2 ; D 48 . 10.17 ; D 48.10.22.4-7-10; D 48.18.11;D 48.18.18.4-6; D 48.19.38. 4 ; D 49.14.45.3 ; D 49.15.29 (Labẻon) ; D 50.16.79.1 ; Ulpien D 1.12 1.1 ; D 2.4.10 pr-1 (Marcellus) ; D 2.9.1.1 ; D 2.9.5; D 2.11.9.1 (Labéon) ; D 2.14.16.1 ; D 2.14.50; D 3.2.4.3 (Ponponius) ; D 4.2.2.5 ; D 4.2.7.1; D 4.2.12 pr ; D 4.2.14.11; D 4.3.7 pr (Julien)-3 (Labéon) $-4-5$; D 4.3.9.1-4 (Labéon) ; D 4.4.11.1 (Scaevola ; constitution de Marc-Aurèle)-4 (Marcellus); D 4.7.4.5; D 5.1.67;D 5.2.8.17; D 5.2. 26 ; D 5.3.5 pr ; D 5.3.20 pr-1 (Julien) -3 ; D 5.3.27 pr ; D 5.3.33.1 (Ju1ien) ; D 6.1.5.5 (Pomponius) ; D 6.1.13 (Labéon); D 6.1.15 pr (Labéon) -3 ; D 6.1 .17 pr (Julien)-1 (Julien) ; D 6.1.22; D 6.2.9.6 ; D 6.2.11.2-3 (Julien)-4 (Julien) -5 (Pomponius) ; D 7.1.12.1 (Labéon) -3 (Julien, Pomponius)-4 (Julien)-5 (Julien) ; D 7.1.15.1-2-3 (Labéon) ; D 7. 1.17 .1 (Cassius, Aristo) $2-3$; D 7.1.21 (Labéon) ; D 7.1.22; D 7.1.23 pr (Julien) -1 (Sabinus, Cassius) ; D $7.1 .25 \mathrm{pr}-1$ (Julien, Mauricianus, Marcellus) $-2-3$ (Julien) $-4-5$ (Julien) -6 (Scaevola) $\rightarrow$ (Pegagus, Julien); D 7.1.27.2-5; D 7.1.68 pr (Brutus) ; D 7.2.1.1 (Julien)-2 (Julien); D 7.3.1.2 (Julien) ; D 7.4.5.1; D 7.4.12.1; D 7.4.15; D 7.6.5. 4 (a) -5 ; D 7.7.2; D 7.7.6.1-2-3; D 7.8.12.5-6 (Labéon, Sabinus, Octavenus) ; D 7.8.14 Pr ; D 9.1.1.14; D 9.2.11.7 (Julien); D 9.2.13.2 (Celsus) -3 (Julien) ; D 9.2.15 pr-1 (Julien) ; D 9.2.17; D 9.2 .19 (Celsus) ; D 9.2.20; D 9.2.27.1 (Proculus, U. Ferox) -2 (Celsus)-34 (Mela) ; D 9.2.35; D 9.4.2.1 (Celsus); D 9.4.5 pr-1 ; 9. 4.7.1 (Pomponius) ; D 9.4.8; D 9.4.11; D 9.4.14.1; D 9.4.21 $\mathrm{pr}^{-1}$; D 9.4 .36 ; D 9.4.38 pr ; D 10.2.8 pr (Pomponius) ; D 10.2.12 pr (Sabinus)
-1 ; D 10.2.16.6 (Ofilius) ; D 10.2.18.1 (Pomponius)-2 (Pomponius) D 10.2.49; D 10.3.6.4 (Sabinus, Atilicinus)-9 (Julien) ; D 10.4.3. 6-7-10-12 (Pomponius); D 10.4.5.6; D 10.4.9 pr (Julien) ; D 10.4. 11 pr-1 (Labéon) ; D 11.1 .17 ; D 11.3 .1 .1 ; D 11.3 .5 .4 ; D 11.3 .9 Pr (Julien)-1; D 11.3 .13 .1 ; D. 12.1 .13 .2 (Papinien); D 12.2.18 Pr (Julien); D 12.2.34 Pr (Marcellus); D 12.3.54; D 12.4.5.1-2-3-4; D 12 6.26 .13 (Celsus, Marcellus) -14 (Celsus); D $13.1 .7 .2 ; \mathrm{D} 13.3 .3 ; \mathrm{D} 13$. 4.26.13 (Celsus, Marcellus) -14 (Celsus); 0 (N.1.7. ; $013.3 .3 ; 013$ lius); D 13.7.24.3; D 14.1.1.22-23;D 14.1.4.2; D 14.3.11.8; D 14.

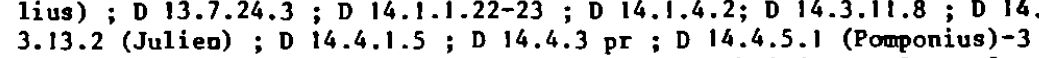
-10 ; D 15.1.3 pr ; D 15.1.7.1 (Julien, Marcellus)-4-6; D 15.1.9.5 ; D 15.1 .11 .4 (Julien)-5 (Julien)-7 (Julien)-8 (Julien)-9 (Julien) ; D 15. 1.13 (Julien) ; D 15.1.15 ; D 15.1 .17 (Servius, Proculus, Atilicinus) ; D $15.1 .19 \mathrm{pr}^{-1}$ (Pomponius, Marcellus, Papinien)-2 ; D 15.1.21.2 ; D 15J.30.1-5; D 15.1.32 pr (Julien)-1-2 (Neratius) ; D 15.2.1 $\mathrm{pr}^{-1}$ -3-4-5-6-7 (Pegasus, Africain)-8 (Scaevola)-9 (Pomponius) ; D 15.3 .3 . 8; D 15.3.5 $\mathrm{pr}^{-3}$; D 15.3 .13 (Julien); D 15.4.1.8 (Marcelius); D 16.3. 1.5-9-10 (Pomponius) $-24-28-29-30-31 ; \mathrm{D} 16.3 .7 \mathrm{pr}$; D 16.3.11 (Sabinus) ; D 17.1 .8 .4 (Julien) -10 ; D 17.1 .10 .1 ; D 17.1.19 (Pomponius) ;D 17.2.49; D 17.2.51.1; D 17.2.58.3; D 17.2.63.9 (Sabinus, Julien, Pomponius) ; D 18.1.7 pr (veteres) ; D 18.1.9 pr ; D 18.1.11.1 ; D 18. 1.29 ; D 18.2.4.1 (Julien) -2 (Julien); D 18.4.2.12 (Julien)-13 (Marcellus)-15 ; D 18.7.1 (Papinien) ; D 19.1.11.5-7 (Neratius)-8 (Neratius)-12 (Neratius)-15 (Julien); D 19.1.13.1-2-3-4-13-18-22 (Trebatius, Labêon) ; D 19.2.9.1 (Marcellus) ; D 19.5.13.1 ; D 20.1.6 ; D 20.1.8; D 20.1.21.1 (Julien) ; D 20.1.27 (Marcellus) ; D 20.2.6 (Pomponius); D 20.4.11.2; D 20.6.4 pr; D 21.1 .1 pr (Labéon) $-1-2-3-4$ ponius) ; D 20.4.11.2 ; D 20.6.4 pr ; D $21.1 .1 \mathrm{pr}$ (Labéon) - - -2-3 -4
$-5-6-7$ (Sabinus) $-8-9$ (Vivianus) -10 (Vivianus) -11 (Vivianus) ; D 21.1 $4 \mathrm{pr}-1-2$ (Pomponius)-3 (Pomponius) $-4-5$ (Pomponius) -6 (Pomponius); D 21.1.6 pr (Pomponius)-1 (Trebatius)-2; D 21.1.7; D 21.1 .8 ; D 21.1 .9 (Sabinus) ; D 21.1.10 pr (Ofilius)-1 (Cato)-2-3-4-5; D 21.1.12 pr-1 (Pedius) -2-3-4 (Trebatius) ; D 21.1.14 pr (Sabinus)-1-2-3 (Trebatius, Caelius Sabinus) -4 (Pedius) -5 (Pedius) $-6-7-8-9-10$ (Caelius Sabinus) D 21.1 .17 pr (Ofilius)-1 (Caelius Sabinus) -2 (Cassius) -3 (Vivianus) -4 (Proculus) -5 (Vivianus) -6 (Caelius Sabinus) -7 (Caelius Sabinus) -8 (Caelius Sabinus)-9 (Caelius Sabinus)-10 (Caelius Sabinus) $-11-12$ (Labêon, Caelius Sabinus) -13 (Caelius Sabinus)-14 (Labéon)-15 (Labéon, Caelius Sabinus)-16 (Caelius Sabinus) $-17-18-19-20$; D 21.1.19 pr (PeCaelius Sabinus) -16 (Caelius Sabinus) $-17-18-19-20$; D $21.1 .19 \mathrm{pr}$ (Peponius); D 21.1.23 pr (Pouponius) $-1-2$ (Pomponius) $-3-4-5-6 \rightarrow 7$ (Julien) -8 (Julien)-9. (Pedius); D 21.1 .25 pr-1-2-3-4 (Pedius)-5-6-7-8-9-10; D 21 . 1.27 ; D 21.1.29 $\mathrm{pr}^{-1-2-3}$ (Julien) ; D $21.1 .31 \mathrm{pr}-1-2-3-4-5$ (Pomponius) -6 (Pomponius) -7 (Marcellus) -8 (Marcellus) -9 (Pomponius) $-10-11-12$ -13 (Pedius) -14 (Pedius) -15 (Pedius) -16 (Ju1ien) $-17-18-19-20-21-22-$ 23-24; D 21.1.33 pr (Pomponius)-I ; D 21.1 .35 ; D 21.1.37; D 21.1.38. 2-3-7 (Ofilius, Caelius Sabinus) $-10-14$; D $21.1 .40 \mathrm{pr}$; D $21.1 .59 \mathrm{pr}-1$; D 21.1 .63 ; D 21.2.4 pr ; D 21.2.21 pr-2 (Julien) -3. (Julien); D 21.2.25; D 21.2.31; D 21.2.32 pr-1 (Julien) ; D 21.2.33; D 21.2.37.1; D 21 . 2.51 .1 (Julien) -3 ; D 23.2.45 pr (rescrit de Sévềre et Caracallia) ; D 23.3.10.2-5; D 23.3.12.1 (Marcellus, Scaevola) ; D 24.1.3.8 ; D 24 . 1.7.8 (Sabinus, Papinien; constitution de Marc-Aurèle)-9; D 24.1 . 9 pr-1 (Julien) ; D 24.1.17.1 ; D 24.1.19 pr (Julien)-1 (Julien, Marce 1lus) ; D 24.1.22; D 24.3.7.10-16; D 24.3.21; D 24.3.24.4-5; D 24 . 3.62 ; D 24.3.64 pr-1-2-3-4-5-6-7-8-9 (Labéon)-10; D 25.3.5.22 (Marcellus) ; D 26.4.3.2 (constitution de Marc-Aurèle) ; D 26.7.5.9 ; D 27. 
2.3.2 ; D 27.3.13; D 28.1.21.1 ; D 28.5.6.2-3; D 29.5.9.16-17-1920 ; D 28.5 .30 (rescrit de Sêvère) ; D 28.5.35.3 (Q. Mucius); D 28. $5.51 \mathrm{pr}$; D 28.6.18 pr (Julien)-1; D 28.7.2 pr-1 (Celsus); D 28.7. 4.1 (Julien) ; D 28.8.1 pr ; D 28.8.5.1 ; D 29.2.20.1 ; D 29.2 25 pr-1; D $29.2 .42 .2-3$; D 29.2.66; D 29.2.67; D 29.2.71.1; D 29.4. 6.10 ; D 29.5.1.1-2-3-4-6-13 (Scaevola) ; D 29.5.3.4-13-15; D 30.32.1 D 30.37 pr (Cassius ; rescrit de Sévère et Caracalla); D 30.39 pr (JuD 30.37 pr (Cassius; rescrit de Sévère et Caracalla); D $30.39 \mathrm{pr}$ (Ju-

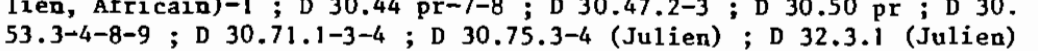
D $32.7 \mathrm{pr} ; \mathrm{D} 32.49 \mathrm{pr}$; D $32.66 ; \mathrm{D} 32.68 .1-2-3 ; \mathrm{D} 32.71 ; \mathrm{D} 32.73$ pr-1-2-3-4-5 (Pomponius) ; D 33.2.7; D 33.4.1.6; D 33.5.2 $\mathrm{pr}^{-1-2}$; D $33.7 .8 \mathrm{pr}-1$; D 33.7 .12 pr (Servius) -2 (Alfenus)-3 (Labéon, Pegasus) -4 (Labéon, Neratius) -5 (Trebatius) $-6-7-8-9-12-13-31$ (Ce1sus) -32 -33-35 (Neratius)-37 (Papinien)-38 (Papinien)-42 (Papinien)-44 (Ce1sus) -45 (Papinien) -46 (Papinien) ; D 33.8.6.2-3; D 33.8.8.4 (Sabinus) -8 (Julien) ; D 33.8.24; D 34.3.7.1 (Julien) ; D 34.4.12; D 35.1 .50 (Antonin le pieux) ; D 35.2.43; D 36.1.11.2 (Antonin le pieux) ; D 36.1.17.10-14-17 (rescrit d'intonin le pieux); D 36.I.23.3 (Neratius); D 36.2 .7 .6 ; D 36.2 .11 ; D $36.2 .12 .7-8 ;$ D $36.2 .23 ; 036.4 .5 .8$; D 37.11 .2 .9 ; D 38.1.13 pr (constitution de Marc-Aurè le) ; D 38.1 .15 1 (Celsus) ; D 38.2.3.3 ; D 38.2.16.1-9; D 38.16.3.2-3 (constitution de Marc-Aurè1e) ; D 39.4.12.2 ; D 39.5.13 ; D 39.5.18.1 (Aristo, Pomponius)-2 (Aristo, Pomponius); D 39.6.37.1 (Julien); D 40.1.2; D 40. 1.4 pr-1-2-3-4-5-6-7-8-9-10-11-12-13 (constitution de Marc-Aurèle et Verus) ; $\mathrm{D} 40.2 .2$; D 40.2.3 ; D 40.2.16.1; D 40.2.20 pr-l (constitutinn de Marc-Aurèle) ; D 40.4.6 ; D 40.4.9 pr ; D 40.5.4.6 (constitution de Marc-Aurèle) ; D 40.5.7 ; D 40.5.24.6 (rescrit d'Antonin.le pieux)-8 (Celsus) -10-12-14-15-16-17-18-21; 0 40.5.26.2 (rescrits d'Antonin 1e pieux et de Sévère et Caracalla) $-6-11 ; 040.5 .28$ pr ; 040.5 .30 .6 (rescrit d'Antonin le pieux); D $40.5 .45 \mathrm{pr}-2 ; \mathrm{D} 40.5 .46 .5 ; \mathrm{D} 40.5 .52$; D 40.7.2 pr-2 (Julien) -3 (Julien) ; D 40.7.3.3-7-8-I7; D 40.7.6.3-5 D 40.7 .2 pr-2 (Julien) -3 (Julien) ; D 40.7.3.3-7-8-17 ; D 40.7.6.3-5 $-6-7$; D 40.7.9.1-2 (Octavenus)-3; D 40.9.4; D 40.9.12.1-2 (Africain)-3-4-6 (Africain)-7 ; D 40.9.14 pr-4-5-6; D 40.9.30 pr (constitution de Marc-Aurèle)-2; D 40.12.8 pr-1-2; D 40.12.12.5; D 40.12 $14 \mathrm{pr}$; D 40.12.16 pr;D 40.12.20.4; D 41.1.17; D 41.1.18; D 41 . 1.22 ; D 41.1.23 pr-1-2-3 (Julien, Scaevola); $D$ 41.1.33 pr (Marce1lus, Scaevola)-2 (Julien) ; D 41.2.4; D 41.2.13 pr-2-3; D 41.2.34.2 (Ce1sus); D 41.2.42 pr; D 41.3.10.2 (Marcellus, Scaevola) ; D 42.1.4.8 (Ce]sus) ; D 42.2.6.1; D 42.5.9.3; D 42.6.1.12; D 42.8.6.5; 042.8 .10 21 ; D 43.16.1.46 (Vivianus); D 43.24.7.1 (Neratius); $043.31 .1 \mathrm{pr}-1$; D 43.32.1 pr ; D 44.2.7.1-3; D 44.2.11.1 (Ce1sus) $-4-8$; D 44.3.8 ; D 43.32.1 Pr; D 44.2.7.1-3; D 44.2.1.1 (Ce1sus) $4-8$; D 44.3 .8 ;

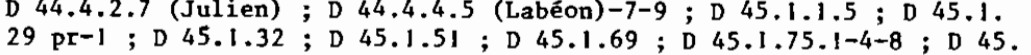
$1.81 \mathrm{pr}$ (Celsus) ; D 45.1.82.1; D 45.3.2; D 45.3.4; D 45.3.5 ; D 45 . $3.7 \mathrm{pr}-1$ (Julien); D $45.3 .9 \mathrm{pr}-1$ (Cassius, Julien) ; D 45.3.11 (Julien) ; D 45.3.13 ; D 46.1.8.8 (Julien, Marce1lus)-9 (Julien)-10-11; D 46.1 .33 ; D 46.2.8 pr ; D 46.2.14 pr (Marcellus) ; D 46.3.9.1 D 46.3.29; D 46.4.8.1-2 (Labêon) ; D 46.4.13.4 (Julien)-6 ; D 46.6. 2 ; D 47.2.14.5 (Papinien)-6 (Pomponius, Papinien)-7 (Pomponius, Papinien)-11-15; D 47.2.17.1-2 (Sabinus)-3 (Julien, Pomponius) ; D 47.2. 43.12 ; D 47.2.46.1-3-4 (Africain) -5 ; D 47.2.48.5-6; D 47.2.52.4-23 (Me1a); D 47.4.1.1 (Labéon); D $47.6 .3 \mathrm{pr}-1$; D 47.8.2.14-18; D 47.10. 1.6 (Julien) -7 (Labéon) ; D 47.10.15.36-37-44 (ordinarius sous-entend $v i$ carius) -47-48-49 ; D 47.10.17 pr-9; D 47.10.24; D 48.5.28.1-2-3-4-6-89-1I-12-13-14; D 48.15.2 pr-1-2-3; D 48.18.1.6 (rescrit de Marc-Aurèle et Verus)-7-8-15 (rescrit de Sévère et Caracal1a)-27 (epistula de Marc-Aurèle et Verus) ; D 48.18.2 ; D 48.18 .3 (constitution de Sévère et
Caracal1a); D 49.17.6; D 49.17.9 (Marcellus); D 50.16.160 (Marcellus); D 50.16.174 ; D 50.16.195.3 ; L.Rufinus D 40.7.32 ; Macer D 48.19.10 pr D 48.19 .14 (Menander); Marcien D 15.1.40.1 (veteres) ; D 18.1 .42 (rescrit de Marc-Aurèle et Verus) ; D 18.1.44; D 18.7.2 ; D 20.1.13.1; D 21.1.52; D 22.1.32.5; D 28.5.39; D 28.5.52.1 (Celsus); D 29.2.52 pr (rescrit $A^{\prime}$ Antonin 1e Dieux) ; D 30.112.1; D 30.114.1; D 32.65 pr(Labéon) $-1-2-3$ (Celsus') ; D 33.7.17.2; D 34.I.2 pr (rescrit de Sévère et

Caraca11a) ; D 34.8.3.2 ; D 35.1.33.4; D 36.1.15.8 ; D 37.14 .3 (rescrit de Sévère et Caracalla); $\mathrm{D} 38.4 .6$. D 39.4 .16 pr-1 et 2 (rescrit de Sévëre et Caracalla); D 39.4.16.3; D 39.4 .16 .4 et 9 (rescrit d'Antonin le pieux); D 39.4.16.10 (rescrit de Marc-Aurèle et Verus); D 40 tonin le pieur); D 39.4 .16.10 (rescrit de Marc-Aurèle et Verus) ; D 40 . I.5 pr (Marc-Aurêle et Verus) ; D 40.2 .14.1 ; D 40.5.50; D 40.5.51 pr-1
$-2-3-8-10$; D 40.5.53.1 ; D 40.5.54; D 40.5.55 pr-1 (Marcellus) ; D 40 . 8.6 (constitution de Marc-Aurèle) ; D 40.9.9.2; D 48.15.3 pr (constitution de Sévère et Caracalla) ; D 49.14.30 (rescrit de Sévère et Caracalla) ; Modestin D 5.2.9 (Paul) ; D 6.1.32; D 16.3.23; D 20.1.26.2 ; D 21.1.62; D 24.3.58; D 27.1.14.3; D 31.31; D 31.34 pr; D 31.35 ; D 32.81 pr-1 ; D 32.82 ; D 35.1.51.1 ; D $37.14 .7 \mathrm{pr}$ (décret de Vespasien) ; D 37. 14.8.1 ; D 40.5.12 pr (rescrit de Sévère et Caracalla)-1; D 40.5. 15; D 40.7.25; D 40.7.27 (Julien) ; D 44.3.3; D 45.1.103; D 45 . 3.35; D 48.8.11.1 ; D 50.16.101.3; Hermogénien D 5.1.53; D 32.22.2; D 35.1.94 pr-1; D 35.2.38.1-2; D 36.4.11 pr; D 40.9.27.1-2 ; D 4l. $1.16 \mathrm{pr}^{-1}$; D 41.2.50.1 ; Auteur anonyme $\mathrm{D} 36.4 .16$. Les textes ou 1 'esclave apparaît simultanément comme objet d'actes juridiques différent (cf. par exemple Julien D 30.76 ; Gaius D 30.69 .1 ; Pomponius D 30.45 pr (ofilius, Aristo, Neratius) ; Ulpien, D 30.44.7) ne compteront que pour une unité, la comparaison ne concernant plus la place d'un acte juridique par rapport a un autre (comme ce fut le cas a propos des modes d'acquisition de la main-d'oeuvre servile), mais 1 'esclave objet par rapport a l'esclave agent. On a procédé de même pour cette deuxième hypothèse.

640- Il convenait d'intégrer dans cette rubrique non seulement les textes à caractère "positif", mais aussi ceux qui ne traitaient de 1 'esclave que de manière négative, comme Ulpien D 50.17.22 pr (In personam servilem nulla cadit obligatio) ou D 50.17.118 (Qui in servitute est, usucopere non potest : non cum possideatur, possidere non videtur). est, usucopere non potest : non cum possideatur, possidere non videtur) 8.14; D 40.1.6; D 40.7.14 pr ; D 50.16.203; Proculus D 4.3.31; D $12.6 .53 ; \mathrm{D} 23.3 .67 ; \mathrm{D} 33.6 .6$; D 46.34 ; Iavolenus D 15.1.33;

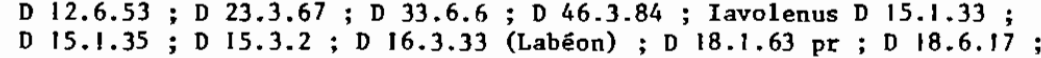
D 19.2.60.7 (Labéon) ; D 24.1.20; D 24.3.66.1 (Trebatius, Labéon) ; D 28.5.65 (Labéon); D 28.8.11 (Trebatius, Labéon) ; D 29.2.62.1 ; D 29.2.64; D 31.38 ; D 31.40 ; D 32.29.4; D 32.30.2 (Trebatius, Labêon); D 33.8.17 ; D 33.8.22 pr (Labéon)-1 (Trebatius, Labéon) ; D 40.7.28 pr-1 (Cassius) ; D 40.7.39 pr (Q. Mucius, Gallus, Servius, ofilius, Labéon) -2 (Trebatius, Labéon) -4 (Trebatius, Ofilius, Labéon) -5 ; D 41.2.24; D 44.3.4; D 45.1.104; D 45.3.34; D 45.3.36; D 46.1.20; Neratius D 15.3.18; D 15.1.55; D 28.5.55; D 40.7.17; D 41.10.5. I ; D 45.3.22; D 45.3.24; Celsus D 15.1.6 (Tubero, Labéon) ; D 19. $1.38 \mathrm{pr}$; D 28.5.6.1 ; D 28.7.2.1; D 29.4.25; D 31.20 (Celsus P., Proculus) ; D 33.8.13; D 33.8.25; D 34.5.25; D $40.7 .23 \mathrm{pr}^{-1}$; D 46.3

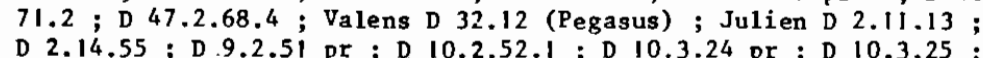

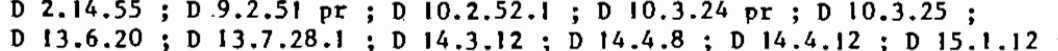


D $15.1 .14 \mathrm{pr}-1 ; \mathrm{D} 15.1 .28 ; \mathrm{D} 15.1 .37 .1-2-3 ; 019.1 .24 \mathrm{pr}-1$ (Sabimus, Cassius); D 21.2.8; D 21.2.39.1; D 21.2.51.3; D 22.1.25.1-2; D 23.3.46 pr ; D 23.3.47; D 24.3.31.4; D 28.1.12; D 28.2.13.1 ; D 28.5.7 pr-1; D 28.5.8 pr-1; D 28.5.22; D $28.5 .38 \mathrm{pr}-1-2-3-4-5$; D 28.5 .41 ; D 28.5 .43 ; D 28.6.19; D 29.2 .43 ; D 29.2.45.1-3; D 29. 4.22 .1 ; D 30.81 .1 ; D 30.86 .2 ; D $30.91 .1-2-3-4-5-6-7$; D 30.94 .1 ; D $30.96 \mathrm{pr}$; D 30.99 ; D $30.101 \mathrm{pr}$; D 30.102 ; D 33.5.10; D 33.5.11; D 33.8.12; D 34.3.12; D 36.1.26.1-3; D 36.1.28.1-17; D 36.2.16. 1-2; D 36.2.17; D 38.2.13; D 38.13.1; D 39.2.42; D 40.4.16.; D 40.4 .17 .1 ; D 40.4 .19 ; D 40.5 .47 .2 ; D 40.7 .12 ; D 40.7.13.1-2-5; D 40.4 .17 .1 ; D 40.4 .19 ; D 40.5 .47 .2 ; D 40.7 .12 ; D $40.7 .13 .1-2-5$; D 40.12 .30 (Sabinus, Cassius) ; D 41.1 .37 pr-1-2-3-4-5-6; D 41.1 ; D 41.2 .38 .2 ; D $41.4 .7 \mathrm{pr}-2-8$; D $41.4 .9 ; \mathrm{D} 41.4 .10 ; \mathrm{D} 44.7 .16$; 45.3 14 (Sabinus); D $46.1 .16 \mathrm{pr}-3-4 ; \mathrm{D} 46.1 .19 ; \mathrm{D} 46.3 .32 ; \mathrm{D} 46.3 .34 .5$ D 47.2.57.2-3; D 49.15.22.1-3; Pomponius D 6.2.15; D 7.4.18; D 7.8.16.2; D 12.1.12; D 13.6.13.2 (Celsus); D 15.1.2; D 15.1.4 pr-1-2-3-4-5-6; D 15.1.22; D 15.1.23; D 15.1.25; D 15.1.34; D 15. $1.49 \mathrm{pr}-1-2 ; \mathrm{D} 17.2 .18 ; \mathrm{D} 18.1 .12 ; \mathrm{D} 18.1 .13 ; \mathrm{D} 18.1 .18 .1$ (A1fenus) ; D 18.1.31; D 23.3.65; D 24.3.67; D 28.1.16 pr ; D 28.5.42. (Tibère); D 28.5.21 pr (Trebatius, Labéon) -1 ; D 29.2 .36 ; D 30.12. 2; D 30.13 (Neratius); D 30.48 pr (Atilicinus) ; D $31.11 \mathrm{pr}$ (Labéon): D $33.7 .15 \mathrm{pr}$ (Servius); D 33.8.10; D 34.4.20; D 35.1 .57 ; beon); D $33.7 .15 \mathrm{Pr}$ (Servius) ; D 33.8.10; D 34.4.20; D 35.1.57; D 35.1 .58 ; D 35.1.110; D 35.1.111 ; D 35.1.112.3 (Neratius); D 38 . $1.8 \mathrm{pr}$ (Labéon) ; D $38.1 .10 \mathrm{pr}$; D $40.4 .4 \mathrm{pr}-1-2 ; \mathrm{D} 40.4 .8 ; \mathrm{D} 40.4$.
$11.1 ;$ D 40.4 .41 .2 (Labéon) ; D 40.4 .46 (Aristo) ; D $40.5 .44 ; \mathrm{D} 40$. 11.1 ; D 40.4 .41 .2 (Labéon) ; D 40.4 .46 (Aristo) ; D $40.5 .44 ; \mathrm{D} 40$.
$7.5 \mathrm{pr}$ (Aristo, Neratius)-1 ; D $40.7 .8 \mathrm{pr}-1$ (Labéon) ; D 40.7 .11 (Aristo) D 40.7.21 pr (veteres, Labéon) ; D 40.7.29 pr-1 (Q. Mucius, Labéon, Aristo, Celsus) ; D 40.14.13.1 ; D 41.1.21 pr (Proculus): ; D 41.1.54. 4 ; D 41.2 .25 .1 ; D. 41.3 .28 ; D 43.26.13;D. 44:2.21.4; D 44.7.

$24 \mathrm{pr}-1$; D 45.1 .40 ; D 45.3 .6 (Ofilius, Sabinus, Cassius) ; D 45.3 . 17 ; D 45.3 .37 ; D 45.3 .38 (Celsus) :D 45.3.39 (Gaius): D 45.3 .40 D 46.1 .2 ;D 46.1 .3 ; D 46.3.19;D 46.3 .83 ; D $50.16 .166 \mathrm{pr} ;$ D 50. 17.18 ; Africain D 4.4.4; D 7.8.17; D 10.3 .9 ; D 12.1 .41 ; D 14.1 .7 $\mathrm{pr}-2$; D 15.1.38.2-3; D 15.3.17 pr-1; D $19.1 .30 \mathrm{pr}$; D 21.1.51 PF-1 ; D 21.2.46.3 ; D 29.2.47; D 30.107.1; D 30.108.1; D 33.8 . $16 \mathrm{pr}-1$; D 34.6 .1 ; D $35.1 .31 ; \mathrm{D} 35.1 .32 ; \mathrm{D} 35.1 .42$; D 39.6 .23 D 40.4.22 ; D 40.7.15 pr-1; D 41.1.40; D 41.2.40 pr ; D 41.4.11; D 46.1.21.2; D 46.3.38.2-3; D 47.2.62.9; D 48.10.6.2; T. Clemens D 29.2.82; D 31.59 ; Gaius D 2.14.18 ; D 2.14.28.2 (Julien) ;.D 2 . 14.30.1 (Julien) ; D 5.3.35 (Julien) ; D 6.1.20; D 7.1.6.3; D 7.1. 74 ; D 12.2.21; D 14.4.11; D 14.5.1; D 15.1.10; D 15.1.27 pr (Julien)-1-2-3 (Julien) 4 (Julien)-5 (Julien)-6 (Julien)-7 (Julien) -8 (Julien); D 15.1.29 pr-1 ; D 15.3.4; D 15.3.12; D 16.1.13 pr . D 20. (Julien) ; D $15.1 .29 \mathrm{pr}-1$; D 15.3 .4 ; D 15.3.12 ; D $16.1 .13 \mathrm{pr}$. D 20

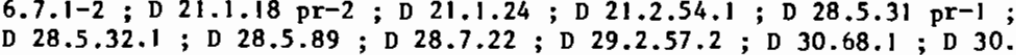
D 28.5.32. ; D 28.5 ; D 35.2.73 pr;D 35.2 .57 .2 ; D 30.68 .1 ; D 30 ;

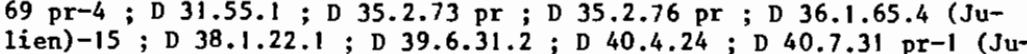
lien); D 40.9.10; D 40.12.9.2 ; D 40.12.25.2; D 41.1 .10 pr-1-3-4-5 D $41.1 .32 ; \mathrm{D} 41.1 .43 \mathrm{pr}-2$; D $41.1 .45 ; \mathrm{D} 45.1 .141 \mathrm{pr} ; \mathrm{D} 45.3 .8$; D 45.3.28 pr-1-2-3-4 (Proculus) ; D 46.1.70.3; D 46.2.34 pr ; D 46. 4.22 ; D 46.6.6 ; D 50.17.107; D 50.17.133; Maecianus D 29.4.28 pr ; D 32.17 .1 ; D 35.1.86 pr (Julien) -1 ; D $35.2 .30 \mathrm{pr}-8$; D 36.1.67 pr; D 40.5.35 (Cassius); D 40.5 .42 (Antonin le pieux); D $49.17 .18 \mathrm{pr}$;

Venuleius D $34.4 .32 .1 ; \mathrm{D} 40.12 .44 \mathrm{pr}-2 ; \mathrm{D} 42.8 .25 .3 ; \mathrm{D} 44.3 .15 .3$;

D 45.2.12.1 (Julien); D 45.3.21 (Julien); D 45.3.25 (Cassius); D 46.
8.8.2 : Marcellus $01.7 .18 ; 07.8 .20 ; 09.2 .36 .1 ; 015.1 .16$ (Julien); D 19.1.23 (Julien); D 23.3.59.2; D 26.8.12 (veteres, Julien); D 28.5.40 (Julien); D 29.2 .63 (Pomponius) : D 29.4.5 ; D 33. 3.2 ; D 35.1 .47 ; D $35.2 .56 \mathrm{pr}-1-4$; D $36.1 .46 \mathrm{pr} ; \mathrm{D} 37.15 .3$; D 38 . 15.5 .2 ; D 39.6 .38 ; D 40.7 .24 ; D 46.3 .68 ; D 49.1.15; D 49.15.1; Florentinus D 15.1.39; D 30.116.3; D 34.4.14 Pr ; D 45.1.65.1; D 45.3.15;D D46.2.16; Scaevola D 3.5.34.2; D 4.3.32; D 10.2.39. 2-4;D 12.6.67 pr ; D 14.3.20;D 15.1.51; D 15.1.54;D 15.1.58; D 15.3.20.1; D 18.5.8; D 20.1.32; D 21.2.69.2-3 (Servius)-4; D 28 $5.84 \mathrm{pr}-1$; D 28.5 .86 ; D $28.6 .48 \mathrm{pr}-1$ (Julien) -2 ; D 31.88.3-6-13 ; D 32.38 .1 ; D $32.41 .2-5$; D 33.1 .18 .1 ; D 33.1 .19 Pr ; D 33.1.21.1; D 33.2.36 $\mathrm{pr}-1$; D 33.7.7; D 33.7.20 pr-1-3-4; D 33.8.21 (Julien); D $33.8 .23 \mathrm{pr}-1-2-3$; D 34.1.18 $\mathrm{pr}-1-2-3$; D 34.1.20 pr-3; D 34.3.31.1 D $34.4 .31 \mathrm{Pr}$; D 34.5 .29 ; D 35.2 .20 ; D 35.2.56.2 (Marcellus) ; D 36 1.80.2-3; D 36.2.27.1; D 39.5.35 pr; D 40.4.59.2; D 40.5.18; D $40.5 .19 \mathrm{pr}-1$; D $40.5 .41 .4-7-8-9-10-11-12-13-14-15-16-17$; D 40.7 . 3.2 (Servius, Labéon, Cassius) ; D 40.7.40 pr-1-3-4-5-6-7-8; D 45 . $1.122 .1-4$; D 45.3.19; D 46.3.89 pr ; D 46.3.102.2 ; D 49.1.28 pr; Papinien D 3.5.30 Pr ; D 5.1.44.1; D 12.6.58; D 14.3.19.1-3; D 15. 1.50.2 3; D 16.1.27.1; D 16.2.19;D 16.3.24; D 17.1.54 Pr; D 20 . 1.1.1; D 22.1.4.1; D 22.1.6 pr; D 22.2.4.1; D 24.3.42.1; D 26.5 . 13 pr (rescrit d'Badrien); D 26.7.37.1 ; D 26.7.39.18; D 28.5.77 (Julien) ; D 30.11 ; D 31.65 pr-2; D $31.76 .4 ; D 32.91 \mathrm{pr}-2$; D 33.1 .10 3 ; D 33.2.2 ; D 33.3.5; D 33.8.19 pr-1; D 34.1.10.1; D 35.1.72.7 (Cassius, Caelius Sabinus) ; D 35.1 .77 pr (rescrit d'Antanin le pieux); D 35.2 .13 ; D 36.1 .54 .1 ; D 36.1 .55 ; D 36.1.57.1 (rescrit d'Antonin. 1e pieux)-2 ; D 39.6.41; D 40.1.19; D 40.3.3; D 40.4.50.i ; D 40.4. 51.1 ; D $40.5 .23 \mathrm{pr}-4$; D $40.7 .34 \mathrm{pr}-1$; D 40.7 .35 ; D 40.7.36 ; D 40 . 7.40 .5 ; D 41.2.44.1-2; D 41.2.45; D 41.2.47 (Nerva f.) ; D 41.2. $49 \mathrm{pr}$; D 41:3.44.3-7; D 41.3.45.1; D 45.1118 pr ; D 45.3.18 pr-1 $-2-3$; D 46.3.94.3; D 46.3.95.7; D 48.19.33 (rescrit de Marc-Aurèle et Verus) ; D 49.17.14.1-2 ; D 49.17.15.3 ; Tryphoninus D 1.5.15; D 12.6.64; D 15.1.57 pr-1-2 ; D 15.3.6; D 26.7.58 pr (Scaevola); D 28.2 .28 .3 ; D 28.5.9.1 ; D 31.88 .12 (Scaevola) ; D 32.80 .5 pr ; D 34. 3.28 .7 (Scaevola); D 38.2.50 pr-1-2-3; D 41.1.63 pr-1-2-3; D 46 . $1.63 .1 ; \mathrm{D} 49.15 .12 .1-11 ; \mathrm{D} 49.17 .19 .1-2-5$; Callistrate D 35.1 .82 ; D $48.10 .15 \mathrm{pr}^{-1}$; Paul D I.18.21; D 2.8.8.2; D 2.14.17.7; D 2.14.21. 5.14 ; D 3.5.17 (Proculus, Pegasus, Neratius); D $3.5 .18 \mathrm{pr}-1$ (Sabinus, Scaevola) ; D 3.5.40; D 3.5.41 ; D 4.3.20 pr (Labêon) ; D 4.3.25 ; D 4.4.24.3; D 4.5.7.2 (Julien); D 4.6.13 pr (Labéon); D 4.8.8; D 4.8. 32.8 (Octavenus) ; D 5.1.12.2; D 5.3.14; D 5.3.32; D 5.3.34.1 (Julien); D 5.3.36 pr (Proculus, Julien, Mauricianus) ; D 6.1 .27 .4 ; D 6.2.10; D 7.1.24; D 7.1.26;D 7.1.31; D 9.4.19.2; D 10.2.15;D 10.3.8.1-4; D 10.4.16; D 11.5.4.1;D 12.1.2.4; D 12.1.29 (Julien); D 12.1.31.1 (Sabinus, Cassius, Julien); D 12.2.20; D 12.2.22; D 12 . $6.13 \mathrm{pr}$ (Pomponius) ; D 12.6.36; D 12.6.65.3; D 13.5.19.2; D 13.7.18 4 ; D 14.1.5 pr-1 ; D 14.1.6 pr-1 ; D 14.3.14; D 14.3.16 ; D 14.3.17 pr-1-3 (Pomponius) -4 (Proculus) ; D 14.4.2; D 14.4.4; D 14.4 .6 ; D 14. 4.10 ; D 14.5 .8 ; D 15.1.8;D 15.1.18;D 15.1.20; D 15.1.26;D 15. 1.31 ; D 15.1.43 (Labéon) ; D $15.1 .47 \mathrm{pr}^{-1}$ (Sabinus)-2-3 (Proculus) -4 (Julien)-5-6 ; D 15.1.48 pr-1 ; D 15.1.52 pr;D 15.1.53; D 15.1.56; D 15.2.2.1; D 15.3.8 (Pomponius) ; D 15.3.11; D 15.3.14 (Ju1ien)

Marce1lus); D 15.4.2 $\mathrm{pr}^{-1-2} ; \mathrm{D} 15.4 .5 \mathrm{pr}-1 ; \mathrm{D} 16.2 .9 \mathrm{pr}, \mathrm{D} 16.3 .21 .1$ (Trebatius) ; D 16.3.27; D 17.1.5.4; D 17.1.22.8 (Neratius)-9 (Me1a); 
D $19.1 .40 .5 ;$ D 18.2.14.3; D 19.1.42; D 20.1.29.3; D 21.1.43.10; D $21.1 .44 \mathrm{pr}$ (Pedius) ; D $21.1 .57 \mathrm{pr}-1$; D 21.1 .58 .1 ; D 21.2.3 D 21.2 .5 (Labéon); D 22.1.11.1; D 24.1.28.5 (Julien, Pomponius) ; D 24. $1.38 \mathrm{pr}$ (A1fenus) ; D 24.3.23; D 26.2.32.2 ; D 26.7.24 pr ; D 28.5 .44 D 28.5.53; D 28.5.56 (Neratius); D 28.5.58; D 28.5 .85 pr-1 (constitution de Marc-Aurèle) -2 ; D 28.5.90; D 29.1.40.1-2; D 29.2.22; D 29.2.26 (Julien, Pomponius, Marcellus) ; D 29.2.58; D 29.2.60 (Labeon, Proculus, Iavolenus) ; D 29.2.65; D 29.2.68 ; D 29.2.74.3-4 (Julien) ; D 29.2.80.2-3; D 29.2.93.1-2; D 29.7.8.5; D 30.7 ; D 30. $52 \mathrm{pr}$; D 31.14 .1 ; D 31.82 .2 (Valens); D $31.83 ; \mathrm{D} 31.84 ; \mathrm{D} 32.4$; D $32.8 .1 ; D 32.61 ; D 32.97 ;$ D 33.1 .11 ; D $33.1 .16 ; \mathrm{D} 33.5 .13 \mathrm{pr}$; D 33.7 .13 pr (Neratius); D 33.7 .18 .4 (Scaevola); D 33.7.22.1; D 33.8 D ; D 33.8.5; D 33.8.9 pr-1 (Marce11us) -2 ; D 33.8.15 (Alfenus) ; D 34 . 1 ; D 33.8.5 ; D 33.8.9 pr-1 (Marce11us) -2 ; D 33.8.15 (Alfenus) ; D 34 .

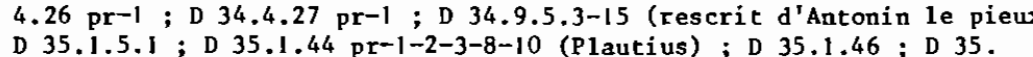
$1.81 \mathrm{pr}$; D 35.2.1.4; D 35.2.21.1 ; D 35.2.22.1-2 ; D $35.2 .49 \mathrm{pr}$ (Atilicinus, Nerva, Sabinus, Cassius, Plautius ; rescrit d'Antonin le pieux) ; D 35.2.63 pr (Pedius) ; D 36.1.41.1 ; D 36.1.42 ; D 36.3.7 ; D 37.11 .10 (Scaevola); D 38.2.45;D 39.5.10;D 39.6.44; D 40.4 . 27 ; D 40.4.28; D 40.4.36; D 40.4.39; D 40.4.53; D 40.5.38; D 40.7.4 pr-I (Julien) $-3-4-6 ;$ D $40.7 .10 ; \mathrm{D} 40.7 .18$; D 40.7 .20 r r -1 (Julien) $-2-3$ (Sabinus, Julien)-4 (rescrit d'Hadrien) -5 ; D 40.7 .22 pr-1-2; D 40.7.41.I (Labéon); D 40.9.18.1; D 40.12.17; D 41.1 .47 Pr-1-2 ; D 40.7.41.I (Labéon) ; D 40.9 .18 .1 ; D 40.12 .17 ; D 41.1 .47 ;
D $41.1 .49 ;$ D 41.1 .57 (Julien) ; D 41.2 .1 .5 (Sabinus, Cassius, Julien) -6-7-8-10-12-13-14 (Cassius, Nerva f., Julien)-15 (Julien)-16 (veteres) $-17-18-19-22$ (Nerva f.) ; D 41.2.3.8-12 ; D 41.2.14 pr; D 41.2 . 32.2 ; D 41.3.4.4-7 (Labéon) -9 (Pomponius) -16 (Sabinus, Cassius) ; D 41. $3.8 \mathrm{pr}$ (Labéon, Neratius, Julien)-1 (Pedius) ; D 41.3 .11 ; D 41.3 . 15 pr (Julien, Marcellus) ; D 41.3.31.2 3 ; D 41.3.34 (Alfenus) ; D 41 . 3.44 .7 ; D $41.4 .2 .10-11$ (Celsus) -12 (Pomponius) -13 (Celsus) -14 (Celsus); D $42.1 .19 \mathrm{pr}$; D 42.5.4;D 43.16.6;D 44.4.3; D 44.4.5.3; D 44.5 . $2.2 ; D 44.7 .14 ; D 44.7 .43 ; D 44.7 .49 ; D 45.1 .39 ; D 45.1 .76 \mathrm{pr}$; $2.2 ; \mathrm{D} 44.7 .14 ; \mathrm{D} 44.7 .43 ; \mathrm{D} 44.7 .49 ; \mathrm{D} 45.1 .39 ; \mathrm{D} 45.1 .76 \mathrm{pr} ;$
$\mathrm{D} 45.1 .126 .2 ; \mathrm{D} 45.3 .12 ; \mathrm{D} 45.3 .16 ; \mathrm{D} 45.3 .20 \mathrm{pr}-1$ (Labéon) ; D 45.

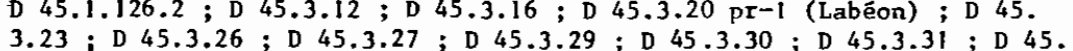

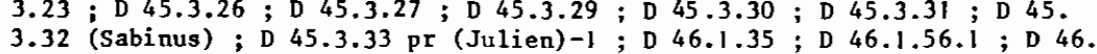
1.66 ; D 46.3.35; D 46.3.62; D 46.3.63; D 46.3.98.7; D 46.4.11 pr-1 $-2-3$; D 47.2.42 pr ; D 47.2.67.3 ; D 48.10.14 pr-1 (Juilien, Marcellus) ; D $48.10 .22 .4-5$; D 48.18.18.5; D 48.19.38.4; D 49.14.13.8 (beneficium Traiani) ; D 49.15.29 (Labéon) ; D 50.17.146 ; D 50.17.175 pr ; Ulpien D 1.12.1.1-8; D 1.19.1.2 ; D 2.4.10 pr (constitution de Marc-Aurōle); D 2.7.3 pr (Pedius); D 2.11.9 pr;D 2.13.4.3; D 2.14.7.18 (V. Verus, Marcellus) ; D 2.14.29 (Celsus) ; D 3.2 .4 .3 (Pomponius) ; 3.3 .33 s Marcellus) $D$ D 2.14 .29 (Celsus) ; 3.2 .4 .3 (Pomponius); D $3.3 .33 \mathrm{pr}$ (Labeon) ; D $3.5 .3 .6 ; 03.5 .5 .7-8$ (Laben, Ponponius) D 3.5 .13 ; D 3.5 .16 ; D 3.5 .44 .1 ; D 4.3 .7 pr (Julien)-8 (Pomponius) ; D 4.3.9.4 (Labéon); D 4.4.3.11 (Marcellus); D 4.4.5; 4.4 .7 .10 (Fapinien ; rescrits d'Antonin le pieux et de Caracalla) ; $04.8 .7 \mathrm{pr}$ (Labéon)-1 (Julien) ; D 4.8.9 pr ; D 4.9.3.3; D 4.9.7.6 ; D 5.1.19.3 (Labéon) ; D 5.1.67; D 5.2.8.10; D 5.3.13.6 (Sabìnus); D 5.3.33 pr ; D6.1.41.1 ; D 6.2.7.10-13 ; D 6.2.9.6 ; D 7.1.12.4-5 (Ju1ien) ; D 7.1. 21 (Labéon); D 7.1.22 ; D $7.1 .23 \mathrm{pr}$ (Julien); D $7.1 .25 \mathrm{pr}-1$ (Julien, Mauricianus, Marce1lus) $-2-3$ (Julien) $-4-5$ (Julien) -6 (Scaevola) -7

(Pegasus, Julien) ; D 7.2.1.1 (Julien)-2 (Julien) ; D 7.3.1.2 (Julien); D 7.4.5.1 ; D 7.6.5.4 (a) ; D 7.8.12.5 ; D 7.8.14 pr ; D 9.2.15.1 ; D 9.2 .23 pr (Neratius)-1 (Julien) $4: D$ 9.3.8; $9.4 .38 .3: D 10.2$.

8 pr (Pomponius); D 10.2.12.1; D 10.2.20.9 (Sabinus); D 11.1.9.
2-8; D 11.3.1.5; D 11.6.3.6 (Pomponius); D 12.1.11.2; D 12.1.13. 2 (Papinien); D 12.2.23; D 12.2.25; D 12.4.3.6 (Celsus) -7 (Ce1sus P., Celsus) -8 (Ce1sus) -9 ; D 12.6.11; D 13.5.1.8; D 13.5.5.10 D 13.6.3.4-5 (Julien) ; D 13.6.14; D 13.7.11.5; D 14.1.1.4-16-20 (Pomponius)-2I-22-23 (Julien); D I4.1.4.2-3-4; D 14.3.1 (Marce11us) ; D 14.3.5.3-7 (Labéon)-8 (Labéon) -9 (Labéon)-15; D 14.3.7.1; D 14.3. 11.7-8; D 14.3.13 pr (Julien)-2 (Julien) ; D $14.4 .1 \mathrm{pr}-1$ (Pedius)-2-3$4-5 ; \mathrm{D} 14.4 .3 \mathrm{pr}$ (Julien)-1 (Pomponius)-2; D 14.4.5.1 (Pomponius) -2 -3-4-5-6-7 (Labéon) -8-9-10-11-12-13 (Labéon)-1/4-15-16-17-18-19; D 14.4 . 7 pr-1-2-3-4-5; D 14.4.9 pr-1-2 (Labéon, Pomponius) ; D 14.5.2 pr ;

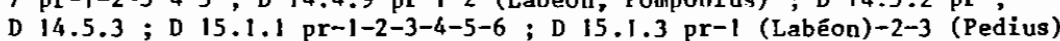
-4-5 (Celsus) -6 (Julien) -7 (Julien) -8 (Nerva $f_{.}$) 9 (Sabinus, Cassius) -12 (Labéon); D 15.1.5 pr-1-2-3-4 (Tubero, Celsus); D 15.1.7 pr (Tubero, Celsus)-1 (Julien, Marcellus)-2 (Pomponius)-3 (Pedius)-4-5 (Labéon) -6-7 ; D 15.1.9 pr-1 (Neratius, Pomponius)-2-3 (Servius) -4 (Pedius) -5 (Julien)-6-7-8; D 15.1 .11 pr-1-2 (Julien)-3 (Nerva, Neratius, Julien) -4 (Julien)-5 (Julien) $-6-7$ (Julien)-8 (Julien) -9 (Julien); D 15.1.13 (Julien) ; D 15.1.15; D 15.1.17 (Servius, Proculus, Atilicinus) ; D 15.1.19 pr-1 (Pomponius, Marcellus, Papinien)-2 ; D 15.1.21 pr (Mela)-1 (Pomponius) $-2-3$; D 15.1.24; D 15.1.30 pr (Proculus, Pegasus) $-1-2-3-4-5-6-7 ;$ D 15.1.32 pr (Julien)-1-2 (Neratius); D 15.1.36 (Pomponius); D 15.1.41; D 15.2.1 pr-1-3-4-5-6-7 (Pegasus, Caelius Sabinus)-8 (Scaevola)-9 (Pomponius) ; D 15.3.1 pr-1 (Labéon)-2 (Julien, Pomponius) ; D 15.3.3 pr-1 (Pomponius)-2-3 (Labéon)-4-5 (Labéon, Pomponius)-6 (Labéon)-7-8-9-10 ; D 15.3.5 pr (Pomponius)-1 (Pomponius) -2-3 D 15.3.7 pr-1-2-3 (Labéon) -4 (Labéon) ; D 15.3.10.5 (Marcellus) -6-7 (Pomponius)-8 (Porponius)-9 (Pomponius) ; D 15.3.13 (Julien) ; D 15.4.1 $\mathrm{pr}-1-2-3-4-5$ (Marcellus) $-6-7-8$ (Marcellus) -9 (Labéon); $\mathrm{D} 15.4 .3 ; \mathrm{D} 15$ 4.4 (Pomponius) ; D 16.3.1.17-t8 (Marce11us)-27-28-29-30-31-32 (Ce1-sus) -33 (Julien)-42 (Julien) ; D 16.3.11 (Sabinus, Pomponius); D 17. 1.10.7; D 17.1.12.2 (Harce11us)-3; D 17.I.19; D 17.2.23.1 (Marce1lus ; Marc-Aurèle) ; D 17.2.24; D 17.2.49; D 17.2.58.3; D 17.2.63. 2-9 (Sabinus, Julien, Pomponius) ; D 18.1.7pr(veteres ; D 18.1.29; D 18.4.2.6-12 (Julien) -13 (Marcellus) ; D 19.1.13.4-13-18; D 21.1 . 23.4-6-9 (Pedius); D 21.1.31.4-7 (Marcellus)-8 (Marcellus); D 21.1. $33 \mathrm{pr}$; D 21.2.51.3; D 21.3.1.4; D 23.3.39 pr; D 24.1.3.3-8; D 24 1.9 .1 (Julien); D 24.1.11.6 (Marcellus, Scaevola); D 24.1.17.1 ; D $24.1 .19 \mathrm{pr}$ (Julien)-1 (Julien, Marcellus); D 24.1.22; D 24.3: 22.13; D 24.3.5.22 (Marce1lus); D 25.4.1.13 (Aristo); D 26.2.10. 4 ; D 26.2.22 ; D 26.7.9 pr (Julien) ; D 26.7.11 (rescrit d'Antonin le pieux) ; D 26.8.1 pr (rescrit d'Antonin le pieux) ; D 27.3.1.3 (décret de Sévère) ; $\mathrm{D} 27.5 .1 .2$ (rescrit de Sévère) ; $\mathrm{D} 27.6 .11 .2$; $\mathrm{D} 27.8$. 1.15-16; D 28.1.20.7; D 28.5.3 pr-1-3; D 28.5.6.2-3 (Julien)-4 (Julien); D 28.5.9.14 (Labéon, Aristo, Neratius)-16-17-18-19-20. D 28.5.30 (rescrit de Sévère); D 28.5.35.3 (Q. Mucius); D 28.5.51 pr ; D 28.6.8.1; D 28.6.10.1-7; D 28.6.18 pr (Julien)-I ; D 28.7. 2 pr-1 (Celsus) ; D 28.8.1pr ; D 29.1.13.3-4 (rescrit de Sévère) D 29.2.6.7 (Celsus) ; D 29.2.20.4 (Julien, Papinien); D 29.2.25 pr-1-2-3;D 29.2.30.7; D 29.2.35 pr ; D 29.2.66 ; D 29.2.67 ; D 29. $2.71 \mathrm{pr}-1-2$; D 29:4.1.3-4; D 29.4.6.10; D 29.6.1.1; D 30.34.9. D $30.37 \mathrm{pr}$ (rescrit de Sévère et Caracalla) ; D 30.39 .1 ; D $30.50 \mathrm{pr}$; D 30.53.2 (Julien, Marcellus) ; D 32.1 .1 ; D 32.7 pr ; D 32.11 .16 ; D 32.49.7; D 33.4.1.10; D 33.6.9.3; D 33.7.8 pr (Sabinus) ; D 33.7. 12.9-38 (Papinien); D 33.8.6 pr-1-2-3-4 (Pegasus, Nerva, Atilicinus: rescrit de Sévère et Caracal1a) $-5 ; 033.8 .8 \mathrm{pr}$ (Pegasus)-1-2-3 (Sabinus) 
-4 (Sabinus) -5 (Labéon)-6-7 (rescrit de Sévère et Caracalla)-8 ; D 33.8.11; D 33.8.24; D 34.3.12; D 35.1.50 (Antonin le pieux); D 35.2 .35 (Scaevola) ; D 35.3.44; D 36.1.1.10: D 36.1 .11 .2 (rescrit d'Antonin le pieux) ; D 36.1.17.10-13 (Maecianus)-14 (Maecianus)-1617 (rescrit d'Antonin le pieux) ; D 36.1.23.1; D 36.2.5.7 : D 36.2.7.6 ; D 36.2 .8 ; D 36.2.9; D 36.2.12.2 (Julien) ; D 36.3.1.20; D $37.1 .7 \mathrm{pr}$; D $37.4 .3 .15-16$; D 37.5.3.2; D 37.10.3.5-11 (Julien); D 37.11.2.9; D 38.1.15.1 (Ce1sus); D 38.2.3.4; D 38.5.1.22-24-25 (Pomponius);

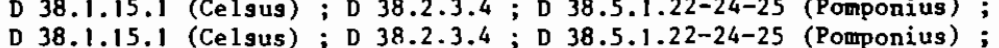
D 38.1 .15 .1 (Celsus) ; D $38.2 .3 .4 ;$ D $38.5 .1 .22-24-25$ (Pomponius) ; D $38.9 .1 .3-14$; D 39.1.5.1-3; D $39.4 .1 .5 ;$ D 39.5.13 ; D 39.5.19.4 ; $11-12$ et 13 (constitution de Marc-Auréle et Verus) ; D 40.2.13 ; D 40 4.2 (Aristo) ; D 40.4.9 pr ; D 40.4.12 pr-1-2 (Julien) -3 ; D 40.4.13. 1-2 (Julien) ; D 40.4.14 ; D 40.5 .2 (constitution de Marc-Aurèle); D 40.5.3 (constitution de Marc-Aurèle) ; D 40.5.4.6 (constitution de Marc-Aurèle) ; D 40.5.24.19; D 40.5.30.5 (rescrit de Caracalla); D 40.5.37 (rescrit de Marc-Aurèle) ; D 40.7.2.3 (Julien)-4 (Celsus) : D 40.7.3.1-2 (Servius, Labéon, Cassius) $-3-4-5-6-7 m-8-9-11$ (Trebatius, Labéon)-12-13 (Pomponius)-14; D 40.7.6.4-5-6-7; D 40.7.19; D 40.12. 34 (constitution de Caracalla); D 41.1 .17 ; D 41.1 .18 ; D 41.1 .22 ; D 41.1.23 pr-1-2-3 (Scaevola); D 41.1.33 pr (Marcellus, Scaevola)-1 -2 (Julien) ; D 41.2.13.3-8; D 41.2.34.2 (Celsus); D 41.4.3.1; D 42

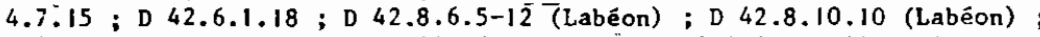
D 43.5.1.7 ; D 43.5.3.4;D 43.16.1.22;D 43.19.3.4;D 43.24.3 pr; D 43.24.5.6 (Servius) ; D 43.26.4.2 ; D 44.4.2.7; D 44.4.4.17; D 44 . 5.1 .4 ; D 44.6.2 (Julien) ; D 45.1.1 pr ; D 45.1.38.6 (Julien)-7-8-17; D $45.3 .2 ; D 45.3 .3 ; \mathrm{D} 45.3 .4 ; \mathrm{D} 45.3 .5 ; \mathrm{D} 45.3 .7 \mathrm{pr}-1$; D 45.3. $9 \mathrm{pr}-1$ (Cassius, Julien) ; D 45.3 .11 (Julien); D $45.3 .13 ; \mathrm{D} 46.1 .3$; D 46.1.33; D 46.3.9 pr; $46.3 .18 ; 046.3 .29$ pr; 46.4 .8 .1 (Octavenus) -2 (Labéon) 4 ; D $46.6 .2 ; \mathrm{D} \mathrm{47.2.14.17;} \mathrm{D} \mathrm{47.2.52.9-15-26-28;}$

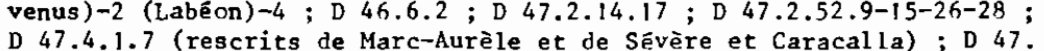
D 47.4.1.7 (rescrits de Marc-Aurèle et de Sévère et Caracal la) ; D 47 .
10.5 .11 ; D 47.10.15.44; D 48.5.18.5; D 49.1 .14 pr (Antonin le pieux)(epistula de Marc-Aurèle et verus); D 49.17.9 (Marcellus); D 50.12.12. 1 ; D 50.16.17 pr ; D 50.16.68 ; D 50.16.99.3 ; D 50.16.182; D 50. $17.22 \mathrm{pr}$; D 50.17 .32 ; D 50.17.118 ; L. Rufinus D 31.62 ; D 34.4 .21 ; D 40.7.32 ; Macer D 48.10.10 pr ; Marcien D 15.1.40 pr (P. Fronto)-1 (veteres); D 20.3 .1 .1 ; D 20.6.8.5; D 22.1.32.1. D 28.5 .39 ; D 28 5.49 .2 (Congtitution de Sevère et Caracalla) D $28.5 .52 \mathrm{pr}$ (rescrit (verer de Marc-Aurele)-1 (Celsus) ; D 28.6.36 pr ; D 28.7 .18 pr (rescrit d'Antonin le pieux); D 30.113 pr (Marcellus)-i (rescrit de Sevère et Caracalla) ; D 30.114 .2 (rescrit de Sevère et Caracalla)-10; D 30.119 ; D $32.65 \mathrm{pr}$ (Labéon) ; D 33.7 .17 .2 ; D $33.8 .18 ; \mathrm{D} 33.8 .20 ; \mathrm{D} 34.8 .3$. 2 ; D 36.1.31.2 ; D 36.1.32.1 (rescrit d'Antonin le pieux) ; D 39.4.16. 2 (rescrit de Sếvère et Caracalla) ; $D$ 40.1.5 pr (constitution de MarcAurèle et Verus)-1 ; D 40.4.26 (rescrits d'Antonin le pieux et de MarcAurèle et Verus); $D$ 48.2.13 ; D 48.10.1.8 ; D 48.10.7 (rescrit de MarcAurèle et Commode) (par a contrario); D 48.12.1; D 49.14.30 (rescrit de Sévère et Caracalla) ; Modestin D $5.2 .12 \mathrm{pr}^{-1}$; D $16.1 .25 \mathrm{pr}-1$; D 22.5 .7 ; D 24.3.58; D 26.7.32.3; D 27.1 .14 .3 ; D 28.1 .19 ; D 28.1 . $28 ; \mathrm{D} 28.6 .3 ; \mathrm{D} 28.7 .25 ; \mathrm{D} 29.2 .50 ; \mathrm{D} 34.1 .4 \mathrm{pr} ; \mathrm{D} 34.3 .19 ; \mathrm{D} 35$. 1.51 .1 ; D 35.1.53;D 35.1.66; D 40.7.26 pr-1;D 40.7.27 (Julien); D 45.3.35;D 48.4.7.2 ; I. Aquila D 26.7.34; D 26.10.12 ; Hermogênien D 5.1.53; D 35.1.94 pr-1; D 37.14.21.3; D 41.1.61 pr-1; D 41 2.50.1; D 49.14.46.8; A. Charisius D 48.18.10.4; Auteur anonyme D 36.4.16. Certains textes font apparaître l'esclave à la fois come

ol l'esclave apparaît à propos d'un acte juridique, par périodes, on parvient au tableau suivant :

\section{L'esclave agent}

d'acte juridique

- analyse des paragrapbes

$\begin{array}{lcll}\text { I } & - & =- \\ \text { II } & 5 / 8 & =62,5 \% \\ \text { III } & 370 / 868 & =43 \% \\ \text { IV } & 136 / 279 & =49 \% \\ \text { V } & 1032 / 2456 & =42 \%\end{array}$

TOTAL $1543 / 3611=43 \%$

- analyse des citations

$\begin{array}{lrl}\text { I } & 42 / 105 & =40 \% \\ \text { II } & 133 / 294 & =45 \% \\ \text { III } & 194 / 441 & =44 \% \\ \text { IV } & 42 / 81 & =52 \% \\ \text { V } & 6 / 22 & =27 \% \\ \text { TOTAL } & 417 / 943 & =44 \%\end{array}$

Il faut une fois de plus noter que les pourcentages obtenus pour la période II dans 1'analyse directe, reposant sur un fondement numérique insuffisant, ne sont pas pertinents. Ils sont d'gilleurs démentis par ceux que nous fournit l'analyse des citations. Ces demiers marquent, conformément à l'étude qui a été menée sur le pécule et plus généralement l'esclave travailleur, que le lersiècle voit croître l'intérêt des juristes pour le rôle d'agent juridique joué par l'esclave.

Cependant, le résultat fondamental auquel nous permet de parvenir ce tableau coneerne la période IV. Cette période se détache en effet irrésistiblement des autres. Dans l'analyse djrecte, on obtient pratiquement un équilibre entre les deux rubriques, le léger avantage des

objet et come agent (par exemple Iavolenus D 31.38 ou Papinien D 17. $1.54 \mathrm{pr}$ où les deux rôles juridiques sont joués par le même esclave ou bien, Ulpien (Julien) D $24.1 .19 \mathrm{pr}$, où les deux rôles sont distincts (servus anciltan acceperit), mais cette espèce est en rọalité plus complexe ; il y est question d'une femme qui fait donation d'un esclave (objet) ; celui-ci (agent) acquiert une ancilia (objet); autrement dit, les rôles (agent) acquiert une ancilla (objet); Ces textes ont été relevés dans les deux rubriques (agent/objet). 
textes concernant l'esclave objet disparaissant dans l'analyse des citations, et se renversant au profit de ceux qui traitent de l'esclave en tant qu'agent d'acte juridique. La période $\Gamma V$, qui correspond aux règnes des derniers Antonins, constitue un tournant important quant à l'image que se font les juristes de l'esclave, et plus globalement quant ̀̀ son utilisaticn. Ce résultat confirme donc pleinement nos conclusions antérieures.

Après cette période, point culminant dans le développement d'une capacité de l'esclave, on en revient à des pourcentages "normaux". La prestation de le période $V$ dans $I$ 'analyse des citations manque de pertinence pour les mêmes raisons que celles exposées plus haut à propos de la période II. L'analyse des paragraphes, reposant sur une base numérique imposante, fournit en revanche des indications beaucoup plus fiables. On relève que le pourcentage réalisé par cette période est sensiblement du même ordre que celui de la période III, correspondant eux règnes des premiers Antonins. Ainsi, malgré la baisse d'intérêt que connaissent alors les ituristes dour l' esclavake (641), ils continuent à se préoccuper de manière jmportante de ses formes les plus achevées.Cela confirme aussi les résultats auxquels nous sommes auparevant parvenu.

C'est là la constatation fondementale. Cependant, si au-delà de ces considérations chronologiques, on se penche sur les deux rubriques de façon globale, on remarque que l'esclave est plus souvent objet qu'agent d'actes juridiques, et ce pour les deux analyses. En est-il de même en matière de faits juridiques ?

\section{Peragraphe 2 : Esclave et faits juridiques}

Attachons-nous à répondre à cette question avant d'envisager plus en détail la situation de l'esclave au regard des différents délits. Opérons de la même manière en distinguant esclave agent (642)

\section{1- Cf. Supra les conclusions de Chapître préliminaire.}

642- Alfenus (chez auteur anonyme) D 9.2.52.1 ; D 11.3 .16 ; D 44.7. 20 ; Iavolenus D 9.2.38; D $11.1 .14 \mathrm{pr}$; D 41.2 .24 ; Neratius D 40.7 . 17 ; D 47.2.65; D 47.2.84.1 ; Celsus D 13.1.15; D 46.3.69; D 47.2. E8.3-4; Julien D 9.4.16; D 9.4.39 pr-1-2-3-4; D 9.4.40; D 9.4.41; D 12.5 .5 (Proculus) ; D 13.6.20; D 17.1.30; D 39.6.18.3; D 41.1. 37.2 ; D 41.3 .33 .6 ; D 41.4 .9 ; D $41.4 .10 ;$ D $43.24 .14 ;$ D 47.2 .57 .
3-5 ; D 47.6.2 ; Pomponius D 9.4.18; D 9.4.33; D 9.4.43; D 10.2.45. 1 ; D 12.4 .15 (Labéon, Proculus) ; D 15.1.4.2-3-4; D 15.1.23; D 21.1 46 ; D 21.2.30; D 30.45.1 ; D $30.48 \mathrm{pr}$ (Atilicinus) ; D $40.7 .29 \mathrm{pr}$; D 40.12.43 (rescrit d'Hadrien) ; D 43.24.21.1; D 46.3.19 (Labéon); D 47.2.35.1; D 47.2.44.2; Africain D 3.5.48; D 9.4.28; D 13.6.21. I ; D 13.7.31 (Julien); D 19.1.30 pr; D 30.110; D 33.8.16 pr ; D 40 . 4.22 ; D 47.2.61 ; D 47.2.62 pr-1-2-3-4-5-6-7-9; Gaius D 2.9.4 (Sabinus) ; D $5.3 .39 \mathrm{pr}$; D $9.2 .4 \mathrm{pr}-1$; D $9.2 .32 \mathrm{pr}$; $\mathrm{D} 9.4 .1 ; \mathrm{D} 9.4 .13$; D 9.4 .15 (Sabinus, Cassius) ; D $9.4 .20 ; \mathrm{D} 9.4 .23 ; \mathrm{D} 9.4 .25 ; \mathrm{D}$. 4.27 pr-1 ; D 9.4.29; D 9.4.30; D 15.1 .27 .1 ; D 29.5.25 pr-1; D 30 , $4.27 \mathrm{pr}-1$; D 9.4 .29 ; D 9.4 .30 ; D 15.1 .27 .1 ; D $29.5 .25 \mathrm{pr}-1$; D 30.
$67 \mathrm{pr} ; \mathrm{D} 30.70 \mathrm{pr}-2-3$; D 39.4 .2 ; D $39.4 .13 .2-3$; D $40.12 .13 \mathrm{pr}-1$; D 41.2.15; D 44.7.5.5; D 47.4.2 ; D 47.10.34; Maecianus D 29.5.14; D 40.5.35 (Cassius); Venuleius D 29.5.13; D 48.2.12.4; D 48.19.16.3 Marcellus D 29.5.16 ; D 45.1.96 ; D 46.3.72.5 ; D 47.6.5 ; Scaevola D 40.5.41.11 ; D 40.7.40 pr-4 ; D 47.6.6 (Labéon) ; Papinien D 1.21.1 pr ; D 19.5.8 ; D 29.5.4; D 29.5.21 pr ; D 35.2.11.4 ; D 40.5.23 pr ; D $43.10 .1 .2 ; \mathrm{D} 48.1 .14 ; \mathrm{D} 48.3 .2 \mathrm{pr}-2$; Tryphoninus D 9.4 .37 ; 11 4.5 (rescrit d'Antonin le pieux) ; D 40.4.59.1 (Scaevola); D 50.16.225; Callistrate D 9.4.32; D 47.9.7; D 47.21.3.1 (L'empereur Nerva) ; D 48.10.15.1-2; D 48,19.28.11; Faul D 1.15.3.5 ; D 2.1.9 (Pouponius; Octavenus); D 2.10.2 (Ofilius, Sabinus); D 2.9.2 $\mathrm{pr}^{-1}$ (V. Verus, Julien, Pomponius) ; D 2.9.6; D 3.2.14; D 3.3.36; D 3.5.40; D 4.4. 24.3 ; D 4.9.6.1-4; D 5.1.24.1-2 (Cassius, Julien) D 5.3.30.4 ; D 6 1.58 ; D 7.4.27; D 9.2.30 pr ; D 9.2.45; D 9.2.48; D $9.4 .4 \mathrm{pr}-1$ $-2-3$; D 9.4.9; D 9.4.10; D 9.4.12; D 9.4.17 pr-1 ; D 9.4.26 pr-1-2-3-4 -5(Labéon, Octavenus)-6;D9.4.19 pr-1-2;D9.4.22 pr-i-2;D9.4.24 (Labéon, Julien); D9.4.31 (Sabinus, Cassius, Jul ien, Pomponius);D 10.2.25.15; D10.3.8.3; D10.3.15;D10.4.16;DII.1.8;DI1.1.20 pr;DI 1 .3.14.2(Sabinus)-3-6-7;D12.6.36; D 13.6 .22 ; D 17.1 .26 .7 (Neratius) ; D 19.1.4 pr; D 19.2.30.4; D 19.2.45 pr-1; D 19.4.2 (Aristo) ; D 21.1.43.4; D 21.1.58 pr-1; D 21.2.3; D 21 2.11 .1 ; D 25.2.3.1 ; D 25.2.21.1 (Pedius) 2 ; D 29.5.22; D 33.8.9.1 (Marcellus); D 39.3.11.3 (Julien); D 35.2.39; D 40.1.9; D 40.1.12; D 40.12.24.4; D 40.12.41.1; D 41.3.4.8-9 (Pomponius)-16 (Sabinus, Cassius) ; D 43.1.5 ; D 44.7.49 ; D 47.2.18 (cassiani) ; D 47.2.42 pr-1 (Sabinus) ; D 47.2.54.I-2-4 (Julien) ; D 47.8.3 (Labéon) ; D 47. 9.4.1 (rescrit d'Antonin le pieux) ; D 47.10.18.1; D 47.10.22.9; D 47.11.1.2 ; D 50.16.215 (Sabinus, Cassius); Ulpien D 1.12.1.5 ; D 2. 1.7.1-3; D 2.7.1.1 (Pomponius) ; D 2.9.1 pr-1 (Ofilius, Sabinus); D 2.9.3;D 2.9.5;D 2.10.1.5;D2.14.50; D 3.2.4.3 (Pomponius); D 4.2 .16 .1 ; D 4.3.7 pr (Julien); D 4.3.9.4 (Labê̆on); D $4.4 .11 \mathrm{pr}$ ( $4.2 .16 .1 ; \mathrm{D} 4.3 .7 \mathrm{pr}$ (Julien) ; $\mathrm{D} 4.3 .9 .4$ (Labêon) ; $\mathrm{D} 4.4 .11 \mathrm{pr}$ (Sévère) ; D 4.9.3.3 ; D 4.9.7 pr-3-4-6; D 7.1.17.2 ; D 9.1.1.14; D 9.2 .11 .4 ; D 9.2 .5 pr ; D 9.2.23.4 (Labéon) ; D 9.2 .27 pr (Celsus, Julien)-1 (Proculus, U. Ferox)-2 (Celsus)-3 (Julien, Marcellus)-11 (Proculus) ; D 9.2.44.1 ; D 9.3.1 pr ; D 9.3.5.6-9; D 9.4.2 pr-1 (Ce1sus, Julien, Marcellus) ; D 9.4 .3 ; D $9.4 .5 \mathrm{pr}-1$; D 9.4 .6 ; D $9.4 .7 \mathrm{pr}^{-}$ 1 (Pomponius) ; D 9.4.8; D 9.4.11; D 9.4.14 pr-1; D 9.4.21 pr-1-3 $-4-5-6$ (Sabinus, Neratius) ; D 9.4 .36 ; D $9.4 .38 \mathrm{pr}-2-3_{0}$; D 9.4 .42 pr-1-2 ; D 10.2.16.6 (Ofilius) ; D 10.2.18 pr (Julien) ; D 10.4.3.7; D 10.4.20; D 11.1.16 pr; D 11.3.1.3-4-5; D 11.3.5.3; D 11.3.11 pr (Neratius) $-1-2$ (Julien); D 11.6.3.6 (Pomponius); 12.5 .4 .4 ; D 13.1 .4 ; D 13.7.24.3 ; D 14.3.5.8 (Labéon)-9 (Labéon); D 15.1.3.12 (Labéon); D 15.1.9.1 (Neratius, Pomponius) -6 ; D 15.1.11 pr ; D 16.3.1.18 (Marcellus); D 16.3.11 (Sabinus); D 18.1.29; D 19.1.11.8 (Neratius)-12 (Neratius) ; D J9.1.13.1-2-3-5 (Julien) ; D 19.2.11 pr (Pomponius); D 21.1.1.1; D 21.1.17.17-1820 21.1.19.1; D 21.1.21.1 (Pomponius); 
D 21.1.23.2 (Pomponius)-8 (Julien); D 21.1.25.4 (Pedius)-7; D 21.1 . 29.3 (Julien); D 21.1.31 pr-1; D 21.2.31; D 27.3.1.3 (décret de SEvvère) ; D 29.5.1 pr (par extension) -5 (rescrit d'Antonin le pieux) $-18-21-22-26-27$ (Sextus) -28 (rescrit d'hadrien) -29 (idem)-30-31-3436-37; D 29.5.3.1-2-4-6-12-14-18; D 30.53.3-4-8; D 39.3.6.7 (Ce1sus) ; D 39.4.1 pr-5-6 ; D 39.4.3 pr-1-2-3; D 39.4.12.1-2 ; D 40.7 . 3.9 ; D $40.7 .9 \mathrm{pr}-2$ (Octavenus) ; D 40.9.12.6 (Africain) ; D 40.12.7.4; D 40.12 .12 .6 ; D 42.1.4.8; D 42.5.9.3; D 43.16.1 pr-11-12-15-1617-18-19-21;D $43.16 .3 .11 ; D 43.24 .5 .6$; D 43.24.7.1 (Neratius): D 43.24.11.7; D 44.7.14; D 46.3.18; D 47.1.2.3; D 47.2.7 Pr (Pơr D 43.24 .11 .7 ; D 44.7 .14 ; D 46.3 .18 ; D 47.1 .2 .3 ; D 47.2 .7 Pr (Pour ; ponius) ; D $47.2 .17 \mathrm{pr}$ (veteres)-1-2 (Sabinus) -3 (Julien, Pomponius);
D 47.2.36.1-2 (Sabinus, Pomponius)-3 (Sabinus); D 47.2.43.12 ; D 47. D 47.2.36.1-2 (Sabinus, Pomponius) -3 (Sabinus) ; D 47.2.43.12 ; D 47 .
$2.52 .3-4-9-15-23$ (Mela)-24 D 47.4.1 pr-1 (Labéon)-2-3-4-5-6-7 (res$2.52 .3-4-9-15-23$ (Mela)-24 i $\mathrm{D} 47.4 .1 \mathrm{pr}-1$ (Labéon)-2-3-4-5-6-7 (res-
crits de Marc-Aurèle et de SEvère et Caracalla)-8-9-10 (Scaevola)-11-12-1314-15(Scaevola)-16-17;D47.4.3 (Labéon);D47.5.1.5;D47.6.1 pr-1-2-3;D47.6.3 pr-1-2;D47.6.4;D47.7.7.4-5(Sabinus);D47.8.2 pr-5-14-15-16-19;D47 :8.4.15: D47.9.3.7 (Labéon, Celsus) ; D47.10.9.3;D47.10.17.3-4-5-6-7 (Labéon) -8-9 ; D 47.12.3.11 ; D 48.2.5 (rescrit de Marc-Aurèle) ; D 48.2.6 ; D 48.2. 7.4 (rescrit d'Antonin le pieux) ; D 48.5.28 pr-1-2-3-4-16;D 48.8.4.2 (rescrit d'Hadrien); D 48.10.8; D 48.18.1.5 (rescrits d'Hadrien et d'Antonin le pieux)-19 (rescrit de Trajan) ; D 48.19.1.1 ; D 48.19.6.1; D 50.16 .174 ; D 50.16.195.3; D 50.17.157 pr ; Macer D 48.5.25 pr ; D 48.7.3; D 48.10.10 pr ; Marcien D 21.1 .52 ; D 28.5.49.2 (constitution de Sévère et Caracalla) ; $D$ 39.4.16.1 (rescrit de Sévère et Caracalla)-11 (rescrit de Caracalla) ; D 48.5.34 pr (rescrit d'Antonin le pieux)-1 ; D 48.6.3 pr ; D 48.8.3.4; D 48.10.1.13; D 48.19.11.1 ; Modestin D 29.5.19; D 48.2.17 ; D 48.18.14 ; Hermogénien D 47.10.45. Malgré ce passage d'Africain (D 47.2.61 ; Ancilla fugitiva quemadnoçum sui furtum facere intellegitur) où le juriste assimile la fuite au furtum il nousa paru plus à propos de considérer les textes sur la

fuite séparément dans une étude sur les manifestations de lutte des esclaves. De même, les textes visant seulement la répression seront vus plus loin.

643- Alfenus (chez auteur anonyme) D 9.2.52 pr-1; D 11.3 .16 ; Iavolenus D 9.2.38; D 24.1.50 pr-1 ; D 47.2.75 ; D 49.15.27 (Trebatius, Ofilius, Labéon) ; Celsus D 47.2.68.2-4; Julien D 1.5.26; D 9.2.47; D 9.2.51 pr-1-2 ; D 13.1.14 pr ; D 19.1.24.1 (Sabinus, Cassius) ; D 41. 1.39; D 41.3.33 pr ; D 41.3.35 (Sabinus) ; D 41.4.7.7; D 41.4.9; D 41 . 4.10 ; D 45.3.14 (Sabinus) ; D 46.3.33.1 ; Pomponius D $21.1 .48 \mathrm{pr}$; D 21.2.16.2 (Proculus); D 21.2.34 pr; D 26.7.61; D 30.38.1; D 41 . $10.4 \mathrm{pr}$ (Trebatius); D 44.7.56 ; Africain D 30.108.11; D 47.2.61 ; Gaius D 6.1.36.1 ; D 9.2.2 pr ; D 9.2.32.1 ; D 11.3.15 ; D 41.3.36. I ; D 41.3.37 pr ; Venuleius D 48.8.6 ; Marcellus D 9.2.34; D 9.2.36 $\mathrm{pr}-1$ (Sabinus) ; $\mathrm{D} 46.3 .72 .3$; Papinien D 13.1.17; D 18.7.6 $\mathrm{pr}$ (Sabinus); D 21.2.64 pr ; D 46.3.95.1; D 47.2.81 pr-1-2 ; D 48.5.6 pr ; Binus Trencesit d Hadrien) $7.66 ; 0$; Pam ponius) ; D 7.1.66; D 9.2.18; D 9.2.22 $\mathrm{pr}^{-1}$; D 9.2.24; D 9.2.26; D 9.2.301-4; D 9.2.33 $\mathrm{pr}$ (Pedius) ; D 9.2.45 $\mathrm{pr}^{-1-2} ; \mathrm{D} 9.2 .55 ; \mathrm{D} 9$. 4.10; D 10.3.8.2; D $11.1 .20 \mathrm{pr}$; D 11.3 .2 ; D 11.3 .4 ; D 11.3 .6 ; D 11.3 .8 ; D 11.3 .10 ; D 11.3 .12 ; D $11.3 .14 \mathrm{pr}-1-2$ (Sabinus) -3-4-5-6-7 -8-9; D 12.1.31.1 (Sabinus, Cassius, Julien); D 12.2.30.1 ; D 13.1. 3 (Julien) ; D 13.1.13 ; D 17.1.22.6-9; D 17.2.56; D 18.1.56; D 18. 7.9 ; D 19.2.42; D 19.2.43; D 21.1.43.2-5; D 24.1.28.1; D 33.8.9.1
L'esclave agent

de fait juridique

- analyse des paragraphes

L'esclave objet

de fait juridique

\begin{tabular}{|c|c|c|c|c|c|}
\hline I & - & $=-$ & I & - & $=$ \\
\hline II &.- & $=$ & II & - & $=$ \\
\hline III & $98 / 135^{\circ}$ & $73 x$ & III & $37 / 135$ & 27 \\
\hline IV & $13 / 18$ & $72 \%$ & IV & $5 / 18$ & 28 \\
\hline v & $375 / 656$ & $=57 x$ & v & $280 / 656$ & $=43 x$ \\
\hline TOTAL & $487 / 809$ & $=60 \%$ & TOTAL & $322 / 809$ & $=$ \\
\hline
\end{tabular}

Marcellus) ; D 36.1.68.2 ; D 37.15 .6 ; D 40.8 .7 (constitution de Sévère et Caracalla) ; D 41.3.4.15-16 (Sabinus, Cassius)-17-18; 42.2.4 ; D 43. 16.6 ; D 44.7.34 pr ; D 45.1.83.7; D 45.1.88; D 47.2.54.4 (Julien) ; D 47.2.67.5 ; D 47.2.83.2; D 47.2.86; D 47.10.4; D 47.10.16 (Pedius); D 47.10 .18 .1 ; D 47.10 .26 ; D 47.10.29; D 47.11.1.2; D 48.7.4.1 (Labéon); Ulpien D 1.12.1.8; D 2.4.10.1 ; D 2.14.50; D 3.2.4.2-3 (Pomponius) ; D 3.2.24 (SÉvère) ; D 6.1.17.1 (Julien) D 6.2 .11 .4 (Ju(Pomponius); D 3.2.24 (Sêvère) ; D 6.197 (Julien) $\mathrm{D} 6.2 .11 .4$ (Julien) ; D 7.1.17.3 ; D 9.2 .3 ; D $9.2 .5 \mathrm{pr}-3$ (Julien) ; D $9.2 .7 .1-3$
culus) $-4-5$ (Labéon) -6 (Celsus)-7 (Celsus); D 9.2 .9 .2 (Neratius) -3 (Ofilius) -4 ; D 9.2.11.1-2 (Julien) -3 (Cel sus, Marcellus) $-6-7$ (Julien) -8 -9 (Julien)-10 (Julien) ; D 9.2.13 pr-1 (Julien)-2 (Celsus)-3 ; D 9.2. 15 pr-1;D9.2.19(Celsus);D9.2.21.1 (Celsus, Julien) -2;D9.2.23pr(Neratius) -1

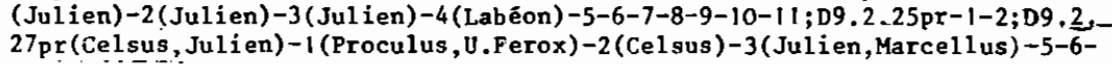

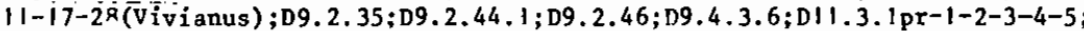
D $11.3 .3 \mathrm{pr}-1$; D $11.3 .5 \mathrm{pr}-1-2-3-4$; D 11.3 .7 ; D $11.3 .9 \mathrm{pr}$ (Julien) $-1-2-3$ (Neratius); D 11.3 .11 pr (Neratius) $-1-2$ (Julien); D 11.3 .13 . $\mathrm{pr}-1$; D $11.4 .1 \mathrm{pr}-2$ (epistula de Marc-Aurale et Commode) ; D 12.4.5. 4 ; D 13.1.7.2 ; D 13.3.3 ; D 15.1 .9 pr-1 (Neratius, Pomponius) ; D 17.2.51.1; D 19.1.13.5 (Julien); D 19.5.14.1; D 21.1.17.7; D 21 . $1.23 \mathrm{pr}$ (Pomponius); D $21.1 .25 \mathrm{pr}-1$ (Neratius)-4 (Pedius)-7-8; D 21.1 31.6 (Pomponius) -9 (Pomponius)-11 ; D 24.3.21; D 30.53.8-9; D 41.1 . 22 ; D 41.3.10.2 (Marcellus, Scaevola) ; D 42.5.9.5; D 43.16.1.34 (Julien)-46 (Vivianus) ; D 45.1.29.1 (Marcellus); D 47.1.2.1-2-3-4-5-6 ; D 47.2.14.5 (Papinien)-6 (Pomponius, Papinien)-7 (Pomponius, Papinien) -11 ; D 47.2.36 pr (Pomponius); D 47.2.39; D 47.2.46 pr-1-2-3-4-5; D $47.2 .48 .2-3-5 ;$ D 47.2 .52 .23 (Mela) $-24-28-29 ; D$ 47.4.1.11; D 47 . 8.2.18; D 47.8.4.13-14; D 47.10.1.3-6 (Julien)-7 (Labéon); D 47.10. 3.4 ; D 47.10.7.1; D 47.10.9.4; D 47.10.11.9; D 47.10.13 pr; D 47 10.15.15-16-34-35-36-37-38-39 (Labéon) -40-41-42 (Labéon)-43-44-45 (Mela)-46 (Labéon)-47-48-49; D $47.10 .17 \mathrm{pr}-1-2$ (Trebatius, Mela); D 47.10 .25 ; D $47.10 .30 \mathrm{pr}$; D 47.11 .5 ; D 48.8 .4 .2 (rescrit d'Hadrien) ; D 48.15.2 pr-1-2-3 ; D 50.16.26 (Scaevola) ; Marcien D 11. 3.17 ; D 22.1.32.5 ; D 47.2.63; D 40.8.6 ; D 48.8.1.2 ; D 48.15.3 pr (constitution de Sévère et Caracalla)-1 (rescrit des mêmes); Modestin D 37.14.1 pr (décret de Vespasien) ; D 48.15.5 ; Hermogénien D 48.15 .7 . 
- analyse des citations

\begin{tabular}{|c|c|c|c|c|c|}
\hline I & $9 / 18$ & $50 \%$ & I & $9 / 18$ & $50 \%$ \\
\hline II & $43 / 67$ & $64 \%$ & II & $24 / 67$ & $36 \%$ \\
\hline III & $53 / 105$ & $50 \%$ & III & $52 / 105$ & $50 \%$ \\
\hline IV & $7 / 14$ & $50 \%$ & IV & $7 / 14$ & $=50 \%$ \\
\hline v & $-/ 3$ & $=$ & v & $3 / 3$ & $=100 \mathrm{~m}$ \\
\hline
\end{tabular}

TOTAL $112 / 207$

Considéré de manière globale, ce tablear montre une prépondérance de la rubrique "esclave agent de fait juridique" par rapport, à la rubrique "esclave objet de fait juridique". C'est déjà un point important; on a obtenu un résultat analogue avec l'étude des mots servus et, surtout, forilia. On se méfie de l'esclave, il fait peur... Et il n'est pas moins intéressant de constater que, selon l'analyse directe, cette crainte liée à l'esclave délinquant est la plus forte sous les Antonins, précisément à une époque où le développement d'une certaine capacité de l'esclave connâ̂t ses formes les plus avancées. On a vu que cette reconnaissance partielle était due à des nécessités économiques, mais ce n'est qu'une partie de l'explication. La présente analyse permet d'aller plus loin en montrant que cette évolution est aussi liée à un besoin de libéralisation. On revienura sur co point, en étudiant les comportements des esclaves, mais on peut dire dès maintenant qu'il và catégoriquenent à l'encontre de la thèse qui voudrait que les mesures prises par les Antonins en faveur des esclaves soient principalement d'origine humanitaire (644).

Cette iđée à laquelle l'analyse quantitative nous permet de parvenir ne nous autorise cefendant pas, malgrá son iuportance, à négliger les aspects plus détaillés de la question.

\section{I - L'esclave objet de fait juridique}

Outre les agressions d'ordre sexuel, dont il a déjè été traité (645); il est possible de ranger les délits dont l'esclave peut être victime, dans trois ruoriques $(646)$ : appropriation punissable,

644- H. WALLON, Histoire de l'esclavage, cit. III, p. $48 \mathrm{~s}$. Sous 1 es Sévères, on remarquera que la délinquance ressort de manière plus faible. Cela ne signifie pas qu'il y ait pour autant rêduction des tensions entre maîtres et esclaves, la délinquance ne constituant pas en soi un moyen de lutte. De nombreux textes concernent ainsi le vol, considéré par les juristes comme un mal quasi institutionnel (cf. Ulpien D 47.5.1.5).

645- Cf. supra, 1'étude sur les pratiques sexuelles : en particulier les notes $463,464,465,481,482$ et 489.

646- Elles s'inspirent des catégories de Th. MOMMSEN, Droit pénal, cit. détérioration et injure.

Il faut en premier lieu distinguer trois modalités d'appropriation répréhensible : le furtion (647), la rapina (648) et le plagizm (649).

Le furtion, déjà prévu par la loi des XII tables (650), va faire l'objet d'une lente construction jurisprudentielle (651). La

647- Les textes du Digeste où l'esclave est objet d'un furtum sont 647- Les textes du Digeste ou 47.2.68.2-4; Julien D 1.5.26; D 13.1. Iavolenus D 47.2.75 ; Celsus D Cl.4s); D $41.1 .39 ; 041.3 .33 \mathrm{pr}$; $14 \mathrm{pr}$; D 19.3 (Sabinus); D 41.4.7.7; D 41.4.9; D 41.4.10; D 45.3.14 (Sa-

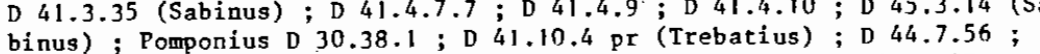
Africain D 47.2.61 ; Gaius D 41.3.36.1 (par extension); D $41.3 .37 \mathrm{pr}$ (idem): Marcellus D 46.3.72.3; Papinien D 13.1.17; D 47.2.81 pr-1-2 ; Callistrate D 48.15.6 pr (rescrit d'Hadrien); Paul D 12.1.31.I (Sabinus, Cassius, Julien) ; D 12.2.30.1 ; D 13.1.3 (Julien) ; D 13.1.13; D 17.1. 22.9; D 19.2.42; D 24.1.28.1; D 41.3.4.15-16 (Sabinus, Cassius)-17-18; D 45.1.83.7; D 47.2.54.4 (Julien) ; D 47.2.67.5;D 47.2.83.2; D 47.2. 86 ; 36; D 11.4.1 pr; D 13.1.7.2; D 17.2.51.1; D 19.1.13.5 (Ju1ien) ; D 24. 3.21; D 41.3.10.2 (Marce11us); D 45.1.29.1 (Marce11us) ; D 47.1.2.1-3 3.21 ; D 41.3 .10 .2 (Marcellus);
$-4-5-6$; D 47.2 .14 .5 (Papinien)-6 (Pomponius, Papinien) -7 (1es memes)
-11 ; D $47.2 .36 \mathrm{pr}$ (Pomponius)-3 (Sabinus); D 47.2 .39 ; D $47.2 .46 \mathrm{pr}$ $-11 ; \mathrm{D} 47.2 .36 \mathrm{pr}$ (Pomponius)-3 (Sabinus) ; D 47.2 .39 ; D 47.2.46 pr
$-1-2-3-4-5 ; \mathrm{D} 47.2 .48 .2-3-5$; D $47.2 .52 .12-23$ (Me1a) $-28-29-$; D 47.4 .1 . 11 ; D 47.10 .25 ; D 50.16.26 (Scaevola); Marcien D 47.2.63. I1 faut assimiler au vol les textes où on voit apparaitre la notion de recipere ¥ propos de l'actio servi corrupti (cF. G. SAUTEL, Actio servi cormpti, in Iura 2 (1960) p. $247 \mathrm{~s}$. qui constitue un c.r. de B. ALBANESE, Actio in Iui corroti, in AUPA 27 (1959), 1-152); il s'agit de U1pien D 11 servi cormeti, in AupA 27 (1959)

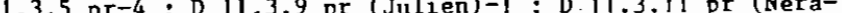
tius)-2 (Ju1ien).

648- Il faut relever Iavolenus D 49.15.27 (Trebatius, Ofilius, Labéon) (par eripuerant) ; Tryphoninus D 43.16.19 (Julien) ; Paul D 21.1.43.5. D 43.16.6; U1pien D $41.122: 043.16 .1 .34$ (Julien) $46, D 47.1 .2 .2$; D $47.2 .39 ; 047.8 .2 .18$.

649 - Callistrate D 48.15.6 pr (rescrit d'Hadrien)-1-2 ; Pau1 D 47.2. 83.2; U1pien D 17.2.51.1 ; D 21.1.17.7; D 47.2.39; D 47.2.48.2; D 48 . $15.2 \mathrm{pr}-1-2-3$; D $48.15 .3 \mathrm{pr}$ (constitution de Sévère et Caracal1a)-1 (rescrit des (âmes); Modestin $\mathrm{D} 48.15 .5$, Hemogénien D 48.15.7. Il faut (rescrit des aussi sigaler, bien que le mot plagium a aparaisse pas, ces deux textes d'Ulpien (D 11.4.1 pr-2 (epistula de Marc-Aurale et Comode) ou il est question de la répression du fait de recueillir chez soi des esclaves fugitifs .

650- A. GUARINO, Diritto privato romano, cit., p. 875. On trouve sur le furtum une abondante littérature : HUVELLIN, Ftudes sur le furthem dans le furtum une abondante littérature : HUVEL.IN, tudes sur a flr then dans le très ancien droit romain, Les sources (1915) ; W.W. BUCKLAND, D 47.2
(De furtis) and the methods of the compilers, in T. $10(1930), \mathrm{p} .117 \mathrm{~s}$.; JOLOWICZ, Digest XIVII 2 de furtis (1940); OLIVECRONA, D 47.2.21, Some question concerning furtum, in Three essays in roman 1 aw (1949) ; J. GAUDEMET, A propos du furtion à l'époque classique, in Labeo 7 (1961), p. 7 в. ; U. BRASIELlO, Furto, (dir. rom.) in NNDI ; B. ALBANESE, Furto (intr. stor.), in ED.

III, dont 1 'ouvrage reste fondamental sur le problème. 
dérinition qui semble refléter le mieux la commonis opinio classique nous est donnée par Paul :

Prom tom est contrectatio rei fraudulosa lucri faciendi gratia vel ipsius rei vel etian ueus eius possessionisve (652).

La rapina est une forme aggravée du vol, c'est le vol avec violence (653). Ce délit troure son origine dans l'édit qu'introduisit en 76 av. J.C. le préteur pérégrin Lucullus, en vue de réprimer les actes de brigandage commis au moyen d'hommes armés ou en bandes, hominibus armatis coactisve (654).

Contrairement au furtum et à la rapina, qui constituent des délits privés du ire civiles le plagion est un crimen, un délit public (655). Il consiste en une appropriation du pouvoir dominical (656). La lex Fabia de plagiariis punit l'instigation à la fuite de l'esclave d'autrui, ainsi que le rait de recueillir chez soi un tel esclave (657).

651- B. ALBANESE, La nozione del frortum fino a Nerazio, in AUPA (1953) et la nozione del furtum da Nerazio a Marciano, in AUPA (1957); cr de J. GAUDEMET, in Iura 2 (1958), p. $192 \mathrm{~s}$.

652- Paul D 47.2.1.3.

653- A. GUARINO, Diritto privato romano, cit., p. 881 ; U.. BRASIELLO, Rapina (dir. rom.), in NNDI ; $M$. BALZARINI, Ricerche in tema di danno violento e rapina nel diritto romano (1969) ; VACCA, Ricerche sulla rapina nel diritto romano, 1 - L'editto di Lucullo e la lex Plautia, in SUC (1965-68) ; U.EBERT, Die Geschichte des Edikts de hominibus armatis coactisve (1968).

654- J. MACQUERON, Histoire des obligations, cit., p. 323. Le préteur a aussi prévu une actio in duplum a l'encontre de ceux qui auraient profitê d'un attroupement (turba) pour conmettre un vol ou causer un dommage à quelqu'un (cf. A. GUARINO, Diritto privato romano, cit., p. 890) Dans ce deuxième sens, il ne s'agit plus d'un vol à proprement parler, mais d'une détérioration (cf. par ex. D 47.8.4.13-14 Ulpien).

655- A. GUARINO, Diritto privato romano, cit., p. 871 ; A. BERGER, Encycl. Diction., cit., p. 552.

656- Th MOMMSEN, Droit pénal, III, cit., p. 90, $92 \mathrm{n} .1$ : d'après C. Th. 9.20.1 (- C.I.9.31.1), en cas de vol d'esclave, on peut agir au civil par 1 'actio furti ou au criminel par 1 'action fabienne. Hadrien rappelle (chez Callistrate D $48.15 .6 \mathrm{pr}$ ) que tout vol d'esclave n'est pas un plagizm; 1a seule différence quant aux élêments du délit consiste dans le fait que le frotum usus n'est pas compris dans le plagivon (cf. Paul D 47.2.83.2).

657- G. LONGO, Lex Fabia de plagiariis, in NNDI ; A. BERGER, Encycl. Diction., cit., p. 552. Du point de vue de 1 'instigation a la fuite, cette lex présente une certaine analogie avec 1 'actio servi compti. Sur la date incertaine de la ler Fabia et son application l'appropriation dolosive d'individus nés libres, cf. supra première partie n. 205 à 212 .
Il est intéressant à ce propos de noter que le plagium pourait s'opsrer avec l'accord de l'esclave (658), ce qui donne une coloration particulière à ce délit.

En second lien, la détérioration de l'esclave peut revêtir deux formes : le daman iniuria datim (659) et la servi cormptio (660).

Le damnon iniuria datum, littéralement domage causé injustement, voit sa répression assurée, au début du ße siècle av. J.C., par la lex Aquilia (661) qui protège le maître en cas de meurtre de l'esclave

658- Th. MOMMSEN, Droit penal, cit., III, p. 91 ; cf. D 21.1 .17 .7 Ulpien.

659- On relève dans le Digeste Alfenus (chez auteur anonyme) D 9.2. 52 pr-1 ; Lavolenus D 9.2.38; D 24.1.50.1; Julien D 9.2.47; D 9.2.51 pr-1 (veteres)-2 ; D 46.3.33.1 ; Pomponius D 26.7.6.1 ; D 44.7.56; Gaius D 6.1.36.1; D 9.2.2 pr;D 9.2.32.1 ; Marcellus D 9.2.34 ; D 9$2.36 \mathrm{pr}-1$ (Sabinus); Papinien D 46.3.95.1; D 48.5.6 pr ; Paul D 4.3. 18.5 ; D 4.9.6.2 (Pomponius) ; D 9.2.22 pr-1 ; D 9.2.24; D 9.2.26 ; D 9.2.30.1-4; D 9.2.33 pr (Pedius) ; D 9.2.45 pr-1-2 ; D 9.2.55; D $11.1 .20 \mathrm{pr}$; D 17.1.22.6 ; D 19.2.43; D 33.8.9.1 (Marcellus); D 36. I.68.2 ; D 42.2.4; D 44.7.34 pr ; D 45.1.88; U1pien D 6.1.17.1 (Julien) ; D 7.1.17.3; D 9.2.3; D9.2.7.1-3 (Proculus) -4-5 (Labéon) 6 (Celsus) -7 (Celsus); 09.2 .9 .2 (Neratius) -3 (ofilius) -4 ; D 9.2.11. $1-2$ (Julien) -3 (Celsus, Marce1lus) $-6-7$ (Julien) $-8-9$ (Julien) -10 (Julien) D 9.2.13 pr-1 (Julien)-2 (Celsus) -3 ; $\mathrm{D} 9.2 .15 \mathrm{pr}-1$; D 9.2.19 (Celsus) : D 9.2.21.1 (Celsus, Julien) -2 ; D 9.2.23 pr-1 (Julien)-2 (Julien) -3 (Julien) -4 (Labéon) $-5-6-7-8-9-10-11 ; 0 \cdot 9.2 .25 \mathrm{pr}-1-2 ; 0$ 9.2.27 pr (Celsus, Julien)-1 (Proculus, U. Ferox) -2 (Celsus) -3 (Julien, Marcel1us) $-5-6-17-28 ; \mathrm{D} 9.2 .35 ; \mathrm{D} 9.2 .44 .1 ; \mathrm{D} 9.2 .46 ; \mathrm{D} 12.4 .5 .4 . ; \mathrm{D} 13.3$. 3 ; D 15.1.9 pr-1 (Neratius, Pomponius) ; D 19.5.14.1; D 47.1.2.1-2-3 -4-6; D 47.10.7.1; D 47.10.25; Marcien D 22.1.32.5; D 48.8.1.2. Sur 1 'exclusion des textes pourvant viser 1 a répression de l'esclave par un autre que le maitre, of infra n. 662 .

660- Alfenus (chez auteur anonyme) D 11.3 .16 ; Gaius D 11.3.15 ; Papinien D 48.5.6 pr ; Paul D 1.18.21; D 7.1.66 ; D 10.3.8.2 ; D 11.3.4; D 11.3 .6 ; D 11.3.8; D 11.3.10; D 11.3 .12 ; D 11.3 .14 pr-1-2 (Sabinus) $-3-4-5-6-7-8-9 ; D 17.2 .56 ; D 21.1 .43 .2 ; D 37.15 .6 ; D 47.10 .26$; Ulpien D 2.14.50; D $11.3 .1 \mathrm{pr}-1-2-3-4-5 ; \mathrm{D} 11.3 .2 ; \mathrm{D} 11.3 .3 \mathrm{pr}^{-1}$; D $11.3 .5 \mathrm{pr}-1-2-3-4 ; \mathrm{D} 11.3 .7 ; \mathrm{D} 11.3 .9 \mathrm{pr}$ (Julien)-1-2-3 (Neratius) D $11.3 .11 \mathrm{pr}$ (Neratius) $-1-2$; D $11.3 .13 \mathrm{pr}-1$; D 30.53.8; D 47.1.2.5; D 47.2.36 pr (Pomponius)-2 (Sabinus, Pomponius)-3 (Sabinus); $\mathrm{D} 47.2$. 52.24 ; D 47.11:5 ; Marcien D 11.3.17. Les textes ou il Etait question d'une incitation la délinquance (par ex. D 30.53.8) où a la fuite (par ex. D 47.2.36 pr), sans qu'apparaissent les termes servi corruptio; ont étê intégrés dans cette liste. Certains textes parlent seulement de détérioration : elle peut thêoriquement avoir été provoquée par une cormptio ou un damum iniuria datum; il s'agit de Iavolenus D 24.1 . $50 \mathrm{pr}$; Pomponius D 21.2.16.2 (Proculus); Africain D 30.108.1! ; Papinien D 21.2.64 pr; Paul D 9.4.10; Ulpien D $21.1 .25 \mathrm{pr}-1$ (Neratius) -4 (Pedius)-7-8; D 21.1.31.6 (Pomponius)-9 (Pomponius); D 42.5.9.5.

661- A. GUARINo, Diritto privato romano, cit., p. 883 ; bibliographie donnée in NNDI par B. ALBANESE, Darmum iniuria datwo et $G$. LONGO, Lex 
ou de blessures qui lui auraient été infligées (662).

Quent à l'action prétorienne servi corrupti, elle punit le fait de favoriser la fuite d'un esclave ou d'exercer sur lui des pressions morales de manière à le corrompre (663). L'esclave n'apparaît donc plus seulement comme une chose à qui l'on peut causer un dommage, mais également come un individu sur lequel est exercée une action psychologique et qui peut, outre se dégrader lui-même, causer des domages à son maître ou à un tiers (664).

Aquilia de damno. On peut signaler W. KUNKEL, Exegetische Studien zur Aquilischen Haftung, in ZSS 49 (1929), p. $128 \mathrm{~s}$. ; B. ALBANESE, Studi sulla legge Aquilia, in AUPA (1950) et Note aquiliane, in AUPA (1953); G. LONGO, Appunti esegetici e note critiche in tema di lex Aquilia, in AUMA (1958) ; F. PRINGSHEIM, The origin of the lex Aquilia, in Mël. LêvyBruh1 (1959), p. $233 \mathrm{~s}$. ; SCHIPANI, Responsabilità ex lege Aquilia, Criteri di imputazione e problemi della culpa (1969) ; PUGSLEY, Dammi iniuria, in T. 36 (1968), p. $371 \mathrm{~s}$.; U. VON LUBTOW, Untersuchungen zar lex Aquilia de dormo dato (1971).

662- Le meurtre est prévu par le Chapître premier de la lex, les blessures par le Chapitre III, cf. A. GUARINO, Diritto privato romano, cit., p. 884 s. ; J. MACQUERON, Histoire des obligations, cit., p. 306 s. ; Sur les extensions de 1 'action legis Aquiliae, ibid., p. $318 \mathrm{~s}$. On trouve une analyse des chapítres de la lex Aquilia chez C. TOMULESCU, On trouve une a Les trois chapitres de la lex Aquilia, in Iura 21 (1970), p. 191 s. En ce qui concerne enfin la date de la lex Aquilia, A. BISCARDI, Sulla dat della lex Aquilia, in St. Giuffrè 1 (1967), p. 75 s., selon lequel la lex aurait été antérieure à 1 'institution du préteur pérégrin (242 av. J.C.). D'un point de vue concret, il faut remarquer qu'il est parfois difficile d'interpréter les textes du Digeste pour savoir si on a affaire à un cas de répression privée ou à une agression pure et simple. La frontière entre ces deux notions est des plus ténues. Lorsque le texte mettait en relation directe le dominant (maître, usufruitier ou tout autre individu détenant un droit sur 1 'esclave) et le dominé, on a penché pour la répression, car, même si le dorinant commet une faute, i1 la comet sous le couvert de la répression ; par contre, lorsque l'esclave se trouvait en relation avec un tiers, on a privilégié

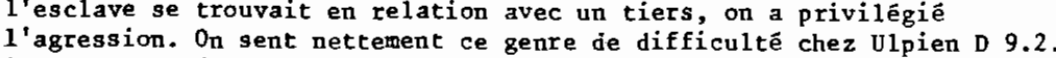
1 'agression. On sent nettement ce genre de difficulté chez Ulpien D
5.3 où un magister blesse 1 'esclave qu'il voulait corriger ; il est vrai qu'en I'espèce, Julien répond qu'il est responsable au regard de la lex Aquilia; il s'agit pourtant concrètement d'un cas de répression à l'échelle privée.

663- A. GUARINo, Diritto privato romano, cit., p. 891 ; B. ALBANESE, Actio servi corrupti in AUPA (1959) ; G. LONGO, D $11.3 .$, in BIDR 64 (1961), p. 199 s. ; G. PROVERA, Actio de servo cormpto, in NNDI.

664- v. G. SAUTEL, Actio servi corrupti, in Iura 2 (1960), p. $247 \mathrm{~s}$.
En troisième et dernier lieu, le délit privé d'iniuria (665) consiste en une atteinte à la personnalité tant physique que morale (666), le deuxième sens ayant surtout été dêveloppé par le droit prétorien (667). Toutefois, cette définition apparement simple de l'iniumia soulève quelques difficultés lorsque c'est un esclave qui en est la victime. On sait en effet que, dans le cas de blessures infligées à un esclave, la lex Aquilia permettait au maître d'obtenir une poena (668). Ainsi, l'actio iniuriarom ne pourait aroir d'ntilité que dans l'hypothèse oul la détérioration de l'esclave avait été effectuée in contumeliam domini, dans le but d'offenser le maitre (669). A ce sujet, un passage de Paul ne manque pas d'intêrêt :

Si servus servo fecerit iniuriam, perinde agendum, quasi si dormino focisset $(670)$.

ce qui signifie : si un esclave fait une injure à un autre esclave, il est censé Ia faire au mâ̂tre de ce dernier...

665- En tenant compte des textes intégrables par a contrario, on relève : Pomponius D 44.7.56 ; Papinien D 48.5.6 pr ; Paul D 7.1.66 ; D 18.7.9; D 21.1.43.5; D 44.7.34 pr ; D 47.10.4; D 47.10.16 (Pedius) ; D 47.10.18.1 ; D 47.10.26 ; D 47.10.29; D 48.7.4. I- (Labéon); Ulpien D 9.2.27.17-28 (Vivianus) ; D 47.1.2.4; D 47.8.4.14; D 47.10. 1.3-6 (Julien)-7 (Labéon) ; D 47.10.3.4; D 47.10.7.1 ; D 47.10.9.4; D 47.10.11.9; D 47.10.13 pr ; D 47.10.15.15-16-34-35-36-37-38-39 (Labéon)-40-4i-42 (Labéon) -43-44-45 (Mela)-46 (Labéon) -47-48-49. D 47.10.17 pr-i-2 (Trebatius, Mela); D 47.10.25; D 47.10.30 pr.

666- J. MACQUERON, Histoire des obligations, cit., p. 277 s. ; sur 1'iniuria, il convient de signaler les ouvrages de EUVELIN, La notion de 1 'iniuria dans le très ancien droit romain, in Mél. Appleton (1903) p. $369 \mathrm{~s}$. ; PUGLIESE, Studi sul1'iniuria, 1 (1941); DI PAOLA, La p. 369 s. ; PUGLIESE, Studi sull iniuria, (1941); DI PAOLA, La
genesi storica del concetto di iniuria, in AUCT (1947); MARRONE, genesi storica del concetto di iniuma, in AUCT (1947) ; MARRONE, p. $475 \mathrm{s.;}$ CRIFO, Diffamazione e ingiuria (dir. rom.) in ED; V. DEVILLA, iniuria, in NNDI.

667- J. MACQUERON, Histoire des obligations, cit., p. 278.

668- A. GUARINO, Diritto privato romano, cit., p. 874.

669- J. MACQUERON, Bistoire des obligations, cit., p. 282. U1pien reconnaît cependant qu'une injure peut être subie par 1 'esclave sans qu'elle ait été faite dans le but d'offenser le maître, (cf. D 47.10.15. 35 ; ... si vero non ad suggizlationem domini id fecit, ipsi servo facta iniuria inulta a praetore relimui non debuit, maxime si verberibus vel quaestione fieret : hanc enim et servim sentire paian est.). Dans ce cas, le préteur ne doit pas la laisser impunie, surtout si l'esclave a été frappé ou soumis a la torture, car, écrit le juriste, il est évident que 1 'esclave en a souffert...

$670-$ D 47.10 .18 .1 
Par ailleurs, on retrouve en matière d'iniuria une discrimination sociale au sein du grcure servile, identique à celle que I'on a pu đégager en matière économique, alimentaire, vestimentaire, ramiliale. Mais laissons la parole à Ulpien :

Itaque praetor non ex omi cousa iniurianon iudicizon servi nomine promittit : nan si leviter percussus sit vel malediction ei leviter, non dabit actionem : at si infamatus sit vel facto aliquo vel curmine scripto,puto causae cognitionem praetoris porrigendam et ad servi qualitatem : etenim multum interest, qualis servus sit, bonae frugi, ordinarius, dispensator, on vero vulgaris vel mediastinus on qualisqualis. Et quid si compeditus vel male notus vel notae extremas? Babbit igitur praetor rationem tam iniuriae, quae admissa dicitur, quam personae servi, in quem admissa dicitur, et sic aut permittet aut denegabit actionem (671).

Le préteur ne doit pas toujours accorder l'actio iniuriarm oevi nomine. Il doit ainsi la reruser si l'esclave a êté frappé légèrement ou $\mathrm{s}^{\prime} i l$ a essưé une injure verbale sans importance. Si, cependant, on a attenté à sa réputation par un fit quelconque ou par un écrit, le prêteur devra, pour se prononcer, prendre en considération le rang de I'esclave, servi qualitatem. Ici, I'assimilation entre bonae frugi, de bonne conduite, ordinarius et dispensator d'une part, et d'autre part entre mediastinus et vulgaire ou quelconque, est intéressante en ce qu'elle opère une fusion entre rang social et qualités proprement dites.

Si l'on s'en tient à ces rubriques (672), on relève une própondérance du thème de la détérioration, à I'exception il est vrai de la période III, mais le pourcentage de cette période n'est pas confirmé par l'analyse des citations. L'esclave est donc avant tout l'objet d'agressions. Quelle est la nature de ces agressions?

671- D 47.10.15.44.

672- Chiffres tirés des notes 647, 648, 649 (appropriation) ; 654, 659, 660 (đétêrioration); 665 (injure).

- appropriation

$\begin{array}{lclllll}\text { I } & - & = & - & \text { I } & 3 / 9 & =33 \% \\ \text { II } & - & = & - & \text { II } & 11 / 27 & =41 \% \\ \text { III } & 20 / 37 & = & 54 \% & \text { III } & 15 / 54 & =27 \% \\ \text { IV } & 1 / 4 & = & 25 \% & \text { IV } & 4 / 7 & =57 \% \\ \text { V } & 88 / 294 & =30 \% & \text { V } & 3 / 3 & =100 \% .\end{array}$

- détérioration

$\begin{array}{lclllll}\text { I } & - & = & & \text { I } & 5 / 9 & =56 \% \\ \text { II } & - & = & & \text { II } & 9 / 27 & =33 \% \\ \text { III } & 16 / 37 & =43 \% & \text { III } & 36 / 54 & =67 \% \\ \text { IV } & 3 / 4 & =75 \% & \text { IV } & 3 / 7 & =43 \% \\ \text { V } & 160 / 294 & =54 \% & \text { V } & 0 / 3 & =-\end{array}$

\section{- injure}

$\begin{array}{lclllll}\text { I } & - & = & & \text { I } & 1 / 9 & =11 \% \\ \text { II } & - & = & - & \text { II } & 7 / 27 & =26 \% \\ \text { III } & 1 / 37 & = & 3 \% & \text { III } & 3 / 54 & =5 \%(673) \\ \text { IV } & 0 / 4 & = & - & \text { IV } & 0 / 7 & =\end{array}$

Une lecture plus précise des textes sur ce point permet d'obtenir le tableau suivant :

Meurtre $(674)$

- analyse des paragraphes

$\begin{array}{llllllll}\text { I } & - & = & - & \text { I } & - & = & - \\ \text { II } & - & = & - & \text { II } & - & = & - \\ \text { III } & 7 / 12 & = & 58 \% & \text { III } & 5 / 12 & =\end{array}$

673- 5,55\% arrondi a $5 \%$ pour obtenir un total "rond" de $100 \%$.

674- La liste des textes sur le meurtre se trouve supra in $n .357$.

675- Les textes visant une blessure dont la gravité entraîne 1a mort de $l^{\prime}$ esclave ont été relevés à propos du meurtre, (cf. par ex. Alfenus (chez auteur anonyme) D 9.2.52 pr.). Cependant, certains textes distinguent les deux cas, ils ont alors été relevés dans chacune des rubriques : meurtre et blessures (cf. par ex. Gaius D 9.2.32.1). Il faut ainsi signaler a propos des blessures Alfenus (chez auteur anonyme) D 9.2.52.1 ; Iavolenus D 9.2.38; Julien D 9.2.51.1 (veteres) ; D 46. 3.33.1 ; Pomponius D 26.7.61; Gaiue D 9.2.32.1; Paul D 9.2.24; D 9.2.30.4; D 9.2.45.1; D 17.1.22.6; D 19.2.43; D 33.8.9.1 (Marce 1Ius) ; Ulpien D 9.2.15 pr-1 ; D 9.2.19 (Celsus) ; D 9.2.21.1 (Celsus, Julien) ; D 9.2.25.1 ; D 9.2.27.17; D 9.2.44.1; D 47.1.2.6. 


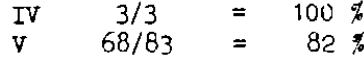

- analyse des citations

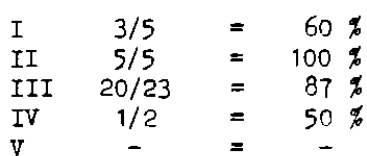

\begin{tabular}{|c|c|c|c|}
\hline I & $2 / 5$ & $=$ & \\
\hline II & $0 / 5$ & $=$ & \\
\hline III & $3 / 23$ & & \\
\hline IV & $1 / 2$ & & \\
\hline $\mathrm{v}$ & - & & \\
\hline
\end{tabular}

En dehors du cas oì l'agression peut être perpétrée sous le couvert d'une activité répressive, cas du dominant qu'on se réserve d'étudier plus loin, l'agression dont l'esclave est victime de la part d'un tiers est généralement radicale. Quelle que soit la période prise en considération, il $y$ survit rarement... L'agresseur de l'esclave peut être esclave lui-même, c'est un trait que $l^{\prime}$ on avait évoqué à propos de l'étude sur la mort $(676)$.

L'injure ne constitue pas, du point de vue de la réalité, une catégorie autonome par rapport à celle que nous venons d'examiner. A l'exception d'un texte, qui évoque un esclave victime d'une iniuria morale, on a toujours affaire à une injure physique, autrement dit ̀̀ une agression, l'atteinte à la pudicitia servi (677) se présentant aussi concrètement comne une atteinte à l'intégrité physique. Il est par ailleurs édifiant de relever que $1^{\prime}$ injure morale n'est considérée corme telle que dans la mesure où l'esclave qui la subit occupe une position privilégiée, par exemple celle d'ordinarius ou de dispensator (678).

Le vol apparait quantitativement en seconde position. Il convient à son sujet de tenter de répondre à plusieurs questions.

En premier lieu, n'importe quel esclave fait-il l'objet d'un vol, ou bien les voleurs s'intéressent-ils à certains esclaves en particulier ? Dans deux hypothèses au moins, il faut pencher pour la deuxième solution. Ainsi, la femme esclave semble être un butin tout

676- L'esclave est tué par un esclave (cf. supra n. 383) ; blessê par un esclave (cf. Iavolenus D 9.2.38 ; Paul D 33.8.9.I (Marcellus); Ulpien D 9.2.44.1).

677- Cf. supra n. 481 et 482 .

678- Ulpien D 47.10.15.44. indiqué, que le tol s'opère dans le but d'en abuser (679) ou dans celui de tirer profit de son rôle de reproductrice (680). Si l'on en croit les textes, elle constitue également une monnaie d'śchange appréciable : c'est ce qui paraît découler du fait qu'un esclave procure une ancilla, volée, à son mâtre pour payer le prix de $\mathrm{sa}$ liberté (681). D'autre part, $l$ ' "élevage" des enfants esclaves étant d'un bon rapport, 1 'enfant est aussi une proie intéressante (682).

En second lieu, covment le vol s'opère-t-il ? Il peut être occasionné par la faute de celui qui avait la garde de l'esclave (683), mais, souvent, il apparâ̂t lié à une hypothèse de fuite. Un texte d'Africain est important à ce propos :

Ancilla fugitiva quemadmodum sui furtum facere intellegitur, ita partum quoque contrectondo furtivion facit (684).

La femme esclave qui s'enfuit de chez son maître cormet son propre vol, et si elle emmène son enfant avec elle, elle en commet un autre. La construction juridique est intéressante, la ferme se trouvant à la fois dans la position d'agent et d'objet. D'autre part, celui qui recueille chez lui un esclave fugitif est considéré conme un voleur. On

679- Par exemple Paul D 47.2.83.2 ; Ulpien D 47.1.2.5; D 47.2.39 (cette dernière hypothèse se distingue des précédentes dans la mesure où la femme dont il est question est une prostiture; dans ce cas, 1 'actio furti n'a pas lieu ; elle a par contre lieu si on vole une ancilla non prostituée libidinis causa, cf. Paul D 47.2.83.2).

680- Par exemple Julien D 1.5.26; Ulpien D 47.2.48.5; D 41.3.10.2 (Scaevola) ; D 50.16.26 (Scaevola). Selon Julien (D 1.5.26), si l'ancilla est volée alors qu'elle est enceinte, son enfant ne pourra pas être proscrit, als qu elle est enceinte, son enfant ne pourra pas bonne foi. Ulpien nous soumet une étude plus élaborée du problème : si l'ancilla a été volée étant enceinte ou qu'elle ait conçu chez le voleur, son partus sera furtivus, qu'elle accouche chez le voleur ou chez un possesseur de bonne foi. Si, par contre, elle a conçu chez le possesseur de bonne foi et qu'elle y ait accouché, son partus pourra être prescrit (D 47.2 .48 .5 ).

681- L'ancilla peut être fournie par l'esclave lui-même (cf. Julien D 41.4.9; Paul D 41.3.4.16 (Sabinus, Cassius) ou par un tiers (cf. Paul D $41 \cdot 3 \cdot 4 \cdot 17)$.

682- I. BIEZUNSKA-MALOWIST, Les enfants-esclaves $¥$ la luniẻre des papyrus, cit., p. 93,95,96; cf. Celsus D 47.2.68.2; Paul D 13.1.13; U1pien D 47.4 .1 .11 .

683- Sur ce problème, Papinien D 47.2.81 pr et 2 (par a contrario); Ulpien D 47.2.14.6 (Pomponius, Papinien).

684- Africain D 47.2.61. 
troure cette idée chez Ulpien :

Is qui fugitirnon celavit fur est (685).

En pratique, cette espèce est intéressante en ce que le vol s'opère avec l'accord de l'esclave, sans aucune violence. Mais celvi qui conseille simplement à l'esclave de s'enfuir peut-il être qualifié de voleur ? Il faut citer un passage d'Ulpien :

Qui servo pereuasit, ut fugeret, fur non est : nec enim qui aliour malum consilizm dedit, furtuon facit, non magis quan $8 i$ ei persuasit, ut se prescipitet aut monus sibi inferret : haec onim furti non admittunt actionem. Sed oi alius ei fugom persucserit, ut $a b$ alio oubripiatur, furti tenebitur is qui persuasit, quasi ope consilio sius furtion factum sit. Plus Ponponius scripsit evon, qui persucsit, quomis interim furti non teneretur, two tamen incipere teneri, cum quis fugitivi fur esse coeperit, quasi videatur ope consilio eius furtum factum (686).

Selon Ulpien, celui qui conseille à un esclave de s'enfuir ne coumet pas de vol, à moins qu'il ne lui ait donné ce conseil dans le but de le faire enlever par un complice. Pomponius, qui écrit un demisiècle auparavant, est plus sévère. Il estime en effet que celui qui persurde un esclave de prendre la fuite doit être tenu de l'actio furti même dans le cas oul l'esclave est enlevé indépendamment de sa volonté. La rigueur de cette solution n'est pas étrengère à l'État de la fuite sous les Antonins, c'est un point sur lequel on reviendra. Le problène de l'incitation à la fuite prend un relief particulier dans le cas ou deux esclaves se sont mutuellement conseillés de quitter leur maittre.

Si duo servi invicem sibi persuaserunt et ambo simul aufugernont, alter alterius fur non est. Quid ergo, oi invicem se celavernon ? Fieri enim potest, ut invicem fures sint (687).

Fidèle à sa position, Ulpien écrit que s'ils se sont seulement conseillés de fuir, l'un n'est pas voleur de l'autre, mais il n'en va plus de même ai invicem se celaverunt, s'ils se sont mutuellement cachés. Cette construction juridique ne manque pas non plus d'interêt,

\section{5- Ulpien D $11.4 .1 \mathrm{pr}$.}

686- U1pien D 47.2.36 pr.

687- Ulpien D 47.2 .36 .3 . chaque esclave étant, par le même fait, à la fois dans une situation d'agent et d'objet. Enrin, un texte de Marcien montre à quel point le vol et la ruite sont liés dans l'esprit des juristes; cet auteur précise en effet que ce n'est pas cammettre un vol que de montrer son chemin à un fugitif (688).

En dehors des bypothèses qui viennent d'être décrites, le vol d'esclave peut s'effectuer de manière violente (689). il en était probablement ainsi lorsqu'il était effectué par des bandes organisées (690). D'ordinaire, le vol ne porte que sur un esclave, mais il arrive $q u^{\prime} i l$ en concerne plusieurs. Ainsi, Upien éroque deux esclaves volés ensemble (691). Ailleurs, il est question d'un individu qui détourne des esclaves d'une succession (692) ou d'un esclave affranchi par testament qui soustrait des partus alors que 1 'hereditas est encore iacens (693).

Que devient l'esclave après avoir été volé ? Il est possible qu'il soit revendu (694), généralement à un acheteur de bonne foi. On relève dans ce sens un passage de Iavolenus :

Furtivan ancillan bona fide duorwn aureorwn emptam (695).

On avait sigaalé, dans l'étude sur le prix de l'escläre, que deux aure $i$ constituaient un prix bas, mais on avait remarqué qu'en l'espèce le voleur était peut-être pressé de se débarrasser de l'ancilla. Quoi qu'il en soit, l'argent de la vente représente en ce cas un bénéfice intégral pour le voleur, car

nummus ergo hic qui redactus est es pretio rei furtivae, non est furtivus (696),

688- Marcien D 47.2.63.

689- Ainsi, Tryphoninus D 43.16.19 (Julien) ; Paul D 21.1.43.5 ; D 43.16.6; U1pien D 47.1.2.2 ; D 47.2.39; D 47.8.2.18.

690- Iavolenus D 49.15.27 (Trebatius, ofilius, Labéon); Paul D 24. 1.28 .1 ; U1pien D 24.3.21.

691- U1pien D 47.2.14.7 (Pomponius, Papinien).

692- Ulpien D 19.1.13.5 (Julien).

693- U1pien D 47.4.1.11.

694- Ainsi, Iavolenus D 47.2.75; Julien D 1.5.26; D 19.1.24.1 (Sabinus, Cassius) ; D 41.1.39.; D 41.3.33 pr ; Paul D 12.1.31.1 (Sabinus, Cassius, Julien); U1pien D 9.4.36 ; D 41.3.10.2 (Marcellus, Scaevo1a).

695- Iavolenus D 47.2.75.

696- U1pien D 47.2.48.7 
c'est-à-dire, l'argent provenant du prix de la chose volée n'est pas considéré corme étant furtif.

Il est possible aussi que l'esclave demeure chez le voleur (697). Celsus traite d'un esclave volé étant enfant qui grandit chez son voleur (698). Paul évoque dans le même sens un enfant élevé par celui qui I'a enlevé (699). Il est question chez Ulpien d'une ancilla qui conçoit chez le voleur (700). Autant dire que les rapports voleurs/volés se présentent sous les auspices les meilleurs dans certains cas. Il est même prévu une protection du voleur :

Quod fum ipsi furtum fecerit furtivus servus, eo nomine actionem cum domino furem habitum placet, ne facinora talium servorw non solun ipsis impunitatem, sed dominis quoque eorron quaestui erunt : plerumque enim eius generis servomom furtis peculia eorundem augentur (701).

Selon Celsus, qui est l'auteur de ce passage, si l'esclave volé dérobe quelque chose au voleur, ce dernier peut s'attaquer au mầtre, car il ne faut pas que les délits de tels esclaves deviennent lucratifs pour leurs meitres. La solution est remarquable, aussi bien pour ce qui est de la théorie que de la pratique...

S'il arrive, comme on l'a vu, que le séjour chez le voleur se passe bien, il $n^{\prime}$ en est pas toujours ainsi. Certains textes, qui ont il est vrai des allures d'hypothèses d'école, envisagent la possibilité d'un meurtre successif au vol (702).

Les délits dont il est question à propos de l'esclave objet rentrent dans trois rubriques. En va-t-il de même en ce qui concerne l'esclave agent ou a-t-on affaire à une plus grande variété de faits juridiques?

697- Cf. Celsus D 47.2.68.2-4 ; Julien D 45.3.14 (Sabinus); Pau1 D 13. 1.13 ; D 17.1.22.9 (Mela); Ulpien D 47.2.48.5. Rentrent bien entendu dans cette catégorie les textes où il est question du plagizon dont on a parlé plus haut.

698- Celsus D 47.2.68.2.

699- PauI D 13.1.13 (Fulcinius).

700- Ulpien D 47.2.48.5 exposé supra n. 680.

701- Ce1sus D 47.2.68.4

702- U1pien D 47.1.2.1-2-3-4.

\section{II - L'esclave agent de fait juridique.}

A l'exception de la castration (703), de l'edultère (704) et du proxénétisme (705), déjà envisagés, on retrouve tout d'abord les rubriques précédentes. On voit l'esclave mêlé, en tant qu'agent, cette fois, à des cas de furtıon (706), de rapina (707), de plagium (708). On relève

703- Ulpien D 48.8.4.2 (rescrit d'Hadrien).

704- Tryphoninus D 50.16.225 ; Paul D 9.2.30 pr ; Ulpien D 1.12.I.5; D 40.9.12.6 (Africain); D 48.2.5 (rescrit de Marc-Aurẽle) ; D 48.5. $28 \mathrm{pr}-1-2-3-4-16$; Macer D 48.5.25 pr ; Marcien D 28.5.49.2 (constitution de Sévère et Caracalla); D 48.5 .34 pr (rescrit d'Antonio le pieux)-1

705- U1pien D 3.2.4.3 (Pomponius).

706- Devant faire plus loin une analyse comparative entre esclave volé et esclave voleur, on donnera dans cette note la totalité des tex$v i$ bonomon roptomom et le plagizen. On peut signaler, a propos de 1 'herevi bonomon raptorum et le plagizon. On peut signaler, à propos de 1 hereditas iacens, que Marc-Aurèle introduisit le crimen expilatae heredita-
tis pour le vol des biens héréditaires avant l'acceptation de la succession, cf. S. SOLAZZI, Sul crimen expilatae hereditatis (1936), in Scr. dir. rom. 3 (1960), p. 563 s. ; A. BERGER, Encycl. Diction., cit., p. 418 ; dans 1e Digeste, v. Papinien D 40.5 .23 pr. Le vol peut aussi donner lieu a des actions spécifiques : 1 'actio rerron amotamom, cf. WACKE, Actio remon amotamum (1963) ; A. GUARINO, Res amotoe, in ANA (1966) ; 1'actio rationibus distrahendis, cf. A. GUARINO, Diritto privato romano, cit., p. 554 ; A. BERGER, Encyci. Diction., cit., p. 343 ; 1 'actio de tigno iuncto, cf. A. GUARINo, o. c., p. 583 ; A. BERGER, o. c., p. 737 ; D 47.3 , mais $i 1$ n'est pas question d'esclaves a son sujet; c., p. 737 ; D 47.3 , mais il n'est pas question d'esclaves a son sujet ; Note intorno alle azioni in factum di danno e di furto contro il caupo e lo stabularius (1909) ; F.M. DE ROBERTIS, Reception nautam, Studio sulla responsabilità dell'armatore in dir. rom., con riferimento alla disciplina particolare concernente il caupo e lo stabulocius, AUBA (1962) ; v. D 47.5.1.5; 1 'actio arbomm furtim caesarun, cf. B. ALBANESE, Actio sorvi cormeti e actio artorm furtim caesorion, in Labeo 5 (1959) P. 325 s i v D 7.7.7.4-5; selon D 47.7.1, cette actiab 5 (195) p. J25 s. ; v. Dh7.7.7.4-5 ; selon d 47.7 , cetre actian po vait être lntentée apres celle de la lex Aquitia poir le supplément

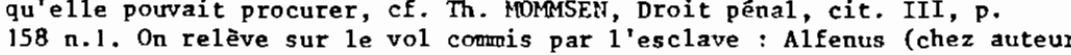

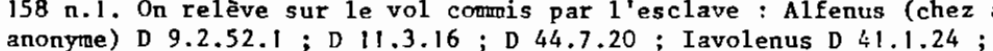
Neratius D 40.7.17; D 47.2.65; D 47.2.84.1; Celsus D 13.1.15; D 47 2.68.3-4; Julien D 9.4.39 pr ; D 9.4.40 ; D 9.4.41; D 12.5.5 (Proculus) ; D 13.6.20; D 41.1.37.2; D 41.3.33.6; D 41.4.9; D 41.4.10; D 47.2.57.3-5; D 47.6.2 ; Pomponius D 9.4.18; D 12.4.15 (Labéon, Proculus) ; D 15.1.4.2-3-4; D 21.1 .46 ; D 21.2 .30 ; D 30.45.1 ; D 30.48 pr (Atilicinus); D 40.12.43 (rescrit d'Hadrien); D 46.3.19 (Labéon). D 47.2.35.1; D 47.2.44.2; Africain D 3.5 .48 ; D 13.6.21.1; D 13.7 . 31 (Julien); D 19.1.30 pr; D $30.110 ; D 40.4 .22 ; \mathrm{D} 47.2 .61 ; \mathrm{D} 47.2$. $62 \mathrm{pr}-1-2-3-4-5-6-7$; Gaius D 9.2.4 pr-1; D 9.2.32 pr; D 15.1.27.1 ; D $30.67 \mathrm{pr}$; D 30.70 pr-2-3; D $41.2 .15 ; \mathrm{D} 47.4 .2$; Marcellus D 47.6 .5 ; Scaevola D40.5.41.11 ; D 40.7.40 pr ; D 47.6.6 (Labếon); Papinien D 19. 
également dans les textes sa présence en matière de domnon iniuria dation (709), de sorvi corruptio (710), ce qui éclaire d'um jour noureau ce délit prétorien. Il en est aussi question dans l'action prétorienne do sepulchro violato, sur la violation de sépulture (711), et

5.8; D 40.5.23 pr ; Tryphoninus D 9.4.37; D 40.4.59.1 (Scaevola): D 50.16.225 ; Callistrate D 47.9.7 ; Paul D 4.9.6.1-4; D 5.3.40.4 ; D 6 1.58 ; D 9.4.9; D 9.4.10; D 9.4.31 (Sabinus, Cassius, Julien, Powponius) ; D 10.4.16; D 11.3.14.6; D 12.6.36; D 13.6.22; D 17.1.26.7 (Neratius) ; D 19.1.4 pr; D 19.2.45 pr-1; D 19.4.2 (Aristo) ; D 21.1 $58 \mathrm{pr}^{-1}$; D 21.2.3; D 25.2.3.1; D 25.2.21.1 (Pedius) 2 ; D 33.8.9.1 (Marcellus) ; D 40.1.12; D 40.12.24.4; D 40.12.41.1; D 41.3.4.8-9 (Pouponius) -16 (Sabinus, Cassius) ; D 47.2 .18 (cassiani) ; D $47.2 .42 \mathrm{pr}-$ J (Sabinus) ; D 47.2.54.1-2 ; D 47.8.3 (Labéon) ; D 47.9.4.1 (rescrit d'Antonin le pieux); Olpien D 2.14.50; D 4.4.11 pr (Sévère) ; D 4.9. 3.3; D 9.2.5 pr ; D 9.2.27 pr (Celsus, Julien); D 9.4.2.1 (Celsus, Julfen, Marcellus) ; D $9.4 .8 ; \mathrm{D} \mathrm{9.4.11;D} 9.4 .36$; D 9.4.38 pr-2-3 ; D 10.2.16.6 (Ofilius); D $10.2 .18 \mathrm{pr}$ (Julien); D $11.3 .1 .4-5 ; \mathrm{D} 11$.

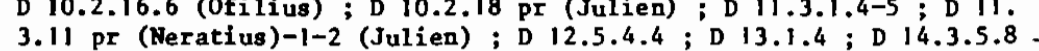

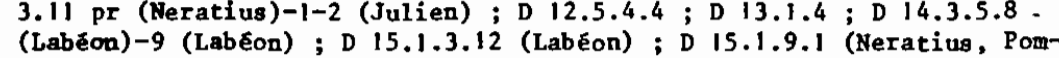
ponius) -6 ; D 15.3.3.9; D 16.3.11 (Sabinus) ; D 18.1.29; D 19.1.11. 8 (Neratius) : D 19.1.13.1-2-3-5 (Julien) ; D 21.1.17.20; D 21.1.19.1; D21.1.21.1 (Poøponius);D21.1.23.8(Julien);D21.1.31 .1;D21.2.31;D39.4.1.5; D 39.4.12,1:D40.7 3.9.D46.3.18.D47.1.2.3:D47.2.7pr(Pomponius):D47.2.17pr

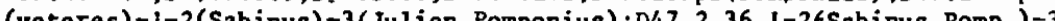
(veteres) - 1 -2(Sabinus) -3 (Julien, Pomponius) ; $047.2 .36 .1-2$ \&Sabinus, Pomp.) pr-1 (Labéón) $-2-3-4-5-6-7$ (rescrits de Marc-Aurèle et de Sévère et Carápr-1 (Labéon)-2-3-4-5-6-7 (rescrits de Marc-Aurèle et de SÉvère et Car
calla) -8-9-10 (Scaevola)-11-12-13-14-15 (Scaevola)-16-17 ; D 47.4.3

(Labếon); D 47.5.1.5; D 47.6.1 pr-1-2-3; D 47.6.3 pr-1-2; D 47.6.4; D 47.8.2 $\mathrm{pr}-5-14-15-16-19$; D 48.18.1.5 (rescrits d'Hadrien et d'Antonin le pieux) ; D 50.16.174 ; D 50.16.195.3 ; Marcien D 21.1.52 ; D 48 19.11 .1

707- Paul D 47.8.3 (Labéon); Ulpien D 47.8 .2 pr-5-14-15-16-19. A ropos de de turba, Ulpien $D$ 47.8.4.15. Il est plus généralement question d'esclaves en matière de violence dans les leges Iuliae ds vi publica et de vi privata, (cf. Marcien D 48.6.3 pr ; Macer D 48.7.3). Dans le titre de vi et ds vi armata, on relève Ulpien $D$ 43.16.1 pr-1i-12-15-16 $-17-18-19-21 ; D$ 43.16.3.11.

708- Paul D 40.1.12 : Lege Fabia prohibetur servus, qui plagizm ad̃misit, pro quo dominus poenam intulit, intra decem annos manumitti; un esclave qui s'est rendu coupable de plagionn ne peut être affranchi avant que s'écoule un délai de dix ans.

709- Par exemple, Julien D 9.4.41; Gaius D 9.2.32 pr ; Paul D 4.9. 6.1 ; U1pien D 39.4.1 pr; D 39.4.12.1.

710- Paul D 11.3.14.2 (Sabinus)-3; Ulpien D 11.3.1.5; D 11.3.5.3.

$711-$ Ulpien D 47.12.3.11. Sur cette action, Th. MOMASEN, Droit pénal, cit. III, p. 127 s. ; A. BERGER, Encycl. Diction., cit., p. 345, 767 ; MOREL, Le sepulchmon violatum (1928); F. DE VISSCHER, Les peines sépulcrales, in Fs. Lewald(I953), p. $175 \mathrm{s.,}$, et Le droit des tombeaux romains (1963). dans l'Edit ds albo corrupto (712) par lequel le prêteur réprimait ceux qui se seraient permis de degrader son texte. Une lex agraria de Nerva punit de mort l'esclave qui se serait livré à un deplacement de borne (713). L'esclave peut enfin être 1'auteur d'une iniuria (714).

Mais, preuve de l'importance de la délinquance servile, on trouve 1 'esclave à propos d'autres actes déliçtueux. Ainsi, en matière dè faux et de dol (715), on le voit coumettre une fraude dans les comptes du maître (716), dans l'hérédité (717) ou dans les comptes des débiteurs $(718)$; on le voit encore commettre un faux en matière de testament (719) ou de monnaie (720). Il apparait enfin dans les textes traitant du meurtre. On a vu que la lex Pompeia do parricidits s'appliquait aux esclaves (721), mais c'est sur l'assassinat du maître (722) ou le refug de le secourir, alors qu'il était en danger (723), que les textes sont les plus nombreux.

712- Paul D 2.1.9 (Pomponius, Octavenus) ; Ulpien D 2.1.7.1-3; A. BERGER, Encycl. Diction., cit., p. 342 ; J. MACQUERON, Histoire des obligations, cit., p. 324 .

713- Callistrate D 47.21.3.1: Alia quaque lege agraria, quam divus Herva tulit, cavetur, ut, si servus servave insciente domino dolo malo fecerit, ei copital esse, nisi dominus dominave miltam sufferre maluerit. fecerit, ei capital esse, nisi dominus dominave multan sufferre maluer en payant l'amende. Sur cette, action, v. Th. MOMMSEN, Droit pénal cit. III, p. $140 \mathrm{~s}$. ; A. BERGER, Encycl. Diction., cit., p. 343.

714- Gaius D 47.10.34; Venuleius D 48.2.12.4; Paul D 2.1.9 (Pomponius, Octavenus) ; D 47.10 .18 .1 ; vlpien D 2.9 .5 ; D 11.3 .1 .5 ; D 47 10.9.3; D 47.10.17.3-4-5-6-7 (Labêon) -8 9; Hermogénien D 47.10.45.

715- v. Th. MOMMSEN, Droit pénal, cit. II, p. $388 \mathrm{~s}$.

716- Ainsi, chez Scaevola D 40.7.40.4; Ulpien D 9.2.23.4 (Labéon); D 11.3 .1 .5 .

717- Par exemple, Ulpien D 10.2.18 pr; et la globalité de D 47.4.

718- Ulpien D 11.3.11.1.

719- Par exemple, Paul D 48.10.22.9.

720- U1pien D $48 \cdot 10.8$.

721- Venuleius D 48.2.12.4.

722- Julien D 17.1.30 (par extension) ; Gaius D 29.5 .25 pr-1 ; Maecianus D 29.5.14; Venuleius D 29.5.13 ; Marcell us D 29.5.16 ; Papinien D 1.21.1 pr ; D 29.5.4; D 29.5.21 pr ; D 35.2.11.4 ; D 48.1.14; Callistrate D 48.19.28.11 ; Paul D 29.5.22 ; D 35.2.39; Ulpien D 29. 5.1 .5 (rescrit d'Antonin le pieux) $-21-30$; D 29.5.3.1-14-18; D 40 . $12.7 .4^{\circ}$.

723- U1pien D 29.5.1.18-22-27 (Sextus)-26-28 (rescrit d'Hadrien)-29 (idem)-31-34-36-37 ; D 29.5.3.2-4-6-12 ; Modestin D 29.5.19. 
La faible représentativité numérique de ces différents délits ne permet pas une analyse statistique valable. Le seul thème qui soit correctement représenté est le vol. C'est pourquoi il convient de s'y arrêter.

Une comparaison quantitative opérée entre les textes où l'esclave est objet de vol (724) et ceux où il intervient en tant que voleur (725) permet de dégager une nette supériorité des seconds. Les totalu réalisés

Esclave volé

- analyse des paragraphes

$\begin{array}{lcll}\text { I } & - & = & - \\ \text { II } & - & = & - \\ \text { III } & 20 / 77 & = & 26 \% \\ \text { IV } & 1 / 5 & = & 20 \% \\ \text { V } & 88 / 229 & = & 38 \%\end{array}$

- analyse des citations,

$\begin{array}{lclllrl}\text { I } & 3 / 8 & =37,5 \% & \text { I } & 5 / 8 & =62,5 \% \\ \text { II } & 11 / 34 & =32 \% & \text { II } & 23 / 34 & =68 \% \\ \text { III } & 15 / 39 & =38 \% & \text { III } & 24 / 39 & =62 \% \\ \text { IV } & 4 / 9 & =44 \% & \text { IV } & 5 / 9 & =56 \% \\ \text { V } & 3 / 3 & =100 \%(726) & \text { V } & 0 / 3 & =\end{array}$

par les périodes III et IV, dans l'analyse directe, confiment les résultats de l'analyse globale sur l'esclave agent/objet de fait juridique. Cette importance de l'esclave voleur est-elle due à une mentalité esclavagiste qui s'exprimerait par les juristes ? Correspond-elle au contraire à une réalité ? Il est difficile de trancher, cela pour la simple raison qu'il y a probablement une part de vérité dens chacune des hypothèses émises. Pour la première, il suffit de faire un bond dans le temps et de constater que la réputation qui est faite aujourd'hui au travailleur immigré en général, au nord-africain en particulier, n'est guère plus reluisante à ce niveau que ne l'était celle de l'esclave jadis. En faveur de la seconde, il faut d'une part renvoyer à Ulpien qui parle des vols commis par des esclaves comme d'un mal domestique (727), et rappeler d'autre part que $1^{\prime}$ on s'efforce de s'en

724- Cf. Supra liste in notes 647,648 et 649.

725- Cf. supra n. 706 .

726- Compte tenu de son faible fondement numérique, ce pourcentage ne peut être considéré conme pertinent.

727- Ulpien D 47.5.1.5: domestico malo. protéger dans les actes juridiques les plus divers : vente (728), louage (729), dépôt (730), commodat (731), gage (732).

Cela dit, il est intéressant de questionner les textes sur le point de savoir comment le vol se déroule dans la pratique. Il semble qu'il y ait des moments privilégiés dans la vie de l'esclave pour se livrer à ce genre d'activité. Hormis le fait que l'esclave qui s'enfuit commette son propre vol (733), la fuite constitue un moment idéal pour emporter quelques effets appartenant au maitre (734). Il est vrai qu'à ce stade, il n'y a plus grand chose à perdre... Autre moment important. 1 'attente de la liberté testamentaire qui voit l'esclave détourner des biens successoraux, de manière fréquente sans doute puisqu'un titre du Digeste (D 47.4 : si is, qui testamento liber esse iussus erit, post mortem domini ante aditam hereditatem subripuisse aut corrupisee quid dicetur) est consacré à ce problème. Ce genre de vol est intéressant dans la mesure où il ne reflète pas, pour user d'un euphémisme, des rapports idylliques, ne serait-ce que posthumes, entre l'esclave et le défunt maître. Le vol n'est pas forcément commis de manière individuelle, il peut l'être de manière collective. C'est ce qui ressort du titre D 47.6 (Si farmilia furtum fecisge dicetur). Là encore, le fait qu'un titre du Digeste traite du vol perpétré collectivcment signifie vraisemblablement que la pratique n'était pas rare. De plus, ce délit ne se déroule pas toujours "en douceur", il peut s'effectuer de manière violente (735).

Par ailleurs, certains textes fournissent des précisions quant à l'esclave voleur : esclave d'un publicain (736), d'un exercitor navis (737), d'un fossoyeur (738). Le vol peut se produire dans une

728- Par exemple, chez Celsus D 47.2.68.3; Africain D 47.2.62 .2-4-5; Ulpien D 47.2.17.2.

729- Par exemple, chez Africain D 47.2.62.5.

730- Par exemple, chez Africain D 47.2.62.5.

731- Par exemple, chez Africain D 47.2.62.6; Paul D 47.2.54.1-2.

732- Par exemple, chez Africain D 47.2.62.1-5.

733- CF. Africain D 47.2.61.

734- Pomponius D 46.3.19 (Labéon) ; Paul D 21.1.58 pr ; Ulpien D 19.1.13. 2 ; D 47.2.36.2 (Sabinus, Pomponius)-3 (Sabinus).

735- Paul D 47.8.3 (Labéon); Ulpien D 47.8.2 pr-5-14-15-16-19; D 50. 16.195.3.

736- Ulpien D 39.4.1.5

737- Ulpien D 4.9.3.3; D 47.5.1.5

738- Ulpien D 14.3.5.8 (Labéon) 
boutique (739), un navire, une auberge (740), ou encore à l'occasion d'un naurrage ou d'un effondrement (741).

La plupart des tertes concernent un vol fait au maître (742). La protection de celui-ci est, il est vrai, bien assurée. Il faut citer à ce sujet un passage de Julien :

Cum catem servus rem suam peculiarem furandi consilio anovet, quandiu ean retinet, condicio eius non mutatur (nihil enim domino abest) : oed oi alii tradiderit, furtum faciet (743).

ce qui signifie : si l'esclave détourne un effet de son pécule dans l'intention de le voler, il a'y a pas vol tant qu'il le conserve, car rien n'est enlevé au maitre; c'est seulement $s$ 'il le livre à un tiers que le vol sera consomée.

Bien que le plus souvent l'esclave décide de commettre un vol. de sa propre initiative, il n'en est pas to:1jourz ainsi. Il est possible, par exemple, qu'il y soit sollicité par un tiers (744). Il arrive même que ce soit le mâ̂tre lui-même qui lui en donne l'ordre (745). On

\section{9- Paul D 19.2.45.1. \\ 740- Paul D 4.9.6.1.}

741- Callistrate D 47.9.7 rapporte un s.c. interdisant aux esclaves et affranchis imperiaux de s'interposer pour recueillir les effets des naufrages ; Paul D 47.9.4.1 (rescrit d'Antonin le pieux).

742- Ainsi, Neratius D 40.7.17; Julien D 41.3.33.6; D 47.2.57.3; Pomponius D 15.1.4.2-3-4; Africain D 3.5 .48 ; D $19.1 .30 \mathrm{pr}$; D 47.2 . $62 \mathrm{pr}-2-4$;Gaius D 41.2 .15 ; Scaevola D 40.5 .41 , 11 ; Tryphoninus D 50.16 . 225 ; Paul D 12.6.36; D 21.1.58 pr-1 ; D 21.2.3; D 40.12.41.1 ; D 41 . 3.4.8-9 (Pomponius) ; U1pien D 4.4.11 pr (Sévère) ; D $11.3 .11 \mathrm{pr}$ (Neratius) -2 ; 15.1 .9 .6 ; D 18.1 .29 ; 21.1 .23 .8 (Julien) ; D 21.1 .31 .1 D 46.3.18;D 47.2 .17 pr-1-2 (Sabinus)-3 (Julien, Pouponius) ; D 47.2 . 43.12 ; D 47.4.1.2 ; Marcien D 21.1 .52 ; D 48.19.11.1.

743- Julien D 47.2.57.3; v. aussi, du même auteur, D 41.3.33.6 : Si rem pignori datam creditoris servus subripuerit, cton ean creditor possideret, non interpellabitur usucapio debitoris, quia servus dominum sum possessione non subvertit.

744- Paul D 11.3.14.6 ; Ulpien D 11.3.1.4-5. Particulièrement intéressant, ce texte d'vlpien (D 2.14.50) : Non impossibile puto in contractibus depositi, commodati et locati 'et ceteris similibus' hoc pactun : ne facias furem vel fugitivion serviom mewn.

745- Par exemple, chez Alfenus (chez auteur anonyme) D 44.7.20; v1pien D 9.4.2.1 (Celsus, Julien, Marcellus); D 21.1.21.1 (Pomponius). D'autre part, le maître peut aider son esclave a coumettre le vol, cf. par exemple vlpien D 47.2.36.1; D 48.18.1.5 (rescrits d'Hadrien et d'Antonin le pieux). possède dans ce sens un terte remarquable d'Al renus, dans lequel un esclave exerce la piraterie iusau domini (746).

Les textes nous renseignent aussi sur les objets volés par l'esclave : or (747), argenterie (748), meubles (749), argent (750). On trouve également des esclaves (751). Puis on remarque des objets de moindre valeur : des vêtements (752), une lanterne (753), ou simplement une boisson (754). Il semble bien au vu de cette énumération que la fonction sociale remplie par l'esclave soit déterminante de l'importance du vol. Il est d'ailleurs évident que le vol d'objets de valeur est chose facile pour celui qui occupe un enploi privilégié.

Que fait l'esclave du produit de son délit ? Les textes fournissent trois sortes de réponses. On a va que l'ancilla volée était parfois remise au maitre par l'esclave en vue de payer le prix de la liberté (755). Il peut aussi vendre la chose volée (756). Enfin, et il s'asit vraisemblablement d'un val d'importance, Julien Evoque un esclave achetant un fonds avec des furtivi numi (757).

Si l'esclave est souvent voleur, sa propension la delinquance $n^{\prime}$ est pas identique pour chaque délit. Prenong par exemple le cas de l'iniuria. La période $\mathrm{V}$, la seule a être suffisament représentée, montrè que l'esclave en est plus souvent victime ( $77 \%$ des textes) qu'auteur (23 s) (758). C'est probablement là le signe d'une mentajité esclavagiste. Cette mentalité ressort également des solutions juridiques proprement

746- Alfenus (chez auteur anonyne) D 44.7.20.

747- Vlpien D 48.18.1.5 (rescrits d'Hadrien et d'Antonin le pieux).

748- Julien D 47.2.57.5; Africain D 40.4.22; D 47.2.62.7; Vlpien D 40.7.3.9; D 48.18.1.5 (rescrits d'Hadrien et d'Antonin le pieux).

749- Scaevola D 40.7.40 pr.

750- Julien D 41.1.37.2 ; Pomponius D 46.3.19 (Labéon); vlpien D 9. 4.38.3; D 40.7.3.9.

751- Par exemple, Julien D 41.4.9; D 41.4.10; Paul D 41.3.4.16 (Sabinus, Cassius).

752- vlpien D 47.2.52.9.

753- Alfenus (chez auteur anonyme) D 9.2.52.1.

754- Pomponius D 47.2.35.1.

755- Julien D 41.4.9; Paul D 41.3.4.16 (Sabinus, Cassius).

756- Ulpien D 9.4.38.2.

757- Julien D 41.1.37.2. 
dites.

On a dit plus haut que lorsque l'esclave en était victime, il existait certains faits dont $1 a$ qualification d'iniuria n'était pas automatique et nécessitait un examen par le préteur de la servi qualitas . C'était le cas de l'injure morale (759). Or, le problème ne se pose pas lorsqu'il en est l'auteur (760). D'ailleurs, Ulpien écrit clairement :

Quaedon iniuriae a liberis hominibus factae leves (non nullius momenti) videntur, enimvero a servis graves sunt. Crescit enim contumelia ex persona eius qui contumeliam fecit (761).

Le caractère grave de 1 'injure dépend de la qualité de celui qui la profère. La même injure peut être considérée comme légère si elle est faite par un homme libre, conme grave si elle est faite par un esclave. La discrimination est donc nette en ce qui concerne la qualification du délit. Elle ne l'est pas moins en matière de peine.

Dans le cas d'une injure proférée par un esclave, on sait que le maître pouvait remettre le coupable à la victime pour être soumis à une verberatio, après quoi il devait lui être restitué (762). C'était une possibilité pour le maître d'échapper au paiement de la peine ou à $1^{\prime}$ abendon noxal de l'esclave (763). C'est un premier point : l'esclave encourt pour ce délit une peine corporelle. Qu'en est-il de I'homme libre ? Un texte d'Hermogénien nous permet de répondre à cette question, mais il convient au préalable de le situer dans son contexte. Sous 1'Mmpire, la victime d'une iniuria peut soit exercer l'action estimatoire pour obtenir une peine pécuniaire (civiliter agere), soit saisir les juridictions répressives pour faire châtier le coupable (crimi-

758- 46 textes sur 60 concernent un esclave injurié, 14 seulement un esclave auteur d'une iniuria. Ces chiffres sont obtenus à partir des listes se trouvant supra in $n$. 665 et 713 .

759- Ulpien D 47.10.15.44.

760- Gaius D 47.10.34; U1pien D 47.10.17.3.

761- Ulpien D 47.10.17.3.

762- M. KASER, RPR $I_{2}$, p. 632 ; A. GUARINO, Diritto privato romano, cit., p. 874 ; T. SPAGNUOLO VIGORITA, Actio iniumiamom noxalis, in Labeo is (1969), p. $33 \mathrm{~s}$. ; Ulpien D 47.10.17.4-6.

763- J. MACQUERON, Histoire des obligations, cit, p. 283 ; T. SPAGNUOLO VIGORITA, o. c., p. 33 . naliter agere) (764). Cette deuxième voie semble être la plus suivie aי 4ème siècle pour les affaires d'une certaine gravité :

De iniuria nunc extra ordinem ex causa et persona statui solet. Et servi quidem flagellis caesi dominis restituuntur, liberi vero homilioris quidem loci fustibus subiciuntur, ceteri autem vel exilio temporali vel interdictione certae rei ccercentur (765).

Pour un délit identique, les esclaves sont fouettés ; les homes libres de basse condition puris de 1 a bastonnade, les autres seulement d'un exil temporaire. Il convient de faire deux remarques. Tout d'abord, le châtiment corporel n'est pas le seul "privilège" de l'esclave,mais sa punition a un caractère plus grave eu égard à l'instrument par lequel la répression s'exerce, le fouet. On notera ensuite, mais c'est un point sur lequel on a déjà insisté $(766)$, la discrimination opérée entre honestiores et humiliores sur le plan de la peine.

Quant à l'apfranchi, il a du mal, même dans ce domaine, à se débarrasser de ses antécédents serviles. Ainsi, l'injure qu'il adresse à son patron est toujours considérée comme atrox (767), la réciproque n'étant évidenment pas vraie.

Levis enim coercitio etian in nuptom vel convici non impudici dictio cur patrono denegetur? (768).

Pourquoi le fait d'infliger un "léger" châtiment ou d'zdresser une insulte non impudique à son affranchie mariée serait-il interdit au patron ? De tels faits ne donnent même pas lieu à poursuite, car

nec enim ferre praetor debet heri servon, hodie liberom conquerentem, quod dominus ei conviciun dixerit vel quod leviter pulsaverit vel emendaverit (769)

764- J. MACQUERON, Histoire des obligations, cit., p. 284 ; 1 a victime a le choix ertre ces deux actions, nais ne peut les ezercer successivement, cf. Pairl $n \Delta 7,10.6$.

765- Hermogenien D 47.10.45.

766- Cf. supra première partie n. 301 et 1 'article fondamental sur le problème de G. CARDASCIA, L'apparition dans le droit des classes d'homestiores et d'hamiliores, cit.

767- Ulpien D 47.10.7.8 : Atrocem autem iniuriam aut persona aut tempore aut re ipsa fieri Labeo ait. Dersona atrocior iniuria fit, ut cum magistratui, cum parenti patrono fiat.

768- Ulpien D 47.10.11.7.

769- Ulpien D 47.10.7.2. 
le préteur ne doit pes admettre qu'un individu, hier encore esclave, se plaigre de ce que le patron lui adresse quelques propos injurieux ou lui inflige quelques coups légers ou quelque correction... Féanmoins tout pouvoir, rût-il absolu, supporte parfois des limitations, et si le patron fouette l'affranchi ou le blesse gravement, le préteur interviendra $(770)$.

Ayant vu que l'esclave apparaissait sourent dans les textes du Digeste en tant qu'agent délictuel, il convient, pour terminer ce tour d'horizon sur les délits, de se demander si le droit l'en considère responsable.

On a brièvement évoqué le système de la noxalité à propos de l'iniuria, et si l'on s'en tenait à la faculté qu'a le mast,re de choisir entre le paiement de la poena et l'abandon du coupable (771), il faudrait répondre par la.négative.Cependant, ce n'en est pas moins l'esclave qui est visé car, en cas d'affranchissement, il est tenu de payer la poena (772). Cette responsabilité de l'esclave pouvait, dans

770- Ulpien D 47.10.7.2 (suite) : Sed si flagris, si verberibus, si vulneravit non mediocriter, aequissimm erit praetorem ei subvenire.

771- Gaius 4,75. Sur I'extension de I'institution, R. MONIER, Droit romain II, cit., p. 74. G. MAC CORMACK, The thievish slave, in RIDA 19 (1972), p. 345 s., soutient que les juristes classiques auraient déja posé les jalons d'une action contractuelle à titre noxal. Sur ce point, cf. W.W. BUCKLAND, Roman law, cit., p. 124 s.; E. LEVY, Die Konkurrenz der Aktionen und Personen im Klass. röm. Recht 2, 1 (1922), p. $70 \mathrm{s.}$; G. PROVERA, Contributi alla teoria dei iudicia contramia, (1951), p. 81 G. PROVERA, Contributi alla teoria dei iudicia contrama, (1951), P. 81 Law (1956), c. 12 ; J.A.C. THOMAS, Pro noxal surrender, in Labeo 17 (1971), p. 16. Contra, B. BIONDI, Iudiciae bonae fidei, AUPA 7 (1918), et Actiones noxales, AUPA 10 (1925), p. 120 ; HELDRICH, Das Verschulden beim Vertragsabschluss (1924), p. 22 s. ; ERBE, Die Fiducia im rôm. Recht (1940), p. $117 \mathrm{~s}$. ; R. MONIER, La garantie contre les vices cachés, Paris (1930), 'p. $199 \mathrm{~s}$. ; F. DE VISSCHER, Le régime romain de la noxalité, Bruxelles (1947), p. $470 \mathrm{~s}$. Le mâ̂tre doit avoir I'esclave en sa puissance, cf. J. MACQUERON, Histoire des obligations, cit., p. 328 ; H. NEHLSEN, Sklavenrecht zwischen Antike und Mittelalter, Göttingen (1972), p. $74 \mathrm{~s}$. Sur la garantie de disponibilité de I'esclave pour 1a (1972), P. $74 \mathrm{~s}$. Sur 1 a garantie de disponibilite de 1 'esclave pour la
nozae deditio, M. BRUTT, II vadimonizm nelle azioni nossali, in RISG, 8. III, 24 (1970), P. 261 s. Sur les modalitês de I'abandon, par mancipatio ou in iure cessio, cf. Z. LISOWSKI, Noxalis actio, in RE. supp1. 7 (1940), p. 619 ; M. RASER, RPR I 2, p. 164 ; H. NEHLSEN, o, c., p. 72 ; par traditio, cf. F. DE VISSCHER, o. c., p. 42 s. et $292 \mathrm{s.;}$ J. MACQUERON, O. C., p. 330.

772- H. NEHLSEN, Sklavenrecht, cit., p. 74 ; M. KASER, RPR I, p. 631. 1a loi des XII tables, faire écran à celle du maître, ainsi en matière de vol (773). La lex Aquilia va prévoir la responsabilité du martre du fait de sa sciontia $\left(77^{4}\right)$. L'utilisation d'esclaves dans les violences de la fin de la République conduit tucullus, en 76 av. J.C., à établìr dans l'édit la responsabilité du maître tout en atteignant aussi directement les membres de la familia l'ayant aidé (775). Ainsi, à côté de celle du maftre, la responsabilité de l'esclave est bien établie en matière de délits privés. Mais le lien de dépendance dans lequel se troure l'esclave ne peut-il pas, en certains cas, faire disparaître sa responsabilité, s'il agit sur un ordre de son maitre? Toute l'ambiguĩté de la situation apparaît ici. En effet, en matière de dommon iniuria datwon, Paul admet que celui qui doit obéir ne commet aucune culpa (776). Cependant, il n'en va pas de même pour l'iniuria ou le furtum, l'esclave pouvant alors se voir réclamer la paena une fois affranchi (777). L'esclave ne doit donc pas obéir en toute circonstance à son maitre, mais en a-t-il le choix en pratique ?

773- J. MACQUERON, Histoire des obligations, cit., p. 328 ; Ulpien D 9.4 .2 .1

774- B. ALBANESE, Sulla responsabilità del dominus sciens per 1 delitti del servo, in BIDR 70 (1967), p. 119 s. D 9.4.2.1 indique la coincidence entre scientia domini et iussus domini. Pour Ulpien, la scientia regroupe le cas ou le dominus est auctor et celui od il ferme les yeux (D 9.4.3; cf. D 9.3.5.10; D 14.1.1.3; D 14.1.4.5; D 47.6.1. 1) ; il $y$ a cependant eu des divergences sur cette notion de scientia, simple patientia pour Paul et Ulpien (cf. D 9.4.2 et 4), instigation pour d'autres. Selon G. TILLI, Dominus sciens e servus agens, in Labeo 23 (1977), p. $16 \mathrm{~s}$., le rapport mâ̂tre/esclave s'analyse ici comme un rapport de complicité; la victime peut exercer tant l'action pénale in solidum contre le maitre que 1 'action noxale. La loi des XII tables aurait reconnu à 1 'esclave la possibilitê de discerner 1 'ordre illicite et de ne pas obéir, tandis que la lex Aquilía, présumant que 1'esclave pouvait facilement y être contraint, sous menace de mort, n'aurait pas reconnu cette possibilité, $V$. SCARANO USSANI, Valori e storia, cit., p. 130. La première punit l'esclave, la seconde le maître, F. CASAvola, Cultura, cit., p. 153.

775- R. MONIER, Droit romain, cit. II, p. 63 ; J. MACQUERON, His toi re des obligations, cit., p. 323.

776- Paul D 50.17.169 pr: Is dammon dat, qui iubet dare : eius vero nulla culpa est, cui parere necesse sit.

777- E.M. SCHTAERMAN, La schiavitù, cit., p. 213 ; Alfenus (chez auteur anonyme) D 44.7.20 : Servus non in omibus rebus sine poena domino dicto audiens esse solet, sicuti si dominus hominem occidere 'aut furtw dicto audiens esse solet, sicuti si dominus hominem occidere 'aut
alicui facere' servon iussisset; Ulpien D 47.10.17.7 : Si iussu domini serrus iniumiam fecerit, utique dominue conveniri poterit etian suo nomine. Sed si proponatwr servus manumissus, placet Labeoni dandam in evm actionem, quia et noxa caput sequitur nec in omnia serrus domino parere debet, cf. aussi Paul D 50.17.157 et Ulpien D 43.24.11.7;

H. NEHLSEN, Sk1avenrecht, cit., p. 78 
Four les délits publics, $I^{\prime}$ ordre du maître ne constitue pas non plus un fait justificatif ( 778$)$. Le principe de la possibilité d'une poursuite de l'esclave est d'ailleurs si bien établi que, si le mâ̂tre refuse de le défendre, I'esclave peut faire appel à un autre homme libre (779). La peine infligée devra certes être adaptée à sa condition, ce qui peut porter atteinte aux intérêts du maître.

Soulever ce problème, c'est renare imminente la nécessité de se pencher sur le phénomène répressif et, plus globalement, sur les sanctions du droit en matière d'esclavage. Toutefois, il est auparavant souhaitable de rappeler les conclusions les plus importantes auxquelles cette étude nous a permis de parvenir.

$$
-0_{-}^{-}
$$

C'est à l'époque de Marc-Aurèle et de Commode que l'esclave apparaît le plus souvent comme agent d'acte juridique. Cette période correspond dans les textes à un changement d'utilisation de l'esclavage, au développement de formes d'exploitation plus douces par la reconnaissance, notament, d'une certaine capacité juridique à l'esclave. Cette évolution est due aux nécessités économiques, les esclaves privilépiós participant, dans une proportion toujours grandissante, à la vie des affaires. Mais elle n'est probablement pas étrangère à la recrudescence de la délinquance servile que les textes situent à l'époque des Antonins.

778- M. KASER, RPR $I I_{2}$, p. 432, n. 54.

779- E.M. SCHTAEPMAN, La srhiavitù, cit., p. 240 ; H. NEHLSEN, Sklavenrecht, cit., p. 97 ; U1pien $D 48.19 .19$ : Si non defendontur servi dominis, non utique statim ad supplicivom deducuntur, sed permittetur eis defendi vel ab alio, et qui cognoscit, debebit de innocentia eorm quaerere.
SECTION 2. LES SANCTIONS DU DROIT VIS-A-VIS DE L'ESCLAVE

L'Etat impérial va de plus en plus intervenir dans les relations privées esclave/maître. Il va lui-même prendre en charge l'exercice de la répression, mais la simple terreur risquant d'être insuffisante pour juguler toute manifestation de mécontentement servile, il va parallèlement développer l'idée de favor libertatis. Favor libertatis et répression ne sont done contradictoires qu'en apparence.

\section{Faragraphe 1 : Répression et torture.}

La fuitc et l'assassinat du maître constituant plus des formes de résistance au pouvoir dominical que de simples activités délictuelles, on les examinera plus tard. Cela dit, on peut distinguer répression et torture : lorsque son ressort n'est pas exclusivement la cruauté, la répression répond directement à un dólit dont l'esclave s'est renđu coupable; la torture s'exerce par contre de manière aveugle, pour un délit dont on ignore le coupable.

\section{I - La rápression de la délinquance servile}

La répression peut revêtir deux formes : tantôt, elle s'exerce de manière privée ; tantôt, elle est assurée par l'autorité publique.

\section{A/ - Le répression privée}

Le vocable "privée" est choisi à dessein. En effet, la répression peut être sssurée à l'échelle privée par un autre individu que le mâ̂tre. C'est tout d'abord le cas du flagrant dólit. Ainsi, selon Gaius, si quelqu'un a tué un esclave qui était en embuscade dans le but de le aépouiller de ses biens, aucune action n'est concédée au maître (780).

De même ne commet pas d'iniuria celui qui tue un esclave qui l'attaquait à main armée (781), mais il est vrai que la légitime défense ne constitue pas un cas spécifique à l'esclave.

In droit de correction est cnncédé au tiers è condition

780- Gaius D 9.2.4 pr.

781 - Ulpien 0.2 .5 pr. 
qu'il n'outrepasse pas les limites des bon mores (782). Ce droit est. bien entendu défini de manière plus large lorsque le tiers est détenteur d'un droit sur l'esclave. L'usufruitier, par exemple, peut verberare l'esclave sans que son maitre puisse $s^{\prime}$ en plaindre (783). Cependant, iI ne peut que le châtier modérément ( 784$)$. Il ne peut ni le fouetter, ai lui raire endurer des tourments (785). Il ne peut pas non plus lui faire des cicatrices qui le rendent difforme (786)... Malgré ces limitations, les textes laissent perplexe, et il est intéressant de relever que quatre des cinq passages traitant d'une castigatio infligée à l'esclave par l'usufruitier envisagent le cas où ce dernier n'a précisément pas fait preuve de modération ( 787$)$. Ne $s^{\prime a g i t-i l ~ q u e ~ d e ~ b a v u r e s ? . . . ~}$

Dans ces cas, le mâtre peut avoir recours non seulement à l'actio legis Aquiliae, mais ausi à l'actio servi corrupti et à l'actio iniuriamon (788). En outre, la lex Cornelia va s'appliquer au meurtre de I'esclave et permettre au mấtre d'exercer des poursuites criminelles $(789)$.

782- U1pien D 47.10.15.38 : Adicitur 'adversus bonos mores', ut non omnis omnino qui verberavit, sed qui adversus bonos mores verberavit, teneatur : ceterion si quis corrigendi animo aut si quis emendandi, non tenetur; M. KASER, RPR I , p. $505 \mathrm{n} .13$, v. aussi Papinien D 43.10 . 1.2 .

783- U1pien D 47.10,15.37.

784- U1pien D 7.1.23.1.

785- Ibid. : etenim modicam quoque castigationem fructuario competere Sabinus respondit et Cassius libro octavo iuris civilis scripsit, ut neque torqueat, neque flagellis caedat ; Paul D 7.1.66:... servrom torquendo deteriorem fecerit.

786- Paul D 7.1.17.1 : ... quamvis usufmetuarius nec contrariis quidem ministeriis aut inusitatis artificizon eius cormompere possit nec servim cicatricibus deformare. Cependant, ce butoir qu'on ne peut dépasser ne nous renseigne pas en tant que tel sur la réalité quotidienne.

787- Ces quatre textes sont D 7.1.66 Paul; Ulpien D 7.1.15.3 (Labéon); D 7.1.17.1 (Cassius, Aristo); D 7.1.23.1 (Sabinus, Cassius). Le seuI texte où il ne soit pas question d'un excès de l'usufruitier est D 47. 10.15.37 de U1pien.

788- Paul D 7.1.66.

789- Gaius 3, 213 ; Marcien D 48.8.1.2 : Et qui hominem occiderit, pumitur non habita differentia, cuius condicionis hominem interemit;

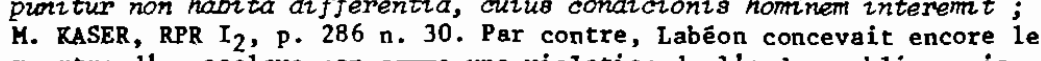
meurtre d'un esclave non conme une violation de l'ordre public, mais seulement comme un đélit privé, cf. D 47.10.7.1; Th. MOMMSEN, Droit pénal, cit. II, p. 329 n. 2.
Quant à la repression domestique proprement dite, elle est, si $l^{\prime}$ 'on en croit les textes, autant varióe que cruelle. A titre de sanction, un esclave peut être transféré de la farrilia urbana dans la farmilia mustica $(790)$, il peut être vendu à l'étranger (791). Ce sont là des peines bien douces, tout du moins physiquement. Dans le cadre d'une simple correction, l'esclave reçoit parfois un coup de poing de san maître (792) ; il est ailleurs battu, caesurus (793) ou soumis à la verberatio (794). Il est jeté dans la prison damestique, vinctus (795), sanction qui peut être lourde de conséquences pour son affranchissement éventuel (796). On lui inflige le port des entraves (797).

Souvent, ces peines sobt sans cosmune mesure avec l'acte répréhensible. Ainsi, dans un but de correction, l'esclave est enchaîné alors

790- Ulpien D 28.5.35.3: ... si servus fuerit missus in villam interim illic futurus, quia dominum offenderat, quasi ad tempus relegatus...; J. MARQUARDT, vie privée, cit. p. 214.

791 - La vente a l'extérieur est aussi un châtiment selon J. MARQUARDT, o. C., P. 214 n. 7 ; cf. Ulpien D 21.1 .17 .19 ; v. aussi D 40.1.4.9 et Papinien D 18.7.7.

792- Paul D 47.10.4.

79.3- Ulpien D 47.10.15.45 (Mela).

794- Ulpien D 47,10.15.36-37.

795- Carcer et vincula sont juridiquement synonymes parce que tout prisonnier peùt être enchẫné en droit strict, Th :MOMMSEN, Droit pénal, cit.III p. 304, cf. Callistrate D 4.6.9. I1 ne faut pas confondre ces vincula, infligées dans le cadre de la discipline damestique, et se déroulant dans 1 'ergastulum (cf. J. MARQUARDT, Vie privée, cit., p. 211, cf. Colum. $\mathrm{I}, 6,3 ; \mathrm{I}, 8,16 ; \mathrm{XI}, 1,22 ; \mathrm{Th}$. MOMMSEN, o.c., p. 306, souligne que $1^{\prime}$ ergastulion est à l'origine une institution écononique et non un lieu de peines), avec les vincula publica (cf. par ex. Papinien D 48.3. $2 \mathrm{pr}$ ), Th. MOMMSEN, o.c., I, p. $352 \mathrm{n}$. i. Les érgastula auraient été abolis par Hadrien, cf. SHA, Hadr., 18, 10 ; J. KOLENDO, Lavoro servile, cit., p. 33.

796- Les vincula ont une importance sur la question de savoir si 1 'esclave peut parvenir au complet affranchissement. Une prescription de la lex Aelia Sentia ne permet pas a l'affranchissement de 1 'esclave qui a Été enchaîné de produire la plénitude de ses effets ; la sinple incarcération ne suffit pas, Th. MOMMSEN, Droit pénal, cit. I, p. 352 , n. I et III p. $304 \mathrm{n}$. I ; cf. Ulpien D 50.16.216 : Verum est evm, qui in et esse, nisi corpori eius vincula sint adribita. En outre, la liberte fidéicomissaire n'est plus due à 1 'esclave que son maître a enchaîné depuis, cf. Paul D 40.5.43.

797- Compeditus, cf. U1pien D 4.3.7.7 (Labéon, Scaevola); D 47. 10.15 .44 . 
qu'il n'avait comnis qu'une levissima offensa (798). Mais il est également fréquent que le seul moteur de la discipline domestique soit le caprice ou la cruauté (799). Il arrive par exemple que le maître refuse d'alimenter son esclave ou lui fasse endurer des violences insupportables $(800)$, ou même le tue sans que la raison de cet acte apparaisse dans le texte (801).

Auguste s'efforce de respecter les prérogatives des maîtres et c'est ainsi qu'il se contente d'user de la persuasion auprès de Vedius Pollio (802). Mais le pouvoir impérial va devoir très rapidement entrer en conflit avec l'autorité absolue des mâtres (803). En 19 après J.c., la lex Petronia soumet au contrôle du nagistrat le pouvoir du maître de livrer ses esclaves aux combats avec des bêtes (804). Claude interdit au maître de tuer son esclave incapable de travailler (805). Hadrien réitère l'interdirsion de tuer son esclave, intimant au maître de s'adresser à un tribunal pour fize punir l'esclave coupable d'avoir commis une faute arave (806). Antonin le ploux prévoit des peines con-

798- Ulpien D 20.1.27 (Marcellus) ; v. D 1.6.2 : ... matronam... ex levissimis causis ancillas atrocissime tractasset, vlpien (- Coll., 3, $3,4)$.

799- Th. MOMMSEN, Droit pénal, cit. I, p. 22 ; v. par ex. Ulpien D 21. 1.23 pr (Pouponius) ; D 40.5.24.8.

800- Ulpien D 1.6 .2 rappelle qu'un rescrit d'Antonin le pieux est intervenu pour réprimer de tels abus, et qu'Hadrien avait condamé a une relegatio de cinq ans une maîtresse trop cruelle avec ses ancillae; E.M. SCHTAERMAN, La schiavitũ, cit., p. 232 .

801- Par exemple, v. Pomponius D 45.1.23; Papinien D 46.3.95.1 Paul D 9.2.12 ; D 45.1.91 pr ; Ulpien D 4.3.7.4; D 6.1.17 pr (Julien); D 9.2 .17 .

Rappelons enfin que l'affranchi est encore soumis à une levis coercitio du maître devenu patron, cf. Ulpien D 47.10.11.7.

802- Sênèque, De ira, 3, 40 ; Pline l'Ancien, H.N., 9, 39, 2 ; Dion Cassius 54, 23, 1-4. E.M. SCHTAERMAN, La schi avitù, cit., p. 211 . Sous la République, le censeur pouvait toujours intervenir pour prendre des mesures contre le mauvais usage de ses pouvoirs par le maître d'un esclave, cf. M. KASER, RPR $I_{2}$, P. 284.

803- Labéon, qui reflète $l$ 'esprit de son époque, déclare que celui qui libère de ses chaînes l'esclave d'autrui n'est pas tenu par l'actio furti s'il a agi par misericordia (chez Ulpien D 4.3.7.7).

804- Modestin D 48.8.11.2 : Post legem Petroniam et senatus consulta ad eam legem pertinentia dominis potestas ablato est ad bestias depugnandas suo arbitrio servos tradere : oblato tamen iudici servo, si iusta sit domini querella, sic poenae tradetur. M. RASER, RPR I, p. 285. Sur $l a$ date de cette $l \in x$, J. GAUDEMET, Institutions de $l^{\prime}$ Antiquité, cit., p. 547, donne avec incertitude 19.

805- Slétone, Claude 25,2 ; Th. MOMMSEN, Droit pénal, cit. II, p. 330 ; M. K: SER, RPR $L_{2}$, p. 286, n. 29 . tre les maitres qui tuent leurs esclaves sine causa (o0-). Cette évolution a lieu parallèlement à celle qui touche le fils (80Tbis). Marc-Aurèle et Verus interdisent de vendre un esclave à un acheteur désireux de lui paire combattre les bêtes (808). Septime-Sévère, malgré une attitude rigoureuse dans la répression de l'adultère, interdit au maitre de tuer son esclave coupable de ce crime avec sa propre femme : l'intervention d'un magistrat est nécessaire (809). Caracalla ne permet qu'exceptionnellement qu'un esclave soit enchâ̂né à perpétuité (810).

Ces limites à l'arbitraire des meîtres peuvent obliger à vendre l'esclave (811) ou aboutir à une sanction pénale (812). Ellles remettent en question les pouroirs du propriétaire d'esclaves, mais qui provoque l'application de ces mesures?

Sans doute connaît-on déjà l'usage pour un esclave de demander la protection du magistrat contre un maître trop cruel, et au début du Principat s'instaure I'usage de permettre aux esclaves de trouver asile au temple de Jules César (813). Sous Tibère, la protection est Etendue, 806- SHA, Hadr. 18,7-11. M. RASER, RPR $I_{2}$, p. 286, n. 29.

807- Gaius I, 53 ; D 1.6.1.2. S. SOLAZZI, in SDHI 20 (1954), p. $304 \mathrm{~s}$.

807 bis- Sur les limitations du ius vitae necisque du père sur.son fils, cf. Trajan chez Papinien D 37.12.5; Hadrien chez Marcien D 48. 9.5. Il ne semble pas cependant que ces mesures extra ordinem dans $l^{\prime}$ intérêt du fils aient précédé la tégislation favorable aux esclaves. On a vu en effet que $C l$ aude avait déjä fait rentrer dans le champ d'application de la loi sur le meurtre la mise a mort de l'esclave par son plication de la loi sur le meurtre la mise a mort de l'esclave par son propre maitre, du moins lorsque celui-ci avait fait mourir un esclave incapable de travailler ( $c f$. supra n. 805 ). Il faudra attendre l'époque post-classique pour que ce droit s'éteigne à l'égard du fils (cf. C.Th. $9.15 .1=$ CI 9.17 .1 . Th. MOMMSEN, Droit pénal, cit. II, p. 332 et n. 1; A. GUARINO, Diritto privato romano, cit., p. 494).

808- Marcien D 18.1.42: Domini neque Fer se neque per procuratores swos possunt saltem criminosos servos vendere, ut cum bestiis pugnarent. Et ita divi fratres reacripsemont; Modestin D 48.8.11.1. H. WALLON, Histoire de l'esclavage, cit. III, p. 57 n. 4.

809- Ulpien D 1.12.1.5: Si quis servum surm adulterium commisiase dicat in uxorem sucon, apud praefectrm urbi erit audiendus. E.M. SCHTAERMAN, La schiavitù, cit., p. 240.

810- c. I. 9.47.6 : Incredibile est, quod adlegas libemon hominem, ut vinculis perpetuis contineretur, esse damatum : hoc enim vix in sola servili condicione procedere poteat. E.M. SCATAERMAN, La schiaviti, cit., p. 238 .

8I1- Rescrit d'Antonin le pieux cité par Ulpien D 1,6.2.

912- Cf. D 1.6.2 : cinq ans de relégation par Badrien (M. RASER, RPR I , p. 286, n. 32) ; application de la lex Comelia de sicorits et veneficiis pour le maitre qui tue son esclave, cf. M. FASCIATO, Note sur l'affranchissement des esclaves abandonoés dans l'île d'Esculape, in RFD (1949), p. 463.

813- Dion Casaius, $47,19,3$ 
Les 1 mages des empereurs constıtuant, une salvegarde, qu'elles soient exposées dans un lieu public ou privé $(914)$. Le préret de la ville a pour tâche d'examiner le cas de ces esclaves, sans que ceux-ci puissent être tenus pour coupables d'avcir accusé leur maître (815).

Quel est 1'impact de ces mesures protectrices dans la pratique ? Prenons un exemple. On a vu qu'Antonin le pieux avait prévu des peines contre ceux qui tuaient leurs esclaves sine cansa, c'est-ì-dire sans raison suffisante. Bien qu'il appartienne au juge de dire s'il y a juste motif $(816)$, on reste perplexe quant au contrôle que celui-ci peut exercer en pratique sur une discipline domestique qui, par définitîn, supporte mal les restrictions (817). Toujours est-il qu'on voit Marcellus et Unpien déclarer que le maitre qui tue avec juste raison, ob facinus (818), hoc eat merentem ( 819 ), un esclave ayant fait l'objet d'we stipulation ou d'un legs, est libre de toute obligation de dommages-intérêts vis-à-vis du créancier. L'affirmation selon laquelle Hadrien aurait interdit aux mâtres de tuer leurs esclaves eux-mêmes est difficilement conciliable avec les espèces précédentes. Peut-être cet empereur s'est-il contenté d'une simple exhortation (820) ? Quci qu'il en soit, des textes postérieurs à 1 'époque des Antonins montrent que ces prescriptions n'empêchent pas le maittre d'avois la main leste dans la pratique (821).

Si les mesures concernant la limitation du droit de mise à mort de l'esclave par son maitre sont le signe d'une certaine amélioration, il n'en ra pas ainsi dans d'autres domaines. Prenons le cas des vincula perpetua. Alors que Caracalla s'efforce d'en limiter l'usage à des cas exceptionnels, Alexandre-Sévère considère que l'esclave qui a été rendu à son maitre sub poena vinculorn, sans que la durée de la peine soit précisée, doit rester enchaîné à perpétuité (822)...

814- Tacite, Ann., 3, 36. C. GIOFFREDI, Ad statuas confugere. SOHI 12 (1946), P. 187 ; M. KASER, RPR I P. 286 ; v. dans le Digeste Ulpien D 1,6.2 (rescrit d'Antonin le pieux) ; D 21.1.17.12 (Labéon, Caelius Sabinus) ; D $21,1.19 .1$

815- Ulpien D 1.12.1.1 : Servos qui ad statuas confugerint, vel sua pecunia emptos ut manumittantur, de dominis querentes, audiet.

816- J. GaVDEMET, Institutions de l'Antiquité, cit., p. 547, n. 7.

817- Th. MOMMSEN, Droit pénal, cit. II, p. 330.

818- Marcellus D 45.1.96.

819- Ulpien D 30.53 .3

820- Th. MOMSEN, Droit pénal, cit. II, p. 330. n. 4.

821 - v. supra n. 801 . tione domino redai iussus sententia praesidis provinciae pexpetuo vinctus esse debet. rette dernière resure soulève le problème te l'utilisaticn to l: repression domestique dans le domaine du droit pénal. L'Etat, aglz: ب́ son intervention croissante, tente en effet de ménager parallèlement la susceptibilité des propriétaires d'esclaves. C'est ainsi que pour atteindre le moins possible le propriótaire innocent, le magistrat lui laisse, lorsque la nature du dólit le pentet, le soin d'assurer la punition du coupable en l'enfemant dans la prison domestique (823). Selon une constitution de Marc-Aurèle et Verus, celui qui, prétendant s'être racheté de ses deniers, porte plainte contro le maitre pour refus de l'affranchir et échoue dans sa demanđe, sera condemné in opus metalli à moins que le maître ne le réclame, euquel cas il ne pourra lui infliger une peine plus forte (824). Paul écrit que celui qui ne parvient pas à prouver qu'il s'est racheté de ses deniers sera rendu au maître aub poena vinculomom, et si le maitre le désire, condamns in metallzm (825). Enfin, pour Macer, si celui qui, devant être rendu à son mâtre sub poena vinculorion, n'est pas rócupéré par lui, il sera vendu, et si aucun acheteur n'en veut, il sera condamné in opus publicion perpetison (826).

Le procédé inverse par lequel, en cas de délit public, le dominant livre l'esclave au magistrat au lieu de le punir lui-rême semble très rare (827).

\section{B/ - La répression publique}

Malgré sa dureté, la répression publique présente au moins pour l'esclave la garantie d'une protection vis-à-vis de l'arbitraire dominical (828). Les différentes feines susceptibles d'être infligées à un esclave, dont fait, état le Digeste, sont la mort, l'esclarage pénal, l'internement et, enfin, divers̀es peines corporelles.

823- Th. MOMNSEN, Drait pénal, cit. III, p. 307

824- Chez Marcien D 40.1.5 pr.

825- Paul D 48.19.38.4.

826- Macer D 48.19.10 pr.

827- Ulpien D 13.7.24.3 : Plane si pro maleficiis suis ccercuit vel vinxit vel optulit praefecturae vel praesidi, dicendum est piareraticia creditorem non teneri.

828- Exemple de dureté de la répression publique : un mấtre remet son esclave à Attius qui le soupçonnait de vol, pour qu'il le fasse soumettre a la quaestio; Attius le remet au préfet des vigiles qui le condamne au summum supplicium, cf. Pomponius D 12.4.15 (Labéon, Proculus). 
La peine de mort ressort des textes par $1^{\prime}$ emploi des termes oupplicium (829) ou poena capitis (830). Le fait que ces textes soient relativement nombreux incite à penser qu'il s'agissait d'une peine d'usage fréquent (831). Cependant,le Digeste est pauvre en renseignements sur les réalités de ces exécutions publiques d'esclaves (832). Callistrate déclare que ceux qui ont attenté à la vie de leur mâ̂tre doivent périr par le feu (833). D'autre part, les condamations au iuctom venatorizm ou ad bestias, bien qu'il faille être plus nuancé pour la première, se présentent aussi comme des formes d'exécution de la peine de mort (834). Ces condamnations font de 1 'esclave un servus poenae. 829- Selon Th. MOYMSEN, ultimom aupplicion oppose la peine de mort
grave a la simple poena copitis (Droit pénal, cit. III, p. $243 \mathrm{n}$. 5), cf. Modestin D 48.9.9.1 ; sur summon supplicium, cf. Callistrate D 48 . $19.28 \mathrm{pr}$. Dans une autre conception, summom aupplicivon et ultimum suppliçizm désignent la peine de mort par opposition aux peines non capitales (Paul D 47.12.11 ; Celsus D 48.19.21).

830- On a vu (supra chapitre préliminaire n. 17) que la peine capitale était graduée (cf. Callistrate D $48.19 .28 \mathrm{pr}$ : eummon supplicium, condamation in metallum, deportatio) ; une condamation capitale entrainne, selon Ulpien (D 48.19.2 pr) soit la mort, soit la perte du droit de citê, soit l'esclavage. Bien que cette condamnation puisse théoriquement entrainer la peine des mines, la poena capitis semble viser en première ligne pour l'esclave la peine de mort $:$. Par ailleurs, il ne faut pas ignorer non plus les termes causa copitalis (par ex. Hermogénien D 1.5.13), copitis accusatio (par ex. Paul D 40.5.33.1) et orimen capitalis (par ex. Papinien D 48.3.2 pr).

831- On relève sur supplicilan : Pomponius D 12.4.15 (Labéon, Procu1us); Maecianus D 29.5.14 ; Callistrate D 48.19.28.11 ; Paul D 35. 2.39 ; D 47.11.1.2 ; Ulpien D 2.1.12 ; D 29.5.1.12 (Scaevola)-13 (Scaevola) $-14-21-28$ (rescrit d'Hadrien) $-30-31-33$; D 29.5.3.4-13-16-17-18; D 29.5 .5 .2 ; D $30.53 .8 ; D$ D $40.12 .7 .4 ;$ D $48.18 .7 ;$ D $48.19 .19 ;$ D 48 . 8.4.2 (rescrit d'Hadrien) ; D 48.10.8; Marcien D 48.10.1.13; Modesti D 29.5.19. Sur poena copitis, Callistrate D 47.21.3.1 (Nerva); Ulpien D 48.18.1.20. Sur crimen capitalis, Papinien D 48.3.2 pr ; Modestin D 48.2.17. Sur causa copitalis, Marcien D 40.9.9.1; D 48.1.9; Hermogénien D 1.5.13. Sur capitis accusatio, Paul D 40.5.33.I.

832- Sur la pratique des supplicia, v. F. DE MARTINo, I aupplicia dell'iscrizione di Pozzuoli, Labeo 21 (1975), p. 211 s.

833- Callistrate D 48.19.28.11 : Igni cremantur plemonque semi, qui saluti dominorw suomum insidiaverint, nonrunquan etiam liberi plebeii et huriles personae. Selon Paul, Sent., 5, 23, 17, 1a mort par le feu est plus rigoureuse que le crucifiement et l'exécution dans une fête populaire, cf. Th. MOYMSEN, Droit pénal, cit. III, p. 243 n. 5.

834- Sur ces peines, cf. supra première partie n. 291, 294, 295.
A ce propos, Upien nous apprend que les esclaves sont ordinairement condamnés in metallum, in opus metalli ou in ludim venatorizm (835), liste à laquelle il faut, courme on vient de le voir, sjouter la condamation ab bestias (836). Les textes sont peu nombreux sur ce problème (837), mais il est possible d'en extraire quelque indications intéressantes. L'esclave condamné à une telle peine est soustrait à la puissance de son maître (838) et devient la propriété du Fisc (839)). Ainsi, si un esclave condamné in metalzzon est libéré par la grâce de l'empereur, un rescrit de Caracalla précise qu'il ne doit pas être restitué à son mâ̂tre (840). En principe, un tel esclave ne peut espérer la liberté. Cependant, si on lui a laissé une liberté fidéicommissaire et qu'il ait été libêré de sa peine par la grâce de l'empereur, il pourra l'obtenir, cela en vertu d'un rescrit du même Caracalla (841). Selon Aristo, rapporté par Pomponius, si un esclave, qui a reçu la liberté pour en jouir lorsqu'il aura trente ans, est condamné in metallum avant cet âge, et qu'il soit parla suite grâcié, il pourra réclamer la liberté qui lui avait été laissée (842).

835- U1pien D 48.19.8.12 : Servos in matall $l_{2 m}$ vel in opus metalli, item in ludion venatoriun dari solere nulla dubitatio est. Sur la distinction entre in metallion et in opus metalli, cf. supra première partie, n. 281 .

836- Modestin D 48.8.11.2; D 49.1.18.

837- Condamation in metallum : Pomponius D 40.4.46 (Aristo) ; Papinien D 48.18.17.3; Paul D 47.9.4.1 (rescrit d'Antonin le pieux); D 48 19.38.4 ; Ulpien D 40.5.24.5 (rescrit de Caracalla); D 48.19.8.12 (rescrit de Caracalla). Condamation in opus metalli': Ulpien D 48.19.8. 12 ; Marcien D 40.1.5 pr (constitution de Marc-Aurelle et Verus). Condamation in Zudum venatorium : Ulpien D 48.19.8.12. Condannation ad bestias : Modestin D 48.8.11.2; D 49.1.18. Un texte d'Ulpien indique seulement qu'une statulibera est devenu serva poenae sans nous renseigner sur la nature de la peine a subir (cf. D $40.7 .6 \mathrm{pr}$ ). Il est également question d'esclavage de la peine a propos d'affranchis, la seule peine qui transparaisse étant celle des mines, cf. Scaevola D 38.2 .48 . Ulpien D 1.12.1.10; D 37.14.1; D 37.14.21 pr.

838- Ulpien D 40.5.24.5 (rescrit de Caracalla); D 48.19.8.12 (rescrit de Caracalla).

839- Cf. supra premiere partie n. 276 .

840- Chez Ulpien D 48.19.8.12.

841- Chez Ulpien D 40.5.24.5. W.w. BUCKLAND, Roman law, cit., p. 278, n. 2 .

842- Pomponius $D$ 40.4.46. La fin du passage montre que la solution est $1 \mathrm{a}$ même si l'esclave a été institué heritier sous condition. 
Si l'esclave peut être enchaîné dans le cadre de la discipline domestique, son enchaînement peut aussi être ordonné par l'autorité publique, que ce soit à titre de détention préventive ou répressive (843). En ce qui concerne le premier cas, Papinien affirme qu'un esclave accusé d'un crime capital, s'il n'est pas défendu, doit être jeté in vincula publica (844). Il admet néanmoins la possibilité pour le mâtre de payer la caution par la suite (845).

Du point de vue répressif, en dehors du cas, le plus fréquent, ol cette peine est purgée dans la prison domestique, vincula apparaît dans deux textes oul il est synonyme de travaux forcés (846). Un rescrit de Marc-Aurèle et Verus nous apprend dans le premier que l'esclave condamné in temporaria vincula peut recevoir la liberté après avoir purgé sa peine (847). Dans le second, Ulpien écrit que l'esclave condrumé in perpetua ou in temporaria vincula continue d'appartenir à celui qui, avant la condamnation, était son maître $(848)$. Ces deux textes sont.

843- Il est également question de détention a propos de la quaestio (cf. Ulpien D 16.3.7 pr) et de la garde des fugitifs (cf. Ulpien D 11. 4.1.7; D 47.2.52.12) : on y reviendra en examinant ces thèmes.

844- Papinien D 48.3.2 pr.

845- Papinien D 48.3.2.1 : Solet itaque tractari, an postea domino permittendrom sit oblata satisdatione servwn surm vinculis liberare. Dubitationem auget edictum Domitioni, quo cautum est abolitiones ex senatus consulto factas ad huiusmodi servos non pertinere. Nom et lex ipsa prohibet ewn absolvi, priusquam de eo iudicetur. Sed haec interpretatio perdura, pernimium severa est in eo, cuius dominus absens fuit vel quoa per inopinian illo momento temporis satisdationem implere non potuit : neque enim pro indefenso derelictus recte dici potest, qui dominum praesentem non habuit vel habuit paratum defendere, pauperem tomen.

Le texte est intéressant à un autre titre : il montre que même un pauvre Le texte est intêressant à un autre titre : il montre que même un pauvre
peut possêder un esclave. Sur la notion d'abolitio, v. A. BERGER, Encycl. Diction., cit., p. 338 s. ; v. également Papinien D 48.16.8; D 48.16.10 pr ; Ulpien D 48.16.12 ; Macer D 48.16.9; et surtout Paul D 48.16.16: Domitianue rescripsit, quod de feriis et abolendis reis dicitur, non pertinere ad servos, qui accusati in vinculis esse iubentur, ne iudicium finiatur.

846- Il s'agit de Papinien D 48.19.33 et U1pien D 48.19.8.13. Sur le fait que, juridiquement, la prison soit la detention avec travaux forcés, cf. Th. MOMSEN, Droit pênal, cit. III, p. $295 \mathrm{n} .4$.

847- Papinien D 48.19.33. Mais si la liberté est laissée a l'esclave alors qu'il est dans les chaînes, il ne pourra en profiter : Si cutem beneficiun libertatis in vinculis eos inveniat, ratio iuris et verba constitutionis libertati refragantur.

848- Ulpien $D$ 48.19.8.13: Sed sive in perpetua vincula fuerit domatus servus sive in temporalia, eius remanet, cuius fuit, ontequam domaretiur. des exceptions au principe en vertu duquel un esclave ne peut ôtro condamné aux travaux forcés (849). Il est rai qu'une telle neine nuit au maitre (850). L'interdiction ressort d'un passage de Papinien selon qui, si l'esclave a été condamné par erreur in opus publicom temporarizon, il doit être rendu à son maître une fois le temps de la peine révolu (851).

L'esclave peut enfin être soumis par l'autorité répressive publique à des châtiments corporels. Le correction qui, ̀̀ l'orig̣ine, n'est qu'une peine accessoire, va devenir pour les esclaves une peine autonome (852). L'esclave est alors soumis à la verberatio (853). Cette correction administrée par l'autorité n'affecte évidement pas le droit de propriété du maître (854). Du point de vue des mentalités, un texte de Callistrate mérite d'être cité :

In metallum damnatis libertas adimitur, cun etian verberibus servilibus coercentur (855),

c'est-æ̀-dire : les condamnés à la peine des mines perdent la liberté car ils subissent même la correction dans les conditions d'usage pour les esclaves. Certes, seuls les hommes libres de basse

849- v. aussi Macer D $48 \cdot 19 \cdot 10 \mathrm{pr}$. Th. MOMMSEN, Droit pênal, cit. III, p. 296 , n. 5 .

850- Th. MOMMSEN, Droit pénal, cit. III, p. 296.

851- Papinien D 48.19.34 pr : Servus in opue publicwm perpoturm ac multo magis temporarium non datur. Cun igitur per errorem in opus temporarizon fuisset datus, expleto tempore domino servion ssse reddenaizm respondi.

852- Th. MOMSEN, Droit pénal, cit. III, p. 334. Il faut aussi signaque la correction remplace les amendes publiques vis-a-vis des esclaves, lorsque le maître ne veut pas les acquitter à leur place, et visa-vis des personnes libres sans ressources (ibid., p. 335); c'est dans ce sens qu'il faut entendre le corpus torquendron de $\mathrm{D} 2.1 .7 .3$ (U1pien), la torture proprement dite ne se rencontrant que dans la procédure de la preuve (ibid. n. 5).

853- Le terme verberatio $a$ propos de la rêpression publique de l'esclave apparaît chez Callistrate D 49.14.12; Paul D 4.4.24.3; Ulpien D 47.10.15.39 (Labéon) ; D 47.10.17.4-5-6; D 48.2.6.

854- Callistrate D 48.19.28.4 : Servi caesi solent dominis reddi. Il est intếressant de noter que Labéon admet même une possibilité de poursuite pour le maître dans le cas où le magistrat a corrigé l'esclave adversus bonos mores, cf. Ulpien D 47.10.15.39.

855- Callistrate D 49.14.12. 
condition peuvent avoir à endurer cette peine (856). II n'en demeure pas moins qu'on voit là le caractère grave de la verberatio servile. Ce caractère ressort également $d^{\prime}$ un passage d'Ulpien :

Verberasse dicitur abusive et qui pugnis ceciderit (857),

ce qui signifie : verberasse est employé de manière impropre à propos de celui qui a frappé l'esclave à coups de poing. La correction doit donc être plus sévère (858). Elle semble généralement s'effectuer par le fouet (859), mais cet instrument de répression connait Iui-même une gradation. Ainsi l'esclave peut être battu au moyen d'un fouet de cuir, lomon (860), ou bien d'un fouet de cordes à noeuds, flagrion (861) ou flagellum (862), assorti de fils de fer et d'aiguillons (863).

Le fouet est l'expression de la discrimination sur le plan répressif entre esclaves et libres (864), ceux-ci subissant pour un délit identique la bastonnede. La distinction ressort expressément d'un passage de Macer :

In servomon persona ita observatur, ut exemplo homiliomon puniontur. Et ex quibus cousis liber fustibus caeditur, ex his servrs flagellio caedi et domino reddi iubetur (865).

\section{6- Cf. supra première partie n. 301.}

857- Olpien D 47.10.15.40.

858- Pour une idée de la barbarie, v. J. MARQUARDT, vie privée, cit p. $214 \mathrm{s.g}$ dont le développement est très intéressant sur les réali tés des supplices.

859- Le fouet devient une, peine indépendante pour les crimina levia, в. NEHLSEN, Sklavenrecht, cit., p. 94 ; cf. Ulpien D 48.2.6.

860- Le terme apparaît chez ulpien D 47.10.15.39 (Labéon). Sur sa définition, J. MARQUARDT, vie privée, cit., p. 214 n. 2 .

861- Ulpien D 47.10.9.3.

862- Paul D 47.9.4.1 (rescrit d'Antonin le pieux); Ulpien D 47.10. 17.2 (Trebatius, Labéon, Mela); D 48.2.6 ; Macer D 48.19.10 pr Bermogénien D 47.10.45.

863- J. MARQQUARDT, Vie privée, cit., p. 214 n. 2.

864- A titre indicatif, il est intéressant de remarquer que, dans le domaine privé, le fait, pour l'affranchi, d'être fouetté par son patron est constitutif d'iniuria, cf. Ulpien D 47,10,7.2. La Beule peine corporelle à être infligée à l'affranchi de manière publique qui ressorte du Digeste est la bastonnade, cf. Ulpien D 1.12 .1 .10 ; D 1.16.9.3 ; Modestin D 37.14.7.1.

865- Macer D 48.19.10 pr. L'inégalité légale des peìnes est un príncipe contraire au droit pénal; elle s'explique en l'espèce par l'incapacitế
Les esclaves sont pumis à I'image des humiliores (866). Dans le cas où l'home libre est soumis à la bastonnade, l'ešclave est fouetté et restitué à son maître. Si l'on en croit UIpien, cela est valable pour les levia crimina (867). Mais la discrimination apparaitt aussi dans des délits plus graves. On l'a évoquée plus haut en matière d'injure (868). Il est possible de donner d'autres exemples.

Ainsi, en cas de vol importent comis par la force à l'occasion d'un naufrage ou de l'effondrement d'un édifice, un rescrit d'Antonin le pieux prévoit que l'homae libre doit être puni de la bastonnade et d'une relégation de trois ans. S'il est de basse condition, il doit être condamné aux travaux forcés pour la même durée. Quent à l'esclave, il doit être founetté et condarmé in metallum (869). En matière de faux, l'homme libre est puni par la déportation et la confiscation de ses biens, tandis que I'esclave est condamné à mort $(870)$.

Ces peines, quoique très dures, sont au moins fondées en droit. Elles répondent à un acte délictuel. On ne peut en dire autant de la torture.

\section{II - La torture}

La torture n'est pas une peine. Elle est considérée en droit romain comme un moyen de preuve. Avant d'examiner les réalités que nous livrent les textes sur ce procédé, il convient de s'attarder sur les principaux problèmes juridiques qu'il soulève.

Il faiut tout d'abord noter qu'en cas d'action délictuelle privée, le propriétaire de l'esclave n'est pas tenu de permettre son inter-

juridique de l'esclave, cependant responsable de ses dellits, Th. MOMMSEN, Droit pénal, cit. III, p. 390.

866- On a déja traité des inégalités de peines entre homes libres (cf. supra première partie $\pi .299,300$ et 301 ), et il n'est donc pas utile d'y revenir ici.

867- Jlpien D 48.2.6.

868- Hermogéniẹ D 47.10 .45

869- Paul D 47.9.4.1

870- Marcien D 48.10.1.13. 
rogatoire (871), de telle manì̀re que, si la victime y procède de sa propre initiative, il y a domage puissable (872). Par contre le consentement du maître n'est pas nécessaire dans le cadre de la procédure pénale publique (873). Cependant, d'autres problèmes juridiques vont surgir.

En premier lieu, cet interrogatoire est interdit s'il doit ettre effectué à l'encontre du maître. On tend de la sorte à faire triompher le respect de l'autorité des maîtres sur les nécessités de la sécurité publique. C'est pourquoi, cette règle souffre des exceptions. Cela est, vrai dès l'époque républicaine, notamment en matière politique (874). Sous les premiers empereurs, elle est fréquemment tournée en matière de lèse-majesté (875) et d'adultère (876), les esclaves torturés passant alors dans la propriété de l'Etat (877). Ces exceptions deviendront légales par la suite (878). En second lieu, l'interrogatoire en faveur

871- Th. MOMMSEN, Droit pénal, cit. II, p. 89.

872- Paul D 48.18.20.

873- Th. MOMMSEN, Droit pénsl, cit. II, p. 89.

874- Th. MOMMSEN, Droit pénal, cit. II, p. 91 , n. 5 ; w.W. BUCKLAND, Roman law, cit., p. 90.

875- Dion, 55,5 (Auguste); Tacite, Ann. 2,30;3, 22 (Tibère).

876- U1pien D 48.5.28.11-14 ; Papinien D 48.18.6 pr. Th. MOMMSEN, Droit pénal, cit. II, p. 91 .

877- En dehors des textes précités (supra n. 876), il convient de relever quelques traits particuliers de la quaestio en matière d'adultère. L'esclave de 1 'accusé, pour ne pas craindre de dire la vérité (ulpien D 48.5.28.11-12) passe automatiquement dans la propriêté de l'Etat, même s'il nie (Ulpien D 48.5.13). Le durcissement de la quaestio en la matière se remarque aussi à d'autres égards : l'esclave peut être interrogé contre le possesseur de bonne foi (Ulpien D 48.5.28.9; alors que ce $n$ 'est pas possible ordinairement, ef. Ulpien $D$ 48.18.1.8), contre $l^{\prime}$ usufruitier (Ulpien $D$ 48.5.28.8). Alors que l'esclave a qui est due une liberte fideicomissaire ne peut être habituellement soumis a la une liberce fideicomissaire ne peutêtre habituellenent soumis à la quaestio qu'autant qu'il est accusé par les esclaves qui viennent d'y
être appliqués (Tryphoninus $\mathrm{D} 48.18 .19$ ), il y est automatiquement soumis être appliqués (Tryphoninus $D$ 48.18.19), il y est automatiquement soum
en matière d'adultère, de même que $\mathrm{I}^{\prime}$ 'esclave affranchi sous condition (U1pien D 48.5.28.10). On observe aussi un durcissement $a$ propos du s.c. silanien, mais on y reviendra en étudiant ce sénatus-consulte.

878- Selon Th. MOMMSEN, Droit pénal, cit. II, p. 91, 92 n. 1 a 3 , ces exceptions ne seraient légales qu'à partir de Septime-Sévère (non seulement en matière de 1 èse-majesté (cf. Modestin D 48.4.7.2 ; Hermogénien D 5.1.53) et d'adultère (cf. Ulpien D 48.5.28.6), mais aussi de falsification de monnaie (Hermogénien D 5.1.53), de meurtre (Paul D 29.5.6. 1), d'accaparement de céréales (Hermogénien D 5.1.53), d'impôts (C.I. 9.41.1 pr) et d'entretien par une femme de relations punissables avec son esclave (C.I. 9.11.1). W.W. BUCKLAND, Roman hw, cit., p. 90 , pense que $l^{\prime}$ exception concernant le meurtre d'un maître est antérieure à du mâtre est-il permig ? Fadrien semble l'admettre (870). Paul l'axclut dans un passage de ses Sententiae (880) mais semble l'autoriser dans un rragment du Digeste (881). Une constitution de $\hat{c}^{4} \mathbf{H}$ ap. J.C. 1 'interdit enfin expressément, traitant de cette règle comme faisant partie depuis longtemps de l'arsenal juridique (882).

L'évolution s'effectue donc de manière nuancée, tout en ménageant la susceptibilité des propriétaires d'esclaves, dans le sens d'un accroissement de l'intervention étatique. Cela est, particulièrement clair dans l'espèce suivante : l'abus s'était introduit de soustraire, par l'affranchissement, à la torture l'esclave dont la déclaration pouvait, nuire au maître (883). Ulpien, renvoyant à un rescrit d'Antonin le pieux, déclare qu'un tel affranchissement est nul (884).

Aucune délimitation juridique ne séparant la torture du témoin de celle de l'accusé (885), des prescriptions restrictives étaient nécessaires.

Sévère. Cela dit, $11 \mathrm{~s}^{\prime}$ agit bien d'exceptions : la déclaration de 1 'esclave contre son maître est punie de mort si elle intervient en dehors des matières énumérẻes, cf. Th. MOMMSEN, o. c., p. $92 \mathrm{n} .5$ ), mais cette mesure a été le fruit d'une évolution difficile. Les Julio-claudiens ont admis la délation d'un maître par ses propres esclaves, en matière politique essentiellement (E.M. SCHTAERMAN, La schiavitù, cit., p. 222). A cette pratique, Pline le jeune oppose celle de vitũ, cit., p. 222). A cette pratique, Pline le jeune oppose celle de Trajan (Paneg. 42). Pourtant, ce principe semble encore admettre 1 in-
terrogatoire des esclaves des personnes condamnes puisque ces esclaves terrogatoire des esclaves des personnes condamées puisque ces esclaves
ne sont plus en la possession de celles-ci (U1pien D 48.18.1.11-19). Quoi qu'il en soit, la règle est bien établie sous Septime-SÉvère (U1pien $D$ 48.18.1.16). En marge de ces considérations, $i 1$ convient de remarquer que l'esclave d'une corporation peut être soumis a la torture a 1 'encontre des membres de celle-ci, car il ne leur appartient pas (Ulpien D 48.18.1.7).

879- Papinien D 48.18.17.2.

880- Paul, Sent. 2, 17, 2 .

881- Paul D 29.5.6.2.

882- C.9.41.6. W.W. BUCKLAND, Roman law, cit., p. 88.

883- Th. MOMMSEN, Droit pénal, cit., II, p. 94.

884- Ulpien D 48.18.1.13 : Si servus ad hoc exit manumissus, ne torqueatur, dumodo in coput domini non torqueatur, posse elom torquer divus Pius rescripsit. v, aussi Paul, Sent., 5, 16,9.

885- Th. MOMMSEN, Droit pênal, cit. II, p. 94. Il faut signaler, en marge du problème qui nous préoccupe, que la torture a parfois été appliquée aux honmes libres aussi. Alors qu'elle était formellement interdite sous la République (ibid., p. 80), elle apparaît au début de 1'Empire pour 1'home libre accusé d'un délit (p. 81 ), le procédé étant plus ou moins utilisé selon les tendances des différents empereurs. On retrouve Evidemment $a$ ce propos 1a distinction entre personnes de rang 
Dans ce sens, Auguste n'autorise la torture que si la gravité du délit 1 'exige (886) et recommande de ne pas commencer par ce dur expédient (887). Hadrien réitère cette recommandation et interdit de recourir à la quaestio si le délit n'est pas assez vraisemblable ou s'il existe d'autres moyens de preure (888). Les juristes vont suivre cette voie. Paul requiert l'audition de plus d'un témoin avant qu'on puisse soumettre à la quasestio ( 889$)$. Le même juriste prescrit de n'y avoir recours que lorsque l'existence du délit est certaine (890) Selon Ulpien, c'est au juge de fixer les modalités de la torture, de manière à ce que l'esclave en sorte sauf, pour jouir de son innocence ou pour être sounis au supplicium s'il est coupable (891). On sent là toute la dureté de la procédure, et au hème siècle Arcadius Charisius juge encore nécessaire de rappeler que les tormenta ne doivent pas être employés selon les voeux de l'accusateur, mais moderatae rationia (892). Si dans ces deux derniers cas, Ia protection relative de l'esclave est vraisemblablement inspirée par la sauvegarde du droit de

supérieur et inférieur (p. 82). Quant à la torture de l'houme libre comme témoin, elle ne semble être apparue qu'à l'époque de Septime-Sévère, et seulement de manière restreinte (p. 83), cf. Callistrate D 48.18.15 pr.

886- Paul D 48.18.8 pr : Edictun divi Auqusti, quod proposuit Vibio Habito et Lucio Aproniano consulibus, in hunc modum exstat : 'Quaestiones neque semper in omni causa et persona desiderami debere arbitror, et, cum capitalia et atrociora maleficia non aliter explorari et investigam possunt quam per servomm quaestiones, efficacissimas eas esse ad requirendam veritatem eristimo et habendas censeo'. esse ad requirendan veritatem existimo et habendas censeo'. On remarquera néanmoins qu'Auguste trouve la torture très efficace pour connaî-
tre la vérité...

887- Ulpien D 48.18.1 pr : In criminibus emendis quaestio adhiberi solet. Sed quando vel quatenus id faciendrom sit, videcums. Et non esse a tormentis incipiendrm et divus Augustus constituit neque adeo fidem a tormentis incipiendam et divus Augustus constituit neque adeo fidem quaestioni adhibendam, sed et epistula divi Hadriani ad Sennium Sabinum continetur.

888- Ulpien D 48.18.1 pr (cité supra n. 887) et D 48.18.1.1 : Verba rescripti ita se habent : 'Ad tormenta servorun ita demum veniri oportet cum suspectus est reus et aliis argumentis ita probationi admovetur, ut sola confessio servorion deesse videatur'; v. aussi D 48.18.1.2.

889- Paul D 48.18.20. H. WALLON, Histoire de l'esclavage, cit. III, P. 61 .

890- Paul D 48.18.18.2.

891- Ulpien D 48.18.7.

892- A. Charisius D 48.18.10.3. Il faut remarquer par ailleurs que s'ils doivent être produits ex domo accusatoris, les esclaves font l'objet d'une protection particulière (cf. Ulpien D 48.18.1.3 (rescrit de Marc-Aurèle et Verus).) propriété de son maître, dans d'autres, c'est la personnalité de l'esclave qui est prise en considération. Ainsi, les fommes enceintes ne doivent pas être soumises à la torture $(803)$. Les enfants en sont pareillement exclus. Ulpien nous apprend qu'on se contente de les épouvanter, les punissant parfois à coups de férule ou de nerf de boeuf (894). Cette exclusion, qui semble due à Antonin le pieux (895), ne s'applique cependant pas à la lèse-majesté (836).

Quelles que soient ces mesures impériales, ces solutions jurisprudentielles, elles ne doivent pas nous voiler l'image concrète d'une procédure qu'elles s'efforcent d'adoucir.

Upien nous donne la définition de la quaestio:

Quaestionem intellegere debormus tormenta it corporis dolorem ad eruendam veritatem. Nuda ergo interrogatio vel levis territio non pertinet ad hoc edictum. Quaestionis verbo etion ea, quan malom mansionem dicunt, continebitur. Cum igitur per vim et tormenta quaestio est, tunc quasstio intellegitur (897).

Par quaestio, il faut entendre les tourments et la douleur du corps infligés pour connaître la vérité. Il ne s'agit pas d'une simple interrogation ou d'une légère terreur. On inclut aussi dans le vocable quaestio le procédé de la mala mansio, par lequel on attache l'esclave tout au long d'une planche jusqu'à ce qu'il avoue (898). Ainsi, la quaestio consiste à décourrix la vérité par la violence et les tourments.

Il est également fait état de la mala mansio dans un autre passage d'Ulpien, qui nous apprend que l'esclave peut parfois être déposé chez un séquestre ut quaestio habeatur (899). Le Digeste ne fournit

\section{3- Ulpien D 48.19.3. \\ 894- Ulpien D 29.5.1.33.}

895- A. Charisius D 48.18.10 pr : De minore quattuoriecim annis quaestio habenda non est, $u t$ et divus Pius Caecilio Iuventiono rescripsit ; v. aussi Callistrate D $48.18 .15 \mathrm{pr}$.

896- A. Charisius D 48.18.10.1 : Sed omnes omnino in maiestatis crimine quod ad personas principum attinet, $8 i$ ad testimonitom provocentur, cum res exigit, torquentur.

897- U1pien D 47.10.15.41.

898- A. BERGER, Encycl. Diction., cit., p. 575.

899- Ulpien D 16.3.7 pr.W.W. BuCKLAND, Roman law, cit., p. 87, n. 14. 
malheureusement fas d'autrog précisions quant alux instruments le corture $(000)$. M $y$ trouve par contro des recomandations inté-essantes sur ce qu'on pourrait appeler l' "art de la torture" (901).

Ainsi, selon Paul, on doit comencer la graestio par celui qui est le plus fragile, timiatior, ou par colui que son âge rend plus rulnérable, tenerae aetatis (902). Ce même juriste écrit encore qu'un osclave peut être soumia une nouvelle fois à la quaestio, car il a pu s'endurcir à l'épreuve des tormenta, et ainsi ne pas dévoiler la vérité (903). On retrouve une idée semblable chez ulpien. Cet auteur rappelle que plusieurs constitutions déclarent qu'il ne faut pas toujours prêter foi aux aveux extorqués par la torture. En effet, écrit-il, la plupart des esclaves s'endurcissent à l'épreuve des tormenta, de telle maniòre que l'on ne peut en obtenir la vérité. D'autres, poursuit Ulpien, ont si peur de souffrir qu'ils préfèrent mentir, voire s':ruser $(\ldots)$. plutôt que de subir les tormenta (904). Ces considérations d'ordre psȳchologique ne sont pas étrangères à Arcadius Charisius selon qui, dans la recherche de la vérité, il faut faire attention même au son de la voix, à la fermeté dans la manière de s'exprimer ou au contraire au manque d'assurance, sans oublier la réputation dont jouit celui qu'on interroge (905).

Mais que pouvaient ces recommandations lorsque l'esclave s'accusait d'un délit qu'il n'avait pas cormis dans le seul but de ne pas retourner chez son maitre? Selon Marc-Aurèle et Verus, un tel esclave devait être absous et vendu (906). Pour en arriver là, encore fallaitil que l'esclave sorte indeme de l'épreuve de la torture. Or, force est de constater que ce n'était pas toujours le cas, des textes de Papinien et Ulpien envisageant de manière pour ainsi dire banale la mort

900- Sur ce point, v. J. MARQUaRDT, vie pívée, cit., p. 214, n. 10. $901-$ La torture est infligée par les subalternes du tribunal, Th. MOMMSEN, Droit pénal, cit. II, p. 93 ; sur sa procédure, ibid., p.93, n. 3.

902- Paul D 48.18.18 pr.

903- Pau1 D 48.18.18.1.

904- Ulpien D 48.18,1.23.

905- A. Charisius D $48.18 \cdot 10.5$.

906- Chez Ulpien D 48.18.1.27. d'esclaves lors de leur sommission à la ruzestio farti.

$$
-3-
$$

Cette étude, compte tenu du faible norbre de toxtes qu'elle implique, ne prête pas à uno investigat,ion statistig̣ue (008). Il

907- Papinien D 48.18.5 pr : Fatre vel marito ds aduiterio agente et postulantibus, de servis rei ut quaestio habeativ, si vere causa percrata testibus prolatis absolutio secuta fuerit, moneipiomon, quae mortua sunt, aestinatio habetur : secuta vero damatione quae suapersunt publisunt, aestimatio habetur: secuta vero dannatione quae suparsunt publi-
cantur ; Ulpien 48.5 .28 .15 : Si reus vel rea absoluti fuerint, aesticantur ; Ulpien $\mathrm{D} 48.5 .28 .15$ : Si reus vel rea absoluti fuemint, aesti-
mari per iudicss lex dommon voluit, sive mortui fuerint, quantas pscuniae ante quaestionem fuerint, sive vivent, quantre pecumiae in his damion dation fuerit faction'e esset. On notera qu'il s'agit dans les deux cas d'une quaestio en matière d'adultère, les esclaves n'ayant ici qu'une seule chose à se reprocher : avoir appartenu a l'accuse...

908- Seuls les textes traitant de 1 a torture sont correctement representés. La torture est parfois indiquée par le terme quaestio (par ex. Ulpien D 9.2.23.4), ou bien par torqueri (par ex. Papinien D 48.18.6.1), ou par tormenta (par ex. A. Charisius D 48.18.10.6). Mais il arrive que le terme référant a la torture soit absent. Dans ce cas, il faut néanmoins tenir coupte du texte lorsque le contexte le commande (le cas est fréquent a propos de D 29.5, sur le s.c. silanien). Ont ainsi été relevés : Pomponius D 12.4.15 (Labéon, Proculus); Gaius D 29.5.9; D 29. $5.25 \mathrm{pr}-1-2$; D $30.67 \mathrm{pr}$; Maecianus D 29.5.14; D 29.5 .23 ; Venuleius D 29.5.13 ; Marcellus D 29.5.16 ; Scaevola D 29.5.26; Papinien D 3.6. 9 ; D 19.5 .8 ; D 29.5.4 ; D 29.5.20; D 29.5.21 pr-1-2 ; D 31.76 .4 ; D 48.5 .40 .8 ; D $48.18 .6 \mathrm{pr}-1$; D $48.18 .17 \mathrm{pr}$ (rescrits de Marc-Aurèle et de Caracalla)-1-2 (rescrit d'Hadrien) -3 ; Tryphoninus D 29.5.11 ; D 48.18.19; Callistrate D 29.5.2 (rescrit de Comode) ; D 29.5.27; D 48. 18.15.1 (rescrit d'Antonin le pieux)-2 (idem); Paul D 1.15.3.2 (rescrit d'Antonin le pieux) ; D 10.3.27 ; D 19.2.55 pr ; D 21.1.43.5 ; D 22.3.7 ; D 29.5.6 pr-1-2-3; D 29.5.7; D $29.5 .8 \mathrm{pr}-1$; D 29.5 . $10 \mathrm{pr}-1$ (constitution de Trajan) ; D 29.5.12; D 29.5.22; D 43.16 .6 ; D 48.7.4.1 (Labéon); D 48.18 .8 pr (édit d'Auguste)-1; D 48.18.11; D 48.18.18 $\mathrm{pr}^{-1-2-3-4-5-6-7-8-9}$; D 48.18.20; Ulpien D 9.2.23.4 (Labéon); D 10.2.18 pr (Julien)-1 (Julien); D 10.4.20; D 16.3.7 pr ; D 27.3.1.3 (décret de Sévère); D 29.5.1 pr-1-2-3-4-5 (rescrit d'Antonin le pieux)-6-7-8 (Marcellus)-9-10-11-12 (Scaevo1a)-13 (Scaevo1s)-14 -15-16 (Marcellus)-17 (Labêon)-18-19-20-21-22-23-24-25-26-27-28 (rescrit d'Hadrien) -29 (idem) $-30-31-32-33-34-35-36-37-38 ;$ D 29.5 .3 pr-1-2 -3-4-5-6-7-8-9-10-11-12-13-14-15-16-17-18-19-20-21-22-23-24-25-26-27 -28-29-30 (Scaevola)-3I-32; D 29.5 .5 pr-1-2-3; D 30.50.2; D 36.1.3.5; D 38.2.37.1 (Julien); D 40.9.12 pr-5-6 (Africain); D 47.10.15.34$35-42$ (Labéon) $-43-47$; D 47.10 .17 .1 ; D $48.5 .28 \mathrm{pr}-1-2-3-4-5$ (Africain) $-6-7-8-9-10-11-12-13-14-15 ; \mathrm{D}$ 48.18.1 pr (constitution d'Auguste) -1 (rescrit d'Hadrien) -2 (idem) -3 (rescrit de Marc-Aurèle et Verus) 4 (idem) -5 (rescrits d'Hadrien et d'Antonin le pieux) 6 (rescrit de MarcAurèle et Verus) $\rightarrow-8-9$ (il s'agit ici d'un affranchi, le passage interdisant qu'un affranchi soit soumis à la torture contre son patron) -10 (rescrit de Sévère et Caracalla)-11 (rescrit de Trajan)-12 (idem)-13 (rescrit d'Antonin le pieux)-14 (rescrit de Marc-Aurèle et Verus)-15 (rescrit de Sevère let paracalla)-16 (rescrit de Sévère)-17 (idem)-18 
faut en retenir deux idées. Tout d'abord, malgré les prescriptions restrictives énoncées, la sanction s'exerce de manière très dure. Ensuite, on remarquera une intervention de plus en plus importante de l'Etat, non seulement par le moyen de la répression publique, mais aussi par le maintien de la discipline domestique dans certaines limites. Outre le domaine répressif, l'Etat va pénétrer les rapports mâtre/esclave dans une matière tout aussi fondamentale, la libertě : mieux vaut prévenir que guérir...

\section{Paragraphe 2 : Les solutions du droit en matière de liberté}

Le droit romain est émaillé de solutions tantôt favorables,

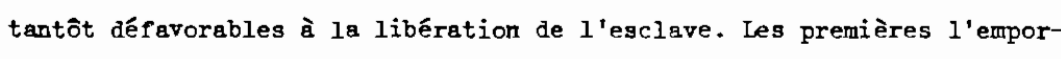
tent-elles sur les secondes, ou bien est-ce l'inverse qui se produit? Du point de vue ehronologique, existe-t-il un tournant, sensible dans une étude statistique, de l'évolution du droit en la matière ? Vcilà les questions auxquelles il s'agit désormais de répondre. Il convient cependant de décrire au préalable le contenu de ces différentes solutions.

On sait que, dès le règne d'Auguste, le pouvoir impérial s'attache à contrôler la concession de la liberté aux esclaves (909). La lex Fufia Coninia (2 av. J.C.) limite le nombre des esclaves que 1 'on peut affranchir par testament, ce nombre variart slon celui des esclaves appartenent au testateur mais ne pouvant jamais dépasser cent $(010)$. Selon la lex Aelia Sentia ( 4 ap. J.C.), l'affranchissement par des maitres de moins de vingt ans ou d'esclaves de moins de trente ans est

(rescrit de Sévère et Caracalla)-19 (rescrit de Trajan)-20-21 (rescrit de Trajan)-22 (rescrit d'Hadrien)-23-25-27 (epistula de MarcAurèle et Verus) ; D 48.18.2 ; D 48.18.3 (constitution de Sévère et
Caracalla) ; D 48.18.4 (Papinien) ; D 48.18.7; D 48.18 .12 (rescrit d'Ha drien); D 48.19.3 ; Marcien D 1.8.6.1 ; D 29.5.15 pr-1 (rescrit de Sévère et Caracalla)-2 ; D 48.5.34 pr (rescrit d'Antonin le pieux); D $48.18 .5 ; D 48.18,9$ pr (rescrits d'Antonin le pieux et de SF́vère)-1 -3; Modestin D 29.5.17; D 29.5.18 (Paul); D 29.5.19; D 48.18.13; D 48.18.16 pr (rescrit de Marc-Aurèle et Verus)-1 (rescrit d'Antonin le pieux); A. Charisius D 48.18 .10 pr (rescrit d'Antonin le pieux) -1 $-2-3-4-5-6$.

909- ¿. GAUDEMET, Institutions de l'Antiquité, cit., p. 559 ; M. RASER, RPR I 2 , p. 297 .

910- Cf. supra première partie n. 83 , deuxième partie, n. 162. contrôlé par un conseil des affranchissements, sous peine de nullité dans le premier cas et sous peine de ne conférer que le statut inférieur de latin junien dans le second (911). Mais le droit va aller plus dans la restriction des affranchissements.

On a vu, à propos de la répression des délits serviles, que l'affranchissement d'um esclave enchaîné postérieurement à la disposition fidéicommissaire de liberté est nul (912), de mêre que celui qui a pour but de soustraire l'esclave à la torture (913). En outre, Marcien nous fait savoir qu'um esclava accusé d'un crime capital et non défendu par son maître n'est pas libre du fait de son absolution (914). Il est una nimement reconnu par les juristes que l'esclave vendu sous la condition de ne pas être affranchi ne peut parvenir à la liberté (915). Wême la volonté populaire est inopérante, Marc-Aurèle interdisant 1'affranchissement ex adclamatione populi (916). Une constitution de Sévère et Caracalla ne reconnaît pas à la maîtresse accusée d'adultère avec son propre esclave la possibilité de lui donner la liberté (917).

911- Gaius I, 18-22; 38-41. A. WILINSRI, Zur frage von Latinern ex lege Aelia Sentia,zSS 80 (1963), p. 378 s.

9!2- Paul D 40.5.43.

913- Ulpien D 48.18.1.13 (rescrit d'Antonin le pieux) cité supra cf. n. 884 .

914- Marcien D 40.9.9.1 : Item nec ille liber fieri potest, qui a domino non eat defensus in capitali crimine posteaque absolutus egt. E.M. SCHTAERMAN, la schiavitù, cit., p. 239.

915- Cf. par ex. Tryphoninus D 49.15.12.16; Paul D 40.1.9; D 40.1.12; Ulpien D 40.1.4.9; Marcien D 40.9.9.2. Il en va de même de 1 'esclave légué a condition de ne pas être affranchi (cf. Paul D 40.5.40.1). Ce dernier cas est sensiblement différent de celui où le testateur lègue quelque chose $¥$ un individu pour que celui-ci n'affranchisse pas oon esclave (cf. Iavolenus $D$ 35.1.67). La différence tient ce que l'esclave victime de la privation de liberté n'est plus l'esclave du testateur, mais celui du lëgataire. Il faut aussi signaler le cas de l'esclave $a$ qui on a ordonné $d$ 'abandonner Rome, et qui persiste néanmoins a y demeurer : il ne pourra pas non plus ètre affranchí (cf. Ulpien D 40.9.2).

916- Ulpien $D$ 40.9.17 pr. Si on peut considérer comme đéfavorable a la liberté le fait de nier tout effet a la volonté populaire, on ne peut interpréter de la même manière le refus de la liberté pour l'esclave qui a contraint son maitre $a$ l'affranchir (cf. Marcien D 40.9 . 9 pr). Il n'y a pas, daas ce dernier cas, de dáfaveur au sens strict, le refus d'avaliser une liberté obtenue de telle manière apparaissant corme normal.

917- Marcien D 28.5.49.2. 
La romige en question des affranchissements peut aussi venir sanctionner ane fraude risquant de porter atteinte aux créanciers ou au putron du manumissor ( 018$)$. Une autre fraude, celle consistant pour le maitre à laisser ses esclaves faire proclamer leur liberté, et en même temps leur ingénuité grâce à un procès fictif, va être déjouée par un sénatus-consulte de l'époque de Domitien, qui permet à celui qui fait décourrir la manoeurre d'entrer en possession de l'esclave coupable (919).

Il faut enfin évoquer le problème de l'affranchi ingrat, bien qu'il ne soit que marginal (920), pour dire que l'autorité impériale se montre très rétive quant à la possibilité pour le patron de faire révoquer des affranchissements (G21). Certes, Claude permet en certains cas des révocations pour ingratitude, mais en 56 . Néron s'oppose à une mesure générale que veut prendre le Sénat pour permettre une telle révocation (922). Il faudra attendre le tème siècle pour que la révocation

918- Gaius, I, 37 et 47 . J. GAUDEMET, Institutions de 1 'Antiquité, cit., p. 560 ; G. IMPALLOMENI, Nota minima in tema di manomissioni fraudolente, Studi Grosso 4 (1971), p. 459 s. A. METRO, La lex Aelia Sentia e le manomissioni fraudolente, Labeo $7(1961)_{p}$. 200, limite la p̈rohibition de la lex aux seuls affranchissements mortis causa; contra, D'ORS, La ley Aelia Sentia y las manumisiones testamentarias, SDHI 40 (1974), p. 425 s.

919- Gaius D 40.16.1. E.M. SCHTAERMAN, La schiavitù, cit., p. 227. 920- C'est un problème marginal car il y a antériorité de la liberté. En outre, il n'est pas certain que la révocation pour ingratitude soit au fond défavorable à l'esclave. Si on ne châtiait pas l'ingratitude, ne risquerait-on pas de fermer la voie des affranchissements? En effet, les maîtres ne les éviteraient-ils pas sinon, de crainte que leur générosité ne soit pas récompensée ? Pour ces raisons, on ne tiendra pas compte de ce problème dans les tableaux à venir.

921- L'accusatio ingrati liberti a été introduite par la lex Aelia Sentia, P. DE FRANCISCI, La revocatio in servitutem del liberto ingrato, in MEl. Cornil 1 (1926), p. 303 ; sur le thème, plus recement, A. WILINSKI, Intorno all'accusatio e revocatio in servitutem del liberto ingrato, in St. Volterra 2 (Milan 1971), p. $559 \mathrm{~s}$.

922- Suétone, Claude, 25, 3 ; Dion Cassius, 60, 13, 2 et 60, 28, 1 : Marcien D 37.14.5 pr : Divus Claudius libertum, qui probatus fuit patrono delatores summisisse, qui de statu eius facerent ei quastionem, servrom patroni esse iussit eum iibertum. Sur l'opposition de Neron, Tacite, Ann. 13,26-27. Ainsi, la revocatio in servitutem a'est pas admise, a 1 'époque de Claude, comme conséquence de 1 'ingrati accusatio; compte tenu de la gravité du cas prévu par Claude, il ne peut s'agir que d'une mesure exceptionnelle, au même titre que celle de Nerva, qui punira de mort les affranchis qui, sous Dotritien, s'étaient faits les délateurs de leurs patrons (cf. Dion Cassius, 68, 13), P. DE FRANCISC o. c., p. 308. Quant a la constitutinn de Coumode (Modestin D 25.3. 6.1 : Imperatoris Eomodi constitutio talio profertur : 'Cum probation oit contumeliis patronos a libertis esse violatos vel illata manu atroci pour ingratitude soit intégrée à 1 arsenal juridique rejarj f : affranchis (923). A l'ápoque classique, le principe du ius orịnarivor reste celui de l'irrévocabilité de la liberté (924), et cela amène à parler des solutions favorables à la libération de l'esclave.

Une politique favorable aux afrranchissements se met en place st se traduit sur le plan juridique tant par des dispositions impériales que par des interpretations jurisprudentielles. Elle s'exprime par l'expression favor libertatis (925). Elle apparait dans le domaine des afrranchissements, ou le maître lui-même donne la liberté, mais aussi en diverses mesures où la volonté du maître est absente de la concession de liberté. esse pulsatos aut etion paupertate vel corporis valetudine laborantes
relictos, primon eos in potestate patronorm redigi et ministerium relictos, primon eos in potestate patronorm redigi et ministerium
dominis prasbers cogi : sin autem nsc hoc modo admoneantur, vel a praeside emptori addicentur et pretium patronis tribuetur") qui prévoit qu'en cas de manquements graves l'obligation d'obsequium, les affranchis doivent être remis aux patrons pour remplir leurs devoirs envers eux, et, si cela est insuffisant, être vendus par ordre du magistrât pour que le prix profite aux patrons, elle ne peut concerner la revocatio in servitutem, si l'on considère le texte comme étant authentique, pour une raison bien simple : si la première mesure visalt en effet une pour une raison bien simple : si la premiere mesure visalt en effet revocatio in servitutem, quel serait donc le sens de la seconde qui nécessite 1 intervention du magistrat pour permettre au patron, redevenu maître, de vendre son affranchi, redevenu esclave ? P. DE FRANCISCI, O. c., p. 309. Il n'est par ailleurs pas exclu que la deuxième partie du passage (a partir de oin autem) ait été interpolée (P. DE FRANCISCI, ibid. ; contra, E. ALBERTARIO, Contributi alla critica del Digesto, (1911), p. 44) Et même $s$ 'il en était ainsi, coment expliquer qu'Ulpien, traitant de l'intervention du préfet de la ville (cf. D 1.12. 1.10) ou des praesides (cf. D 37.14.1) ₹ propos d'hypothèses d'ingratitude de 1'affranchi, ne siongle pas la constitution de Commode, si celle-ci btait devenue une norme générale? Cette dernière interprêtation du passage conduirait donc à penser qu'il s'agit d'une mesure exception du passage conduirait do, comme celle de Claude, P. DE FRANCISCI, o.c., p. 310.

tionnelle, comme celle de Claude, P. DE FRANCISCI, O.C., P. 310 . P. DE FRANCISCI, O. c., p. 314 s. et M. AMELOTTI, Per l'interpretazione della legislazione privatistica di Diocleziano, Milan (1960), p. $115 \mathrm{~s}$. Sous Constantin, la revocatio in servitutem deviendra norme générale et Sous Constantin, la revocatio in servitutem deviendra norme gênérale et s'appliquera même ea
CI, o.c., p. 315 s.

924- P. DE FRANCISCI, o.c., p. $306 \mathrm{~s}$. ; J. GAUDEMET, Institutions de 1 Antiquité, cit., p. 565 ; N.w. BUCKLAND, Roman law, cit., p. 566 .

925- A titre indicatif, on relève $1^{\prime}$ expression chez Iavolenus $\mathrm{D} 40.7$. $28 \mathrm{pr}$; Julien D 36.1.26.2 ; D 40.2.4 pr ; D 40.4.16; D 40.4.17.2 ; Gaius D 4.7.3.1 ; D 50.17.122 ; Maecianus D 35.2.32.5 ; Marcellus D 28. 4.3.1 ; Scaevola D 40.4.29; Tryphoninus D 49.17.19.5; Paul D 4.8.32.7; D 18.7.9; D 31.1.4 pr ; D 40.4.10.1; D 40.7.4.6; D 40.7.20.3; D 40 . D 18.7.9; D 31.14 Pr ; D 40.4.10.1; D 40.7.4.6; D 40.7.20.3; D 40. D 40.5 .2 ; D 40.5.24.10; D 40.5.26 pr; D 40.5.30.12 ; D 40.7.9.3; D 40.5.2; D 40.5.24.10; D 40.5.26 Pr; D 40.5.30.12; D 40.7.9.3; sique du favor libertatis a été contesté par E. AlberTario, gchiavitù e favor libertatis, BIDR $33(1923)$, p. $50 \mathrm{~s}$.; et Studi di diritto romano 
L'apparition de procédés d'affranchissement non solennels et du fidéicommis de liberté, dont il a été traité, est en soi favorable à la libération de l'esclave (926). Elle constitue cependant un critère trop large pour une átude statistique din favor libertatis, qu'il convient d'entreprendre sous un angle plus ponctuel (926 bis).

En matière d'affranchissement par vindicta, on va alléger les formalités par la suppression de la nécessité des paroles sacramentelles que devait prononcer le maitre devant le magistrat (927). Ainsi, le muet peut đésormais affranchir (928). En certains cas, il est même suppléé à l'absence du mâttre (929). En matière de testement, l'affranchissement est reconnu valable si, à défaut de l'héritier désigné, un autre recueille la sucession $a b$ intestat (930). Si personne ne se présente, Marc-Aurèle permet de faire adjuger les biens pour que les libertés pro-

Milan (1933), p. 63 s., ainsi que par M. NICOLAU, Causa liberalis, Paris (1933), p. 175 s. ; Contra, F. LANFRANCHI, Il diritto nei retori romani (1938), p. 186 s., 606 s., et J. DMBERT, Favor libertatis, RHD (I949), p. $274 \mathrm{~s}$. Ce dernier relève que Iavolenus, son élève Julien, Maecianus, Gaius et Marcellus font partie de l'école sabinienne qui aurait donc, contrairement aux proculiens, été fortement influencée par les théories humanitaires des stoíciens (o.c., p. 277-278). Sur le favor libertatis, v. v. aussi E.M. SCHTAERMAN, La

926- Cf. supra Section l Chapitre II Titre I, deuxième partie : Le passage de l'état d'esclave à celui d'affranchi.

926 bis- La présence de ces procêdês dans le Digeste révèle certes le souci chez les juristes d'une interprétation favorable à l'esclave. Ils constituent un ensemble trop large pour une investigation statistique car, tout d'abord, les textes concernant par exemple l'affranchissement fidéicomissaire sont trop nombreux (370), ce qui risque de provoquer un désequlibre au profit des solutions favorables à la liberté (risque ' 'autant plus inportant que (risque fidéchté fidêiconmisaire ne contienorables a l esclave, ce. dans ce sens Paul D 40.5 .43 ); ensuite, parce que la distinction liberté testamentaire directe/fidéicommissaire, on 1' vu, ne ressort pas toujours de manière claire. L'intégration de tels textes manquerait donc assurément de "sérieux statistique".

927- H. WALLON, Histoire de l'esclavage, cit., III, p. 64.

928- U1pien D 40.9.1 (Celsus). L'affranchissement peut se faire par le ministère de licteurs sans que le maître ait à parler, cf. Hermogénien D 40.2.23.

929- Cf. supra n. 137. En outre, on déroge au principe selon lequel un proconsul ou tout autre gouverneur ne peut affranchir son propre esclave, cf. Julien $D$ 40.2.5

930- Ulpien D 40.4.25. H. WALLON, O.c., III, p. 65.

931- Uipien D 40.5.2. Un rescrit de Marc-Aurèle et Comode pernet à 1 'ssciàn d'attaquer le maître qui lui aurait caché le testament sur la tẩe íquel lui était laissée la liherté, cf. Marcien D 48.10.7.

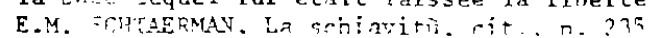

duisent leur effet (931). Les cläuses"d'affranchissement incertaines sont validées. Dans le cas aù il ressortirait de la lettre du testament que la liberté est donnée puis reprise, Paul déclare qu'il faut 1 s donner (932). De même, il faut affranchir celui dont le nơm est mal écrit lorsqu'il n'y a pas de doute sur son identité (933). La liberté laissée à un esclave captif est valable, bien qu'il n'sit été sous la puissance du testateur ni au moment du testament, ni à celui de la mort (934). Quant aux libertés fiđéicommissaires, elles sont protégées par un rescrit de Marc-Aurèle (935). Si le fiduciaire meurt sans successeur avant d'avoir affranchi l'esclave, Marc-Aurèle et Verus assurent néanmoins à celui-ci sa liberté (936). Le favor libertatis triomphe même $i c i$ des intérêts du fisc : en cas de confiscation de la succession, Septime-Sévère et Caracalla prescrivent de laisser libres ceux qui devaient obtenir la liberté par legs ou fidéicomanis (937). La jurisprudence fixe des limites au pouvoir d'appréciation de la personne chargée du fidéicommis : il existe une sorte de présomption selon laquelle l'esclave remplit les conditions exigées (938).

On prend systématiquement à la lettre toutes les conditions favorables à l'esclave et, inversement, on cherche à faire prévaloir la

932- Paul D 40.4.10.1. Si un esclave est légué et reçoit une liberté fidéicomaissaire, Scaevola pense qu'il faut prendre en considération le premier acte intervenu, qu'il g'agisse du legs ou de la liberté. Mais Marcien écrit que si on ne couprend pas pourquoi le testateur a voulu léguer un esclave auquel il avait précédemuent laissé la liberté, il faut se prononcer pour la liberté : in obscuro libertatem praevalere, faut se prononce
cf. $D 40.5 .50$.

933- Scaevola D 40.4.54.

934- U1pien D 40.4.30. Il faut ausi souligner qu'un tel esclave ne revient pas au maître $s$ 'il a été racheté par un individu qui lui a donné la liberté, cf. Tryphoninus D 49.15.12.9.

935- U1pien D 40.5.30.16 : Divus etiam Marcus rescripsit fideicommissas libertates neque aetate neque condicione neque mora non praestantivm taraiusve reddentium corrumpi aut in deteriorem statum perduci.

936- Ulpien D 40.5.30.13. A défaut d'héritier, c'est le fisc qui hérite et qui doit accomplir les fidéi commis de liberté, cf. Marcien D $40.5 .51 \mathrm{pr}$.

937- Modestin D 40,5,12.2 : Divus Antoninus et (Fertinax) rescripsemut hereditate fisco vindicata, quoniam tacite quis rogatus erat ei, qui capere non poterat, restituere hereditatem, et directo et per fideiqui capere non poterat, restituere hereditatem, et directo et per fide commission datas libertates deberi. E.M. SCHTAERMAN, La schiavitù, cit. p. 244, pense qu'il s'agit d'un rescrit de Sêvère et Caracalla : 1'interprétation paraît valable dans la mesure où aucun Antoninus n'a régné avec Pertinax et où D 40.5 .12 pr fait état d'un rescrit de Caracalla.

938- Dn fait appel à la notion de irir bonus, cf. Julien D 40.5.47.2. Scaevola D 40.5.4I.4. Cette notion apparaît également dans un rescrit de Septime-Sêvère rapporté par Ulpien (D 40.5.46.3). H. HALLON, Histoire de l'esclavage, cit., III, p. 69. 
volonte du testateur favorable à l'esclave et contraire à la lettre de l'acte (939). Les plus grandes facilites sont données à l'esclave pour accomplir les conditions requises. Par exemple, selon paw et Modestin, l'esclave affranchi à condition de promettre quelque chose doit être libre, non quand la chose est faite, mais dès l'instant où il promet $(940)$. Ulpien affirme que l'esclave derant rendre ses comptes dans les trente jours du décès de son mâtre est libre si ses comptes sont en règle, alors même que l'beritier n'a recueilli la succession qu'après les trente jours ( 941$)$. Si trois esclaves ont été légués à quelqu'un à condition de ne servir que lui, et que les biens de celuici soient confisqués, Caracalla estime qu'ils doivent obtenir la liberté $(942)$.

D'autre part, de justes causes permettent de passer outre les prescriptions de la lex Aelia Sentia (943). Dans un autre domaine,

939- Ainsi, 1a condition post annos est interprétée "après deux ans", cf. Julien D 40.4.17.2. Toujours en matière de délai, si un esclave est affranchi "lorsque Titius aura trente ans", et que Titius vienne a mourir avant d'atteindre cet âge, il sera libre immédiatement, cf. Julien $D$ 40.4.16.

940- Paul D 40.4.36; Modestin D 40.4.44. H . HALLON, O.C., III, p. 70. 941- U1pien D 40.7.3.11.

942- Modestin D 40.5.12 pr.

943- U1pien (D 40.2.11, 12 et 13) donne des causes d'affranchissement par des maîtres de moins de vingt ans : $\mathrm{I}^{\prime}$ affranchissement est admis si le mineur est parent avec l'esclave auquel il veut donner la liberté, ou bien si ce dernier est son frère de lait, son précepteur, sa nourrice, ou les enfants d'un d'entre eux, ou encore s'il s'agit de son alumms ou de son secrétaire; il peut également affranchir un esclave pour en faire son secretanter faire son procurator, a condition que celui-ci ait au moins dix-huit an et que ce ne soit pas le seul esclave du mineur; il peut enfin donner la liberté à une esclave, en exigeant d'elle le serment de 1 'épouser dans un délai de six mois. Outre les hypothèses prêrues par Ulpien, Paul et Marcien font aussi allusion a de justes motifs d'affranchissement. Marcien (D $40.2 .9 \mathrm{pr}$ ) estime que le fait, pour l'esclave, d'avoir évité a son maître la perte de la vie ou de 1 'honneur, est une juste cause d'affranchissement. Quant a Paul, il déclare (D 40.2.15 pr) qu'on doit autoriser un mineur de vingt ans $a$ affranchir un esclave pour remplir la condition qui lui a êtê imposée : ainsi, s'il a étê institué héritier a condition de donner la liberté a un esclave. S'il existe de justes causes pour affranchir il en existe jalent causes pour affranchir, il en existe egalement pour ne pas le faire : il en est ainsi lorsque 1 esclave a falsifié les comptes de son maître ou a cherché a attenter à sa vie (Julien D 17.1.30), mais il ne s'agit pas dans ce cas d'une solution défavorable a la liberté au sens strict

puisqu'elle exprime la répression d'un délit commis par 1'esclave, tout comme la disposition par laquelle la lex Fabia interdit d'affranchi avant dix ans l'esclave qui s'est rendu coupable de plagiat (Paul D 40.
Marc-Aurèle permet à l'esclave vendu sous la condition d'être affranchi dans un certain délai de parrenir à la liberté en cas d'inexécution' de la condition ( 944$)$.

En tout cas la volonté du mâtre est à la base de la concession de liberte, bịen qu'il ne s'agisse pour la dernière hypothèse Evoquée que de la volonte du maître initial (945). Mais l'Etat va aller plus

2.12). A propos de la prescription de la lex Aelia Sentia qui interdit 1 'affranchissement en fraude des créanciers, Aristo, rapporté par Paul (D 40.9.16.3), préroit un allègement tout-a-fait relatif : il déclare que l'esclave affranchi par un débiteur du fisc ne peut voir sa liberté réroquée s'il en a joui depuis plus de dix ans. L'interdiction est levée si l'esclave est institue heres necesscrius (cf. Scaevola D 28.5.84 pr ; Gaius D 36.1.15.15), mais il ne s'agit pas, au vola D 28.5.84 pr : Gaiug D 36.1 . Is. I5), mais il ne s'agit pas, au sens strict, dune solution favorable la liberte, cet esclave ne pouvsnt refuser la succession et devant en supporter les dettes, $M$. RASER, RPR $\mathrm{r}_{2}$, p. $684 \mathrm{n} .33,714$; c'est pourquoi ces deux textes. renvoyant à la lex Aelia Sentia, ont été relevés, d'un point de vue plus global, dans les solutions defavorables.

944- Paul D 40.12.38.1. Un rescrit du même Marc-Aurêle prévoit aussi qu'un tel esclave acquerra la liberté dans le cas ou le vendeur et $1^{\prime}$ acheteur mourraient tous deux sans heritiers, cf. Paul $D$ 40.8.1. Si le délai de 1 'affranchissement n'est pas fixé dans la vente, Paul nous fait savoir qu'il peut être ramené à deux mois seulement, cf. $D 40.8 .9$.

945- $\mathrm{I} 1$ faut évoquer, en marge de ces considérations, deux autres problèmes qui n'ont pas leur place dans ce développement, compte tenu de leur particularitê, maís dont 1 'importance $n$ 'en est pas moins évidente. On a vu, à propos de la répression, que I'esclave devenu servus poenae pouvait, en certains cas, espérer la liberté (cf. supra n. 841, 842), qu'une simple incarcêration ne suffisait pas pour empêcher I'affranchissement de produire la plénitude de ses effets (cf. supra n. 796), mais c'est un autre point qu'il convient de souligner désormais. Un rescrit d'Antonin le pieux prêvoit que, du point de vue des peines, le statuliber doit être traité en houre libre (chez U1pien D 48.19.9.16). Bien que la matière semble tout d'abord avoir été sujette à discussion (Pomponius D 40.7.29 pr), U1pien (texte précité) et Modestin (D 48.18.14) nius D $40.7 .29 \mathrm{pr}$ ), U1pien (texte précité) et Modestin (D 48.18.14)
attestent son application au 3ème siècle. Il ne s'agit pas à proprement attestent son application au Jème siècle. Il ne s'agit pas à propreme
parler d'une mesure favorable a la libération de l'esclave, mais il n'est pas possible d'ignorer $1^{\prime}$ 'impact qu'a, a travers elle, l'attente de la liberté sur la condition de 1 'esclave. En ce sens, c'est une solution favorable à la liberté, au même titre que celle, dont Modestin se fait 1 'écho, qui interdit de joindre une condition aggravante à la vente d'un statuliber (D 40.7.25). Le deuxième problème qui doit être signale ne concerne pas non plus la libération de $I^{\prime}$ esclave au sens strict. II s'agit de 1 'interdiction d'elever une contestation sur l'état d'un individu décédé depuis plus de cinq ans (cf. D 40.15: Ne de statu defunctorum post quinquennium quaeratur). Si cette mesure, due selon Callistrate (D 40.15.4 pr) a Nerva, tend au respect de la mémoire du défunt, elle protège aussi le statut de son enfant (Marcien D 40.15.1. 4 , cite Marcellius). Cette règle peut être levée si le but de la demande est $I^{\prime}$ 'obtention d'un jugement plus favorable, s'i.1 s'agit par exemple de faire déclarer affranchi celui qui avait êtể jugê esclave (D.40.15.1. 
Son intervention va s'effectuer en ronction de deux sxes, se présentant tantôt corme une récompense de l'esclave, tantôt comme une punition de son maitre. L'empereur peut vouloir récompenser l'esclave, le préteur procédent alors à la concession de liberté. Il en est ainsi lorsque l'esclave dénonce le meurtrier de son maître (946). Mais il peut aussi vouloir pénaliser le maître. C'est ainsi que se présentent tout d'abord l'édit de Claude, qui autorise $I$ ' affranchissement des esciaves âfés et malades que celui-ci a abandonnés (947), et ensuite les mesures de Vespasien, d'Hadrien et de Sévère et Caracalla faisant don de la liberté aux esclaves que leur maître prostituerait (948). Quant à l'esclave qui a payé sa liberté après accord avec son maître, Marc-Aurèle et Verus Iui pernettent de devenir libre, dans le

4). Si on tient compte de cette mesure parce qu'il y a un risque évidént que celui dont il est interdit de contester le statut ait été esclave, on ne peut intégrer a $I^{\prime}$ analyse les solutions facilitant la vindicatio in libertatem (par ex. D 40.12.1 pr-1-2; $040.12 .3 \mathrm{pr}-1-2$ ), car on a alors affaire a un individu qui se trouve de fait dans un état: servile, mais qui, en droit, est libre.

946- Il est question d'un esclave dénonçant le meurtrier de son maítre chez Marcellus D 29.5.16 ; Tryphoninus D 28.5.91 ; D 37.14 .23 pr ; Pau1 D 29.5.12; D 38.2.4 pr; U1pien D 29.5.3.13-14-15; D 38.16.3.4; D 40.8.5. C'est le préteur qui délivre alors la liberté a l'esclave, et celui-ci n'a pas de patron (cf. D 38.2.4 pr ; D 40.8.5). L'esclave peut aussi obtenir la liberté, comme nous I'apprend Ulpien (D 47.10 . 5.10-11) a propos de la ler Comelia de iniumis s'il denonce ceux qui ont diffamé publiquement quelqu'un par des inscriptions ou autrement. Sur I'affranchissement par acte d'autorite publique, J. GAUDEMET, InstiSur I'af franchissement par acte d'autorité publique, J. GAUDEMET, Insti
tutions de 1 'Antiquitê, cit., p. $560 ; \mathrm{F}$. D'IPPolIto, Concessioni pubbliche di liberta, Labeo 10 (1964), p. $38 \mathrm{~s}$.

947- Modestin D 40.8.2 : Servo, quem pro derelicto dominus ob gravem infirmitatem habuit, ex edicto divi Claudii competit libertas, of. C. I. 7.6.1 ; Suétone, Claude, 25. E. VOLTERRA, Intorno a un editto dell'imperatore Claudio, in Acc. Lincei, Rendic. sc. morali, VIII, (1956), p. 205 s. ; C. CASTELlo, La libertà claudiana senza manomi ssione, in Ann. Fac. Giur. Genova, I (1962), p. $213 \mathrm{~s}$. ; M. KASER, RPR I 2 , p. 286 a. 28 ; J. GAUDEMET, Institutions de 1 'Antiquité, cit., p. 547. Un tel esclave devient latin junien, $M$. FASCIATO, Note sur 1 'affranchissement des esclaves abandonnés dans I'île d'Esculape, RHD (1949), p. 458. En affranchissant 1 'esclave malade abandonné, I'empereur 1 'empêche de passer, s'il guérit, en une puissance autre que celle de son précédent maître. L'atteinte portée par claude aux droits du maître apparaît donc come fort reduite porte par claude aux droits du maitre apparait donc donne la vente de 1 'esclave maltraité (M. FASCIATO, o.c., p. 460-46I). 948- Le détail de ces mesures a été prêsenté supra, dans 1'étude sur les pratiques sexuelles (v. n. 467 a 472). cag oul he maitre refuserait ensuite de donnor la liberté à l'asclava $(g+s)$.

Ce tour d'horizon des prircipales solutions du droit en matière de liberté étant fait, il est désormais possible de passer à la comparaison statistique des deux types de solutions, ravorables (950) et

949- Ulpien D 40.1.4 pr : Ts qui suis numis emitur epistula divorn fratum ad Urbium Maximum in econ condicionem redigitur, ut libertatem adipiscatur ; v. aussi Marcien D 40.1.5 pr. M. KASER, RPR $\mathrm{I}_{2}, \mathrm{p} .286$, n. 33.

950- Ont été relevés en tant que solutions favorables à la liberté les textes suivants : Iavolenus D 32.77 ; D $40,1.26$ (Labeon) ; D 40.7.28 pr ; D 40.7.39.4 (Trebatius) -3 (Labéon) -5 ; Celsus D 40.7.23.1 ; $\mathrm{Va-}$ lens D 35.1.87; Julien D 7.1.35.1 (Sabinus); D 28.2.13.1; D 30.81. 10 ; D 33.5.9.1; D 35.2.87.3; D 36.1.26.2; D 40.2.4 $\mathrm{pr}^{-1}$; D 40.2. 5 (Iavolenus); D 40.2.6; D 40.4.15;D 40.4.16; D 40.4.17.2; D 40. 4.18 pr (Sabinus) -1; D 40.5.47.1-2-3-4; D 40.5.48; D 40.12.30 (Sabinus, Cassius); D 42.8.15 ; Pomponius D 21.2.34 pr; D 28.6.16 pr ; D 35.1.110; D 40.1.13 (Octavenus) ; D 40.2.1; D 40.4.5; D 40.4. II pr-i ; D 40.4.40 pr (Julien)-1 (Ofilius); D 40.4.46 (Áristo); D 40.4.61.2 (Octavenus) ; D 40.5.20 (Aristo, Octavenus) : D 40.5.34 pr-1 (Campanus)-2 (Sabinus) ; D $40.5 .44 ; \mathrm{D} 40.7 .5$ pr (Aristo, Neratius) D 50.17.20; Africain D 35.1.32; D 40.4.20; D 40.5.49; D 40.7.15 pr T. Clemens D 40.9.24 (Julien); Gaius D 4.7.3.1; D 35.1.88; D 40.1. 18 ; D 40.1.25; D 40.4.57 (Sabinus, Cassius); D 40.12.9.2; D 40.12.25. 1 ; D 49.14.14; D 50.17.122 ; Maecianus D 29.4.28.1 ; D 35.2.32.5 ; D 40.4.55 pr-1; D 40.5.35; D 40.5.36 pr-2 ; D 40.5.42 (Antonin le pieux) ; D 40.5.54 ; Marcellus D 28.4.3 pr (rescrit de Marc-Aurèle)-1 (idem) ; D 29.1.31; D 29.5.16 ; D 40.1.15; D 40.5.9; D 40.5.10 pr-I ; D 40.7.24 ; Scaevola D 2.15.3 pr (rescrit de Marc-Aurèle et Verus); D 32.37 .3 (constitution d'Antonin le pieux): D 40.4.29: D 40 rus) ; D 32.54 Fr-1 ; D 40.4.59 pr; D 40.4.60; D 40.5.18; D $40.5 .19 .1 ;$ D 40. $4.54 \mathrm{pr}-1 ; \mathrm{D} 40.4 .59 \mathrm{pr} ; \mathrm{D} 40.4 .60 ; \mathrm{D} 40.5 .18 ; \mathrm{D} 40.5 .19 .1 ; \mathrm{D} 40$.
$5.41 \mathrm{pr}-1-2-3-4-5-7 ; \mathrm{D} 40.7 .40 .1-3-7 ; \mathrm{D} 42.8 .23 ; \mathrm{D} 45.1 .122 .2 ; \mathrm{D} 48$

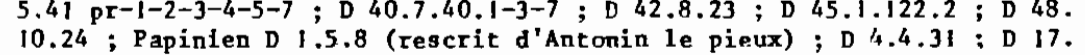
I.54 pr ; D 18.7.8; D 26.5.13.1; D 31.76 pr ; D 31.77.29; D 35.1.78 pr ; D 37.7.6 ; D 38.2.41; D 40.1.19; D 40.1.20 pr (2itterae de MarcAurè̀le)-2 (constitution du même) ; D 40.1.21; D $40.4 .47 \mathrm{pr}-1 ; \mathrm{D} 40.4$. 50 pr (constitution de Marc-Aurèle) ; D 40.4.5I pr ; D 40.5.2I ; D 40.5 $22 \mathrm{pr}-1-2$; D 40.5.23 $\mathrm{pr}^{-1-2-3-4 ; D} 40.7 .33$; D 40.7.34.1; D 40.7 .36 (prudentes) ; D 40.8.8 (constitution de Marc-Aurèle) ; D 40.15.2 pr-J-2 ; D 44.2.29 pr; D 48.19.33 (rescrit de Marc-Aurèle et Verus): Tryphoninus D 18.7.10 (Hadrien) (Scaevola) ; D 28.5.91; D 37.14.23pr-1 (Hadrien) D 40.4.59.1 (Scaevola); D 48.18.19; D 49.15.12.9; D 49.17.19.3 (constitution d'Hadrien) -5 ; Callistrate $D$ 40.8.3 (rescrit de Marc-Aurèle et Coumode) ; D 40.15.4 pr (edit de Nerva)-1 (rescrit de Claude) ; $D 48$. 10.15.2; Paul D 1.5.7; D 4.4.48.1; D 4.8.32.7; D 5.2.28 (dêcret d'Hadrien) ; D 18.7.3; D 18.7.9; D 26.2.32.2; D 28.5.85.2 (constitution de Marc-Aurèle); D 29.1.40.1 ; D 29.5.12 ; D 31.14 pr ; D 35 . $1.81 \mathrm{pr}$; D 35.1.96.1 ; D 38.2.4 pr ; D 40.1.10 (constitution de MarcAurèle) ; D 40.1.23; D 40.2.4.2 (Julien) ; D 40.2.15 pr-1; D 40.2.22 ; D 40.2 .24 ; D 40.4.10 pr-1;D 40.4.36; D 40.4.52; D 40.4.56 (rescrit de Marc-Aurèle) ; D 40.5.5 ; D 40.5.6 ; D 40.5.27; D 40.5.31.2-4 (rescrit

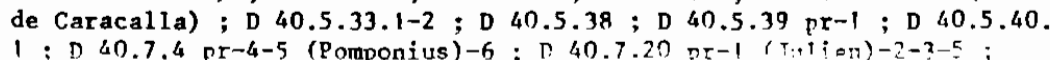


D 40.7.38; D 40.7.41.1 (Labéon) : D 40.8.1 (rescrit de Marc-Aurè le); D 40.8 .7 (constitution de Sévère et Caracal la); $\mathrm{D} \mathrm{40.8.9;} \mathrm{D} 40.9 .15$ 1 (Julien); D 40.9.16.1-3 (Aristo) ; D 40.9.26 (Scaevola); D 40.12. $38 \mathrm{pr}^{-1}$ (constitution de Marc-Aurèle)-2-3; $\mathrm{D}$ 40.13.4; $\mathrm{D} 41.7 .8$; D $42.1 .38 \mathrm{pr}$ (constitution d'Antonin le pieux) ; D 42.5.4; D 48.10 . 22.7 ; D 49.17.20 ; D 50.17.179; Ulpien D 1.5.18 (rescrit d'Hadrien) ; D 1.5.25; D 1.7.19 pr ; D 2.4.10.1 (Marcellus); D 2.12.2 ; D 2.12.3.2 ; D 4.3.7 pr (Julien); D 4.3.24; D 4.4.5; D 4.4.7.10 (Papinien ;-rescrits d'Antonin le pieux et de Caracal1a); D 4.4.9.6 : D 5.1.67; D 5. 2.8.17 ; D 5.2.29.2; D 12.4.5.1 ; D 20.2.6 (Pomponius) ; D 24.1.7.8 (constitution de Marc-Aurèle) ; D 25.6.1.11 ; D 26.2.10.4; D 26.4.1.3 (rescrit d'Antonin le pieux) ; D 26.4.3.2 (constitution de Marc-Aurèle) -3 ; D 28.3.12.1 ; D 28.5.6.4 (Julien) ; D 29.1.13.4 (rescrit de Sévère) D 29.2.71 pr ; D 29.4.12 pr; D 29.4.29; D 29.5.3.13-14-15; D 34.4. 3.9 ; D 34.5.10.1; D 35.1.92; D 36.1.15.3; D.36.1.23.1; D 38.1.13 pr (constitution de Marc-Aurèle) ; D $38,16.1 .1$ (rescrits de MarcAurèle et Verus et de Caracalla) i D 38.16.3.3 (constitution de Marc-Aurèle) 4 Verus et de Caracalla) $D$ (

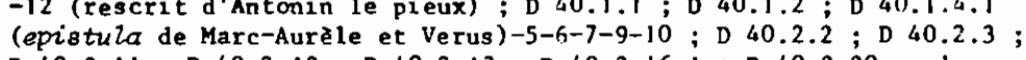
D 40.2.11; D 40.2.12; D 40.2.13; D 40.2.16.1; D 40.2.20 pr-1 (constitution de Marc-Aurèle)-2 ; D 40.4.1; D 40.4.2 ; D 40.4.9 pr ; D 40.4.13 pr-1-3; D 40.4.25;D 40.4.30; D 40.4.32; D 40.5.1; D 40 5.2 (constitution de Marc-Aurèle); $\mathrm{D} 40.5 .3$ (idem) ; D $40.5 .4 \mathrm{pr}-\mathrm{I}-2-3-$ 4-15-16-17 et 18 (idem) ; D 40.5.7; D 40.5.24.5 (rescrit de Caracal1a) -10-12 (Marcellus) $\rightarrow 13$ (idem)-14-15-16-19-21 (rescrits d'Hadrien et d'Antonin le pieux) ; D 40.5.26 $\mathrm{pr}-1-2$ (Antonin le pieux ; rescrit de Sêvère et Caracalla)-3 (rescrit de Sevère et Caracalla)-4 (rescrits d'Antonin le pieux et de Sévère et Caracalla)-6-7-8 (rescrit de Sévère er Caracalla) $-9-10-11$; D 40.5 .28 pr (Marcellus)-1-2-3-4; 0 de 40.5 .30 . $1-2-3$ (rescrit de Marc-Aurèle et Verus) $-4-6$ (rescrit d'Antonin le pieux) -7 (idem) $-8-9-10-11-12-13$ (rescrit de Marc-Aurèle et Verus)-16 (rescrit -7 (idem) $-8-9-10-11-12-13$ (rescrit de Marc-Aurèle et Verus) -16 (rescr
de Marc-Aurèle) -17 (rescrit de Sévère et Caracalla); D 40.5 .37 (resde Marc-Aurèle)-17 (rescrit de Sévère et Caracalla); D 40.5.37 (res-
crit de Marc-Aurèle) ; D 40.5.45.1 ; D 40.5.46.3 (rescrit de Sévère) D 40.7.2.1 (Julien)-2 (idem)-3 (idem) ; D 40.7.3.2 (Servius, Labéon, Cassius) $-3-4-7-8-9-10-11-12-15-16-17 ; 040.7 .6 \mathrm{pr}-1-2 ; 040.7 .9$ $\mathrm{pr}-1-3$; D 40.7.19; D 40.8.4 ; D 40.8.5; D 40.9.1 (Celsus) ; D 40.9. $14.3-4$; D 40.9.30 pr (constitution de Marc-Aurèle) ; D 40.12.27.1 ; (rescrit d'Hadrien) ; D 40.12.31; D 43.29.3.9; D 44.2.1; D 47.4. 1.7 (rescrits de Marc-Aurèle et de Sévêre et Caracalla); 0 II; D 48.19.9.16 (rescrit d'Antonin le pietix); D 49.1.14 pr (Antonin le pieux)-1 (epistula de Marc-Aurèle et Verus) ; 49.17 .6 ; D 49 . nin le pieux 17.9 ; D 50.16.216; D 50.17.161; L. Rufinus D 40.7.32; Marcien D I. $5.5 .2-3$; D $29.2 .52 \mathrm{pr}$ (rescrit d'Antonin le pieux); D $36.1 .32 \mathrm{pr}$; $5.5 .2-3$; D $29.2 .52 \mathrm{pr}$ (rescrit d'Antonin le pieux) ; D $36.1 .32 \mathrm{pr}$;
D $40.1 .5 \mathrm{pr}$ (constitution de Marc-Aurèle et Verus); D $40.2 .9 \mathrm{pr}-1$ (res crit d'Antunin le pieux); 0 40.4.26 (rescrits d'Antonin le pieux et de Marc-Aurèle et Verus) ; D 40.5.50; D 40.5.51 pr-1-3-4 -6-8-10-11 (Antonin le pieux) ; D 40.5.53 pr-1; D $40.5 .55 \mathrm{pr}-1$ (Marcellus) ; D 40.8. 6 (constitution de Marc-Aurèle) ; D $40.15 .1 \mathrm{pr}^{-1-2}$ (constitution d'Hadrien) -3-4 (Marcellus) ; D 48.10.7 (rescrit de Marc-Aurèle et Conmode); D 49.1.5.1 (rescrit d'Antonin le pieux); Modestin D J.5.22; D 35.

$1.51 \mathrm{pr} ; \mathrm{D} 35.1 .53$; D $37.14 .7 \mathrm{pr}$ (décret de Vespasien) ; D 40.1.16. D 40.4 .45 ; $\mathrm{D} 40.5 .12 \mathrm{pr}$ (rescrit de Caracalla)-2 (rescrit de Sévère et Caracalia) ; D 40.5.13;D 40.5.14;D 40.5.15;D 40.7.25;D 40 . 7.26 .1 ; D 40.8.2 (édit de Claude) ; D 48.18.14; Hermogénien D 5.1 .53 D $35.1 .94 \mathrm{pr}^{-1}$; D 36.4.11 pr ; D 40.1.24 pr-1; D 40.2.23; D 40.15.3. Un texte de Gaius ( $\mathrm{D} 40.2 .25$ ) n'a pas été mentionné car seule la citation défavorables (051). Si l'on opère cette comparaison par rapnort au

qu'opère ce juriste par renvoi a Fufidius est favorable a la liberté. Par contre, la solution qu'il donne ne l'est pas. Fufidius, en effet, admet l'affranchissement d'un esclave par un mineur de vingt ans pour en faire son tuteur. Au contraire, Nerva filius et Gaius s'y opposent. Il convient donc en l'espece de ne comptabiliser que la citation graphes, devant être intégré dans les solutions défavorables a la ligraphes.

951- Ce même problème (supra n. 950 in fine) se pose également en ce qui concerne cette rubrique. Dans certains textes, seul le renvoi est drfavorable la liberté, alors que l'auteur direct du passage donne defre solution favorable. une solution favorable. On ae doit done pareillenus 0 40.7.39.3; 0filius

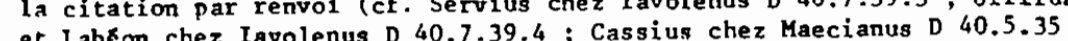
et Gaius D 40.4.57. Scaevola chez Marcien D 40.5.50). On a vu (supra $n$ 946) que selon ; rescrit d'Antonin le pieux (chez Ulpien D 48.19 . $9.16)$, le statuliber doit être puni comme un home libre : les textes. donnant des solutions inverses ont donc étê relevés ici (v. Pomponius D 40.7.29 pr; Ulpien D 29.5.1.4; D 48.5.28.10 sur le statuliber et l'esclave attendant sa liberté d'un fidéicommis ; Venuleius D 29. 5.13 et ulpien $D$ 29.5.1.13 et D 29.5.3.16 sur des esclaves dans 1 'attente d' tente doune solutions défavorables a la liberté, outre lavolenus D 35.1 .67 Al enus (chez auteur anonyme) D 40.12 .42 (Labéon); Julien D $40.5 .47 \mathrm{pr}$; D 40.7.13.2, D 40.9.5 pr-2 ; D 40.9.7 pr-1 (Proculus) ; D 40.12.30 (Sabinus, Cassius) : Pomponius D 40.1.13;D 40.4.3; D 40.5.34.1 (Campanus) ; D 40 7.29 pr ; D 40.9.22 ; D 40.9.23 ; Africain D $40.9 .8 \mathrm{pr}-1$; $T$. Clemens D 40.6.1 ; D 40.9.24 (Julfen); Gaius D 24.1.8; D 26.8.9.1 ; D 35.1 . 90 (rescrit d'Antonin le pieux) ; D 36.1.65.15; D 40.2.25 (Nerva filius) : D 40.7.31.1 ; D 40.9.3; D 40.9.10; D $40.9 .29 \mathrm{pr}-1 ; D 40$. 16.1; Venuleius D 29.5.13; Marcellus D 18.7.4; D 29.1.29.1; D 40 . 6.1 ; Venuleius 28.54 ; $D 40.4 .592$; 41.6 .5 ; Papinien $D 28$ 13.2 ; Scaevola D $28.5 .84 \mathrm{pr}$; D 40.4 .59 .2 ; D 40.1 .20 .1 D $40.7 .35 ; \mathrm{D} 40.9 .25$, Tryphoninus D 49.15 .12 .16 ; D 49.17 .19 .4 ; Callistrate D 40.16.3; Tryphoninus D 49.15.12.16; D 49.17.19.4 ; Callistrate D 40.16.3; Paul D 4.4.10 ; D 28.5.58; D 34.9.5.15 (Antonin le pieux) ; D 35 ; 40. 37 (Neratius) ; D 40.1.9; D 40.1.11; D 40.4.31 ; D 40.5.31.3 ; D 40.
$5.40 \mathrm{pr} ; \mathrm{D} 40.5 .43 ; \mathrm{D} 40.7 .1 .1 ; \mathrm{D} 40.9 .13 ; \mathrm{D} 40.9 .15 \mathrm{pr}$ (rescrit de Caracalla)-1 (Julien) ; D 40.9.16.1-2-4-5; D 40.9.17 pr (Marc-Aurêle) $-1-2$; D 40.9.18 pr-1; D 40.9.26 (Scaevola) ; D 40.9.28; D 40.13.4 ; D 42.8 .16 ; D 45.1.66; D 49.14.45.3; Ulpien D 4.4.11.1 (Scaevola); D 5.2.8.16; D 21.1.17.19; D 24.1.7.8 (Sabinus, Papinien)-9 ; D 29. 5.1.4-13 (Scaevola) $-16 ; D 29.5 .3 .15-16 ; D 30.44 .7 ; D$ 34.4.3.9 (Ju1i.4 Papinien); $34.5 .10 \mathrm{pr} ; \mathrm{D} 5.1 .7 \mathrm{pr} ; \mathrm{D} \mathrm{40.1.1;0} 40.1 .4 .9$ lien, Papinien) $\mathrm{D} 34.5 .10 \mathrm{pr}, \mathrm{D} 35.7 \mathrm{pr} ; \mathrm{D} 40 . \mathrm{D}, \mathrm{D} 40.1 .4$ (constitution de Marc-Aurele et Verus) ; $D$ 40.4 .32 ; 40.5 .4 .19 ; D 40.5.24.9 (Marcellus; rescrit de Sévère et Caracalla) $-11-16$; 40.9 .

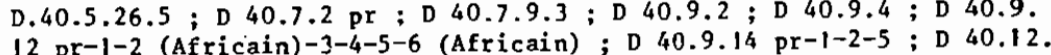
$12 \mathrm{pr}-1-2$ (Africain) $-3-4-5-6$ (Africain) ; D $40.9 .14 \mathrm{pr}-1-2-5 ; \mathrm{D} 40$.
$7.4 ; \mathrm{D} 40.16 .2 \mathrm{pr}-1-2-3-4 ; \mathrm{D} 42.8 .6 .5 ; \mathrm{D} 48.5 .28 .10 ; \mathrm{D} 48.18 .1$. 7.4 ; D 40.16.2 pr-I-2-3-4 ; D 42.8 .6 .5 ; D 48.5 .28 .10 ; D 48.18.1. 33 8.20 ; D 40.1 .8 .2 (rescrit d'Antonin le pieux) -3 (rescrit d'hadrien); D 40.9.9.1-2; D 40.9.11 pr-1 (rescrit de Marc-Aurèle et Verus) ; Modestin D 34.5 .27 ; D 40.4.45;D 40.9.19; D 40.9.20; D 40.9.21 (Julien) 
nombre total de textes, ou de citations par renvoi, relatifs à chaque période en matière de liberté, on obtient :

Solutions fevorables à la liberté

\section{Solutions défavorables} à la liberté

\section{- analyse des paragraphes}

\begin{tabular}{|c|c|c|c|c|c|c|}
\hline I & - & $=\quad-$ & I & - & $=$ & - \\
\hline II & - & $=$ & II & - & $=$ & - \\
\hline III & $63 / 94$ & $67 \%$ & III & $31 / 94$ & $=$ & $33 \%$ \\
\hline IV & $40 / 47$ & $85 \%$ & IV & $7 / 47$ & $=$ & $15 \%$ \\
\hline$\nabla$ & $331 / 432$ & $77 \%$ & $\mathrm{~V}$ & $101 / 432$ & $=$ & $23 \%$ \\
\hline TOTAL & $434 / 573$ & $76 \%$ & TOTAI & $139 / 573$ & $=$ & \\
\hline
\end{tabular}

- analyse des citations

\begin{tabular}{|c|c|c|c|c|c|}
\hline I & $4 / 7$ & $57 \%$ & I & $3 / 7$ & $=$ \\
\hline II & $13 / 22$ & 59 & II & $9 / 22$ & $=$ \\
\hline III & $23 / 33$ & $70 \%$ & III & $10 / 33$ & $=$ \\
\hline IV & $8 / 13$ & $62 \%$ & IV & $5 / 13$ & $=$ \\
\hline $\mathrm{v}$ & $1 / 2$ & $50 \%$ & $\mathrm{y}$ & $1 / 2$ & $=$ \\
\hline TOTAL & $49 / 77$ & $64 \%$ & TOTAL & $28 / 77$ & $=$ \\
\hline
\end{tabular}

D'un point de vue global, il ressort nettement de ce-tableau que les solutions favorables à la liberté l'emportent largement sur les solutions défavorables. Plus précisément, 1 'ang.lyse des citations par renvoi montre que la poussée du favor libertatis s'effectue sous les premiers Aatonins. Grâce à l'anslyse directe, on voit, que cette tendance atteint son point culminant sous les derniers Antonins. C'est un point fondamental. La seconde moitié du zème siècle marque à cet ésard encore un tournant de l'óvolution in l. jurisprulence. Le pourcentape peu important réalisé par cette période dans l'analyse des citations ne remet pas cette assertion en cause. Les juristes écrivant durant la première moitié du Zème siècle sont certainement plus cités que leurs successeurs immédiats dans la mesure où ce sont les premiers à avoir résolument poussé le droit dans cette direction.

Le développement des solutions favorables à la liberté coincide avec la reconnaissance d'une certrine capacité à I'esclave. On trouve

Hermogénien D 37.5 .23 (constitution d'Antonin le pieux) ; D 40.9.27 $\mathrm{pr}^{-1}$. Certains textes ont été relevés dans les deux rubriques car ils contiennent les teux types de solutions (Eavorable/défavorable) (cf. par ex. Paul D 40.13.4; Ulpien D 40.5.24.19). aussi une preuve de cela dans le fait que le favor libertatis reste important au 3ème siècle. Conme en matière de capacité áconomique et juridique, les juristes de l'époque des Sévères $s$ 'efforcent de rester sur la voie tracée par leurs prédécesseurs.

Si les solutions du droit en matière de liberté se scnt probablement inspirées, au moins dans une certaine mesure, des idées stoiciennes, il n'est pas possible de leur donner principalement un fondement moral, qui aurait correspondu à une sorte de sentiment culpabilisateur (952). Il ne faut tout de même pas oublier que l'affranchi restè exploitable, et puis les faits sont là. On ne peut s'empêcher de relever que c'est précisément à l'époque où les courbes du favor libertatis et de la capacité sont les plus élevées que les textes parlent le plus de la délinquance servile. Il semble donc que la libéralisation du discours réponde beaucoup plus à une recherche de compromis qu'à une inspiration de type philosophique.

L'explication de la libáralisation par la propression de la délinquance servile est toutefois insurfisante. On pourrait, en effet objecter que la recrudescence de la délinquance servile est, une conséquence et non une cause du dévoloppement. de la "caparíté" de l'esclave, l'esclave privilégié se trouvant, de par ses foncticns, dans une situation favorable au détournement a'effets appartenant au maître. Bien que nos résultats démentent cette allékaticn, la progression statistique du favor libertatis et de la "canacité" de l'esclave (période IV) étant postérieure à la montée de la délinquance (périodeIII), nn voit là la faiblesse de la thèse qui consisterait à fonder l'explication de la libéralisation sur l'unique phénomòne félinquant. Il nous faut donc chercher ses causes profondes ailleurs.

$-0^{-}$

952- Sur l'idée d'un fondement philosophique du favor libertatis, v. J. MARQUUARDT, Vie privée, cit., p. $224 \mathrm{~s}$; ; J. MMBERT, Favor libertatis, cit., p. 278 ; sur les idées des stoiciens en matière d'esclavage, P.A. MILANI, La schiavitù nel pensiero politico dai freci al Basso Medio Evo, Milan (1972), p. $204 \mathrm{~s}$. M. MESLIN, L'borme romain, cit., p. 231 , note que le stoícisme n'a jamais songé a abolir la concit., p. 231 , note que le stoicisme n'a jamais songé a abolir la con-
dition servile. V. également S. RICCOBONO, L'i dea di homanitas come fonte di progresso del diritto, in Studi in onore di B. Biondi, 2 (Milan 1963), p. 585 s. : F. CASAVOLA, Cultura e scienza giuridica nel II sec. D.C., ANRW, II, 15 (1976), F. 160 . 
CBAPITRE III : ESCLAVAGE ET COMPORTEMENTS

Cette étude sera organisée en deux volet.s. Il s'agira tout d'abord d'observer la nature des rapports dans lesquels on relève la présence d'un esclave ou d'un affranchi. On examinera ensuite les seuls comportements dysfonctionnels des esclaves car, remettant en cause le système, ils revêtent une importance particulière.

\section{SECTION I. DEPENDANCE ET RAPPORTS SOCIAUX}

Il convient d'envisager non seulement les rapports sociaux ol des esclaves sont impliqués, mais aussi ceux où apparaissent des affranchis. Il est en effet d'un granni intérêt de savnir comment l'esclave est accepté par la société, une fois affranchi.

\section{Paragraphe 1: L'esclave dans les rapports sociaux}

En vue de mener une analyse statistique de ces rapports, ils ont été rangés en deux rubriques : bons ou mauvais.

\section{I - Bons rapports.}

Deux rapports sont à prendre en considération : esclave/maître et esclave/tiers libre.

\section{A/ - Les bons rapports esclave/maitre}

C'est parfois l'esclave qui préside à ce rapport. On le voit défendre son maître dans une rixe (953), commettre une iniuria dans un même but de défense (954), le protéger contre des brigands, le guérir (955), l'avertir d'une conspiration fomentée contre lui (956). On le voit ailleurs emprunter une somme pour constituer une dot à la fille de son mâtre (957). C'est cependant le maître qui ressort des textes conme étant le plus souvent à la tête d'un tel rapport.

953- Alfenus (chez auteur anonyme) D 44.7.20.

954- Ulpien D 47.10.17.8.

955- Paul D 40.2.15.1.

956- Paul D 35.2.39.

957- Paul D 15.3 .8 (Pomponius).
Ainsi, il est question de son affection pour l'asclave (958), de son indulgentia (959), de sa confiance en lui (960). Il se port.e caution pour son esclave 961$)$. Un maître va même jusqu'à lui conseiller de s'enfuir, pour échapper il est rrai à une action noxale, mais un tel conseil n'est concevable que dans le cadre d'un certain rapport (962). Un autre prévoit dans une clause de la vente que son ancilla ne devra pas être prostituée (963). Certains esclaves sont recoumandés par le testateur à son fils ou, plus généralement, à l'héritier (964).

L'affranchissement d'esclaves constitue un critère trop général pour la détermination d'un bon rapport, la problématique juridique $l$ 'emportant alors sur le cas concret. N'cublions pas non plus que, comme on l'a vu, l'affrenchissement est rarement gratuit, et que même dans ce cas l'affranchi reste exploitable. Il faut s'en tenir à la même position en mat,ière de donations, legs ou fidéicommis laissés par le maître à l'esclave (965). En effet, ces situations nécessitent un bon -

958- Papinien D 17.1.54 pr ; D 18.7.7 ; Callistrate D 29.5 .2 (rescrit de Comode) ; Paul D 9.2.33 pr (Pedius) ; D 40.5.38; Ulpien D 7.7.6.2 ; D $20.1 .6 ; \mathrm{D} 40.2 .16 \mathrm{pr}-1$. Un de ces textes est particulièrement intêressant, celui de Callistrate : un maître a été blessé mais a absous ses esclaves : ceux-ci ne seront pas punis,estime Conmode : nec-pietas pro servis, nec sollicitudo heredis obtinere debet, ut ad poenam vocentur, quos absolvit dominus ipse.

959- Gaius D 40.16.1.

960- Africain D 47.2.62.7.

961- Ulpien D 15.1.9.8 (Julien, Marcellus) ; D 15.4.1.5 (Marcellus); Modestin D 16.1.25.1.

962- Paul D 9.4 .24 .

963- Papinien D 18.7.6 pr (Sabinus) ; Paul D 18.1.56; D 18.7.9; Marcien D 40.8.6 ; Modestin D 37.14.7 pr (décret de Vespasien).

964- Scaevola D 40.5.41.4-15; Paul D 40.5.39.1.

965- Rappelons tout d'abord quelques points juridiques : un legs sine libertate fait par le testateur $a$ son esclave est nul (cf. vlpien D 30 . 34.9. W.w. BUCKLAND, Roman law, cit., p. 144). Si la liberté est accordée pure, le legs peut être conditionnel ou simple; il se peut aussi que le legs soit simple et la liberté conditionnelle (w.w. BUCKLAND, o.c., p. 471). Si le testateur fait un legs à l'esclave qu'il lègue, le legs fait à l'esclave est acquis au légataire de celui-ci (Afric.D 30.107.I); s'il fait semblablement un legs à l'esclave qu'il lègue, et qu'il le vende, le legs ira. 1 'acheteur (cf. Julien D 30.91.3). Cependant, même dans ces cas, le legs est le signe d'un bon rapport car, contrairement a l'institution d'un heres necessarius, il ne comporte aucun caractere utilitaire. Signalons enfin que la donation est assimilable du point de vue des rapports qui nous de vue des rapports qui nous préoccupent, au legs (cf. par ex. Julien clave de la part de son maitre : Iavolenus D 32.29 .4 (Tubero, Labéon); 
rapport, mais ne peurent ètre retenues ans une analyse quantitative sous peine d'sboutir à un déséquilibre au profit des problèmes soulevant le plus de difficultés juridiques, par rapport ミ̀ la réalité quotidienne des relations maître/esclave. Si les legs nécessitent cet éclaircissement méthodologique, il $n$ 'en va pas de même de l'institution de l'esclave comme hores necessarius, celui-ci n'étant alors qu'un domestious herse, sans ius atstinendi, غ̀ moins qu'il ne soit apranchi par le testateur de son rivant, auquel cas il devient heres voluntarius (966).

D 32.30.2 (Trebatius, Labeon) ; D 33.5.15; D 35.1.40.3 (Servius, Namusa) ; Celeus D 34.5.25; Valens D 32.12 (Pegasus); Julien D 28.5.38.4; D $30.91 .1-2-3-4-5 ;$ D $30.96 \mathrm{pr}$; D 30.102 ; D 33.5.11; D 36.2.17. D 40.4.16; D 40.4.19; D 41.1.37.1-2; Pomponius D 3i.11 pr (Labécn) ; D $34.4 .20 ;$ D $40.4 .4 \mathrm{pr}^{-1-2} ; \mathrm{D} 40.4 .46$ (Aristo) ; D 40.7.11 (Aristo); D $40.7 .21 \mathrm{pr}$ (veteres, Labeon); Africain D 30.107 .1 ; D 35.1 .31 ; D 40.7.21 pr (veteres, Labeon); Africain D 30.107 .1 ; D 35.1 .31 ;
D 48.10.6.2; Gaius D $30.69 \mathrm{pr}$; D 40.7.31 pr; Maecianus D $35.1 .86 \mathrm{pr}$ (Julien)-I ; D 35.2.30.8; Venuleius D 34.4.32.1 ; Marcellus $D 35.1 .47$; Plorentinus D 15.1.39; Screvola D 31.88.3-6-13; D 32.38.1; D 33.1. 18.1; D 33.1.19 pr ; D 33.1.21.1; D $33.2 .36 \mathrm{pr}-1$; D 33.8.23.1-2-3.; D 34.1.18.2-3; D 34.1.20 pr ; D 36.2.27.1; D 40.7.40.3 ; Papinien D 12.6.38; D 31.65.2; D 31.76.4; D 35.1 .77 pr (rescrit d'Antonin le pieux) ; D 40.7.34.1; D 48.19.33 (rescrit de Marc-Aurêle et Verus) ; Tryphonimus D 31.88 .12 (Scaevola); D 34.3 .28 .7 (Scaevola); Callistrate Tryphonimu: D 31.88.12 (Scaevola); D 34.3.28.7 (Scaevola); Cal1 D $32.97 ;$ D $33.1 .16 ;$ D 33.5.13 pr; D $34.4 .26 \mathrm{pr}-1 ;$ D $34.4 .27 \mathrm{pr}-1$; D $35.1 .81 \mathrm{pr}$; D 35.2 .1 .4 ; D $35.2 .49 \mathrm{pr}$ (Sabinus, Nerva, Atilicinus, Cassius, Plautius ; rescrit d'Antonin le pieux); D 40.4.39; D 40.5.38; D 40.7.11; D 40.7.20.3 (Julien) ; D 48.10.22.3-4-5; Ulpien D 17.2.63.9 (Sabinua, Jullen, Pomponius) ; D 30.34.9; D 32.49 .7 ; D 35.2 .35 (Scaevola); D 36.1.11.2 (rescrit d'Antonin le pieux); D 36.2.7.6: D 36 . 2.8 ; D 40.4.9 pr ; D 40.4.12.1-2 (Julien) -3 ; D 40.4.13.1 ; D 40.5.30. 15 (rescrit de Caracalla): D 40.7 .19 . Marcien D 28.7.18 pr (rescrit d'Antonin le pieux); D 40.4.26 (rescrits d'Antonin le pieux et de Marc Aurale et Verus); Modestin D 35.1 .66 . Le contenu de ces legs ou fideicommis consiste essentiellement en une soume d'argent (cf. par ex. Iavolenus D 32.30.2 (Trebatius, Labéon); Julien D 30.91.2 ; Pomponius D 40 . $7.21 \mathrm{pr}$ (veteres, Labéon) ; Africain D 35.1 .31 ; Scaevola D 33.1.21.1 D $34.1 .20 \mathrm{pr}$ (ici, outre une somme d'argent, 1 'esclave affranchi reçoit le legs de sa femme et de ses enfants) ; Paul D 35.1.81 pr)ou un fonds (cf. Iavolenus D 32.29.4 (Tubero, Labeon); Julien D 33.5.11; D 40.4 . 16 ; Scaevola D 31.88 .6 : D $33.2 .36 \mathrm{pr}^{-1}$ (il s'agit dans ces deux paragraphes de 1 'usufruit d'un fonds); Tryphoninus D 31.88 .12 (Scaevola); Paul D 32.97,; D 35.2.49 pr (Sabiaus, Cassius, Nerva, Atilicinus, PlauPaul D 32.97 . D $35.2 .49 \mathrm{pr}$ (Sabinus, Cassius, Nerva, Atilicinus, Pla
tius).). Le rejet de ces textes s'explique aussi par ce que ces legs

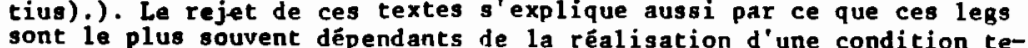
nant soit directement au legs, soit a la liberté.

966- W.W. BUCXCAND, Roman law, cit., P. 505; P. BONFANIE, Corsi di diritto romano, vol. VI, Milan (1974). Le successioni, p. 378 ; A. GUAdiritto romano, vol. VI, Miritan (1974). Le successioni, P. 378 ; A. GUA ou testateur ;'il est affranchi inter vivos, il peut acceptar la succession lui-même, il est alors herss coilurtirius (c $\overrightarrow{\mathrm{f}}$. Neratius D 28.5.55; Scaevila D 28.6.48.2 ; Tryphoninus D 28.5.91; U1pien D 28.5.9.19 : 028. 6.18 pr ; 029.2 .71 .1 ) (c'est la seule hypothèse qui soit statistiquement intégrable come bon rapourt); si l'esclave institué hẹritier par son maírre of aliáné. l'institution est assimilable a celle d'un gerruo alienus,en faveur du nouveau maître (cf. par ex. Julien D 28.5.7.1. P. BONFANTE, o.c., p. 379). En droit classique, un servus proprius ne peut être institue heres necessarius que s'il est affranchi dans le testament; c'est seulement a l'époque de Justinien que l'institution d'héritier emportera implicitement concession de la liberté, P. BONFANTE, o.c., p. emportera implicitement concession de la liberté, P. BONFANTE, o.c., p.
378, n. 4. Le choix du rapport (esclave/maítre ou esclave/tiers) déri$378, n$. 4. Le choix du rapport (esclave/maître ou esclave/tiers) déri
vait donc, en l'absence des termes heres neassaarius, de l'institution d'un esclave avec la liberté. Cependant, certains textes contiennent ces deux rapports, que ceux-ci soient seulement possibles (cf. Papinien D 48.19.33) ou apparents (cf. Celsus D 28.7.21 ; Marcellus D 29.2.63 (Pomponius) ; Ulpien D 46.1.33). En présence de deux rapports, on a privilégié, lorsque cela était possible, le rapport initial : ainsí, un testateur aliène l'esclave qu'il avait institué héritier ; celui-ci accestateur alizer quilert la successlon par ordre de 1 'acheteur. Cette espace, rapportée . maf tre et esclave/tiers, car lesclave, une fois aliéne, se trouve visa-vis du testateur dans la situation d un servus alienus. Néanmoins, le premier rapport, celui od l'esclave est institué par son maître, reste prédominant et 'c'est lui qu'il convient de faire ressortir. On a opéré de même pour Julien D 28.5.38.2 3; Marcellus D 35.1 .47 ; Scaevola D 28. 6.48.2 ; Paul D 32.6.1 ; Ulpien D 28.5.9.16; D 28.5.51 pr ; D 42.8. 6.5 ; Marcien D 28.5.52.1. Le fait que la disposition de la lex Aelia Sentia, selon laquelle est nul l'affranchissement effectué en fraude des créanciers, ne s'applique pas ici (cf. supra n. 943), montre à quel point cette institution d'heritier nécessai ne point cette institution d'heritier necessaire ne met pas en présence d'un bon rapport. In est aussi intéressant de relever a ce propos que l'esclave qui a payé son maître pour parvenir a la liberté peut s'en abstenir (cf. Ulpien D 29.2.71.2). Si l'esclave est institure ex parte. il est libre et heres necessarius avant que 1 'autre heritier accepte la succession (cf. Paul D 29.2.58 : Ulpien D 40.7.2.3. W.W. BUCKLAND, Roman law, cit., p. 506). Il n'est pas obligatoirement institué au premier degré : il peut s'agir d'un substitué, que la substitution soit vulgaire (cf. Marcien D 28.6.36 pr) ou pupillaire (cf. Scaevola D 28. 6.48.2) ; sur ce probleme, R. MONIER, Droit romain, cit. I, p. $471 \mathrm{~s}$. Il peut recevoir la succession par un fidéicommis d'hérédité (cf. v1pien D 29.1.13.4. W.W. BUCKCAND, O.c., p. 509 ; R. MONIER, o.c., I, p. $528 \mathrm{s.)}$. L'esclave peut être institué pure et affranchi sous condition, ou affranchi pure et institue conditionnellement; il peut aussi être affranchi ex die et institue sous condition, (sur ces differents cas, W.W. BUCKLAND, o.c., p. $511 \mathrm{s.}$ ). Ont été relevés dans cette rubrique : Neratius D 28.5.55; Celsus D 28.5.61; D 28.7.21; Julien D 10.2.52. I ; D 21.2.7; D 28.1.12; D 28.2.13.1; D 28.5.7 pr-1; D 28.5.8 pr-1; D 28.5.22; D 28.5.38 pr-1-2-3; D 28.5.43; D 28.6.19; D 29.2.43; D 29.4.22.1 ; D 30.91.7 ; D 30.98 ; Pomponius D 28.5.21 pr (Trebatius, Labéon)-1 ; D 40.4.46 (Aristo) ; Africain D 12.1.41; Gaius D 28.5.89;. D 28.7 .22 ; D 29.2.57.2; D 36.1.65.15; Maecianus D 35.1.86.1; D 40 . 5.42 (Antonin le pleux) ; Marcellus D 9.2.36.1 (Sabinus); D 29.2.63
(Pomponius); D 35.1.47; D 36.1.46 pr; Scaevola D 28.5.84 pr-1; D 28. 5.86 ; D 28.6.48.1 (Julien) -2 ; Papinien D 26.5.13 pr (rescrit d'Hadrien) ;

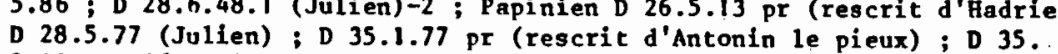
D 28.5.77 (Julien) ; D 35.1.77 pr (rescrit d'Antonin le pieux) ; D
2.13 ; D 36.1.54.1 ; D 36.1.55; D 36.1.57.1 (rescrit d'Antonin le pieux)-2 ; D 40.4.50.1 ; D 40.7.36 ; D 48.19.33 (rescrit de Marc-Aurèle et Verus) : Tryphoninus D 28.5.91 ; Paul D 3.2.14; D 4.6.13 pr (Labeon); D 28.5.44; D 28.5 .56 (Neratius) ; D 28.5.58; D 28.5 .85 pr-1 (constitution de Marc-Aurèle)-2; D 29.1.40.2 ; D 29.2.58 ; D 29.2.60 (Labéon, Proculus, Iavolenus)：D 32.6.1 ; D 35.1.44.10 (P1autius); D 40.4.27; 
On doit encore ajouter qu'il n'y a pas à proprement parlor bons rapports, walgré les apparences, quand l'esclave se porte au secours de son maitre en danger de mort, is y est contraint (367), nil quand il Jénonce les assagins de son maître, ce geste pcuvant ne pas ôtre inspiré par l'attachement qu'il aveit pour lui, mais simplement par l'espoir d'une liberté prochaine (968). En sens inverse, on ne peut paraillement interpréter come bons rapports le fait que le maitre se charge de faire instruire l'esclave (960) ou de lui faire prodiguer des soins (970), dans un cas combe dans I'autre, ces actions pouvant être liées à un souci de rentabilité. La défense en justice par le maitre constitue semblablement un rapport normal, celui-ci y ayant un intérêt patrimonial (971). Enfin, le mâ̂tre qui déclare faussement aux magistrats que son esclave est libre, pour que celui-ci ne soit pas châtié, peut adopter cette attitude non pas pour éviter à l'esclave le châtiment, mais de crainte qu'il avoue quelque chose contre lui (972).

Dans des espèces semblables, il en est. en revanche qui sont déterminantes $d$ 'un bon rapport lorsque la relation associe un esclave, non plus à son maittre, mais à un tiers.

D. 40.4.28; D 42.5.4; U1pien D 2.14.7.18 (V.Verus, Marcellus) ; D 4.4. 7.10 (Papinien; rescrits d'Antonin le pieux et de Caracalla); D 9.2. 15.1 ; D 9.2.23.1 (Julien) ; D 15.1.3.1 (Labêon); D 15.1.30.2.; D 24.1. 22 ; D 25.4.1.13 (Aristo); D 28.5.3.1 ; D 28.5.6.3 (Julien)-4 (Julien); D 28.5.9.14 (Labéon, Aristo, Neratius)-16-17-18-19-20; D 28.5.30

(rescrit de Sévère); $D 28.5 .51 \mathrm{pr}$; D 28.6.10.1-7; D 28.6.18 pr (Julien)-1; D 28.7.2 pr-1 (Celsus); D 29.1.13.3-4 (rescrit de Sévère); D 29.2.66; D 29.2.71 pr-1-2; D 36.1.11.2 (rescrit d'Antonin le pieux) . D 36.1.17.16-17 (rescrit d'Antonin le pieux); D 37.1 .7 pr; D 36.1.23. D 36.1 D 37.10 .3 .11 (Julien): D 40.4.2 (Aristo); D 40.4.14; D 40.7.2.3 (Julien) 4 (Celsus); D 42.6.1.18; D 42.8.6.5; D 42.8.10.10 (Labéon); D 46.1.33; D 49.17.9 (Marcel1us); Marcien D 28.5.49.2 (constitution de Sévère et Caracalla); $\mathrm{D} 28.5 .52 \mathrm{pr}$ (rescrit de Marc-Aurèle)-1 (Ce1sus); D 28.6.36 pr ; D 28.7.18 pr (rescrit d'Antonin le pieux) ; D 36. 1.32.1 (rescrit d'Antonin le pieux); D 40.4 .26 (rescrits d'Antonin le pieux et de Marc-Aurèle et Verus).

967- U1pien D 29.5.1.34-35-37.

968- Cf. supra n. 946.

969- Par exemple, Ulpien D 19.5.13.1 (Julien); D 21.1.17.3 (Vivianus). 970- Par exemple, U1pien D 9.2.27.17; D 15.1.9.7; D 19.1.13.22 (Labéon).

971- Par exemple, Paul D 47.2.54.4 (Ju1ien) ; Ulpien D 48.2.7.4 (rescrit d'Antonin le pieux); Modestin D 48.2.17.

972- Pau1 D 40.9.17.1.

\section{B/ - Lea bons rapports esciqve/tiers}

Il en est ainsi lorsque l'escrlave ost dórendu on ivstion nar un tiers (973) ou est institué hóritior par lui, l'institution n'ayent plus le même caractère obligatoire qu'anparavant, 1 'esclave n'accepttant la succession que si son maître lui en donne le iusgsum (974). On

973- Paul D 3.5.40; Ulpien D 9.4.21.1.

974- L'institution d'un servus alierus correspond $\Rightarrow$ l'institution indirecte de son maitre, et exige que celui-ci ait la testomenti factio avec le testateur (cf. Gaius D 28.5.31 pr ; Paul 28.5.53. P. BONFANTE, o.c., . . 379). L'esclave ne peut accepter la succession sans le iussum domini (cf. Gaius D 41.1.10.1). Une simple ratification ne suffit pas (cf. Ulpien D 29.2.25.4. W.W. BUCKIAND, Roman law, cit., p. 138). C'est cependant la personne de l'heres, c'est-dire de l'esclave, qui est prise en consideration (cF. Paul D 35.1 .44 pr ; Papinien D 33.3.5 ; L. Rufinus D 34.4.21. P. BONFANTE, O.c., p. 379). Si l'esclave est affranchi avant l'adition d'hérédi tê, il nourra accepter la succession luimême (cf. Modestin D 5.2.12 pr). Cette institution peut enfin être conditionnelle (cf. Paul D 12.6.65.3; D 29.2.80.2-3; U1pien D 28.5 . 6.2). I1 faut citer dans cette rubrique : Iavolenus D 28.5.65 (Labéon). 6.2). Il faut citer dans cette rubrique : Iavolenus D 28.5 .65 (Labéon)
D 29.2.62.1; D 29.2.64; Celsus D 28.7.21; D 29.4.25; Julien D 9 . $2.51 \mathrm{pr}$; D 21.2 .8 ; D 21.2 .51 .3 ; D 24.3 .31 .4 ; D 28.5.38.5; D 28.5.41; D 29.2.45.1-3; D 30.91.7; D 30.94.1; D 36.1.26.1-3; D 36.1.28. $1-17$; D 38.2.13; D 38.13.1; D 49.15.22.1; Pomponius D 23.3.65; D $28.1 .16 \mathrm{pr}$; D 28.5 .42 (Tíè̀re) ; D 29.2.36 ; Africain D.29.2.47; D 34.6 .1 ; D 46.1 .21 .2 ; T. Clemens D 29.2 .82 ; Gaius D 6.1 .20 ; D 7.1. 6.3 ; D 28.5.31 pr-I ; D 28.5.32.1 ; D 36.1.65.4 (Julien) ; D 38.1.22. 1 (Julien) ; D 41.1.10.1-3 ; Maecianus D 29.4.28 pr ; D 35.2.30 pr ; D $36.1 .67 \mathrm{pr}$; D $49.17 .18 \mathrm{pr}$; Marcellus D 28.5 .40 (Julien) ; D 29.4 . 5 ; D 38.15.5.2 ; D 29.2.63 (Pomponius) ; Florentinus D 28.5.50 pr ; D 30.116.2 ; Scaevola D 28.6.48 pr; D 35.2.20 (Maecianus); D 36.1.80. 2-3; Papinien D 30.11 ; D 48.19.33 (rescrít de Marc-Aurèle et Verus); Tryphoninus D 38.2.50, pr-1-2-3; D 46.1.63.1; D 49.17.19.1 ; Pau 1 D 12.6.65.3 ; D 24.1.28.5 (Julien, Pomponius); D 28.5.53; D 29.2. 26 (Julien, Pomponius, Marcellus) ; D 29.2.65; D 29.2.68; D 29.2.74. 3-4 (Julien) ; D 29.2.80.2-3; D 29.2.93.1-2;D 31.14.1;D 32.4 ; D 35 . 1.5 .1 ; D $35.1 .44 \mathrm{pr}-1$; D 35.2.21.1; D 35.2.22.1-2 ; D 35.2.63 pr (Pedius); D 36.1.41.1 ; D 36.1.42 ; D 37.11.10 (Scaevola) ; D 38.2.45; D 41.1 .47 ; D 41.3 .4 .4 ; D 43.16.6; D 48.20.22.4; Ulpien D 1.19.1.2; D 7.1.21 (Labéon) ; D9.2.23 pr (Neratius); D 15.1.7.5 (Labéon); D 15. 1.19.1 (Pomporius) ; D 15.3.7.4 (Labéon); D 19.1.13.18; D 21.1.23.9 (Pedius); D 21.2.51.3; D 28.5.3 pr-3; D 28.5.6.2; D 28.6.8.1 ; D 28 .

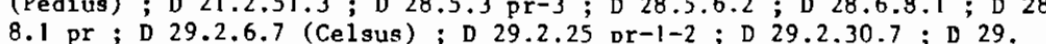
$2.35 \mathrm{pr} ; \mathrm{D} 29.2 .67 ;$ D $29.4 .1 .3-4 ; \mathrm{D} 29.6 .1$; D $30.39 .1 ; \mathrm{D} 36.1$

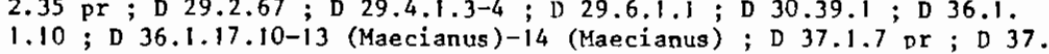
4.3 .15 ; D 37.11 .2 .9 ; D 38.9.1.14; D 46.1.33; D 47.2.52.28; L. RuFinus D 31.62 ; Marcien D 28.5.39; D 36.1.31.2; Modestin D 5.2.12 pr ; D 24.3 .58 ; D 28.6.3 ; D 28.7.25; D 29.2.50; Hermogénien D 37.14.21. 3 ; D 41.1.16 pr ; D 49.14.46.8. Il est question d'un rapport esclave/ tiers $a$ propos d'une heroditatis petitio chez Gaius D 5.3 .35 (Julien) et chez Paul D 5.3.34.1 (Julien) et D 5.3.36 pr (Proculus, Julien, Mauricianus) ; sur le probleme, $v$. A. GUARINO, Diritto privato romano, p. 433 ; il faut rappeler $a$ ce sujet (cF. supra n. 634) que le droit ne 
peut nérnnoins sunoos‘r. ̀̀ l'égard d'une t,elle ingtitution, que l'esclave était rentré en contact d'affaires avec le testateur qui voyait en lui un débiteur valable. Conme dans les relations maître/esclave, on a affaire à un bon rapport lorsque l'esclave reçoit une donation, un legs ou un fidéicommis (975). Cependant, en matière de droit successoral, il faut formuler les mêmes réserves que celles exposées plus haut, une intégration statistique de ces problèmes présentant le risque de privilégier le cas juridique au détriment de la réalité.

reconnaît pas à l'esclave la faculté d'être possessor pro herede. II faut signaler que $1^{\prime}$ institution d'un servus poenae est regardée comme non écrite (cf. un rescrit d'Antonin le pieux chez Ulpien D 29.2.25.3). $D^{\prime} u n$ point de vue quantitatif, on peut enfin remarquer que, à propos des institutions d'héritiers, la relation tiers/esclave est légèrement majoritaire (136 textes) par rapport a la relation maître/esclave (122 textes).

975- Cơme pour l'institution, le legs fait a un servus alienus dépend de la testonenti factio du maitre et est en principe équivalent $a$ un legs fait à celui-ci (cF. Pomponius D 30.12.2. W.W. BUCKLAND, Roman 1 aw, legs fait a celuíci (cF. Ponpros cit., p. l qui détermine le sort du legs (cf. Julien D 30.91 .6 ; Ulpien $D 36.2 .5 .7$ W.H. BUCKIAND, O.c., p. 145) ; on tient néanmoins compte de la "personnalitê" de l'esclave (cf. Valens chez Paul D 31.82.2. W.W. BUCRLAND, o.c., p. 147). Un tel legs peut aussi être conditionnel (cf. T. Clemens D 31.59 ; Paul D 35.1.44.8). Ont été relevés dans cette rubrique : Iavolenus D 24.3.66.1 (Trebatius, Labéon); D 31.40 ; Celsus D 31.20 (Proculus, Celsus p.) ; Julien D 23.3.47; D 24.3.31.4; D 30.81 .1 ; D 30 . 86.2 ; D 30.91.6-7; D 30.99 ; D 30.101 pr ; D 33.5.10 ; D 36.2.16.1-2 D 41.1 .37 .1 ; D 49.15.22.1; Pomponius D 7.4.18; D 7.8.16.2; D 23.3.

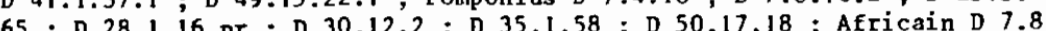
17; D 35.1 .42 ; D 39.6 .23 ; T. Clemens D 31.59 ; Gaius D 7.1 .74 . D $6.1 .20 ; D$ D $30.68 .1 ; D$ D $31.55 .1 ; D 41.1 .10 .1-3$; Maecianus D 32.17 . I ; D 35.2.30 pr; Marcellus D 23.3.59.2; D 35.2.56.4; D 49.15.1;

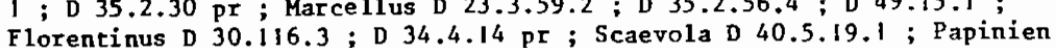
D 30.11 ; D 33.3.5 ; D 48.19.33 (rescrit de Marc-Aurèle et Verus) ; D 49.17.14.2; Tryphoninus D 15.1.57.2; D 41.1.63.1; D 49.17.19.5; Paul D 7.1.24; D 7.1.31 ; D 10.2.15 ; D 24.1.28.5 (Julien, Pomponius) D 30.7 ; D 31.82 .2 (Valens) ; D 31.83 ; D 32.8 .1 ; D 33.1 .11 ; D 34.9 . 5.3 ; D $35.1 .44 \mathrm{pr}-1-8$; D 35.2.21.1 ; D 36.3.7 ; D 39.6.44; D 41.1.49; D 45.3 .26 ; D 48.10.14 pr-1 (Julien, Marcellus); D 48.10.22.4;D 49 . 14.13 .8 (beneficium de Trajan); Ulpien D 7.1 .21 (Labeon) ; D 7.1 .22

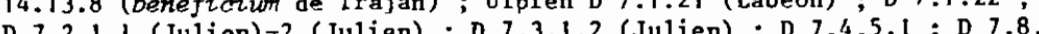

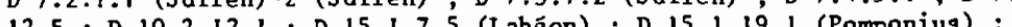
D 17.512 .10 ;

D 17.1.12.2 (Marcellus); D 19.1 .13 .18 ; D 21.1 .23 .9 (Pedius) ; D 23.

$3.39 \mathrm{pr}$; D 30.39 .1 ; D $30.50 \mathrm{pr}$; D 30.53 .2 (Julien, Marcellus) ;

D 32.7 pr ; D 32.11 .16 ; D 36.2 .5 .7 ; D 36.2.12.2 (Julien).; D 36.3.1.20 D 37.4.3.16 ; D 37.5.3.2 ; D 40.5.24.19; D 41.1.33 pr (Marcellus, Scaevola) ; L. Rufinus D 34.4 .21 ; Marcien D 30.113 pr (Marcellus); D 30.114 .10 ; D 34.8.3.2 ; Modestin D 5.2.12 pr ; D 31.34 .2 ; Auteur anonyme $D 36,4,16$. On constate un équilibre parfait en la matière, 103 textes visant une relation esclave/maître, 103 une reiation esclave/ tiers.
Il g'agit également d'un bon rapport dans le cas ol un individu se porte caution pour un servus alienus (976), ou inversement dens ceiui où un esclave se porte caution pour lui (97T); de même dans le cas ol une femme intercède en faveur $d$ 'un esclave qui ne lui appartient pas (978). Il est ailleurs question de la gratia du préteur enversun esclave (979).

Certains textes sur la liberté ont un relief particulier et méritent d'être signalés : il s'anit de ceux où la soume servant à payer la liberté est fournie par un tiers (980). Néanmoins, pour les raisons indiquées plus haut, il convient de ne pas les retenir dans une analyse statistique, à l'exception de deux textes où la sorme est fournie par un ami de l'esclave (981), le thème de l'amitié tranchant alors sur celui de la liberté. Ce thème apparait à d'autres égards : on voit par exemple un esclave se rendre chez des amis, noti (982). C'est par amitié pour l'esclave qu'un tiers gère les affaires dépendant de sonpécule (983). Un patron a de l'affection pour l'esclave de son affranchi (984). Alors que dans le cadre d'une relation esclave/maitre, on ne peut présumer un bon rapport en cas d'union conjugale, le premier étant par trop dépenđant du second, l'union entre un (ou une) esclave et une (ou un) ingénue est le signe d'un bon rapport, au moins lorsqu'il n'y a pas erreur sur le status de l'esclave (985).

976- Proculus D 46.3.84; Neratius D 15.3.18; Pomponius D 44.2.21. 4 ; Africain D 46.3.38.2 ; Paul D 46.1.35; Ulpien D 4.3.7.8 (Pomponius).

977- Julien D 46.1.19; Paul D 46.1.66; U1pien D 15.1.3.5 (Celsus); D 17.1 .12 .3 .

978- Pomponiug D 16.1.32.5; Pau1 D 16.1.9.

979- Ulpien D 40.5.24.17.

980- Le maître reçoit une somme pour affranchir son esclave (cf. supra n. 172) chez Julien D 12.1.19 pr ; Marcellus D 37.15.3; Scaevola D 22.2.5 Pr ; Papinien D 19.5.7; D 40.1.19; Paul D 19.5.5.2 (Julien) ; $\mathrm{D} 40.12 .38$.I (constitution de Marc-Aurè̀le) ; D 41.3 .4 .17 ; v1pien D 12.4 .1 pr ; D 12.4.3.2-3 (Proculus) -4 ; D 12.4.5.2-3-4; D 38 . 2.34. L'aide d'un tiers ressort aussi à propos d'un statuliber (Iavolenus D 40.7.39.5) et d'une suis nummis redemptio (Ulpien D 40.1.4.1).

981- Marcellus D 37.15.3; U1pien D 40.1.4.1.

982- Scaevola D 33.7.20.5.

983- Ulpien D 3.5.5.8 (Lahếon, Pomponius).

984- Iavolenus D 38.2.36.

985- Tryphoninus D 28.2.28.3; Parl D 16.3.27; D 41.7.8; $050.2 .9 \mathrm{pr}$ (Sévère) ; Ulpien D 38.16.1.1 (rescrits de Marc-Aurèle er Verus, et de Caracalla). 
On voit quelqu'un verser une somme pour qu'un esclave ne soit pas tué (986). Un particulier cache chez lui un esclave coupable du meurtre de son maître (987). Contrairoment aux juristes, il faut admettre que l'incitation à la fuite (988) n'est pas un maurais conseil pour l'esclave qui se trouve cbez un maitre trop dur, et qu'elle occasionne même, avec le fait de cacber chez soi l'esclave fugitif (989), un bon rapport entre l'esclave et le tiers. Il est vrai qu'un tel. rapport n'est pas toujours désintéressé. Ainsi, celui qui conseille à l'esclave de fuir peut avoir projeté de le faire enlever par un complice (990). D'autre part, recueillir un esclave fugitif, c'est accroître sa main-d'oeuvre, et la protection exercée par un vilicus sur cet esclave, dont fait état un sénatus-consulte rapporté par Ulpien (991), vise sans doute un tel but et non une solidarité à l'intérieur du groupe servile. Il n'y a par contre aucun risque d'ambiguité lorsqu'un particulier facilite l'évasion d'un fugitif qui devait être rendu à son maitre (992). Il en va de même lorsque l'esclave s'enfuit chez un ami du maitre pour que celui-ci obtienne son pardon (993).

Le tiers peut aussi agir par pitié. Labéon évoque un esclave ainsi débarrassé de ses chaînes (994). Ulpien cite le cas d'un individu abritant chez lui un esclave par homitas ou misericordia, ce qui en dit long sur les rapports vécus par cet esclave avec son maître (995). On voit même un sequester, à qui l'esclave avait été remis pour subir

\section{6- Ulpien D 3.5.5.10 (Pomponius)}

987- Ulpien D 29.5.3.12.

988- Callistrate D 48.15.6.2; Paul D 21.1.43.2; Ulpien D 11.3.1.4-5; D 47.2.36 pr (Pomponius) -2 (Sabinus, Pomponius); D 47.11 .5

989- Callistrate D 48.15.6.2 ; Ulpien D $11.4 .1 \mathrm{pr}-1-2$ (epistula de Marc-Aurèle et Conmode); Modestin D 48.15.5. Il est également question d'un individu qui offre un refuge $a$ un esclave, à propos de l'actio servi corrupti, chez Ulpien D 11.3.1.2. Sur le rapport existant entre le recipere de 1 'actio servi corrupti et le celare de la lex Fabia, v. H. BELLEN, Studien zur Sklavenflucht im röm. Kaiserreich, Wiesbaden (1971), p. 48 ; G. LONGO, cr sur Bellen, Iura 23 (1972), p. 177. 990- Ulpien D 47.2.36 pr (Pomponius).

991- Ulpien D 11.4.1.1. P. JAL, cr sur BeIlen, Latomus 33 (1974), p.

454 .

992- Modestin D 48.3.14.7 (Venuleius).

993- Paul D 21.1.43.1.

994- Chez U1pien D 4.3.7.7 (qui cite aussi Scaevola, cf. O.LENEL, Pal, II, p. 1229 n. 1 Quintus;).

995- Ulpien D 11.3 .5 pr. 1a quaestio, le délier pour des raisnns semblables ( 996$)$.

Cela dit, on ne peut voir un bon rapport dans le cas où le tiers rend un service à l'esclave moyennant une contrepsrtie : ainsi, si le fugitif a remis de l'argent à un particutier pour qu'il le cache (997) ou si le tiers accepte une somue pour ne pas dóvoiler le vol commis par l'esclave (998). De même, le commodataire ou le créancier du gage ne font pas oeurre de cbarité en assurant des soins à l'esclave malade, dans la mesure ou lis peuvent obtenir le remboursement des frais occasionnés par cet,te maladie (999).

Farmi ces rapports esclave/maitre et esclave/tiers ont une coloration particulière ceux qui mettent en cause un esclave et un affranchi, ce dernier pouvant aussi bien intervenir come tiers que comme maître. On relève de telles associations en matière successorale (1000), mais un texte d'Ulpien nous livre une espèce plus intéressante. Il y est question d'un maître assassiné par ses esclaves. Ce maître est un affranchi (1001)...

\section{II - Mauvais rapports}

Comme précédemment, il convient d'examiner successivement les relations esclave/maitre et esclave/tiers.

\section{A/ - Les mauvais rapports esclave/maître}

De teḷ rapports apparaissent fréquemment en matière de liberté. Ils se traduisent par le refus du maître d'affranchir son esclave, celuici prenent des formes diverses. Il peut s'agir de la vente ou du legs d'un esclave à condition qu'il ne sera pas affranchi (1002). Un tel

996- Ulpien D 16.3.7 pr.

997- Ulpien D 12.5.4.4.

998 Julien D 12.5 .5 (Proculus).

999- Paul D 13.6.22; Pomponius D 13.7.8 pr.

1000- L'affranchi institue héritier l'esclave d'autrui, du patron en 1 'occurrence, chez Marcellus D 29.4.5 ; Tryphoninus D 38.2.50 pr-1-2-3; Paul D 38.2.45. Il lui laisse un legs (Paul D 31.83 ; Ulpien D 38.2.8.2), lui fait une donation (Ulpien $D$ 38.5.1.22). L'affranchi intervient en tant que waittre chez Scaevola $D$ 32.38.1 ou, dans son testament, il laisse la liberté à son ancilla et lui lègue la partie d'un fonds.

1001- Ulpien D 38.2.37.1 (Julien).

1002- Cf. supra n. 915. 
refus g'exprime parfois brusq'ement : c'est ainsi qu'on voit un mâtre qui désirait vendre son esclave pour qu'il soit affranchi changer d'avis (1003). En matière testamentaire, la liberté peut être anéantie far une ademptio libertatis, qu'elle soit expresse ou tacite (1004). Marcellus évoque un testrateur rayant, le nom d'un esclave du testament où il lui avait laissé la liberté (1005). Il cite ailleursle cas d'un maître qui, dans le but de faire obstacle à un éventuel affranchissement de l'ésclave, interdit dans son testament qu'il soit vendu (1006). La liberté peit aussi être laissée sous une condition irréalisable, du type : "j'affranchis tel esclave s'il donne mille fois cette sonme à mon héritier" ou encore "lorsqu'il mourra" (1007). Et même en présence d'une condition réalisable, l'esclave ne parvient pas toujours facilement à la liberté.

II n'est pas rare en effet, si l'on se fie ł̇ la diversité des périodes auxquelles le problème apparât, que l'hóritier empêche sciemment l'esclave de remplir cette condition (1008) : ainsi cet héritier qui fait prencre à une femme, affranchie sous 1 a condition d'avoir trois enfants, une potion qui la rend stérile (1009) ; cet autre qui, alors qu'un ezclave doit se rendre en Hispanie pour obtenir sa liberté, le retient à Rowe (1010); cet autre encoro qui, four faire obstacie à la liberté, ne rembourse pas ce qu'il doit à l'esclave ou n'rctionne pas

1003- Papinien D 18.7.8.

1004- Sur ce problème, W.W. BUCKl,AND, Roman law, cit., p. $466 \mathrm{~s}$. L'ademptio tacite consiste dans le fait d'aliéner l'esclave ou de le iégler (cf. Paul D 40.4.10.I. A. BERGER, Encycl. Diction., cit., F. 349). L' $x d-$ emptio de la liberté implique l'ademptio du legs (cf. Venuleius 034.

4.32. I) fait à 1 'esclave. Il faut noter que si un maître vend un esclave et lui ôte la liberté ensuite, 1 'ademptio libertatis ne produit pas d'effer car alieno inutiliter adimatur (cf. Paul D 34.4.26 pr) ; cependant si, fet car alieno inutiziter adimatur (cf. Paul D $34.4 .26 \mathrm{pr}$ ) ; cependant $\mathrm{si}$,
dans la même hypothèse, le testateur avait fait un legs à 1 'esclave, cedui-ci serait annulé.

1005- Marcellus D $28.4 .3 \mathrm{pr}-1 \cdot$ (rescrit de Marc-Aurèle).

1006- Marcellus D 40.5.10 pr.

1007- Paul D 40.7.4.1 (Julien); Julien D 40.4.17 pr ; Pomponius D 40.

$4.61 \mathrm{pr}$ (Julien).

1008- Le problème est évoquế par Celsus D 40.7.23.1 ; Scaevola D 40.7.40.7; Papinien D 40.7.34.1 (reserit de Caracalla); D 40.7.35 ; Paul D 40.7 . 4.4 ; D 40.7.20 pr-1 (Julien) -5 ; D 40.7 .38 ; Ulpien D 40.7.3.1-2 (Servius, Labéon, Cassius) $-3-4-7-8-1 n-13$ (Pomponius)-16 (Julien)-17; Movius, Labéon, Cassi,

1000- Ulpien D 40.7.3.15 (Julien).

I010- Papinien D 40.7.34.1. le débiteur de celui-ci (1011); cet autre enfin qui vend un statuliber sans son pécule pour l'empêcher de payer le prix de la liberté (1012). Un héritier vend même un esclave sans l'informer de la liberté fidéicommissaire qui lui a été laissée (1013) ou aggrave sa condition en déclarant, par exemple, qu'il doit payer dix au lieu de cinq (1014). Il peut aussi différer l'affranchissement d'une femme pour que les enfants de celle-ci lui appartiennent (1015). Il arrive enfin qu'il se cache porr ne pas délivrer à l'esclave la liherté fiđéicommissnire qui lui est due $(1016)$.

Cependant conformément au critère d'interprétation restrictif défini précédemment (1017), il convient de ne pas tenir compte de ces textes dans un calcul statistique. Quels sont donc les rapports dont l'intégration soit ici possible?

Même exercée sous le couvert du drcit, la rópression domestique vise nécessairement un mauvais rapport, ontre le maitre et l'esclave. Cela est narticulièrement clair lorsqu'elle se solde par la mise ì mort. de l'esclave. Ce thòme ayant ét,é traité (1018), il suffit, je ranpeler que le seul ressort do cette forme he rópressicn reut ôtre le caprice ou la cruauté. On a ainsi ru tei maitre priver de nourriture ses esclaves, leur faire endurer des violences insupportables (1019)."

10I1- v1pien D 40.7.3.2 (Servius, Labéon, Cassius).

1012- vipien 0 40.7.3.7.

1013- Taul D in.1.43.

1014- Africain D 21.2.46.3.

1015- Marcien D 40.5.53.1.

1016- Julien D 40.5.47.2 ; Papinien D 40.5.22.1.

1017- Cf. supra texte sur note 965.

1018- Cf. supra étude sur répression (paragr. I Section 2 , chapitre II, titre II, deuxième partie). On retiendra Julien 0 46.3.33.1 ; Pomponius D 21.1.48.3-4; D 30.48.1; D 36.1.72.1; D 45.1.23; Marce11115 D 45.1. 96 ; Papinien D 18.7.7 ; D 46.3.95.1; Paul D 5.3.36.2 (Pompronius); D 9. 2.12 ; D 9.2.14; D 10.3 .3 .2 ; D 40.5 ; Pa ; D 45.1 .91 fr $^{-2-5}$ (Pomponius) 6 D 17.40 .4 ; D 48.19.38.4 ; Vlpien 0 1.6.2 (Madrien, Antonin le pieux) D 1.12 .1 .8 ; D 4.3 .7 .3 (Labéon) $-4-5-7$ (Labéon, Scaevola) ; D 6.1 .13 ;

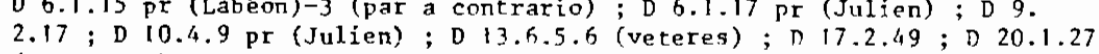

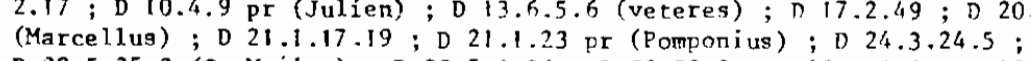
D 28.5.35.3 (Q. Mucius) ; D 29.5.1.26;D 30.53.3; D 40.1.4.9; D 40.5.24. 8 (Celsus) ; D 47.10.3.4; D 47.10.15.36-37-44-45; D 50.26.216;: Marcien D 18.7.2; D 40.1.5 pr ; Modestin D 48.8.11.1-2.

1019- Ulpien D $1,6.2$. 
L'action du dominant peut être plus pernicieuse encore et reposer uniquement sur la volonté de nuire à un tiers. Avant de vendre l'esclave, le maître lui fait absorber du poison (1020) ou lui fait exécuter des travalıx dangereux (1021). On relève ailleurs un possesseur qui, alors que l'esclave est revendiqué, lui fait sciemment emprunter des passages peu sûrs (1022). On voit encore un héritier inciter l'esclave légué à conmettre un délit pour qu'il soit tué par un tiers ou condamné à mort (1023). Quelquefois, le texte indique simplement que l'esclave est mort par la culpa de celui en la puissance duquel il se trouve (1024).

Un testateur interdit à son héritier d'aliéner un esclave pour qu'il ne puisse parvenir à un maître plus doux que lui (1025). Des esclaves sont prostituées par leur mâtre, pratique qui, si elle est vraisemblablement d'un bon rapport financier, óvoque par contre un piètre rapport humain (1026). Des esclaves sont abandonnés (1027). D'autres ne sont pas défendus en justice par leur maŝtre (1028), que ce soit par pure négligence ou bien parce qu'il estime cette attitude moins coûteuse.

De tels faits impliquent forcément une méfiance de l'esclave visà vis du maître. Il est intéressant à ce sujet de rappeler que l'esclave "interrogé" en matière d'adultère est soustrait à la puissance dominicale pour qu'il ne craigne pas de dire la vérité (1029). Mais deux autres cas 1020- Ulpien D 4.3.7.3 (Labéon).

1021- Paul D 19.1.54 pr (Labéon).

1022- Gaius D 6.1.36.1.

1023- Ulpien D 30.53.8. L'esclave est capturé par mauvaise foi (dolus) de l'héritier (cf. D 30.53.9) L'esclave légué est livré par l'héritier dans un état de détérioration physique (cf. Pomponius $D$ 30.108.11).

1024- Pomponius D 21.1.48 pr; D 26.7. 61 (blessures) (par a contrario) Paul D 6.1.27.2 (par a contrario); Ulpien D 19.1.13.22 (par a contrario); D $21.1 .31 .11-12-13 ; \mathrm{D} 30.114 .19$ (par a contrario). Il est aussi question de dolus (Ulpien D 21.2.21 pr) et de simple negligentia (Alfenus chez auteur anonyme $D 9.2 .52 \mathrm{pr}$ ).

1025- Ulpien D 40.5.24.8 (Celsus).

1026- Pomponius D 21.2.34 pr ; Papinien D 18.7.6 pr ; Paul D 18.1.56 ; D 18.7.9; D 40.8.7 (rescrit de Sévère et Caracalla); Ulpien D I.12.1. 8 ; D 2.4.10.1; D 3.2.4.2 ; Marcien D 40.8.6; Modestin D 37.14.7 pr (décret de vespasien).

1027- Iavolenus D 45.3.36; Pạul D 41.7.8; Modestin D 40.8.2 (êdit de Clauce).

1028- Pomponius D 9.4.33 ; D 9.4.43; Gaius D 9.4.29; Marcellus D 49.1. 15 ; Papinien D 48.3.2 pr; Ulpien D 2.1.7.3; D 43.16.1.19; Marcien D 40. 9.9.1; 048.1 .9 .

1029- Ulpien D 48.5.28.13-14. sont encore plus éloquents : celui de l'esclave qui s'sccuse faussement d'un délit pour ne pas retourner chez son maître $(103 n)$ at colui du fugitif qui préfère combattre dans l'arène plutôt que d'être restitué à son propriétaire (1031).

Les réactions de l'esclave peuvent se borner à un simple refus, refus d'obéissance (1032), refus d'accomplir la volonté du testateur (1033), refus pour l'esclave affranchi d'aroir un tel come patron (1034). Mais l'esclave peut aussi proférer une offensa (1035), mópriser son maître (1036), vouloir le dénoncer (1037). On est proche à ce stade de la rupture.

Si $l^{\prime}$ on en croit les text,es, cette rupture est fráquente, et se présente sous les formes de la fuite (1038), de l'assassinat du

1030- Ulpien D 48.18.1.27 (epistula de Marc-Aurèle et Verus).

1031- Tryphoninus D 11.4 .5 (rescrit d'Antonin le pieux).

1032- Paul D 11.3.2; Ulpien D 29.4.1.3 (il refuse d'accepter une succession alors que son maitre le lui ordonne).

1033- Paul D 40.4 .52 (l'esclave refuse de demeurer chez l'hêritier comme le lui intimait le testament de son maitre).

1034- Paul D 19.1 .43 (Ulpien); D 40.5 .25 (Valens).

1035- Papinien D 40.4.51.1; Ulpien D 28.5.35.3 (Q. Mucius).

1036- Gaius D 11.3.15.

1037- Callistrate D 49.14.2.6 (constitution de Sévère) ; Paul D 48.18. 18.5; Ulpien $D$ 4.2.7.1. Un esclave accusê $d$ 'un délit avoue quelque chose a l'encontre de son maître (cf. Ulpien D 48.18.1.19 rapportant sur ce point un rescrit de Trajan).

1038- Alfenus (chez auteur anonyme) D 40.7.14.1; Julien D 30.84.10; D 46.3.34.5; Pomponius D 1.14.3; D 6.2.15; D 17.2.60.1 (Labéon);

D 18.1 .13 ; D 21.1.48.4; D 21.1.64.2 (Labëon); D 21.2.16.2 (Proculus) ; D 24.1 .31 .5 ; D 30.56 ; D 41.1 .54 .4 ; D 46.3.19 (Labéon); Africain D 13 $6.21 .1 ; \mathrm{D} 30.108 \mathrm{pr} ; \mathrm{D} 47.2 .61$; Gaius D 6.1.36.1; D 13.6.18 $\mathrm{pr}^{-2}$; D 18.1.35.3; D 21.1.22; D 29.5.25 pr-1 ; D 39.4.13.2 ; D 40.12.11 ; D 40.12.25.2; D 41.2.15; Papinien D 21.1 .54 ; D 21.1.55; Tryphoninus D 11.4 .5 (rescrit d'Antonin le pieux) ; D 50.16.225; Menander D 49. 16.4.14-15; Callistrate D 11.4 .2 ; D 48.15.6.1 (rescrit d'Hadrien)-2 ; Paul D 6.1.21 (Julien, Pomponius) ; D 9.4.24; D 10.3.19.3; D 11.4 .4 ; D 14.5.8; D 15.1.48 pr ; D 17.1.22.9 (Mela); D 18.7.9; D 19.4.2 (Aristo); D 21.1.43.1-2-3; D 21.1.44.2; D 21.1.58 pr-2 ; D 22.3.4; D 22.3.7; D 24.3 .25 .3 ; D $31.8 \mathrm{pr} ; \mathrm{D} 33.8 .9 .2$; D 40.7.4.8; D 40.12. $38.3 ;$ D 41.2 .1 .14 (Nerva filius, Cassius, Julien); D $41.3 .15 .1 ; \mathrm{D} 47$. 10.22; D $49.15 .19 .5-6$; Ulpien D 1.6 .2 (rescrit d'Antonin le pieux) ; D $1.12 .1 .1 ; D 1.15 .5 ; D 2.14 .50 ; D 4.2 .14 .5$ (Vivianus) -11 ; D 4.3 .7 . 7 (Labếon, Scaevola); D 4.4.11.5 (Julien, Marcellus); D 6.1.22 ; D 7 . 1.12 .3 (Julien, Pomponius); $09.2 .13 \mathrm{pr}$; D 9.2.27.3 (Julien, Marcellus) ; D 9.4.21.3 ; D 10.4.5.6; D 11.3.1.4-5; D $11.4 .1 \mathrm{pr}-1-2$ (epistuza de Marc-Aurèle et Commode)-3-4-5 (Labêon)-6-7-8; D 11.4.3 (rescrit 
maitre $(1059)$ on du refus ie le secourir aiors qu'il est en danger de mort (1040), du suicixe (10i4), du sabotage (10i2), autant de formes sur lesquelles on revienżra plus lnin. Il n'est pas étonnant que de tels actes suscitent la peur des maîtres. Cette peur ressort de manière

d'Antonin le pieux) ; D 12.1.11.2 ; D 12.4.5.3; D 12.5.4.4; D 12.6.5. 13 (Cartilius); D 17.1.8.10; D 19.1.11.7 (Neratius); D 19.1.13.t-2; D 19.5.15; D 19.5.18; D 21.1.1.1 ; D 21.1.4.3 (Pomponius) ; D 21.1.

17 pr (Ofilius) -1 (Caelius Sabinus) -2 (Cassius) -3 (Vivianus) -4 (Proculus) -5 (Vivianus) -6 (Caelius Sabinus)-7 (idem)-8 (idem) -9 (idem) -10 (idem) $-11-12$ (Labéon, Caelius Sabinus)-13 (Caelius Sabinus)-14 (Labéon)-15 (Labéon, Caelius Sabinus) 16 (Caelius Sabinus); D 21.1.19.1; D 21.1.21.3

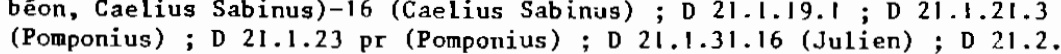
21.3 (Julien); D 21.2.32 pr-1 (Julien) ; D 29.5.1.31 ; D 29.5.3.17; D $30.39 \mathrm{pr}$ (Julien, Africain) ; D 30.47.2-3; D 39.4.12.2 : D 40.12 .10 (Alfenus); D $41.2 .13 \mathrm{pr}$; D 44.3.8; D 44.4.4.5 (Labéon); D 47.2.17.3 (Julien, Pomponius) ; D 47.2.36 pr (Pomponius)-2 (Sabinus, Pomponius)-3 (Sabinus) ; D 47.2.46 pr (par extension : res/servus) ; D 47.2.52.12. D $47.8 .2 .18-25 ; 047.14 .5 ; D 48.15 .2 \mathrm{pr}-1-2-3 ; \mathrm{D} 50.17 .23$; Marcien D 39.4.16.4 (rescrit d'Antonin le pieux); D 47.2.63; Modestin D 32.82 . D 48.3.14.7 (Venuleius); D 48.15.5; Hermogénien D 4i.2.50.1.

1039- Julien D 17.1.30 ; Gaius D 29.5.25 pr-1 ; Maecianus D 29.5.14; Venuleius D 29.5.13 ; Marcellus D 29.5.16 ; Papinien D 1.21.1 pr ; D 29.5 4'; D 29.5.21 pr ; D 35.2.11.4 ; D 48.1.14 ; Callistrate D 48.19.28.11; Paul D 29.5.22; D 35.2.39; Ulpien D 29.5.1.5 (rescrit d'Antonin le pieux) $-21-30$; D 29.5.3.1-14-18; D 38.2.37.1; D 40.12.7.4. Il faut également mentionner ce passage d'vipien selon lequel la vie des maitres est mise en péril $a b$ domesticis (D 29.5.1 pr).

1040- Ulpien D 29.5.1.18-22-26-27 (Sextus)-28 (rescrit d'Hadrien)-29 (idem)-31-34-36-37; D 29.5.3.2-4-6-12 ; Modestin D 29.5.19. Ce dernier texte nous apprend que $l^{\prime}$ esclave qui, pouvant porter secours à son maître ne l'aura pas fait, sera condamné au sicpplicium. Il convient donc d'intégrer les textes mentionnant le supplicium aux mauvais rapports esclave/ tégrer les textes mentionnant le suppticium aux mavais rapports esclave/
maître, ceux-ci visant soit l'assassinat du maître par l'esclave, soit maître, ceux-ci visant soit l'assassinat du maître par l'esclave, soi le refus de ce dernier de lui porter secours (cf. Ulpien D 29.5.1.12 (Scaevola)-13 (Scaevola)-14-33; D 29.5.3.16-17; D 29.5.5.2), les autres esclaves étant simplement, si 1'on peut dire, soumis à la quaestio. On reviendra sur ce point à propos du s.c. silanien. On peut déjà dire néanmoins que la distinction entre quasstio et supplicivem apparait chez Utpien (D 29.5.1.33: D 29.5 .3 .18 ), et, dans un aurre contexte que celui dus.c. silanien chez Ulpien (D 48.18.7).

1041- Paul D 21.1.43.4; D 33.8.9.2; D 14.2.2.5; Ulpien D 15.1.9.7; D 21.1.1.1 ; D 21.1.17.4 (Proculus)-6 (Caelius Sabinus) ; D 21.1.23.3; D 47.2.36 pr (Pomponius)

1042- Paul D 19.2.30.4; Ulpien D 9.2.27.11 (Proculus); D 19.2.11.4 (Labéon), ces textes visant un incendie allumé par l'esclave. évidente dans les espèces suivantes : Pap̣inien signale in esclave vendu à condition qu'il ne restera pas dars un endroit déterminé, cela pour la sécurité du vendeur (i043) ; Marcien évoque d'autre part un mâ̂tre qui, par crainte de son esclave, lui écrit qu'il l'affranchit. (1044).

Signalons enfin qu'il n'est pas possible, à l'exception du damnum d'assimiler à ces réactions los délits, pour ainsi dire habituels, commis par l'esclave envers son maitre (1045). L'exemple du vol est explicite (1046). Le vol domestique est en effet quasiment institutionnel si I'on en croit Ulpien (1047). Il n'implique pas pour autant de mauvais rapports entre maître et esclave.

Avant de passer à l'analyse statistique, il nous reste encore à examiner les rapports esclave/tiers lorsqu'ils sont dégradés.

\section{B/ - Les mauvais rapports esclave/tiers}

Un tel rapport apparait dans des espèces diverses. On a évoqué précédemment un esclave remettant une somme $\grave{z}$ un tiers pour que celui-ci ne dêvoile pas un vol qu'il avait commis ; or,il le dénonce (1048). In esclave s'enfuit de chez un précepteur où il était en apprentissage, car il 1'exploitait immoderate (1049). In tiers retient, à l'insu du maître, un esclave enchaîné (1050). On apprend qu'un esrlave est mort

1043- Papinien chez Ulpien D 18.7.1.

1044- Marcien D 40.9.9 pr.

1045- Darmum causé par 1'esclave au maitre (cf. Pomponius D 15.1.4.3. Pank D 11.3 .14 .7$)$; le dommum pent attssi être causé par le maître à l'esclave (cf. Ulpien D $15.1 .9 \mathrm{pr}$ ).

1046- Cas de vol fait par l'esclave au maitre par exemple che $z$ Neratius D 40.7.17; Jalien D 41.3.33.5; Pomponius D 15.1.4.3; D 15.1.4.2-4; Africain D 47.2.62 pr ; Gaius D 41.2 .15 ; Screvola D 40.5.41.11; Tryphoninus D 50.16.225; Paul D 12.6.36 ; D 21.1.58 $\mathrm{pr}^{-1}$; D 41.3.4.8-9 (Pomponius); U1pien D 11.3.1I pr (Neratius)-2; D 15.1.9.6; D 21.1.23.8 (Julien); D 21.1 .31 .1 ; D 46.3.18; D 47.2.17 pr-I-2 (Sabinus); D 47.2. 43.12 ; Marcien D 21.1.52 ; D 48.19.11.1. Jlpien fait même état d'un vol fait par le maître à l'esclave (cf. D $15.1 .9 \mathrm{pr}$ ) : il s'agit bien entendu d'une hypothèse juridique d'école. Du point de vue des rapports, il faut assimiler à ce type de délit la fraude cormise par l'esclave dans les comptes de son maitre (cf. par excmple Ulpien 09.2 .23 .4 ).

1047- Ulpien D 47.5.1.5.

1048- Julien D 12.5 .5 (Proculus). On vnit aussi le fils d'un patron porter une accusation calomieuse contre un esclave irf. Paul D 39.2.51 Laterin? ?

1049- Jipien D 21.1.1:.3: vierianis 
par la faute d'u prociurateur (1051), d'un tuteur ou d'un curateur (105?).

Far ailleurs, il raut considérer ln répression privée, au même titre que la répression donestique, come le signe d'un mauvais rapport (1053), d'autant que la cruauté n'y est pas non plus étrangòre (1054). Mais, le plus souvent, c'est de véritables agressions dont l'esclave est. l'objet (1055). Si parfois ces agressions ne se soldent que par des blessures (1056), elles ahoutissent fréquemront, on l'a vu, à la mort de l'esclave (1057). Il arrive que le texte indique seulerrent qu'il a ét.é victime d'une détérioration physique (1058), rais on possède des renseionements plus prócis. Ainsi, on voit un tiers faire soumettre un esclave à

\section{0- Callistrate D 48.15.6.2.}

1051- Pomponius D 21.1.48 pr (par a contrario); Ulpien D 21.1.31.11. On notera, grâce à ces textes, que l'esclave peut aussi mourir par la faute de la fanilia.

1052- Ulpien D 21.1.31.14 (Pedius).

1053- Gaius D 9.2.4 pr-1; Paul D 7.1.66; D 9.2.18; D 9.2.30 pr ; D 19 2.43 ; Ulpien D 7.1.15.3 (Labéon) ; D 7.1.17.1 (Cassius, Aristo) ; D 7.1 . 23.1 (Sabinus, Cassius) ; D 9.2.5 pr-3 (Julien); D 12.4.15 (Labéon, Proculus) : D 13.7.24.3; D 24.3.24.5; D 30.53.8; D 47.10.15.37-38.

1054- CE. supra n. 785, 786 .

1055- A l'exception des dêlits sexuels, dont le caractère est spécifique, l'esclave est l'objet d'une agression de la part d'un tiers chez Alfenus (chez auteur anonyme) $\mathrm{D} 9.2 .52 \mathrm{pr}^{-1}$; Julien $\mathrm{D} 9.2 .47$; $\mathrm{D} 9.2 .51 \mathrm{pr}^{-1}$ (veteres)-2 ; D 46.3.33.1 ; Pomponius D 26.7.61; D 44.7.56 ; Gaius D 6.1. 36.1 ; D 9.2.2 pr ; D 9.2.32.1 ; Marcellus D 9.2.34; D 9.2.36 pr-1 (Sabinus) ; Papinien D 46.3.95.1; Paul D 4.3.18.5; D 9.2.22 pr-1; D 9.2.24; Dus) ; Papinien D 46.3.95.1 ; Paul D 4.3. 9.2 .26 ; D 9.2.30.1-4; D $9.2 .33 \mathrm{pr}$ (Tedius); $\mathrm{D} 9.2 .45 \mathrm{pr}^{-1}$ (veteres) $\mathrm{D} 9.2 .26 ; \mathrm{D} 9.2 .30 .1-4 ; \mathrm{D} 9.2 .33 \mathrm{pr}$ (Pedius) $\mathrm{D} 9.2 .45 \mathrm{pr}^{-1}$ (veter
$-2 ; \mathrm{D} 9.2 .55 ; \mathrm{D} 11.1 .20 \mathrm{pr} ; \mathrm{D} 11.3 .4 ; \mathrm{D} 17.1 .22 .6 ; \mathrm{D} 21.1 .43 .5 ;$ D $42.2 .4 ; D 44.7 .34 \mathrm{pr}$; D 45.1 .88 ; D 47.10 .16 (Pedius) ; D 47.10.29; D 48.7.4.1 (Labéon); Ulpien D 6.1.17.1 (Julien); D 7.1.17.3; D 9.2.3; D 9.2.7.1-3 (Proculus) -4-5 (Labéon)-6 (Celsus)-7 (Celsus); D 9.2.9.2 (Neratius)-3 (Ofilius)-4 ; D 9.2.1I.1-2 (Julien)-3 (Celsus, Marcellus); D 9.2.13.2 (Celsus)-3 (Julien) ; D 9.2.15 pr-1 (Julien) ; D 9.2.19; D 9.2.21.1 (Ce1sus, Julien)-2; D 9.2.23 pr (Neratius)-1-2-3 (Julien) -4 (Labêon)-5-6-7-8-9-10-11 (Julien) ; D 9.2.25 pr-1 ; D 9.2.27.5-6-17; D 9.2.35; D 9.2.46; D 12.4.5.4;D 13.3.3; D 19.5.14.1; D 47.1.2.1-2 -3 (Pomponius) $4-6 ; \mathrm{D} 47.8 .4 .13-14 ; \mathrm{D} 47.10 .13 \mathrm{pr} ; \mathrm{D} 47.10 .1 .3-6-7$ (Labếon); D 47.10.7.1; D 47.10.15.34-35-43-44-45 (Mela)-46 (Labéon) 47 $-48-49$; D 47.10.17 pr ; D 47.10.30; Marcien D 22.1.32.5; D 48.8.1.2. 1056- CE. supran. 675 .

1057- Cf. supra n. 357.

1058- U1pien D 11.3.9.3 (Neratius); D 42.5.9.5 (par manque de cura du créancier). La détérioration intellectuelle, par contre, ne vise pas nécessairement un mauvais rapport entre le tiers et 1 'esclave : c'est par exemple le cas de l'incitation à la fuite. On ne peut donc intégrer à l'analyse les textes qui en traitent, de même que ceux qui indiquent seulement que 'l'esclave a fait l'objet d'une dêtérioration, sans autre précision (cf. par ex. Ulpien D $21.1 .25 \mathrm{pr}-1$ ), ceux-ci pouvant théoriquement concerner la quaestio sans 1 'ordre du maitre (1050) ou, plus simplement, sans raison (1060) ! L'esclave est souvent hattu, at ce ne sont pas les ndjectifs qui ront téfaut : verberntus (1061), caesus (1062), pulsatis (1063), percussus (1064). De raçon plus subtile, on peut persuater 1'esclave de monter sur un toit ou de descendro dans un puits pour qu'il se hlesse (1065). L'esclave reste-t-il insensible à ces traitement,s ?

Bien que peu de textes fassent allusion à une ráation servile, on voit néanmoins plusieurs esclaves battre un individu ou 1 'insulter (1066), un esclave injurier son usufruitjer (1067). Sur un plan difrórent, on le voit tuer par ordre du majt,re (1068) ou comnettre une iniuria pour dófendre celui-ci (1069).

Il est désormais possible de passer à l'analyse quantitative de ces textes, en confrontant les textes traitant des bons ou des mauvais rapports au nombre total de textes concernant les rapports esclave/ maître ou esclave/tiers pour chaque période.

une détérioration intellectuelle. Il est tout de même intéressant de noter que la détérioration de l'esclave peut être une forme de divertissement pour le tiers (cf. Ulpien D $1 / .3 .3 \mathrm{pr}$ ).

1059- Ulpien D 47.10.15:34.

1060- Paul D 21.1.43.5.

106I- Paul D 44.7.34 pr; U1pien D 47.8.4.14; D 47.10.15.34-46-47.

1062- Pau1 D 21.1.43.5; U1pien D 47.10.15.48-49.

1063- Ulpien D 47.8.4.13; D 47.10.15.48.

1064- U1pien D $47.10 \cdot 15.44$.

1065- UIpien D 11.3.3.1.

1066- Gaius D 47.10.34. Iniuria commise par plusieurs esclaves chez Pau1 D 2.1.9 (Pomponius, Octavenus).

1067- Ulpien D 47.10.17.9.

1068- Ulpien D $47.10 \cdot 17.7$.

1069- U1pien D 47.10,17.8. On remarque aussi un esclave auteur d'iniuria chez Venuleius $D$ 48.2.12.4; Ulpien D 2.9 .5 ; D 11.3 .1 .5 : D 47.10 9.3 ; D 47.10.17.3-4-5-6; Hermogénien D 47.10.45. 
ichs fapports

Mauvais rapports

- escieveitantre (10

- analyse des paragraphes

$\begin{array}{lc}\text { I } & - \\ \text { II } & - \\ \text { III } & 3 / 43 \\ \text { IV } & 3 / 5 \\ \text { V } & 26 / 262\end{array}$

TOTAL $32 / 310$

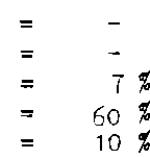

$10 \%$

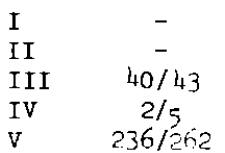

$93 \%$

$90 \%$

TOTAL $278 / 310=90 \%$

- analyse des citations

$\begin{array}{ll}\text { I } & 1 / 7 \\ \text { II } & 1 / 34 \\ \text { III } & 3 / 33 \\ \text { IV } & 2 / 8 \\ \text { V } & 0 / 2\end{array}$

$=14 \%$
$=\quad 3 \%$
$=\quad 9 \%$
$=\quad 25 \%$
$=\quad-$

TOTAL $7 / 84$

$\begin{array}{lc}\text { I } & 6 / 7 \\ \text { II } & 33 / 34 \\ \text { III } & 30 / 33 \\ \text { IV } & 6 / 8 \\ \text { V } & 2 / 2\end{array}$

TOTAL $\quad 77 / 84$
- analyse des citations

$\begin{array}{lllllll}\text { I } & 0 / 5 & & - & \text { I } & 5 / 5 & =100 \% \\ \text { II } & 3 / 19 & = & 16 \% & \text { II } & 16 / 19 & =84 \% \\ \text { III } & 6 / 30 & = & 20 \% & \text { III } & 24 / 30 & =80 \% \\ \text { IV } & 2 / 3 & =67 \% & \text { IV } & 1 / 3 & =33 \% \\ \text { V } & - & & - & & & \end{array}$

D'un point de vue général, on peut tout d'abord remarquer que les rapports sociaux dans lesquels on relève la présence d'un esclave sont mavais dans leur écrasante majorité. Ce n'est pas surprenant : le Digeste est avant tout une source de la "réalité clinique" et les bons rapports ne soulèvent pas de difficultés juridiques. Il faut donc se contenter d'observer les variations des pourcentages concernant les mauvais rapports. On peut ensuite noter que les juristes se próoccupent davantage du rapport esclave/mait,re que du repport, esclave/tiers. Cette suprématie s'explique par une intervention étatique croissante dans les. relations esclave/maître, l'Etat, limitant l'arbitraire dominical, finissant par prendre lui-même en cbarge l'exercice de la répression domestique.

Du point de vue chronologique, il faut avant tout, émettre une réserve. Les pourcentages réalisés par les périodes I et IV, reposant sur un nombre trop faible de textes, manquent de pertinence. Cela dit, l'analyse des citations indique une poussée des mauvais rapports esclave/ maître dès le ler siècle (période II). Mais il faut, attendre 1 'apogée de l'Empire (période III) pour que ces rapports attoipnent, leur fópradation maximale, comme le montre l'analyse des paragraphes. Cela détruit définitivement toute explication de la législation, favorable alux esclaves, des Antonins par un fondement avant tnut humanitaire. Le système souffre, il faut à tout prix le soulager. C'est la voie dans laquelle se dirigeront les juristes de la seconde moitié du zème siècle, aussi bien par la reconnaissance croissante d'une certaine capacité servile que par le développement important du favor libertatis, ces solutions étant symptomatiques de la crise du système esclavagiste dans la mesure où elles altèrent les rapports sociaux traditionnellement fontés sur lui. Il est intéressant enfin de relever que le discours des juristes du 3ème siècle, loin de donner une meilleure image des rapports esclave/ maître, montre qu'un relatif adoucissement, de l'exploitation n'est pas la solution au problème. Sous les Sévères, l'analyse des paragraphes indique en effet une persistance des mauvais rapport,s esclave/maîtro.
1070- Les textes servant de fondement à 1a rubrique "bons rapports pour heres "sont tirés des notes 953 à 964 incluse et de la note 965 rapports esclave/maitre" des notes 1018 à 1044 incluse et de la note 995 . Les textes apparaissant plusieurs fois n'ont été comptabilisés qu'une seule fois (cf. Pomponius D 21.1.48.4 : Gaius D 6.1.36.1 ; Paul D 18.7.9 ; D 33.8.9.2; Ulpien D 1.6.2; D 1.12.1.8; D 4.3.7.3-7; D 21.1.1.1; D $21.1 .17 .4-6$; D $21.1 .23 \mathrm{pr}$; D 28.5 .35 .3 ; D 29.5.1.26-31;D 29.5.3.17; $40.5 .24 .8 ; D 47.2 .36 \mathrm{pr}$ )

1071 - Les textes servant de fondement a la rubrique "bons rapports esclave/tiers" sont tirés des notes 973, 97f, 977, 978, 979, 981, 982. $983,984.985,986,987,988,989,992,993,994,995,996$; ceux servant de fondement à la rubrique "mauvais rapports esclave/tiers" des notes 1048, 1049, 1050, 1051, 1052, 1053, 1055, 1058, 1055, 1066, 1067, 1068,

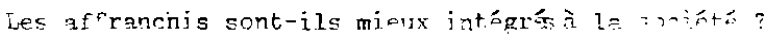


Paragraphe 2 : Laffranchi dans les rapports sociaux

En ce qui concerne la relation affranchiftiers, on peut tout d' abord relever la présence de maurais rapports. Ainsi, on voit un affranchi être enlevé (1072), 1'affranchi d'un praeses essuyer une injure (1073), un tuteur intenter au nom du pupille une accusation capitale contre l'affranchi paternel (1074), un autre empeccher l'affranchi de réaliser la condition sous laquelle lui a été laissé un fidéicommis (1075).

Contrairement à la relation affranchi/patron où le consentement de 1'affranchie manque sérieusement d'autonomie (1076), l'union conjugale entre affranchi (ou affranchie) et ingénue (ou ingénu) est le signe évident d'un bon rapport (1077). On a sussi affaire à un rapport de ce type lorsqu'un tiers se porte caution pour un affranchi (1078) ou, inversement, lorsqu'un affranchi se porte caution pour un tiers (1079); de même, quand il est adrogé par lui (1080). L'affranchi peut recevoir un legs de la part d'un tiers (1081). Il peut pareillement en laisser un à celui-ci, lui faire une donation (1082) ou encore l'instituer héritier (1083). Un texte de Julien a un relief particulier, le juriste y

\section{2- Callistrate D 48.15.6.2.}

1073- Macer D 1.18.16.

1074- Tryphoninus D 34.9.22.

1075- Modestin D 31.34.4.

1076- Cf. Supra n. $557 \mathrm{~s}$.

1077- A l'exception des textes concernant 1'interdiction d'unions entre affranchis (ou affranchies) et personnes appartenant à l'aristocratie sénatoriale, on relève, en tenant compte des textes a portée négative : Proculus D 23.3.67; Celsus D 23.2.23; Marcellus D 23.2.41.1; Papinien D 23.2.62.1; Callistrate D 23.2.64 pr; Paul D 23.2.37; D 23.2.66.1; Ulpien D 24.2.11 pr (Julien) ; D 25.7.1 pr ; D 38.16.3.5; D 47.10.11. 7 (Marcellus) -8 ; Marcien D 25.7.3 pr.

1078- Scaevola D 38.1.44; Ulpien D 44.5.1.8.

1079- Africain D 46.1.21.2; Ulpien D 38.5.1.19.

1080- Marcellus D 22.1.32.

1081- Africain D 38.2.26; Scaevola D 33.1.20.1; D 35.2.94; Papinien D 31.79 ; Ulpien D 38.5.1.6. Ou être institué héritier : Ulpien D 44.5.1.8.

1082- A propos de legs, fidéicomanis ou donations, on relève Iavolenus D 38.5.12 ; Julien D 38.5.6; D 38.5.9; Africain D 38.5.10; Paul D 38 . $2.44 \mathrm{pr}$; D 38.5.11; D 38.5.5 pr ; Scaevola D 38.5.7; D 38.5.8 ; Ulpien D 38.5.1.1-11-14 (Pomponius)-15-16-17-27 (Pomponius, Papinien); D 38.5 . 3 pr-5 (Papinien).

1083- En Ecartant les textes où l'affranchi institue son fils, qui ne peut être assimilé a un tiers (cf. par ex. Ulpien D $38.2 .6 \mathrm{pr}-2$ ), on note : évoquant un affranchi faisant une donation à des anis (1084). Ménnmoins, ces hypothèses ne sont pas comptabilisables, les problèmes juridiques qu'elles soulèvent nécessitant des développements qui faussent sans doute l'image de la réalité. Par ailleurs, les rapports entretenus par l'affranchi avec des tiers' $n$ 'ont pas la même valeur que ceux qui nous mettaient en présence d'un esclave, celui-ci étant désormais libre. Pour ces raisons, il convient de s'attacher uniquement à une analyse statistique des rapports affranchi/patron.

Sur le plan des bons rapports, Scaevola évoque un patron ayant une bonne opinion de ses affranchis (1085). Un patron se porte caution pour un affranchi (1086). Un autre l'adopte (1087), ou encore consent a ce qu'il obtienne la restitutio natalium (1088). Papinien rappelle un testateur désirant que ses affranchis soient enterrés au mêne endroit que lui (1089). On voit aussi des testateurs faire des recomandations à leurs héritiers, les charger d'avoir une affection paternelle (1090) oú des rapports amicaux avec les affranchis (1091), leur défendre d'aliéner un effet de la succession pour protéger les affranchis (1092). Un testateur prie son héritier de laisser trois affranchis, vieux et infirmes, terminer leur carrière à l'endroit où ils se trouvent (1093). Si le patron

Iavolenus D 38.2.34; D 38.2.35; D 38.2.36; Julien D 38.2.20.2 ; D 38 . $2.23 \mathrm{pr}$; Africain D 38.2.26; Scaevola D $32.35 \mathrm{pr}$; Papinien D 38.2.42.

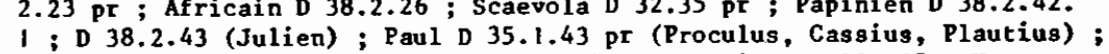
D 38.2.4.3; U1pien D 38.2.37.1 (Julien); R. Maximus D 30.125; Hermogênien $D$ 37.14.21.3.

1084- Julien D 38.5. 9 : Vivus libertus donare bene merentibus amicis potest.

1085- Scaevola D 32.39 pr (constitution de Marc-Aurèle).

1086- Ulpien D 16.3.1.14 (Labéon).

1087- Ulpien D 1.5.27.

1088- L'assentiment du patron étant nécessaire pour l'obtention de la restitutio natalium (cf. Marcien D 40.11.2. J. GAUDEMET, Institutions de 1 'Antiquité, cit., p. 567), il convient d'intégrer toutes les hypothèses de restitutio natalizm, 1 'assentiment du patron étant toujours présumé : de restitutio natalizm, 1 assentiment du patron étant toujours présumé D 40.10.4; D 40.11.4; Marcien D 40.11.2 ; Modestin 0 40.11.5 pr-1. 1089- Ulpien D $11.7 .6 \mathrm{pr}$ (Papinien).

1090- Scaevola D 33.1.21.4.

1091- Scaevola D 33.2.32 pr ; Modestin 34.1.5.

1092- Marcien D 30.114.14 (rescrit de Sévère et Caracalla).

1093- Scaevola D 33.2.33.2. 
fait preive de bons sentiments envers son affranchi, celui-ci peut aussi s'attacher à la personne de son patron ou à celle de ses enfants.

Ainsi, un affranchi désire venger la mort du patron (1094). Ailleurs, il prouve sa gratitude en accusant un tuteur pour sa mauvaise gestion des affaires du patron on des enfants de celui-ci (1095). Il conseille à une mère de donner un tuteur à son fils (1096). Il témoigne attachement et fidélité au fils du patron (1097), il va même jusqu'à 1 'adroger (1098).

Fn matière successorale, si l'institution de l'affranchi par son patron (1099) ou le legs reçu de ce dernier (1100) rentre dans le domaine

1094- Pomponius D 48.2.1; Ulpien D 48.2.4.

1095- Ulpien D 26.10.3.1. Dans une autre hypothèse, v. Papinien D 26. 5.13.2.

1096- Ulpien $D$ 38.17.2.24. I1 prévient une mère absente de demander des tuteurs pour ses enfants (cf. Ulpien $D 38.17 .2 .27$ ). On $n$ 'a pas retenu en tant que bon rapport le cas où 1 'affranchi est nomé tuteur d'un enfant du patron : il s'agit en effet d'une charge (cf. Papinien D 27.1.24 ; ulpien 0 26.7.3.4 (rescrit de Sévère et Caracalla) ; D 27.3.1.6 (Mela); Modestin D 27.1.10.7 ; D 27.1.14.1-2-4; Hermogénien D 27.1.43). De même n'a pas été retenu le cas où le patron se porte tuteur de son af franchi : il peut avoir intêrêt à surveiller les affaires de celui-ci (cf. D 26.1.8; aílleurs, il en charge quelqu'un, cf. Papinien D 26.2.28.2; D 27.1.30. 3).

\section{7- Paul D 38.2.47.2.}

1098- Tryphoninus D 38.2.50.5.

1099- Iavolenus D 38.2.35; Scaevola D 32.37.2; D 32.38.1; D 33.7.27. 3 ; D 34.4.30.1 (il s'agit ici de la soeur de lait, collactanea, de la patronne) ; D 36.1.80.13 ; D 39.5.35 pr ; D 40.7.40.2 ; Papinien D 28.5. 79 pr ; Paul D 17.2.71.1 ; U1pien D 38.4.3.5 ; Modestin D 37.14.12.

1100- Il faut excepter le legs des alimenta (cf. supra n. 402), le rapport alimentaire entre patron et affranchi ayant un caractère obligatoire (cf. R. ORESTANO, Alimenti (dir. rom.), in NNDI). On relève par ailleurs : Celsus D 31.29 pr ; Valens D 32.94 (Julien) ; Julien D 34.5 .11 ; D 42.8 . 17.1 ; Africain D 30.108.3-5-15; Marcellus D 31.17.1; D 31.25; Scaevola D $31.88 .6-9-14$; D 31.89 .6 ; D $32.35 \mathrm{pr}-2$; D 32.37.2; D 32.38.2 ; D 32 . $39 \mathrm{pr}$ (constitution de Marc-Aurèle); D $32.41 .1-3$; $\mathrm{D} 32.93 .2$; $\mathrm{D} 32$. 102.3 ; D $33.1 .18 \mathrm{pr}$; D $33.1 .21 \mathrm{pr}-1-4 ; \mathrm{D} 33.2 .32 \mathrm{pr}$; D 33.2.33.2;

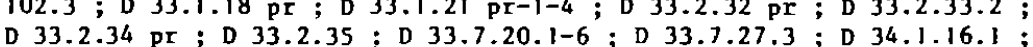
D $33.2 .34 \mathrm{Pr}$; D 33.2 .35 ; D $33.7 .20 .1-6 ; \mathrm{D} 33.7 .27 .3$; D $34.1 .16 .1 ;$ 80.13 ; D 40.7.40.2 ; Papinien D 18.6.19 Pr; D 31.67.7; D 31.77.13$15-27-28-32$; $\mathrm{D} 33.7 .3 \mathrm{pr} ; \mathrm{D} 34.1 .9 \mathrm{pr} ; \mathrm{D} 35.1 .72 .1$ (Sévère) ; $\mathrm{D} 35$. 1.101 .4 ; Tryphonims D 32.38.5 (Scaevola) ; Paul D 17.2.71.1; D 31 . 87.2 ; D 33.1 .9 (Papinien) ; D 34.4 .29 ; U1pien D 31.24 (Marcellus, Scaevola) ; D 38.1.7.1 (Celsus) ; D 39.5.16 ; Marcien D 34.9.1 (rescrit de Sêvère et Caracalla) ; D 35.1.33.2 (rescrit des mêmes) ; Modestin D 31. 34.4. Sur le contenu de ces legs, on remarque principalement des fonds (cE. Ear ex. Valens D 32.94 (Julien) : Sraevola D 31.88.6-9: D 32.35 .2 ) des bons rapports, on ne peut en dire autant du cas oì le patron est institué héritier par son affranchi, ou reçoit un legs ou une donation mortis causa de la part de celui-ci (1101). La deuxième hypothèse a en effet un caractère obligatoire, le préteur reconnaissant au patron une bonomon possessio portant au moins sur la moitié du patrimoine de l'affranchi (1102). Cette règle s'applique même dans le cas ờ l'affranchi a disposé par testament de plus de la moitié de ses biens ou a passè son patron sous silence (1103), mais on a alors affaire à un mauvais

quelquefois assortis des esclaves qui y travaillent (cf. Scaevola D 32. 93.2 ; D 33.7.20.1-6; D 33.7.27.3), des maisons (cF. Scaevola D 31.88 . 14 ; D 32.38.2 ; D 32.41.1-3; D 33.2.34 pr ; D 35.1.108); un testateur lègue à ses affranchis leurs fenmes et leurs enfants (cf. Scaevola $D 32$. $37.7 ; D 32.41 .2)$. I1 peut enfin $s$ 'agir de legs aussi variés que celui d'une some (cf. par ex. Scaevola D 33.1.21.4), d'une place dans une tribu (cf. Scaevola D 32.35 pr), d'une militia (cf. Scaevola D 32.102.3), de cinquante bouteilles de vin vieux (cf. Scaevola D 32.37.2). Ces legs peuvent être conditionnels : ainsi, on voit un affranchi recevoir un leggs ou un fidéiconnis s'il n'abandonne pas les enfants du patron (cf. Papinien D 35.1.72.1; Modestin D 31.34.4).

1101- Le patron peut recevoir sa part dans la succession de l'affranchi par le moyen d'une institution, cas le plus courant, mais aussi d'un legs (cf. Ulpien D 38.2.3.15), d'un fidéiconanis (cf. Ulpien D 38.2.3.16) ou d'une donation mortis causa (cf. Vlpien D 38.2.3.17). Suit la liste des textes où le patron reçoit, par un de ces moyens, de son affranchi la part que lui accorde le droit : Iavolenus D 38.2.34; Julien D 30.81 .4 ,

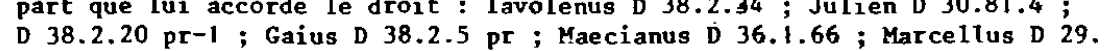
D $38.2 .20 \mathrm{pr}-1$; Gaius D 38.2 .5 pr ; Maecianus D 36.1 .66 ; Marcellus D 29.
4.5; D $32.52 \mathrm{pr}$; D $39.5 .20 \mathrm{pr}$; Papinien D 24.3.61; D 38.2 .41 ; D 38 . 2.42.3; Paul D 31.83 ; D 36.1.62; D $38.2 .44 \mathrm{pr}-1-2$; D 38.2.45; U1pien D 24.3.64.5; D 38.2.3.11-12-13-14-15-16-17-18-19; D 38.2.6.1 (MarcAurèle); D 38.2.10 pr ; D 38.2.19.1 ; D 38.5.1.5; D 38.5.3.5 (Papinien). Des hypothèses telles que celle où un patron, instituể pour la part à laquelle il a droit, 1 'a acceptêe, ignorant que son affranchi avait fait des aliénations en fraude de son droit (cf. Ulpien D $38.5 .3 \mathrm{pr}$ (Papinien)) ou celle où le patron, institué seul héritier, est chargé de legs qui amputent la part a laquelle il a droit (cf. Ulpien D 35.3.1.14) n'ont évidemputent la part à laquelle
ment pas été retenues ici.

1102- Cettebonorum possessio dimidige partis n'est en principe invocable que par le patron et ses descendants mâles. Toutefois,dans le cadre des préoccupations natalistes, la lex Papica Popprea (9 ap. J.-c.) reconnaît cette faculté à la femme qui a le ius liberorum (ingénue ayant trois enfants, ou affranchie en ayant quatre), cf. J. GAUDEMET, Institutions de l'Antiquité, cit. P. 563, n. 4. Cette même lex améliore aussi la situa1'Antiquité, cit., p. $563, \mathrm{n} .4$. Cette même lex améliore aussi la situa-
tion du patron en cas de succession $a b$ intestat : pour les successions d'affranchis dépassant 100.000 sesterces, elle lui donne droit à une part d'enfant lorsqu'il est en concours avec un ou deux fils de l'affranchi, J. GAUDEMET, o. c., p. 564 .

1103- Le patron est institué pour une part inférieure a celle que lui donne le droit ou est passé sous silence chez Iavolenus D 38.2.34; D 38 2.35 ; D 38.2.36; Julien D 36.1.26.13; D 38.2.11; D 38.2.13; D 38.2. $20.2-3$; D 38.2.2i ; D 38.2.23 pr ; D 38.2 .25 ; D 38.6 .2 ; Pomponius 
rapport, type de rapport qu'il convient d'envisager maintenant.

Dans le sens patron-afranchi, on remarque un patron chassant deux affranchis de chez lui (1104), un autre injuriant son affranchi, parfois très durement (1105), un héritier exerçant un pouvoir tyrannique qui provoque le départ des afrranchis (1106). Un patron va jusqu'à simuler I'indigence pour contraindre son affrunchi à lui verser des alimentadiot: ou bien il le gêne dans l'exercice de sa liberté (1108). Le problème est différent de celui qu'on a exariné précédemment à propos de l'esclave, le mauvais rapport $s^{\prime}$ instaurant une fois $l^{\prime}$ esclave afrranchi et consistant, par exemple, ̀̀ lui faire promettre une scume, non dans l'intention de l'exiger, mais dans celle de se faire craindre et obéir (1109). Servius éroque des patrons demandant des travaux très pénibles ̀̀ leurs affranchis, durissimas res $(1110)$. On voit encore un patron refuser les alimenta à son affranchi (1111), le déposséder violenment (1112), le revendiquer

D 38.2.2 pr-1 ; Africain D 38.2.26; T. Clemens D 37.4.10; Gaius D 38 2.7 ; Marcellus D 38.2.31; D 38.2.32; Papinien D 38.2 .41 ; D 38.2 .43 (Julien); Tryphoninus D $38.2 .50 \mathrm{pr}-1-2-3-4-5-6$; Paul D $35.1 .43 \mathrm{pr}$

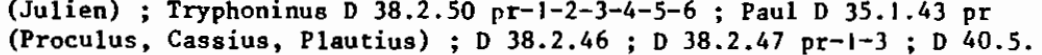
(Proculus, Cassius, Plautius) ; D 38.2.46; D 38.2.47 pr-1-3 ; D 40.5.
31.3 ; D 48.10.14.2 (êdit de Claude) ; Ulpien D 35.3.1.14; D 35.1.19.2; D $37.14 .17 \mathrm{pr}$ (Proculus, Julien, Maecianus ; Marc-Aurèle et Verus) -1 (Proculus, Julien) ; D 38.2.3 pr-1-2-3-4-5-6-7-8 (constitutión d'Hadrien) $-9-10$; D 38.2.6.4; D 38.2.8 pr-1-2-3-4-5; D 38.2.10 pr-1 (Julien) ; D 38.2.14 pr-5 ; D 38.2.16.5-10-11 ; D 38.2.19 pr ; D 38.2.37 pr (Julien) -1 (Julien) ; Marcien D 38.2.29 pr ; Modestín D 38.1.32 ; R. Maximus D 30. 125 . Hermogenien D 37.14 .21 pr-1-2. Il est question dialienations faites par l'affrogenien D 37.014 .21 prpar taffranchi en fraude du droit du patron chez lavolenus D 38.5 .12 Julien $D$ 38.5.6 ; 38.5 .8 ; D 38.5 .9 ; Africain D 38.5.10 ; Scaevola D 22.3.6 ; D Ulpien D 5.3.16.6 ; D 37.14.16 pr (Julien)-I-2 ; D 38.2.3.20; D 38.5.1 pr-1-2 (par a contrario)-3-4-5-6-7-8 (par a contrario)-9-10 (par a contrario)-11-12-13-14 (Pomponius) -15-16-17-18-19-20-21-22-23-24-25 (Pomponius)-26-27 (Pomponius)-28; D 38.5.3 pr (Papinien)-1-2-3-4-5 (Papinien) (par a contrario) ; D 38.5.4 pr-1 ; Marcien D 38.5.2.

1104- Scaevola D 31.88 .11 .

1105- Julien D 37.15.2 pr ; U1pien D 2.4.10.12 (Pedius) ; D 47.10.7.2; D $47 \cdot 10.11 .7$ (Marcellus)-8 (Labéon). Le patron qui commet un adultère avec la femme de son affranchi est censê infliger à celui-ci une iniuria atrox, la femme de son affranchi
cf. Papinien D 48.5.39.9.

1106- Scaevola D 34.1.13.2

$1107-$ Papinien D 38.1.41.

1108- Paul D 38.1.39 pr ; D 44.5.2.2; U1pien D 44.5.1.5-6-i-8-9-10

(Cassius, U. Ferox)-11-12; Modestin D 38.1.32.

1109- Pau1 D 44.5.2.2.

1110 - Chez U1pien D 38.2.1 pr; v. aussi U1pien D 38.1.2 pr.

111 1- Marcien D 37.14.5.1 ; Modestin D 25.3.6 pr ; D 38.2.33.

1112- Julien D 37.15.2.1; Ulpien D 43.16.1.43; Macer D 48.2.11.1. coame esclave (1113), l'accuger gans fondement (1114). On voit mêtue un mari contester le status de sa reme, qui est son effranchie (1115)...

On peut ausi déceler de maurais repports posthumes : il en est ainsi lorsque le patron ne cherche pas à ce que la lumière soit faite sur l'assassinat de son afrranchi (1116). En erfet, même g'il g'agit d'une simple dégligence, la mort de l'arrranchi ne semble pas beaucoun le peiner. Inversement, dans le sens affranchi-patron, l'affranchi qui, après la mort de son patron, dénonce le colmerce prohibé que celui-ci pratiquait, ne fait guère preuve de respect pour ga mémoire (1117), de même, à un niveau moindre, que celui qui refuse d'élever un monument comme le souhaitait le testateur (1118).

Dans le même sens, on voit l'affranchi d'un pupille refuser de

1113- Ulpien D 38.2.14 pr ; D 38.2.16 pr-1-2-3-4 (rescrit de Marc-Aurèle et Verus). Un fils revendique comme esclave-1'affrancht de son pêre, cf. Paul D 38.2.9; Ulpien D 2.4.10.11; D 40.12.31.

1114- Scaevola D 38.2.48 ; Papinien D 2.4.14. Le plus souvent, cette

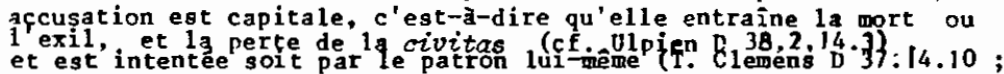
Ulpien D 25.3.5.23 (Marcellus) ; D 37.14.11; D 38.2.14 pr-1-2-3-4 (par extension)), soit par son fils (Paul D 38.2.5I (Labéon); Ulpien D 2. 4.10.11 ; D 37.14.17 pr (Proculus, Julien, Maecianus; Marc-Aurèle et Verus)-1 (Proculus, Julien); D 38.2.3.9; D 38.2.14.7 et 8 (par extension): D 38.2.16.4 (rescrit de Marc-Aurèle et Verus) ; Modestin D 37.14.9.1). Dans son testament, un patron accuse son affranchi d'avoir voulu attenDans son testament, un patron accuse son affranchi d avoir voulu attenaffranchi dans une accusation capitale intentée contre lui (Ulpien D 38. af $\mathrm{franchi}$ dans une accusation capitale intentee contre lui (Ulpien $D 38$ 2.14.5) ; de même, le fils prête son ministêre à 1 'accusateur de 1 'affranchi (cf. Ulpien D 38.2.14.9). Celui qui intente 1 'accusation calownieuse perd ses droits a la bonorum possessio de $I^{\prime}$ affranchi (cf. par ex. Scaevola D 38.2,48). Il n'en va évidenment pas de même lorsque 1'accusation est fondée (cf. Tryphoninus D 38.2.15; Ulpien D 38.2.14.6-11). Toutefois, on a encore affaire dans cette dernière hypothèse $a$ un mauvais rapport, l'accusation, même fondée, n'évoquant pas, de manière évidente, une entente cordiale entre patron et affranchi.

1115- Paul D 40.12.39.3, Les textes concernant une rupture de la vie commune entre patron et af franchie, posant un problàme tout-a-fait spécifique, n'ont pas été retenus dans cette analyse.

1116- Paul D 34.9.21; Ulpien D 38.2.37.1 (Julien).

1117- Marcien D 34.9.1 (rescrit de Sévère et Caracalla).

1118- Julien D 12.4.11. Sur un autre plan, ne fait pas preuve de sympathie pour le patron l'affranchi qui vend une terre ou ses parents sont inhumés, cf. Ulpien'D 38.5.1.15. 
le défendre alors qu'il est menacé de saisie (1119), un autre faire preuve de négligence ou de malice (1120). Un testateur affirme que son affranchi n'a aucun mérite (1121), est très mauvais, pessimus libertus (1122). Un affranchi se laisse vendre comme esclave à l'insu de son patron (1123) ou, toujours à son insu, se fait déclarer ingénu par un jugement (1124). Un autre nie être l'affranchi du patron (1125) ou, plus glohalement, nie être affranchi (1126). Les operae ne sont pas rendues au patron (1127), il ne perçoit pas les alimenta (1128), est laissé dans Ia misère ou la maladie (1129). Des esclaves médecins abandonnent une femme après avoir obtenu d'elle la liberté (1130). L'ingratitude peut prendre des formes plus radicales encore (1131).

\section{$1119-$ Ulpien D 42.4.5.1. \\ 1120- Modestin D 26.6.2.1. \\ 1121- Scaevola D 32.37.2.}

1122- Marcien D 34.4.13 (rescrit de Sévère et Caracalla).

1123- Gaius D 40.12.4.

1124- Papinien D 40.14.5.

1125- Ulpien D 25.3.5.18.

1126- Paul D 12.2.30.4; Ulpien D 12.2.13 pr.

1127- Alfenus (chez auteur anonyme) D 38.1.26.1 ; Julien D 38.1 .24 ; Gaius D 38.1.22 pr ; Paul D 38.1.39.1 ; Ulpien D 38.2.1.1.

1128 - Ulpien D 25.3.5.18-25.

1129- Ulpien D 1.12.1.2 : Sed et patronos egentes de suis libertis querentes cudiet, maxime si aegros se ess dicant desiderentque a libertis exhiberi; c'est au préfet de la ville de recevoir les plaintes formulées exhiber ; c'est au préfet de la ville de recevoir les plaintes formulées chis. Sur la constitution de Commode rapportée par Modestin (D 25.3.6.1), cf. supra n. 922 .

$1130-$ Scaevola D 40.5.41.6.

1131- En dehors de toute autre indication, i1 est question d'un libertus ingratus chez Marcellus D 37.15.3 ; Papinien D 23.3.69.6. (il s'agit ici d'une affranchie) ; 037.15 .11 (idem) (par a contrario : une affranchie ne devient pas ingrate si elle exerce une profession contre la volonté de sa patronne) ; Paul D 4.2.21 pr (affranchie) ; D 34.4.29; D 37.14.19; D 50.16.70; Ulpien D 3.3.35.1; D 31.24 (Marcellus, Scaevola) (indigras); D $40.9 .30 \mathrm{pr}-1-2-3-4-5$; Marcien D 37.15 .4 (rescrit de Sévère et Caracalla); Modestin $D$ 37.14.7.1. On doit également retenir, d'un point de vue plus général, les textes où il est question d'un contentieux judiciaire entre 1'affranchi et le patron ou sơ fils (cf. Paul D 2.4.11 (Labéon); D 2.4.15; D 2.7.2 pr ; D 37.15.6 ; D 42.1.19 pr ; Ulpien D 2.4.4.1 ; D 2.4.8.2 (Celsus, Julien) : D 2.4.10 pr (constitution de Marc-Aurèle) -5 $-7-8$; D 2.4.12 ; D 2.8.2.2 ; D 4.3.11.1 (Labéon); D 4.6.26.2 ; D 26. 10.3.1 ; D 37.15.5.1 ; D 37.15.7 pr-1-2-3-4; D 44.4.4.16; D 44.7.25.1; Marcien D 2.4.23 ; Modestin D 2.4.25), malgré I'interdiction pour I'afiranrai de les assigner en justice sans l'autorisation du oréteur. sous
L'affranchi ne respecte pas le patron (1132), I'injurie (1133), suscite la délation à son encontre (1134), conspire (1135), va jusqu'à lever la main sur lui (1136). La dégradation du rapport peut d'ailleurs connâttre un dénouement radical. Paul évoque des affranchis maltraitant leur patron, ils sont soupçonnés de l'avoir assassiné (1137). Dans son testament, rappeile Ulpien, un patron accuse son affranchi d'avoir voulu l'empoisonner (1138). Au-delà de la simple suspicion ou de la tèntative, le meurtre est parfois consommé, ainsi que nous l'apprennent Venuleius et Marcien (1139). Un passage de Papinien est enfin digne d'intérêt : il y est question d'un affranchi qui reçoit un legs à condition de ne pas abandonner les enfants du patron; on pourra exiger de lui, la caution mucienne car :

fuit enim periculosum ac triste libertwo coniunctum patroni liberis eorundem mortem exspectare (1140),

il serait triste et dangereux qu'il souhaite leur mort... C'est dire si la confiance règne.

Avant de passer à l'analyse statistique de ces rapports, précisons encore qu'il convient, comme on I'a fait pour I'esclave, de délaisser d'une part les questions successorales pour les raisons alors exposées, et d'autre part, les furta domestica, comme, les appelle Marcien,

peine de verser une indeumité de cinquante aurei et, s'il n'a pas cette somme, d'être condamé par le préfet de la ville à une peine corporelle somme, d'être condamé par le préfet de la
(cf. Ulpien D 2.4.12; Modestin D 2.4.25)

I132- Ulpien D 1.16.9.3; D 38.2.1.1.

1133- Ulpien D I.12.1.10; D 37.14.1; D 47.10.7.8. Les plaintes des patrons contre leurs affranchis sont portées devant les praesides; si $l^{\prime}$ affranchi a injurié son patron, il est condanmé a l'exil temporaire (D 37.14.1). v. aussi Modestin D 25.3.6.1 (constitution de Comode).

1134- Callistrate $D$ 49.14.2.6 (constitution de Sêvère); U1pien $D 1$. 12.1.10; Marcien D 37.14 .5 pr (Claude) (cf. supra n. 922). I1 1 'accuse de lèse-imajesté (cf. Ulpien D 38.2.14.6 ; Modestin D 48.4.7.2).

1135- Ulpien D 1.12.1.10.

1136- Ulpien D 37.14.1 ; Modestin D 25.3.6.1 (constitution de Comanode).

$1137-$ Paul D 38.2 .47 .1

$1138-$ Ulpien D 38.2.14.10.

1139- Venuleius D 48.2.12.4; Marcien D 48.9.1.

I140- Papinien D 35.1.72.1. Sur la caution mucienne, A. BERGER, Encycl. Diction., cit., p. $384 \mathrm{~s}$. ; A. GUARINO, Diritto privato romano, cit., p. 377 et bibliographie n. 33.5 .4 . 
le vol de l'affranchi envers son petron ne soulevant pas, d'un point de nue concret, des problèmes différents de celui comis par l'esclave envers son maftre (1141). Remarquons néanmoins, en sens inverse, ce passag̣e de Paul, selon lequel les patrons, ou plus généralement ceux qui est dû le respect, magna verscundia, ont couture de commettre des vols au détriment de leurs arfranchis (1142).

$$
-\circ
$$

$\mathrm{Si}$ on confronte les textes visant un bon ou un maurais rapport au nombre total de ceux qui traitent de la relation affranchi/patron pour chaque période, on parvient au tableau suivant :

Bons rapports (1143)

- analyse des paragraphes

$\begin{array}{lcll}\text { I } & - & = & - \\ \text { II } & - & = & - \\ \text { III } & 1 / 8 & =12,5 \% \\ \text { IV } & 5 / 12 & =42 \% \\ \text { V } & 20 / 143 & =14 \% \\ \text { TOTAL } & 26 / 163 & =16 \%\end{array}$

- analyse des citations

$\begin{array}{lll}\text { I } & 0 / 2 & = \\ \text { II } & 1 / 8 & =12,5 \% \\ \text { III } & 0 / 7 & =- \\ \text { IV } & 0 / 5 & =- \\ \text { V } & 1 / 1 & =100 \% \\ \text { TOTAL } & 2 / 23 & =9 \%\end{array}$

1141- Marcien D 48.19.11.1; v. ausBi Paul D 47.2.90; Ulpien D 47.2. 17.1. Néanmoins, conformément a $l$ 'attitude adoptée en matière d'esclavage, il convient d'intégrex un texte plus général de Callistrate (D 48.19.

28.8) qui noua apprend que les méfaits, admisea, perpétrés à I'encontre des patrons sont punis plus sevèrement.

1142- Paul D 47.2.92 (Labéon).

1143- Les textes servant de fondement à la rubrique "bons rapports" sont tires des notes 1085 a 1098 .

1144- Les textes servant de fondement a la rubrique "wavais rapports" sont tires des notes 1104 a 1141 . Ceux qui apparaissent plusieurs fois ne sont tiress des notes comptes que pour une seule référence (Cf, Paul D 44.5 .2 .2 ; 1 pien D 1.12 . $1.10 ;$ D $2.4 .10 .11 ;$ D 25.3 .5
16.4 ; Modestin D 25.3.6.1
Corme en matière d'esclavage at mur les mêmess rnisons, on constate une nette prépondérance des mauvais rapport.s dans lns deux anglyses entreprises.

Certaines periodes étant insuffisamant reprósentées, il faut énettre des réserves a leur Égard. Ainsi, le purcentage obtenu au moyen de $l$ 'analyse des citations pour la période $V$ n'est sbsolument pas confirmépar l'analyse des paragraphes. Inversement, le pourcentage assez faible de maurais rapports relatif à la période IV n'est pas étayé par les citations.

Un résultat mérite en particulier notre attention, il s'agit de celui obtenu pour la periode $V$. Il est en effet remarquable que 123 textes sur un total de 143 mettant en relation affranchiet patron visent un mauvais rapport. On notera d'ailleurs que le pourcentage réalisé par cette période en la matière n'est guère inférieur à celui qui arait été dégagé des rapports esclave/maître. L'esclave n'est donc pas mieux intégré à la société une fois affranchi. Il faut dire que le droit permet a son ancien maitre de le soumettre, dans une certaine mesure, d̀ des rexations. Rappelons à ce propos ces paroles d'Ulpien : pourquoi un individu, hier encore esclave, aujourd'hui affranchi, pourrait-il se plaindre de ce que son patron lui a adressé quelque insulte ou infligé quelque correction (1145)?

Dans ces conditions, on voit mal pourquoi, même au jème siècle, ces rapports connaîtraient une amélioration. C'est d'ailleurs plutôt de rupture qu'il convient de parler.

\section{SECTION 2. LA RUPTURE}

On a observe des oppositions d'affranchis au pouvoir patronal, mais c'est en matière $d^{\prime}$ oppositions serviles que les textes sont les plus prolixes. L'étude sur la main d'oeuvre a permis de constater que les esclaves ne constituaient pas un ensemble homogène, seuls les esclaves ruraux semblant être exploités de manière uniforme (1146). Cette variété

1145- U1pien D 47.10.7.2.

1146- Dans les campagnes, E.M. SCHTAERMAN (Die Blütezeit der Sklavenwirtschaft in der römiachen Republik, Wiesbaden (1959), p. 108) soutient que l'ordre servile, ignoré des Romains en tant que catégorie, repose sur une classe. Sur le sens de ordo, v. J. HELEGOUARC'H, Le vocabulaire 
de conditions de vie des esclaves empêche une prise de conscience de la profonde solidarité qui devrait les unir, c'est un fait qu'on a eu maintes fois l'occasion de souligner (1147). Toutefois, manque de conscience ne signifie pas absence de luttes. Après les grandes révoltes serviles de l'époque républicaine, la résistance va revêtir des formes inorganiques (1148). Ces formes ont déjà été évoquées : il s'agit du suicide, du sabotage, de la fuite et de la participation au meurtre du maître. Il est inutile de revenir sur les deux premières. Le suicide a été étudié à propos de la mort de l'esclave (1149). Quant au sabotage, il se présente dans nos textes sous la forme de l'incendie valontaire (1150). Ces deux formes sont difficiles à atteindre. Il n'en va pas de même de la fuite ou de la participation plus ou moins active au meurtre du mâtre.

\section{Paragraphe 1 : La fuite.}

Arrêtons-nous tout d'abord sur la définition juridique de la fuite. D'après ofilius, qui écrit au ler siècle avant notre ère, le fugitif est un esclave qui se trouve hors de la maison de son maitre dans le but de se cacher de lui (1151). Cette définition ve êt,re précisée sous l'Empire.

latin des relations et des partis politiques sous la république (1963), p. 428,449$)$. M. BARTOSER, Le classi sociali nella Roma antica, in Etudes Macqueron, Aix-en-Provence ( 1970$)$, p. $78 \mathrm{n}$. 196, rappelant que le transfert d'un esclave de la fomilia urbona dans la fomitia rustica repose sur la volonté du maître, estime que les esclaves privilégiés ne peuvent être volonté du maître, estime que les esclaves privilégiés ne peuvent être
détachés de la classe des esclaves ; contra, E.M. SCuTAERMAN, o.c., p. 168 ; v. aussi E. DENLAUX, cr. sur Schtaerman, in REA (1970), 72, p. 221.

1147- Par exemple, ce servus ordinarius prostituant les esclaves de son pécule, cet esclave đénonçant 1'assass in de son maître, etc. Ce sont surtout les esclavagistes qui ont conscience de l'existence d'une "classe" servile, M. BARTOSEK, o.c., p. 77. Sur la notion d'esclavagiste, ibid., p. 79 .

1148- La reconnaissance de certains "droits" aux esclaves, la limitation de $1^{\prime}$ arbitraire dominical, en d'autres termes 1 'affaiblissement apparent dea contradictions entre esclaves et maitres ne doivent pas voiler 1 'existence de luttes, dont ces relatives améliorations ne sont que le résultat, E.M. SCHTAERMAN, La schiavi tù, cit., p. 252. Malgré les tentatives d'assimilation, 1 'antagonisme fondamental demeure, $D$. MUSTI, Per una ricerca sul valore di scambio nel modo di produzione schiavistico, cit., p. 168.

1149- Cf. Supra section 3 , chapitre III Titre I, Deuxième partie.

$1150-$ Cf. supra n. 1042 .

1151- Chez Ulpien D $21.1 .17 \mathrm{pr}$.
Selon Caelius Sabinus, est fugitif celui qui a quitté la maison du maître dans le but de ne plus y revenir, car même son retour ne suffirait pas ¿े I'innocenter (1152). Pour Vivianus, on est fugitif plus par l'intention de fuir que par la fuite proprement dite (1153). Ainsi, l'esclave qui s'enfuit de chez un précepteur pour retourner chez son maître, celui qui se réfugie chez un ami du mâttre pour qu'il intervienne en sa faveur, le puer qui, ayant fui le magister qui le traitait trop durement, va implorer sa mère d'obtenir le pardon, ne sont pas des servi fugitivi (1154).

Si ces applications sont favorables à l'esclave, il n'en est pas toujours ainsi. Pour Proculus, qui semble être allé le plus loin sur cette voie, l'esclave qui se cache dans la maison du maitre dans l'intention de prendre la fuite doit être considéré comne fugitif, mêne s'il n'y parvient pas (1155). Toutefois, Tryphoninus estime la seule intention insuffisante. Selon ce juriste, un esclave n'est pas censé être fugitif pour avoir simplement eu l'intention de s'enfuir, même s'il l'a divulguée ;il doit avoir mis ses idées en pratique (1156). Voilà quels sont les traits juridiques généraux de la notion de fuite, mais, d'un point de vue plus concret, qui est fugifif ?

Une réponse logique voudrait que les esclaves tentés de s'enfuir soient, ceux qui sont traités le plus durement, les esclaves rurrux notam ment. Malheureusement, le Digeste est paurre en lá matière. Far contre, on voit des esclaves dont une association faisait le commerce blesser un des associós et s'enfuir (1157), d'autres profiter de ce que le maitre est attaqué lors d'un voyage (1158) ou fuir avant qu'sit lieu la quaestio sur son assassinat (1159). Ces fuites s'expliquent aisément. Le Digeste nous ofrre aussi des exemples plus inattendus .

Des esclaves fuient en effet alors qu'ils sont dans l'attente de la liberté, tel cet esclave affrenchi à condition de fournir ses operze

\section{2- Chez Ulpien D 21.1.17.1.}

1153- Chez U1pien D 21.1.17.3.

1154- Vivianus chez v1pien D 21.1.17.3-5.

1155- Chez Ulpien D 21.1.17.4.

1156- Tryphoninus D 50.16.225.

1157- Pomponius D 17.2.60.1.

1158- Ulpien D 29.5.1.31.

1159- Gaius D 29.5.25 pr. 
à l'héritier pentant sept ans, ou cet autre simplement tenu de servir pendant un an (1160). Ulpien nous apprend que ces esclaves sont soumis à une surveillance moins stricte, ce qui facilite leur fuite (1161). Ignorent-ils la liberté testamentaire qui leur a át.é laissée ? Rien dans les espèces évoquées ne le laisse supposer. D'autres hypothèses ne laissent place à aucun doute et montrent que la fuite n'est pas l'apenage des plus défavorisés. Ainsi, avant de fuir, un esclave exerçait des attributions à caractère financier (1162). Le maître avait concédé à un autre l'administratio peculii (1163). On voit même un ordinarius prendre la fuite arec san vicarius (1164).

Force est donc de constater que les couches serviles privilégiées subissent une polarisation des couches défavorisées. Si brutalité (1165), avaritia (1166) des maîtres sont souvent des causes de fuite, ce ne sont pas les seules. Il est d'ailleurs remarquable à cet ógard que les juristes envisagent la fujte comme un défaut moral latent, un vitium animi latens (1167). Quoi qu'il en soit, la fuite doit être interprét́́e comme une protestation contre le maître, mais contribue-t-elle effectivement à remettre en cause l'esclavage ?

Aucune équivoque n'est possible dans le cas du passage du fugitif chez les Barbares (1168). On ne peut cependant en dire autant de cet ancien esclave fugitif, Barbarius Philippus, dont Pomponius rappelle qu'il

1160- A1fenus (chez auteur anonywe) D 40.7.14.1; Pau1 D 40.7.4.8 (Cassius).

1161 - Ulpien D 12.4.5.3.

1162- Paul D 14.5.8.

1163- Ulpien D 12.1.11.2

1164- U1pien D 21.1 .17 .7 (Caelius Sabinus).

1165- Ulpien D 21.1.23 pr (Pomponius).

1166- P. JAL, cr sur Bellen, Sklavenflucht, cit. in Latomus 33 (1974), p. 453 ; H. BELLEN, o.c., p. 66 : saevitia, libido, avaritia.

1167- Papinien D 21.1.55. H. BELLEN, Sklavenflucht, cit., dont l'ouvrage est fondamental sur le thème, p. 128.

1168- Sur 1'esclave transfuge, Africain D 13.6.21.1 ; Paul D 49.15. 19.5-6 (il s'agit ici d'un statuliber); Ulpien D 47.2.46 pr (par extension : res... serros). Un texte de Paul (D 11.4 .4$)$ est aussi intéressant : le juriste évoque les limenarchae, chargés d'assurer la surveillance des ports, ce qui sous-entend une tentative de fuite par la mer. Sur le passage aux Barbares, E.M. SCHTAERMAN, La schiavitu, cit., p. 257. devint préteur (1169). De même en sst-il ì un moindro ni reau de ces esclaves qui se retrouvent comme travailleurs sur les grands dcmines, royant plus dana l'espérance de trouvar des conditions de fie plus supportables que dans le but de s'élever contre un systène sosial (117C). Des fugitifs sont revendus à des acbeteurs de bonne foi (1171). D'autres sont utilisés par lies publicains (1172).

Ces faits prouvent que la fuite est souvent "récupérée"en pratique. Cette récupération de la part du système est parfoisjinutile. Ainsi, des rugitirs se conduisent en propriétaires d'esclaves, à l'instar de celui qui, ayant acquis de l'argent chez son détenteur, 1 'investit dans 1 'achet d'esclaves (1173) ! Si le mimétisme donne à la fuite un caractère quelquefois original, elle $n$ 'en demeure pas moins im fait fondamental, ccrme le montre une analyse chronologique.

Si l'on dégage un pourcentage du nombre de paragraphes concernant la fuite par rapport au nombre total de ceux qui traitent d'esclavage, on obtient le tebleau suirant $(1174)$ :

1169- Chez Ulpien D 1.14.3.

$1170-\quad v$. notament Ulpien $D 11.4 .1 .1$. Les fui tes ont été facilitées par I'incitation qu'exerçaient les grands propriétaires aux époques où la main-d'oetrore faisait défaut, H. BELLEN, Sklavenflucht, cit., p. 133 s. ; I'auteur (p. 134) parle de travail noir. C'est la selon lui un caractère essentiel de la fuite. Cette vision, bien que méritant d'être prise en considération, cantonne l'esclave dans un rôle par trop passif, ne correspondant pas avec la réalité que nous livrent les textes du Digeste.

$1171-$ L'esclave peut se trouver chez celui qui l'a enlevé (Paul $D 17$. 1.22 .9 (Mela)) mais il peut également être revendu à un acheteur de bonne foi (Ulpien $D 4.2 .14 .5$ ). Il est auss $i$ question de possession de bonne foi d'un esclave fugitif chez Pomponius $D 41.1 .54 .4 ;$ Ulpien D 47.2.17.3 (Julien, Pomponius) ; $D$ 47.8.2.18. Selon Caelius Sabinus (chez Ulpien D 21.1.17,10), la fuite procure à l'esclave une espèce de liberté car, en fait, Il n'est plus sous la puissance dominicale. Il n'en demeure pas moins qu'il continue a être possédé par son waître, cf. P. BONFANTE, Corso (3) (1933), p. 333 s. ; PEROZZI, IDR 2 I (1928), p. 850 ; RABEL, Zum Besitzverlust nach Klass. Lehre, in St. Riccobono (4) (1930), p. $220 \mathrm{s.}$; CARCATERRA, Il servus fugitivus ed il possesso, in A.G. 120 (1938), p. $158 \mathrm{~s}$.; P. BONETTI, In tema di sexvus fugitivus, in Synte-

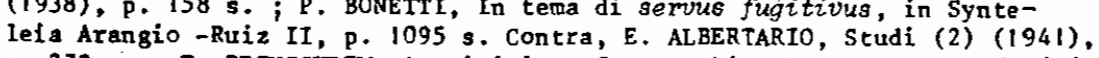
p. $273 \mathrm{~s}$.; F. PRINGSHEIM, Acquisition of ownership through servus fugitivus, in St. Solazzi (1948), p. $603 \mathrm{~s}$. ; G. LONGO, Animo retinere possessionem, in AUMA 20 (1956), p. $111 \mathrm{~s}$.

1172- Ulpien D 39.4.12.2. E.M. SChTAERMAN, La schiavitù, cit., p. 256.

1173- Paul D 17.1.22.9 (Meln).

1174- La liste des textes sur la fuite se trouve supra n. 1038. 
Analyse des paragraphes

Analyse des citations

\begin{tabular}{|c|c|c|c|c|c|c|}
\hline I & - & $=$ & - & I & $3 / 128$ & $2 \%$ \\
\hline II & - & $=$ & - & II & $28 / 347$ & $8 \%$ \\
\hline III & $27 / 985$ & $=$ & $3 \%$ & III & $24 / 519$ & $5 \%$ \\
\hline IV & - & $=$ & - & IV & $4 / 105$ & $=$ \\
\hline $\mathrm{v}$ & $128 / 3840$ & $=$ & $3 \%$ & $\mathrm{v}$ & - & $=$ \\
\hline
\end{tabular}

On réalise sans peine que les fuites d'esclaves ont constitué un mal endémique du système esclavagiste. On remarquera, dans l'analyse des citations, le bond effectué par la période II, correspondant au premier siècle de l'Empire, bond peut-être dû à 1 a disparition des grandes révoltes serviles. Mais on notera que la fuite reste pour les juristes du 3ème siècle un sujet de préoccupation aussi important que pour les juristes écrivant sous les premiers Antonins. Impuissance du pouvoir ?

Pour prévenir la fuite, des usages s'établissent, comme celui d'onchaîner certains esclaves ou de leur faire porter des médailles avec indication du nom de leur maitre (1175). Le vendeur d'un esclave doit s'engager à déclarer qu'il n'est pas fugitions (1176). Les juristes distinguent à ce propos le fugitif du simple vagabond. Selon Labéon,

Erronem... pusilium fugitivim esse, et ex diverso fugitivrom magnum erronem esse (1177).

L'esclave vagabond ne fuit pas, mais il sort fréquemment sans raison et rentre chez son maître plus tard qu'il ne doit. Le fait que ce même Labéon opère une comparaison à ce sujet entre un esclave et une bête de somme n'est pas sans intérêt (1178)... On relève une clauss remarquable en vertu de laquelle le vendeur s'engage à ce que l'esclave ne cherche pas asile auprès d'une statue de l'empereur, servum ad̀ staticam numquan confugisse (1179). D'ailleurs, le tiers qui donnerait à l'esclave

1375- G. LONGO,cr sur Bellen, in Iura 23 (1972), p. 174.

1176- V. par exemple dans le titre consacré à l'édit des édiles : Pomponius D 21.1.48.4; D 21.1.64.2 (Labéon); Papinien D 21.1.55; Paul D 21.1.44.2; U1pien D 21.1.1.1; D 21.1.4.3 (Pomponius); D 21.1 .31 . 16 (Julien). Sur le thème, H. BELLEN, Sklavenflucht, cit., p. $31 \mathrm{~s}$.

$1177-$ Chez Ulpien D 21.1.17.14.

1178- Chez Pooponius D 21.1.64.2 : Tbidem ait errare et fugere iumentum posse, nec tomen erronem aut figitivum esse agi posse.

1179- Ulpien D 21.1.19.1. H. BELLEN, Sklavenflucht, cit., p. 72-73. Sur le thème, C. GIOFFREDI, Ad statilas confugere, SDHI 12 (1946), p. $187 \mathrm{~s}$. un tel conseil "pour déshonorer son mâtre" serait tenu pour responsable de sa fuite $(1180)$.

On voit le maître, à qui incombe la recherche du fugitif, promettre une récompense à celui qui le ramènerait (1181) ou qui indiquerait où il se trouve (1182), infliger des sanctions aux esclaves ainsi repris, dans le cadre de la familia (1183). Mais l'Etat va ausi intervenir.

La lex Fabia, du ler siècle avant notre ère, punit celui qui fait passer sous son autorité l'esclave d'autrui (1184). Toutefois, un sénatus-consulte rapporté par Ulpien, prévoit la venia in ante actum en faveur de ceux qui rendent l'esclave dans un délai de vingt jours à son maître (1185). A côté de la pénalité de la lex Fabia se développe, sur le plan civil, une application de l'sction de servo corrupto à ces espèces, l'incitation à la fuite causant un préjudice patrimonial au mattre $(1186)$.

1180- Ulpien D 47.11.5. E.M. SCHTAERMAN, La schiavitu, cit., p. 239. 1181 - Ulpien D 19.5.18. Sur le fugitivarius, qui ne va pas sans rappeler le chasseur de primes des temps plus proches, v. F. GUIZZI, Professionisti e no : il fugitivarius, Synt. Arangio-Ruiz I, p. 237-239.

1182- Ulpien D 19.5.15. Un maître charge son ami, qui part _pour un long voyage, de retrouver son esclave fugitif et de le'vendre (Gaius D 18.1. 35.3). L'acheteur doit donner caution de poursuivre le fugitif si le vendeur l'avait averti qu'il était sujet à s'enfuir (Ulpien D 21.1.21.3 (Pomponius).) Si quelques esclaves dotaux se sont enfuis, le mari doit donner caution de les rechercher et de les ramener a sa femme (Paul D 24. 3.25.3). Il est intéressant de noter que la fulfe des esclaves qu'on n'avait pas coutume de surveiller est considérée comme un cas fortuit, au même titre que la mort des animaux, les inondations, l'irruption de brigands (Ulpien D 50.17.23).

1183- H. BELLEN, Sklavenflucht, cit., p. $17 \mathrm{~s}$. Auguste déclare dans ses Res gestae $(V, 25,2)$ qu'il a rendu à leur maître, ad supolicium sumendum, les esclaves fugitifs qui avaient combattu contre lui, faisant exécuter les autres.

1184- H. BELLEN, Sklavenflucht, cit., p. 45. La lex Fabia punit également selon $l$ 'auteur le fait de vendre ou d'acheter un esclave fugitif dolo malo (p. 45, p. 53 n. 365 et 367 ), mais la référence (D 48.15.2) sur laquelle il se fonde est interpolée selon G. LONGO, in Iura 23 (1972), p. 179 .

1185- Ulpien D 11.4.1.1. H. BELLEN, O.c., p. 52 ; G. LONGO, o.c., p. 175.

1186- G. LONGO, Ricerche romanistiche, Milan (1968), p. $571 \mathrm{s.}$ et Iura 23 (1972), p. 177. L'esclave est rendu vilior par sa fuite : s'il a un pécule, on en déduira ce qu'il vaut de moins (Paul D 33.8.9.2). Sur les liens entre plagium et actio furti, H. BELIEN, Sk lavenflucht, cit., p. $47 \mathrm{s.,} 50 \mathrm{~s}$. ; sur celx existant entre plagizm et actio servi. cormeti, ibid., p. $46 \mathrm{~s} ., 50 \mathrm{~s}$. ; critique de G. LONCO in lura 23 (1972), p. 175 s. 
Tout en alourdissant les peines próvues contre ceux qui contribuent à la fuite des esclaves (1187), l'Etat va surtout intervenir en matière de recherche $(1188)$. Selon un rescrit d'Antonin le pieux, celui qui veut rechercher son esclave sur la terre d'autrui doit se présenter devant le praeses qui lui fournira un ordre de perquisition et, si besoin est, le fera accompaner d'un apparitor (1189). Les domaines des particuliers, les domaines sénatoriaux et même impériaux ne constituent plus des asiles $(1190)$. Un sénatus-consulte de l'époque d'Antonin le pieux ordonne aux magistrats de prêter main forte aux maîtres dans leurs recherches (1191). Une epistula de Marc-Aurìle et Cormode étend cette injonction aux praesides et aux militaires (1192). Il n'est pas inintéressant de relever que, dans un passage de Menander, la poursuite d'esclaves fugitifs est citée aux côtés des raisons de santé ou d'amour comme une excuse valable à l'absence d'un militaire (1193). SEvère et caracalla vont aller plus loin que leurs prédécesseurs en faisant assumer cette fonction de recherche à l'Etat lui-même, par le biais de sa police, plus précisément du préfet des vigiles $\left(1191_{4}\right)$.

Quiconque arrête un fugitif doit le conduire devant l'autorité publique (1195). Magistrats municipaux, limenarchae et stationarii doivent les garder sous une surveillance étroite, les enchaîner en cas de besoin, jusqu'à ce qu'ils soient remis au oréfet des vigiles ou au gouverneur (1196). Dans leur signalement il est fait mention de leur nom,

1187- Ainsi, 1'amende prévue par la lex Fabia atteint 50.000 sesterces au 3ème siècle, E.M. SCHTAERMAN, La schiavitù, cit., p. 239 ; H. BELLEN, Sklavenflucht, cit., p. 45.

1188- Cet aspect de la lutte contre les fuites d'esclaves semble s'être intensifié sous les Antonins, H. BELLEN, Sklavenflucht, cit., p. $10 \mathrm{~s}$.

1189- Chez Ulpien D 11.4.3.

1190- Sénatus-consulte rapporté par Ulpien D 11.4 .1 .2 ; oratio Marci également rapportée par Ulpien D 11.4 .3 .

1191-Chez Ulpien D 11.4.1.2. Le refus d'exécuter cet ordre entraîne une peine de 10.000 sesterces (cention solidi), cf. H. BELLEN, Sklavenflucht, cit., p. 10, n. 49 .

1192- Chez Ulpien D 11.4.1.2.

1193- Menander D 49.16.4.15.

1194- Chez Ulpien D 1.15.5. E.M. ScHTAERMAN, La schi avitù, cit.,

p. 239 ; H. BELLEN, Sklavenflucht, cit., p. 13.

1195- Ulpien D 11.4.1.3-6.

1196- Paul D 11.4.4; U1pien D 11.4.1.4-7-8. du mâttre auquel ils declarent éventuellement appartenir, sinsi que de leurs cicatrices (1197)...

Antonin le pieux affirme que ces escleves ne sont pas confisqués, mais restitués à leurs mâttres (1198), à la puissance desquels ne peut même pas les soustraire une activité aussi peu enviable que celle de bestiarius (1199). Quant à ceux qui se sont fait passer pour libres, Callistrate nous apprend qu'ils sont punis plus sévèrement (1200).

Ces mesures montrent à quel point la fuite, quels que soient ses motifs, est ressentie par les propriétaires d'esclaves comme une remise en cause dangereuse du système. Mais cela n'est rien comparativement à la peur viscérale que leur inspire la possibilité d'être un jour assassinés par ces esclaves qui les côtoient et souvent les haissent, ou de les roir spectateurs passifs à leur assassinat.

\section{Paragraphe 2 : Le sénatus-consulte silanien}

Les textes visant l'assassinat du maître par ses esclaves ne sont pas en nombre suffisant pour permettre une analyse statistique (1201). Néanmoins, le s.c. silanien donne à lui seul une idée de la fréquence de cette pratique (1202).

Datant de 10 après J.C. (1203), ce s.c. prévoit qu'en cas de

\section{7- Ulpien D 11.4.1.8.}

1198- Chez Marcien D 39.4.16.4 : ne inquit, in potestate servorum sit invitis vel ignorantibus dominis fugae se tradendo potestati daminorum se subtrahere. H. BELLEN, Sklavenflucht, cit., p. 42.

1199- Chez Tryphoninus D 11.4.5.

1200- Callistrate D 11.4 .2 ; Eugitivi simplices dominis reddendi sunt : sed si pro libero se gesserint, gravius coerceri solent. Si l'on en croit la diversité chronologique des juristes qui font allusion a ce problềme, il est fréquent que des esclaves fugitifs se fassent passer pour des hommes libres (cf. Julien D 46.3.34.5 ; Pomponius D 46.3.19 (Labéon); Paul D 41.3.15.1). Relevons enfin cette espèce originale : selon Alfenus (chez Ulpien D 40.12.10), si un individu, se trauvant de fait dans un êtat servile et se sachant libre, s'enfuit de chez son prétendu maître, il est considêré coume esclave par le seul fait qu'il est en fuite, et ceci même, poursuit Gaius (D 40.12.11), s'il se comporte pendant sa fuite en home libre !

1201- V. supra n. 1039 et 1040.

1202- E. VOLTERRA, Senatus consulta, in NNDI (1969), p. 1064 ; E.M. SCHTAERMAN, La schiavitù, cit., p. 255.

1203- v. ARANGI0-RUIZ, Storia del diritto romano, Naples (1977), p. 238 A. GUARIN0, Storia del diritto romano, Naples (1975), p. 422; E. VOLTERRA, o.c., p. 1064, n ${ }^{\circ}$ 73. E.M. SCHTAERMAN, La schiavitù, cit., p. 211, 
neurtre du maitre par un membre de la comus ou far un tiers, tous les esclaves vivant sous le même toit que la victime, ̀̀ une distance à laquelle ils ont pu entendre ses cris, seront soumis à la torture et éventuellement condamnés à mort $(1204)$. Si certains esclaves ne se trouvaient pas sous le même toit mais dans un autre endroit đu même domaine, le s.c. ne s'appliquera que s'ils ont eu connaissance du meurtre (1205). Si. la terre sur laquelle le maître a été tué est très étendue, seluls les esclaves qui étaient avec lui à ce moment-1à, ceux qui sont soupçonnés de l'avoir tué, et enfin ceux qui en ont eu connaissance seront, soumis aux dispositions du s.c. (1206). Lorsque le meître est tué en voyage, les esclaves qui formaient sa suite subiront le supplicium (1207).

On touche ici un point essentiel du s.c. Le seul fait a'avoir vécu sous le même toit que le maître ou d'avoir été en rapport avec lui suffit à justifier l'usage de la torture. Mais la quaestio n'est pas une punition, c'est un préliminaire au supplicizon qui attend le coupable (1208). Or, la culpabilité de l'esclave ne repose pas seulement sur une participation active au meurtre du maître, mais aussi sur le refus de porter secours à son maître en danger (1209).

situe ce s.c. en 1 'an 9 après J.C. Se fondant sur Tacite, Ann. 13, 32, L. HERRMANN, La genèse du s.c. Sizanianum, in RIDA (1952), p. 49 s., soutient qu'en 57 après J.C., sur proposition de M. Iunius Silanus Torquatus, aurait été pris un s.c. sur les esclaves et affranchis testamentaires, du noru de Silanien, et qui devrait être identifié au s.c. Neronianum et au s.c. Fisonianum (ibid., p. 500) ; un déplacement des chapitres des Annales aurait été la source d'une date erronée. Contra E. VOLTERRA, o.c., p. 1065 .

1204 - W.W. BUCKLAND, Roman 1 aw, cit, p. 95 ; A. GUARTNO, Storia de 1 dirit to romano, cit., p. 422. Ulpien D 29.5.1.27. Le s.c. n'a en principe lieu qu'en cas de meurtre perpétré de manière violente et manifeste (cf. Ulpien D 29.5.1.17 (Labéon)-20) et non en cas d'empoisonnement et de meurtre secret (cf. Ulpien D 29.5.1.18). Cependant, $s i$ on a contraint le maître à absorber le poison, le s.c. aura lieu (UIpien D 29.5.1.19). De même, en cas d'empoisonnement sans violence, seront soumis aux dispositions du s.c. ceux qui en ont eu connaissance (cf. Ulpien D 29.5.1.21). Ulpien nous apprend enfin que le suicide du maître ne restera pas impuni si les esclaves ont pu 1 'empêcher (cf. D 29.5.1.22).

1205- Ulpien D 29.5.1.26.

1206- Ulpien E 29.5.1.30.

1207- Ulpien D 29.5.1.31.

1208- Uipien D 29.5.3.18. W.N. BUCKLAND, Roman law, cit., p. 96.

1209- Tlpien D 29.5.1.28 (rescrit d'Hadrien); Modestin D 29.5.19.
Secourir le maître, c'est, repousser les agresseurs

soit au moyen d'armes, soit à mains nues, soit par des cris, soit enfin en interposant son corps entre ceux du maître et de son (ou ses) agresseur (1210). Ulpien admet toutefois que l'esclave est censé avoir secouru son maitre lorsqu'il a fait t,out son fossible pour le sauver, même si cela n'a pu enpêcher la mort (1211). Par aillours, on excuse l'esclave commun qui a volé au secours d'un seul de ses deux maîtrës, alors que les deux étaient attaqués, ainsi que l'esclave de la ferme qui a secouru le mari plutôtt que sa domina (1212).

L'héroisme domestique étant requis, il n'est pas étonnant de voir des esclaves se faire volontairement enfermer (1213) ou même aller jusqu'à se mutiler pour ne pas avoir à risquer leur vie pour celle d'un maître qu'ils haissent (1214). Cela soulève des problèmes de preuve intéressants. Seuls échappent aux terribles dispositions du s.c. les esclaves qui, physiquement, n'ont pu venir au secours de leur maître :ainsi, l'esclave gravement malade (1215), sourd (1216), aveugle (1217), muet par l'impossibilité dans laquelle il est, d'appeler à l'aide (1218). De même sont excusés l'esclave dément, (1219) et celui dont, l'âge trop avancé ne pexmet aucune intervention (1220). Inversement, Ulpien nous apprend que les impubères échappent au suppliciım et même à la quaes tio (1221).

Pour qu'aucun autre esclave $n$ 'b́chappe, en dehors de ces cas

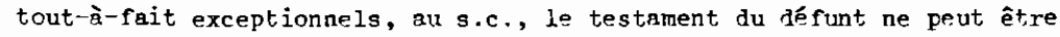

\section{0- Modestin D 29.5.19.}

1211 - Ulpien D 29.5.1.35.

1212- U1pien D 29.5.3.4-5.

1213- U1pien D 29.5.3.6. Même les esclaves vincti seront reconnus responsables si leurs liens pouvaient être rompus pour leur permettre de secourir leur maître (cf. Ulpien D 29.5.3.6).

1214- Ulpien D 29.5.I.37. Plus simplement, des esclaves font semblant de secourir leur maitre (cf. Ulpien D 29.5.1.34).

1215 - Ulpien D $29.5 .3 \mathrm{pr}$.

1216 - Ulpien D 29.5.3.8.

1217- Ulpien D 29.5.3.9.

1218- Ulpien D 29.5.3.10.

1219- v1pien D 29.5.3.11.

1220- U1pien D 29.5.3.7.

1221- Ulpien D 29.5.1.32-33. 
ouver: avant cette procédure répressive, sous peine d'indignitas (1222). On veut éviter que l'héritier ne tente de sauver les esclaves lui appartenant désormais (1223). Dans l'intérêt des propriétaires d'esclaves en général, le s.c. vient de la sorte causer un grave préjudice patrimonial à un maitre en particulier (1224). On relève aussi dans ce sens les dispositions prévoyant aux dépens de la succession des réccmpenses pour ceux qui dénonceraient les esclazes qui se seraient cachés (1225), ou donnant la liberté à l'esclave qui dénoncerait les assassins de son maître (1226). Il est intéressant, à propos de cette dernière mesure, de noter que même l'esclave vendu par son mâ̂tre sous la condition ut ne manumitterstur peut parvenir à la liberté de cette manière (1227) : ce n'est pas en l'espèce par attachement pour le défunt, avec lequel il entretenait certainement de mauvais rapports, que l'esclave dénonce les assasains, c'est par le seul appât de la liberté qui représente alors, dans le cas ou les assassins font également partie de la familia, un facteur grave de désolidarisation.

A côté du droit de propriété du maître est également remise en cause la situation des atatuliberi, eux aussi soumis au s.c. (1228). L'application du s.c. va d'ailleurs être étendue à plusieurs reprises jusqu'au milieu du 2ème siècle.

1222- V. ARANGIO-RUIZ, Storia del diritto romano, cit., p. 239 ; A. GUARINO, Storia del dirit to romano, cit., p. 422 . D 29.5 : De s.c. Silaniono et Claudiono : quomom testamenta ne aperiantur.

1223- E.M. SCHTAERYAN, La schiavitù, cit., p. 212.

1224- F. BENEDER, A senatus consultum Silanianum, Budapest (1963), résumé in Iura 15 (1964), p. 556, note que le s.c. n'a pas su surmonter l'opposition d'intérêts d'un maître en particulier et de l'ensemble des propriétaires d'esclaves.

1225- La lex Cornetia prévoit cinq ourei par esclave retrouvé ; si les biens du défunt sont insuffisants, la récompense est a la charge de l'seramizon (cf. Gaius D 29.5.25 pr). E.M. SCHTAERMAN, La schiavitü, cit., p. 212. Inversement est puni celui qui cache l'esclave (cf. Ulpien D 29. $5.3 .12)$.

1226- Cet affranchi n'a pas de patron, cf. supra $n$. 946. Le fait que le s.c. silanien ait prévu la libertê pour les esclaves coopérant a la découverte de $1^{\prime}$ assassin est discuté, cf. F. D'IPPOLITo, Una presunta disposizione del s.c. silaniano, in Synteleia Arangio-Ruiz II (Naples 1964), p. 717 s. ; 1 'auteur pense (p. 721) que la mesure aurait été introduite par le préteur pour tempérer la rigidité des normes répressives du s.c.

1227- U1pien D 29.5.3.15.

1228- U1pien D 29.5.1.4.
C'est ainsi qu'un nouveau s.r. vient franoer los esslave onpartenant au conjoint du propriétaire assassiné (1220) et. átendro on outre les dispositions du s.c. silanien aux esclaves afranchis tans le testament du defunt (1230). Trajan ra plus loin encore en y sounettant les affranchis déjà libres lors du meurtre, y compris ceux ayant obtenu le ius oureorm anuloron (1231). Hadrien confirme par un rescrit les cruelles dispositions du s.c. silanien (1232). L'espèce traitéè est digne d'intérêt : si une ancilla, se trouvant dans la même pièce que sa maîtresse, n'a pas appelé de secours car l'assassin la menaçait, elle sera condaranée d̀ mort pour que les autres esclaves sachent qu'ils ne doivent pas préférer leur vie à celle de leur maître.

Le s.c. va s'appliquer à la mort d'un enfant, légitime ou adoptif, vivant avec le paterfomilias, qu'il soit ou non sous sa potestas (1233). Selon Scaevola, si le fils est tué alors que son père est en captivité, le s.c. aura lieu pour les esclaves du père (1234). L'interpretation dés textes va aussi atteindre les esclaves appartenant au pécule du fils du défunt (1235) et même les esclaves jeunes. Sur ce dernier point, il faut évoquer une espèce rappelée par Maecianus : tout en rappelant que les esclaves impubères échappent en principe aux dispositions du s.c. silanien, le juriste estime fondée la décision d'un certain légat, du nom de Trobius Germanus, condamnant à mort un enfant qui, ayant assisté au me urtre de son mâtre, $n$ 'en avait pas par la suite dénoncé l'auteur

1229- Vlpien D 29.5.1.15. W.H. BUCRLAND, Roman law, cit., p. 95, n. 9. Selon E. VOLTERRA, Senatus consulta, cit., p. $1069, n^{\circ} 114$, il s'agirait du s.c. Neronianum, mais 1 'auteur reconnaît qu'on pourrait avoir affai re a une disposition du s.c. Pisonianton, qui prévoit, en ce qui concerne les esclaves devant être torturés déja vendus, que l'acheteur a droit à la restitution du prix (cf. Paul D $29.5 .8 \mathrm{pr}$ ). A. GUARINo, Storia del diritto romano, cit., p. 422, traite de ces deux dispositions comme relevant du s.c. Pisonianum (57 ap. J.C.).

1230- U1pien D 29.5.3.16. E. VOLTERRA, o.c., p. 1070.

1231- Pau1 D 29.5.10.1; Tryphoninus D 29.5.11.

1232- Chez U1pien D 29.5.1.28.

1233- U1pien D 29:5.1.7. Marcellus émet un doute quant aux enfants qui in potestate non sunt (chez U1pien D 29.5.1.8). Le s.c. $n$ 'a en revanche pas lieu par rapport aux enfants donnés en adoption (cf. Ulpien D 29.5.1.9).

1234- Chez U1pien D 29.5.1.12. De même, si le fils, institué héritier par son père, est tué avant 1 'adition d'hérédité, les esclaves du père, qui ont été légués ou affranchis sans condition, pourront être soumis à la quaestio et êventuellement condamnés au supplicium ( $\mathrm{cf}$. Scaevola chez Ul.pien D 29.5.1.13)

1235- Esclaves du pécule castrense, esciaves deg enfantz non 
(1236).

Seuls Antonin le pieux et Commode apportent quelques timides atténuations, le premier ordonnant de ne soumettre alu s.c. un esclave à qui est due une liberté fidéicommissaire pure que $s^{\prime}$ il est convaincu d'avoir participé au meurtre (1237), le second accordant le pardon aux esclaves que le maître, blessé, aurait absous (1238).

Le s.c. silanien va constituer une arme redoutable dans les mains de l'Etat. Du point, de vue des sanctions qu'il prévoit, on retiendra sa cruauté : il faut en outre bien être conscient du fait que la distinction entre quaestio et supplicium peut n'être que théorique, l'esclave ne survivant pas toujours à la torture (1239). On est loin des mesures favorables à l'esclave, notarment du favor libertatis.

$$
-{ }^{\circ}-
$$

L'affaiblissement des contradictions entre esclaves et propriétaires d'esclaves, dû à la reconnaissance de certains "droits" sux premiers et à la limitation de l'arbitraire des seconds, n'est qu' apparent (1240). Il est significatif de noter que l'ápoque des Antonins, qui voit une poussée du favor libertatis, connsît également un pourcentage de fuites non négligeable. Ces fuites, bien que leurs motivations évoquent parfois un manque de conscience sociale, sont toujours ressenties par les maîtres comme une atteinte grave à leur intégrité patrimoniale. Le s.c. silanien se passe quant à lui de tout commentaire, mais il est tout de même remarquable que son extension à de jeumes esclaves soit contenue dans un texte de Maecianus, qui écrit précisément sous les Antonins.

(cf. Ulpien D 29.5.1.14). W.W. BuCKuAND, Roman law, cit., p. 95. Bien (cf. Ulpien D $29.5,1.14)$. W.W. BUCKLAND, Roman law, cit., P. 95 , Bien
que Marcellus se prononce pour 1 'affirmative, Ulpien estime que le s.c. que Marcellus se prononce pour $1^{\prime}$ affirmative, Ulpien estime que le $s . c$.
ne $s$ 'applique pas aux esclaves du beau-père (socer) en cas de meurtre du ne s'applique pas aux esclaves du beau-per
mari ou de la feme (cf. D 29.5.1.16).

1236- Maecianus D 29.5.14.

1237- Chez Ulpien D 29.5.1.5.

1238- Chez Callistrate D 29.5.2.

1239- Cf. supra n. 907 .

$1240-$ Il faut rapprocher ce phéncmène de l'attén:lation des différences esclave/libre, due, on l'a vu, à la difficulté de distinguer la naissance libre de la naissance servile, à la fréquence des unions mixtes, des affranchissements. Cette atténuation ne va cependant pas, il faut le souligner, à l'encontre de l'id̄́e d'une crise. Si certains affranchis parviennent à être intégrés (ce qui ne va pas sans difficultés si 1 'on en croit les rapports affranchi/patron que nous livre le Digeste), ne tendentils pas à devenir à lenr tour des propriétaires d'esclaves? En ce sens, 1 'intégration n'est pas nécessairement réductrice des tensions qui affectent la société.
Conclusion de la deuxième partie

L'analyse menée sur les pratiques sociales confirme et complète les conclusions tirées de l'examen des textes sur la main-d'oeuvre servile. Le fossé séparant les privilégiés des autres esclaves n'apparaît. plus comme étant seulement économique, mais aussi vestimentaire, alimentaire et môme familial.

Le ler siècle voit crốtre l'intérêt des juristes pour ces privilégiés, institores, actores, sortes de "cadres moyons" antiques. Les nécessités économiques incitent certes à leur reconnaît,re une certaine capacité. Cependant, le bond qui s'effectue dans nos chiffres en matière de fuite n'est, sûrement pas étranger non plus à cett,e reconnaissance. Les juristes écrivant sous les premiers Antonins vont, dóvelopper les solutions du droit dans le même sens et aussi dans la direction du favor libertatis. Cette évolution ne ropose pas seulement sur un fondement philosophique, elle répond à un besoin de libéralisation. C'est en effet à cett,e époque que les rapports esclave/maitre semblent atteindre leur dégradation maximale. C'est également alors qu'il est le plus question dans les textes de délinquance servile. Il faut donc aller plus loin dans le sens̄ de la libéralisation.

Ce bond en avant serz le fait des juristes de la seconde moitié du zème siècle. Le discours atteint son point culminant aussi bien dans la reconnaissance d'une certaine capacité servile que dans le développement di favor libertatis. Ces solutions snnt symptomatiques de la crise qui secoue alors le système esclavagiste, précisément dans la mesure ò̀ elles altèrent, les rapports traditionnellement fondés sur lui. Les résultats obtenus pour l'époque des Sévères montrent clairement l'inefficacité de ce "ompromis". Les rapports sociaux ne s'améliorent pas. Le nombre de fuites, loin de diminuer, sembje au contraire persister. Jlpien commente amplement le s.c. silanien qui, deux siècles après sa parution, reste d'actualité...

Bien aue,sous les Sévères, le discours des juristes ne connaisse pas d'évolution qualitative jmportante, les juristes se préoccrpant des formes les plus "achevées" de l'esclavage, il accuse une baisse quant,itative. On avait supposé, au début de ce travail, que l'esclavage orcupait. cependant toujours à cet.te époque une place non négligåble du pcint de vue idéologique. Il est temps désormais de passer du plan des hypothèses à celui des ráalités. 
TROISIEME PARTIE

LES JURISTES PORTEURS DE

L'IDEOLOGIE ESCLAVAGISTE 
Hormis les quelques textes signalés plus haut sur le thème de l'anitié, on ne relève pas dans le Digeste de manifestations d'une idénlogie dépendante (1). En revanche, le droit romain se fait, l'écho de l'idéologie dominante, ne serait-ce que par le fait qu'il rend possible l'exploitation de l'esclave, quelle que soit in forme de celle-ci (2).

On trouve des traces de cette idénlogie à deux niveaux : dans la représentation de l'esclave par les juristes, et dans son utilisation en tant qu'élément patrimonial interchangeable.

\section{TTTRE I}

\section{LA REPRESENTATION DE L'ESCLAVE}

\section{PAR LES JURISTES}

L'i déologie dominante apparaît à travers la prise en considération de l'esclavage, mais surtout elle nous apporte, de manière plus précise, un portrait-type de l'esclave.

1- Sur les manifestations d'une idéologie dépendante, v. P. PETIT, $L^{\prime}$ esclavage antique, cit., p. $24 \mathrm{s.}$, qui donne un rếsumé des travaux de E.M. SCHTAERMAN. L' historienne (ibid., p. 26) soutient, que cette idéologie existe et qu'elle est commune à toutes les "classes" exploitées, esclaves, affranchis, paysans ou artisans libres et même colons; cependant, il ne faut pas leur attribuer une conscience de classe aussi éveillée qu'à 1 'époque moderne.

2- M. BARTOSEK, Le classi sociali, cit., p. 83
On sait que, pour Gaius et Marcien, l'esclavage, dérivant de la guerre, était considéré come une institution fin ius gentium (3). Plus nuancée était la position d'Ulpien, selon qui je ius naturale ne connaissait pas l'esclavage cum iure naturali rmes liberi nascerentur (4). Distinction fragile, il est vrai, car le rapport ertre ces deux iura n'est pas un rapport de valorisation mais d'antériorité (5). L'affirmation en vertu de laquelle 1 'esclavage a áté introduit par le ius gentium, alors que, sous le ius naturale, tous les homnes naissaient libres, ne tend pas à porter atteinte à l'institution de l'esclavage, mais, bien au contraire, à en justifier l'existence actuelle (6).

Au-delà de ce débat sur 1a justitication de l'esclavage, le discours des juristes est éloquent quant à sa réalité.

L'esclavage est assimilé à la captivité (7), à la détention (8); mais également à la honte, ignominia (9). L'homme libre pris pour un esclave subit une injure (10). L'esclavage est même comparé à la mort (11).

3- Gaius D 41.1.5.7; Marcien D 1.5.5.1. Selon Gaius (D 1.1.9), le ius gentium est un droit originaire, universel, rationnel et équitable.

4- Ulpien D 1.1.4. M. KASER, RPR $\mathrm{I}_{2}$, p. 284, n. 7.

5- P.A. MILANI, La schiavitù nel pensiero politico dai Greci al Basso Medio Evo, Milan (1972), p. 227.

6- P.A. MILANi, o.c., p. 229. Selon W.L. WESTERMANN, Slave Systems, cit., p. 113,116 , la société serait parvenue à une conviction humanitaire en vertu de laquelle l'institution de l'esclavage aurait infligé a l'être humain des dommages bien plus graves que les dommages simplement matériels. P.A. milnini, o.c., p. 232, critique cette idée et soutient que les améliorations sporadiques de la condition servile $n$ 'ont jamais visé que la $1 \mathrm{i}-$ mitation de l'arbitraire dominical.

7- Paul D 24.2.1; Ulpien D 4.6.1.1.

8- Callistrate D 4.6.11; Ulpien D 4.6.1.1.

9- Ulpien D 40.12.1.1.

10- Paul $D$ 47.10.22. Le fait d'avoir un de nos parents en servitude nous fait subir une iniuria (cf. Gaius D 40.12.2). Retenir de mauvaise foi un homme libre comme esclave constitue anssi une iniuria (cF. Ulpien D 43. 29.3.11). Soutenir que le descendant du défunt est esclave, c'est encore lui faire injure (cf. llpien (Julien ; rescrit d'Antonin le pieux) $D 37$. $10.1 .5)$.

11- Qau? D $24.2 .1 ;$ Ulpien D $24.1 .32 .6 ; 055.59 .2: 048.20 .575$; D 50.17 .200 
Il n'est pas surprenant, dans ce contexte, de lire chez Paul que la liberté est une chose inestimable (12). Cette mentalité persiste après les Sévères. Modestin parle encore de l'esclavage comme d'une tache, macula (13). Au Lème siècle, Hermogénien définit la liberté par rapport à $1 a$ servitude come un status honestior (14).

Sur un autre plan, les associations pratiquées par les juristes avec le terme manumissus, bien qu'étant dues aux nécessités de développements juridiques, ne sont pas moins intéressantes. On voit ainsi, dans un même texte, l'affranchissement en relation avec la vente (15), l'aliénation (16), le legs (17) d'un esclave. Un esclave affranchi intervient, dans le développement $d$ 'une espèce, aux côtés d'un esclave fugitif (18). A propos de la libération du débiteur, Pomponius écrit même :

manumissio morti similis est (19).

12- Paul D 50.17.106.
13- $\quad$ Modestin D 40.11.5.1.
14- Hermogénien D 40.15.3.

15- Julien D 43.24.14; Gaius D 39.4.13.2 ; Tryphoninus D 38.2.50.1 ; Paul D 46.3.35 (Alfenus); Ulpien D 47.6.3 pr.

16- Iavolenus D 32.29.4 (Tubero, Labéon); Julien D 28.5.38.4; D 30. 91.5 ; Pomponius D 36.1 .72 .1 ; D 44.7.56; D 45.3.40; Africain D 47.2 . $62 \mathrm{pr}$ (Liber factus); T. Clemens D 29.2.82 ; Gaius D 26.8.9.1; D 33.8.4 ; D 39.4.13.2 ; Maecianus D 35.1.86 pr (Julien) ; Paul D 9.4.24 (Labéon Julien) D15.1.26;D33.8.1;D33.8.3;D47.10.29;U1pien D9.2.15.1(Julien);D9.4.2.1(Celsus, Julien, Marcellus);D9.4.5.1; DI1 .3.5.4;D15.1.32pr(Julien);DI5.2.1 pr-1-4;DI6.3 1.30; D16.3.11 (Sabinus, Pomponius);D28.5.9.17-19;D29.2.25.1;D38.5.1.25 (Pomponius);D40.7.2pr;D40.9.12.1-3-4-6(Africain)-7;D40.9.14pr-4-5;D 47.2.17.1: D 47.4.1.I (Labéon) (ad Zibertatem pervenerit) ; Marcien D 49.14.30 (rescrit de Sévère et Caracalla).

17- Ulpien D 15.1.9.5 (Julien) : D 29.5.1.13 (Scaevola). Libertaset liber interviennent aussi souvent en comparaison avec un legs, cf. Iavolenus D 32.77 ; Valens D 35.1 .87 ; Julien D 35.2 .87 .3 ; D 40.5 .47 pr ; D 40. 7.13.5; Pomponius D 50.16.120; Gaius D 29.4.17; D 40.7.31.1 (Julien); D 49.14.14 ; Marcellus D 28.5.54 ; Scaevola D 33.8.23.2 ; Papinien D 20. $1.3 \mathrm{pr} ; \mathrm{D} 28.3 .17 ; \mathrm{D} 35.1 .78 \mathrm{pr} ; \mathrm{D} 48.19 .33$ (rescrit de Marc-Aurèle et Verus) Paul 5.2 .28 (deret d ; verus) ; Paul D 5.28 (decret d'hadrien); D 6.1 .66 ; D 28.6 .38 .3 (ScaeD 29.1 , 48.10 .22 .7 ; D 29.1.19.1 ; D 34.5.10 pr ; D 35.1.6.5 ; D 36.1.15.3; D 44.2.1 ; D 49 . $1.14 \mathrm{pr}$ (Antonin le pieux)-1 (epistuta de Marc-Aurèle et Verus) ; D 50.17. 161 ; Marcien D 49.1.5.1 ; Modestin D 31.31; D 31.34.6.

18- Pomponius D 21.2.16.2 ; Gaius D 39.4.13.2.

19- Pomponius D 46.3.92 pr. Sur le rapprochement opéré par les juristes entre la trort et $\mathrm{I}^{\prime}$ 'affranchissement de 1 'esclave, cf. aussi Pomponius D 15.2 .3 ; D 21.2.16.2 (Proculus); D 36.1.72.1; D 44.7 .56 ; Africain D 47. $2.62 \mathrm{pr}$ (íber factus) ; Gaius D 33.8.4; Paul D 5.3.36 pr (Proculus, Julien, Mauricianus) ; D 33.8.1; D 33.8.3; Ulpien D 9.4.5.1 ; D 11.3 .5 .4 ; D $15.1 .32 \mathrm{pr}$ (Julien) ; D 15.2.1 pr-1-4; D 16.3.1.30; D 28.7.8.7; D 38. 5.1 .25 (Pomponius); D $47.2 .46 \mathrm{pr}$.
En dehors de ces hypothèses de liberté, vente (20), aliénation (21), détérioration (22), fuite (23) apparaissent aux côtés de morts d'esclaves. On voit un esclave malade ou jeune assimilé à un esclave fugitif car, écrit Ulpien, il n'est pas possible de tirer des operae de tels esclaves (24).

Il est clair que les manifestations idéologiques du langage juridique sont intinement liées aux conditions matérielles de l'exploitation esclavagiste (25). Ce rapport est frappant si 1 'on tente de dégager des textes une typologie de l'esclave.

\section{CHAPITRE II : PORTRAIT D'ESCLAAVE}

Dans quelle - proportion les qualités d'esclave sont-elles représentées par rapport à ses défauts ? Fst-il davantage question de défauts physiques que moraux, ou bien est-ce le contraire ? Avant de répondre, il est nécessaire de retracer la réalité de ces qualités et défauts et de donner quelques précisions quant à l'analyse à mener sur ce thène.

D'un point de vue moral, est un bon esclave celui qui est sage (26), honnête, obéissant (27), fidèle (28), se conduisant, bien ${ }^{-}$(29),

20- Africain D 12.1.23 (Julien).

21- Africain D 15.3.17.1; Ulpien D 14.1.4.3; D 15.2.1.3; D 43.24. 7.1 (Neratius).

22- Iavolenus D 24.1.50 $\mathrm{pr}-1$.

23- Gaius D 6.1.36.1 ; D 13.6.18 pr

24- Ulpien D 7.1.12.3.

25- Sur le lien entre facteurs idéologiques et méthodes d'exploitation, v. E.M. SCHTAERMAN, La schiavitù, cit., p. 261 s. H. BIRNBAUM, L'Etude sociologique de 1 'Idéologie (1940-1960), Coll, La sociologie contemporaine, vol. IX, Londres (1962), P. 193, écrit : "Les idées dominantes ne sont rien d'autre que $I^{\prime}$ 'expression idéale des conditions matêrielles dominantes, les conditions matérielles dominantes prises conme des idées".

26- U1pien D 21.1.4.3 (Pomponius).

27- Ulpien D $21.1 .19 \mathrm{pr}$.

28- Ulpien D 4.9.7.4; D 19.1.13.3.

29- Bonae frugi, cf. Ulpien D 9.2.23.5; D 11.3 .1 .4 ; D 11.3.9.1 ; D 19.1.13.3; D 21.1.19 pr. 
constant, aimant son travail (30), vigilant, capable d'accroître son pécule (31). Plus généralement, le texte nous apprend qu'il est possible de se fier à l'esclave, c'est un serus idoneus (32), ou encore qu'il mérite la liberté (33), qu'il est. le meilleur parmi les esclaves de la forilia (34). D'un point de rue physique, Gaius mentionne combe une qualité le fait d'être bon coureur (35). Une bonne santé est également souhaitée (36).

Inversement est défectueux l'esclave infirme ou malade, la maladie étant, selon Sabinus cité par Ulpien, une affection qui rend le corps moins apte à remplir les fonctions auxquclles la nature, il serait plus convenable ici de dire le système, l'avait destiné (37). Ayant déjà consacré une étude aux vices affectant le corps de l'esclave (38), il est inutile de les énumérer à nouveau. Il convient cependant de préciser, à propos de la maladie, que seuls les textes en traitant expressément conme d'un défaut sont intégrables dans l'analyse statistique à venir (39).

De même, la détérioration à l'origine d'une dévnlorisation de l'esclave ne peut être prise en considération que si le texte indique qu'il s'agit d'une détérioration physique ou morale (40).

30- Gaius D 21.1.18 pr. Cette qualité implique un jugement valorisateur dépassant la simple aptitude professionnel le de l'esclave, le fait, par exemple, qu'il soit un excellent cuisinier (Gaius D 21.1.18.1), talentueux (Venuleius (Caelius Sabinus) D 21.1.65.2), très habile (Ulpien D 21. 1.19.4), ou, au contraire, incapable (Ulpien (Mela) D 9.2.27.34), complètement ignorant (U1pien D 21.1.19.4). Ces hypothèses ne seront pas retenues dans 1 'analyse quantitative, au même titre que les aptitudes intellectuelles de l'esclave au travail (cf. supra première partie n. 484 et $\mathrm{s}$.).

\section{1- Gaius D 21.1.18 pr.}

32- Julien D 13.6.20.

33- Pomponius D 40.9.23.

34- U1pien D 4.3.9.1. V. aussi Ulpien D 30.37 pr (Cassius ; rescrit de Sévère et Caraca1la) : optimus.

35- Gaius D $21.1 .18 \mathrm{pr}$.

36- Pomponius D 21.1.48.7; D 30.45.1; Paul D 17.1.26.8 (Mela); D 19. 4.2 (Aristo); Ulpien D 17.1.10.1; D 21.2 .31 .

37- D 21.1 .1 .7 .

38- Cf. supra section 2 , chapitre III, titre I, deuxiēme partie.

39- Exemples de textes ne pouvant être intégrés : Ulpien D 24.3.7.16 (Pomponius) et D 29.5.3 pr. Le choix doit parfois s'effectuer au détriment de la solution juridique : ainsi, bien que l'esclave ayant un gốtre de de la solution juridique : ainsi, bien que l'esclave ayant un goître de naissance soit considéré comme sain, la réalité oblige à reteni
physique mentionné conme prếdominant (cf. Ulpien D 21.1.12.2).

40- La détérioration en tant que telle constituerait un critère d'intégration trop gênéral. Exemples de textes ne pouvant être retenus :
Si on adopte un critère d'interprétation restrictif on mat.iǹro physique, il convient, pour ne créer aucun déséqquilibre quant.itat, if entre les deux rubriques, d'en faire autant en matière morale. Ainsi, on ne doit pas intégrer dans notre analyse la totalité des textes sur la fuite, mais seulement ceux la mentionnant comane un défaut, le vendeur s'engrgeant par exemple à déclarer que l'esclave n'est pas fugitious (41). C'est évidement dans le comentaire de l'édit des śdiles que cette pratique est la plus fréquente. Il est intéressant à ce propos de remarquer que fugitious vise alors un esclave ayant fui au moins une fois de la maison du vendeur (42). Le fait que l'acheteur actionne le vendeur mose donc le problème de la récidive, ce qui donne un relief supplémentaire au phénomène de la fuite, relief assez surprenant compte tenı de la répression mise en oeuvre pour juguler ce "mal" (h3).

Par ailleurs, le vendeur d'un esclave doit également s'engager à déclarer qu'il est noxa solutus, qu' aucune poursuite délictuelle n'est en cours contre lui (44). Cette garantie d'une qualité présente vise indirectement un risque de défaut antérieur.

Ces quelques précisions de méthode étant apportées, voyons quels sont les défauts signalés par les juristes. Leur liste est impressionnante et certains d'entre eux sont, pour l'observateur moderne, inattendus. Parqii les défauts "attendus", on relève la paresse, la somnolence, l'oisiveté (45), l'indocilité (46). Sont semblablement vitiosi les esclaves insolents (47), séditieux (48), effrontés (49), coléreux (50),

Iavolenus D 24.1.50 pr ; Papinien D 21.2.64 pr; U1pien D 21.1.25 pr-1-3 (Neratius) -4 (Pedius) $-5-8$.

41- Cf. supra deuxième partie n. 1176.

42- Papinien D 21.1.5.4. W.W. BUCKLAND, Roman 1 aw, cit., p. 55 ; H,

BELLEN, Sklavenflucht, cit., p. 36, n. 253.

43- v. par exemple Paul D 22.3.4.

44- Par exemple, chez Pomponius D 21.1.46: D 21.2.30; Marcellus D 46. 3.72.5; Paul D 21.2.3; D 21.2.11.1; Ulpien D 19.1.11.8; D 21.1.1.1;

D 21.1 .17 .17 .

45- Gaius D 21.1.18 pr.

46- Paul D 11.3 .2 .

47- Gaius D 21.1.3.

48- Ulpien D 11.3 .1 .5$.

49- Ulpien D 21.1.1.9.

50- U1pien D $21.1 .1 .9-11$ (Vivianus). 
menteurs (51), querelleurs (52), enclins au jeu (53), à la boisson (54), à la débauche (55), sujets à s'enfuir ou à vagabonder (56), voleurs (57). Cupidité et avarice (58) sont consiaérées comme des vices, mais il en est de même de la prodigalité (59). De manière moins évidente, des esclaves goumands (60), gros mangeurs (61), volages (62), timides (63), superstitieux (64), lunatiques (65), tristes (66), déprimés (67) sont de mauvais esclaves. Il est vrai que l'esclave qui tente de se suicider, et surtout qui y parvient, cause un préjudice patrimonial à son meître (68). venuleius écrit qu'aimer les spectacles, la peinture est un vitium ani$m i$ plus qu'un vitium corporis (69) I On note encore, d'un point de vue

51- Venuleius D 21.1.65 pr ; U1pien D 21.1.4.2 (Pomponius).

52- Ulpien D 21.1.4.2 (Pomponius).

53- Tryphoninus D 50.16.225; Ulpien D 21.1 .4 .2 (Pomponius) ; D 21.

1.19 .1 ; D 21.1 .25 .6 .

54- U1pien D 21.1.4.2 (Pomponius); D 21.1.25.6.

55- Pau1 D 11.3.2; Ulpien D 9.2.23.5; D 11.3.1.5.

56- Cf. par exemple U1pien D $21.1 .17 \mathrm{pr}$ a 16 . Sur la distinction entre fugitivus et erro, cf. supra deuxième partie n. 1177 .

57- Il s'agit ici aussi de ne retenir que les textes où le fait pour l'esclave d'être voleur ressort expressément comme un défaut (cf. par exemple, Africain D 47.2.62.3-5; Tryphoninus D 50.16.225 ; Paul D 17.1.26. 7 (Neratius); D 19.1.4 pr ; U1pien D 19.1.13.1-3).

58- Ulpien D 21.1 .1 .11 (Vivianus).

59- Paul D 11.3.2.

60- Ulpien D 21.1.4.2 (Pomponius) : ce défaut ne tomberait pas snis le coup de l'édit, conme le fait de mentir ou de chercìer querolle.

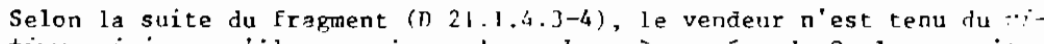
tium animi que s'il a fromis quelque chose à son égard. Duel que soit le vitium animi, il n'y a pas lieu à reinhibitio à moins que le vendeur ait déclaré que l' esclave n'avait pas ce défaut et qu'en réalité il l'ait.

61- Gaius D $21.1 .18 \mathrm{pr}$.

62- Gaius, ibid.

63- Vlpien D 21.1 .1 .11 (Vivianus).

64- Ulpien D 21.1 .1 .9$.

65- Paul D 21.1.43.6. Au sens strict, la folie concerne un plan plus intellectuel que moral : néanmoins, 1 'attitude du droit permet d'en tenir compte dans la catégorie des vitii animi. Sur la folie, cf. par exemple Iavolenus D 40.1.26 (Labéon) ; Pomponius D 41.2.25.1; Paul D 21.1. 43.6 ; D 41.2.1.10; U1pien D 29.5.3.11.

66- Melancholici, Paul D 21.1.2.

67- Paul D 21.1.43.4.

68- U1pien D 21.1.23.3.

69- Jenuleius D 21.1 .55 pr. plus général, la présence de servi non idonei (70) ou de manvais esclaves, indignes de la liberté (71).

Si l'on dégage un pourcentage du nombre de paragraphes traitant de qualités (72) ou de défauts (73) d'esclave par rapport au nombre total de textes concernant ces rubriques pour chaque période, on obtient :

70

71- Africain D 40.4.20.

72- Julien D 13.6.20; Pomponius D 21.1.48.7; D 30.45.1; D 40.9.23; Gaius D 21.1.18 pr ; Paul D 17.1.26.8 (Mela) ; D 19.4.2 (Aristo) ; Ulpien D 4.3.9.1; D 4.9.7.4; D 9.2.23.5;D 11.3.1.4; D 11.3.9.1; D 17.1 . 10.1 ; D 19.1.13.3; D 21.1.4.3 (Pomponius); D 21.1.19 pr; D 21.2.31; D 30.37 pr (Cassius ; rescrit de Sévère et Caracal1a); D 39.4.3 pr ; D 47.10 .15 .44 .

73- Alfenus (chez auteur anonyrne) D 11.3 .16 ; Lavolenus D 21.1.53; D 40.1.26 (Labéon); Celsus D 17.1.50.1; Julien D 13.6.20; D 21.1.50; D $30.84 .4 ;$ D 44.2 .25 .1 ; Pomponius D 10.2.45.1; D 18.1.13; D 21.1 .16 ; D $21.1 .46 ; D 21.1 .48 .1-4-7 ; D 21.1 .64 .1$ (Labéon) -2 (Labéon); D 21.2 . D 1 .1.46; D 21.1.48.1-4-7 ; D 21.1.64.1 (Labêon) -2 (Labéon); D 21.2 . 2.25.I ; Africain D 21.1.34 pr-1 ; D $21.1 .51 \mathrm{pr}-1$; D 30.108.11 ; D 30 . 110 ; D 40.4.20; D 47.2.62.3-5; Gaius D 11.3.15; D 21.1.3; D 21.1 . 13 ; D $21.1 .18 \mathrm{pr}$; D 21.1 .22 ; D 21.1 .32 ; D 39.4.13.2; Venuleius D 21. $1.65 \mathrm{pr}-1$; Marcellus D 46.3.72.5 ; F1orentinus D 18.1.43.1 ; Papinien D 21.1.54: D 21.1.55; Tryphoninus D 50.16.225; Paul D 6.1.21 (Julien, Pomponius ) ; D 6.1 .79 pr ; D 11.3 .2 ; D 11.3 .4 ; D 11.3.14.6-7-9; D 17 . 1.26 .7 (Neratius) ; D 19.1.4 pr ; D 19.1.21 pr; D 19.4 .2 (Aristo) ; D 21 . $1.2 ;$ D $21.1 .5: D 21.1 .11 ;$ D $21.1 .15: 021.1 .39: 021.1 .43 .1-2-3-4-6$; D 21.1 .44 .2 ; D $21.1 .58 \mathrm{pr}-2$; D 21.2 .3 ; D 21.2 .11 .1 ; D 29.2.93.2 ; D 33.8.9.1 (Marcellus)-2; D 34.9.5.15;D 40.7.4.5; D 41.2.1.10; Ulpien D 4.9.7.4; 03.2 .23 .5 ; D 3.2.27.17; D $10.4 .17 ; n 11.3 .1 \mathrm{pr}^{-3}$ $-4-5$; D I1.3.3.1; D I1.3.7; D $11.3 .9 .1-3$ (Neratius) : D $11.3 .11 .1-2$; D $12.4 .5 .3 ; D$ 13.3.3; D 15.3.3.9; D 17.1.10.1; D 13.1.11.7-8-12 (Neratius) ; D 19.1.13.1-2-3; D 21.1.1.1-6-7 (Sabinus)-8-9 (Vivianus) -10 (Vivianus) -11 (Vivianus); D $21.1 .4 \mathrm{pr}^{-1-2}$ (Pomponius) -3 (Pomponius) -4-5 (Pomponius) -6 (Pomponius) ; D $21.1 .6 \mathrm{pr}$ (Pomponius)-1 (Trebatius) ; D 21.1.9 (Sabinus); D 21.1 .10 pr (Ofilius)-1 (Cato)-2-3-4-5; 021. $1.12 \mathrm{pr}-1$ (Pedius) $-2-3-4$ (Trebatius) ; D $21.1 .14 \mathrm{pr}$ (Sabinus)-1-2-3 (Trebatius, Caelius Sabinus) -4 (Pedius) -5 (Pedius) $-6-7-8-9-10$ (Caelius Sabinus) ; D 21.1.17 pr (Ofilius)-1 (Caelius Sabinus)-2 (Cassius) lius Sabinus) ; D 21.1 .17 pr (Ofilius) -1 (Caelius Sabinus) -2 (Cassius)
-3 (Vivianus) -4 (Proculus) -5 (Vivianus) -6 (Caelius Sabinus) -7 (Caelius -3 (Vivianus) -4 (Proculus) -5 (Vivianus) -6 (Caelius Sabinus)-7 (Caelius
Sabinus) -8 (Caelius Sabinus) -9 (Caelius Sabinus) -10 (Caelius Sabinus)-11 Sabinus) -8 (Caelius Sabinus) -9 (Caelius Sabinus) -10 (Caelius Sabin
-12 (Labéon, Caelius Sabinus)-13 (Caelius Sabinus) -14 (Labéon) -15 (Labéon, Caelius Sabinus)-I6 (Caelius Sabinus)-17-18-20; D 21.1.19.1; D 21.1 .21 .3 (Pomponius) ; D $21.1 .23 \mathrm{pr}$ (Pomponius)-3 ; D 21.1.25.6; D 21.1.31.16 (Julien)-21; D 21.1 .35 ; D $21.1 .38 .3-7$ (Ofilius, Caelius Sabinus)-10-14 ; D 21.2.31 ; D $21.2 .32 \mathrm{pr}-1$; D 25.4.1.13 (Aristo) ; D 29.5.3.8-9-10-11; D 30.37 pr (Cassius ; rescrit de Sévère et de Caracalla) ; D 30.53.8; D 44.4.4.5 (Labéon) ; D 47.5.1.5; D 47.6.1 pr ; D $47.6 .3 \mathrm{pr}$; D 47.10 .15 .44 ; D 50.16.174 : Marcien D 21.1.52 ; Modestin 26.7 .32 .3 . 
Le "bon"esclave

le "meuvais" esclave

- analyse des paragraphes

\begin{tabular}{|c|c|c|c|c|c|}
\hline I & - & - & I & - & $=$ \\
\hline II & - & $=$ & II & - & $=$ \\
\hline III & $5 / 43$ & $12 \%$ & III & $38 / 43$ & $88 x$ \\
\hline IV & $0 / 4$ & - & IV & $4 / 4$ & $100 x$ \\
\hline v & $15 / 163$ & $9 x$ & v & $148 / 163$ & 918 \\
\hline TOTAL & $20 / 210$ & $10 x$ & TOTAL & $190 / 210$ & $90 x$ \\
\hline \multicolumn{6}{|c|}{ - analyse des citations } \\
\hline I & $0 / 7$ & $=$ & I & $7 / 7$ & 1008 \\
\hline II & $2 / 30$ & $7 \%$ & II & $28 / 30$ & $93 x$ \\
\hline III & $2 / 26$ & $8 x$ & III & $24 / 26$ & 927 \\
\hline IV & $0 / 1$ & $=$ & $\Gamma V$ & $1 / 1$ & $100 \%$ \\
\hline v & - & $=$ & v & - & $=$ \\
\hline TOTAL & $4 / 64$ & $6 x$ & TOTAL & $60 / 64$ & $94 \%$ \\
\hline
\end{tabular}

On observe une très nette prépondérance des défauts, quelle que soit la période envisagre. C'est une chose "normale". L'intérêt du droit en général, de l'édit des édiles en particulier, principale source en la matière, est de protéger l'acheteur contre les défauts éventuels de l'esclave qui lui est vendu. Il ne s'agit nullement de dresser un tableau élogieux des "bons"esclaves, ceux-ci ne soulevant pas de difficultés juridiques. Dans la même lignée, il faut remarquer qu'il est beaucoup plus question de "mauvais" affranchis que de "bons" affranchis (74). C'est là une pièce. à conviction supplémentaire apportée à l'idée d'une continuité entre la condition de l'esclave et celle de I'affranchi.

Il convient encore d'interroger les données sur le fait de savoir quels sont les défauts le plus fréquemment représentés. s'agit-il des defauts physiques ou moraux ? La démarche à suivre est statistiquement identique à la précédente. Elle consiste à confronter les textes traitant de défauts physiques (75) ou noraux (76) au nombre total de ceux

74- Affranchis malades ne pouvant acquitter les operae (cf. Julien D 38.1.23.1; Paul D 38.1.16.1; D 38.1.17 : Ulpien D 38.1.15 pr), affranchis ingrats (cf. supra n. |131), affranchi pessimus (cf. Marcien D 34.4 .13 (rescrit de Sévère et Caracalla)), affranchie ignominiosa (T. Clemens D 23.2.48.1). On ne releve en revanche que trois textes visant expressément de bons affranchis (cf. Scaevola D 40.5.41.6-15; U1pien D 42.4.5.1).

75- Iavolenus D 21.1.53; Celsus D 17.1.50.1; Julien D 21.1.50; D 30. concernant ces défauts, ceci pour chaque période. Signalons encore que le nombre total ne correspond pas exactement à celui atteint auparavant, les textes contenant simplement la mention vitium ou vitiosus n'ayant pas pu être retenus, le vitium pouvant aussi bien affecter le corps que l'esprit (TT).

84.4; Pomponius D 21.1.16; D 21.1.48.1-7; D 21.1.64.1 (Labéon); D 21. 2.16 .2 (Proculus) ; D 30.45 .1 ; Africain D $21.1 .34 \mathrm{pr} ; \mathrm{D} 21.1 .51 \mathrm{pr}^{-1}$; D 30.108.11; Gaius D 21.1 .3 ; D 21.1 .13 ; D 21.1.32; Venuleius D 21.1. $65 \mathrm{pr}-1$; Paul D 11.3.4; D 19.1.21 pr; D 21.1.5; D 21.1.11; D 21.1. 15 ; D 21.1.44.2; D 33.8.9.1 (Marcellus); D 40.7.4.5; vipien D 9.2. 27.17 ; D 10.4.17; D 11.3.3.1; D 11.3.9.3 (Neratius); D 13.3.3; D 21 . 1.1.1-6-7 (Sabinus)-8; D 21.1.4.1-4-5 (Pomponius)-6 (Pomponius) ; D21.1.6pr (Pomponius)-1 (Trebatius)-2;D21.1.7;D21.1.8;D21.1.9(Sabinus); D21.1.10pr (Pomponius) - (Trebatius) -2;D21.1.7; D21.1.8;D21:9(Sabinus); D21.1.10p (Ofilius) - 1 (Cato)-2-3-4-5; D $21.1 .12 \mathrm{pr}-1$ (Pedius)-2-3-4 (Trebatius) ;
D 21.1 .14 pr (Sabinus)-1-2-3(Treb.,C.Sab.)-4 et 5(Pedius)-6-7-8-9-10(Caelius D 21.1.14 pr (Sabinus)-1-2-3(Treb.,C.Sab.)-4 et 5(Pedius)-6-7-8-9-10(Caeli
Sabinus); D 21.1.31.16 (Julien); D 21.1.35; D 21.1.38.3-7 (Ofilius, . Caelius Sabinus)-10; D 21.2.32.1; D 29.5.3.8-9-10.

76- Alfenus D 11.3.16 (chez auteur anonyme); Iavolenus D 40.1.26 (Labên) ; Celsus D 17.1.50.1 ; Julien D 13.6.20; Pomponius D 10.2.45.1; D 18.1.13; D 21.1.46; D 21.1.48.4; D 21.1.64.2 (Labéon); D 21.2.16. 2 (Proculus); $21 ; 30 ; \mathrm{D} 24.1 .31 .5 ; \mathrm{D} 30.45 .1 ; \mathrm{D} 30.56 ; \mathrm{D} 41$ 2.25.1; Africain D 40.4.20; D 47.2.62.3-5; D 30.110 ; Gaius D 11.3 .15 ; D 21.1 .3 ; D $21.1 .18 \mathrm{pr}$; D 21.1.22; D 39.4.13.2; Venuleius D 21.1 .65 pr ; Marcellus D 46.3.72.5; Papinien D 21.1.54; D 21.1.55; Tryphoninus D 50.16.225; Paul D 6.1.21 (Julien, Pomponius); D $11.3 .2 ; \mathrm{D} 11.3$. 14.6 ; D 17.1.26.7 (Neratius); D 19.1.4 pr ; D 19.4.2 (Aristo); D 21.1 . 2 ; D 21.1.43.1-2-3-4-6; D 21.1.44.2; D 21.1.58 pr-2 ; D 21.2.3 ; D 21.2.11.1; D 34.9.5.15 (Antonin le pieux); D 41.2.1.10; U1pien D 4. 9.7.4; D 9.2.23.5; D $11.3 .1 \mathrm{pr}-3-4-5$; D $11.3 .1 ; \mathrm{D} 11.3 .9 .1-3$ (Neratius) ; D 11.3.11.1-2 (Julien); D 12.4.5.3; D 15.3.3.9; D 19.1.11.7$8-12$ (Neratius); D 19.1.13.1-2-3; D $21.1 .1 .1-9$ (Vivianus) -10 (Vivianus) -11 (Vivianus) ; D 21.1.4.1-2 (Pomponius) -3 (Pomponius)-4; D $21.1 .17 \mathrm{pr}$ (Ofilius)-1 (Caelius Sabinus)-2 (Cassius) -3 (Vivianus) -4 (Proculus) -5 (Vivianus) -6 (Caelius Sabinus) -7 (Caelius Sabinus)-8 (Caelius Sabinus) -9 (Caelius Sabinus) -10 (Caelius Sabinus) $-11-12$ (Labeon, Caelius Sabinus) -13 (Caelius Sabinus)-14 (Labéon)-15 (Labêon, Caelius Sabinus)-16(Caelius Sabinus)17-18-20;D21.1.19.1;D21.1.21.3(Pomponius); D21 . 1.23pr(Pomponius)-3;D21.1.25. 6-7:D21.1.31.1-16(Jul ien);D21.2.31;D21.2.32pr-1;D25.4.1.13(Aristo);D29.5.3. 11: D 30.53.8 ; D 44.4.4.5 (Labéon);D47.5.1.5;D47.6.1pr;D 47.6.3pr; D 47. 10.15.44; D S0.16.174; Marcien D 18.1.42 (Marc-Aurele et Verus); D 21.1.52; Modestin D 26.7.32.3.

77 - v. par exemple venuleius D $21.1 .65 \mathrm{pr}$. 
- analyse des paragraphes

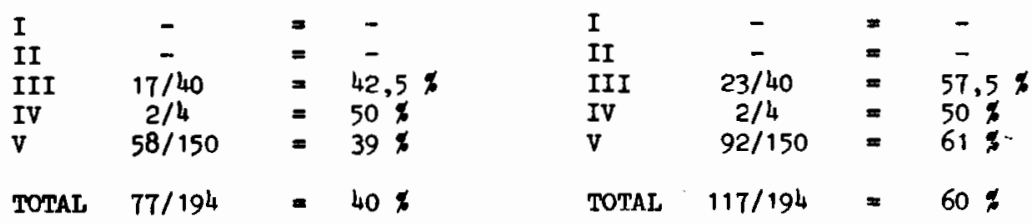

\section{- analyse des citations}

$\begin{array}{lllllll}\text { I } & 6 / 8 & =75 \% & \text { I } & 2 / 8 & =25 \% \\ \text { II } & 8 / 27 & = & \text { II } & 19 / 27 & =70 \% \\ \text { III } & 8 / 26 & =30 \% & \text { III } & 18 / 26 & =69 \% \\ \text { IV } & 1 / 1 & =100 \% & \text { IV } & 0 / 1 & = \\ \text { V } & - & = & \text { V } & - & = \\ \text { TOTAL } & 23 / 62 & & & & \end{array}$

Le tableau ci-dessus montre $1 a$ prédominance globale des défauts moraux par rapport aux défauts physiques. Il est vrai que ces derniers préoccupent moins les juristes dans la mesure ou ils sont apparents. Cependant, à 1 'exception des réserves que 1 'on peut émettre pour la période IV, insuffisamment représentée dans les deux types d'analyse, on remarque une évolution. C'est à partir de l'Empire que crôt l'intérêt des juristes pour les défauts moraux de 1 'esclave. Cet intérêt atteint son point culminant au 3ème siècle, ce qui s'explique tant par le changement opéré dans l'utilisation de l'esclave que par l'importance prise par les privilégiés dans la vie quotidienne.

Le discours des juristes exprime ici très clairement les problèmes vécus par les propriétaires d'esclaves. Le portrait de l'esclave qu'il nous livre est nettement tracé en fonction de l'échelle de valeur dominante. Au-delà des solutions qu'il préconise, il se fait le tremplin d'une idéologie dominante qu'il contribue d'ailleurs lui-même à forger (78).

78- Il y a souvent identité physique entre dirigeants et juristes:faut-il rappeler que Iavolenus, Valens, Vindius Verus, Julien, Maecianus, Marcellus, Scaevola, T. Paternus ont fait partie du Consiliun principis que Papinien, Paul et Ulpien ont été préfets du prétoire (cf. supra introduction)?
Cette ideologie ressort Egalement de l'utilisation de l'esclave coume élément patrimonial interchangeable.

\section{TITRE II \\ L'ESCLAVE \\ ELEMENT PATRTMONIAL \\ INTERCHANGEABLE}

L'esclave est fréquenment associé par les juristes à d'autres éléments patrimoniaux. Il convient tout d'abord de dégager quels sont ces éléments. Mais ceci ne constitue qu'une analyse indicative. Est-il possible, à travers une analyse statistique de cette utilisation de l'esclave par le langaxe juridique, de déterminer le maintien ou l'affaiblissement de 1'idéologie esclavegiste ?

\section{CHAPIIRE I : ASSOCIATIONS D'ESCLAVES A D'AUTRES ELFMENTS PATRIMONIAUXX}

Ces associations peuvent s'effectuer à dés degrés divers. L'esclave peut intervenir come un objet direct, le texte se présentant de la sorte : "si je te vends un fonds ou un esclave" (79) ; ou bien il apparaît dans une énumération de biens, du genre : "dans le legs de l'instrumention fundi sont inclus les esclaves, les animaux, les outils" (80). L'association peut aussi être pratiquée de manière plus nuancée : Paul, dens le titre De inpensis in res dotales factis, compare la plantation d'arbustes $\mathrm{a}$ l'instruction des esclaves ( 81 ) : Ulpien, donnant des exemples de cas fortuits, assimile la mort, d'animawx aux fuites d'esclaves (82). Enfin, l'association opérée par le juriste peut n'être qu'indirecte : les operae servi sont ainsi comparées au prix de transport des marchandises par bateau ou au moyen de bêtes de somme (83).

79-. Par exemp1e, Callistrate D 21.2.72.

80- U1pien D 33.7.8 pr (Sabinus).

$81-\quad$ D 25.1 .6

82- U1pien D 50.17.23 (Celsus).

83- Par exemple, U1pien D 19.1.13.13. 
Cependant, quel que soit le degré de l'association, ces hypothèses sont indissociables, l'esclave ressort,ant toujours explicitement des textes sur le même plan que d'autres éléments patrimoniaux. Un tableau chronologique permettra de mieux se rendre compte de la fréquence de ces associations (84).

\begin{tabular}{|c|c|c|c|}
\hline $\begin{array}{l}\text { juriste } \\
\text { auteur } \\
\text { du passage }\end{array}$ & $\begin{array}{l}\text { juriste } \\
\text { cité }\end{array}$ & autre élément patrimonial & source \\
\hline Proculus & & vinum & D 33.6 .6 \\
\hline Iavolenus & & praedia & D 38.2 .36 \\
\hline Neratius & & $\begin{array}{l}\text { (cibaria... mancipiis)/modifica } \\
\text { aedificiorum... refectio/agrorum } \\
\text { cultura. }\end{array}$ & D 25.1 .15 \\
\hline Celsus & & frument,um & D 17.1 .50 .1 \\
\hline Julien & & $\begin{array}{c}\text { fundus } \\
\text { fundus } \\
\text { (servi operis)/fructus } \\
\text { ex fundo } \\
\text { fundus } \\
\text { aedes...servitute/ } \\
\text { (debilitatum...servum) } \\
\text { mulorum, mulae } \\
\text { fundum }\end{array}$ & $\begin{array}{l}\text { D } 10.4 .8 \\
\text { D } 12.4 .7 .1 \\
\text { D } 22.1 .25 .1 \\
\text { D } 22.1 .25 .2 \\
\text { D } 30.84 .4 \\
\text { D } 32.62 . \\
\text { D } 34.5 .13 .2\end{array}$ \\
\hline
\end{tabular}

84- N'ont été relevées que les associations effectuées à l'intérieur d'un même paragraphe. Ces associations sont parfois opérées dans deux paragraphes successifs (ainsi chez Celsus D 32.79 .2 (area legata) et D 32.79 .3 (servus legatus); Pomponius D 44.2.21.1 (si petiero gregem) et D 44.2.21.2 (si Stichwn et Famphilum twos esse Fetieris) ; Gaius D 22.1. $28 \mathrm{pr}$ (fetus pecudion) et D 22.1.28.1 (partus); Ulpien D 30.53.3-4 (hominem) et D 30.53 .5 (animal) ; D 23.3.10.2 (semsi subolem) et D 23.3.10.3 nem) et D 30.53.5 : Modestin D $32.81 \mathrm{pr}(-1)$ (semis) et D 32.81 .2 (pecu(fetus pecorwn); Modestin D 32.81 pr (-1) (sempis) et D 32.81 .2 (pecu-
dibus) ) ou deux fragments (ainsi chez Ulpien D 47.2 .36 .3 (sorvi) et Pomponius D 47.2.37 (pavonem) ; chez Ulpien D 25.1.1.3 (Labéon, Fulcinius) (aedificizm... refecerit/oliveta... restauraverit) et Paul D 25.1 .2 (valetudinem semorom impenderit). )

\begin{tabular}{|c|c|c|c|}
\hline $\begin{array}{c}\text { juriste } \\
\text { auteur } \\
\text { du passage }\end{array}$ & $\begin{array}{c}\text { juriste } \\
\text { cité }\end{array}$ & autre slément patrimonial & sourcs \\
\hline $\begin{array}{l}\text { Julien } \\
\text { (suite) }\end{array}$ & & $\begin{array}{l}\text { frumentum } \\
\text { praedia } \\
\text { fundo } \\
\text { fundum } \\
\text { fundum }\end{array}$ & $\begin{array}{l}\text { D } 36.2 .11 . \\
\text { D } 37.4 .13 \mathrm{pr} \\
\text { D } 39.6 .18 .3 \\
\text { D } 1, \mu_{1} .7 .16- \\
\text { D } 46.3 .33 \mathrm{pr}\end{array}$ \\
\hline Porpponius & $\begin{array}{l}\text { Labéon } \\
\text { Labéon } \\
\text { Q. Mucilis }\end{array}$ & $\begin{array}{l}\text { fundus/insula } \\
\text { pecorum fetu } \\
\text { animali } \\
\text { rundug } \\
\text { vestimentis/aedibus } \\
\text { iumentis } \\
\text { fundo } \\
\text { (50) olei (... libras) } \\
\text { vino/fetus } \\
\text { praedium } \\
\text { fundus } \\
\text { vina/vestem } \\
\text { frui fondo/(operas Stichi) } \\
\text { caballos } \\
\text { vas/vestimenta } \\
\text { grex/fundus } \\
\text { fundum }\end{array}$ & $\begin{array}{ll}\text { D } & 13.7 .8 \mathrm{pr} \\
\text { D } & 15.2 .3 \\
\text { D } & 21.1 .48 .6 \\
\text { D } & 21.1 .64 \mathrm{pr} \\
\text { D } & 24.1 .18 \\
\text { D } & 24.1 .31 .10 \\
\text { D } & 26.7 .61 \\
\text { D } & 30.8 .2 \\
\text { D } & 30.24 \mathrm{pr} \\
\text { D } & 30.38 .1 \\
\text { D } 30.45 .1 \\
\text { D } 32.85 \\
\text { D } 33.1 .2 \\
\text { D } 33.7 .15 \mathrm{pr} \\
\text { D } 34.2 .10 \\
\text { D } 41.3 .30 \mathrm{pr} \\
\text { D } 46.3 .26\end{array}$ \\
\hline Africain & & $\begin{array}{l}\text { quadrigas, mulas } \\
\text { fundum } \\
\text { fund!m }\end{array}$ & $\begin{array}{l}\text { D } 21 \cdot 1 \cdot 34.1 \\
\text { D } 23 \cdot 5 \cdot 9.2 \\
\text { D } 41.1 .40^{2}\end{array}$ \\
\hline Gaius & Julien & $\begin{array}{c}\text { fundus/vestis/ } \\
\text { aurum, argentum } \\
\text { reficiendorm aedificiorum } \\
\text { gratia/(aut, cum servorum } \\
\text { gratia litis aestimatio } \\
\text { solvitur) } \\
\text { navem } \\
\text { fundo/aedibus/iumentis } \\
\text { pecus, quadrupes } \\
\text { fetus pecorum } \\
\text { fundum } \\
\text { fetu pecorum } \\
\text { fetus pecudum } \\
\text { aedibus } \\
\text { fundus/vini/tritici }\end{array}$ & $\begin{array}{l}\text { D } 1.8 .1 .1 \\
\text { D } 5.3 .39 \mathrm{pr} \\
\\
\text { D } 6.1 .36 .1 \\
\text { D } 7.1 .3 .1 \\
\text { D } 9.2 .2 \mathrm{pr}-2 \\
\text { D } 20.1 .15 \mathrm{pr} \\
\text { D } 35.1 .17 \mathrm{pr} \\
\text { D } 35.2 .73 \mathrm{pr} \\
\text { D } 41.3 .36 .1 \\
\text { D } 44.7 .9 \\
\text { D } 45.1 .74\end{array}$ \\
\hline
\end{tabular}




\begin{tabular}{|c|c|c|c|}
\hline $\begin{array}{l}\text { juriste } \\
\text { auteur } \\
\text { du passage }\end{array}$ & $\begin{array}{l}\text { jurist,e } \\
\text { cité }\end{array}$ & autre élément patrimonial & source \\
\hline Maecianus & Aristo & $\begin{array}{c}\text { praedia/vestem /argentum } \\
\text { (morte servorum)/ } \\
\text { animalium } \\
\text { praediorum } \\
\text { praedia }\end{array}$ & $\begin{array}{l}\text { D } 32.95 \\
\text { D } 35.2 .30 \text { or } \\
\text { D } 36.1 .71 \\
\text { D } 49.17 .18 .3\end{array}$ \\
\hline Florentinus & & domus & D $18.1 .43 \mathrm{pr}$ \\
\hline Scaevola & & $\begin{array}{c}\text { fundum } \\
\text { praedia } \\
\text { ornements } \\
\text { proedia } \\
\text { gregibus } \\
\text { praediorum/s'spellectilis } \\
\text { supellectili/horreum/vinarium/ } \\
\text { vasis. } \\
\text { supellectile/pecore } \\
\text { pecore } \\
\text { pecoribus } \\
\text { praela } \\
\text { species argenti }\end{array}$ & $\begin{array}{l}\text { D } 21.2 .69 .5 \\
\text { D } 24.1 .58 \mathrm{pr} \\
\text { D } 31.88 .7 \\
\text { D } 32.37 .3 \\
\text { D } 32.101 \mathrm{pr} \\
\text { D } 33.2 .37 . \\
\text { D } 33.7 .7 \\
\text { D } 33.7 .20 \mathrm{pr} \\
\text { D } 33.7 .20 .3 \\
\text { D } 33.7 .20 .9 \\
\text { D } 33.7 .27 .4 \\
\text { D } 34.2 .18 \mathrm{pr}\end{array}$ \\
\hline Papinien & & $\begin{array}{c}\text { urbana praedia/navis/ } \\
\text { (mercedibus servorum) } \\
\text { fundus } \\
\text { fetus } \\
\text { purpuris } \\
\text { fetus } \\
\text { fetus pecorum } \\
\text { urbanorum praediorm }\end{array}$ & $\begin{array}{l}\text { D } 12.6 .55 \\
\text { D } 21.2 .64 \mathrm{pr} \\
\text { D } 22.1 .8 \\
\text { D } 32.91 .2 \\
\text { D } 33.7 .3 \mathrm{pr} \\
\text { D } 36.1 .60 .4 \\
\text { D } 36.1 .60 .6\end{array}$ \\
\hline Tryphoninus & Julien & $\begin{array}{l}\text { fetus pecudum } \\
\text { pecoribus }\end{array}$ & $\begin{array}{l}\text { D } 15.1 .57 .2 \\
\text { D } 43.16 .19\end{array}$ \\
\hline Callistrate & & fundi & D 21.2 .72 \\
\hline Peul & $\begin{array}{l}\text { Octavenus } \\
\text { Erocuius }\end{array}$ & $\begin{array}{c}\text { iumenta, pecora } \\
\text { vestimenta/vasis/ } \\
\text { fundum } \\
\text { ores } \\
\text { aren } \\
\text { ager }\end{array}$ & $\begin{array}{l}\text { D } 5.3 .40 \mathrm{pr} \\
\text { D } 6.1 .6 \\
\text { D } 6.1 .23 .5 \\
\text { D } 6.1 .27 .5 \\
\text { D } 7.4 .25\end{array}$ \\
\hline
\end{tabular}

\begin{tabular}{|c|c|c|c|}
\hline $\begin{array}{l}\text { Juriste auteur } \\
\text { du passage }\end{array}$ & Juriste cité & autre élément patrimonial & Source \\
\hline $\begin{array}{l}\text { Paul } \\
\text { (suite) }\end{array}$ & $\begin{array}{l}\text { Sabinus } \\
\text { Labéon } \\
\text { Labéon } \\
\text { Pedius } \\
\text { Pomponius } \\
\text { Nerrtius } \\
\text { Screvola } \\
\text { Fescrit d'Anto- } \\
\text { nin le pieux } \\
\text { Nerva filius } \\
\text { Ponponius } \\
\text { Neratius } \\
\text { Fedius } \\
\text { Fulcinius }\end{array}$ & 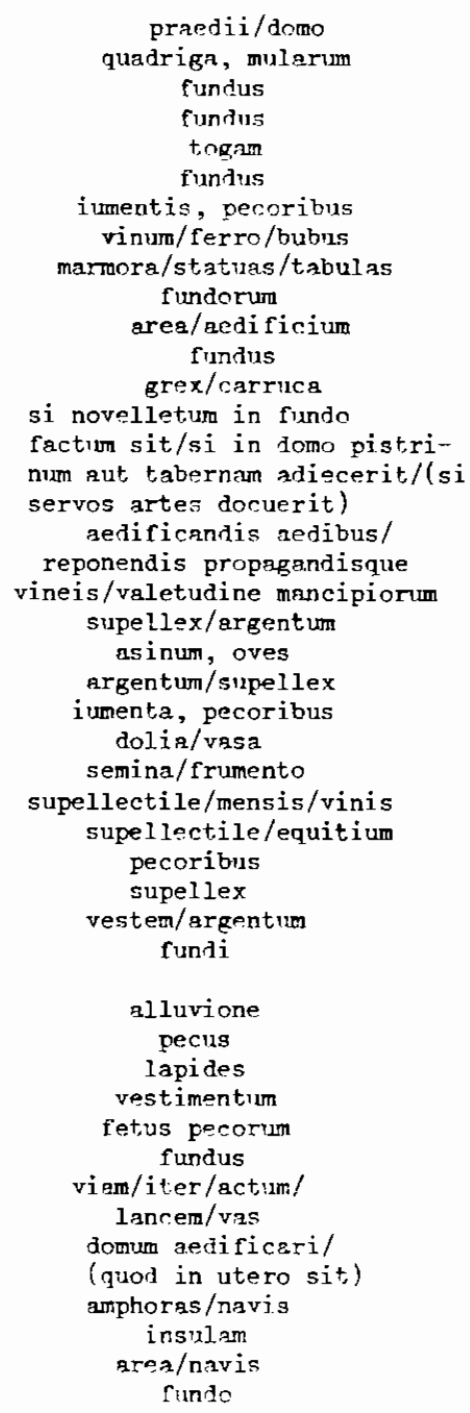 & $\begin{array}{ll}\text { D } & 7.8 .15 \mathrm{pr} \\
\text { D } & 9.2 .22 .1 \\
\text { D } & 10.3 .8 .2 \\
\text { D } & 12.1 .31 \mathrm{pr} \\
\text { D } & 12.6 .21 \\
\text { D } & 13.7 .18 .2 \\
\text { D } & 14.3 .17 \mathrm{pr} \\
\text { D } & 18.1 .1 .1 \\
\text { D } & 18.1 .34 \mathrm{pr} \\
\text { D } & 19.1 .42 \\
\text { D } & 19.1 .45 .1 \\
\text { D } & 21.1 .44 \mathrm{pr} \\
\text { D } & 24.1 .28 .4 \\
\text { D } & 25.1 .6 \\
& \\
& \\
\text { D } & 25.1 .12 \\
& \\
\text { D } & 31.86 \mathrm{pr} \\
\text { D } & 32.60 .3 \\
\text { D } & 32.78 .1 \\
\text { D } & 32.92 \mathrm{pr} \\
\text { D } & 33.7 .13 \mathrm{pr} \\
\text { D } & 33.7 .18 .9 \\
\text { D } & 33.7 .18 .13 \\
\text { D } & 33.7 .19 .1 \\
\text { D } & 33.7 .22 .1 \\
\text { D } & 33.10 .12 \\
\text { D } & 34.2 .7 \\
\text { D } & 34.3 .25 \\
\text { D } & 38.2 .44 .2 \\
\text { D } & 41.2 .3 .13 \\
\text { D } & 41.2 .13 \mathrm{pr} \\
\text { D } & 41.2 .30 .4 \\
\text { D } & 41.3 .4 .5 \\
\text { D } & 41.4 .2 .6 \\
\text { D } & 45.1 .2 .1 \\
& \\
\text { D } & 45.1 .73 \mathrm{pr} \\
\text { D } & 45.1 .83 .5 \\
\text { D } & 46.3 .98 .6 \\
\text { D } & 46.3 .98 .8 \\
\text { D } & 50.16 .79 .1\end{array}$ \\
\hline Ulpien & $\begin{array}{l}\text { Labéon, Frocu- } \\
\text { lus, Celsis, } \\
\text { julien }\end{array}$ & 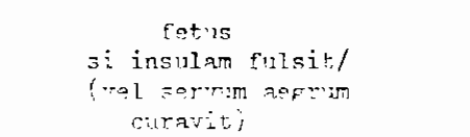 & $\begin{array}{l}3.5 .3 .6 \\
\mathrm{D} 3.5 .9 .1\end{array}$ \\
\hline
\end{tabular}




\begin{tabular}{|c|c|c|c|}
\hline $\begin{array}{l}\text { juriste } \\
\text { nuteur } \\
\text { du passage }\end{array}$ & $\begin{array}{l}\text { juriste } \\
\text { cité }\end{array}$ & qutre élément patrimonial & Source \\
\hline $\begin{array}{l}\text { Upien } \\
\text { (suite) }\end{array}$ & $\begin{array}{l}\text { Pomponius } \\
\text { Labéon } \\
\text { Julien, } \\
\text { Marcellus } \\
\text { Pomponius } \\
\text { Julien } \\
\text { Prutus } \\
\text { Julien } \\
\text { Celsus } \\
\\
\text { Trebatius, } \\
\text { Labéon } \\
\text { Marcellus } \\
\text { Julien } \\
\text { Pomponius } \\
\text { Pomponius } \\
\text { Ofilius, } \\
\text { Caelius Sabinus }\end{array}$ & 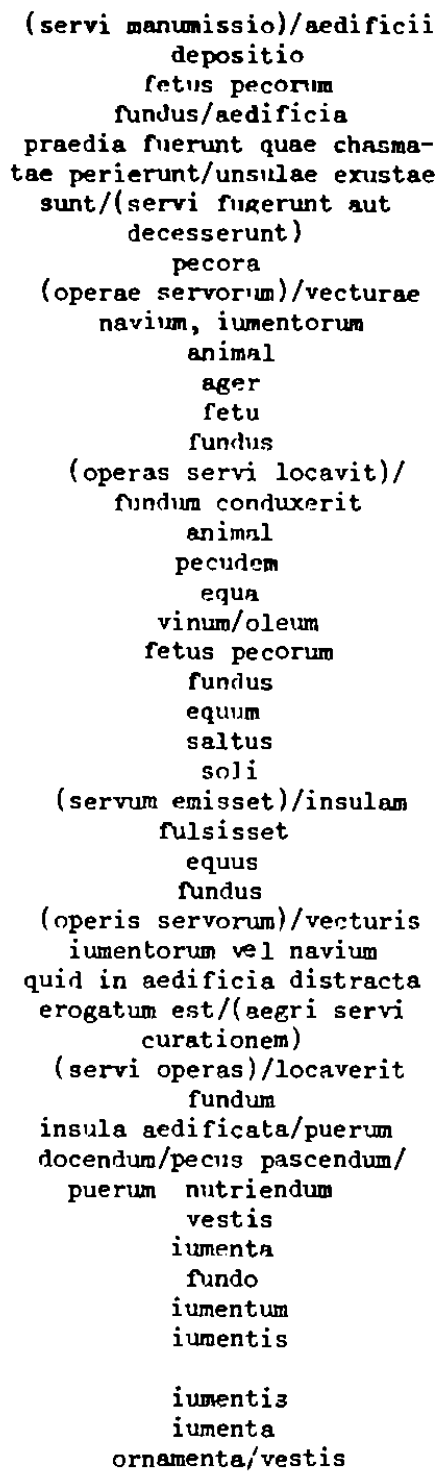 & $\begin{array}{l}\text { D } 4.2 .9 .2 \\
\text { D } 4.2 .12 \mathrm{pr} \\
\text { D } 4.3 .7 .3 \\
\text { D } 4.4 .11 .5 \\
\text { D } 5.3 .20 \mathrm{pr} \\
\text { D } 5.3 .29 \\
\text { D } 6.1 .15 .3 \\
\text { D } 7.1 .12 .3 \\
\text { D } 7.1 .12 .5 \\
\text { D } 7.6 .5 .5 \\
\text { D } 7.8 .12 .6 \\
\text { D } 9.1 .1 .14 \\
\text { D } 9.2 .27 .5-6 \\
\text { D } 9.2 .27 .22 \\
\text { D } 10.4 .9 \mathrm{pr} \\
\text { D } 12.2 .11 .1 \\
\text { D } 12.6 .26 .12 \\
\text { D } 13.6 .5 \\
\text { D } 13.7 .25 \\
\text { D } 15.1 .7 .4 \\
\text { D } 15.3 .3 .8 \\
\text { D } 16.3 .1 .5 \\
\text { D } 18.1 .9 \mathrm{pr} \\
\text { D } 19.1 .13 .13 \\
\text { D } 19.1 .13 .22 \\
\text { D } 19.2 .9 .1 \\
\text { D } 19.2 .1 \\
\text { D } 19.5 .13 .1 \\
\text { D } 20.1 .6 \\
\text { D } 21.1 .4 .3 \\
\text { D } 21.1 .33 \mathrm{pr} \\
\text { D } 21.1 .38 .3 \\
\text { D } 21.1 .38 .7 \\
\text { D } 21.1 .38 .10 \\
\text { D } 21.1 .38 .14 \\
\text { D } 21.2 .37 .1\end{array}$ \\
\hline
\end{tabular}

\begin{tabular}{|c|c|c|c|}
\hline $\begin{array}{c}\text { juriste } \\
\text { auteur } \\
\text { du passage }\end{array}$ & $\begin{array}{l}\text { juriste } \\
\text { citê }\end{array}$ & autre bláment patrimonial & Sonurce \\
\hline $\begin{array}{l}\text { Ulpien } \\
\text { (suite) }\end{array}$ & $\begin{array}{l}\text { Sabinus } \\
\text { Sabinus, Cas- } \\
\text { sius, Neratius } \\
\text { Papinien } \\
\text { Julien } \\
\text { Sabinus } \\
\text { Ofilius } \\
\text { Neratius } \\
\text { Marcellus, } \\
\text { Scaevola } \\
\end{array}$ & 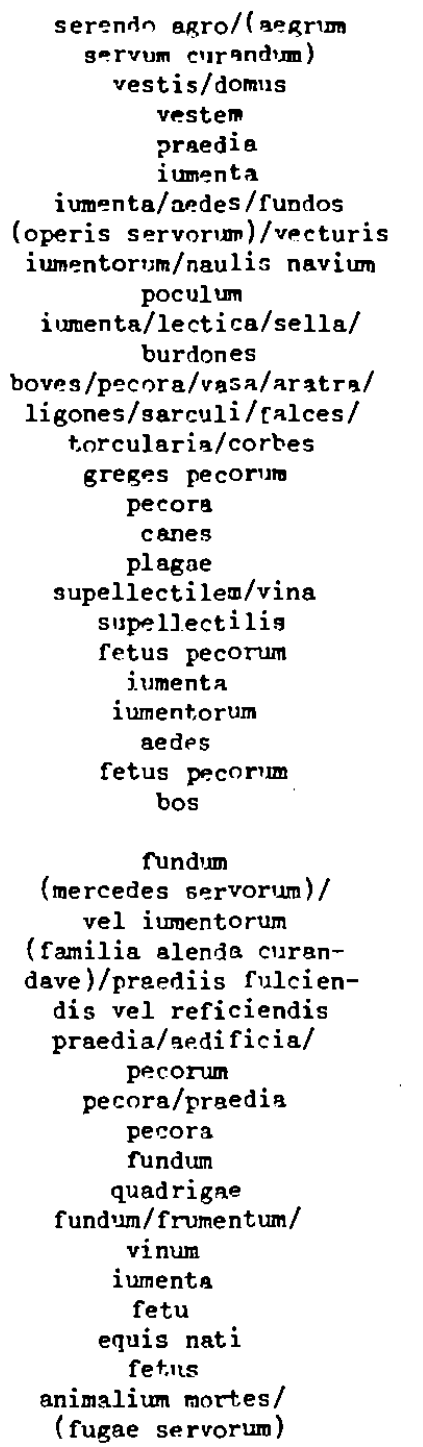 & $\begin{array}{l}\text { D } 24.3 .7 .16 \\
\text { D. } 26.7 .5 .9 \\
\text { D } 27.2 .3 .2 \\
\text { D } 27.3 .13 \\
\text { D } 28.8 .5 .1 \\
\text { D } 29.2 .20 .1 \\
\text { D } 30.39 .1 \\
\text { D } 30.71 .4 \\
\text { D } 32.49 \mathrm{pr} \\
\text { D } 33.7 .8 \mathrm{pr} \\
\\
\text { D } 33.7 .8 .1 \\
\text { D } 33.7 .12 .8 \\
\text { D } 33.7 .12 .12 \\
\text { D } 33.7 .12 .13 \\
\text { D } 33.7 .12 .35 \\
\text { D } 33.7 .12 .46 \\
\text { D } 33.8 .8 .8 \\
\text { D } 33.9 .3 \mathrm{pr} \\
\text { D } 33.9 .3 .8 \\
\text { D } 36.1 .23 .3 \\
\text { D } 35.4 .5 .8 \\
\text { D } 41.3 .10 .2 \\
\text { D } 42.2 .6 .1 \\
\text { D } 42.5 .8 .2 \\
\text { D } 42.5 .9 .3 \\
\text { D } 42.5 .3 \\
\text { D } 42.5 .9 .5 \\
\text { D } 42.6 .1 .12 \\
\text { D } 43.16 .1 .34 \\
\text { D } 44.2 .11 .4 \\
\text { D } 45.1 .29 \mathrm{pr} \\
\text { D } 45.1 .75 .1 \\
\text { D } 47.2 .14 .15 \\
\text { D } 47.2 .48 .5 \\
\text { D } 47.2 .48 .6 \\
\text { D } 47.4 .1 .11 \\
\text { D } 50.17 .23\end{array}$ \\
\hline Macer & & fundum & $D 42.1 .63$ \\
\hline
\end{tabular}




\begin{tabular}{|l|c|c|c|}
\hline $\begin{array}{c}\text { Juriste } \\
\text { auteur } \\
\text { du passage }\end{array}$ & $\begin{array}{c}\text { muriste } \\
\text { cité }\end{array}$ & autre élément patrimonial & Source \\
\hline Marcien & $\begin{array}{c}\text { rescrit de Marc } \\
\text { Aurèle et Verus }\end{array}$ & $\begin{array}{c}\text { theatra/stadium } \\
\text { (operas servi)/paenulas, } \\
\text { tunicas }\end{array}$ & D 1.8 .6 .1 \\
\hline Modestin & $\begin{array}{c}19.5 .25 \\
\text { praediis } \\
\text { Sundum }\end{array}$ & $\begin{array}{l}\text { D } 4.3 .3 \\
\text { D } 45.1 .10 .3\end{array}$ \\
\hline
\end{tabular}

Malgré la situation privilégiée de certains, l'esclave reste une chose, une res mobilis (85). On notera, dans les deux colonnes de gauche, la variété des juristes représentés. Quelle que soit d'ailleurs la période envisagée, les éléments patrimoniaux arec lesquels l'esclave est mis en rolation sont deg plus variés. On trouve au premier plan des fonds de terre et des animaux, mais on relève aussi des édifices, des outils, des meubles, de l'or ou de l'argenterie, des vases, et même des vêtements et des denrées alimentaires $(86)$.

85- Papinien D 41.2 .47 (Nerva filius); Paul D 4!.2.3.13 (Nerva filius). Res est souvent associé par le discours juridique à servus ou àhomo (cf. par exemple, Alfenus (chez auteur anonyme) D 10.3.26; Julien D 5.1 .25 ; D 30.82 pr (Stichus) ; D 41.3.35 (Sabinus) ; D 41.4.4.7; Africain D 12.1.23 (Julien) : D $21.1 .34 \mathrm{pr}$ (comoedi vel choms); D $30.108 \mathrm{pr}$; Gaius D 6.1.76.1 ; D 16.3 . 14.1 (Sabinus, Cassius); D 30.69 .5 ; Vunuleius D $46.2 .31 \mathrm{pr}$; Marcellus D 19.2 48.1 : Scaevola D 32.93.2 ; Paul D 33.7.16.2 (Alfenus) ; D 35.2.63 pr ; D 41 . 2.5 (Stichus); D 41.3.4.18; D 45.1.83.4 (Stichus, Pamphilus); D 46.3 .

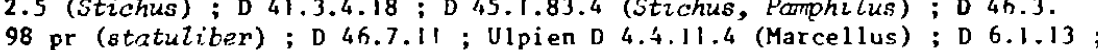
$98 \mathrm{pr}$ (statuliber) ; D 46.7. Il ; Ulpien D 4.4.11.4 (Marcellus); D 6.1.13 ;
D 10.2.12.2 (statuliber); D 10.4.3.6; 018.2 .4 .1 (Julien) (ancilla); D i9. 1.13 .5 (Julien) (mancipia); D 21.1 .31 .9 (Pomponius); D 21.1.63 (mancipia): D 30.71.1-3 ; $043.32 .1 \mathrm{pr}$; D 44.2.11.1 (Celsus) ; D 47.2.46 pr ; D 47.2. 52.29 (statuliber); D $48.5 .28 \mathrm{pr}$; Modestin D 3.3.63).

86- L'esclave est aussi fréquetrment en relation avec une somme d'argent, que le montant de cette some soit indiqué (cf. supra première partie, étude sur le prix de l'esclave) ou non (cF. par exemple chez Papinien D 41.2 .44 pr; Paul D 45.1.91.6 (Julien). La formule fomilio peruniaque, que l'on trorve chez Ulpien (D 36.1.15.8) dôsigne L'universalité du patrimoine (cf. tronve chez Ulpien (D 36.1 .15 .8 ) désigne L universalité du patrimoine (cf.
R. MONIFR, Droit romain, cit. I, p. 528 s. ; P. BOHFANTE, Corso di diritto romano, cit. VI, p. I01; A. BERGER, Enrycl. Diction., cit., p. 468 ; B. ALBAPESE, Surcessione ereditaria, AllPA $20(1949)$, p. $134 \mathrm{s.})$.
L' ampleur toujours importante de ce champ associatif all jème siècle indique une persistance de l'idéologie esclavagiste (B7). Peut-on la faire apparaître de manière chifrrée ?

\section{CHAPITRE II : LA PERSISTANCE DE L' IDEOLOGIE ESCLAVAGISTE}

On a montré, au début de ce travail, qu'il existait une continuité du discours des juristes en matière d'esclavage $(88)$. On relevait ainsi dans une mêne catégorie Neratius et Macer, ou A. Charisius ; Pomponius, Gaius et Papinien, Paul, Ulpien, Marcien, Modestin, Hermogénien, et dans les citations, Servius, Labéon, Aristo, Ponponius et Menander. On avait expliqué ce phénomène par le fait que les juristes, souvent ne traitaient pas de l'esclavage pour trouver une solution à un problème le concernant, mais s'y ráféraient de manière seulement secondaire pour illustrer d'un exemple un cas ne le concernant pas. Seule une étude comparative peut prouver le bien-fondé de cette allégation.

Pour des raisons déjì évoquées plus haut, c'est, le fonds de terre qui semble être l'élément de comparaison le plus pertinent. (8o). Avant d'en venir à l'analyse proprement dite, il est, cependant indispensable-d'apporter quelques éclaircissements méthodologiques. Quand esclave et ronds peuventils être retenus cóme éléments patrimoniaux interchangeables ? Grossièrement, l'interchangeabilité colle à la rubrique "objet d'acte juridique", mais à plusieurs áfards, sa vocation est plus rest.rictive.

En ce qui concerne l'esclave, il fant, tout d'abord écarter de cette étude les textes apparaissant dans un titre concernant de manière directe, ou même geulement accessoire, l'esclavage, c'est-à-dire les titres classés en début $d$ 'ouvrage dans les ensembles $A$ et $B(O 0)$. Toutefois, en dehors $d$ 'un tel contexte so posait un problème $d$ 'interprétation plus délicat, l'esclave

87- Sur la notion de champ associatif en linguistique, Ch. BALLY, Larbitraire du signe, Le Français moderne 8 (1940), p. 193-206; W. VON WARTBURG et $S$. ULlMANN Problèmes et méthodes de la linguistipue, (tr. fr. Paris 1969) , 229. L. LANTELIA, Il lavoro sistemarico, cit dernier dernier note en fonction de celles des autres termes avec lesquels on l'associe dans l'usage.

88- Cf. supra Chapitre préliminaire.

89- Cf. supra première partie, n. 32.

90- Cf. supra Chapître prétiminaire n. \& et 9. 
pouvent en effet ressortir d'un même texte en qualité d'agent et d'objet de droit. Si la coexistence entre esclave ngent de fait juridique objet d'acte juridique ne soulève pas de dirficultés particulières (91), il n'en est pas de même lorsque l'esclave apparait simultanément, comene agent et comme objet d'sctes juridiques. On a alors arfaire à deux hypothòses : soit le contexte du titre permet de trancher ; soit il ne le permet pas.

La première de ces hypothèses peut elle-même se décomposer en deux cas. Premier cas, l'esclave objet est relié au titre : ainsi, dans le titre D 7.1 (De usu fructu), on voit un esclave objet d'usufruit se faire livrer une chose (92). Le lien entre esclave ob.jet et titre étant iumédiat, il est permis de considérer l'esclave cơme un ólément patrimonial int.erchangeable et de l'intégrer à l'analyse (93). Inversenent, deuxième crs, ce peut-être l'esclave agent qui est directement relié au contexte du titre et non l'esclave en tant qu'objet : ainsi, dans le titre D 2.14 (De pactis), on voit un esclave objet d'usufruit, faire un pacte (94). Le lien s'effectuant ici entre titre et esclnve agent, il n'est pas possible d'adopter la même attitude que précédemment. Il faut exclure le texte de l'analyse (95).

91- Que l'esclave apparaisse come agent de fait juridique n'empôche pas de privilégier son rôle d'objet d'acte juridique, les deux rôles pouvant aisément être dissociês. Ont donc été retenus pour l'analyse à venir les textes suivants : Celsus D 47.2.68.3 ; Africain D 47.2.62 pr-1-2-3-4-56-7 : Paul D 47.2.18 (cassiani); Ulpien D 47.2.17.2 (Sabinus)-3 (Julien Pomponius) ; D 47.2.43.12; D 47.8.2.14;D 47.10.17.9;D 48.5.28.1-2-3-4.

92- Ulpien D 7.1.12.5 (Julien).

93- Doivent ainsi être intêgrés Lavolenus D 24.1.20; D 31.38 ; Julien D 10.3.24 pr ; D 10.3.25; D 23.3.47; D 24.3.31.4; Pomponius D 18.1.13; D 23.3.65; Africain D 10.3.9; Gaius D 6.1.20; Marcellus D 7.8.20; Paul D 7.1 .24 ; D 7.1.26; D 10.3.8.4; D 24.1.28.5 (Julien, Pomponius) ; D 24.1 . $38 \mathrm{pr}$; Ulpien D 7.1.12.4 (Julien)-5 (Julien) ; D 7.1.21 ; D 7.1 .22 ; D 7.1. $23 \mathrm{pr}$ (Julien) ; D 7.1.25 pr-1 (Julien, Mauricianus, Marce1lus)-2-3 (Julien) -4-5 (Julien)-6 (Scaevola)-7 (Pegasus, Julien); D 7.6.5.4 (a) ; D 7.8 .14 pr ; D 7.8.16.2 (Pomponius) ; D 24.1.17.1 ; D 24.1.19 pr (Julien)-1 (Julien, Marcellug); D 42.8.6.5; D 46.1.33.

94- Julien D 2.14.55.

95- Ont donc été écartés Julien D 2.14.55; D 30.94.1; D 30.102 ; D 36 1.28 .1 ; D $41.1 .37 \mathrm{pr}-1-2-3-5-6$; D 41.1 .39 ; D 41.2 .38 .2 ; D 45.1.54.1; Pomponius D 41.1 .21 pr (Proculus) ; D 46.3.83 ; Africain D 41.1.40 ; Gaius D 36.1.65.4 (Julien) ; D 41.1.10 pr-3-4-5; D 41.1.43 pr-2 ; D 41.1.45; Marcellus D 26.8.12 (veteres, Julien) ; Florentinus D 30.116.3 ; Papinien D 41.3.44.3; D 45.1.118 pr; Tryphoninus D 41.1.63 .1-2-3; Faul D 2.14 27.10 ; D $34.4 .26 \mathrm{pr}$; D $35.2 .63 \mathrm{pr}$ (Pedius) ; D 41.1 .47 ; D $41.1 .49 ; \mathrm{D} 41$.

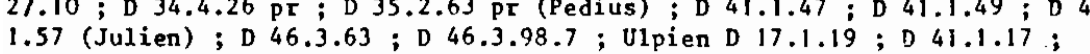
1.57 (Julien) ; D 46.3 .63 ; D 46.3 .98 .7 ; U1pien D $17.1 .19 ; D 41.1 .17$;
D $41.1 .18 ; D 41.1 .22 ;$ D 41.1 .23 pr-1-2-3 (Julien, Scaevola) ; D 41.1 .33 pr (Marcelius, Scaevola)-2 (Julien); Marcien D 34.8.3.2; Modestin D 41.1 54.4 ; Hermogënien D $41.1 .61 \mathrm{pr}^{-1}$.
Le rontexte offert par 10 tit.re ne pormat pas touinura to trancher. ¿'est la deuxième hypothèse. En effet, agent at objat, sant. rolín all tit.r. (96). Se prósentent deux cas : soit los rñles d'akent. et. d'nbìt. sant, nasurós dans le texte par le même esclave, il en est ninsi lorso'se l'esclare légué reçoit un legs (97) : soit ces rôleq incombent ì daryx esclaves dis-

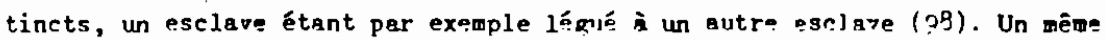
individu ne pouvant être divisible au aré de l'ana\}yse, dans la premier cas, agent l'emporte gur objet à moins de ne ressnrtir explicitement, que de manière secondaire ( 99$)$. La divisibilitś, átant rendue possible far la présence, dans le second cas, d'un agent et. $d$ 'un objet distincts, autorise en revanche à considérer l'esclave objet coume un ạlóment patrimonial intẹrchangeable $(100)$.

En ce qui concerne le fonds de terre, les présautions máthodologiques sont moins nombreuses, mais il est tcutefois nécessaire de faire trois remarques. Rappelons, tout d'abort, que le critère d'intéaration terminologique des textes, tel qu'il a été retenu dans l'étude comparative mené = sur l'acquisition de la main d'oeurre servile, est extensif (101). Il faut ensuite, comme en matière d'esclavage, exclure de l'aralyse les textes qui apparaisgent dans un titre concernant, ne serait-ce que de manière acceasoire, le fonds (102). Enfin, pour que la compnraison puisse valablement s'opérer,

96- Le cas selon lequel agent et objet ne se rapporteraient ni $1^{\prime}$ 'un ni 1 'autre au titre ne se présente pas.

97- Julien 030.91 .3 .

98- Pomponius D 30.13 (Neratius).

99- Le même esclave est à la fois agent ot objet chez Iavolenus $D 33.5$. 15 ; Julien D 30.91.2-3-4-5-6 ; D 30.98; D 33.5.11 ; D 36.2.16.1 ; D 36.2. 17 ; Africain D 30.107.1 ; Gaius D 30.69 pr ; D 41.2 .15 ; Florentinus D 30. I16.2 ; Papinien D 31.65.2 ; Paul D 20.1.29.3 ; D 33.5.13 Fr; D 34.4 .27 $\mathrm{pr}^{-1}$; D $35.2 .49 \mathrm{pr}$ (rescrit d'Antonin le pieux); auteur anonye D 36.4 . 16. Le rôle d'agent ne se limite pas au contexte du titre et apparait plus diffus par rapport au rôle d'objet chez Julien 030.86 .2 ; 030.91 .7 ; Maecianus D 35.2.30 pr ; Ulpien D 30.91.

100- On doit ainsi retenir Julien D 21.2.39.1; D 30.99 ; D 30.101 pr; D 35.5.10 ; D 44.7.16; Marcellus D 35.2.56.4 ; Pomponius D 30.13 (Neratius); Paul D 35.2.21.1; U1pien D 15.3.5 pr (Fomponius) ; $D$ 36.2.7.6.

101- Cf. supra première partie n. 34 .

102- I1 en est par exemple ainsi de D 6.3 (Si ager vectigalis), de D 8 qui porte intégralement sur les servitudes, de D 11.6 ( $\mathrm{Si}$ mensor falswn modum dixerit), de D 23.5 (De fundo dotali), de 033.7 (De instructo vei instrumento legato) où le fonds apparaît sur-représenté, etc. En dehors du contexte de $\mathrm{D} 33.7$, les textes traitant du legs, de la vente ou de la donation d'un fonds črom cervis doivent être relevês aussi bien dans la rubrique concernant le fonds que celle concernant l'esclave. 
il convient d'écarter les textes. où le fonds est objet d'un droit qui lui eat spécifique. C'est par exemple le cas lorsque le texte traite du legs d'un droit d'extraire des pierres de tel fonds (103).

Ces précisions données, opérons une comparaison chronologique de nos deux éléments patrimoniaux par rapport au nombre total de textes et de citations, concernant chaque période, oul ils apparaissent.

Esclave pris patrimonial interchangeable (104)

\section{- analyse des paragraphes}

\begin{tabular}{|c|c|c|c|c|c|}
\hline $\begin{array}{l}\text { I } \\
\text { II } \\
\text { III } \\
\text { IV } \\
V\end{array}$ & $\begin{array}{c}- \\
2 / 9 \\
268 / 539 \\
91 / 224 \\
646 / 1322\end{array}$ & $\begin{array}{l}=22 x \\
=50 \% \\
=41 \% \\
=49 \%\end{array}$ & $\begin{array}{l}\text { I } \\
\text { II } \\
\text { III } \\
\text { IV } \\
\text { V }\end{array}$ & $\begin{array}{c}- \\
7 / 9 \\
271 / 539 \\
133 / 224 \\
676 / 1322\end{array}$ & $\begin{array}{l}=\quad-78 \\
=50 \\
=59 \\
=51\end{array}$ \\
\hline TOTAL & $1007 / 2094$ & $48 \%$ & TOTAL & $1087 / 2094$ & 52 \\
\hline
\end{tabular}

103- Cf. par exemple UIpien D 30.39.4. Le problème se pose dans les mêmes termes lorsqu'on se trouve en présence d'une servitude grevant le fonds, la référence étant néanmoins intégrable si elle contient une autre indication d'objet (cf. Pouponius D 18.1.66.1 ; Venuleius (Q. Mucius, Sabinus) D 21.2.75), même sous-entendue (cf. Ulpien D 7.1.27.4 : usufruit ; Vlpien D 18.1 .47 : vente).

104- AIfenus (chez auteur anonyme) D 10.3.26; D 44.1.14; Proculus D 31 . $48 \mathrm{pr}$; D 33.6.6 ; Iavolenus D 18.6.17; D 19.2.60.7 (Labéon) ; D 21.2.58; D 24.1 .20 ; D 28.7.20.1 (Labéon); D 31.37 ; D 31.38 ; D 32.29.3; D 32 . $100 \mathrm{pr}$ (Cascellius, Labéon); $\mathrm{D} 33.4 .6 .1$ (Servius, Cascellius Ofilius, Namusa, Labéon); D 33.5.14; D $34.5 .28 ; D 38.2 .36 ; D 41.3 .16$; D 41.3 . 19 ; D 45.1.105 (Sabinus); D 47.2.75; Neratius D 19.1.31.1 ; D 25.1.15. Celsus D 12.4.16; D 17.1.50.1; D 19.1.38 pr-1 (Sextus Aelius, Drusus); D 23.3.58.1; D 30.63 (Servius); D 31.15; D 31.18; D 31.19; D 32.79 pr-3; D 47.2.68.3; Valens D 35.1.89; Julien D 5.1.25; D 7.i.35.1 (Sabinus) ; D 10.3.24 pr ; D 10.3.25; D 10.4.8; D 12.4.7.1; D 12.6.32 pr-3; D 12.6. 37 ; D 17.1.30; D 18.2.17; D 18.5.5.2;D 19.1.24.1 (Sabinus, Cassius); D 21.2 .7 ; D 21.2 .8 ; D 21.2.39.1-3-4-5; D 22.1.25.1-2; D 23.3.47; D 23 . 4.21 ; D 24.3.31.4;D 30.6; D 30.76 ; D 30.81.2-4-6-9-10; D $30.82 \mathrm{pr}-4-6$; D 30.84.3-4-5-8-9-10-11-12; D $30.86 \mathrm{pr}-1-2$; D 30.91.7; D 30.94.2-3 ; D 30 . 96.2 ; D 30.97 ; D 30.98 ; D 30.99 ; D $33.5 .9 \mathrm{pr}-1-2$ (Q. Mucius) ; D 33. 5.10 ; D 33.5.12 ; D 34.4.11; D 34.5.13.1-2; D 36.2.11; D 36.2.12.7; D $36.2 .16 \mathrm{pr}$; D 37.4.13 pr;D 39.5 .2 .7 ; D 39.6.18.3; D 41.2.38 Pr; D $41.3 .33 \mathrm{pr} ; \mathrm{D} 41.3 .35: \mathrm{D} 41.4 .7 .7 ; \mathrm{D} 41.4 .8 ; \mathrm{D} 41.4 .9 ; \mathrm{D} 41.4 .10$; D $41.3 .33 \mathrm{pr}$; D 41.3 .35 ; D 41.4 .7 .7 ; D 41.4 .8 ; D 41.4 .9 ; D 41.4 .10 ; D $43.26 .19 .1 ; \mathrm{D} 43.33 .1 \mathrm{pr}$; D $44.2 .25 .1 ; \mathrm{D} 44.7 .16 ; \mathrm{D} 44.7 .18$; D 45. $1.54 \mathrm{pr} ; \mathrm{D} 45.1 .56 .8 ; \mathrm{D} 46.3 .33 \mathrm{pr}-1 ; \mathrm{D} 46.3 .34 \mathrm{pr}-1-2-10 ; \mathrm{D} 46.4 .17 ;$
Pouponius D 3.5 .10 (Proculus); D 6.1.29; $\mathrm{D} 7.1 .55 ; \mathrm{D} 7.8 .16 .2 ; \mathrm{D} 11.1 .15$ $\mathrm{pr} ; \mathrm{D} 12.6 .19 .3$; D $13.7 .8 \mathrm{pr}-1$; D 17.1 .47 .1 (Julien) ; D $18.1 .8 \mathrm{pr}$; D 18 . 1.13 ; D 18.1.31 ; D 19.1.55 (Octavenus) ; D 21.2.16.2 (Proculus) ; D 21.2. 30 ; D 21.2.34 pr; D 23.3.18 (Labéon); D 23.3.65; D 24.1.18; D 24.1.
29 pr (Fulcinias) ; D 24.1.31.1-3-5-10;D 24.3.67; D 26.7.61; D 28.6. 16 pr ; D 30.8.2 ; D 30.12.1; D 30.13 (Neratius) ; D $30.20 ; D 30.24$ pr ; D 30.36 pr (Labéon) $-1-3$; D $30.38 .1 ; 030.45 \mathrm{pr}$ (Ofilius, Aristo, Neratius) $-1-2$; D 30.46 ; D 30.48.1 ; D 30.56; D 31.11 .1 (Julien); D 32.74 ; D 32 . 85 (Labéon); D 33.1.2; D 33.2.20; D 33.5.6; D $33.5 .8 \mathrm{pr}-2$; D 33.7 . $15 \mathrm{pr}$ (Servius)-2; D 34.2.10; D 35.1.1.3; D 36.1.72.1; D 41.3.30 pr; D 41.4.6.1-2; D 41.10.3 (Neratius); D 41.10.4 pr (Trebatius); D 43.26.

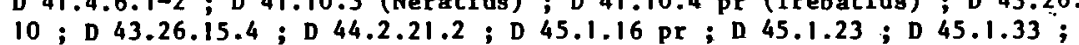

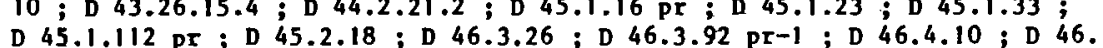

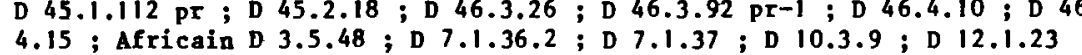

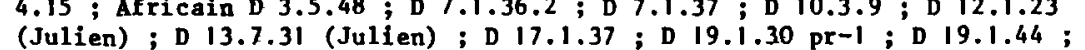
D $20.4 .9 \mathrm{pr}$; D 21.2 .24 ; D $21.2 .46 .2-3$; D 21.2 .47 ; D 23.5.9.2 ; D 30.108 Pr-2-4-5-9-11; D 30.110 ; D 41.2.40.2-3;D 44.3.6.1 ; D 46.3.38.3; D 47.2.62 pr-1-2-3-4-5-6-7; T. Clemens D 33.5.17; Gaius D 4.7.3.1 ; D 6.1 . 18 ; D 6.1.20;D 6.1.28; D 6.1.30; D 6.1.36.1; D 6.1.76.1; D 7.1.3.1; D 7.1.45; D 7.8.13;D 10.3.11; D 12.6.63; D 13.6.18.2 ; D 16.3.14.1 (Sabinus, Cassius) ; D 17.1.27.1 ; D 20.1.15 pr (Julien) ; D 20.4.11.2-3 ; D 21.2 .54 .1 ; D 22.1.28.1 ; D 24.1 .8 ; D $30.65 \mathrm{pr}-1$; D $30.67 \mathrm{pr}$; D 30.68 2-3; D 30.69.1-4-5; D $30.70 \mathrm{pr}-2-3$; D 35.1.17 pr-1 ; D 35.2.73 pr ; D 39.5.11; D 41.3.36.1; D 44.7.1.9; D 45.1.74; D 45.2.15 (Julien); Maecianus D 32.95 ; D $35.2 .30 \mathrm{pr}$; D 36.1 .71 ; D 49.17 .18 .3 ; Venuleius D 41 . 1.66 ; D 42.8.25.4-5 (Proculus) ; D 45.1.137.1-4; D 46.2.31 pr ; D 48 . 8.6 ; Marcellus D 7.8.20; D $17.1 .49 ; D 19.1 .23$ (Julien) ; D 19.2.48.1; D 29.1 .31 ; D 30.83 ; D 31.26 ; D 31.50 .1 ; D 32.69.1; D 35.2.56.3-4; D 45.1 .96 ; D 46.1.38 pr; D 46.3.67; D 46.3.72 pr-1-2-3-4-5-6; D 46.8. 17 ; Florentinus D 18.1.43 pr-1; Scaevola D 2.14.54; D 3.5.34.2; D 4.3. 32 ; D 10.2.39.4; D 16.1.28 pr; D 16.2.22; D 20.1.32; D 20.1.34.1; D 21.2.69 pr-1-2-3 (Servius)-4-5; D 23.4.29.2; D 24.1.58 pr-1-2; D 31 . 88.7 ; D 32.37.3-7; D 32.41.2-4-5-10; D 32.93.2; D 32.101 pr-1; D 33.2 . 37 ; D 33.5.18 (Neratius) ; D 33.5.21; D 33.7.7; D 33.7.20 pr-1-2-3-4-56-9; D 33.7.27 $\mathrm{pr}-1-3-4 ; D 33.7 .28 ; \mathrm{D} 34.1 .17 ; \mathrm{D} 34.2 .18 \mathrm{pr} ; \mathrm{D} 34.4 .31$ Pr ; D 36.1.80.12;D 41.6 .5 ; D 41.9.3; D 44.3.14.2-4; D 45.1.122.2; D 45.1.127; D 45.1.135.3 ; Papinien D 3.3.66;D 4.8.42; D 6.1.65.1 ; D 10 2.31 ; D 10.2.34; D 12.6.55; D 17.1.57; D 19.5.8; D 20.1.1.1; D 21.2 . $64 \mathrm{pr}$; D 22.1.4 pr-1 ; D 22.1.2 ; D 22.1.8; D 23.3.69.9; D 24.3.61 ; D 29. 2.86 .2 ; D $31.65 \mathrm{pr}-3$; D 31.66 .3 ; D $31.73 ; \mathrm{D} 31.77 .17$; D 32.91 .2 ; D 33 . 2.24 .1 ; D 33.4.8; D 33.7.3 pr-1; D 34.4.24.1 ; D 35.2.9.1 ; D 36.1.60. 4-6; D 36.2.25.1; D 39.5.31.2; D 41.2.44 Pr;D 41.2.48; D 41.3 .44 pr-2; D 45.1.115.2 (Pegasus, Sabinus); D 45.1.117; D 46.3.95 pr-1; D 47. 2.81 Pr-1-2; 49.1715 .4 ; trate D 21.2.72; Paul D 2.14.27.6; D 3.6.7.2; D 4.3.18.1-5; D 4.4.48.1; trate D 21.2 .72 ; Paul D 2.14 .27 .6 ; D 3.6 .7 .2 ; D $4.3 .18 .1-5 ;$ D 4.4 .48 .1
D $4.7 .9 ;$ D $4.8 .32 .5 ;$ D $4.9 .6 .1-2 ;$ D 5.3 .36 .2 (Pomponius); D 5.3 .40 pr-4 (Proculus, Cassius) ; D 6.1 .6 ; D 6.1 .16 pr ; D 6.1 .21 (Julien, PonpoPr-4 (Proculus, Cassius) ; D 6.1.6; D 6.1.16 pr ; D 6.1.21 (Julien, Poupo nius) ; D 6.1.23.5 ; D 6.1.27.2-5; D 6.1.31; D 6.1.58; D 6.1.79 (Labéon) ; D 6.2.12 pr (rescrit d'Antonin le pieux) $-5 ; \mathrm{D} 7.1 .24 ; \mathrm{D} 7.1 .26$;

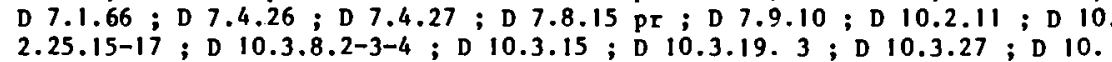
4.12.4; D 10.4.16; D 12.1.31 pr; D 12.2.30.1; D 12.4.12; D 12.6.21; D 12.6.65.5-8; D 13.5.12; D 13.6.22; D 13.7.18.2; D 14.3.17 pr (intégré malgre le contexte du titre, celui-ci visant le rôle d'agent joué par 1'esclave et non l'esclave en tant qu'objet); D 17.1.5.5; D 17.1.22.9; D 17. 1.26 .8 (Mela) ; D 17.1.46; D 18.1.1.1 (Sabinus); D 18.1.34.6; D 18.1.56; D 18.4.21; D i8.6.8.1 (Juíien); D i (9.1.4 pr; D 19.1.21 pr; D 19.1.42; D 18.4 .21 ; D 18.6 .8 .1 (Julien) ; D $19.1 .4 \mathrm{pr}$; D $19.1 .21 \mathrm{pr} ; \mathrm{D} 19.1 .42$; $54 \mathrm{pr}$ (Labéon); D 19.2.42; D 19.2.43; D 19.2.45.1; D 19.2.54.2; D 19 . 
4.2 (Aristo) : D 19.5.5.1-2 (Julien) : D 20.1.29.1; D 20.2.9; D 21.2.3; D 21.2 .5 (Labéon); D 21.2.9; D 21.2.11.1; D 21.2.26; D 21.2.35; D 21.2 . $41 \mathrm{pr}-1$; D 21.2.42; D 21.2.56.2-3; D 22.1.10; D 22.1.14 pr-1 (Neratius) D 22.3.4; D 23.3.25; D 23.3.56 pr ; D 24.1.28. 1-2-3 (Flautius)-4 (Fomponius) -5 (Julien, Pomponius); D 24.1.38 pr; D 24.1.67 (Labéon); D 24.3.25. 3 ; D 24.3.26; D 24.3.63;D 25.1 .2 ; D 25.1.6;D 25.1.12;D 25.2.21.2; D $30.5 \mathrm{pr}-1$ (Trebatius, Labéon, Cassius) : D $30.35 ; \mathrm{D} 30.52 \mathrm{pr}$; D 30.62 D 31.5 .1 ; D $31.8 \mathrm{pr}-1$; D $31.14 \mathrm{pr}$; D $31.86 \mathrm{pr}$; D 32.60 .1 (Alfenus)-3 (Alfenus); D 32.66 ; D $32.78 \mathrm{pr}-1-2-3 ; \mathrm{D} 32.92 \mathrm{pr} ; \mathrm{D} 32.99 \mathrm{pr}-1-2-3-4-5$; D 33.2.21 ; D 33.5.7 ; D 33.5.13.1 (Pomponius) ; D 33.7.13 pr-1 ; D 33.7.14 ; D 33.7.16.2 (A1fenus) ; D 33.7.18.1-4 (Scaevola)-5-6-7-8-9-10 (Cassiug)-1113 (Scaevola) ; D 33.7.19 pr-1 ; D 33.7.22.1; D 33.9.4.5 (Labéon) ; D 33.10.

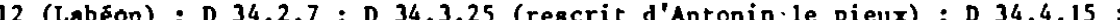

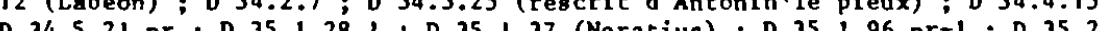

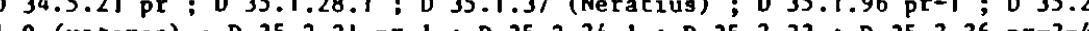
(Africain); $\mathrm{D} 35.2 .48 ; \mathrm{D} 36.1 .68 .2 ; \mathrm{D} 38.2 .44 .1-2 ; \mathrm{D} 39.6 .39 ; \mathrm{D} 41.2 .1$. 16 (veteres) ; D $41 \cdot 2 \cdot 3 \cdot 10-13$ (Nerva filius) ; D 41.2 .5 ; D $41.3 .4 .5-15-$ 16 (Sabinus, Cassius)-17-18; D 41.3.15.1; D 41.4.2.6 (Neratius)-7 (Trebatius) ; D 41.10 .2 ; D 42.1 .8 ; D 42.2 .5 ; D 42.2 .6 .1 ; D $42.5 .38 \mathrm{pr}$; D 44.4.5.4;D 44.7.44.4;D 44.7.45;D 45.1.2.1-3; D 45.1.8 (Sabinus, Proculus, Marcellus) ; D 45.1.24;D $45.1 .49 \mathrm{pr}-3$; D 45.1.73 pr-1-2 ; D 45.1: 83.1-2-4-5-7; D 45.1.85.4; D 45.1.88; D 45.1.91 pr-I-2 (Julien)-3 (Celsus, Julien)-5 (Posponius)-6 (Julien); D 45.1.93; D 45.1.128; D 45. $3.20 \mathrm{pr} ; \mathrm{D} 46.1 .34 ; \mathrm{D} 46.1 .58 .1 ; \mathrm{D} 46.2 .32 ; \mathrm{D} 46.3 .60 ; \mathrm{D} 46.3 .98 \mathrm{pr}-$

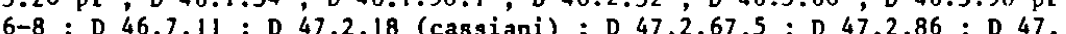
10.16 (Pedius); D 48.10.17; D 50.16.79.1 ; Ulpien D 2.4.10 pr (constitu10.16 (Pedius) ; D 48.10.17; D 50.16.79.1 ; Ulpien D 2.4.10 pr (constitu-
tion de Marc-Aurèle)-1 (Marcellus); D 2.14.6.1; D 2.14.7.2 (Julien, Mauri-

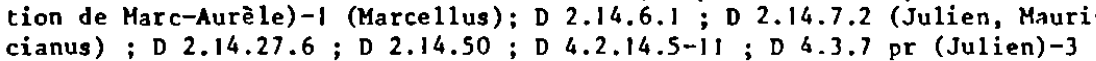

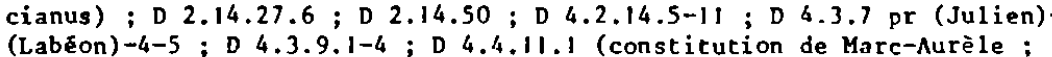
Scaevola)-i (Julien, Marcellus) ; D 4.7.4.5 ; D 5.3 .5 pr ; D 5.3 .20 pr-1 (Julien)-3 ; D 5.3.27 pr ; D 5.3.29 ; D 5.3.33.1 (Julien) ; D 6.1.5.5 (Pomponius) : D 6.1.13; D 6.1.15 pr (Labéon)-3; D 6.1.17 pr (Julien)-1 (Julien); D 6.1 .22 ; D 6.1.45; D 6.2.11.3 (Julien)-4 (Julien)-5 (Pomponius) lien) ; 6.1 .22 ; D 6.1 .49 ; D 6.2 .11 .3 (Juilen) (Ju1ien) 5 (Pomponius) Den béon); (Julien)-1 (Sabinus, Cassius) 0 pr (Julien)-1 (Sabinus, Cassius) ; D $7.1 .25 \mathrm{pr}-1$ (Julien, Mauricianus, Mar ce1lus) $-2-3$ (Julien) $-4-5$ (Julien) -6 (Scaevola)-7 (Pegasus, Julien) ; D 7 ;
$1.27 .2-5 ; \mathrm{D} 7.1 .68 \mathrm{pr}$ (Brutus); $7.4 .5 .1 ; \mathrm{D} 7.4 .12 .1 ; \mathrm{D} 7.4 .15 ; \mathrm{D} 7$. 6.5 .4 (a)-5; D 7.8.12.5-6 (Labéon); D 7.8.14 pr; D 7.8.16.2 (Pomponius); D 7.9.5.3 ; D 10.2.8 pr (Powponius) : D 10.2.12 pr-2; D 10.2.16.6 (Ofilius); D 10.2.18 pr (Julien)-2 (Pomponius); D 10.3.6.4 (Sabinus, Atilicinus)-9 (Julien); D 10.4.3.6-7-10 (Julien)-12 (Pomponius) ; D 10.4.5.6; D 10.4. $9 \mathrm{pr}-7 ; \mathrm{D} 10.4 .11 \mathrm{pr}-1$ (Labéon) ; D 10.4.17; D 10.4.20; D 11.1.17; D 12.2.11.1; D 12.2.34 pr (Marce11us); D 12.4.5.1-2-3-4; D 12.6.26.12 (Ce1sus) -13 (Celsus, Marcellus) -14 (Ce1sus) ; $D$ 13.4.2.2 ; D 13.6.5.6 (veteres) -7 (Namusa, Mela)-13 (Cartilius) ; D 13.7.24.3; D 13.7.25; D 15.3.3.8; D 15.3.5 pr (Pomponius); D 16.3.1.5-9-10 (Pomponius); D 16.3.7 pr; D 17. 1.8.4 (Ju1ien) -10 ; D 17.1 .10 .1 ; D 17.2.49; D 17.2.51.1; D 18.1 .7 pr (veteres) ; D 18.1.9 pr;D 18.1.11.1; D 18.1.29; D 18.2.4.1 (Julien)-2 (Julien) ; D 18.4.2.12 (Julien)-13 (Marce1lus)-15; D 19.1.11.5-7 (Neratius)-8 (Neratius)-12 (Neratius) 15 (Julien) ; D 19.1.13.1-2-3-4-5 (Julien)-13-18 -22 (Trebatius, Labéon); D 19.5.13.1 (Julien); D 20.1.6; D 20.1.8 D 20.1.21.1 (Julien) ; D 20.1.27 (Marcellus); D 20.2.6 (Pomponius) ; D 20. $6.4 \mathrm{pr} ; \mathrm{D} 21.2 .4 \mathrm{pr} ; \mathrm{D} 21.2 .21 \mathrm{pr}-2$ (Julien)-3 (Julien); D 21.2.25; D 21 $2.31 ; D 21.2 .32 \mathrm{pr}-1$ (Julien); D 21.2.33; D 21.2.37.1; D 21.2.51.1

(Julien)-3;D 23.3.10.2-5;D 23.3.12.1 (Marcellus, Scaevola); D 24.1.7.8
(Sabinus, Papinien ; Marc-Aurèle)-9; D 24.1.9 rr-1 (Juliañ); 024.1 .17 .1 ; D 24.1 .19 pr (Julien)-1 (Julien, Yarcellus); D $24.1 .22 ; 024.3 .7 .10-16$ (Poraponius): D 24.3.24.4-5; D 24.3.62; D 24.3.64 pr-1-2-3-4-5-6-7-8-9 (Labéon) $-10 ; D$ 26.4.3.2 (congtitution de Marc-Auréle); D 26.7.5.9; 0 27.3.13; D 28.1.21.1 ; D 28.5.35.3; D 28.7.4.I (Julien) : D 28.8.5.1 ; n 29.2.20.1; D 29.2.42.2-3 : 030.32 .1 ; D $30.37 \mathrm{pr}$ (Cassius : rescrit de Sîvère et Caracalla) : D $30.39 \mathrm{pr}$ (Julien. Africain)-1; D $30.44 \mathrm{pr}-7-8$ : D 30.47 . 2-3 ; D 30.53.3-4-8-9; D 30.71.1-3-4; D 30.75.3-4 (Ju1ien) : D 32.3 .1 (Julien) ; D $32.49 \mathrm{pr}$; D $32.68 .2-3 ; \mathrm{D} 32.73 \mathrm{pr}-\mathrm{I}-2-3-4-5 ; \mathrm{D} 33.4 .1 .6$; D $33.5 .2 \mathrm{pr}-1-2 ; \mathrm{D} 33.7 .8 \mathrm{pr}$ (Sabinus) -1 ; $\mathrm{D} 33.7 .12 \mathrm{pr}$ (Servius)-2 (A1fenus) -3 (Labéon, Pegasus)-4-7-8-9-10-12-13-31 (Celsus)-32-33-35 (Neratius) -37 (Papinien)-38 (Papinien)-42 (Papinien)-44 (Papinien)-45 (Papinien)-45 (Papinien):D 34.3 .7 .1 (Julien) : D 34.4.12:D $35.2 .43 ; \mathrm{D} 36.1 .23 .3$ : D 36.7 ; D 36.2.23; D 39.5.18.1 (Aristo, Pomponius)-2 (Aristo, PompoD $36.2 .7 .5 ; D$ D 36.2 .23 ; D J9.5.18.1 (Aristo, Pomponius)-2 (Aristo, Pomponius) ; D 39.6.37.1 (Julien) ; D 41.2.13 $\mathrm{pr}-2$; D $41.2 .42 \mathrm{pr}$; D 41.3 .10 .2 (Marce1lus, Scaevola) ; D 42.1.4.8 (Ce1sus) ; D 12.5.8.2; D 42.5.9.3-5 : D 42.6.1.12; D 42.8.6.5; D 42.8.10.21; D 44.2.7.i-3; D 44.2.11.1 (Ce1sus) $-4.8 ; D 44.3 .8 ; D 44.4 .2 .7$; D $44.4 .3 ; 044.4 .4 .7$ (I.abéon); D 45.1.1.5; D $45.1 .29 \mathrm{pr}-1$; D 45.1.32; D 45.1.51;D 45.1.69; D 45.1.75.1-4-8; D 45.1.82.1; D 46.1.8.8 (Julien, Marcellus)-9 (Jullen)-10-11; D 46.1.33 , D $45.2 .8 \mathrm{pr} ; \mathrm{D} 45.2 .14 \mathrm{pr}$ (Marce1lus) ; $\mathrm{D} 46.3 .9 .1 ; \mathrm{D} 46.4 .13 .4$ (Ju1ien)-6; D 47.2.14.5 (Papinien)-6 (Pomponius, Papinien) 7 (Fomponius, Papinien)-11-15; D 47.2.17.2 (Sabinus)-3 (Julien, Pomponius); 47.2 . P3.12; D 47.2.46.1-3-4; D 47.2.48.5-6; D 47.2.52.23 (Me1a); D 47.8.2. $43.12 ; D 47.2 .46 .1-3-4 ; D 47.2 .48 .5-6 ; D 47.2 .52 .23$ (Mela); D 47.8 .2 14 ; D 47.10.1.6 (Julien)-7 (Labéon); D 47.10.15.35-37-48-49; D 47.10 $17 \mathrm{pr}-9$; D 48.5.28.1-2-3-4; D 49.17.6; D 50.15.4.9; D 50.16.160 (Marcel1us); Macer D 48.19.10 pr ; Narcien D 12.3.5.4; D 18.1.42; D 18.1.44; D 20.1 .13 .1 ;. D 22.1.32.5; D 30.112.1; D 30.114.19; D 32.65 pi-1-2-3; ; D 33.7 .17 .2 ; D 35.1 .33 .4 ; D 39.4 .16 pr(rescrit de Sêveòre et Caracalla)-1 (idem)-2 (idem)-3-4 (rescrit d'Antonin le pieux)-9 (idem)-10 (rescrit de Marc-Aurèle et Verus) ; Modestin D 6.1 .32 ; D 16.3.23; D 20.1.26.2 ; D 24. 3.58 ; D $31.31 ; \mathrm{D} 31.34 \mathrm{pr}$; D $31.35 ; \mathrm{D} 32.81 \mathrm{pr}-1$; D 32.82; D 44.3.3; D 45.1.103; D 48.8.11.1; D 50.16.101.3; Hermogénien D $32.22 .2 ; 035.2 .38$. $1-2 ; 036.4 .11 \mathrm{pr}$

105- Alfenus (chez auteur anonyme) D $6.1 .57 ; \mathrm{D} 15.3 .16 ; 019.1 .26 ; 019$ 2.29 ; Proculus D 2.14.36; D $18.1 .68 \mathrm{pr}-2 ; \mathrm{D} 19.5 .12 ; \mathrm{D} 41.2 .27 ; \mathrm{D} 46$. 3.82 ; D 50.16.126; Iavolenus $\mathrm{D} 7.1 .53 ; \mathrm{D} 7.1 .54 ; 07.4 .24 \mathrm{pr}$ (Labson):

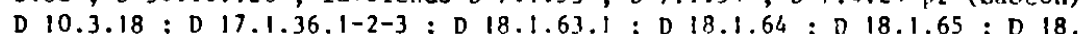
1.77 (Tubero, Labéon) ; $\mathrm{D} 18.1 .78 \mathrm{pr}$ (Labẻon)-1 (Labéon)-2 (Labéon); $\mathrm{D} 18$. 1.79 (Trebatius, Labéon); $018.1 .80 \mathrm{pr}$ (Labéon)-2 (Servius, Labéon); $\mathrm{D} 18$. 2.19 ; D 19.1.51.1 (Labéon); D 19.2.21; D 19.2.51 pr; D 19.2.57 (Labéon); 019.2 .60 .1 (Labéon)-5 (Labêon); D 20.6.14 (Labécn); D 23.4.32 rr (Labéon) ; D 31.10 ; D 31.39 ; D $31.41 \mathrm{pr}$; D 32.29 .4 (Tubero, Labéon) ; D 32. 30.1 (Labẻon) -3 (Labéon) -4 (Labéon); D 33.2.30.1 (Labéon); D 33.2.31 (Labéon) ; D 33.2.4! (Labêon) ; D 33.2.42; D 35.1.40.1 (Cascellius, ofilius Cinna) -4 (Trebatius, of ilius, Labêon) ; D 35.1 .55 ; D 35.3 .56 ; D 35

$1.67 ; D 35.2 .61 ; D 38.2 .35 ; D$ D $38.2 .36 ; D 38.5 .12 ; 041.3 .21 ; 041.4 .4$ pr-1: D 45.1.106; Neratius D 17.1.35;D 18.3.5;D 20.2.4 pr;D 21.2.48: D 25.1.15; D 25.1.16 ; Celsus D 6.1.38; D 18.1.59 (Q. Mucius); D 21.2.10; D 21.2 .62 .2 ; D 31.27 ; D 32.79 .2 ; D 34.7 .1 .2 ; D 41.2 .18 .2 ; D 47.2.68.5 ; D 50.16 .88 ; Julien $D 2.14 .56 ; 03.3 .75 ; 04.4 .41 ; 05.3 .54 .2$; 06.1 . $34 ; 06.1 .52 ; 06.1 .55 ; D 7.1 .34 .1-2 ; D 7.2 .4 ; 07.4 .7 ; 07.4 .17$; D 7.5.6 pr ; D 7.6.4;D 10.2.51 pr;D 10.4.8;D 12.4.7.1; D 12.6.34;D 13. $1.41 \mathrm{pr}$; D 19.1.22 ; D 19.1.28; D 19.2 .32 (Cassius) ; D $21.2 .39 \mathrm{pr}-2$; 
D $21.2 .40 ;$ D 22.1.25 $\mathrm{pr}^{-2}$; D 23.4.13; D 23.4.22; D 27.10 .7 .3 ; D 29.2 . $44 ; \mathrm{D} 30.81 \mathrm{pr}-3-5-7-8 ; \mathrm{D} 30.82 .2 ; \mathrm{D} 30.84 .2-10 ; \mathrm{D} 30.86 .3-4 ; \mathrm{D} 30$. 104.4 ; D 33.2.10; D 33.5.11; D 34.5.13.1-2; D 35.1.23;D 35.1.25;D 35 . 1.30 ; D 35.2.86 ; D 35.2.87 pr-I ; D 39.6.14; D 39.6.18.3 ; D 39.6.20; D 40.4.16;D 41.2.36; D 41.2.38.1 ; D 41.3.33.1-2-3; D 41.4.7 pr-1-2-4-6; D 41.5 .2 .1 ; D 44.2 .8 ; D 44.2 .25 .2 ; D 44.7.16; D 45.1.56.7; D 45.1.58; D 45.3.10; D 46.3.33 pr ; D 46.8.22.8; Valens D 36.4.15; Pomponius D 6 . $1.53 ; \mathrm{D} 7.1 .10 ; \mathrm{D} 7.1 .19 .1 ; \mathrm{D} 7.1 .32 ; \mathrm{D} 7.1 .73 ; \mathrm{D} 7.4 .6 ; \mathrm{D} 7.4 .23$; D 7.6.2 ; D 7.8.16 pr-1 ; D 7.8.22 pr (Hadrien) ; D I0.2.47 pr ; D 10.3. 20 ; D 12.6.22.1 ; D 13.7.6 pr-1 ; D 13.7.8 pr ; D 16.1.32.2; D 18.1.6.1; $D 18.18 .1$ (Alfenus) $D 18.1 .20$ (Sabinus) D $18.33: D 18.1 .66 \mathrm{pr}^{-1-2}$ (Q. Mucius) ; D 18.2.15 pr-1 ; D 18.3.2 ; D 19.1.6 pr-2-3-6; D 19.1.16; D 19.2.3 (Proculus); D 19.2.52; D 21.1.64 pr (Labéon); D 21.2.16 pr; D 21.2 .20 ; D 21.2.22.1; D 21.3.2; D 23.3.32; D 23.3.66; D 24.1.31.2; D 24.3 .9 ; D 26.7 .61 ; D $30.8 \mathrm{pr}$; D $30.24 .2-3-4$; D 30.38 .1 ; D 30.45 .1 D 32.16 ; D 32.44 ; D 32.54 ; D $34.4 .2 \mathrm{pr}-1-2$; D 35.1 .105 ; D 35.1 .112 . I-2;D 36.1.74 (Aristo) ; D 41.2.25.1; D 41.2.26;D 41.2.31; D 41.2.33; D 43.16.14;D 43.26.17; D 44.2.21.3; D 45.1.9; D 46.3.26; D 46.8.16. 1 (Julien); Africain D $3.5 .45 \mathrm{pr}$; D $7.1 .36 \mathrm{pr}-1$; D 7.2 .9 ; D 16.1.17.2 D 17.1.34.1; D 18.2.18; D 19.2.33 ; D 19.2.35 pr (Servius)-1 (Servius); D 20.4 .9 .3 ; D 21.2.46 pr ; D 23.3.50 pr-1; D 23.5.9.2; D 30.108.6 D 30.109 .1 ; D 32.64; D 38.2.26;D 41.1 .40 ; D 41.2.40 pr-1; D 44.1.16; D 44.1 .18 ; D 44.2.26.1; D 47.2.62.8; T. Clemens D 31.54 ; Gaius D 1.8 . I.I ; D 4.7.3.2-3; D 7.1.31; D 7.8.11; D 15.3.12; D 17.1.2.6; D 17.1.13; D 18.1.35.8; D 18.6.9; D 19.2.25.1-2-3-4-5-6;D 19.2.34;D 20.1.15 pr (Julien); D 20.4.11.2;D $21.1 .32 ; D 21.2 .6 ; D 29.1 .17 \mathrm{pr} ; \mathrm{D} 30.65$; ,D 30.69.3; D 30.70.1; D 33.1.8; D 33.2.29; D 35.1.17.2-3; D 41.3. 37.1 ; D 43.16.10 ; !laecianus D 32.95 (Aristo) ; D 35.2.30.1 ; D 36.1.7I D 49.17.18.2-3; Venuleius D 21.2.75 (Q. Mucius, Sabinus); D 42.8.25. 6 ; D 43.24.12; D 43.26.21; Marcellus D 7.1.71; D 13.7.34; D 18.I.60 D $24.1 .49 ;$ D 24.3 .57 ; D 30.82 .3 (Julien); D 30.92 pr (Julien); D 31 $23: D 33.2 .15 .1 ; 035.1 .35 .1 ; 035.2 .54 ; 038.2 .31 ; 041.2 .19 \mathrm{pr}$ D 41.3 .17 ; D 43.16.12; D 46.3.68; P. Iustus D 50.8.11.2 (rescrit de MarcAurèle et Verus) ; Florentinus D 30.116.1-4 ; Scaevola D 2.14.47 pr ; D 4.4 $39.1 ; \mathrm{D} 4.4 .47 .1 ; \mathrm{D} 7.1 .58 \mathrm{pr}-1$; D $10.2 .39 .5 ; \mathrm{D} 13.7 .43 \mathrm{pr} ; \mathrm{D} 15.1 .54$; D 15.1.58; D 16.1.28.1; D 17.1.62 pr ; D 18.1.81 $\Gamma^{-1}$; D 18.3.6 pr-1; D 18.3.8;D 18.5.8;D 18.5.9; D 18.5.10 $\mathrm{pr}^{-1} ; \mathrm{D} 18.6 .11$ (Julien): D 19.1 $48: D 19.1 .52 \mathrm{pr}-1 ; \mathrm{D} 19.2 .61 \mathrm{Fr} ; \mathrm{D} 20.1 .31 \mathrm{Fr}-1 ; \mathrm{D} 20.1 .32 ; 020.6$. 15 ; D 21.2 .12 ; D 21.2.69.5-6; D 23.3.85; D 23.4.29; D 24.1.58 pr D 26.7.57.1; D 31.88.6-9; D 31.89.4-7; D 32.34.3; D 32.35.1-2 ; D 32.37 $\mathrm{pr}^{-3}$; D $32.38 \mathrm{pr}-1-3-4-7-8 ; \mathrm{D} 32.39 .2 ; \mathrm{D} 32.40 \mathrm{pr}-1 ; \mathrm{D} 32.41 \mathrm{pr}-3-5-9$; D $32.93 .2-4-5 ; \mathrm{D} 32.101 \mathrm{pr}-1 ; \mathrm{D} 32.102 \mathrm{pr} ; \mathrm{D} 33.1 .19 \mathrm{pr} ; \mathrm{D} 33.1 .21 \mathrm{pr}-1-$ 5 ; D 33.2.17; D 33.2.32 $\mathrm{pr}^{-2-3-5-7} ; \mathrm{D} 33.2 .34 \mathrm{pr}-1 ; \mathrm{D} 33.2 .35 ; \mathrm{D} 33.2$. $36 \mathrm{pr}^{-1}$; D $33.2 .37 ; \mathrm{D} 33.4 .10 ; \mathrm{D} 33.5 .22 ; \mathrm{D} 33.8 .23 .3 ; \mathrm{D} 34.1 .16 .1$. D 34.1 .20 .2 ; D $34.3 .31 \mathrm{pr} ; \mathrm{D} 34.4 .30 .1 ; \mathrm{D} 34.4 .31 \mathrm{pr}^{-1} ; \mathrm{D} 35.1 .108$ D $35.2 .19 ;$ D 35.2 .23 ; D 35.2.25.1 ; D 36.1.80.13-15; D 36.2.28; D 39 $5.35 .1 ; \mathrm{D} 40.7 .40 \mathrm{pr} ; \mathrm{D} 41.4 .13 ; \mathrm{D} 41.4 .14 ; \mathrm{D} 42.8 .21 ; \mathrm{D} 46.4 .15 ; \mathrm{D} 45$ 1.131 .1 ; D 45.1.135.2; D 46.1.45; Papinien D 2.14.42; D 3.3.67; D 3 5.30 .7 ; D 5.1.48; D 5.1.65 Fr ; D 7.1.5; D 7.1.33.1 ; D 7.1.57 pr (Julien)-1;D 10.2.33;D 10.2.35; D $16.1 .27 .1 ; D$ 18.1.58; D 18.1.7? $\mathrm{pr}^{-1}$; D 19.2 .53 ; D 20.1.1.2-4; D 20.4.2 ; D 20.4.3.1-2 ; D 20.5.1 ; D 20.5.2 ; D 20.6.1.1; D $21.2 .64 \mathrm{pr}^{-1-2-3-4}$; D $21.2 .66 \mathrm{pr}-1-3 ; \mathrm{D} 22.1 .3 .3$; D 23.3. $69 \mathrm{pr}$; J 24.3 .42 .1 ; D 31.66 pr (Julien)-1-2-4-6; D $31.67 .3-4-5-6-7 ; \mathrm{D} 31$ 67.1 ; 31.75 .2 ; D 31.77 pr-5-7-8-10-11-13-15-27-28-32; D 31.78.1 Sévòre)
$-3 ; \mathrm{D} 32.91 \mathrm{pr}-1-3-5 ; \mathrm{D} 33.2 .25 ; \mathrm{D} 33.4 .9 ; \mathrm{D} 34.4 .24 .1 ; \mathrm{D} 34.4 .25$; D $34.5 .1 ;$ D 35.1 .71 pr (Africain); D $35.1 .72 .4-5 ; \mathrm{D} 35.1 .73 ; \mathrm{D} 35.1$. $77 \mathrm{pr}$; D $35.2 .15 .6 ; \mathrm{D} 36.1 .60 .3+5 ; \mathrm{D} 36.2 .25 .1 ; \mathrm{D} 40.5 .22 \mathrm{pr}$; D 41 . $2.44 .2 ; D 41.2 .45 ; D 41.2 .46 ; D 41.2 .48 ; D 41.3 .42 ; D 41.3 .45 \mathrm{pr} ; \mathrm{D} 43$. $16.18 \mathrm{pr}$; D $45.1 .115 \mathrm{pr}$; D 46.2 .28 ; D $50.8 .5 \mathrm{pr}$; D 50.15.5 pr-1 (2i.tterae d'Antonin le pieux) -2 ; Tryphoninus $\mathrm{D} 7.1 .62 \mathrm{pr}-1$; $\mathrm{D} 23.3 .75$; D 23. $3.78 \mathrm{pr}^{-1-2-3-4}$; D 31.88 .12 (Scaevola) ; D 32.38.5; D 34.3.28.7 (Scaevola); D 36.1.80.6 ; D 41.1.63.4; Callistrate D 6.1 .50 pr ; D 21.2.72; D $33.10 .14 ;$ D $35.1 .82 ;$ Paul D 2.14.27.8;D 3.5.12; D 4.2.21.2 (Pomponius) ; D 4.4.24.4;D 4.4.38 pr; D 5.2.23.1; D 6.1.6 (Octavenus) ; D 6.1.8 (Pomponius); D 6.1.27.5.'(Proculus); D 6.1.35.1 (Mauricianus); D 6.1.37 (Julien); D 6.1 .74 ; D 6.1 .78 (Labéon); D 6.2 .12 .2 ; D 7.1.11; D 7.1.16; D J.1.18; D 7.1.48.1; D 7.1 .50 (Scaevola) ; D 7.1.59 pr-1-2; D 7.1.60.1; D 7.1 .18 ; D 7.1.48.1 ; D 7.1.50 (Scaevola) ; D $7.1 .59 \mathrm{pr}-1-2$; D 7.1 .60 .1 ; D 7.9.6; D 10.2.25.8 (Pomponius)-18; D 10.2.44.3 (Julien) ; D 10.3.8.2 D 10.3.14.1 (Julien); D 10.3.29 pr; D 11.1 .20 .1 (Iavolenus)-2; D 12.1 . $31 \mathrm{pr}$; D 12.2.26.8; D 12.6.12; D 13.7.16.2; D 13.7.18.2-3 (Cassius); D 13.7.2 I D 17.1.5.3; D 17.1.15; D 17.1.45 pr ; D 17.2.46; D 18.1.34 pr ; D 18.1.40 pr-1-2-3-4-5; D 18.1.51; D 18.2.1; D 18.2.14.1 (Labéon, Sabinus, Nerva)-2 ; D 18.4.25 (Labéon) ; D 18.6.7 pr-1 ; D 19.1.4.1 ; D 19.1.8 pr-1:D 19.1.21.1-4-j; D 19.1.27; D 19.1.42 (Labéon); D 19.1.45.1;D 19.1. Pr-1; D 19.1.21 DIg.25 1-2;D20.4.15;D20.4.16;D20.4.17;D20.5.10;D20.6.11;D21.1.44pr(Pedius); D $21.2 .11 ; 021.213$ (Proculus) ;D $21.2 .15 \mathrm{pr}^{-1} ; \mathrm{D} 21.2 .41 .2 ; 021.2 .45$ (Alfenus); D $21.2 .53 \mathrm{pr} ; 021.2 .71 ; D 21.2 .73 ; \mathrm{D} 22.1 .38 .1-2-3-14 ; \mathrm{D} 23$. 3.56.3 (Nerva, Scaevola); D 23.4.12.3 (Mela)-4;D 24.1.55; D 24.3.6; D $24.3 .8 \mathrm{pr}-1$; D 24.3.49.1 ; D 25.1 .6 ; D 25.1 .12 ; D 25.1.13; D 26 . $7.46 \mathrm{pr} ; \mathrm{D} 27.9 .13 \mathrm{pr}$ (rescrit de Sévère et Caracalla)-1 (Papinien) D 27.9 .14 ; D 28.5 .10 ; D 28.5 .11 ; D 29.1 .16 ; D. 30.5.2; D 30.72 ; D 30. 85 ; D 30.122 .2 ; D 31.49 .2 (Trebatius, Labéon, Fulcinius)-3 (Proculus) ; D $31.82 \mathrm{pr}-1$; D 31.86 .1 ; D $31.87 .2-4$ (rescrit d'Alexandre Sévère); D 32.

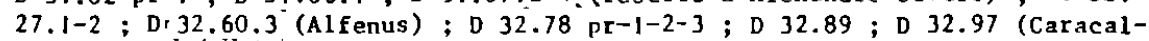
1a); D 33.1.9 (Papinien); D 33.1.12: D 33.2.12 (Alfenus); D $33.2 .26 \mathrm{pr}$ -I ; D 33.2.28 ; D 34.1.12 ; D 34.3.25 (rescrit d'Antonin le pieux) D $34.4 .6 \mathrm{nr}^{-2}$; D 35.1 .13 ; D $35.2 .49 \mathrm{pr}$ (Sabinus, Atilicinus, Nerya, Cassius, Plautius) -1 ; D 35.2 .65 ; D 36.1 .83 ; D $36.2 .24 \mathrm{pr}$; D $38.2 .44 \mathrm{pr}$; D 39.3.5 (Labéon); D 41.1.62; D 41.2.1.17; D 41.2.3.1-8-12;D 41.2.30. 5-6.; D 41.2.41; D 41.3.4 22-23-25 (Cassius) ; D 41.3.13.2; D 41.3.31.3; D 41.4 .2 .6 (Neratius) ; D 42.1.41.1;0 42.5.12.2; 442.8 .7 (Proculus); D 43.16.20 (Labéon) ; D 43.24.19 (Sabinus) ; D 44.1.17; D 44.2.30.1; 44. $7.34 .2 ; 045.1 .78 .1 ; 045.1 .85 .6 ; 043.1 .89 ; 045.1 .126 .1 ; D 45 ; .136$. I: D $46.1 .58 \mathrm{pr} ; \mathrm{D} 46.3 .98 .8 ; \mathrm{D} 47.2 .26 .1 ; \mathrm{D} 47.2 .83 .1 ; \mathrm{D} 47.2 .86$; D $48.10 .22 .11 ; \mathrm{D} 48.14 .40 \mathrm{pr} ; \mathrm{D} 49.14 .50 ; \mathrm{D} 50.16 .79 .1$ (Fulcinius); 50 16.169 ; D 50.16.205; U1pien D 1.21.2.1; D 2.14.52.1; D 2.15.8.15; D 3 . 5.7.1 ; D 3.5.16 ; D 5.3.20.2 ; D 5.3.27.1 ; D 5.3.29;D 6.1 .37 (Julien) D 6.1 .72 ; D 6.1 .77 ; D 6.2.11.7 (Fomponius) 9 (Pomponius) ; D $7.1 .7 \mathrm{nr}$; D $7.1 .9 \mathrm{pr}$ (Celsus) $-1-2-3-4$ (Fegasus) $-5-6-7 ; 07.1 .12 \mathrm{pr}$ (Labéon) -3 (Fomponius) ; D 7.1.13.2 (Julien)-4-5-6; D 7.1.15.6-7 (Julien); D 7.1.17 pr;n7.1.20; D 7.1.27 pr-3-4 ; D 7.1.34.1 ; D 7.2.1.3 (Celsus, Julien, Plant ius) ; D 7. 2.3.1 (Celsus) ; D 7.2.6.2 ; D 7.4.1 pr-2; D 7.4.5.3; $7.4 .8 ; \mathrm{D} 7.4$. $10 \mathrm{pr}-2-3-1$; D 7.4.29 pr (Pomponius)-1; D 7.6.1 pre (Labéon, Nerva, Julien, Marcellus) $-1-2-3-4 ; 07.6 .5 \mathrm{pr}^{-1}$ (Julien) $-5 ; 07.8 .10 .4$ (Labên) D 9.2 .27 .9 (Neratius)-11 (Proculus) 14 (Ce1sus); D 10.2.10; D 10.2.14.1; D 10.216 (Neratius) -11 (Proculus) -14 (Celsus); D

2 (Papinien) -6 (Trelatius, Labéon)-8 (I11ien)-10; D 10.3 .7 rr-1 (Neratius) 
-11-13; D 10.3.21; D 11.7.14.1 (Pomponius) ; D 12.1.4.1; D 12.2.13.1 (Julien)-5 (Venuleius, Marcellus) ; $D$ 12.2.26.4 (Marcellus)-6 (Marcellus) -7 (Julien)-12 (Celsus) ; D 13.3.1 pr ; D 13.3.2 (Sabirus, Celsus) : D 13. 6.1.1 ; D 13.7.11.4; D 13.7.15; D 13.7.22.2 ; D 13.7.25; D 16.1.8.1 (Papinien) $D$ D 17.1.8.6 $; 0$ 17.1.10 pr-2 ; D 17.1.12.8 (Papinien) ; $D$ 17.2.

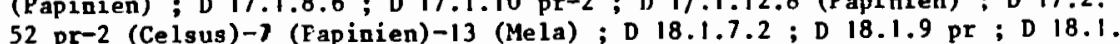
$52 \mathrm{pr}-2$ (Celsus)-7 (Fapinien) -13 (Mela) ; D 18.18 .2 ; D (Marcellus) -4 $25.1 ;$ D 18.1 .37 ; D 18.1 .47 ; D $18.2 .2 \mathrm{pr} ; \mathrm{D} 18.2 .4 .3$ (Marcellus) -4 .
(Julien) ; D $18.2 .11 \mathrm{pr}$ (Sabinus, Julien) ; D $18.2 .13 \mathrm{pr}$ (Q. Mucius, Brutus, Labéon, Sabinus, Celsus) ; D 18.3.1 ; D 18.3.4 pr (rescrit de Sévère et Caracalla)-3 ; D 18.4.2.19; D 19.1.1.1 ; D 19.1.13.6 (Julien)-10 $-11-14$ (Neratius)-15-17 (Celsus)-24-30 (Servius, Tubero); D 19.1.15; D 19.1.17 pr-2 (Trebatius)-6 (Gallus Aquilius, Mela)-11 ; D 19.1.34;D 19. 1.35 ; D 19.2.9 pr (Pomponius)-1 (Marcellus ; rescrit de Sévère et Caracal1a)-2 (Julien)-3 ; D 19.2.13.11 ; D 19.2.14 (Marcellus); D 19.2.15.1-2 (Serviuse)-3-4 (Papinien)-8-9 (Julien) ; D 19.2.19.1 (Servius, Labêon, Sabinus, Cassius)-2 (Aristo, Neratius)-3; D 19.5.19.1; D 20.1.21 pr ; D 20. 4.6 .2 ; D 21.1.33 Pr (Pomponius); D 21.1.61; D 21.2.1; D 21.2.14; D 21 . 2.6.2 ; D 21.1.33 Pr (Pomponius) ; D 21.1 .61 ; D 21.2 .1 ; id 1 : D 23.3.27; D 23:4.4 (Marcellus) ; D $24.1 .5 .8-9-11-12 ; D 24.1 .7 .6 ; D 24.3 .5 ; D 24$. D 23.4 .4 (Marcellus) ; D 24.1.5.8-9-11-12; D 24.1 .7 .6 ; D 24.3.5 ; D 24.
$3.7 \mathrm{pr}$ (Scaevola)-1 (Papinien)-2-3-8-9-12-13-14-15-16 (Pomponius) ; D 25.1 .3 Pr ; D 25.1.5 pr (Pomponius) ; D 26.7.5 pr; D 26.7.46 pr ; D 26.9.2 ; D 27.3.13; D 27.9.1.2 ; D 27.9.3 pr-1-2-3-5-6; D 27.9.5. I-2-3-4-6-78-9-10-14-16; D 27.9.6 ; D 27.9.7 pr ; D 27.9.9; D 27.9.10 ; D. 27.9.11; D 28.5.1.4 : D 28.5.9.13 (Sabinus) : D 28.5.35 pr-1 (Julien) : D 28.8.7.3 ; D29.2.20.1; D30.4pr (Pomponius); D30. 14pr (Cel sus, Aristo, Marcell lus); D30.34.4-1213-14-15;D30.37.1;D30.39.3(Julien)-5-6-8-10;D30.44.4-9;D30.47.6(Labéon); D30.49. 8-9;D30.53.1-7;D30.71.5(Jul .)-6;D30.120.2;D32.11.4-9;032.68pr(Jul.)-3; D 33. 4.1.8-14 (Mela)-15 (Mela) ; D 33.4.2.1 ; D 34.4.3 pr-4-6-8; D 36.1.1.16 (Julien); D 36.2.14.1 (Ju1ien); D 36.4.5.7-22; D 37.6.1. I ; D 40.5 .24 . 20 ; D 41.2.10.1 (Pomponius) ; D 41.2.34 pr (Celsus, Marcellus); D 41.3. 26 ; D 42.1.15.12 ; D 42.2.6.1-2 ; D 42.4.7.17 (Celsus) ; D 42.5.9.3-5; D 42.6.1.12;D 43.16.1.3-10-22-24-25 (Proculus)-32 (Julien) : D 43.16.3. 14-15; D 43.19.1.7 (Pedius)-11; D 43.19.3.4 (Julien) ; D 43.24.13.6-7 (Julien) : D 43.26.6.2; D 43.32.1.1; D 43.33.2; D 44.2.7 Pr ; D 44.2.9.1 (Neratius); D 44.2.11.2-3 (Julien)-4-9; D 44.4.4.12-32; D 44.5.1.2; D 45. 1.38 .21 ; D 45.1.72 Pr (Tubero, Celsus); D 45.1.75.1-3-10 ; D 45.1.114; D 46.3.27; D 46.4.13.1-2-5; D 47.2.14.2 ; D 47.2.25.1 (Labéon, Celsus); D 47.2.52.8; D 47.10.5.4; D 50.8.2.1; D 50.8.3.2 ; D 50.15.4.8; L. Rufinus D 28.5.75 ; Macer D 2.8.15 pr-1 (U1pien) -3-6 ; D 42.1.63; Marcien finus D 28.5.75; Macer D 2.8.15 pr-1 (U1pien) $-3-6$; D 42.1 .63 ; Marcien D 20.1 .16 pr ; D 20.4.12.8; D 20.6.8.3-4-6-7-9;D 23.3.52; D 30.112 pr-2 D 30.114.3 (Marcellus)-4 (Marcellus)-15-16 (Marcellus)-17; D 32.13 ; D 32. 67 ; D 35.2.91; D 39.4.16.11; D 41.2 .37 ; D $41.2 .43 \mathrm{pr}$ (Julien) ; D 41.. 3.39 ; D 44.4 .10 ; D 46.3.46.1-2 ; Modestin D 3.3 .63 ; D 3.5 .26 pr ; D 10 2.30 ; D 10.3.17; D 13.7.39; D 18.1.62 pr ; D 19.1.39;D 20.1.26.2 ; D $20.6 .9 \mathrm{pr}-1$; D 21.2 .63 .1 ; D 22.1.42 ; D 23.3.62; D 26.7.32.4-6; D $31.32 .1-2$; D $31.34 .1-7$; D 32.82 ; D 33.2.19; D 34.1 .4 Pr ; D 41.3.18; D 44.3 .3 ; D 45.1 .103 ; D 50.1.36.1 ; Hermogénien D 18.1.75; D 19.1.49 pr ; D 35.2.40.1.

\section{- analyze des citations}

\begin{tabular}{|c|c|c|c|c|c|}
\hline I & $29 / 59$ & $=49 \%$ & I & $30 / 59$ & $=$ \\
\hline II & $63 / 140$ & $45 \%$ & II & $77 / 140$ & $=$ \\
\hline III & $113 / 194$ & $58 \%$ & III & $81 / 194$ & $=$ \\
\hline IV & $20 / 40$ & $50 x$ & IV & $20 / 40$ & $=$ \\
\hline $\mathrm{v}$ & $11 / 20$ & 558 & $\mathrm{v}$ & $9 / 20$ & $=$ \\
\hline TOTAL & $236 / 453$ & $52 \%$ & TOTAL & $217 / 453$ & $=$ \\
\hline
\end{tabular}

Bien que les solutions relatives à la propriété du fonds soient liées au développement de l'esclavage, on avait observé; lors de l'étude comparative menée sur les modes d'acquisition de la main-d'oeuvre servile, une prédominance des textes traitant de $\mathrm{I}^{\prime}$ esclave par rapport à ceix :concernant un fonds. Dásormais détachés de ce contexte, ne tenant compte que des paragraphes où ces éléments patrimoniaux n'interviennent pas de manière indispensable au développement de la question traitée, les résultats obtenus sont de nature differente.

On remarque, đun point de vue général, un équilibre entre les deux éléments, la légère suprématie du fonds dans 1 'analyse des paragraphes cédant le pas à celle de l'esclave dans l'analyse des citations: Plus précisément, l'analyse des citations montre que l'utilisation de l'esclave par les juristes comme élément patrimonial interchangeable est déjà importante à l'époque républicaine. Loin de connaître une baisse avec le développenent d'une certaine capacité servije, elle semble au contraire progresser scus les premiers Antonins, comme l'indique l'analyse des paragraphes, mais le fait est sans doute dû à la meilleure représentation de cesjuristes dans le Digeste. Le recul accusé par la période IV peut s'expliquer, conformément aux résultats antérieurs, par l'importance de l'intervention directe des juristes à cette époque. Il ne s'agit nullement d'un recul de l'ideologie esclavagiste. Cette apparente accalmie n'est d'ailleurs pas confirmée par la période $V$ : l'idéologie esclavagiste n'est absolument pas affectée par la baisse d'intérêt que connaît alors le discours des juristes pour l'esclave. 
Le portrait de l'esclave que permettent de tracer les textes du Digeste montre clairement que le disccurs des juristes est le tremplin de l'idéologie dominante (106). Est un bon esclave l'esclave fidèle, obéissant. Est mauvais celui qui ne se plie pas aveuglément à la volonté de son meître.

Cette idéologie, loin de s'estomper avec la baisse de l'intérêt porté à I'esclavage par les juristes du Jème siècle, persiste. On a beau reconnầtre une certaine capacité à l'esclave, se préoccuper au 3ème siècle enccre des formes les plug achevées de l'esclavage, un esclave regte un esclave.

106- Selon L. LOMBARDI, Dalla fides alla bona fides, Milan (1961), p. 54, le discours juridique $n$ 'est mystifiant qu'a un certain niveau de complexité sociale ou de "progrès" culturel. L. LANTFLLA, Il lavoro sistematico, cit., p. 278 , pense en revanche que mystification ne signifie pas forcếment tromperie, stratégie de persuasion, mais aussi représentation des chnses de son propre point de vie p̣lutôt que di point de vue de la tọtalité sociale, et $(p .280)$ qu'à ce titre, le discours des juristes romains est un discours mystifiant. L'auteur ajoute (p. 283) que le modêle de la contrnunication, très proche de celui de la conversation par le fait de l'ógalité sociale de ceux pri 'changent les aessages, n'inplique pas l'absenga de propagande ideologique.

\section{CONCLUSIOK}

Les constitutions impériales relevées dans le Digeste attestent une baisse d'intérêt pour l'esclavage dès la seconde moitié du zème siècle. Or, c'est à cette époque que le discours des juristes est quantitativement le plus important en la matière. Cette distorsion n'ast toutefois qu'apparente. Par la "remise en cause", même limitée, de certains principes, le droit impérial nécessite en effet des développements jurisprudentiels accrus (1). Le sursaut de la production jurisprudentielle sous les derniers Antonins correspond ainsi "ì sa manière" à la crise qui secoue alors le système esclavagiste (2). Comment, le Digeste permetil $d^{\prime}$ analyser cette crise ?

En tant que source spécifique, image d'une "réalité clinique", le Digeste incite à la prudence. Certains rósultats sont áloquents à cet égard : par exemple, on ne trouve qu'une proportion infime de bons rapports entre esclave et, maître, l'assassinat apparait comme la mort d'esclave la plus fréquente. Cela ne correspond sans doute pas à la réalité.Mais le Digeste fournit par ailleurs des renseignements particulièrement dignes d'intérôt. Ainsi en est-il du manque de solidarité au sein du groupe servile, du mimétisme social des esclaves privilégiés.

On décòle la crise chez ces esclares par le iéveloppement d'ue capacité économique et, juridique "anormale". C'cst surtout de ceux-ci dont les juristes se préoccupent, dans la mesure sil leur exploitation soulève de toute évidence plus de difficultós juridiques que colle des esclaves ruraux travaillant, dans dos snmitions tròs dures. Il faut ce-

1 - Selon E.M. SCrtaErman, La chute du régime esclavagiste, cit., p. 140. le fait que la situation juridique des esclaves reste inchangée ne doit pas induire en erreur. En effet, les excepcions introduites graduellement réduisent pratiquement à néant les principes gênéraux inchangés.

2- Date également la crise de l'esctavage sous les derniers Antonins, E.M. SCHTAERMAN, La chute du rágime esclavagiste, cit.. p. 137, 158 ; ta Schiavitù, cit., p. 336. A. CARANDinI, la anatomia dell.t scimmin, cit., note que les ville eaclavagistes sout geniralement abantenncẹa ver:l lis lin de l'époque des Antonins ou au début de relle des Sévéres (p. 129). Il signalo aussi une crige des ateliers et de la production artistique (p. 132). 
pendant attendre la seconde moitié du ème siècle pour les voir se tourner résolument vers eux. C'est indubitablement 1 à un sigre de la crise. mêne $s^{\prime}$ il n'est que frngmentaire (3), tout d'abord parce que chercher de la sorte des appuis chez res "cadres moyens" contribile à dénaturor l'exploitation esclavagiste, ensuite et surtout parce que l'esclavage perd ainsi de sa spécificité économique. L'esclave qui peut se trouver dans la situation de possédant, voire de propriétaire d'esclaves, n'èst déjà plus un esclave au sens absolu de ce terme (4).

Ce changement a été conditionné dans une certaine mesure par des raisons économiques, notamment la nécessité de faire participer de tels esclaves à la vie des affaires, mais il faut rechercher ses causes profondes ailleurs. Pourquoi reconnaitre à certains esclaves une caprcité démesurée ? Pourquoi la crise de l'esclavage ?

Le Digeste se fait l'écho d'une grande st,abilité : prix, acquisition et utilisation de la main-d'oeuvre servile ne connaissent aucune variation importante. Il est possible que cette permenence soit due une fois encore à la nature de notre source, le droit étant une représentation spécifique de la réalité, et pouvant en constituer un prisme déformant, surtout lorsque celle-ci est exclusivement économique. Toujours est-il que rien dans le Digeste ne permet de vérifier l'hypothèse d'une diminution brutale du nombre des esclaves consécutive à la cessation de la conquête systématique (5).

On observe par contre un recoupement des courbes statistiques concernant d'une part le développement d'une capacité servile, le favor $l i$ bertatis et, d'autre part, la recrudescence de la délinquance servile et l'aggravation des tensions entre maîtres et esclaves. Cela ne peut tout de mêtme pas relever du seul domaine des coincidences. Les mesures "favorables" à l'esclave, plus précisément à la frange privilégiée du groupe servile, apparaissent ainsi comme une réponse à la déaradation des raoports sociaux.

3- Il ne s'agit pas forcément d'un reflet de l'ensemble. On notera cependant que c'est à la même époque qu'augmente l'intérêt des textes juridiques pour les colons. L'apparition plus précoce du colonat en Afrique, décrite par J. KOLENDO, La formation du colonat, cit., p. 129-157, semble due à des conditions particulières. La genèse du colonat est d'ailleurs différente en Afrique (p.148). Sur le point, v. M. CLAVEL-LEVEquE, Impérialisme, développement et transition, cit., p. 20.

4- E.M. SChTAERMAN, La chute du régime esclavagiste, cit., p. 137.

5- Réfutent notament cette explication de la crise M.I. FINLEY, L'économie antique, cit., p. 113 et E.M. SCHTAERMAN, La schiavitũ, cit., p. 329.

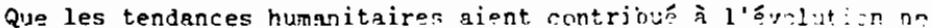
fait pas de doute, mais elles sont insuffisantes à l'explique anglàtement (6). On en trouve a nreuve non seulement dans cotte convergence statistique, mais aussi dans la progressinn de la politique répressive.

N'est-ce pas en effet sous les Antonins que s'intensifie la lutte contre la fuite ? N'assiste-t-on pas, sous ces mêmes empereurs, qux extensions les plus remarquables du s.c. silanien, qui en vient à toucher les esclaves déjà affranchis du vivant du testateur, y compris ceux ayant obtenu le ius ourecmom anulorum, et même, en cortains cas, les esclaves impubères (7) ? De telles mesures ne symbolisent-elles pas une crainte liée àl'esclave (8), simple inquiétude patrimoniale du maitre, en cas de fuite, ou, plus sérieusement, peur de celui-ci d'être assassiné, crainte qui apparaîtrait de la sorte comme une explication plus sûre de l'évolution?

\section{Toutefois, la libéralisation ne sera pas la solution au} problème, comme l'attestent les écrits des juristes du 3ème siècle. D'une part, les tensions sociales ne faibliront pas ( 9 ). D'autre part, cet adou-

6- Selon E.M. SCHTAERMAN, Die Rrise, cit., p. 48 s, ces tendances, ne créant qu'un intérêt illusoire, sous-rentabilisent l'activité de l'esclave et poussent a le remplacer par le colon. J. KOLENDO, La formation du colonat, cit., p. 149 , pense qu'on peut voir une preuve de la rentabilité du travail des colons dans l'installation de certains esclaves sur des parcelles.

7- Les timides atténuations apportées a ce s.c. par Antonin le pieux et Conmode (cf. supra p. 505) sont tout a fait occasionnelles et, en tout cas, bien pâles par rapport a ces extensions ( $v$. sur ce point E.M. SCHTAERMAN, La schiavitü, cit., p. 230).

8- Le mécontentement servile, qui se trouve à l'origine de cette crainte, n'opère plus de manière ouverte, comme à l'époque républicaine ; il revêt des formes inorganíques (cf. E.M. SCHTAERMAN, La schiavitù, cit., p. 252) et se ramène souvent, on 1 'a observé à propos de la fuite, a la volonté de profiter du système et non à celle de l'abolir (cf. E.M. SCHTAERMAN, 1.c.). La crainte qu'il inspire aux propriétaires d'esclaves n'en est pas pour autant plus faible ainsi qu'en témoigne de manière éclatante le s.c. silanien.

9- On peut être surpris de ce que la libéralisation de l'exploitation n'apaise pas les tensions sociales, mais, au fond, à qui profite-t-elle. siuon essentiellement aux esclaves privilégiés, souvent eux-mêmes propriésinon essentiellement
taires d'esclaves? 
cissement, limitant le poluvoir des maîtres, portera un coup à l'une des conditions principales de l'explnitation (10). La crise de l'esclavage, irréversible selon le Digeste dès la seconde moitié du zème siècle, entraînera avec elle l'Et,at esclavagiste (11).

Au Jème siècle, tout en restant tournée vers les formes les "plus "achevées" de l'esclavage, la jurisprudence diminue nettement d'intensité à son égard. Cependant, l'idéologie esclavagiste, dont elle est le tremplin, persiste.

10- E.M. SChTAERMAN, La chute du régime esclavagiste, cit., p. 139

11 - E.M. SChTAERMAN, La chute du régime esclavagiste, cit., p. 154. Selon $P$. DOCKES, La libération, cit., p. $45-46$ et $278 \mathrm{~s}$., la crise de l'Etat, que l'auteur situe au début du jème siècle, serait la cause de la fin de l'esclavage, l'Etat ne parvenant plus à assurer sa fnnction répressive du fait de sa dégénérescence. Cotté thèse, pour sejuisante répressive du fait de sa degánerescence. Cotto thase, pour seduisante réalité si l'on peut "reprocher" a la jurisprudu d'etre realite. si lon peut "reprocher" a la jurisprudence d'etre en retard sur la pratique, on ne peut sérieusement lui faire grief d'être en avance sur celle-ci ! Or, elle dénonce l'existence de la crise dès la seconde moitié du zème siècle, date antérieure à celle à laquelle l'auteur situe la crise de l'Etat. 
ANNEXE

Relevé des paragraphes et citations

concernant I'esclavage

dans Ie Digeste. 


\begin{tabular}{|c|c|}
\hline 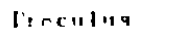 & 0 \\
\hline $\ln v a|r| 119$ & 0 \\
\hline $\begin{array}{l}\text { Neratiug } \\
\text { inglis }\end{array}$ & $\begin{array}{l}0 \\
\text { I }\end{array}$ \\
\hline Julien & I \\
\hline Inmponiug & 2 \\
\hline (inius & B \\
\hline Venuleius & 0 \\
\hline Marcellus & I \\
\hline Flnrentinus & .] \\
\hline Tapinion & 3 \\
\hline Trgphinninus & I \\
\hline Callistrate & 0 \\
\hline Tertullien & $\mathbf{0}$ \\
\hline Paul & 6 \\
\hline Ul pien & 25 \\
\hline
\end{tabular}

Ul pien

L. Rufinus

Macer

Marcien

Moriegtin

lle rmngénien

A.Chnriaius

Tntal.....
D1.18.17.

D1.5.26.

D1.2.2.7-2.4.

D1.5.3; D1.5.6; BI.13.1 pr-I-2; DI.8.1.1.

D1.7.18.

DI.5.1 $\mathrm{pr}-1-2-3$.

D1.5.8; DL.9.9; (11.2I.I F.

D1.5.15. D1.5.7; D1.5.I1; Dt.7.36.1; D1.15.3.2-5; D1.18.21;
D1.1.4; D1.5.10; D1.5.18; D1.5.25; D1.5.27; D1.6.2; D1.6.7; DL.7.I5.3; D1.7.I9pr; D1.7.16; D1. In.I pr-I-2 DI.12.1.1-2-5-8-10; D1.I1.1; D1.I1..3; D1.15.5; D1.16.9.3; D1.18.2; D1.I8.13pr; D1.19.2.2.

D1.18.16.

D1.5.5pr-I-2-7; DI.R.B.1; D1.16.2pr-I; D1.22.2. D1.5.21; D1.5.22. DI.5.11. 


\begin{tabular}{|c|c|c|c|c|c|c|}
\hline $\begin{array}{l}\text { Procul lue } \\
\text { Invol enue } \\
\text { Veratius } \\
\text { Cel sus }\end{array}$ & $\begin{array}{l}0 \\
0 \\
0 \\
0\end{array}$ & $\begin{array}{l}1 \\
1 \\
2 \\
2\end{array}$ & & $\begin{array}{l}\text { Ierol enus } \\
\text { Jali en } \\
\text { Pomponiue } \\
\text { Africain }\end{array}$ & $\begin{array}{l}0 \\
0 \\
1 \\
1\end{array}$ & $\begin{array}{l}\mathbf{3} \\
\mathbf{0} \\
\mathbf{3} \\
\mathbf{5}\end{array}$ \\
\hline Julien & 2 & 9 & D2.11.13; D2.14.55 & $\begin{array}{l}\text { Astickin } \\
\text { Gaius }\end{array}$ & 1 & 27 \\
\hline Pouponiue & 0 & 1 & & Hercellus & 0 & $\mathbf{1}$ \\
\hline Africaia & o & 1 & & Scaerola & 1 & 6 \\
\hline Maurictenus & $\mathbf{0}$ & 1 & & Papinien & 4 & 21 \\
\hline $\begin{array}{l}\text { Gaiue } \\
\text { P. Inetus }\end{array}$ & $\begin{array}{l}4 \\
0\end{array}$ & $\begin{array}{r}32 \\
2\end{array}$ & D2.9.4; D2.11.18; D2.14.38.2; D2.14.30.1. & $\begin{array}{l}\text { Tryphoninus } \\
\text { Calliotrate }\end{array}$ & $\begin{array}{l}0 \\
0\end{array}$ & $\begin{array}{l}3 \\
1\end{array}$ \\
\hline Florentinus & 0 & 2 & & Paul & 14 & 95 \\
\hline $\begin{array}{l}\text { Scaerole } \\
\text { Pepiniea }\end{array}$ & $\begin{array}{l}2 \\
1\end{array}$ & $\begin{array}{r}8 \\
13\end{array}$ & $\begin{array}{l}\text { D2.14.54; D2.15.3pr. } \\
\text { D2.4.14; }\end{array}$ & & & \\
\hline Tryphoni nus & 0 & 1 & & Ul pi en & 17 & 187 \\
\hline $\begin{array}{l}\text { Calibetrate } \\
\text { Paul }\end{array}$ & $\begin{array}{r}1 \\
19\end{array}$ & 3 & & & & \\
\hline & & 113 & $\begin{array}{l}\mathrm{D} 2.1 .9 ; \mathrm{D} 2.4 .9 ; \mathrm{D} 2.4 .11 ; \mathrm{D} 2.4 .15 ; \mathrm{D} 2.6 .1 ; \mathrm{D} 2.7 .2 \mathrm{pr} \\
\mathrm{D} 2.8 .8 .2-5 ; \mathrm{D} 2.9 .2 \mathrm{pr}-1-6 ; \mathrm{D} 2.10 .2 ; \mathrm{D} 2.11 .7 ; \mathrm{D} 2.14 .17 . \\
7 ; \mathrm{D} 2.14 .19 \mathrm{pr} ; \mathrm{D} 2.14 .21 .1 ; \mathrm{D} 2.14 .27 .6-7-10 ;\end{array}$ & Hodtetin & $\boldsymbol{I}$ & $\boldsymbol{T}$ \\
\hline Ul pien & 43 & 236 & $\begin{array}{l}\mathrm{D} 2.1 .7 .1-3 ; \mathrm{D} 2.1 .10 ; \mathrm{D} 2.1 .12 ; \mathrm{D} 2.4 .4 .1-3 ; \mathrm{D} 2.4 .8 .1-2 ; \\
\mathrm{D} 2.4 .10 \mathrm{pr}-1-2-3-4-5-6-7-8-9-10-11-12-13 ; \mathrm{D} 2.4 .12 ; \\
\mathrm{D} 2.7 .1 .1-2 ; \mathrm{D} 2.7 .3 \mathrm{pr} ; \mathrm{D} 2.8 .2 .2 ; \mathrm{D} 2.9 .1 \mathrm{pr}-1 ; \mathrm{D} 2.9 .3 ; \\
\mathrm{D} 2.9 .3 ; \mathrm{D} 2.10 .1 .5 ; \mathrm{D} 2.11 .9 \mathrm{pr}-1 ; \mathrm{D} 2.12 .2 ; \mathrm{D} 2.12 .3 .1 ; \\
\mathrm{D} 2.13 .4 .3 ; \mathrm{D} 2.14 .7 .7-18 ; \mathrm{V} 2.14 .16 .1 ; \mathrm{D} 2.14 .29 ; \\
\mathrm{D} 2.14 .50 ; \mathrm{D} 2.15 .8 .7 .\end{array}$ & Total....... & 40 & 365 \\
\hline Kec er & $\mathbf{0}$ & 9 & & & & \\
\hline Marcien & 1 & 1 & 02.4 .23 . & & & \\
\hline Modeetin & 1 & B & $D 2.4 .25$. & & & \\
\hline Anthienue & 0 & 1 & & & & \\
\hline Hernog ǵni en & $\mathbf{0}$ & 2 & & & & \\
\hline Total ....... & 74 & 447 & & & & \\
\hline
\end{tabular}

DJ.5.10.

D3.5.48.

D3.5.21.

D3.3.34.2.

D3.3.60; D3.5.30 pr-4; D3.6.9.

D3.2.14, D3.3.30，D3.3.43.5，D3.4.10; D3.5.14

$\begin{array}{llll}\text { D3.5.17; D3. } & \text { D3 }\end{array}$ D3.5.41, D3.6.7.2.

D3.5.41; D3.6.7.2.
D3.1.1.11; D3.2.4.2-3; D3.2.24, 85.3.33pr-1;

D3.3.39.5; D3.3.35.1; D3.5.3.6; D3.5.5.7-8-9-10; D3.5.9.1; D3.5.13; D3.5.16; D3.5.44.1.

D3.3.63. 
Proculus Iavnlenus Celsus

Julien

Valena.

Pomponius

Africaill

Gaius

Marcellua

Scaevolo

Papinien

Tryphoninus

Calliatrace

Paul

Ul pi en

5

313

D.4.4.33)

124.4.4.

D.1.7.3.1.

D4.3.32.

$0.1 .1 .31 ;$ D.8.4.2.

D-1.6.11.

D4.2.1; D4.2.8.1; D.t.2.21 pr; D4.3.18.1-5; D.1.3.20pr D4.J.25; 1.4.4.10; B.4.24.3; D.4.18.1; D4.5.3.1; DH.5.7.2; D4.6.13pr; D.t.7.9; D.R.R; DL.8.32.5-7-8; D.1.9.6.I-2.

D4.I.B; D.1.2.7.1; D.1.2.9.2; M.2.12pr; D4.2.14.5-11;

D4.2.16.1; D4.3.7pr-3-4-5-7-8; D4.3.9.1-4; D1.13.11.1 D. .3.24; D4.4.3.11; D.4.1.5; D.4.4.7.5-10; D4.1.9.1-6; D..4.11 pr-I-4.5; D.t.1.22; D.t.6.1.1; D.6.10; D..6.12;

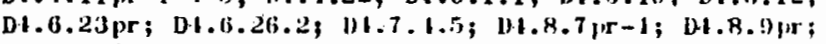
D1.9.I.3-5; 134.9 .3 .3 ; DI.9.7pr-3-1-6.6.

H.5.1.

$\begin{array}{crrr}\text { Invalenus } & 0 & 1 & \\ \text { Neratius } & 0 & 1 & \\ \text { Celsus } & 0 & 2 & \\ \text { Julien } & 1 & 11 & \text { D5.1.25. }\end{array}$

Africuin

Gaius

Marcellus

Scaerola

Papinien

Trjphoninas

Paul

vlpien

I. Rurinus

Marcien

Hndestin

Hermongénien

Total......
D5.3.3; D5.3.35; D5.3.39pr.

D5.1.44.1.

D5.1.12.2, L5.1.24.1-2; D5.2.19; D5.2.28, D5.3.14;

D6.3.32, D5.3.34.1; D5.3.36 pr-2; DK.3.40 pr-4.

D6.1.19.3, D5.1.52.1, D5.1.67, D5.2.8.9-10-16-17,

D5.2.26, D5.2.29.2, D5.3.5 pr; D5.3.7 pr-C-2; D5.3.13.6; D5.3.16.6; D5.3.20 pr-1-3; D6.3.27 pr; D5.3.29;

D5.3.33pr-1; 06.4 .6 .1 .

D5.2.0, D5.2.12pr-1

Ds.1.53.
Macer

Marcien

inchianu

IIertingenien

$\operatorname{Tn} \ln (1+\cdots$
378 


Nerutius
Celsus
Julien
Pomponius
Gaius
Scaerola
Papinien
Callistrate
Paul

ulpien

Mndegtin

enthianus

Intal......
Di.1.29; D6.2.15.

D6.1.18; D6.1.20; D6.1.28; D6.1.30. DG.J.36.I; D6.1.78.1.

D6.1.65.1.

D6.I.6; D6.I.J6pr; D6.I.2I; D6.I.23.5; V6.I.2\%.2-4-5; Dt.1.3I; D6.1.58; DG.1.66; D6.1.79; D6.2.6; D6.2.10; DA.2.12pr-5.

D6.1.5.5; D6.1.13; D6.1.15 pr-3; D6.1.17 pr-1; D6.1.22; D6.1.41.1; D6.1.45; D6.2.5; D6.2.7.10-13; D6.2.9.6; D6.2.11.2-3-4-5, D6.1.32.
Iarolenus

Neratius

Celsur

Julien

Pomponatus

Africain

T. Clemens

Gaius

Veuuleius

Harcellus

Florentious

Scaevole

Papiniea
Tryphoninus

Paul

UI pieu

tien

Marcien

Hodeatio

Total......
D7.1.35.1.

D7.1.55; D7.4.18; D7.4.22; D7.8.16.2 DT.1.38.2; D7.1.37; D7.8.17. D7.1.5.

$$
\text { D7.7.5. }
$$

D7.1.3.1, D7.1.6.3, D7.1.45, DT.1.74; D7.7.3; D7.7.4; DT.8.13.

D7.8.20.

\section{D7.1.57.1.}

D7.1.24; D7.1.26; D7.1.31; D7.1.66; D7.4.26; D7.4.27; D7.7.1; $77.8 .15 \mathrm{pr} ; \mathrm{DT} .8 .10$

D7.1.12.3-4-5; D7.1.15.1-2-3, D7.1.17.1-2-3; D7.1.21; D7.1.22; D7. 1.23 pr-1; D7.1.25 pr-1-2-3-4-5-6-7,

D7.1.27.2-5; D7.1.68pr;D7.2.1.1-2; D7.3.1.2; D7.4.5.1; D7.4.12.1; D7.4.15; D7.6.5.4(a) $-5 ; \mathrm{D} 7.7 .2 ; \mathrm{DT} .7 .6 \mathrm{pr}-$ 1-2-3; DT.8.2.1; D7.8.4pr; D7.8.6; D7.8.10.4; D7.8.12.5-6; DT.8.14 pr; D7.9.5.3. 


\begin{tabular}{|c|c|c|c|}
\hline Praculun & 0 & 1 & \\
\hline lavolenuen & 1 & 15 & DA. 1.12. \\
\hline Neratiun & 0 & .3 & \\
\hline Celsun & 0 & 6 & \\
\hline Julien & 1 & 12 & D8.5.18. \\
\hline Fnofunius & 0 & 38 & \\
\hline Afrirain & 1) & 3 & \\
\hline Gaius & 0 & 10 & \\
\hline Marcellun & 0 & 5 & \\
\hline P. luntun & 0 & 2 & \\
\hline Scaevala & $n$ & 1 & \\
\hline Fapioien & 0 & 8 & \\
\hline Calliatrate & 0 & 1 & \\
\hline Faul & 1 & $\pi$ & D8.3.19. \\
\hline U1 pien & 0 & 67 & \\
\hline Marcien & 0 & 3 & \\
\hline Modestin & 0 & 2 & \\
\hline Total....... & 3 & 255 & \\
\hline
\end{tabular}

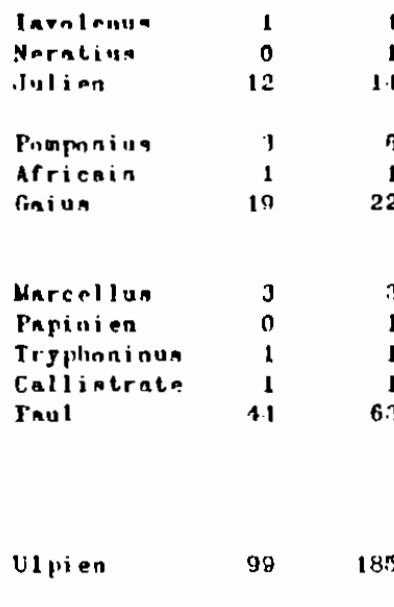

Marcien

In. 2. 3R.

D2.2.47; $19.2 .51 \mathrm{rr}-1-2 ; \mathrm{m} .1 .1 \mathrm{~A} ; \mathrm{m} .1 .3 \mathrm{pr}^{-1-2-3-1 ;}$ เn. 1. 10; $107.1 .+11$.

M.1.18; M.1.13; 0.1.1.1.

0.9. 1.28 .

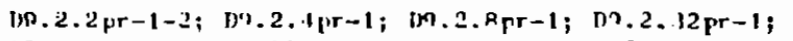
บ9.4.1; 19.4.13; D9.1.15; 112.4.2n; D9.1.2:3; D9.1.2.5; 09.4.27 pr-1; $199.1 .29 ; \mathrm{dn} .8 .30$.

D9.2.3Ipr; $\mathrm{Dn} .2 .36 \mathrm{pr}-1$.

va.1.37.

D?. 4.32 .

D9.2.12; De.2.11; 19.2.18; D9.2.22pr-1; DQ.2.21;

DQ.2.30 pr-1-1; D?.2.31; $199.2 .33 \mathrm{pr} ; \mathrm{Dg} .2 .45 \mathrm{pr}-1-2-3-1 ;$ D9.2.18; DP.2.55: [10.4.4pr-1-2-3; D9.4. o, DQ.4.10; ng.4.12; D9.4.17pr-1; n9.1.19pr-1-2; DQ.1.22pr-1-23-4 D..1.2.1; D9.4.2A $\mathrm{pr}-1-2-3-4-5-6 ;$ D9.1..31.

Do.1.1.11; DR.2.3; D9.2.5pr-1; 59.2.7.1-2-.3-4-3-6-7-8 D9.2.9pr-1-2-3-4; D9.2.11 pr-1-2-3-1-6-7-8, D9.2.13 pr1-2-3; D9.2.15 pr-1; D9.2.17; D9.2.19; D9.2.20;

v9.2.21 pr-1-2; $\mathrm{pg} .2 .23 \mathrm{pr}-1-2-3-1-3-6-7-8-8-10-11$;

D9.2. $25 \mathrm{pr}-1$; D9.2.27pr-1-2-3-5-B-0-11-17-22-24-28-31, D9.3.35; D9.2.14.1; D9.2.46; D9.3.1 pr-8; D9.3.5.1$6-10 ; 09.4 .2 \mathrm{pr}-1 ; \mathrm{D} 9.4 .3 ; \mathrm{D} 9.4 .5 \mathrm{pr}-1 ; \mathrm{D} 9.4 .6 \% \mathrm{D} 9.4$. $7 \mathrm{pr}-1 ; \mathrm{vg.4.8}$; D9.4.11; v9.4.14pr-1; D9.4.21 pr-1-2$3-1-5-6 ; 09.4 .36 ;$ D9.4.38pr-1-2-3; D9.4.42 $\mathrm{pr}-1-2$. 
I avol enus Noratiue Juliea Ponponius

Africain

Geius:

Venuleius Scaerola Papinien Paul

Ul pien

DI0.3.24 pri D10.3.25, D10.4.8.

$010.2 .+5.1$

010.3 .9$.

D10.3.11; D10.4.13.

D10.2.39.2-1.

D10.2.31; D10.2.34,

D10.2.11; D10.2.15; D10.2.25.15-17; D10.2.41; D10.3.8. 1-2-3-4; D10.3.15; D10.3.10.3; D10.3.27; D10.3.31;

D10.4.12 $\mathrm{pr}-4 ; \mathrm{D} 10.4 .16$.

D10.2.8pr; D10.2.12pr-1-2; D10.2.16.6; D10.2.18pr-1-2; D10.2.20.9; D10.2.49; D10.3.6.4-8; D10.4.3.6-7-10-12-

14; D10.4.5.6; D10.4.9.7; D10.4.11 pr-1; D10.A.17;

D10.4.20.

307

\section{Ierolenus}

Jalien

Pomponias

Geine

Mrecienus

Marcellus

Florentinue

Scaerole

Pepinien

Tryplooninue

Calliatrate

Paul

Ulpien

Hacer

Marcien

Total......
1
0
3
1
0
0
0
2

\section{$2011.1 .14 \mathrm{pr}$}

D11.1.15 pr-1; D11.7.15.

D11.3.15.

\section{D11.4.5.}

D11.4. 2.

D11.1.8; D11.1.13.1; D11.1.20 pr; D11.3.2; D11.3.1 D11.3.8; D11.3.8; D11.3.10; D11.3.12; D11.3.14pr$3-4-5-6-7-8-9$; D11.4.4; D11.5.4pr-1-2; D11.7.25.

11.1.9.2-8; D11.1.16 pr-1; D11.1.17, D11.3.1 pr-1-2-34-5; D11.3.3pr-1; D11.3.5 ur-1-2-3-4; D11.3.7; D11.3.0 pr-1-2-3; D11.3.11 pr-1-2; D11.3.1:3 pr-1; D11.4.1 pr-1-2-3-4-5-6-7-8; D11.4.3; D11.6.3.6; D11.7.2pr; D11.7.6pr3 DI1.7.31.1.

D11.3.17. 
Proculue tavolenua

Nerntiun

Celsus

Julien

Fomponius

Afrirain

Gein.

Garcellua

Pcaerol.

Scaerala

Fopinien

Fou!

Ulpien

Marcien

Modestin

Totel ........
DR.1.12.

D8.5.18.

D8.3.1!.

Marcien

Total.....
Inenlenue

Noracina

Prominiva

Africain

Giniun

Marcellun

Fapinien

Tryphoninu

rnul

Ulpien

185
D?.2.3R.

09.2.17; 19.2.51;r-1-2; Mn. 1.1A; DO. 1. 30pr-1-2-.3-1; Bn. $4.10 ; 10.4 .+11$

m.1.18; m.1. 1.3; m.1.13

on.1.28.

19.2.2pr-1-2; $D^{0} .2 .+1 \mathrm{pr}-1 ; \mathrm{t}^{2} .2 .8 \mathrm{pr}-1 ;$ n7.2. $12 \mathrm{pr}-1$;

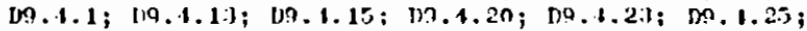
D9.1.27 $\mathrm{pr}-1 ; 109.1 .29 ; 19.1 .30$.

D9.2.31 $\mathrm{pr} ; 00.2 .16 \mathrm{pr}-1$.

ป9. 1.37.

Dn. 1.32

DQ.2.12; De.2.11; v9.2.18; D8.2.22 pr-1; D9.2.21; $109.2 .30 \mathrm{pr}-1-1 ; \mathrm{DS} .2 .31 ; 199.2 .33 \mathrm{pr} ; 09.2 .15 \mathrm{pr}-1-2-3-13$ D9.2.18; DQ.2.55; D9.4.4pr-1-2-3; 19.4.9; D9.4.10; D9.4.12; $\mathrm{D} 9.1 .17 \mathrm{pr}-1 ; \mathrm{M} .1 .19 \mathrm{pr}-1-2 ; \mathrm{D} 0.4 .22 \mathrm{pr}-1-2-$ $3-1 ; D 9.1 .21 ;$ DQ.4.2n pr-1-2-.3-1-5-6; DQ.1.11.

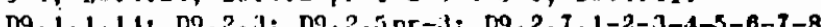

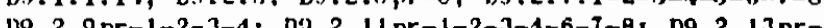

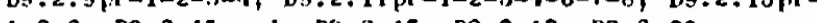

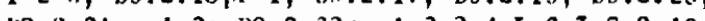
U9.2.21 pr-1-2; 199.2.23pr-1-2-3-4-5-6-7-8-9-10-114 D9.2. $25 \mathrm{pr}-1 ;$ D $.2 .27 \mathrm{pr}-1-2-3-5-6-9-11-17-22-24-28-34$; D9.2.35; v9.2.14.1; D9.2.46; D9.3.1 pr-8; 09.3.5.16-10; D9.1.2pr-1; D9.4.3; D9.4.5pr-1; D9.1. B; D9.4. $7 \mathrm{pr}-1 ; \mathrm{V0.4.8}$; D9.4.11; D9.4.14 pr-1; D9.4.21 pr-1-2$3-1-5-6 ; 09.4 .36 ; 09.4 .38 \mathrm{pr}-1-2-3 ; 09.1 .12 \mathrm{pr}-1-2$. บ?.2.16. 
Iavol enus Neratiue Juliea

Ponponiue

Africalo

Gaiue

Venuleios

Scaerola

Papinien

Paul

Ol pien

D10.3.24 Pr D10.3.25, D10.4.8.

D10.2. 15.1 .

D10.3.9.

D10.3.11; D10.4.13.

D10.2.39.2-4.

D10.2.31; D10.2.34.

D10.2.11; D10.2.15; D10.2.25.15-17; D10.2.41; D10.3.8.

1-2-3-4; D10.3.15; D10.J.19.3; D10.3.27; D10.3.31;

D10.4.12pr-4; D10.4.16.

D10.2.Rpr; D10.2.12pr-1-2; D10.2.16.6; D10.2.18pr-1-2; D10.2.20.9; D10.2.49; D10.3.6.4-8, D10, 3.6-7-10-12-

14; D10.4.5.6; D10.4.9.7; D10.4.11 Pr-1; D10.4.17;

D10.4.20.

Total....... 5 51 307

Jalied

Poponias

Gasins

Hracianus

Narcellus

Florentinus

Scaevole

Papinien

Trjphoninum

Calligtrate

Ulpien
Macer

Harci eo

Total.......

$\mathbf{1}$
$\mathbf{0}$
$\mathbf{3}$
$\mathbf{1}$
0
$\mathbf{0}$
$\mathbf{0}$
$\mathbf{0}$
$\mathbf{0}$
1
1
$\mathbf{2 1}$

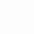

11

9

1

1

4

D11.4.5

46

D11.1.8; D11.1.13.1; D11.1.20 pr; D11.3.2; D11.3.4; D11.3.8; D11.3.8; D11.3.10; D11.3.12; D11.3.14pr-1-23-4-5-6-7-8 - ; D11.4.4; D11.5.4pr-1-2; D11.7.25.

D11.1.9.2-8; D11.1.16 pr-1; D11.1.17; D11.3.1 pr-1-2-31-5; $111.3 .3 \mathrm{pr}-1 ; \mathrm{D} 11.3 .5 \mathrm{pr}-1-2-3-4 ; 111.3 .7$; D11.3.0pr-1-2 3, D11.3.11 pr-1-2; D11.3.1:3 pr-1; 011.4.1pr-1-2-3-4-5-6-7-8; D11.4.3; D11.6.3.6; D11.7.2pr, D11.7.6pr, B11.7.31.1.

D11.3.17. 


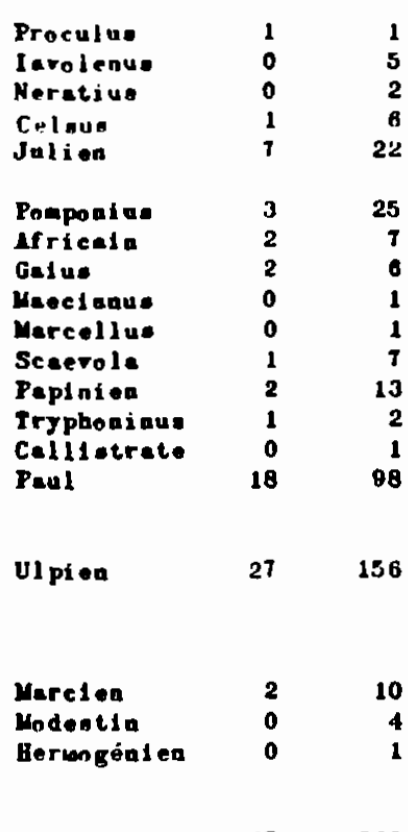

Tntel......

\section{D12.6.5.3.}

D12.4.16.

V12.1.19pr; D12.4.7.1; D12.4.11; D12.5.5; D12.6.32pr-3; D12.6.37.

D12.1.12, D12.4.15, D12.6.19.3

D12.1.23; 012.1 .41

D12.2.21; D12.6.63.

\section{D12.6.67 pr.}

D12.6.55, D12.6.58.

D12.6.64.

D12.1.2.4; D12.1.29; D12.1.31 pr-1; D12.2.14; D12.2.20; D12.2.22; D12.2.24; D12.2.30.1-1; D12.4.12; D12.6.13Pr; D12.6.15 Pr; D12.6.21; D12.6.36; D12.6.65.3-5-8.

D12.1.11.2; D12.1.13.2; D12.2.11.1; D12.2.13pr;

D12.2.16, D12.2.23; D12.2.25, D12.2.34pr, D12.4.1 pr;

D12.4.3.1-2-3-4-5-6-7-8-9; D12.4.5.1-2-3-4; D12.5.4.4;

D12.6.11, D12,6.26.12-13-14.

D12.3.5.1, D12.6.10.2.

\begin{tabular}{|c|c|c|}
\hline Celaun & 1 & 1 \\
\hline Juljen & 1 & 11 \\
\hline Inmponius & 3 & 20 \\
\hline Africnin & 2 & \\
\hline Gaiun & 2 & 12 \\
\hline Narcelling & $n$ & $\mathbf{3}$ \\
\hline Flenrentiuss & n & 2 \\
\hline Scnernla & 0 & $\mathbf{5}$ \\
\hline Papinien & 2 & B \\
\hline Tryplonninus & 0 & 2 \\
\hline Paul 1 & 10 & 56 \\
\hline Ul pien & 15 & 1.1 .1 \\
\hline Mnrcien & $n$ & 1 \\
\hline Mindestin & 0 & 2 \\
\hline Tolal $\ldots \ldots$. & 39 & 274 \\
\hline
\end{tabular}

D1:3.1.15.

DI3.1.A.1pr; DI.J.5.2.3; D13.6.2n, DI3.7.28.1. D13.6.1:1.2; D13.7.8 $\mathrm{pr}-1$.

Dis.

D13.6. $18 \mathrm{pr}-2$.

D1.3.1.17; 013.7 .16 .1

D1.3.1.3; D1.3.1.13; D1.3.5.63 D13.5.12; D13.5.19.2, D13.5.21 mr; D1.3.6.2.; D1.3.7.18.2-4; D1.3.7.25.

D13.1.4; D1:1.1.7.2; DI.3.3.3; DI3.1.2.2-3; D1.3.5.1.8; D13.5.5.10; D13.6.3.1-5; D13.6.5.6-7-13; D13.6.11; D13.7.11.5, D1:3.7.24.3. 


$\begin{array}{lr}\text { Juliea } & 3 \\ \text { Pomponiua } & 0 \\ \text { Africain } & 2 \\ \text { Gaius } & 2 \\ \text { Maecianus } & 0 \\ \text { Venuleiug } & 0 \\ \text { Scaerola } & 1 \\ \text { Papinien } & 2 \\ \text { Callistrate } & 0 \\ \text { Paul } & 19\end{array}$

Ul pien

Marcien

Hermogénien

Total......

\section{D14.3.12; D14.4.8; D.t.1.12.}

D14.1.7 pr-2

D1.4.4.11; D14.5.1.

D14.3.20.

D14.3.19.1-3.

D14.1.5 pr-1; D14.1.6pr-1; D14.2.2.2-5; D1 1.2.10 pr; D1.4.3.1.4; D14.3.16; D1.t.3.17 pr-1-3-1-5; D14.4.2; D14.4.4; D1.4.4.6; D14.4.10; D14.5.8.

D14.1.1.4-16-20-21-22-2.3; D14.1.4.2-3-4; D14.3.1; D14.3.5.3-7-8-9-15; D14.3.7.1; D1.4.3.11.7-8;

D14.3.13 pr-2; D14.1.1 pr-1-2-3-4-5; D14.4.3pr-1-2; D14.4.5 $\mathrm{pr}-1-2-3-1-5-6-7-8-8-10-11-12-13-14-15-16-17-$ 18-19; D14.4.7 pr-1-2-J-4-5; D14.4.9pr-1-2; D14.5.2pr; D14.5.3. larolenns

Neratius

Juliea

Pomponius

Arricaio

Gaing

Marcellus

Florentinus

Scaerola

Papinien

Tryphonina:

Paul

Ul pi en

230
D15.1.33; D15.1.35; D15.3.2.

D15.1.55; D15.3.18

D15.1.B.

D15.1.12, D15.1.11pr-1，D15.1.29; D15.1.37.1-2-3.

D15.1.2; D15.1.tpr-1-2-3-1-5-8, D15.1.22; D15.1.23; D15.1.25; D15.1.34; D15.1.49 pr-1-2; D15.2.3.

D15.1.38.2-3; D15.3.17 pr-1.

D15. $1.38 .20 \%$ DIS. J.17pr-1. D15.3.4, D15.3.12.

D15.1.16.

D15.1.16.

D15.1.51; D15.1.54; D15.1.58; D15.3.20.1; D15.3.21. D15.1.50.2-3.

D15.1.57 pr-1-2, D15.3.6.

D15.1.8; D15.1.18; D15.1.20; D15.1.26; D15.1.31,

D15.1.43; D15.1.47 pr-1-2-3-4-5-6; D15.1. 18pr-1;

D15.1.52Pr\} D15.1.5.3; D15.1.56; D15.2.2.1; D15.3.8,

D15.3.11; D15.3.14, D15.4.2pr-1-2, D15.4.5 pr-1.

D15.1.1 pr-1-2-3-1-3-6; D15 1.2pr-1-2-3-1-5-8-7-8-9-12 D15.1.5 pr-1-2-3-1; $015.1 .7 \mathrm{pr}-1-2-3-4-5-6-7$; $015.1 .9 \mathrm{pr}$ 1-2-3-4-5-6-7-8, D15 . 1, 11 pr-1-2-3-4-5-6-7-8-8,

D15.1.13; D15.1.15; D15.1.17; D15.1.19pr-1-2; D15.1. $21 \mathrm{pr}-1-2-3, \mathrm{D} 15 \cdot 1.24, \mathrm{D} 15 \cdot 1.30 \mathrm{pr}-1-2-4-3-\mathrm{g}-7, \mathrm{D} 15.1$. 32pr-1-2; D15.1.316; D15.1.41; D15.2.1 pr-1-3-4-5-6-78-9, D15.3.1 pr-1-2; D15, 3. 3pr-1-2-3-4-5-6-7-8-8-10; D15.3.5 pr-1-2-3; D15.3.7 pr-1-2-3-1; D15.3.10.5-8-78-8; D15.3.13; D15.4.1 pr-1-2-3-4-5-6-7-8-9; D15.4.3; D15; 1.4 . D15.1.10 pr-1. 
Ievol eaue Neratius Celsus

Jaliea

Pomponi us

Pricei

Gains

Marcellue

Floreatian

Scaerola

Pepiaien

Tryphoninus

Paul

olpie.

Modestin

Total $\ldots . .$.

\section{D16.3.33}

D16.1.32.5.

D16.1.13pr; D16.3.14.1.

D16.1.28 pr; D16.2.22.

D16.1.27, D16.2.19; D16.3.24.

D16.3.31.1.

D16.1.9; D16.2.9Pr; D16.3.21.1; D16.3.27

D16.1.26; D16.3.1.5-8-10-14-17-18-24-27-28-29-30-31-

32-33-12; D16.3.7 pr; D16.3.11;

D16.1.25 $\mathrm{pr}-1 ;$ D16.3.23.

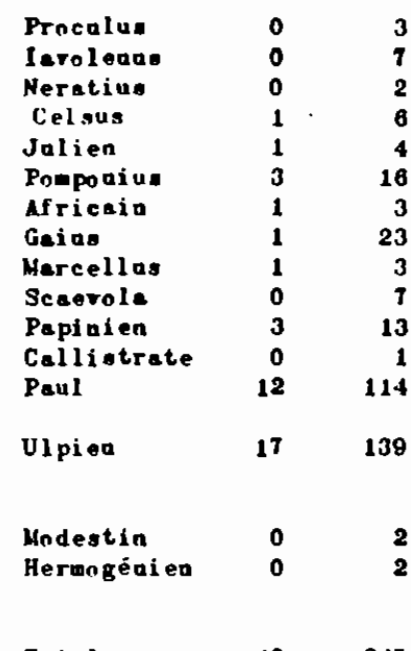

D17.1.50.1

D17.1.30.

D17.1.47.1; D17.2.18, D17.2.60.1.

D17.1.37.

D17.1.27.1.

D17.1.49.

D17.1.54pr-1; D17.1.57.

D17.1.5.4-5; D17.1.22.6-8-9; D17.1.26.7-8; D17.1.46; D17.2.10; D17.2.56; D17.2.6.5.5, D17.2.71.1.

D17.1.8.4-5-10; D17.1.10.1-7, D17.1.12.2-3-8,

D17.1.19; D17.2.23.1; D17.2.24; D17.2.49; D17.2.51.1; D17.2.52.4; D17.2.58.3; D17.2.63.2-9. 


\begin{tabular}{|c|c|c|c|}
\hline Proculas & $\mathbf{0}$ & 4 & \\
\hline Ierol eans & 3 & 19 & D18.1.63pr; D18.1.78; D18.6.17. \\
\hline Neratius & $\mathbf{0}$ & 1 & \\
\hline Celsus & $\mathbf{0}$ & 1 & \\
\hline Jalien & 2 & 12 & D18.2.17; D18.5.5.2. \\
\hline Pomponius & $\mathbf{T}$ & 31 & $\begin{array}{l}\text { D18.1.4; D18.1.6pr; D18.1.8pr; D18.1.12;D18.1.13; } \\
\text { D18.1.18.1; D18.1.31. }\end{array}$ \\
\hline Mfricain & $\mathbf{0}$ & 3 & \\
\hline Gai us & 1 & 16 & D18.1.35.3. \\
\hline Marcellas & 1 & $\mathbf{3}$ & D18.7.4. \\
\hline P. Inatas & $\mathbf{0}$ & 1 & \\
\hline Florentinas & 2 & $\mathbf{3}$ & D18.1.43pr-1. \\
\hline Scaevole & 1 & 12 & D18.5.8. \\
\hline Papinien & 6 & 14 . & D18.6.19pr; D18.7.5; D18.7.6pr-1; D18.7.7; D18.7.8. \\
\hline Tryphoninus & 1 & 1 & D18.7.10. \\
\hline Paul & 12 & 76 & $\begin{array}{l}D 18.1 .1 .1 ; D 18.1 .5 ; D 18.1 .34 \mathrm{pr}-2-6 ; 018.1 .40 .5 ; \\
\text { D18.1.56;D18.2.14.3;D18.4.21;D18.6.8.1; D18.7.3; } \\
\text { D18.7.9. }\end{array}$ \\
\hline Ul pi en & 10 & 84 & $\begin{array}{l}\mathrm{D} 18.1 .7 \mathrm{pr} ; \mathrm{D} 18.1 .9 \mathrm{pr} ; \mathrm{D18.1.11.1;} \mathrm{D} 18.1 .29 ; \mathrm{D} 18.2 .4 .1 ; \\
\text { D18.4.2.6-12-13-15; D18.7.1. }\end{array}$ \\
\hline L. Rufinas & 1 & 1 & D18.1.70. \\
\hline Marcien & 3 & 5 & D18.1.42; D18.1.44; D18.7.2. \\
\hline Hodestin & 0 & 3 & \\
\hline Hermogéni en & $\mathbf{0}$ & 5 & \\
\hline Total...... & 50 & 298 & \\
\hline
\end{tabular}

\begin{tabular}{|c|c|c|}
\hline Prnculus & 0 & 1 \\
\hline Iavolenus & 1 & 28 \\
\hline Neratius & 1 & 5 \\
\hline Celsug & 2 & 5 \\
\hline Julien & 3 & 11 \\
\hline l'nuponius & 1 & 28 \\
\hline Africain & $\mathbf{3}$ & $\boldsymbol{\tau}$ \\
\hline Gaius & 0 & 17 \\
\hline Marcellus & 2 & 5 \\
\hline Finrentinus & 0 & 1 \\
\hline Screvala & $\mathbf{0}$ & $\boldsymbol{T}$ \\
\hline Papinien & 2 & 8 \\
\hline Tryphoninug & 0 & 1 \\
\hline Paul & 21 & 79 \\
\hline Ulpien & 21 & 147 \\
\hline Marcien & $\mathbf{l}$ & 1 \\
\hline Mndestin & $n$ & 1 \\
\hline llermngenien & 0 & 1 \\
\hline
\end{tabular}

D19.2.60.7.

D19.1.31.1.

D19.1. $.38 \mathrm{pr}-1$.

D19.1.24 pr-1-2.

D19.1.55.

D19.1.30pr-1; D19.1.4.1.

D19.1.23; D19.2.18.1.

D19.5.7; D19.5.8.

D19.1.1pr; D19.1.21pr-3; D19.1.12; D19.1.13; D19.1.15 pr-1-2; DI9.1.51pr; D19.2.30.1; DI9.2.12; D19.2.13; D19.2.15pr-1; D19.2.51.2; D19.2.55pr; D19.1.2; D19.5.5pr-1-2- 5 .

D19.1.11.5-7-8-12; D19.1 13 1-2-3-1-5-13-18-22; D19.2.9.1; D19.2.11 pr 1; D19.2.13pr-7; D19.5.13.1; D19.5.1.1.1; D19.5.15; D19.5.18.

D19.5.25. 
Larnlenus Nerutius Pnampinius Africain

\section{Gaius}

Scaern1a

Papinien

Puul

UI pien

Tryphnninus

Marcien

Mndestit

Total

23
D.n.t.9pr.

D20.1.15 pr; D20.1.11.2-3; Din.6.7.1-2.

D:2n.1.32; D:20.1.31.1.

D20.1.20.1-13; D20.2.0; D20..3.5; D20.6.10.1.

D20.1.6; D20.1.8; D20.1.21.1; D20.1.27; D20.2.6; D20.6.4pr.

D20.1.13.1; D20.3.1.1; I)20.9.8.5. D2n.1.26.2.

Ulpi en

Marcien

Modestin

Hermogéni eo

Total....

Mricain

Gajus

13

Venuletus Scaevola

Papinieo

Callinirnte

Paul
Iarolenus

Celsus

Jalien

$$
10-2
$$

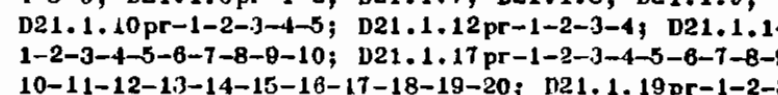
10-11-12-13-14-15-16-17-18-19-20; n21.1. 19 pr-1-2-3$4-5-6$; D21.1.21 pr-1-2-3; D21.1.23pr-1-2-3-4-5-6-7-8-9; D21.1.25 pr-1-2-3-4-5-6-7-8-9-10; D21.1:27; D21.1.29 pr$1-2-3 ; \mathrm{D} 21.1 .31 \mathrm{pr}-1-2-3-4-5-6-7-8-9-10-11-12-13-14-$ 15-16-17-18-19-20-21-22-23-21; D21 .1.3.3 pr-1; D21.1.35; D21.1.37; D21.1.38.2-3-7-10-1.1; D21.1.40pr; D21.1.42; D21.1.59 pr-1; D21.1.633 D21.2.4pr; D21.2.19.1; D21.2.21 pr-2-3; D21.2.25; D21.2.31; D21.2.32pr-1; D21.2.33; D21.2.37.1; D21.2.51.1-3; D21.3.1.4. $\mathrm{D} 21.1 .52$.

D21.1.62.
$021.1 .53 ; 021.2 .58$.

21.1.50; D21.2.7; D21.2.8; D21.2.38.1-3-4-5. D21.1.16; D21.1.36; D21.1.46; D21.1.18 pr-1-2-3-4-5$\mathrm{D} 21.2 .34 \mathrm{pr}-1$

D21.1.34p

$021.2 .69 \mathrm{pr}-1-2-3-4-5$.

D21.1.54; D21.1.55; D21.2.64 pr; D21.2.66.2; D21.2.67

1.1.2; D21.1.5; D21.1.11; D21.1.15; D21.1.30 pr-1; $1-2$; $\mathrm{D} 21.1 .17 \mathrm{pr}-1 ; \mathrm{D} 21.1 .5 \mathrm{~B} ; \mathrm{D} 21.1 .57 \mathrm{pr}-1$;

D21.1.58 pr-1-2; D21.1.60; D21.2.3; D21.2.5; D21.2.9; D21.2.11.1; DE1.2.15.1; D21.2.26; D21.2.35 21.2.41 pr-1; D21.2.42; D21.2.56.2-3.

.1.1 pr-1-2-3-1-5-6-7-8-8-10-11; D21.1.4 pr-1-2-3-

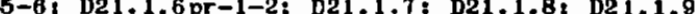




\begin{tabular}{|c|c|c|c|}
\hline Iavolenua & 0 & 1 & \\
\hline Neratius & 0 & 1 & \\
\hline Celsus & $\mathbf{0}$ & 5 & \\
\hline Jalien & 3 & 5 & D22.1.25.1 2; D22.3.20. \\
\hline Povponius & $\mathbf{0}$ & $\boldsymbol{T}$ & \\
\hline Africain & $\mathbf{0}$ & 1 & \\
\hline Ter.Clewent & $\mathbf{0}$ & 2 & \\
\hline Gaiug & 1 & 6 & D22.1.28.1. \\
\hline Venuleiug & 0 & 3 & \\
\hline Harcellas & $\mathbf{0}$ & 1 & \\
\hline Scaetola & 2 & 11 & $\mathrm{D} 22.2 .5 \mathrm{pr} ; \mathrm{D} 22.3 .0$ \\
\hline Papinien & 6 & 29 & $\mathrm{D} 22.1 .2 ; \mathrm{D} 22.1 .4 \mathrm{pr}-1 ; \mathrm{D} 22.1 .6 \mathrm{pr} ; \mathrm{D} 22.1 .8 ; \mathrm{D} 22.2 .4 .1$. \\
\hline Calliatrate & $\mathbf{0}$ & 8 & \\
\hline Paul & 9 & 81 & $\begin{array}{l}\mathrm{D} 22.1 .10 ; \mathrm{D} 22.1 .11 .1 ; \mathrm{D} 22.1 .14 \mathrm{pr}-1 ; \mathrm{D} 22.3 .4 ; \mathrm{D} 22.3 .7 ; \\
\mathrm{D} 22 . \mathrm{J} .8 ; \mathrm{D} 22.5 .4 ; \mathrm{D} 22.6 .1 .2 .\end{array}$ \\
\hline Ulpien & $\mathbf{3}$ & 27 & $\mathrm{D} 22.3 .14 ; \mathrm{D} 22.3 .18 \mathrm{pr} ; \mathrm{D} 22.4 .6$ \\
\hline L. Rafinus & 0 & 1 & \\
\hline Marcien & 2 & 9 & D22.1.32.1-5. \\
\hline Modeatin & 1 & 14 & D22.5.7. \\
\hline A.Ciruriaius & 0 & 8 & \\
\hline
\end{tabular}

Total ..... $27 \quad .220$

Proculas
Iavoleuus
Neratius
Celsus
Julied
Pampnius
Arricain
Ter.Clemens
Gaius
Marcellus
Florentiaus
Scaevola
Papinien
Tryphoninus
Caliogtrate
Paul
Ulpien

\section{I..Rufinus \\ Mlarcien \\ sindestin}

Hermogénien
D23.3.67.

D23.2.23; D23.3.58.1

D23.3.46 Pr; D2J.3.47; D2J.4.21

D23.2.8; D23.3.18; D23.3.65.

D23.5.9.2.

D23. $2.48 \mathrm{pr}-1-2$

D23.2.18

D23.2.32; D23.2.41.1; D23.2.50; D23.3.59.2.

D23.4.29.2.

D23.2.34.J; D23.2.62.1; D23.3.69.6-9.

D23.2.64 pr.

D23.2.11.2-3; D23.2.1Bpr; D23.2.J7; D2:3.2.44pr-1; D23.2.47; D23.2.66.1; D23.3.25; D23.3.56pr; D23.5.3pr D23.2.13; D23.2.27; D23.2.29; D23.2.31; D23.2.45 pr-12-3-1-5-6; D23.2.56; D23.3.10.2-5; D23.3.12.1; D23.3..39pr; D23.5.2.

D23.2.51 pr-1.

D23.2.28; D23.2.57; D23.2.58。 D2.3.2.12.1. 
Proculus Invol onus Neratiu.

Celsus

Jelien

Pomponius

Africein

Ter.Clement

\section{Geiu.}

Marcellus

Sceevola

Papinien

Trjphoninus

Cellistrate

Paul

Ulpien

178

.. Rufinus

Marcien

Hodeatin

Hermogénien

Total.....
D24.1.203 D24.1.50 pr-1, D24.3.66.1-3.

Neratius

Joli ien

Pomponius

Africain

Gaius

D24.3.31.4.

D2.4.1.18; D24.1.29 pr, D24.1.31.1-3-6-10; D24.3.67.

D24.1.8.

D21.1.58 pr-1-2.

D24.1.54, D24.3.42.1, D24.3.61.

D24.1.28.1-2-3-1-5; D24.1.38pr; D24.1.67; D24.2.1; D2. $4.2 .9 ;$ D24.3.23; D24.3.25.3; D24.3.26; D2.4.3.54 D24.3.56, D24.3.63.

D24.1.3.1-3-8; D2.1.1.7.8-9; D2.1.1.9pr-1; D2t.1.11.63 D24.1.17.1, D24.1.19pr-1, D24.1.22; D24.1.12.6-28; D2 1.2.11 pr-1-2; D24.3.7.10-16; D24.3.21; D24.3.22.13,

Scerrole

Scaerole

Tryphnninus

Paul

Ulpi en

tiarci en

Modeatin

Hermogéni en

Tntel......
D25.1.15.

D25.1.2, D25.1.6; D25.1.12; D25.2.3.1; D25.2.21.1-2; D25.3. $;$; D25.7.2

D25.3.5.18-19-20-21-22-2.3-24-25-2A; D25.4.1.10-13,

D2A.6.1.11; D25.7.1 pr-3.

D25.7.3pr.

D25.3. 6 pr-1; D25.3.7. $0-10$.

D24.3.35.

D24.2.10; D24.3.58.

D24.1.62.1. 


\begin{tabular}{|c|c|c|c|}
\hline $\begin{array}{l}\text { Iavoleaus } \\
\text { Neratius }\end{array}$ & $\begin{array}{l}\mathbf{0} \\
\mathbf{0}\end{array}$ & $\begin{array}{l}2 \\
4\end{array}$ & \\
\hline Cel sus & $\mathbf{0}$ & $\mathbf{1}$ & \\
\hline Julien & o & $\mathbf{3}$ & \\
\hline Pomponius & 1 & 15 & D28.7.B1. \\
\hline Africatn & $\mathbf{0}$ & 2 & \\
\hline Gatue & 2 & 19 & $\mathrm{D} 26.7 .13 \mathrm{pr}\} \mathrm{D} 26.8 .9 .1$ \\
\hline Venuleius & $\mathbf{0}$ & 1 & \\
\hline Harcellus & 1 & $\mathbf{5}$ & D26.8.12, \\
\hline Scaerola & $\mathbf{0}$ & 25 & \\
\hline Papinien & 8 & 49 & $\begin{array}{l}\text { D26.2.28.1-2; D26.5.13pr-1-2; D26.5.14; D26.7.37.1; } \\
\text { D26.7.39.18. }\end{array}$ \\
\hline $\begin{array}{l}\text { Tryphoninus } \\
\text { Calliotrate }\end{array}$ & $\begin{array}{l}1 \\
0\end{array}$ & $\begin{array}{r}16 \\
6\end{array}$ & D'26.7.58 pr. \\
\hline Paul & $\mathbf{3}$ & 64 & 1)26.2.32.2; D26.7.12.3, U26.7.2.1pr. \\
\hline Ul pien & 26 & 215 & $\begin{array}{l}\text { D26.1.8; D26.1.11.1; D26.2.10.4; D26.2.22; D26.4.1 pr- } \\
1-2-3 ; \mathrm{D} 26.4 .3 \mathrm{pr}-1-2-3-1-5-6-7-8 ; \mathrm{D} 26.1 .5 .1-4 ; \\
\text { D26.7.3.4; D26.7.5.9; D26.7.9pr; D26.7.11; D26.8.1 pr; } \\
\text { D26.10.2; D26.10.3.1. }\end{array}$ \\
\hline Jarcien & $\mathbf{0}$ & $\mathbf{5}$ & \\
\hline Nodegtin & 3 & 36 & D2甘.6.2.1; D26.7.32.3; D26.10.9. \\
\hline I. Aquil la & 2 & 2 & D26.7.34; D26.10.12. \\
\hline Hermogéni en & 1 & 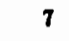 & $\mathrm{D} 26.5 .27 .1$ \\
\hline Total...... & 48 & 477 & \\
\hline
\end{tabular}

Iaroleaus
Neratius
Celsus
Julien
Pomponius
Gaius
Marcellus
Scnevnln
Papinien
Tryphoninus
Callistrate
Paul
Ulpien
Marcien
Mindestin
Herwogénien

Total......
D27.10.17.

D27.1.24; D27.1.30.3.

$\mathrm{D} 27.1 .44 \mathrm{pr}-1-2-3 ; \mathrm{D27} \cdot 1.45 \cdot 4$.

D27.2.1.2; D27.2.3.2; D27.3.1.3-6-8; D27.3.13; D27.5.1.2; D27.6.11.2; D27.8.1.15-16; D27.9.5.11.

9 D27.1.1.1; D27.1.10.7; D27.1.14pr-1-2-3-4; D27.1.15.8.
D27.1.43.

432 
Proculus Iarnlenus Neratiu: Celaus Julieo"

Pomponi us Africain Ter.Clemens Gatus

\section{Maecianus} Marcellua Florentinus Scaevola Papini on Tryplenninua Paul

Ul pi en

L. Rufinus Hacer Marcien

Hodestin Hermogéni en

1
17
1
13
32

D28.5.65; D28.5.60, D28.7.20.1, D28.8.11. D28.5.55.

D28.5.60 pr; D28.5.61; D28.7.21.

D28.1.12; D28.2.13.1-2; D28.5.7 pr-1; D28.5.8pr-1; D28.5.22, D28.5 :38pr-1-2-3-4-5; D28.5.11; D28.5.43;

27

D28.1.16 pr

D28.6.16 pr.

D28.1.8.4; D28.3.31 pr-1; D28.5.32.1; D28.5.89, n28.7.22.

D28.4.3 pr-1; D28.5.40; D28.5.54

D28.5. $00 \mathrm{pr}$.

D28.5.84 pr-1; D28.5.86; D28.6.48pr-1-2

D28.3.17; D28.5.77; D2R.5.79pr.

D28.2.28.:1; D28.5.91.

D28.1.14; D28.5.44; D28.5.53; D28.5.56; D28.5.58;

U28.5.85 pr-1-2; D28.5.90; D28.5.38.3; D28.6.4J.3.

D28.1.20.7; 028.1.21.1; D28.3.6.5-6-7-10; D28.3.12.1; D28.5.3pr-1-3; 128.5.0.1-3-4; D28.5.8 pr-14-16-17-18-

19-20; D28.5.30; D2B.5.35.J; D28.5.51 pr; D28.6.8.1;

D28.6.10.1-T, D28.6.18pr-1; D28.7.2 pr-1; D28.7.4.1;

D28.7.8.7, D28.8.1 pr; D28.8.5.1.

D28.5.39; D28.5.49.2; D28.5.52pr-1; U28.6.36pr; D28.7. $18 \mu \mathrm{r}$.

D28.1.19; D28.1.28; D28.6.3; D28.7.25.

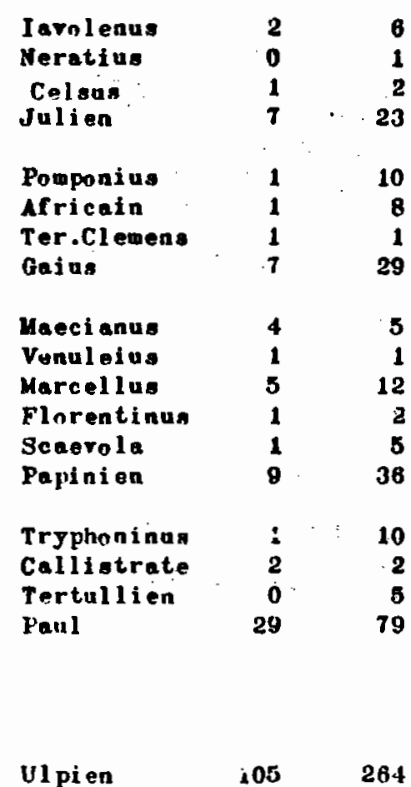

Macer Marcien Hodestia
D29.2.62.1; 029.2 .64 .

D29.4.25.

D29.2.43; D29.2.15.1-3-4; D29.4.22.1, D29.7.2.2, D29.7.4.

D29.2.36.

D29.2.47.

D29.2.82

D29.2.57.2, D29.4.17; D29.4.19; D29.5.9, D29.5.25 pr $1-2$.

D29.4.28pr-1, D29.5.14, D29.3.23.

D29.5.13.

D29.1.29.1，D24.1.31，D29.2.63; D29.4.5，D29.5.16.

D29.1.24.

D29.2.88.2, D29.5.4; D28.5.20; D29.5.21 pr-1-2;

DEQ.7.1.1, D29.7.12, n29.7.13 pr

D29.5.11.

D29.5.2, D29.5.27.

D29.1.37; D20.1.40.1-2; D29.2.22; D29.2.26; D29.2.58; D29.2.60; D29.2.65; C29.2.68; D29.2.73; D29.2.74.2$3-4$; D29.2.80.2-3, D29.2.93.1-2; D29.5.6 pr-1-2-3, D29.5.7, D29.5.8pr-1; D29.5.10pr-1; D29.5.12;

D29.5.22; D29.7.8.5

D29.1.13.2-3-4; D29.1.18.1, D29.2.6.7, D20.2.20.1-2-1; D29.2.25 pr-i-2-3; D29.2.30.7; D20.2.35 Pr; D29.2.42.2-3: D29.2.66; D24.2.67; D29.2.71 pr-1-2; D29.1.1.3-4; D29.4.6.8-10 ; D29.t.12pr; D29.4.29; D29.3.1 pr-1-2-3$1-5-6-7-8-9-10-11-12-13-1 \cdot 4-15-16-17-18=19-20-21-22-23-$ 24-25-26-27-28-29-30-31-32-33-34-35-36-37-38; D29.5 . J pr-1-2-33-4-5-65-7-8-9-10-11-12-13-1 4-15-16-1718-19-20-21-22-23-2.t-25-26-27-28-29-30-31-32; D29.5.5 pr-1-2-3; D29.5.24; D29.6.1.1.

D29.2.52 $\mathrm{pr} ; \mathrm{D} 29.5 .15 \mathrm{pr}-1-2$

D29.2.50; D29.5.17; D29.5.18; D29.5.19. 
Neratius

\section{Celsus}

Julien

Pomponius

Africain

Gaius

Narcellue

Florentinu

Papinien

Paul

Ulpier

Marcien

n.Maximu.
D:3n. (i3).

D.30.6; D:H. i6; D30.R1.1-2-1-6-9-10; D.30.82pr-4-6;

D3n.8. . 1-1-5-8-9-10-11-12; B:30.86 pr-1-2; D3n.91 pr-1-

2-3-4-5-6-7; Din.02. 1; D:30.9.4.1-2-3; Di3n.98 pr-2-4;

D:30.97; D30.98; D30.98; D30. InIpr; b:30.1n2.

D30.8.2; D.30.12.1- 2; D330.13; D30.2n; D.30. $34 \mathrm{pr}$

D30.36 pr-1-3; D.30.38. 1; D30. $15 \mathrm{pr}-1-2$; D.30.16

Di3n. $18 \mathrm{pr}-1$; Di3n.5.4.2; Di30.56.

D30.107.1; D30.108pr - 1-2-3-1-5-0-11-14-15; D30.109

D30.110.

D330.65 pr-1; D3n.67pr; D3n.68.1-2-.3; D3n.69pr-1-4-5;

D30.70 pr-2-3.

D3n . 83.

D30.116.2-3.

D30.11.

D30.5 pr-1; D:3n.7; D30. 35; D:30.52pr; D:30.62.

D30.32. 1; D3n. 34.9 ; D3n.37 pr; D30.30 pr-1; D30.44 pr-

$7-8 ;$ D.30. 17.2-3; D30.50,rr-2; D30.5.3.2-3-1-8-9

D30.71.1-3-4; D30.75.3-1; D3n.95

D30.112.1 ; J:10.113pr-1; D30.114.1-10-12-14-19,

D30. 119

D30 . 125.
Prnculus

Iarol enus

Celsus
Julien

Pomponitus

Pomponius

Haurictanus

Ter.clewen

Gaius

Marcellins

Scaevola

Papinien

Tryphoninus

Callistrate

Fanl

vipien

L. Rufinus

Modestin

Tntal.....
D:34.48pr.

D31.37; D.J1.38; D3i.4n.

D31.15; D31.18; D31.19; [31..20; D31.29pr.

D31. $11 \mathrm{pr}-1$.

D31.59.

D31.55.1.

D31.17.1; D31.25; D31.26; D31.29; D31.50.1

131.88.3-6-7-9-11-13-14; D31.89.6.

D31.65 pr-2-3, D31.66.3; D31.67.7; D31.73; D31.76pr-4; D31.77.11-13-15-17-21-27-28-29-32; D.31.79. D31.88.12.

D31.5.1; D31.8pr-1-5; D31.1.4pr-1; D31.82.2; D3i.83; D31.8.; D31.83pr, D31.87.2.

D3t. 21

D3i.9; D31.31; D31.34yr-2-1-6; D31.35. 
Proculus Iavol enua
Heratius Celaus

A. Valena

Jalien

Porfoniua

Africai

Gaius

Neecianua

Marcellu:

Scaevola

Pepiuien

Trypioninus

Fuul

Ul pi en

Marcien Indestin Hermengéni en

Total......
D32.29.3-4; v32.30.2, D32.77; D32.100 pr.

D32. $7 \theta_{\mathrm{p}} \mathrm{rr}-3$

D32.12; D.32.94.

E32. 22.

D32. iif D.32.85.

D32.17.1, D32.85.

D32.68.1

D32.35 pr-2; D.32.37.2-3-7; D32.38.1-2; D32.39 pr

D32. $11.1-2-3-4-3-10$; D32.93.2; D32.101 pr-1; D32.102.3. D32. $91 \mathrm{pr}-2$.

D32.38.5.

D32.1; D32.5.1; D32.8.1; D32.60.12-3; D32.61; D32.60;

D32.78pr-1-2-3; D32.92pr; D32.97; D32.99 pr-1-2-3-4-5.

D32. $\mathrm{pr}-1-10$; D32. 3.1 ; $332.7 \mathrm{pr}$, D32.11.16, D32.49pr-7 D.32.6A.1-2-3; D.32.7; $332.73 \mathrm{pr}-1-2-3-4-5$.

D32. $65 \mathrm{pr}-1-2-3$

D32.81 pr-1; D32.82; D32.83.1

D32.22.2.
Proculus
Iavol enus

Neratius

Celsus

A.Valens

Julien

Pompolus

\section{Africain}

Laurici nnus

Ter.Cl emens

Galus

Venuleius

Marcellus

Florentiuus

Scaevnla

Tryploninus
Callistrate
Paul

Ul pien

51

197

Harcien

Hindestin

Mermogéni en
- LIVRE xodIII -

D.33.6.B.

D33.4.6.1, D33.5.14; D33.5.15; D33.7.25.1-2; D33.8.17, D33.8.22pr-1.

D3:3.8.13; D33.8.25.

D33.5.9pr-1-2, D.33.5.10; D33.5.11; D33.5.12; D33.8.12. D33.1.2, D33.2.20, D33.5.6; D33.5.8pr-2, D33.7.15 pr-2; D33.8.10.

D33.8.16 pr-i.

D33.5. 17 .

D33.8.2; D33.8.4.

D33.3.2.

D33.1.13.1; D33.1.18ur-1; D33.1.19pr; D.3.3.1.20.1, D33.1.21 pr-1-4; D33.2.32pr; D33.2.33pr-1-2; D33.2.34 pr; D33.2.35, D33.2.3Apr-1; D33.2.37; D33.5.18, D33.5.213 D33.7.7; D.33.7.20 pr-1-3-3-4-5-6-9, D33. $7.27 \mathrm{pr}-1-3-4$; DJ3.7.28, D33.8.21; D33.8. 23pr-1-2-3.

D33.1.10.2, D33.2.2, D33.2.24.1; D33.3.5, 133.4.8, D3:1.7.3 pr-1, D.33.8.10 pr-1-2.

D33.1.9, D33.1.11; D33.1.16; D33.2.21; D33.5.7, D33.5.13pr-1; D33.7.:3pr-1, D33.7.14; D33.7.16.2, D33 .7 .18.1-4-5-6-7-8-0-10-11-13; D33.7 .19 pr-1;

D.3.7.7.22.1; D:33.R.1; D3i.8.3, D:3.8.5, D33.8.9pr-1-2, D3:.8.15, D.3J.9.2, $1-5$, D33.10.12.

E33.2.7; 0.33.1.1.6-10; D33.5.2pr-1-2, D33.6.8.3, D.33.7.8pr-1, D3.3.7.12 pr-2-3-1-5-6-7-8-8-10-12-13-3132-33-35-:37-38-42-41-45-43; D33.8.6pr-1-2-.1-4-5, D33.8.8 pr-i-3-3-4-5-6-7-8; D33.8.11; D33.8.24; D33. $9.3 \mathrm{pr}-6-8$.

D33.7.17.2; Di33.8.18; D33.8.20. D33.2.18. 
Iarnl enus Neratius Celous

Celous

A. Val en

Julien

Pomponiue

Mrricain

Gaius

Maecianus

Marcellue

Scaerni.

Papinien

Tryphnninus

Calliatrute

Ul pien

Marcien

Mndestin

Hermongéni en

.......

Tntal......

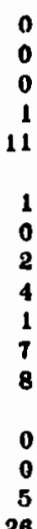

5

3
0
1
1
D36.4.15.

D36.1.20.1-2-3; D36.1.28.1-I3-17; D36.2.113

D36.2.16 pr-1-2; D36.2.17.

D36.1.72.1.

D36. 1 . B5.4-15.

D36.1.66.3; D36.1.67 pr-3; D36.1.71.

D36.1.16 pr.

D36.1.80.2-3-12-13, D36.2.27.1, D36.3.18pr-1.

D36.1.5.1.1; D.36.1.55; D36.1.57 pr-1-2, D36.1.60.4-6,

D36.2.25. 1 .

D.36.1.41.1; D36.1.42; D 36.1.62; D36.1.68.2; D.36.3.7.

D36.1.1.10; D36.1.3.5; D36.1.6.5; D36.1.11.2;

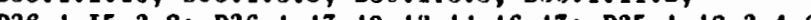

D36. 1.

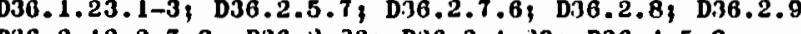

DJ6.2.12.2-7-8; D36.2.23; D36.3.1.20\} D36.4.5.8

D36.1.3I;2; D36.1.32pr-1.

Neratius

Celsus

D36. $4.11 \mathrm{pr}$

D36.4.16.
Marcien

Godestin

Julien

Africain

Ter.Clemens

Gaius

Karcellus

Senerola

Papinien

Tryphoninus

Pau 1

ul pi en

33

290

D37.4 - 13pr, D37.10,7.3-7; D37. 13.2pr-1.

D37. 14.10

D37.14.22.

D37.15.3.

D37.14.18

D37.1.78pr; D37.7.6, D37.10.11; D37.15.11.

D37.14.23 pr-1, D37.15.10.

D37.1.6pr; D37.4.4pr; D37.9.4.1, D37.10.6.3; D37.11.10 D37.14.6 pr-1-2-3-4; D37.14.15

D37.1.7 pr; D37.4.1.9, D37.4.3.15-16; D37.5.3.2,

D37.5.8.2; D37.10.1.5-6; D37.10.3.5-11; D37.11.2.9;

D37.12.1 pr-2-5; D37.14.1; D37.14.2; D37.14.11;

D37. 14.14, D37.14.16 pr-1-2; D37.14.17 pr-1; D37.15.1.1; D37.15.5 pr-1, D37.15.7pr-1-2-3-1-3; D37.15.9.

D37.14.3, D37.14.4, D37.14.5pr-1; D37.15.4

D37.14.7 pr-1; D37.14.8pr-1; D37.14.9pr-1; D37.14.12

DST . 14.13.

Hermogéni en 7

D37.4.2, D37.5.23, D37.14.21 pr-1-2-3-4.

Total..... $85 \quad 494$ 


\begin{tabular}{|c|c|c|c|c|c|c|}
\hline Iarolenü & 6 & 6 & $\begin{array}{l}\text { D33.1.21; D38.1.33; D38.2.34, D38.2.35; D38.2.36, } \\
\text { D38.5.12. }\end{array}$ & $\begin{array}{l}\text { Ierol enue } \\
\text { Neretiue }\end{array}$ & $\begin{array}{l}0 \\
0\end{array}$ & $\begin{array}{l}5 \\
2\end{array}$ \\
\hline $\begin{array}{l}\text { Neretive } \\
\text { Cel gus }\end{array}$ & $\begin{array}{l}2 \\
2\end{array}$ & $\begin{array}{l}2 \\
3\end{array}$ & $\begin{array}{l}\text { D38.1.60 pr-1. } \\
\text { D38.1.30 pr-1. }\end{array}$ & Colvus & $\mathbf{0}$ & 2 \\
\hline A.Velous & 2 & 2 & D38.1.46; D38.1.4T. & $\begin{array}{l}\text { Julien } \\
\text { Pouponius }\end{array}$ & 0 & $\begin{array}{l}31 \\
19\end{array}$ \\
\hline Julien & $\mathbf{3 0}$ & 38 & $\begin{array}{l}\text { D38.1.11; D38.1.23pr-1; D38.1.24; D38.1.25 pr-1-2-3-4; } \\
\text { D38.1.27; D38.2.11; D38.2.13; D38.2.20 pr-1-2-3-4-5-6; } \\
\text { D38.2.21; D38.2.23pr-1-2; D38.2.24; D38.2.25; D38.5.6; }\end{array}$ & $\begin{array}{l}\text { Africain } \\
\text { Gaiue } \\
\text { Marcellus }\end{array}$ & $\begin{array}{l}1 \\
\mathbf{5} \\
\mathbf{2}\end{array}$ & 25 \\
\hline Pomponiug & 18 & 29 & $\begin{array}{l}\text { D38.5.8; D38.5.9; D38.6.2; D38.13.1. } \\
\text { D38.1.3pr-1; D38.1. } 1 \text {, D38.1.8pr-1; D38.1.10 pr-1; } \\
\text { D38.1.12; D38.1.34; D38.2.2pr-1-2; D38.4.2; D38.4.4; } \\
\text { D38.4.12; D38.4.13pr-1-2-3. }\end{array}$ & $\begin{array}{l}\text { P. Iuntua } \\
\text { Scaerola } \\
\text { Papinien } \\
\text { Peul }\end{array}$ & $\begin{array}{l}0 \\
1 \\
2 \\
5\end{array}$ & $\begin{array}{r}2 \\
18 \\
132\end{array}$ \\
\hline $\begin{array}{l}\text { Africeln } \\
\text { Ter.clemen. }\end{array}$ & $\begin{array}{l}3 \\
7\end{array}$ & $\begin{array}{l}3 \\
7\end{array}$ & $\begin{array}{l}\text { D38.2.26; D38.2.27; D38.5.10, } \\
\text { D38.1.14; D38.2.38pr-1; D38.2.39; D38.2.40; D38.4.10 } \\
\text { pr-1. }\end{array}$ & U1 pi on & 20 & 284 \\
\hline Gatus & 10 & 25 & $\begin{array}{l}\text { D38.1.19; D38.1.22pr-1-2; D38.1.49; D38.2.5pr-1; } \\
\text { D38.2.1; D38.2.30; D38.8.2. }\end{array}$ & $\begin{array}{l}\text { Harcien } \\
\text { Modestio }\end{array}$ & $\begin{array}{l}8 \\
0\end{array}$ & \\
\hline $\begin{array}{l}\text { Mercellus } \\
\text { Floreutinas }\end{array}$ & $\begin{array}{l}3 \\
2\end{array}$ & $\begin{array}{l}\mathbf{5} \\
\mathbf{2}\end{array}$ & $\begin{array}{l}\text { D38.2.31, D38.2.32, D38.15.5.2. } \\
\text { D38.2.28pr-1. }\end{array}$ & Hermogéni en & o & \\
\hline $\begin{array}{l}\text { Sceerol } 3 \\
\text { Pepinien }\end{array}$ & $\begin{array}{r}5 \\
10\end{array}$ & $\begin{array}{r}7 \\
20\end{array}$ & $\begin{array}{l}\text { D38.1.44; D38.1.45; D38.2.48; D38.4.7; D38.5.7. } \\
\text { D38.1.40; D38.1.41; D38.1.42; D38.1.43; D38.2.41; } \\
\text { D38.2.42-1-2-3; D38.2. 13; D38.4.11. }\end{array}$ & Total...... & 47 & 572 \\
\hline $\begin{array}{l}\text { Trjphoninua } \\
\text { Calliatrate }\end{array}$ & $\mathbf{8}$ & 8 & $\begin{array}{l}\text { D38.2.15, D38.2.50 pr-1-2-3-4-5-6. } \\
\text { D38.1.38 pr-1. }\end{array}$ & & & \\
\hline Paul & 44 & 74 & $\begin{array}{l}\text { D38.1.1; D38.1.16pr-1; D38.1.17; D38.1.18; D38.1.20pr-; } \\
\text { D38.1.28; D38.1.35; D38.1.37 pr-1-2-3-1-6-6-7-8; } \\
\text { D38.1.39pr-1; D38.1.5 1; D38.2.4pr-1-2-3; D38.2.0; } \\
\text { D38.2.18; D38.2.42pr; D38.2.44pr-1-2; L38.2.45; } \\
\text { D38.2.46; D38.2.47 pr-1-2-3-4; D38.2.49; D38.2.51; } \\
\text { D38.5.5 pr-1; D38.5.11; D38.10.10.5. }\end{array}$ & & & \\
\hline Ul pi en & 183 & $\mathbf{3 1 7}$ & 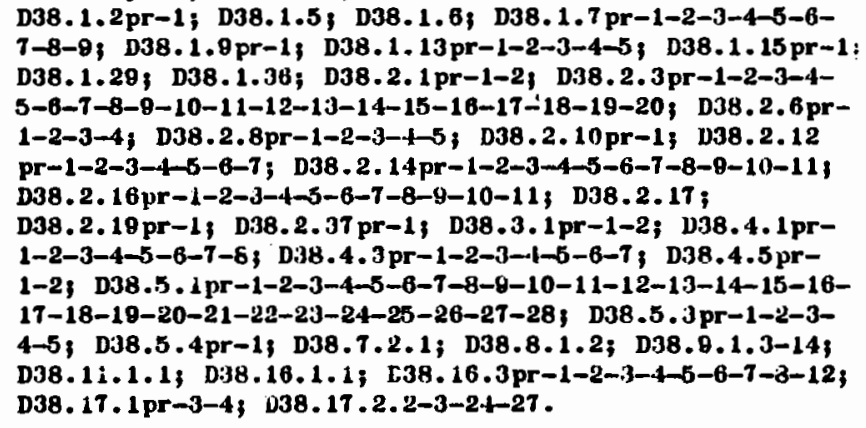 & & & \\
\hline Macer & $\mathbf{0}$ & 1 & & & & \\
\hline Marcion & $\mathbf{5}$ & 6 & D38.2.22; D38.2.29pr-1，D38.1.6; D38.5.2. & & & \\
\hline Modeatia & $\boldsymbol{T}$ & 27 & $\begin{array}{l}\text { D38.1.31; D38.1.32; D38.2.33; D38.4.8; D38.4.8; } \\
\text { D38.8.7; D38.10.4.8. }\end{array}$ & & & \\
\hline Hermogónieu & $\mathbf{3}$ & 4 & D38.1.48 pr-1-2. & & & \\
\hline
\end{tabular}

D39.2.42; D39.5.2.7; D39.6.18.3.

D39.6.23.

D39.4.2, D39.4.13.2-3; D39.5.11, D39.6.31.2. D39.5.20 pr; D39.6.38.

D39.5. 35 pr

D39.5.31.2; D39.6.41

D39.3.11.3; D39.5.8; D39.5.10; D39.6.39; D39.6.44. D39.4.12.1-2, D.9.5.13; D39.5.16; D39.5.18.1-2; D39.5 -19.4, D39.6.8pr; D39.6.36, D39.6.37.1

D39.4.16pr-1-2-3-4-9-10-11. 
D40.1.13; D40.2.1; D40.4.3; D40.4.4 pr-1-2; D40.4.5; D10.4.8; D40.4.11 pr-1-2; D40.4.40 pr-1; D40.4.41 pr-1-2; D40.4.46; D40.4.61 pr-1-2; D40.5.8; D40.5.20 D40.5.34 pr-1-2; D40.5.4.4; D40.7.5 pr-1; D40.7.8 pr-1 D 40.7.11; D D $0.7 .21 \mathrm{pr}-1 ; \mathrm{D} 10.7 .29 \mathrm{pr}-1 ; \mathrm{D} 40.7 .30$ D40.8.22; D40.9.23; D40.12.28; D40.12.43; D 10.13 .3 ; D40.14.3pr-1.

Africain

6 D 40.6 .1 ; D 10.9.24; D 10.9.31; D40.9.32 pr-1-2.

27 D.10.1.18; D $10.1 .25 ; \mathrm{D} 40.2 .7 ; \mathrm{D} 40.2 .25 ; \mathrm{D} 40.1 .21 ;$ D-40.4.57; D.10.7.31 pr-1; D.40.7.37; D40.9.3; D10.9.1n; D40.9.29 $\mathrm{pr}-1$; D40.12.2; D10.12.4; D40.12.6; D 10.12 . $9 \mathrm{pr}-1-2 ; \mathrm{D} 40.12 .11 ; \mathrm{D} 40.12 .13 \mathrm{pr}-1 ; \mathrm{D} 10.12 .25 \mathrm{pr}-1-2$; Opr-1-2; D40.12.11;
D40.12.26; D.10.16.1.

D.40.4.55 pr-1-2; D.10.4.58; D 10.5.32 pr-1-2; D40.5.35; D40.5.36 pr-1-2; D4n.5.42; D40.5.54

Maecianue

$6 \quad$ D40.12.44 $\mathrm{pr}-1-2 ; \mathrm{D} 40.11 .2 \mathrm{pr}-1-2$.

Marcellus D40.7.2.4; D.0.13.2; D40.14.1

$39 \quad \mathrm{D}+0.4 .29 ; \mathrm{D} 10.4 .54 \mathrm{pr}-1 ; 040.4 .59 \mathrm{pr}-1-2 ; \mathrm{D} 10.4 .60$ D40.5.18; D.0.5. $19 \mathrm{pr}-1$; $\mathrm{D} 40.5 .41 \mathrm{pr}-1-2-3-4-5-6-7-8-9-$ 10-11-12-13-1 1-15-16-17; D40.7.40 $\mathrm{gr}-1-2-3-4-5-6-7-8$; D.0.9.6; D 40.11 .3 .

$\mathrm{D} 40.1 .19 ; \mathrm{D} 40.1 .20 \mathrm{pr}-1-2-3 ; \mathrm{D} 40.1 .21 ; \mathrm{D} 40.1 .22 ;$ D40.3.3; D40.4.47 $\mathrm{pr}-1$; D40.4.48; D40.4.19; D40.4.50 $\mathrm{pr}-1 ; \mathrm{D} 40.4 .51 \mathrm{pr}-1 ;$ T10.5.21; D 10.5.22 pr-:-2; $\mathrm{D} 40.5 .23 \mathrm{pr}-1-2-3-4 ; \mathrm{D} 49.7 .33 ; \mathrm{D} 40.7 .3 . \mathrm{pr}-1 ; \mathrm{D} 40.7 .35$; D.10.7.38; D40.3.8; D40.9.25; D40.10.1pr-1; D40.10.2; D.10.12.35; V40.12.36; D40.14.4; D40.14.5; D $40.15 .2 \mathrm{pr}-$ $1-2$.

$\mathrm{P} 10.4 .59 .1 ; \mathrm{D} 40.5 .17$

$\mathrm{D} 40.12 .29 \mathrm{pr}-\mathrm{i}$

D.10.8.3; D10.12.37; D40.15.4pr-1; D.10.16.3.

D.10.1.3; D $10.1 .9 ;$ D:0.1.10; D.40.1.11; J10.1.12;

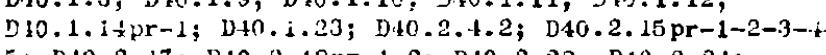
5 ; D10.2.17; D.10.2.18pr-1-2; D.10.2.22; D-40.2.2.t; D4n.4.10 pr-1; D.10.4.27; D 40.4.28; D40.4.31; 1) 10.4.33; D.40.4.34; D40.4.35; D40.4.36; D40.4.37; D40.4.38; $\mathrm{D}: 40.4 .39 ; \mathrm{D} 10.4 .52 ; \mathrm{DA0.4.53}$; D40.4.56; D40.5.5; D4h.5. 6 ; D $40.5 .25 ; D 40.5 .27 ; D 40.5 .29 ; D 40.5 .31 \mathrm{pr}-1-$ $2-3-4$; D10.5.33 $\mathrm{rr}-1-2 ; \mathrm{D} \cdot 10.5 .38 ; \mathrm{D} 40.5 .39 \mathrm{pr}-1$;

D $10.5 .40 \mathrm{pr}-1 ; 1) 40.5 .43 ; 0+0.7 .1 \mathrm{pr}-1 ; 0.40 .7 .4 \mathrm{pr}-1-2-3-$ $1-5-6-7-8$; D In.7.7; 5.40.7.10; v40.7.18; D 10.7.20 pr-1$2-3-4-5-8 ; 010.7 .22 \mathrm{pr}-1-2 ; \mathrm{D} 10.7 .38 ; 240.7 \cdot 11 ;$

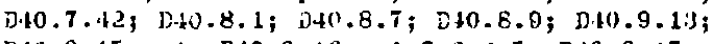
D.40.9.15 pr-1; D.10.9.16pr-1-2-3-t-5; D.10.9.17 pr-1-2; D $40.9 .18 \mathrm{pr}-1 ; \mathrm{D}) 10.9 .26$; D $40.9 .28 ; \mathrm{D} 4 \mathrm{4} .10 .5 ; \mathrm{D} .10 .11 .4$; D40.12.15; D 10.12 .17 ; D 40.12.19; D 10.12.23pr-1-2;
D40.12.24 pr-1-2-3-4; $240.12 .32, D 40.12 .33,040.12 .38 \mathrm{pr}-$ $1-2-3 ; 240.12 .39 \mathrm{pr}-1-2-3 ; \mathrm{D} 40.12 .4 \mathrm{pr}-1 ; \mathrm{D}-40.13 .4$; D40.13.5.

D40.1.1; D40.1.2, D40.1.4 pr-1-2-. 13-14; D40.2.2; D 30.2 .3 ; D40.2.8, D-30.2.11; D40.2.12, D+0.2.13, G H0.2.16 pr-1; D-30.2.20 pr-1-2-3-4; [40.3.1; D4G.3.2, D 40.4 .1 ; D.40.4.2, D40.4.6; D40.4.7, D40.4.8pr$13 \mathrm{D} 40.4 .12 \mathrm{pr}-1-2-3$; $\mathrm{D} 40.4$. $\mathrm{i3pr}-1-2-3$; $\mathrm{D40}-4$. 14; D40.4.25; D40.4.30; D4n.4.32; D40.5.1; D40.5.23 D-40.5.3; D40.5 . $4 \mathrm{pr}-1-2-3-4-5-6-7-8-9-10-11-12-13-14-15-$ 16-17-18-19-20-21-22-23; D40.5.7; D40.5.24 pr-1-2-35-6-7-8-8-10-11-12-13-14-15-16-17-18-18-20-21, 040.5 . $26 \mathrm{pr}-1-2-3-1-5-6-7-8-9-10-11 ;, \mathrm{J} / 40.5$. $28 \mathrm{pr}-1-2-3$ 4-5; 040 .5 .30 pr-1-2-3-4-5-6-7-8-9-10-11-12-13-14-1516-17; D40.5.37; D40.5 . $45 \mathrm{pr}-1-2 ;$ D40.5 .46 pr-1-2-3-4-5; D40.5.52; D40.7.2pr-1-2-3-4; D40.7.3 pr-1-2-3-4-5-6-7$8-9-10-11-12-13-14-15-16-17$; $040.7 .6 \mathrm{pr}-1-223-4-5-6-7$ D40.7.9pr-1-2-3; D40.7.16; D40.7.19; D40.8.4; D40.9.1; D $10.9 .2 ; \mathrm{D} 40.9 .1 ; \mathrm{D} 10.9 .12 \mathrm{pr}-1-2-3-4-5-6-7 ; \mathrm{D} 40.9 .14 \mathrm{pr}-$ $1-2-3-4-5-6 ; \quad D 40.9 .30 \mathrm{pr}-1-2-3-1-5 ; \mathrm{D} 40.10 .4 ; \mathrm{D} 40.10 .63$ D10.11.1; D40.12.1 pr-1-2; D40.12.3pr-1-2-3; $\mathrm{D} 40.12 .5 \mathrm{pr}-$ 1; $040.12 .7 \mathrm{pr}-1-2-3-4-5 ; \mathrm{D} 40.12 .8 \mathrm{pr}-1-2 ; \mathrm{D} 40.12 .103$ D40.12.12 pr-1-2-3-1-5-6; J H 0.12.14 pr-1-2; DH0.12.16 pr 1-2 -3-4; D40. 12.18pr-1; D40.12.20 pr-1-2-3-1; D40.12. $22 \mathrm{pr}-1-2-3-4-5-6 ; \mathrm{D} 44.12 .27 \mathrm{pr}-1-2 ; \mathrm{D} 40.12 .3 \mathrm{i}$; $\mathrm{D} 40.12 .34 ; \mathrm{D}+10.13 .1 \mathrm{pr}-1 ; \mathrm{D} 40.14 .6 ; \mathrm{D} 40.16 .2 \mathrm{pr}-1-2-3-4$; D40.10.4

L.Rufinua $22 \quad 2 \quad$ D40.5.16; D 40.7.32

Harcien $\quad 46 \quad 46 \quad 040.1 .5 \mathrm{pr}-1 ; \mathrm{D} 40.1 .8 \mathrm{pr}-1-2-3 ; \mathrm{D} 40.2 .9 \mathrm{pr}-1 ; \mathrm{D} 40.2 .10$; D.10.2.14pr-1; D40.4.23 $\mathrm{pr}-1 ; \mathrm{D} 40.4 .26 ; \mathrm{D} 4 \mathrm{n} .5 .50$; D40.5.51 pr-1-2-i3-4-5-6-7-8-9-10-1i-12; D40.5 .5 3 $\mathrm{pr}-1$, D.40.5.55 pr-1; D $10.8 .5 ; \mathrm{D} 40.8 .6 ; \mathrm{D} 40.9 .9 \mathrm{pr}-1-2 ;$

D40.9.11 $\mathrm{pr}-1, \mathrm{D} 40.10 .3 ; \mathrm{D} 40,11.2 ; \mathrm{D} 40,15,1 \mathrm{pr}-1-2-3-4$ D.40.1.16; D4h.1.17; D40.2.21; D40.4.13; D40.4.44; D40.4..15; D.10.5.11; D40.5.12pr-1-2; D40.5.13; $D 40.5 .14 ; D 40.5 .15 ; D 40.7 .25 ; \quad 040.7 .26 \mathrm{pr}-1 ; \quad D 40.7 .27$ $\mathrm{D} 40.8 .2$; $1 \mathrm{D} 40.9 .19$; D 40.9 .20 ; D 40.9 .21 ; D $40.11 .5 \mathrm{pr}-1$; D40.12.21 pr-1 


$\begin{array}{lrr}\text { Q.Ilucius } & 0 & 1 \\ \text { Prnculus } & 0 & 1 \\ \text { Iarolenus } & 4 & 23 \\ \text { Neristius } & 1 & 9 \\ \text { Celsus } & 0 & 7 \\ \text { Julien } & 20 & .19\end{array}$

Fompnnius

Africain

Gaius

Venuleius

Marcellus

Florentinus

Florentinus

Pepinien

Tryphnninus

Callistrate

Tertullien

Peul
D.1.2.23.2; D.1I.2.24; D.11.3.16; D 11.3.19

D.11.10.5.1.

D41.1.37pr-1-2-3-1-7-6; b.1.1.39; D11.2.38pr-2; D.H.:1.33pr-6; D.I.3.35; D.1.1.7pr-2-7-8; D.1.1.8; D11.1.9; D.11.1.10. D.1.1.19; D-11.1.21pr; D 11.1.5.1pr-1-2-3-4; D.11.2.25.1; D11.3.28; D41.3.31.pr; D11.4.6.1-2; D41.10.3; D.11.10. $1 \mathrm{pr}$.

D.11.1.10; D.11.2.10 pr-2-3; D 11.1 .11

D.1.1.10pr-1-3-1-5; D41.1.32; D11.1.13pr-2; D11.1.45 D.11.2.15; D41.3.36.1; D $11.3 .37 \mathrm{pr}$.

D11.1.66

D.11.6.5; D.1I.9.3.

D4I.2.14pr-1-2; D4I.2.15; D4I.2.17; D.1I.2.48;

D1I.2.49pr; D41.3.1.1 pr-2-3-7; D 1I.3.45.I.

D.11.1.63 $\mathrm{pr}-1-2-3$.

D41.1.47; D $11.1 .19 ;$ D H 1.1.57; D 11.2.1.5-6-7-8-10-1213-14-15+16-17-18-19-22; D4I.2.3.8-10-12-13; D41.2.5 DAI .2.14 pr; D.41.2.30.1-4; D.11.2.32.2; D.11.3.4.1-5-78-9-15-16-17-18; D.11.3.8pr-1; D4I .3.11; 0.1 .3. $15 \mathrm{pr}-1$; D+1.3.31.2-3; D41.3.34; D-1.4.2.6-7-10-11-12-13-14;

$68 \quad$ D $11.7 .8 ; \mathrm{D}+41.10 .2$.

DH1.1.17; D+1.1.18; DH1.1.22; D 11.1.23pr-1-2-3;

D41.1.3.Jpr-1-2; D.1.2.4; D 11.2.13pr-2-3-8; D 11.2.34.2; D.11.2.42 pr; D 11.3 .10 .2 .

D+1.1.61pr-1; D 41.2 .50 .1
Iavol enus

Celsus

Julien

Pomponius

Africain

Gaiun

Venuleius

Marcellus

P.Iustus

Scaevola

Papinien

Tryphoninus

Callistrate

Paul

U I pi en

L. Rurinus

Macer

hiarcien

Sodestin

Hermogén i en

rntal $\ldots \ldots$
D42.6.6.1; D42.8.15; D.42.8.17.1

$042 \cdot 8 \cdot 25.3-4-5$.

D12.8.23.

D42.1.8; D42.1.19pr; D.12.1.38pr; D42.1.19; D42.2 D42.5.4; D.12.5.38pr; D.12.8.16.

D42.1.4.8; D-4.1.17; D.12.2.5; D42.2.6.1; D12.4.3. D42.4.5.1; D42.4.7.15; D42.5.8.2; D42.5.9.3-5; D42.6.1.12-18; D42.8.6.5-12; D42.8.10.10-21.

D.42.1.63. 


\section{- LIVIE XLIII -}

Iavolenug
Neratius
Celsus
Julien
Pomponius
Africain
Geiua
Venulaius
Marcolius
Scaernla
Papinien
Tryphoninus
Paui
Ulpien

Ul pi en

Mermosénien

Total ......

$\begin{array}{rr}0 & 2 \\ 0 & 1 \\ 0 & 8 \\ 3 & 15 \\ 4 & 28 \\ 0 & 1 \\ 0 & 5 \\ 5 & 24 \\ 0 & 1 \\ 0 & 3 \\ 1 & 8 \\ 1 & 1 \\ 3 & 50 \\ 45 & 602\end{array}$

D43.16.19:

$62 \quad 751$
243.24.14; 043.26349.1; 043.33 .1 pr

D43.24.21.1; D43.26.1n; D43.20.13; D43.28.15.4,

D.43.29.2; $0+3.29 .4 \mathrm{pr}-1-2-3$.

D43. 10.1.2.

D43.1.2.1; D13.1.5; D.13.16.B.

D43.4.1.7; D4.3.5.1.7; D.13.5.3.4; D.3.16.1 pr-11-12-1516-17-18-19-21-22-34-43-4B; D13.16.3.11; D4.3.19.3.4; D.43.24.3ur; D43.24.5.6-11; D43.24.7.1; D.3.24.11.7; D43.26.4.2; D13.29.1 pr-1; D.13.29.3 $\mathrm{pr}^{-1-2-3-4-5-6-7-6-8}$ 9-10-11-12-13-14-15; D4.3.31.1pr-1; D.13.32.1 $\mathrm{pr}$.
- LIVIE XIJIV -

$\therefore \because \cdots$

Iavnlenus

Neratius

Celsus

Julien

Pomponius

Africain

Gaius

Venulęius

Marcellus

Scaevola

Papinien

Paul

Ulpien

L. Rurinus

Marcien

Mndestin

Hermngéni en

Tntal

54
26

1
0
0
3
5
1
2
1
0
3
1
0
10

o 1
D4.4.2.25.1; D.4.7.16; D44.7.18.

D4.2.21.2-4; D44.7.24pr-1; D44.7.56

D4.4.3.6. I.

D4t.7. I: $9 ;$ D 44.7 .5 .5

D $44.3 .15: 3$.

D.4.3.14.2-4; D4.1.7.30.

D4. 2. $29 \mathrm{pr}$.

D4.1.1.7 pr; D4.1.1.3; D4.4.5.3-4; DA4.5.2.2; D44.7.3.4pr; D.4.7.13; D41.7.41.4; D4.7.15; D44.7.10.

D44.2.I; D.4.2.7.I-3; DH.1.2.II.I-4-8; D.4.3.8 DH4.4.2.7; D44.4.4.5-7-9-16-I7-3I; D14.5.I.4-5-67-8-9-10-11-12; D4.6.6.2; DA4.7.I4; DI1.7.25.I.

D.14.0.3. 


\begin{tabular}{|c|c|c|c|}
\hline Proculas & $\mathbf{0}$ & 2 & \\
\hline Iarolenus & + & $\mathrm{g}$ & D45.1.104;D45.1.105;D D5.3.34; D45.3.3A. \\
\hline Neratius & 2 & 2 & $D+5.3 .22 ; 1045.3 .24$. \\
\hline Celsus & 0 & 5 & \\
\hline Julien & 13 & 32 & $\begin{array}{l}\mathrm{D} 45.1 .54 \mathrm{pr}-1 ; \mathrm{D} 45.1 .56 .8 ; \mathrm{D}-15.1 .62 ; \mathrm{D} 45.3 .1 \mathrm{pr}-1-2-3- \\
\text { 4-5-6; D.5.3.10; } 445.3 .14 .\end{array}$ \\
\hline \multirow[t]{2}{*}{ Pomponius } & 12 & 39 & $\begin{array}{l}\text { D45.1.16pr; D45.1.23; D.45.1.33; D45.1.10; D45.1.112pr; } \\
\text { D45.2.18; D45.3.8; D45.3.17; D45.3.37; D45.3.38; }\end{array}$ \\
\hline & & & $\mathrm{D} 45.3 .39 ; \mathrm{D} 45.3 .40$ \\
\hline Africain & $\mathbf{0}$ & 2 & \\
\hline Gatua & 9 & 19 & $\begin{array}{l}\text { D45.1.74; D45.1.141 pr; D45.2.15; D45.3.8; D45.3.28pr- } \\
\text { 1-2-3-4. }\end{array}$ \\
\hline Venuleius & 5 & 17 & D45.1.137.1-4; D.15.2.12.1; D45.3.21; D45.3.25. \\
\hline Marcellug & 1 & $\mathbf{5}$ & D.15.1.86. \\
\hline Florentinug & 2 & 4 & D45.1.65.1; D45.3.15. \\
\hline Scaevola & 6 & 18 & D45.1.122.1-2-4; D 45.1 .127 ; D45.1.135.3; D45.3.19. \\
\hline Papiuien & $\boldsymbol{7}$ & 27 & $\mathrm{D} 45.1 .115 .2 ; \mathrm{D} 45.1 .117 ; \mathrm{D} 45.1 .118 \mathrm{pr} ; \mathrm{D} 45.3 .18 \mathrm{pr}-1-2-3$. \\
\hline Paul & 42 & 102 & $\begin{array}{l}\text { D45.1.2.1-3; D45.1.8; D45.1.24; D D .1.39; D45.1.49pr-3 } \\
\text { D-15.1.66; D45.1.73pr-1-2; D } 15.1 .76 \mathrm{pr} ; \mathrm{D} 45.1 .83 .1-2-3- \\
\text { 4-5-7-B; D45.1.85.4; D45.1.88; D45.1.91 pr-1-2-3-5-6; }\end{array}$ \\
\hline & & & 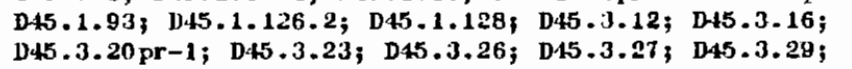 \\
\hline Ulpi en & 25 & 105 & $\begin{array}{l}\text { D } 15.3 .30 ; \text { D45.3.31; D45.3.32; D 15.3.33pr-1. } \\
\text { D45.1.1pr-5; D45.1.29pr-1; D45.1.32; D45.1.38.6-7-8-17 } \\
\text { D15.1.51; D45.1.69; D45.1.75.1-8; D45.1.81pr; }\end{array}$ \\
\hline & & & $\begin{array}{l}\text { D45.1.82.1; D45.3.2; D45.3.3; D+5.3.4; D45.3.5; } \\
\text { D45.3.7pr-1; D45.3.9pr-1; D45.3.11; D45.3.13. }\end{array}$ \\
\hline Modestin & 2 & 6 & D45.1.103; D 15.3 .35 \\
\hline
\end{tabular}

Total..... 130

\begin{tabular}{|c|c|c|}
\hline Proculus & 1 & \\
\hline Iavnlenus & 1 & \\
\hline Neratius & 0 & \\
\hline Celsus & 2 & \\
\hline Julien & $1: 3$ & 51 \\
\hline Pomponiug & 8 & 35 \\
\hline Africuin & 4 & 19 \\
\hline Gaius & 1 & 17 \\
\hline Laeciunus & 0 & 2 \\
\hline Venuleius & 2 & 13 \\
\hline Marcellug & 11 & 16 \\
\hline Flnrentinus & 1 & 5 \\
\hline Seaevala & 2 & 20 \\
\hline l'spinien & 4 & 52 \\
\hline Tryploninus & $n$ & -1 \\
\hline Calligtrate & 0 & 1 \\
\hline Pau 1 & 22 & 96 \\
\hline U I pi en & 19 & 181 \\
\hline Murcien & 0 & 9 \\
\hline Mndestin & 1 & 11 \\
\hline Hermnyóni en & $n$ & \\
\hline$\ldots \ldots$ & 0 & 1 \\
\hline Tat:1 1 & 05 & 550 \\
\hline
\end{tabular}

D46.3.8.4.

$0.16 .1 .2 n$

D16.3.69; D 16.3 .71 .2

D46.1.16pr-3-4; D.6.1.19; D 16.3 .12 ; D.6.3.33pr-1; D.16.3.3.1pr-1-2-5-10; D16.1.17.

D46.1.2; D46.3.19; D16.7.20; D16.3.80, D46.3.02pr-1; D.16.1.10; D.16.1.15.

D.16.1.21.2; D16.3.38pr-2-3.

D.16.1.70.3; D16.2.31 pr; D16.4.22; D.16.6.6

D46.2.31 pr; D.6.8.8.2.

D.16.1.38pr; D16.3.67; D 16.3.68; D.16.3.72pr-1-2-3$1-5-6 ; D 16.8 .17$.

D.16.2.16.

D 16.3.89 pr; D16.3.102.2.

D)6.3.91.3; D $16.3 .95 \mathrm{pr}-1-7$.

D.18.1.34; D46.1.35; D 16.1.56 pr-1; D.16.1.58.1; D.16.1.66; D.16.2. 32; D 16.3.35; D.16.3.51; D.6.3.80; D 16.3.62; D1G.3.6.3; D16.3.98pr-1-6-7-8; D46.1.11pr$1-2-3 ; \mathrm{D} 16.7 .11$

D.16.1.3; D16.1.8.8-9-10-11; D4t.1.33; DIf.2.8pr; D16.2.14pr; D.16.3.9pr-1; D16.3.18; D16.3.29; D.16.1.8.1-2-1; D.16.1.13pr-1-6; D.16.6.2.

D46.3.77. 


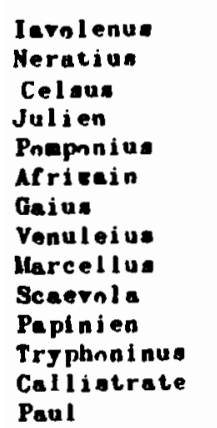

Callis
Paul

Ui pien

110

D47.2.75.

D47.2.05; D47.2.84.1.

D+7. $2.08 .2-3-1-5$

D47.2.57.2-3-5; D.17.A.2; D 17.6.1.

D17.2.35.1; D47.2.14.2.

D.7.2.61, D47.2.62 pr-1-2-3-4-5-6-7-8.

D47.1.2; D47.10.12; D47.10.34.

D47.6.5.

D47. B.6.

D47.2. $11 \mathrm{pr}-17^{2}$

$047.4 .7 ; 1047.21 .3 .1$

M7.2.18; D17.2.12pr-1; D)17.2.54.1-2-4, D-17.2.67.3-5; D47.2.83.2; D17.2.86; D47.2.90; D17.2.92, D47.8.3; D47.9.1.1; D.47.10.4; D.47.10.16; D.17.10.18.1; $047.10 .26,047.10 .29 ;$ D47.11.1.2; D47.12.11 1):37.18.2.

D.17.1.2.1-2-3-4-5-6; D.7.2.7pr; D.17.2.14.5-6-7-1115-17, $1247.2 .17 \mathrm{pr}-1-3-3$; D47 .2.36 pr-1-2-.3; D47 .2.39; D) 170 D.17.2.52.3-4-90-12-15-2:-24-26-28-29; D.17.4.1 pr-I-2-3-

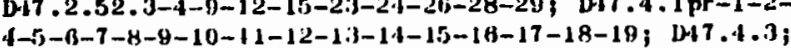
157 .5.1,5; D.17.6.1 pr-1-2-3; D47.6.3 pr-1-2; D47.7.7. W47.5.1,5; D.17.6.1pr-1-2-3; D47.6.3pr-1-2; D47.7.7.

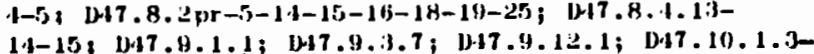
14-15; 154.H.1.1; H17.9.3.7; J-17.4.12.1; D47. [217.10.9.33-1; D47.19.11.7-8-45; D47.10.1:1, ; D47.10. $15.15-16-314-315-36-; 177-33-39-40-41-42-43-44-15-46-47$ 48-49, 017 . $10.17 \mathrm{pr}-1-2-3]-1-\{1-63-7-8-9 ;$ D. 7 . 10-22; 047 10-21; $147,10,25,017,10.30 \mathrm{pr}, 047.11 .5$ D47.11.10; D47.12.3.7-11; D47.14.1.3; D-17.20.3.2.

D27.2.H3; 1.17 .22 .3 .2$.

1047.10.15.

Mndestin
D-18.2.1.

D48. 10.6.2-3.

D48.15.1; D48.19.29.

D-48.1.11.

D48.2.12.3-4; D48.8.6; D-18.19.16.3.

D48.10.24.

D-18.1.14; D.18.2.2pr; D.18.3.2pr-1-2; D.18.5.6pr; D 18.5.39.9; D48.5.10.8; D.48.18.6 pr-1; D18.18.17 pr2-3, D48. 19.33; D48.19.34 pr.

D18.5.4.3; D 18.18.19.

D18. I0. I5 pr-1-2-3-4; D48.15.6pr-I-2; D48.18.15.1-2, D48. 19.28 pr-4-5-6-8-10-11-14-15-16; D.18.20.1 pr.

D-18.7.4.1; D 18.10.14 pr-1-2; D 18.10.17; D48.10.22.34-5-7-8-9-10; D48.16.16; D.18.18.R.1; D.18.18.11,

D $18.18 .18 \mathrm{pr}-1-2-3-1-5-6-7-8-9 ;$ D $18.18 .20 ;$ D48.19.38pr 2-3-4-5-7-8-9; D48.20.7.1; D48.22.3; D-18.22.13,

D-18.2:1.4.

D48.1.5.1; D18.2.1; D48.2.5; D 18.2.6; D 18.2.7.4; D18.5.14 pr; D48.5.18.5, D48.5. $28 \mathrm{pr}-1-2-3-1-5-6-7-8$ 9-10-11-12-13-1.1-15-16; D48.6.19pr; D.18.8.4.2,

D-48.10.8; D 18.13.7; D-18.13.8.2; D48.15.1; D48.15.2 pr1-2-3, D48, 18.1 . 1-2-3-4-5-6-7-8-9-10-11-12-13-14-1316-17-18-19-20)-21-22-23-25-27; D48.18.2; D48.18.3; D.8.18.4; D.48.18.7; D48.18.12; D18.19.1.1; D48.19.2 pr; D+8. 19.3; D $18.19 .5 \mathrm{pr}$; D 48 . 19.6.1-2, D.8.18.8.4-5-67-8-0-10-11-12-13; D.8.10.9.11-16; D48.19.18; D.48.20.5 pr; D D $18.23 .1 \mathrm{pr}-1$.

D48.2.11.1; D18.5.25 pr; D18.7.Jpr; D48.10.10 pr D18.11.7.3; D18.19.10 pr-1; D48.19.12; D48.19.14; D48.20.8pr-I $-2-3$.

D.8.1.9; D48.2.13; D48.4.3; D48.5.3.1 pr-1; D48.6.3 pr; D48.8.1.2; D.18.8.3.4-5; D48.9.1; D.8.10.1.8-13; D.8.10.7, D.18.12.1; D18.15.3pr-1; D.18.17.1.19

D.18.18.5; D $48.18 .9 \mathrm{pr}-1-3 ;$ D $48.19 .11 .1-3 ;$ D 18.19.17pri D18.22.2; D48.22.15; D48.22.16

D18.2.17, D.8.3.11.2-7, D48.4.7.2; D.18.8.11.1-2;

D48.9.9 pr, D-18.15.5; D18.18.13, D18.18.14; D18.18. $16 \mathrm{pr}-1$; D48.19.22; D.18.19.23; D.8.19.3I pr D 18.4 .9$, D 48.15 .7 ; D.8.19.36. D-18.18. $10 \mathrm{pr}-1-2-3-1-5-1$. 


\begin{tabular}{|c|c|c|}
\hline Prnculus & 0 & 3 \\
\hline Iavnlenus & 1 & 2 \\
\hline A. Valens & $\mathbf{0}$ & 2 \\
\hline Julien & 2 & 6 \\
\hline Promponius & 1 & 12 \\
\hline Mauricianus & 0 & $\begin{array}{lll} & \\
\end{array}$ \\
\hline Gaius & 1 & 1 \\
\hline Maeciunus & 2 & 6 \\
\hline Venuleius & 0 & 1 \\
\hline Hurcellus & 2 & 6 \\
\hline P.Iustug & 0 & + \\
\hline Flarentinus & 0 & 1 \\
\hline Scaevnla & 1 & $\theta$ \\
\hline T. Puternus & 0 & 1 \\
\hline Papinien & 5 & 32 \\
\hline Tryplinninus & 17 & 25 \\
\hline Mlenander & 5 & 38 \\
\hline Calligurate & 3 & 28 \\
\hline Tertullien & 0 & 3 \\
\hline Paul & 10 & $7 i$ \\
\hline Ul pi en & 5 & 100 \\
\hline Macer & 2 & 35 \\
\hline Harcien & $t$ & 34 \\
\hline Mindestin & 1 & 37 \\
\hline Hernagyéui en & 2 & is \\
\hline
\end{tabular}

$049 \cdot 15.27$

D-19.15.22.1-i].

349.15.5.3.

D19.14.1.4.

D.9.17. $18 \mathrm{pr}-3$.

D49.1.15; D.19.15.1.

D-19.1.28pr.

D19.17.13; D19.17.11.1-2; D19.17.15.3-1.

D.19.15.12. 1-7-8-9-1n-11-12-13-14-16-17-18; D.19.17. $19,1-2-3-4-5$.

D19.16.1.1-10-14-15; D 19.18.1.

D49.14.2.6; D.4.14.3.9; D.19.14.12.

D49.1.25; D.19.1.4.13.8; D49.14.17.1; D49.11.15.3; D 19.15.19.5-6-10; D.19.15.29; D19.15.30; D 19.17.2n. D19.1.14pr-1; D19.16.8; D19.17.6;D19.17.9. D $19.1 .9 ; \mathrm{D}+9.1 .2 .2$.

D 19.1 .5 .1 ; D.9.11.30; D $19.16 .11 ;$ D.19.18.3.

D.19.1.18; D 19.11.8; D.19.16.3.1-10.

D.19. $11.16 .7-8$.

477

\begin{tabular}{|c|c|c|}
\hline Q-Mluci ius & 0 & 6 \\
\hline Álius Gallus & $\mathbf{0}$ & 2 \\
\hline Prnculus & 0 & 3 \\
\hline Iavnl enus & 0 & 23 \\
\hline Neratius & 0 & 2 \\
\hline Cel sus & $\mathbf{0}$ & 22 \\
\hline A. Valens & 0 & I \\
\hline Julien & $\mathbf{0}$ & 8 \\
\hline Pnmpnnius & 7 & 52 \\
\hline Africain & $\mathbf{I}$ & 3 \\
\hline Ter.Clemens & $\mathbf{0}$ & 4 \\
\hline Gaius & 4 & 60 \\
\hline Venuleius & 0 & 2 \\
\hline Marcellus & $\mathbf{0}$ & 8 \\
\hline P. Iustus & o & 24 \\
\hline Flnrentinus & 0 & 2 \\
\hline Scuernla & 1 & 11 \\
\hline T. Paternus & 0 & 1 \\
\hline Papinien & 4 & 63 \\
\hline Tryplonninus & 1 & I \\
\hline Callistrate & 3 & 39 \\
\hline Paul & 14 & 246 \\
\hline Jl pien & 32 & \\
\hline
\end{tabular}

L. Rufinus

Mncer

Marcien

Mndestin

liermngénien

A. Charisius

Total $\ldots . .$.
D.50.18.120; D50.16.162.1; D50.16.166 pr；D50.I6.17I; D50.16.239.1; D50.17.18; D50.17.20.

D50.16.207.

D50.16.58.1; D50.17.107; D50.17.122;D50.17.13:3。

D.50.16.2.43.

D50.1.17 pr-1-8; D50.17.77.

D50.16.225.

D50.1.37.1; D50.6.6.1; D50.13.5.3.

D50.1.22 pr-2; D50.2.9pr; D50.16.53 pr-1; D50.16.70; D50.16.79.1; D50.16.204; D50.16.215; D50.17.106;

D50.17.146; D50.17.175pr; D50.17.179; D50.17.2II.

D.50.1.1 pr; D50.1.6..3; D50.1.7; D50.1.27pr;

$2-8 ; 1250.12 .2 .1 ; 1050.15 .4 .5-9 ; 1550.16 .17 \mathrm{pr} ;$ D50.16.68; D50.16.09.3; D50.16.160; D50.16.174;

D50.16.182; D50.18.195.1-3; D50.16.216; D50.17.22 pr D50.17.23; D50.17.32; D50.17.118; D50.17.126.1; D50.17.157 pr; D50.17.16I; D50.17.200.

D50.16.210.

D50.16.10L.3; D50.16.105; D50.16.107.

$050.1 .23 \mathrm{pr}$. 
Citations jar renvoi

à des juristes. 


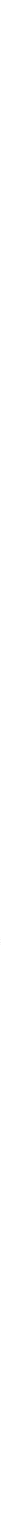


Serrius

Alfenus

Labóon

Sabinus

Proculus

Atilicinas Nerra filiu Casaius

Pegasue

Plautias

Neratiu

Cel gus

v. Severus

Juli on

Pediue

Pomponius

chez Paul D3.5.14; chex Ul pi en D3.2.4.3; D3.5.5.8-9.

chez Paul D3.5.18.1.

17

chez Paul D3.5.18.2, chez Ulpien D3.3.33pr D3.5.5.8; D3.5.9.1.

1 chez Paul D3.5.18.1. chaz Paul D3.5.17.

chez Paul D3.3.43.5

chez Paul D3.5.17.

chez Paul D3.5.17. chez U1 pi err D3.5.9.1.

1 chez Ulpien D3.5.9.1.

Marcellu

Scaerol

UI pi en

Total.....
1 chex Paul D3.5.20 pr

chez Paul $13.5 .20 \mathrm{pr}$

chex Pomponius D3.5.10; chez Paul D3.3.43.6, chez Ul pien D3.1.1.5; D3.2.2.5; D3.2.4.4;

D3.2.8, 03.5.3.5-9, D3.5.5.5-6.

3 chez Paul D3.3.45 pr; chez Ulpien D3.2.1 pr.

1 chez U1pion D3.1.1.3; D3.2.2.5.

chez Ul pien D3.2.4pr.

chez Ul pi en D3.2.2.5.

chez Paul D3.3.61.

chez Ul pi en D3.2.11.3; D3.3.27.1. 1

2 chex Jullen D3.5.29; chex Ulpien D3.3.8pr.

12 chex Paul D3.3.45.2; D3.5.12; chez Ul pien D3.3.8pr-3; D3.3.27.1 D3.3.31.2, D3.3.35.3, D3.3.37.1 D3. 30.48 D3.5.5.21 D3.5.7.3.

cbez Ulpion D3.5.5.11-12-13.

16 chez Scaerola D3.5.8; chez Ulpien

D3.1.1.B-10; D3.1.3.2; D3.2.2pr; D3.2.11.2; D3.3.1.1; D3.3.37.1; D3.3.39.1-6, D.3.3.10 pr; D3.0.1.1.

4 chez Ulpien D3.3.27.1; D3.3.39.3; Di .5 .3 .11 ; D3.5.7pr.

1 chez Papinion DJ.5.30.2.

\begin{tabular}{|c|c|c|}
\hline Serviua & 0 & \\
\hline Trebatius & 0 & \\
\hline $\begin{array}{l}\text { Alfenus } \\
\text { Ofil lius } \\
\text { Labéon }\end{array}$ & $\begin{array}{l}0 \\
0 \\
7\end{array}$ & $\begin{array}{l}\text { chez Paul D4.3.20pr; } \\
\text { D4.6.13pr, chez U1 pien } \\
\text { D4.3.7.3-7, D4.3.8.4, } \\
\text { D4.3.11.1, D4.8.7 pr. }\end{array}$ \\
\hline Sebinus & 0 & \\
\hline Proculus & $\mathbf{0}$ & \\
\hline $\begin{array}{l}\text { Atilicinus } \\
\text { Cagafue }\end{array}$ & $\begin{array}{l}\mathbf{0} \\
\mathbf{0}\end{array}$ & \\
\hline $\begin{array}{l}\text { Pegasus } \\
\text { Ariato }\end{array}$ & $\begin{array}{l}0 \\
0\end{array}$ & \\
\hline Neratiua. & 0 & \\
\hline Virianus & 0 & \\
\hline Cel aus & o & \\
\hline $\begin{array}{l}\text { Octerenus } \\
\text { Julien }\end{array}$ & 1 & $\begin{array}{l}\text { cliez Paul D4.8.J2.8. } \\
\text { chez Valeng D4.4.33; } \\
\text { chez Paul D4.5.7.2; } \\
\text { chez U1 pi en D.4.1.6; } \\
\text { D..3.7pr, D4.4.11.4-5, } \\
\text { D4.8.7.1. }\end{array}$ \\
\hline
\end{tabular}

Pedius

Pomponiua

4 chez Paul D1.9.6.2;
1 chez Ulpien $04.8 .7 \mathrm{pr}$. shez Ul pi en D4.2.8.2; D4.3.7.8; D4.8.7 pr.
3 chez Prmponiug D4.8.40; chez Ulpien 04.3.1.2; D4.6.26.4.

chez Paul D4.3.18.3-4; chez Ul pien D4.8.21.1-2.

2 chez a-a D4.6.42; D4.8.50.

2 chez Ulpien D4.4.16.1; D4.8.21.1.

35 chez Callintrate D4.4.45 pri D4.6.9; chez Paul D4.6.22.2; D4.8.19pr; chez Ul pien D4.2.5; D4.2.9pr; D4.2.14.6-9; D4.3.1.2-6; D4.3.7.63 D4.3.9.3; D4.3.13.1; D4.4.13.1; D4.4.16.1; D4.6.17.1; D4.6.26.4-6 D4.6.28.3-1; D4.8.3 pr; D4.8.15; D4.8.17.1; D4.8.25 pr-2; D4.8.27.1; D4.9.1.13 D4.9.3.1.

3 cbez Paul D4.3.29; D4.8.19.2; cbez Ul pi en D4.8.29.

3 chez Paul Di.6.35.9; chez Ulpien D4.8.21.9; D4.8.23.1.

1 chez Ul pi en D4.8.21.9.

3 chez Poming 8.40 , chez Paul D.t.8. 19.2; chez U1 pien D4.6.26.7

1 chez Ulpiell D4.8.21.10

. D4.4.16.2.

2 chez Papinien D4.3.19; chez Ulpien D4.6.15.3.

5 cber Paul D4.6.35.9; chez Ulpien D4.2.11.5; D4.8.21.11; D4.9.1.6; D4.9.4.2.

9 chez U1pien D4.4.3.1-3; D4.8.17.7, 0.4.8.21.11-12; D4.8.23 pr-1-2-3.

chez Gnius D4.8.35, chez Marcellus D4.6.41; chez Papinion D4.3.19; chez Paul D4.3.20.1, D4.8.19rr; D1.8.32.6-16; thez Ulpien D4.2.9.57-8; D t.2.12.2; D.1.2.14.9-12-14; D4.4.11.7; D4.6.17 pr-1; D4.0.26.7; D4.8.9.4, D4.8.11.4-5, D4.8.17pr D1.8.21.9-10; D4.8.27.3-5

8 chez Paul D4.8.32.16-20; chex Ul pien D1.2.7pr; D4.2.14.5; D..3.1.4; D.7.4.2; D.1.8.13.2.

50 chez Paul D4.2.2;.2; D..4.1.4 chez Ul pien D4.2.3.1; D4.2.7.1; D4.2.9pr-1-3; D4.2.19.1; D4.2.14.13; D.1.3.1.4t-5-5; D.1.3.7 pr-2-10; D4.3.9.3; D4.3.21; D4.4.3.9; D4.4.7.2-3-7; D.t.4.11.4-6; D..4.13.1 D1.A.16.2-1; DR.B.17.1; DA.6.26.1; D.4.6.28.6; D 1.8.11.2-3-5; D4.8.13pr-1 D4.8.17.3-4; D1.8.21.3-4; D4.8.25 pr2 ; $14.9 .1 .2-7$; D.1.9.3pr-1-1-K. 


\section{Marcellua Scaevola Pepini en Marci en} Total $\ldots$ ohes Uliptea m.4.3.11 D4.4.11.4-6.

2 chez U1 pion D4.3.7.7, D4.4.11.1.

2 chez U1 pi en D4.4.7.10 D4.4.9.4.

27
7 chez Paul DH.4.23, ches U1 pion D. 2.9.8, D4.3.21, D4.4.11.7. 3 elies Paul D4.4.24.2.

5 ches Ul pi en D4.4.20pr; D4.6.23.2, D4.8.21.5.

1 chez Calliatrnte D4.2.13.

183

\begin{tabular}{|c|c|c|}
\hline $\begin{array}{l}\text { Servius } \\
\text { Alfenus } \\
\text { Labónn } \\
\text { Wols } \\
\text { Sabinus } \\
\text { Proculus }\end{array}$ & $\begin{array}{l}0 \\
0 \\
1 \\
0 \\
1 \\
2\end{array}$ & $\begin{array}{l}\text { chez U1 pi en D5.1.24.2 } \\
\text { chez UL pi en D5.3.13.6. } \\
\text { cbez Paul Do.3.36pri } \\
\text { D6.3.40 pr. }\end{array}$ \\
\hline $\begin{array}{l}\text { Attlicinus } \\
\text { Cassius }\end{array}$ & $\begin{array}{l}0 \\
2\end{array}$ & $\begin{array}{l}\text { chez Paul D5.3.40pr; } \\
\text { chez Ul pi en D5.1.24.2. }\end{array}$ \\
\hline $\begin{array}{l}\text { Peganus } \\
\text { Neratius } \\
\text { Celsus }\end{array}$ & $\begin{array}{l}0 . \\
0 \\
0\end{array}$ & \\
\hline $\begin{array}{l}\text { Octavenus } \\
\text { Jul ten }\end{array}$ & $\begin{array}{l}0 \\
8\end{array}$ & $\begin{array}{l}\text { chez Paul D5.1.24.23 } \\
\text { chez U1 pi en DS.3.16.63 } \\
\text { D5.3.20.1-5, D5.3.33.1; } \\
\text { D5.3.34.1; D5.3.35\} } \\
\text { D5.3.36pr. }\end{array}$ \\
\hline
\end{tabular}

v. Verus

L.Felix

Pomponius

Murici anu

Marcellus

Scaevnla

rapinien

Arri inus

Paul

0
0
1
1
0
0
0
0

Total $\ldots$

chez Paul D5.3.36.2.
1 chez Pomponilua D5.1.80.

chez a.a D5.1.76.

chez Ulpien D5.3.18pr/ D5.4.1.4.

chez Ulpien Do.1.2.6.

chez Paul D5.1.28.5, D5.3.36.5.

chez Ulpien D6.3.11 pr.

I chez Paul D5.3.43.

a chez Paul DS.1.26; DE.1.28.5, chez U1 pi en D5.3.13.4, D5.3.31.5.

chez Ulpien D5.3.13.3

chez Ulpien D5.1.2.3, D5.1.61 pr, D5.3.13.13; D5.3.16.3, D5.3.18.1.

2 cluez Ul pien D5.3.16pr; D5.3.18pr.

31 chez Gaius Do.3.41.1, chez Callistrate D5.1.36.1; chez Paul Do.1.75 D5.3.30; chez U l pien D5.1.2pr-4-5, D5.1.18; D5.1.17, D5.1.18.1, D5.1.19.1 D5.3.13.1-13, D5.3.16.2-3-4-5-7, D5.3.20.4-18; D5.3.25.17; D5.3.3I pr5.

chez Ulpien D5.1.5.

chez Paul D5.3.43, D5.4.3.

3 chez Ulpien D5.1.5; D5.3.16pr. cliez Paul D5.3.i36pr D5.1.75, chez til pien D5.1.2.5-6; $155.1 .11 ; \mathrm{D} 0.3 .13 .10-13$ 1.t; D5.3.25.12-15.

2 chez Pul1 D5.1.19.1; D5.2.13.

9 clrez Ul pien 105.2 .6 .2 ; D5.2.8ur5-8-1.1; D6.3.13.5-6; 155.3.20.14. chez Uipien D5.J.11pr. chez Ul pi en D5.4.1.3. 


\begin{tabular}{|c|c|c|}
\hline $\begin{array}{l}\text { Trebetive } \\
\text { Alfenus }\end{array}$ & $\begin{array}{l}0 \\
0\end{array}$ & \\
\hline Labion & 2 & $\begin{array}{l}\text { chez Paul D6.1.79, } \\
\text { chez Vlpien Do.1.15 pr. }\end{array}$ \\
\hline $\begin{array}{l}\text { Merra p: } \\
\text { Procula: } \\
\text { Canalua } \\
\text { Pegasua } \\
\text { Neratias } \\
\text { Octavenus }\end{array}$ & $\begin{array}{l}0 \\
1 \\
0 \\
0 \\
0 \\
0\end{array}$ & Chez Paul D6.1.27.5. \\
\hline Jalien & 5 & $\begin{array}{l}\text { chez Poul D6.1.21, } \\
\text { chez Ul pi en D6.1.17 pr-1, } \\
\text { De.2.11.3-4. }\end{array}$ \\
\hline $\begin{array}{l}\text { Pedius } \\
\text { Pomponius }\end{array}$ & $\begin{array}{l}0 \\
3\end{array}$ & $\begin{array}{l}\text { chez Paul D6.1.21; } \\
\text { chez U1 Pien D6.1.5.6; } \\
\text { D6.2.11.5. }\end{array}$ \\
\hline $\begin{array}{l}\text { Mauricianus } \\
\text { Marcellus }\end{array}$ & $\begin{array}{l}0 \\
0\end{array}$ & \\
\hline Pepinien & 0 & \\
\hline
\end{tabular}

chez Paul D6.1.33. D6.1.5. 3 .

chez Paul De.1.78, chez Ulpten D6.1.19, D6.1.13.

1 ches V1 pi ea D6:1.5.3.

2 chez Paul D6.1.27.1.

chez Paul D6.1.23.5.

chez Ulpien D6.1.9.

chez Ulpten D6.2.9.3.

chez Paul D6.1.6.

12 chez Papinien D6.1.62.1; chez Paul D6.1.33; chez Ul pien D6.1.37, D6.1.39.1; D6.2.7.1-17; D6.2.9.4

1 chez Paul D6.1.T.

17 chez Paul D6.1.8; D6.1.33; chez U1 pi en D6.1.1.2-3; D6.1.3.2; D6.1.5 pr-1-2; D6.2.7.14; D6.2.11.6$7-8-9-10$.

1 chez Paul D6.1.35.1.

3 chez Ul pien D6.1.3pr, D6.1.25, D6.2.7.2.

2 chez Peul D6.2.16; chez Ulpten D6.2.14.

51
1 chez UIpien Dr.1.68pr.

2.Mucias

Tubero

Labfan

Meln

Sabinas

Nerva p.

Proculus

Nerva 1 .

Cassius

Peganus

Peganus

Ariato

Neratiug

1

U. Ferox

Celsu:

Octerenus

Julien

Posponins

Maniciciang

Maeciunas

Harcellua

ches Ul pi en D7,1.25. i.

chez U1 pien V7.1.25.1.

Scaevola

1 cbex Ulpien D7.1.25.6.

Paptnien

Marcien

chex Ul pi en D7.8.2.1.

chez Gaius 07.8 .13 ,

chez Ulpiea D?.1.15.3, D7.1.21; DT.8.2.1;

D7.8.10.43 D7.8.12.6.

1 chez Ul pien D7.7.6 pr.

chez Julien D7.1.35.13 chez Ulpien M.1.23.1;

o

chez Ulpien D7.1.17.13 D7.1.23.1.

1 catex U1pien D7.1.25.7.

2 chez Ulpien D7.1.1T.1; D7.8.6.

1 chez Ulpien Dr.8.2.1.

1 chez Ulpien of.8.12.6.

chez T.Cleweng DT.T.5; chez Ul pi en D7, 1, 12. 3-4 5 ; DT.1.23pr; D7.1.25.1 $3-5-T_{1}$ DT.2.1.1-23 D7.3:1.2.

1 ches Ulpien DT.1.t2.3.

Total..... chez Ulpien $\mathrm{DT} .8 \cdot 10.3$. chez Ulpien pr.8.4.1. cliez Ul pien D7.1.9.7.

1

chez Iarolenus of .4.24pr-1-2; chez Paul DT.4.13; chez Ul pien DT.1.7.1, D7.1.12pr; D7.1.13.7: D7.6.1 pr: DT.8.12.1-2.

10

chez Julien $\mathrm{D}$.1.35 pr 3 cluez Ul pien D7.1.9.2; D7.1.68.1; D7.4.10.7 D7.5.5.1, D7.8.12.1.

3 chez Ulpian of.5.3; DT.6.1 prs D7.8.12.1.

6 chez Pasponius Dr.1.19pry chez Uipie 17.5.3; $\mathrm{M} .8 .4 \mathrm{pr} ; \mathrm{D7.8.10.2}$; D7.8.12.1; D7.9.7 pr.

1 chez Ul pien $\mathrm{M} .1 .1 .7 .7$.

11 chez Ulpien 17.1 .7 .3 ; D7.1.9.5; D7.1.12.2; D7.1.68.1; D7.1.70pr-2; D7.4.10.5; DT.5.3, DT.8.12.1.

3 chez Ul pien D7.1.9.4 DT.1.12.2.

chez UIpien $D 7.2 .1 .3$.

6 chez Ulpien D7.1.7.3; D7.2.3.2, D7.8.14.1.

6 chez Papinien D7.1.33pr; chez Pau DT.8.23; chez Ul pi en DT.1.T.3; DT .2.3pr-2; $\mathrm{DT} .8 .10 .2$.

1 chez Ul plen D7.4.10.5.

11 chez Ulpien D7.1.7.23 17.1.9pr D7.1.13.3\} D7.1.29; D7.2.1.3; D7.2.3 pr-1; D7.5.5.1; D7.8.10.3; Di.8.12.1.

1 chez Gaius DT.2.5, chez Papinien D7.1.33.1; chez Paul D7.4.13; cliez. Ul pi en $07.1 .12 .23 \mathrm{DT} .1 .13 \mathrm{pr}-2-3$ D7.1.15.7, D7.1.29, D7.1.70.1-5; D7.2.1.3-4, D7.2.8, D7.5.5.2; D7.6.1pr, DZ.6.5.1.

15 chex Uipien DT.t.12.23 D7.2.3.23 D7.2.8; DT.t.29pr-2; DT.5.5.2; DT.6.1.J3 DT.6.5.4, D7.8.4.1; 1 D7.8.8.1; D7.8.14.1-2; D7.9.9.2-3.

2 chez Ulpien DT.1.72, D7.t.3pr. chez Paul 17.1 .30 ; ches UI pi en D7.1.7.2, D7.1.12.5; D7.3.1pr; DT.6.1 pr.

3 ches Paul 07.1.50; chez U1 pi en D7.3.1.4.

5 chez UH pien DT.4.3.1-2, D7.8.4ts DT.8.10pr-1.

1 chez Paul or.9.8. 
Trebatius

Alf enus

ofilius

Labéon

Mela

Sabinus

Nerva p.

Proculus

Atilicinue

Cassius

Nerating

U. Fernx

Celsus

Julien

Powponius

Marcellus

Scaevola

Papinien

\section{0}

1 chex a-a D10.3.28.

1 chez Ulpien DiO-2.16.6.

cher Ulpien D1O.t.11.1.

chez Ulpien $010.2 .12 \mathrm{pr}$ D10.3.6.4.

chez Ulpien D10.3.6.4.

o

0

5 chez Ulpien D10.2.18pri D10.3.6.9; D10.4.3.10-14; $D 10.4 .9 \mathrm{pr}$.

4 chez Ul pien D10.2.8pr; D10.2.18.1, D10.4.3.12, D10.4.8.7.

\section{o}

chez U1pien D10.2.20.9.
1 chez Ulpien D10.3.6.6.

1

8 chez Pouponius D10.4.15; chez Paul D10.3.19.4, chez Ul pien DIO.2.4.3, D10.2.22pr; D10.3.6.8-7, D10.4.7.7.

1 chez Ulpien D10.3.4.1.

1 chez Papinien DIO.3.28, chez Ul pien

D10.3.6.5; D10.4.9.3.

1 chez Jlplen D10.3.6.3.

chez Julien D10.2.52pr3 D10.3.5.

chez Ulpien D10.3.6.5.

chez Panl D10.2.44.7.

chez Ulpien D10.2.20.7; D10.3.7.13 D10.4.3.11; D10.4.5.4; D10.4.9.8

1 chez Ulpien D10.3.6.19.

1 chez Ulpien D10.3.

15 chez Panl D10.2.44.8; D10.3.14.1; chez Ul pi en Din.2.16,1-4-5, D10.3.4. D10.3.6.8, D10.3.7.5; D10.4.5.1-2.

21 chez Paul D10.1.4.7; D10.2.25.7, D10.3.19.2; D10.3.29.1; chez Ulpien D10.2.6 ; D10.2.8.1-2; D10.2.16.4; D10.2.22.1; D10.4.3.4-8-13; D10.4.7.1-5-6-7, D10.4.9.1.

3 chez Ulpien D10.2.20.2; D10.2.22.5; D10.4.9.4.

1 chez Paul D10.2.48.

10 chex Ulpien D10.2.18.6; D10.2.20 pr3-5-6-8; D10.2.22.5; D10.2.24pri D10.3.6.2
Trebatius

Alfenas

Hela

Proculua

Atilicinus

Fulcinius

Aristo

Neratios

Cel wus

Julien

Pomponius

Larcellue

Papini en

Total .......
1 chez a-a D11.3.16.

cher Ulpien D11.t.1.5.

o

1 chex U1pien D11.T.2pr. chez Ul pien D11.3.9.3, D11.3.11 pr.

1 chez Ulpien D11.3.0pr.

1 cbez Ulpien D11.6.3.6.

1 chez Ulpion D11.7.6pr. chez Ulpien D11.T.14.11. (1) Chez Ulpien D11.7.14.3-12-13-15-16.

chez Ul pi en D11.7.14.2

chez Julien D11.1.18; chez Ul pien D11. 14.11 .

1 chez Pomponius D11.7.28.

cliez Gaius D11.7.29pr.

chez Paul D11.1.20.1.

chez Ul pien D11.7.20pr.

5 chex Olpien D11,1.9.4, D11.7.2.5, D11.7.20.2.

4 chez Ul pi on D11.1.0.7, D11.7.2.7, D11.7.24.

12 chex Paul p11.7.5; chez Ulpien D11.5.1.3-4; D11.6.3.2-3-4-5; D11.6.5 pr-1; D11.6.7.2; D11.7.14.1. 
Servius

reteres

Labón.

Sabi nue

Nerve p.

Proculua

Atilicinus

Casaiva

Pegaaus

Celeus p.

Arioto

Neretiue

Celsus

Julien

Pedius

Pomponius

Venul ei us

Marcellus

Scaerola Popinien

\section{o}

1 chez Pomponius D12.4.15.

1 chez Paul D12.1.31.1.

3 chez Juli en D12.5.5; chez Pomponiua D12.4.15 o 1 chez Paul D12.1.31.1.

1

chez Ulpien D12.4.3.7.

2 chez Gaius D12.6.63; chez Ulpi en D12.4.3.5.

+ chez Ulpien D12.t.3.6-7, D12.6.26.12-13.

5 chez Africain D12.1.23; chez Paul D12.1.29, D12.1.31.13 chez U1pien D12.4.3.5 D12.3.26.12.

0 chez Paul D12.6.13pr.

0

3 chez Ul pi an D12.2.3.tpr; D12.6.26.12-13.

o

1 chez Ul pi en D12.1.13.2.
1 ches Nerstius D12.4.8.

1 ches Ulpien D12.5.6.

ches Pomponiue D12.2.41; chez Paul D12.6.6 $\mathrm{pr}-1$; chez U1 pien D12.B.4.3.

4 cher Julien D12.1.22; chez vlpien D12.5.tpr: D12.5.6.

3 cher Juli en D12.4.7pr, ches bipieu D12.1.0.9; D12.1.11 pr.

cher Ul pten D12.1.9.9.

1 chez Julien D12.1.7pr.

chez Paul D12.2.26.1; D12.2.28.1; chez Ulpien D12.7.2.

1 chez Ulpien D12.5.4pr.

chez Ulpien D12.1.9.8.

2

11 chez Pomponiug D12.6.19.4, chez Paul ग12.6.6pr-2, chez Ulpien D12.1.1; D12.5.4.2; D12.5.6; D12.6.17

21 Chez Pomponius D12.1.8, D12.2.42pr; D12.1.12; chez Paul D12.2.28.1; D12.4.1. \& D12.6.6.3; D12.6.60pr; chez U1 pi en D12.1.9.8, D12.1.18pr; D12.2.9.6; D12.2.13.1-2; D12.2.18, D12.2.34.3; D12.5.2pr; D12.6.26.T

2 chez Paul D12.1.6; D12.2.30pr.

5 ćliez Paul D12.2.28.9; D12.5.9.1;

chez Ulpien D12.2.9.4; D12.6.23pr.

1 chez Ulpien D12.2.13.5.

13 chez Ulpien D12.1.9.8, D12.1.14; D12.2.3.3, D12.2.13.5, D12.t.6; D12.5.4.3) D12.6.26.4-5 6;

chez Marcien D12.4.13.

1 chez vipien D12.1.17.

4 chez Paul D12.1.10; chez Ul pien D12.1.13.1; D12.3:7.
Servias

Nernes.

veteres

Labóon

ches U1 pi an D13.6.5.T. chez U1 pl en D13.6.5.6.

Mela

Cartilius

Atilleinas

Casaius

Fulciniua

Ariato

Neratius

Vivianun

Celsus

Julien

Pedius

Pomponius

Scaerola

Papiniea

Pacnniue

o

86

2 chez Africala DiJ.7.31; chez Ul pien D13.u.s.7. chez Vlpien D13.6.5.13.

2

1

$\mathbf{3}$

1 cliez Paul D13.1.13.

chez Ul pien D13.1.12.2.

1 ches Ul pien D13.1.12.2.

2 chez Paul D13.6.17.4; chez U1 pien

Chez Pomponius D13.6.13.2. T chez Ulpien D13.1.10.3; D13.3.2; chez Paul D13.1.3;

16

chez Ul pi en D13.5.3.2.

8 chez Scaerola D13.1.18; chez Paul

5 chez Paul D13.7.16.1; cluez Ulpien

1 chez Ulpien D13.6.1.1. (Lenel- PAL I
p603-4- "incertae aetatis". n1 p80369 ches Ponius D13.7.6pr.

D13.7.18.39 chas U1 chez Paul D13.6.1.1. D13.3.3; D13.5.18.1; D13.6.5.15; D13.6.7 pr.

choz Paul D13.1.19, cher Ul pien D13.1, 19 pr-1; v13.4, 2.6-7-8; D13.5.5.1-3-6; D13.5.7.1; D13.5.18. D13.6.5.8; D13.7.11.5; D13.7.13 pr. D13.5.19.1; D13.6.17.4; chez U1 pien D13.5.3.1; D13.5.1.t.2, D13.5.18pr; D13.5.27; D13.T.36pr. D13.1.12pr; D13.3.3; D13.4.2.7; 113.5.18.2. chez Ul pien D13.7.22pr-1.

U1pien D13.5.1803 cher

chez Pomponius D13.7.3; chez Paul D13.6.17.5; chez Ulpien D13.4.2.8; D13.5.J.2; D13.5.27; D13.6.1.1; D13.6.5.12-14。

inius F., Pacuvius dett.) 


\begin{tabular}{|c|c|c|}
\hline $\begin{array}{l}\text { Brutus } \\
\text { Serriua }\end{array}$ & $\begin{array}{l}0 \\
0\end{array}$ & \\
\hline oftlius & $\mathbf{0}$ & \\
\hline Labéon & 8 & $\begin{array}{l}\text { chez Paul D14.2.10pr; } \\
\text { chez Ul pi en D14.3.5.7- } \\
\text { 8-9; D14.4.5.T-13; } \\
\text { D14.4.7.4; D14.4.9.2. }\end{array}$ \\
\hline 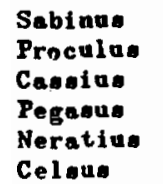 & $\begin{array}{l}0 \\
1 \\
0 \\
0 \\
0 \\
0\end{array}$ & cbez Paul D14.3.1T.4. \\
\hline Juliea & 4 & $\begin{array}{l}\text { chez U1 pien D14.1.1.23; } \\
\text { D14.3.13pr-2; D14.4.3pr. }\end{array}$ \\
\hline $\begin{array}{l}\text { Pedius } \\
\text { Pomponius }\end{array}$ & 1 & $\begin{array}{l}\text { chez Ulpien D14.4.7.1. } \\
\text { chez Paul D14.3.17.31 } \\
\text { chez U1 pien D14.1.1.20, } \\
\text { D14.4.3.1; D14.4.5.1; } \\
\text { D14.4.9.2. }\end{array}$ \\
\hline $\begin{array}{l}\text { Marcellus } \\
\text { Papinien } \\
\text { P.Fronto }\end{array}$ & $\begin{array}{l}1 \\
0 \\
0\end{array}$ & chez U1 pieu. D14.3.1. \\
\hline
\end{tabular}

20 chez Ul pi en D14.3.5.1.

chez Paul D14.2.2pr-3; chez Ul pien D14.3.5.1.

2 chez Paul D14.2.2.3; chez Ulpien D14.1.1.9.

12 chez Paul D14.2.2.3; D14.2.10.1-2; ches Ulpien D14.3.5.2.

2 chez Callintrate D14.2.4pr-1.

2 chez Ulpien D1A.5.4.5.

2 chez Ulpien D1A.5.4.5.

chez Ul pi en D14.1.1.8.

1 ches Ulpien D14.6.7pr.

2 chez Ul pi en D14.5.4.5; D14.6.7pr.

4 chez Gaius D14.6.13; chez Porponiue D14.6.19; cher Ul pi en D14.1.1.5 D14.6.3.1-2, D14.6.7pr-1-11; D14.6.9.1, D14.6.11.

2 chez Ulpien D14.1.1.8.

6 chez Ulpien D14.4.5pr.

3 chez U1 pien D14.5.4.1; D14.5.6.

1 chez Ul pi en D14.5.4.3.

1 chez Calliatrate D14.2.4.2.

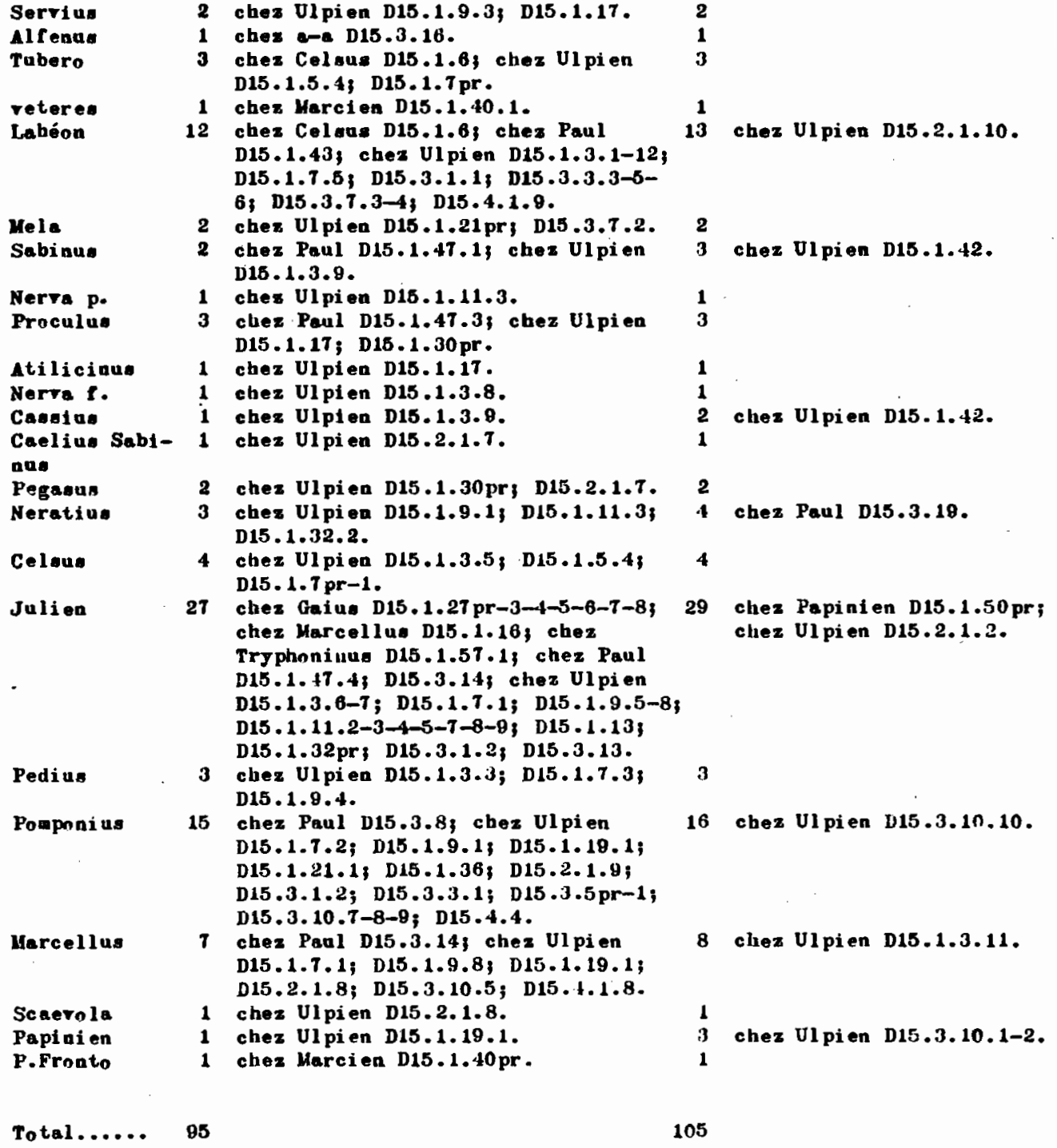

Servius

Alfenus

Tubero

reteres

Labéon

Mele

Sabinus

Nerta p.

Proculus

Atilicinus

Nerra $\mathrm{I}$.

Cangius

Caeliue Sabi-

nus

Pegauun

Neratius

Celsu:

Julien

Pedius

Pomponi us

Marcellus

Scmerola

Papinien

P.Fronto

Total .....

2 chez Ulpien D15.1.9.3; D15.1.17.

chex a-a D15.3.16.

chez Celsus D15.1.6; chez Ul pien D15.1.5.4, D13.1.7 pr.

1 ches Hercien D15 1010.1

12 chez Celans D15.1.63 chez Paul chez Celand D15.1.6; chez Paul D15.1.T.6; D15.3.1.1; D15.3.3.3-66 ; D15.3.T.3-4; D15.4.1.9.

2 cher U1 pien D15.1.21 pr; D15.3.T.2.

2 chez Paul D15.1.47.1; chex Ul pien D15.1.3.8.

1 cher Ulpien D16.1.11.3.

chex Peul D15.1.47.3, chez U1pien D15.1.17; D15.1.30 pr.

chez Ul pien D15.1.17.

chez Ul pien D15.1.3.8.

chez Ulpten D15.1.3.

chez Ul pien D15.2.1.T.

2 chez Ulpien D15.1.30pri D15.2.1.T.

3 chez Ulpien D15.1.9.1; D15.1.11.3; D15.1.32.2.

4 chez Ul pi en D15.1.3.5, D15.1.5.4; D15.1. $7 \mathrm{pr}-1$.

27 chez Gaium D15,1.27 pr-3-4-5-6-7-8; chez Harcellug D15.1.16, chez Tryphoniuus D15.1.5T.13 chez Paul D15.1.47.4; D15.3.14; chex U1 pien D15.1.3.6-7; D15.1.T.1; D15.1.9.5-8; D15 - 1.11.2-3-4-5-7-8-9; D15 - 1.13; D15.1.32pr; D15.3.1.2; D15.3.13.

3 chez Ul pien D15.1.3.3; DI5.1.7.3 D15.1.9.4.

15 chez Paul D15.3.8; chez Ul pi en D15.1.T.2; D15.1.9.1; D15.1.19.1; D15.1.21.1; D15.1.36; D15.2.1.9; D15.1.21.1; D15.1.36; D15.2.1.9; D15.3.1.2; D15.3.3.1; D D

D15.3. 10.7-8-9; D15.4.4. chez Paul D15.3.14; chez Ul pien
D15.1.T.1; D15.1.9.8; D15.1.19.1; D15.2.1.8; D15.3.10.5; D15.t.1.8

chez Ul pien D15.2.1.8.

chez Ul pi en D15.1.19.1.

1 chez Marcien D15.1.40 pr.

chez Ulpien D15.2.1.10.

chez Ulpien D15.1.+2.

chez Papinien D15.1.50 pr; chez Ul pien D15.2.1.2.

chex Ulpien D15.1.3.11.

chez Ulpien D15.3.10.1-2. 
1 chez tlpien D16.3.21.1. chex Iarol enus D16.3.3:3 chez Ulplen D16.3.1.14. ches Gaius Dio.3.14.1 o

chez Gafue D16.3.14.1.

1 chez Ul pien D16.3.1.32. chez Ul pi en D16.3.1.3342.

3 chez Ulpien D16.3.1.1014; D16.3.11.

2 chez Tryphoninus D16.3. 31.1 , chez Ulpien D16.3.1.18.

0
2 cliez Ulpien D16.3.1.41.

chez Paul D16.3.34; cliez Ul pien

2 . D16.2.13; D16.3.1.38 41.

1 chez Col aue D16.3.32.

1 chez Celong D16.3.32.

2 chez Julien T16.1.16.1.

cliez Paul D16.2.43 chez Ul pi on D16.3.1.21.

1

1 chez Africain D16.1.19.13 chez Ulpion D16.1.8pr-2-12; D16.2.10.3; n16.3.1.22-35-37; Di6.3.19.

8 chez Paul D16.1.22, Dib.2.4, chez Ul pl en D1B.3.1.11-12-13, D16.3.5.2.

chez Ulpien D16.1.8.2-9; D10.3.1.2239, D16.3.18.

2 chez Ulpien D16.1.6; D16.1.8.1.

46

$\begin{array}{ll}\text { Q.Hucius } & 0 \\ \text { Serrius } & 0 \\ \text { Trebatios } & 0 \\ \text { Alfenus } & 0 \\ \text { Ofilius } & 0 \\ \text { A.Namugs } & 0 \\ \text { Labéon } & 2\end{array}$

Niola

Sabinus

Nerva p.

Proculus

chez Paul D17.2.65.5.

Atilicinue 0

Cassiun 0

Celous p. o

Neratius

Celsus

Julien

Pomponiug

Marcellus

Papini en
0

5 D17.1. 17.1; chez ul pien D17.1.3.4-5; D17.2.52.43 D17.2.63.9. 2 chez Ulpien D17.1.19;
D17.2.63.9.

2 chez Dlpion D17.1.12.2; D17.2.23.1.

1 chez Ulpien D17.1.12.8.
3 chez Celaus D17.1.48pr; chez Panl D17.2.30; chez U1 Pi en D1T.2.11.

3 cliex Paul D17.2.30; D17.2.65.8; chez Ulpian D17.2.52.18.

1 chez Paul D17.1.22.10.

2 chez Taul $517.2 .65 .8 ; D 17.2 .71 \mathrm{pr}$.

chez Paul D17.1.22.10.

1 chez Ulpien D17.2.5:18.

11 chez Iavolenus D17.2.84; chez Proponiug D17.2.60pr; chez Paul D17.1.22.10; D17.2,65.1-2; chez Ul pien D17.1.8pr; D17.1.10.8-9-10.

4 chez Paul bi7.1.22.11; chez Ulpien D17.2.52.13.

7 chez Pnwonilus D17.2.59pr; chaz Paul D17.2.38 pr; chez U1 pi en D17.2.9; D17.2.29.1; D17.2.52.18; D17.2.63pr.

1 chez Paul D17.1.15.7.

- chez Gaius D17.1.4; chez Paul D17.1.22.7; D17.2.38.1; D17.2.65 pr; D17.2.67 pr.

2 chez Faul D17.1.45.7, chez U1pien D17.2.52.18.

5 chez Paul nit.2. 35.3; chez vipien D17.2.29.1 2; D17.2.82.:2-18.

1 chez Neratiue D17.1.39.

3 chez Neratius D17.1.39; chez Pomponius D17.2.62; chez Ulpien D17.2.29.2.

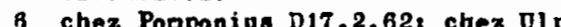
D17.1.12.5, 017.2.52.16-17.

6 chez Ul pien D17.1.6.4; D17.1.16; D17.2.47.1; D17.2.52.2; D17.2.58pr-1

18 chez Pomponius D17.1.47 pr; cliez Paul D17.1.22.4; D17.1.26pr; D17.2.65.4; chez Ul pi en D17.1.6.6; D17.1.1R.12, D17.1.14pr; $117.1 .29 .6 ; D 17.2 .47 .1$; D17.2.52pr; D17.2.58.2; D17.2.61; D17.2.03.1.

7 chez Paul D17.1.40; chez Ul pien D17.2.14; D17.2.23pr; D17.2.47.1, D17.2.57.

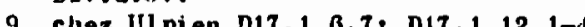
16-17; D17.2.26; D17.2.63.3.

13 chez Ul pien D17.1.10.3-4-3-6; D17.1.12.14-15; D17.1.28; D17.2.52.6-7-8-9-10. 


\begin{tabular}{|c|c|c|}
\hline $\begin{array}{l}\text { Bratus } \\
\text { Q.Mucius }\end{array}$ & $\begin{array}{l}0 \\
0\end{array}$ & \\
\hline $\begin{array}{l}\text { Serviug } \\
\text { Trebatius }\end{array}$ & $\begin{array}{l}0 \\
0\end{array}$ & \\
\hline Alfenus & 2 & $\begin{array}{l}\text { chez a-a D18.6.12; chez } \\
\text { Pnoponius D18.1.18.1. }\end{array}$ \\
\hline $\begin{array}{l}\text { Tubero } \\
\text { reteres } \\
\text { Lebéonon }\end{array}$ & $\begin{array}{l}0 \\
1 \\
2\end{array}$ & $\begin{array}{l}\text { chez U1 pi en D18.1.7 pr. } \\
\text { chez Iavolenus D18.1.78.1;2 } \\
\text { chez Paul D18.1.34 pr. }\end{array}$ \\
\hline
\end{tabular}

Sebinus

3 chez Papinien D18.7.6pr-13 chez Paul D18.1.1.1.

Nerve p.

Proculus Casatus

I avol enus Iavole
Arioto

Neratius

Celsua

Octarenus Julien

Pnompnius

Marcellus Scaevola Papinien Paul

Total, ....

0

chez Paul D18.6.8.1; chez Ul pi en D18.2.4.1-2 D18.4.2.12.

o

1 chez Ulpien D18.4.2.13.

1 chez Tryphoninue D18.7.10. 1 chez Ulpien D18.7.1. chez Ulpien D18.2.13pr.

Sex.Aelius chez Celsus D18.1.59; chez Pomponius Drusus 1.63 .2 ; chez Ul pi en D18.2.13pr. chex Iavol enus D18.1.80.2. 18.1.79; chez Ulpien Gallus D18.6.1.2; chez Marcien D18.1.45.

chez Iarnlenus D18.1.77.

chez Iarol enus $018.1 .77,018,1.78 \mathrm{pr}$

2-3; D18.1.79, 018.1.80pr-1-2-3; D18.t.24; chez Pomponius D18.6.18; chez Paul D18.2.14.1; D18.4.25; chez Paul D18.2.14.1; D18.4.25;
chez Ul pi en D18.1.50; $018.2 .13 \mathrm{pr}$; D18.4.2.17, D18.6.1.2; chez Marcien D18.1.45.

14 chez Pouponius D18.1.20, chez Gaidus D18.1.35.5; chez Paul D18.1.27; D18.1.57 pr; D18.2.14.1, D18.5.63 chez Ulpien D18.2.4.5; D18.2.9, D18.2.11 pr-1; D18.2.13 pr

a chez Paul D18.1.1.1; D18.1.57pr; D18.2.14.1.

2 chez Puul D18.1.1.1, D18.6.8pr.

1 chex Pomponius D18.1.6pr. J chez Pomponius D18.5.1, chez Ulpien D18.2.4pr-6, D18.2.6.1, chez Harcien

Pedius

Pomponius
3 chez taius D18.1.35.5; chez Faul D18.1.1.1; D18.1.57 pr.

1 chez Ulplen D18,4.2.17.

2 chez Neratius D18.3.5, chez Foeponius D18.5.1.

2 chez Peul D18.1.57 pr; chez Ulpien D18.3.4.1. D18.2.13pr 318.4 .4

1 chez Paul Dis, $6.8 \mathrm{pr}$

13 chez Scaernla D13.6.11; chez Paul D18.5.3; chez Ulpien Di8.2.2.1 D18.2.4.4; D18.2.6 pr; D18.2.11 pr; D18.4.2. T-15; chez larcien D18.1.45

8 chez Paul D18.1.15.2, D18.1.343 D18.6.8pr, chez Ul pien D18.1.40; D18.1.45.

4 chez Ulpien D18.1.9.2, D18.2.4.3, D18.3.4.4.

Nela 0

Sabinas

1 chez Julfen D19.1.24.1.

Minicius

Proculus

Casoivo

Aristo

Neratius

1 clrez Paul D19.4.2.

3 chez Ulpien

D19.1.11.7-8-12.

Vivianus

Celsus

Octerenus

Julien

2 chez Ulpien D18.3.4.2.

1 chez Papinien D18.1.79pr.

chez Pomponius D19.1.55,

chez Harcellus D19.1.23; chez Paul D19.1.t3; D19.5.5.2; D19.1.45 pr; chez Ulpien D19.1.11.15r cher Celsus D19,1.38,1. cher Celsu D19.1.38.1.

chez Iarolenua D19.1.13.22, D19.2.11.4 D19.1.13.5; D19.5.13.1.

1 chez Ulpien D19.2.11 pr.
1

chez Pomponing D19.1.40.

1 chez Ul pien D19.1.17.6.

5 chez Africaia Di9.2.35 pr-1; chez Ulpien D19.1.13.30; D19.2.15.2; D19.2.19.1.

3 chez Paul D19.1.21.6; chez Ulpien D19.1.13.22, D19.1.17.2.

11 chez a-a D19.1.26; D19.2.27 pr-1; D19.2.29; chez Paul D19.2.30pr-1-2J-1; D19.2.31; D19.5.23.

1 chez Ul pien D19.1.13.30.

2 chez Gaius D19.1.19, chez lodeatin D19.1.39. Chez Iavolenas 019.1 .503 $010.1 .51 \mathrm{pr}-1$ D19.2.28pr-1-2; D19.2.57, D19.2.58pr $1-2 ;$ D18.2.60 pr-1-2-3-4-5-6-8-9; cher Pomponius D19.1.0.4; chez Papiuien D19.5.1.1; chez Paul D19.1.42; D19.1.53pr-1-2; D19.1.54 pr1; D19.2.62, chez U1 pi en D19.1:11.3; D19.1.17.T, D19.2.13.1-7-8; D19.19.19 D19.2.19.1-43
D19.5.20 pr-2.

4 cirez Ulpien D19.1.1T.6; D19.2.13:8; D19.2.19.6; D19.5.20.1.

7 chez Iavol enus D19.2.59; chez Poroponius D18.1.6.4, chez Ulpien D19.1.11.3; D19.2.13.8; D19.2.19.1; D19.5.17.5.

1 chez Pompanius D19.1.6.4.

3 chez Celsus D19.1.38.2; chez Powprnius D19.2.3, chez Ulpien v19.2.15.8.

4 chez Julien D19.2.32, chez Ulpien D19.1.11.14; D19.2.19.1.

4 chez Pomponius D19.5.16.13 chez Ulpien D10.2.19.2; v19.3.14.3.

10 chez Pomponius D19.1.6.1; chez Ul pien $019 \cdot 1 \cdot 11 \cdot 9-10-11-13$; D19.1.13.14; D19.2.19.2.

1 chez U1 pi en $019.5 .17 \mathrm{pr}$

chez Ul pien D19.1.13.16-17;D19.2.9.5 1

chex U1 pien D19.1 11, B-13-11-18-18 D19.1.13 pr-6; D19.2.9.2; D19.2.13.4, D19.2.15.9; D19.2.19pr; D19.2.41.

1 chez Paul DL9.4.1.J.

chez Paul D19.1.5.1; chez Ul pien i) 19.1 .32 ; 1)19.2.9pr; D19.5.14.2; D19.5.17.1. 
- LIVE XX -

Arricala Yarcellu,

Ulpiea

Total
1 chez Paul D19.1.45 pr.

1 chez vipi on D19.2.9.1.

-

1 chez Paul D19.1.43.

23
1

3 chez Ulpten D19.2.14, D19.2.41.

chez Ulpten D19.1.13.25-26,

D19.2.15.4) D19.2.19.10; D19.5.17.2.

142

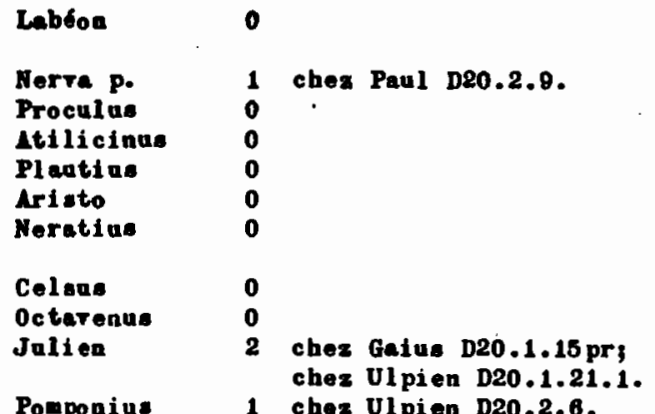

Pomponius

Marcellus

Scserola

Papinien

o

chex Ulpien D20.1.27.

Total
2 chez Iarolenus D20.6.14, chez Paul D20.1.35.

chez Paul D20.4.13.

chez Paul D20.4.13.

chez Ul pien D20.6.B.2.

chez Paul D20.4.13.

1 chez Paul D20.3.3.

chez Paul D20.3.3; chez Dlpien D20.2.3.

1 chez U1 piea D20.1.14pr.

1 chez Marcien D20.3.1.2.

3 chez Marcien D20.5.7 pr.

T chez Paul D20.1.12; D20.5.9.1; chez Marcien D20.1.13.2; D20.2.2; D20.2.5 pr-1.

3 chez Ulpien D20.4.7.1; D20.6.3.

2 chez Iryphouinue D20.5.12.1,

chez Iiarcien DRO.3.1.2.

- chez Marciou D20.1.11.2,

D20.4.12.5-6-9. 


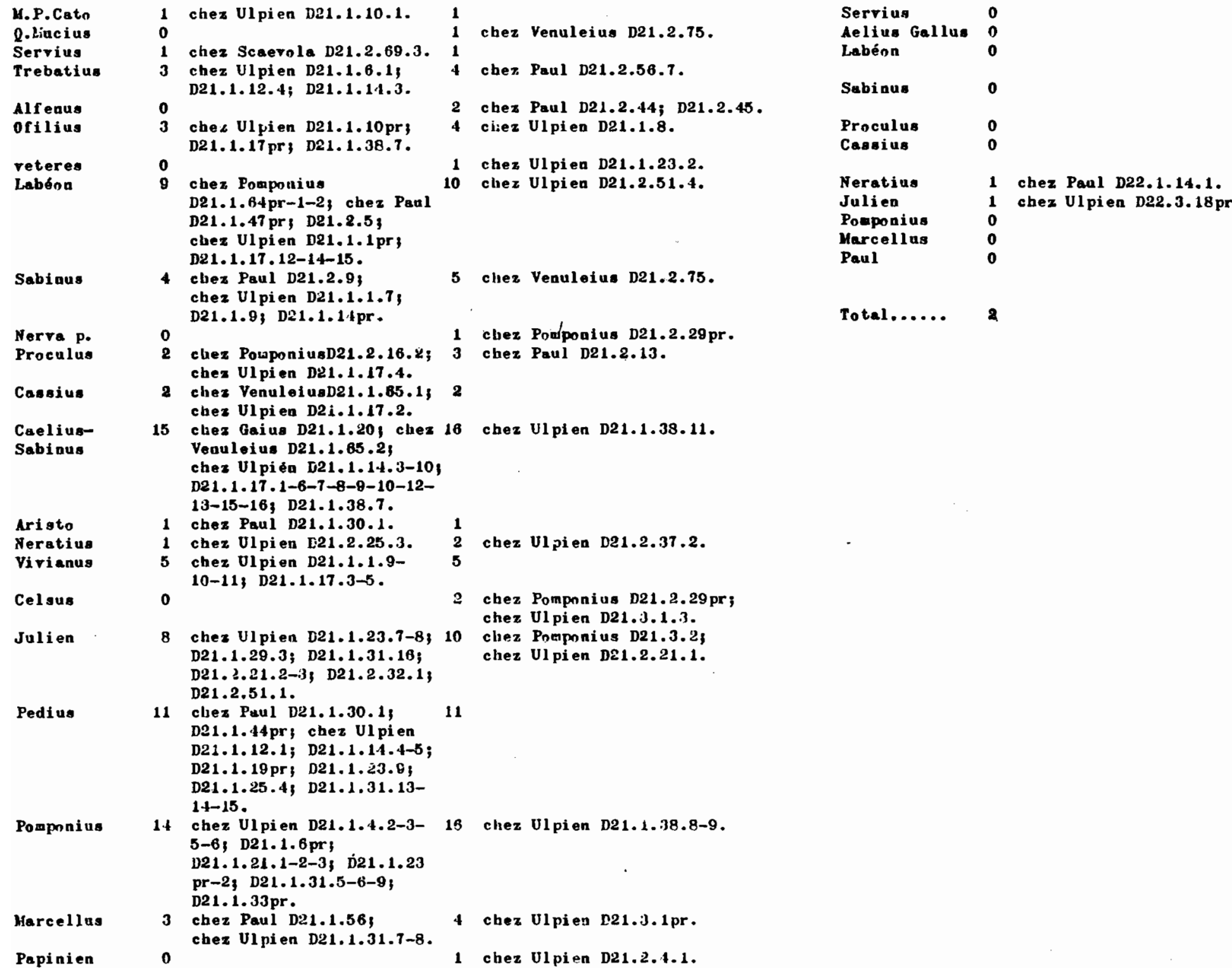

M.P.Cato

Q.Lincius Q. Liacius Servius

Alfenus ofilius

reteres Labéna

Sabinus

Nerva p. Nerva p.
Proculus

Cassius Caelius-
Sabinus

Aristo Neratius Vivianus

Celsus

Julien

Pedius

Pomponius

Harcellus

Papinien

1 chex Ul pi en D21.1.10.1.

1 chez Scaerola D21.2.69.3.

chez Ulpien D21.1.6.1, D21.1.12.4; D21.1.14.3.

0

chec U1pien D21.1.10pr D21.1.17pr; D21.1.38.7. chez Pomponius D21.1.64pr-1-2; chez Panl D21.1.47 pr ; D21.2.5, chex Ulpien D21.1.1pr; D21.1.17.12-14-15.

4 chez Paul D21.2.9; chez U1 pien D21.1.1.7, D21.1.9; D21.1.14pr.

5 cher Venuleius D21.2.75.

1 chez Podponius D21.2.29pr. chez Ul pi en De1.1.17.4.

2 chez VenuleiusD21.1.85.1;

chez Ul pi en D2i.1.17.2.

15 cliez Gaius D21.1.20; chez 16 chez U1pien D21.1.38.11. Venuleius D21.1.65.2, chez Ul pién $\mathrm{J} 21.1 .14 .3-10$ chez U1 pi én t21.1.14.3-10, 13-15-16; D21.1.38.7.

1 chez Paul D21.1.30.1.

1 chez Ulpien E21.2.25.3. 2 chez U1 pien D21.2.37.2.

5 chez Vlpien D21.1.1.9$10-11 ; \mathrm{D} 21 \cdot 1 \cdot 17 \cdot 3-5$

8 chez Ulpien D21.1.23.7-8; 10 D21.1.29.3; D21.1.31.18; D21.1.29.3; D21.1.31.18; D21.3.21.2-3, D21.2.32.1 D21.2.51.1.

11 clsez Paul D21.1.30.1, D21.1.44pr; chez U1 pien D21.1.12.1; D21.1.14.4-5; D21.1.19Pr; D21.1.23.6 D21.1.25.4, D21.1.31.1314-15.

14 chez U1pien D21.1.4.2-35-6; D21.1.6pr: 121.1.21 1-2-3, Dं21 1 D21.1.21.1-2-3\} D21.1. pr-23 D21.1.31.5-6-9 D21.1.33pr.

3 chez Paul D21.1.56; chex U1 pien D21.1.31.7-8

chez Venuleius D21.2.75.

chex Paul D21.2.56.7.

2 chex Paul D21.2.44; D21.2.45

cíez U1 pien D21.1.8.

chez Ulpien D21.1.23.2.

3 chez Paul D21.2.13.

2 chez Pomponius D21.2.29pr; chex U1 pien D21.J.1.3.

chez Pnopnius D21.3.2 chez Ul pien D21.2.21.1.

11

16 chez U1pien D21.1.38.8-9.

4 chez vlpien n21.j.1pr.

1 chez Ul pien D21.2.4.1.

$\begin{array}{ll}\text { Servius } & 0 \\ \text { Aelius gallus } & 0\end{array}$

Labéon

Subinus 0

$\begin{array}{ll}\text { Proculus } & 0 \\ \text { Cassius } & 0\end{array}$

Neratius

Julien

Pomponius

Marcellus

Paul

Total......

chez Paul D22.1.14.1.

1 chez Ulfien D22.2.8.

chez Gaius D22.1.19pr.

chez Pomponius d22.2.2; chez Paul

2 chez Pomponiug D22.6.3.1; chez Paul D22.1.38.7.

1 Chez Gaius D22.1.19pr.

chez Powponius D22.6.3.1; chez Paul D22.1.38.T.

1

chez Mlarcien D22.1.32pr.

chez Paul D22,1.11.1.

l chez Papinien D22.1.1.2. 
Serviue

ilfenua

teius

Clane

Labéon

Capito

Yela

Sebinue

Nerve p.

Proculus

Atilicinus

iriato

Neratiua

Cel aus

Octarenue

Julien

Pomponias

Marcellus Scnerola

Papinien chez Pomponiue D23.3.18.

chez U1pien D23.2.29.

Chez Gaius D23.2.46.

2 chex Ulpien D23.2.45.6, cher L.Rufinuad23.2.51.1.

chez U1 pien D23.3.12.1. chez Uipien D23.3.12.1.
2 chez Iavolenes D23.3.79pr-1.

chez Peul D23.4.19, D23.5.8

chez Tarol Denue D.79.1

chex U1 pien D23.2.6.

1 chez Iavolenue D23.3.19pr-1, D23.3.80; D23.4.32 pr: D23.5.18pr-1 chez Peul D23.3.41, D23.3.84

1 chez U1 pien D23.1.9, D23.3.40.4.

2 chez Paul D23.4.12.2-3.

1 ohez Ulpien D23.3.33.

ches Uplon

chez Jult on D23.3.48.1.

chez Proculua D23.1.17.

1

2 chez Pomponilus D23.2.40; chez Peul D23.3.20.

chez Paul D23.3.20, chez vipien D23.3.5.6-8.

2 chez Pomponiue D23.4.10; chez Ulpien D23.3.7.2.

2 chez Poul D23.2.14.3, chez Ul pion D23.2.43.3.

16 chez larcellus D23.3.44.13 chez TrJphoninus D23.3.78.4, chez Panl D23.1.7.1, D23.3.20, D23.3.41.4, chez Ul plen D23.1.9, D23.2.12.3 D23.3.5.5-9-13-14; D23.3.33; D23.3.40.4, D23.5.5

4 chez Paul D23.2.3; D23.2.44.5; D23.3.20, ches U1 pl on D23.4.6.

chez U1 pien D23.3.5.

4 chez Ulpien Das D23.3.43pr; D23.5.13.4.

5 Chez Ulpien D23.1.9; D23.3.5.12; D23.4.2; chez Marcien D23.2.57.

66
M.Porciue Cato O

P.Yucins Cato

.Mucius

Sorriua

Trebetius

Alfenue

of iliue

Labóon

Mela

Sabinus

Nerve p.

Proculus

Cassius

Ceelius-

Sabinus

Plantius:

Falcinus

abinien.

Aristo

Neratius

Celsus

Julien

Pedius

Pomponilue

Africal n

Iarcellus

Senerola

Papiaion

o

0
0
0
1 chez Iarol enuaD24.3.66.1.
chez Peal D24.1.38pr.
0 chez Iarol enus
D24.3.66.1-3;
chez Paul D24.1.67,
chez U1 pien D24.3.64.8.

chez U1 piea D24.1.T.8.

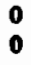

chez Paul D2t.1.28.3.

chez Pomponiued24.1.29pr.

Paul D24.1.28.5 chez Ulpien D24.1.9.1; D24.1.19pr-1; D24.2.11pr.

chez Paul D24.1.28.4-5; chez Ulpien D24.3.7.16.

chez Ulpien D24.1.11.6 D24.1.18.1.

chez Ulpien D24.1.11.6.

chez Ul pien D2A.1.7.8. chez Paul D24.3.44pr.

chez Ievolenue D2t.3.66pr.

chez Pomponiue D24.1.51.

chen Iarolenue D24.3.00

D24.1.04, D24.3.66.2. chez Paul D24.1.38.1.

1 chez Pomponius D24.3.18.1.

15 cttdz Iavolenus D24.1.64, D24.1.65, D24.3.66.2-4-5-6-7\} chez Pomponiue D24.1.29.1, D24.3.18pr, chez Vlpien D24.1.32.27; D24.3.22.6.

1 chez Ul pien D24.3.24.2.

chez Julien D24.3.59; chez Peul D24.3.17.2; D24.3.25.4; chez Ulpien D24.3.2.1; D24.3.22.12

2 chez Paul D24.1.36.1; D24.3.44pr.

5 chez Iavolenue D24.1.64; chez Pomponiua D24.1.31.7, D24.3.10.1, chez Paul D24.3.17 pr-1. -

2 chez Jull on D24.3.59; chez vlpien D24.3.22.12.

1 chez Iorolenus D24.1.64.

1 chez U1 pilen D24.1.11.3.

1 chez Paul D24.3.44pr.

chez Paul D24.1.26.1; D24.3.17 pr-2, chez U1 pi en D24.1.5.5, D24.1.13.2.

4 chez Paul D24.1.28.T; chez U1 pi en D24.1.3.12, D24.1.5.15, D24.3.21.

21 chez U1pien D24.1.3.13; D24.1.3.1-4 5-13, D24.1.11.1-3-10-11, D21.1.15.1; D24.1.17 Pr; D24.1.21.1, D24.1.32.27; D24.2.4, D2.4.3.2.2, D24.3.22.3.

1 chez Paul D21.1.36pr.

$T$ chez Paul D24.3.t4pri chez Ulpien D24.1.5.5; D24.1.7.5, D24.3.14.1.

1 ches Paul D24.1.2.

9 chez U1 pi en D24.1.5.17, D24.1.7.1-3; D24.1.11.2-3-7, D2.1.1.46.

chez Ulpien D24.3.T pr.

8 chez U1 pien D24.1.21 pr, 324.1.23;

D24.1.32.16-27, D24.3.7.1. 


Ofilius
Labéon
Mela
Sabinus
Nerrap.
Proculus
Atilicinus
Casaius
Fulcinius
Arigto
Julien
Pedius
Ponponius
Africain
Marcellus

Mericain

chez Paul D25.2.21.1. 0

chez Ulpien D25.3.5.22-23.
1 chez Paul D25.2.3.3.

3 chez Ulpien D25.1.1.3; D25.2.13 J25.2.19.

1 chez Paul D25.2.3.4.

4 chez Pomponius D25.2.8.1; D25.2.10; chez Paul D25.2.1, chez Ul pien D25.1.11.1.

1 chez Paul D25.2.1.

2 chez Paul D25.2.1; D25.2.3.4

2 chez Paul D25.2.6pr; chez Ulpien D25.7.1.1.

1 chez Paul Das.2.1.

- chez Pail D25.2.3.4; D25.2.6pr; chez U1pien D25.1.1.3; D25.1.3pr.

chez U1pien D25.4.1.13.

3 chez Paul D25.2.6.5; chez U1 pien D25.1.11 pr.

10 chez Paul D25.2.1, cbez Ul pien D25.2.17.2; D25.3.1.10-12-13-14; D25.3.3.4-5, D25.6.1.8-9.

1

chex Ul pien D25.1.5 pr.

chez Ul pi en D25.3.3.4.

7 chez Uipien D25.1.5.2; D25.2.11pr; D25.3.1.11; D25.3.3pr; chex Marcial D25.7.3.1.

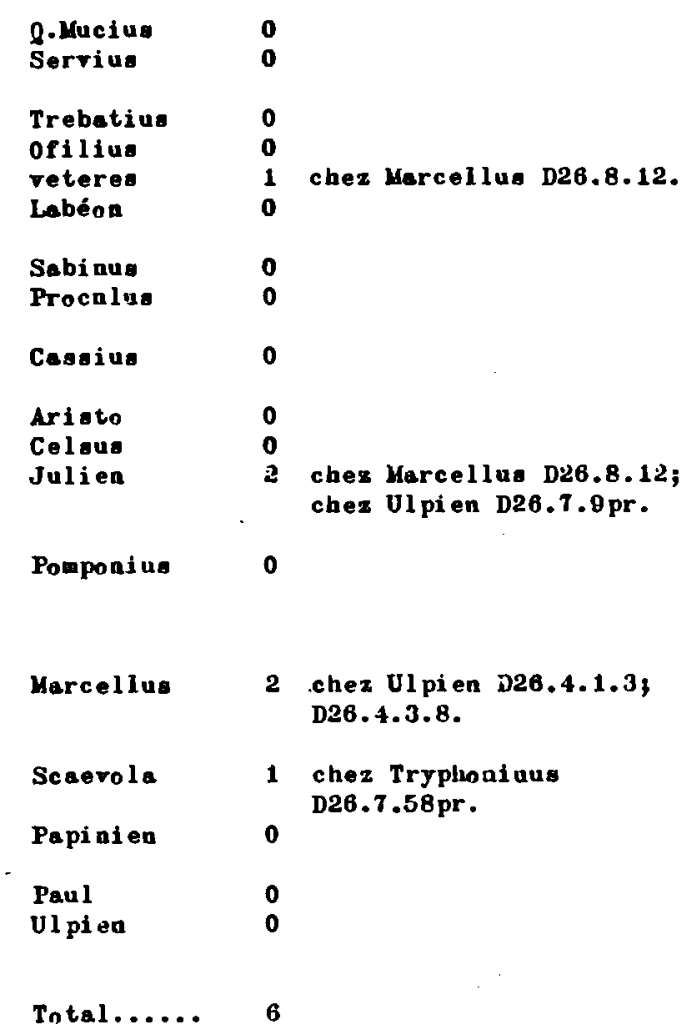

chez Ulpien D26.1.3pr.

chez Paul D26.1.1pr; chez Ulpien D26.1.3.4.

1 chez Iarolenus D26.2.33.

1 cher Pampaniug D20.8.4.

1

chez Iarnlenus D26.2.33; chez Paul D26.8.22; chez U1 pi en D26.4.5.2.

chez Fapinien D26.7.37 pr-2, D26.9.3 2hez Iarnlenus D26.2.33; chez Ulpies D26.2.10.3.

3 chez Papiniea D26.7.37 pr-2; chex Ulpien D26.1.3.2.

2 chez Pomponiug D26.7.6.1; D26.9.1.

2 chez Ul pien J26.1.3.4; D26.5.12.1.

8 cluez Tryphnninus D26.2.27.1; chez. U1 pien D26.1.3pr; D26.1.6pr;

U1 pi en D26.1.3pr; D26.1.6pr;

9 chez Paul D2A.1.1.3; chez Ui pi en D26.1.3.3, D26.1.6.1; D26.2.10.2-3; D26.2.16.1; D26.5.17; D26.7.3pr

chez Marci en D26.8.15.

7 chez Paul Da6.7.16; chez Ulpien D26.2.19.1; D26.7.2.1; D26.7.9.7; D26.7.28.1.

chex Ulpien D26.7.5.8; D26.7.7.14; D26.7.25; D26.10.4.2.

1 chez Modestin D26.6.2.5.

1 chez Modestin D26.B.2.5. 


$\begin{array}{ll}\begin{array}{l}\text { Servius } \\ \text { Lebion }\end{array} & 0 \\ \text { Mele } & 0 \\ \text { Sabinas } & 1 \\ \text { Yeratias } \\ \text { Jaliea }\end{array}$

1 chez Ul pi en $D 27.7 .4 \mathrm{pr}$.

chez U1pien D27.3.1.6.

1 5 chez Paul D27.6.2; cbez U1pien
D27.3.1.4-6; D27.4.3pr; D27.6.9.1.

chez Peul D2T.6.8; chez Ul pien D27.4.1.2.

1 chez Ulpion D27.3.9.1.

chez Paul D27.3.4.1, D27.6.6, chez Ulplen D27.3.1.1-2; D87.3.11; D27.6.11.3-4; D27.8.1.17; D27.10.10p

5 chez UIpI en D27.6.1.3-4-5, D27.6.7.3 D27 $B$ Pr

1 chez Ul pien D27.3.1.15.

2 chez Paul D27.1.32; chez Modeatin D27.1.13.2.

2 chez Paul D27,0.13.1; chez ulpien D27.3.1.22.

4 chez Mndestin D27.1.6.5-10-19; D27.1.13.2.

9 chez Mndestin D27.1.2.9; D27.1.4.1; D27.1.6.6-13; D27.1.8.9; D27.1.10.8; D27.1.12 pr; D27.1.13.2; D27.1.15.16.

12
Q.Mucina
Gellus
1 ches Ulpien D28.5.35.3.
Servius
o
Cascelline
Trebatius
$\mathbf{0}$
Alfenus
0
$\begin{array}{ll}\text { ofilins } & 0 \\ \text { reteres } & 0\end{array}$
Labéon
6
chez Iavol enut D28.5.65 D28.7.20.1; D28.8.11; chez Celous D28.5.60 pr; chez Pompouius D28.5.21 pr chez Ulpien D28.5.9.14.
Sabinus $\quad 0$
$\begin{array}{ll}\text { Cartilius } & 0 \\ \text { Proculuas } & 0\end{array}$
$\begin{array}{ll}\text { Cassius } & 0 \\ \text { Pegesnas } & 0 \\ \text { Ierolenus } & 0\end{array}$
Aristo
Nerstius
Cel sus
Julien
Pomponius
o
Mauricianus 0
Maecianus
1 chez U1pien D28.5.9.14.
2 chez Paul 028.5.56, chez Ul piea D28.5.9.14.

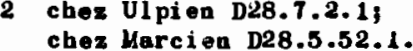
8 chez Marcellun D28.5.40; 32 chez Scaerole D28.6.48.1; chez Papinien D28.5.77; chez Ul pi en D28.5.6.3-4; D28.6.18pr; D28.7.4.13 chez Harci en D28.5.39.
5 chez Africain D28.6.33.1; chex chez Africain D28.6.33.1; chez
Sceornla De8.2.29pr-2-13; chez Sceernla D28.2.29pr
3 chez Alfenus (a-a) D28.5.45; chez Iavnlenus D28.1.25, chez Ul pien D28.5.17.1.
2 chez Iavolenus D28.6.39pr-2. chez Proculus D28.5.70; chez
Iarolenus D28.6.39pr; chez Ul pien D28.5.13.7.
3 chez a-a D28.5.45; chez Iarolenus D28.1.25; chez Paul D28.5.46.
2 chez Iavolenus $D 28.6 .39 \mathrm{pr}-2$.
3 chez Gaius D28.5.32pr; chez Papinien D28.5.78.1; chez U1 pi on D28.1.20.8.
24 chez larnlenus D28.1.2; D28.1.25; D28.6.9; D28.6.39 pri D28.7.20 pr-2; chez Pomponius D28.5.29; chez Paul D28.5.20.1; chez UI pi en D28.2.6 pr; D28.8.7.2.
6 chez Paul D28.3.10, D28.5.10, D28.5.18; chez Ulpien D28.2.3.6, D28.5.8.13; D28.5.17 pr.
1 chez Proculus 028.50 chez Iarolenus D28.5.11; chez Colsus D28.5.60.2; chez Ul pien D28.2.6.1; D28.5.9.3.
2 chez U1pien D28.2.3.6; D28.2.6pr.
1 chez Ulpien D28.5. $1 \theta$.
3 chez Ulpien D28.2.6pr; D28.5.17.5, D28.5.19.
4 chez U1pien D28.5.17.5; D28.5.19; D28.8.5 pr.
11 chez Julien D28.2.13pr; chez Pomponius D28.5.27 pr; chez Ul pi en D28.5.2pr; D28.5.9.2-3-13 D28.5, $17.1-2-4$.
2 chez T.Clemens D28.5.73; D28.6.6; chez Marcellus D28.5.5; chez Screvola D28.2.29.15-16, chez Paul D28.2.9.2; V28.5.20.1; chez UI pien L28.2.3.1-2-6; D 28.2 .3 .1 ; E28.3.5; D28.5.1.5-7; D28.5.3.2; D28.5.4 pr; D28.5.6 pr; D28.5.15 pr-1; D28.d.2.2-4 D28.6.10.7, D28.7.4pr; chez blarcien D28.7.16.
5 chez Marcellus bi28.1.16.1; chez Paul D28.2.9.2-3; chez Ul pien D28.4.1.5; D28.5.19.
1 chez Ul pi en D28.2.3.5.
1 chez Paul D28.6.44. 
Marcellus

1 chez Harcien D28.5.39.

1 chez Paul D28.6.38.3.

Sceerola

Pepinien

Tertullien

Arrianus

Paul

Marci en

époque incertaine
Aulua

Totel..... 2
10 chez Paul D28.2.9.2; chez vipien D28.1.20.2; D28.3.6pr; D28.5.1.63 D28.5.2pr-1; D28.5.9.5; D28.6.20 pr; D28.7.4pr.

4 chez Faul p28.2.193 chez Ulpien D28.2.3.4, D28.6.10.8.

2 chez Ul pien D28.5.35.1, chez Harcien D28.7.18.1.

1 chez Ulpien D28.5.3.2.

1 chez ulpi en D28.5.19.

1 chez Papinieu D28.4.4.

1 chez Ulpien D28.1.5.

1 chez Ulpien D28.5.17.5.

143
Labéon

Sabinus

Proculus

Casaius

Iaroleaue

Ariato

Virianus

Celous

Juli en

Pomponius

Maecianus

Marcellus

Scaevola

Papinien

Tertullien

Peul

Sextua
3 chez Paul D29.2.60 D29.2.73; chez Ul pien D29.5.1.17.

0

chez Panl D29.2.60.

o

1. chez Paul D29.2.60.

cher U1 pien D29.2.6.7, D29. H.6.8.

chez Peul D29.2.26; D29.2.74.4; ches Uipien D29.2.20.4.

3 cnez Marcellua D29.2.63, chez Paul D2G.2.22, D29.2.26. chez Peul Dag.2.26;

chez Ulpien D29.5.1.12-13; J D29.5.3.30.

2 chez Ulpien D29.2.20.t; chez hlarci Qn D29.5.15 pr.

0

1 chez Hodestin D29.5.18.

5 chez Paul D29.2.97; chez Ul pien 1.15.83 D28.2.20.3.

1 chez U1 pi en D29.2.30.6.

6 chez Iavolenus $\mathrm{D} 29.2 .62 \mathrm{pr}$ chez Pomponius D29.2.27, chez Ul pi en D29.4.1.12.

2 chez Scaerola Da9.7.14pr; cluez Ul pi en 029.2 .71 .0$.

3 chez Iarnleaus D29.2.62pr; chez Scaerola D29.1.1.tpr.

5 chez Pomponiug D29.2.99, chez Scaevola D29.7.14pr; chez Ul pien D29.2.21.3; D29.2.25.4-5. D29. $2.28 ; 029,7$.

1 chez Scaerola D29.7.14pr.

chez Gaius D29.1.17.13 chez Papini D29.4.26 pr-1; cliez Paul D29.7.20; chez Ul pi en D29.2.6.4; D29.2.20 prD29.2.21.3; D29.2.30.6; D29.2.40; D29.2.42pr-1; D29.4.6pr-1-2,

D29.4.10 pr.

1 chez Papinien D29.2.86pr.

chez U1 pi en D29.1.13.1; D29.2.40; $\mathrm{D} 29.2 .42 \mathrm{pr} ; \mathrm{D} 29.2 .56 ; \mathrm{D} 29.4 .10 \mathrm{pr}$; 29.7.9; D29.7.19.

1 ckez Ulpien D28.5.1.27. 


\section{Gallas 0}

Servius

Trebatius

Alfenue

ofilius

Lebéon

Sabinus

Nerva p. Proculua

Atilicinas Casaius

Aristo

Neratius

Celsus

Octarenus:

Julien

\section{Pomponius} Africain Marcellus

Screvola Pepinien

P.Fronto

Sextus

Total ....

o

\section{o}

\section{0}

chez Celsus D30.63.

1 chez Paul D30.5.1.

1 Chez Pomponius D30.45 pr.

cliez Pouponiua D30.36pr chez Paul D30.5.1.

chez Pomponius D30.12.1.

1 chez Powponius D30.48pr.

chez Paul D30.5.1, chez Ulpien D30.37 pr.

1 clez Pomponius D30.45 pr.

2 Chez Pompouius D30.13 D30. 45 pr.

4 chez Gaius D30.68.3, chez Ul pi en DJ0.38 pr D30.53.2; D30.75.4.

1 chez Ul pien D30.39 pr.

2 chez Ul pi en D30.53.2; chez Harcien D30.113pr.
2 chez Paul D30.127; chez Ul pien D30.30.7.

2 chez Ulpien D30.30.2.

chez U1 pi en D30.30.5-7.

chez Paul D30.106.

2 chez Ul pi en D30.30.7.

5 chez Pomponius D30.12pr, cleez

Ulpi en D30.30.2, D30.47.6.

- chez Julien D30.104.2-7, chez Pomponius D30.26.2, chez Marcellua D30.80; chez Paul D30.122.1; cbez U1 pi en D30.50.1.

1 chez Fomponius D30.26.2.

chez Pomponius D30.26.21 chez Faul D30.15 pr.

chez Julien D30.104.1; chez Pomponius D30.26.2.

chez U1 pi en Di30.14pr, D3o.28pr; chez Narcien D30.88.

chez Ulpien D:30,1.1pr.

1 chez Pompaning D30.0.

16 chez Harcellus D30.80; D.30.82.3, D30.92pr; chez Papinien D30.11; cliez Paul D30.10, chez Ul pi en chez Paul D30.10) chez Ul pien D30.34.10; D30.39.2-3; D30.4.t.

2 chez Ulpien D30.tpr; D30.32pr.

10 chez U1pien D30.1.tpr D 30.28 .1 , D30.39.4; D30.43.1; D30.49pr; chez Vercien $D 30.111 .3-1-16$.

1 chez Marcien D30.114.7.

b chez Ul pien D30.1.1; D30.19 pr-1; D30.34.5; D30.41.5; chez Marcien D30.113.5.

1 chez Marcien D3O.114.7.

1 chez Ul pien D30.32pr.

Trebatius

dlfenu:

Lebénn

Proculue

Pegasue

Fulcinius

Celoun p.

Neratius

A. Valens

Julien

Publicius

Marcellus

Scaevola

Total.....

1 chez Ponponiua D31.11 pr. chez Celsus D31.20.

cliez Cel aus D31.20; D31.29 pr.

0

chez Paul D31.82.2.

chez Powponius D31.11.1, chez Paul D31.82.2.

1 chez U1 pi ea N31.24.

2 chez Tryphoninus

D31.88.12; chez Ulpien D31.2.1.
C chez Paul D31.49.2.

chez Papini en D31.74.

cher Paul D31.19.2.

chez Pomponius D31.43.2

cleez Paul D31.49.2.

chez Papini en D31.67.8.

chez Ter.Clemens D31.53pr; chez papiuien D31.6öpr; chez UI vi en D31.60; D31.61.1.

1 chez liarcellua D31.50.2. 


Q.Mucius
Gallus
Servius
Cascellius
Trebatius
Alrenus
orilius
Ateius
Tubero
veteres
Labéon

Sabinus
Nerva p.
Proculus
Atilicinus
Casaius
Pegasus
Aristo
Celsus
A.Valens
Julien
Panponius
Maecianus
Marcellus
Scaevola

Scaevola
Scarcellus
0

chez Julien D32.62.

chez Iavolenus $132.100 \mathrm{pr}$ chez Iavolenus D32.30.2.

chez Paul D32.60.1-3; D32.61.

chez Iavolenus D32.29.4.

chex Iaralenus D32.29,3-4, 22 D32. 30.2; D32.100 pr; chez Pompnnius D32.85; chez Harcien D32. $05 \mathrm{pr}$.
0

0

chez Valens v32. 12.

chez Mrecianus D32. 95 .

chez hiarcien D32.65.3.

2 chez Valens D32.94; chez Ulpien D32.3.1. chez Ulpien D32.73.5. 0

1 chez Tryphoninus D.32.38.5.
3 chez Ievolenus D32.29.1; chez Ul pi en Di2.55 pr-2.

- cher Iavolenus DJ2.29.1-2; chez Pomponius Di2.5 7 .

3 ctiez Iarnlenus D32.29pr; D32.100.1. chez Iavolenus D32.29pr; D32.30 pr-5: D32. 100.1-2-3-4.

6 chez Iavolenus D32.29.2; chez Paul

6 chez Iavolenus D32.29.1; D32.100.1; chez Ul pi en D32.55 . 1-2-4-7.

1 cliez Iavolenus D32.30.6.

chez Celsus D32.13.

chez U1 pien D32.70.12.

chez Proculus D32.86; chez Iavolenus D.32. 29pr-1-2; D32. J0 pr-1-3-4-5-6; D.32.100.1-3-1; chez Cel sus D32.43; chez Paul D32.31; cbez Ul pien D32.52.8.

7 cuez Julien D32.63; cher Ulpien D32. 15 ; D32.50 pr-3; D32.52.3-i; D32.70.2.

2 chez Valens D32. 19; chez U1 pi en D32.52.7.

4 chez Iuvolenus D32.100.2-3; chez Celsus D32.79.1; chez Ul pi en D32.49.3.

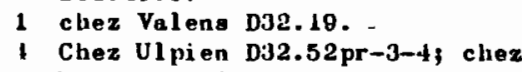
1 Marcien 032.65 .4 .

1 chez Faul D32.78.6.

chez Paul D32.6pr.

2 chez Ulpien D32.52.8.

2 chez Ulpien D32.11.1-15.

chez Ulpien D32.7.1; D32.11.20

3 chez Tryphoninus D32.36; D32.37.4.
1 chez I arolenus b32.29.1. c32.60 pr-2.

Ser.Aelius
Rutilius
2.Hucius

Servius

Cascellius

Trebatius

Alfenus

A. Nemusa

Tubero

20

o

2 cher Julien D33.5.9.2, chez Ul pien D33.9.3.6,

3 chez Iavolenus D33.4.6.1; cliex Pomponiue D33.7.15 pr; chez Ul pien D33.7.12pr-6, D33.9.3.6.

1 chez Iavolenua D33.4.6.1.

3 chez Iarolenns D33.8.22.1; 13 D33.7.25.13 chez U1 pien D33.7 12.5.

4 chez a-a D33.8.14 chex Paul D33.7.16.2 D33.8.15; chez Ulpien D33.7.12.2.

3 chez Iavolenua DJ3.4.6.1; D33.7.25.2; chez Ul pien D33.9.3.8.

2 ciez Iavolenus D33.4.6.1; crez Ul pien D33.7.12.6.

Labénn

Blaesus

Hela

Sebinus

Nerva p.

Proculus

Atilicinus

Casaius

Pegasus Iavol enus D33.4.6.1; D33.7.25.13 D33.8.22pr-1; chez Paul p33. 4.5 ; D33.10.12; chez Ul pien D33.7.12.3-4; D33.8.8.5.

chez Ul pien D3J.7.8pr; D33.8.8.3-4; D33.9.3pr.

chez Ul pien D33.8.6.4. chez Ulpien D33.8.6.4. 1 chez Paul D33.7.18.10.

3 chez Ul pien D33.7.12.3; D33.8.6.4; D33.8.8pr

Iavolenus Aristo

Neratius

o

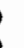

5 chez Scaernla D;13.5.18; chez Paul D33.7.13pr-1; chez Paul D33.7.13 pr-1;
chez Ulpien D33.7.12.t-35.
1 chez Ul pien D33.9.3.9.

chez Ul pien b33.9.3.9.

chez Ponponius D33.1.7, chez Paul D33.4.11; chez Ulpien D33.9.3pr-9. chez Iavolenus D33.4.6pr; chez Celsus D33.10.T.2; chez Paul

D33.T.16.1; chez Ulpien D33.9.3.10.

chez Iarolenus D33.6.7pr; D33.7.4; D33.7.28.1; D33.10.10

chez Priculus D33.6.15; D33.6.16pr; chez Iavnlenus D33.1.17 pr; D33.2.31 D33.6.7 pr; D33.7.4; D33.7.26 pr-1;

D33.10.11; chez Ulpi en D33.9.3.2.

2 chez Iavnlenus D33.4.6pr; chez Paul D33.1.22; D33.2.12; D33.2.40

D33. T. $10 \mathrm{pr}-1$, D33.10.6 pr-1.

9 chez Is vol enus D33.6.7 pr-1; D33.7.26 D3:3.10.10; chez Ul pi en D33.9.3.5-9.

3 chez Iavolenus D33.5.20.

chez Ierolenus D33.6.7pr; D3J.7.25 p chez Celsus b.33.10.7.1-2.

chez Proculug D33.6.10pr-2; chez

Iavol enus D33.1.17 pr-1; D33.2.30 pr; D33.2.31; D33.2.41; D33.4.6pr; D33.5.20; D33.6.7 pr-1; D33.7.4;

D33.7.25 pr; D33.7.26 pr-1; D33.10.10 D33.10.11; chez Cel aua D33.10.7pr; chez Paul D33.4.13; D33.7.5; D33.7.29; chez Ulpi en D33.9.3.2-3.

1 chez Involenus D33.2.31.

chez U1 pien D33.1.14; D33.4.1.1.t-15 D33.9.3.10.

12 Chez Paul D33.1.4; D33.7.18.12; D33.9.4.2; chez Ul pi en D33.6.9pr D33.6.13; D33.7.12.13-20-27.

2 chez Ulpien D33.1.14.

1 chez Ulpien Di3.9.3.2.

chez Paul Da3.9.5 pr; chez Ulpien D33.7 .12, 11-13-17-16-20-27.

5 chez Ulpien D33.7.12.16-18.

1 chez Valens D33.1.15.

t chez Ulpien D33.2.22; D33.9.3.1-11; chez Marcien 133.7 .17 .1

7 chez Paul D:33.7.18.2; chez Ul pi en D:3:3.7.12.4:3. 
Cel nue

Julten

Pediue

Powponing

Marcellu.

Seserola

Papiaien

3 chez U1pien D33.7.12.3144 , D33.8.6 pr.

5 chez Scaernila D33.8.21, chez U1pi en D33.4.1.10, 0 D33.8.6

1 chez Paul D33.5.13.1.

chez Paul D33.8.9.1.

2 chez Panl D33.7.18.4-13.

6 chez Ulpien D33.7.12.37$38-42-15-46$,

chez Paul D33.1.9.

182
C chez Ul pi en D33.4.1.8, D33.4.2prs D33.6.3.1, D33.7.12.20-30.

10 chez Ulpten D33.4.1.11-12-13, D33.4.2pr; D33.9.1.

1 chez Paul D33.7.18.3.

3 chez Paul D33.7.29; D33.9.4.6.

2 chez Vlpien D33.9.1.

chez Trypboninue D33.2.32.1, chez Paul D33.4.16; D33.7.18.5

12 chez Ulpi ea D33.7.12.23-39-40-4143-47. $\begin{array}{ll}\text { M.Porcias Cato } & 0 \\ \text { Q. Nuctas } & 0\end{array}$

Gellus

Serrius

0

Cascelliua

Trebatius

Alfenus.

ofilius

Steius

Tubero

Labéon

Mela

Sabinus

Nerva p.

Procul us

Atilicinus

Caseitus

Pleutins

Fufidius

Iarolenus

Aristo

Neratiue

Celsus

Julien

Yomponius

Yenul oi us

Harcellus

Scaerola

Papinien

Total

0

:

0

o

o

o

0

0

0

0

0

3
1 chez Paul D34.2.4.

chez Ulpien D34.3.7.1, ע34. 4 . 3.9.

chez Tryphouinue

D34.1.15.1; 13.1.1.13.2; D34.3.28.7.

1 chez Ul pien D34.4.3.y.
1 chez Cel sus D34.7.1 pr.

7 chez Pomponius D34.2.10, D34.2.33, D34.2.34 pr-1-2, chez Ulpien D34.2. 19.9, D34.2.27 pr.

1 chez Paul D34.2.32.1.

4 chez levolenus 034.2 .39 .2 ; chez U1 pi en D34.2.18.17, D34.2.27.3.

1 chez Iavolenus D34.2.39.1.

1 chez Harcien D34.5.14.

2 chez a-a D34.2.28, D34.8.2.

2 cbez Iarolenus D34.2.39 pr-1.

chez Iarnlenus D34.2.39.2.

1 cbez Paul D34.2.32.1.

5 chez Iarnl enus D34.2.31, D34.2.39pr1) chez Paul D34.2.32.1-6.

1 chez Ul pien D34.1.14pr.

4 chez Tryphoninus D34.8.22, chez U1 pien D.34.2.19.13-17-18.

1 chez Paul D3.4.3.13.

1 chez Paul D34.2.32.5.

2 cbez Africain D34.2.5; chez Paul DJ4.3.16.

3 chez Pomponius 134.2.21.2; chez Paul D34.3.18, chez Ulpien D.94.2.19. 19.

1 Ghez Paul D34.2.8.

1 chez Africain D34.2.5.

1 cinez Paul D34.2.8.

1 chez Ui pi en DJ4.2.25.1.

cuez Pomponiua D34.3.8.2; cbez Paul D34.2.32.5.

5 chez Yomponiug D34.3.2pr-1; chez U1 pi en D3.1.2.19.2-3-6.

17 chez Gaius D34.5.5 pry chez Ter. Clemens D34.3.21.1; chez Ul pien D34.3.1.13 D34.3.3.2-3-5; D34.3.5 pr1-2-3-4; D34.3.7 pr-2-5; D34.8.4.1.

2 cirez Ulpien Di34.2.1Y.5; D34.2.25.9.

1 cuez Ulpien Di34.2.19.7.

J clez Ul pi en D34.3.3.5; D34.3.5.2, chez wiarcien D34.9.3. chez Paul D34.2. J2.3-7-8-9.

2 chez Ulpien D3.1.9.4. 
Servius Cascellius Trebatius

Alf enus ofilius A.Nemua Cinar

reterea

Labéon

Sabinus

Nerra p. Procula:

Atilicinus Casaiua

CaeliusSabinas Plautius

Ariato

Neratius

Celsus Julien

v. Verus Pedius Pomponius Africain Naecianus Publicius Harcellag

\section{Screvola} Papinien vl pi en

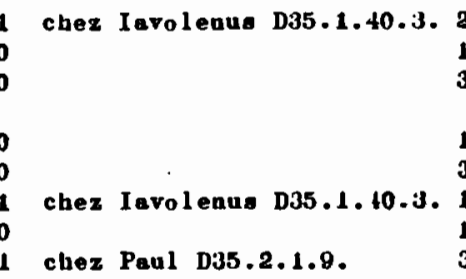

1 chez Paul D35.2.49pr.

1 chez Paul D35.2.49pr.

2 chez Pomponius L35.1.58, chez Panl D35.1.43pr.

1 chez Paal D35.2.49pr.

3 chez Papinien D35. 72 . 9 chez Paul D35 . 1.43pr? J35:2.49pr.

1 chez Papinien D35.1.72.7. 1

3 chez Paul D35.1.43pr; $\mathrm{D} 35.1 .44 .10 ; \mathrm{D} 35.2 .49 \mathrm{pr}$.

3 chez Pomponius D35.1.6.13 Da5.1.112.3; chez Paul D35.1.37.

0

2 chez Haecianus D35.1.80pr; 17 chez Marcellus D35.2.34.

\section{0}

chez Paul D35.2.63pr.

chez Paul D35.2.36.1. chez Screvole D35.2.20.

1 chez Scaerola v35.2.56.2.

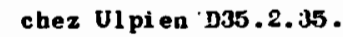

1

\begin{tabular}{ll}
$\begin{array}{l}\text { Ofilius } \\
\text { Labéon }\end{array}$ & 0 \\
& 0 \\
\hline Sabinus & 0 \\
Cassius & 0 \\
Aristo & 0
\end{tabular}

Neratius

Celsus D35 1.9 .18 D35 chez Pompnnius D35. 1.68 .

6 cliez Pomponius Das.1.6.1; D36.1.14; cidez Papinien D35.1.71.3; D35.1.72. chez Paul D35.2.1.19.

7 chez Iarol enus D35.1.40.5; chez Pomponius D35.1.14; chez tiaius D35.1.69, chez Paul D35.2.1.14; D35.2.45.1. chez Iarnlenus D35.1.54 pr; chez Powponius D35.1.6.1; D35.2.31; ctez Paul D35.2.1.14.

2 chez Paul D35.2.1.9, chez Ulpiea D35.1.7 pr.

chez Paul D35.1.43.3, chez Ul pien D35.1.7 pr, D35.2.82.

1 chez Maecianus Da5.2.30.7. chez Ter.Clewens D35.1.82.1-2; D35.1.64pr; chez Waecianus D35.2.30.7; D35.2.32.2; chex Harcellus Di35.1.20, chez Papinien D35.2.11.8; chez Paul D35.1.45, D35.2.1.9-1+-17, chez Ulpien D35.1.7 pr D35.2.62pr; D35.3.1.7-8.

1 cbez Haecianus D35.2.32.4.

2 chez Ul pi en D35.3.3.2.

2 chez Ulpien D35.3.1.2; D35.3.3.10. chez Papinien D35.1.71pr.

chez Nodentin Da5.1.51.1. chez Paul D35.2.1.19; D35.2.3.2 chez Ul pien D35.1.1.3.

2 chez Tryphoninus D35.1.109.

1 chez Ul pien D.35.1.92.

2 chez liecer $\mathrm{D} 35.2 .68 \mathrm{pr}-1$

1 chez Ul pi en D36.3.1.15.

chez Iarnlenus D36.2.30; D36.4.143 chez Pomponiua D38.2.22.1; chez Ul pi en D36.2.12.1.

2 chez Pomponius D36.3.10; chez Ulpien D36.2.12.1.

1 chez Ul pien D36.2.12.1.

chez Neratius D36.3.13, chez Pomponius D36.1.22; D36.1.7ł; chez Paul D36.1.20.1, chez Ul pi en D36.1.3.2.

3 chez Gaius DJ6.1.85.12; chez Ulpien D36.1.1.19.

3 chex Ul pien D36.2,12,1-3; chez Harcien D36.1.34.

Octarenns Jalien

chez Gaius D36.1.05.4; chez Ulpien D38.2.12.2.

Pomponius

Haecianus

Harcellus

Scaevola

Papiaien

1 chez Valens D36.1.69 pr.

31 chez Gaius D36,1.65.9-10-13; chez Haecianus DJf.1.67.1; chez Marcellus D36.1.26pr; D36.1.28.6-7-11; chez Paul D36.1.68.1; D36.2.6.1; chez Ul pi en D36.1.1.8-16-17-20-21,

D.36.1.3pr; D36.1.6.1-6; D36.1.9pr; D36.1.11pr-1; D36.1.15.3; D36.1.17. D36.1.18.1; D36.2.12.1-3; D36.2.14. D36.4.5.29; chez Liarcien D36.2.20.

3 chez Paul D36.2.21.1; chez Ulpien D36.1.3.2; D36.2.12.6.

chez Ulpien D38.A.18.4.

chez Ulpien D38.1.17.1314.

D36. $1.17 \mathrm{pr}-3-6-8-9$ chez Ul pien D36.1.3 pri D36.3.1.17, D36.4.5. 29.

5 chez Tryptoninus D36.1.79.1;

D36.1.80.6-9; D33.1.82; chez Ulpien D:36.1.23pr.

t chez Glpi en Das.1.17.5; D.36,1.18.6; Da6.1. 1.4. 


\begin{tabular}{|c|}
\hline $\begin{array}{l}\text { Q. Yucius } \\
\text { Servius } \\
\text { Cascellius } \\
\text { Trebatius }\end{array}$ \\
\hline Alfenus \\
\hline ofilius \\
\hline A. Namuga \\
\hline $\begin{array}{l}\text { Ateius } \\
\text { Tubern } \\
\text { Labéon }\end{array}$ \\
\hline
\end{tabular}

\section{0

0 D39.3.6.T.

3 chez Paul D39.3.11.3, chez Ulpien D39.B.BFr; D39.6.37.1 cliez Ulpien D39.5.18.1-2.

2 ches ULpien p:39.3.1.3-4.

J chez Ul pien D39.2.2.4.4-5; Di39.3.1.6. Q.Mucius

chez Ulpien nj9.3.1.17,

Chez laul D39.2.25; D39.3.11.6; chez Ulpien D39.2.9.1; D39.2.24.12; DE9.3.1.3; D39.3.3pr-1.

10 chez a-a D39.2.43pr-1-2; D39.4.15, chez Paul D39.3.2.5; D39.3.21 pr-1-2-3; chez Ulpien D39.2.9.2.

5 chez Paul D39.3.2.10; chez Ulpien D39.3.1.5-21; D39.3.3.2; D39.3.11.5.

2 cluez Paul D39.3.2.6; chez Ul pi en D39.3.1.6.

2 Chaz Paul D39.3.2.1; D39.3.14pr.

1 cliez Ulpien Dag.3.1 pr.

18 chez Neratius D39.2.47, D39.3.1.7-17-2021-22-23; chez Powponius D39.3.19; chez Paul D39.3.2.1-2-5-6-7-8-9-10; D39. 1.4pr; chez Ul pi en D39.1.3.2, D39.1.20.8; D39.1.21.5-6-7; D39.2.9.2; D30.2.13.5; D39.2.15.10-15-20-24-32-33$34-35 ; D 30.2 .21 .2-3-4-5-11$; D39.2.30.1, D.39.3.1.7-20-21-22; D39.3.3.3; D39.3.4 pr D39.3.5; D39.3.10.2, D39.5.18.3 D39.5.19.1.

12 chez Paul D39.2.18.11, chez Ulpien D39.2.13.6; 039.2.15.12-32; D39.2.37; ก39.2.10.1-3; D39.3.1.8-9-10-11; D39.3.6pr.

2 chez Paul D39.j.11.2; chez Ulpien D39.2.26.

11 chez Paul D39.2.10; D39.3.2.3; D39.3.11.1 chez Ulpien D39.2.15.35; D32.2.24.8, D39.2.28; D39.3.1.8-9-10-11-19.

1 chez Ulpien D39.5.19.8.

1 chez Paul D39.2.22.1.

1 chez Nerstiua D39.6.13.

1 chez Paul D39.6.35.3.

5 chez Paul D39.2.18.10; chez Ulpien D39.2.28; D39.5.18pr.

3 chez Julien Djo.t.21; chez Ulpien D39.2.9.3; D39.3.1.2.

1 chez Paul D39.3.11.2.

2 chez U1 pi en D39.2.24.9-10.

1 chez Ulpien D39.1.1.10, D39.2.9.5; D39.2.15.25.

25 chez Gaius D39.6.31.1-3; chez Harcellus D39.6.13.1; chez Paul D39.2.10;D39.2.18.5 D:39.6.15; chez UIpi en D39.1.5pr-16; D39.2.7.2; D39.2.9pr-43 D39.2.13.1D-11; D39.2.15.16; D39.2.40.2-3; D39.3.4.1-2-3;

1 chez Ulpien D39.1.5.9.

8 chex Paul D39.2.18.1-5; cluez Ulpien D39.2.4.5; D39.2.40.4; D39.1.1.t; D39.5.18pr.

- chez Paul Dí.6.15, chez Ulpien Dis9.2.11; D39.2.12.9; D39.3.1.12.
Gallus Servius

Trebatiua Alfenus

ofilius

veteres

Labénn

Mela

Sabinus

Proculus Nerva fils.

Cassiug

Fufidius

Iavolenua

Ariato

Neratius

Celaus

Campanus

Octavenus

A. Valens Pact.clemen Julien

Pomponiu Africain

Scaerala

L. Huf i nus

Total
3 chex Iavolenus D40.7.39pr; chez Pomponius D40.7.29.1; chez Paul D40.12.23 pr.

1 chez Iavolenus $040.7 .39 \mathrm{pr}$

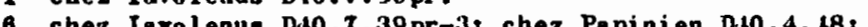
chez Paul D40.4.35; D40.12.24.1; chez U1 pien D40.7.3.2.

4 chez Iavolenus D $40.7 .39 .2-4$; chez Ulpien D $40.7 .3 .11-12$.

5 chez $D 40.1 .6 ; D 40.1 .7 ;$ D40.7.14pr-1; chez Ulpien D40.12.10.

4 chez Iavalenus D40.7.39pr-1-4; chez Pouponius . D40.4.40.1.

1 cluez Pomponiua D40.7.21 pr.

17 chez Involenus Dt0.1.26; D.40.7.39pr-1-2-3-4; D40.12.42; 17 chez Pomponius D.40.4.41.2; D40.7.8.1; D40.7.21 pr; D40.7.29.1; chez Paul D40.7.41 pr-1; D 40.7 .42 ; cluez Ul pi en D40.7.3.2-11; D40.12.22.5.

1 chez Paul Di0.12.21.4. D40.12.30, ches Gaius D40.4.57; chez Paul D40.7.20.3.

1 chez Gaius D40.2.25.

7 chez I avol enus D40.7.28.13 chez Julien D40.12.303 chez Gaius D40.4.57; chez hiaecianus D.40.5.35; chez Peul D40.7.4.8; chez U1 pi en D.40.7.2.1; D40.7.3.2.

1 chez Gaius D40.2.25. Chez Pomponing D40.4.46; D40.5.20; D40.7.5 pr; D40.7.11; W40.7.29.1; chez Faul D-10.9.16.3; chez Ulpien D40.4.2.

2 chez Pomponius D40.7.5pr; chez Ul pien D40.4.7.

4 chez U1pien D40.5.24.8; D D 0.7.2.4; D40.7.29.1; D 40.9 .1 .

1 chez Fomponius D40.5.34.1.

5 chez Pomponius D40.1.13; D40.4.61.2; D.t0.5.20; chez T.Clemens D.0.9.32.2; chez Ul pien DH0.T.9.2.

1 chez Paul D40.5.25.

1 chez Pnoponius D40.7.21.1.

poniug D40.7.21.1.

D4n.4.40pr; D40.4.61pr; D10.5.20; chez 25 T.Clemens D40.9.24; D40.9.31; chez Gaius D40.4.57; D.10.7.27; D40.7.31.1; chez Scaevola D+0.9.6; chez Paul D40.2.4.2; D40.7.4.1; D40.7.20.1-3; D40.7.22.23 D 10.9.15.1; chez UI pien D40.t.12.2; D40.4.13.2; D40.7.2.1-2-3; D40.7.3.16; D40.9.30.5; D40.12.10 chez Modestin D40.7.27; D40.9.21.

3 chez Paul D 10.7.4.5; chez U1pien D40.7.3.13; D40.7.6.7. 3 2 chez Ulpien D40.9.12.2-6.

7 chez Ulpien D40.2.20.3; D D $0.5 .24 .9-12-13$; $040.5 .28 \mathrm{pr}$; chez Marci en D40.5.55.1; D D 0.15.1.4.

4 cbez Tryphoninus D40.4.58.1; D40.5.17; chez Paul D40.9.20; chez Marcien 140.5 .50 .

1 chez Paul D40.13.4.
5 clsez Ponponiun D40.5.34.2; chez Julien D40.4.18pr;

1 chez Julien D40.9.7.1.

(1)




\section{Alfenus} ofilius reteres

Labéou chez Pomponiue D41.1.19; 241.10.4pr; chez Paul D11.4.2.7.

1 chez Paul D+1.3.j4.

chez Paul DH1.2.1.16.

3 chez Pomponius D.11.1.19; chez Paul D41.3.1.7; D+1.3.8pr.

3 chez Julien D41.3.35 chez Paul D\&1.2.1.5; D41.3. 1.18.

0 chez Powponius D-1.1.21pr.

4 chez Papinien D41.2.47; chez Paul D.1.2.1.i.t-22; D.1.2.3.13

3 clez Paul D41.2.1.5-14; D+1.3.t.16.

0

0

chez Poraponius D.11.1.19. chez Pomponiun DH1.1.19.

3 chez Ponponius D41.10.3; chez Ponponius DH1. D.11.1.2.6.

4 chez Piul D41.4.2.11-13l+3chez Ul pi en D41.2.34.2.

8 chez Paul Dit.1.57;

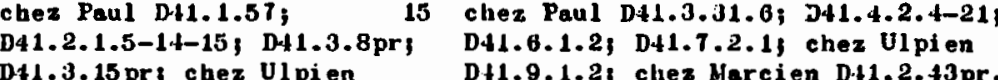
D41.1.23-3, C4ं1,1.33-2.

1 chez Paul D41.3.8.1.

3 chez Paul D41.1.49, D41.3.4.9, D41.4.2.12.

0

3 chez Paul D $41.3 .15 \mathrm{pr}$ chez Ul pi en D11.1.33pr D11.3.10.2.

3 chez Ulpien D11.1.23.3; D41.1.33pr; DH1.3.10.2.
1 chez Paul Dy1.2.3.3.

1 chez Paul D41.2.3.3.

1 chez Paul D41.2.3.23.

chez Julien D41.5.2.2, chez Pasl D.1.1.26pr; D.11.1.2.8.

Chez Pompnius D41.6.3; chez Galus D.11.1.5.1; chez FlorentiausD41.1.16; chez Paul DA1.2.3.6, chez Ulpien

1

chez Yaul U\$1.2.1.3.

chez Gaius 141.3.38; chez Marcellus D11.2.19.1; chez Paul D41.2.3.18-19.

21 chez Iarolenus D41.2.51, chez Pomponius D41.1.28; D41.3.30.1, D11.3.32.2, chez Paul D41.1.26pr-2; D.11.1.65 pr-1-2-3-1; D41.2.1 pri D+1.2.J.5-17; D-41.2.30.2;1341.3.4.10; D11.3.49, chez U1pien D+1.2.6.1.

11 Chez Pomponius D\$1.1.28; chez Gaius D.11.1.7.7, chez Paul 1041.2.3.3-5-18; D41.4.2.2-3; cliez U1 pien D41.3.10 pr.

Chez Gaius D41.1.7.7.

chez Phaponius D41.1.27.2\} D41.1..26, chez deius D41.1.7.7, chez Paul D.1.3.31 pr; D41.7.2.1.

8 chez Paul Di1.2.1.1-3, D41.2.3.1.1-17

12 chez Pomponiug D+1.1.27.2; chez Gaiu DH1.1.7.7; chez Paul D41.2.3.18; D+1.3.4.21-25; DH1.6.1.2; chez

U1 pi en D.1.3.10pr; D.11.9.1.3-4.

2 chez Pomponius D41.1.27.2; chez U I pi en DHI.1.41.

1 chez Marcien DH1.1.11. 1

chez Paul E41.2.1.21; D41.2.3.34 C.11.2.7.

7 chez Pomponius D+1.1.30.1; chez

11 chez Paul D41.1.48.1; chez Ul pien D.1.1.4t; D+1.2.6pr; DH1.2.10pr-1, D.1.2.13pr; chez MarcienD.11.2.43.1-2

1 chez U1 pien DH1.10.1.1.

I chez Ul pien D41.2.34 pr.

\section{0}

2 chez U1pien 042.8 .6 .12 ; D42.8.10.10.

\section{Mela} Sebinus

Proculus

Cassius

Fufidiue

Fulcinius

Neratius

\section{Celsus}

Julien

Pomponius

Marcellus

Scaerola

Sextus

Total.....
1 chez Venulaius o D42.8.25.5.

o

0

1 chez Ulpien D42.1.4.8.
1 chez Paul D12.1.62.

1 chez Ulpien D42.4.7.13. Paul D42.3.12pri chez Ul pien D42.1.t.3; D42.4.7.10; D-12.3.15.1, D42.8.6.6-1C.

1 chez Venuleius D42.8.25.7

2 chez Paul D42.8.9, ches Ul ipien chez Paul Dit

3 chez Paul D42.8.T, chez Ul pien D42.8.0.23.

eius D12.8.11; chez Ulpi on D42.3.4.1, D42.7.2.3-5.

1 chez Paul D42.5.29.

1 chez Ulpi en D42.4.7 pr.

2 chez Paul Di2.1.21; chez Ulpien $042,4.7 .16$.

4 chez Ul pien D42,4,7,17-18,D42,7.2.20

7 chez Paul D 42.1 .38 .1 ; D 42.2 .3 ; D 12.5.6.2, chez Ulpi en D42.4.3pr D+2.8.6.4-7; 142.8.10.16.

2 chez Paul D.12.1.36; chez U1pien D42.4.7.7.

3 chez Ulpien D42.4.3pr-3;D42.8.10.1.

1 chez Paul Dit.s.6.2.

1 cher Ulpien D42.4.7.17. 


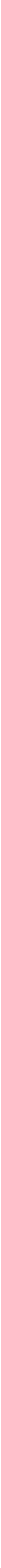


M. Porcius Cato Q.Hucins
oftlius Tubero

reteres

Lahéon

Sebinus

Froculus

Atilicinas Caseivas

Peganus

ariato

Cel aus

Julien

(1)

Pedius

Pomponi

Gaius

Harcellus chez paul DH5.3.20.1.
1 chez Pomponius 145.3.6.

1 chez Paul Dis.1.4.1.

chez Papinien D45,1.115.2.

chez Ulpien D45.1.72pr.

chez Paul D45.1.91.3; V45.1.140.1.

chez Venulelus Di5.1.137.7; chez

Ul pi en D.5. $1 . B 7.1$.

7 cbez Iarolenus D45.1.105; 12 ches Venuleius D+5.1.137.5; chez Julien D45.3.14, J45.1.138pr, chez Peul D45.2.17,

chez Pnoponius D45.3.63

chez Papinien i45.1.115.23

chlez Paul D45.1.8,

D45.1.83.7; D45.3.32.

2 chez Gaius D-15.3.28.4, chez Paul D45.1.8.

4 chez Posponing D+5.3.6, chez Geius D45.3.28. 13 chez Ul pi en D45.3.8.1.

1 chez Papiniea D45.1.115.2.

0 chez Ul pis.1.91.34

14 chez Gaius D45.2.15, D.45.3.28pri chez Venuleius D-45.3.28pri cliez Venul chez Papinien Da5.3.18.3, chez Paul Di5.1.01.1-2-36) D45.3.33 pr; chez Ul pi en D45.1.38.6; D45.3.7.1 D $45.3 .9 .1 ; 045.3 .11$.

1 chez Paul D45.1.91.5.

1 chez Pomponius D $\$ 5.3 .39$.

2 chez Paul Dis.1.8;

cluez Ul pi en D.5.1.29.1. chez Geius D45.3.28.13 chez Paul D45.1.83.1.

chez Pomponius D 45.1 .14 , cirez Ul pien

5 chez Pomponiug D45.1.21; D45.1. 112.1 chez Venuleiue D+5.1.138pr.

chez Paul D 45.2 .17

5 chez Paul D15.2.17.

D 15.1 .67 .1 ; D $45.1 .72 \mathrm{pr}$.

16 chez Scaerola D45.1.131pry chez

Paul D45.1.2.5.

Poeponius

Mercellus

chez Paul D45.1.2.2-5; D 15.1.91.A.

chez Paul D.15.1.4.1; D.45.1.8,

chez Ulpi en D\&5.1.38.20.

$\begin{array}{ll}\text { Gellus } & 0 \\ \text { Serrius } & 0 \\ \text { Alfenus } & 1 \\ \text { reteres } & 0 \\ \text { Labén } & 3 \\ & \\ \text { Mela } & 0 \\ \text { Sabines } & 0 \\ \text { Minicins } & 1 \\ \text { Proculus } & 0 \\ \text { Nerra fils } & 0 \\ \text { Casaius } & 0 \\ \text { Pegasus } & 0 \\ \text { Aristo } & 0 \\ \text { Celsus } & 1 \\ \text { Octarenus } & 1 \\ \text { Julien } & 0\end{array}$

chex Paul D48.3.36.

chez Pomponius D+6.3.19, chez Venuleins D-16.8.8.8 chez Ul pi en D46.4.8.2.

chez Julien D46.1.19.

chez Paul D+6.3.98.8.

chez Ulpien D46.4.8.1.

chez U1pien D46.1.8.8-9;

D46.t.13.4.

37

67
Scaerola

Paul

ches Ulpien D46.1.8.8,

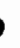

Totel......

12
1 chez Flnrentinus D46.4.18.1. chez larcellus D46.3.67. ches Harcellue D46.3.67.

chez Papinien D46.3.97.

chez Papinien D46.3.95.7, chez Paul D46.3.81; D46.4.23; D46.8.15.

1 chez Africain D46.3.39.

2 chez Venuleius D46.8.8pr; chez Sceerole D46.3.83.3.

ches Sceotol. D46.3.03.3.

1 chez Venuleius D46.4.21.

chez Ievolenus D46.3.78; chez Pomponius D46.3.17.

chez Papinien Dib.3.95.7.

1 chez Pompnnius D46.3.16.

3 chez Ul pien D.6.2.8.2-3.

Arez Pomponils D+6.8.16.1; cher Africain D46.3.38.4, chez Gaius D.46.7.7, Chez Maecianns D46.3.103; chez viarcellus D46.8.22.5; chez Papinien Di6, 1.47pri chez Paul D46.1.34, D46.3.59; D*6.5.2.23 chez UI Ui en DH6.1.5, D46.1.8.3; D46.3.58pr; D46.8.12.2.

5 chez Paul D46.1.56.2, D46.3.8; chez Ul pien D46.1.27 pr-3; D46.3.7.

6 chez Ulpien D46.1.25, D46.2.8.4; D46.3.5.3, D46.4.20.

3 chez Tryphont aus D46.3.88; D46.7.20; chez Marci en 046.3.47.1.

1 chez Papinien D46.5.8pr.
57 
Q.Mucius Servius

Trebatius

Alfenus

ofilius

veteres

Labénn

Mela

Sabinus

Proculus

Atilicinus

Cassius

"cansiani"

Neratius

Cel sus

0ctarenus Julien

Pedius

Pouponius

Venuleius Marcellua Scaevola Papinien

Ulpien
Modestin

Total....
0

0

1 chez Ulpien D47.2.17pr.

14 chez Scaevola D47.B.B; chez Paul DAT.2.92, D.17.8.3; chezUl pien D.17.8.3; chezUl pi en $\begin{array}{ll}\text { D47.4.1.1; } & \text { D47.4.3\} } \\ \text { D47.9.3.7, } & \text { D47.10.1.7 }\end{array}$ D47.10.7.8, D47.10.15.16$39-42-46 ;$ D47 . 10.17.2-7

3 chez U1pi en DA7.2.52.23, D47.10.15.45; D47.10.17.2.

6 chez Payl D47.2.42.1, D.7.2.54.1; cinez Ulpien D47.2.17.2; D47.2.36.2-3; D47.7.7.5.

0

o

chez Paul D47.2.18.

1 chez U1pien D47.9.3.7.

chez Paul D47.2.54.4 D47.2.17.3; D47.10.1.6.

I chez Paul vit.10.16.

1 chez Ulpien D.17.1.2.3; D47.2.7 pr; D47.2.14.6-7; 104.2.17.3; D47.2.36pr-2. 1 chez U1pien D47.10.11.7. 2 chez U1 pi en D.7.4.1.10-15. 2

chez Ul pi en D47.2.14.5-6-7.6 chez Ul pien D47.2.12.2; 047.10.15.29 30 .

chez Paul D47,2,21,2.

cliez U1 pi en $\mathbf{P}+\mathbf{7} . \mathbf{2} 2.20$

chez Pomponius D47.2.77.1.

U1 pi en DA7.10.15.32.

chez Paul Di7.2.21pr; D47:7.1.

1 chez Panl D4T.2.58.

Paul D47.2.21, D47.10.23;

Paul D-4T.2.67.2; chez Ul pien

$.7 .3 \mathrm{pr} ; \mathrm{D} \cdot 17.10 .13 .7$

chez Iavolenus D47.2.81pr; D47.10.44; chez Paul D47.2.1pr; D47.7.7:1; D47.10.27; chez U1pien D47.2.23; D47.2.25.1; D47.2.31.1; D47.2.50.43 D.17.2.52.1;, D47.7.3.5-6;D47.8.2.20, D.17.8.4.2-3; D47.0.3.2; D47.10.1.1; D47 . 10.5.5; D47 . 10.7.1-4; D47 .10.13.4-5; D47. 10.15 pr-3-7-17-26 D47.12.3.9; D $47.15 .1 \mathrm{pr}$.

10 chez Panl D47.2.1prz D47.2.21.83 chez Ul pi en D47.2.43.5, D47.10:5.8.

1 chez UIpien D47.10.11.4.

1 chez Ulpien D47,10.11.6.

2 chez U1 rien D47.2.5 pr; D D 77.2.43.5.

4 chez Ulpien D.17.2.43.1; D47.10.1.8-8 D47.10.7.5.

chez Ul pi en D47 .2.7.2-3; D47 .2.14pr10 ; D $17.2 .25 \cdot 1 ;$ D D T .2.43.10

D47.2.50.1; D:17.12.2.

1. chez Paul D.47.2.67.1.

21 chez Marcellug D47.2.69; chez Paul D47.2.24; D47.2.67 pr; chez Ulpien D47.2.3.2; D47.2.12.2; D47.2.14pr-14-10; D47.2.19.6, D47.2.23; D47.2.52.16; D47.8.2.10; D47.10.5.7 D47 . 10.17.18-10-20.

chez U1pien D47.2.50.2; D.17.7.7.1; D47.9.4pr.

2 chez Paul D47.2.15.2; D47,2.21.2-410 , D47.10.18.2, chez Ulpi en

D.7.2.7.1; D47.2.12.2; Di7.2.14.8-9; D47.10.9pr; D47.10.13.7
1 ehez Ulpien M47.10.17.2. D47.2.67.3; chez Ulpien chez Ulpien D47.10.8.1. D.7.2.41.3; D47.2.46.8; D17.7.3.3-4;
Alf enum
Labéon

Julien

Powponius

Africain

Hecianus

Yenul efus

Marcellus

Papini en

Menander

Total..... 8
1 chez Paul D.8.22.3.

1 chez Peul D48.10.14.1.

chez Ul pien D 48.5 .28 .5 .

chez Hocestin D48.3.14.7.

chez Paul D48.10.14.1.

chez Ul pi en D48.18.4.

1 chez Macer D48.19.1.4. chez Paul D 18.13.11.2-63 chez Ulpien D $48.5 .24 \mathrm{pr}$.

chez Gaiue D18.5.44; chez Paul D.8. 10.22.6.

Chez Ul pi en D.18.5.16pr-5; D48.5.24pr. chez Ulpien $018.5,14.1$.

chez Ulpien $D .48 .9 .6$.

ciez U1 pi en D48. 20.5 .1 .

chez Niarcien D48.5.8; D48.16.1.4-510-1:3; D48.17.1.4; D18.21.3pr. 


\begin{tabular}{|c|}
\hline $\begin{array}{l}\text { Bratus } \\
\text { Q.Mucius } \\
\text { Jervius } \\
\text { Trebatius } \\
\text { Ofilius } \\
\text { veteres }\end{array}$ \\
\hline Labéon \\
\hline Sabinu. \\
\hline Julien \\
\hline $\begin{array}{l}\text { Pomponius } \\
\text { Marcellus }\end{array}$ \\
\hline $\begin{array}{l}\text { Scaevole } \\
\text { T.Paternus } \\
\text { Papinien }\end{array}$ \\
\hline $\begin{array}{l}\text { Mesaius } \\
\text { Tryphontnus } \\
\text { Menander } \\
\text { Paul }\end{array}$ \\
\hline
\end{tabular}

0

cbez Inrolenus DeO.15.27. crez Ievolenus D 4 -15.15.27.

chez Tryphonin

D49.15.12.9.

3 chez Ievolenus D49.15.27; chez Peul D49.15.29,

$$
\text { D49. } 15.30 \text {. }
$$

1 chez Tryphoninue

D48. 15.12 .9$.

1 chez Ul pien 049.17 .0 .

o

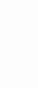

chez Hodentin D40.15.4.

chez Modestin D.99.15.4

cuez Tryphnninus 049.15.12pr.

chez Macer D49.16.13.4.

chez Callistrate Dig.14.1.1\} chez Peul D19.15.28.

4 chez Powponius D49.14.35; chez TrJphoninus D 19.15 .12 .2 ; chez Callistrate D49.14.3pri chez Ul pien D49.4.1.1.4.

1 chez Harcellus D+19.17.10.

4 chez Tryplioninus D49.15.12.2; chez Peul D49.14.21, chez liarcien D49.14.18.10.

1 chez Tryphnninus D49.17.19pr.

1 chez Tryphnninus D49.17.18

1 chez liacer U49.16.12.1. D49.1.10.3, DH9.14.28, chez Marcien D.9.14.18.10.

1 chez Paul D49.14.50.

1 chez Peul D40.14.50.

2 chez Hacer Et9.16.13.5-6.

2 chez Macer D48.4.2.3; D49.16.13.5.

34
Total $\ldots .$.

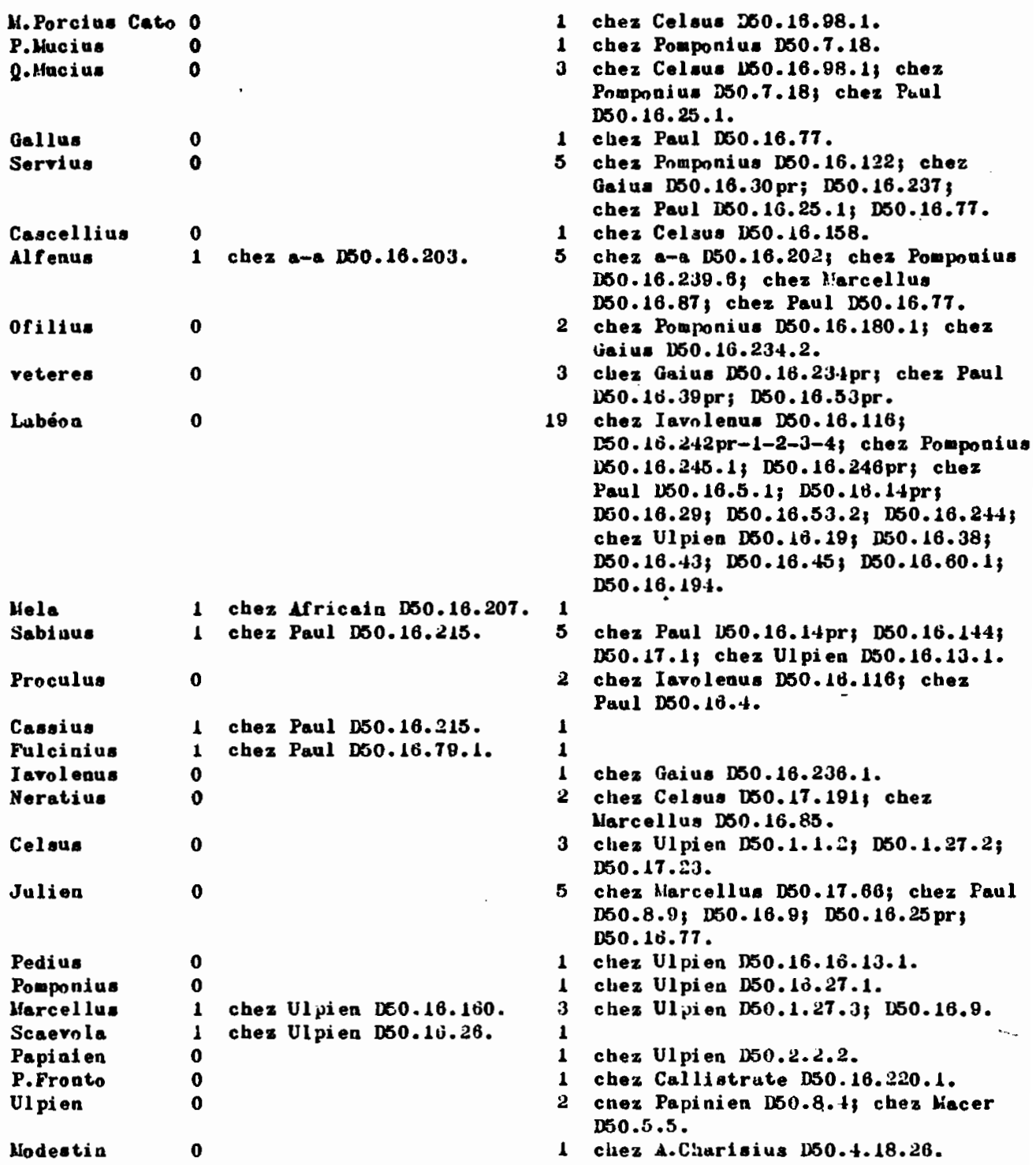

M. Porclue Cato 0

$\begin{array}{ll}\text { P.Hucius } & 0 \\ \text { Q. Muciuas } & 0\end{array}$

$\begin{array}{ll}\text { Gellus } & 0 \\ \text { Servius } & 0\end{array}$

Cascelliug

Alfenus

1 chez a-d D50.16.203.

Ofilius $\quad 0$

veteres 0

Lubéon

o

Hele

Sabiuus

1 chez Africatn D50.16.207.

chez Paul D50.16.215.

Proculus

Cassius

Fulcinius

Iavol enu:

Neratius

Celsua

Julien

Pedius:

Pomponius

Mercellue

Scaernia

Papiaten

P.Fronto

Ul pien

Modestin

1 chez Celsus \$60.16.98.1.

chez Pomponius D50.7.18.

3 chez Celsus W60.16.98.1; chez Pomponiue D50.7.18; chez Puul D50.16.25.1.

1 chez Peul 050.16 .77$.

5 chez Pomponius D50.16.122; chez Gaius D50.16.30 pr; D50.16.237; chez Peul D50.16.25.1; D50.16.77.

1 chez Celsus Do.16.158.

cher Celavo D50.10.158. D60.16.239.6; chez liarcellus D50.16.87; Chez Paul DSO.16.77.

2 chez Pomponius D50.16.180.1; chez Leiue 050.16 .234 .2 .

3 chez Gaius D60.16.23.4pr, chez Paul $160.16 .39 \mathrm{pr}$; D50.16.3 pr.

19 chez Iavnleaus D50.16.116,

D50.16.242 pr-1-2-3-4; chez Pomponius W60.16.245.1; D50.16.246pr; chez Paul U50.16.5.1; D50.18.14pr;

D50.16.29; D50.16.53.2; D50.16.244; Chez U1 pi en D60.18.19; D50.16.38; D50.16.43; D50.16.45; D50.16.60.1; D50.16.194.

chez Paul L50.16.14pr; D50.16.1443 D50.17.1, chez Ulpi en D50.16.13.1.

chez Paul D50.16.215. chez Paul D50.16.79.1.

2 chez Iavolenus D50.18.116; chez

1 Paul D50.10.4.

1 chez Gaius D50.16.236.1.

2 chez Celsus Do0.17.191, chez

Marcellus DSO.16.83.

3 chez U1pien D50.1.1.2; D50.1.27.2, D50.17. 23.

5 chez Marcellus D50.17.66; chez Faul D50.8.0; 050.16 .9 ; D50.16.25 pr; D50.16.77.

1 chez Ulpien $\$ 50.16 .16 .13 .1$.

1 chez U1pien D50.10.27.1.

chez Ulpien DE0.16.160. chez Ulpien D50.1ن.26.

1

1 chez Ulpien 050.2 .2 .2 .

1 chez Callistrute D50.16.220.1.

cnez Papin

1 chez A.Clzurisius D50.4.18.26. 


\section{Citations par renvoi}

à des constitutions impériales. 


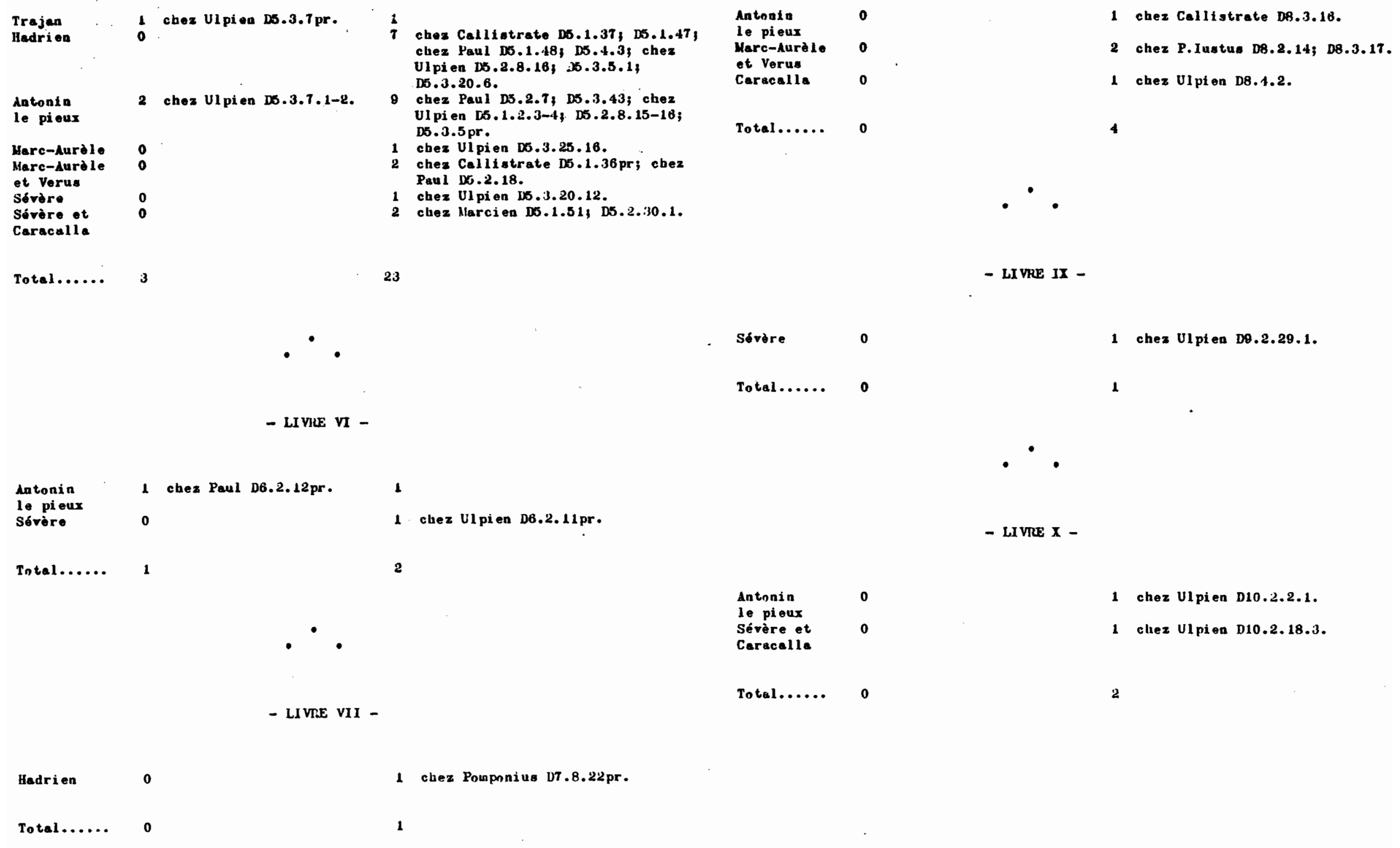


1 chez Macer D11.7.37.1.

chez Tryphoninus D11.4.5, chez Ul pien D1l.4.3.

chez Ul pi en D11.4.1.2.

1 chez Ulpien D11.7.14.14.

chez Ulpien D11.7.6.14

chez Marcien D11.7.39.

1 chez Ulpien D11.6.7.3.

1 chez Ulpien D11.7.12pr.

1 chez U1 pien D11.T.14.7.

10

- LI VIE XIV -

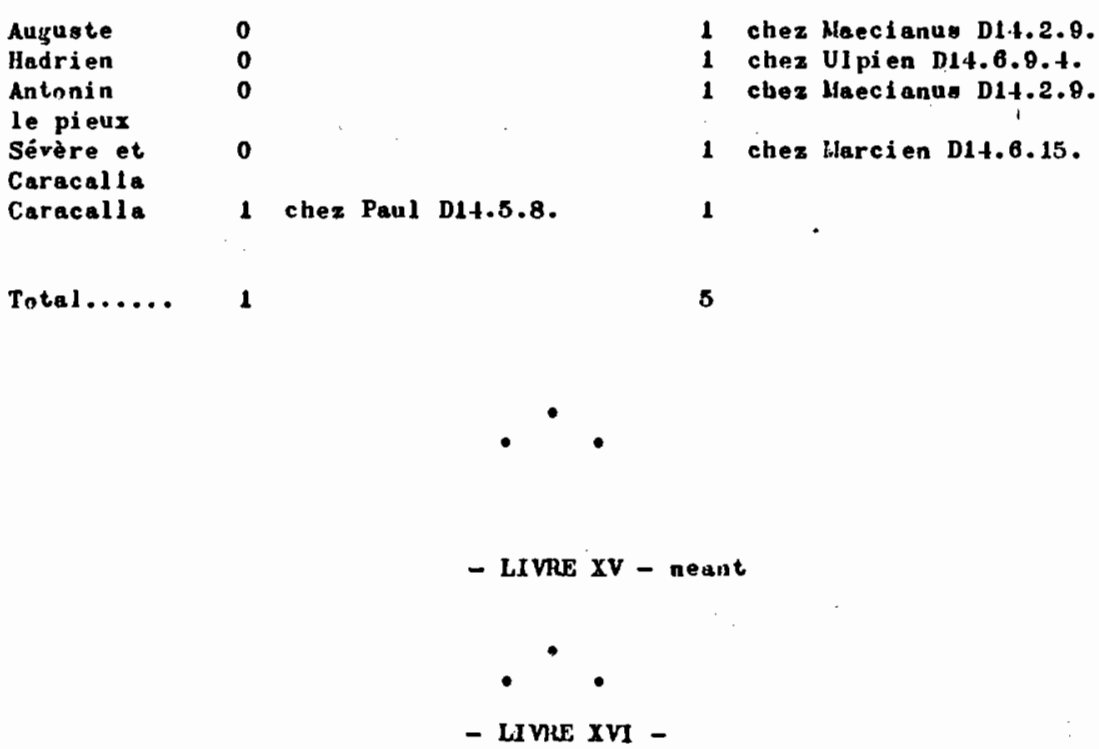

Auguate

Claude

Antonin

le pieux

Sévere

Caracalla
1 chez Ulpien D13.6.3pr.

1 chez Harcien D13.7.17.

1 chez UIpien D13.7.11.6.

\section{- LIVRE XII -}

- LIVIE XVI -

Total $\ldots$

1 chez Ulpien D1B.1.2pr.

1 chez Ulpien D16.1.2pr.

2 chez UI plen D16.1.2.3; $216.1 .4 \mathrm{pr}$.

2 chez U1 pien D16.1.2.3; D16.2.11.

chez Paul D16.2.2.t; chez Ul pien chez Paul Dit. 


\begin{tabular}{|c|c|c|c|c|c|}
\hline $\begin{array}{l}\text { Marc-Aurèle } \\
\text { Harc-durè le } \\
\text { et Verus } \\
\text { Sévere } \\
\text { Caracalla }\end{array}$ & $\begin{array}{l}1 \\
0\end{array}$ & cbez Ulpien D1T.2.23.1. & $\begin{array}{l}1 \\
2 \\
2 \\
1\end{array}$ & $\begin{array}{l}\text { chez Ul pi en D17.1.\$.7; D17.1.8.8. } \\
\text { chez Ulpi en D17.1.12.10; D1T.2.52.5. } \\
\text { chez Paul D17.2.25. }\end{array}$ & $\begin{array}{l}\text { Antonin } \\
\text { lo pieux } \\
\text { Sévère } \\
\text { Sévère et } \\
\text { Caracalla }\end{array}$ \\
\hline Total...... & 1 & & 6 & & Total.... \\
\hline
\end{tabular}

......
1 chex TryphoninusD18.7.10. 1

chez TryplioninusD18.7.10.

chez Narcien D18.1.42. 2 chez P.Iustus D18.1.71.

Marc-durele 1 chez karcien D18.1.42。

Harc-Aure

\section{Sévàro}

Séràre ot

Caracalla

Total $\ldots$.

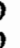

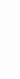

3

- LIVRE XIX chez Marcien D18.1.46.
1 cbez Ulpien D18.2.16.

chez Ul pien $018.3 .4 p r$

7

$\begin{array}{ll}\text { Hadrien } & 0 \\ \text { Antonin } & 0 \\ \text { le pi eux } & \\ \text { Harc-Aurdele } & 0\end{array}$

biarc-Aurele le 0

et Verus

Commonde

Sévère

Sévère et

Caracalla

Totel......
1 chez Harcien D20.3.1.2.

1 chez Tryphnninus nio.5.12pr.

1 chez Harcien D20.1.16.9.

- LIVRE jXII -

5 chez Callistrate D22.5.3.1-2-3-4-6. cluex Paul D22.1.17.1-2-3; D22.6.9.5; chez Marci en D22.1.32pr.

2 chez Papini en D22.1..3pr;

chez Paul D22.1.17 pr.

2 chez Scaerola D22.3.29pr; chez Callistrate D22.5.3.6.

chex Papinien D22.3.26.

1 chez Papinien D22.1.6.1.

1 chez Paul D22.6.9.5.

$\begin{array}{ll}\text { Antonin } & 0 \\ \text { le pieux } & \\ \text { Séràret } & 0 \\ \text { Caracalla } & \\ \text { Caracalla } & 0 \\ & \end{array}$

Total......
2 chez Clpien D19.2.15.5.

3 chez Ulpien $119 . \dot{a} .9 .1-t ;$; 19.2 .19 .9$.

1 ehez vilpien U19.2.15.0. 
Auguate Ti bère Trajan Hadri en Antonin le pi eux

Mare-Aurèle

Harc-Aurèle et Verus Sévère Séròre et Caracalla Caracalla

Total .....

1 chez Pomponius D28.5.42.

2 chez Ul pi en W28.3.6.6-7. chez Marci en $028.7 .18 \mathrm{pr}$.

4 chez Marcellug 028.4.3 pr-137 chez Marci eu D28.5.52pr

o hez Paul D28.5.85.1.

chez Marcien D28.5.49.2. 2 chez Modestin D28.6.4.2.

2 chez Ulpi en D28.3.12pr, D28.6.2.4.

26

\section{- LIVILE XXIX -}

Titu

Domitien

Nerra

Trajen

Hadrien

dncouin

le pieux

Mare-Aurdèle Marc-Aurè l

et Verus

Coumode

Sévère

Sévère et

Caracalla

Caracalla

chez Paul p28.2.26.

cbez U1 pi ea D28.5.1pr.

D28. 1.15 , D28.5.1.5-6, D28.6.10.6

chez Hodertin D:28.6.4.1.

chez Marcellus D28.1.3pr; cbez

U1 pi en D28.1.20.9; D28.3.3.2.

1 chez hodegtin D28.6.4pr.

. chez Ul pi en D29.1.1 pr.

cher Ulpien D29,1.1 pr.

o

3 chez Ulpien D2G.1.1pr.

2 chez Floreatin D29.1.24 clez Paul D29.5.10.1;

chez Tryphoni nus D29.5.11.

1 chez U1 pi on D29.5.1.28-29. 5 chez Yapinien D29.1.34pr; chez $029.4 .2 \mathrm{pr}$; $\mathrm{D} 29.6 .1 \mathrm{pr}$.

3 chez Ul pi en D29.2.25.3; D29.2.52pr. D29.1.30; chez Ul pien D29.1.9.1; D29.1.15.2; D29.2.6.3; D29.2.30 pr D29.1.10.1.

1 chez Ulpien 029.1 .3$.

1 chez Ul pien D29.1.28.

chez Calligtrate D29.5.2. 1

chez Callistrate D29.5.2.

2 chez llacer D29.2.61.

3 chez Harcien D29.7.6pr. chez Marci en D29.5.15.1.
1 chez UL pien D28.5.30.

chez Marcellus D28.4.3pr; chez Ul pien

- LI VRE XXXI -

Hadrien

Antnni

Le Pi eux

Marc-Aurèle

Jiare-Aurèle

et Coumnde

Tryphoniaus D 29.1 .11 .1 ; chez Ulpien D29.5.1.5; chez Harcien

10 chez Papinien D29.2.86pr; chez Paul
3 chez Gaius D30.73.1, chez Ulpien D30.34.3, D30.77.

1 chez Ul pí en Djo.41.7.

1 chez Murcien D30.112pr.

1 chez Ulpien Dao.41.5

11 chez Ulpien D30.41.J-5, D30.74 prs chez Marcien D30.111, D30.112.43 D3n.114.11.15.
Sévère

Caracalla

Al exindre

Sévère

Tntal chez Ul pi en $030.37 \mathrm{pr}$
1 chez Paul D3I.8.5.

o

0

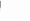

2 chez Mauricianus D31.57.

chez Mauricianus DJ1.57. chez Gaius D31.56.

I chez Papinien Da1.67.10.

1 chez Papinien D31.64.

3 chez Papinien DJ1.67.9; D3I.78.1, chez Ul pien D31.61.1.

1 chez Papinien D31.70pr.

1 chez Paul D31.87.3-4. 


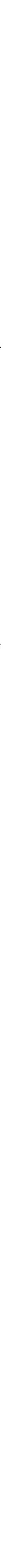


Claude Vesposi en Trajan

Badrien

Lntonin

le pieur

Marc-Aurèle

Marc-iurèle Sévàre et Caracalla

Total $\ldots .$. et Verua
1 chez Liarcien D37.14.5 pr. 1 1. chez Modestin D37.14.7pr. 0 .

2 cbez Trjplioniaus D37.14.23.1; cluez liodestin D.37.1.1.8pr.

2 chez Ulpien D37.10.1.5, chez Hermogeni en D37.5.23.

\section{0}

3 chez Marcien D37.14.3; D37.14.4, D37.15.4.
1 chez U1pien D37.14.17 pr.

chez Papinien 137.12.5. chez Paul D37.9.8; che 2 Ul pi eo D57.9.1.14; D37.10.3.5.

10 chez Marcellua D37.8.3; chez Tryptioninu, D37.5.7; D37.8.7, chez Tryphonin U37.7.1 pr; D37.10.3.1; D37.12.1.4

2 chez Tryplioninua E37.7.9;

chez Undestin 037.8 .4

3 chez U1pien D37.6.1.14; D37.6.5pr.

3

26

- LIVRE XXXVIII -

Hadrien

Antonin

le pi eux

liarc-kuròle

Larc-iurèle

et Verus

Sévère ot

Caracalla

Caracall

Total $\ldots \ldots$
3 chez Ulpien D38.1.7.4; D38.2.3.8; chez Harcien L38.2.22.

5 cher Pepini en D38.2.42.3; chez Ul pi en D3B.1.13 pr-1; D38.2.6.13 D38.16.3.3.

2 chez Ulpien D38.2.16.4, D38.16.1.1.

0

2 chex Ulpien D38.16.1.1; D38.17.2.2.
1 chez Ulpien D38.16.3.12.
3 .

4 chez Paul D38.5.13; chez Ulpien 38.16.2.7; D38.17.2.9.

2 chez Ulpien Dị8.17.1.3, D3B.1T.2.47.
Hadrien

Antonin

le pieux

Marc-Aurèle

et Verus

Marc-Aurèle

et Comminde

Sévère et

Caracalla

Caracalla

Total .....
, cher

4-9.

chez Harcien D39.4.16. 10

o

$1-2$

l chez Harcien D39.4.16.11.
2 chez Paul D30.4.4.1, chez Harcten D39.4.16.5.

3 chez Ulpien D39.5.12.

1 chez P.Iustus D39.4.7pr-1; chez Mercien D39.4.16.8.

1 chez Marcien D30.4.16.6.

7 chez Marcien D39.4.16.12-14; D39.5.15, chez lndestin 039.4.6.

Marc-Aurèle et Verus

Murc-Aurè le et Commonde Counande Pertinax Sévère Sévàre et Caracalla Caracalla

Auguste

Nerva

Hadrien

Antonin

Marc-Aurè le.
$\boldsymbol{T}$

18.
- LIVRE XI -

1 chez Mndestin D40.2.21.

2 chez Callistrute D0.15.4.13 chez Undeatin D40.8.2.

1 chez Callistrate D.40.15.4pr.

chez Pomponiue D40.12.43; chez Venuleius D40.14.2pr, chez Papinien D40.4.47pr; chez Paul D.40.7.20.4, D.10.12.83.2; chez Ul pi en D40.5.24.21, D40.10.6, D40.12.27.1; chez Marcien D40.1.8.3, D40.15.1.2.

13 chez Pompnnius Di0.7.21.1, chez laecianus D40.5.42, cliez U1 pi en D40.5.24.6-21, D\&0.5.26.2-1, D40.5.30.5-6-7, chez Harcien D40.1.8.2, D40.2.0.1, D40.4.26; D40.5.51.9-11.

16 chez Papinien Dio.1.20 pr; D40.4.50 pr, D40.8.8, chez Pnal 16 D40.1.10, D40.4.56, D10.8.1; D 10.9.17 pr; DHO.12.38pr-1, chez Ui pien D40.2.20.1, D.10.3.1, D40.5.2, D40.5.3\} D40.5.4 pr à 23, D40.5.30.16; D40.5.37, D10.9.30 pr ; D10.16.2pr, chez Marcien Di0.8.6.

6 chez Paul D10.5,31.1, chez Ul pien D40.1.4pr ì 14, D40.5.30.3-13; D40.12.27 pr; chez Harcien D40.1.5pr; D.10.4.26, D.10.9.11.1.

1 chez Callistrate 040.8 .3

1 chez Marcien D40.10.3.

1 chez Undestin D.10.5.12.2.

2 chez Ul pien D40.5.26.1, D40.5.46.2.

8 chez Paul D 10.4.52, D10.8.7, chez U1 pien D.10.5.24.9, D40.5.20.2-3-t-B, D40.5.30.17, chex Mndestin D40.5.12.2

9 chez Papinien D40.T.J4.1, chez Paul D40.5.31.4,D40.5.38, D40.9.15 pr, chez Ul pien D40.5.2.1.5; D40.5.30 pr-15, D40.12.34; chez Undestin D10.5.12pr. 


Trajen
Intouin
10 pieux
Total.......

Total.......

- LIVHE XLII -

Hadri en $\quad 0$

Antonin 1. pieux

Marc-Aurd 1. 0

Liarc-Aurèl l

ot Vorue

Sovere et

Caracalla

Caracalla

Total......
1 ches Paul DH1.t.2.8.

chez Florentin D41.1.16. chez Harcien DH.3.8

- LIVRE XLV - néent -

- LIVRE XLVI -

2 chez Callietrate D42.1.3:3; chez Ulpien $D+2.4 .7 .16$.

6 chez Callistrate D.ti.1.j1; chez Ul pi an D12.1.15 pr: D 12.4.7.19; Det2. 1 . is chez Hodegtin 0.12 .1 .20

cheo U1 ui on D.t2.5.21.13 D42.8.10.17

chez P.Iugtus D-12.1.35; D.2.5.30 D+2.7.4.

+ cliez Ulpien D42.1.15.1-3, D.2.6.1.3 to.ti2.8.10.1.

1 chez Ulpien D.2.1.15.4.
Hadrien 0

Antonin 0

le pieux

Sévère t 0

Ceracalle

Total......
1 chez Gaius D46.1.263 chez Ulpien D.16.1.27.1.

1 chez Ulpt on D46.3.3.2.
1 chez Parinien D46.1.tU.1.

\section{- LIVRE XIVII -}

Nerva

Trajan

Hedrien

chez Ulpien D43.4.3.3.

2 chez Ulpien D43.30.1.3, D43:30.3.3.

chez Ulpien D43.30.1.3.

chez Ulpien 0.43 .30 .1 .3$.

1 chez Ulpien Dij.4.i.i.

Antonia

\section{chez Collistrate} Dit.21.3.1.

1 chez Ulpien D47.14.1.3.

1. pi eux

lierc-Aurè le

et verua

Sévàre

Sévère et

Caracalla

Caracalla

Total
1 chez Paul D47.9.t.1.

chez VI pien $D 47.4 .1 .7$.

1 chez Ulpien D+1.12.3.7.

1 chez Ulpien DH7.4.1.T.
1

2 chez Callistrate D.47.1.4.3.3; chez Ulpien D.17.11.6.1.

6 chez Callistrate D47.9.7; D-47.21.2; chez Ulpien D.17.11.6.2; D17.12.3.5; D.17.14.1 pr.

clese Ulpien D17.12.3.1, D47.18.1.80 cher Ulpien $0.17 .11 .8 ;$ D+7.18.1 pr.

4 chez Ulpien D47.12.3.43 chez Hacer 047.10.40; chez Marcien D47.22.1pr.

t clsez Ulpien D47.9.12pr; chez Marcien D.17.11.4; v.17.19.3.

1 chez Uluien $0+7.12 .3 .3$. 
I chez Marcien D48.10.7.

5 chez Ulpien B18.18.1.1617) D48. 10.8.5; chez Marcien D48.18.0pr, chez Verméni en D48.4.H.

0

chez Callistrute D.8.10.15 pr; chez Panl D+8.10.14.2.

2. cluez Papinien D48.3.2.1 chez Paul D48.16.16.

4 chez Ulpien Di8.8.1.1112-19-2I. D48.15.6pr-1; D48.19.28-6D48.18.12; chez Hndestin D+8.9.9 pr.

14 chez Callitrate D48 . 10 . 15.3; D48. 18 . 15 .I2) chez Uipien 1048.2.7.4; D.8.13.8.2; D.8.18.1.5-13; D48.19.9.16; chez Murcten D48. .34 pr; D18.18.9 pr ; D.8.18.16.1; D 18.19.22; chez A. Charisius Di8.18. $10 \mathrm{pr}$. chez Ul pi en D.18.2.5. 7 chez Papinion D48.19.33; 1-6-14-27; chez Hndestin Di8.18.16 pr.
13 chez Papinien D48. I8. 17.2, chez Callistrate 14 ; chez Ulpien D 48.5 .28 .6 ;

2 chez Papinien D48.18.17pr; 3 chez Callistrate B48.7.7.

24 chez P.Iustus $1+8 \$ 2.3 \mathrm{pr}-1$; D48.16.18pr-1-2; chez Papinien D+8.5.30.4-5-6; chez Callistrate D48.2.19pr; D-8.10.3I, D48.19.26 D48.19.27 pr; D18.20.1.3; chez Ul pien D-18.19.8.1; D48.22.7.10-18; chez Marcien D48.10.11.

3 chez Papinien D48.5.39.8; chez

Hacer D48.5.33pr.
chez U1 pi en D48.5.2.6, D48.5.14.8, D48.22.6.1; chez Harcien D48.10.1.4.

D48.20.2; chez Prul D48.8.5

chez Ulpien D48.8.4.1; D48.16.7 pr;
4 chez Ulpien D48.5.1; D48.18.1 pr; D48.24.1.

chez Papinien D48.2.2.1.

Chez Pnopnnius D48.22.1; chez Papinien D48.16.10.2, chez Ul pien chez Hopestin 048.17 .5 .2 .

chez Venuleius D48.2.12.1; D48.19.15; DA8.2.19.1; D48.3.12pr;

D48.10.21; D48.18.21; D.8.20.7.3; D48.3.6pr; D48.8.1.3-1; D48.13.5.4; D48.21.3.5. Calistrate D48.10.31; D.48.10.28.7, D48.2.7.2-3-5; Di8.3.3; Di8.6.6 Chez Marcien D-48.3.6.1, D18.6.5.1, D48.8.1.5; D48.18.9.2; D48.21.3.1-28; chez Indestin D.8.8.11 pr.
Sévere et Caracalla

7 chez Ulpien D48.1.5.1; D.8.18.1 $10-15-18$, D48.18.3; chez Marcien D.48.15.3 pr-1.

Caracalla

Al exendre Sévè re chez Paul M48.18.20
24

eliez Ulpien D48.5.14.3, D.18.18.1.10, chez Mncer D 18.16.15.4; D48.21.2pr; chez Marcien D.18.2.13; D D.4.5.1-2; D48.10.1.9-11, M8.13.B; D48.13.12.1, D48.17.1 pr; D.8.17.3; Di8.24.2; chez Mndestin D48.2.20; D48.17.5.1, D.8.19.31.

3 chez Papinien D.8.18.17 pr; 8 chez Ul pi en D48.19.8.12. hez Paul D48. 19.43pr-1; chez Ul pien D48.1.5.1; chez Marcien D\$8.21.3.1; D48.22.16. 
cirez Callisurate D49.14.2.0.

2 chez Tryphoninu V19.15.12.17; cuez ilarci en D.19.14.:30.

0

1 chez Faul D49,1.25. chez Tryphnninus

chez Callistrate W49.14.12; chez Ulpien D+9.1.5.1.
1 chez biacer D49.16.12.1.

Vespasien

o

chez Callistrate D49.14.1.3.

chez A.Valeng D 19.14.12pr-1; chez Mauricianus Dt9.14.15.3) cirez

Henander $149.16 .+\mathrm{pr}-5-12-13$; chez Paul D+9.14.13pr-1-6; D49.14.49; chez Ul pien D+9.14.16.

3 ciex Papinien 049.17.13; 15 chez Llauricianus D49.1.4.15.2; chez Callistrute b.19.14.3.9. D49.1.

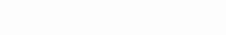

1 cuez il pi en biy.1.1+.1. Papinien D49.17.16pr; cher Menander D.49.16.5.8; D-19.16.6.7; chez Callistrate D49.14.2.1-4; D.19.14.i3.1 6; chez Paul 149.5.7pr; D.19.1t.13.45-10.

12 chez Callistrate D.19.14.1.2;

D49.14.2.5; D49.14.3.4; chez Paul D+9.14.13.10; D.19.14.49; chez Ul pien D+9.1.1.1; chez macer D.19.18.13.6;. chez liarcien $D 40.1 .5 .2$; D 49.1 .2 .22 .2 .

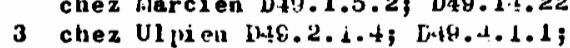
D49.1.1.7.

12 cuez f.Iustus $149.1 .21 \mathrm{pr}-1$; cluez

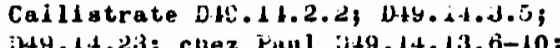
Cuez Ul pi en D49.1.8; D.18.9.1; D.19.11.1; chez harcien j49.1.4.12.

1 chez liarcien $14+1.1$ t.31.

1 chez liurcien b49.14.31. chez liarcien D 49.1 .7 .

13 cliez Paul Dig.18.5pr; cliez Ulpiel D.19.15.9; chez Mlacer D.49.33.1.1; D.19.14.34; $240.30 ̈ .13 .0$; chez inarcien D 18.1t.22pr-1-33; D-19.15.25; 2-69.16.9 chez londestin D.6.10.3pr.

3 cirez Ul pien D+9.4.43; D+4.5.5.3; cllez Niacer D ty.1.4. 2 .

2 cuez Hacer $D+Y .1 J .1 \mathrm{pr}$.

Titus

Trajan

Hadrien

Intonin

le pieux

1e pi eux

Marc-Aurèle 0

et Verus

Pertinax

Súvère

o
(1)

Jlarc-Aurèle 0

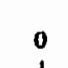

1 chez Paul D50.2.8pr.

Sévère ot

Caracalla

71
3 chez Paul D50.15.8.7; chez Marcien D50.T.5.6; chez A.Charisius D50.4.18.30

1 cuez Paul D50.15.8.7.

1 chez Paul D50.15.8.7.

1 chez Pomponius D50.12.14. cliez Callistrate D50.1.37 pr;
D50.4.1.4.6; D50.6.6.5-8; D50.9.5; chez Marcien D50.7.5.5, chez A.Charisius D50.t.18.30.

15 chez Pomponius D60.12.14; chez Papinien D50.1.11pr; D50.1.17.9; D50.15.5.1; chez Callistrate D50.6.6.1-9-12; D50.10.7pr; chez Pau D50.2.11; D60.13.4; 150.15.8.5; chez Ul pi en D50.10.5 pr; $D 50.17 .28$; chez llarcien D50.7.5pr; chez Mlndestin $1050.4 .11 \mathrm{pr}$.

3 chez Callistrate D50.16.220.2; chez llodestin D50.4.11; D50.10.6.

35 chez P.lustus $150.1 .38 \mathrm{pr}-1-2-3-4-5-6$ D50.2.13 pr-1-2-3; D60.8.11 pr-1-2, $560.8 .12 \mathrm{pr}-1-2-3-4-6-6$; D50.8. 133 D50.12.13pr-1, Chez Scaevnla D50.1.2 chez Callistrate D50.1.37.2,

chez Caillatrate Don D50.4.Bpr-1; D60.12.8; D50.13.1.9; chez llarcien 050.1 .8 ; chez Mndestin D50.1.11.2.

2 chez Callistrate $150.6 \cdot 6.2-13$.

13 chez Papinien D60.5.73 D50.5.8pr; chez Callistrate D50.4.14.4; chez Paul D50.1.18; D50.12.7; chez Ul pl on D50.13.1.13, D50.15.1.2-3-7-9, chez lfarcien 050.4 .7 .1 ; chez lodestia $1250.12 .12 \mathrm{pr}$.

14 chez Paul D50.1.21.6; D50.15.8.113 DEN.1:. In:; chez Ulpien D50.2.3.3; D50.4.6.2; D50.7.7; D50.12.1.5; D50.12.6.2-3; D50.13.1.10-12; chez liarcien 1500.7 .5 .1 ; chez Hodestin $150.4 .11 .3 ; 250.12 .9$.

9 chez Paul L50.7.12.1; D50.15.8.6; crez Lipi en 050.2 .3 .1 ; D50.12.1.0; i50.12.6.i; $250.25 .1 .+$; $50.150 .3 .1 ;$ chez hiodestin D50.t.11.4; DEC.12.12 


\section{BIBLIOGRAPIIIE SIMPLIFIEE (1)}

\section{Ouvrages et articles}

ANDERSON P., Les passages de 1'Antiquité an féodalisme, Paris, 1977 , 325 p.

ARARGIO-RUIZ V., Storia del diritto romano (7ème éd.), Naples, 1977, $\mathrm{XDX}+458 \mathrm{p}$.

ARAKG IO-FUIZ V., Istituzioni di diritto romano (14ème éd.), Naples 1976, XV + $605 \mathrm{p}$.

BARROW R.H., Slavery in the Roman Empire, Londres, 1928, XVI + 259 p.

BAPTOSEK M., Le classi sociali nella Roma antica, Etudes J. Macqueron, Aix-en-Provence, 1970, p. 43-88.

BETLEN H., Studien zur Sklavenflucht im römischen Kaiserreich, Forschungen zur antiken Sklaverei, 4, Wiesbaden, 1971, XII + 179 p.

BERGER A., Encyclopedic Dictionary of Roman Law, Philadelphie, 1953, The American Philosophical Society, p. 333-808.

BISCARDI A., La capacitì processuale dello schiaro, Labeo 21, 1975, p. 143-171.

BIEZUNSKA-MALOWIST I., Les enfants esclaves à la lumière des papyrus, in Hommages à M. Renard 2, Coll. Latomus 102, Bruxelles, 1969, p.91-96.

1. Ne sont repris ici que les titres les plus souvent cités. En ce qui concerne les articles et ouvrages apparaissant moins fréquenment, se reporter aux notes les mentionnant. Pour une bibliographie plus se reporter aux notes les mentionnant. Pour une bibliographie plus
étoffée sur 1 'esclavage, voir celle dressée par G. BOULVER dans un article, dont la conception nous est commune, a paraître in ANRW article, dont la conception nous est commune, a paraitre in
sous le titre "Le droit de l'esclavage sous le taut-Empire".
BOULVERT G., Esclaves et affranchis impériaux sous le Haut-Empire romain, rôle politique et administratif, Naples, $1970,499 \mathrm{p}$.

BOULVERT G., Domestique et fonctionnaire sous le Haut-Empire romain, Annales littéraires de l'Université de Besançon, vol. 151, Paris, 1974,379 p.

BRETONE M. , Statuliber, NNDI, XVIII, 1971, p. 380-383.

BRETONE M., Tecniche e ideologie dei giuristi romani (2ème éd.), Naples, 1975, $270 \mathrm{p}$

BROGGINI G., Obligatio naturalis, SDHI 31, 1965, p. 362-384 (= Studi di dir. ran., Milan, 1966, p. 487-523).

BUCKIAND W.W., The Roman Lay of Slavery. The condition of the Slave frow Augustus to Justinian, Cambridge 1908 (réimpr. 1970), XIV + 735 p.

BURDESE A., La nozione classica di naturalis obligatio, Turin, 1955, 163 p.

BURDESE A., Dubbi in tema di naturalis obligatio, in Studi Scherillo, Milan, 1972, p. 485-513.

BUTI I., Studi sulla capacità patrimoniale dei servî, Pubbl. Fac. Giur. Camerino, 13, Naples, 1976, 299 p.

CARANDINI A., L'anatomia della scimia. La formazione economica della società prima del capitale, Turin, 1979, XXI + 348 p.

CARCOPINO J., La vie quotidienne à Rome à l'apogée de 1'Empire, Paris, 1939 (réimpr. 1978), 351 p.

CARDASCIA G., L'apparition dans le droit des classes d'honestiores et d'homiliores, RHD 28, 1950, p. 305-337 et 461-485.

CIULEI G., Liber homo bona fide serviens, Paris, 1941, 98 p.

CLAVEL-LEVEQUE M. , Impérialisme, développement et transition : pluralité des voies et universalisme dans le modèle impórial romain, la Pensée no 196, déc. 1977, p. 10-27. 
CORNIOLEY P., Naturalis Obligatio. Essai sur l'origine et l'évolution de la notion en droit ramain, Genève, 1964, vIII + 333 p.

cosmrrmI c., Studi sui liberti. Contributo allo studio della condizione giuridica dei liberti cittadini, Univ. Catania,Pubbl. Fac. Giur. 11 , Catagne, 1948, X +272 p. : 14, Catagne, 1950, 197 p.

CROOK J., Consilium principis, Cembridge, 1955, XI + 197 p.

DIDIBR Ph., Evolution des notions de liens naturels dans la jurisprudence romaine classique (thèse dactyl.), Paris, 1967, 329 p.

DIDIER Ph., Les obligations naturelles chez les derniers Sabiniens, RIDA 19, 1972, p. 239-273.

DOCKES P., La libération médiévale, Paris, 1979, 321 p.

DE DOMInICIS M., Les Latins Juniens dans la pensée du législateur ramain, RIDA 20, 1973, p. 311-324.

DONATUTI G., Lo statulibero, Milan, 1940, 338 p.

DUFF A.M., Freedmen in the early Roman Empire, Oxford, 1928 (rééd. Londres 1958), XII + $252 \mathrm{p}$.

ERMAN H., Servus vicarius. L'esclave de l'esclave ramain. Recueil publié par la Faculté de Droit de l'Université de Lausanne à l'occssion de l'exposition nationale suisse, Genève et Lausanne, 1896, vII + 41-532.

ERNOUT A. et MEILLET A., Dictionnaire étymologique de la..langue-latine, Paris, 1959 (Lème éd.) (2ème tirage 1967), XVIII + 827 p.

FAVORY F., Présentation de l'Index thématique de Besançon consacré à l'esclavage et aux formes de dépendance, in Schiavitì, manomissione e classi dipendenti nel mondo antico, L'Erma di Bretschneider, Rome, 1979, p. $143-159$.
PInLI M.I., L'Econonie antique, tr rr. de M.P. HIGGS, Paris, 1975, 242 p.

DE FRANCISCI P., La revocatio in servitutem del liberto ingrato, in Melanges Cornil 1, Gand, Paris, 1926, p. 297-323.

GAUDENBT J., Institutions de l'Antiquite, Paris, 1967, XDX + 909 \$.

GIRARD P.F., Manuel Elementaire de, droit romain, Paris, 1924 (7ème éd.), $X I N+1158 \mathrm{p}$.

GUARIro A., Storia del diritto romano (5ème ê.), Naples, 1975, XDX + 766 p.

GuARINo A. , Diritto privato romano (5ème éd.), Naples, 1976, XV + 933 p.

HALKCN L., Les esclaves publics chez les Romains. Biblio. de la Faculte de Philosophie et des Lettres de l'Université de Liège, I, 1897,251 p.

HONCRE T., "Imperial" rescripts A.D. 193-305: authorship and suthenticity, JRS, 1979, p. 51-64.

HORVAT M. , Note intorno allo ius respondendi, in Synteleia Arangio-Ruiz 2, Naples, 1964, p. 710-716.

HORVAT M., Legatum: fundi e servi, in Studi Volterra 5, Rome, 1971, p. $89-97$.

DMBERT J., Eavor libertatis, RID 27, 1949, p. 274-280.

D'IPPOLITO F., I giuristi e la città, Naples, 1978, 136 p.

JACOTA M., Les pactes de l'esclave en son nom propre, RIDA 13, 1966, p. $205-230$.

JACOTA M., L'esclave créancier, in Studi Grosso 2, Turin, 1968, p. 213-238. 
JACOTA M.,Les trensformations de l'éconamie romaine durant les premiers siècles de notre ère et la condition de l'esclave agriculteur, Etudes Macqueron, Aix-en-Provence, 1970, p. 375-383.

JAUBERT M., Le Lex Aelia Sentia et la locatio conductio des operae liberti, RHD 43, 1965, p. 5-21.

KASER M., Das römische Privatrecht (cite sous la forme RPR), $I_{2}$ Handbuch der Altertumswissenschaft 10,3,3, Munich, 1971, $100 \mathrm{X}+833$ p. ; $\mathrm{II}_{2}$, Munich, 1975, XXX $+680 \mathrm{p}$.

KOLENDO J., Il lavoro servile e $i$ mutamenti delle tecniche agrarie nell' Italia sntica dal I secolo a.c: al I secolo d.c., in Storia sociale ed econconica dell' età classica negli studi polacchi contemporanei, Milan, 1975, p. 9-53.

KOLFADO J., Le formation du solonat en Afrique, Recherches Internationales 3,1975 , p. 129-157.

KOVALEV S., Le tournant social du IIIe au Ve siècle dans I'Empire romain d'Occident, Recherches Internationales 2, 1957, p. 159-178 (tr. fr. de J. RAARE).

KUNKKLL W. , Herkunft und soziele Stellung der römischen Juristen (2ème éd.), Graz, vienne, Cologne, 1967, XI + 415 p.

LAMBERT J.R., Les operae liberti, Contribution I'histoire des droits du patronat, Paris, 1934, 351 p.

LAMBRECHIS P., La composition du génat romain de l'accession au trône d'Hadrien à la mort de Comode (117 - 192), Anvers, 1936, 234 p.

LAKFRANCHI F., Ius exponendi e obbligo elimentare nel diritto romano classico, SDHI 6 , 1940, p. 5-69.

LANTELCA L., Il lavoro sistematico nel discorso giuridico romano, Turin, 1975, 289 p.
LAPICKI B., La transformation de la nature juridique du colonat ramain, in Studi volterra 3, Rome, 1971, p. 359-373.

IAVAGGI G., Muovi studi sui liberti, in Studi De Francisci 2 , Milan, 1956, p. 75-111.

LECRIVATN Ch., Vicarius, DSDA, v, p. 823-825.

LEVEQUE P., Esclavage et exploitation du troveil dans 1 'Egypte hellénistique et romaine, Revue des Etudes Grecques, XCII, 1979, p. 231-238.

LOMBARDI L., Saggio sul diritto giurisprudenziele, Milan, 1967, XXXVI + 619 p.

LONoO G., Il concetto classico e il concetto giustinianeo di administratio peculii, AG 100, 1928, p. 184-203 (= Ricerche romanistiche, Milan, 1966, p. 367-385).

LONGO G., Libera administratio peculii, I limiti e lo spirito di una innovazione giustinianea, BIDR 38, 1930, p. 29-52 ( = Ricerche ramanistiche, Milan, 1966, p. 387-404).

LoNco G., Actio exercitoria, actio institoria, actio quasi institoria, in Studi Scherillo 2, Milan, 1972, p. 581-626.

LOlGo G. .. , Concetto e limiti deli'obligazione naturale dello schiavo, SDHI 16, 1950, p. 86-122.

LOnco G.E., Ricerche sull' obligatio naturalis, Rome, 1962, 302 p.

MACQUERON J., Droit romain (cours polycopie)). Les personnes, Aix-enProvence, 1954, VI + 309-565.

MACQUEROI J., Le travail des homes libres dang l'Antiquite romaine (2ème é.), Aix-en-Provence, 1958, 238 p.

MACQUBRON J., Histoire des obligations. Le droit romain (2ème éa.), Aix-en-Provence, $1975,498 \mathrm{p}$

MARQUARDT J., La.vie privée des Romains, I (tr. fr. de V. hLARY), Paris, 1892, XIV + 455 p. 
DE MARTIEO F., Storia della costituzione romane (2ème éd.) ; II, Naples, 1973, 546 p. ; III, Naples, 1973, 405 p. ; IV, vol. 1, Naples, 1974, 685 p. ; v, Maples, 1975,607 p.

DE MARTINO F., Storia economica di Rome antica, II, Florence, 1980 , p. $217-582$.

MAZIA M., Lotte sociali e restaurazione autoritaria nel III secolo d.c. (2ime Ed.), Rome, 1973, VIII + 714 p.

MEIILN G., Economia e giurisprudenza a Roma, Naples, 1978, 124 p.

MESLIS M. , L'home romain des origines au ler sięcle de notre ère, Paris, 1978, 296 p.

METRO A., La lex Aelia Sentia e le manomissioni fraudolente, Labeo 7 , 1961 , p. $147-200$.

MICOLIRR G., Pécule et capacité patrimoniale, Lyon, 1932, 874 p.

MIIAKI P.A., La schiavitu nel pensiero politico dai Greci al basso Medio Evo, Milan, 1972, 402 p.

MOASEM Th., Le droit public romain (tr. fr. en 7 vol. de P.F. GIRARD), Paris, 1889-1896, I, XXXVI +436 p.

Manswa Th, te droit pénal ramain (tr. er. en 3 vol. de J. DUQUESNE), Paris, 1907 ; I, XVI + 401 p. ; II, 443 p. ; III, 420 p.

MONIRR R., Manuel élémentaire de droit romain (6ène éd.), Paris, 1947 ; I, 551 p. ; II, 345 p.

NEHISEN H., Sklavenrecht zwischen Antike und Mittelalter, germanisches und römisches Recht in der germanischen Rechts aufzeichnung, I, Ostgoten, Westgoten, Franken, Langobarden; Göttinger Studien zur Rechtsgeschichte 7, Göttingen, 1972, 432 p.

NICOLAU M. , Causa liberalis. Etude historique et comparative du procès de liberté dans les législations anciennes, Paris, 1934, 344 p.
FESCANI P., Operae Libertorum. Saggio storico romanistico, Trieste, 1967, $159 \mathrm{p}$.

PETIT P., Le paix romaine (2ème éd.), Paris, 1971, 412 p.

PETIT P., Histoire générale de l'Mnpire Romain, Paris, 1974, 799 p. .

PELAUM H.G., Les carrières procuratoriennes equestres sous le HautFnpire romain, Paris, 1960-1961, 3 rol., XII + 1469 p.

FUGLIESE G., In tema di actio exercitoria, Labeo 3, 1957, p. 308-343. REGGI R., Liber homo bona fide serviens, Milan, 1958, vIII + 481 p.

DE ROBERTIS F.M., Lavoro e lavoratori nel mondo romano, Bari, 1963, $X I+436$ p.

SAUTEL G. et M., Note sur l'action quod iussu et ses destinées postclassiques, in Mélanges Lévy-Bruhl, Paris, 1959, p. 257-268.

SCARANO USSANI V., Valori e storia nella cultura giưidica fra Nerva e Adriano, Studi su Nerazio e Celso, Naples, 1979, 229 p.

SCHLAVONE A., Nascita della giurisprudenza (2ème હd.), Bari, 1977 , VII +161 p.

SCHTAERMAN E.M, La chute du régime esclavagiste, in Recherches internationales 2, 1957, p. 113-158, (tr. fr. de J. MACORIGH).

EADEM et TROF IMOVA M.K., La schiavitu nell' Italia imperiale (tr. it.), Rame, 1975, 347 p.

TREGGIARI S. , Roman freedmen during the Late Republic, Oxford, 1969, XII + 293 P.

VAZNY J., Naturalis obligatio, in Studi Bonfente 4, Milan 1930 , p. $130-180$.

VISKY K., Esclavage et artes liberales ì Rome, RJDA 15, 1968, p. $473-485$. 
VISKY $K$. , Le operae dei liberti, cr de P. PESCANI (cité supra), Index 1, 1970, p. 221-230.

VOLTERRA E., Senatus consuZta, NNDI, XVI, 1969, p. 1047-1078.

WALLON H., Histoire de l'esclavage dans l'Antiquité, I et II, Paris, $1847,492+523$ p. ; III $_{2}$, Paris, 1879, 567 p.

WESTERMANN W. L., Sklaverei, RE Supplt VI, 1935, p. 894-1068.

WESTEPAANN W.L., The Slave Systems of Greek and Roman Antiquity, Memoirs of the American Philosophical Society 40, Philadelphie, 1955, $X I I+180$ p.

\section{INDEX DES ABREVIATIONS}

AESC

AG

AHDE

AHR

AIV

ANA

ANRW

АTO

AUBA

AUCA

AUCT

AUMA

AUPA

BIDR

BGU

CIL

CRAI

DS (DA)

ED

EP (3)
= Annales Economie, Société, Civilisation, Faris.

= Archivio Giuridico, Modène.

= Anuario de Historia del derecho espanol, Madrid.

= American Historical Review, Washington.

= Atti dell' Istituto Veneto di Scienze, Lettere ed Arti.

$=$ Atti dell' Accademia di Scienze morali e politiche di Napoli.

$=$ Aufstieg und Niedergang der römischen Welt, Berlin, New-York.

= Atti dell' Accademia delle Scienze di Torino.

= Annali della Facoltà di Giurisprudenza dell'Università di Bari.

= Annali dell' Università di Camerino.

= Annali del Seminario Giuridico dell' Università di Catania.

= Annali dell' Università di Macerata.

= Annali del Seminario Giuridico dell' Università di Palermo.

= Bollettino dell' Istituto di diritto romano, Milan.

= Agyptische Urkunden aus den Staatlichen Museen zu Berlin, Griech.Urkunden, Berlin.

= Corpus Inscriptionum Latinarum, Berlin.

= Comptes-rendus de l'Académie des Inscriptions et BellesLettres, Paris.

= Dizionario epigrafico di" antichità romane, E. DE RUGGIERO, Rome.

C.v. DAREMBERG et E. SAGLIo, Dictionnaire des Antiquités grecques et romaines, Paris.

= Enciclopedia del diritto, Milan.

= O. LENEL, Das Edictum perpetzum, Leipzig, 1927 (réimpr. 1956). 
Historia , Zeitschrift für alte Geschichte, Wiesbaden.

Index , Quaderni camerti di studi romanistici, International 'survey of roman law, Naples, Camerino.

Index itp. = Index interpolationem quae in Iustiniani Digestis inesse dicuntur, E. LEVY et E. RABEL. A également été utile, dans le même ordre d'idées, le Vocabularium iumisprudentiae Romanae de GRADENWTTZ, KUBLER, puis SCHWARZ et autres.

Iura

, Rivista internazionale di diritto ramano e antico, Naples, Catagne.

JRS = Journal of Roman Studies, Londres.

Klio , Beiträge zur alten Geschichte, Leipzig.

Labeo , Rassegna di diritto romano, Naples.

Latọus , Revue d'Etudes Latines, Bruxelles.

NNDI = Novissimo Digesto Italiano, Turin.

PAL $\quad=0$. LENEL, Palingenesia Iuris Civilis, I, II, Leipzig, 1889.

PIR = Prosopographia Imperii Romani I, II, III, 1ère éd. en 3 vol. par H. DESSAU, E. KLEBS et P. VON ROHDEN ; 2ème éd. par E. GROAG et A. STEIN, Berlin.

RAL

= Rendiconti dell' Accadenia dei Lincei, Rome.

RBPH

= Revue belge de Fhilologie et d'Histoire, Bruxelles, Paris.

= PAULY-WISSOWA, Real Encyclopädie der classischen Altertumswissenschaft, Stuttgart.

= Revue des Etudes Anciennes, Bordeaux.

REL $\quad=$ Revue des Etudes Latines, Paris.

RHD = Revue historique de aroit français et étranger, Paris.

RIDA = Revue internationale des droits de l'Antiquité, Bruxelles.

RIL = Rendiconti dell' Istituto Lombardo di Scienze e Lettere, Classe Lettere.

RISC

= Rivista italiana par la Scienze giuridiche, Rame.

SHIA

= Scriptores Historiae Augustae.
SIFC

SDHI

= Studi italiani di filologia classica, Florence.

Studii Clasice, Bucarest.

SUCC = Studi economici-giuridici dell' Università di Cagliari.

T (ou Tija.) = Tijdschrift voor Rechtsgeschiedenis. Revue d'histoire du droit. The legal history review. Leiden.

VDI = Vestnik drevnej istorii, Moscou.

zSS $(\mathrm{RA})^{\circ}=$ Zeitschrift des Savigny Stiftung, Römanistiche Abteilung, Weimar. 
Section 2. Les réalités de 1 'acquisition d'esclaves $\ldots \ldots \ldots \ldots \ldots 78$

Paragraphe 1. Le nombre d'esclaves possédés par un

maître $\ldots \ldots \ldots \ldots \ldots \ldots \ldots \ldots \ldots \ldots \ldots \ldots \ldots \ldots \ldots \ldots \ldots$

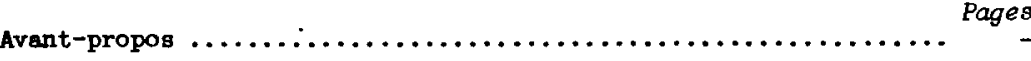

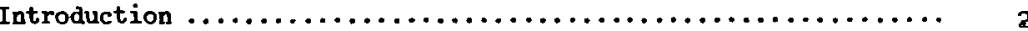

Chapitre préliminaire. L'importance de l'esclavage dans le

discours des juristes romains ......... 15

Section 1. Eléments de méthode : 1a classification des textes ... 17

Paragraphe 1. L'analyse directe ................ 18

I. Le choix de l'indice : le paragraphe ........ 18

II. Construction d'un critère permettant

l'intégration des paragraphes litigieux ..... 19

Paragraphe 2. L'analyse des citations par renvoi ..... 27

I. Les renvois operés sur des juristes ........ 28

II. Les renvois à la législation impériale ...... 29

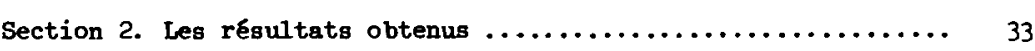

Paragraphe 1. Les tableaux .................... 33

I. L'analyse directe des paragraphes $\ldots \ldots \ldots \ldots \ldots 34$

II. L'analyse des citations par renroi ........ 35

A. des juristes $\ldots \ldots \ldots \ldots \ldots \ldots \ldots \ldots \ldots, 35$

B. à des constitutions impériales $\ldots \ldots \ldots \ldots$... 39

Paragraphe 2. Les conclusions $\ldots \ldots \ldots \ldots \ldots \ldots \ldots \ldots, 40$

I. Le discours des juristes en matière d'esclavage par rapport à la conjoncture .... 40

II. La continuité du discours des juristes en matière d'esclavage

PRFMIERE PARTIE

\section{LA MAIN-D'OEUVRE SERVILE}

Titre I. L'esclave, slent patrimonial ........

Chapitre I. Les formes d'acquisition de la main-d'oeuvre servile moyennant transaction juridique .............. 48

Section 1. Analyse comparative des divers modes d'acquisition ... 48

Paragraphe 1. L'acquisition de l'esclave objet .... 49

Paragraphe 2. L'utilisation de 1a force de

travail servile

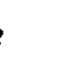

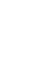

8

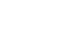

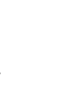

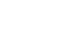

3

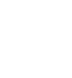

(2)

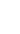

Paragraphe 2. Le prix de l'esclave ............. 86

I. Le prix de l'esclave proprement dit ........ 86

A. Il apparaît seul $\ldots \ldots \ldots \ldots \ldots \ldots \ldots \ldots \ldots, 86$

B. Il ressort d'une comparaison ........... 88

II. Le prix de l'esclave ressort de la somme due pour l'obtention de sa liberte ............ 90

Chapitre II. La reproduction du groupe servile.............. 98

Section 1. La reproduction interne, par la naissance ......... 98

Section 2. La reproduction externe, à partir d'hommes libres .... 108

Paragraphe 1. La force de la pratique ............ 108

I. L'home libre retenu de mauraise foi ........ 109

II. Le liber hamo bona fide serviene .......... 112

A. Ignorance du status ................ 11 i

B. Servitude volontaire ................... 117

Paragraphe 2. Le droit intervient pour consacrer 1a

réduction en esclavage d'homes libres .. 120

I. La reproduction privée du groupe servile à partir d'homes libres : la vente de soi-même.. 121

II. L'esclavege de la peine ............... 126

Titre II. L' esclave travailleur

Chapitre I. La nature du travail servile : de la dépendance a

l'autonomie relative $\ldots \ldots \ldots \ldots \ldots \ldots \ldots \ldots \ldots \ldots, 135$

Section 1. Une hiérarchie de la dépendance (analyse des activités

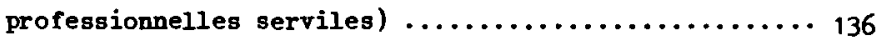

Paragraphe 1. Fonctions d'exécution ............. 138

I. Fonctions d'exécution rurales ............. 138

A. Indicateur d'activité explicite ......... 138

B. Indicateur implicite ................ 140

II. Fonctions d'exécution urbaines ............ 144

Paragraphe 2. Fonctions privilégiées ............ 162

I. Fonctions de direction $\ldots \ldots \ldots \ldots \ldots \ldots \ldots, 162$

A. A caractère financier et de gestion ....... 162

B. Fonctions de surveillance $\ldots \ldots \ldots \ldots \ldots \ldots, 168$ 
II. Fonctions intellectuelles et fonctions de

prestige 170

A. Fonctions intellectuelles ............ 170

B. Fonctions de prestige $. \ldots \ldots \ldots \ldots \ldots \ldots, 174$

1. Liées aux plaisirs du maître ......... 175

2. Autres fonctions $\ldots \ldots \ldots \ldots \ldots \ldots \ldots \ldots, 176$

Section 2. Une indépendance graduée (analyse des actions auxquelles

donne lieu 1 'activité économique de l'esclave) ....... 179

Paragraphe 1. L'indépendance de fait de l'esclave :

actions exercitoria, institoria,

quad iussu

Paragraphe 2. L'autonomie relative de l'esclave :

actions de peculio, tributoria, de in

rem verso $\ldots \ldots \ldots \ldots \ldots \ldots \ldots \ldots \ldots \ldots . \ldots \ldots$

I. La signification de l'autonomie relative ..... 183

II. Les progrès de la notion ................ 192

Chapitre II. L'esclave à la tête de biens matériels .......... 199

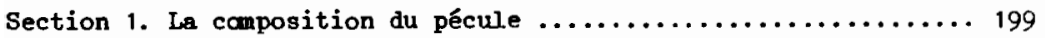

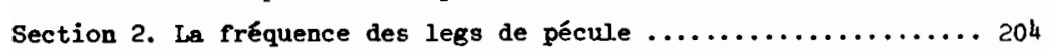

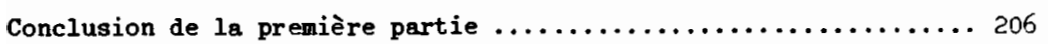

\section{DEUXIEME PARTIE}

ESCLAVAGE ET PRATIQUES SOCIALES $\ldots \ldots \ldots \ldots \ldots 210$

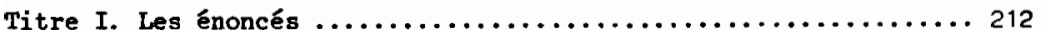

Chapitre I. La terminologie de l'esclavage et de l'après-

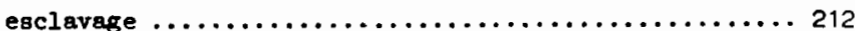

Section 1. La terminologie de l'esclavage $\ldots \ldots \ldots \ldots \ldots \ldots \ldots \ldots, 212$

Paragraphe 1. La résonance juridique des termes

désignant l'esclave ................ 213

I. La désignation impersonnelle de l'esclave .....2 213

A. La désignation individuelle de l'esclave ... 213

1. Les termes servant à désigner 1'homme

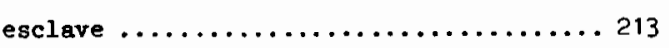

a. serrus $\ldots \ldots \ldots \ldots \ldots \ldots \ldots \ldots \ldots . \ldots . \ldots . \ldots 213$

b. homo $\ldots \ldots \ldots \ldots \ldots \ldots \ldots \ldots \ldots \ldots . \ldots 229$

c. mancipium .................... 232
2. Les termes servant à désigner 1 a femme

esclave ....................... 234

a. anciliza...................... 23

b. serva $\ldots \ldots \ldots \ldots \ldots \ldots \ldots \ldots \ldots \ldots . \ldots \ldots \ldots$

c. autres termes $\ldots \ldots \ldots \ldots \ldots \ldots \ldots \ldots 237$

3. Les termes servant à désigner l'enfant esclave $\ldots \ldots \ldots \ldots \ldots \ldots \ldots \ldots \ldots \ldots . \ldots \ldots$

4. Autres indications terminologiques de

1a présence d'un esclave .............. 240

B. La désignation collective par familia ..... 242

II. L'onomastique de 1 'esclavage .............. 245

Paragraphe 2. Les relations terminologiques ......... 257

I. Servus/dominus .......................... 257

II. Servus/filius $\ldots \ldots \ldots \ldots \ldots \ldots \ldots \ldots \ldots \ldots . \ldots \ldots 1$

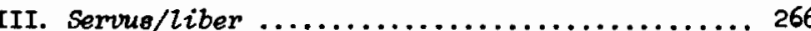

Section 2. La terminologie de 1 'après-esclavage $\ldots \ldots \ldots \ldots \ldots \ldots$. 275

Paragraphe 1. Les termes désignant l'affranchi ....... 275

I. Statuliber : une situation transitoire ........ 275

II. Monumissus ........................ 277

III. Libertus et libertinus .................. 280

Paragraphe 2. Les relations terminologiques .......... 283

I. Libertus/patronus ................... 283

II. Libertus/servus et Libertinus/ingenuus....... 285

Chapitre II. Le statut du dépendant . . . . . . . . . . . . . . . . . . . . 292

Section 1. Le passage de l'état d'esclave à celui d'affranchi .... 292

Paragraphe 1. Affranchissement entre vifs et

af franchissenent à cause de mort. ........ 294

Paragraphe 2. Affranchissement gratuit ou onéreux et

affranchissement simple ou conditionnel . 306

I. Affranchissement gratuit ou onéreux ........ 306

II. Af franchissement simple ou conditionnel ..... 312

Section 2. Formes particulières de 1a dépendance ............. 325

Paragraphe 1. Les esclaves et affranchis publics ..... 325

Paragraphe 2. Les esclaves et affranchis impériaux .... 328

Chapitre III. Les données des juristes sur la population servile . 331

Section 1. La situation géographique de 1 'esclave $\ldots \ldots \ldots \ldots \ldots . \ldots 332$ 
Section 2. Maladie et mortalité : l'esclave, capital périssable .. 334 Paragraphe 1. L'esclave malade $\ldots \ldots \ldots \ldots \ldots \ldots \ldots \ldots, 334$ Paragraphe 2. La mort de l'esclave ............ 337

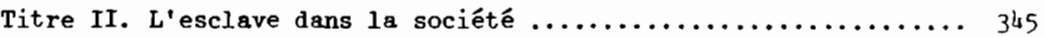
Chapitre I. Des signes sociaux ......................... 345 Section 1. La pratique soeiale au niveau de I'individu ......... 345 Paragraphe 1. Alimentation, habillement et logement ... 345 Paragraphe 2. Pratiques sexuelles et formes d'unions .. 355

I. Les pratiques sexuelles ................ 355

II. Les formes d'unions .................. 361

A. Les unions nouées à l'intérieur du groupe des esclaves et affranchis ............. 361

1. Unions entre personnes de même condition. 361

a. entre esclaves ................. 361

b. entre affranchis ............... 365

2. Unions entre personnes de conditions inégales ......................... 366

B. Les unions nouées en dehors du groupe des esclaves et affranchis ............... 366

1. Unions ayant trait à des esclaves ...... 366

a. le maître a pour concubine une de ses esclaves ................... 366

b. autres cas $\ldots \ldots \ldots \ldots \ldots \ldots \ldots \ldots \ldots, 368$

2. Unions ayant trait ̀̀ des affranchis .... 369

a. l 'union est nouée avec le patron ..... 370

b. autres cas $\ldots \ldots \ldots \ldots \ldots \ldots \ldots \ldots \ldots . \ldots \ldots$

Section 2. La pratique sociale au niveau de la société ......... 375 Chapitre II. Esclavage et droit ...................... 378

Section 1. La place de 1 'esclave dans les opérations juridiques .. 378 Paragraphe 1. Esclave et actes juridiques ......... 378

I. L'esclave objet $d$ 'acte juridique ............ 378

II. L'esclave agent d'acte juridique $\ldots \ldots \ldots \ldots \ldots 38$ Paragraphe 2. Esclave et faits juridiques .......... 401

I. L'esclave objet de fait juridique $\ldots \ldots \ldots \ldots .405$

II. L'esclave agent de fait juridique ......... 418
Section 2. Les sanctions du droit vis-à-vis de l'esclave........ 430 Paragraphe 1. Répression et torture............... 430

I. La répression de la délinquance serrile...... 430

A. Répression privée ................... 430

B. Répression publique $\ldots \ldots \ldots \ldots \ldots \ldots \ldots \ldots, 436$

II. La torture.............................

Paragraphe 2. Les solutions du droit en matière de liberté ..................... 449

Chapitre III. Esclavage et comportements ................. 463

Section 1. Dépendance et rapports sociaux ................. 463

Paragraphe 1. L'esclave dans les rapports-sociaux ..... 463

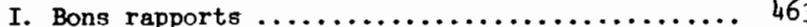

A. Esclave/maitre ..................... 463

B. Esclave/tiers ..................... 468

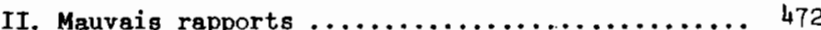

A. Esclave/maître .................. 472

B. Esclave/tiers ...................... 478

Paragraphe 2. L'affrenchi dans les rapports sociaux ...' 483

Section 2. La rupture $\ldots \ldots \ldots \ldots \ldots \ldots \ldots \ldots \ldots \ldots \ldots \ldots \ldots \ldots, 492$

Paragraphe 1. La fuite $\ldots \ldots \ldots \ldots \ldots \ldots \ldots \ldots \ldots, 493$

Paragraphe 2. Le sénatus-consulte Silanien .......... 500

Conclusion de la deuxième partie $\ldots \ldots \ldots \ldots \ldots \ldots \ldots \ldots \ldots \ldots, \ldots \ldots \ldots$

\section{TROISIEME PARTIE}

LES JURISTES, PORTEURS DE

L' IDEOLOGIE ESCLAVAGISTE

Titre I. La représentation de l'esclave par les

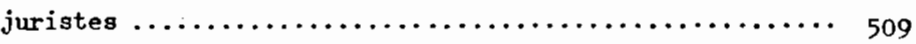

Chapitre I. L'esclavarge dans les mentalités ................ 510

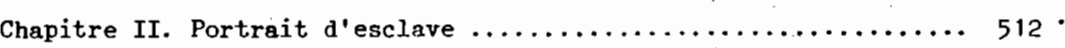

Titre II. L'esclave, élément patrimonial interchangeable ....... 520

Chapitre I. Associations d'esclaves à d'autres éléments

patrimoniaux 
Chapitre II. La persistance de L'idéningie esclavgiste ........ i.

Conclusion de la troisième partie $\ldots \ldots \ldots \ldots \ldots \ldots \ldots \ldots \ldots \ldots, 539$

Conclusion générale

Annexe : relevé des paragraphes et citations concernant

l'esclavage dans le Digeste $\ldots \ldots \ldots \ldots \ldots \ldots \ldots \ldots \ldots, 544$

Bibliographie simplifiée ......................... 675

Index des abréviations $\ldots \ldots \ldots \ldots \ldots \ldots \ldots \ldots \ldots \ldots \ldots \ldots, 684$

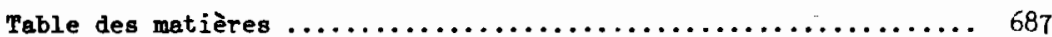

